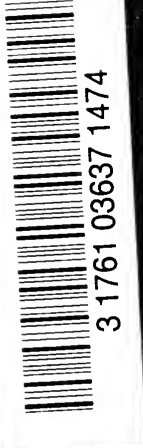



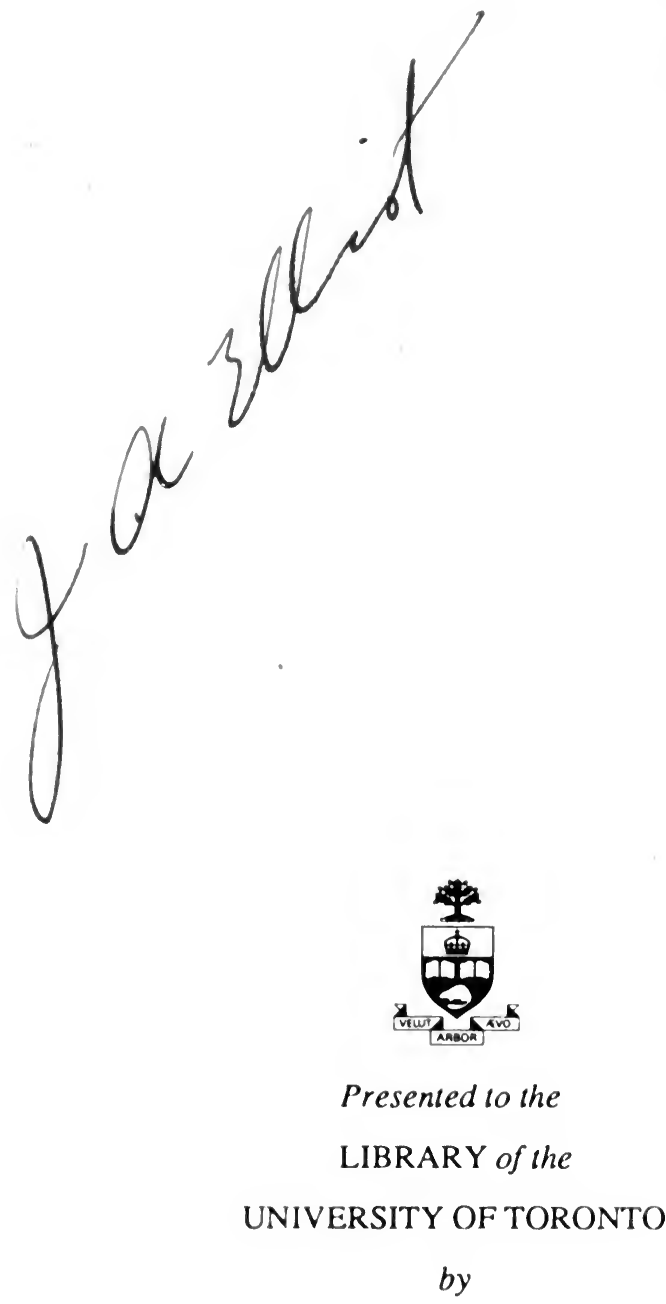

J.W. PUDDY 


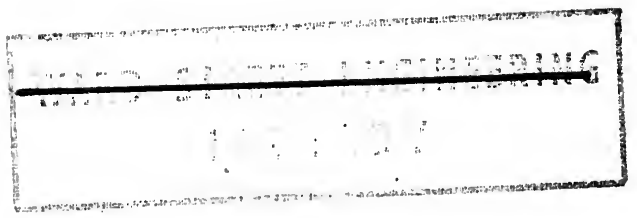




\section{Digitized by the Internet Archive in 2007 with funding from Microsoft Corporation}




\title{
HOW TO MAKE AND USE GRAPHIC CHARTS
}

BY

\author{
ALLAN C. HASKELL, B.S.
}

Principal Assistant Engineer, Construction Service Company

\author{
WITH AN INTRODUCTION BY \\ RICHARD T. DANA \\ Consulting Engineer
}

First Edition

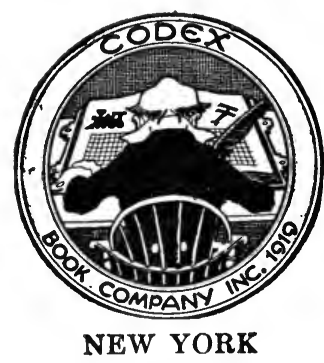

1919 
Copyright, 1919, by

Construction Service Company

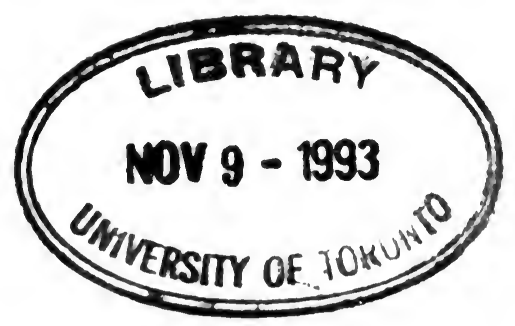




\section{PREFACE}

Graphic methods are rapidly superseding older methods because they are superior in two ways. They ean accomplish the same results more quickly and with less chance of error than was formerly possible, and they often produce results which are very valuable and which have not been achieved at all by other means. As examples out of many that could be cited, note the almost incredible labor-saving features of the logarithmic chart whereby curves of many forms and any degree may be plotted as straight lines. The tabulation of data following parabolic laws, which formerly required days of laborious calculation, ean now be done in a few minutes by a draftsman who does not even have to understand the mathematics of the process. Consider further what charts have done to visualize figures; relative quantities can be grasped through optical means with a comprehensiveness that is not even approached in any other manner, for to our instinctively accurate conceptions of magnitude as they appear to the eye a chart appeals with unique force. In the analysis of certain statistics the work that has been done with semi-logarithmic and trilinear diagrams has proved to be of the greatest value.

Strange to say, nearly everyone who uses charts does so on a very limited acquaintance with the general subject. Most people are familiar with only a few kinds of charts, but there are many forms of which they could avail themselves and which offer extraordinary opportunities for saving their labor while multiplying their achievements. As an instance, the trilinear chart is almost unknown in the United States, yet its principles, and many applications thereof, were announced over twenty-five years ago. This book is the result of an effort to correct this general situation.

The ultimate theory of charts is mathematical, and often deeply mathematical; but, without in the least attempting to decry the theory, or knowledge of profoundest theory, it is a fact that, once given the properly ruled paper, nine-tenths of the practical work done with charts may be accomplished without knowledge of its true mathematical inwardness. If mathematics were necessary to the use of charts most of the people who now employ them would have been without their help.

Charts, then, enable us to do a great deal of mathematical work without the mathematics. With a view to the requirements of the semitechnical reader, the author in preparing these pages has kept the explanations of how to make and use charts largely free from heavy theory. Nevertheless, whenever it has seemed necessary to go into theory for the purposes of clear exposition this has been done.

If this book results in a wider knowledge of this superb method and tends to broaden the field of graphic application its purpose will be amply fulfilled and will have succeeded here and there in causing "two blades of grass to grow where one grew before." 
The author desires to make the most grateful acknowledgment of his indebtedness to those writers in engineering periodicals, and in the proceedings of technical societies, who have presented to their profession within the last few years such a wealth of material. He also feels that he owes a very special debt of gratitude to the editors of the technical magazines who so generously allowed him the use of several articles from their most excellent publications.

It has been possible here to utilize but a very small part of the enormous quantity of material available, and therefore, in justice to the reader, a bibliography of the principal articles that have come to the author's notice has been included in this volume for the benefit of those who desire to pursue the various subjects further.

In abstracting data that have been published in the periodical press, full acknowledgment has been made in the text, and so far as possible the language of the original writer has been followed, in order to avoid any chance of mistaking his meaning. 


\section{CONTENTS}

('HAPTER I INTRODUCTION

Growth of Graphic Methods.-Chart Plotting Quicker than Arithmetical Methods.-Mathematical Knowledge Unnecessary to Successful Graphical Computation.-Graphs are Time-savers.-Principal Functions of Charts.-Greater Precision of Charts.-For Illustration, Charts Are Cheaper and More Accurate than Tables.-Charts Most Compact Form for Filing Records.-Principal Kinds of Charts.-Each Type of Chart Has Special Uses. Daily or Monthly Records.-Advantages of Logarithmic Ruling.-Semi-logarithmic Ruling for Statistical Prediction.-Polar Charts.-Isometric Charts.-Triangular Co-ordinate Charts.-Alignment or Nomographic Charts.-Millimeter Charts Convenient.-General Characteristies of Charts.-Correct Designing of Paper Necessary.-Accuracy Obtainable with Logarithmic Ruling.-Size of Sheets and Quality of Paper.

\section{Chapter II Rectilinear Charts}

Rectangular Co-ordinates.-Locating a Point.-Design of Rectilinear Charts.-Selection of Scale.-Illustration of Use of Rectilinear Charts.

\section{Chapter III Logarithmic Charts}

Knowledge of Logarithms Not Necessary.-Object of Logarithms.Definitions of Terms.-Arithmetical Progression.-Geometrical Progression.-Natural or Naperian System.-Common or Briggs System.-Deductions.-Design of Logarithmic Chart.-Reading of Chart Automatic.-Interpolation.-Wide Range Obtainable.

\section{Chapter IV Semi-Logarithimic Charts . . . . . . . . . .}

Description of Ruling.-Useful for Daily Record Chart.-Advantage Over Rectilinear Chart.-Example of Superiority for Obtaining Comparisons.Value for Cost and Record Analysis.

\section{Chapter V Polar Charts}

Description of Ruling.-Uses of This Ruling.-Locating a Point.-Plotting Relative Values.

\section{Chapter Vi Isometric Charts}

Mechanical Drawing.-Perspective Drawing.-Showing How Isometric Drawing Combines Principles of Both.-Example of Isometric Ruling.Use of Isometric Chart.-Advantage Obtained by Use of Isometric Drawing.

\section{Chapter ViI Trilinear Charts}

For Investigation and Demonstration of Problems Involving Three Variables.-Computations Can Be Made Without Figuring.--Speed and Accuracy Obtainable.-Trilinear Charts Useful for Determining Balanced Food Ration.-Useful to the Metallurgist.-Useful in Concrete Problems.-Trilinear Method Applied to Gas Mixtures.-Trilinear Charts for Coal Analysis. 


\section{CONTENTS}

Chapter VIII Nomographic or Alignuent Charts

Comparison with Other Forms.-Differences Shown.-Mathematical Method of Spacing and Valuing Scales.-Graphic Method of Spacing and Valuing Scales.-Methods of Dividing a Scale Logarithmically.

\section{Chapter IX General Principles Pertaining to the Use of Charts .}

Aim of Book.-Benefits to Be Derived from Use of Charts.-Sphere of Lsefulness.-Charts Save Brain Fatigue.-Charts Are the Blue-prints of Business.-Wethod of Business Charting Simple.-Engineering and Chemical Charting More Complex.- Selection of Right Method.-Types of Charts to Avoid.-Hints to the Beginner--Advantage of Standard Size.-Quality of Paper Should Be High.-Advantages of Pad Binding.-Olive-green Color Recommended.-Diagrams Should Be Keyed or Lettered.-Lines May Be Varied.- - 'se of Different Scales.-Examples of Plotting of Records. - Standards for Graphic Presentation.- Graphic Charts that Mislead.- Relative Values of Various Kinds of Charts.- Suggestions for Filing Charts.-Suggestions for Blue-printing Charts.

\section{Chapter $\mathrm{I}$ Organization and Management Charts.}

Business Records Only Valuable When Analyzed. Graphs Simplify Analysis.-The Essential Facts to Investigate.-Graphs that Place the Business Before the Executive and the Cost Accountant.-Graphic Control Facilitates the Fixing of Profits.-Organization Chart.-Charting the Stenographer's Ability.-To Prevent Overbuying.-Chart Analysis as an Aill in Buying.-Keeping in Touch with Demand.- What and When People Buy.-Keeping the Cost of Selling Down.-Knowing Just What Your Salesmen Are Doing.-To Interest Salesmen and Increase Sales.-Plotting the Performance and Efficiency of Salesmen.-Keeping Tabs on Collections. - Labor Turnover.-New Method for Determining Stability of Working Force-Cost of Labor Turnover.-Chart Records for the Employment Department.-Employment Methods.-Letting the Men Know What They Are Doing.-Fatigue of the Worker.-Charts in Time Study Work.-Labor Output.-Bonus and Piecework Rates.-Piecework Rates.-Bonus Record and P'rogress.-Employees' Benefits.-Accident Prevention.-Fatigue and Accidents.—Wages and cost of Living._Output.-Reducing "Time out."Truck Performance.-Results of Trucking Operations.

\section{Chapter XI Costs ani Cost Analysis Charts .}

Cost of coal for Generating Steam.-Cost of Coal per $1000 \mathrm{lb}$. of Steam. -Fuel Cost of Heat and Power.-Cost of Leaking Steam.-Installing Feed Water Heater.-Cost per lb. of Electrical Machinery.-Cost of Steam Pumps.-Cost of Generating Plants.-Construction Costs.-Costs in Railway Maintenance Work.-Cost Chart for Reinforced-Concrete Factory Building.-Cost Per Siguare Foot of Floor.-Cost of Placing Steel Reinforement.-Cost of Driviug Piles.-Cost of Repairs.-Auto Costs.-Analysis of Managerial Problems.-Analysis of Manufacturing Costs.-Manufacturing Costs.-Cost of Poles per Mile.-Relation Between Electric Railway Investment and Traffic Density.-Cost of Combinution of lilectric Service.-C'oal cost and Selling Price Analysis.-Analysis of Motor-Truck Transportation.-Cost of Plowing.

\section{Chapter XII Singdelng ani Progress ('harts}

Constrution Work.-Railroad Work.-Scheduling Jabor Requirements. - Combination Sichedule and Progress Chart.-Another liorm of Progress Chart.-Fiwer Work.-Transmission Line Progress Chart.-Railway Scheduling.-Goheduling Equipment Requirements.- Scheduling Merchant Ships.-Ciraphic: 'roduction Control. 
Operating Characteristics of Distributing Transformers and Squirrelcage Motors.-Operating Characteristics of Centrifugal Pumps.-Belt Conveyors.-Comparison of Stokers.-Operating Characteristics of Plants.Refrigerating Plant.-Electric Railway Operation.-Hotel Operating Characteristics.

\section{Chapter XIV Charts Showing the Results of Tests and Experi-} MENTS

Strength of Materials.-Soil Pressures.-Testing Concrete Under Freezing Conditions.-Artificial Light for Hens.-Light Distribution.--Tests of Efficiency of Train and Team Drawn Dump Wagons.-Traffic Census. Rail Wear.-Gasoline Engine Test.-Friction of Lineshaft Bearings.

\section{Chapter XV Trends, Tendencies and Statistical Prediction Shown} BY Charts

Trend of Bond and Stock Markets.-Prices of Commodities.-Trends in Design and Manufacture.-Membership Trends.-Steel Prices Reflect Demand.-Business Death Rate.-Predicting Yearly Earnings.-Probable Population.-Probable Future Traffic.-Predicted Water Consumption.Going Value.-Determination of Unit Prices of Material for Purposes of Valuation.

\section{Chapter XVI Computation, Arithmetical and Geometrical by Charts}

Plotting an Equation.-Description of Method.-Diagram for Safe Loads on Piles.-Resistances in Parallel.-Logarithmic Paper for Computation.-Locomotive Horsepower.-Logarithmic Paper for Finding Formulas to Fit Given Curves. Logarithmic Charts for Hydraulic Formulas. -Logarithmic Diagram for Determining Cutting Time.-Isometric Plotting.-Nomographic, or Alignment Chart Computations.-What Chart to Use.-Diagrams for Obtaining Number Feet B. M. in Various Timbers.Some Uses for the Polar Chart.-Diagrams in Chemical Calculations.Solution of Pulp Problems.

\section{Chapter XVII Charts as an Aid to Designing and Estimating.}

Concrete Bridge Floors.-Column Design.-Boiler Design.-Engine Design.--Sizes of Pipes.-Relation Between Number of Trains and Passing Points.-String-Lining Curves.-Economical Size of Electrical Conductors.-Pulley Sizes.-Calculating Conveyor Belts.-Size of Rope Required.Approximate Cost of Mill Buildings.-Cost of Retaining Walls.-Printing Estimates.-Cost of Transportation Estimates.-Determining Time of Performance of Work (Grading).-Manholes.-Estimating Highway Bridges.

Chapter XVIII Miscellaneous Uses of Charts . . . . . . . 509

Corrections for Sag in Long Steel Tapes.-Stadia Corrections.-Designing, Drawing, etc.-Use of Rectilinear Chart in Mechanical Drawing.Mapping.-Plotting Stadia Notes.-Reconnaissance Mapping.-Zones.Chart of Angles.-Notations.-Demonstration.-Reduction in Cost of Electric Lighting.-Comparison of Fare Schedules.-Electric Car Versus Jitney Operation.-Growth of a Company.-Cost of Living.-Conversion Chart.-Integration. 
2 


\section{CHAPTER I}

\section{INTRODUCTION}

The use of graphic methods for accomplishing a great many functions of the engineer has developed, within recent years, to a most astonishing degree,- - and while this is recognized by a great many persons who have had to make a special study of the subject in their work, it remains a fact that most engineers, and practically all other professional men are largely ignorant of a great deal that may be accomplished by these methods.

Professor Mr. d'Ocagne, of the Ecole des Ponts et Chaussees, published in 1908 his admirable treatise on the mathematies of charts, and this was followed by Professor Peddle in 1910 ("The Construction of Graphical Charts") and by Professor Lipka in 1918 ("Graphical and Mechanical Computation"), while Brinton, in 1914 published his valuable book, "Graphic Methods for Presenting Facts." Each of these works has performed a valuable function in connection with this interesting subject, and certainly the first three may be considered indispensable for any one investigating the theory of chart construction, while $\mathrm{Mr}$. Brinton's book is valuable as furnishing a collection of illustrations pertaining to the use of this method for demonstration purposes.

There are, however, at least seven distinct and most important functions that may be performed by graphic charts, several of them simultaneously, and each of them susceptible of being accomplished by this method, always as well as, and generally more perfectly than, in any other way.

It is for the purpose of showing this peculiar adaptability to all these functions that Mr. Haskell has written the present volume, and also to show, with the minimum of mathematical elaboration, how best and most effectively to make use of the method for the current engineering problems of today. The writer is frank to confess that, while he claims to be not entirely innocent of aptitude with figures, he has found irresistible the temptation to make his computations by the simple drawing of straight lines on variously ruled paper rather than developing his weary mind through the exercise of Algebra, and mayhap the calculus, in twice the time. It may be an admission of mental indolence, or worse, but he has always preferred the simple and easy methods, especially where mental effort is involved, and he has never been able to appreciate the attitude of a very dear friend of his who declined to use a slide rule for the reason that it might spoil his arithmetic.

Now, it is a fact that an enormous amount of computation is done graphically without the computer knowing anything whatever about the mathematics of the proceeding, all that is necessary to instruct the novice being to show him a similar chart and indicate the results to be achieved. In selecting illustrations from the vast mass of valuable data, the author has been guided by the above principle, having in mind the 
ordinary way in which curves are plotted and charts are made while at the same time recognizing the theoretical principles which cannot fail to be of help in practice. The engineer, the mechanic, the doctor, the salesman, - all are pressed for time; they all have to get results in the shortest possible way and, if possible, they all want their results in such form that they can be roughly ehecked by visual comparison with facts of experience. For this purpose graphic charts are an admirable instrument, and the effort here has been to accomplish it with the minimum of labor.

The Principal Functions of Charts. These fall into the following classes :-

1. Computation (Mechanical),

Arithmetical and Geometrical Calculations,

Interpolation,

Calibration,

Integration,

2. Tabulation,

Statistical Comparisons.

3. Plotting,

Sketehes,

Maps,

Stadia Surveys,

Organization plans, Location of Buildings and Machinery.

4. Recording,

Filing,

Blue Printing,

Photographic Reproduction,

Statistical Comparison.

5. Demonstration,

for Instruction,

for Advertising.

6. Statistical Prediction,

Weather,

Population,

Vital Statisties, ete.

7. Notations,

Field Notes.

For enmputations the graphic method with charts has all the advantages of graphic statios without the limitations of the slide rule in regard to presision. this being a matter of the size of the chart, the accuracy of its ruling, and the skill of the draftsman. A great many "urves ean be exoressed as st raight lines on one or another of the various kinds of charts. so chabling all of the facts in a given class to be established by the location of two points and a line drawn through them. Practically any mathematical equation and almost any number of variables can be expressed in this way and interpolated mathematieally without any computation at all. The obvious advantages of this fact hardly need be pointed out. With a proper chart such computations can be made not only more rapidly than by the older methods, but 
they can be entrusted to persons who are unfamiliar with the arithmetical or algebraical methods of computation, and they can be checked directly from the charts in the same manner.

Since a table is a collection of certain sets of data, a chart with one curve representing each set of data can be made to take the place of the table. Wherever a chart can be plotted by straight lines, the speed of this process is infinitely greater than making out a table, and where the curvilinear law is known, or can be approximated by the use of the empyric law, the speed is but little less. For purposes of book illustration, the plotting of such data and their photographic reproduction upon cuts is much less costly and less liable to errors than is the process under the old-fashioned method of tabulation. In plotting maps and stadia surveys to scale, a very large part of the draftsman's time is spent in manipulating the scale, T-square and triangle; and it is a fact of experience that such plotting upon paper ruled in squares that correspond to the units of the scale desired can be done very much more rapidly than by the scale and triangle method. The same principle applies to the making of sketches which are ordinarily not done to scale. The additional element of accuracy contributed by making them to scale is always an asset, and when the properly ruled paper is used, it is secured with no additional expenditure of time.

For purposes of record, charts have the merit of being blue-printable, or photographically reproduced, and, if they are originally made on sheets of convenient size, they can be filed in a standard manner. Since a great deal more information can be placed on a square foot of chart than by other methods of record, the facts thus recorded are in the most compact form, and where the charts are made on good paper they are also in the most durable shape.

The best known function of charts is for demonstration purposes, to show up facts. When so presented they do not require a trained mind for their appreciation, since the spatial sense through the optic nerve is among the commonest of the human attributes. A child, or a hod-carrier, can appreciate relative quantities shown on a chart just as well as any one, and it requires infinitely less mental effort to grasp quantities when shown in this manner than by their expression through the use of figures. The advertising value of this method is therefore tremendous, and is being recognized more and more.

Charts form an admirable means for predicting the growth of population, vital statistics, etc., which are not readily worked out by tabular methods, especially where the law involved is not known. The curve that fits the facts plotted up-to-date can be prolonged mechanically, and give a much more accurate index of the future than the ordinary methods of assumption.

A good deal of time may be saved in taking notes such as, stadia observations, for instance, by marking the points directly upon the prepared sheet, the process being analogous, though not very similar, to the use of a plane table in surveying; and in a great many cascs much time may be saved by filling in blank forms with conventional signs. Since notation can be accomplished in this way with fewer mental operations than with the ordinary note book, greater speed as well as greater accuracy can be attained. 
The Principal Kinds of Charts. These are divisible into the following classes:-

1. Rectilinear co-ordinates, equally spaced, decimal divisions.

2. Reetilinear eo-ordinates, equally spaeed, other than decimal divisions.

3. Reetilinear co-ordinates, unequally spaced, various divisions.

4. Iogarithmic.

5. Semi-logarithmic.

6. Polar.

7. Isometrie.

8. Trilinear.

9. Nomographic or Alignment Charts.

10. Special Forms, ruled and partly printed.

Of the first class the prineipal rulings in general use are those in which every fifth line is heavier than the other four, and every tenth line still heavier, the space between the nearest lines being respectively $1 / 20 \mathrm{in.}$, 1/10 in., one millimeter, and $1 / 2 \mathrm{in}$. For reasons that will appear below, the 1, in. spacing lends itself to a greater variety of uses than the others. but each of these rulings has eertain special uses for which it is peenliarly adapted. For the preparation of eharts to be photographically reproduced for book and advertisement cuts the 1/10 in. ruling is very well adapted, since, when skillfully plotted and lettered, charts of this seale can be reduced $50 \%$ in linear dimensions with great resultant sharpness.

Other divisions in general use with eecual spacing in both direetions are $12,16,8,6$, and 4 divisions por inch. The six divisions ruling brings the lines just about the right distance apart to form guides for the taking of long hand notes and for the arrangement of figures in columns, etc. For book-keeping and aceounting work this stationery gives a sort of automatic columna ruling alaptable to various wilths of column, which is a very considerable labor saver in such work as making appraisal inventories and the like. The $1 / 40 \mathrm{in}$. and $1 / 8 \mathrm{in}$. rulings are very convenient for architectural skotehes that have to be mate to seale, and the 3/2 in. ruling is used a great deal for machine designing and meehanical sketrones that require exactness, together with 3 in in. ruling, - the latter for sketehing the smaller details.

For various purposes such as, for instance, plotting weekly performancere recerdse it is desirable to have the ruline aceorling to the decimal scale in one direction and areording to a different seale the other way. Thus a platre ruled to 12 divisions per inch the long way and 20 the shere way. each both line of the short way hoing extrat heary, is well arlapted to the abere purpose. This size may also be used for monthly reenork. Various sorts of diagrams can be madre on paper ruled in this manner to make the docimal and duodecimal systems interchangeable. A form that has been wery mudh used consists of 366 vertieal lines, one for each dav in the rear, with a heary line for the first of each month -and 1.0 horizontal lines doeimally arranged for plotting all sorts of statistical data.

The logarithmic ruling lends itself to an extraordinary number of 
uses, and probably has resulted in more saving of labor to those making use of it than any other form of chart with the possible exception of the decimal. The author has presented a considerable amount of material illustrative of what can be done with this sort of diagram. Most of the logarithmic sheets that are on the market run for only one complete cycle in each direction, or from one to ten, or ten to one hundred, or one hundred to one thousand, etc. For practical purposes it is very desirable to have at least two cycles in one direction and three or more in the other, thus allowing plots to be made from 1 to 100 , and 1 to 1000 , - the amount of data that can be placed upon such a sheet as compared with a one-cycle sheet being in the ratio of 100,000 to 100 . This explains the extraordinary advantages derived from the use of sheets covering several logarithmic cycles as compared with the single cycle sheet on which, theoretically, any curve can be plotted.

Semi-logarithmic, which is the name given to a logarithmic ruling in one direction and a plain ruling in the other, is extremely useful for the plotting of all sorts of statisties of which proportionate variations appear to corresponding scale on the chart,-which is not true of the decimal chart. Take, for instance, a city with a growing populationstatistics of population, school attendance, crime, sickness, etc., can all be plotted on the same chart, and a $10 \%$ variation at the top of the chart will show up just as markedly as the same percentage of variation near the bottom or any other part of the chart. For giving a true index of conditions of this sort, such a chart is invaluable, and this semilogarithmic is the only ruling that admits of such results. A further peculiarity of semi-logarithmic paper is that a chart can be made in this way covering-say-two logarithmic cycles in the vertical direction and enabling as much plotting to be done in that direction as would be the case with a rectilinear sheet five times as large, making it most convenient for many kinds of data. This extraordinary peculiarity of the logarithmic scale is worthy of a good deal more attention than has been paid to it heretofore.

There are a number of so-called polar charts on the market for the purpose, not only of plotting eurves with polar co-ordinates, but of making various kinds of percentage charts-the complete area indicating unity. They are also useful for dividing up the working day in organization plans and have been used a good deal for showing railroad and other statistics. Charts of this kind are valuable also for plotting a logarithmic spiral, and for the solution of numerous problems of illumination in electricity. For plotting stadia notes, either in the field or office, polar charts are admirably adapted. Isometric charts are coming into general use for making sketches to this scale, and such sketches are very useful in explaining to workmén machine details of which a single projection sketch is not adequate.

Triangular co-ordinate charts are a valuable means for plotting curves of the form $\mathrm{X}+\mathrm{Y}+\mathrm{Z}=1$ or $100 \%$.

The alignment or nomographic chart on which the results are read by the application of the straight edge, or the straight line ruled on transparent celluloid, has become quite generally used and has a wide variety of applications. One disadvantage of it is that it has no pictorial value, but for obtaining its results it depends upon the mechanical 
operation rather than the visual operation performed by the person using it. Prcfessor Lipka says that no charts ave so rapidly constructed, nor so easily read, as those of this type, with which statement the writer can hardly concur, although enthusiastically acknowledging the great value of these charts for many purposes.

Ruled and partially printed forms, being more or less a eombination of charts with printed matter, are used in tremendous quantities for many purposes, but the function of most of these is rather outside of the present work. Such forms, for instance, as those for traverse eomputations in eomputing surveys, conveniently arranged for making these computations, are useful, just as are special rulings for computation of rule work by the end area method,-but this searcely eomes within the classification of graphic eharts.

General Characteristics of Charts. Graphic representation by means of charts depends upon the super-position of special lines or eurves upon base lines drawn or ruled in a standard manner. For the economic construction of these eharts as well as their correct use it is neeessary that the standard rulings be eorrectly designed.

A draftsman with a sharp pencil can locate points on a smooth paper approximately to the nearest $1 / 100 \mathrm{in}$. and by inspection he ean divide a space less than $1 / 2 \mathrm{in}$. in extent into 5 parts with considerable accuracy. He cannot divide the same space into 10 parts with anything like the same accuracy. These two facts seem to be dependent upon some peculiarity of physiology or psychology and they may be used as a general basis for desimning charts. Thus, sub-divisions of $1 / 20 \mathrm{in}$. on the chart may be further subdivided by inspection to $1 / 100 \mathrm{in}$. which is practically the limit of accuracy in plotting, whereas when lines are $1 / 10 \mathrm{in}$. apart, or more, the most preeise work necessitates the use of the scale.

This seems to be the reason why the decimal charts are plotted to a seale of $1,20 \mathrm{in}$. with an extra heavy line every half inch, and why millimeter charts in the Inited States are found to be as convenient as those of rather larger size. It has been the eustom for many years to plot railroad cross sections to a scale of $1 \mathrm{in} .=10 \mathrm{ft}$, reading both distances in the field to $1 / 10$ font and endeavoring to plot 1/100 in: In taking off the areal quantities with a planimeter or by dividing into triangles, scaling perpendiculars, and computing by slide rule, it is apparent that much greater convenience with approximately the same accuracy can he obtained by plotting to a seale of 1/2 $\mathrm{in.}=10 \mathrm{ft}$. on paper which is ruled to 16 on in. or else, ecfual convenience and much more accuracy may be secured by the use of a finer ruling.

The most accuratr plotting on logarithmic paper will oceur where the lines are rather less than about 1/2n in. apart, but when the space is even as great as $1 / 3 \mathrm{in}$. no particular inaceuracy will result therefrom since logarithmic spacing varies continuonsly which necessitates dropping ont various lines of sub-division in different parts of the seale. 'This naturally gives rise to a little (onfusion until one has become aceustomed to the arrangement, just as in the case of the slide rule. In making up logarithmie sheets the guestion at once arises as to how many eyeles of the logarithmie seale ran be applied on a sheet of given size without violating the best practice in regard to the physiological facts above 
referred to and yet to accomplish as much as possible on the available area of paper. The writer's experience would seem to indicate that for one logarithmic cycle a space of nearly 4 in. is necessary, that more than 4 in. is unnecessarily large and, unless extra lines are inserted, involves some inaccuracy of plotting especially at the lower part of the scale. Therefore, he has found it practicable to use about $6 \mathrm{com}$ plete cycles in a space of about the size of a letterhead, 2 in one direction by nearly 3 in the other. This gives arithmetical range in one direction of from 10 to 1,000 and in the other direction from 7 to 4,000 , which covers most of the requirements of his work while allowing the greatest convenience in ruling.

A word may be added in regard to the standard size of sheets and the quality of paper. The writer has found a good, strong, rather thin, tough bond paper superior to tracing cloth for most purposes, much more durable, much less expensive, and almost equally blueprintable. After much experimenting a sheet $8 \frac{1}{2}$ by 11 in. has been adopted as standard in his office. This, when punched with three holes in the margin, may be filed in a loose-leaf ring binder with other standard sheets or may go into the letter file in the ordinary way. Where larger sheets are necessary one 11 by $17 \mathrm{in}$. has been found to file with one fold most conveniently in the same sort of binder. 


\section{CHAP'TER II}

\section{Rectilinear Charts}

The purpose of this book is twofold: (1) by giving many illustrations, to sugges $\hat{\imath}$ to those already familiar with the functions of graphic charts new ideas for the uses of the various rulings, and (2) to show those who have never used charts at all, or but little, the prineiples of their use and to explain as simply as possible the many advantages to be derived by becoming familiar with this method of presenting facts. The following description aims to fulfill the second purpose and engineers, statisticians, ete., familiar with the theory of reetangular co-ordinates need not take the time to read this elementary material, but are urged to turn to the later chapters where they may expect to find many valuable suggestions.

Rectangular Co-ordinates. It may be said that a straight line has two directions distinguished one from the other by the sequence of the letters. In Fig. 1 when we move from left to right, we read the line $a b$, when we go from right to left we have the line $b a$.

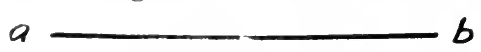

Fig. 1

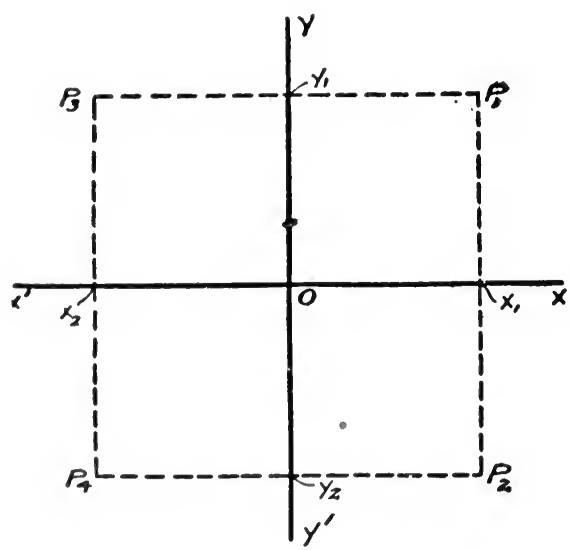

Fig. 2

This being true, in order to determine the position of one point with respect to another point, it is necessary to know not only its ristane but also the flirection in which the distance is taken. For axample. if we wish to locate point $b$ one inch from point $a$ we must know its definite direction with respect to $a$, otherwise it might be 
any point in the circumference of a circle of radius one inch, whose centre is $a$.

To simplify the locating of a point, it has been generally accepted that a distance measured in one direction shall be positive and in the opposite direction negative, designated by the well-known signs + and - Lines drawn from left to right and from down to up are called positive, and those from right to left and up to down, negative. The fixed point is called the origin, $O$. In general practice a vertical line is drawn through $\mathrm{O}$, known as the $y$-axis, and distances measured along this axis are called ordinates; at right angles to the $\mathrm{y}$-axis a horizontal line is drawn through $\mathrm{O}$, known as the $x$-axis, and distances measured along this axis are called abscissas. Thus the abscissa is positive or negative according as it is measured on $\mathrm{Ox}$ or $\mathrm{Ox}^{\prime}$, Fig. 2, and the ordinate is positive or negative according as it is measured on $\mathrm{Oy}$ or $\mathrm{Oy}^{\prime}$.

The abscissa and ordinate of a point are the co-ordinates of the point.

Thus it will be seen that it is possible to locate any point definitely by the algebraic values of its co-ordinates $x$ and $y$, and it is generally agreed, for convenience, to always name the $\mathrm{x}$ value first and the $\mathrm{y}$ value second. For example, in Fig. 2 let $p_{1} p_{2} p_{3} p_{4}$ be any points.

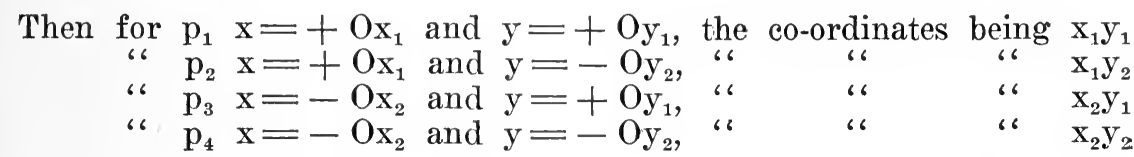

If the point $p$ were on the $x$-axis, $y$ would equal 0 and if on the $y$-axis, $x$ would equal 0 and if at the origin $O$ both $x$ and $y$ would equal 0 .

Without going into greater detail this should give the reader an understanding of the theory of rectangular co-ordinates. In practice, the quantities dealt with are usually both positive ( + ) and hence the upper right hand quadrant, Fig. 2, where both $\mathrm{x}$ and $\mathrm{y}$ are plus, is used.

Rectilinear Charts. In order to assist in locating or plotting a point, as it is called, by rectangular co-ordinates, the rectilinear chart has been devised. This consists of a series of horizontal lines drawn parallel to and at equal distances from one another, and at right angles to them a series of vertical lines similarly drawn. Any spacing between lines may be used, but for convenience either a decimal division is employed such as 5, 10, or 20 divisions per inch, or some other non-decimal division which will be of advantage for special work, as 4, 6, 8, 12, or 16 divisions per inch. The uses for these will be discussed later.

It is customary, in order to assist the eye in following the divisions on the paper, to make the lines at each inch heavy, those at the half and quarter inch somewhat lighter, and the intermediate divisions light.

This cross sectional arrangement also obviates the necessity of using a scale which would be required in plotting a point where only the $\mathrm{x}$ - and y-axes were used.

On rectilinear paper the origin may be taken at any convenient place depending upon whether + or - quantities or both are to be plotted. Since, as mentioned above, the quadrant where $\mathrm{x}$ and $\mathrm{y}$ are both + is most generally used, the origin is taken at the lower left hand 
corner of the paper, the outside ruling on the left representing the $y$-axis and the outside ruling on the bottom representing the $\mathrm{x}$-axis, thus making the whole sheet equivalent to the + or upper right hand quadrant.

Any convenient seale may be used with magnitudes increasing from the origin to the top. It is advisable, where practicable, to so select the scale that the zero point shall appear at the origin, as a clearer conception of relative values is obtained. This is not feasible, however, if the figures plotted are large.

It is also well to so solect the seale that the increase in graduations shall be uniform, both vertically and horizontally, but the rate of inerease need not necessarily be the same. That is, one eonvenient seale may be used vertically and an entirely different scale, chosen for convenienee also, may be used horizontally.

The best method of illustrating the practical use of rectilinear charts is to give an example. Suppose that a concern is engaged in the mannfacture and sale of note books. There are available monthly records of the gross earnings. These have acemmulated for a period of years, but eventually a now manager is appointed and he wishes to obtain a graphie pieture of the "ups and downs" in the past history of the business to determine whether there is any fixed relation between the volume of sales and the season of the year, with the idea of working in some other product during the dull season. IIe arranges his records of sales in order and then tabulates them as follows:

\begin{tabular}{|c|c|c|c|c|c|c|}
\hline & Jan. & Fels. & March & April & May & June \\
\hline 1910 & $.2,516$ & 2,433 & 2,626 & 2,650 & 2,819 & 3,042 \\
\hline 1911 & $\ldots \ldots \ldots \ldots$ & 2,287 & 2,780 & 2,837 & 3,091 & 3,260 \\
\hline 1912 & 2,870 & 2,354 & 2,042 & 2,916 & 3,250 & 3,478 \\
\hline & July & Aug. & Sept. & Oct. & Nov. & Dec. \\
\hline 910 & $2,956 \mathrm{j}$ & 2.475 & 2,830 & 2,773 & 2,652 & 2,497 \\
\hline 1911 & $\ldots \ldots \ldots \ldots \ldots 3,14.5$ & 2,273 & 2.966 & 3,111 & 2,822 & 2,587 \\
\hline 1912 & $\ldots \ldots \ldots \ldots \ldots 3,3 \wedge 6$ & 2,3992 & $3,0.47$ & $3,2: 25$ & 2,918 & 2,733 \\
\hline
\end{tabular}

Then with a sheet of decimal rectilinear paper, $812 \times 11 \mathrm{in}$. in size, Fig. 3, he starts at the lower left corner letting each $1 / 4$ inch space represent a month, progressing horizontally. Beeause he requires 36 spaces (3 years (a) 12 months) he makes the long edge of the paper the horizontal axis, the short edge allowing only 30 spaees. He then marks off a seale on the vertical axis to represent "dollars." If he starts with (), in ord(cr to get $\$ 3500$ on the sheet, he must let each inch represent siono. H" then proceeds to plot or locate his points. Ilis months are abscissas and his dollars are ordinates. Therefore, as he progresses from the origin horizontally, above each month he seales off on the paper the oorresponding sales in dollars; i.e. when he reaches Jan., 1910, he soales vertically $\$ 2516$ with as great preceision as possible. He then prooeseds horizontally to Feb., 1910, and above this point he seales vertionlly $\$ 24: 3$, and so on until he has empleted his figures. Ifo then takes a straight erlge and comnects with lines the points that he has located, and when this is done he has before him a complete pieture of the variations in his business. He notes at a glance what are the "peaks" or high months and what the low months. He sees that 
the low points consistently oceur during July, August, December, January and February each year and he immediately starts an investigation to determine the reasons.

It will be noted in this illustration, where $1 / 4 \mathrm{in}$. spaces were made to represent months, that the points were plotted on the right hand boundaries of the spaces. They could have been plotted on the left hand boundaries just as well, but it seems a little more logical to use the right hand ones as they represent the ends of the months and it is at the end of the month that we know the total sales during the month. Another method is to consider the space as a month's time and to erect the points midway between the two boundary lines, to

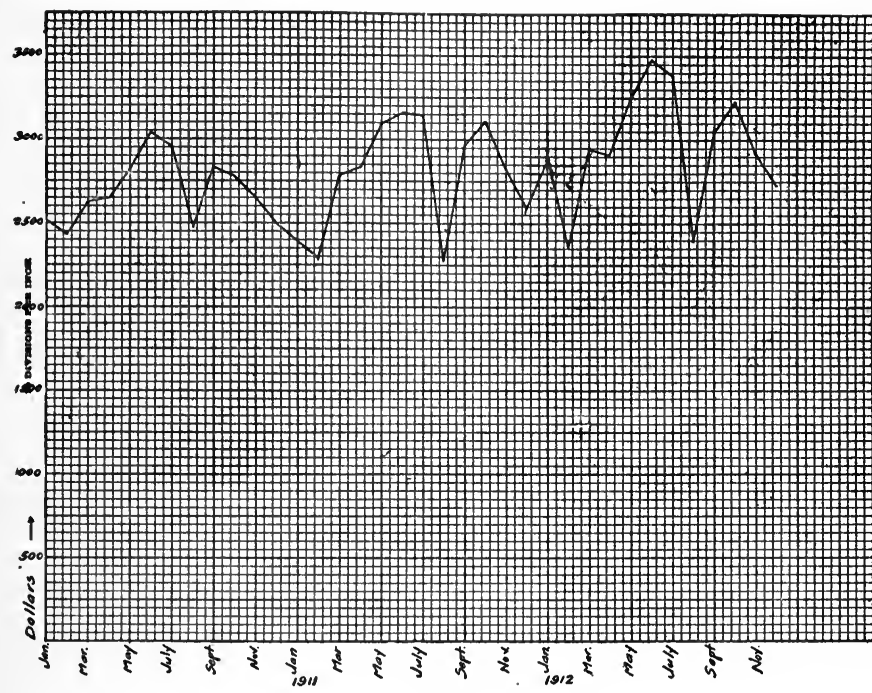

Fig. 3

represent an average of irregular occurrences during the months. Still another way is to draw a horizontal line through the point and terminated by the boundaries, the purpose being to show that the results plotted occurred during the entire month. See Fig. 6, Chapter IX.

Some prefer not to connect the points with lines at all, but to erect vertical "bars," either heavier lines or solid spaces, obtaining their mental picture by the relative heights of the bars. The author has always been in favor, personally, of the connecting line method as it presents to him a more pronounced picture of the high and low spots, with the corresponding inclines and declines. In Chapters $\mathrm{X}$-XVIII many examples of both methods are given and the reader will doubtless find that for certain uses each method has its advantages. 


\section{CHAPTER III}

\section{Logarithmic Charts}

For many purposes the logarithmic ehart, Fig. 1, is unequalled by any other ruling, but unfortunately its worth is realized by comparatively few persons. This may be due to the name, eoupled with the belief that an understanding of logarithms is necessary to use the paper. On the eontrary, one need know nothing of the theory to make logarithmie eharts, but it is the author's opinion that most people wish to know a little something of the why and wherefore of the tools they employ. With this end in view as simple a mathematieal exposition as possible will be given, which need not be followed through by the initiated.

Logarithms. The object of logarithms is to facilitate and shorten calculations involving multiplication, division, and the finding of roots. and powers.

Before continuing further it may be well to give definitions of such mathematical terms as it is neeessary to use.

Any number multiplied by itself is said to be raised to its second pover, or squared; any number multiplied by itself twice, is said to be raised to its third power, or cubed, etc. The exponent of a number, written to the right and a little above the number, indicates how many. times the number is repeated as a factor. For example, to express in abbreviated form that the number 3 is raised to the fourth power, write $3^{4}$ instead of $3 \times 3 \times 3 \times 3$. The first power of a number is the number itself, which may be considered as having the exponent 1 . The rules governing exponents are easily understood by the following examples :

$$
\begin{aligned}
a \times a \times a & =a^{3} \text { dividing this by a, we have } \\
a \times a & =a^{2} \text { dividing this by a, we have } \\
\mathbf{a} & =\mathbf{a}
\end{aligned}
$$

Thus, it is seen that dividing any power of a by a substracts unity $\mathrm{a}^{2}$ from the exponent. Therefore since, as above, $-=a^{2-1}=a$, so.

$$
\text { a }
$$$$
-=\mathrm{a}^{1-1}=\mathrm{a}^{0}=1
$$

a

$$
\begin{aligned}
& \frac{a^{0}}{a}=a^{0-1}=a^{-1}=\frac{1}{a} \\
& \frac{a^{-1}}{a}=a^{-1-1}=a^{-2}=\frac{1}{a^{2}}, \text { etc. }
\end{aligned}
$$


The square root of a given number is that number which, when multiplied by itself, will give a product equal to the given number. The third, (cube root), fourth, fifth, etc., roots of a given number are those numbers which, when repeated as factors three, four, five, etc., times will give as a product the given number. The square root of 16 (written $\sqrt{10})=4$, because $4 \times 4=16$; the fifth root of $32(\sqrt[5]{32}$, the 5 being called the index of the root) $=2$, since $2 \times 2 \times 2 \times 2 \times 2=32$.

Arithmetical progression is a series of numbers in which every successive term is increased or decreased by a constant amount, as $2,4,6$, 8,10 , etc., or $12,9,6,3$, etc.

Geometrical progression is a series of numbers, in which every successive term is multiplied or divided by a constant factor, as $2,4,8$, 16,32 , etc., or $192,48,12$, 3 , etc.

If we use any number, 2 for example, as the base or multiplier of a geometrical progression the exponents form an arithmetical progression

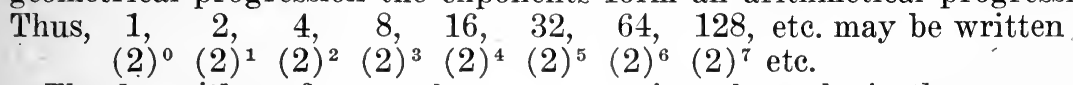

The logarithm of a number, $\mathrm{a}$, to a given base, $\mathrm{b}$, is the power, $\mathrm{n}$, to which that base, $b$, must be raised in order to equal the number, $a$. In the above, therefore, each term of the arithmetical progression (exponent) is the logarithm of the corresponding term of the geometrical progression, the base being 2. For example: the logarithm of 16 to the base 2 is 4 , written $\log _{2} 16=4$.

In general practice 2 is not used as the base. There are two systems, one known as the Natural or Naperian, used in pure mathematical discussion and in steam engineering, having as the base, e $=2.71828 \ldots$; the other, the Common or Briggs system, generally used for all practical purposes, and having as base 10 . In this latter system the two progressions are replaced by:

$1 / 10000,1 / 1000,1 / 100,1 / 10, \quad 1, \quad 10,100,1000,10,000$ $(10)^{-4}(10)^{-3}(10)^{-2}(10)^{-1}(10)^{0}(10)^{1}(10)^{2}(10)^{3}(10)^{4}$, and the logarithm of 1,000 to the base $10=3$, written $\log _{10} 1,000=3$; and $\log _{10} 1 / 1,000=-3$

If the above series were indefinitely extended in both directions the exponents in the arithmetical series, or the logarithms, would increase or decrease by whole numbers; but for all numbers in the geometrical series, between 100 and 1,000 for instance, there are corresponding numbers in the arithmetrical series with exponents between 2 and 3 ; i.e., the logarithm of 500 is more than 2 and less than 3. Since the intermediate numbers in the geometrical progression may be taken so as to differ from one another by an infinitely small amount and since the corresponding powers in the arithmetical progression will vary by infinitely small amounts, we may say that any positive number has a logarithm, and any number, positive or negative, is the logarithm of a positive number.

In practice, tables are calculated in which the logarithms are worked out to varying degrees of accuracy and the logarithms of any numbers not given in the tables are found by interpolation.

Referring to the geometrical and arithmetical series above, it will be noted that: 
(1) The logarithm of the base 10 is 1 .

(2) The logarithm of 1 is 0 .

(3) The logarithm of a number greater than 1 is +

(4) The logarithm of a number less than 1 is -

(5) A negative number has no logarithm.

(6) The logarithm of the product of several factors, $10^{-2}=1 / 100,10^{1}=$ 10 , and $10^{4}=10,000$ is equal to the sum, $-2+1+4=3$, of the logarithms of the factors: $\log \left(10^{-2}+10^{1}+10^{4}\right)=\log 10^{-2}$ $+1+4=\log 10^{3}=-2+1+4=3$. The logarithm 3 corresponds to $10^{3}=1,000$, which is the product of $1 / 100 \times 10 \times 10,000$. That is, multiplication is effected by aid of addition.

(7) The logarithm of the quotient obtained by dividing one number, $10^{5}=1000,000$, by another, $10^{3}=1000$, is the logarithm 5 of the $10^{5}$

dividend minus the logarithm 3 of the divisor; $\log \frac{1}{10^{3}}=\log$ $10^{5-3}=5-3=2$, and 2 is the logarithm of 100 , the quotient 100,000

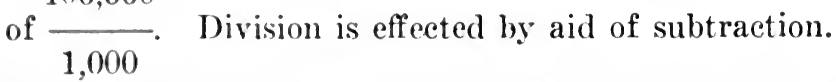

(8) The logarithm of a power, $\left(10^{2}\right)^{3}$, of a number, $10^{2}=100$, equals the logarithm 2 of the number multiplied by the degree 3 of the power: $\log \left(10^{2}\right)^{3}=\log 10^{2 \times 3}=2 \times 3=6$, and the logarithm 6 eorresponds to $10^{3}=1,000,000$ which equals $100^{3}$. A number is raised to a power by multiplication

(9) The logarithm of a root of a number, $\sqrt{10}$, is equal to the lowarithm 6 of the number divided by the index 3 of the $\operatorname{root} ; \log \sqrt[3]{10^{\circ}}=$ $\log 10^{0} / 3=\log 10^{2}=\% / 3=2$, and the logarithm 2 corresponds to 100 which is the cube root of $1,000,000$. Roots are extracted by division.

While the above is not a complete exposition of the theory of logarithms it should suffice to give the reader an understanding of what a logarithm is and to enable him to comprehend the theory of the logarithmic chart.

Logarithmic Chart. The divisions on the logarithmic chart are proportional, not to the numbers thenselves, but to the logarithms of the numbers. In other words the chart is divided logarithmically, but instead of the logarithms being lettered on, the numbers corresponding to the logarithms are put on. That is why the base line is 1 . The logarithm at the base line would be 0 , but the number which eorresponds to that logarithm is 1 . In the same way note that + is over halfway between 1 and 10. That is bereause the logarithm of 4 is 0.6021 , but instearl of the logarithm 0.6il)el being put on the chart the corresponding number 4 is put on. Therefore any calculations marle up of multiplieation, division. roots and powers may be solved by straight lines, in accordance with thre principles ontlined in 6, 7, 8 and 9. above. For example, in multiplieation the logarithm of the product of two numbers is the sllm of the legarthms of the mumbers. Therefore, if we wish to multiply any number. 2. by another number 3, by (6) $\log 6=\log$ $3+\log 2$. From 2 on the $x$-axis, Fig. 1, we draw a line at an angle of 
$45^{\circ}$. Then from 3 on the $y$-axis we follow across until we hit the $45^{\circ}$ line, then we drop to the $\mathrm{x}$-axis and the answer is 6 .

The distance $\mathrm{AC}=\mathrm{BD}=\log 2$.

The distance $\mathrm{CE}=\mathrm{CD}=\log 3$.

$\mathrm{AC}(\log 2)+\mathrm{CE}(\log 3)=\mathrm{AE}$, and $\mathrm{AE}=\log 6$.

Therefore, it will be seen that what takes place when two numbers are multiplied together is the graphic addition of their logarithms, but since on the chart the numbers corresponding to the logs are lettered, instead of the logs, we are able to read the correct answer automatically.

In the same manner the other three functions,-division, raising to powers and extracting roots,--reduce to simple graphic additions and substractions or combinations of the two, on the logarithmic chart.

When interpolating between two lines, with a given scale, it should

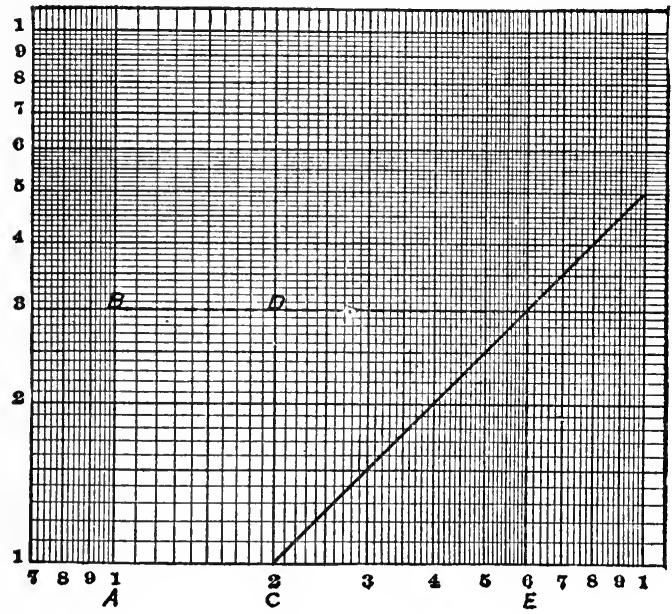

Fig. 1

be remembered that the divisions are not uniform, and proper account be taken of this fact.

As mentioned at the beginning of this chapter a person may advantageously use the logarithmic chart without any knowledge whatever of the principles of its construction. It is sufficient to remember that for all operations involving multiplication, division, powers and roots, the lines plotted will be straight and hence easier to deal with.

On a logarithmic chart one complete cycle is usually numbered from 1 to 10 , and the decimal point may be so placed that the scale range will be suitable for plotting the data in hand. That is, one cycle may be 0.01 to $0.10,0.10$ to 1,1 to 10,10 to 100,100 to $1,000,1,000$ to 10,000 , etc. It is possible to obtain a wide range by drawing several lines on a single cycle chart or by drawing one line on a multiple cycle chart. The former method is explained on page 352, but the latter method entails considerably less work, and the author has found that a logarithmic chart having two cycles in one direction and two and a half or three cycles in the other gives ample range for most problems. This permits 
variations of from 1 to $100 ; 100$ to 10,$000 ; 10,000$ to $1,000,000$; etc., one way, and 1 to 1,$000 ; 1,000$ to $1,000,000$; etc., the other. Thus it will be seen that a logarithmie chart $812 \times 11$ ins. having two cycles one way and three the other will permit the plotting of data covering a range 1,000 times as great as a single eycle. Also, if we were to seale a uniform chart, 81\% $\times 11$ ins., in the same manner starting at unity, we could cover only 1 to 10 the long way and 1 to 7.5 the short way. 


\section{CHAPTER IV}

\section{Semi-Logarithmic Charts}

The semi-logarithmic chart, Fig. 4, is a combination of the logarithmic and rectilinear charts, usually having the logarithmic ruling vertically along the $y$-axis and the rectilinear ruling, preferably decimal, as 20 divisions per inch, horizontally along the x-axis. For certain statistical comparisons a very useful chart is the Semi-logarithmic-Daily Record Chart, which has the logarithmic ruling vertically and one division for each day of the year horizontally.

The semi-logarithmic chart has a wide use for the plotting of comparative statisties of similar kind but dissimilar magnitude, in that the rectilinear horizontal ruling allows the uniform spacing for periods of time as days, weeks, months, years, etc. while the vertical logarithmic scale gives the true relative comparison between variations. On a chart $8 \frac{1}{2} \times 11$ ins. in size (plate size $7 \frac{1}{2} \times 10$ ins.) with two logarithmic cycles vertically the short way of the paper and 20 divisions per inch the long way, there would be 200 divisions horizontally against which might be plotted quantities varying from 1 to 100,10 to $1,000,100$ to 10,000 , etc. on the double log scale. Thus the range is very nearly seven times that of the rectilinear chart, 20 divisions per inch.

Furthermore, on the rectilinear chart the percentage of variations of any curve shows up equally to the eye regardless of where the curve is on the paper. For example, on a rectilinear chart with a single scale, 0 to $1,000,000$, the population curve of a city of 800,000 people would be near the top of the sheet, and the curve showing the total number of school children would be near the bottom. The lines, or graphical pictures, indicating percentage variations in the number of children attending school will correspond in size to those indicating variations in the population, although the variation in actual numbers in the first case is very considerably less than in the second. On the semi-logarithmic chart these percentages of variation would automatically show up in their true relation to one another.

An excellent illustration of the superiority of the semi-logarithmic chart to the rectilinear for obtaining relative comparisons is given in the following, from an article by John Wenzel published in Scientific American Supplement, April 4, 1917. Mr. Wenzel speaks of the charts as "logarithmic," but to conform to the classification used here and to differentiate between those charts which have logarithmic ruling in both directions and those having it in one direction only, this has been changed in each case to read "semi-logarithmic."

On arithmetically ruled paper as in Fig 1, the horizontal rulings are the same distance apart, so that the distance on the scale at the left is the same from 10 to 20 as it is from 20 to 30 and 30 to 40 . A curve which rises in ten years from 10 to 20 represents an increase of $100 \%$. 
A curve which starts at 100 and rises in ten years to 110 represents an increase of only 10\%. In Fig. 1 a $10 \%$ inerease is shown by the same angle as a $100 \%$ inerease. It is therefore evident how impossible it is to get the correct comparison of the rate of inerease between a large quantity and a small quantity when eharted on ordinary arithmetically ruled paper.

This is shown very clearly by eharting the same data on both arithmetically and semi-logarithmically ruled paper, as has been done in Figs. 1 and 2. Here the rate of growth of an investment of $\$ 1$ was compared with a similar investment of $\$ 6$. Both investments doubled every ten years. The growth of the two investments was as follows:

$\begin{array}{rrr}1870 & \$ 1.00 & \$ 6.00 \\ 1880 & 2.00 & 12.00 \\ 1890 & 4.00 & 24.00 \\ 1900 & 8.00 & 48.00 \\ 1910 & 16.00 & 96.00\end{array}$

The rate of increase was the same in both cases and yet the angles at which the two curves rose in Fig. 1 were so unlike that it was hardly conceirable that there was any relationship whatever between the two eurves. This shows how misleading it is to attempt to compare large and small quantities on the ordinary arithmetically ruled paper.

The correct relationship between the two curves ean be shown by charting them on semi-logarithmically ruled paper as in Fig. 2. The rate of increase is the same in both the upper and the lower eurves, therefore the two curves are parallel. Because the rate of increase is uniform throughout the whole period from 1870 to 1910 , the curves representing the two investments are straight. The eurves in Fig. 1 give the erroneous impression that the larger quantity increases much more rapilly. especially between 1900 and 1910 .

The mistake in attempting to eompare large and small quantities on the same arithmetically ruled ehart is shown by a comparison of Figs. 3) and 4. IIfre are charteol for a period of years the total sales and sales cost which includes the salesmen's salaries, salesmen's expenses and advertising expronses. The details of these fignres are shown in the tahulation in rig. i.

In Fig. "s the data are charted on arithmetically ruled paper. The "Total sales" curve being much larerer than all the other eurves stands out prominently at the top of the (hart, while the salesmen's salaries, salesments expenses and advertising exponses, which added together make up the sales rost, are all so near the base of the diarram that it is impossibe on compare the latter with the former. When these data are charted on semi-logarithmically ruled paper as in Fig. 4 the ratio of increase or of derepase beromes instantly apparent. For the first time it is posihle to sore that in the advertising expenses and salesmen's expense courves thepe has really beren more fluetuation than there has been in the Total sales curre, the growth of which is much more speetamelar in Fig. :3 than it is in Fig. 4.

The cost Index which appears in Fig. 4 is a very useful addition to the chart. It shows how many cents out of each sales dollar go to 


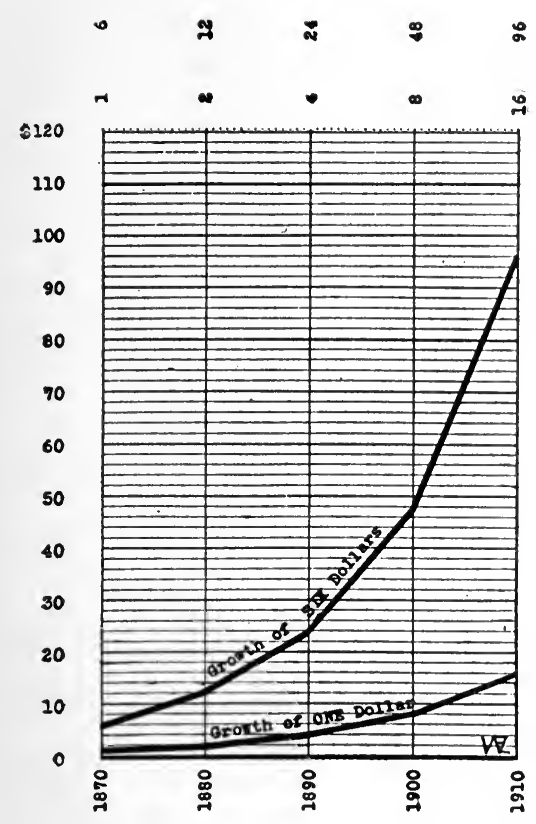

Fig. 1

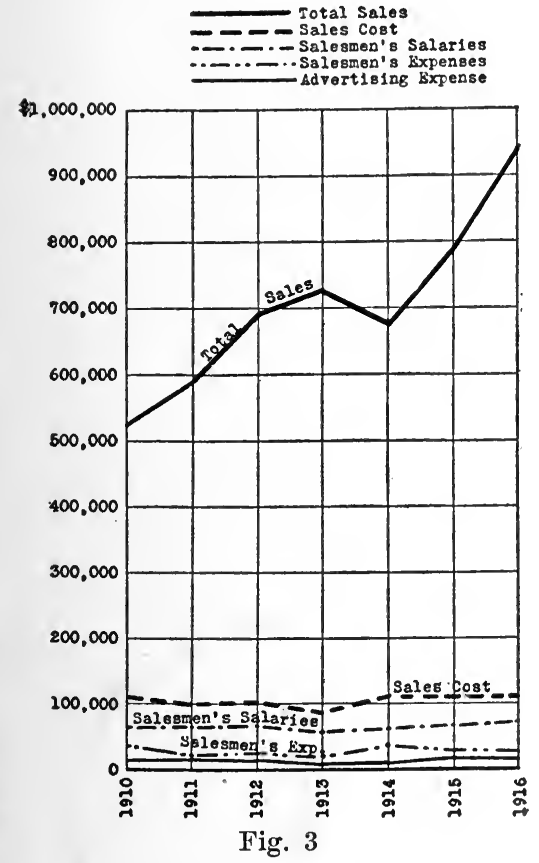

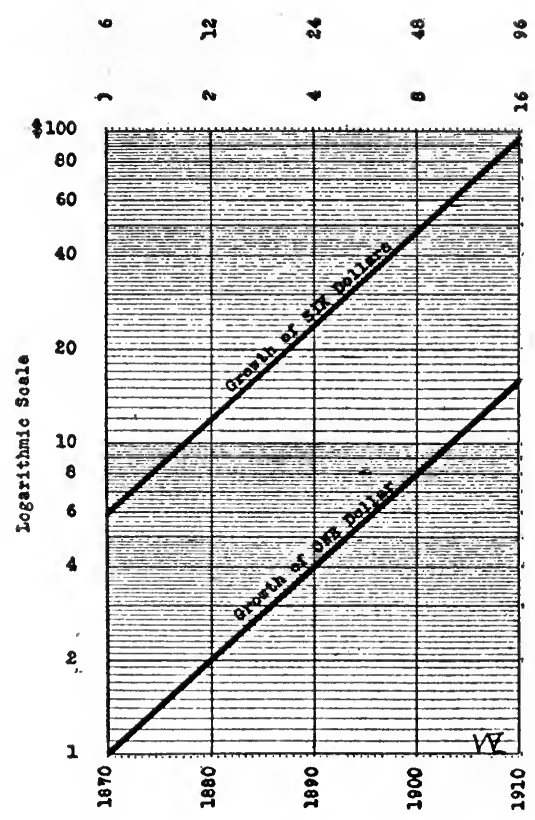

Fig. 2

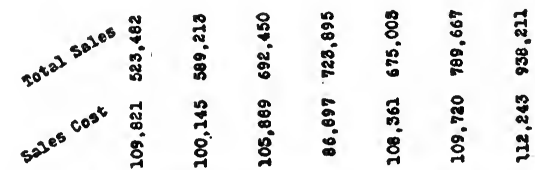

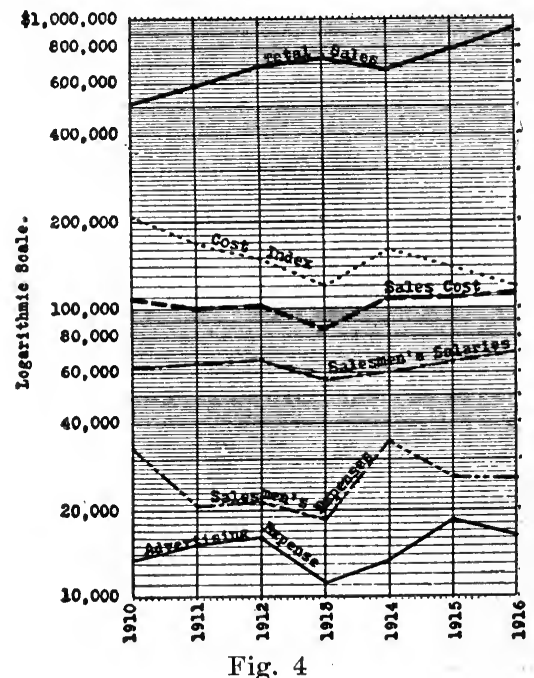


sales cost. This is read from the bottom of the chart as though the base line were one, leaving four ciphers off each number in the scale. In 1910 the Cost Index was 21 cts; in 1911 it had dropped to 17 ets.; in 1912, to 15 ets., while in 1913 it had reached 12 ets. In 1914 it had gone back to 16 ets. Since then it has been going down until it was again 12 ets. in 1916. The Cost Index line which is usually plotted in red ink is one of the most helpful features of this form of chart, as it enables one to get the exact situation at a glance.

\begin{tabular}{|c|c|c|c|c|c|c|}
\hline Your & Setel & $12 \log c$ & sele: & sale: & Mevense & $\begin{array}{l}\text { Cons: } \\
\text { Indic. }\end{array}$ \\
\hline 2310 & 1423,4t2 & 1203,021 & (6), 2s3 & 193, ,008 & 913,560 & 21 \\
\hline 2921 & 508.218 & 200,248 & 68,218 & 20.630 & $25.10 \mathrm{~s}$ & 27 \\
\hline 1822 & 698.450 & 208.809 & 86.490 & 21.490 & 36.212 & 20 \\
\hline 1113 & 723,006 & a6,e91 & 89.223 & 28,866 & 13.130 & 12 \\
\hline 2014 & 673.008 & 200.362 & 80.696 & 34.378 & 23.408 & 26 \\
\hline 2818 & 708.667 & 204.720 & 68,110 & 26.023 & 18.509 & 24 \\
\hline 2316 & 930,211 & 328.248 & 90,344 & 28567 & 26.232 & 12 \\
\hline
\end{tabular}

Fig. 5

Recently a sales manager charted all the expenses that went into the sales cost and because the chart looked like Fig. 3 he concluded that the business was in prime condition. He showed the charts to a friend who suggested that he might get a new viewpoint of his business problems by using semi-hogarithmically instead of arithmetically ruled paper. The result was startling; he found that many of the curves presented an entirely different aspect, and that his business was not as prosperous as he had supposed.

The value of semi-logarithmic paper for cost and record analysis is clearly shown in the following, from an article by W. J. Cunningham, published in the Railroad Age Gazette, June 25, 1909.

'The sir-alled "practical" officer (as distinguished from his technical associate) is learning to appreciate the superiority of diagrams over tabular work, when different factors are to be compared with each other, with preceding periods, or both.

No other form of indiating the trond of receipts, expenses or work done as expresserl in train, engine, ear or ton miles, cars handled, tons handled, etc.) erfuals the graphic method. By its use the high spots in expenses stand ont like danger signals, and gradual but eontinued losses in cofterency are much more apparent at a glance than a careful study of the figures, by themselves, will disclose. It is trite to say that statistics in their newal form are dry, oven repellant, to the busy officer. He prefers on deal with affairs of the hour or the morrow rather than study pont mortem tabulations. But when the same information is put beforro him uraphioally it beromes attractive, and is replete with suggestions, both eorrective and productive.

Inder certain conditions, however, the ordinary form of graphic chart is slightly mislealing. It will be conceded that its true function is to portay comparative fluotuations. This result is practically secured whon the factors or guantities compared are nearly of the same value or volume, hut analysis will show that this is not accomplished when the amounts comparen differ greatly in value or volume. The fluct- 
uations shown are absolute rather than relative. The extent or degree of the fluctuation, as indicated by the ordinary chart, depends in a measure on the proximity of the curve to the top or bottom of the chart.

As an illustration: if the seale has a range of 1 to 100, a curve beginning at 90 and affected by an increase of $10 \%$ would move upward nine units on the scale to 99 , while a curve beginning at 10 and affected by the same percentage of increase $(10 \%)$ would move upward but one unit on the scale to 11 . The extent of the change in the curve near the top of the scale is nine times greater than in the curve near the bottom of the scale, yet in each case the relative change was the same$10 \%$. The chart registers the actual change in value, rather than the ratio or percentage of change. The wider the range of the scale the greater the variation between actual and relative changes.

The same criticism applies to charts which employ two or more scales for various curves. If the different scales are in proper proportion, the result is the same as with one scale, but when two or more scales are used which are not proportional an indication may be given with respect to comparative fluctuations which is absolutely false.

The percentage scale is used to some extent in graphic charts and corrects the deficiency in the ordinary chart by showing the changes in percentages of increase or decrease. But in correcting one deficiency, another is introduced. The percentage scale gives no clue to the magnitude of the quantity represented by any curve. The true proportion of relative changes is shown, but the actual value is not indicated. The use of the percentage scale also involves considerable labor in computing percentages of change.

As a substitute for the ordinary (or natural) scale and the scale of percentages, as well as compound scales, the semi-logarithmic scale, or scale of ratios, practically meets the requirements. It permits the exhibition of both actual and relative values, and actual and relative fluctuations.

To illustrate the features to which reference has been made, two charts are presented, each showing graphically the same figures. The data are taken from the quarterly accident bulletins of the Interstate Commerce Commission and the annual reports of the same body. The subject of train accidents is of sufficient importance in itself to make either chart of interest, showing as each does the close relation between accidents and volume of traffic. The "passenger miles" and "ton miles" for fiscal year ending June 30, 1908, are estimated from the preliminary report of the commission showing passenger and freight revenue.

Fig. 6 uses the ordinary or natural scale; Fig. 7 has the semi-logarithmic scale. The advantages of the latter are apparent by comparison. On the natural seale fatal injuries reflect little change, while non-fatal injuries fluctuate within wide bounds. Fatal injuries, being so much smaller in number, appear very near the bottom of the chart, and it takes a very marked increase or decrease to register an appreciable ehange in the curve. On the other hand, non-fatal injuries, being very much greater in number, are near the top of the scale, and a very slight percentage of increase makes the line shoot upward.

Fig. 7 shows the two lower lines with their trua ratio of change and 
indicates that relatively they fluetuated more than the upper lines. Compare, for instance, the third quarter of 1904 . Fatal injuries to passengers increased from 2-3 to 228 (nearly $900 \%$ ), while non-fatal injuries increased from 1,134 to $2,154(90 \%)$. The percentage of increase in fatalities in that quarter was ten times greater than the percentage of increase in non-fatal injuries, yet the chart with the natural scale does not give that indication. Fig. 7, with the semi-logarithmic scale, shows the relative changes in true proportion.

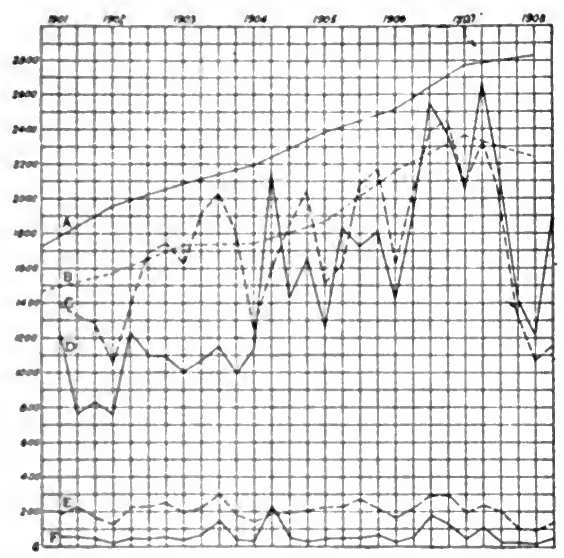

Fig. 6-Natural Sicale

Passengers and rmujloyens killed and injured in train aceilents. (From quarterly reports of the Interstate Commerce Commismion.)

$A=$ Pasminger miles $(2,000$ on seale $=20$ billion pasnenger miles).

$B=$ Ton-miles (2.000 on scale $=20$ hillion ton-miles).

$\mathrm{C}=$ Emploveres injured.

I) = l'amerigers injured.

$\mathrm{F}=$ Employees killed.

$\mathrm{F}=\mathrm{i}$ assongers killeol.

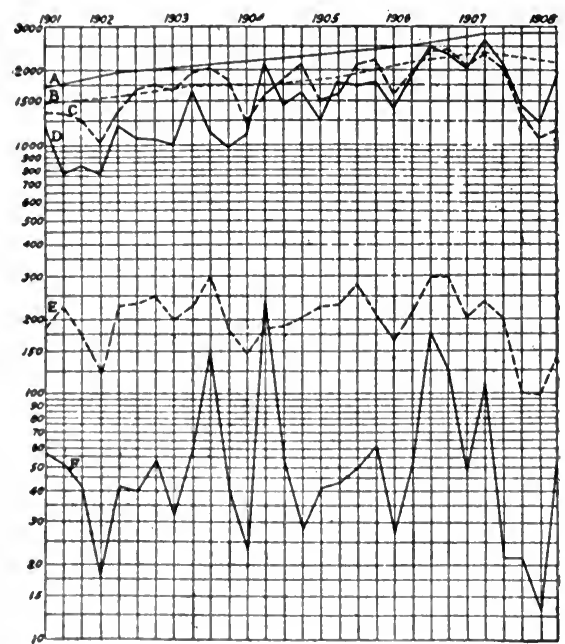

Fig. 7-Semi-Logarithmic Scale

Passengers and employees killed and injured in train acidents, all railroads in Inited states. (From quarterly reports of the Interstate Conmerce Commission.)

$A=$ Passengers carried 1 mile $(2,000$ on scale $=20$ billion passengermiles).

$\mathrm{B}=$ Ton-miles $(2,000$ on seale $=20$ billion ton-miles).

$\mathrm{C}=$ Employees injured.

I) $=$ P'asseligers injured

$\mathrm{k}=$ Fimployees killed.

$\mathrm{F}=$ Passengers killed.

As another instance, note the decrease in the last quarter of 1907. Passenger: injureol dropped from 2,66:3 to 2,125 (about $25 \%$ ); passengers killed, from 261 to 21 (92\%). The pereentage of decrease in fatal injuries was noarly four times greater than in non-fatal injuries, yet an oppositn indiogtion is given by Fig. 6 . The true ratio of change is shown on Fig. 7

Is a further "xamplo of the practical utility of the semi-logarithmic scale, where a singlo chart with the ordinarv seale could not have been nserl, Fig. 8 is presentorl, showing various froight statisties. With the natural sealo the flurtuations in "fons hauled one mile," "average rate 
per ton mile" and "total tons hauled" would be out of proportion with the other curves lower on the scale. In fact, the curves on the lower half of the scale would be so close together as to make them indistinguishable.

No matter on what location on the chart, when the semi-logarithmic scale is used the angle of upward or downward inclination is the same in all curves affected by the same percentage of change. A curve having an upward inclination equalling the distance from 100 to 200, 200 to 400,300 to 600 , or the distance between any number on the scale and double that number, has had an increase of $100 \%$. In other words,

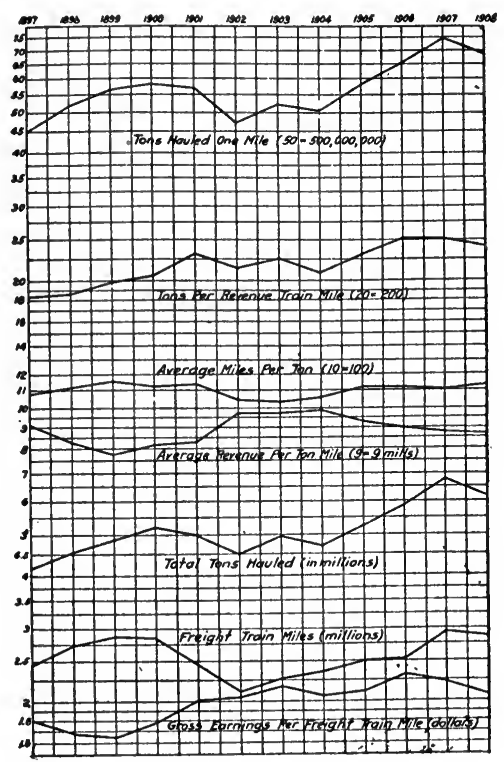

Fig. 8-Semi-Logarithmic Scale

Freight statistics of the Boston \& Albany; year ending June 30.

the quantity has been doubled. It will be noted that the distances on the scale from 200 to 400,300 to 600 , etc., are equal, the ratio in each case being $1: 2$, or $100 \%$. A curve having an upward inclination from 100 to 300,200 to 600,300 to 900 , or the distance between any number on the scale and three times that number, has had an increase of $200 \%$. The ratio in the latter cases is $1: 3$, or $200 \%$.

The scale for decreases works somewhat differently. A curve having a downward inclination from 100 to 80 , or any distance equal to it from any starting point, has had a decrease of $20 \%$. A downward inclination equal to the distance from 100 to 60 equals $40 \%$; from 100 to 40 , the percentage of decrease is $60 \% ; 100$ to $20,80 \% ; 100$ to $10,90 \%$. A decrease of $99 \%$ would be reflected by a curve with a downward inclination equalling the distance from 100 to $1 ; 99.9 \%$ by the distance from 100 to $0.1 ; 99.99 \%$ by the distance from 100 to 0.01 , and so on, ad infinitum. 
To summarize: With the ordinary seale, fluctuations in large factors are very noticeable, while relatively greater fluctuations in smaller factors are barely apparent. The semi-logarithmic scale permits the graphic representation of changes in every quantity on the same basis, without respect to the magnitude of the quantity itself. At the same time, it shows the actual value by reference to the numbers in the scale column. By indicating both absolute and relative values and changes to one seale, it combines the advantages of both the natural and percentage seale, without the disadvantages of either. 


\section{CHAPTER V}

\section{Polar Charts}

The Polar Chart, Fig. 1, consists of a series of concentric circles 1/10 in. apart. The larger, or outside circles are divided into 360 degrees, half-degree marks being shown. The chart is numbered both clockwise and counter-clockwise from $0^{\circ}$ to $360^{\circ}$. The smaller circles are not

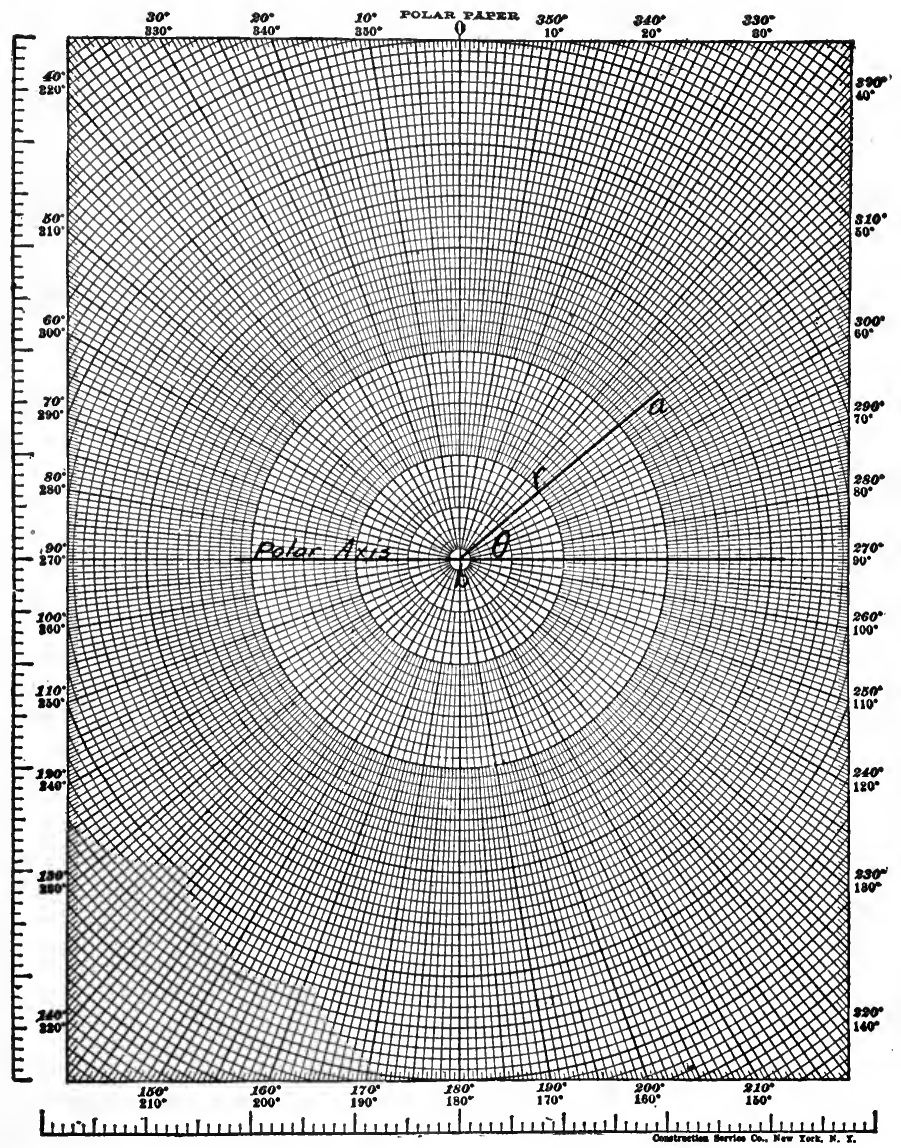

Fig. 1

as finely divided, being marked off every two, five, or ten degrees as they approach the center.

This paper was designed primarily for plotting curves by polar coordinates, for which purpose it is as suitable as the rectilinear chart is 
for rectangular co-ordinates. Many other uses have developed, such for example as the graphic picturing of ratios, where the entire area bounded by one of the circumferences equals unity or $100 \%$, and the component parts are represented by sector areas, suitably lettered, shaded, etc. Hourly variation for a single day may be best shown on this chart, taking each degree equivalent to 4 mimutes of time. Flow diagrams so plotted may be planimetered. Spherical and hemi-spherical eandle powers of sorirees of light are plotted on this paper.

It may be used for a protractor and for this purpose is much cheaper than the metallie, celluloid or eard-board ones.

Its use for stadia notes is deseribed on page 521 .

In Chapter II it was explained how the position of a point with respect to another could be located by rectangular co-ordinates, thus determining the distance and direction. The same thing may be accomplished by polar co-ordinates, having given the length, $r$, which one point, $a$, is distant from another, $b$, and the angle $\theta$, which the line connecting the two points makes with the horizontal through b, known as the polar axis, Fig. 1.

Any function, $r$, of a single variable, $\theta$, may be represented by a curve in polar co-ordinates. From the horizontal polar axis lay off the given values of $\theta$ as angles, taking as positive the counter-elockwise direction about the origin. Along the terminal side of each angle $\theta$, neasure off to the seale best adapted for the range in magnitudes, the corresponding lengths of $r$, forward for positive values and backward for negative ones. Through the points thus determined draw a smooth curve.

Where it is desired to plot relative values the use of the rectilinear or semi-logarithmic chart is to be preferred to the radial plotting for which the polar chart is sometimes used. 


\section{CHAPTER VI}

\section{ISOMETRIC Charts}

Isometric drawing combines the principles of mechanical drawing and perspective drawing.

In a mechanical drawing each separate view of an object-front, top, bottom and sides-is shown just as it is and not as it appears to an observer. One view may show any two dimensions-length, breadth or thickness-but it requires a second view to show the third dimension. All parallel lines of the object are drawn parallel.

Perspective drawings are like photographs, showing an object as it appears and three separate faces-front, top and side, for exampleare shown in one view, parallel lines in the object not being. parallel in the view but converging toward a vanishing point.

In an isometric drawing all parallel lines of an object are drawn parallel and at the same time three separate faces of the object are shown in one view. The principal lines are vertical, horizontal, and 30 or 60 degrees to the horizontal.

In mechanical drawing the center, reference and dimension lines are vertical and horizontal, while in isometric drawing these lines are drawn at an angle of 30 or 60 degrees with the horizontal and vertical.

Fig. 1 is an illustration of a rectangular block, 4 by 4 by 8 ins., $\mathrm{I}_{1}, \mathrm{I}_{2}$, and $\mathrm{I}_{3}$ being isometric drawings. The latter show the block in different positions, and without the aid of isometric paper the construction of each would proceed as follows:

For $I_{1}$, draw center lines 1-2, 3-4, with T-square and 30 degree triangle each line being 30 degrees with the horizontal. From $\mathrm{O}$, the intersection of 1-2, 3-4, lay off 2 ins. along $01,02,03$ and 04 , and cail the points $5,6,7$, and 8 . Through the points $b, c, d$, draw the vertical lines be, cf, dg, 8 ins. long and connect the points $\mathrm{c}$, f, and $\mathrm{g}$.

For $\dot{\mathrm{I}}_{2}$, draw center lines 1-2, 3-4 as above, using 30 and 60 degree angles. From their intersection, $\mathrm{O}$, lay off $\mathrm{O} 5$ and $\mathrm{O} 64$ ins. long; $\mathrm{O} 7$ and 082 ins. long. Through points $5,6,7,8$, draw lines parallel to $3-4,1-2$, and the view abcd is formed. Through b, c, d, draw the vertical lines be, ef, and dg, 4 ins. long and connect points e, f and g.

For $I_{3}$, draw center lines 1-2, 3-4, 30 degrees and vertical. From their intersection, $\mathrm{O}$, lay off $05, \mathrm{O} 6, \mathrm{O} 7,-$ and $\mathrm{O} 8,2$ ins. long. Through points $5,6,7$ and 8 , draw lines parallel to $3-4,1-2$, and the view abed is formed. Through $\mathrm{O}, 30$ degrees with the horizontal, draw $\mathrm{OO}^{\prime}, 8$ ins. long. Through $\mathrm{O}^{\prime}$, draw center lines, and construct efg in the sane method as abed.

The isometric chart has been devised for the rapid construction of isometric drawings. It has lines, vertical, horizontal and at angles of 60 degrees to the horizontal. When the chart is turned 90 degrees, it gives verticals, horizontals and 30 degrees to the horizontal. See Fig. 1. 
The use of such a ehart obviates the necessity for T-square and 30-60 degree triangle, and with little practice one may become very adept in presenting views by this method.

To those who earry note books for jotting down the results of their observations, a few sheets of this paper are a great aid and time saver.

Fig. 1 shows the views, $I_{1}, I_{2}$ and $I_{3}$ on an isometrie chart. A straight edge was used to rule the lines, but they might have been drawn free hand just as well, and one great advantage is that the side of a building or the back of a book may be the drawing board, the correct 30 and 60 degree angles still being obtainable.

The following notes by D. A. Hampson, published in Power, June 8, 1915, show the value of isometrie drawings for the purpose described. While Fig. 16 is not a true isometric drawing as we should define it,

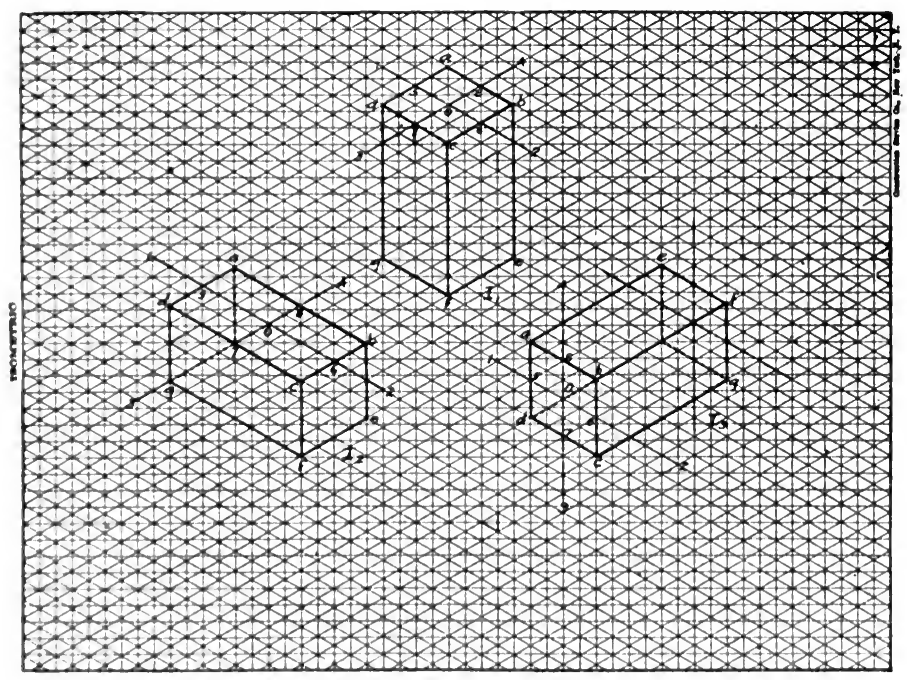

Fig. 1

get it serves the purpose of illustration and could be drawn on isometric paper rasily.

Iost power-plant engineers can make peneil sketehes of the general appearance of parts of machinery, showing the dimensions and thus convering the desired information to others. Most men would probably draw frechand two or three views on the back of a report sheet and write in such information as they thought the drawing failed to give. A sketch in isometric: perspeetive, or sort of a bird's-eye view, neessitates but on view and is easy to make, yet engineers generally think it begond their ability. Sum a sketeh shows the parts in their true relation loest and is really easier to draw.

In a number of eases repair parts made from drawings eapable of being misinterpreted have resulted in expensive mistakes. One instance will illustrate. A Corliss engine broke one of the bell eranks. The engineer took the gear apart and sent a helper in with a sketeh of 
the broken piece, Fig. 2. I could see that he had laid the broken crank on the paper and drawn a line around it, and then put on such dimensions and notes as he thought were necessary. The hub was shown dotted and there was a chance for a misunderstanding as to which side the hub should be on, so I phoned to him for the old casting. For some reason he refused to part with it and told me to make it "just as it says on that paper-and be quick about it," for he had to start up at $6: 30$ o'elock.

It was forged out, finished and delivered after working hours. Next morning there was a phone call for another crank made with the hub on the other side, "where it belongs." The second crank was made as

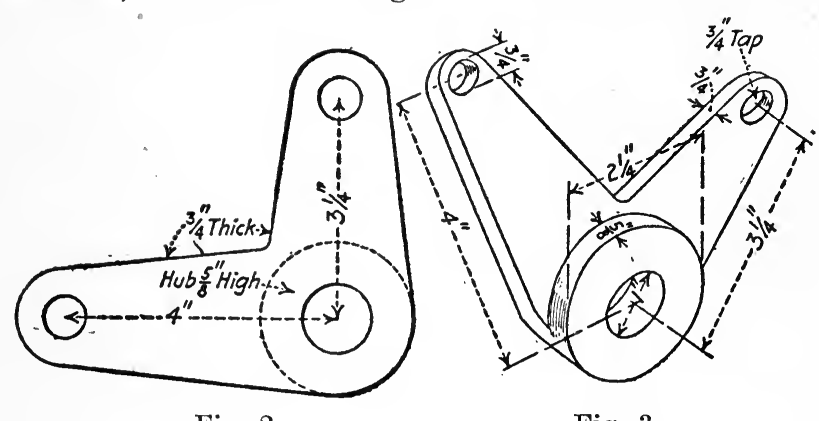

Fig. 2

Fig. 3

shown in Fig. 3, but there was trouble when the bill for two cranks was presented. Fortunately, we had retained the engineer's drawing and the first erank.

The bill was not paid until a competent person had passed on the correctness of the work. Of course, the intentions were all right, but the mistake was in showing the hub by dotted lines, indicating a lower or invisible surface according to the rules of drawing. The engineer had not known this and had committed the error.

A little time spent in practice will make anyone fairly proficient in sketching. It is interesting and is helpful even when correct threeview drawings are at hand. 


\section{CHAPTER VII}

\section{Trilinear Charts}

The trilinear form of ehart lends itself admirably to the investigation and demonstration of such problems as those involving alloys containing three metals, chemical compounds eontaining three elements, conerete mixtures containing three ingredients, food rations containing three dietetic elements, etc. By means of a trilinear chart computations can be made graphically without the necessity of figuring, with a speed and aecuraey that is very remarkable. After plotting upon a trilinear ehart the various mixtures or compounds to be investigated, these ean be grouped as to special characteristies by the lines drawn upon the chart, suggrestive of contour lines upon a topographical map, with facility and dispatch.

The method was suggested by Professor Ferèt in the Annales des Pont et Chanssees, 1892, for the purpose of investigating the strength of eonerete mixtures containing sand of different grades.

In any equilateral triangle the sum of the perpendiculars from any point to the three sicles is equal to the altitude of the triangle. Therefore, if we ennsider that the altitude of the triangle represents any number, say $100 \%$, and place a point at such distances from the three sides that those distances represent the pereentages of the three elements or ingredients contained in the body represented by the point, the location of this point on the triangle will give a rigid representation of the three ynantities or culalities considered. Thus, in Fig. 1 we let the triangle A B C represent the pronerties that we desire to investigate in a balaneerl food ration. Let the side a represent the line of zero carbohydrates; the side $b$ the line of zero proteids; the side e the line of zero fats. Ior us, therefore, rule the triangle in parallel lines for convenionce in plotting, separating each side from its opposite apex by 50 divisions. Then, ateh division will renresent $2 \%$ in any mixture of foods. We shall find, for example, milk containing $19 \%$ proteid, $29 \%$ carbohydrate, $52 \%$ fat. by a definite point, and only one, on the triangle, these propentages representing the proportions of food value measured in ablories obtainable from the respective elements in a given quantity of milk. Wo "an likewise plot another point expressing $29 \%$ carbohydrate. 49\% protrid, $22 \%$ fat, which will represent an oyster. In a similar manner we can represent any food by its constituents, and if we further make a note. preforably on the chart, indicating the duantity neopsary to develop 100 calories of nutritive energy, we have a restly mons of eacily computing a balanced ration, or of obtaining at sight a purview of the various fools to be considered in making up such rations that is of immonse help both hygienically and economically.

Without the aid of such a chart those who have attempted it appreciate the fact that the enmputation of a balanced ration for soldiers, 
babies, pigs or chickens is a matter of cutting and trying, with much arithmetic, to say nothing of the wear and tear upon one's vocabulary and temper. These computations can be made with extra rapidity as a result of a peculiarity of the trilinear chart to which it appears that Professor Ferèt was the first to call attention. For example, take two points on the chart, one representing $1 / 12 \mathrm{lb}$. of bread, the other $1 / 3 \mathrm{lb}$. of chicken, each the equivalent in fuel value of 100 calories. If we draw a straight line joining these two points, a point on this line midway between them will represent the composition of a mixture of an equal

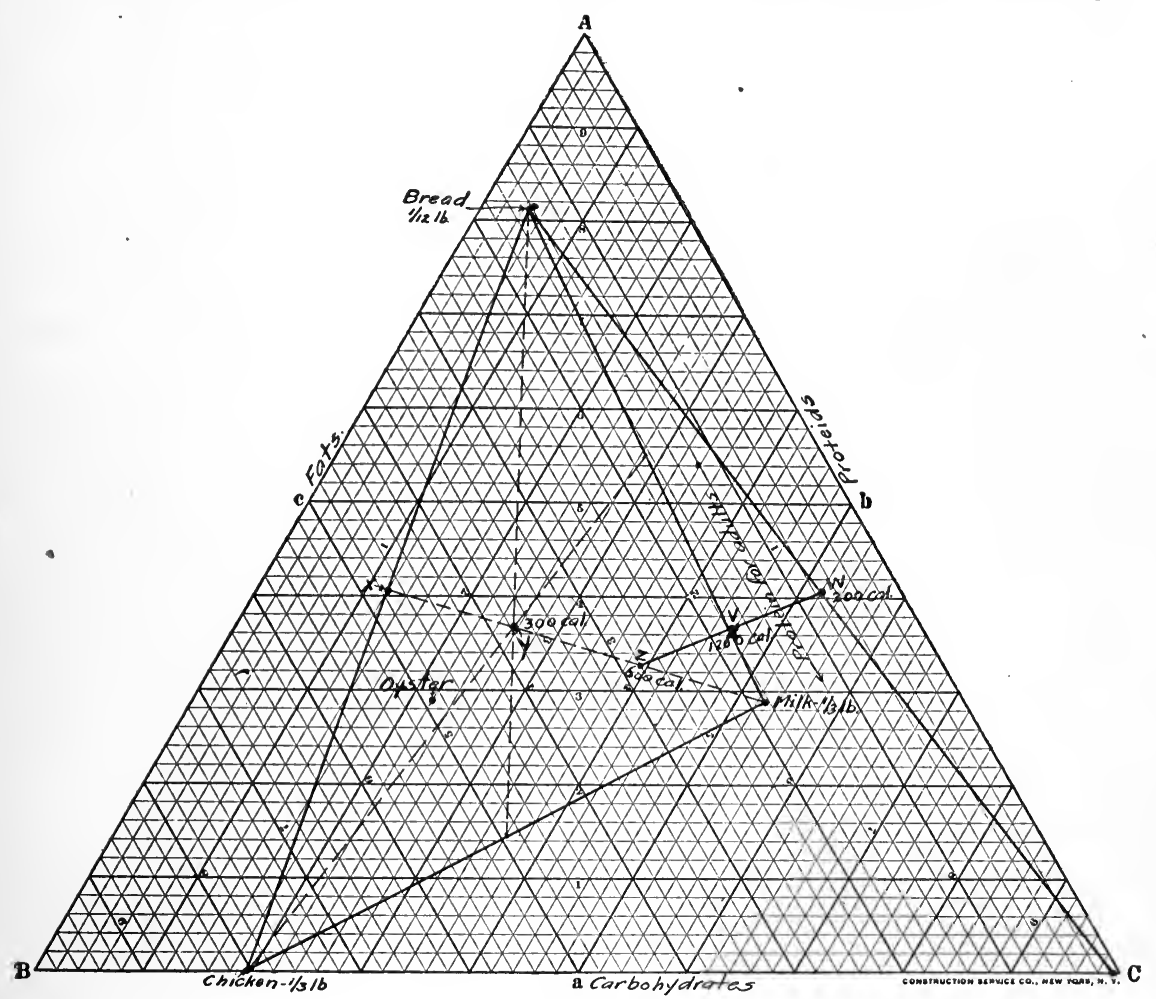

Fig. 1

number of calories of each. Thus, the point $\mathrm{x}$ will indicate a bread and chicken mixture containing $40.5 \%$ carbohydrate, $47.5 \%$ proteid, and. the balance, $12.0 \%$ fat. This line indicates, moreover, that by mixing bread and chicken in various proportions, we can make a ration indicated by various points along this line. But no mixture of bread and chicken will enable us to put up a ration indicated by any other point than one on this line. If we wish to keep our proteid element near to $15 \%$ we must make the mixture mostly bread, or if we wish to keep our carbohydrate quantity low we must put in a large amount of chicken and a smaller amount of bread. If, now, we wish to add another element to the ration we can do so graphically. Let us add enough milk 
for 100 calories, or, say 1/3 lb. and if we take 100 calories each of chicken, bread and milk we may determine the food value of our niixture by drawing a smaller triangle connecting the three points and finding the center of gravity of this triangle by drawing the median lines (lines joining each apex to the middle of the opposite side) as indicated by the point $y$ in Fig. 1. We ean then read directly from the chart that such a mixture will contain $37.6 \%$ proteid, $36.8 \%$ earbohydrate and the balance $25.6 \%$ fat.

We know that for an adult it is desirable to have a food ration containing about $12 \%$ proteid which we can indicate by drawing a line $12 \%$ from the side b of the large triangle. It is apparent that the mixture indieated by the point $y$ is too far to the left of this line for proper economy, and in order to properly balance it we should add more elements low in proteid. Point y indicates 300 calories. We ean add more milk if we so desire by drawing a line connecting the point $\mathrm{y}$ and the point representing milk and, remembering that the point $y$ has a weight of 300 calories and the milk point a weight of 100 ealories, by adding say 300 more calories of milk we will obtain another point $\mathrm{z}$ containing 600 calories, represented by $1 \frac{113}{3} \mathrm{lbs}$. of milk, 1/3 lb. of chicken, and 1,12 lb. of bread. In order to come still eloser to our balanced proteid line we may ard more bread and butter by taking a bread and butter portion amounting to $1 / 2$ ounce of butter and $1 / 12 \mathrm{lb}$. of bread, thus adding the element $w$ containing 200 calories which we can eombine with the z point above determined by drawing a line between them, and since $w$ has a value of 200 calories and $z$ a value of 600 calories the point represented by the mixture of $w$ and $\%$ will be $1 / 4$ of the distance between them from the $z$ end. 'Two more portions of bread and butter added to the above figures would locate our final point halfway between $w$ and 2. and give us a point $v$ which is quite close to our economic proteid line and contains enough fuel value for an ordinary workman's dimer. It is also well balanced as to fat and carbohydrate. This final point $v$ contains approximately $17 \%$ of proteid, $37 \%$ carbohydrate, and the balance of fat. 'This entire computation can be made on the chart in less than two minutes, and the anthor is not familiar with any other method of getting the same results in less than half an hour.

If we desire to analyze the ration with respect to its proteid and fat elements alone we mag draw a line from $A$ through the point $v$ and entting the line l' (' at a point which shows us that of the proteid and fat elements the ration contains $28 \%$ of the former and $72 \%$ of the latter.

To the motallurgist there will at once oecur many ways in which such a form of chart can be used with aenomy of time. Another very useful pecouliarity of this form of chart is that lines may be drawn upon the rhart indicatiug prints of similar characteristies. 'Thus, Professor Feret showed how mixtures containing different grades of sand of the same strength or of the same capacity for taking up water could be expressed by lines through these points upon the chart, enabling mixtures to be made up by the interpolation of certain predetermined characteristics.

The following article by Eslward .J. Brady is from the Journal of the. Franklin Institute, Fobriary, 1918.

Trilinear co-ordinates to express the characteristies of alloys consist- 
ing of three different metals were first used by Professor Thurston in 1877.

For a better illustration of their value, we may cite their use in the theory of color where any integral color may be represented by a point on a triangle, each corner representing $100 \%$ of one of the three primary sensations.

The fact that this method of graphical representation can be admirably applied to the determination of the characteristies of gas mixtures seems to have been overlooked.

In a mixture of three gases the heating value of the mixture is equal to the sum of the products of the percentage of each constituent multiplied by its respective heating value. This is also true of specific gravity, as well as the amount of air necessary for complete combustion.

It follows from the above that if on each of the three corners of a "gas mixture triangle" we erect perpendicular lines proportional to the heating value of the constituent at that corner, the upper ends of these lines will determine a plane. This plane, which we may call the heating value plane, will, in general, make an angle with the plane of the paper. Contours, or lines of equal heating value, drawn upon this plane will be straight parallel lines, making angles with the sides of the triangle, depending upon the heating value of the particular gases mixed. Such lines are the loci of an infinite number of mixtures of the three gases represented, all having the same heating value.

Similar systems may be drawn for specific gravity and air required for complete combustion.

The latter system will, in general, be almost parallel with the heating value contours, because of the fact that heating value is roughly proportional to the number of $\mathrm{O}_{2}$ atoms required for complete combustion.

Specific gravity contours or lines of equal gravity generally cross the heating value lines at certain angles. It is thus possible to eliminate one of these variables from experiments on gas mixtures.

Travelling along one of the gravity contours changes the heating value but not the gravity, and vice versa.

These lines are all straight because of the linear relation between the particular characteristics so chosen, viz., heating value, gravity, etc., and the quantity of gas involved.

However, other characteristics of the mixture may also be represented, as velocity of inflammation by curved lines.

The "gas mixture triangle" is not limited to pure gases. We may determine the characteristics of such gases as coal gas, water gas, and oil gas in the ordinary way, using these values to construct a triangle, and proceed as above. The quality of a mixture may be decided upon and its position on the diagram located at a point. A circle drawn with the point as a center will establish a tolerance outside of which the mixture must not fall. Other uses will suggest themselves.

Trilinear Chart for Coal Analysis. Technical Paper 93 of the Department of the Interior by Oliver C. Ralston is entitled: "Graphic Studies of Ultimate Analyses of Coals." Without quoting in detail the results of these studies the following has been abstracted and explains the application of trilinear co-ordinates to Mr. Ralston's methods. 
This paper presents the results of a study of the most reliable published analyses of coal. The analy'ses are studied from several different angles, a special type of trilinear co-ordinates being used for plotting the ultimate analy'ses of coals in terms of the recaleulated percentages of carbon, hydrogen, and oxygen, and the nitrogen and sulphur being ignored, as they are known to be, to some extent, organic constituents of the coal substance, but in very small amounts and varying aceording to no known laws. The analyses are calculated to a moisture-free, ash-free, nitrogen-free, and sulphur-free basis, so that $\mathrm{C}+\mathrm{H}+\mathrm{O}=$ $100 \%$.

By the use of such a system of trilinear co-ordinates it is possible to represent these three variables by one point on a diagram, and a fourth variaable ean be plotted on an axis perpendicular to the plane of the diagram. As a fourth variable there have been used such entities as time, pressure, volatile matter, and ealorific value, and the results have revealed certain faets and laws that have not been suspected, as well as showing graphically much information that has been expressed in extensive tables of figures and in long statements that have been difficult of interpretation.

Former workers have not been able to get a definite line of division between the different kinds of eoals, most of them having been able to plot with only two variables at one time, and none of them having been able to plot all three of the fundamental constituents-carbon, hydrogen, and oxyen. The lines of division between the fields that separate different kinds of coals on the ternary diagram would have to bear some simple relation to the axes of eo-ordinates if any of the two-variable or three-variable methods of plotting heretofore used were to be successful, but they do not. The statement that thece boundary lines would have to hear come simple relation to the axes of en-ordinates means that they would have to be either parallel or perpendienlar to them. The mathematies of this statement will beeome evident after the use of the diagram has been explained.

All the conal analyses plotted fall in a rather narrow band aeross the diagram, with only one distinct curve in it. The width of this band is considerably more than that due to the total error of analysis, so that it can not be assimmerl that the band would narrow to a line if all of the errors were oliminated. 'This gives one more reacon why former investigators have failed to reach comclusions that apply to all coals from the consideration of sum ratios as that of earbon to oxygen or of hydrogen to oxyen, as the coals on the uppere side and those of the lower side of the band differ distinctly, but otherwise are close together in composition. The ability to plot all three of these variables arainst one another has allowed much more rigid and valuable comclusions to be drawn. Alse, the amomin of avalaho hodrogen can be read directly from the diagram, and this entity ran hence lo used to advantage when so desired.

The fundamental theorem in gerometry upon wheh the equilateral system of prilinear oopordinates is based ean be stated as follows: "In ange erguilateral triangle. (Fig. 2) the sum of the three perpendienlars from any print, $p$, th the three sides of the triangle is a eonstant." This constant canl he made $100 \%$, and each perpendivolar ean be made to represent the pereontage of some one eomstituent of a ternary mixture. Thus, in the case of oxyen, hydrogen, and carbon the percentage of 
each in a coal can be represented by the perpendicular distance of the point representing the coal from the respective bases of the triangle. It is easily seen that any specific ternary mixture of this kind is represented by one point only within the triangle. The composition of cellulose, $\mathrm{C}_{6} \mathrm{H}_{10} \mathrm{O}_{5}$, calculated to percentages by weight of each constituent, is plotted in Fig. 2. The analysis of a ternary mixture must be calculated to percentages by weight, which should total $100 \%$.

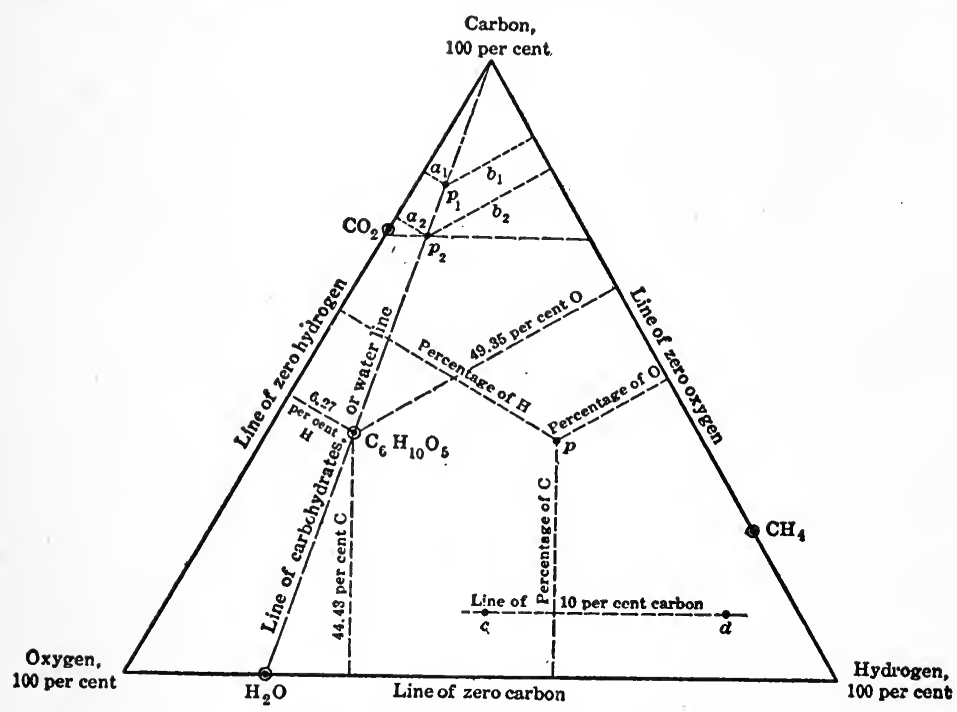

Fig. 2-Triangle to illustrate equilateral theorem

For convenience in plotting, the triangle is often ruled off into spaces for every $10 \%$ or for every $1 \%$, or subdivisions of $1 \%$, if the scale used is large enough (Fig. 3). An examination of Fig. 3 will show how a point is plotted, and anyone unfamiliar with this kind of co-ordinates would do well to pick out the points on this diagram representing $10 \%$ and multiples of $10 \%$, such as the point representing a compound containing $70 \%$ carbon, $10 \%$ hydrogen, and $20 \%$ oxygen.

Several important properties of the co-ordinates are as follows:

First, a point on one of the side lines, say the one designated " $\mathrm{H}_{2} \mathrm{O}$ " (Fig. 2), is on the zero line of the element that reaches $100 \%$ at the vertex opposite, and hence represents a compound that contains only two elements. Consequently, compounds containing only two elements, such as $\mathrm{CO}_{2}, \mathrm{CO}, \mathrm{CH}_{4}$, and $\dot{\mathrm{H}}_{2} \mathrm{O}$, will all be on one of the edges of the triangle. In the same way pure oxygen is represented by one of the vertices of the triangle where the zero lines of the other two elements meet.

Next, if a line be drawn from one vertex to an opposite side, such as the one from the carbon vertex to the point $\mathrm{H}_{2} \mathrm{O}$ (Fig. 2), then a certain simple relation exists between the co-ordinates of any series of points along this line. For example, consider the points $p_{1}$ and $p_{2}$, for which the ordinates representing the percentages of hydrogen and oxygen are $a_{1}, a_{2}, b_{1}$, and $b_{2}$, respectively. It is evident that by the application 
of the theorems of similar triangles the following ratio holds: $a_{1} \div a_{2}=b_{1} \div b_{2}$, and the same ratio of hydrogen to oxygen holds for the points representing cellulose and water. That is to say, for all the points along this particular line the ratio of oxygen to hydrogen is eonstant, which, in the line ehosen, happens to be the right ratio for them to form water. In other words, all of the so-called "earbohydrates" fall on this line. The same constaney of ratio holds for one of the pairs of components whenever a straight line is drawn through a vertex of the triangle.

Also for a series of points along a line parallel to one of the sides of

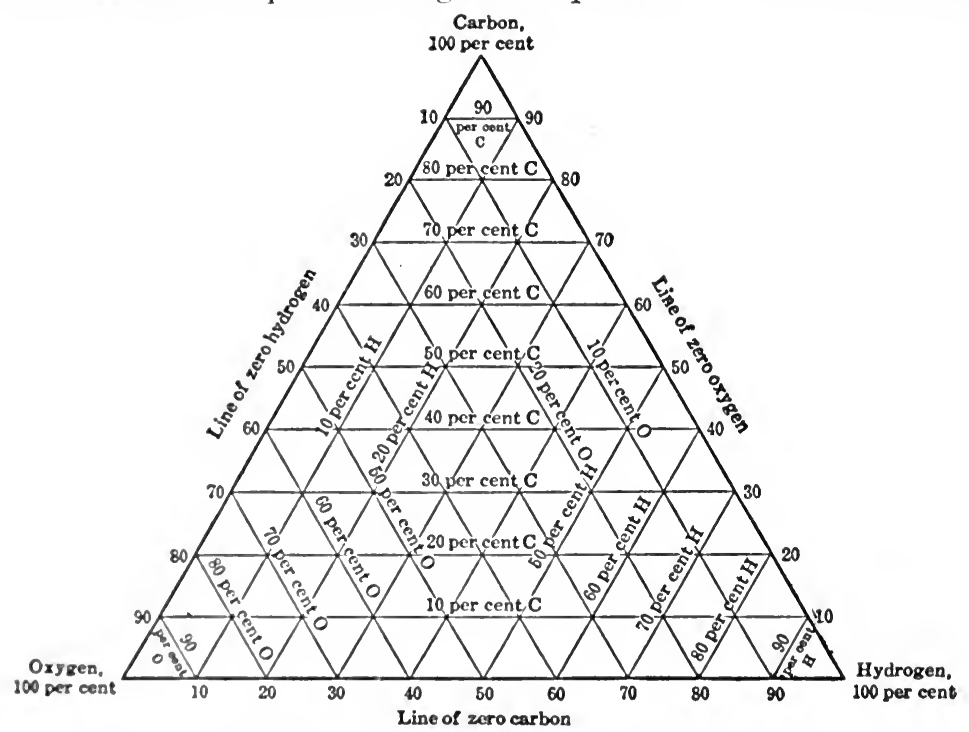

Fig. 3-Trilinear co-ordinates on an equilateral triangle

the triangle there is a emstant percentage of the constituent whose zero line is represented by that side. Such is the line $c d$ in Fig. 2, where proints $c$ and $d$ and all other points along this line would represent a constant pereentage of earbon- $10 \%$ in this case.

Lastly, if a compomnd, such as cellulose (Fig. 2), loses some such constitucnt as $\mathrm{H}_{2}()$, the composition of the residue will have to ehange along a straight line drawn through the two points representing $\mathrm{H}_{2} \mathrm{O}$ and cellulose to some point like $p_{1}$ or $p_{2}$ on the side away from the point representing $\mathrm{H}_{2} \mathrm{O}$. (Or, if cellulose were to gain water the composition of the complex would be changed along this same straight line toward the print $\mathrm{H}_{2} \mathrm{O}$. 


\section{CHAPTER VIII}

\section{Nomographic or Alignment Charts}

The nomographic or alignment chart differs from the rectilinear and logarithmic ones in that it consists of axes which are usually vertical and parallel, whereas the others have axes which cross. Further, scaled or cross-sectioned paper is of little advantage in the construction of nomographic charts as the scales of all of the axes may be different.

The main difference between the two types of charts is described in the following abstract from an article Nomography, by F. L. Martineau, assisted by A. M. Arter, in Automotive Industries, May 16, 1918:

It is usual to represent two variable formulæ by a graph in cartesian co-ordinates, the abscissæ representing the values of one variable and the ordinates the values of the other. In such a case a definite curve is

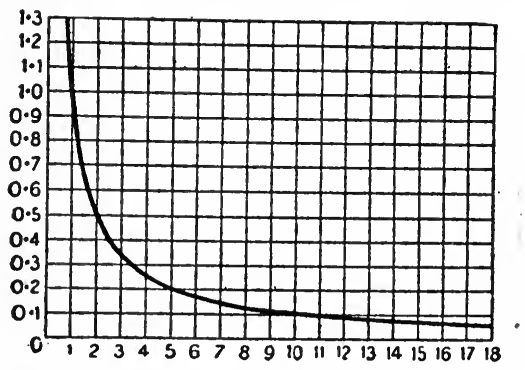

Fig. 1-Graph of numbers and reciprocals

formed (see Fig. 1) in which the abscissa $a$ and the ordinate $b$ of any point on the curve represent corresponding values of the variables.

In this instance

$$
b=1 / a
$$

i.e., $b$ is the reciprocal of $a$.

Here the intervening values have to be read by the intersection of the two lines representing abscissa and ordinate, and as a consequence interpolation is difficult. The corresponding "nomogram" becomes one line with two scales, one reading on each side.

Two different nomograms are given in Fig. 2 to show this, and are both converted from Fig. 1.

In each instance $a$ is the right-hand scale, i.e., the number, and $b$ is the left-hand scale, i.e., the reciprocal. In (I.) the number scale is regular and the reciprocal scale varied to suit it, while in (II.) both scales are logarithmic scales, the one reversed on the other. It will be noticed that in this instance interpolation for intermediate values is quite easy, 
and, further, that the diagram with the logarithmic scale renders this more simple than the regular seale.

When a graph is made to represent three variables in cartesian coordinates, it is necessary that two should be indicated by abscissa and ordinate respectively, and that a series of eurves should be drawn representing definite known values of the third variable.

The diagram given in Fig. 3 shows such a graph, in which the ordinates indieate the fuel used in pounds per horsepower per hour by an engine and the abscissa the thermal effieiency per cent, while a series of curves are drawn representing known values of fuel in British thermal units per pound.

If, as an example, it is desired to find the efficiency of an engine using fuel of value $17,700 \mathrm{~B}$.t.u.'s per $1 \mathrm{~b}$. and consuming $0.54 \mathrm{lb}$. per hp. per

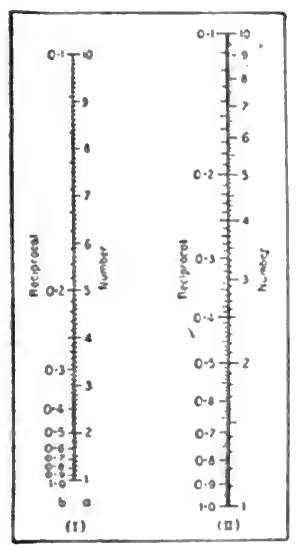

Fig. 2-Siales of numbers and reciprocals

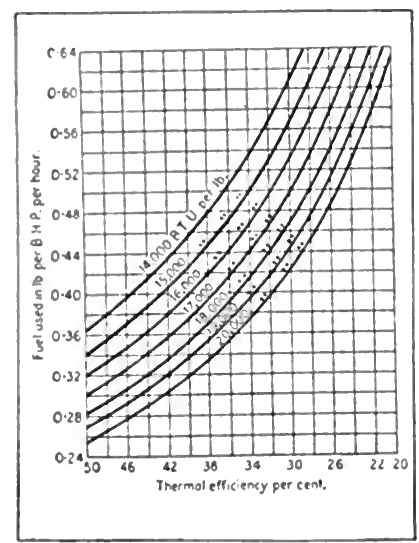

Fig. 3-ihermal efliciency "intersection" diagram

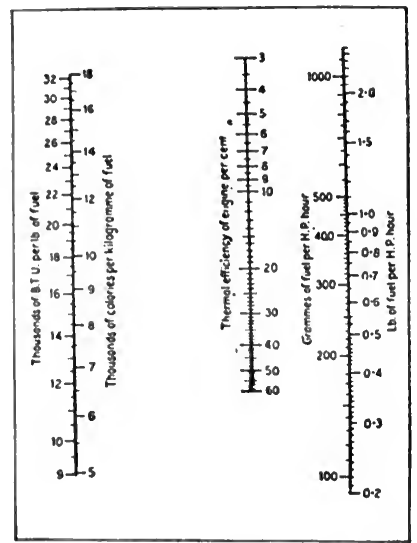

Fi". 4-Thermal effeiency "alignment" diagram

hour, then it is necessary to interpolate the value of the fuel and afterward refor to the base line for the efficiency. This operation is extremely difienlt in a diagram of this type, and one likoly to lead to error. When, however. this "intersection", diagram is changed to a "nomogram" or "alignment" diagram, all these diffienties disappear and the realing is romblered much more areollate.

Fig. 4 shows a nomogram, from which be the simple drawing of one straight line the thermal efficioney of any eluerine ean be at onee obtained. The diagram ran obvinsly be used for oither indicated or brake efficiency as may he desiruel.

It will be nofieed that it has heen possible to make this diagram over a much lareor ranere than the other so large, in fact, as to make it include almost all usual fun values and all reasomable consumptions.

A further roforenofo foig. 3 will show that, shomld it be required to read this diagram in mofriv measurements, it can be arranged with the ordinate seale graduated also in grammes of fuel per horsepower per hour, but the eurves drawn across the diagram to represent fuel values 
will not then read in round numbers for calories per kilogram of fuel, so that the question of interpolation will become still more difficult.

In the nomogram Fig. 4, however, both scales are easily graduated to read in British and metric units; under these conditions it is therefore possible to read either or both and make a comparison between tests carried out under both units of measurement.

In the construction of a nomographic or alignment chart it requires more or less calculating to determine the most suitable scales for the different axes and also to compute the correct distances apant which the axes should be. A method of doing these things mathematically is described by Geo. L. Hedges in Machinery, March, 1918, as follows:

An alignment chart, often called a "straight-line diagram," is a form of calculation diagram that is often much simpler to make and to use than is the ordinary curve plot on rectangular axes. In its usual form, such a chart consists of three or more parallel axes, usually vertical, the number depending on the number of variables in the formula to be plotted. To use such a chart, known values on two axes are joined by a straight line, the intersection of which with the third axis gives the desired value. With a chart of more than three axes, this intersection is joined to a known value on a fourth axis by a straight line, and its intersection with a fifth axis gives the desired value. If there are more than five variables, the straight-line operation is again repeated.

Alignment charts are additive charts; therefore, a formula to be plotted with this type of chart must be expressed as the algebraic sum of functions of the variables, that is, in the form:

$$
F(Y)=F^{\prime}(X)+F(Z)+\ldots
$$

The functions of the variables may be the variable itself, its products, logarithmic functions, trigonometric functions, etc. The axes are named for the variables and are graduated to the functions of the variables. The usual engineering formulas consist of the product of functions of the variables, as :

$$
F(Y)=F(X) \times F(Z) \times \cdots
$$

These may always be expressed as the sum of functions of the variables, as :

$$
\log F(Y)=\log F(X)+\log F(Z)+\ldots
$$

in which form they are adapted for an alignment chart. The axes are named for the variables and graduated to the logarithmic functions of the variables, the scales used depending on the formula being plotted and on the chart size desired.

To graduate the axes, decimal-divided scales with a table of logarithms are generally used. However, if many charts are to be made, a set of special logarithmic scales on different bases, say from 2 to 10 inches, will be found convenient. Decimal scales are divided into parts of an inch and numbered continuously every ten divisions; that is, one unit on the scale equals ten divisions.

Let $S=$ scale number and

$l=$ length of unit on scale, in inches. 
Then the relation between the length of unit and the scale number of a decimal-divided scale is:

$$
S=\frac{10}{l}, \text { or } l=\frac{10}{S}
$$

That is, on the 50 seale of an engineer's scale the length of one unit is $l=10 / 50=1 / 5$ inch. There are, of course, any number of possible scales, each with its corresponding value of $l$ : but generally the 10,20 , $30,40,50$ and 60 seales, and the 10 or 0.1 multiples thereof, are used, as these may be plotted with the ordinary triangular engineer's seale. The values of the seale number and the scale corresponding to different values of $l$ are given in Table 1.

\section{TABLE 1. VAILES OF SCAIE NUMBER AN!) SCALE ON ENGINEERS SCALE CORRESPONDING TO DIFFERENT VALUE OF I}

$\begin{array}{ccccccc}1 & S & 1 & S & 1 & S & \text { Scale to Use } \\ 100 & 0.1 & 10 & 1 & 1 & 10 & 10 \\ 50 & 0.2 & 5 & 2 & 1 / 2 & 20 & 20 \\ 331 / 3 & 0.3 & 31 / 3 & 3 & 1 / 3 & 30 & 30 \\ 25 & 0.4 & 21 / 2 & 4 & 1 / 4 & 40 & 40 \\ 20 & 0.5 & 2 & 5 & 1 / 5 & 50 & 50 \\ 1693 & 0.6 & 135 & 6 & 1 / 6 & 60 & 60 \\ & & & & & & \text { Machinery }\end{array}$

The principal labor in the making of an alignment chart is in arranging the lengths of the axes, or the distance along the axis between extreme praduations, so that available deeimal seales may be used.

Let $G=$ length of axis, in inches;

$d=$ function difference, or numerieal difference between extreme values of function plotted on axis.

Then :

$$
G=l d
$$

The value of $f$ for the longest axis determines the height of the ehart. I erraduated axis is used for each variable: with formulas of more than three variables, "dummy" axes, which usually are not graduated, are requirm. These dummy axes represent the partial additions in the formula, and aro three less in number than the number of variables.

The procedure in the making of an aligmment chart can best be shown by axamples. The example given in Fig. 5, the ehart for a simple formula of throe variables, shows the general scheme of procedure; the wharts given in Fies. 6 and 7 , for more complicated formulas, illustrate sperial fratures of the work. It is desirable to make use of a tabular form as shown in the examples. With a formula of three variables, the first two lines of the tabular form are used for the known variables and the third line for the unkuown variable. With a formula of more than three variables, the first two lines of the form are used for two known variables and the third for a dummy axis, which represents the sum of the functions of these two known variables. The fourth line is used for a third known variable, and the fifth line for the resultant of it 
and the first dummy axis; and so on for additional variables. The tabular columns of the form should be filled in in the following order:

1. Name of axis.

2. Symbol. That is, the symbol of the quantity whose function is plotted. This quantity must be some function of the variable represented by the name of the axis. Constants in the formula are neglected

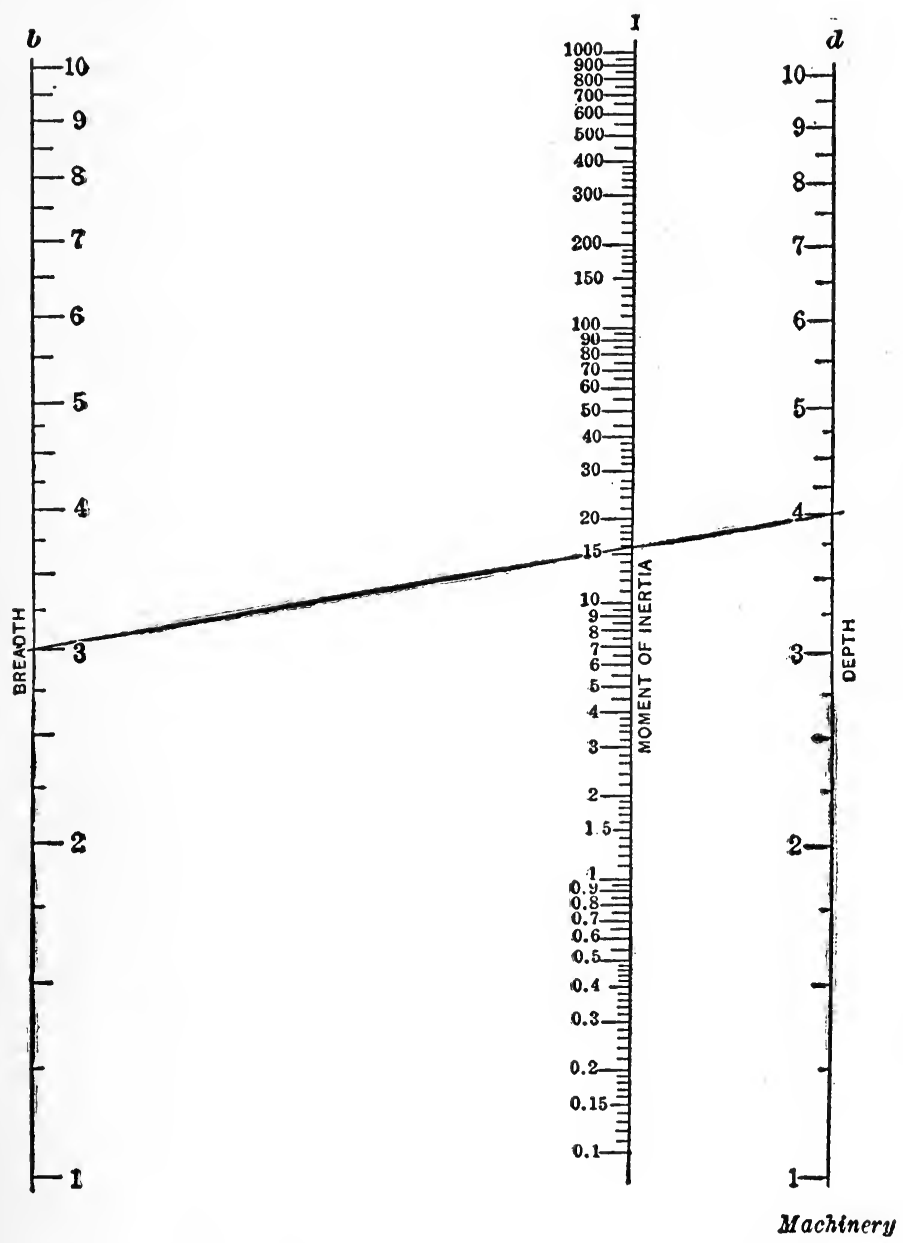

Fig. 5-Chart for determining Moment of Inertia of a Rectangle

in the tabular form; the constant serves merely to locate the position of the graduations on the intermediate axis, and this position will be determined in a more direct manner, which is explained later.

3. Number limits. Assume maximum and minimum values of the known variables and find the corresponding values of the unknown variable. 
4. Function limits. The values of the functions of the variables given by their number limits.

5. Function difference. The arithmetical difference, for each variable, between its function limits.

6. Length of unit $l$ on a decimal-divided seale, or the factor by which to multiply the function difference to get the actual length $G$ between extreme graduations on the axis.

7. Actual length $G$, in inches. between extreme graduations on an axis.

8. Seale number. 'The seale number for an unknown variable axis is the sum of the scale numbers of the axes of which it is a resultant.

9. Equivalent seale. This is the seale which, used with the symbol with exponent omitted, will give the identical graduation as by the given seale and given symbol. The equivalent scale number is obtained by dividing the given siale number by the exponent of the symbol.

Let $x=$ exponent of symbol;

Then :

$E=$ equivalent scale number.

$$
E=\frac{S}{x}
$$

In the chart in Fig. 7, for the axis "Gallons per Minute," the symbol is $Q^{2 / 3}$ and the scale number is 2 ; henee the equivalent seale number is 2 divided by $25=5$. This means that $Q$ plotted with the 5 seale (the 50 scale should be nsed) gives the same graduation as $Q^{2 / 5}$ plotted with the 2 scale (using the 20 scale), and is much easier to plot.

10. Sicale to use, shown by Table 1.

A slietch should now be made showing the positions of the axes and the directions of graduations thereon, and the values of maximum and minimum numbers whose functions are plotted. The width of the chart is determined by the fact that for accurate reading the intersecting line used in reading the chart should never make an angle much greater than 4.5 degrees with the axes. Where it may be necessary to draw a line from the nuper end of one axis to the lower end of the other, the chart should be approximately scinare; where the line will usually be drawn nearly horizontal, the width may be eonsiderably less than the height. In constructing the chart, the axes should be drawn first and the limit values markend thereon.

For a formula of three variahles, the axis of the unknown variable is the intermediate axis. Its listances from the outer axes are inversely proportional to the scalo numbers of those axes; for example, if $S_{1}$ and $S_{2}$ are the seale numbers of the outer axes, $S_{3}=S_{1}+S_{2}$ is the seale number of the intermediate axis. 'Then, if $I$ ) is the distance, in inches, between the outer axes, the position of the intermediate axis is:

$$
\left.\frac{s_{1}}{S_{3}} \times I\right) \text { inches from the } S_{2} \text { axis }
$$

and

$$
\left.\frac{S_{2}^{\prime}}{S_{3}^{\prime}} \times I\right) \text { inches from the } S_{1} \text { axis }
$$


For a formula of more than three variables, it is necessary first to consider two known vasiables and find the position of the first dummy axis. Then this dummy axis is considered with another of the known variables, and the position of the second dummy axis is determined in the same manner as the first. Finally, from the last dummy axis and the last known variable, the position of the axis of the unknown variable is found.

To find the directions and positions of graduations on the axes, consider the axes in successive sets of three. The outer axes of a set will have known values, and the intermediate axis will have the unknown values. The corresponding unknown and known limit values should be at the same end of the chart. The graduation for the maximum unknown value at one end of the intermediate axis should be at the intersection of this axis with a line joining the corresponding known limit values on the two outer axes; similarly, the graduation for the minimum unknown value at the other end of the intermediate axis should be at the intersection of this axis with a line joining the other two limit values on the outer axes.

In graduating an axis, it is well first to check the chart layout by seeing if the distance between the extreme graduations on the axis, measured with the scale from Table 1 corresponding to the scale number for the axis, equals a 10 multiple of the function difference for the axis; then lay off the graduations for 0.1, 1, 10, etc., and, lastly, fill in the intermediate graduations desired.

\section{Example 1-Construct a chart for the formula $I=\frac{b d^{3}}{12}$,}

which is the formula for the moment of inertia of a rectangle of breadth $b$ and depth $d$. In logarithmic form, it becomes $\log I=\log b+\log d^{3}$ - $\log 12$. The functions of the variables are logarithmic functions. As there are three variables, the chart has three axes; the known variables $b$ and $d$ are plotted as the outer axes, and the unknown variable $I$ as the intermediate axis.

The first step is arranging the matter in tabular form, as shown in "Table 2.

TABLE 2. TABULATED VALUES FOR EXAMPLE 1

\begin{tabular}{|c|c|c|c|c|c|c|c|c|c|}
\hline 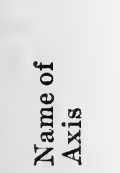 & 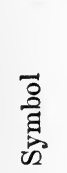 & 总: & 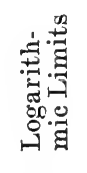 & 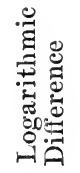 & & 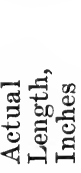 & 岕 & 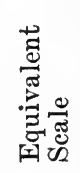 & 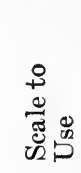 \\
\hline Breadth & $\mathrm{b}$ & 1 to 10 & 0 to 1 & 1 & 10 & 10 & 1 & 1 & 10 \\
\hline Depth & $d^{3}$ & $1^{3}$ to $10^{3}$ & 0 to 3 & 3 & $3^{1 / 3}$ & 10 & 3 & 1 & 10 \\
\hline $\begin{array}{l}\text { Moment } \\
\text { of } \\
\text { Inertia }\end{array}$ & I & $\begin{array}{c}0.083 \\
\text { to } 833\end{array}$ & $\begin{array}{c}\bar{z} .921 \\
\text { to } \\
2.921\end{array}$ & 4 & $2^{1 / 2}$ & 10 & 4 & $M a$ & 40 \\
\hline
\end{tabular}

The names of the axes are Breadth, Depth, and Moment of Inertia, and the symbols are $b, d^{3}$, and $I$. Maximum values of $10 \mathrm{in}$. and minimum values of 1 inch are assumed for both breadth and depth: hence the number limits will be 1 to 10 for breadth and $1^{3}$ to $10^{3}$ for 
depth, the corresponding logarithmie limits will be 0 to 1 and 0 to 3 , and the logarithmie differences will be 1 and 3 . An inspection of the formula for the example shows that the minimum limit value of $I$, the unknown variable, will be obtained from $b=1$ and $d=1$, for which values $I=0.083$; similarly, the maximum limit value of $I$ is found to be 833 . The logarithmic limits are, then, $\log 0.083=\overline{2} .921$ and $\log 833$. $=2.921$ : hence the logarithmic difference, which is the numerieal difference between these quantities, equals 4 .

For the axis Breadth, the logarithmic difference of which is 1 , aecording to Formula (2) and Table 1 when $l$ is 5,10 , or $162 / 3$, the length $G$ of the axis is 5,10 , or $162 / 3$, respectively. Adopting the value $l=10$, giving a length of axis of 10 inches, the chart may be plotted on paper $81 / 2$ by 11 inches. For this value of $l$, Table I gives seale number $=1$ and Formula (3) gives the equivalent seale number $=1$, for which Table 1 gives 10 as the scale to use. Similarly, for the axis Depth, the logarithmie differenee of which is 3 , a value of $l=31 / 3$ will be used to give a length of axis of $10 \mathrm{in}$.; Table 1 gives the seale number 3 and Formula (3) gives the equivalent scale number 1. for which Table 1 gives 10 as the seale to use. The scale number for the unknown variable $I$ is then the sum of these scale numbers, or $1+3=4$. Formula (3) gives the equivalent scale number 4, for which Table 1 gives as the scale to use 40 .

The distance between the outer axes of the ehart is made $7.2 \mathrm{in}$., which is about as great as can be used on a letter-size sheet; hence the position of the intermediate axis is, from Formulas (4) and (5):

$1 / 4 \times 7.2=1.8 \mathrm{in}$. from depth axis, and $3 / 4 \times 7.2=5.4 \mathrm{in}$. from the breadth axis

The minimum limit value of $I$ is 0.083 , and the values of $b$ and $d$ corresponding thereto are $b=1$ and $d=1$. These values of $b, d$ and $I$ must be, therefore, at the same end of the chart, say the lower end, and a line joining the value of $b=1$ on one outer axis to $d=1$ on the other outer axis will intersect the intermediate axis at $I=0.083$. Laying off on the outer axes their length of $10 \mathrm{in}$. determines the points $b=10$ and $d=10$, and a line joining these points intersects the intermediate axis at the maximum value of $I=833$. If the ehart is correct so far, the distance between these points for $I$ on the intermediate axis, measured with the 40 scale, should equal a 10 multiple of 4 , the logarithmie difference for the axis. These points determine the position of the graduations on the axis. The logarithm of 0.083 is $\overline{2} .921$, and of 833 is 2.921. Place the 40 scale so that 9.21 on the scale coineides with 0.08 .3 on the axis and 49.21 on the scale coineides with 833 on the axis, and mark off the points 10, 20, 30 and 40 on the scale corresponding to values of $I$ of $1,10,100$ and 1000 , respectively, on the axis. Intermerliate graduations may now be marked off as desired. The alignment chart for Example 1 is shown in Fig. 5.

Example 2-Construct a rhart for the formila:

$$
\log \left[\frac{P}{p-\left(\frac{P}{p}-1 k\right)}\right]
$$

$n=$

$\log k$ 
in which $P=$ given sum, in dollars;

$p=$ amount of each monthly payment, in dollars;

$n=$ time to pay out, in months;

$\boldsymbol{r}=$ yearly rate of interest;

$k=1+\frac{r}{12}$, constant for a given rate of interest.

The interest rate will be taken as $7 \%$, for which the value of $k$ is 1.005833 .

This is the formula for the time, in months, required to pay a given sum, with interest compounded monthly, by monthly partial payments made at the beginning of each month and at the end of the last month. This formula is often used in selling real estate on the pay-like-rent installment plan. This formula is not in the proper form for an alignment chart, but solving for $P / p$ gives:

whence:

$$
\frac{P}{p}=\frac{k^{(\mathrm{n}+1)}-1}{k^{\mathrm{n}}(k-1)}
$$

$$
\log P-\log p=\log \left|\frac{\left[i^{\mathrm{n}}+1\right)-1}{k^{\mathrm{n}}(k-1)}\right|
$$

The two known variables are $P$ and $p$ and their functions in the formula are logarithmic; the unknown variable is $n$ and its function is logarithmic and is the quantity making the right-hand half of the formula. In plotting the alignment chart for this formula, the value of this function of $n$ must be calculated for all points desired on the $n$ scale and used for laying off these points. Table 3 gives the tabulated figures for this

\begin{tabular}{|c|c|c|c|c|c|c|c|c|c|}
\hline 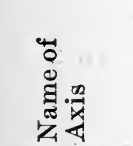 & 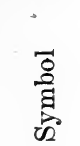 & 总总 & 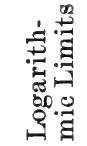 & 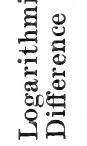 & $\sim$ & 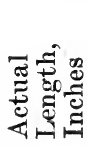 & 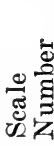 & 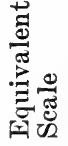 & 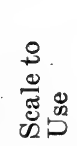 \\
\hline $\begin{array}{l}\text { Principal } \\
\text { sum }\end{array}$ & $\mathrm{P}$ & $\begin{array}{r}\$ 1,000 \text { to } \\
\$ 10,000\end{array}$ & 3 to 4 & 1 & 10 & 10 & 1 & 1 & 10 \\
\hline $\begin{array}{l}\text { Monthly } \\
\text { payment }\end{array}$ & $\mathrm{p}$ & $\underset{\$ 50}{\$ 10 \text { to }}$ & $\begin{array}{r}1 \text { to } \\
1.699\end{array}$ & 0.699 & 10 & 6.99 & 1 & 1 & 10 \\
\hline $\begin{array}{l}\text { Time to } \\
\text { pay out }\end{array}$ & 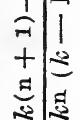 & $\left(\begin{array}{c}24 \text { to } 300 \\
\text { months } \\
23.25 \text { to } \\
142.6\end{array}\right)$ & $\begin{array}{c}1.366 \\
\text { to } \\
2.154\end{array}$ & 0.788 & 5 & 3.94 & 2 & 2 & 20 \\
\hline
\end{tabular}
formula and Fig. 6 is the alignment chart.

TABLE 3. TABULATED VALUES FOR EXAMPLE 2

The formula gives the time in months, but the chart is marked in years. The position of the graduations on the intermediate axis of this heart were determined by finding values of $P$ and $p$ corresponding to 


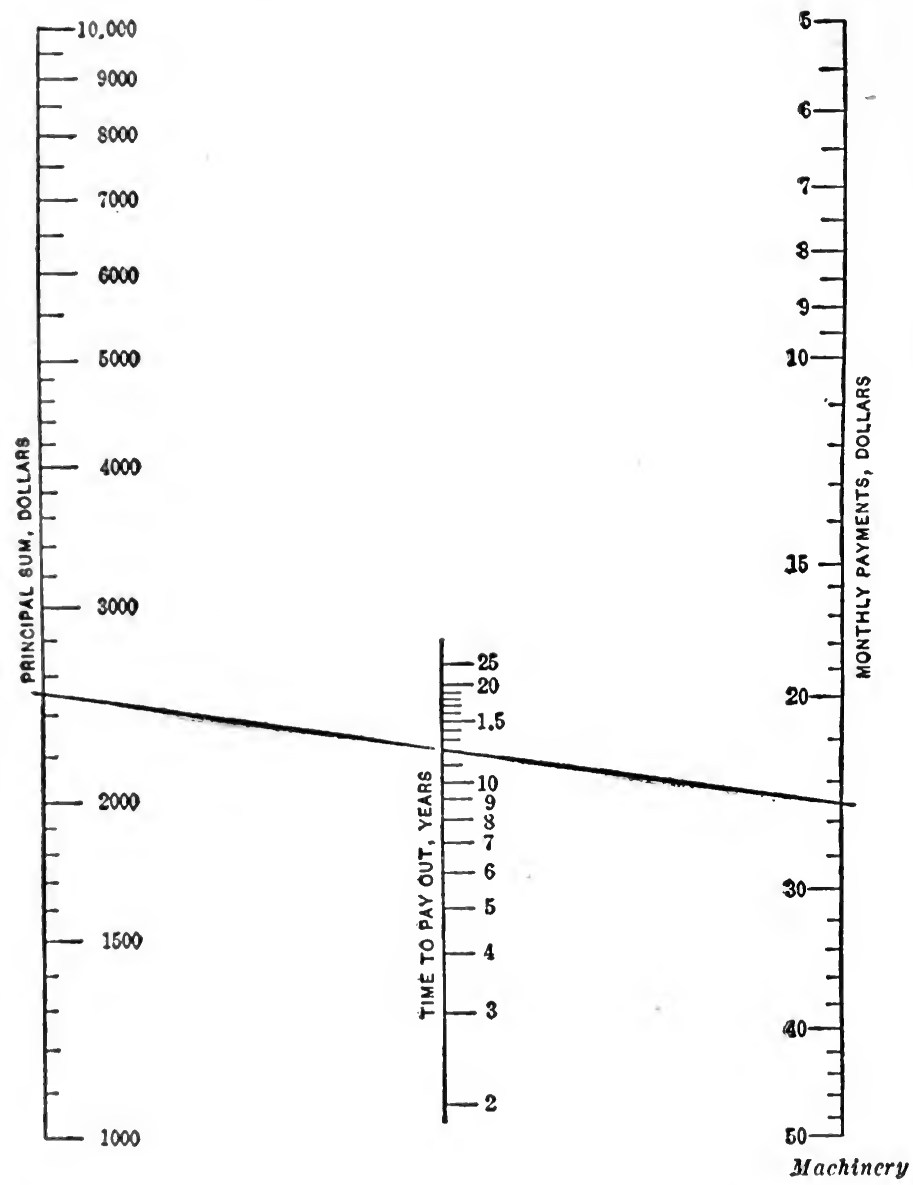

Fig. 6-Chart for determining Time required to pay, by.Monthly Payments, a Given sum with Interest

the value of the function of $n$ for maximum and minimum assumed values of $n$; that is, when $n=24, \frac{P}{-}=23.25$, and when $n=300, \frac{P}{P}=142.57$. p) $p$

As is shown in the chart, it is not necessary to graduate an axis full length-only such graduations as are desired need be used.

Example 3- Construet an alignment chart for the formula for finding the diameter of pipe for a given flow of water, viz.:

$$
d=\sqrt[5]{\frac{Q^{2} L}{333333 F}}
$$

in which $Q=$ gallons of wator per minute;

$F=$ pressure lost by friction, pounds per square inch; 
$L=$ length of pipe, in feet;

$d=$ actual inside diameter of pipe, in inches.

Arranging this into the proper form for constructing an alignment chart gives :

$$
\log d=\log Q^{2 / 5}-\log F^{1 / 5}+\log L^{1 / 5}-\log (3333)^{1 / 5}
$$
chart.

Table 4 gives the tabulated values for this example and Fig. 7 the TABLE 4. TABULATED VALUES FOR EXAMPLE 3

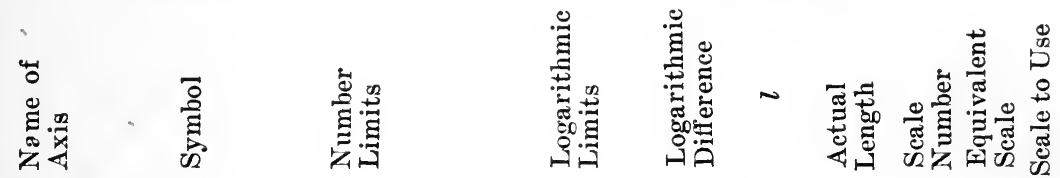

Gallons per min-

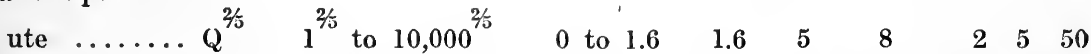

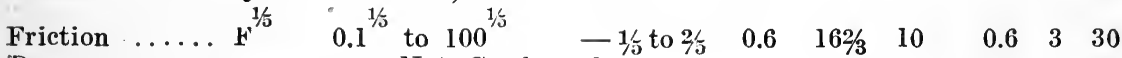

Length of pipe. $L^{1 / 5} \quad 1^{1 / 5}$ to $100^{1 / 5} \quad 0$ to $\%$ \% $\begin{array}{llllllllll}\text { Diameter of pipe } & \mathrm{d} & 0.0786 \text { to } 31.3 & \bar{z} .8954 \text { to } & 2.6 & 31 / 3 & 82 / 3 & 3 & 3 & 30\end{array}$ 1.4954 Machinery

This chart is handled in exactly the same manner as the others. From Formulas (4) and (5) and from the scale numbers of the $Q, F$

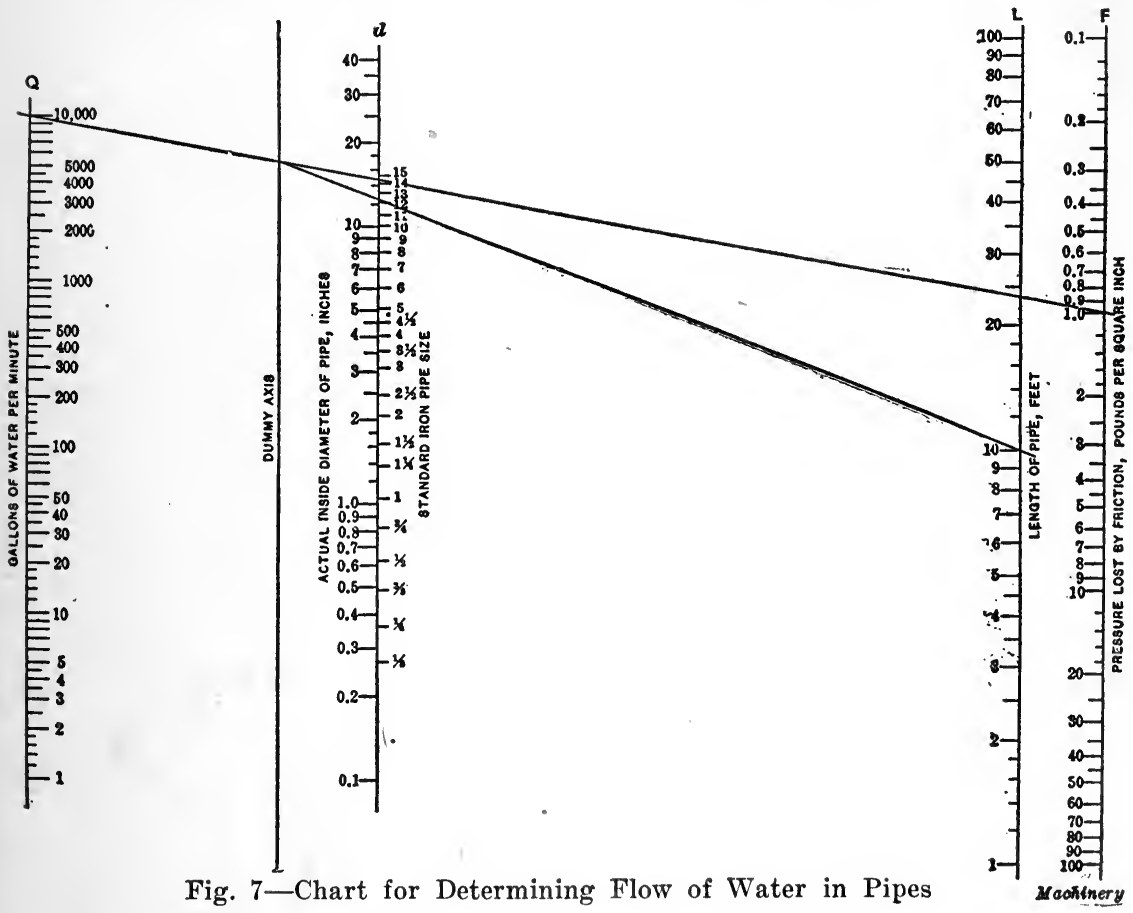


and dummy axes is found the position of the dummy axis. Then the position of the $L$ axis is assumed, and from the scale numbers of the $L$ axis, dummy axis and $d$ axis the position of the $d$ axis is found. The $d$ axis has an additional set of graduations, showing standard ironpipe sizes. This use of two seales on an axis will often be found convenient; for example, an axis for velocity may be graduated both in feet per second and in miles per hour.

The lines drawn on the ehart to indicate the order in which the axes are used show that $Q$ and $F$ values, say 10,000 and 1 , are joined by a straight line, and from the interseetion of this line with the dummy, axis a line is drawn to the value of $L$, say 10 ; the intersection of the latter line with the $d$ axis, which is at 12 inches, gives the required inside diameter of pipe.

The size and number of graduation marks used on the axes of a chart depend eonsiderably on the particular chart eonsidered. In general, it will be found desirable to have the graduations between 1 and 10 repeated between 10 and 100 and between 100 and 1000 , ete. The longest graduation marks should be used for the digit numbers 1,2 , 3, . . 9, and their ten multiples; the marks for half divisions should be about two-thirds as long, and the marks for 0.1 or 0.2 divisions should be about one-third as long.

Graphic Method of Spacing and Valuing the Monographic Scales. In Vol. IAXVII, Page 1359, of the Transactions of the American Society of Civil Engineers is published a paper by R. C. Strachan, which was presented to the Society on Feb. 3, 1915, entitled "Nomographie Solutions of Formulas of Various Types" and which describes a graphic method of spacing and valuing the seales. This follows in part:

A nomographic representation, or, more concisely, a nomograph of a formula, may be defined as a plot or chart on which appear seales for the variables involved in the formula, their relative magnitudes and relative positions being such that corresponding values of the variables are found on a line crossing the scales.

B. the expression "corresponding values" is meant a set of values which satisfy the equation represented by the nomograph; and the crossing line, therefore, is properly termed an isopleth.

The aim is to substitute the simplest possible mechanical operation for the labor of solving the formula by computation; and, with this in viow, the nomograph should preferably be designed so that its isopleths are rectilinear.

Take, as a single illustration, three parallel scales of equal parts, starting from a rommon base line, $c d$, Fig. 8, and suppose the $L$ line to be erguidistant from the other two. Draw any straight line $a b$, across the scales. Then $f g=f k+k g=\frac{1}{2} M+\frac{1}{2} N$.

The figure is a nomograph of the expression $L=\frac{1}{2}(M+N)$ and the line, $a b$, is an isopleth, its intersection showing by the seale readings a set of values which satisfy the equation. 


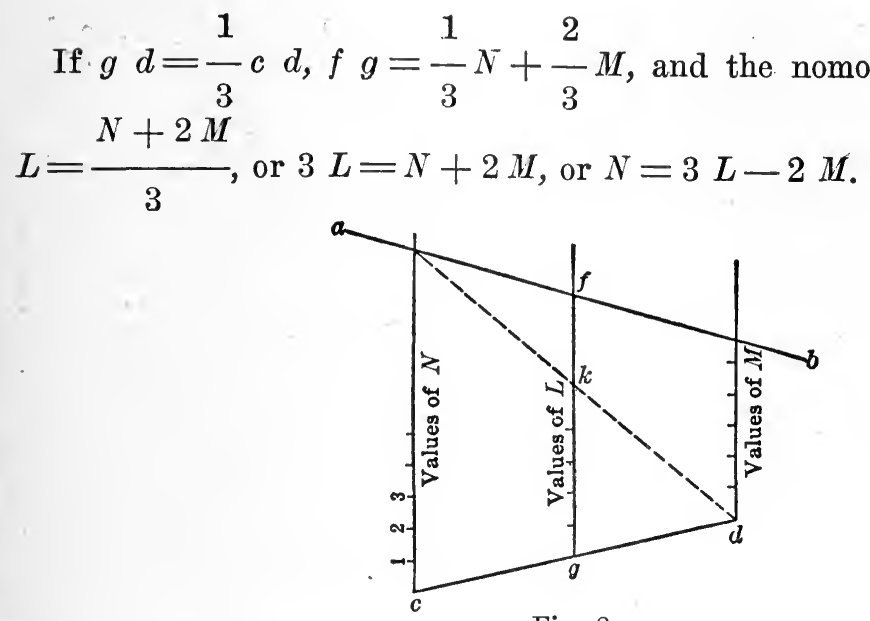

Fig. 8

If the units on the $L$ scale are made one-half as great as those on the $2 N+4 M$

other scales, the nomograph represents $L=\frac{2 N}{3}$, or $3 L=2 N+4 M$.

As another illustration, in Fig. 9, let $c d=d e$, and lay off the parallel scales for $P$ and $Q$ as shown. This is a nomograph of $P=\frac{1}{2} Q$, and any straight line drawn from $c$ through the scales is an isopleth.

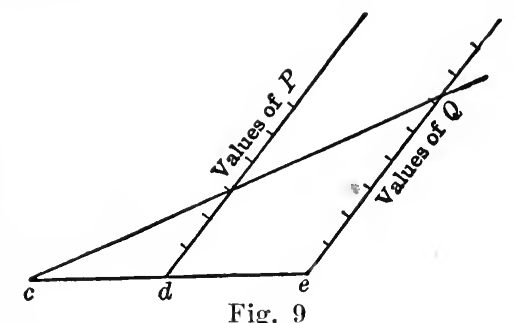

Fig. 9

Evidently, changes in the positions and magnitudes of the scales may also be made in this diagram, with corresponding effects on the expression represented.

If in place of the numbers we lay off their logarithms, we may read products instead of sums; exponents may be affected by changing the distance between scales, and numerical coefficients may be introduced by varying longitudinally the position of one or more scales.

A straight-edge may be used to indicate the position of an isopleth, or, for greater ease in reading the scales, a strip of transparent celluloid having a line scratched on it, will be found very convenient.

The mathematical principles involved are very simple, and their application is easy when the right clue has been discovered. Many ex- 
pressions which in their usual forms appear to be hopeless can be adapted to nomographic representation by a proper transformation; but the necessary change may not suggest itself until the equation has been studied somewhat thoroughly.

It will be found that, as a rule, scales of natural numbers are suitable for formulas in which the signs + and - appear; and logarithmic seales are adapted to those in which quantities affected by exponents must be multiplied or divided. In many cases, however, a formula in which there is an integral exponent may be transformed so as to permit the use of natural numbers for its nomographic seales.

The nomograph is easily read with precision on account of having few lines; it saves much valuable time, providing in effect a perfect tabulation of all possible values within the limits of the chart; and it enables one to see instantly the effect of a change, whether small or great, in any of the variabies. Furthermore, the solutions are equally simple, whichever quantity in the formula is unknown.

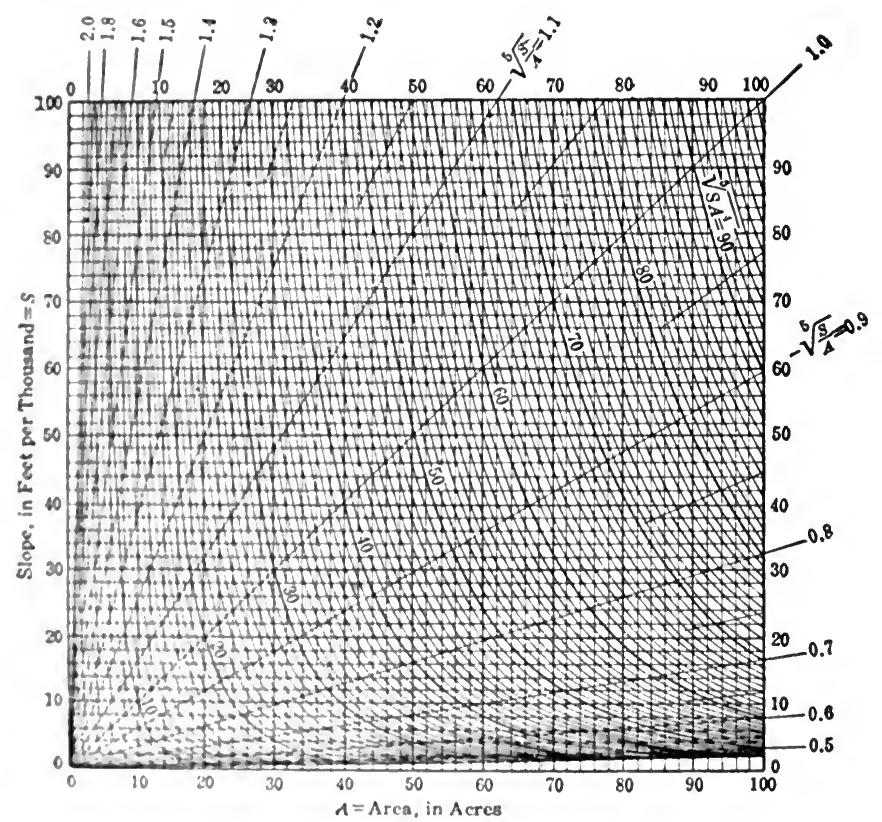

Fig. 10

The following comparison will illustrate these points: In a recent is-

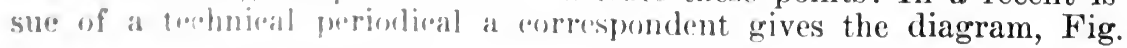

10, for finding is... in the Mc. Math run-off formula.

He explains that, to construct this, it is necessary to compute $\sqrt[5]{\frac{S}{A}}$

and darive from this $" A^{1}$ for a number of cases sufficient to give all the points required for plotting the curves. 
The finished diagram contains four sets of carefully plotted lines, and the intersections near the lower and left-hand edges are very indefinite.

A nomograph of this radical consists of three scales, and may be constructed with great ease and without computation, as follows:

Let $N=\sqrt[5]{D^{1} A^{4}}$.

Then log. $N=\frac{1}{5} \log . S+\frac{4}{5} \log . A$.

Lay off three parallel scales of the logarithms of numbers from 1 to the limit desired (Fig. 11), making all three with logarithmic units of the same magnitude. (The minor subdivisions are omitted from Fig. 11.)

Place the $N$ scale at a distance of $\frac{1}{5} D$ from the $A$ scale as shown. Then, for any position of $a b$,

$$
f g=\frac{1}{5} k l+\frac{4}{5} m n \text {. }
$$

The reading on the $N$ scale, therefore, is the number corresponding to 1

$$
\frac{1}{5} \log . S+\frac{4}{5} \log . A \text {, }
$$

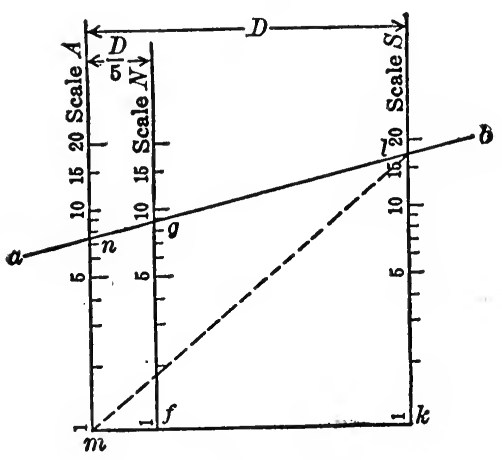

Fig. 11

and $a b$ is an isopleth, giving three scale readings, $S, A$, and $N$, which satisfy the equation,

$$
N=\sqrt[5]{S^{\prime} A^{4}} .
$$

A Method of Dividing a Scale Logarithmically. The explanation below is taken from an article by G. G. Stevenson, published in Machinery, June, 1918.

A simple and easy way to divide an alignment chart axis logarithmically where logarithmic paper is available is shown in Fig. 12. The logarithmic paper is placed at such an angle that the two extreme 
divisions desired just coineide with the horizontal extensions of the ends of the line to be divided; then with the 'T-square the points needed are projected to the line. If more convenient, a slide-rule may be used. This is done by multiplying the logarithm of each number to be located by the length of the whole line. In other words, the logarithms found are for a line one inch in length; then the points can be easily located by the use of a decimal scale.

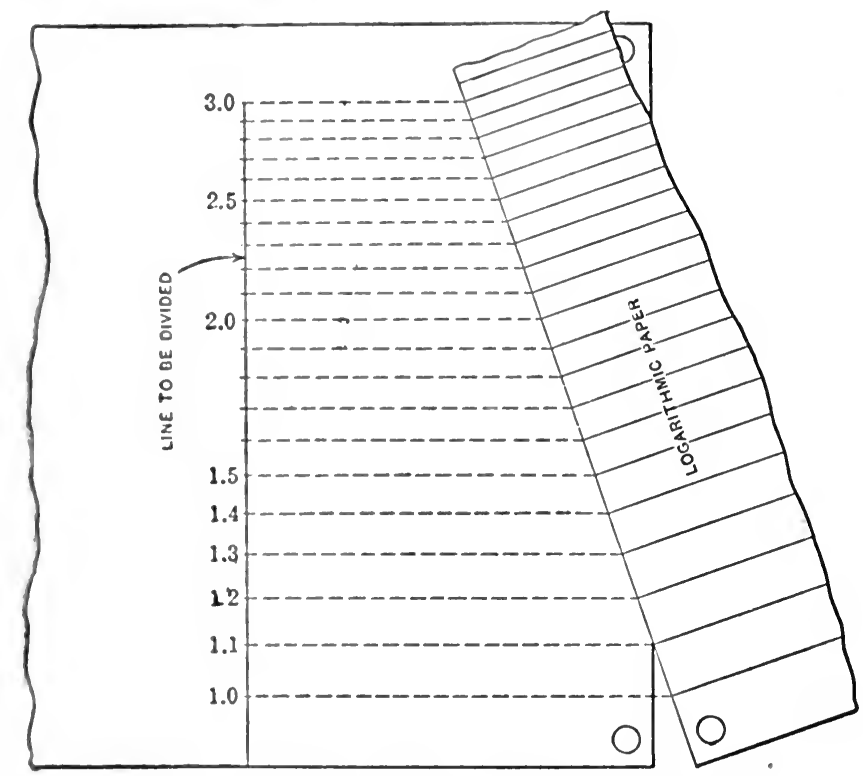

Fig. 12-Method of dividing a Line logarithmically

Construction for Logarithmic Graduation. The following is from Wachinery, January, 1917.

The following construction may be used for dividing a line for logarithmic graduation, when the line is of an odd length.

I) raw a square $A O)^{\prime} Y$, Fig. $1: 3$, on the line $A Y$, which it is desired to divide logarithmically, ancl take on $O A$ a point $a$, such that $O a=$

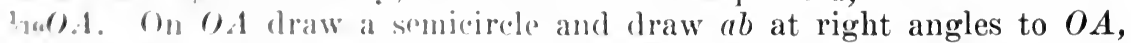
rutting vhre semirirele at $b$ : then with $O$ as a center inseribe the are $b b_{1}$, making $O b_{1}=() b$, and draw a verticle line through $b_{1}$ (assuming that $O A$ is horizontal, in mon in $I^{2}$ a horizontal line through $b_{2}$, which is such that $\left(1 b_{2}=160 . X\right.$. I) raw $b_{1}$ c vertivally to meet the semicirele in $c$ and inseribe are ere from $O$ ) as a eenter; biseet $b_{2} X$ in $c_{2}$ and draw horizontal and rertical lines through $c_{2}$ and $c_{1}$ to meet in $C$. The point I) is noxt fonmd hy finding $d_{1}$ similarly, and bisecting $c_{2} X$ in $d_{2}$ and projecting horizontal and rortical lines as shown. We thus get points BC'IDE upon a curve; to obtain intromediate points, draw a semicirele on Oc $c_{1}$ and draw the ares gg, and $h h_{1}$ from the eenter $O$. We then bisect $O C_{2}$ in $g_{3}$ and draw horizontal and vertical lines through $g_{2}$ and $g_{1}$, respectively, to meet in $f_{i}$; and reprat the construction for $H$ by 
bisecting $b_{2} c_{2}$ in $h_{2}$. The point $J$ is obtained by drawing a semicircle upon $O b_{1}$. In this way any desired number of points may be obtained, the resulting curve being a curve of logarithms. To save confusion with the construction lines, this curve is redrawn in Fig. 14, and the man-

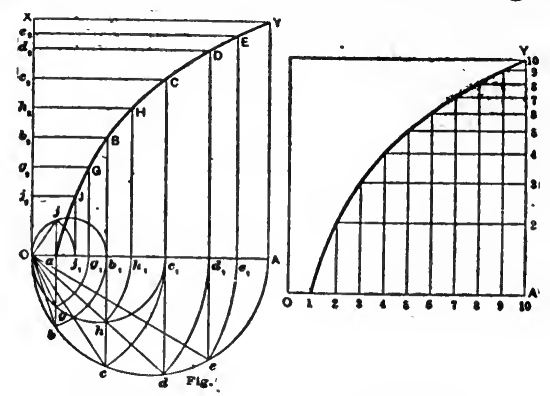

Fig. 13

Fig. 14

ner in which it is employed to divide $A Y$ into logarithmic graduations is shown. The base $O A$ is divided into ten equal parts, and the resulting points are projected vertically to the curve, the points of intersection being then transferred to the vertical line $A Y$ by horizontal projections as shown. 


\section{CHAPTER IX}

\section{General Principles Pertainlyg to the Use of Charts}

The first part of the book has dealt with the theory and eonstruction of the various types of eharts and now it is purposed to illustrate many of the uses to which these charts may be put. It has not been the intention to gather here as eomplete a collection of examples of the many uses of graphic charts as possible, for, first, it would be a stupendous task and require too huge a volume and, again, much of the material would be very special in nature and of interest only to the few to whom it applied; but rather it has been the aim to group under a few general heads typieal illustrations that will be of universal value and interest, and instead of trying to show the man in every business or profession definitely what he can do, to suggest to him ideas and principles which he can most advantageously adapt to his individual requirements. One of the great benefits to be derived from the use of charts is the development of analytical thinking and investigation. Invariably a chart fairly bristles with interrogations-why is this sudden decrease? or what does that rapid increase portend at this time? etc.-which in many cases require thought, study and eareful research to obtain the correct answer.

A few years back it was only the technical man who was trained to use graphic methods and he not very extensively. Today engineers in constantly increasing numbers use this device for computations, designing, estimating, cost analysis, etc., and, in addition, men in nearly every business and profession have found uses for graphic presentation. Industrial executives and managers, advertising men and salesmen, manufacturers, bank officials, lawyers, physicians, farmers, etc., all have learned that graphie methods are vastly superior to any other for a thorough analysis of certain records.

Today managers of all up-to-rlate plants are presented with daily reports for comparison, and while a good manager may know at a glance whether the record he receives this morning is above or below the average and very likely remembers what it was yesterday and possibly the day before, the bist one alive eamot remember the figures for six months past: and it is grenerally the results over a period which tell the real story. Also, it is difficult for any man to so visualize figures over a back perion as more pronounced than have been former ones, whether they extend over a longer time than they shomld, ete.

The rhart which is plotforl daily presents as perfect a picture of the events of a business as the rorrecetness and accuracy of the data from which it is made up allow. No (hain is stronger than its weakest link and no chart will give facts about a business when compiled from incorrecet reports. Provided, however, the material is dependable, the chart presents a graphic picture of the facts as readily analyzed and 
capable of suggesting constructive ideas as the engineer's or architect's layout of a building, and just as it would be difficult to comprehensively suggest the necessary alterations and revisions if the engineer came in and told what he was doing, or proposed to do, so it is equally perplexing for a manager to understandingly make the essential recommendations when he has to deal with a conglomerate mass of heterogeneous data. Just as the tracing or blue-print collects into a form that is quickly and easily understood all the facts concerning the building, so does the chart collect the facts concerning a business, and both pressent a record which may be studied and analyzed.

For the purposes of comparing and analyzing statistics, records, etc., it is not necessary that the user of graphic charts be schooled in higher mathematics or the manipulation of drafting materials. He need only understand the method of charting and be able to handle a straightedge and pencil or pen. The method is simple. It is hoped that the preliminary explanation has been sufficiently clear to demonstrate this

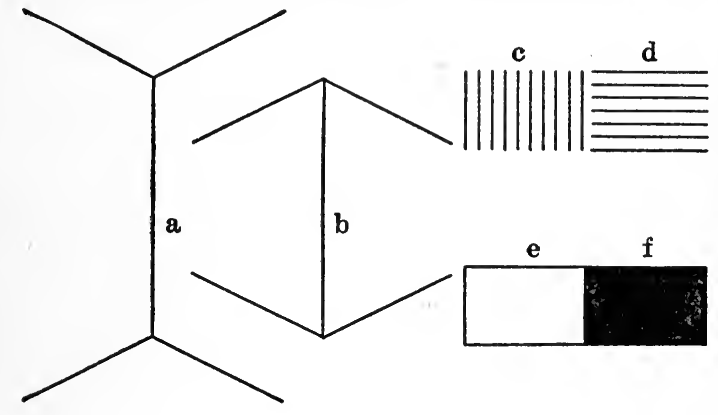

Fig. 1

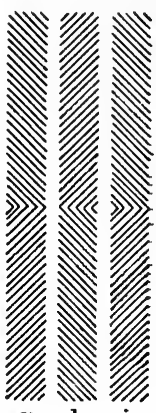

g h i

fact, but if there still remains any doubt in the mind of the reader it is believed the examination and study of those following chapters which are not descriptive of mathematical analysis, will dispel it.

There are many more complex uses for charts than the comparison and analysis of records, but most of these require at least an understanding of the principles of mathematics and many of them a fairly comprehensive knowledge of higher mathematics.

The engineer uses charts for arithmetical and geometrical computations, for the graphical solution of design formulas, for the plotting or mapping of surveys, etc., chemists and physicists use charts to show the results of their tests and experiments, and in fact, it is safe to say that everyone who makes a study of mathematics recognizes the value of graphic methods.

For those who are about to undertake the use of charts the one thing to be avoided is the selection of any method which gives a false picture of the facts presented. There are certain optical illusions, due to methods of shading and cross-hatching sometimes used in the above mentioned diagrams. In Fig. 1 the line a looks longer than $b$, whereas they are equal. The shaded area $c$ appears to be wider than $d$, and they are the same. The white area e looks larger than the black one $\mathrm{f}$, but they are equal. Of the bars $\mathrm{g}, \mathrm{h}$, and $\mathrm{i}, \mathrm{g}$ and $\mathrm{h}$ seem to be 
nearest together at their ends; $\mathrm{h}$ and $\mathrm{i}$ at their middles; whereas all are straight and parallel.

Another type of ehart which it is well to avoid is that portraying relative quantities by pictures, as, for example, where the sizes of the armies of different nations are eompared by pictures of soldiers. One man, representing an army three times the size of another, is made three times as tall as the man representing the smaller army. But as the man grows tall, he grows broad as well, and the eye, in eomparing one with the other, includes breadth with height-in other words areaand the tall man looks and is, considerably more than three times the size of the other. There are other similar fallaeies and it is well for the person undertaking the use of graphic methods to train himself at the outset to employ those charts which give a eorrect analysis of the facts.

For the beginner in the use of graphic eharts a few general hints as to arrangement, taken from the experience of the author, may be helpful.

It is best to standardize as far as possible-size of sheet, lettering, titles, etc. After considerable experimenting the author uses sheets of standard letter-head size, $81 / 2 \times 11$ ins., or multiples thereof, as $17 \times 11$ ins., etc.. which may be folded to the letter-head size. The ruling on the $81 / 2 \times 11 \mathrm{in}$. sheets usually measures $7 \frac{1}{2} \times 10$ ins., leaving margins of $3 / 4 \mathrm{in}$. at the top and left and $1 / 4 \mathrm{in}$. at the bottom and right, which allows a sufficient binding edge and room for lettering on the scale figures, marginal notes, ete. Sheets of these sizes may be filed either with the correspondence to which they refer, or they may be punched and filed in a loose-leaf book.

To obtain eopies of a chart, blue-printing is generally the most satisfactory and the cheapest means. Therefore, the ruled sheets should be translucent. Onion-skin paper, either glazed or unglazed, may be obtained, but the author prefers his charts on a light-weight, high-grade bond paper, as it prints perfectly and has the advantage of standing erasure better and does not erack with age. If the sheets are bound in pad form this adds to the convenience in handling and keeps the paper clean and fresh.

Experiment also proved an olive-green ruling the most satisfactory. It is easier upon the eyes, especially in artificial light, than some of the more brilliant yellows, oranges, and reds. The latter will make better bluc-prints theoretieally, but the olive-green has proved entirely satisfactory in practice.

At the time a chart is drawn explanatory notes should be used unsparingly and all curves or diagrams should be so keyed or lettered that there will be no guestion as to just what each represents when the ehart is referred to later. When it is not necessary to make blue-prints, different colored inks or water-eolor paints may be used in a drawingpen to distinguish one eurve from another and a key showing what each color represents put on the sheet, as for example:

Gross earnings:

Expenses:

Net revenue: ('red line)

(blue line)

(green line) 
It is also well to letter neatly on each curve just what it is. A chart is for clearness and any notes which tend to increase this are to be recommended.

When blue prints are made it is not easy to distinguish colors and it is then better to use black drawing ink and to vary the lines, as, for example: heavy solid, light solid, heavy broken, light broken, and many combinations of dots and dashes, with a key to show what each represents.

Each scale should show what it represents-dollars, days, years, ete. This may usually best be lettered in the margins directly alongside the scale figures. Each sheet should have a title telling what the chart is and include any reference numbers, as file number, job number, etc.

When points are plotted they should be made plainly evident. One method is to inscribe tiny circles about the points with a bow-pen.

Different scales may be used on the same chart for different curves, or one scale may be used on one portion of the diagram and different ones on other parts. See Fig. 13, Chapter XI.

The following is from an excellent article by Stanley C. Tarrant, published in Gas Age, January 1, 1917. While Mr. Tarrant's remarks and illustrations pertain specifically to gas company statistics they are applicable to other industries just as well and the general principles outlined and the suggestions contained in the examples are well worth a careful reading.

Accurate comparative statistics are always of value to those responsible for the policies of a gas company, but when it comes to driving the facts home, the manner in which they are presented is of as much importance as the facts themselves-a proper presentation enhances their value.

Graphic methods convey to the mind a more comprehensive grasp of essential features than do written reports, because one can naturally gather interesting details from a picture in far less time than from a written description. Further than this, the examination of a picture. allows one to make deductions of his own, while in the case of a written description the reader must, to a great degree, accept the conclusions of the author.

Many people imagine that graphic charts cannot be understood except by expert mathematicians who have devoted years of study to the subject. This is a mistaken idea, and if instead of passing over charts as if they were something beyond their comprehension more people would make an effort to read them, much valuable time would be saved. It is true that some charts covering technical data are difficult even for an expert mathematician to understand, but this is more often the fault of the person preparing the charts than of the system.

When it is more generally realized that charts are not mathematical problems and that data can be presented in the most concise manner when they are used, their use will become more general, and considerable time which is now lost in the study of complicated figured reports will be saved.

A study of charts and chart-making should be made by every gas clerk who desires to be rated first class, because the use of charts is being adopted more and more every year and the time is not far dis- 
tant when a knowledge of graphic methods will be required of clerks taking charge of important comparative reports. Charts are not difficult to prepare, and a little patience and practice will bring forth surprising results, not only in neatness of drafting, but also in originality of plan.

The different combinations of charts that ean be made are almost. unlimited, and every gas man, no matter what his position may be, will be able to find use for them. If this paper does no more than start. the gas man thinking graphic methods its purpose will have been served, for the thought is father to the deed, and once adopted as a part of the record system there is no question that the graphic chart will remain and become of more constant use, as familiarity with it proves its increasing value.

Unlike the system of accounting used by most gas companies, graphic methods of presenting data have not been standardized, and if several persons are given the same data to chart the probabilities are that each will go about it in a different manner. Although the same data can usually be charted correctly in several different ways, there is only one best way, and before preparing charts the gas man should carefully consider which particular form of chart will bring out the desired points the most graphically.

In order to illustrate a few of the many different ways in which the same data ean be shown graphically, several different styles of charts have been applied to the following simple report:

\section{REPORT OF CONSUMERS CONNECTED-1915-1914}

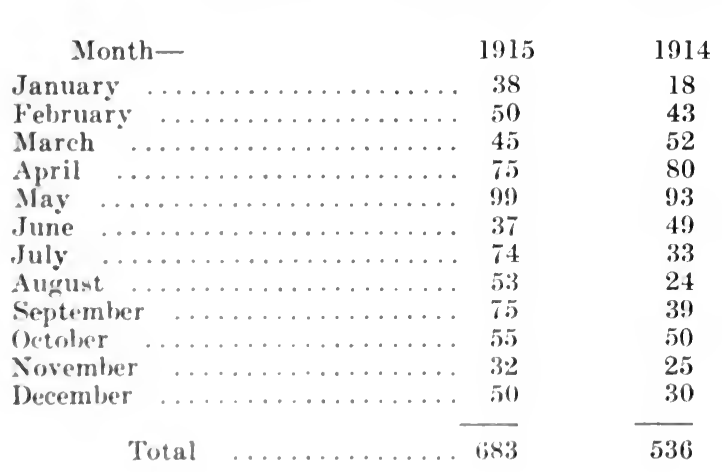

\begin{tabular}{cc}
\multicolumn{2}{c}{ Variation } \\
Increase & Decrease \\
20 & $\cdots$ \\
7 & $\ddot{7}$ \\
$\cdots$ & 5 \\
3 & $\cdots$ \\
41 & $\cdots$ \\
29 & $\cdots$ \\
36 & $\cdots$ \\
5 & $\cdots$ \\
7 & $\cdots$ \\
20 & 24
\end{tabular}

A horizontal-bar methed of charting is shown in Fig. 2 in which the scale for one year runs in the opposite direction to that for the other.

This form of chart makes a very spectacular showing, but is not the best form to use when it is desired to make a comparison of one year with the other. In order to draw such a comparison it is necessary for the eye to look to the right and left at the same time, and such comparison ean only be obtained when the chart is drawn to so small a scale that the ents of the bars for both years can be seen when looking straight down on the chart. When, however, each year is studied 
separately, a fairly clear picture of the monthly fluctuations is shown by a chart of this kind.

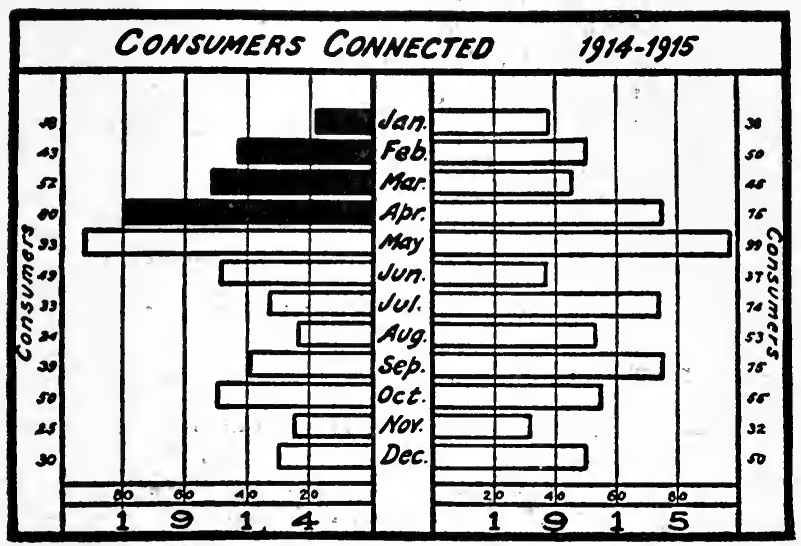

... Fig. 2-Horizontal Bar Method

The best form of horizontal-bar chart to use when monthly comparisons are sought is shown in Fig. 3. On this chart the bar for each

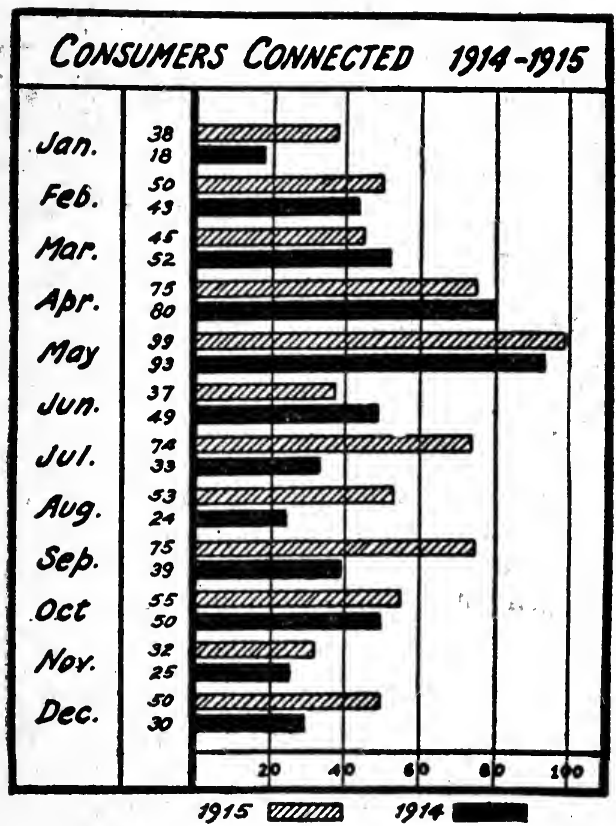

Fig. 3-Paired Horizontal Bars

month of the current year is shown over the bar for the same month of the previous year, thus rendering possible a very accurate and rapid. 
comparison. It will be noticed, however, that this form of chart does not show as graphically as does Fig. 2 the general trend of affairs for each individual year. In the illustration the bars for the different years have been designated by varying the shading, but in praetice it is better to use different colored inks-say, black for 1915 and green for 1914 , as by so doing the bars for the two years will stand out in greater contrast than in the illustration. The comparisons on a chart of this kind need not be confined to two years, as it is possible to make comparison of as many years as space and the number of different eolored inks available will allow. It must be borne in mind, however, that with every additional year the chart becomes more complex and the difficulty of studying the general trend of affairs for any individual year becomes greater.

A vertieal-bar chart is shown in Fig. 4 , and is in some ways prefer-

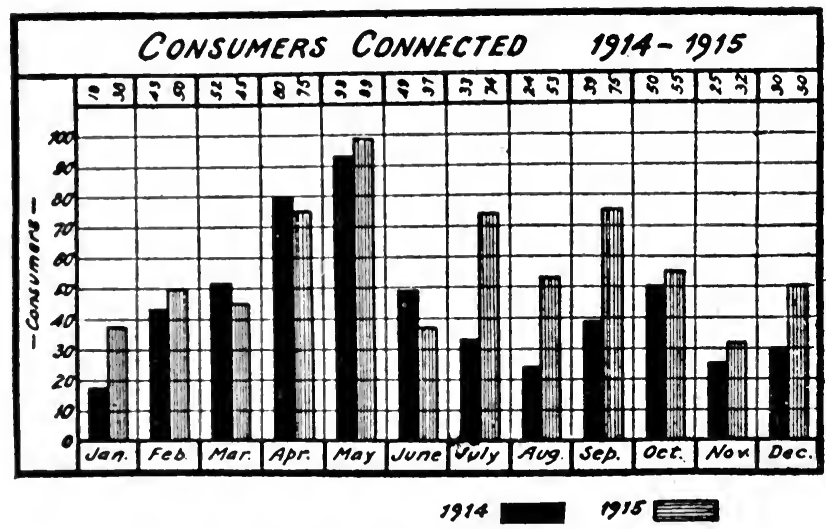

Fig. 4-Vertical Bars Arranged in Pairs

able to the horizontal-bar method. With this form of ehart a better idea of general ennditions during each year ean be obtained, as with a vertical arrangement of the bars an up-and-down effeet is obtained, thus making the monthly fluetuations stand out plainly. By turning Fig. 2 sideways, so that the bars for 1915 are in a vertical position, it will be seen that a far better idea of the general trend of affairs is obtained than when the hars are horizontal. It will be noticed that the two bars for each month in Fig. 4 are joined together, the only advantage of this arrangement being saving of space and a little less drafting.

The bar method of charting is perhaps the most popular form of graphic presentation, and for that reason most widely understood by the general publie. Owing to this fact it will be found a very excellent mothorl for display or advertising purposes, but it has little to recommend it for aceneral application to the records of a gas eompany. It requires more drafting, uses more space, and cannot be so generally applied as other forms of graphice charts.

An excellent method of charting the cumulative growth where it is desired to make a comparison between two years is shown in Fig. 5 . 
This chart shows very graphically that there were not a great many -more consumers added during the first six months of 1915 than during the same period of 1914, and that the increase ran along steadily in about the same proportion. From July to the end of the year the 1915 curve branches away from the 1914 curve, indicating that most of the comparative increase of consumers added during 1915 occurred in these months. In the illustration a dotted line has been used to indicate the curve representing the year 1914, but it is better to use different colored inks when more than one curve appears on the same chart. It is believed that there is no possible combination of figures that will convey as much information to the mind in a given time as a curve of this kind.

Although the chart in Fig. 5 gives a very excellent comparison of the cumulative growth, it does not show very plainly the fluctuations

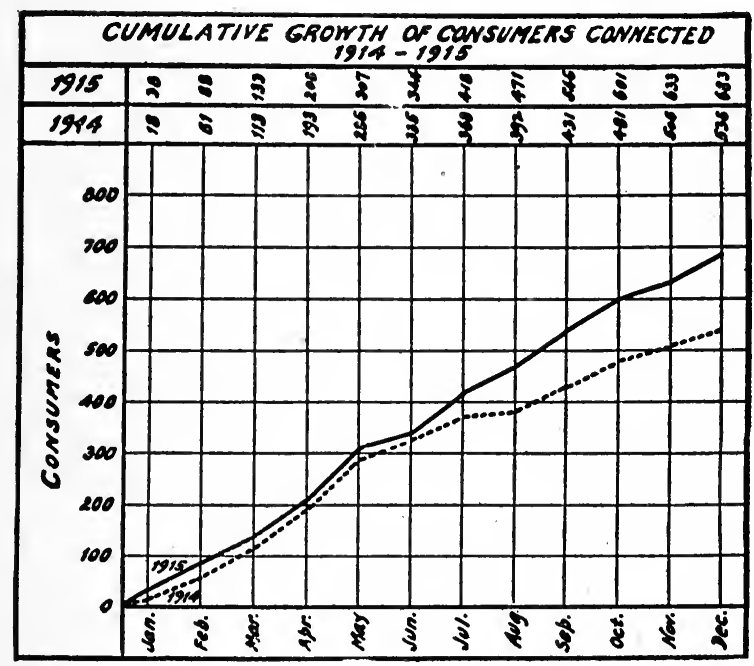

Fig. 5-Cumulative Growth Curve

from month to month. This comparison can be better obtained from a chart similar to those shown in Figs. 6 and 7 .

The flat top form of chart shown in Fig. 6 has been much used in railroad accounting, and although it shows the fluctuations in a graphic manrer, it is fast falling into disuse owing to its several objectionable features. In the first place, it requires more drafting than the chart shown in Fig. 7; there being three lines to draw for every point instead of two. Another disadvantage is when two or more curves intersect one another on the same sheet, as shown in Fig. 6. It will be noticed that the vertical lines must coincide, and it requires considerable care to draw one curve over another so that the vertical lines do not run into each other. The horizontal lines will cause a like trouble whenever it happens that the figure to be plotted for a month of the current year is exactly the same as that for the same month of the previous year. Apart from disadvantages in drafting, this form of chart is not so 
convenient for comparative purposes as the peak top chart, as the reader may prove to his own satisfaction by making a comparison of Figs. 6 and 7.

The peak top method, Fig. 7, is a typical example of the form of chart which can be the most generally applied to the records of a gas

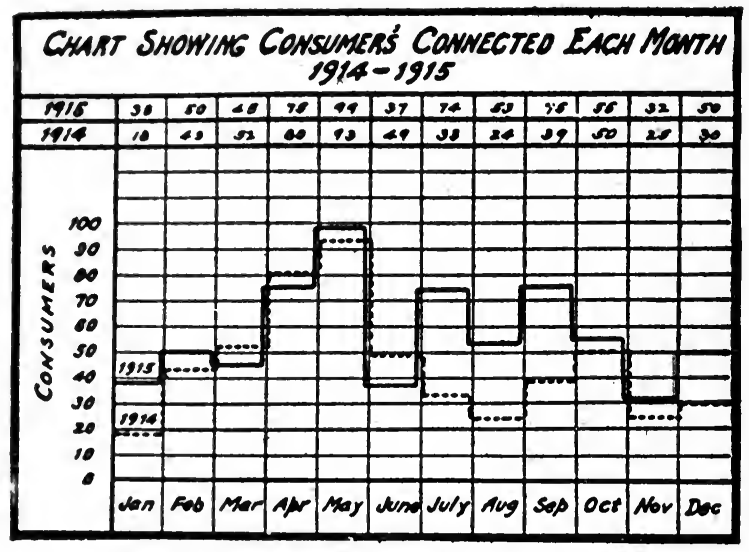

Fig. 6-Stepped or Flat Top Chart

company. Although the bar method of charting has many advantages for display and advertising purposes, the peak top curve is far better for every-day use, and should be used in preference to any other form of ehart when practicable. It gives a more understandable picture of conditions and also has the advantage of simplicity in drafting.

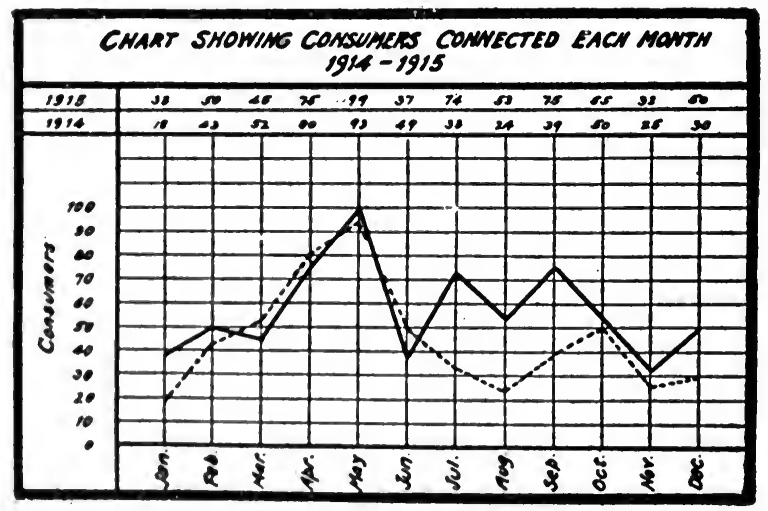

Fig. 7-Peak Top Curve

Having shown a few of the many forms of eharts that can be used, we will now turn our attention to a few practical applications to the gas business.

Fig. 8 shows a method of charting the gas made each month for com. parison with the previous year, the previous year's results being shown 
by a dotted curve line. Note that actual figures for each point on the 1916 curve are shown at the top of the chart, thus combining the graphic

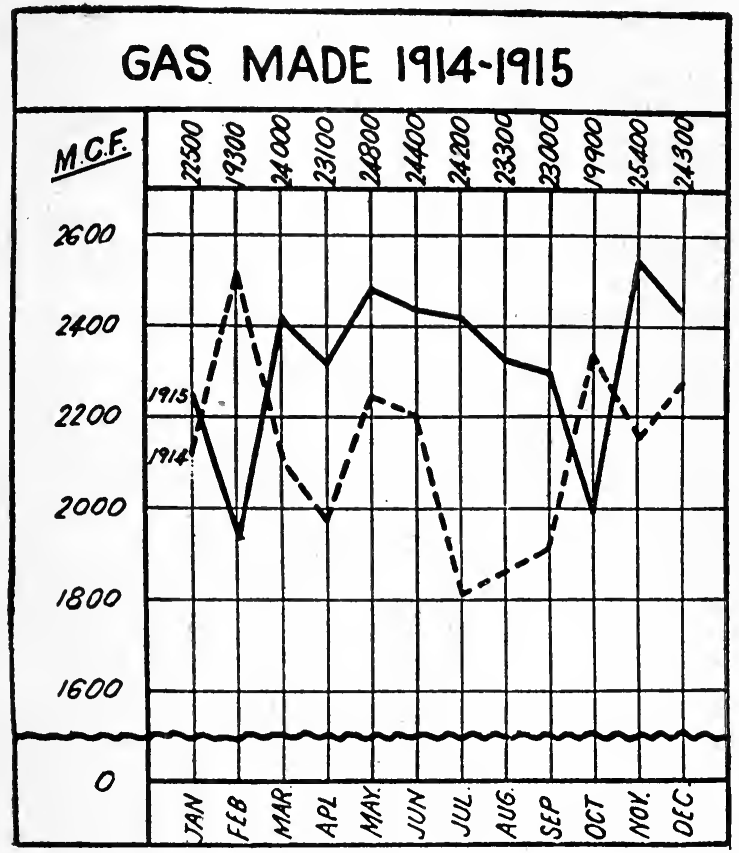

Fig. 8-Actual Figures and Abbreviated Chart

value of the chart and the accuracy of the manufacturing record book. Figures for the 1915 curve can be shown in a similar manner if so desired.

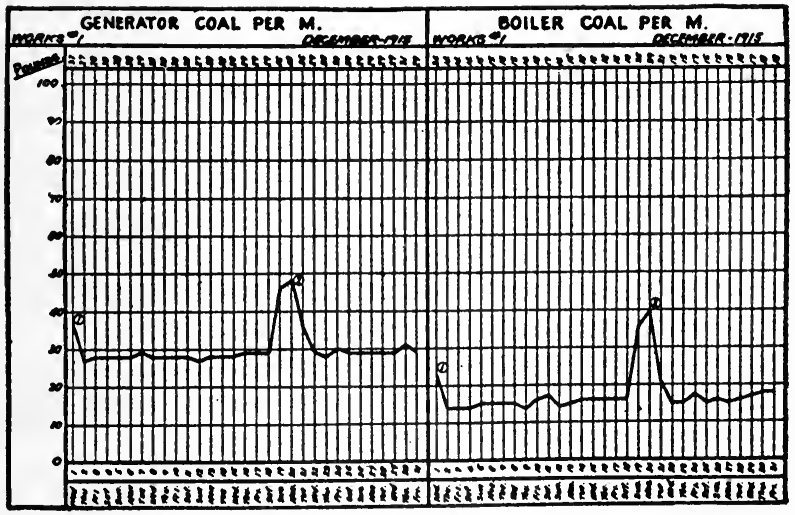

Fig. 9-Showing Abnormal Conditions

Fig. 9 shows a chart covering the quantity of generator and boiler coal used each day, per $1,000 \mathrm{cu}$. ft. of gas made. It is interesting to 
note how vividly a chart of this kind shows unusual conditions. It will be seen that on the 1st, 19th and 20th of the month the amount of fuel used was very much higher than usual. When unusual points of this kind oceur on a chart, notes of explanation should be written on the back of the chart, and should be indexed on the curve by means. of a small number in a circle, as shown in the illustration.

It will be seen that the word "pounds" at the top of the scale column has been printed at an angle. This is the best method to use when it is desired to indicate that the figures in the vertical scale column and those in the horizontal column at the top of the sheet, as well, represent pounds.

Fig. 10 shows a graphic cost report covering two years by months.

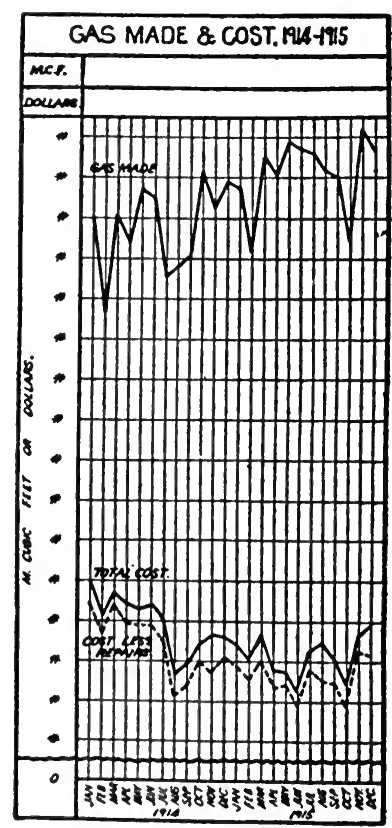

Fig. 10-Comparison of Cost and Production

The curves are all plotted from the same scale which can be read in $1,100()$ "u. ft. on the "gas male" eurve, or in dollars on the "cost" "urve A rhart of this kind gives a very graphic picture of the general tronl of affairs, as with the scalc arrangement used the comparative curves are shown in their true proportion.

$A$ study of the illustration will show an unusual condition. While the trend of the "gas mate" curve is upward, that of the "cost" curve is downward. This unusual condition has been purposely introducerd to show how clearly it stands ont on a chart. It could be caused by a substantial reduction in the unit price of an item of manufacturingmaterial of which a large quantity is used.

A graphie method of analyzing production costs month by month is. 
shown in Fig. 11. This chart shows very graphically the items responsible for variations in total cost. The illustration is of course very much reduced in size, but is sufficiently clear to give the idea of how such a chart can be arranged.

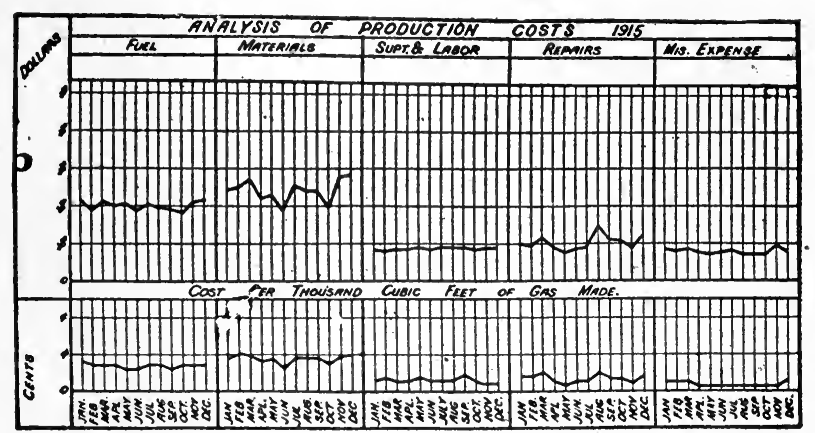

Fig. 11-Comparison of Cost Items

Fig. 12 shows a block method of comparing the percentage of varia. tion in gas made and cost.

Fig. 13 shows a chart which can be conveniently used for keeping track of gas unaccounted for month by month. The dotted curve linc shows the results for the previous year.

A method of keeping track of growth in mains is shown in Fig. 14.

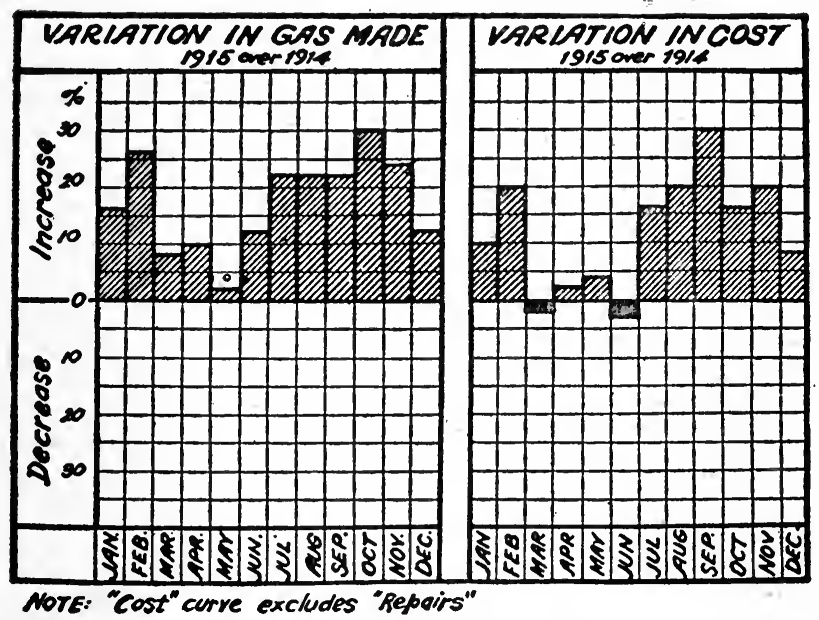

Fig. 12-The Increase-Decrease Chart

"This chart shows the monthly fluctuations and also the cumulative growth.

An interesting chart showing the growth of appliances as compared with the growth of meters during seven years is shown in Fig. 15. Note 
that in 1915 there are nearly as many applianees as meters, indicatinga very efficient appliance department.

Fig. 16 shows three forms of growth charts, the block, step and peak

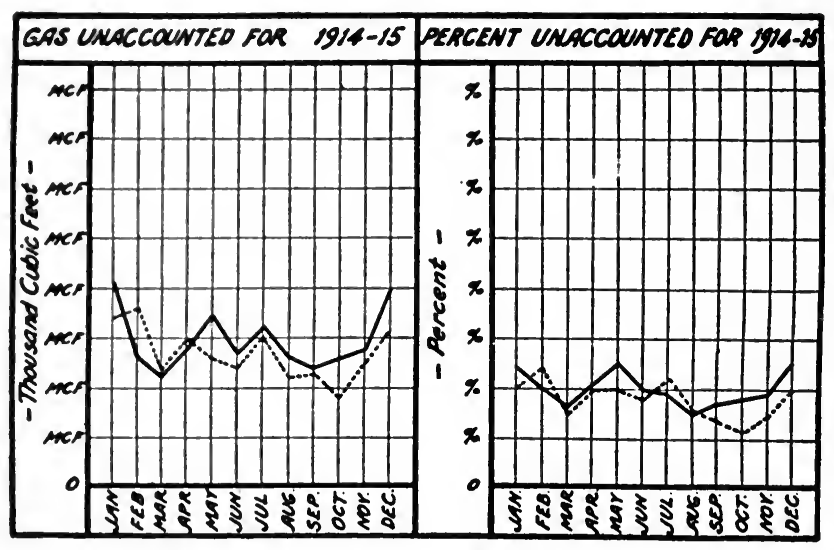

Fig. 13-Comparison with Previous Year

top eurve. It will be observed that chart " $\mathrm{C}$ " gives the most graphic: results, and requires less drafting than "A" or " $\mathrm{B}$ ".
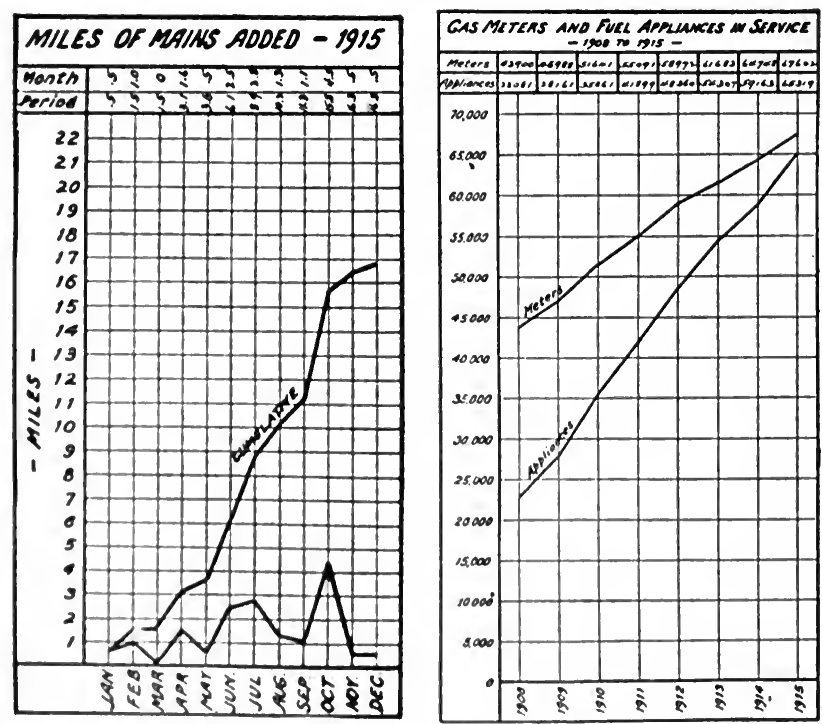

Figs. 14 and 15-Growth of Mains and Appliances

Another chart of intrest to the commercial gas man is shown in Fig. 17. It shows graphically the growth in the use of appliances. The heavy line shows the per cent of the consumers using appliances, while: 
the dotted line shows the per cent of population using appliances. This chart was made from the actual statisties of a certain company.

Fig. 18 shows a useful chart for advertising purposes. Such a chart can be readily printed on the back of the monthly bills, or can be used
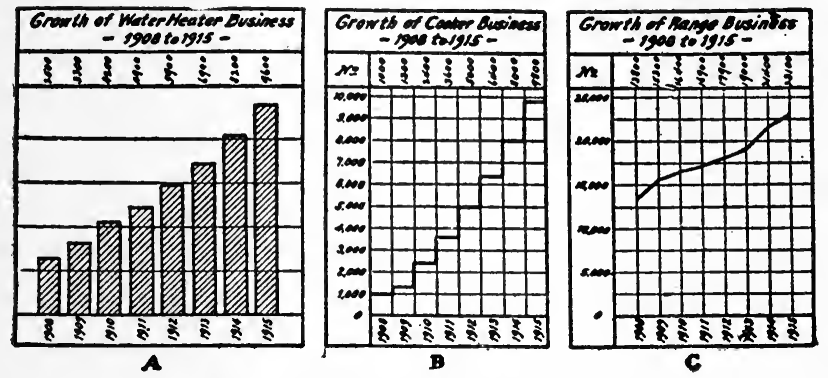

Fig. 16-Forms of Growth Charts

for newspaper advertising. The chart is very simple to understand, and may help to convince the complaining type of consumer that he is not paying too much for gas.

Before closing this paper it may be well to eall attention to a few simple rules that should be observed when preparing charts.

When preparing charts the foremost aim of the designer should be

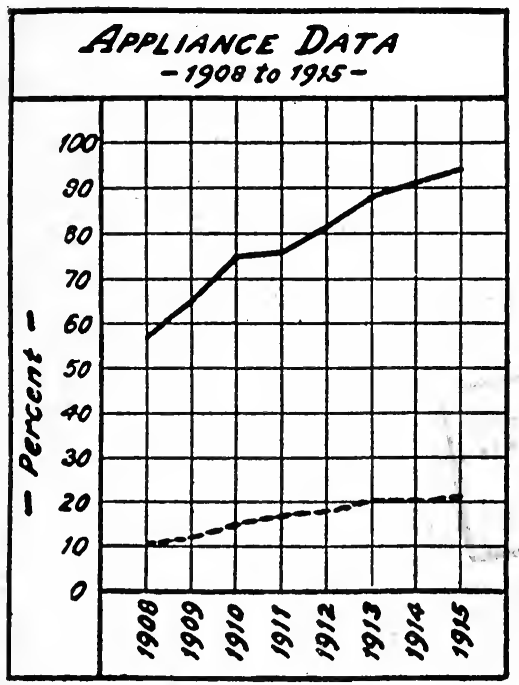

Fig. 17-Percentage of Consumers and Population

toward uniformity of method. It is very annoying to have to study a series of charts in which uniform methods have not been used, where, for example, the vertical scale of one chart is on the left, while on a second it is on the right hand side, or where the horizontal scale runs 
across the top of the sheet on one chart, and across the bottom on another. These details are small in themselves, but uniformity in the nethod of preparing them adds greatly to the value of the charts as reference records, and is very desirable.

All charts should be given a comprehensive title, that there may be no doubt as to what data the chart is intended to cover. This is especially neeessary in comnection with separate exhibits used for illustrating statements in spceial reports, as when the charts are not given distinct titles they are valueless as records when detached from the reports.

The vertical scale on all charts should be arranged on the left hand side, as the reader will find this most convenient when studying the

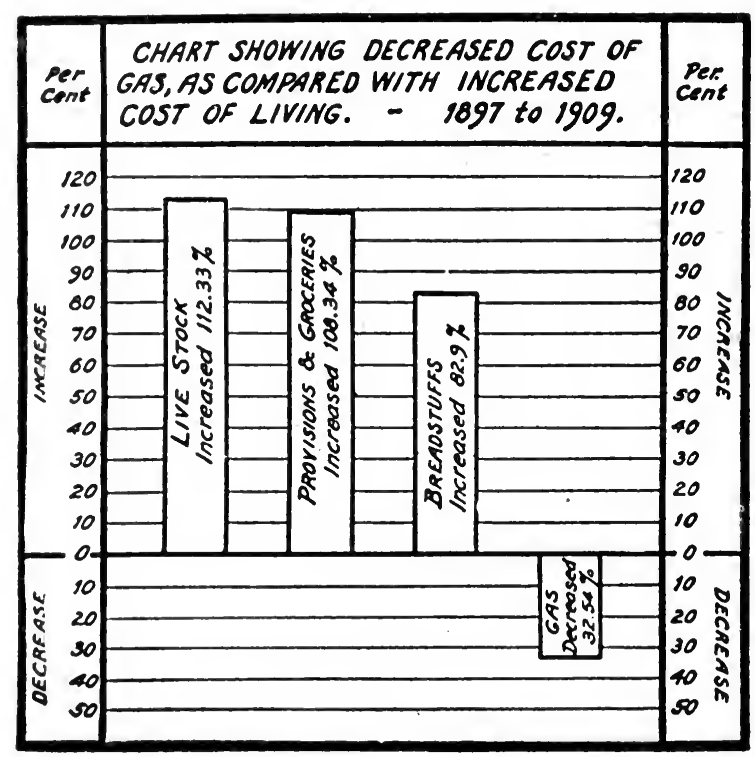

Fig. 18-Favorable Publicity Chart

"hart. In graduating this scale, considerable thought and care are required, as a badly arranged seale often means that the resulting eurve will give a distorted picture of eonditions.

The zero of the seale should appear on every chart, and should be shown by a heary line carried across the sheet. If this is not done the realer may assime the bottom of the sheet to be zero and so be misled. The suale should be graduated from zero to a little over the maximum figure to he plotted on the charts, so that there will be a space between the highes peak on the enrve and the top of the chart.

When draling with very large quantities it is not always practicable to use a seale which starts at zero, and is carried up by even steps to a figure representing the highest peak on the curve. Such a chart would either be ton large for envenient handling, or else the scale would have to be endensed so that only very large fluctuations would be indieated on the curve. In a case of this kind the best practice is to start the 
scale at zero, and just above this point draw a wavy line across the sheet to indicate that the scale is broken at this point. This line can be very easily drawn with an ordinary serrated edge ruler as used by many accountants. The scale starts again on the upper side of the wavy line at a figure a little lower than the lowest point on the curve, and is carried up by even steps to a figure a little above the highest point to be shown on the curve.

On many charts it is not necessary for the curves to show a fluctuation of less than 5 or $10 \%$, and it is sometimes more preferable to make smaller charts on which the curves will only indicate fairly large fluctuations, than to make very large charts on which the curves can

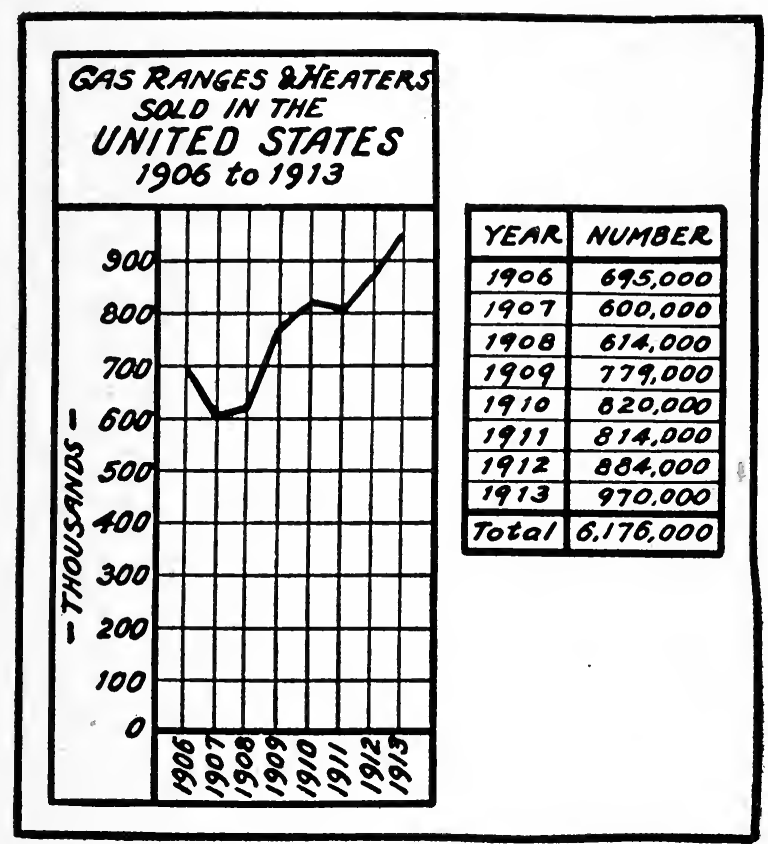

Fig. 19-Separate Table of Actual Figures

be read on the scale within $1 \%$. This is a matter which should receive careful consideration before a chart is drawn, as very large charts are inconvenient to handle and, owing to this fact, are sometimes not given the attention they deserve. On every chart the numeral value of each point on the curve should be shown at the top of the sheet, as this avoids the necessity of estimating from the scale when numeral values are required. In cases where the numeral value cannot be shown conveniently at the top of the chart, a separate statement should be made on the same sheet, as shown in Fig. 19. The curve will show the general trend of affairs, and if the executive wants exact figures they are available on the same sheet, thus combining a graphic and a written record. The figures have to be gathered for plotting, and it represents very 
little additional work to write them across the top or to list them by the side of the chart.

The practice of drawing several curves on the same sheet is not to be commended except in eases where the curves will not intersect. A crowded chart on which the eurves frequently intersect resembles a Chinese puzzle more than a graphic record, and a report submitted in figures is to be preferred to a chart of this kind. Even when the curves do not intersect, they should be made in different colors in order that they may be readily distinguished, one from the other. It should be borne in mind that charts are sometimes studied under artificial light and for this reason it is advisable to use colors that will stand out distinctly by virtue of contrast. For example, lines drawn in blue or violet ink appear, under artificial light, very much the same as lines drawn in black ink, therefore these colors should never be used on the same chart. On the other hand, a chart carrying black, red, green and yellow eurves can be very easily read under any form of light. In cases where it may be necessary to draw more than four curves on the same chart, it is better to distinguish the curves by using lines of various forms than to use a colored ink that will not be a distinct contrast to the other colors on the chart. Thus, with four colors available, it is possible to draw eight distinctive curves by making two curves of the same color, one being shown by an unbroken line, the other by a dotted line.

In using different colored inks to show different items, or the same item for different years, it is a good plan to standardize the colors so that the same item or year on all charts is shown in the same color. This arrangement will avoid much confusion when constant reference has to be made to a large number of different charts.

When designating on a chart what items the different colored curves represent, it is not sufficient to make a key showing that black represents one item and red another. In all cases where more than one item appears on the same chart, whether different colored inks are used or otherwise, each curve should have its title plainly printed over it near the scale column. Those who have had experience in reading charts will know how annoying it is to have to consult a key in order to learn what a certain curve is intended to represent.

Wherever unusual peaks or valleys occur on a curve it is a good plan to mark these points with a small figure inside a circle. This figure should refer to a note on the back of the chart explaining the reason for the unusual condition. It is not always sufficient to show that a certain item is musually high or low ; the executive will want to know why it is that way. An efficient chart elerk will not wait to be questioned about unusual points on his eurves, but will endeavor to find and note the reason for them before the chart is submitted to his superior.

When (harts are to be prepared for illustrating printed reports, or for advertising purposes, consideration must be given to the fact that although the cosordinate lines on the original chart may be blue, green or red, they will be black when reproduced. Therefore, unless a very heavy eurve line is 1 sed, there will be insufficient contrast between the curve and the co-ordinate lines. The best plan to follow when prepar- 
ing charts from which euts are to be made is to make the original on co-ordinate paper and from this original make a tracing, showing only those lines that are absolutely necessary as guides. The curve can be plotted more accurately on co-ordinate paper, but the finished chart stands out more clearly in print when the cut is made from a tracing. This method has been used in preparing all the illustrations accompanying this paper.

At the invitation of the American Society of Mechanical Engineers, a number of national associations have appointed representatives to serve on a joint committee on "Standards for Graphic Presentation." This committee is making a study of the methods used for presenting data in graphic form. The value of this work cannot be overestimated, for, if simple and convenient standards are adopted and made generally known, it will result in a more universal use of graphic methods.

Following are the Applicable Principles of Elementary Graphic Presentation from the Preliminary Report of the Joint Committee on Standards for Graphic Presentation, of which Mr. W. C. Brinton was Chairman, mentioned by Mr. Tarrant in the last paragraph.

1. The general arrangement of a diagram should proceed from left to right.

2. Where possible represent quantities by linear magnitude as areas or volumes are more likely to be misinterpreted.

3. For a curve the vertical scale, whenever practicable, should be so selected that the zero line will appear in the diagram.

4. If the zero line of the vertical seale will not normally appear in the curve diagram, the zero line should be shown by the use of a horizontal break in the diagram.

5. The zero lines of the scales for a curve should be sharply distinguished from the other co-ordinate lines.

6 . For curves having a scale representing percentages, it is usually desirable to emphasize in some distinctive way the $100 \%$ line or other line used as a basis of comparison.

7. When the scale of the diagram refers to dates, and the period represented is not a complete unit, it is better not to emphasize the first and last ordinates, since such a diagram does not represent the begining and end of time.

8. When curves are drawn on logarithmic co-ordinates, the limiting lines of the diagram should each be at some power of 10 on the logarithmic scale.

9. It is advisable not to show any more co-ordinate lines than necessary to guide the eye in reading the diagram.

10. The curve lines of a diagram should be sharply distinguished from the ruling.

11. In curves representing a series of observations, it is advisable whenever possible, to indicate clearly on the diagram all the points representing the separate observations.

12. The horizontal scale for curves should usually read from left to right and the vertical scale from bottom to top.

13. Figures for the scale of a diagram should be placed at the left and at the bottom or along the respective axes. 
14. It is often desirable to include in the diagram the numerical data or formulae represented.

15. If numerical data are not included in the diagram it is desirable: to give the data in tabular form accompanying the diagram.

16. All lettering and all figures in a diagram should be placed so as to be easily read from the base as the bottom, or from the right hand edge of the diagram as the bottom.

17. The title of a diagram should be made as elear and complete as. possible. Sub-titles or descriptions should be added if necessary to insure clearness.

To illustrate Prineiples 3 and 4 the following abstract from an article by John Wenzel, "Graphic Charts That Mislead," 'Scientific American Supplement, June 16, 1917, has been made.

During the last few years there has been a marked tendency towards greater correctness in the form and style of diagrams which have appeared in books, magazines and papers, but many have not conformed to the accepted standards for the making of graphic charts, and are positively misleading.

That the zero line should be shown whenever possible, is a fundamental rule that has been laid down for the making of graphic charts, and yet this rule is frequently broken by those who are engaged in the graphic presentation of facts. Cutting off all the horizontal rulings below the curve line. as is often done, may save space, and the cost of the plate may be reduced if the chart is intended for publication, but by taking away the zero line the correct interpretation of the chart is hindered, and impressions may be ereated which are misleading, Failure to understand the true significance of the curve line is undoubtedly responsible for the frequent omission of the zero line. If it is desired to show graphically the population of the United States by decades during the last 50 years,

\begin{tabular}{|c|c|c|}
\hline Yea & & \\
\hline & & \\
\hline & & 8,371 \\
\hline & & 50 \\
\hline 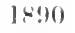 & $\ldots \ldots \ldots \ldots \ldots$ & \\
\hline & 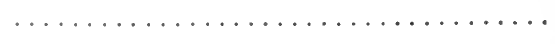 & $75,994,575$ \\
\hline & & $91,972,266$ \\
\hline
\end{tabular}

one of the simplest methods is to use vertical bars as in Fig. 20, the heights of the respective columns representing the population at different periods acoording to the scale at the left. As the eye travels along the tops of these columns, it unconsciously judges their relative heights and constructs an imaginary eurve which coincides with the tops of the columis.

Insteal of a heavy black column representing the population of each decade, a line oould be used, the tops of these lines being joined with a curve, Fig. 21, which by visualizing the population for each respective decade, helps the mind to grasp at a single glance the comparative increase of population.

The next step in the development of the curve line is shown in Fig. 22. Ilere upon a field with both vertical and horizontal rulings, points marked with an $X$, representing the population of each successive ten- 
year period, are located on the vertical rulings according to the scale at the left and correspond with the heights of the respective columns and lines in Figs. 20 and 21. By joining these points the curve line becomes the most prominent feature of the diagram.

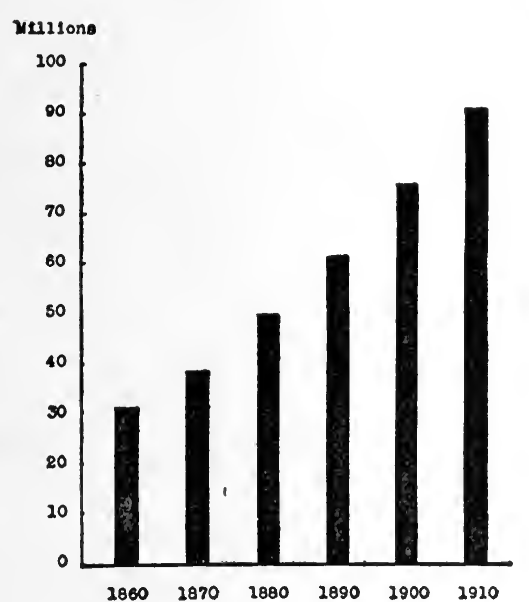

Fig. 20

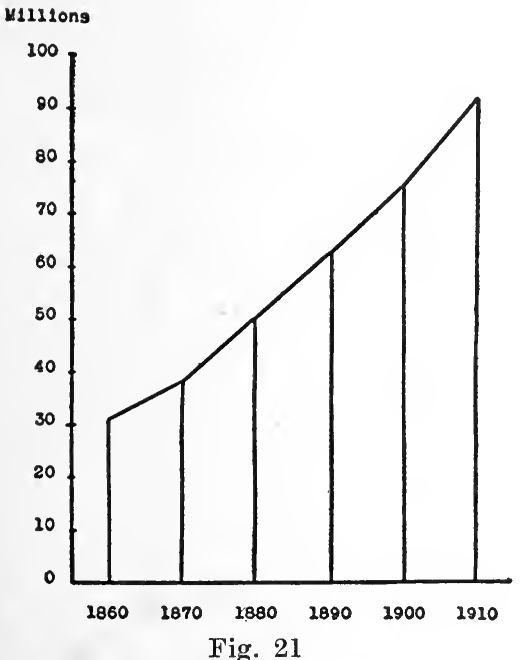

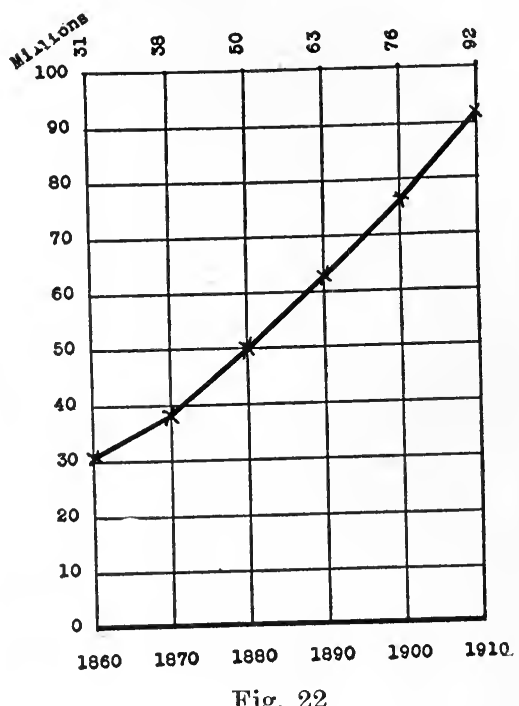

Fig. 22

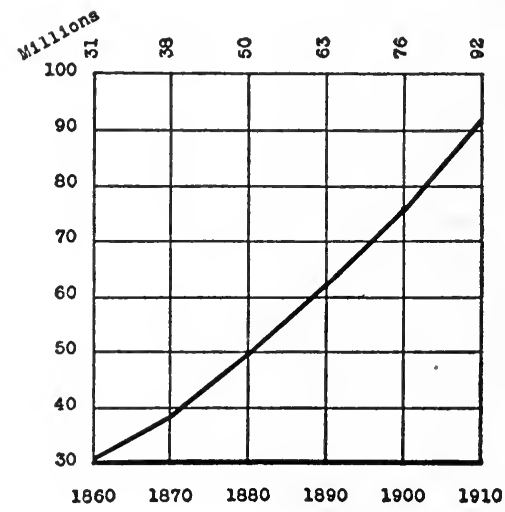

Fig. 23

As the population in 1860 was $31,000,000$ and in $1910,92,000,000$ the former was approximately one-third of the latter. Measurement shows the distance from $\mathrm{X}$ to the zero line in 1860 to be very nearly one-third of the distance from $\mathrm{X}$ to the zero line on the 1910 ruling. The ratio 
expressed mathematically is approximately $31: 92:: 1: 3$. The length of the bars in Fig. 20 and the vertical lines in Fig. 21 are relatively correet and convey to the reader a truthful impression of the number of persons who were living in the United States at the times when the census enumerations were made. Likewise the distance from the zero line to the respective $\mathrm{X}$ 's gives a correct presentation of the actual population, and thus shows the relative growth from decade to decade.

If in Fig. 22 the three lowest horizontal rulings were omitted and the thirty million line were used as the base line as is shown in Fig. 23, the curve would be identical with that in Fig. 22, but there would be no accurate means of eomparing quickly the 1860 population unless an imaginary zero line were constructed as far below the thirty line as the thirty line is below the sixty line, as in Fig. 24. Thirty millions have been eut off of each column thereby practically destroying the measure for comparing accurately the population of one decade with that of another, for few people will take the trouble to construct an imaginary zero line as in Fig. 24.

Fig. 23 has been mutilated and distorted as much as Fig. 20 would be, were 30,000,000 cut from the bottom of each column, making Fig. 20 look like Fig. 25. Returning to the proportion $31: 92:: 1: 3$; if thirty is subtracted from the first two numbers, the result obtained 1:62: $: 1: 3$, is incorrect, the last figure being almost twenty times too small. These figures emphasize the importance of the zero line and give conclusive proof of its necessity for the correct interpretation of a graphic chart.

It seems hardly credible that a financial house which sells industrial securities should wilfully misrepresent the sales of one of the companies whose stock it was trying to sell. Nevertheless, if anyone saw the diagram shown in Fig. 26, he would be given an entirely erroneous impression of the difference between sales for January, 1913, as shown in the left-hand column and the sales of October, 1916, as represented by the column on the extreme right.

Only after referring to the scale at the left will it be seen that instead of starting at zero, the seale begins at 130 millions and ends with 240 millions so that the short column at the left which is about one one-hundredth as long as the column at the extreme right, really stands for 1:31 millions or more than half as much as the column at the right which stands for 238 millions.

Although the volume of sales for each quarter appears in figures at the top of each column the reader may get the real facts only by a most carpul examination of the scale and the figures for each quarter. The real function of the graphic chart is to present facts so that the whole story may be fold at a glance, and a hasty glance at this diagram would hardly fail to ereate a wrong impression.

In Fig. $2 \frac{7}{6}$ the diagram has been redrawn so as to show the correct method of presenting the facts. In this diagram the proper relationship betwen the sales of Jamuary, 1913, and Oetober, 1916, is shown and the impression enveyed is very different from what Fig. 26 would have you believe. In Fin. 27 the seale has been made about half as large so that the $t$ wo diacrams might be approximately the same size. In this the base line begins at zero instead of at 130 millions. The bar representing the January, 1913, sales is more than half as long as the bar 


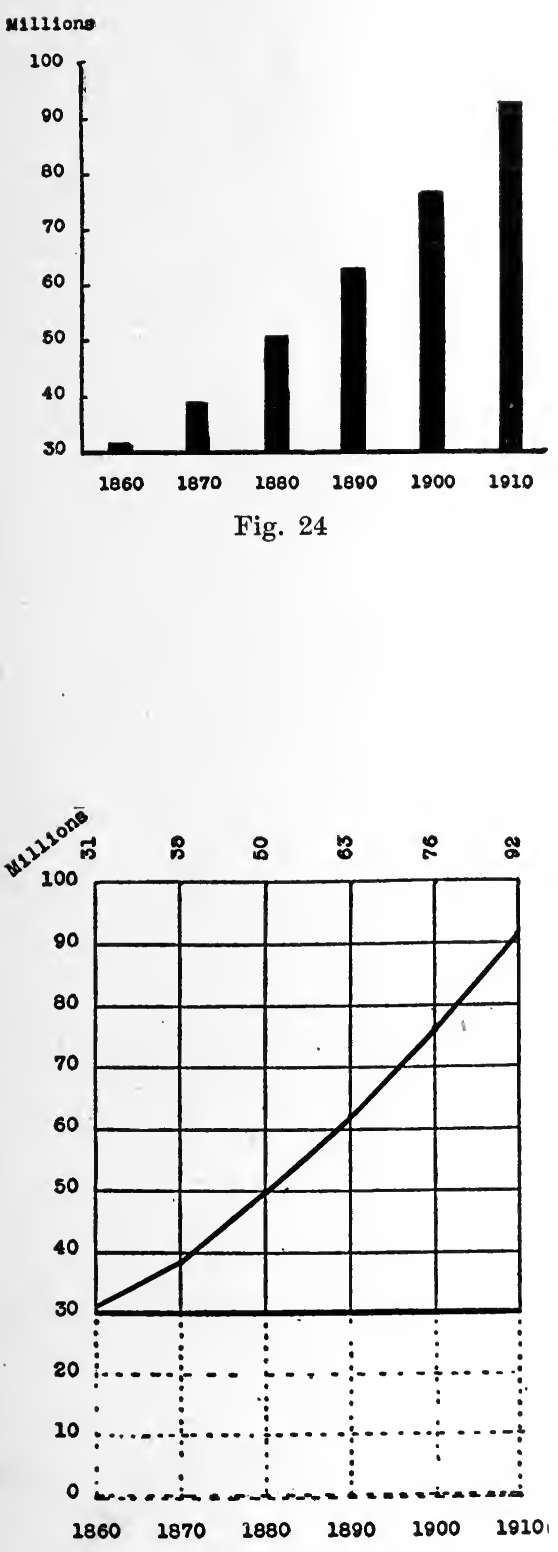

Fig. 25

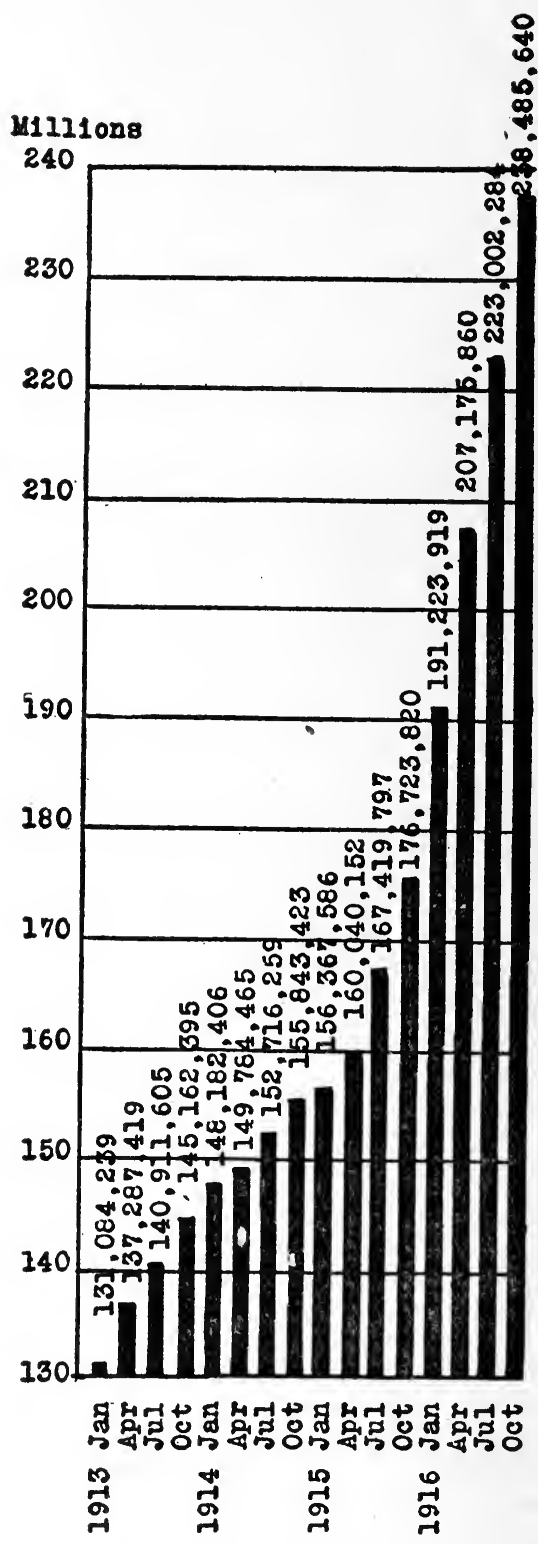

Fig. 26 


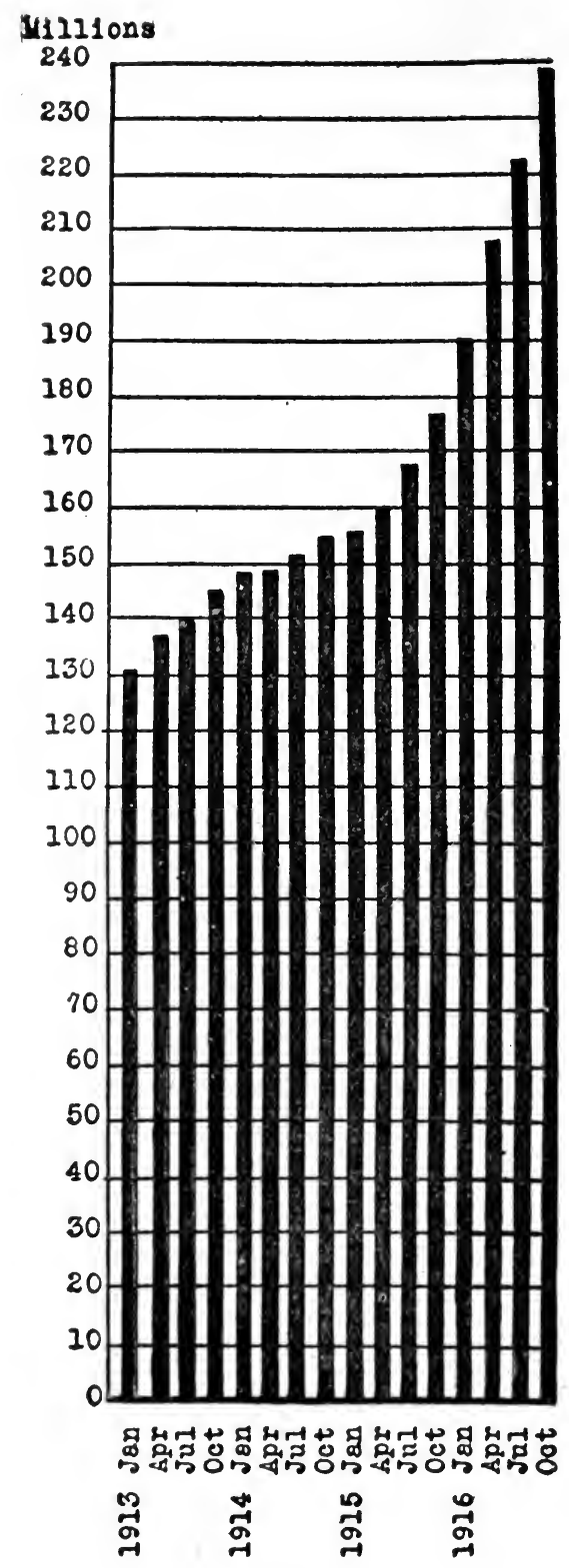

Fig. 27 
representing the October, 1916, sales which conforms with the actual facts. At first glance the growth as shown in Fig. 27 does not appear to be so great as it appeared to be in Fig. 26, and yet both diagrams present graphically exactly the same data. Fig. 26 shows it in a way which, if not intended to mislead, is likely to do so unless examined with great care. Instead of presenting the real facts so they can be easily and instantly understood, this diagram shows them in a way very likely to be misinterpreted and this is far from the proper function of the graphic chart.

If it will be borne in mind that it is the distance from the top of the bar or from the curve to the zero line that is really significant, then the zero line will be considered as important as the length of the bar or the curve itself, and in drawing a diagram a scale will be chosen that will permit the whole field to appear within the space allowed for the diagram.

It will be observed that, in a great many of the diagrams which follow, the lines are far apart. This is usually so because the diagrams are photographically reproduced from drawings and tracings, and, were the lines in the original too close together, the reproduction, usually considerably reduced in size, would be illegible. Generally speaking, when making charts the scale should not be any finer than is necessary to plot the data; i.e. if it were required to plot on a sheet variations from 0 to 200 to the nearest 5 points, it would not be necessary to use a paper 20 divisions per inch, but 5 divisions per inch would be the proper sheet, allowing each inch to represent 25 points; but if it were desired to plot the same variation to the nearest unit or less, 20 divisions per inch paper would be superior.

Where the $8 \frac{1}{2} \times 11 \mathrm{in}$. sheets are punched and filed in a loose-leaf book, if the charts are in constant use it is suggested that they be reinforced with small circular gummed cloth patches. These may be pasted, one over each hole in the paper, and will prevent it from tearing indefinitely.

When the typewriter is used to put titles, tabulations, etc., on charts a much clearer blue-print may be obtained if a piece of carbon paper is placed with the inked side next the back of the chart so that a double printing is obtained. 


\section{CHAPTER X}

\section{Organization and Management}

Management engineers find that as time goes on business concerns are less apt to wait until they are in difficulties before ealling for outside assistance. Not many years ago it was an unusual thing for the services of a management engineer to be requested until finaneial losses, either immediate or impending, demanded some reactionary course on the part of the concern affected. Now, however, the more progressive business and industrial people realize the value of managerial economies and spend large sums annually for the maintenance of staffs for this very branch.

The recults which these management departments aim to achieve are obtainable only from research. Research means records, and for the reeords to be valuable they must be thoroughly studied and analysed. Any method which facilitates and simplifies this analysis is certainly well worth while. The use of graphic eharts is one method which accomplishes this very thing and hence its importance. Charts are closely identified with the backbone of a business-the data which enable the manager to know-not to guess, or think, or hope-but to know what his business is doing and to predict with a very fair degree of precision what it is going to do. The following, from an editorial in Engineering and Contracting, Jan. 17, 1912, entitled "Systematic Research as a Principle of Managerial Economies," if read and heeded by all those who feel inclined to belittle the importance of such things, would prove well worth while.

Although it is evident that the object of all management should be to increase profits, both by increasing seales and by reducing unit costs, it has not been the general practice to investigate existing methods and machines systematically with a view to redueing unit costs. The Taylor scheme of timing the elements of operation of machines has merited all the praise that it has received. But it seems not to have been perceived by many of the exponents of "scientific management" that unit timing of processes is itself only one method of conducting systematic research for the purpose of devising more economic methods of production. Nor has it been generally recognized that unit cost keeping-which is a much older devien - is also but a method of eonducting a continuous research whose ultimate aim is the reduction of unit eosts.

Like many another principle whose merit is conceded, the prineiple of systematic researeh does not as yet receive a fraction of the attention that it deserves. The majority of business firms are small, and the manager of a small firm is apt to say: "Systematic researeh is all very well for the big business encern, but we, being small, have neither the money nor the time to devote to such things. It keeps us busy enough doing what we have to do now." 
In this argument we have one of the main reasons why most small concerns remain small. Unless, the manager of a small business takes the time himself or assigns someone else to study the problems of economic production and selling, he will always be extremely busy-busy trying to keep from going to the wall under the stress of competition. Systematic and continuous research is the secret of the rise of many a small firm into prominence. Such research usually begins with unit cost keeping and a careful study of the elements of cost with a view to cost reduction. Then it may be followed by the Taylor method of unit timing of operations. Then laboratory and field research may follow, leading to a number of small improvements, which, in the aggregate, however, add very substantially to the economy effected. Finally some radical improvement, so radical as to merit being called an important invention, gives the business a great forward impetus. The invention need not necessarily consist in a mechanical combination or in a chemical combination, for it may be a greatly improved process or product that is not even patentable.

Failure to succeed greatly in management usually occurs not so much from lack of knowledge of the important principles of the science of management as from failure to apply them. Most of the principles of successful management are old, and many of them have received sufficient publicity to be well known, but managers are curiously prone to look upon managerial success as a personal attribute that is slightly dependent on principles or laws. In fact, however, managerial success is but an evidence of skill in applying the laws of management. Systematic research involves: (1) Unit cost keeping and cost analysis; (2) unit timing of operations; (3) laboratory testing and experimenting; (4) systematic investigation and experimenting in shop and field under working conditions.

In every one of these four, the use of charts greatly helps to make the records easily understood, and to reduce the time required to analyze them.

The Essential Facts to Investigate. For any manager to utilize graphic methods for visualizing the vital facts of his business, in the first place it must be impressed upon his consciousness that the method will produce the results for him and then he must know how to get up a chart correctly, and last, but far from least, he must know what the essential facts of his business are. Charts, in themselves, mean little and like many another force for the accomplishment of good, if misdirected, may result unprofitably. The author has frequently been asked by some proud originator to look over a multitude of charts, excellent as productions of a draftsman's skill, but of no real value, in that the data they pictured were relatively unimportant. Dwight T. Farnham, in a series of three articles published in The Engineering Magazine, August, September and October, 1916, entitled "Visualizing the Essential Facts of a Business," "Scientific versus Intuitive Admin istration," and "How Graphic Control Facilitates the Fixing of Profits," points out what he considers to be the facts which the executive should receive in simple and effective form. From these the following has been quoted:

An exact knowledge of conditions, and consequent timely application 
of praise or of constructive criticism, is one of the chief forces of the executive in securing satisfactory results. Undeserved criticism is unjust and destroys initiative, while unmerited praise tends to render the executive ridiculous in the eyes of his subordinates; both are detrimental to discipline and weaken the power of the executive.

Cost figures may have historical interest but they are not worth onetenth the expense of assembling unless they aid actively in the administration of the business. The problem of the executive, then-once his organization is perfected-is to secure live data covering the exact conditions of the business at all times. These data should be arranged so as to give him all the facts, subordinated according to their relative bearing upon net earnings, and do so with the least demand upon his time. Furthermore, these facts must be so exhibited that the general laws underlying the business may be easily and aceurately deduced and standards of aecomplishment set which will be a continual incentive to greater accomplishment.

If a pieture can be arranged which will give him at a glance the exact state of his whole business, with detail subordinated in the order of its importance, so that the large and important things stand boldly in the foreground where they cannot be overlooked, and the less important facts, though present, receive attention only when necessary, the executive is in a position to grasp and to direct his whole business with an intelligence and sureness of tonch which insures his stockholders getting the fullest return on their investment.

A few years ago some of our more nrogressive corporations began showing the fluetuations of their sales in different districts by means of graphs. Later the same method was applied to costs, but it is only recently that anything like a comprehensive scheme eovering the whole business has been worked out and put into effeet. Within the last two years several large corporations have gone a step further. They have not only shown by means of graphs what they had done and were doing, but also what they proposed to do. And the most remarkable thing about these prophecies by graphs is the regularity and exactness with which the ideals aimed at have been realized.

It is absolutely necessary, first of all, to analyze existing eonditions. This is extremely difficult for the executive to do with any degree of preeision where the usual types of balance sheets and eost records are in use. When these are placed on his desk at the end of the month, he glances through them cursorily and if, in the light of what he happens to remember about past performances, the showing seems satisfactory, he drops the sheets and returns to his routine. A sudden drop in profits or a rise in costs presages a call on either the sales or the manufacturing department, or both, for an explanation. Usually these departments know what kind of an answer will satisfy the "old man." The ancient bogeys of eompetition or of interrupted production, are paraded forth and the "old man" groes back to his desk. This is all that happens unless a prolonged periof of low profits ensues. In this event the directors come in and hold a post mortem. After a critical review of the office rent, the amomint spent for postage and telegrams and the salaries of the stenowraphers (I have seen board meetings over exaetly this sort of trash), a period of retrenchment is considered vital. The 
office force is thereupon cut down and the department heads are deprived of their stenographers. A man here and a man there, on the "non-productive" side of the ledger, is dismissed. When they get through they don't know any more about the real cause of the falling off in business than they did before. Worst of all, the retrenchment has applied the axe, perhaps, to the only part of the organization which could by any chance tell them.

For such summary action, however, the financial managers cannot be wholly censured. The statistics prepared by only too many costkeeping departments are misleading rather than enlightening to the executive, and it may be the less of them the better. Masses of figures come to him each month which eannot possibly be remembered until the next month. As a result conditions each time have to be considered largely by themselves or as contrasted with some other single month. The very volume of the figures makes it a physical impossibility to lay out on a table and to compare the statisties for more than a few months at a time. It is not in this way that the laws underlying fluctuations in costs, in sales prices, or in profits can be deduced. Yet predictions as to future conditions are an impossibility unless these laws are known.

The present purpose, therefore, is to indicate by conerete illustrations just how the facts-and all the facts-over an extended period, may be brought before the executive at one time in the exact order of their importance. The methods to be deseribed here were first employed by the writer in executive work in a large corporation and have demonstrated their practicability over a number of years. The same methods have since been adopted by several other large and successful corporations in various parts of the country. For obvious reasons hypothetical figures only will be given and the discussion of underlying tendencies will be of a general nature.

Conditions are, of course, different in every business, but the underlying principles are identical in all. Were this not so, bankers would not be able day after day, by a few well put questions, to lay hold, as they are accustomed, of the essentials of countless business ventures.

Under the following heads the facts which an executive should have before him at all times in order to administer the business efficiently are given in skeleton form in the order of their greatest importance.

I-Dividends-It goes without saying that dividends-large, regular and frequent-are the raison d'etre for the existence of any business, since it was the hope of an adequate return upon their money which induced the stockholders in the first place to invest. Dividends are directly dependent upon earnings, so that the first leaf in the "Bible" of the executive should show graphically the fluctuations in earnings over the period of the concern's existence.

II-Profits-Since profits consist in the difference between the selling price and cost price, the second section should show by means of curves the average monthly selling price per unit of the major products as compared with their total cost sold. The space between these two curves at all times represents the profit. Low profits may be due to a low selling price or to a high manufacturing cost, By watching the fluctuations in the two curves the executive at once can determine which 
of the two halves of his organization needs his attention and assistance, and he ean throw his strength to the weakest point.

III-Sales-Graphs showing the total quantity of sales, the distribution of quantities by districts and subdistricts, show the executive and the sales manager just how well each portion of their organization is doing as compared with past performances and just which portions of the territory need attention. The prices obtained in each section of the country are averaged each month and the data so presented that every effort may be intelligently and consistently made to hold prices to a maximum. In a similar manner sales expense is kept track ofsubdivided as common sense suggests.

In concerns which have applied the principles of scientific management to their sales department-rewarding their salesmen and salesmanagers-exactly in proportion to what they accomplish, quantity sold, price obtained, and expense saved-the "efficiency," or percentage of attainment of the standard set on these points in each territory is platted so that the executive may see at a glance just what is being accomplished in each territory in proportion to what eareful analysis of local conditions in each district has determined should be accomplished. This standardization simplifies the work of the executive considerably, since the same results obtained from the application of scientific management to the factory - such as the increase in the employees' interest in their work, the introduetion of team play, etc., etc., renders less active supervision upon the part of the ehief execntive necessary, and the percentage system of recording results boils down all results to a common denominator so that much less of his time is required to grasp the exact state of affairs.

IV-Manufacturing Costs-Should on examination the sales graphs show that low profits during a given period were in no way attributable to avoidable faults in the sales department, the executive would naturally next turn his attention to the manufacturing department. It is not our purpose to indicate in detail how a complete cost system may be reproduced graphically. These, however, are the principal questions which the executive probably wonld be prompted to ask: (a) Was the total cost of manufacture high or low as compared with past costs? (b) As compared with the standard costs? (c) Was the output unusually high or unusually low? (d) For how much of the variation in costs was the output responsible? (e) Was a period of low output followed by merely a rise in the indirect labor, supervision, rent, general expense and various other sorts of overhead or did it extend to the direet labor, showing, that men were kept on unnecessarily in slack times by foremen "just to be a good fellow"? The answer would at once determine the executives' course of action. (f) Was the variation in cost due to labor or material?

With such questions as these answered-in fact with the answers so arranged in advance that they strike the executive forcibly, unavoidably, relentlessly, and regularly every month-the necessary action is taken or the executive cannot avoid the admission, even to himself, that he is incompetent.

$V$-Material Costs- $\Lambda n$ increase in the cost of raw material often takes the rise in costs outside the executive's control. At the same time 
he should know exactly to what extent this rise affects his cost of production. Such knowledge either drives him to raise the selling priceand for a legitimate reason-or to attack with renewed vigor some department which seems capable of reducing its operating costs sufficiently to offset the increased material costs. In other words he is driven to make an extracrdinary effort to meet the changed condition by a knowledge of its exact seriousness and his stockholders can rest assured that everything possible has been done to safeguard their interests.

VI-Elimination of Waste-Every business has its own particular sort of rat holes, through which its profits are carried piecemeal, and in quantities hardly noticeable at the time, but which aggregate thousands every year. The best way to plug these sources of loss is by accumulating data in regard to them and then keeping this data prominently before the executive. Possibly a graph showing the total rejections by customers is required; or a graph showing the number of pieces spoiled in each department. Knowledge of this sort ends very shortly in a raid upon a particular department and a general reorganization along more efficient lines. The result is a sharp drop in the rejection curve. Sometimes it is the production of seconds which needs watching, or perhaps the scrap produced, shown by departments. Frequently a curve showing the number and causes of shutdowns leads immediately to action which saves the company thousands of dollars. The mere reduction of such matters to figures is invariably beneficial. Couple such tabulation with an effective graphical presentation and the executive cannot fail to note the consequences of the losses in dollars and cents. It is his fault, then, if he fails to act at once and strike straight at the root of the trouble.

VII-Inventories - Very often, too, the accumulation of manufactured stock becomes a serious menace to a business. The executive may know in a general way that a large quantity of a certain variety of product is on hand; he may know furthermore approximately what the total investment in finished stock aggregates. The important matter, however. may be the size of the stock of each variety as compared with the stock at the same season previous years, or at a period when market conditions or manufacturing conditions were similar. Certain grades of product have a way of accumulating in many plants, especially where the market demand is not as great as the production of these grades. With the facts all before him the executive is in a position to dispose -by bargain sale or special attention - of all grades to good advantage as they are manufactured; while if the facts do not come to his attention until the accumulation of stock is large, a forced sale at a sacrifice may be the only remedy.

VIII-Labor Turnover-This is another matter which few executives watch closely. The figures are sometimes available in the office of the employment department or can be assembled from the records of the timekeeper. In most cases, however, executives do not know how many men they are hiring annually to maintain their force. When this insidious source of loss is called to their attention they will usually admit that it costs them $\$ 50$ to $\$ 125$ to break in a new man. Especially will they make this admission if they can be induced to recall a few of the -mistakes costly to their employers which were made when they were 
serving their own apprenticeships. The figures are usually appalling when first compiled. It is not at all unusual for a business to have a labor turnover of from $100 \%$ to $600 \%$ ! A concern employing a thousand men, whose normal turnover should not exceed $50 \%$, has one of $250 \%$. Assuming that it costs them fifty dollars to break in each new man-and fifty dollars is low if you consider the spoiled material, and the time of foremen and fellow employees taken up in teaching a new hand the ropes-the ammual loss amounts to $\$ 100,000$ !

An examination of the labor turnover often brings unexpected inefficiencies to light. One concern which the writer investigated had a turnover of over six hundred per cent-about four hundred per cent above normal, considering the conditions in the local labor market. It was thus losing about fifty thousand dollars a year unnecessarily. The searching investigation which followed disclosed the fact that a superintendent was receiving a rake-off from an enterprising employment agency for every man he hired! The sudden and enforced exit of the superintendent resulted in the turnover percentage dropping to normal. Thousands of dollars are being saved every year in consequence. A graph which keeps the labor turnover before the executive makes such occurrences as this impossible.

IX-Safety First-The "safety-first" movement has ceased to be a humanitarian fad and is now generally recognized as fully justified for sound business reasons. The cost of damage suits, hospital bills and pensions to say nothing of the cost of "finding a place' for disabled employees during convalescence, breaking in a new employee on short notice, having the crew disorganized by the absence of an employee for a day or two and the general fall in morale in a factory where accidents are frequent, is sufficient to make the reduction of accidents a matter of considerable importance to the executive. Tabulations showing the causes of all acciclents each month and a graph to keep the state of affairs before the executive continually just as inevitably leads to the elimination of the causes of aceidents as the elimination of the accidents leads to increased earnings in the business.

$\mathrm{X}$-Daily r'urues-Finally, every business has certain vital operations -it may be the pomnds of coke refinired to produce a ton of steel or the specific gravity of a chemical-upon which depends to a large extent the success of the husiness. Graphs which keep such quantities or such pereentages daily hofore the executive bring his attention and assistance at once in case of need. The correction is immediate and the saving therohy offereted may be far-reaching.

The pages immodiatrly following, illustrated by Fig. 1, showing the first series of typical graphs, describe the method of placing a hypothetical business before the executive in sufficient detail to enable an intelligent cost areonutant to proceed with the work, once the executive has decifled upon the points of his particular business which it is important for him to know. The second series of eurves, Figs. 2, 3, and 4, show the inethor of setting graphic standards in the same detail.

Fig. 1 shows the "fotal net profit" aceruing from the business each month, in eomparison with the standard of $10 \%$ set as a proper retum upon the investment. 'The average for the year is also shown in eomparison with the average net profit for the year previous. The lower 
broken line represents the danger point-below which the net profit curve should not fall if adequate return is to be earned.

The sources of the net profit are shown by the three central curves marked Works No. 1, Works No. 2, and Works No. 3. These represent the three factories of the company whose earnings are given in terms of gross profits. From the aggregate of these is subtracted each month the amounts represented by the lowest curve, marked "Deductions," which includes interest, diseount, commissions, and any other amounts not easily pro-rated to the proper factory, leaving as a balance the amounts shown in the "total net profit" curve.

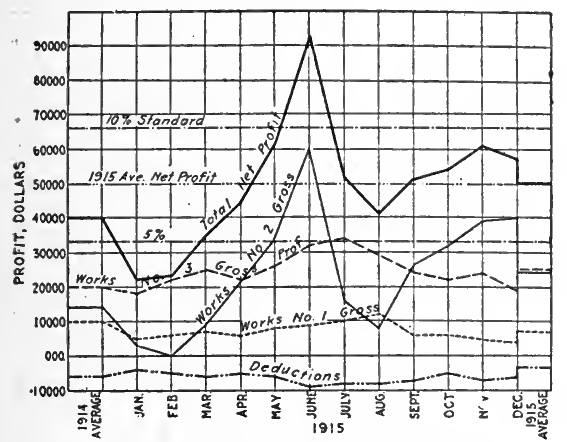

Fig. 1-Profits and Deductions
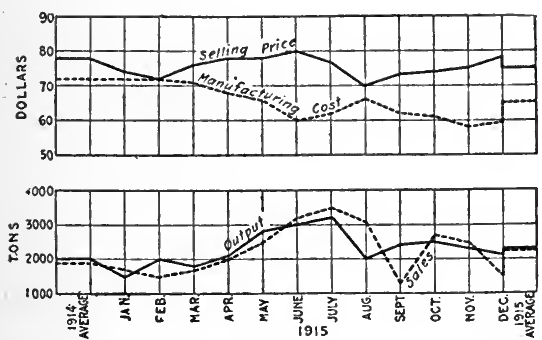

Fig. 3

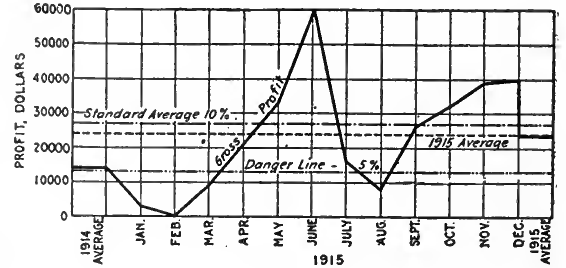

Fig. 2
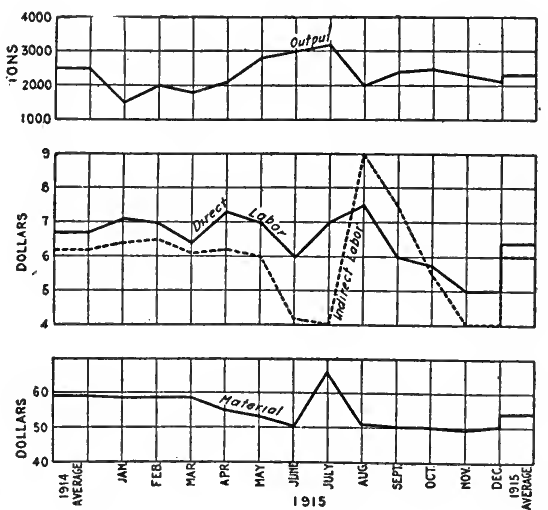

Fig. 4

The executive, in making use of this graph, as it is filled in monthly during the year, would have the variations in the net profit forcibly and unavoidably brought to his attention, in such a way that investigation and the consequent maximum improvement under the circumstances would inevitably ensue. If the net profit curve dropped suddenly, as in August, it is simply necessary to follow the August line down to note that the profits from Works No. 3 were normal, being well above the 1914 average; that profits from Works No. 1 were also normal; but that there was a sharp falling off in the profits at Works No. 2. He need not, therefore, concern himself for the present with conditions anywhere except at Works No. 2.

Similarly, since all abnormal conditions must be carefully analyzed in order to avoid in future those that are unfavorable and to make permanent those that are favorable, the rise in the net profit curve in 
June, when investigated, would show that Works No. 2 was the principal source of the additional profit, conditions at Works No. 1 being normal and at Works No. 3 only slightly above normal. The executive may, therefore, conclude that there is greater need for careful analysis of operating conditions at Works No. 2 than at the others.

Since the great rise in net profits for June and fall in August were at Works No. 2. their causes must be obtained by turning to Fig. 2 in the second series.

The graph therein shown keeps the executive informed at all times just how closely the profits at this particular factory approximate the standard earning previously set-in this case $10 \%$ on the investmentand warns him at once in case the earnings fall below the 5\% danger line. This is merely a refinement of the graph shown for Works No. 2 in Fig. 1, and is valuable principally because it shows more exactly just how serious the variation from the normal is, what its effect will be on the year's earnings, and emphasizes the urgency for attention.

It will be noted that the June profit reached $\$ 60,000$, more than twice the $\$ 28,000$ required to earn the $10 \%$; while in August this factory made a return of only $\$ 8,000-\$ 5,000$ below the $5 \%$ danger line.

The causes for these fluctuations must be sought on the next graph of the series. Fig. 3.

There the upper pair of curves show the "selling price" by months as compared with the "total manufacturing cost" at Works No. 2.

The lower eurves show the output of the factory by months in tons as compared with tons sold. In each case the final monthly average for the year is shown at the right, and the monthly average for the year previous is shown at the left.

The profit earned at any factory is, of course, the difference between the prices received for each unit when it is sold and the cost to manufacture this unit, multiplied by the total number of units made, or the factory output. The gap, then, between the "selling price" and " manufacturing cost" curves, multiplied by the number of units shown any month, gives the "factory profit" for that month.

It should be noted that there are of course, certain adjustments which must be made to suit each business, since very few eoncerns would sell during the month all goods made that month, while still fewer concerns would have no goods in process of manufacture at the end of each month. We are assuming, however, that such would be the case in this instance in order to avoid a long digression on methods of inventorying.

Carrying the analysis of the abnormal profits in June and in August to this graph, the executive is at ouce able to determine whether the variation is due to market conditions or to manufacturing conditions, and is thus enabled to throw his support to the sales department or to the factory.

The "selling price" "urve in June reaches the high point for the year, $\$ 80$, although only two dollars above the average for the previous year. This showing is favorable, althomgh not suffieiently removed from the normal to demand immediate attention. The "manufacturing cost" curve shows a decided drop below any point reached so far during the year, 1915, and twelve dollars below the 1914 average. Evidently the increased profit is due to manufacturing conditions which must be in- 
vestigated in order that the gain may be made permanent if possible. Analyzing August conditions in a similar manner, it is found that the cause for the profit falling $\$ 19,000$ below the standard set is due about equally to the drop in the selling price and to the rise in manufacturing costs. The first suggests to the executive an investigation of selling conditions, and the sales manager may be asked to report fully on the reasons for the fall in prices. The second causes the executive to turn to the next graph, Fig. 4, which deals with operating costs at the factory. (Note: The "sales" curve in Fig. 3 is shown to indicate how sales keep up with production. Sales-analysis graphs are described elsewhere.)

Fig. 4 comprises three sets of curves: the curve at the top shows the monthly "output" of the factory in tons; the two curves in the center show the "direct labor cost" per ton and the "indirect labor cost" per ton; while the curve at the bottom shows the "material cost" per ton. Monthly averages for the year and for the previous year are shown at the right and left respectively.

On this sheet we are getting down to fundamentals, and by careful analysis of the conditions and their effects the executive may deduce the general laws underlying his own particular business.

For instance, take the low manufacturing cost in June shown on the previous chart. The output is 500 tons, or $20 \%$ above the average. We would naturally expect this to cause a drop in the indirect cost per ton, since the fixed charges would remain nearly constant while the divisor (the output) increased. We find this to be the case on reference to the dotted curve which shows a reduction of $\$ 2.20$ per ton, $33 \%$ below the: average indirect cost per ton for the previous year.

It is to be noted also that other factors in the cost reduction were a drop of about $\$ 0.70$ in the direct labor and of $\$ 9$ in the material cost. This latter cost, quite likely, is beyond the control of the executive, but it must be shown in order that he may not congratulate himself on a low cost when the saving is not the fault of his organization but of market conditions. Otherwise he might naturally neglect certain departments in which the cost of production is rising and which consequently need attention. In other words, such matters as are the business of the executive must be put squarely up to him in such a way that he cannot overlook or neglect them.

Having reached the conclusion, then, that the causes of the June cost reduction under his control are due to indirect and direct labor, the executive investigates, first, the methods of maintaining a large output. This eventually leads him into the sales department and to an investigation of market conditions. Incidentally, graphs would be consulted to show the increase or decrease in such items as general expense, sales expense, etc., in order that the assurance may be gained that there is no waste, or that some extraordinary economy may not be overlooked and the chance lost to make it permanent.

The drop in direct labor in June leads to a perusal of the graphs covering the various departments and the cost of each operation therein. If direct labor costs are low or high there is a reason for it, and the executive must not stop until he has made the gain permanent or corrected the fault responsible for the rise in cost. 
We have not attempted to carry our illustrations into further subdivisions because we should very shortly emerge from the field of prineiples into the diseussion of a particular business, which is not our purpose at this time. We believe enough has been said, however, to illustrate the method-more would be merely a refinement.

But to illustrate the analysis of a month in whieh costs are high, we will take the month of August, when the costs were some seven dollars higher than in June. What strikes us first is, of course, the jump of about five dollars in indirect labor cost. This we may lay to the decrease of a thousand tons in the output-caused, let us assume, by a fire in one of the larger departments. Direct labor costs rose also. The reason for this may be determined by reference to the department graphs. The executive might find that the rise in eost was due to the disorganization incidental to. and following, the fire; or that labor due to repairing and eleaning up was eharged against operation instead of to extraordinary expense as it should have been. Such matters must be investigated and from the conclusions drawn exact methods evolved for future guidance. The economy is effeeted through the knowledge of exactly what the disaster cost in operating costs, in loss of output, and so in profits. Complete analysis in this ease would lead the executive, when he discovered that his loss of output had cost him $\$ 52.000$ profit to steps of the most strenuous nature. (Compare June and August, Fig. 2, after noting that material costs were nearly constant, with the same months in Fig. 4.) As a result of the fire extinguishers or the sprinkler system installed, or the employees fire denartment organized, or the fire-proof building erected, such a loss would be avoided in the future. It is much easier for an executive to obtain appropriations for needed preventatives when he an show his directors exactly where the disaster touched the pockets of the stockholders, than when he has to rely upon mere arguments.

We have indicated the economies to which an analysis of particular months may learl. There remains to be shown the advantage to be gained by analyzing one eurve eovering a eonsiderable period, and noting the effect of other conditions upon it. $\Lambda$ s an illustration, take the effect of the ontput upon the indireet labor. During 1914, the factory had a moderate output of 2,500 tons per month and a moderately high indirect labor cost of $\$ 6.20$ per ton. During the early months of 1915 , the low output incidental to the season of the year resulted in the charge of indirect labor hovering around $\$ 6.50$. As the output jumper in June and July, the indirect labor dropped over $\$ 2$ per ton. The arecident in August took the indirect labor cost up to $\$ 9$, and it was not until October that it approached $\$ 4$ again. The output apparently was not enough larger, however, since it was 700 tons less than in June, to account for all of this drop. Reasons for that would have to be someht in graphs covering various sorts of overhead expense. The tendeney, however, is very clear and the executive may determine exactly the effecot output has upon his profits, and plan ways and means to carry the business over seasonal slumps. After studying this phase for a yor or two, he is in a position to determine exactly how much he can afford to cut prices at eertain seasons of the year in order to keep the factory busy to capacity at all times. It was eon- 
sideration of just such factors as this which led some of our larger corporations to dump a certain portion of their product in foreign countries a few years ago-an item obtaining wide publicity and causing caustic comment by such economists as were socialistically inclined. The principle, however, is a sound one, but if disaster is to be avoided exact knowledge of the limits permissible is essential.

The "direct labor" curve must be examined in connection with the departmental curves. Careful study of this curve over a period leads almost inevitably to the standardization of labor conditions. The fall in the "direct labor" eurve shown on Fig. 4 would have to be ascribed to some such cause as that.

The general tendency downward in the "material" curve would be caused to a great extent by a fall in the cost of raw materials, although a considerable percentage of it might be due to the standardization of materials and a bonus to employees to encourage the elimination of wastes.

In general, we can say of these various curves that the fluctuations of one are indefinably associated with the fluctuations of each of the others, and that it is only by close study of the effect of one upon the other over a considerable period of time that the executive can be sure of obtaining maximum results in the way of low operating costs, large sales, and ultimate profits.

It is only after a thorough and detailed study of past conditions that the executive is in a position to set standards to be attained. The following graphs, Figs. 5, 6 and 7, illustrate a method of effecting this standardization. The standards set in each case were based upon an hypothetical analysis of conditions assumed to prevail during 1915. In it will also be noted that we show the actual 1915 accomplishment. This is done merely for convenience to illustrate the method of procedure. Thorough standardization has, in the writer's experience, almost without exception resulted in a much greater degree of attainment than that shown.

Fig. 5 shows the profit which must be earned each month at Works No. 2 in order that it may bear its share of the $10 \%$ return on the investment which has been set as a standard net profit for the corporation as a whole. The broken line, marked "standard," represents the cumulative earning in dollars which an analysis of selling and manufacturing conditions has determined to be a reasonable attainment each month. Thus the gross profit from this factory should be $\$ 24,000$ for January. By the end of February this profit should have grown to $\$ 52,000$; by the end of March to $\$ 81,000$, etc.

The solid line, marked "actual," shows how nearly the 1915 conditions would have allowed the actual profit for that year to approach the prescribed standard. It will be noted that at the end of February the actual was $\$ 49, \mathrm{C} 00$ below the standard, and by May the actual was $\$ 76,000$ short of the standard, etc.

In order to set a standard profit it is obvious that it is only necessary to determine for each month a standard selling price and a standard manufacturing cost per unit, and to multiply the difference between these by a standard output. The difficulty lies in the standardization of factors composing the cost, selling price, and other items. The 
method of determining these will be discussed as we proceed. It should be noted, however, that the profit has been standardized to bring in, allowing for seasonal fluctuations, at least enough to secure the $10 \%$ return on the investment which has been set as the standard earning for the company.

Fig. 6 shows, by means of the diagonal broken line marked "standard returns," the eumulative amount by months to be received from sales. The solid line shows the actual receipts for 1915 . The standard sales price per ton set for each month is shown by the curve at the top of the chart-the cumulative average being shown by the broken line.

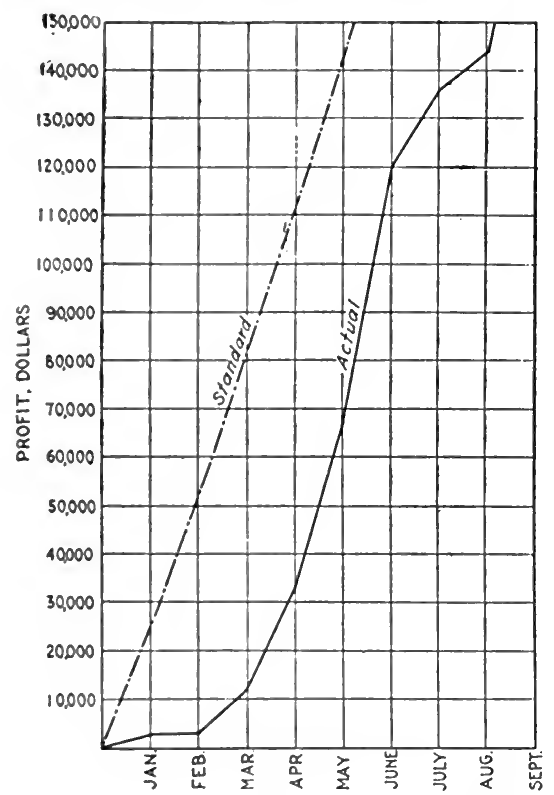

Fig. 5-Standard Versus Actual Profits - C'umulative by Months

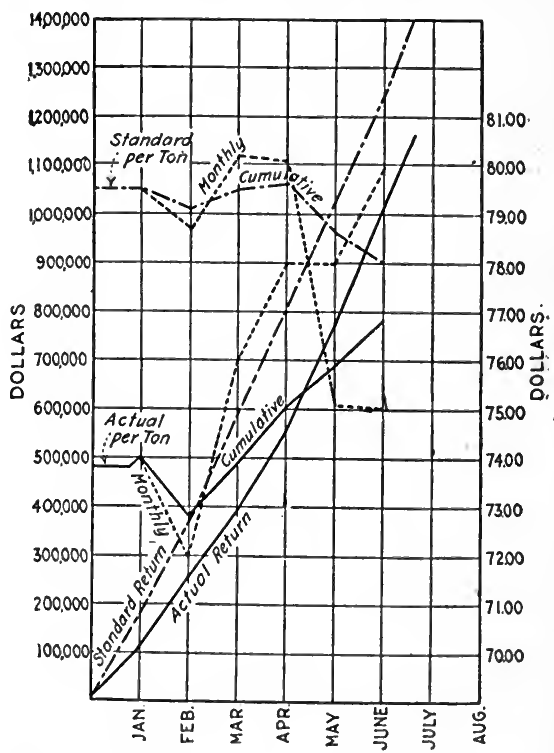

Fig. 6-Selling Price-Cumulative and Monthly

The scale at the left indicates monthly and cumulative tons. At the right, prices per ton.

and the individual months being shown by the dotted line. Similarly, the curve below shows how nearly the monthly selling priees for 1915 reached these standards.

The matter of standardizing selling price is a difficult one. In some cases it is manifestly impossible to set a price any length of time in advance at which the goods should be sold. We must remember, however, that in every business there is some one in authority who does set the selling prim. Further, that he sets this price before the sale is made. And finally, that the more past data-eall it experience or what you will-he has in his possession, the more intelligently he ean set this price, the longer in advance he can prediet market conditions, and the more he will, therefore, obtain for his goods.

Such is the principle. The application varies with every business. It is sufficient to say that the writer has in mind at the present writing 
instances of three widely diverging types of business-business where the keenest competition prevails, where there are considerable general and seasonal market fluctuations, and where the selling price is set months in advance; yet these concerns are all living up to the standards set with a regularity and precision which to the uninitiated is, at times, positively uncanny.

The standardization of the selling price is greatly assisted by the "bonus-for-salesmen" system, which makes it directly to the financial interest of each salesman and sales manager to maintain the selling price, the quantity of sales, and the amount of sales expense, by rewarding him exactly in proportion to the effect his acts in regard to these matters have upon the ultimate profits of the firm. This system tends to diminish materially the many and specious reasons for cutting prices which are advanced by salesmen on commission or by salesmen who think their promotion depends upon the quantity of their sales alone; it also tends very materially to steady the market.

Just how far ahead the sales price can be standardized depends upon the particular business, and must be determined in each case. The further the executive tries to look ahead, however, the more proficient he becomes. The more exact his data on past performances, the more likely he will be to predict correctly. The mere fact that he has set up a mark to shoot at will raise the average of his bullseyes. A rise in the average selling price, other things being equal, is inevitableand an increase in the net profits is equally unavoidable.

It will be noted that Fig. 6 shows a standard selling price of about $\$ 79$ for January and February, which is raised (it is assumed) for March and April to cover an anticipated rise in the price of certain raw materials, and is later lowered to cover a drop in the market. Actual conditions in 1915, shown in curve marked "actual," do not even approximate the conditions predicted, either in the selling price per ton or in the return from sales.

In standardizing the selling price it should be understood that by so doing the firm places no restraint upon prices, unless it is decided that it is to the interest of the firm in the long run not to exceed a certain maximum price. In the particular instance covered by the graphs the selling price was standardized simply at a figure sufficiently above the standard of production so that the difference would, when multiplied by the standard output for the year, yield sufficient profit to earn the standard return of $10 \%$ on the investment. By comparing the actual selling price, then, with this standard, the executive knows at any time, at a glance, how nearly he is earning his standard profit.

To illustrate, take the month of May: Reading the cumulative diagonals, the executive observes that he has received $\$ 770,000$ from sales during the five months where he should have taken in $\$ 1,200,000$. Further, that so far he has sold his product at an average price of $\$ 75.90$, while he should have averaged $\$ 78.60$ for the five months. In addition, he notes that his May sales brought him $\$ 78$ per ton, while he only expected to receive $\$ 75.10$ per ton. He can thus tell at a glance just how well he is doing in any particular month and just how much he is behind schedule-and how much he must manage to boost prices during 
the remainder of the year if he is to make his standard profit. There is no question about the spur of such knowledge.

Fig. 7 shows, in cumulative form, the standard output determined upon, and how nearly the 1915 output approaehed this standard.

In order to establish a standard profit a standard output must be determined. The executive must know how many units must be madeand sold-as well as the standard selling price, in order to attain the standard profit.

An examination of former production eurves has shown him that the

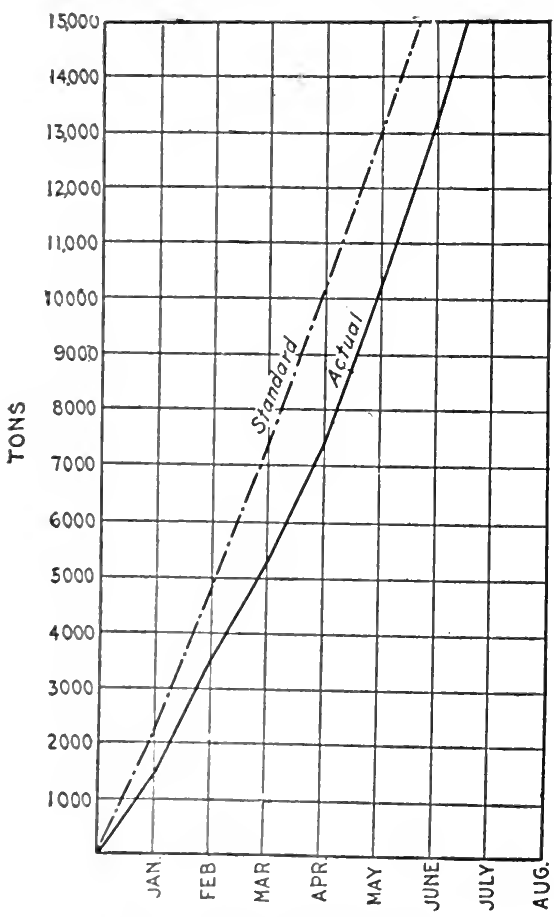

Fig. 7-Output-Cumulative

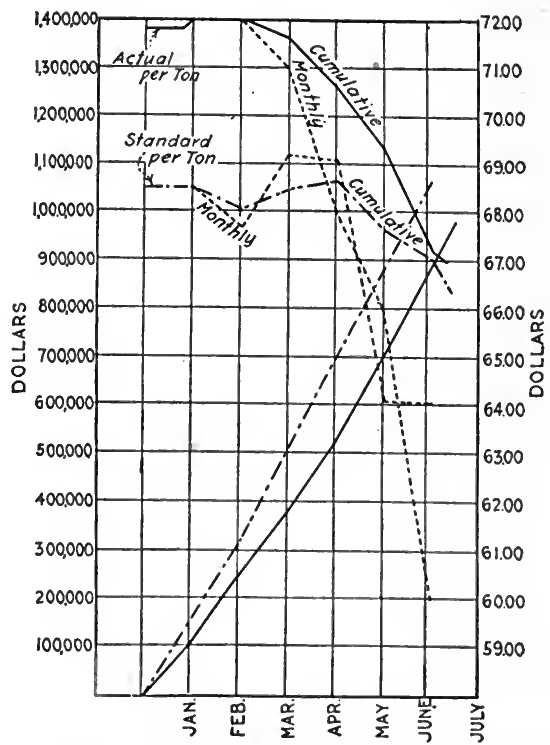

Fig. 8-Total Manufacturing Cost

The scale at the right shows total expenditures; that at the left, expenditures per ton.

factory can, when pushed, turn out, say, 3,200 tons a month (Fig. 4). He has further determined from his sales eurve that, unless he is prepared to stork heavily, it is unwise to make more than 2,200 tons in Jannary, 2,50) tons in February, 2,700 tons in Nareh, 2,800 tons in April, efe. He standardizes his output, then, aceordingly, so that his cumulative production at the end of April should amount to 10,200 tons. Reference to his standard output graph (Fig. 7), then, would show him that hy the enel of $\lambda$ pril he has made 7,400 tons and is 2,800 tons behind schedule, which must be made up by the end of the year if he is to attain his standard merofit.

In Fig. 8, the diagenal hroken line shows the standard preseribed as the cumulative expense to be incurred for manufacture at Works No. 2. The solid line shows how nearly manufacturing expense for 1915 reached this predentermined budget as the year progressed. 
The curve at the top shows the actual cost per ton for 1915, by months and in terms of cumulative average. The lower curve shows the standards set. The other factor in determining the profit, besides selling price and quantity sold, is the manufacturing cost. The standardization of manufacturing cost implies usually the introduction of scientific management in the factory. It is not our purpose to enter into a lengthly discussion of this phase of the matter at this time; but assuming, however, that the executive has determined what his product should cost, he would find by reference to this graph in May, for instance, that if this factory had turned out the standard output at the standard cost there would have been expended $\$ 880,000$. Actually, however, the solid diagonal line shows that only $\$ 700,000$ has been spent. We have already seen that the factory failed on the output. Reference to the cost curves at the top of the chart, which show the cost

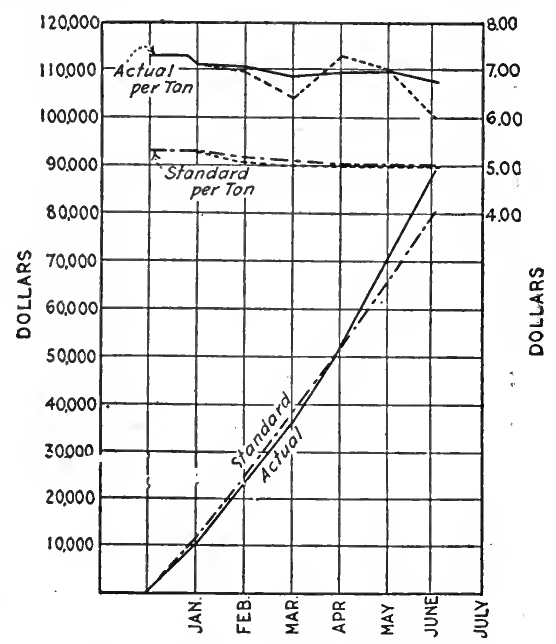

Fig. 9-Direct Labor Expense

per ton to manufacture, discloses the fact that the average cost per ton during the first five months of the year was $\$ 69.30$, when it should have been $\$ 67.65$; and that the May cost per ton was $\$ 66$ as against a standard May cost of $\$ 64.10$. Again the executive knows just how much behind he is, and just how much must be accomplished if the business is to attain the standard profit.

This manufacturing expense, and cost per ton to manufacture, is shown, subdivided into its elements, in Figs. 9 and 10.

Fig. 9 shows by the diagonal broken line the monthly expenditure, in cumulative form, set as the standard for "direct labor" at Works No. 2. The solid line shows the expenditure for 1915 .

The curve at the top of the page shows the actual cost per ton of direct labor by months, and also the cumulative average as the year progressed. The lower curve shows the standard cost per ton for direct labor.

As has been stated, the standardization of manufacturing costs is 
dependent largely upon scientific management, and this is particularly true of the direct labor costs. The cost of each operation must be standardized by the use of analytical time study, and it must be made to the interest of each employee to attain this standard by the introduction of a bonus reward.

This graph brings out the apparently anomalous fact that it is quite possible to obtain a satisfactory standard cost per unit of manufacture, even though the profits are unsatisfactory, when too little money is being spent for manufacture. It will be noted that by June the actual cost per ton, cumulative, has reached $\$ 67.20$, within $\$ 0.10$ of the standard set (to earn $10 \%$ profit), but that only $\$ 900,000$ has been spent where $\$ 1,600,000$ should have been spent for manufacture. The reason for this apparent inconsistency would later be found to lie in a drop in the cost of material. This reduced the manufacturing cost and necessitated a cut in price, so that the margin of profit did not increase sufficiently to sustain the profit when the output did not reach stand-

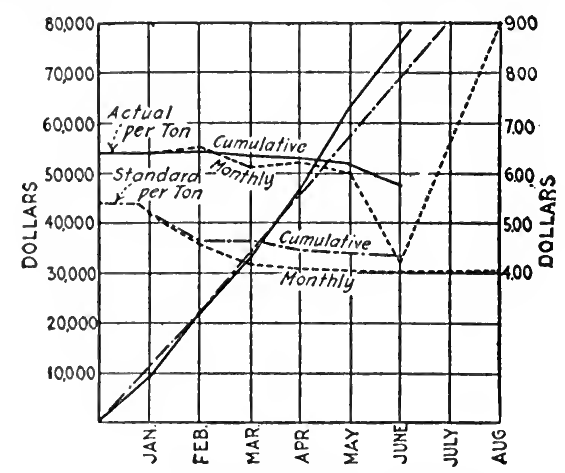

Fig. 10-Indirect Labor Expense

ard. It is just such obscurities as this that are made clear by the use of granhs.

Fig. 10 shows by the diagonal broken line the monthly expenditure, in cumulative form, set as the standard for "indirect labor" at Works No. 2. The solid line shows the actual expenditure for 1915.

The curve at the top shows the actual cost per ton of indirect labor by months, and also the cumulative average. The lower curve shows the standard cost per ton set for direct labor.

Indirect labor cost, when all possible inefficiencies have been eliminated, is largely a matter of output. The executive in this case, after studying the curve, would perhaps conclude that, since the low mark of $\$ 4$ per tom harl been reached in July with an output of 3,200 tons, it would be reasomable to suppose that such a cost could be maintained continuously, if the farcory could turn out 3,200 tons a month. As a matter of fart he knows from a study of past years that the market will not take over 2,20() tons in January, that by Mareh they should be taking 2,700 toms a month, by . June they should be taking 2,900 tons, ete. Ile feels, however, that by thoroughly overhauling the works, introducing more efficient methods of organization, etc., he can reduce 
this overhead to some extent and, therefore, places his indirect labor standard at $\$ 4.05$ for the busy season when he believes the market should absorb 2,900 tons. In the Spring, when the market is good for only 2,800 tons, the indirect labor cost is raised proportionately and is standardized at $\$ 4.10$, rising to $\$ 4.20$ with 2,700 tons standard output in March, to $\$ 4.60$ in February with 2,500 tons, and reaching the high point of $\$ 5.20$ for January with its 2,200 tons standard.

Once the indirect labor standard has been figured out in this way, the executive is in a position to decide whether to cut prices and maintain output, or to maintain price and let the output fall, since he is in a position to know almost to the dollar just what every ton drop in output will cost him.

The setting of accurate and attainable standards depends upon accurate and easily assimilated records, and records in greater detail than it is possible to show in an article of this sort. It is necessary to go much further into departmental subdivisions to predict with exactness. We have attempted only to indicate the way with a few generalities, since the illustrations can only be general unless a specific business is discussed.

Given the organization, the executive who has his business before him-as a modern general has his battlefield before him accurately mapped to scale-is much more likely to predict the outcome, and to realize the outcome he desires, than the man who depends upon an uncorrelated mass of recollection and upon his intuition. And the executive who, like the modern general, is prepared is the one who will win his fight upon the battlefields of business.

Organization Chart. Cross-section papers, especially the $8 \frac{1 / 2}{2}$ by $11 \mathrm{in}$. sizes, are very handy for drawing up organization charts. Fig. 11 is an example of a general case. It is customary to write in the names of the individuals filling the various positions, either in place of, or together with, the names of the positions. The lines of the paper offer guides both for the small rectangles making up the chart and for writing or printing within the rectangles. The chart shown is reproduced from one published in Metallurgical and Chemical Engineering, November 15. 1916.

Charting the Stenographer's Ability. The direct application of a series of tests to stenographers, from these the setting of standards and the resulting efficiencies of those tested is described by Geo. A. Ricker, in System, the Magazine of Business, February, 1916, as fol. Jws:

Studies resulted in a practical plan for standardizing our stenographic department. The following factors were easily obtainable:

1. Average number of words in a typewritten line $\ldots \ldots \ldots \ldots \ldots \ldots \ldots \ldots \ldots \ldots \ldots$

2. Average number of lines to a typewritten page $\ldots \ldots \ldots \ldots \ldots \ldots \ldots \ldots \ldots \ldots \ldots$;

3. Average number of stenographic pages to a typewritten page $\ldots \ldots \ldots \ldots \ldots$;

4. Average speed typewriting (words per minute) $\ldots \ldots \ldots \ldots \ldots \ldots \ldots \ldots \ldots \ldots \ldots \ldots \ldots$

5. Average time required to typewrite one page (minutes) $\ldots \ldots \ldots \ldots \ldots \ldots .5$.

Interruptions during dictation made it impossible for us to estimate the time required to typewrite from the time consumed in dictating. The obvious method, therefore, was to count the number of stenographic pages 


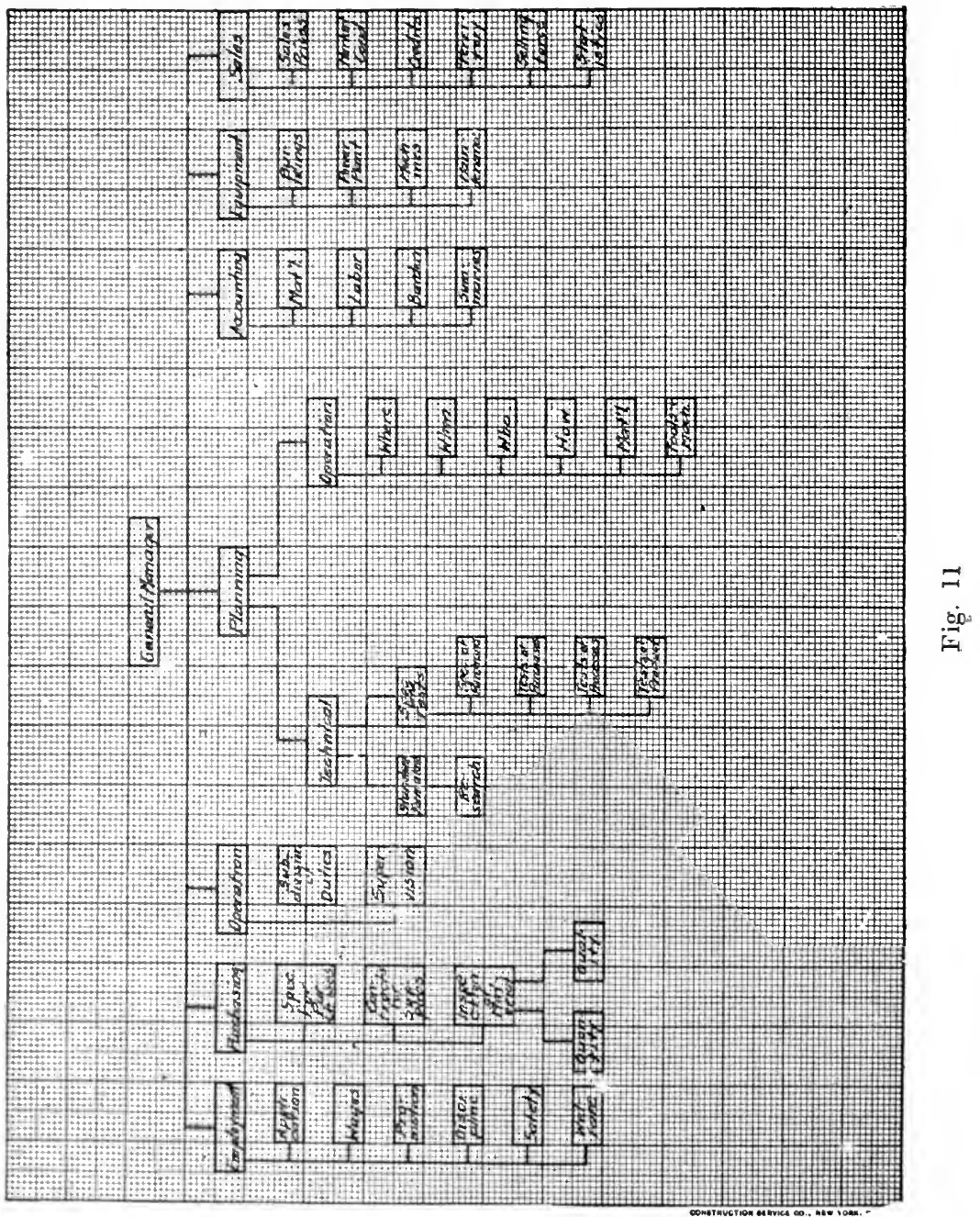

in the dietation. anlenlate the number of typewritten pages this would make. and from that figure the time resuired for transeription.

W" have two fixol pepmirements for our stemographers: first, they

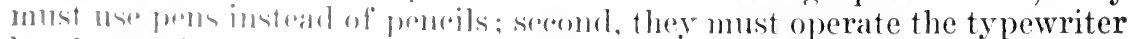

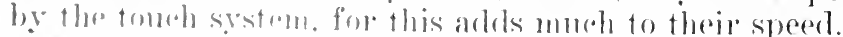

1 fail comprarison of the variations is shown in Fig. 12 . Stenographer "I," mate this record which may be too high, however, for an average.

1. Sprol while typowriting from stenograplice notes; 30 words per minute.

2. Cuend while typersitine from straight printod copes: 54 words per minute.

3. Speed while typewriting from the sperinl sentence. "Now is the time for all good men to come to the airl of thrir party "; 115 words per minute. 
Applicants for stenographic positions are given the three tests. Then their records are filed. We thus know how proficient stenographers are at the beginning of their service; and we have a basis for observing whether they are getting better as time goes on.

Fig. 12 represents the records of two applicants.

1. Stenographer " $A$ " is $100 \%$ proficient.

2. Stenographer " $\mathrm{B}$ "' is only $50 \%$ efficient as a stenographer, but is $90 \%$ effective as a typist. It would, therefore, be more economical to use " $\mathrm{B}$ " on copy work than on transcription, because of her difficulty in reading her notes.

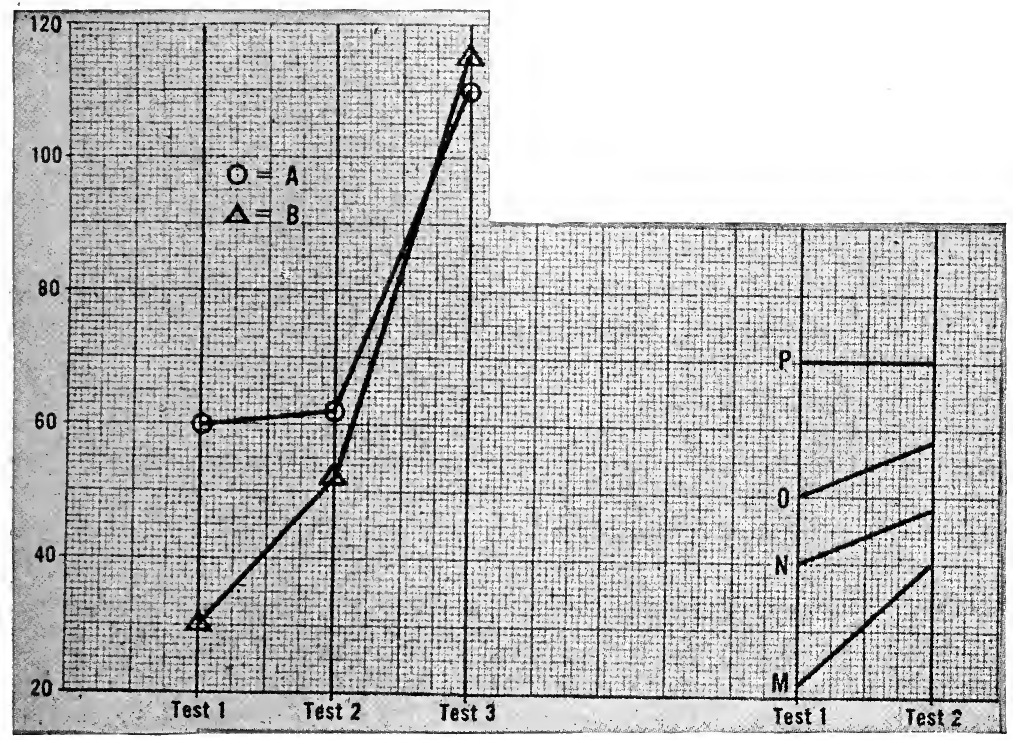

Fig. 12-Results of Stenographers' Tests

3. Stenographer "B" is capable of greater ultimate maximum efficiency than "A." This is indicated by the greater speed on test 3.

Stenographers, fresh from training, plot a curve similar to that of "B." As they increase their effectiveness, they change the angle until it becomes an actual attainment plot, as shown in the "A" curve.

The curves shown to the right represent the records of a stenographer upon entering our employ, and at later periods when tests are made to determine her wage value. Notice the decreased angle of inclination as greater proficiency is attained.

For the standard, or $100 \%$ proficiency, we pay $\$ 18$ a week.

To Prevent Overbuying. The following, from an article, "Nutshell Reports for the Boss," by James H. McCullough, published in System, the Magazine of Business, May, 1918, illustrates the value of a graphic chart to prevent overbuving.

Fig. 13 was designed by one factory manager to help him control better the purchase and use of a certain class of materials. The heavy 
straight line at $\$ 3,250$ shows the ideal inventory-the amount with: which an excellent rate of turnover could be secured. This quantity is just about enough to run the department for a month and a half. The heavy upper line shows the real inventory. The solid lower line. shows the month's purchases, and the dotted line the material used.

Owing mainly to uncertain market conditions, the actual inventory has been consistently well above the "ideal." Efforts to force it downward are evident, however, in the latter part of 1915 and throughout. 1916, and again in the latter part of 1917. In March, April, May, June, and July of 1917, purehases were unusually heavy, and since the amount used did not keep pace, the actual inventory line ascended rapidly.

The chart clearly shows that immediately after this, purchases were:

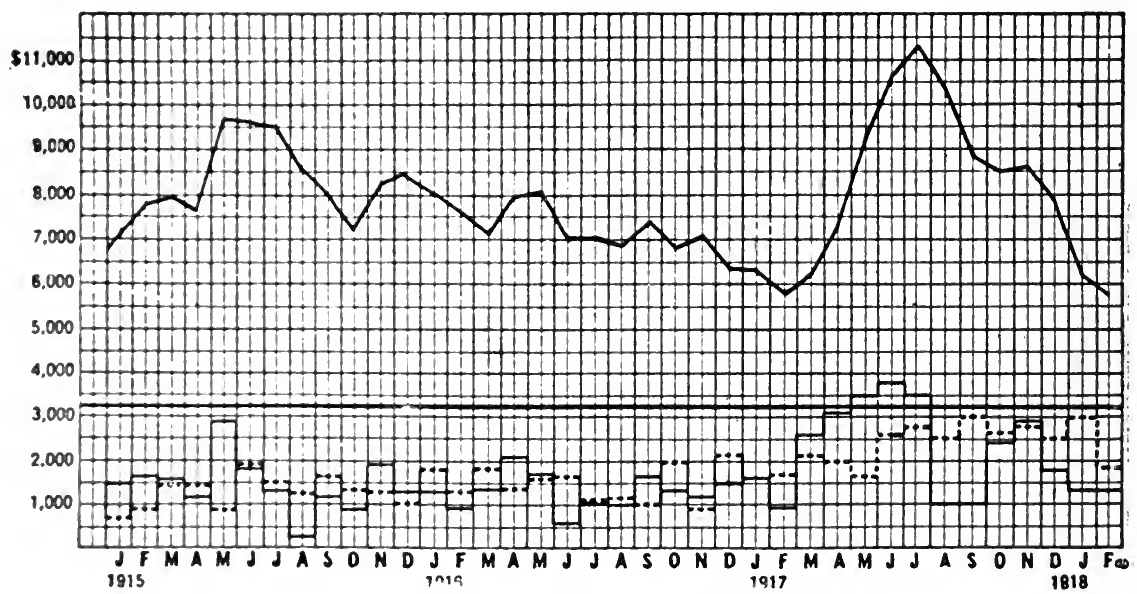

Fig. 13-To Prevent Overbuying

redneed while the amount used increased. As a result, by February of 1918 the actual inventory is as low as it has ever been.

Chart Analysis as an Aid in Buying. The following from an article "What's Bohind a Good Buyer's Guess?" by A. W. Douglas, published in S'ystem, the Magazine of Business, May, 1916, shows the value of charts as an aid in the analysis of conditions relating to buying. See Fig. 14.

The ordering of goods is usually held to be a very simple, elemental affair largoly homnd up in a want book. But in truth it is one of the most eomplex propositions imaginable and requires, if it is to be done suceessfully and correctly, the utmost thought and intelligent study.

A well efuipurel retail hardware store in a large town may have anywhere from 10 to 15 thousand items in stock. The problem is to have as many of these as necessary on hand all the time-and no moreso that there may be no shortages when orders are to be filled, no dead stocks, and no overstocks of seasonable goods to be carried beyond the season.

The real difficulty lims in the fact that each of these items in stock, or each line of gorols, is constantly affected in price and demand-or both-by practically revything that happens in the world. There- 
fore, the quantities that should be ordered or kept in stock are constantly changing.

Of all the causes which affect sales none is more puzzling than the weather. This is for the simple reason that the weather can not be foretold with any degree of accuracy more than 36 hours in advance.

I went back over the weather bureau records for all parts of the country for over 50 years, and the only conclusion I reached was that the weather runs in irregular, but none the less certain, recurring cycles, each of about 50 years. Also, that in these periods there occur the same extremes of heat and cold, of wet and dry seasons.

And it is upon these related phenomena-the reappearance of past experiences-that most of the guesses in ordering goods have to be

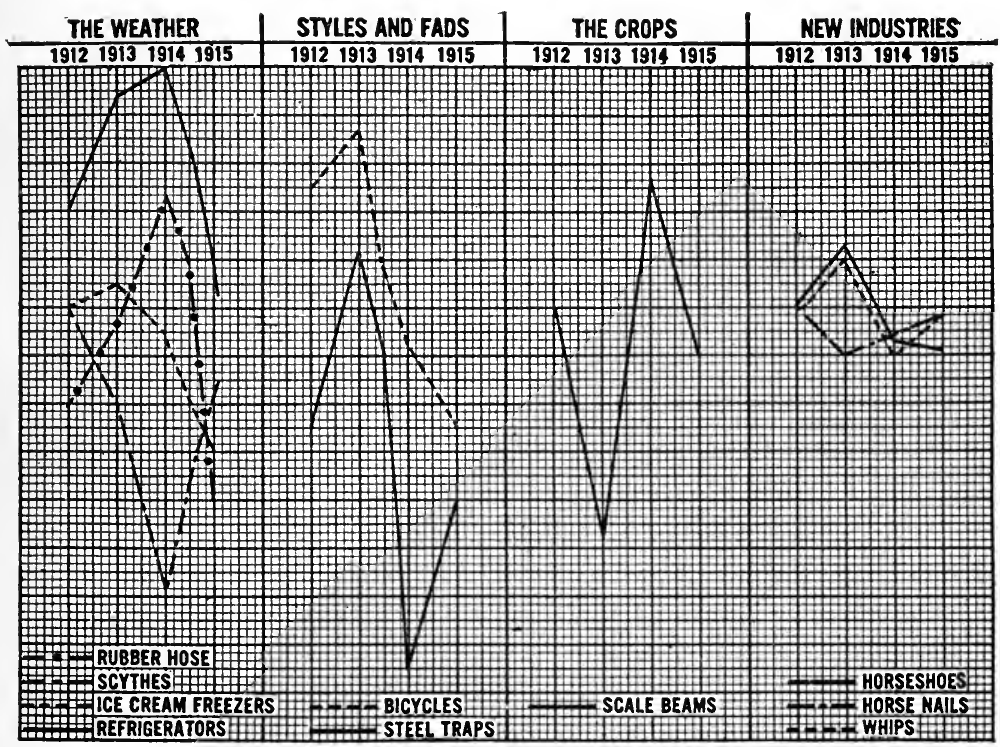

Fig. 14-Keeping in Touch with Demand

founded. Whether you will or no, you have to do some kind of guessing as to the quantities needed. You must base your guesses on past experiences.

Here are some sales figures for typical seasonable goods:

$\begin{array}{rrrr}\text { wet } & \text { dry } & \text { dry } & \text { wet } \\ 1912 & 1913 & 1914 & 1915 \\ 40,000 & 48,000 & 62,000 & 31,000 \\ 1,000 & 800 & 400 & 850 \\ 2,000 & 2,100 & 1,900 & 1,400 \\ 600 & 720 & 780 & 510\end{array}$

Rubber Hose-Feet ............... 40,000

Grass and Grain Scythes (Dozens) ....... 1,000

Ice Cream Freezers . . . . . . . . . . . . . 2, 2,000

Refrigerators

The year 1912 was characterized by abundant rainfall. 1913. and 1914 were extraordinarily hot and dry. 1915 was the wettest year known for a long time.

The figures cover the first six months of each year-the period when 
these goods sell heavily. Rubber hose is, of course, mostly used in dry weather, for sprinkling lawns. So the sales in that line are just what might have been expected from the prevailing weather in each year. See Fig. 14.

Botl grass and grain seythes are used principally in wet weather. The comparatively large sales in 1913 are due to a curious phenomenon which illustrates the necessity of detailed study of each year. The spring and early summer of 1913 had the usual amount of rainfall. The drought did not commence until early in July when the scythe season was over. Conversely, in 1914 the dry spell commenced about the first of May and lasted all through the scythe-selling season.

These conditions likewise influenced the sales of ice cream freezers and refrigerators. They are hot, dry weather goods, and sell best in years like 1913 and 1914, and slowest in wet, cool years like 1915.

Yon ean easily and logically comnect the sale of seasonable goods with the prevailing weather conditions. You can make an intelligent study of past cause and effect as influencing your present action under similar conditions. You are no longer relying on your memory, which is usually short and treacherous in such matters.

If. for instance, one year an abnormally wet season during grain harvesting resulted in unusually large sales of grain scythes, the graphical presentation of that fact on a chart, as in Fig. 14, should prevent your orders for the following year from blindly counting on the same conditions.

Of course good or bad times are always a constant factor one way or the other. This factor in bad times tends to decrease sales, and in good times to increase sales beyond the effect of weather conditions.

Another factor which must be constantly watched by the forehanded is that of the fashions in some goods. Steel traps for eatching furbcaring animals sell best when the price of skins is high. The price of skins is in turn set by the demand for finished furs. This is in turn set by the fashions.

The years of 1912 and 1913 were years of high-priced skins and much wearing of furs. But when the summer of 1914 eame, the forehanded buyer found out from some fur dealer, or from the Burean of Foreign and I) omestic Commerce at Washington, that our exports of furs to Europe were about $50 \%$ of our total production. IIe therefore knew that the demand for furs from this country would be cut in two. It was then olvious enough to him that the price of skins would decline accorlingly, that trapping would be unprofitable, and that the sale of steel traps would fall off. And that is just what happened.

Another important sonrce of demand variations is the wide divergence in the yield of the crops. This results in a corresponding divergence in the demand for agricultural implements of all kinds.

Information regarding the promise of the erops and estimates of the yields ean asily he secured from the free bulletins of the Department of Agrioulture at Washington.

In all things it is necessary to check general prevailing impressions by facts and common semse, lest you go off on a tangent.

Now observation, study and thought of this sort have two purposes: In the beginning they add much pleasure and interest to a part of busi- 
ness usually regarded as dry and grinding in its monotony. Their second and more practical purpose is to enable you intelligently to order and handle your stock of merchandise so that you will be able to fill your orders promptly, avoid unnecessary and costly overstocks, turn over your stock often, anticipate both the coming demands for new goods and the dying out of old demands, and have seasonable goods when you need them and be entirely out of them when the selling seasons end. And the net result is that the merchandising of your stocks will become a living and profitable part of your business.

What and When People Buy. The following is from an article by Theodore G. Morgan entitled, "I Discover Why My Store's Advertising Pays," published in System, the Magazine of Business, January, 1916. Mr. Morgan secured the assistance of the head of the psychological de-

\begin{tabular}{|c|c|c|c|c|c|c|c|c|c|c|c|c|}
\hline \multirow{2}{*}{ DEPARTMENTS } & \multicolumn{12}{|c|}{ YEARLY CHART OF BUYING HABITS } \\
\hline & JaRUaRY & FEBRUARY & MARCH & APRH & maY & UUNE. & HKY & August & SEPTEMBER & OCTOBER & HOWEMBER & OECEMAER \\
\hline Hockweat & 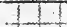 & 7111 & $1--6$ & $-1-=$ & $-1--$ & -21 & & & $\triangle 1 / 1-$ & $-1-$ & $--1-$ & --7 \\
\hline Hosiery & & & $1--$ & $1--\infty$ & $-\infty$ & $1 \quad 1$ & & & $11-0$ & $-2+$ & $1---$ & at \\
\hline Gleves. & & & $1 / 1-$ & $1--$ & --11 & I & & & $\angle 1 / 1-$ & $-1-$ & $-1--$ & -15 \\
\hline Boots & & & $1=-\cdots$ & $1--\cdots$ & ---1 & Na! & & & $1 / 1-$ & --1 & 1112 & --1 \\
\hline Whitewear & --1 & 11 & & \& i & & $-(--1)$ & & & $1---$ & --11 & & \\
\hline Corsets & & $1---$ & --- & Al & & $D: 1$ & & & $\square+$ & $\square 1$ & & $\because$ \\
\hline Malinery & & & $1 / L=$ & $-1-$ & -111 & $(--1)$ & 1 & & $1--$ & -211 & N & $\mathrm{W}$ \\
\hline Women's Suits & & & $V---$ & $1-|1\rangle$ & $D=1$ & a & & & --- & $N 1$ & 213 & 11 \\
\hline Men's furnistings & & & & 111 & --- & --- & & & 161 & --- & $110 / 4$ & -- \\
\hline Men's Clothing & & & 11 & $-1--$ & Ni $/$ & $|-|-11$ & & & & --- & $-\infty-$ & 11 \\
\hline Salks & & 1 & 1117 & $y---\cdots$ & -1.1 & & & 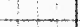 & $11-$ & --- & $-11<1$ & -1 \\
\hline Wash Goods & & & $1---$ & $-1-1$ & I : & & & & $\therefore \quad 1$ & $\square \quad$ & & DI \\
\hline Celored Dress Goods & & & $-\cdots$ & $-1 \times 1$ & & 1 & & 1 & $1--$ & --- & $\sqrt{11}$ & \\
\hline Linens and Cottons & - & & ---- & as 1 & 1 & & & & $1--7$ & --11 & 16 & 1. \\
\hline Fannels and Blankets & & & & & 1 & & & & $11--$ & --- & ---- & No! \\
\hline Ant Embroidery & & & $1: 1$ & 5 & 1 & 1 & & & $11:-$ & --- & $=--$ & -- \\
\hline Baby Carriages & & 1 & $-1-$ & ili. & $1+5$ & H. & & & & 51 & 43 & 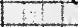 \\
\hline Toys & & & 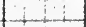 & $T=1$ & $1--$ & -11. & & 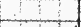 & & 11 & $11-$ & $-=$ \\
\hline Hardware & & & 1 & 1 & $--1-$ & --11 & & & ---7 & 111 & 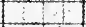 & \\
\hline Faney China & & & I & & $111-$ & $1--1.1$ & & & & $2 \quad 3$ & 11 & $-2-1$ \\
\hline Trunks and Bags & $=-1$ & & is & & $1 / K-$ & --- & -1 & & & 11 & & $1-1$ \\
\hline Wali Paper & & & & $-\angle L$ & $F--7$ & $\Rightarrow \quad 1$ & & & ---- & ---1 & & \\
\hline Draperies aod Curtains & & & 4 & 111 & $1---$ & W. & & 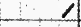 & ---- & --- & $(-\infty)$ & \\
\hline Carpets by the Yard & & & I & $11=-$ & ---1 & 1 & & 7 & $1 / 1 /$ & ---- & 111 & \\
\hline Rugs & & & $1-0$ & $--=$ & --11 & & & & & --- & -1 & 51 \\
\hline Furniture & & & & $1--$ & $-1--1$ & $1] 1$ & & 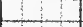 & $0+1$ & 111 & --- & $\mathrm{N}$ \\
\hline Books & & 1 & & $m_{i}$ & 111 & $1---$ & $y$ & $\square$ & 11 & 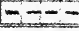 & ---1 & $1-1-1$ \\
\hline & & & 8 up to & & $-=$ & & & & Wn from of & & & \\
\hline
\end{tabular}

Fig. 15

partment of a university who gave him the theories and between them they worked out some practical and profitable results.

Our idea was that if we could define the habits of the shopping public, we could time our appeals to appear at the moment when they would be most effective. If we could do this we might greatly reduce such waste as had occurred in our advertisements.

So we made a careful study of the various departments, by personal observation, by reports from managers and salespeople, and through records of actual sales. What we wanted to do was find on what days and at what seasons the greatest number were in the habit of purchasing particular lines.

We uncovered many interesting facts. For example, we learned that materials sold by the yard, such as silks, dress goods, trimmings and 
wash fabries, were purchased on Mondays and Tuesdays in larger quantities than on any other days of the week. The most opportune time to present announcements of these materials, therefore, was naturally on Monday and Tuesday. By doing this, our tests showed that we greatly increased the pulling power and reduced waste.

In the same manner we discovered when the greatest number of people purchased dress patterns, trunks and bags, and wedding gifts; and we classified and reseheduled our appeals in these lines, as well as in other lines, to reach the public at times when habits made the greatest number of people naturally think of these particular articles.

The time to advertise wedding gifts for June weddings, for instance, is not in the middle of June, when the weddings are actually taking place, but during the month of May when people are receiving wedding invitations and deciding what they ought to give.

Likewise, the time to advertise fine trunks is not when the vacation season is in full swing, but from three weeks to a month previous, when preparations for travel are being made.

Careful study showed us, again, that the public expects bargains on Friday, and may come to stores on that day with no particular aim except to hunt bargains. We recognized the importance of this habit, and made it a point to place our incomplete assortments on sale on that particular day eaeh week.

It was easy for us to note, further, that on Saturdays there were more children in the store with their parents than on any other day in the week. The answer was simple-Saturday was the only day in the week that the children were free from sehool. By assembling all our children's advertising in the Friday papers we reached the homes when mothers were wondering where they should go to purchase things for their children the following day.

We noticed, too, that furniture is most keenly sought on Saturday afternoons and on Mondays. Saturday afternoons the husband can accompany his wife. The reason for Monday buying, we determined, was the Sunday holiday, luring whieh the husband and wife ean discuss the desirability of so important a purchase as furniture. It is perhaps the most important item purchased for the home, and is not usually bought without deliberation. Furniture appeals reaching the public, then, for Saturday and Monday shopping, were directly in accord with a clearly defined habit.

Still another discovery : there is great activity in ready-to-wear apparel and dress aceessories during the latter part of each week. Many buy then in preparation for a week-end outing, or in order to meet the requirements of Simday. And it requires so little time to select these things, that purchasing them can safely be put off until the last moment.

In "arrying out our plans, we first of all made a large "chart of demand." "This was a visual chart, based upon the experience of former years. It chabled nis, at a glance, to determine the volume of future demand, and to flan and prepare our advertising appropriation to coincide to the best alvantage.

The secret of sureessul luying and selling lies in judging this trend and strength of future clemand. With our ehart, the merchandise de- 
partment knows in just how many weeks demand will be at its maximum for a given line, and buying is permitted or curtailed accordingly.

For instance, actual merchandise figures show that a department is overbought. The manager of that department is anxious to go into the market for several new lines, and argues that a four weeks' season is just beginning, which will develop a strong demand for the new line. Often a buyer, in his desire to buy, is overoptimistic in this way. But the chart is a definite restraining guide, Fig. 15.

It will be seen how this shows the length of maximum demand, and how permission to buy can be given or refused largely by finding how long a period of maximum demand still remains.

Keeping the Cost of Selling Down. Edward Corrigan in an article "What It Should Cost to Sell," published in System, the Magazine of Business, October, 1917, tells of the method his concern has adopted to standardize and reduce selling costs. The following is taken from this article :

In the first place sales work is based upon an intangible commodity: human nature, in both salesman and customer. It is impossible to know exactly to the penny whether a salesman is producing at the minimum cost in a given territory. Again, some buyers are quick to decide while others will take a day to transact business that could be finished in an hour. Some customers are small buyers, and it is necessary to see them frequently; others buy heavily and need to be visited only two or three times a year.

We found, therefore, that we could devise no hard and fast system of cost, as we could in the factory. All that we could do was to work out a fairly accurate system.

Our first step was to fix for each territory what we considered an equitable standard of cost. In one territory the standard might be double that in another. Where selling costs by territories were already kept, they formed the basis of the standard. Where the costs as a whole only were available, it was necessary to work out an approximate cost for each territory. As the cost system developed, we adjusted the standard for each territory.

Unit of product, we found, afforded us the best basis in fixing this standard.

In addition to the standard selling cost, we established a quota of sales for each territory.

In figuring the actual cost, we give the salesman eredit for all business from his territory, whether he actually sends in the order or we receive it by mail.

In fixing both standards of sales cost and quotas, we felt justified in figuring the cost a little low and the quotas a little high. Of course, we had to be careful not to put the cost too low, or the quota too high, and thus discourage the salesman.

We try always to keep the idea of reducing the unit cost of sales before each salesman in the most convincing form. Any increase in the quota, of course, is a step tending toward the reduction of sales cost. We plot curves to visualize the facts. In Fig. 16 the heavy line is the quota for a territory covering a period of twelve months, and the light line shows the actual sales in the same period. 
With these figures so graphically before us, it is simple to make up new quotas each year. We usually plan for a certain percentage of increase in sales over the same months of the previous year. You will notice in the curve shown that allowance is made for the usual dull.

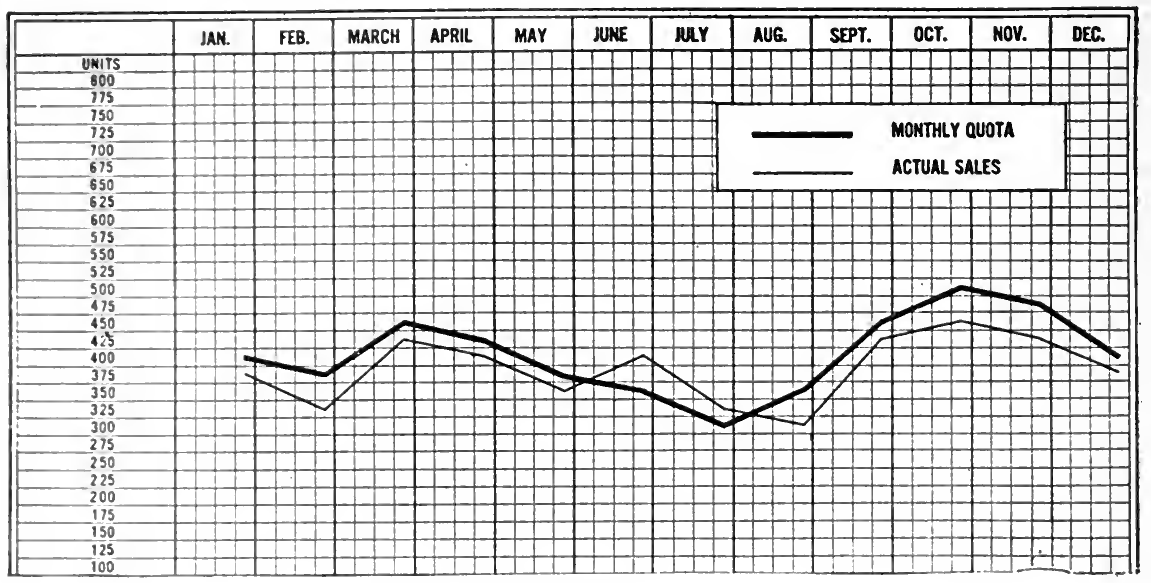

Fig. 16-Keeping Up to Quota

months of summer. As a rule, we find that a standard of sales eost or a sales quota should be based on the same month of the preeeding year rather than the previous month of the same year.

Fig. 17 shows the standard sales cost for a eertain territory. The

\begin{tabular}{|c|c|c|c|c|c|c|c|c|c|c|c|c|}
\hline $\begin{array}{l}\text { COST } \\
\text { PER UNIT }\end{array}$ & JAN. & FEB. & MARCH & APRIL & MAY & JUNE & JULY & AUG. & SEPT. & ост. & NOV. & DEC. - \\
\hline CENIS & & & & & & & 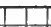 & & 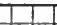 & & 1 & \\
\hline-90 & & & & & & & & & & & 79 & \\
\hline 86 & & & & & & & & \multirow{2}{*}{\multicolumn{5}{|c|}{ STANDARD SALES COST }} \\
\hline & & & & & & & & & & & & \\
\hline & & & & & & & & \multirow{2}{*}{\multicolumn{4}{|c|}{ - ACTUAL SALES COST }} & \\
\hline & & & & & & & & & & & & \\
\hline & & & & & & & & & & & & \\
\hline & & & & & & & & & & & & \\
\hline-14 & & & & & & & & & & & & \\
\hline 12 & & & & & & & & & & & & \\
\hline 70 & & & & & & & & & & & & \\
\hline 69 & & & & & & & & & & & & \\
\hline$=66$ & & & & & & & & & & & & \\
\hline-64 & & & & & & & & & & & & \\
\hline & & & & & & & & & & & & \\
\hline-60 & & & & & & & & & & & & \\
\hline$=53$ & & & & & & & & & & & & 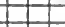 \\
\hline$\square 56$ & & & & & & & & & & & & \\
\hline $\begin{array}{l}54 \\
52\end{array}$ & & & & & & & & & & & & 1 \\
\hline $\begin{array}{r}52 \\
-50\end{array}$ & & & & & & & & & & & & \\
\hline & & $T$ & $T$ & & 11 & & & & & & & \\
\hline is & & & & & & & & & & & & \\
\hline$=4$ & & 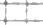 & & & & & & & & & & \\
\hline$=12$ & & & & & & & & & & & & \\
\hline & & & & & & & & & & & & \\
\hline & & & $t$ & & & & & & & & & \\
\hline & & & & & & & & & & & & \\
\hline
\end{tabular}

Fig. 17-Above or Below Standard

heary line represents the standard cost fixed for the territory, and the light line is the arotual reost.

On both of these charts the lines representing the quota and the standard cost are drawn in when the charts are made up at the begin- 
ning of the year. At the end of each month the curves showing the actual sales, or the actual cost to date, are drawn in and sent to the salesman.

Knowing Just What Your Salesmen Are Doing. Mr. Stanley C. Tarrant, in the course of an article published in System, the Magazine of Buisiness, December, 1915, entitled "Graphs for the Sales Manager," tells how a sales manager uses charts to keep a record of his salesmen. See Fig. 18.

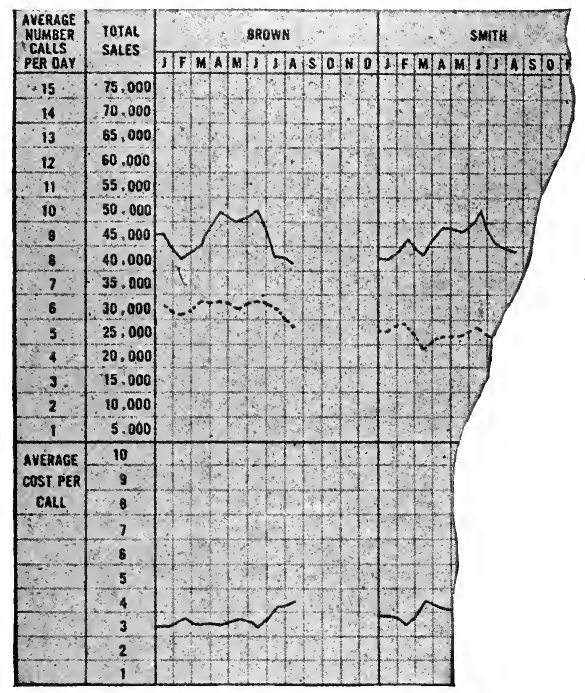

Fig. 18-For the Sales Manager

The chart is drawn on millimeter paper. A six-foot length of this paper takes care of the records of 27 salesmen. It shows graphically 7 facts about every salesman. In the upper portion, the following facts appear :

1. Number of orders obtained: black line;

2. Value of orders obtained: black dotted line;

3. Number of calls made: green line;

4. Average value per order: green dotted line.

On the lower portion of the chart, these facts are shown:

1. Average cost per order (salary, commission and expenses) : black line;

2. Average cost per call: black dotted line;

3. Average cost per $\$ 100$ of orders: green line.

These 7 points are plotted and drawn on the one chart to show the records of 27 salesmen in less than 4 hours a month.

To secure the information which goes on the chart, each salesman is provided with a supply of post cards. He fills out one of these every day and mails it to the office. On it, in the convenient spaces it provides, he shows the number of calls he made during the day, the number of orders he obtained and the value of these orders. 
When the cards are received at the office, the information each carries is transferred to the salesman's individual summary and at the end of each month the figures on this form are totaled and the averages figured.

To Interest Salesmen and Increase Sales. A form of chart intended to excite interest and speed up sales is shown in Fig. 19. This and the following article, "Taking Salesman's Selling Temperature," appeared in Motor World, April 25, 1917. The Grasser Motor Co., Hupmobile dealer, Detroit, is speeding up spring sales by holding a salesman's contest during the month of April.

First, G. Edward Bleil, branch manager, estimated how many cars

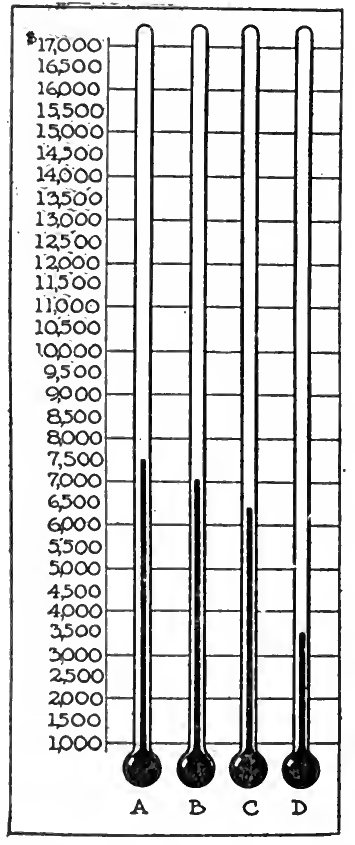

Fig. 19-Salesman's Selling Temperature

(onght to be sold in the month. He knew that all his salesmen were not mual, and hemere could not sell proportional parts of this estimate. So (1) put the (ontest on a fair basis, and to prevent diseouragement, he sot an definite number of cars the men must sell to qualify for a prize.

To win first prize, $\$ 100$, the salesman must sell at least 12 ears; to win second prize, $\$$ is, 10 wars, and third prize, $\$ 50,7$ cars.

The preored of thr salesmen is kept on a chart, the amounts sold being shown by the hoight of the eolumn in the thermometer representing the individual salesmant so sales.

In creder fo prevent the men helding back on their sales and to induow them to make a geosed record through the contest, Bleil did not annomere the antest mit April 1. Ilence the men all started equal.

The contest was precedon by an advertising campaign that tended to plongh up the prosusen ficld and stimulate the sales, but the main effort 
was concentrated in creating a sporting spirit among the salesmen themselves. The sales contest did this.

"Curves That Plot the Performance and Effciency of the Salesman," following, was published in the Electrical World, August 12, 1916.

The Elmira Water, Light \& Railroad Company of Elmira, N. Y., employs a graphical method for following the work of its salesmen. As explained by F. H. Hill, general manager of the company, the salesmen's efforts are reduced to a curve, Fig. 20.

This particular salesman, it will be noted, is an extremely active man, making a very large number of calls per day during the month for which the curve was compiled. The percentage of his sales and his calls, however, is also quite high, and the cost per sale is extremely low. By the word "sale" is meant the securing of a meter contract. The man whose work is indicated on the curve sheet is exclusively on residence work.

At the end of each month each salesman's report is made up on this

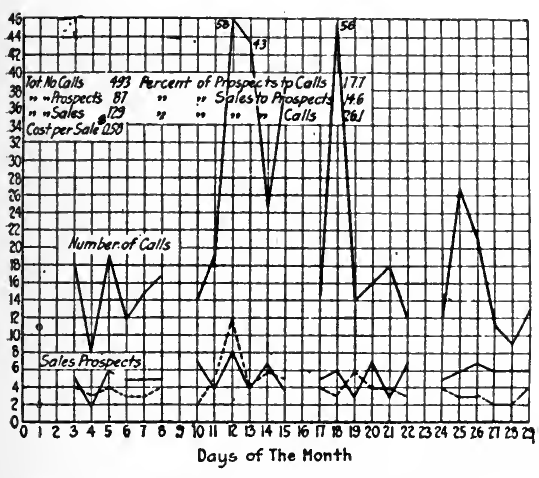

Fig. 20-The Efficiency of the Salesman

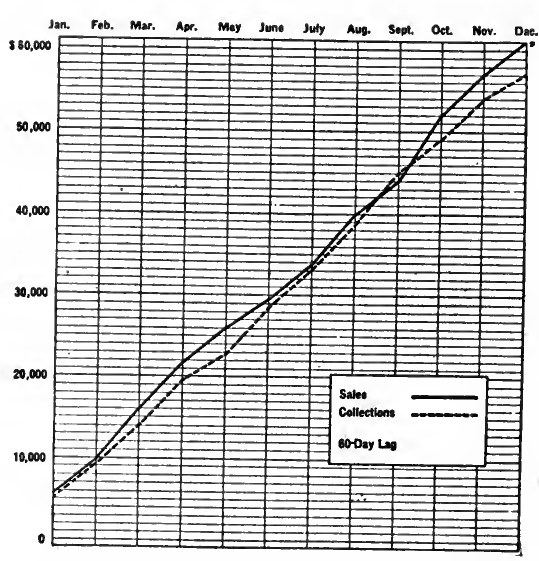

Fig. 21-How Collections and Sales Agree

form and each salesman is given a copy, not only of his own but of every other man's record. "We find," comments Mr. Hill, "that each salesman spends more time in studying the other man's record than he does his own, endeavoring to find out how his competitor does it."

Keeping Tabs on Collections. The following, from an article in System, the Magazine of Business, June, 1918, "Getting Your Money When It's Due," illustrates the value of the chart in his connection. Mr. Horace S. Griswold, who uses the chart, says: (See Fig. 21).

"Here are plotted cumulatively, from month to month, sales and accounts received; using the accounts received not of the same date as the sales, but two months later. January sales are compared with March collections. January plus February sales are compared with March plus April collections. The same relation holds throughout. Working on a 60-day basis if accounts are collected up to date, the accounts-collected line should nearly parallel with the sales line.

"Seasonal lags and spurts in collections are thus clearly shown, and 
the general relations between collections and sales can be properly maintained. The objection might be offered by some that moneys collected during March might apply on sales in March or any prior month. That is true, but in spite of some hits and some misses the general relation holds nearly enough true to make the chart effective.

"I find that the use of the graph for general conditions, and cards for individual variations, serves to warn me of dangerous conditions and tends to keep collections where they should be."

- Labor Turnover. E. Goldberger, in an article, "Labor TurnoverDiseussion," published in Industrial Management, November, 1918, states, in part, that the Packard Motor Car Company, of which he is efficiency engineer, have investigated this proposition repeatedly and

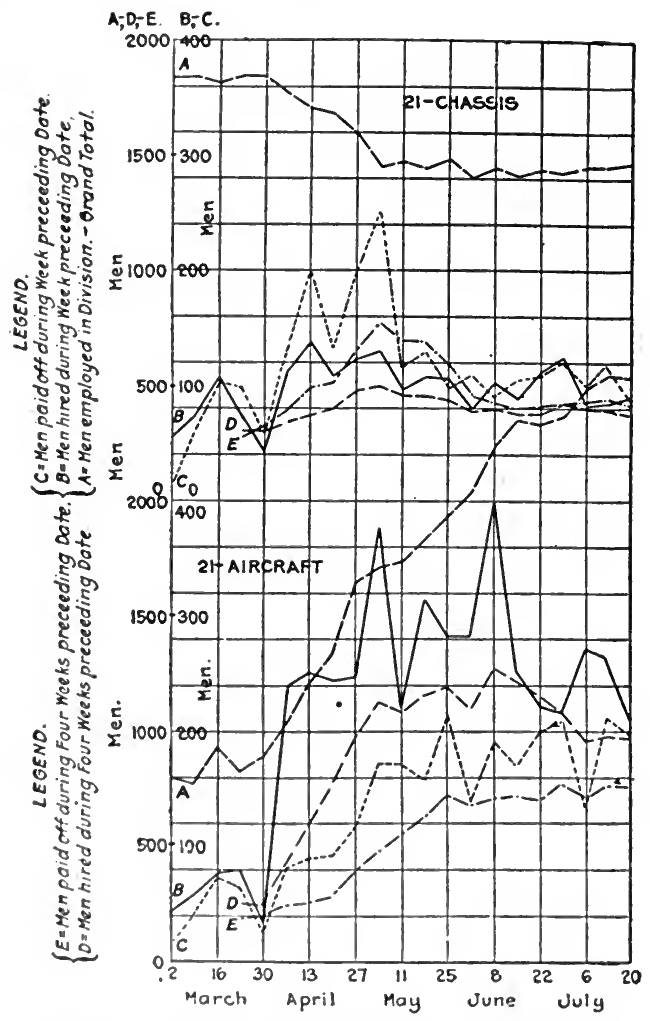

Fig. 22-('hart of the Vital Statistics of Employment

that they have adopterl and applied the following method, which two charts, Figs. 220 and '2:3, help to make clear.

Fig. 220 shows:

A. Frand total of men employed in the division.

13. Total number of men hired during one week preceding the date markerl on the horizontal co-ordinate (this includes new men, rehired, re-instated and transferred in). 
C. Total number of men paid off during one week preceding date marked (includes, resigned, laid off, discharged and transferred out).

D. Total number of men hired during four weeks preceding date.

E. Total number of men paid off during four weeks preceding date.

For figuring the labor turnover I have adopted a new standard that gives a better index as to the labor condition in a division.

1. Instead of one week's figure for hired or paid men, I take the total of four consecutive weeks, which then gives an evener slope in the curves expressing turnover tendencies.

One week is too short a period from which to draw any intelligent conclusion, since the variation between two following weeks might represent a couple of hundred per cent.

2. The total number of men that have been hired and been paid off, $i$. e., actually replaced, represents a complete turnover, and if there be an excess of men hired over paid off, they actually are to be accounted to increase in force, while if there be an excess of men paid off, they represent a decrease in force.

The term "fluctuation of labor" ( + if increasing the force, - if decreasing) is used to express these figures, since it leaves the term "turnover of labor," to represent only what its name implies.

It is important to make this distinction, since avoiding a higher labor turnover calls for one type of preventatives in the employment and factory departments, while a high fluctuation is either unavoidable or the preventative means must be taken in the production and sales departments.

3. To avoid using the information after it has become one month old, from month to month I figure the turnover and fluctuation every week for the past four weeks; this gives 52 chances for intervention instead of only 12 per year.

The labor turnover, then, is the ratio between the total replacements in a division during the four weeks preceding the date of figuring, and the grand total of men employed (on our books) at the end of that period. This figure multiplied by 13 gives the labor turnover percentage reduced to a yearly basis.

The above rule naturally implies that if a man has left for certain reasons, and another one has been hired during the four weeks following his departure, a replacement has taken place which has to be accounted into turnover, but if he be not replaced at all, or replaced only after more than four weeks, it is accounted as fluctuation.

Fig. 23 gives both the turnover and the fluctuation (above the o line if increasing, below if decreasing the department's force). They are both figured in per cent (i.e., taken over the total number of men employed in that division during that week). The figures on the left side of the column read the percentage for the elapsed four weeks (almost a month) and those on the right side the same, reduced to a yearly basis.

The prevailing practice in figuring labor turnover is: Multiply the number of men hired (or leaving) each week by 52 and divide the result by the grand total of employees. This leads to wrong conclusions since it is neither the hircd, nor the leaving men that constitute the basis of labor turnover. 
Both methods (hired or leaving men as basis for turnover) and their error appear in Fig. 22, where in two different divisions the conditions are directly opposed. Either way of figuring would affect, considerably, the factor representing turnover. Actually, the true turnover cannot be so different in two divisions under the same management and same scale of wages.

Of course, the same conclusions could be drawn if, instead of two divisions, we would consider two plants.

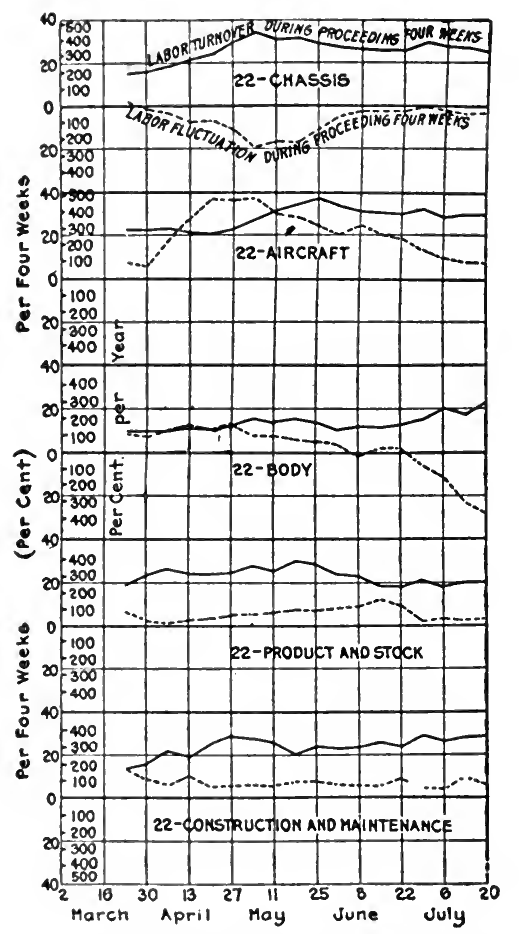

Fig. 23-Chart of Jabor Turnover and Labor Fluctuations-Two Departments

The following remarks amplify the preceding:

1. Transfors in or out are alway's counted as hirings or leavings, keeping in mind, of course, that a man transferred from one department to another one of the same division is to be figured in the departmental turnover but not in the divisional turnover.

2. Cumber of m'n on the books or men actually working. a. There are more reasons in favor of counting the men on our books rather than those really attonding, as some men leave without notice, passing through a transitory stage of not attending and still being on the books; so that, when they leave and are eonnted in the turnover, they do not represent the turnover of the force working, but of that on our books.

b. It is much rasier to count the men on the books than those attending, as the first information can be made available quickly in the em- 
ployment office, while the last one has to be made available by many clerks in different departments or by the time office.

c. The disturbance of the balance of forces in the industrial conditions of the country has compelled us to consider the problem of labor turnover. It is very likely that high wages, where they are unwarranted, will force us to face the problem of absentees, and to study a standard coefficient of attendance. There can be no doubt but that this is represented by the ratio of elapsed straight man hours ( $i$. e., not including overtime) over total available man hours on the books (similar to the ratio of output over input in any power machine). This is, in my opinion, a good enough reason to keep the same denominator for all three coefficients, labor turnover, labor fluctuation and labor attendance.

3. Standard period. A day or a week are too short periods from which to draw intelligent conclusions (see the figures), and a fourweeks' period has an advantage over a monthly period since the last one varies almost $10 \%$ in length, which would also affect our coefficients. It takes about one hour per week to plot the curves shown for all 15 divisions of our plant.

4. Unavoidable replacements. Employment managers like to see the turnover figures as low as possible and favor eliminating the "unavoidable replacements" from the figures expressing turnover. There are, however, two reasons why we have preferred not to make this elimination. First, the unavoidable replacements cost just as much money as the other ones, and, second, how are we going to agree upon a standard deciding what is and what is not an unavoidable replacement?

5. Average number of men on payroll. It would be more correct, from a mathematical standpoint, to take as the denominator of the ratio expressing the labor turnover the average number of men on the payroll during the four weeks preceding the computing of the turnover percentage. To do this, we would have to take one-fourth of the sum of all men on the books on every one of the four weeks. This would be a trifle more complicated than to take the number on the books at the end of the four weeks period (as we now do) and would be only a trifle more correct for practical purposes. A standard should be agreed upon in this case.

New Method for Determining Stability of Working Force. In an article "Labor Maintenance and Its Indices," published in Industrial Management, February, 1919, Winthrop Talbot offers a new method for calculating indices to show the stability, maintenance and replacement of the working force. The value of charts in helping to make new ideas clear is well demonstrated.

Labor maintenance is keeping a required force of workers steadily at work.

When a laboring force is maintained at a required number without change, labor maintenance is effective, for a steady force, on a steady joh, at steady pay, helps to keep lahor costs at a minimum. Employment changes occurring naturally and inevitably in industry are not only to be expected, but in general are not wasteful. In essentially seasonal occupations like the canning industry they contribute essentially to economy and profit. 
It is the unnecessary changes in personnel oceasioned through faulty employing methods, poor foremanship, bad working conditions, inequitable pay, defieient plamning and scheduling, which may often cause enormous and needless expense for supervision, training, time, material, power, machine upkeep, and wages.

Such expense is largely avoidable, and is often so great that much time and thought has been devoted to devising ways and means for expressing the relative number of such useless changes in personnel. In so doing emphasis has been laid on the changes themselves, rather than on the means of prevention. We have been interested more in labor turnover than in labor maintenance. We have concerned ourselves rather with negatives than with positives; with the effects of destruction more than with the means of eonstruction; but in this period of industrial reconstruction the time is at hand to turn ourselves to the processes of building up rather than to the effects of tearing down.

In sharp contrast with labor maintenance, labor turnover denotes merely change of personnel without reference to causes. It is commonly expressed in terms of percentage. This percentage is ealculated by various methods, usually by dividing the number of exits by the number at work. These percentages of labor turnover are misleading when used for purposes of comparison, but unless they may be so used they are almost valueless. The main reasons why they are misleading are beeause the base or number of workers, the amount or number of exits or entrances, the time or payroll period such as days, weeks, months, or years are all variables, or quantities whose value changes in the same discussion, and as a result the percentage becomes a variable also. If the value of a quantity changes, the value of a function of the quantity will change. These changing values arouse endless debate.

We may justly state in terms of pereentage the number of persons employed, or the number of persons discharged on any given day compared with the whole number at work or on the payroll on that day.

We may compare by a percentile figure the number of persons employed or discharged during any given week with the average number at work or on the payroll during that week.

Similarly, we may give the percentage for ins and outs for one month or one year, but when we try to extend our percentile figures from one day to many days, or from one week to many weeks, or from one month to many inonthis, or from one year to many years, it is evident that owing to the variables, and especially the time variable, our percentile reclationship becomes misleading.

Thus we are forcect to the obvious conchusion that the term percentage of labur turmover may be used correctly only for the relationship of ins and onts to the total number of workers for one day, for one wrok, for uns momih. for onc year, and in general for one plant, in one industry, in one locality.

The faretors afferefing the stability of the base, i. e. the total number of workers are so varion and so intimately dependent on: Expansion of the business, seasinal fluctuation, special produetion; as well as such important matters as: Formanship, rate of pay, hours of labor, season of year, nature of industry, work conditions, health of workers, training of workers, common language locality, source of labor supply, race, 
age, sex, that the usefulness of the term, labor turnover percentage, reaches the vanishing point.

There is a fashion in the use of language, just as there is a mode in the fashioning of clothes, which passes with the season or the period. The vague term, percentage of labor turnover, is destined to disappear from our industrial vocabulary. Its present use is unscientific.

In any case, what we are afțer is labor maintenance rather than labor turnover, for the former determines costs, and costs are the main issue of expanding industry and commerce.

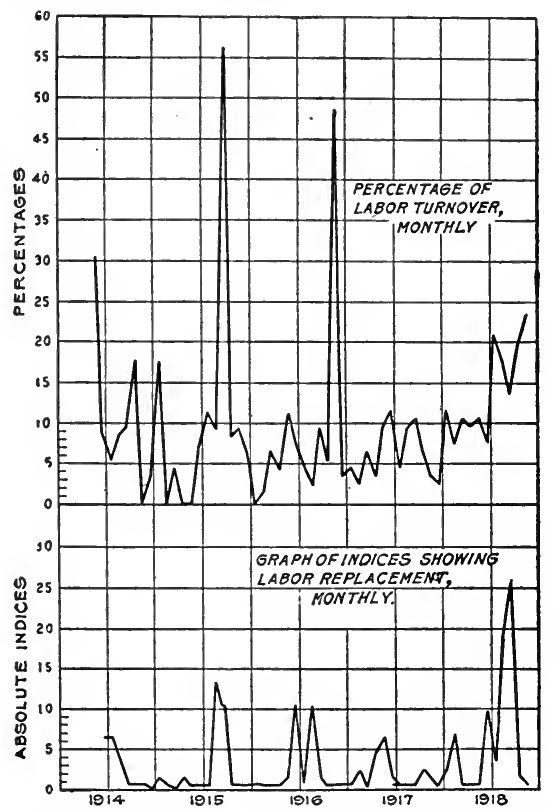

Fig. 24-Labor Turnover-Indices of Labor Replacement

To discuss labor maintenance with profit, it is important to establish, if possible, a method of determining accurately and simply the degree and type of labor stability in any business throughout any period of time.

The accompanying maintenance of labor chart, Fig. 25, shows such conditions as taken from the payrolls of a successful producing concern, manufacturing a line of goods which sells more freely in the summer than in the winter; in other words, a partially seasonal industry. It presents the annual growth of this newly established plant, as well as its seasonal fluctuations in employment, taking on relatively large numbers in the early spring and laying them off in the late autumn. It also demonstrates clearly the unnecessary changes taking place during the height of production.

The chart includes a period of nearly five years, since the inception of the business in 1914: In the background are sketched the numbers of persons at work month by month. Above the base line in heavy 
black is the number newly employed each month, and below the base line is the number who have left during each month.

Beginning in May, 1914, with 29 workers, 9 left during the month, and in June, 12 were employed, thus making a net gain of 3 for June. During June, 3 left, and 4 were employed in July, thus making a net gain of 1 for July. As we follow the ehart from month to month we note in June, 1915, a largely increased number of workers taken on with a consequent falling off in September. In March, 1916, again came a busy season ending in November. In 1917, the busy season commenced in Mareh and fell away gradually in the autumn. Again in Narch and April, 1918, there was not only the seasonal growth, but the growth of the business itself is shown as in the two preceding years by the greatly inereased number of operators.

With each seasonal fluctuation it is plain that large numbers of new

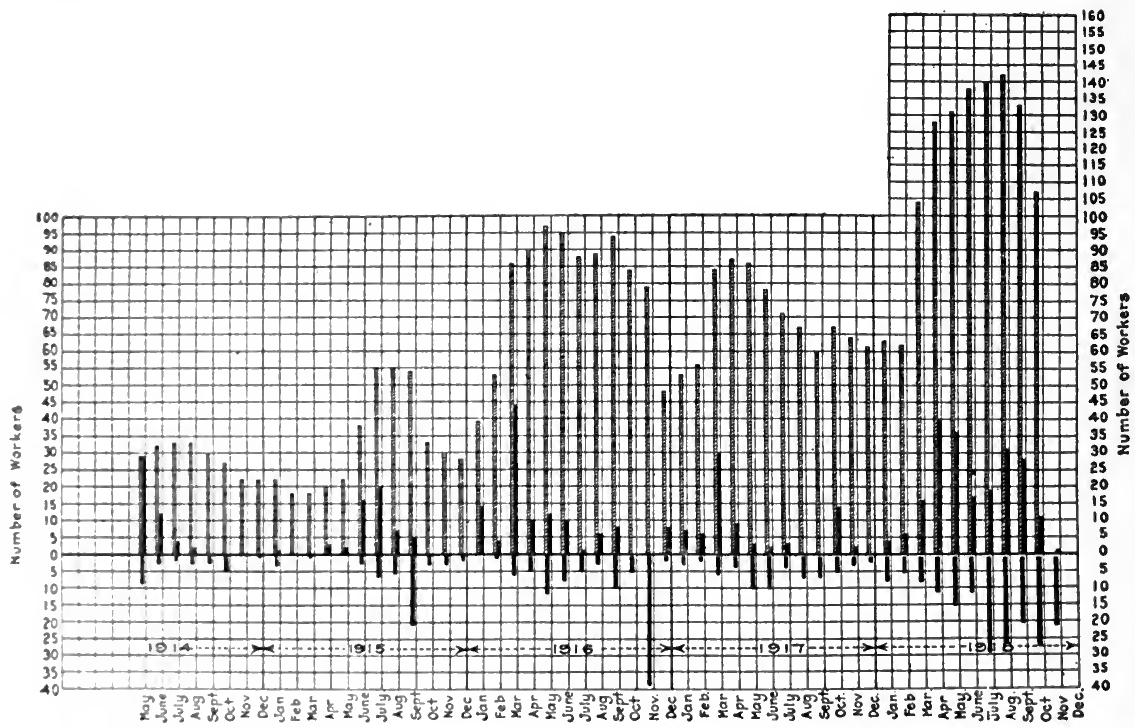

Fig. 25-Lahor Replacement Chart Showing Number at Work, Number of Entrances and Number of Exits, Monthly

workers had to be employed, and at the elose of each season many had to be laid off. This is especially evident in 1915 and 1916. Sueh recpuired increase and reduction in numbers are distinctly in the line of eeonomy and shomkl not be placed in the same eategory with those changes of a regrotable type oceurring in the busy intervening summer months of greatest production. In 1918. for instance, at the time of need for strady workers on steady jobs at steady pay the replacements totalleel more than the full forcee.

To Lain a fairly areourate "onception of change of personnel throughout other divisions of the same organization, similar eharts should be made for each. Worked out for divisions employing large numbers 
of foreign laborers and having much greater labor fluctuation, the results are striking and illuminating. In the present case it was thought to be most helpful to discuss a well-managed type of concern, rather than to use as an illustration the shortcomings of a plant poorly supervised or disadvantageously situated.

With this picture of the monthly entrances and exits before us, we are impressed with the need for disinguishing between necessary and unnecessary additions, as well as between necessary and unnecessary separations. Then too we ought to be kept informed how much the working force is increasing or diminishing from one payroll period to another. No labor turnover percentage is capable of indicating this distinction.

It is desirable to fix some standard or index of comparison which shall not be misleading and shall be expressive of what we need to know, namely :

Requisite fluctuation in personnel

Number of changes required to meet these fluctuations.

Total number of changes which actually take place.

Index of stability. If no workers are needed and no changes are made from one payroll to another, our index is $\frac{0}{0}$, the index of stability.

Index of maintenance. From day to day or week to week the number on the payroll varies, the number increases or diminishes. The amount of variation may be termed the fuctuation factor. To meet these variations and supply the necessary increase or decrease, workers must be hired or laid off. These-constitute the requisite change of the total changes made, and are identical with the fluctuation factor. The relation of the requisite changes to the fluctuation factor, or

\section{Fluctuation factor}

Requisite change

is an index which may be expressed as an index number, i. e. $\frac{1}{1}$.

Example: If three more workers are needed and three more are added, our numerical index is $\frac{3}{3}$, which, reduced to lowest terms is 1 an absolute index of $\frac{1}{1}$. This is our index of maintenance.

Index of replacement. The numerical index of replacement is an exact statement in numerals of requisite changes related to total changes, and is of direct value to the industrial manager or employment director as a check on employing costs.

The absolute index of replacement is of special statistical value for comparing the worth of employing and management methods of one concern with another, when it is not desired to state the actual numbers 
employed. It serves every purpose of a labor turnover percentage and is preferable in that it is accurate.

The closer the replacement index comes to the maintenance index of 1

-, or to the index of stability, -, the lower are the indieated employing 1

costs. A high replacement index such as $\frac{5}{1}$ or $\frac{7}{1}$, that is, where the total changes are five or seven times the requisite changes (or the fluctuation factor) the indicated employing costs are five or seven times as large.

Such changes made for the purpose of maintaining the force are in the line of economy of administration. We are not concerned particularly with such requisite changes, and yet such changes are heavily represented in so-called labor turnover percentages. We are concerned mightily with any other ehanges, all of which are costly.

The relation of requisite changes or the fluctuation factor to total changes is of prime importance. This relation expressed by

$\frac{\text { Total changes }}{\text { Fluctuation factor }}$ or $\frac{T . C .}{F . F .}$

is our index of replacement.

Example: If we require seven new workers during a payroll period, and twenty-one total changes of exits and entrances are made, the 21

numerical index of replacement is - and the absolute index of replace7

3

ment is $\frac{-}{1}$. This index of replacement holds good for any payroll period of time, whether it be a day, week, two weeks, half a month, month, or year. The numerical divisor 1 is retained to indicate maintenance as contrasted with the 0 of stability.

'The table gives the indices of replacement for each month in the maintenance of labor chart showing how the index may be applied to indieate the measurement of efficiency in employment methods and managrement in any plant.

It is necessary to stress the meaning of total changes. We should $r$ member that the main value of replacement figures is their relation to cost. There is expense incurred whenever a name is removed from a payoll and whenerer a name is added to a payroll. Therefore, in establishimg an index figure of replacement which shall aid in determining labor cost wo must (alculate with the sum of exits and entrances or the total number of changes. The torm replacement is held to include the whole number of changes made in addition to and excepting those fluctuation changes marle neressary by the increase or the decrease of the business.

The following table covers a period of five years, and is ealeulated on the number of exits as related to average number on the pavrn". 


\section{INDICES OF LABOR REPLACEMENT, 1914-15, BY MONTHS}

\begin{tabular}{|c|c|c|c|c|c|c|c|c|c|c|c|c|c|}
\hline & & Jan. & Feb. & Mar. & Apr. & May & $\underset{7}{\text { June }}$ & $\underset{7}{\text { July }}$ & $\underset{4}{\text { Aug. }}$ & Sept. & $\begin{array}{c}\text { Oct. } \\
\text { I }\end{array}$ & $\begin{array}{c}\text { Nov. } \\
1\end{array}$ & $\begin{array}{r}\text { Dec. } \\
\text {. }\end{array}$ \\
\hline \multirow[t]{2}{*}{1914} & $\ldots$ & $\cdots$ & . & $\ldots$ & $\cdots$ & .. & - & - & - & - & -- & -- & - \\
\hline & & 2 & 1 & 0 & 2 & 7 & 1 & 1 & 0 & 1 & 1 & 1 & 0 \\
\hline \multirow{3}{*}{1915} & $\ldots$ & - & - & - & $z$ & 1 & 1 & 1.3 & 14 & 11 & 1 & 1 & 1 \\
\hline & & 0 & 1 & 0 & 1 & 1 & 1 & 1 & 0 & 1 & 1 & 1 & 1 \\
\hline & & $1.2 \tilde{5}$ & 1 & 1.04 & 1.25 & 2.4 & 11 & 1.8 & 11 & 2.4 & 1 & 1 & 1.5 \\
\hline \multirow{2}{*}{1916} & ... & 1 & $\overrightarrow{1}$ & $\overline{1}$ & 1 & 1 & $\overline{1}$ & $\overline{1}$ & $\overline{1}$ & -1 & $\overline{1}$ & 1 & 1 \\
\hline & & 1.8 & 3 & 1.15 & 5 & 7 & 1.5 & 1.8 & 1 & I & 3 & 2.3 & \\
\hline \multirow{2}{*}{1917} & $\cdots$ & 1 & 1 & 1 & 1 & I & $\overline{1}$ & 1 & $\overline{1}$ & $\overline{1}$ & $\overline{1}$ & $\overline{1}$ & \\
\hline & & 3 & 7 & 1.9 & 1.5 & 1.8 & 10.6 & 4.3 & 20 & 27 & 2.8 & 1.07 & \\
\hline 1918 & * & 1 & $\overrightarrow{1}$ & 1 & 1 & 1 & 1 & 1 & $\overline{1}$ & $\overline{1}$ & 1 & $\overline{1}$ & \\
\hline
\end{tabular}

\section{LABOR TURNOVER PERCENTAGE}

The following table covers a period of five years, and is calculated on number of exits as related to average number on the payroll.

For the whole period of five years:

Average number on payroll ................ 61

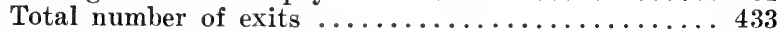

Percentage of labor turnover ............... 710

For each year .................... 1914

$\begin{array}{rccr}1915 & 1916 & 1917 & 1918 \\ 32 & 77 & 69 & 101 \\ 60 & 96 & 65 & 186 \\ 187 & 125 & 91 & .184\end{array}$

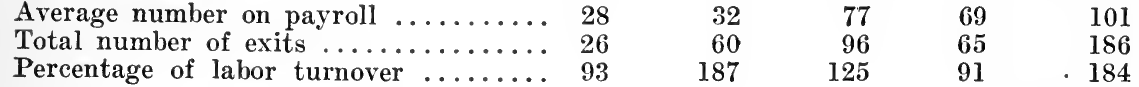

For the whole period of five years:

Death, accident, disease, or removal are commonly regarded as necessary causes for replacement. To a limited extent this is true, yet often death itself may be averted, accident prevented, disease avoided, and removal obviated by better methods of management and administration. But whether these factors play a large or a small part, whether they may be regarded as avoidable or unavoidable, is not pertinent-because every replacement due to these or any cause costs money, and as an expense is bound to be one of the essential elements of cost. For these reasons they are included in the index of replacement.

The common method of estimating percentage of labor turnover upon exits or entrances alone is clearly fallacious. It is manifestly an absurdity to reckon upon exits alone when heavy expense is incurred through hirings, and if as may easily occur a week is chosen for calculating the turnover percentage when the exits are heavy, as for instance at the closing of an industrial season, and the labor turnover for the year is calculated upon the figures for that week, absurd conclusions are reached. It may be said that a typical week or payroll period is to be selected, but who can say what is a so-called typical week in a constantly changing industry?

It is not enough to be concerned with exits or entrances alone. Each replacement involves both an exit and an entrance. A certain expense is incurred in putting each name on a payroll as well as remowing each name from the payroll. Therefore, replacement costs must 
deal with the whole cycle of employing, and include both the person coming and the person going.

The foregoing study of replacement has been made wholly from actual payrolls and with care, to discover if possible a simple, practical, time-saving, accurate method for the industrial manager to ascertain daily, weekly, monthly, and yearly, the relationship in requisite changes to total changes of personnel with the view of quickly ascertaining data necessary to reduce costs and maintain a steady organization.

To fix responsibility for poor foremanship or administrative shortcomings in keeping a steady force, it is necessary to determine the relative replacements of different departments. By the use of this index of replacement the spirit of emulation may be aroused in all the departments of a plant to maintain efficient employment methods, very much as similar emulation has served to reduce the accident hazard.

It is believed that in the index of replacement a means of accuracy in statement is reached, and in the replacement chart a means of graphic illustration is advaneed, both of which through their simplicity and accuracy may become of essential value as means of finally enmeshing that elusive object of our constant industrial search, the Cost of Labor.

Cost of Labor Turnover. E. H. Fish, in the March, 1919, Industrial Management, illustrates graphically his computation of the cost to train a new worker.

The problem is to diseover what it eost the Blank Manufacturing Company to allow John Smith to leave and to fill his place with John Doe. It is a simple case in that only the one attempt was necessary

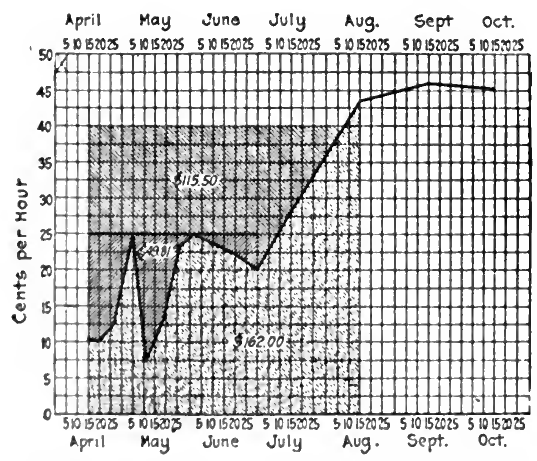

Fig. 26-Craphic Representation of Wages

to fill the job. The wages paid to Doe are shown graphically in Fig. 26. We are neglecting any mention of the actual eost of handling the separation of sinith from the company, and discovering and hiring Doe. Each of these onrations on the average costs a little less than a dollar. so may well bre neglected, seeing that we are by no means sure within many follars of the other costs.

It will be sern from the chall that during the time Doe was learning to go alone wre paicl him $\$ \$ 9.81$ more at day rates than he would have received if we had haid him at piece rates for the work which he accom- 
plished well enough to pass inspection. This we are sure is a part of the cost of replacing Smith.

At the time this change took place we found that we made an acceptable profit when our piece workers were making $40 \mathrm{cts}$. per hr. The only excuse for retaining men making less than that was that they were learners and could be expected to make more later on, so that they would repay us for the loss in production. During the time before Doe reached the 40 -ct. rate we would have paid him $\$ 115.50$ beside the $\$ 49.81$ mentioned above, if he had been a normal producer.

During all this time our manufacturing overnead was practically $\$ 1$ an hour. From the time he came with us until he passed the 40 -ct. rate of productivity the overhead charge totalled $\$ 800$ which, however, has actually to be spread over the $\$ 162$ of work which he actually did, or practically $500 \%$ overhead against $250 \%$ for the average man. From this we eonclude that one-half of this $\$ 800$ for overhead charges has been wasted, and add to the account against the change, $\$ 400$ less the $\$ 115.50$ which we did not pay him for services which he might have rendered but did not.

The total cost for the training period by this method of computation then would be:

Paid hour rate above piece rate earnings $\ldots \ldots \ldots \ldots \ldots \$ 49.81$

Excess overhead charge incurred ..................... 248.50

Total cost

$\$ 334,31$

Another way in which this may be figured, but which seems to make it still more expensive to change help is as follows:

Total wages paid Doe while reaching normal productivity.......

Overhead paid during that time per $\operatorname{man} \ldots \ldots \ldots \ldots \ldots \ldots . . . . . .$.

$\$ 211.75$

800.00

Value of work done

$\$ 1,011.75$

Overhead which would have been paid if it had been done by

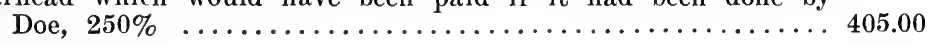

Net total cost

567.00

$\$ 444.75$

Possibly, however, the second method is not merely simpler but more nearly correct.

It has seemed to the writer that it was better to put all the expenses outside of actual training under the general charge of overhead as that is where they show in most accounting systems. If we attempt to pick out all the items of spoiled work, damaged tools and machinery, additional expense for accidents and so on we get into a maze of uncertainty. Neither of the methods suggested above may be the best possible but it seems that if we wish to evaluate the work of service departments we must do it on some approximate basis which uses the material offered by the regular accounting systems of the factory, and which can be applied both to individual cases and to department turnover as a whole. This case cited is one where the payment of piece rates with an hourly rate during part of the learning time simplifies the problem of earnings, but except in new jobs it should be possible under any modern system of cost-keeping to compare a man's actual 
earnings with those which are average, or neeessary to the running of the shop on a fair profit.

Chart Records for the Employment Department. The following is from an article by Russell Waldo, published in Machinery, February, 1919, entitled "Use of Charts in the Employment Department":-

Fig. 27 is shown merely as an illustration, but the full set of charts for keeping records of the employment department may consist of five or more similar to the following:

Chart 1 should be similar to the one shown in the accompanying illustration and gives the number of machines idle, operating, and new machines. The yellow line shows the new machines added; the red line, the number of machines idle from lack of operators; and the green line, the number of machines in operation. The total number of machines is recorded by the black line.
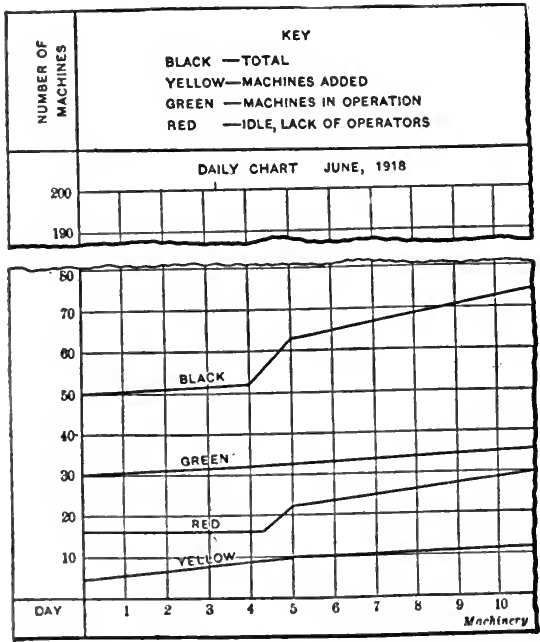

Fig. 27-Chart Used in Employment Department

Chart 2 shows the status of employes. The full black line represents the iotal number of men on the payroll and the black dash line, the number of women. The full yellow line shows the number of men sick, and the dash yellow line, the number of women. The number of men present is represented by a full green line and the number of women present, by a green lash line, while the full red line represents the number of men absent, and the dash red line, the number of women absent.

Chart :3 should recort the weekly labor eonditions. The number of employes dischared should be shown in black lines, and the number leaving voluntarily, in red lines. Green lines should be used to show the number of persons hired. In all cases the full lines refer to men employes and dash lines to women employes.

Chart 4 should record the work of the employment department. It should contain a record of the number of men interviewed, which 
should be shown by a full yellow line. The number of men hired should be shown with a full green line, while the number of men needed should be shown in a full red line. The same color lines should be used to record the conditions in the women's employment department.

Chart 5 is of importance in that it shows the attitude of employes toward their work, and is more in the nature of a personal record than any of the other charts described. A record of the number of employes receiving promotion should be shown upon this chart with yellow lines. The number of employes studying for promotion should be shown with green lines, while the number of employes not studying for promotion should be shown in red. Some interesting percentage figures can be obtained by comparing the total number of employes, shown by a black line, with the number of employes studying for promotion as shown by the green line. While the set of charts described forms a complete record for the employment department, variations can, of course, be adopted to meet special requirements.

Employment Methods. In an instructive and interesting article published in Industrial Management, January, 1919, entitled "Installing Employment Methods," Wm. A. Sawyer presents some excellent suggestions for the use of charts in connection with the employment and health department of the American Pulley Company. Excerpts follow:

One of the vital questions of the day in connection with industrial employment is that of adequate compensation. The higher the wages, other things being equal, the more attractive the position. To some, wages are the controlling factor; to others (and this is particularly true of the finer type of worker), a place may not be attractive even at high wages, especially where the surroundings are unpleasant or where there is dissension and ill feeling. Obviously, some firms cannot pay as high wages as others; these, in order to compete in the labor market, must offer steady employment, good working conditions, and that consideration of the individual which accords with the more modern practice.

The American Pulley Company, Philadelphia, Penn., employs between 500 and 600 men. A concern making a staple commodity in the face of vigorous competition, cannot pay such wages as can be given in cases of emergency. It is difficult to keep pace with the golden offerings of some of our war industries, whêre a man after receiving a week's pay, feels his affluence to the extent of taking a week's vacation. However, since 1914 there has been a general increase of wages amounting to about $70 \%$, which is commensurate, we believe, with the increased cost of living. In spite of the well recognized difficulties all manufacturers have encountered in the last two or three years, we have been so fortunate as to maintain a fairly stable force. We attribute this principally to the fact that we have been consistently trying to make the plant a comfortable place to work in; to give a square deal individually, and to educate the men to a better viewpoint of work in general, as well as train each man in his own particular job. We have tried to make the contact between the directing staff and each man as close and cordial as practicable. We hope, by special education of all those who represent the management in its contact with the man, to arrive at a general co-operative spirit, so that all hands will 
work together with a feeling that suggestions and impersonal criticism. are always weleome.

We employ some 10 or more nationalities, of which $60 \%$ are American born, $23 \%$ Polish, $8 \%$ British, $4 \%$ Italian, and $5 \%$ of mixed nationalities. Although, as in every such working force, there are men of all

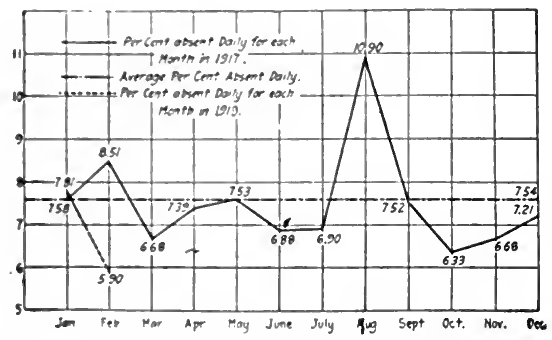

Fig. 28-Chart of Absences by Month

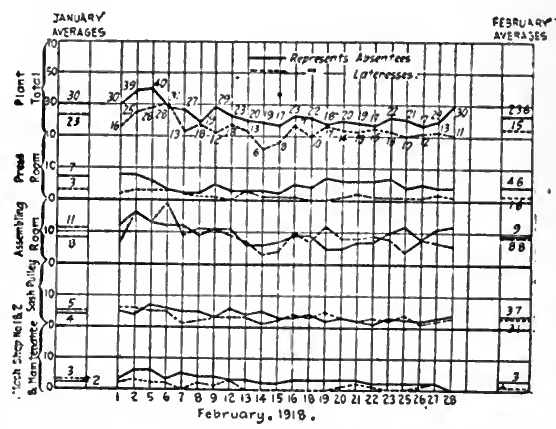

Fig. 30-Turnover by Departments for One Month

kinds, we feel that as to proficiency and character our men are considerably above the average. Narried men form $75 \%$ of our force; $10 \%$ have been with us over 10 years, and $25 \%$ over five years.

The IIealth and Employment Department was started with a staff of three men on full time. During the year previous, most of the directing staff of the plant attended some of the meetings of the

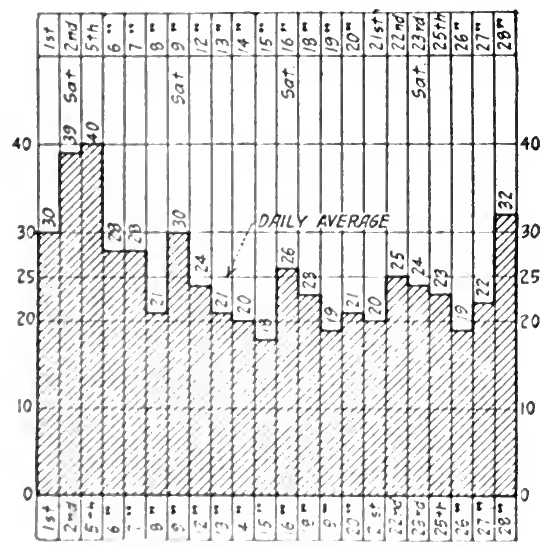

Fig. 29-Chart of Employees for One Month

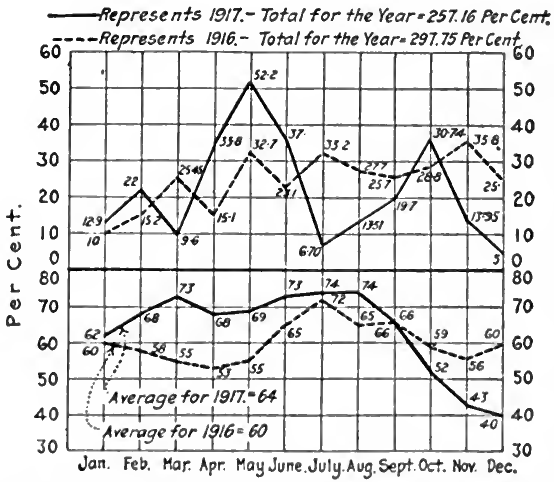

Fig. 31-Chart Showing Turnover in Sash Pulley Department

Philarlelphia Assoriation for the Diseussion of Employment Problems, securing thereby to the Employment Department in its beginnings intelligent (o)-operation and support.

Formerly the gremeral factory foreman did the employing for all departments except the maehine shop, engineering, maintenance, and 
sash pulley departments. In each of these new men were selected by the foreman in charge.

In preparing this paper on our first year's experience we have tried to select such things as may be of real use to others who have

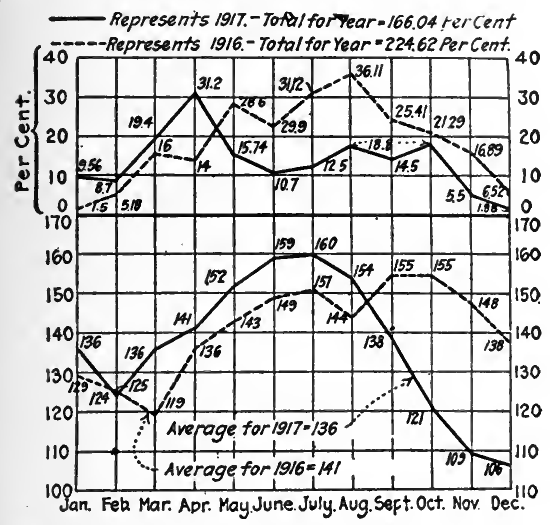

Fig. 32-Effect of Reduction of Force Shown

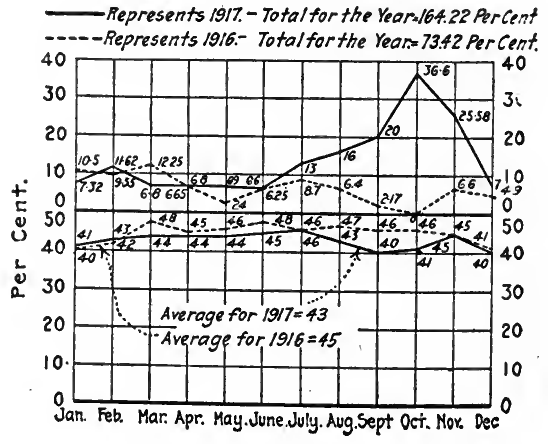

Fig. 33-High Turnover in Machine Shops

begun or are about to begin to travel the same road. We think it best to be quite frank in discussions of this character, relating mistakes and failures as well as successes. One cannot fail to be impressed at meetings of scientific men by their freedom of discussions of disappoint-

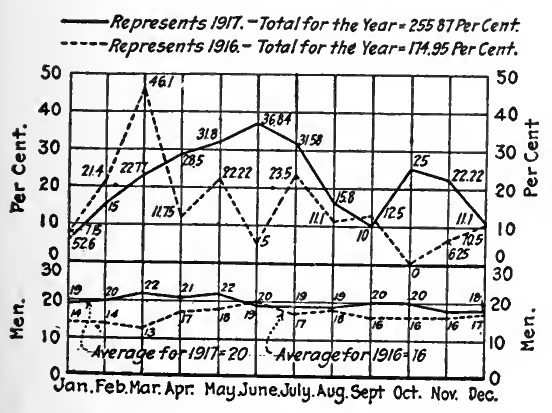

Fig. 34-Chart Showing Turnover

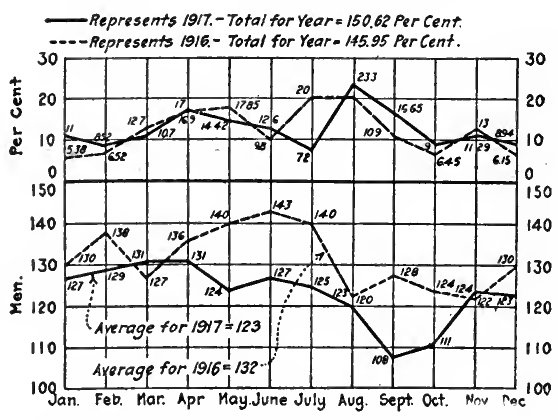

Fig. 35-Pickling Department and Stock Gangs

ments as well as achievements. I believe the same spirit is pervading the discussions of industrial leaders with a growing tendency toward mutual helpfulness.

The subjects which we purpose to discuss are:

1. Initiation of new men.

2. Measures directed to making men contented with their jobs.

3. Absenteeism and home visiting.

4. Dischargings and transfers.

5. Turnover. 
6. Hearing the other side and why men leave.

7. Health supervision.

INitiation of NEW MEN-When the preliminaries of physical and mental requirements have been satisfied and an agreement has been reached with the man respeeting his job, the necessary records and forms are filled out and the man is told to report at the Employment Department the next morning an hour after the regular starting time;

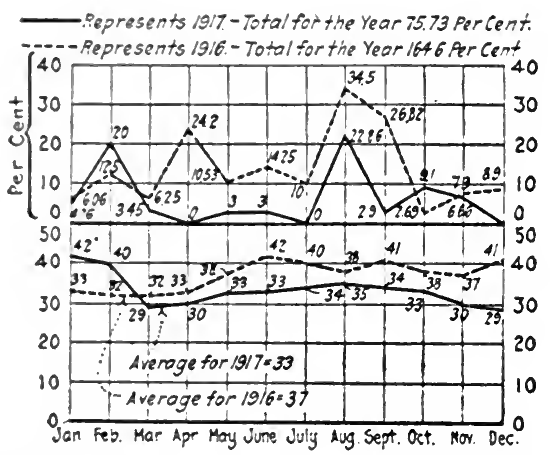

Fig. 36--Chart Showing Results in the Shipping Room

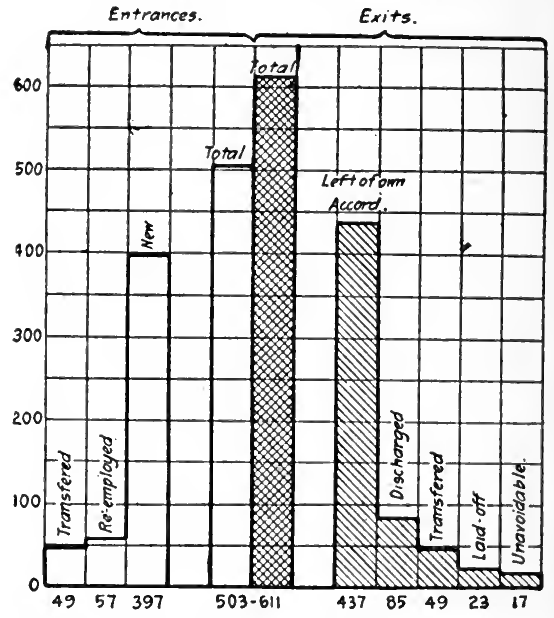

Fig. 38-Chart Showing Why Men Leave

he is paid for this hour. We think this lessens confusion-both for the man and the department to which he goes. There is always constraint in a new environment among new faces; we believe in the psychological importance of first impressions.

The new man is given a locker and then taken to the place where he

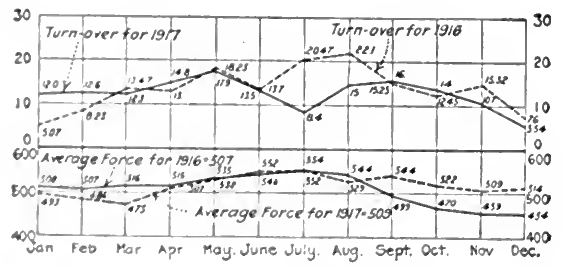

Fig. 37-Turnover and Average Force

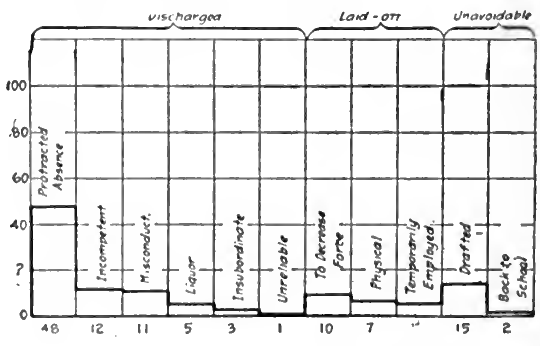

Fig. 39-Causes of Dismissal

is to berin work. At first we made an effort to introduee him to his foreman, but it appears that a foreman is a hard man to introduce. This is no less true of the average worker. We are not satisfied with omr progress in finding the best procedure at this point. Personally, I believe in a corns of instructors and that they should be attached to or have relations with the Employment Department, report- 
ing periodically the progress of the difficulties of the new man.

In the assembling room, where we have the best instructors, an honest effort is made to watch each new-comer and to determine his greatest usefulness. When a man is hired for this department, he is not selected

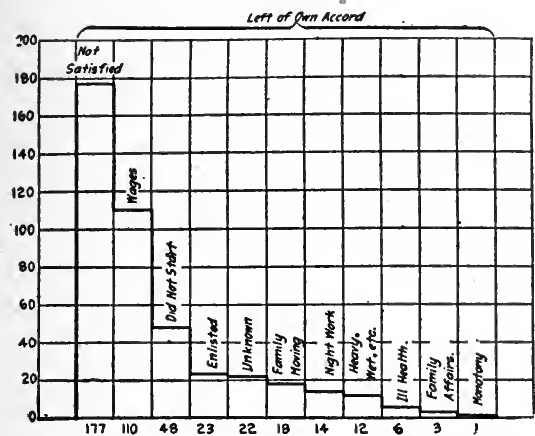

Fig. 40-Record of Causes of Voluntary Leaving

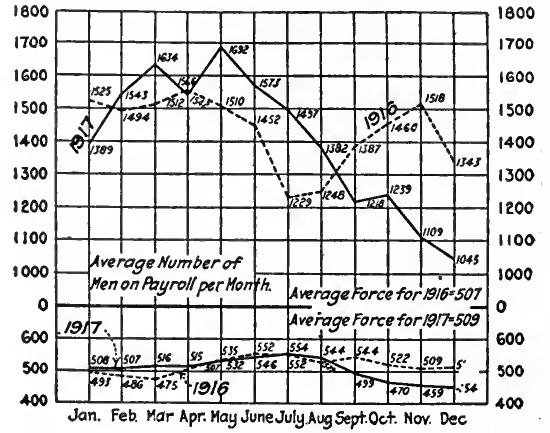

Fig. 41-Effect of Systematic Coöperation

with any one operation in view, but chiefly with respect to his general fitness and adaptability. He commences as a "knockabout" worker, filling in wherever needed until a vacancy occurs in a regular operation. If he does not succeed at one operation, he may make good at

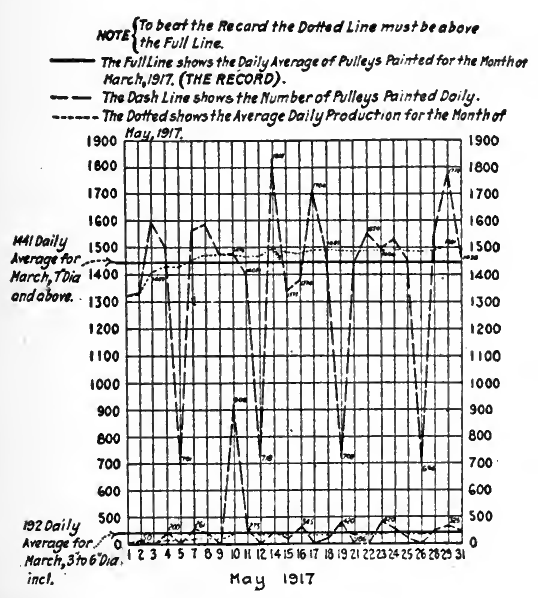

Fig. 42-Effect of Specials Inducement on Production

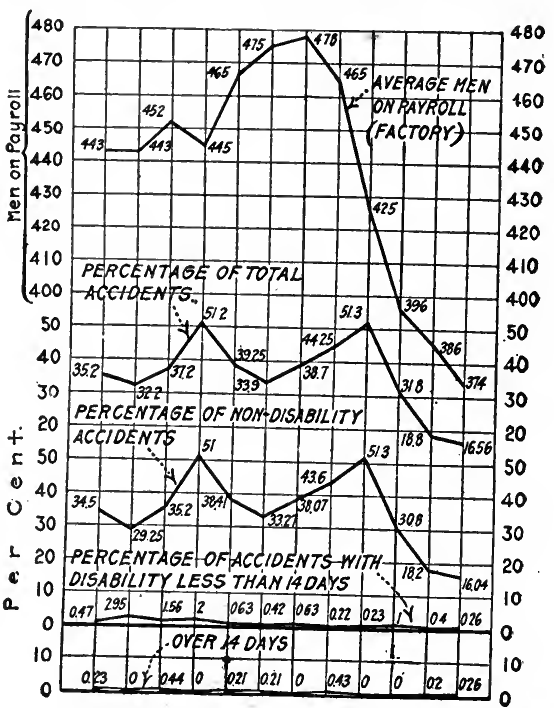

Fig. 43-Chart Showing Accidents in the Plant

another. Should a busy shop foreman attempt to shift a man from one job to another until the one in which the newcomer proved most efficient is found, it would occupy a large part of his time at the expense of other duties. 
When the Employment Department was first opened, all workers except skilled mechanies started at a flat rate of 26 cts. an hr., which has since then been increased to 32 ets. A full week eonsists of $53 \frac{3}{4}$ hrs., 9\% hrs. daily, exeept Saturday, which is a half day. On many jobs, as in the press and assembling rooms, as soon as a man is able to make more than his day rate, he goes automatically on pieee work. Over $77 \%$ of the factory force is on piece work part or all of the time. Great care is exercised in setting piece-work rates, and once set they are never lowered unless eonditions under which the work is done are radieally changed. This rule has obtained now for 20 yrs.

Measures Directed to Making Men Contented With Their JobsFirst of all, we have an employees' service building. It was erected in 1916 for the health and comfort of the men in the factory. It is construeted of brick and concrete and is sanitary throughout. It contains steel loekers, shower baths, lavatory, play roof and solarium. It is mueh appreciated and in constant use by the men. Applicants for work frequently mention that they have heard what a splendid plaee the men have, and we surmise that it is a factor in making the plant more attractive to those on the outside as well as to those within.

On .June 1, 1917, the management put into effect entirely at the expense of the eompany, a group insurance plan offered by one of the standard insuranee companies, whereby every man, after three months of consecutive employment is presented with a life insurance certificate for $\$ 250$; this is increased to $\$ 500$ after. 6 months, and goes on to $\$ 1,000$ after 5 years. This appeals strongly to those having dependents.

At the December, 1917, meeting of the board of directors a resolution was passed authorizing the sale of a certain amount of the eapital stoek of The American Pulley Company to employees in good standing, who had been in the continuous service of the company one year prior to that date. This was sold at par (50). Not more than 20 shares were sold to one person. Employees who had eredits in the reserve fund for six years or over were permitted to use their accumulations to purchase this stock.

We also encourage the men to save, believing it is a good habit for a man to accuire, and that it reaets definitely on his energy.

Last June a plant paper was founded and duly ehristened Pulley Truth, its policy being to develop plant spirit and enthusiasm, and be a means of interesting the employees along eertain lines such as employment matters, health, safety, and the like. This has proven a worth while venture from every point of view.

We comply with the "safety first" measures as required by the State and adrised by the National Safety Couneil. Every eare has been taken to protect the workers and we are convineed that the effort and the expense are well worth while-worth while not only for the sake of our own reputation but also for the efficieney gained through uninterrupted labor, and last, but by no means least, no business has the right to expect, either through negligence or indifference, that any man's ereatest asset, his eapacity for future earning, be placed needlessly in jenparly.

Ansmitemism a.Ni HIome Visits-What does it profit if you have 
a fine, well trained force-absent? The "come and go" attendance so common to the laborer is quite the logical thing in the light of the rest of his hand-to-mouth, hit-or-miss way of living. The laborer plans ahead but little, and recognizes few obligations, because he sees a problem only on its surface. If anything unusual occurs inclining him to "stay out" and he has enough in the house, it's "his business" if he forfeits a day's pay. There is a gap between a sudden jump in wages and the inevitably slower follow up of a better plane of living, in which our man has a tendency toward a feeling of affluence.

The Management could and should, through education, lend a hand until a readjustment is reached. What can we learn from this? The man is not to blame; he is to be taught and interested in a new idea of work and relationship. To mention a few factors: You cannot teach without confidence. To inspire confidence in the person taught, persistence, reliability, sympathy and acquaintance are required. You never know much about a man till you see where he hangs his hat and until you realize that back of him there is usually "her." The plant is where he works, but "Home" is where he lives, and there you can meet him on a man-to-man basis. There is a definite value in home visiting. Very often a call would reveal illness, family troubles of numerous shades, or just plain indifference due to laziness, opportunity for an outing, etc., and sometimes the "gentleman"' was found to be or to have been "cupped" (not medically speaking). In some instances, the visitor was able to bring a man back with him; others reported for work at noon or the next morning, in many eases saving much time and, we believe, often frustrating a half formed whim to "look around a bit."

We can state unreservedly that the home visits obviate much of the persistent loss due to absence, making it necessary to replace the offender. They are, therefore, worth while as tending to reduce labor replacement.

When we make a call and are unable to see the absentee or any of his family, we leave a printed slip which reads as follows:

Our records show you to be absent from work to-day. As we wish to keep in touch with all our men, we have called to see you. We regret that we did not find you in. It is to our mutual advantage that you let us hear from you promptly. Can we help you in some way?

Friday, being pay day, absentees do not trouble us. Saturday, "the day after" and a half day at that, has the poorest attendance, with Monday, also in many cases "the day after," running a close second.

DISCHARGES AND TRANSFERS-After employing with care, every effort is made to give a man unsuccessful at one operation an opportunity to see what he can do at another.

In fact, we have eliminated the word "discharge" from our plant vocabulary so far as possible, preferring the attitude expressed by the word "release." We realize that an unsatisfactory worker may be an unsatisfied one, or one constitutionally unfit in some way.

TURNOvER RECORDS-Since April, 1917, records have been kept of departmental and plant turnover, on sheets devised by the Boston Employment Managers' Association, though careful records on other forms had been kept for some time previous. We can see by our "hind 
sight" that in using turnover forms it is better to use one sheet for the reeord of each small unit or department, and that the experience of any plant can be of more assistance to all coneerned by following standard forms, but adapting them to a partieular organization.

The Sash Pulley Department employs mostly boys to the number of about 50, and up to 21 years of age. The $257.16 \%$ rate of turnover for 1917 is by no means satisfactory to us, though it is $40.59 \%$ lower than in 1916 (Fig. 31). In May we rather suddenly discovered that the rates in this department which had originally been made for younger boys, were not satisfactory to the older ones then employed. The mountain peak on the chart arose as the result of the dissatisfaction at this time. The October high mark is accounted for by the reduction of foree (Fig. 32).

The assembling and bushing departments show a decided improvement over last year though the figures are modified by the high water mark in April. For 1916 it was $224.64 \%$. For 1917, 166.04\%.

The machine shops (Fig. 33) show a turnover of $164.22 \%$ as compared to $73.42 \%$ in 1916 . This was largely due to the abnormal demand for machinists and the high wages to be had, making these men very independent.

The press room, pickling department, stock and yard gangs (Fig. 35) were grouped together on one sheet because of their inter-relation. We now see that had each unit had a record of its own we could have better diagnosed the malady. As bulked, these represent a force of about 127 men and the turnover remained about the same as that of last year.

The shipping room (Fig. 36) had nearly the same experienee. With the introduction of piece work in March the force was redueed by 11 men, the remaining force being able to accomplish the same amount of work by means of the added impetus of being able to earn more money.

The whole plant turnover for the year 1917 was $152.9 \%$, an improvement over the preceding year of $27.4 \%$. By eliminating the departments not served by the Employment Department, the advanee. over last year is brought up to $62.2 \%$.

IIfaring the Other Side and Why Men Leave-In attempting to record why men leave their jobs, as we did by these same turnover sheres, it is naturally necessary to find out from eaeh quitter what the diffienlty with his job inay be, and this means seeing and talking with each man as he leaves. To secure such an interview, we have proreserlert as follows:

Each Friday morning, the day the men receive their weekly pay (three day's overdue), the Employment Department sends a list to the paymaster of the men whom we wish to see for one reason or another. When any of these men appear at the pay window for their envelopes, they aro told to report to the Employment Department for a slip authorizing fayment to them of their wages, which is always necessary in sirch rases.

We have atfomptorl an analysis since last $\Lambda$ pril of the reasons why mon leave and have plofterl the chart of Fig. 38.

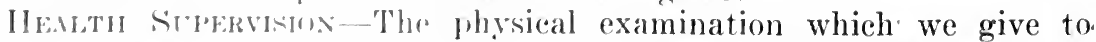
all likely applicants is in no sense so eomplete as that made by insur- 
ance companies or the army or navy, but while the man we pass might not be a good policy risk or fighting man, we aim to accept only good working risks.

We believe that a plant which examines physically soon establishes a reputation in the community among the workers, and that only those apply for work who are reasonably sure they have nothing seriously wrong. On occasions men and boys have bolted from the employment office upon discovering that a physical examination was necessary.

This perhaps explains why we have not seen in the past year a single case of venereal infection and comparatively few other abnormal conditions. For this and other self-evident reasons, the effort is worth all that it casts.

Any move that tends to bring together individuals in a community and to draw ont their co-operative forces-usually talent-whatever the immediate occasion, develops a feeling of mutual responsibility of the sort that is felt as opportunity rather than duty. Mere might cannot demand loyalty and steadfastness; these are voluntarily given and must be won. To paraphrase:

\section{"All who success would win}

Must share it-Success was born a twin."

Production-Last year's production was no greater than that of the year preceding, but one notable thing occurred which demonstrated conclusively how much can be done with a little co-operation and enthusiasm. (Fig. 41.)

However, in March of 1917, with a little urging and stimulation of interest through a daily production chart which we kept posted, production reached the height of 1634 . This astonished every one and at the same time gave enough encouragement to warrant making a try for a still higher mark, so after a little relaxation in April, due to one or two holidays, the men were offered a full day off-June 2-if the production record of May exceeded that of March. As you will see by Fig. 42, which we kept posted, adding daily, the production soon went above the March record and remained there. And, let it be noted, that this was accomplished without increasing the force by a single man.

Fig. 43 gives an idea of what a large percentage of our accidents are minor ones, and we believe it also demonstrates how safety first propaganda along proper lines will bring men to the dispensary with the simplest conditions, thereby, often preventing more serious trouble. In many concerns, a record of a large number of minor injuries would not be found, because the employees are not always taught to report them.

Letting the Men Know What They are Doing. In the course of an article "Developing Pride and Interest in the Job," published in Factory, April, 1919, W. R. Bassett states that the elements of bad work are:

(1) A sense of economic insecurity-low or high, unsteady wages and frequent firing. 
(2) Arbitrary setting of wages and imposing of penalties without explanations-and it makes little differenee in result whether the arbitrary actions are just or unjust.

(3) Lack of a standard of product.

(4) Lack of knowledge of the place and the effect of the particular operation in the whole scheme of fabrication.

(5) Lack of knowledge of the cost of the material which is being used,

and with respect to (5) Mr. Bassett says the knowing of eosts is a step in the direction of becoming master of the job-that is of the machine and the material. To quote: In comparatively simple manufacturing it may be that the only steps required for the other operations in the factory are so few that any worker knows his relation to. the whole. But most manufacturing has so many and such varied processes that the individual worker does not know what kind of link he is in the long chain of fabrication.

To let him know is a very difficult problem where the subdivision. of labor is great, or where he deals with forces rather than tools-as when he uses heat. In every job of that sort the worker needs some record of quality or uniformity which can satisfy the ereative spirit. that the work itself cannot satisfy.

You will note that I say quality or uniformity record; a quantity record is worse than useless because produetion alone is not a stimulus. to good workmanship but is in fact opposed to it, for it springs from. a desire to make money and not from a desire to do good work.

A concrete example of what quality records mean is given by $R$. B. Wolf out of his experience in wood-pulp paper making in several faetories. An important operation is the "cooking" of the pulp and the uniformity of the product depends to a very large degree upon the "cooking" control. The work is monotonous and had formerly been governed entirely by rule of thumb.

Mr. Wolf arranged that weight and moisture tests be taken frequently and the results for each squad plotted and kept on eontinuous exhibition at a point near the machines. Fig. 44.

The men could not see what they were doing by watching the machine but the graph showed them exactly what they were doing. In the first month taking the standard as 100 , they averaged from $71 \%$ to $80 \%$, but in less than six months they were hitting $90 \%$ and, at the same time, had made a large increase in the quantity of product.

The pulp of that mill rose from a poor grade to a special standard of its own. That increase in quality was made without finaneial incentive of any kind; the incentive came from the stimulation of the desire to do good work.

Fatigue of the Worker. The following is from an article, "Time. Studies for I way Allowances in Rate Setting," by Dwight V. Merriek, published in American Machinist, June 21, 1917.

Studies on the offen of fatigne were made by Dr. Taylor soon afterhe had bermu his management work. These studies originally were made to determine thr proint at which fatigne began to affect the output of the operator. They were similar to that shown in Fig. 45, which 
represents a study made recently to demonstrate the value of fatigue allowances to an operator who was skeptical as to the effect of fatigue on his work. While this study indicates, the value of rest periods. in certain classes of work, in the opinion of the writer it is not of sufficient scope to give information for making allowances on a broad line of work. The study illustrated extended over but two days and was on a single operation. To be possible of general use, it should have covered a wide range of work with different operators and extended over many days and under varying conditions.

The method followed in this experiment consists in having the operator work straight through from starting time in the morning until noon, without any stops for rest. The fatigue incident to the work was compensated for by allowing him to set his own pace as he became tired and taking longer for each operation as he desired and as the amount of fatigue increased. The length of time required for each com-
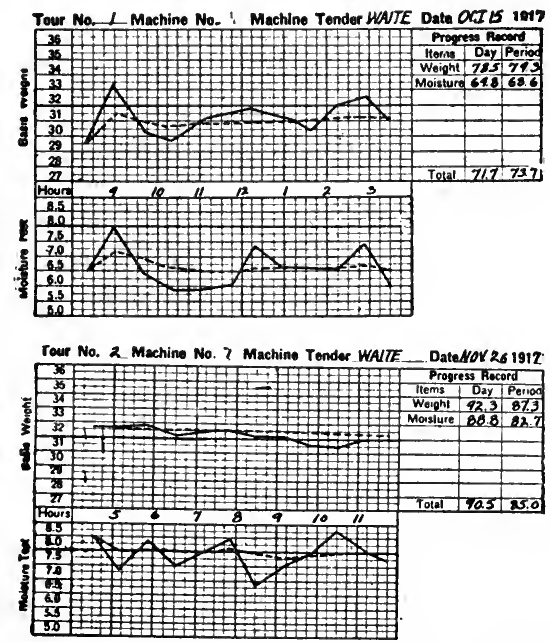

Fig. 44-Records of Results Before and After Showing Man His Performance

plete production operation was noted and recorded as the work progressed, and later plotted as in Fig. 45. After a rest of one hour at noon the experiment was continued and carried forward to quitting time at night.

It should firmly be borne in mind that a procedure of this character - that is, working the operator through the day without any rest except that at noon-is not recommended except as an experiment made to learn facts. In the experiments conducted under the direction of Dr. Taylor it was early discovered that an adequate provision for rest to overcome the fatigue incident to labor was the governing factor in promoting the efficiency of the workman. It was found that, if proper provision was not made to overcome fatigue, the output of the worker would drop and at the end of the day would be seriously redueed. On the other hand, it was found that, when an adequate number 
of rest periods of the proper length were introduced, the workman maintained his efficiency and output at the desired high point throughout the day. No greater misstatement has ever been published than that the work of Taylor aimed at speeding a workman to the point where his ability was sapped and his health ruined. The conservation of the health and therefore of the efficiency of the workman was one of the prime objects of Dr. Taylor's investigations.

The method of making the fatigue study of Fig. 45 was as follows: A job comprising all handling time was selected, for which a minimum
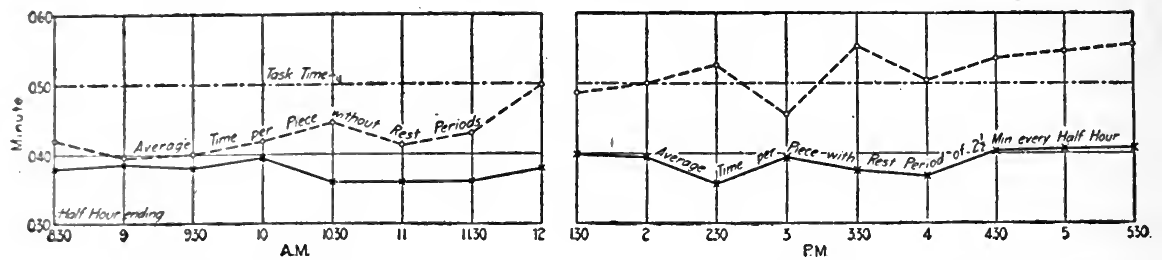

Fig. 45--Effect of a Rest Period on the Time of Production

selected time of slightly under $0.50 \mathrm{~min}$. was fixed by time study. A machine was used; but as it was hand operated, the work falls in the classification of all handling time. Since the minimum selected time is an especially severe task and one not expected to be reached by the average operator, the minimum selected time was slightly modified; and a task of $0.5 \mathrm{~min}$. was set as the standard which was desired and on which the fatigue allowance should be based.

The dotted lines represent the performance of the operator working steadily without any artificial rest periods. The full line represents the performance of the worker on the same kind of work (but possibly

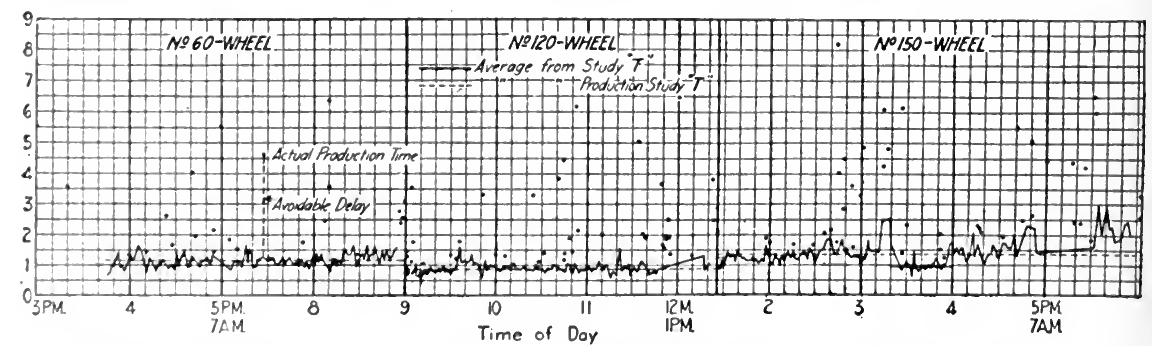

Fig. 46-Graphic Reeord of Fatigue Study Extending Over Several Days

of slightly more difficult character) on the following day, with a rest period of $21 / 2$ min. every half-hour. The curves were obtained by rlividing the number of pieces produced every half-hour into $30 \mathrm{~min}$., thus getting the average time refuired per piece during the period. The points plotted represent the performanee of the half-hour preceding the time given; that is, the reeord at $8: 30$ shows the average time of production per piece for the half-hour between 8 and $8: 30$.

A study of the curve of performance without rest periods reveals several interesting facts, and it is particularly interesting to note that 
any extra exertion after fatigue has begun to make itself apparent is always accompanied by still greater. fatigue and coincident falling off in efficiency. This is clearly shown by the record between 3 o'clock and 4:30 in the afternoon.

The effect of the $2 \frac{1}{1} 2$-min. rest period every half-hour, as shown by the full line, is so obvious as hardly to require comment. Not only did the time per piece remain below the standard throughout the day, but it was maintained at a steady rate, even improving as the day wore on. While the operator was thoroughly tired out at the end of the first day, the effects of fatigue were hardly apparent at all on the second day. Despite the fact that $40 \mathrm{~min}$. was taken for rest and that nearly $30 \mathrm{~min}$. additional was lost, due to machine breakdown on the second day, the actual number of pieces produced by the operator

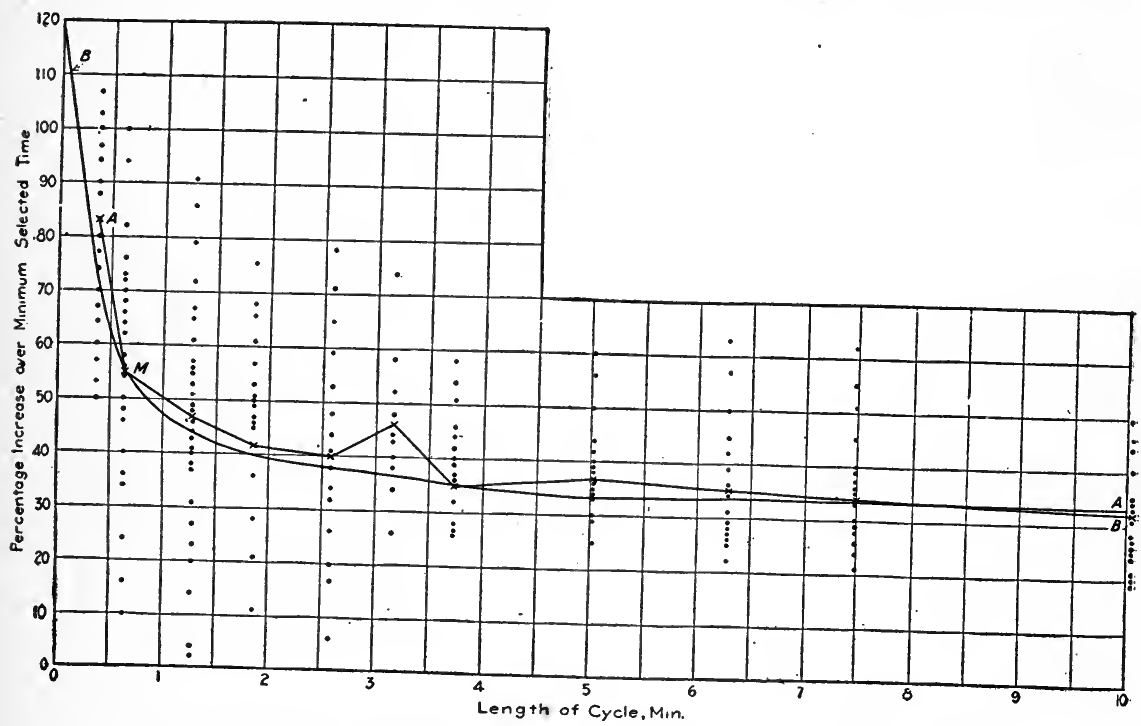

Fig. 47-Method of Making Fatigue Curve from Production Study

was over $10 \%$ greater than the number produced the previous day, when there were no interruptions for rest or machine trouble.

From a casual study of the curves of Fig. 45 it would seem that all the information needed regarding fatigue allowances could be obtained by a set of studies of the character described. This, however, is not the case; and another method is used by the writer, as will be shown later. The method just described does give information as to the maximum rate of speed of the worker and the length of time he could maintain this speed; it also indicates the point at which the first rest period should be applied. In addition, it shows the diminution in output. that may be expected if rest periods are not provided. What the method does not show, however, is how long the rest periods should be, how often they should be applied and what relation they should bear to the character of the work. If these facts are known, allowances can be predetermined even for jobs that have never before been performed, 
and they will work out in practice in very close accordance with the underlying theory. It is quite evident that the determination of the proper interval between, and the length of, the rest periods can only be determined by trial and error with the methods illustrated in Fig. 45, repeating the study over and over again with rest periods of varying lengths and at different intervals. This is at best a cumbrous, expensive and time-consuming proposition.

Instead of providing rest periods, a change in the monotony of the job may effect the same result. In actual practice it may prove unwise from the standpoint of discipline actually to stop production for the purpose of providing foreed rest periods. The same object may be accomplished by introducing a rest period under the guise of a nonproductive operation. Thus, an operator on a high-speed machine may be required at certain intervals to move his finished product to a different location or to go some little distance for his supply of raw material. The change in the nature of the work involved in this procedure provides for the muscles employed in the productive operations the necessary relaxation to overcome the fatigue produced by the work. The introduction of rest periods in this manner is a matter for the man who prepares the instruction cards, and considerable ingenuity may be exereised by him in this respect.

In certain elasses of work, as the operation of automatic machinery, it is often desirable to provide an additional operator to each group of six to twelve workers. This operator takes the place of each of the workers successively, thus providing an opportunity for rest or for attending to their personal needs without stopping production. This additional operator may be the instruetor or supervisor for the group.

Another method of producing the necessary change in monotony is interchanging operators every hour or two on two machines doing different jobs of the same general character of work.

In the method now used by the writer all the necessary information for fatigne and delay allowances can be obtained from the production sturlies that should be carried on as a part of the time-study routine.

Fig. 46 is a plotted record of a production study of several different jobs of the same character, extending over several days The ordinates represent the time of production per piece, while the abscissas are the time of day at which the successive pieces were completed. In plotting the time of production, only the net time is used, avoidable delays being subtracent. It will be observed that above the curve of produetion there are a number of points plotted. These represent the actual time of production, and the distance between them and the corresponding point on the curve reperesents the avoidable delay that has been dedueted. It will be observer also that the time of production per piece has a temlency to incroase somewhat as time passes and the end of the day approarhes.

()ne of the orliest attompts to establish a scientific basis for fatigue allowance was the makiner of a formula to govern this feature. In one of the first shope to $11 \mathrm{se}$ time study, data were gathered as to the pererntage by whoh the artual time of performing jobs on the heavier tools exceederl the minimum selected time, and the following allowances were deduced. 


\section{Type of Machine \\ Size}

Lodge and Shipley lathes..24 to $30 \mathrm{in}$.

Lodge and Shipley lathes.. $48 \mathrm{in.}$

Vertical boring mills...... $120 \mathrm{in.}$

Vertical boring mills....... 36 in.

Vertical boring mills..... 30 in.

Horizontal boring mills. . No. 74 Bennse

Planer ............... 36 in.
Percentage to

Be Added to Minimum Time

35 to 50 On 24- to 30 -in. lathes, the 30 allowance is $35 \%$ when 25 handling time is more than 358 min. and the machine 40 time double handling time; $40 \quad 50 \%$ when handling time is 40 . less than 8 min. and machine time about equal to it.

Similar data on light tools, such as vertical drilling machines, etc., were incorporated into a formula by Carl G. Barth, as follows:

$$
P=\frac{125}{1.20+\sqrt{1}}+20
$$

in which $P$ is the percentage by which the minimum selected time is to be increased and $T$ is the minimum selected time.

The allowances, as given by the table and formula above, while fairly satisfactory for the particular shop in which they were made at the time, proved to be inaccurate when applied to shops in different lines of work.

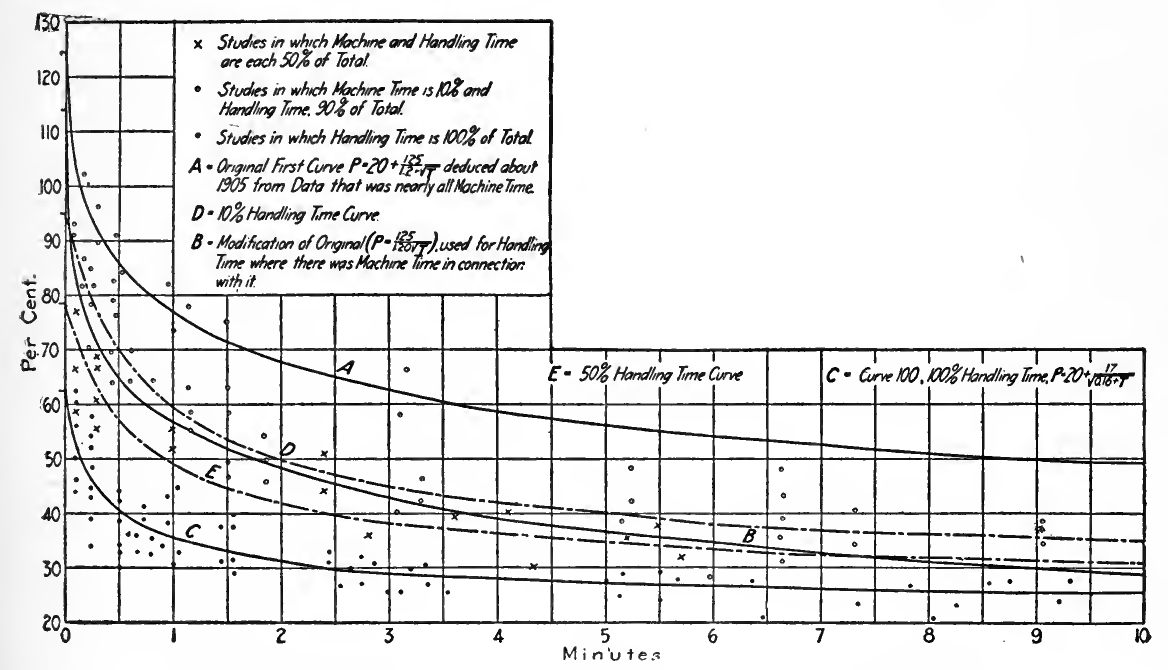

Fig. 48-Comparison of the Early and Recent Fatigue Curves

It was evident that a broader method of ascertaining allowances was necessary, which could be applied along more general lines.

A hypothetical case will be presented to illustrate the method now used. Production studies are made of a series of jobs requiring various lengths of time for their completion, but in which the percentage that the handling time bears to the total time is carefully noted and separated from the machine time, and the total of the handling time in each cycle is expressed as a percentage of the minimum selected time of that 
eycle. For example, if the total minimum selected time for a job was: 1.06 min., made up of a machine time of $0.54 \mathrm{~min}$. and a handiing time of $0.52 \mathrm{~min}$., we might obtain the following figures for handling time in several successive eycles: $0.56,0.64,0.67,0.63,0.65,0.68 \mathrm{~min}$. These would then be expressed as percentages of increase over the minimum selected handling time of $0.52 \mathrm{~min}$., as $26.9,23.1,28.8,21.1,25.0,30.7$ per cent.

The percentage increases of the actual handling time over the minimum selected handling time are plotted with pereentages as ordinates and the length of eycles in minutes as abscissas. A curve that will represent the mean of all the points is then drawn through the field, and from it values may be taken which will be a fair allowance for all work with the same percentage of handling time as the jobs on which the curve was based.

The method of laying out the eurve is shown in detail in Fig. 47, which is a hypothetical case representing the results of production studies on jobs in which the handling time is $50 \%$ of the total time of the eycle. The jobs have eycles varying from $0.30 \mathrm{~min}$. up to $8 \mathrm{~min}$. in length, and the percentages excess of the actual handling time over the minimum selected handling time for the several cycles in each of the jobs is shown as the plotted points.

It is usually eonvenient to determine the average value of the plotted points for each length of cycle, as due weight will then be given to values that recur several times. If a eurve is simply struck through the mean of all the points, values that recur several times in the same cycle will have no greater weight than those which occur but once.

The average for each cycle is indicated by the crosses in Fig. 47. These are connected by the straight lines that give the rough curveAA. With this rough curve as a guide, an even curve $B B$ may then be drawn, which will approximate the average conditions and smooth out the variations in the first rough curve.

Similar curves are plotted for all the jobs with different percentages of handling time, and the shop is then prepared to set tasks and fix allowances with a certainty that the tasks can be accomplished. The final step is to superimpose the eurves for the different percentages of handling time and aseertain if they agree with one another. If the work has been carefully done, it will be found that the several eurves are approximately parallel and that it is possible to derive a mathematical formula to which they will all conform. It is usually advisable, where the mathematical ability is present, to derive this formula and to replot the curves in aceorlance with it.

Fig. 48 represents developments of several classes of eurves that finally resulted in the series shown in Fig. 49. Curve A, Fig. 48, represents the curve obtained by plotting Barth's original formula.

$$
P=20+\frac{125}{1.20+\sqrt{ } T}
$$

This curve, hased on comparatively few observations and a limited number of machine types, gave allowances far in excess of those neeessary for certain classes of work. It was later modified to curve $B$, which 


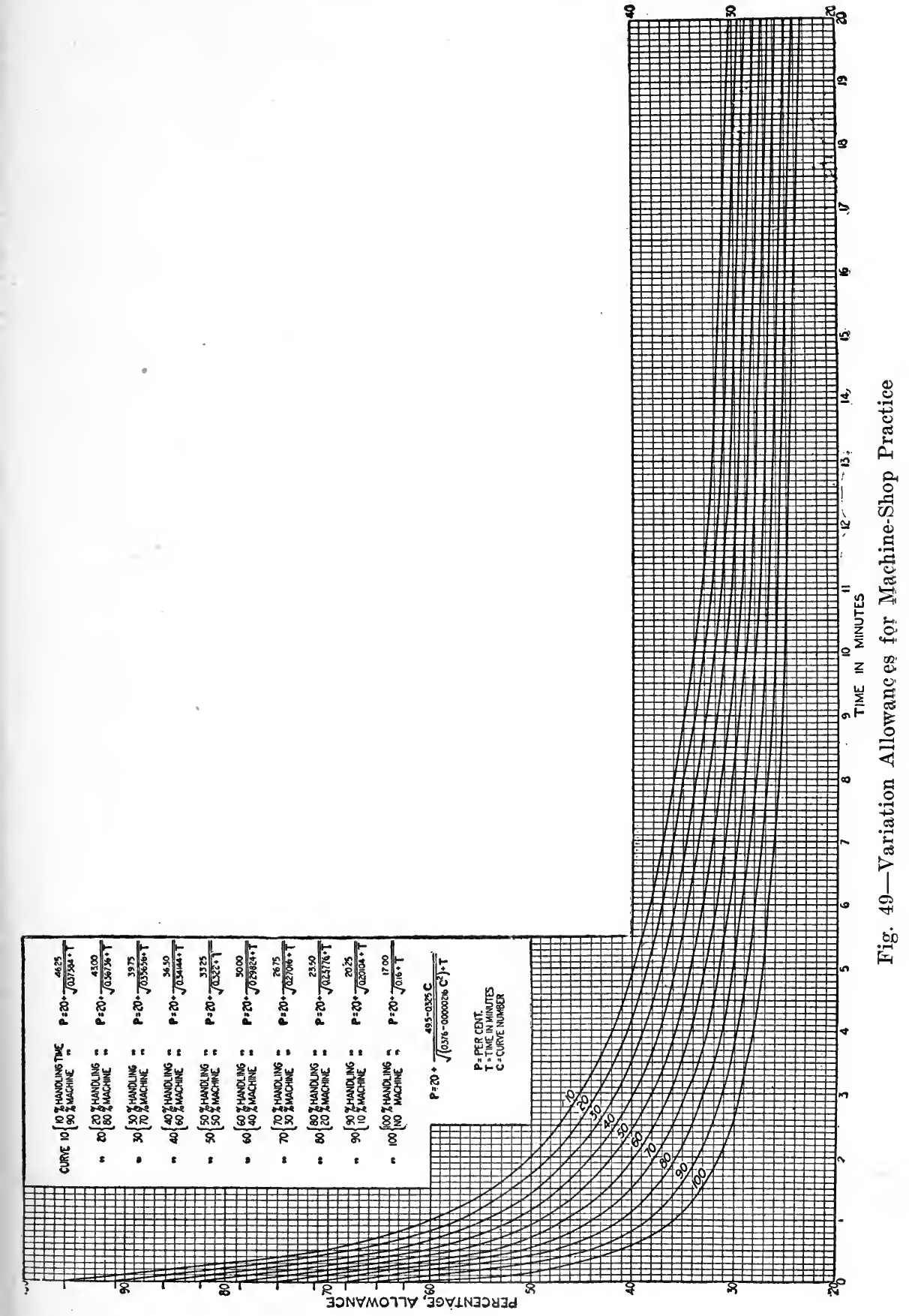


was used where machine time and handling time oceurred in combination. It was later found that still more differentiation should be made between classes of work that varied greatly in the percentage of handling time involved in them, and the curve representing the work involving pereentages of handling time, ranging all the way from zero to 100 . was plotted from observations on work of the above character.

This is the procedure that has been adopted with the curves given in Fig. 49. They represent the result of thousands of actual studies in many different shops, extending over a long term of years. It is thought that in the present form they will be more available for use in the machine shop.

The formula for these curves, which was derived by Mr. Barth, is

$$
P=20+\frac{49.5-0.325 C}{\sqrt{(0.376-0.00002166)^{2}+T}}
$$

in which $P$ is the percentage allowance, $C$ the percentage of handling time, and $T$ the minimum selected time.

The eurves are applicable to the average machine-shop practice in the ordinary well-lighted, well-ventilated and properly heated shop. It should be emphasized that they may not apply to other industries or where temperature and ventilation conditions are such as to tend to the enervation of the workers. The allowances given by the curves are applicable to studies taken and analyzed according to the methods used by the writer, which have been described in previous articles. They cannot be guaranteed as applicable to studies taken and analyzed by any other method.

In using the curves, the particular curve is selected which corresponds most nearly to the percentage of handling time in the eycle on which allowance is to be made. Thus, when there is no machine time involved, curve 100 , representing $100 \%$ handling time, is used. If the cycle represented $50 \%$ handling time and $50 \%$ machine time, then curve 50 is nused. The percentage allowance is made upon the total of the handling time: that is, if a job comprised $3 \mathrm{~min}$. machine time and $2 \mathrm{~min}$. handling time, the $40 \%$ curve would be used and the intersection of the "-min. ordinate with this curve would determine the allowance that would be added to the handling time in making up the instruction ard. For marhine time with power feed a flat allowance of $5 \%$ is adderl. and for machine time with hand feed an allowance of $20 \%$ is adrled to the machine time. The method of marking allowances by means of curves derived from data furnished by production studies takes into (onsideration the delays to the work due to other considerations than fatigure. It is so obvious as hardly to require comment that, eren in the most highly organized and best managed shops, occurrenees aro boum to take place which will delay the work more or less. Sume of these are avrirlable, and others are not, as has been exflained in the article on production studies. The avoidable delays are climinater from the resord before the percentages that are plotted as in Fig. 47 are calcolatan, and only the net productive time, the delay due to fatigur and the mavoirlable delays that in all fairness should be allowed for are taken into consideration. 
Inasmuch as the curves illustrated include other factors than fatigue, the term "variation allowance" has been adopted as a better expression than the term "fatigue allowance," which has been widely used. Fatigue does play a large part in slowing down certain classes of work, particularly where the cycle is short, necessitating frequent and rapid movements on the part of the operator, and where the handling time or period of actual physical exertion on the part of the operator is a large percentage of the total cycle. Its influence is relatively less as the length of the cycle increases and the percentage of handling time diminishes. In such cases the influence of the unavoidable delays may be greater than that of fatigue. These features are clearly shown by the curves, in which the allowance for the short cycles, where there is little opportunity for the operator to recover from fatigue, calls for higher percentage of allowance, while the long cycles, which offer rest periods in the cycles themselves, eall for much lower percentages of allowance.

Charts in Time Study Work. In another article, "Time Studies on Automatic Machines," published in American Machinist, April 12, 1917, Mr. Merrick gives a detailed explanation applying to time-studies of automatic press work, but says that the principles involved apply to practically every class of automatic machinery and summarizes the procedure as follows:

1. Take a study of one or several machines of the same character, extending over several days, noting the production at regular intervals and recording the time of the beginning and ending of each interruption or delay, together with a notation of the nature of such interruption or delay.

2. Analyze the delays and interruptions, noting the number, total and individual times of each class of delay for each size of machine or size of work.

3. Examine the delays to ascertain which are avoidable by correction of existing improper conditions and discard these from consideration, after taking steps to have the improper conditions rectified.

4. Plot the remaining delays to ascertain whether or not any relation exists between them and also to ascertain what effect one character of delay has upon another.

5. Subdivide as minutely as possible these delays and examine them to see if any portion of them can be avoided. If so, discard these items from further consideration.

6. Plot the average time, in minutes, of each class of delay and draw a smooth curve that will represent the average performance of the group of machines or several sizes of work under consideration, and read from the curves the allowable time per delay.

7. Plot in a similar manner the average number of delays per day for each class and determine the allowable number of delays per day.

8. Multiply the number of allowable delays per day by the time per delay, to ascertain the total length of each class of delay per day.

9. Determine the required production per day by means of the formula,

$$
\text { Production }=\frac{Q}{1.05}(M-125 H-W-P)
$$


10. Divide the production per day by the number of delays per day of each class (as found in 7), and divide the quotient into the allowable time per delay (as found in 6), to prorate the total delay to the individual piece.

11. Ascertain the machine time per piece by dividing the total production per day into the number of minutes in the working day.

12. Add the machine time per piece to the total of all the delays per piece, and add to the sum an allowance of $5 \%$ of the machine time per piece and $25 \%$ of the sum of all the delays per piece.

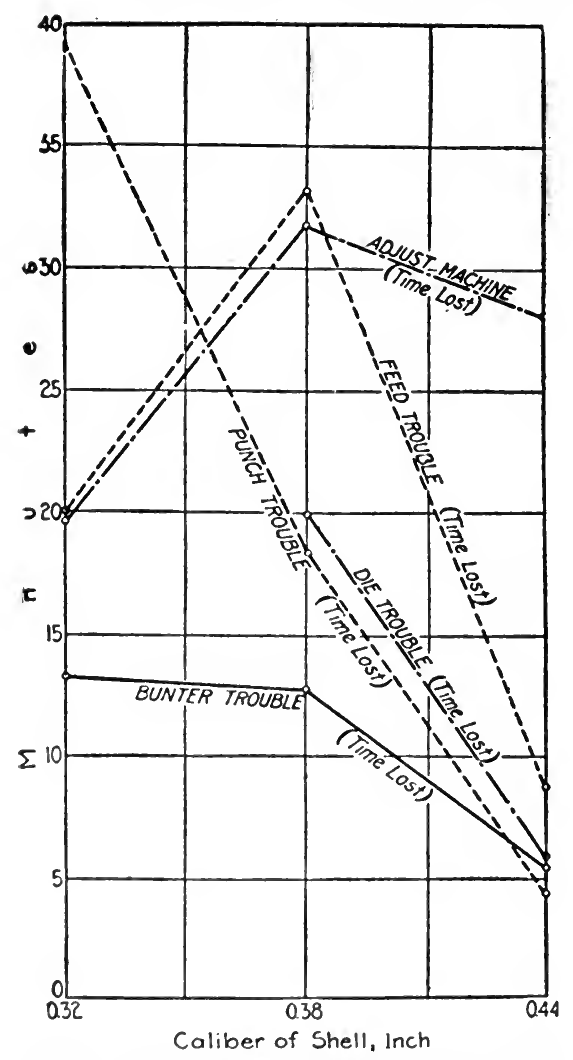

Fig. 50-Craphic Representation of Time

lost in Heading-Press Operation

13. Add to the sum obtained in (12) the prorata allowance per piece for the various personal necessities and washing.

14. Divide the sum olstained in (13) into 60 to find the hourly produetion rerguired.

15. Fix hase rates and task for daily or hourly production.

Fig. 50) illustrates (4), and Mr. Merrick says,

Plotting the delays is an exeellent method of studying the relation that the several delays in the different sizes of work bear to each other. The curves reveal some valuable facts: (1) There is an evident relation 
between the punch, die and bunter trouble and the size of the work, the trouble being less serious with the larger sizes. (2) There is no apparent relation between the size of the work and the adjustment of the machine or the feed trouble. (3) There is apparently a very definite relation between the time lost due to feed trouble and the time lost in adjusting the machine. (4) On account of the steep slope of the curves it is evidently impossible to select a factor for delays that will apply to all sizes of shells alike, but it will be necessary to select different factors for each class of work.

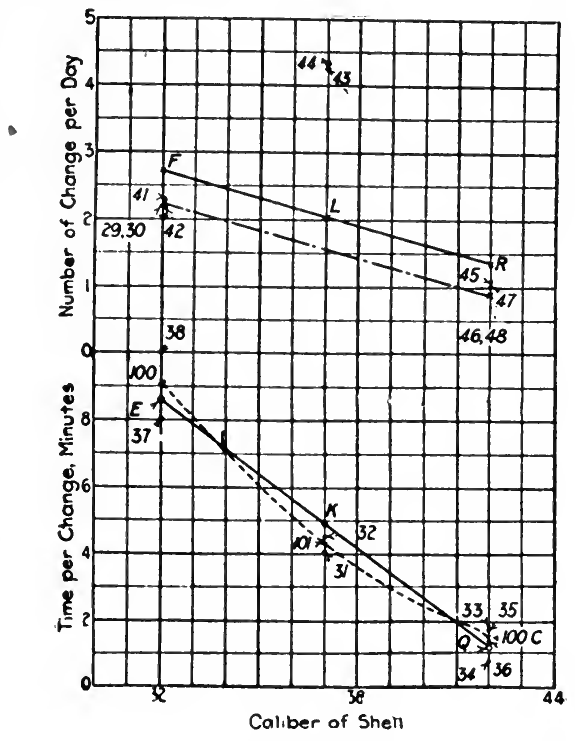

Fig. 51-Graphical Analysis of Necessary Delays

The fact that the delay due to feed trouble is irregular in character indicates that it is due to conditions that are not inherent in the manufacturing rrocess, and that they, therefore, probably are subject to correction which would eliminate this source of delay altogether. As a matter of fact, an investigation of the equipment after the time study had indicated the irregularity of the feed revealed that the feed pipes through which the shells were fed to the presses from the magazines were too small and clogged easily. The substitution of larger feed pipes removed practically all trouble from this source and automatically eliminated this particular item of delay. With respect to the apportionment of delays, he states that in the case of a series of studies on a single type of machine it is probably safe to take the average value, both of the number of changes and of the minutes required per change, and to use this average as a base upon which allowances for delay, fatigue, etc., are figured.

Where several machines are involved, however, or several sizes of work, it is often profitable to plot curves from the results obtained from the time studies and from these curves select more or less arbitrary 
values for the delays which are permissible for the various sizes of work and the several sizes of machine. Fig. 51 shows the plotting of one such set of curves in regard to changing of punches. Points 37 and 38 represent the average time per ellange as determined by two separate studies on 0.32 ealiber shells; 31 and 32 represent similar points for 0.38 caliber shells, while $33,24,25$ and 36 represent the average times for changes for 0.44 ealiber shells, as determined by a corresponding number of studies. Points 100,101 and 102 represent respeetively the mean of the several plotted points for $0.32,0.38$ and 0.44 ealiber shells. A curve drawn from these three points will, it is recognized, be quite flat, and a straight-line approximation of it would probably be sufficiently correct for all practical purposes. Aceordingly, a straight line has been drawn through it in such a position as to divide the error equally on either side of it. Values selected for the delay allowances for changing punches are the points of intersection $E K Q$ of the straight line with the ordinates representing the several sizes of shell. Were there intermediate sizes other than the three, the given delay allowance taken from this straight line would be sufficiently eorreet for the setting of tasks.

Similarly, the curve FLR is laid out to give the average number of changes of the punch per day. Approximate curves are drawn in the same manner to obtain values for all of the necessary and allowable delays-namely, die and hunter trouble and machine adjustment.

Labor Output. In the following, from an article, "Negroes a Source of Industrial Labor,' by D. T. Farnham, published August, 1918, in Industrial Management, it is shown how eharts may be used to compare the output of different kinds of labor and of the same kind under different conditions. Fig. 52 gives a comparison of labor turnover by races, but Mr. Farnham remarks that while the percentage of absentees among negroes is about twice that of Americans and $40 \%$ greater than among Italians, there are some of them who work steadily, hard and loyally as any white man.

Labor turnover among the unskilled is about $50 \%$ greater among negroes than among the whites. This can be reduced by selecting your negroes, but certain other considerations are very important. The ne'gro is fond of family life and is much more likely to remain if he ean lent a house near the plant and move in his women folks and the various pets which seem necessary to his existence. He profers to live near the plant because he enjoys a family party at the noon hour and, where cilcumstances permit, he appreciates an opportmity to aequire the various hack-door perquisites such as kindling wood and the like which residrune (olse to the factory makes convenient.

Another foible of his is the desire for frepuent pay. One public utility company in a mildle western city took advantage of this last summer and secolured sufficient men to do eonsiderable grading at $\$ 1.75$ per day as against current rates of $\$ 2.25$ per day, by paying off every evening. I would not advise any such procedure on manufacturing work, however, as the men so secured are of the most shiftless and transitory class and camnot be depended upon to remain, even while they are learnine their joh.

Fig. 53 shows the premium earned by two colored crews on semi- 
skilled work as compared with the average premium earned by four Southern European crews and by a mixed crew. It will be noted that the first half of the year the negro crews earned no premium. In fact they not only earned no premium during the second quarter of the year, but they took so long to perform their task that the departmental

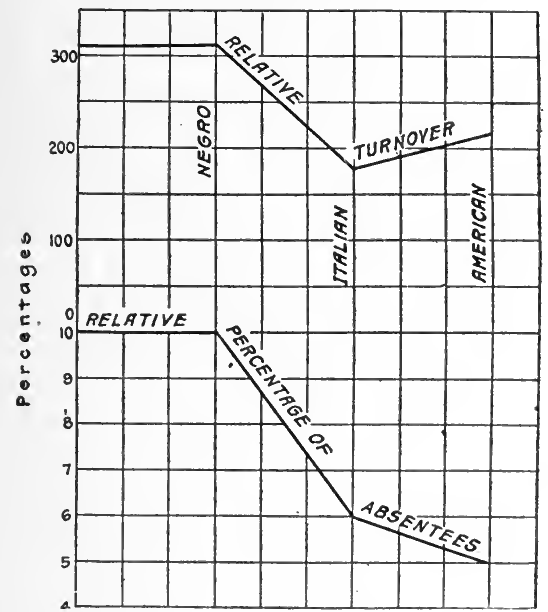

Fig. 52-Comparison of Labor Turnover by Races

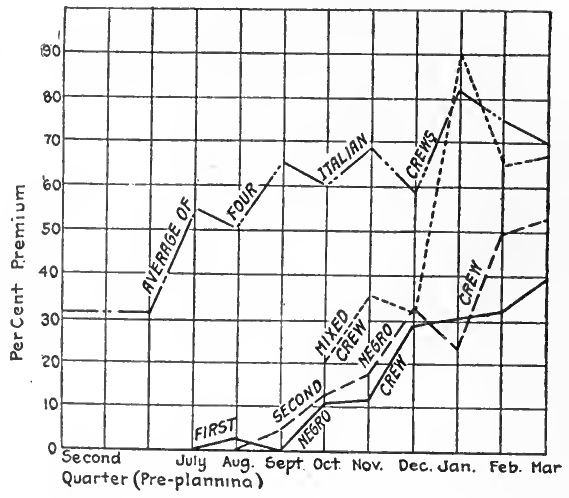

Fig. 53-Comparison of Negro, Italian and Mixed Crews on Same Work

production was seriously menaced at times. Their first premium earnings in July were very small and incidentally very spasmodic and discouraging both to the men and to the foremen who were trying to beg and belabor them into premium earning speed. In October constructive supervision began and from then on the improvement in earnings is much more encouraging. There were occasional set-backs due more

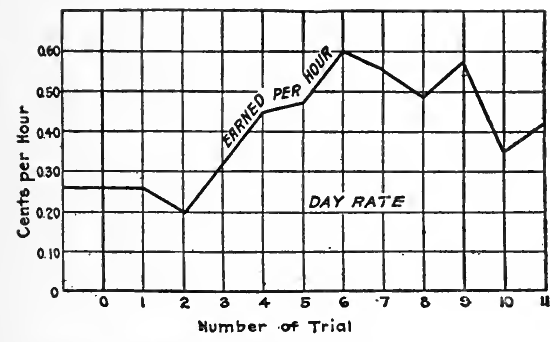

Fig. 54-Negro Labor Under an Experienced Overseer

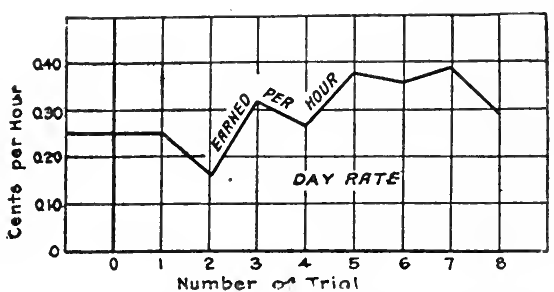

Fig. 55-Results Obtained by the Second Foreman with Negroes on Piece Rates

than anything else to negro leaders becoming slack and having to be replaced, but the progress is, on the whole, steady. The work of the mixed crews is surprising. It began to earn premiums almost at the start and, while given to violent fluctuation at times, finally closely approximated the earnings of the Southern Europeans. The chart is 
of further interest in that it shows how the introduction of scientific planning during the second quarter of the year increased the average preminm earned from 31 to nearly $70 \%$ in excess of the day rate simply by making it possible for the crews to "let themselves out."

Fig. 54 shows the results obtained by an experienced overseer of negro labor during his first attempts to get his erews on the piece rates.

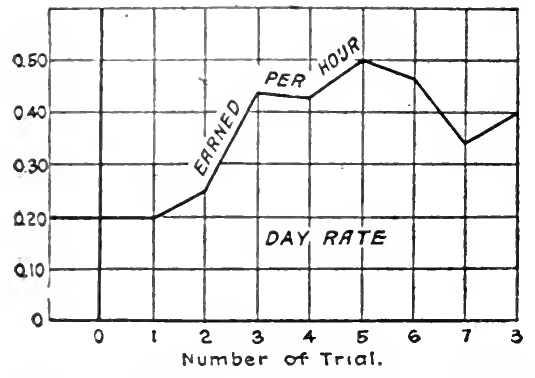

Fig. 56-What the Third Foreman Accomplished

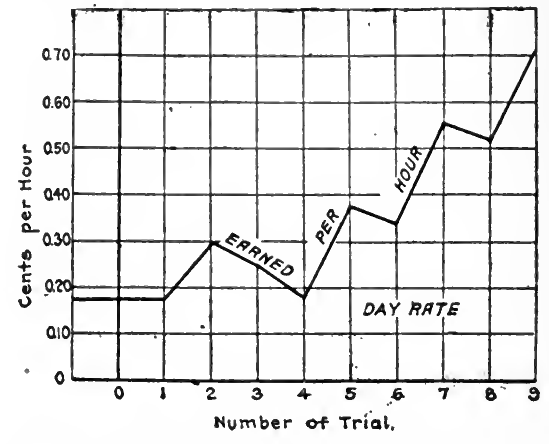

Fig. 57-Results Obtained by the Fourth Foreman

It will be noted that the first trial resulted in day rate being earned. The second trial was a failure. At the third trial they really got their teeth in and from then on earned almost double the amount secured on the first attempts.

Fig. 55 shows the first attempts made by a foreman of unusual intelligence and initiative but inexperienced with negro labor. The premium earned was less but perseverance and resource resulted in the crews

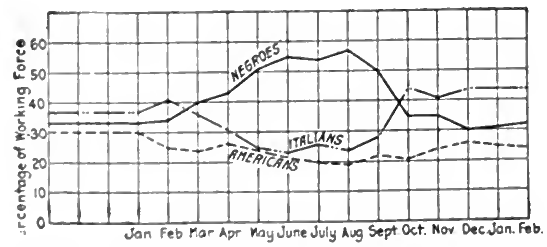

Fig. 58-Example of Deliberate Seasonal Racial Replacement

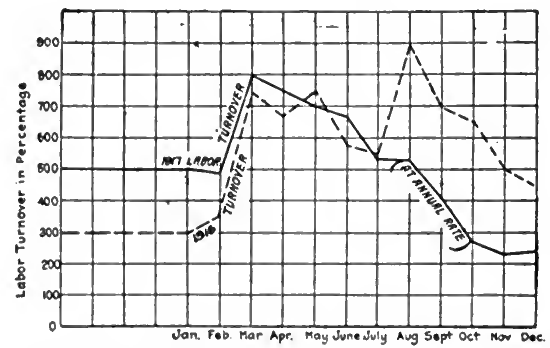

Fig. 59-Effect of Deliberate Seasonal Replacement

earning enough to keep them eontented and to prevent the eompany losing them.

Fig. 56 shows the result obtained by a third foreman who did not propose to be left behind in the race for getting the negroes to respond to piece rates. II studied the methods of the experienced overseer and applied them very suceessfully.

Fig. 5i shows what an Irish boss could do when he set his mind to it. It is apparent that he made his mistakes, but he kept everlastingly at it and jollied and bluffed his erews into doing very well for themselves. 
Figs. 58 and 59 show what may be done by means of deliberate seasonal replacement. The racial tendencies of different classes of labor have so far been insufficiently studied in America.

In the spring the negro is willing to move north. In the autumn he goes south for the cotton picking, which begins in October. He does not like the northern winter and even if he sticks it out through November he generally cannot stand the idea of missing the Southern Christmas.

As the Italian takes the road in the Spring the negro can be induced to fill his place, and when in the Autumn the negro goes south for the cotton picking and Christmas possum the Italian can be encouraged to "dig in" for the winter.

Fig. 58 shows the deliberate replacement in one plant of Italians by negroes in the Spring, and the replacement of negroes by Italians again in the Fall. It will be noted that the Americans on the most skilled operations remained close to $25 \%$ of the working force while the negroes increased from $33 \%$ in January to $57 \%$ in August and decreased to $35 \%$ in October and to $30 \%$ in Decemberr. The curve which represents the Italians began the year at about $40 \%$, fell off to $25 \%$ in the summer and rose again to $45 \%$ in the Autumn. It will be noted that throughout the seasonal fluctuations a nucleus of each race amounting to at least $20 \%$ of the working force was retained.

The result of the adoption of this deliberate policy of seasonal racial replacement is shown by Fig. 59. In fact it may be more accurate to state that the 1916 turnover curve was one of the most moving reasons for the adoption of the replacement policy. It will be observed that the 1916 turnover, figured each month in terms of annual turnover, began the year at about $300 \%$. As soon as railroad work opened in March it jumped to over $700 \%$ where it remained until the most necessary reconstruction work was accomplished, dropping down to about $500 \%$ in June and July. In August construction work was rushed in order to get ready for the winter and the turnover went up to $900 \%$. By December the turnover was shown about to normal.

Conditions at the beginning of 1917 were even worse than they had been the year before on account of the greater general labor shortage throughout the country. January began with 500\%. March reached $800 \%$. It was, therefore, decided to enedavor to avoid the conditions of the previous year by using negroes to fill the gaps in the working force. The balance of the curve tells the story. By August the turnover had dropped to $530 \%$ as compared with $900 \%$ the year before. By September it was down to $400 \%$ and in October it was below that of any month for two years. The Italians came back in the Fall. The negroes went south. One replaced the other almost without friction and the plant's output increased steadily.

Bonus and Piecework Rates. Charts may be used to great advantage in laying out bonus and piece-rate curves, not only for figuring what amounts shall be paid but also as an aid in determining a uniform and consistent rate of payment.

Fig. 60 is from an article by C. E. Knoeppel, "The Bonus Plan of Wage Payments," published in Engineering Magazine, November, 1914. Mr. Knoeppel says: Fig. 60 will show the bonus curve used and ad- 
vocated by me. The heavy curved line is the bonus line starting at $67 \%$ efficieney, which means the workman is expected to attain twothirds of a fair standard before he begins to earn anything additional in bonus. In other words, the man can take $50 \%$ more time than that ealled for by the standard, for which he receives day wages only. Any reduction in time under this $50 \%$ margin would be accompanied by a proportionate amount of bonus.

The bonus line is divided into three sections, A, B and C. Men of low efficiency do not become men of high efficiency over-night. They sometimes feel that they cannot attain the standard determined upon. The aim is therefore to induce the men to work up the " $A$ " incline from $67 \%$ to $85 \%$ efficiency. They then have the incline " $B$ " ahead of them, with promise of additional earnings if they get into this class. Men are not satisfied with standing still, nor do they want to be considered as low-efficieney men. Finally when men are well along towards the $100 \%$ mark they are attracted by the additional $5 \%$ premium for qualifying as $100 \%$ men. A bonus of $20 \%$ plus $5 \%$ premium

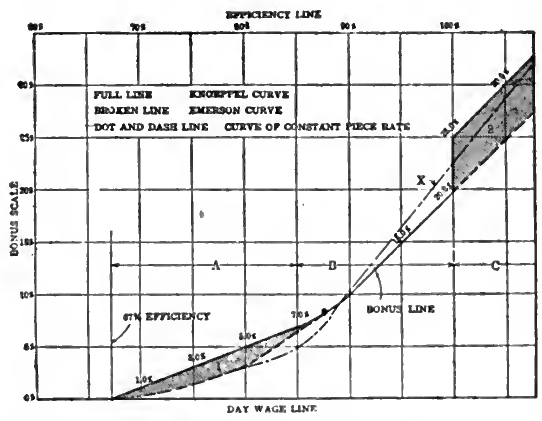

Fig. 60-Bonus Curves

scems worth the additional effort to the man who is within $3 \%$ or $4 \%$ of the goal.

For comparative purposes the Emerson eurve has been shown by a dotted line where it varies from the curve recommended. The extra amount indieated by the shadcd zone 1 is to warrant men, who might otherwise beeome discouraged in making the effort necessary, in attempting the attainment of efficiencies greater than $67 \%$, and the amount measured by the shaded zone 2 is a premium for those who average $100 \%$ of the standards or better.

In one case the client eomplained beeause bonus did not appeal to the worker's as it had on the start. The $20 \%$ in bonus for $100 \%$ efficiency did not seem to justify the effort, and as a result workers were content to earn from $5 \%$ to $15 \%$. When the bonus plan was first eonsidered in this asse, the engineer urged the additional premium of $5 \%$ for $100 \%$ workers, as a means of eliminating this very tendeney. The basic theory was that $5 \%$ in one lump would attract the worker who might otherwion be satisfied with an ordinary bonus. The elient eould not see it. Who was to blame for the ultimate condition, the engineer or the elient? 
The chart in question shows a third line (dot and dash) which may be interesting to the student of bonus plans. The claim has been made that because the Emerson and Knoeppel bonus lines mean slightly decreasing costs per piece, they are unfair to the workmen; that the rate per piece should remain constant as in the straight piece work plan. Bonus paid on the basis of the " $\mathrm{X}$ " line does this, and its comparison with the other two lines will be found interesting.

Mr. Knoeppel, at some length, interestingly and thoroughly develops his various principles for bonus payment and says:

The matter of an intelligent and comprehensive control of the entire work is most important. To take care of this feature properly a number of charts can be used to decided advantage.

Fig. 61 is a record of the bonus earned per man per period. I was once bitterly accused of being too anxious for the men to earn bonus. I am. When men earn bonus it means that efficiency, and therefore

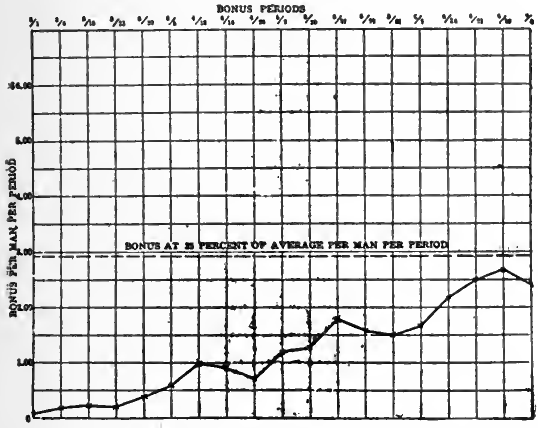

Fig. 61-Average Bonus Earned Per Man Per Period

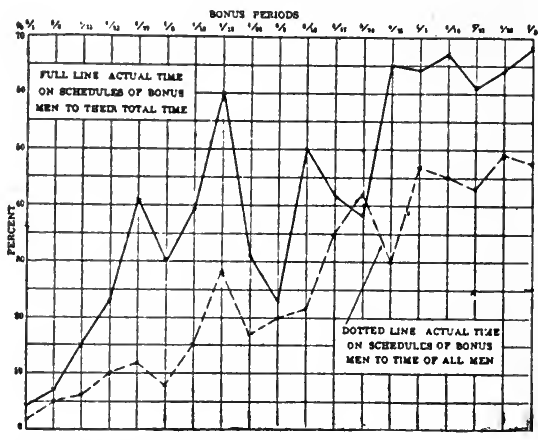

Fig. 62-Ratio of Bonus Time to Possible Time

production, is higher than if they were earning no bonus. It is a good plan to know what the standard earnings should be, which on the chart are shown by a dotted line. The actual bonus earnings come within 20 cts. of the standard in period ending $6 / 28$. The chart shows a healthy condition in that bonus earnings per man show a steady increase.

In order to keep in close touch with the progress of bonus men, the chart shown in Fig. 62 is suggested. Two things are essential-

(1) There should be a steady increase in the number of men put on bonus.

(2) Those on bonus should have as much of their time covered by schedules as is possible.

On the chart the heavy line shows ratio of the time of bonus men on schedules to the total time they work, while the dotted line shows the ratio of time of bonus men on schedules to the time of all men in the department or plant. Take the period ending 5/24 for example; more men were put on bonus, but the time on schedules was less than in the previous period. The dotted line for period $5 / 31$ shows a falling off in the number of men on bonus, although those who were on worked on them $65 \%$ of their time. The heavy line for period $4 / 26$ and $5 / 3$ 
shows such decided drops as to warrant rigid investigation. Both lines, however, show an upward tendency, which is, of course, encouraging.

The "inefficiency chart," Fig. 63, is decidedly necessary. My claim is and has been that inefficiency is the element to analyze, for we increase efficiency only through eliminating inefficiency. Further, the inefficiency of management should be shown as distinct from that of the men. If this is not done there can be no true conception of what is at fault and who to blame. This is accomplished by adding the allowances to the actual hours, after the man-efficiency for a department has been determined, and aividing the same figure for standard hours that was used in figuring the man efficiency, by the increased divisor in the form of actual hours. The full line, or man inefficiency, shows a constant decrease although in period 5/10 it increased noticeably. This increase might be due to putting new men on bonus, or old bonus men on new work, or other causes, like cutting a rate or arousing the opposition of the men. From period $4 / 26$ both inefficiency of manage-

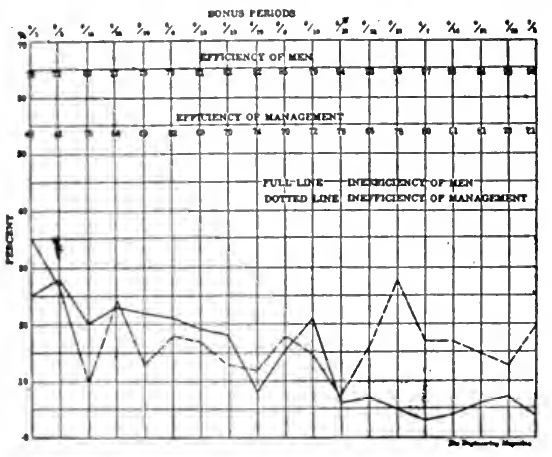

Fig. 63-Inefficiency Chart

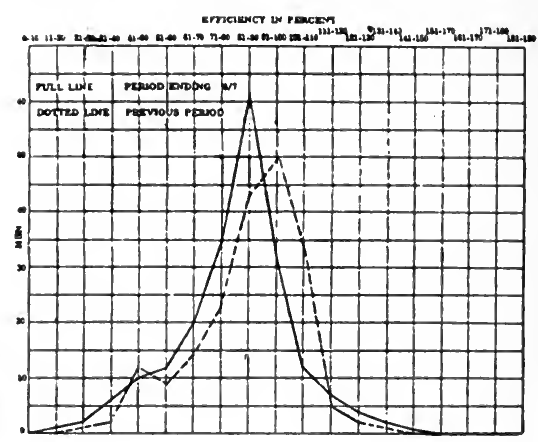

Fig. 64-Number of Men at Various Efficiencies

ment and men increased after several periods of decreases. Then comes the surden drop in both for period 5/17. The significant fact in connection is that following the period 5/17 the man inefficiency decreased while the inefficiency of the management took two upward spurts. Further the lines show that the men are making faster progress in eliminating their inefficiency than the management, the moral of which is-"get after the management."

Fig. 64 is important in showing the number of men at classified efficiencies. For the period in question the chart shows that 127 men attained efficiencies varying from $71 \%$ to $100 \%$, while in the previous periol only 116 men attained these efficiencies. $\Lambda$ t the same time the general showing for the previous period is better than for this period, in that there were less men showing efficiencies from $51 \%$ to $90 \%$ and more men from $91 \%$ to $110 \%$. The value of the chart lies in the ability to concentrate attention on the men showing efficiencies of $80 \%$ and under, and ascertaining what interferes with their attaining greater efficiencies. Further, this chart is especially valuable in connection with Fig. 62 showing relative times. The work of getting more men on schedules, keeping those who are thus employed on schedules for the 
greatest part of their time, and getting the men showing one class of efficiency into the next higher, can be planned from these two charts.

To assist further in the work of eliminating inefficiency a sheet should be prepared covering the efficiency of the workers for a period and posted in a place where it can easily be seen, Fig. 65 .

Piecework Rates. The following is from an article by Otto M. Burkhardt, published in American Machinist, August 29, 1918, entitled "Determining of Piecework Rates from Charts."

In an able speech before the British House of Commons Dr. Christopher Addison, head of the Ministry of Munitions, refers to piecework rates as follows: "Nothing in the relations between capital and labor gives rise to more difficulties and distrust than two customs which are dependent upon one another. The first is the cutting of rates of pay on

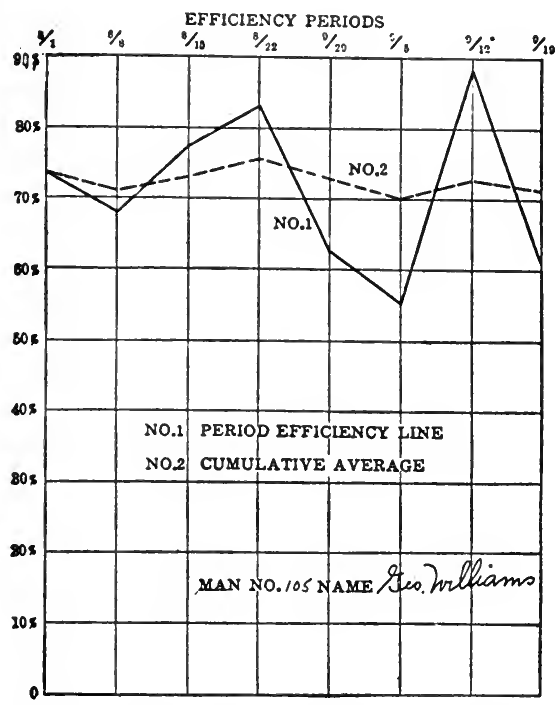

Fig. 65-Graphic Efficiency Record

piecework so as to limit the rise of earnings when improved methods of manufacture leading to greater output are introduced. It is not the practice of the best employers, but it is adopted by many. This practice, or the fear of it, has inevitably led to the second and retaliatory practice of the restriction of output. The influence of these two practices in our industrial life is thoroughly poisonous."

The importance of the above-named problem warrants the closest consideration. The Pierce-Arrow Motor Car Co., of Buffalo, N. Y., for instance, for many years has observed the rule that the only condition under which the rates of piecework can be changed is by the entire change in the mode of manufacture. In this concern all piecework rates are determined by one supervisor who follows certain fixed rules.

First, obtain the actual time per cycle of operation in minutes. Let this be represented by $t$.

Second, select the base rate pertaining to the machine on which the 
operation is performed. This may be denoted by $B$. The above two factors determine the theoretical minimum piece-rate price $P$. Mathematically this may be expressed as follows:

$$
P=t \frac{B}{60}
$$

We have, however, further to consider unavoidable losses of time incident to all operations, as, for instance, the time necessary to change tools. In order to eompensate for these losses a definite amount must be added to the piece-rate price obtained with the equation 1 . Let us suppose the operator is losing $h$ hours per 10-hour workday. It is evident then that his time available for doing piecework may be represented by the fraction $t_{1}=\frac{10-h}{10} t$. In order now to include the monetary compensation for the lost time in the piece-rate price we shall have to arrange our equation so that the operator may earn in the time $t$, as much as obtained from equation 1 for the time $t$. This suggests the following equation for determining the new piece-rate price which we shall denote with $P_{1}$. It is:

$$
\begin{gathered}
P=\frac{10-h}{10} P_{1} \\
\text { Hence: } P_{1}=\frac{10}{10-h} P
\end{gathered}
$$

This equation may be resolved to read:

$$
P_{1}=P+P \frac{h}{10-\mathrm{h}}
$$

After substituting for $P$ the value given in equation 1 , we obtain

$$
P_{1}=t \frac{B}{60}+\frac{h}{10-h} \times t \frac{B}{60}
$$

The first item of the aggregate at the right-hand side of our equations represents the amomint of money which the operator should be paid per piece for performing a certain operation; the seeond item of the same aggregate represents the necessary compensation per piee for time losses.

To stimulate production it is necessary to add to the first item a certain incentive, say, for instance, $10 \%$. The intrcduction of this considB eration expands this item so that it reads $1.1 \times t \frac{-}{60}$. Substituting this in equation 4 we obtain 


\section{ORGANIZATION AND MANAGEMENT}

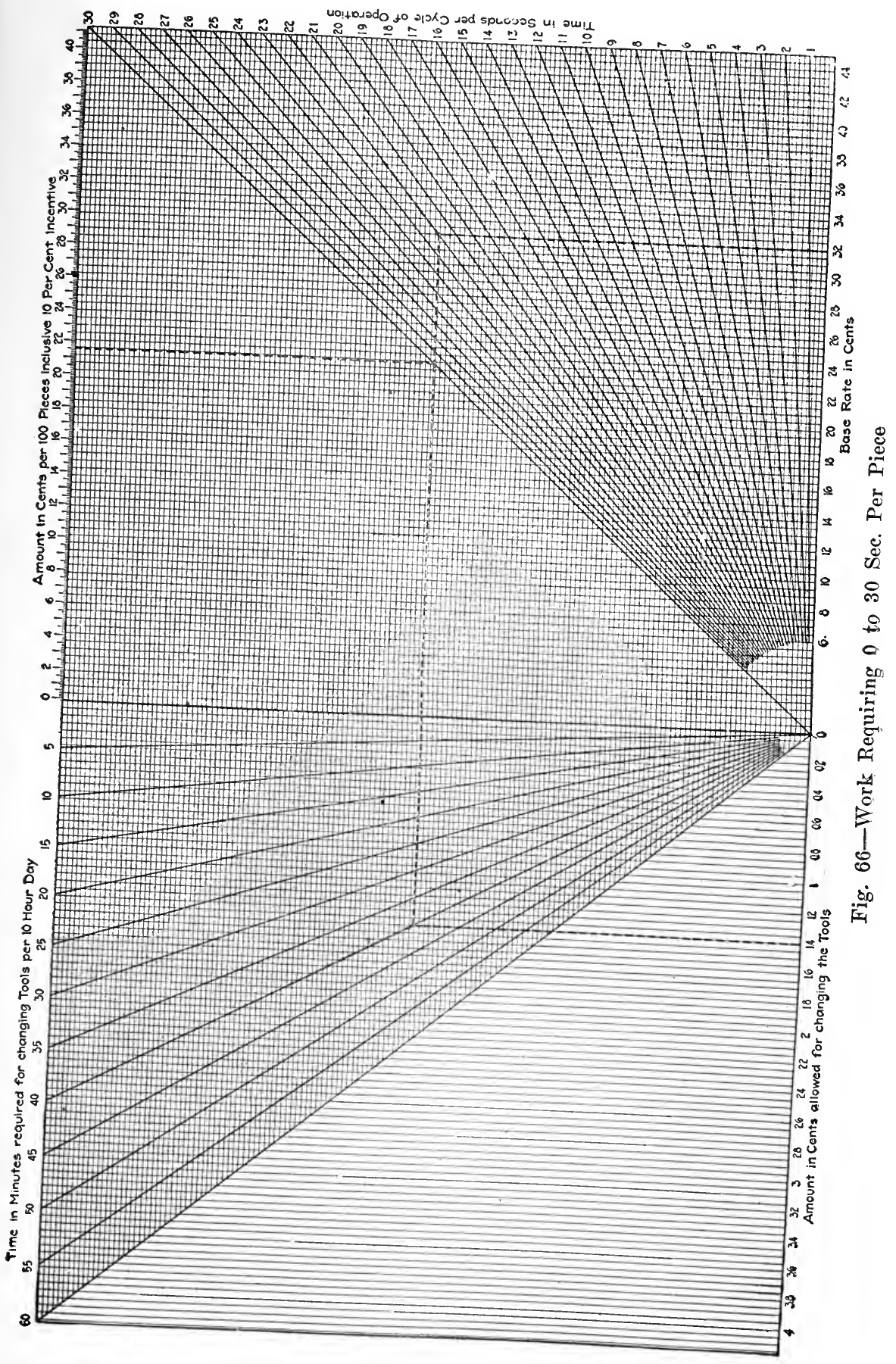




$$
P_{1}=1.1 \times t \frac{B}{60}+\frac{h}{10-h} \times t \frac{B}{60}
$$

It should be noted that no incentive is provided in the item which is introduced to compensate for changing tools. This method has been found most satisfactory in many concerns.

This equation for different values of $t, B$ and $h$, is graphically represented on charts, one for work requiring 0 to 30 sec., one for work requiring 30 to 90 sec., and so on up to 30 to $90 \mathrm{mins}$.

To determine piece-rate prices from the charts we proceed as follows:

Select the base rate from the horizontal axis marked Base Rate. From this figure follow vertically upward until you intersect the radial line which represents the time per cycle of operation. From this intersection follow horizontally to the left until intersection with the last radial line in this quadrant. Vertically above this intersection read the value of the first item of the aggregate at the right-hand side of equation 5 . This value represents the piece-rate price for all operations which involve no time losses. However, if tool changes and other delays are involved we continue to the left from the intersection with the last radial line until intersection with the radial line which represents in hours and minutes the time lost through tool changing per 10 -hour workday. Vertically below this intersection read the amount which must be added to the previously obtained piece-rate. The sum of both is the aggregate piece rate, including $10 \%$ incentive for all productive work and an allowance, derived from the base rate, which is necessary to compensate for tool changing.

We may now select a numerical example:

$$
\begin{gathered}
t=22 \text { seconds }=\frac{22}{60} \text { minutes. } \\
B=32 \\
h=40 \text { minutes }=\frac{40}{60} \text { hour per 10-hour day. }
\end{gathered}
$$

On Fig. 66 select 32 at horizontal axis marked Base Rate. Follow the vertical line upward until interseetion with radial line marked 22, which represents the chosen time per eycle of operation. Follow horizontally to the left until intersection with the last radial line. Vertically above this intersection read 21.5. Now eontinue from the intersection with the last rallial line to the left until intersection with the radial line marked 40, which represents the time required for changing tools. Vertically bolow this intersection read 1.4. Add both values obtained, which gives $21.5+1.4=22.9$ ets. per 100 pieces or 0.229 ets. per one piece.

Now in order to check our charts with equation 5 we shall first simplify this equation. We may write this: 


$$
P_{1}=t \frac{B}{60}\left[1.1+\frac{h}{10-h}\right]
$$

If we substitute in this the above chosen values we obtain

$$
P_{1}=\frac{22}{60} \times \frac{32}{60}\left[1.1+\frac{4 \%, 60}{\left.10-{ }^{40}\right]}\right]
$$

From this follows $P_{1}=0.229$ ct. per piece or 22.9 cts. per 100 pieces. This shows that the chart is correct within the third place.

Bonus Record and Progress. After bonus systems have been installed it is generally desired to keep a record of individuals or groups so that the progress of the new methods may be observed. One chart for this purpose is shown in Fig. 67. This and the following remarks pertaining to it are abstracted from an article by Walter N. Polakov, "Mastering Power Production"' published in Industrial Management, July, 1918. The bonus record chart, Fig. 67 shows the progress in

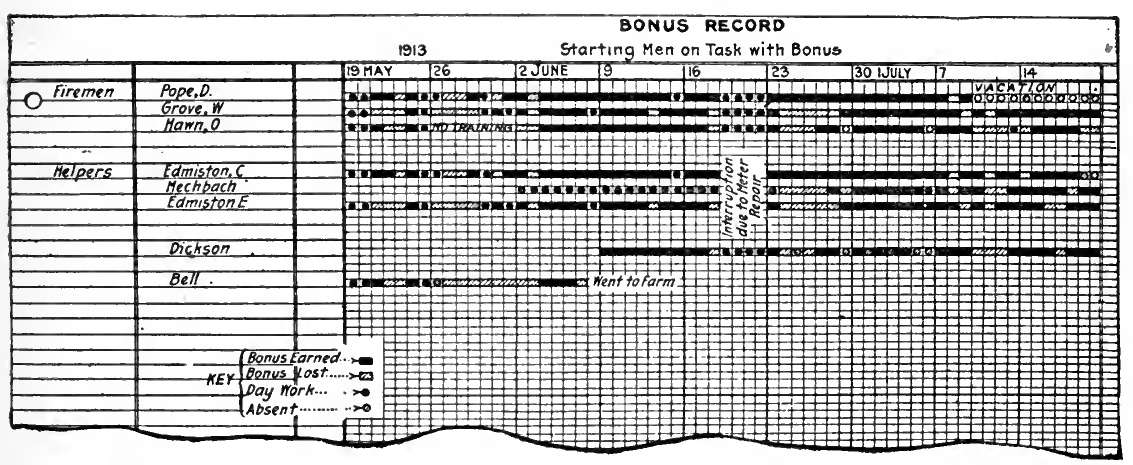

Fig. 67-Chart Showing Bonus Record and Progress After Starting Task Work

starting on task work with bonus in a boiler house of a public utility plant, showing remarkably quick progress in learning, especially considering that most of the firemen had no previous experience.

Employees' Benefits. Charts are a great help in explaining the methods and features of various employees' benefits, such as insurance, pensions, loan funds, etc. A typical example is Fig. 68, which, with the following explanation, is taken from The Automobile, April 5, 1917. There are other benefits not mentioned here, this being taken merely to show the figure.

Joint payment by manufacturer and worker is the feature of the Fell plan, which provides for old age and other times of need. The joint-payment factor makes continuance of relations between the manufacturer and the worker essential to the working out of the plan, so that the system is in essence turnover insurance with the granting of pensions, death, accident, and sickness features as a by-product.

Preliminary to the paying in of any funds, an association is formed 
in the factory composed entirely of employees. The membership is divided into departments, each department selecting its own local secretary. The local secretaries seleet the board of directors for the insuring system, and the direetors appoint such standing eommittees as may be required. This type of organization is adopted to put the control of the plan in the hands of the employees.

Each worker pays a flat rate of $5 \%$ of his wages into the insurance fund. The employer contributes to the same fund on a graduated scale which hinges upon the length of time the employee has been with the

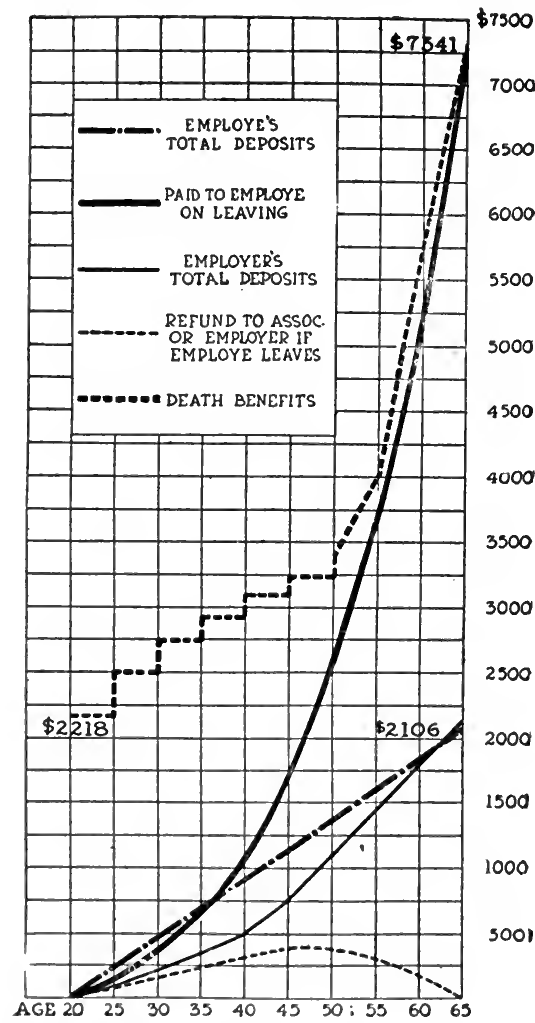

Fig. 68-Showing Working of Fell plan

When Employee Enters at 20, Wages $\$ 18$

eompany. The man who is a new employee pays a larger amount than the manufaeturer, but the eonverse beeomes the ease as the term of service lengthens. The worker is paid for his investment by the varions insuranos mivileges to be deseribed, and the manufaeturer by the saving of waste in changing the personnel of the force.

The men are divided into 5-year groups. The employer pays into the fund $1 \%$ of the wages of those who have been with the company less than 5 years, $2 \%$ in the case of those who have been employed from 5 to 10 years, and so on. The following table shows the schedule: 
Years' service of employee:

$1-5 \quad 5$

10

15

20

25

30

Inasmuch as the employer has no actual cash claims on the fund and derives therefore no direct financial benefit, his payments from the first year represent $20 \%$ interest on the total invested, and constantly increase. A large number of employee's privileges can therefore be paid for under this system.

The easiest way to see how the plan works is to take a typical case. A young man starts work with a company when he is 20 years old. $\mathrm{He}$ gets $\$ 18$ a week, and retires at 65 years of age. Without figuring that the young man might get a raise during 45 years of service, his financial situation from time to time is as follows:

He will have paid in $\$ 234$ at the end of the fifth year. For this he gets: 1-A life insurance policy for $\$ 2,217$ during the period. 2-The right to borrow $\$ 168$ in case of sickness at the end of the fifth year. 3 -A surrender value of his contract of $\$ 126$.

At the end of 20 years the privileges are proportionately much greater, due to the compound interest which the money may have earned and the graduated scale of payments for the employer. The young man at the age of 40 then has paid in $\$ 936$.

$\mathrm{He}$ is carrying a life insurance policy worth $\$ 2,977$, can borrow in case of sickness $\$ 1,268$, or surrender the contract for $\$ 1,008$ eash.

When this employee has reached the age of 65 he can retire from active service with a pension of $\$ 677$ a year for the rest of his life, and if he should die before he had received the pension for 10 years it will nevertheless be paid until a 10-year period has elapsed to his estate. In lieu of a pension he may have a paid-up insurance policy of $\$ 7,341$, and may withdraw this sum if he so desires.

Accident Prevention. Every industrial plant which professes to have the welfare of its employees at heart has some sort of safety work in progress. In this connection charts are a very helpful asset.

The following, from an article by D. E. Charlton, "Utilization of Mine-Accident Reports," published in Engineering and Mining Journal, November 30, 1918, presents some interesting charts and shows what may be done in this line.

The standardization of mine-accident statistics has frequently been discussed, and through the excellent work of the Bureau of Mines most mining companies have followed a general form in the classification of accidents. Compensation laws of various states have made it compulsory that records of casualties be carefully kept, and mining companies in general have adhered to some plan or other which has enabled the public to know the manner in which their operations have been conducted and what efforts they have made toward providing safe working conditions for their men.

Filling out accident reports involves considerable labor, regardless of whether the information is secured in a careful or a careless fashion, but, assuming that average care is used in compiling an accident report, it is certain that the information could be used to more advantage than as a mere history of a specific accident, and that a careful analysis 
of the data secured would show that a number of reports, covering various aceidents and carefully compiled, would constitute a basis for systematic aceident prevention.

There is no doubt whatever that accident prevention pays, and that its cost must necessarily be a part of the total operating expense, and the same eare and study should be applied to the curtailment of accident cost as to economy in other branches of mine operation.

A careful study of a standard aecident report will disclose latent possibilities. To many operators, a complexity of statistics is an abhorrence, and doubtless an overdoing in this direction will lead to indifference, but, with diseretion, there is no reason why carefully prepared data eamnot be elassified in such a way as to be of great value to the mine manager and to those intrusted with the safety of the men.

The modern idea of having professional medieal attendanee for all aceilents, no matter how trivial, is generally accepted, as good policy. not only because it insures a complete understanding of the nature of the injury, but for the additional reason that it often prevents developments which may result seriously. Further, it offers an excellent medium for the collection of aceurate data, as most of the information required can be secured at the time of the injury. All aceidents should be reported, regardless of the extent of injury, for, unless this is done, certain oceurrences which appear trivial may go unrecorded, frequently developing eomplications long after there is apparent reason to look for them, and beeause lack of continuity in the records will be an obstacle to the institution of a safety eampaign.

A further argument in favor of carefully kept statistics is the psyehologieal effect upon the men. No eonseientious miner is content merely to blast his holes and then maintain indifference as to the manner in which the round has broken. He usually finds out what the results are, and not infrequently determines his plans for the next round or for future work by the manner of breaking. Assuming that safety and accident prevention have been given prominenee, the men will naturally show interest in the progress which is being made, and the mine safety bulletin board may be supplemented to excellent advantage by the posting of plats and charts showing the progress or retardation.

Fig. 69 shows a "relative hazard" chart, based on the number of serious accidents which occurred during a two-year period, and considers only the occupations which were affected by those accidents. In preparing the chart, the total number of men in each oceupation was used in eomparison with the number injured in that ocempation. The graph represents the number of times greater or less than their proportion of employment that the men in each division were injured. In view of the fact that a comparatively short period is represented, and that the number of arecidents is small, the graph camnot be said to be representative or comelusive, but nevertheless it is an argument for the continuance of aceoilent statisties, as each additional year will show a better average of the figures represented.

The time, plare and ranse of aceirlent are subjects that should receive special sturly, not only in the individual ease but as covering a number of areirlints as weill. Fig. 70 represents the hours at which accidents oecur and is lased on a three-year period. It may be men- 


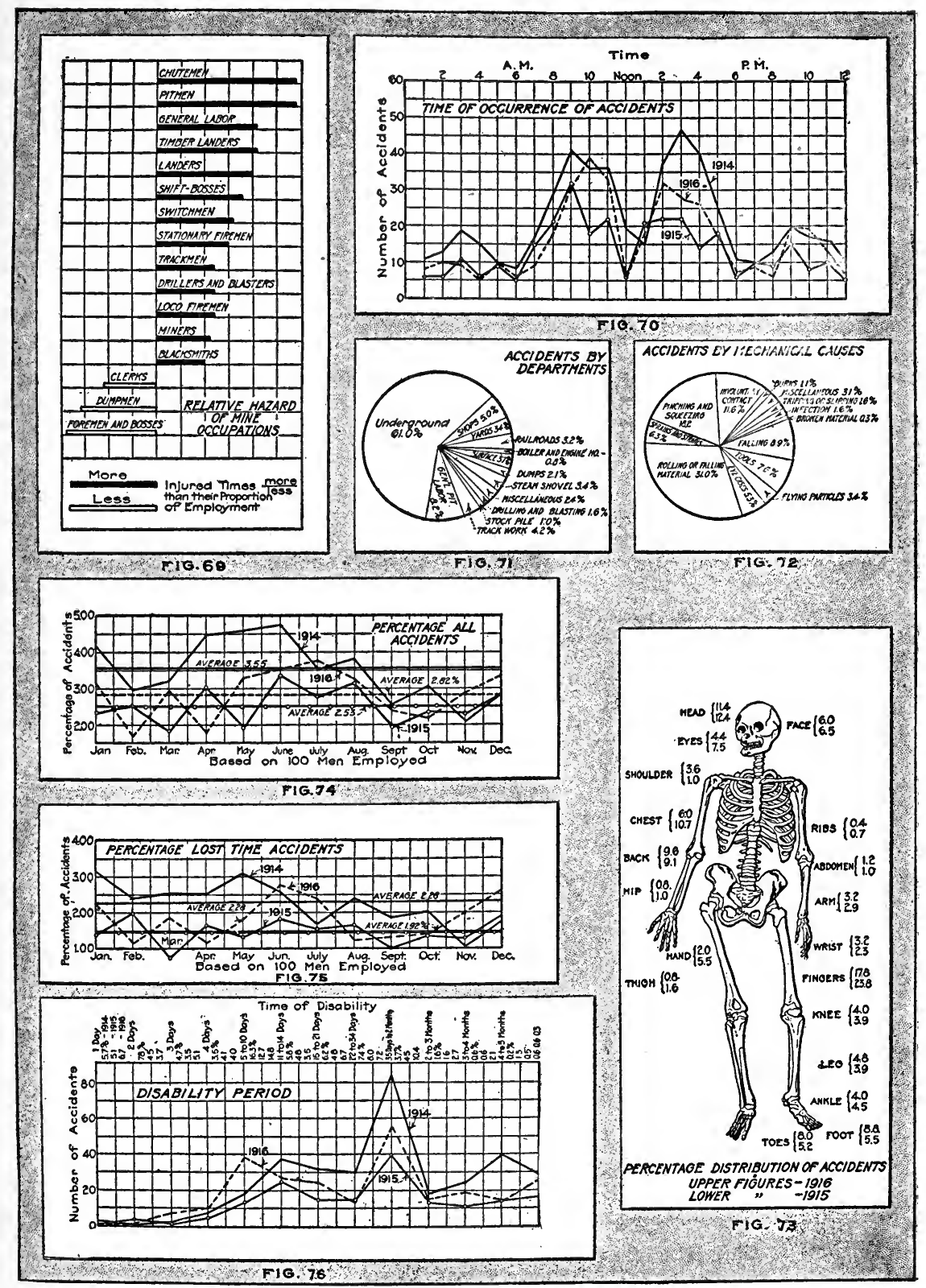


tioned, however, that the results obtained in a graph of this sort may be scattered unless some attempt is made at a separation of the two and three-shift basis. That the graph maintains such continuity is accounted for by the fact that the ratio of the two-shift to the three-shift phase remains fairly constant during the three-year period and that the relation of the underground accidents to those occurring on the surface was approximately the same. Fig. 71 shows another method of comparing accidents, and the varying percentages of different departments are expressed. Only a one-year period is considered, and the percentage is based on the total number of accidents oceurring. The mechanical causes, as found in the answers to question 13 of the report, expressed in a chart similar to the one used in Fig. 71, are shown in Fig. 72.

It has been $m y$ experience that the answers to the questions in the group relating to "safety" are subject to doubt as to their reliability, because not infrequently too much personal opinion is likely to be recorded. The report is usually made out by a foreman, a shift boss or a timekeeper, and though the intention, in making the report, may be good, judgment is sometimes made in haste and without taking account of all the circumstances involved. However, this part of the report may be changed, if necessary, in making up the figures to show comparative results in this group.

It may not be amiss at this time to emphasize an important factor that is worth consideration in accident prevention. That is the interpretation of the division which it is possible to make between "preventable" and "unpreventable" accidents. This is a matter which is dependent largely on the degree of thoroughness with which accidents and their causes are studied. If it be to natural occurrences, or preventable, there is then afforded a tangible basis on which to build up the manner of prevention. whereas if all accidents be attributed to "hazards of the industry," and are assumed to be unpreventable, further research, frequently of value, will be discouraged. Each accident should be made a special object of study, and a practical method of preventing its recurrence should be suggested and put into application. Several companies have arlopted the practice of making a periodical examination of all accident reports, preparing a reeord of their conclusions and carrying out the suggestions made. This policy, supplemented by other preventive methods, has attained excellent results in eutting down the number of accidents.

The value of the diagram presented in Fig. 73 may be questioned, but the figuress show comparatives, and the representation is such as to draw attention on the mine bulletin board. In this figure the percentagres of accidents are presented as to their distribution in various parts of the body and represent the comparison of injuries for two years, the information being secured from a eompilation of the answers given to question 25 of the report.

Figs. 74 and 75 were plotted from caleulated percentages based on accidents and the number of men employed. In Fig. 74, all accidents were considererl, whereas in Fig. 75 only those which entailed the loss of time. The pereentages shown in Fig. 76 were computed on periods of lost time which resulted from accidents, and, necessarily, only those 
involving loss of time were set down. The total percentage for any one year, as expressed in the graph, will equal the percentage represented by the lost-time accidents as compared to all accidents which occurred during that year. The mine report does not provide for a statement of the time lost on each accident, but this is always kept as a supplementary record, and should be available.

Fatigue and Accidents. Mary L. Morris, in connection with her article "Beginnings of Labor Maintenance Service in a Snall Plant,", published in Industrial Management, September, 1918, shows two interesting charts Figs. 77 and 78, and says: "The charts made from our weekly accident sheet, which we keep daily, show how the fatigue element operates to bring about more accidents at the end of the week

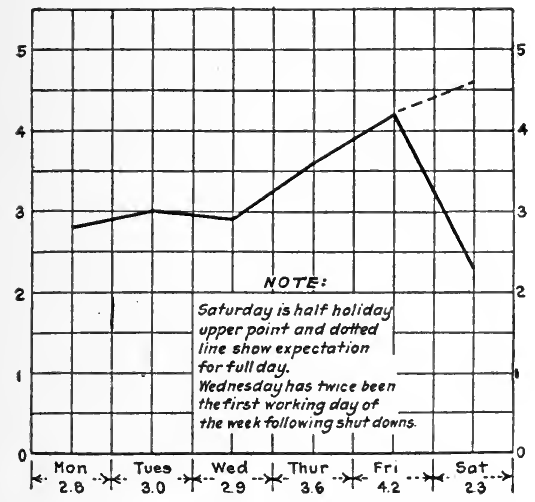

Fig. 77-Average Number of Accidents Per Day for 20 Weeks

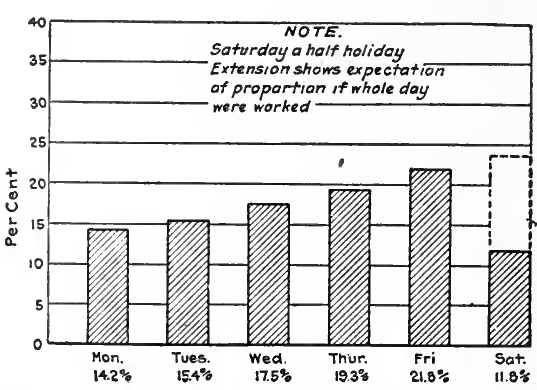

Fig. 78-Percentage of Total Number of Accidents on Each Day of the Week, September 1, 1917-January 18, 1918

than at the beginning. Saturday being a half holiday in the plant accounts for the lower point on that day, but were the corresponding five afternoon hours worked as on the other days, the resulting point would be higher than on any other day.'

Wages vs. Cost of Living. Frequently investigations are made relative to the cost of living of employees in an industrial community. The object is to find out whether wages should be increased proportionately. The graphical chart shows the relative comparison better than any other method. As an example Figs. 79 and 80 are taken from an article, "How to Determine Cost of Living in an Industrial Community," by R. M. Hudson, published in Industrial Management, September, 1918.

Output. The following is from an article by J. B. Conway, "An Accumulative Graphic Record,"' published February 6, 1919, in The American Machinist.

Several years ago I was industrial manager for a well-known gas-engine manufacturer, and after certain changes in the method of production had been made I found it necessary to devise a chart for recording the weekly output of certain departments. Prior to this a daily record was maintained, which was totaled at the end of the week. While 
this served its purpose in a measure it did not portray a vivid record for comparison, and after several weeks of continuous entries the executives were compelled to work over a maze of figures in order to com-pare one week with the next.

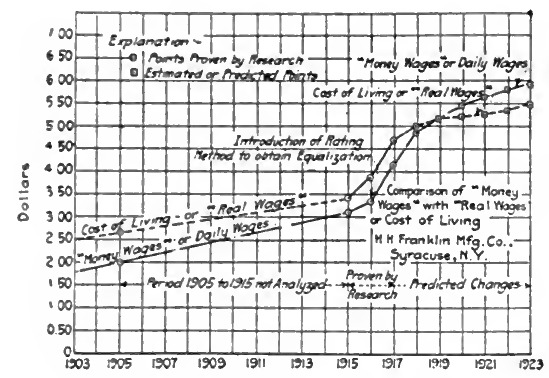

Fig. 79-Chart Showing Trend of Cost of Living

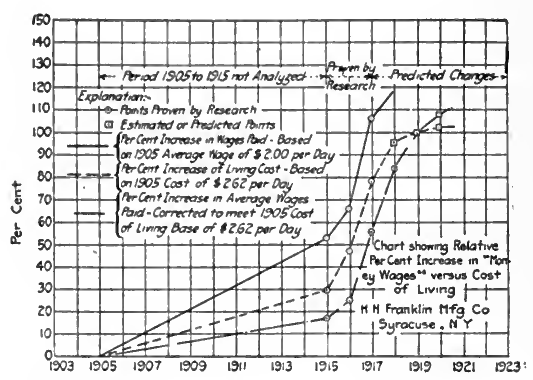

Fig. 80-Increase of Living Costs by Percentages

To grasp quickly the essential facts the ehart, Fig. 81, was used with the exception of the several colors employed in the original. Along the left-hand margin is given in figures the required monthly output of

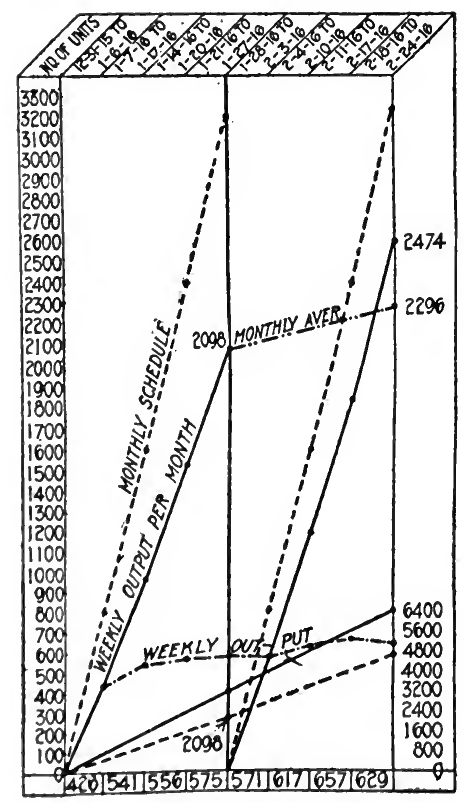

Fig. 81-Graphic-Record Chart

units, which was 3,300 in this instance. Each square represents 100 units, rearling from bottom to top. The weekly periods are shown at the top of the chart. II ravy vertical lines divide the weekly periods into periods of four weeks. 
The monthly schedule is also shown by a "curve" representing the normal weekly output expected. The actual weekly output is shown for the month by the line beneath. It will be readily seen how any deviation from the normal-output curve is brought into prominence when compared in this manner. In the first month the output was only 2,098, or a decrease of 1,102 from the normal, which was 3,200. To the foreman or superintendent responsible for getting production the figures $3,200,2,098$ and 1,102 do not have the same significance that the gap shown on the chart between the two curves has, and they are not so readily comprehended.

Across the lower margin is entered in figures the actual number of units manufactured per week, the first week producing 426 , the second 541 , and so on. These figures not only amplify but verify the points on the curve for the weekly output. The curve marked "Weekly Output" is for convenience in comparing one week's output with another, and was found useful as an object lesson in talking over the results of the past week with the foremen who were more able to appreciate the fluctuations in the curve than the difference in the totals. This statement in no way casts reflections on the ability or intelligence of the foremen. If the reader has any misgivings he should himself make a few comparisons numerically.

The two curves at the bottom of the chart indicate the expected output per week for a period of two months and the actual output per week compared graphically. The scale for these curves is shown at the right-hand side of the chart extending from 0 to 6400 , each square representing 800 units. Attention is called to the use of figures again to verify the points on the curve, which for the first month are 2098 and for the second month 4572 , the latter being the accumulated total, or the sum of the first and second month's output. The first figure checks that represented by the curve showing the actual output per month and the latter is the sum of 2098 and 2474 .

It will be noticed that a considerable gain was made during the second month over that preceding and is readily recognized by the wide divergence of the two curves for the first month and the greater parallelism of the two for the second month. This is seen at a glance in making a comparison, even though no figures were available.

The curve starting with the end of the first month and making its path across the chart is the "Monthly Average," which is the sum of the output for the two months divided by the number of months. This, like the weekly output curve, serves as a comparison of one month's work with another. When earried for only two months as shown the curve does not show up to its best advantage, but it must be borne in mind that this entire chart carried a record for a six months' output and at the end of 12 months the two six-months' charts were fastened together, thereby giving a complete and comprehensive record for the year. In this respect the average monthly curve was an important factor of the chart.

The underlying principles of the chart may be employed to advantage for recording the units of production of almost any article, the only changes necessary being the dates and the substitution of the name 
of the article, if desired, for that of the "units" on the left, and the quantity required.

Reducing "Time Out." The following article by this title, together with Fig. 82, by Rudolph Appel, was published in Factory, September, 1917 :

The presses of a large printing establishment had a capacity of 9,500 impressions per day. The daily average for a half month, however, was only 4.500 per day. This loss of $52.6 \%$ in the possible production was too great to pass by unnoticed.

The manager endeavored to find the source of this loss by a close study of the time eards. The cards for the first two or three days of the month were very easy to compare, but by the time the sixth or

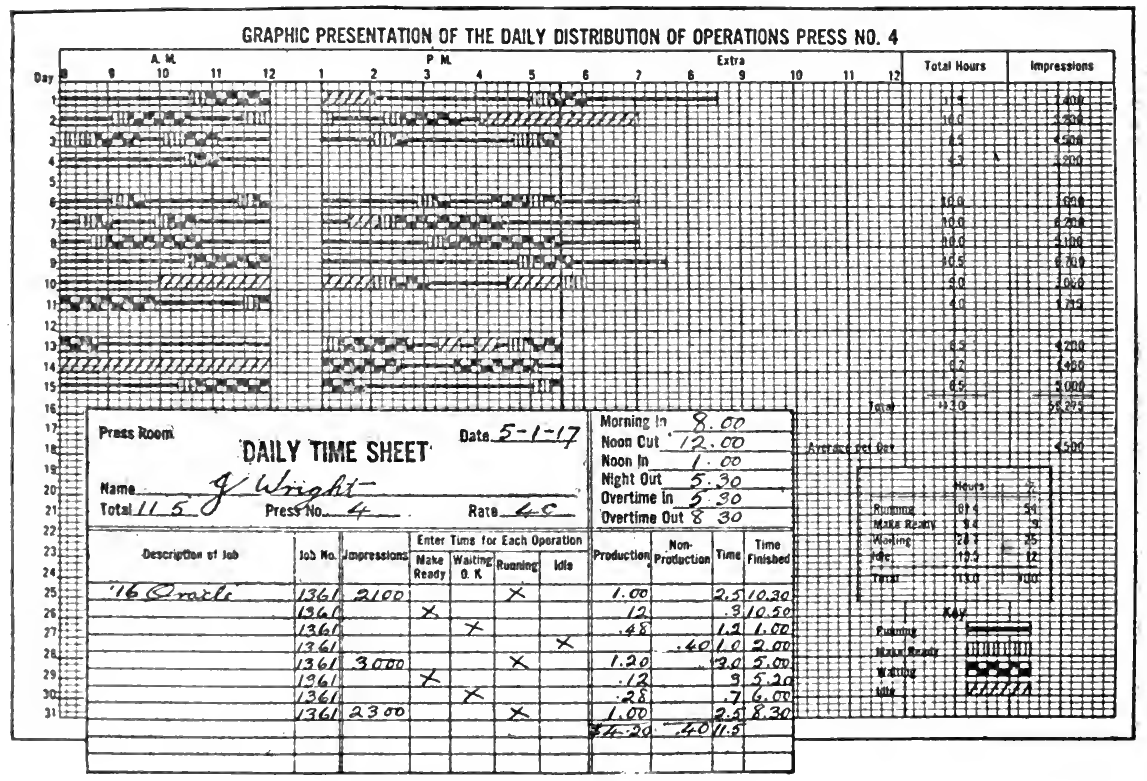

Fig. 82-Reducing Time Out

seventh card was reached they began to look alike. So many items were involved that it became a hopeless jumble. One of these cards filled out for the first day of the month is shown in Fig. 82.

Finally the manager decided to ehart these cards for each press and in this way see if he could locate the somrce of the loss.

Fonr keys as shown wore ehosen to emphasize the different operations-rmming, waiting, make ready and idle. When the time cards. were blotferl the wating time showed up partienlarly strong as the source of a larege proportion of the loss. The first question asked was, "Why all this wating time?" Investigation disclosed that a large portion of it was due to the slowness in returning the $O$. $K$.'d proofs. to the press rom.

Up to this time if had been known that there was an inevitable loss. of time on this operation but until this picture showed up the loss 
so plainly it had been very difficult to speed up this $\mathrm{O}$. K.-ing process. After this was so emphasized the percentage of time lost in O. K.-ing proofs was reduced by $9 \%$. At the same time daily impressions increased to such an extent that a great proportion of the overtime previously considered necessary was eliminated. The make ready and running times, however, were not changed to any marked extent. These, however, were production operations and of course would not be emphasized in a chart of this sort.

During the month, on the second, tenth and fourteenth considerable idle time is shown by the chart due to the power being cut off by severe storms. This one point would be shown up on that day's time card and then forgotten about. The graph, however, makes this so clear that it can be noticed almost at a glance and taken into consideration when making a comparison.

After this chart had been in operation for some time, the firm was able to take on considerable extra work which previously had been refused because it would necessitate overtime. With the additional cost due to overtime they could not compete with other firms at a profit.

A plan of this sort may be used for charting the operation of practically any machine. For instance, a punch press goes through practically the same cycle of operations although the waiting time for inspection is not likely to be so long as was the case at this printing establishment. There may be a waiting time for material or a waiting time for the die-setter which would, of course, be emphasized in a chart like this.

Truck Performance. Figs. 83 and 84, together with the following descriptions, are from an article "Charting Motor Truck Performance," published in Textile World Journal, June 1, 1918.

3
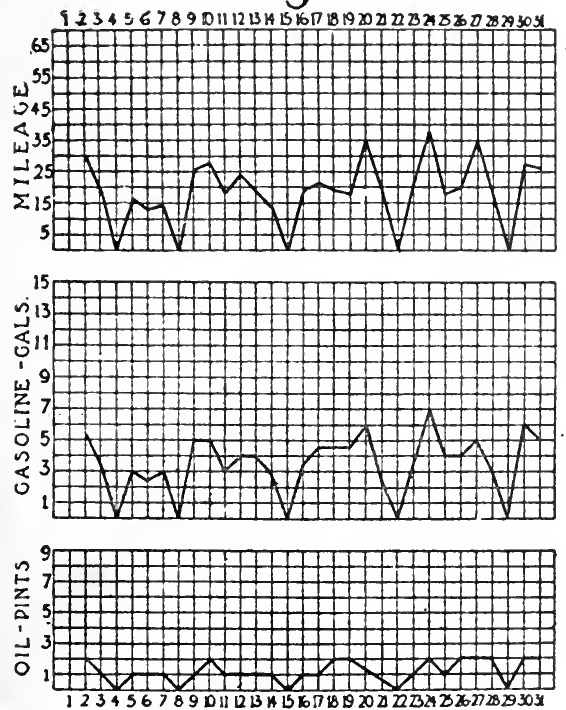

4
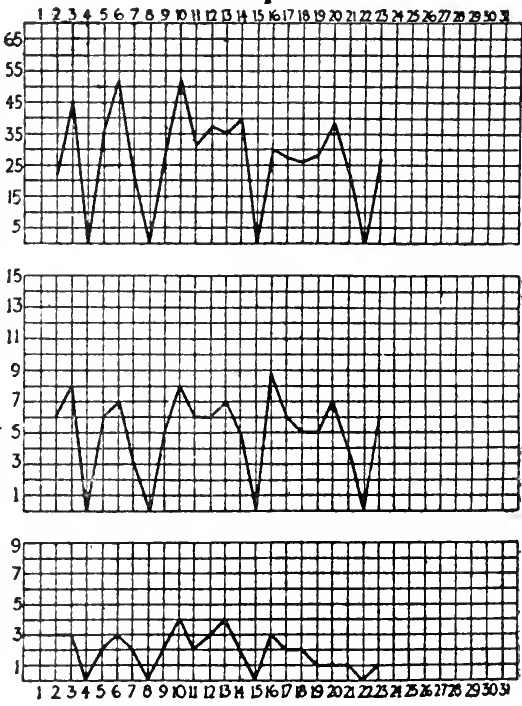

Fig. 83-Truck Performance 


\begin{tabular}{|c|c|c|c|c|c|c|c|c|c|c|c|c|c|c|c|c|c|c|c|}
\hline \multicolumn{20}{|c|}{ 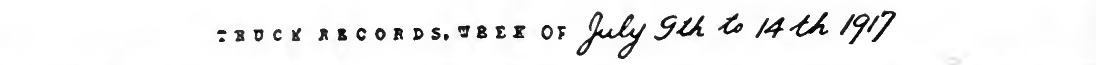 } \\
\hline \multicolumn{4}{|c|}{ zBJCX $\$ 0 . /$} & \multicolumn{4}{|c|}{ rauck so. 2} & \multicolumn{4}{|c|}{ zence no. 3} & \multicolumn{4}{|c|}{$\operatorname{racc} 20.4$} & \multicolumn{4}{|c|}{ 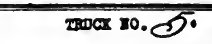 } \\
\hline Dis & GAS & $02:$ & $x: D s$ & DATS & GLS & OL & XILSS & Dasts & ans & OUL & nums & DAF & OLS & OI & Nus & 2458 & ass 10 & \begin{tabular}{|l|l|} 
Out & 12 \\
\end{tabular} & $x u B s$ \\
\hline 9 & II & & 13.5 & 2 & 2 & 1 & 2 & 9 & 5 & 1 & 26.3 & 9 & 5 & 2 & 28 & 9 & 6 & 2 & 7.4 \\
\hline 10 & $p$ & & 85 & 10 & 3 & 1 & 3 & 10 & 5 & 2 & 28.6 & 10 & 8 & 4 & 52 & 10 & 45 & 3 & 10.4 \\
\hline II & 5 & & 4 & II & 3 & 1 & 9 & II & 3 & 1 & 15 & II & 6 & 2 & 315 & $/ 1$ & 4 & 2 & 8 \\
\hline 12 & 6 & & 6.5 & 12 & 3 & 1 & 43 & 12 & 4 & 1 & 24 & 12 & 6 & 3 & 32 & 12 & 4 & 3 & $4^{3}$ \\
\hline 13 & 5 & & 65 & 13 & 2 & 4 & 4 & 13 & 4 & 1 & 19 & 13 & 7 & 4 & 35 & 13 & 5 & 8 & 11.5 \\
\hline 14 & 4 & $\gamma$ & 4.2 & 14 & 4 & 1 & 3.6 & 14 & 3 & 1 & 14.3 & 14 & & 2 & $4 c$ & 14 & 2,1 & 2. & 10.6 \\
\hline \multicolumn{4}{|c|}{$\operatorname{srocos} 50.6$} & \multicolumn{4}{|c|}{ Istox xo. 7} & \multicolumn{4}{|c|}{ mocex } & \multicolumn{4}{|c|}{ IRUCE 50.99.} & \multicolumn{4}{|c|}{ IROCE 30.10} \\
\hline 9 & Es & & We & 9 & 3 & & 7 & $e$ & 5 & & & 9 & & 2 & 12 & 9 & 101 & & Wa. \\
\hline
\end{tabular}

Fig. 84-Records from which Fig. 83 is Made

One of the easiest methods of checking up on the eare which drivers give their trucks and of comparing the care given by different drivers of a large fleet of trucks is by charting the mileage and the gasoline and oil consumptions. A simple method of charting these records worked out by the superintendent of motor truck equipment of a large concern, was given in a recent number of The Commercial Vehicle.

The method followed is shown in the two charts at Fig. 83 which represent the records of trucks Nos. 3 and 4 for a month. The large figures at the top of the chart represent the truck numbers, while the smaller figures on the cross-sectional paper read for the days of the month in a horizontal direction and for the three items of mileage, gasoline consumption and oil consumption when read vertically.

The weekly chart shown at Fig. 84 is of the ordinary ledger type and is filled out by the garageman who has charge of serving out the gasoline and oil to the driver every morning. From the figures shown on this record, the garage superintendent was enabled to prepare the graphic chart shown.

Reading vertically for each truck, it is seen that the gasoline mileage secured should show up in a chart in much the same kind of curve as the mileage curve in the topmost of the three blocks of the cross-sectioned areas in Fig. 83. Similarly, the oil consumption in pints should bear a corresponding ratio to the mileage eovered each day by the truck.

By comparing the records of one truck with those of the other trucks on the same large sheet of paper, the comparative operating efficiency of the trucks on any one sheet may be regularly ganged.

C'arrying ont the idea further, average lines may be drawn through the curves for each item. These average lines would be horizontal and by comparing the average of one truck with that shown next to it on the chart, the relative eeonomy may be judged by the vertical distance between the two horizontal lines.

Results of Trucking Operations. Fig. 85 is from an article by .J. M. Van Harlingen, "The Economy of Centralized Trucking," published March, 1918, in Industrial Management. The trucks were principally hand ones with a few electric industrials. They were equipped with recording devices to show "time in motion" and "idle time." The 
charts were made up from the records and illustrate a method of comparing performance.
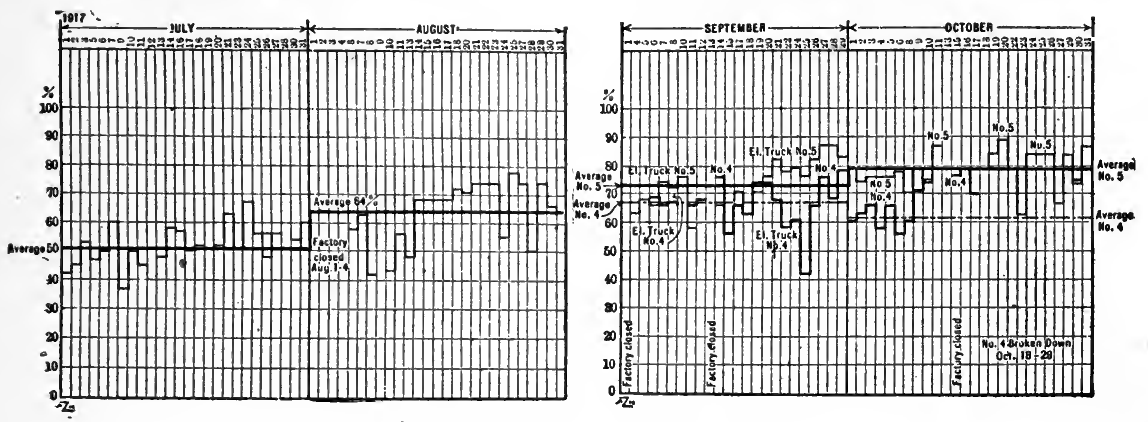

Fig. 85-Trucking Operations

Following, is a list of articles containing further illustrations of the use of graphic charts of the class discussed in this chapter and pertaining: to kindred subjects.

Upon request, the Codex Book Co., Inc., 19 William Street, New York, will be glad to procure, where possible, copies of magazines containing the articles needed, only charging the "old magazine" price, plus postage.

"Mastering Power Production," Series I-X, Walter N. Polakov, Industrial Management, May, 1918.

"The Organization of Manufacturing Plants," A. D. C. Parsons, Iron Age, Dec. 21, 1916.

"How We Rebuilt an Unprofitable Department," M. Bridgman, System, Feb. 1918.

"What is the Remedy for Diminishing Profits?" Prof. Ralph E. Heilman, System, Feb. 1918.

“'This Plan More Than Doubled Our Typists' Output,' W. H. Leffingwell, System, Nov. 1916.

"My Plan for Applying Scientific Management in an Office," W. H. Leffingwell, System, Oct. 1916.

"Methods of Arriving at Labor Turnover," J. M. Van Harlingen and T. J. Dwyer, Industrial Management, April, 1918.

"A New Problem in Employment,"' Merrill Richards, Industrial Management, Feb. 1919.

"Is It Wise to Hire the Repeater?" Leonard Blakey, Industrial Management, May, 1919.

"Time Studies for Rate Setting on Machine Tools," Dwight V. Merrick, Industrial Management, June, 1918.

"Establishing Basic Rates Saves Time Study Work," Carle M. Bigelow, Industrial Management, Jan. 1919.

"Efficient Employees Are an Element in Costs," Fred. Nicholas, Electrical World, Aug. 26, 1916.

"Basing Wages on a Standard Curve," Factory, Jan. 1914.

"Raising Wages With Cost of Living," L. K. Comstock, Electrical World, March 6, 1918.

"Increased Wages and Decreased Efficiency in the Clifton-Morenci District," Walter Douglas, Mining \& Scientific Press, Sept. 8, 1917. 
"Instructions for Unskilled Labor," J. A. De Turk and G. H. Radebaugh, Industrial Management, July, 1917. 1914.

"How I Applied a Piecework Policy,"' W. F. Hamont, Factory, Jan.

"The Human Factor in Task Setting," W. E. Camp, Industrial Management, Nov. 1918.

"Fatigue," in Factory, June, 1917.

"Intemperate Men Cause Tonnage Loss," Coal Age, Aug. 15, 1.918.

“'Increased Output, Basil R. Medley," Factory, June, 1917.

"Elimination of Idleness by Systematic Study," Chas. Whiting Baker, Engineering News-Record, Sept. 5, 1918.

"Aceident Prevention Work in Commonwealth Edison Organization," Electrical World, May 8, 1915.

"Who Gets Hurt and When?" Coal Age, Sept. 12, 1918.

"Better Lighting of Machine Shops," F. H. Bernard, Electrical Review, June 1, 1918.

"Relation of Lighting to Industrial Safety," John A. Hoelveler, September 1, 1917.

"Cost of Industrial IIealth Supervision," Reginald Trautschold, Industrial Management, Jan. 1918.

"Employees' Benefit Association," Series I-VI, W. L. Chandler, Industrial Management, Jan. 1918 to June 1918.

"How to Prevent Freight Car Demurrage," D. T. Farnham, Industrial Management, Nay, 1918.

"Pointing Out by Curves a Handtruck's Worth," Factory, Jan. 1914.

“'A Machine's Advantage Graphically Shown," Factory, Jan. 1914.

"Fuel Department of Rock Island,"' Railway Age Gazette, July 20, 1917.

"Buying for Seasonal Production," letter from J. S. H., in Factory, August, 1917.

"'Right Time to Buy,"' Neil M. Clark, System, Nov. 1916.

"Graphs That Short-cut Your Work," Carrol Dean Murphy, System, Sept. 1916.

"Ciraphic Control of Production and Cost," C. W. Starker, Industrial Management, April, 1918.

"Cutting Ont Little Wastes," II. O. Wood, system, March, 1918. 1918

"Making Pictures of Facts," James W. Morrisson, System, March,

"Would it Pay to XIove," Prof. Ralph E. IIeilman, System, Dee. 1917.

"Six Simple Ways to Picture Facts," Stanley C. Tarrant, System, April, 1918.

"Knowing, in a Small But Growing Business," D. S. Johnston, System, .July, 1917 .

"Graphs, ('harts and Statisties as Aids to Administration," E. C. Stothart, Elertric Riailua!j Journal, Oct. 2, 1915.

"What Perrentage?" J. W. Brussel, Factory, Nov. 1917.

"The One-Nan Business," J. A. Van Brakle, s'ystem, Nov. 1917.

“The IBusiness 'Front Door,', J. A. Van Brakle, System, July, 1918.

"Rising ('osts." ('lyde B. Marston, System, Sept. 1915.

"Keeping Busy All the Time," Ralph E. Heilman, System, August, 1918. 
"Take Off Your Wraps and Feel at Home," Sidney S. Wilson, System, July, 1918.

"Chart With Which to Make Various Records for Coal," Walter N. Polakov, Electrical World, Nov. 10, 1917.

"Graphic Presentation of Gas Co. Statisties," Stan. C. Tarrant, Gas Age, Jan. 1, 1917.

"Visualizing the Vital Facts," Stanley C. Tarrant, System, Dec. 1914.

" Graphs That Watch for Danger Points," Stanley C. Tarrant, System, March, 1916. 


\section{CHAP'TER XI}

\section{Costs and Cost Analysis}

Charts may be used to show costs and prices, in which capacity they are really price lists in diagrammatic form, and also to serve as a means for analyzing costs with the intention of figuring out therefrom a most. economical arrangement, combination or design.

In the first case, in addition to showing the prices of different sizes, kinds, etc., of any given material, they present a picture of relative increases in price for increases in sizes, etc., so that very frequently, if it is not absolutely necessary to select a piece of apparatus of a certain given size, one may note by a chart the point where the price begins to increase at a less rate than the size, and by purchasing at that point can procure more units,-power, pounds, etc.--per dollar, for his expenditure. Also, charts are unequalled for showing comparisons of prices, as the mind is better able to grasp the relative differences between any two articles through the medium of the eye's perceiving this difference in pictorial form than in any other way.

It is often desired to find out whether there is any relation between the price of equipment per piece and the price per pound or per unit of power, etc., and for this sort of analysis no method compares with that of plotting on charts. The article entitled "Cost per Pound of Electrical Machinery," on page 174 is an excellent illustration of this.

For the purpose of analysis the scope of charts is almost limitless, and the examples given here do not begin to cover the possibilities of this graphic method but were selected as typical cases in the belief that they would offer valuable suggestions for a similar use in other directions. There is not a business concern, large or small, but could use to advantage the graphic method of cost analysis.

Cost of Coal for Generating Steam. The following description is taken from Electrical Review, December 7, 1918. Fig. 1 gotten up by the Westinghouse Electrie \& Manufacturing Co. furnishes the power plant operator accurate information on the cost of coal for generating steam. Knowing the price, and the amount of coal burned and the quantity of water evaporated the solution is easy. Start with the cost of coal per tom and project vertically, intersecting the oblique line corresponling to the evaporation from and at $212^{\circ} \mathrm{F}$. being obtained from the boilers, then move horizontally to the left margin, where the cost of eoal to (evaporate one thousand pounds of water from and at $212^{\circ} \mathrm{F}$. is given. The chart can also be used to determine the evaporation required to generate steam at a certain cost, by reversing the above process. By replotting 1 pon an $81 / 2 \times 11 \mathrm{in}$. sheet the seale is very easily read and the chart may be put bencath a sheet of glass for easy reference.

Cost of Coal per $1000 \mathrm{Lb}$. of Steam. W. H. Schott is the authority for Fig. 2 and the explanatory matter, published in Electrical World, 
October 2, 1915. Fig. 2 has been devised to ascertain quickly the cost of any fuel required to produce a thousand pounds of steam. If the fuel is given in the column at the right, it is only necessary to follow the arrow opposite the particular fuel to where it intersects the diagonal line representing the cost per ton and then drop vertically to the scale giving the cost of coal per $1000 \mathrm{lb}$. of steam from and at $212^{\circ} \mathrm{F}$. Refer next to the evaporation chart above and follow the horizontal line from the feed-water temperature until it intersects the diagonal line representing the steam pressure. Directly above will be the evaporation factor. Multiply this factor by the cost of fuel per unit of equivalent evaporation, and the actual cost of coal to produce $1000 \mathrm{lb}$. of steam is determined.

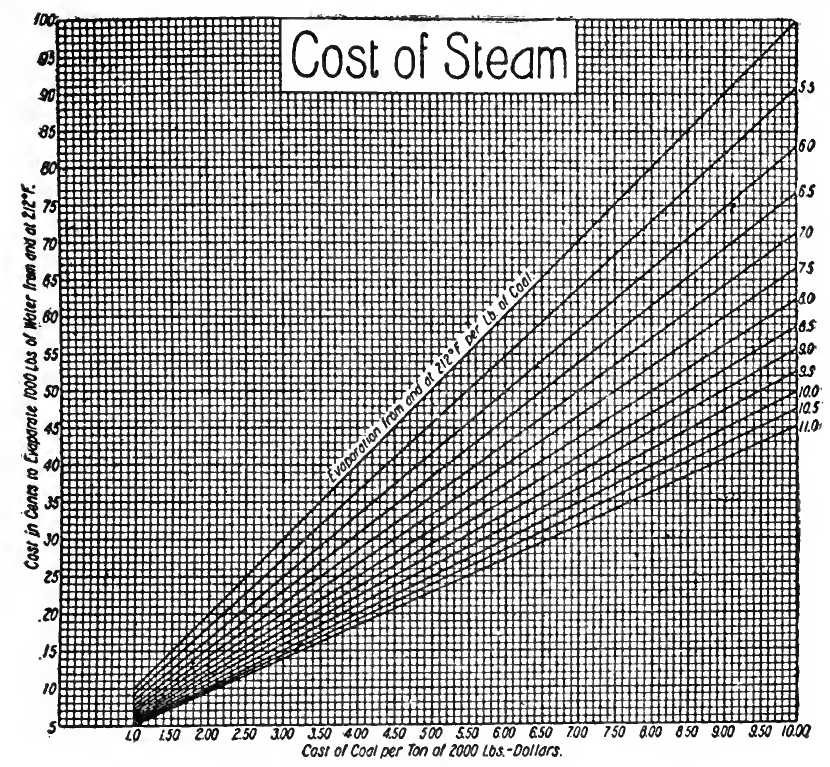

Fig. 1-Chart for Determining Cost of Steam

The arrows opposite the fuel in the column at the right of the chart point to the amount of evaporation which can be expected by burning $1 \mathrm{lb}$. of the particular fuel.

When the fuel used or considered is not listed in the column at the right the proportions of combustible and fixed carbon must be known and the evaporation per pound of fuel estimated. After this is found the procedure is the same as for fuels listed in the chart.

The diagram on the left is to facilitate ascertaining the equivalent evaporation obtainable with a pound of fuel. The method of using it is as follows :

Start with the percentage of combustible matter in the fuel (at the top of the left-hand diagram) and follow the vertical line downward to the horizontal line representing the percentage of fixed carbon, thence upward along the diagonal line to the left-hand margin, where the percentage of carbon in the combustible will be obtained. From this point proceed upward along the diagonal line to the top margin, then down- 


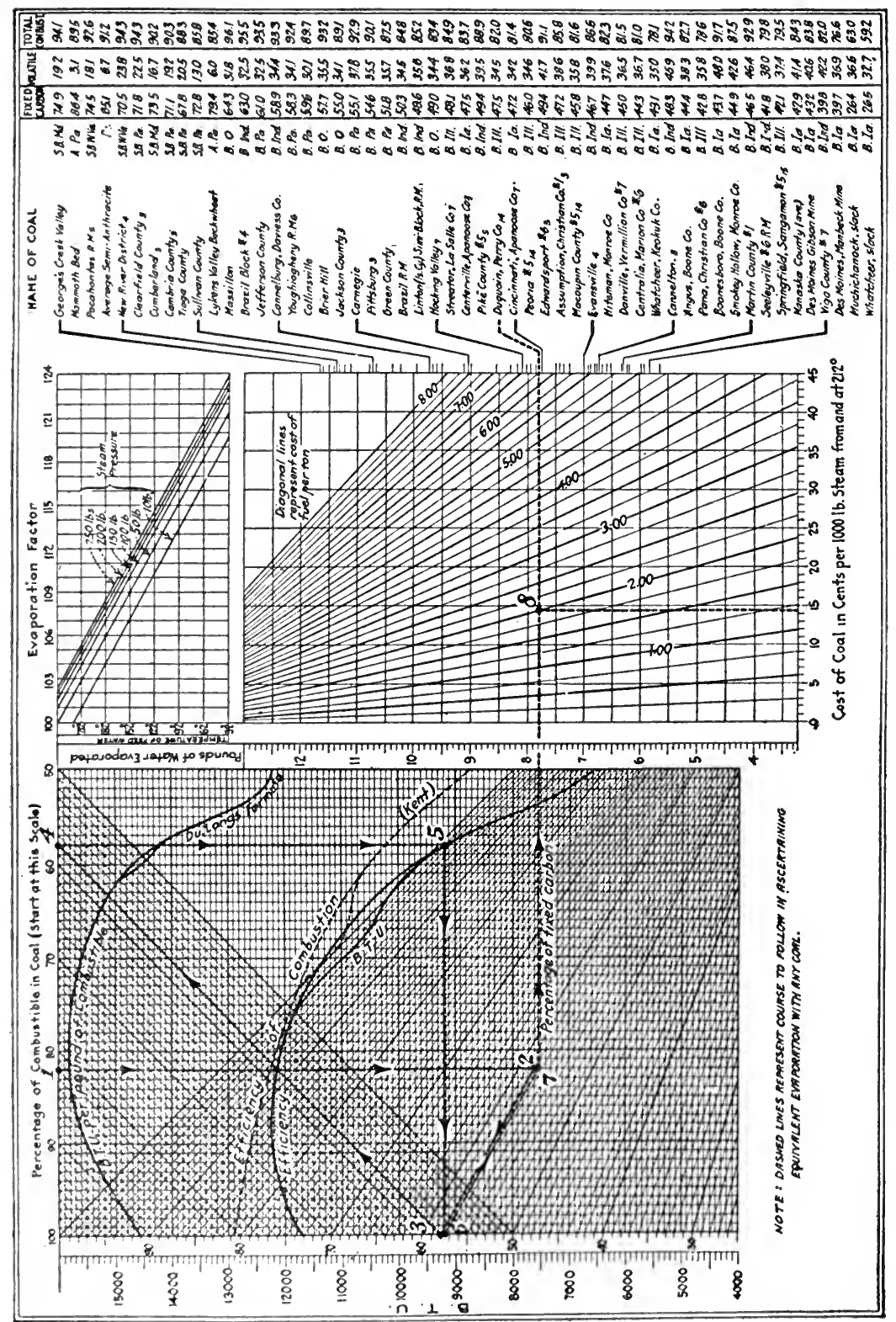


ward along the vertical line to the efficient B.t.u. curve and horizontally to the scale marked B.t.u. (which gives the calorific value on $1 \mathrm{lb}$. of combustible). From this point again follow the diagonal line downward until it intersects the vertical line marked with the percentage of combustible in coal and thence horizontally to the scale marked "pounds of water evaporated."

These charts are based on the efficiency which should be obtained with high-class operation. The upper curve in the left-hand diagram is based on Du Long's formula for figuring the B.t.u. in the combustible. The dotted curve is for determining the efficiency which should be obtained when burning coal under good conditions.

The Fuel Cost of Heat and Power. F. H. Rosencrants is author of the following, published November 12, 1918, in Power. The logarithmic chart, Fig. 3, is composed of 8 panels numbered for convenience. The method of using the chart may be best illustrated by the solution of a problem.

Beginning at the top of panel No. 1, with the Price per ton of coal in storage $(\$ 3.25)$ drop down to the Heating value per pound fuel $(12,000$ B.t.u.) and proceed to the left to Cost of coal per million B.t.u. (13.6c.) or proceed to the right to Efficiency of boiler or gas producer (70\%), panel No. 2. From this point proceed vertically to Cost in cents per million available B.t.u. (19.2) or drop down to Heat supplied per pound of steam in B.t.u. (1000), panel No. 3. From this point proceed to right to Cost per thousand pounds of steam in cents (19.2) or proceed left to Steam consumption per 1hp. in pounds (25), panel No. 4. From this point proceed vertically to Cost per $1 \mathrm{hp}$. in cents (0.48) or drop down to Mechanical efficiency of engine $(90 \%)$, panel No. 5. From this point proceed to the left to Cost per b.hp. in cents (0.53) or to right to efficiency of generator pump, or other driven machine $(90 \%)$, panel No. 6. From this point drop down to Cost per available $\mathrm{hp.}(0.58)$.

Panels Nos. 7 and 8 are for use in case of a producer-gas plant and with an understanding of the foregoing explanation I believe the method of use is clear.

Cost of Leaking Steam. The nomographic chart, Fig. 4, was copyrighted by W. F. Schaphorst and published with this description in Engineering and Mining Journal, February 10, 1917.

To determine the cost of leaking steam with any degree of accuracy at least four factors must be known: (1) The number of pounds of steam evaporated by one pound of coal (the greater this factor the less the cost of leaky pipes, other conditions being the same); (2) the total area of leakage; (3) the absolute steam pressure, which is equal to the gage pressure plus atmospheric pressure (at sea level this is usually gage pressure, plus 14.7 ); (4) the cost of coal per ton.

For example, how much money is lost every $10 \mathrm{hr}$. under the following conditions? Nine pounds of steam is generated by each pound of coal; the total area of leakage is 1/2 sq.in.; the gage pressure is $105 \mathrm{lb}$. per sq. in. (making the absolute pressure approximately $120 \mathrm{lb}$.) ; and the cost of the coal is $\$ 3.50$ per ton.

Simply lay a straight-edge across Fig. 4 three times as indicated by the dotted lines, and the problem is solved. Run the first line from the 


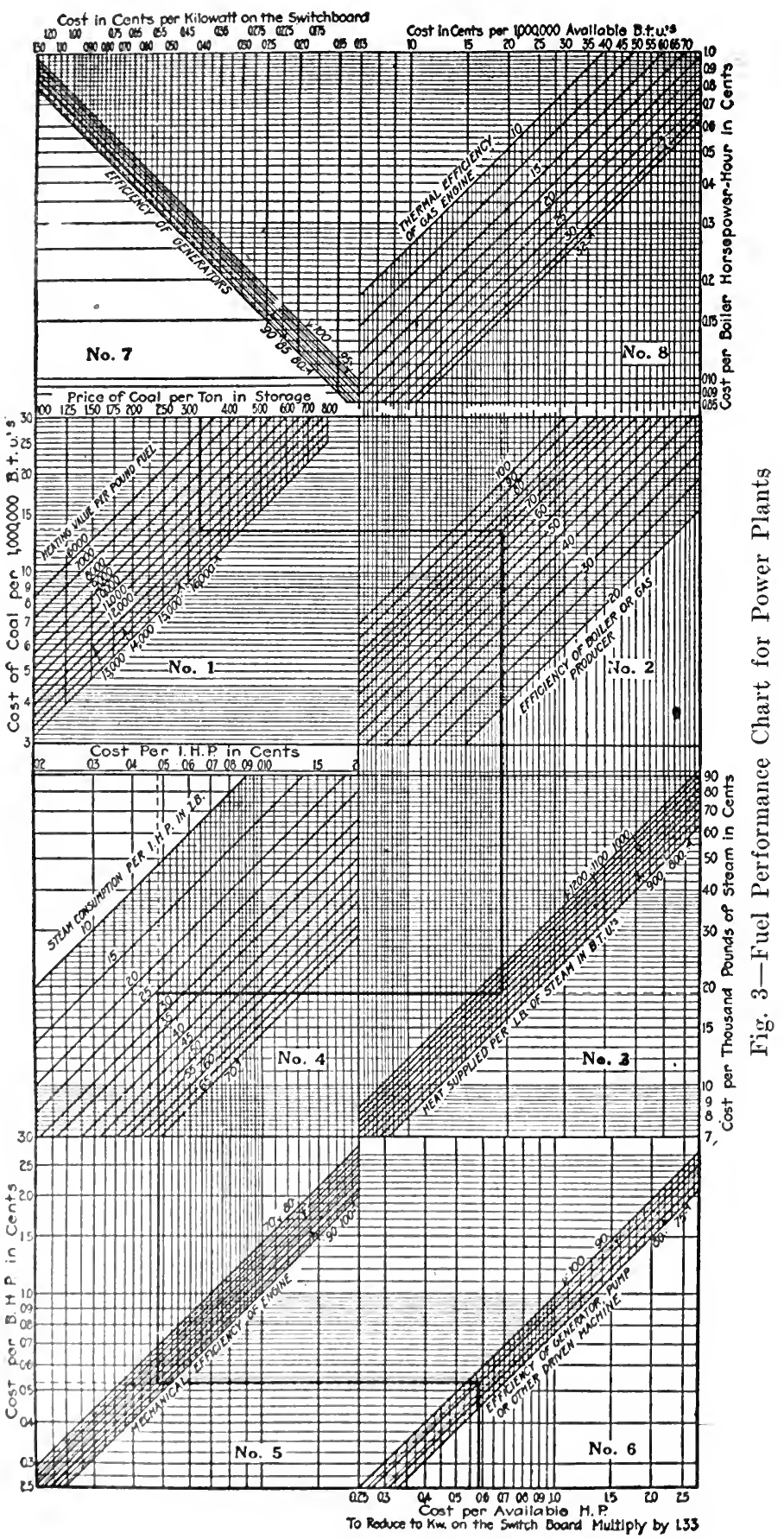


9 (column $A$ ) through the 120 (column $C$ ) and locate the intersection in column $E$. Run the second line from that point of intersection to the 0.5 (column $B$ ) and locate the intersection in column $C$. From that point of intersection run the third and last line to the $\$ 3.50$ (column $E$ ), and the answer is found at the intersection with column $D$. The answer is $\$ 6$ loss per $10 \mathrm{hr}$.

In case it is found that the total leakage area is less than shown in column $B$, the chart may be used just as easily by merely shifting decimal points. For example, if in the problem given the leakage area had been 0.05 instead of 0.5 sq.in. the answer would be $60 \mathrm{c}$. instead of $\$ 6$ per 10 -hr. day.

When using the chart the steps must follow in the order shown and

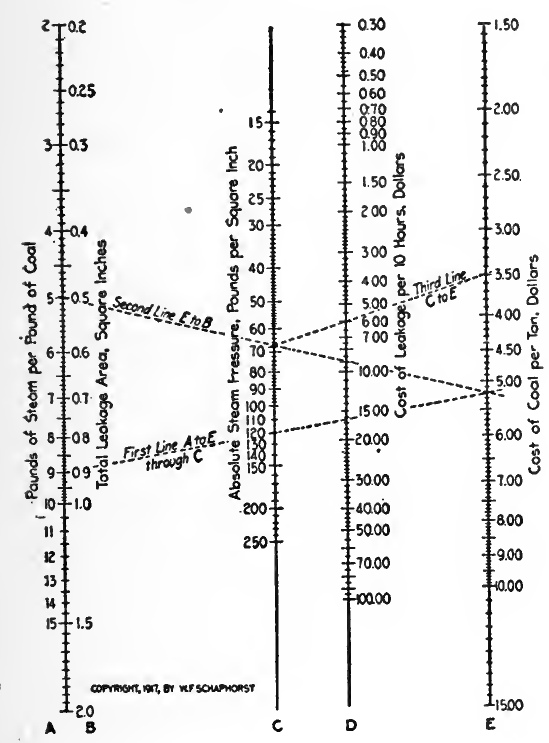

Fig. 4-Diagram for Determining the Cost of Leaking Steam

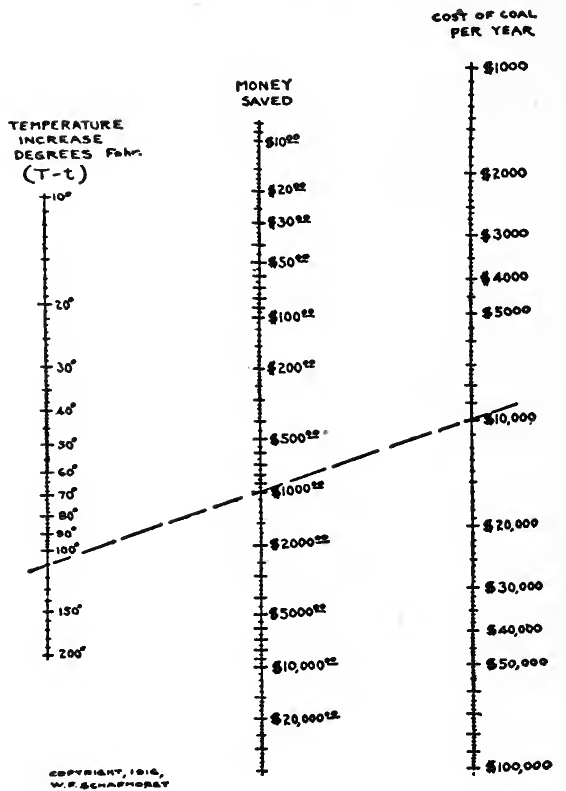

Fig. 5-Cost of Installing Feed Water Heater

described: First line, $A$ to $E$ through $C$; second line, $E$ to $B$; third line, $C$ to $E$.

Whether it Would Pay to Install a Feed Water Heater. Mr. Schaphorst also produced Fig. 5. This, together with the data pertaining to it, is taken from Minirg and Engincering World, December 2, 1916. Fig. 5 shows the money saving that accompanies the installation of a feed water heater. The old rule on which the chart is based is this: "Every eleven degrees temperature increase of the feed water saves one per cent. of the coal pile.'

For example, assuming that you spend $\$ 10,000$ per year for coal, and that by installing a feed water heater you could raise the temperature of the feed.water $110^{\circ} \mathrm{F}$. by using exhaust steam that is now going 
to waste, the money saved would amount to $\$ 1000$ per year, which is a pretty good "salary" for a single piece of apparatus.

The dotted line drawn aeross the chart shows how the problem is solved. Just lay a straight edge across and it's "done." Or, stretch a thread across from known point to known point. The answer is always found in the middle column.

To find the increase in temperature subtract the temperature of the water "entering" the heater from the temperature of the water "leaving" the heater. The difference ('T-t) is the value to be used in the left-hand column.

Cost per Pound of Electrical Machinery. The article of this title by Leonard A. Doggett, part of which follows, was published in Electrical World, October 2, 1915. It is an admirable description of a method applicable to many other types of equipment.

The cost of any article is not as a rule a fixed quantity, but varies more or less widely. It varies with the prosperity of the country, it varies with the business relations between the eustomer and manufacturer, it varies with the size of the order, it varies with the reputation of the manufacturer, it varies with the ncwness of the machine, and, of course, with the many elements entering into the original manufacturing cost.

All these variables render any accurate estimate impossible. The best that can be done is to set limits within which the cost figure will lie. Of course, to a certain limited number in the offices of the manufacturing companies, accurate information is available, but such data are not generally accessible: Ilowever, there are other sources of information which serve to indicate the limits of the eost figure. The figures given below are based upon (1) a paper entitled "Prime Movers," by H. G. Stott, R. J. S. Pigott and W. S. Gorsuch (Proceedings, A. I. E. E., June, 1914); (2) cost figures for a recent line of direct-current machines of one of the largest manufacturing companies, the prices being those offered to a small contractor by a local office of this company; (3) cost data in Pender's "American IIandbook for Electrical Engineers"; (4) miscellaneous data gathered by the writer within the last two years; (5) data on second-hand machines as published in the "Searchlight Section" of the Electrical World and in the "Curb Narket" of the Electric Journal.

It is a well-known fact that a 1-hp. motor having a rated speed of 2000 r.p.m. is much cheaper than, and about one-half as heavy as, a 1-hp. motol having a rated speed of 1000 r.p.m. Therefore, the rational way to tabulate either eost or weight data is in terms, not of dollars or poinds per kilowatt, but of dollars or pounds versus kilowatts divided by speerl. The term (kw.- - r.p.m.) is really torque, and of any machine it can be said that the greater the torcule the greater the necessary size. Woight and cost. Therefore, in this paper the independent variable is taken as (kw. :- r.p.m.). In Figs. 6 and 7 the accumulated data are plotterl.

From these curves Table 1 has been prepared, showing the average cost in dollars at varions values of $\mathrm{kw}$. - r.p.m. From some weight data published by the author in the Electrical World for Nay 3, 1913, Table II was obtained. Table III is obtained from Tables I and II. 
From curves, such as Figs. 6 and 7 , it is possible to obtain formulas which will represent the average result in a compact form and also be serviceable for a certain amount of extrapolation. For example:

\section{Name of Machine}

New direct-current generators and motors......

Second-hand direct-current generators and motors

3,600-r.p.m. turbo-alternator sets

1,800-r.p.m. turbo-alternator sets

\author{
Cost in Dollars \\ $4,500\left(\frac{\text { Kw. }}{\text { R.p.m. }}\right)^{06}$ \\ $1,800\left(\frac{\text { Kw. }}{\text { R.p.m. }}\right)^{06}$ \\ $33,500\left(\frac{\text { Kw. }}{\text { R.p.m. }}\right)^{0768}$ \\ $29,500\left(\frac{\text { Kw. }}{\text { R.p.m. }}\right)^{0685}$
}

However, the curves present the information in the best form, for the limits between which the cost of a machine lies can easily be seen. In using Figs. 6 and 7 it should be remembered in the case of new machinery that these figures represent standard or stock machines, and that ma-

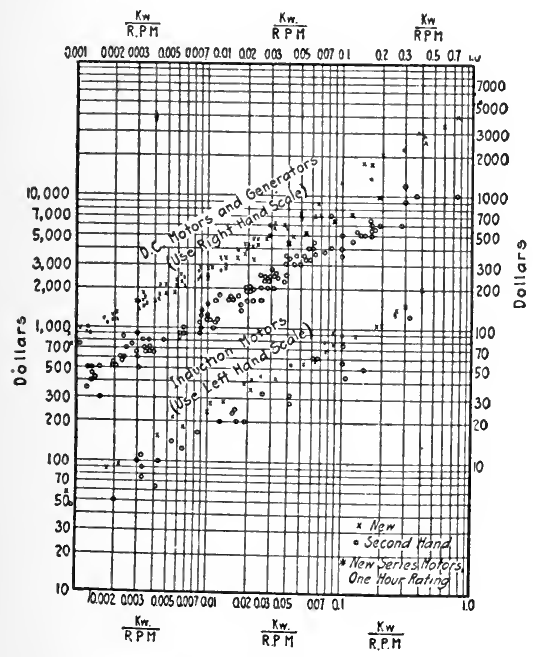

Figs. 6 and 7 -Charts Plotting Cost

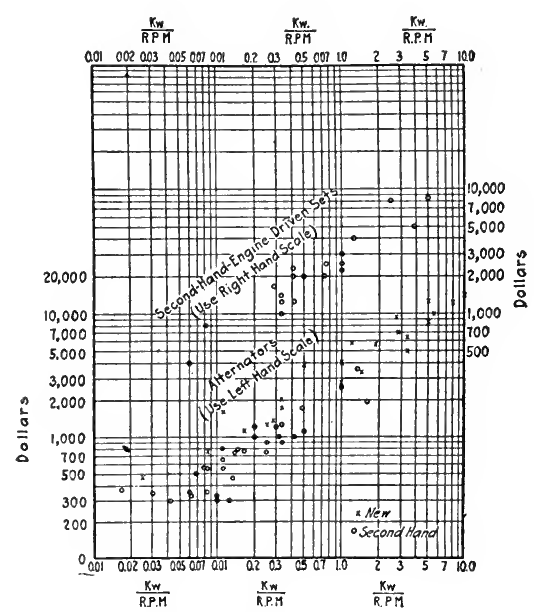

Data for Electrical Machinery

chines with unusual specifications will lie above any data there plotted. In gathering and plotting the information interesting facts developed, many of which could be explained. For example, cost figures on Edison bipolars, Stanley inductor alternators and 133-cycle alternators, if they had been plotted, would always have fallen below the general trend of the plotted points. That is, obsolete types of machines lie between the curve for second-hand machines and the scrap value of the machine. In Fig. 6 are plotted some data on new one-hour-rating series motors, these points being represented by \#. As would be expected, these points 


\section{TABLE I-COST IN DOLLARS}

\section{Name of Machine}

Direct-current generators and mo-

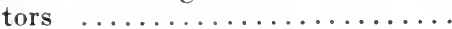

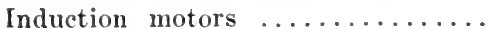

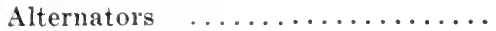

Turbo-alternators $\ldots \ldots \ldots \ldots \ldots$.

Low-speed engines ............

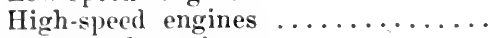

Low-speed engines .............

High-speed engines
New or

Second-

hand

New

New

New

New

New

New

New

New

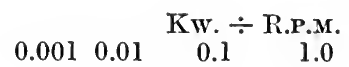

$\begin{array}{rrrr}85 & 280 & 1,150 & 5,500\end{array}$

$\begin{array}{rrrr}100 & 260 & 850 & 3,500\end{array}$

$\begin{array}{llll}\ldots & \ldots & 1,200 & 4,600\end{array}$

$\begin{array}{llll}\ldots & \ldots & \ldots & 37,000\end{array}$

Compound

Compound

Simple

Simple

... 6,000

$1,600 \quad 4,900$

3,300

$680 \quad 2,530$

$\begin{array}{rrrrr}40 & 120 & 450 & 1,600 & \cdots \\ 45 & 170 & 550 & 2,500 & \cdots \\ \cdots & 140 & 450 & 2,200 & 8,000\end{array}$

Direct-current generators and motors ..................... Second-hand Induction motors ............. Second-hand

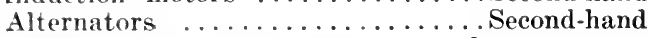
Engine-driven direct-current and al-

ternating-current generators .... Second-hand

․ $200 \quad 700 \quad 3,000$
10.0

16,000 136,000

17,700

$13, \ddot{500}$

....

13,000

\section{TABLE II-WEIGHT IN POUNDS}

\begin{tabular}{|c|c|c|c|c|c|}
\hline \multirow[t]{2}{*}{ Name of Machine } & \multicolumn{5}{|c|}{ Kw. $\div$ R.P.M. } \\
\hline & 0.001 & 0.01 & 0.1 & 1.0 & 10.0 \\
\hline Direct-current generators and motors .... & 130 & 810 & 4,200 & 22,000 & 110,000 \\
\hline Induction motors $\ldots \ldots \ldots \ldots \ldots \ldots \ldots$ & 80 & 510 & 2,800 & 15,000 & 81,000 \\
\hline Alternators .... & 130 & 810 & 4,200 & 20,000 & 90,000 \\
\hline Turbo-alternators & .. & ... & & 170,000 & 640,000 \\
\hline Low-speed engines .... & .. & .... & 2,400 & 19,000 & 140,000 \\
\hline High-speed engines $\ldots \ldots \ldots \ldots \ldots \ldots \ldots$ & ... & $\ldots$ & 4,500 & 31,000 & , \\
\hline 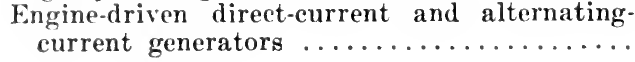 & & 1,400 & 8,000 & 50,000 & 250,000 \\
\hline
\end{tabular}

TABLE III-CENTS PER POUND

\begin{tabular}{lllllll} 
& & \multicolumn{4}{c}{ Kw. $\div$ R.P.M. } \\
Name of Machine & $\begin{array}{c}\text { New or } \\
\text { Second- } \\
\text { hand }\end{array}$ & 0.001 & 0.01 & 0.1 & 1.0 & 10.0
\end{tabular}

Direct-current generators and mo-

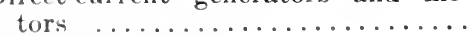

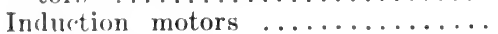

Alternators $\ldots \ldots \ldots \ldots \ldots \ldots$

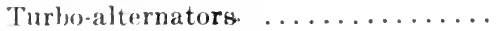

Jow-spoer engines ..............

High-sperd engines $\ldots \ldots \ldots \ldots \ldots$

l.ow-speed engines $\ldots \ldots \ldots \ldots \ldots$

Iligh-speed engines..$\ldots \ldots \ldots \ldots$ New

$\begin{array}{lrrrrr}\text { New } & 65 & 35 & 27 & 25 & \ldots \\ \text { New } & 125 & 51 & 30 & 23 & \text {. } \\ \text { New } & \ldots & . . & 29 & 23 & 18 \\ \text { New } & \ldots & . & \ldots & 22 & 21 \\ \text { New } & \text { Compound } & . & 28 & 11 \\ \text { New } & \text { Compound } & 36 & 15 & \ldots \\ \text { New } & \text { Simple } & . & 17 & 9 \\ \text { New } & \text { Simple } & 15 & 8 & \ldots\end{array}$

Direct-rurrent generators and mo-

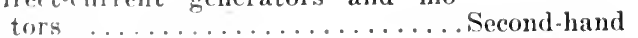

Induction motors .............. Second-hand

Alternators ................. Second-hand

$\begin{array}{ll}31 & 15 \\ 56 & 33 \\ \cdots & 17\end{array}$

11

Engine-driven direct-current and al-

ternating-current generators ... . Second-hand

14

20

lie between the points for new and those for second-hand direct-current machines.

It is interesting to note that the average eost of all the new machinery tabulated is :3 cts. por pound, and of the seeond-hand machinery 17 cts. per pound, representing a depreciation of machinery which is sec- 
ond-hand but not obsolete of about $50 \%$. Some dealers specifically advertise used machines at $40 \%$ of the new price, so that 40 to $50 \%$ may be taken as a reasonable figure.

Estimated Cost of Steam Pumps. Fig. 8, together with the short abstract, is from an article "Economical Duty of Pumps," by Frank H. Carter, published December 5, 1914, in Engineering Record to illustrate the use of the logarithmic chart for plotting prices, where the formula is of the form given.

The cost of a pump must of necessity vary according to some function of $p$, the water pressure on the water and that exerted by the pump. According to a designing engineer employed by one of the leading pump manufacturers, the cost of pumps may be assumed to vary roughly as the 0.8 power of the total dynamic water pressure, including suction, friction and static heads. By tentative trials it is found that the aboye

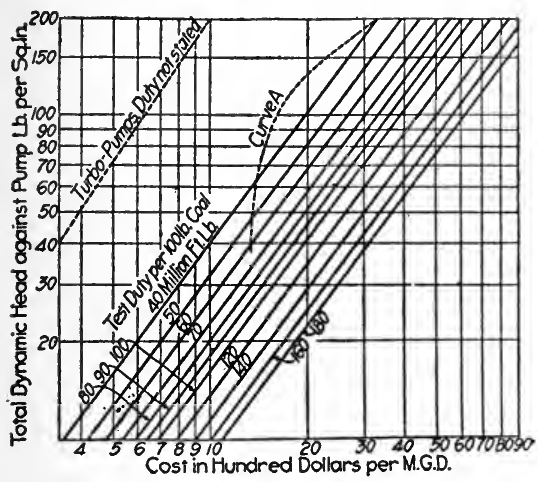

Fig. 8-Estimated Cost of Steam Pumping Engines

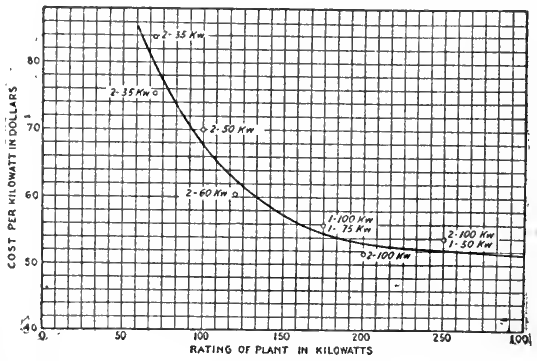

Fig. 9-Cost of High-Speed, Direct-Connected Generating Plants per Kiloof Capacity

costs, exclusive of a consideration of any variation with respect to the dynamic head may be expressed by formula, using the maximum duty allowance under the head "test duty," $C=115 \mathrm{x}{ }^{0.75}$; or, adding the function expressing the variation of cost of the pump as a power of the total water head $p$, assuming $p^{0.75}$ for ease in computation, $C=$ $115 \mathrm{x}^{0.75}(p / 90)^{0.75}$, as the costs given are for $p=90 \mathrm{lb}$. Reducing this formula to simpler terms, $C=3.94(x p)^{0.75}$.

This is graphically shown in Fig. 8 , where $C=$ cost per million gallons capacity; $x$, test duty in million ft. lb. per $100 \mathrm{lb}$. of coal; $p$; total head, including suction, friction and static pressure.

High Speed, Direct Connected Generating Plants. To illustrate the method of drawing a smooth eurve through a few points plotted from known data, Fig. 9 is taken, with the following, from an article, "How to Select Prime Movers for Industrial Electrical Generating Plants" $b_{j}$ H. T. Luscomb, published in Engineering Magazine, August, 1916.

The steam economy which may be expected from units comprising engines of the ordinary piston or slide valve type and the small and medium size turbine has been found to average 60 to 80 pounds of steam per kw-hr. generated, depending upon the size of the engine. The load on the engine has a decided effect upon the economy also. 
Such units, including engine and generator, together with necessary piping, wiring, switehboard, foundations and erection charges, cost from $\$ 50$ to $\$ 90$ per $\mathrm{kw}$. of generator eapacity. Fig. 9 drawn from data secured at a number of actual plants, shows the variation of cost with the eapacity of the plant. Naturally the cost is governed to a certain

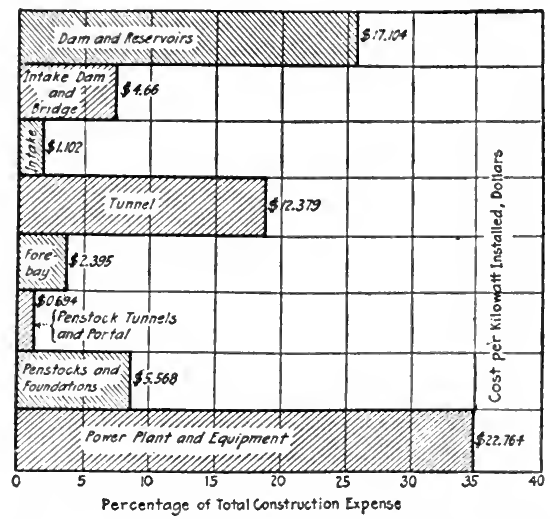

Fig. 10-Relative Costs of Constructing Each Part of the Hydroelectric Plant

extent by such factors as the foundation work necessary, accessibility of the engine room and market conditions. Costs include engines, generators, piping, foundations, switchboard and erection, but no boilers.

Comparison of Construction Costs. Excellent examples of the value of the polar chart for sector analysis are shown in Figs. 10 and 11.
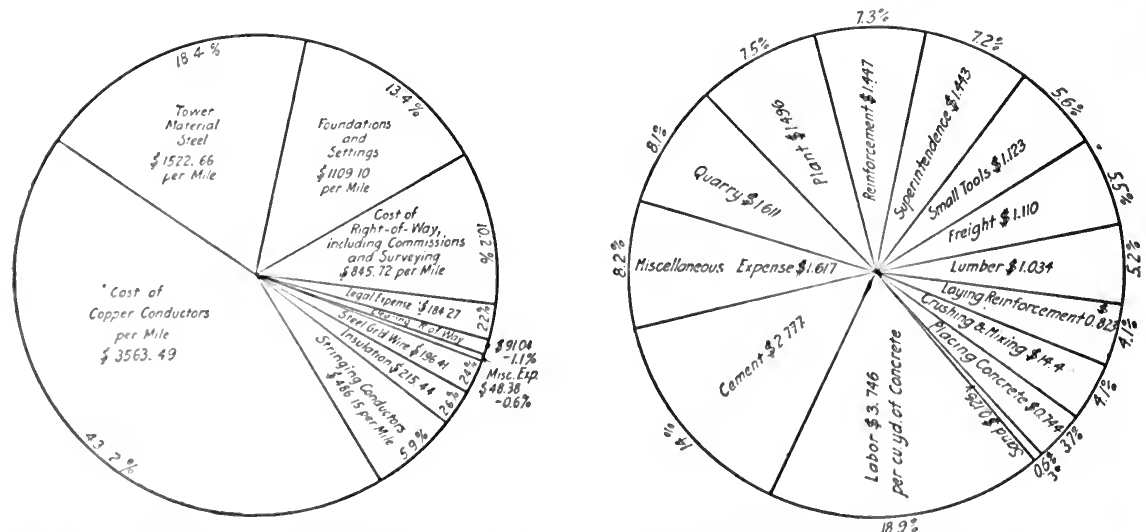

Fig. 11-Init costs of Constructing the Tallulah-Atlanta Transmission Line and (iravity-Type Dams

These, with the descriptive matter are from an article entitled "Unit Construction ('osts for Hydroelectric System" published in Electrical World. October 2:?. 1915.

A graphical analysis of eonstruction eosts is presented which shows the relative magnitudes of the different expenses as well as the unit 
costs represented by the construction work called for by the Tallulah Falls hydroelectric plant and transmission system of the Georgia Railway \& Power Company, which serves large areas in northern and central Georgia.

First, the total expenses due to constructing each part of the system, including dam, tunnel, power house and lines, are compared, Fig. 10. Then each expense is itemized and further compared to show the relative magnitudes of labor, material, transportation and miscellaneous items. Fig. 11 is reproduced as an example.

Costs in Railway Maintenance Work. One of the many uses of graphical charts in connection with railroad work is shown in Fig. 12,

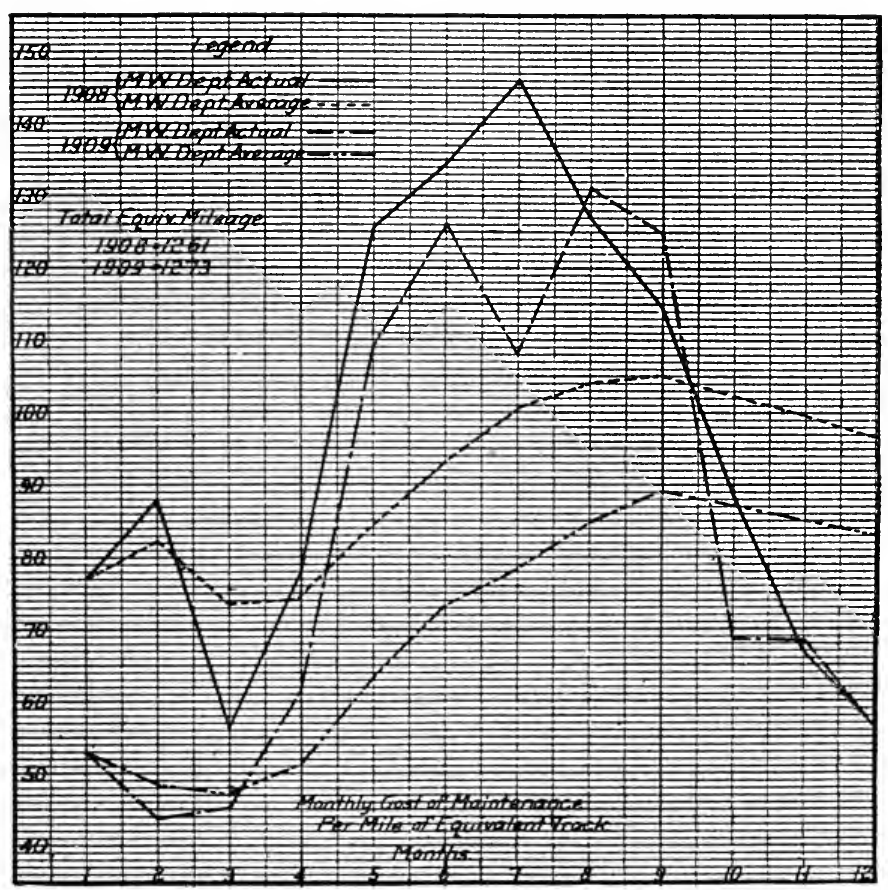

Fig. 12-Showing Costs in Maintenance Work

taken with the following notes from an article, "Efficiency in Track Maintenance," by C. E. Lindsay, published in Railway Age Gazette, May 17, 1912.

A graphical means of showing costs per equivalent mile comparatively month by month and year by year is afforded. The actual monthly expenditure per mile for each particular month is plotted as a point and the points are joined by lines of peculiar form or color for each year. The cumulative monthly average is shown by the dotted lines at the bottom of the figures, and this average is a most valuable index of the trend of expenses and a guide to the engineer as to his future expenditures. 
Maintenance Cost Data in Graphical Form. This was printed in Electrical Railway Journal, February 24, 1917.

Through the courtesy of a company operating a number of railway properties in cities of moderate size, it has been possible to give the data plotted in Fig. 13, which has been cut down for want of space. These bring the record up to the end of 1915. The 1916 data are not in-

PROPERTY A PROPERTY B PROPERTY C

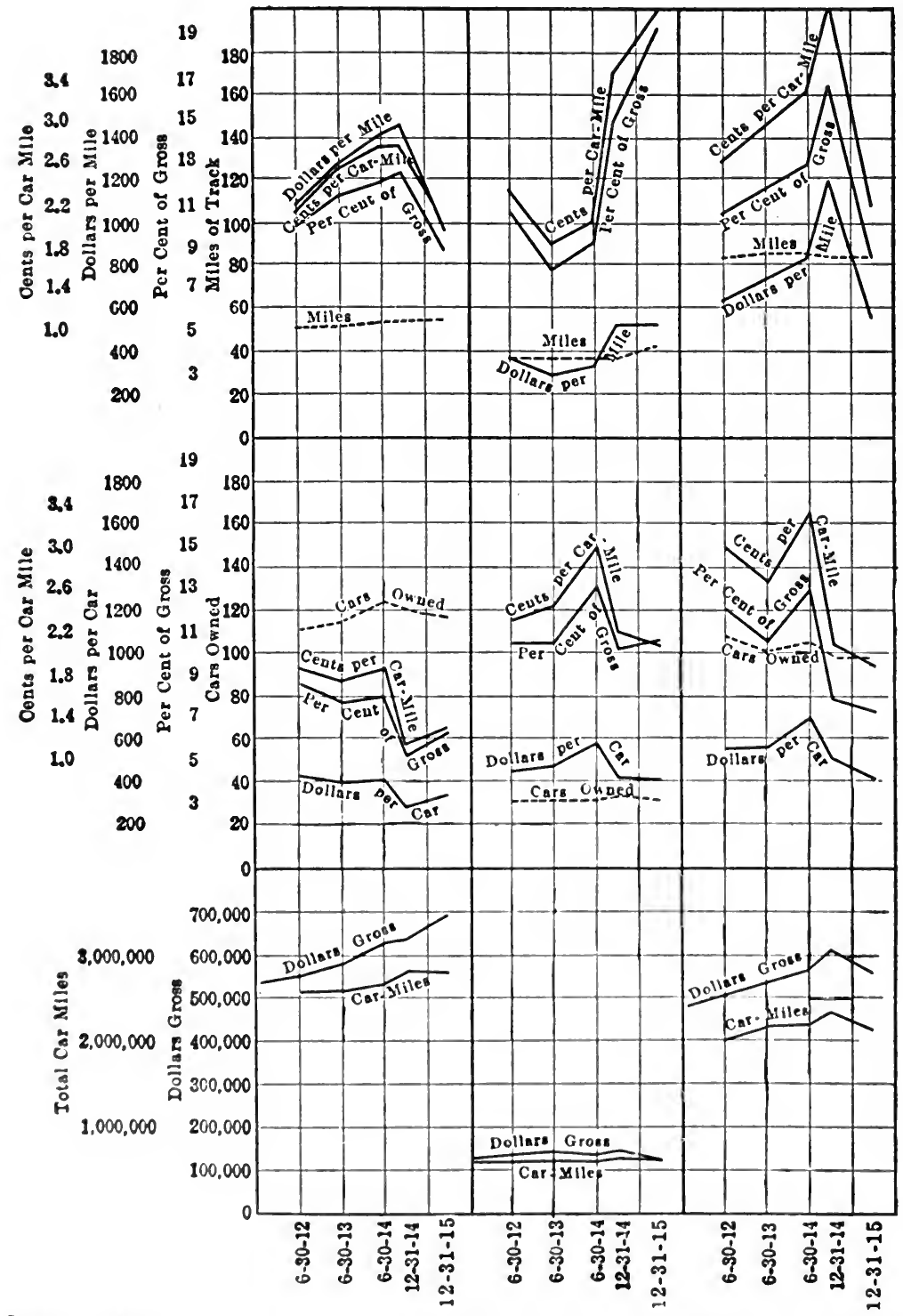

Fig. 13-Maintenance Costs on a Number of Electric Railway Properties of Moderate Size 
cluded as they cannot be considered typical in view of the prevailing high prices of materials and scarcity of labor.

It will be noted that the cost data are stated in several ways, giving an opportunity for comparison based on income, car-miles operated and miles of track for the way and structures, and on income, car-miles operated, and cars operated for the equipment.

Cost Chart for Reinforced-Concrete Factory Building. Fig. 14 gives a graphical analysis of the elements of expense in constructing a fourstory reinforced-concrete machine shop at Jowell, Mass. We have taken this and the description from Engineering Record, February 14, 1914.

It was prepared by the Aberthaw Construction Company, of Boston, and is said to represent a fairly typical case, both as regards distribution and as regards the character of the building itself.

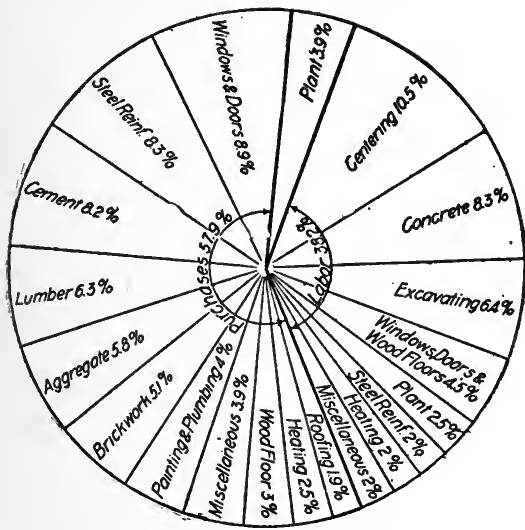

Fig. 14-Cost Chart for Con'rete Building

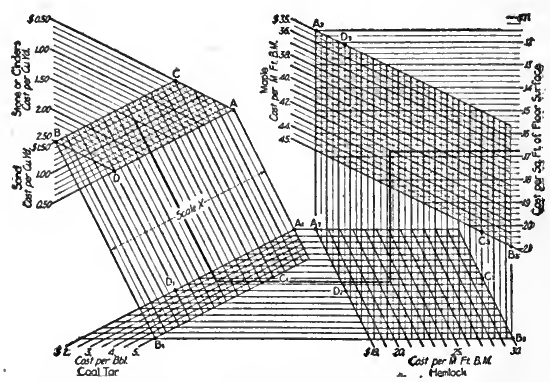

Fig. 15-Cost of Materials in Coal-TarHemlock-Maple Floor

The building is $150 \times 50 \mathrm{ft}$., with brick curtain walls. The general type of interior construction is beam and girder, the height between finished floors being $12 \mathrm{ft}$. $4 \mathrm{in}$. The two lower floors were designed for live loads of $250 \mathrm{lb}$. per square foot and the upper floors for 150-lb. loads. Floorbeams are carried on a single row of columns $10 \mathrm{ft}$. on centers, running the length of the building midway between side walls. Steel sash was used throughout.

Cost Per Square Foot of Floor. Engineering Record, December 25, 1915, published Figs. 15 and 16 and the accompanying explanation.

Fig. 15 gives the cost per sq. ft. of a typical mill-building floor composed of 4 in. of broken stone, $1 \mathrm{in}$. of sand and tar, $3 \mathrm{in}$. of hemlock and a $7 / 8$-in. tongue-and-groove maple finish. Fig. 16 gives the cost per sq. ft. for the material in a 4-in. concrete slab for three different mixes. "The construction of the first chart will be explained, as the method of procedure is applicable to any other case where the unit costs of the component parts are variable and a fixed amount of each enters into the final unit, the cost of which is desired.

Four combination costs for the stone and sand at the limiting values of the costs assumed for these quantities per cu. yd. are first computed 
in cts. per sq. ft. of floor, knowing the quantities of each. Selecting a convenient seale and direction, ealled seale $X$ on the diagram, these combination values are plotted, and four parallel lines, $A A_{1}, B B_{1}, C C_{1}$ and $D D_{1}$ are drawn through the plotted points. The point $A$ is selected arbitrarily and the point $C$ chosen to give sufficient length $A C$ for the

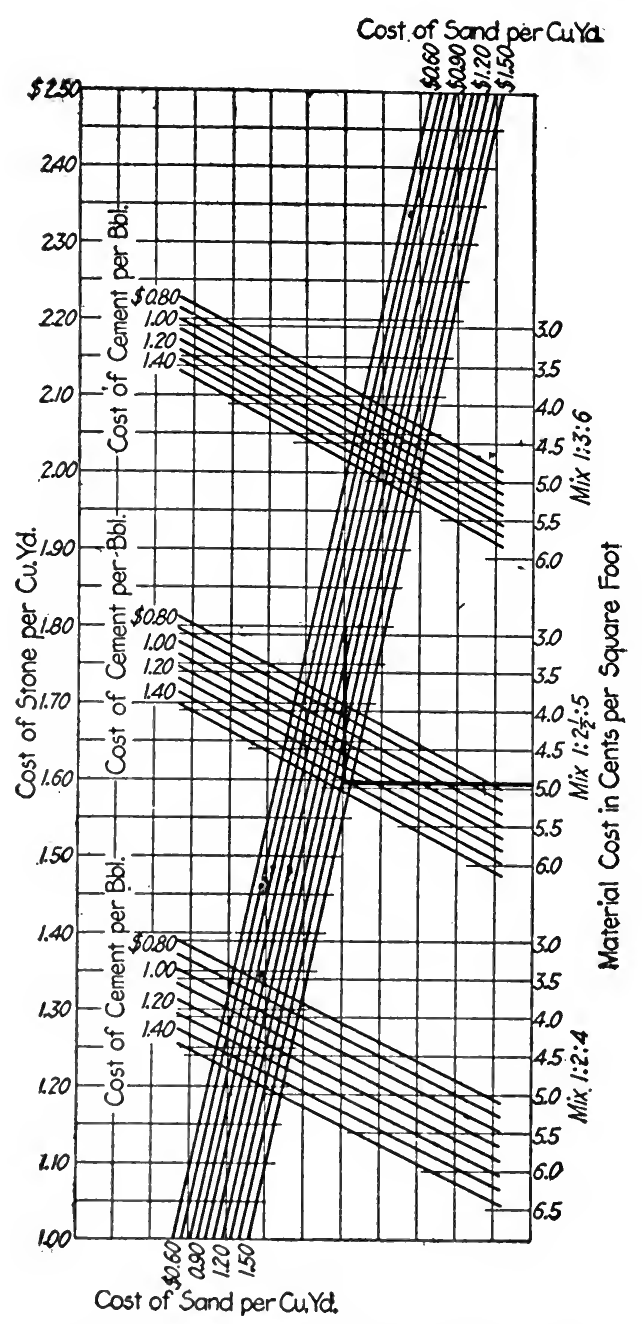

Fig. 16-Cost of Materials in 4-Inch Concrete Slab Floor

sand scale and at the same time a convenient diagonal direction $A C$ for locating the stone scale. The latter is found between the diagonal sides $A C$ and $I) B$ of the parallelogram $A B C D$, the sides $A D$ and $B C$ in this case being conveniently made parallel to the assumed direction of the combination scale $X$. The lines $A D$ and $A C$ are easily divided 
into the desired number of parts by the use of any convenient diagonal line and scale.

Using the previous four combination costs for the first two items, a second set of four combination costs including the cost of the tar used per square foot is computed. The amount of tar is based on an assumption of $1 \mathrm{gal}$. of tar to 51/2 sq. ft. for the stone and 1 gal. to 10 sq. ft. for the sand, with $3 \%$ waste. The four costs are then plotted to a convenient scale in a vertical direction, beginning at any selected point $A_{1}$, and the four horizontal lines $A_{1} A_{2}, B_{1} B_{2}, C_{1} C_{2}$ and $D_{1} D_{2}$ are drawn through them. The lines $A_{1} D_{1}$ and $C_{1} B_{1}$ through the resulting intersection points then give the direction of the scale for the coal tar as shown. This can be divided into the desired number of parts in the usual manner.

Similarly, the four combination costs per sq. ft., including the 3-in. hemlock flooring at limiting prices, with $12 \%$ added for waste and 0.02 cts. for nails, are computed. These are plotted horizontally to an assumed convenient scale, beginning at the point $A_{2}$. The resulting intersections $A_{2}, B_{2}, C_{2}$ and $D_{2}$ where the vertical lines cut the preceding horizontal lines determine the slopes $A_{2} D_{2}$ and $C_{2} B_{2}$ for the hemlock scale.

The final costs, including the $\overline{8}$-in. maple floor faced from 1-in. material and allowing $25 \%$ for the tongue and groove with 0.1 ct. for nails, are then computed for the four limiting prices and plotted in the final vertical scale shown, giving points of intersection $A_{3}, B_{3}, C_{3}$ and $D_{3}$, and fixing the seale for maple cost by the lines $A_{3} D_{3}$ and $B_{3} C_{3}$. Any number of variables can be included by continuing the process of right-angled turns between the scales used for combination costs.

The use of the diagram is explained in the chart, Fig. 15, by the heavy lines, which indicate the successive steps in reading for the case of stone or cinders at $\$ 1.50$ per eu. yd., sand at $\$ 1.20$ per cu. yd., coal tar at $\$ 3$ per barrel, hemlock at $\$ 22$ per thousand and maple at $\$ 42$ per thousand, giving a final total cost of $16^{3 / 4}$ ets. per sq. ft.

Fig. 16 is constructed and used in a similar manner, noting that the quantity of sand and of stone for the given mixes is practically constant. The heavy lines indicate the solution for the case of stone at $\$ 1.80$, sand at $\$ 1$ and cement at $\$ 1.45$ for a $1: 2 \frac{1}{2}: 5$ mix, giving a total cost of nearly 5 cts. per sq. ft. for the materials.

This and similar charts have been prepared by A. Pearson Hoover, engineer with John W. Ferguson Company, engineers and building contractors, Paterson, N. J.

Cost of Placing Steel Reinforcement. Dan Patch is author of the article from which the following is taken. It was published in $E n$ gineering Record, August 26, 1916.

Labor cost in placing steel is usually rated in dollars per ton, although it is recognized that such unit costs increase when light steel is being placed. In order properly to include the effect of size of rods, a graphical method based on weight per running foot of steel has been devised.

The unit costs are usually obtained by dividing the labor cost figured from the timekeeper's sheets by the tons of steel reported placed by the quantity man. In order to obtain data for studying the effect of 
size of bars, only one more item must be recorded-the total length of bars placed. This is easily done by the use of a listing adding machine, by which the total rumning feet of each diameter of rods placed ean be obtained. The daily totals are tabulated in terms of rod sizes and linear feet placed, the total length and total weight eomputed, and

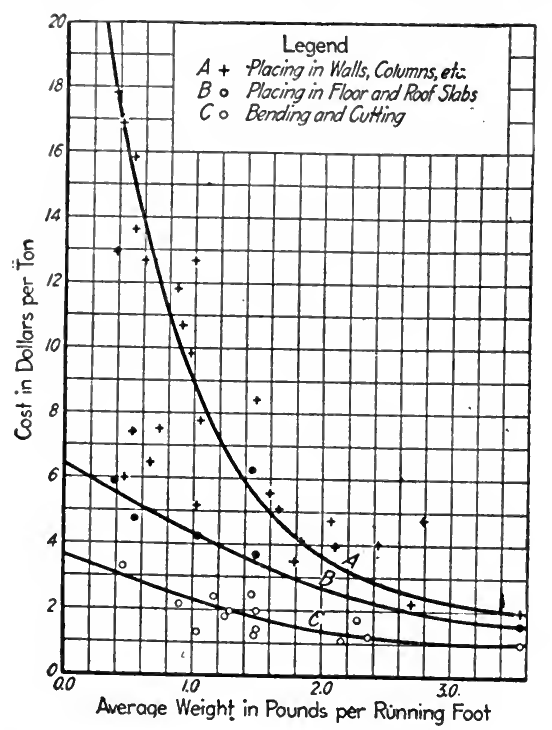

Fig. 17-Cost of Reinforeing Steel Based on Weight Per Foot

the average weight per running foot easily obtained. Knowing total cost and total tonnage, the cost per ton is found, and plotted in Fig. 17.

The curves $A, B$ and $C$, which are drawn through the fields of plotted points obtained for costs of placing in wall, columns, stairs, ete., in floor and roof slabs, and of bending and cutting respectively, indicate the large effects of average weight upon the cost of labor per ton.

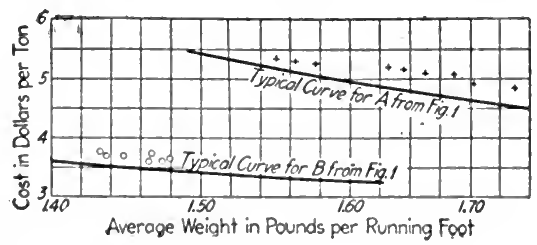

Fig. 18-Typical Examples of Costs at Various Dates Compared with Curves in Fig. 17

If additional argument in favor of aceounting for the weight variable is necessary it will be found in Fig. 18. This diagram shows on a larger scale the plotferl costs for the same kind of work, but for different dates, the sterel growing lighter as the roof is approached. Sections of the typical curves of Fig. 17 to this enlarged scale are shown, 
the costs of placing in walls, columns, etc. (Curve $A$ ), giving the clearer illustration.

As an example of the value of these curves, consider the figures on the work recorded in Fig. 18. On Nov. 23 the cost per ton was $\$ 4.83$. By Jan. 19 this cost had risen to $\$ 5.34$ per ton. With these figures only and no knowledge of the weight of steel it would be assumed that the work was being less efficiently done, but with the typical curve as a basis of comparison it will be noted on Fig. 18 that while there has been a $10 \%$ increase in the cost per ton, the typical cost curve $A$ has been more nearly approached, indicating the increased efficiency that can reasonably be expected as a job progresses and the men become more accustomed to their work.

The results obtained in this study lead to speculation as to whether similar curves would not be valuable in eliminating the variation in floor-form costs resulting from differences in thickness of slab and floor heights; in the costs of laying and jointing drains and sewers on account of differences in diameter; in the cost of setting sash as a result of differences in size of openings, etc.

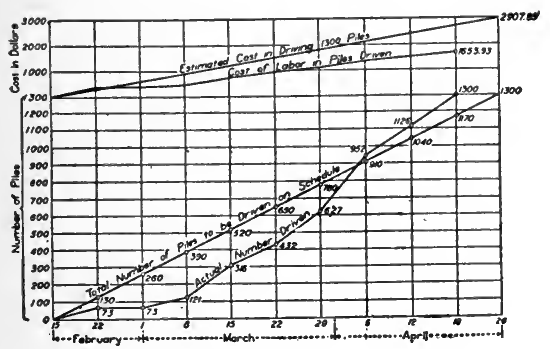

Fig. 19-Diagram of Speed and Cost of Driving Piles with Gasoline Hoist

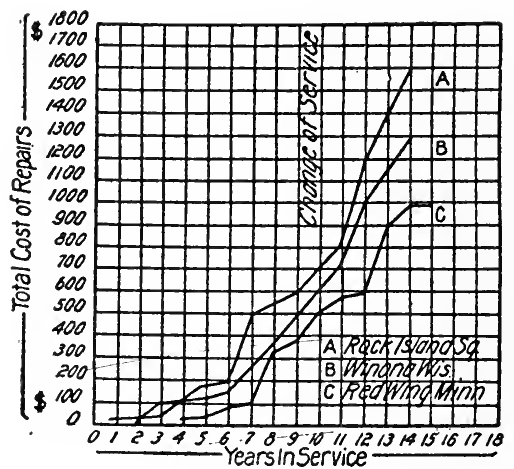

Fig. 20-Cost of Repairs and Life for Barges

Cost of Driving Piles. Fig. 19 and the following are from an article, "Cost of Driving Piles with a Gasoline Hoist," published in Engineering Record, July 18, 1914.

A reversible gasoline hoist with a $6 \frac{1}{2}$-hp engine and operating a 1650-lb. drophammer has been used for driving 1,300 piles to support a stage for 7,000 singers during the St. Louis pageant. These piles were driven from a scow about $6 \mathrm{ft}$. deep in the bottom of the Mississippi River at Forest Park. The niggerhead of the hoist was used to pull the piles in place and the drum was utilized for hoisting the ram. In addition, a pulley on the flywheel ran a centrifugal pump for keeping the scow dry.

In Fig. 19 are shown the total number of piles to be driven on schedule and the actual number of piles driven, the estimated cost of driving 1,300 piles and the actual cost of labor on piles driven.

Cost of Repairs. Fig. 20 is an example of a very valuable use for charts-that of showing repair expenses. It is applicable to almost every kind of work where plant is used and frequently such a graphic 
record has been of great assistance in the selection of the most economical types of equipment. This figure and data are from an article "The Comparative Costs of Repairs to Barges of Treated and Untreated Timbers," published in Engineering and Contracting, April 24, 1912.

The repair costs used in Fig. 20 have been obtained from the plant records of the U. S. Engineering office at Rock Island, Ill., covering Douglas fir barges in use for the past $20 \mathrm{yrs}$. in connection with the work of improving the Upper Mississippi River, between St. Paul and St. Louis. In the past untreated fir barges were kept in service 10 to 17 yrs., the average life being $15 \mathrm{yrs}$. The diagram is intended to show the usual cost of repairs from year to year during the life of an untreated Douglas fir barge. New barges are, as a rule, used for riprap rock transportation, this service requiring a substantial craft. From the diagram it will be noted that during the sixth and seventh years the barges required extensive repairs, the cost ranging from $\$ 200$ to $\$ 300$ per barge; that with repairs costing about $\$ 75$ per year they continued in hard service to the tenth or twelfth year; that they then required large repairs and had to be taken from rock work and placed in the brush earrying service, which is much less severe on account of the large decrease in weight per cubie foot of load. From this time on to the end the cost of repairs per barge is largely increased; and it is debatable whether it would not be fully as economieal to abandon the barge at about the tenth to twelfth year.

Auto Costs. The following, taken from an article, "A Graphic Record of Auto Costs," by Stanley C. Tarrant, published in The Gas Age, December 15, 1916, is a valuable suggestion for all users of motor vehicles.

The superintendent of transportation of a large gas company finds that with the use of graphs he is enabled to keep in closer touch with cost conditions than he ean when he has to study a mass of figures. He has, therefore, installed the following system, which he finds very valnable for his purpose:

When a ear is purchased by his eompany it is given a serial number, and is known by this number throughout its useful life. For comparative purposes a separate record of the cost of operating each car is carefully kept.

Fig. 21 shows the form used for comparing the cost in cents per mile of the different ears. All ears of the same make are shown together on the chart, thus giving a very graphic comparison of eosts. The monthly cost for each car is shown by means of a dotted line, while the cost for the period is shown by a solid line. For example, Ford No. 9 cost 9 cts. per mile to operate during August and 8 cts. per mile during the first eight months of 1916 . This ehart is made up every month, there being one form for each month in the year.

Fig. 22 shows the eost in cents per mile, and the iniles per gallon of gas. A separate form is used for each ear, each form covering a year's record by months. This chart shows very graphieally the fluctuations in eosts, month by month, and readily points out unusual operating conditions.

The charts are drawn on sheets $8 \frac{1 / 2}{2} 4$ in. so that they can be eon- 
veniently carried in the pocket or filed in the pigeon-hole drawers found in most roll-top desks.

In order to avoid the necessity of preparing new forms for the charts every month, the drafting department made negatives from a tracing of the printed matter and co-ordinate lines. From these negatives as many black-and-white prints can be made as desired. This leaves only the curve lines to be drawn in each month.

Fig. 22 shows a rather complete graphic record of auto costs. A separate chart is made up for each car in operation and covers a year's record by months.
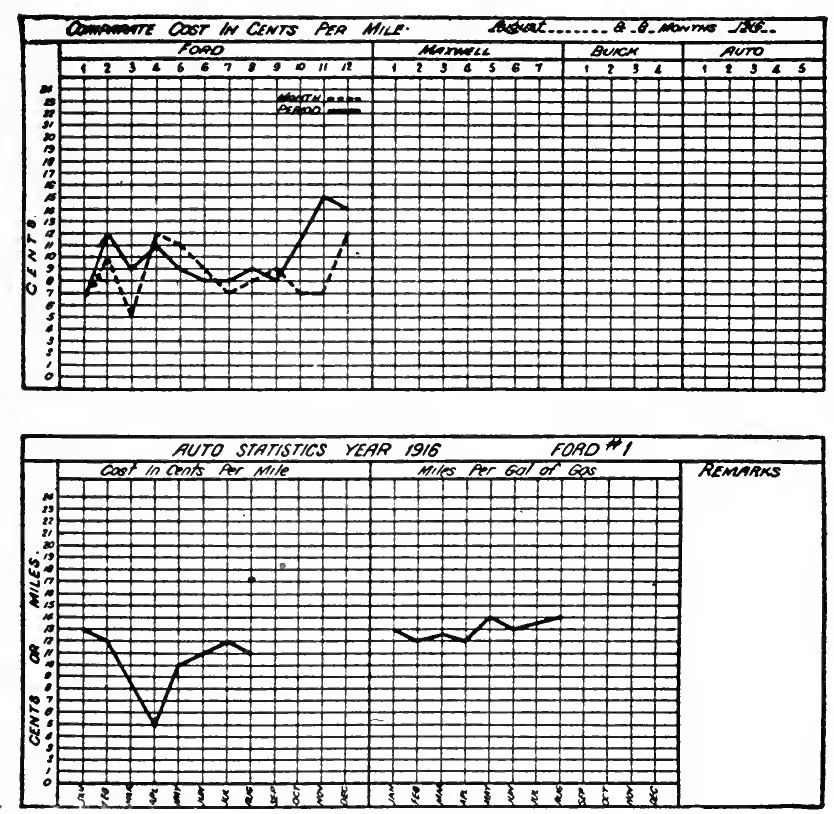

Fig. 21-Comparison of Costs of Operating Motor Cars

Costs are divided into three parts as follows:

Garage cost, including-

Garage superintendence

Mechanics' time.

Washers' time.

Chauffers' time.

Repairs, including-

Labor and material used.

Operating cost, including-

Gasoline.

Batteries.

Lubricants.

Sundries.

Tires and tubes.

The idea of dividing the costs this way was to get at a fair comparison of the actual operating and repair cost of the different cars. It is ob- 
viously unfair to eompare the eost of operating two cars of the same make, when one has a paid chauffeur to drive it and the other has not.

The first half of the ehart shows curves for the cost in dollars, divided as explained above, and also the total cost. The figures at the top of the sheet represent the miles operated during the month. The other half shows the eost in eents per mile for the different divisions.

The figure for the month is shown by means of a solid eurve line, while that for the period is represented by a dotted curve line.

The period curve on the "dollars" chart is purposely omitted, as it

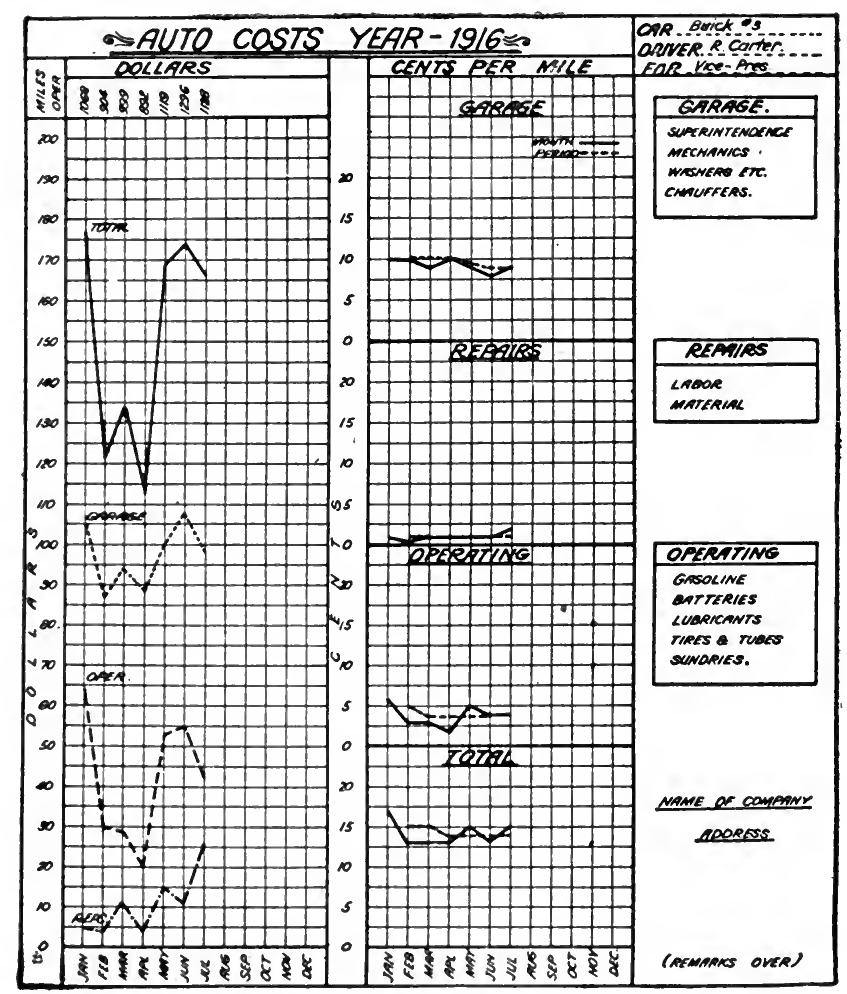

Fig. 22-Monthly Summary of Costs Classified

would necessitate a very long seale column, otherwise the readings would be inaccurate.

In the right hand top eorner of the form the make and serial number of the ear is shown, together with the driver's name, and the name of the individual or department for whose use the ear is maintained. The reasons for unusual peaks of valleys in the eurves are noted on the back of the form.

The forms are punched to fit a loose-leaf binder, and are so filed that all the sheets for the same make of ear are together. The charts are plotted each month as the figures become available in the accounting department. 
Graphic Analysis. One of the most valuable uses to which charts may be put is that of analysis-the analytical study of facts which many times do not adhere to any known mathematical law, but which are nevertheless capable of being reduced to a workable form. An article by Edwin D. Dreyfus, "Graphic Analysis of Managerial Problems," published in Electrical World, October 7, 1916, gives several examples. of the application of the analytical method and graphic charts. An editorial in the same magazine contains the following helpful suggestions.

Mr. Dreyfus' discussion shows how methods which appeal at once to the eye and the intelligence can be utilized in co-ordinating apparently intricate data so that their true relations may be seen, even when these do not apply strictly to engineering matters but to the complex data of business. Not only can facts which to the casual observer seem unrelated be made amenable to graphical methods, but these often serve to bring out correlations which are quite unsuspected. Things apparently independent when plotted on co-ordinate paper often show a systematic trend which points the way to a generalization of real value.

In working through graphics one has, however, to be exceedingly cautious in certain particulars, for instance, when a set of figures, dynamical or financial, are available they are, so long as they are tabulated, instinctively taken merely at their face value. When plotted, however, there is a temptation to extrapolation which is well nigh irresistible to the untrained mind. Sometimes the process can be safely employed, but it requires a rather comprehensive knowledge of the facts that lie back of the data to tell when to go ahead and when to stop.

For example, take the curve which represents the increase of train resistance with increasing speed. The curve is a rather gentle one and as the experimental data are generally not very precise the points secured from a moderate range of speed can be represented almost equally well by a straight line or by several types of curve. If continued in either direction both line and curves are likely to lead to exceedingly wild results, as experience has amply shown, merely from departure of the real data from the extrapolated value. Again, a curve may apparently go on from the plotted data in a beautifully systematic manner with every indication that it will presently become asymptotic, only to pass through a maximum and turn downward to the utter confusion of hypotheses drawn from its early course. Plenty of such instances can be found in engineering data.

Again, even interpolation is not always safe, since a curve not infrequently undergoes a radical change of curvature at some particular point or points, resuming afterward an orderly progression. In this case groups of points taken too far apart and apparently quite consistent may lead to astounding blunders. Instances of this sort may be found in the curves connecting the velocity and air resistance of projectiles, speed and driving power in ships, and in numerous other instances, some of them of a very unexpected character. Finally the student of graphics must watch cautiously lest he be deceived into drawing a smooth curve which may conceal some of the very facts that he sought to discover.

Averages, in other words, have a wonderful facility at covering up 
signifieant variations from them. These warnings do not in any sense detract from the value of IIr. Dreyfus's paper, but are merely presented for their bearing on graphieal practice. Curves should be used, even much more than at present, to put in usuable form the practical data of engineering, yet they need careful scrutiny in cases where the data are ineomplete or where it is difficult to see from theory what the general form of the eurve is likely to be. Oftentimes significant facts turn up in plotting data to polar or logarithmic eo-ordinates which are not at all obvious by the ordinary methods.

A portion of Mr. Dreyfus' article follows:

To appreciate the inherent advantages of linear relationships, serious study and thoughtful attention must be given the matter, in order that the perspective and inter-relation displayed by a family or group of interdependent curved and rectangular lines may be fully grasped. The eye and mind are readily trained to operate in this realm eonsciously at first and later intuitively, with the result that the business man and engineer will aequire a power of judgment and insight in making business introspection and prophetic analyses of a more aceurate and certain nature than obtainable in any other way.

In various branches of mathematies, graphic construction of formulas provides a very instructive study, and in astronomy, in dynamies, and in many of the practical sciences this means furnishes a most profitable guide to the solution of important technical problems. While business operations do not ordinarily oceur with sufficient regularity to permit their results being reduced to formulas or otherwise being represented by fixed conditions, such as a series of curves and lines would convey, averages in the usual rum of business do, however, bear relations in such ways that with the aid of diagrams important deductions may be made.

On account of the diverse interests met with on every hand, and the great complexities of modern business and engineering, every facility is required to open up avenues for a quick perception of new situations that may be confronted. Nothing is so illuminating as a set of properly proportioned diagrams. Business in the past has contented itself in the main with tabular presentation of the trend of its affairs, and habit is no mean force to reckon with. IIowever, graphics have such practical significance in engineering work when extensively used that the regular routine of related business is made much more intelligible by the adoption of this important aid. In addition to the significanee of graphics in analytical work, it is likewise a valuable aid to the memory. A picture is manifestly more readily retained in mind than a description of the same subject, no matter how vividly it may have heen expressed. A pictorial or diagrammatic illustration usually prodnces a firmer and more lasting impression than any composition of words or tabulation of figures, however well they may be arranged or set forth.

The following exhibits taken at random are illustrations of the employment of graphies and emphasize their utility in pursuing a line of reasoning and in experliting the reaching of eonclusions on eertain dubitable and unsolved problems. The examples do not begin to reveal the wide use to which diagrams may be applied and are merely unique 
cases, in which it is believed much work was compassed within notably small space.

Example I.-There are instances where speedy substantiating or else disproving of contentions may have a far-reaching effect. Suppose a witness is placed upon the stand in behalf of a company opposing before a public service commission the granting of a competitive franchise. Assume also that the witness is qualified and prepared to give testimony on the condition of saturation of business of the territory involved. The average present revenue per consumer of the different classes (domestic, commercial and power) of the existing company should have been secured first, and likewise a complete canvass of all prospective consumers should have been made. If, then, the petitioners should claim that 500 domestic, 395 commercial and fifty power consumers were to be obtained, quick calculation of the probable gross revenue is possible, as will be seen by following the dotted guide lines $a, b, c, d, e, f$, reaching an aggregate of $\$ 70,000$, Fig. 23 .

As an illustration, start at (a) with 500 domestic customers, from

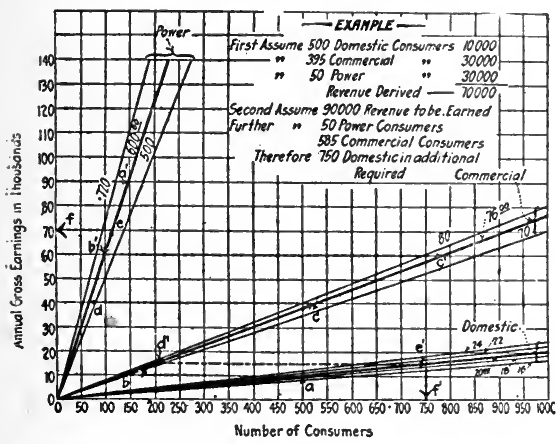

Fig. 23-Revenue Chart for Various Combinations of Domestic, Commercial and Power Consumers

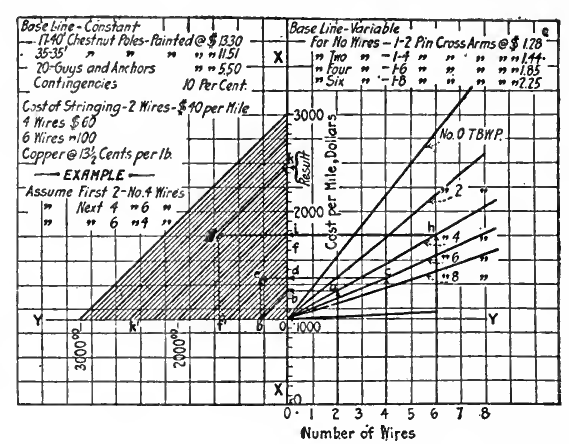

Fig. 24-Chart for Determining Cost of Pole Line Construction with Different Combinations of Circuits

which a $\$ 10,000$ revenue would be secured, the point of intersection of commercial diagonal line with the $\$ 10,000$ line of ordinate indicates an equivalent of 125 commercial consumers, to which is to be added the 395 already specified which, by ready mental operation, shows 520 commercials, which extended to $(c)$ sums up to $\$ 40,000$ revenue. Similarly an intersection with the power diagonal at $(d)$ determines an equivalent of sixty-six power consumers (approximate), and adding the fifty already given makes a total of $116(e)$, which corresponds to $\$ 70,000$ revenue. Now by reversing the process somewhat, and assuming that $\$ 90,000$ annual revenue is essential for the security of a "paralleling" investment, and, further, that it had been so far claimed that fifty power consumers and 585 commercials could be booked, by employing the diagram, and as indicated by guide lines $a^{\prime}, b^{\prime}, c^{\prime}, d^{\prime}, e^{\prime}, f^{\prime}$, it is at once apparent that 750 domestic consumers in addition would be necessary to provide the required $\$ 90,000$ revenue. (The method of subtraction and division on the chart is the opposite of that just described for multiplication and addition, as will be evident.) The impossibility 
of attaining the $\$ 90,000$ revenue would promptly prove the venture to. be unprofitable. Numerous other conditions could be likewise worked out rapidly when charts showing graphical relations are employed.

Example 1I.-In such cases as the above, numerous other diagrams. could be developed to enable quick analysis and estimating of the amounts involved in a proposed second utility's system, and Fig. 24 is a striking example. Approximate costs of the overhead distribution system could be figured immediately, as the extent of the line to be built was stated. An assumed mile of two No. 4 wires, four No. 6 and six No. 4 might be taken for illustration as being under discussion. The pole line cost, to some extent, is practically the same, irrespective of the number of wires strung. Then by equilateral triangulation centered

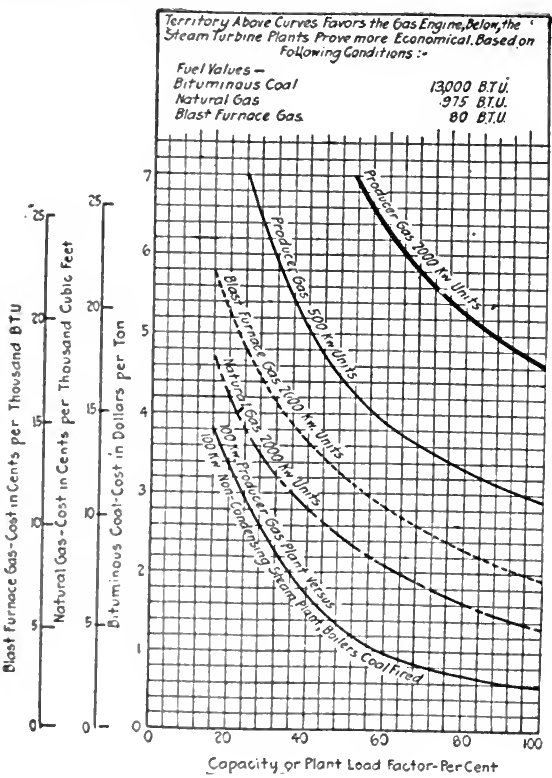

Fig. 2.j-Balance of Total Generation rosts in Fis and Steam Plants with Variable Fuel Prices and Load Factors

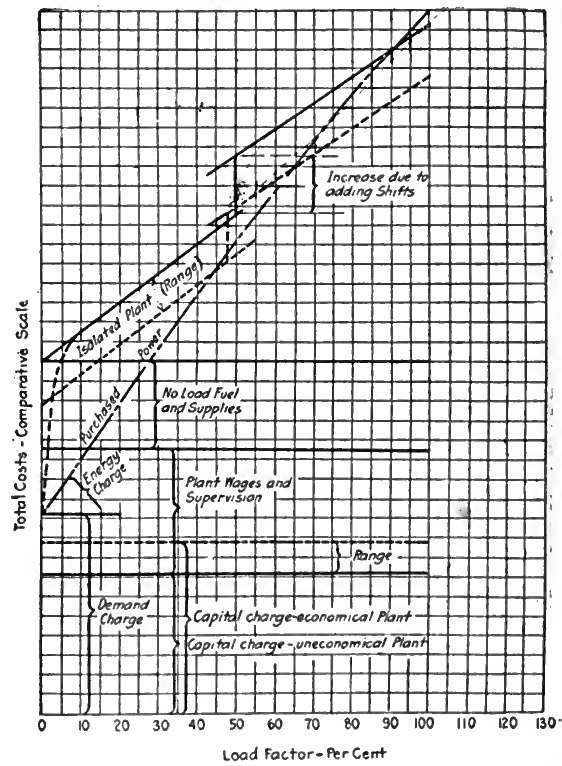

Fig. 26-Diagrammatic Comparison of Purchased Power and Isolated Plant Costs

about the origin or axis $O$, the results are obtained for any combination of wires.

For example, assume the equivalent to be two No. 4 , four No. 6 and six No. 4 wires. For two No. 4 wires as at $(a)$ a value at $(b)$ is found on the vertical dollar scale $(O X)$, or by $45 \mathrm{deg}$. diagonals the same value at $\left(b^{\prime}\right)$ on the identical horizontal dollar seale $\left(O Y^{\prime}\right)$. 'T'o add for four No. 6 at $(c)$, which corresponds to $(d)$ on the $O X$ scale, and avoid duplicating cost of common pole supports, the line $(c d)$ is projected horizontally until it intersects the vertical line $b^{\prime} e$ ) and as will be observed on close inspection the chord, $\left(b^{\prime} e\right)$ is the increment of cost due to the four No. 6 wires. By projecting the point $(c)$ on the diagonal line: to either $(f)$ on seale $(O Y)$, or $\left(f^{\prime}\right)$ on seale $(O Y)$, the cost of the line is obtained. Procererling in the same way to add the additional six 
No. 4 wires, we take $(h)$ and project through $(i)$ to the intersection of $\left(f^{\prime} j\right),\left(f^{\prime} j\right)$ being the increment due to the six No. 4 wires, and running diagonally from $(j)$ to either $\left(k^{\prime}\right)$ or $(k)$ the final result for this example is derived. Any scheme of wire lines may be thus speedily solved for cost by referring to such a chart.

Example III. About five years ago the economic position of the gas power plant was not fully appreciated. Owing to the difference in in-. vestment and operating costs between the steam turbine and gas power plants, the respective size, load factor and cost of fuel influenced their comparative ultimate economies. An exhaustive study was made of the subject and conclusions as reproduced in Fig. 25 were obtained, which

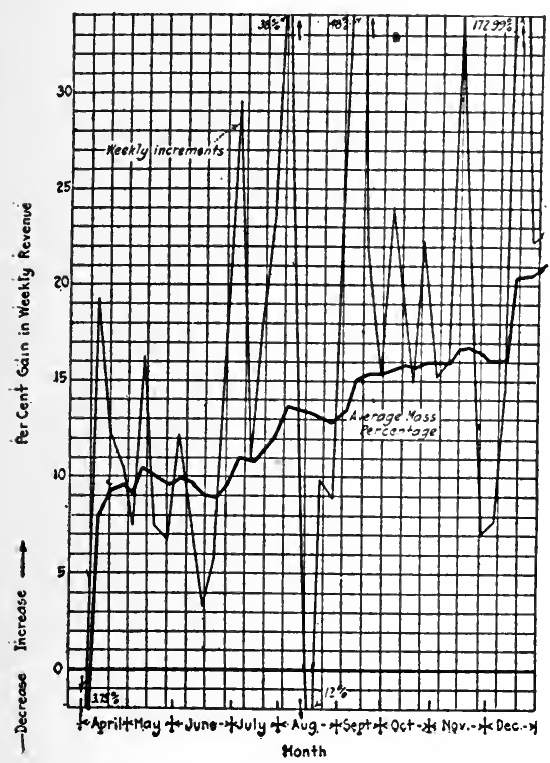

Fig. 27-An Estimate of Net Weekly Increments in Revenue from Light and Power Service for a System

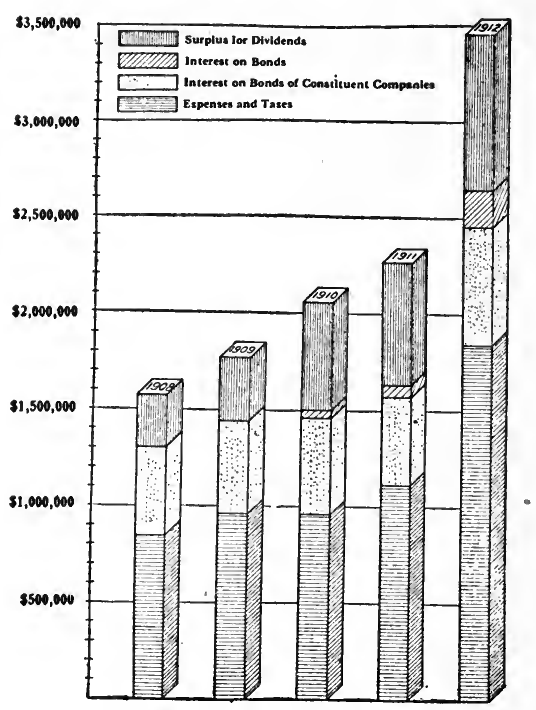

Fig. 28-Pictorial Diagram Suitable for Representation of Certain Data in This Case Comparative Earnings

were given in detail by the writer in the 1911 proceedings of the Association of Iron and Steel Electrical Engineers. Curves are drawn for different sizes of plants, and plotted against the ordinate scale for cost of fuel and the abscissa scale for load factor. The territory lying above the respective curves shows the economical field for the gas-producer power plant and below for the steam turbine plant. In this way the results of a variety of conditions are concentrated in one diagram in what is believed to be an entirely clear manner that permits ready interpretation.

Example IV. The diagram of Fig. 26 brings out the relative situation of isolated power plant costs versus purchased energy under certain assumed conditions. The discussion here centered about the question. as to whether it was advisable to install machinery to minimize the peak load created on the power company's system. It would appear from 
this chart that the additional investment and complications would not be justified under the assumed eircumstances.

Example V. In Fig. 27 a simple case of plotting fluctuations of business returns from week to week is presented, with the addition, however, of an auxiliary curve which shows the accumulated or mass average and brings out clearly the trend either in the direction of an improvement or a decline in results.

Example VI. Often a pictorial diagram or chart such as shown in Fig. 28 produces a quicker perception of the conditions than the average mind may obtain from a mere line diagram. Variations in charts of this kind can be devised in great numbers according to nature of information and features it may be desired to illustrate.

Graphic Analysis of Manufacturing Costs. The following is from an article by C. W. Starker, "Analyzing Manufacturing Costs to Increase Profits,"' published August, 1917, in Industrial Management. In mapping out a campaign intended to increase profits from the manufacture and sale of apparatus by analyzing factory costs my experience indicates the desirability, first, of limiting the seope of the investigation. This is particularly true in the ease of large corporations manufacturing a varied product. It means to concentrate your attack upon a specific line of apparatus, a specific class of product at a time. The next general rule is to begin the investigation with a broad survey of the field and gradually to narrow down the issue point for point to smaller and smaller parts or details, until the smallest operation has been segiregated and put under the mieroscope.

Following this procedure with the product of a company manufacturing, for example, steam turbines, gas engines and air compressors, it would be advisable to limit the scope of an investigation according to the elass of product to, say, steam turbines, and within this elass to a certain range of sizes and capacities. The logical sub-division of sizes usually indieates itself quite naturally, being defined by groups of manufacturing sections, which depend on tool equipment and handling facilities. Another example, a company manufacturing alternating and direct current electrical apparatus, motors, switches, are lamps, and what not. Before beginning an investigation I would suggest taking one class of the product at a time, say direct current motors, and limit this further, for example, to sizes from 2 to 100 horsepower.

The next step, a broad survey, involves answering the questions: What is the amount of business done by the eompany per year in this line, and what is the total business available in this field; that is, the total buying capacity of the market? Sales department records are expected to supply the answer to both of these questions and with such figures it can readily be shown what percentage of the business has actually been secured in the past, as well as what should be the company's share, that is, what may justly be expected or at least aimed at. These data furnish a general indication of the status of affairs and are desirable knowlerlge in starting an analysis of manufacturing costs, even though the cure or improvement may partly lie outside the province of manufacturing costs, being influenced by sales policy or financial conclitions of the company. The pereentage of profit from sales should be registered in a similar way giving an average, for the: 
range of product under analysis, as actually secured profits and as profits which may reasonably be expected. Competitor's prices, costs and profits, if available, may also be used for comparison.

The next step in narrowing down the field is to segregate the different sub-divisions of the product further. They may be divided, for instance, according to service characteristics or performance characteristics of the apparatus. To illustrate what is meant take again the example of electric motors. I would suggest sub-dividing the main division of direct current motors further according to service characteristics in machine-tool motors, erane motors, elevator motors, etc., to find out what relative importance attaches to each group, what percentage of available business is secured, what costs, profits and selling prices prevail. Similarly, according to performance characteristics they might be divided in various ways, for instance, according to voltage or speed

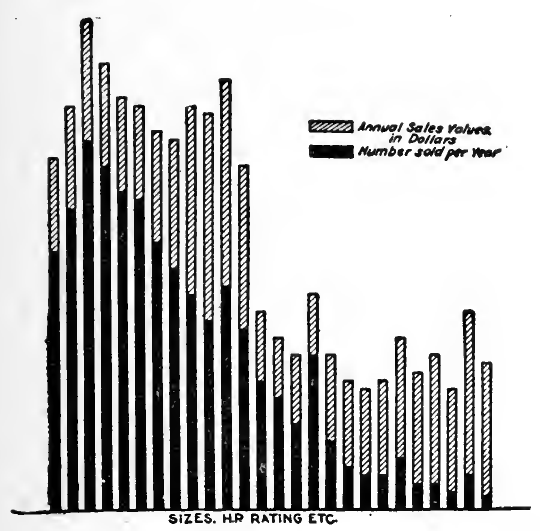

Fig. 29-Relative Importance of Various Sizes by Numbers Sold Per Year and by Annual Sales Value

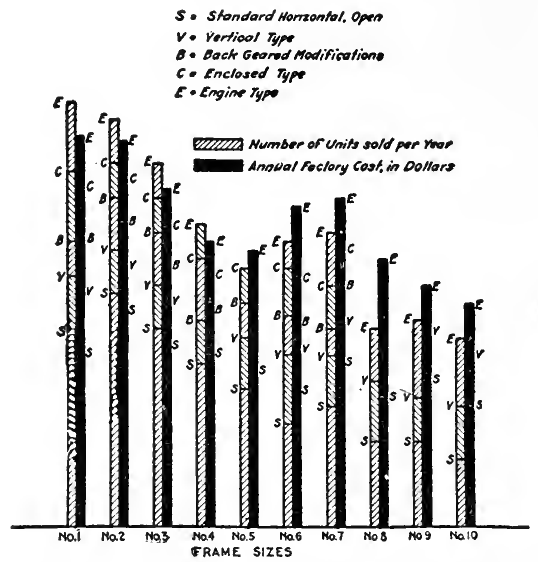

Fig. 30-Relative Importance of Various Modifications on Each Frame Size by Numbers Sold Per Year, and by Annual Factory Cost

characteristics, that is, constant-speed, adjustable-speed, varying-speed motors, etc. In each case the purpose in mind is to bring out the relative importance and the relative need for improvement so as to direct our efforts in the coming detailed analysis to the point most in need and most profitable.

This relative importance is best brought out in graphic form as indicated in Fig. 29 in which the different sizes of a line of apparatus are plotted, first according to number of units sold per year for each size, then according to their annual sales value or annual factory cost. The annual profits for each size may well be shown on the same curve. In a similar manner Fig. 30 is given to illustrate the relative importance according to classes of service of apparatus, giving again the number of units sold and the annual factory cost of these units for each curve. (The values used in the curves are, of course, fictitious and merely used as illustration). The story told by these curves will be 
understood without much explanation. There may be some surprises in what such eurves reveal; it may be shown that in some instances the investments made for certain sizes are not warranted by the sales, or that other sizes of which great quantities are sold would warrant much better tooling-up, necessitating quite different arrangements in manufacture more in keeping with the selling possibilities.

After data such as these have been secured the first general survey may be considered completed. We have secured a general outlook over

WORKS DEPARTMENT-SUMMARY COST SHEET

\begin{tabular}{|c|c|c|c|c|c|c|c|c|c|c|}
\hline \multicolumn{2}{|c|}{ Apparatus-Commutator } & \multicolumn{5}{|c|}{ Piece: No. 143,291} & \multicolumn{4}{|c|}{ Date: May 1st, 1917} \\
\hline \multirow[b]{2}{*}{ Part-Operation } & \multirow{2}{*}{$\begin{array}{l}\text { Dwg. and } \\
\text { Item Cost } \\
\text { Refer. }\end{array}$} & \multirow[b]{2}{*}{ 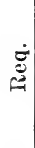 } & \multirow{2}{*}{ 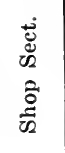 } & \multirow{2}{*}{$\begin{array}{c}\text { Labor } \\
\$\end{array}$} & \multicolumn{2}{|c|}{ Overhead } & \multicolumn{3}{|c|}{ Material } & \multirow{2}{*}{ 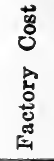 } \\
\hline & & & & & $\%$ & $\$$ & $\begin{array}{l}\text { Weight } \\
\text { Kind }\end{array}$ & $\begin{array}{l}\text { Cost } \\
\text { rer } \\
\text { lb. }\end{array}$ & $\underset{\$}{\text { Value }}$ & \\
\hline $\begin{array}{r}\text { Bush, } P \text { No. } 145,437 \ldots . \\
\text { Bore and Face..... }\end{array}$ & $\begin{array}{c}325,617-3 \\
\text { OP No. } 76,32\end{array}$ & 1 & S.51 & -.079 & 100 & -.079 & 5.5M.1. & .04 & -.220 & \\
\hline & DF & & S. 51 & .100 & 100 & .100 & . & . & -.200 & \\
\hline $\begin{array}{l}\text { Key } \\
\text { ing, }\end{array}$ & $\begin{aligned} & \text { “ } 76,7 \\
& 212,597-5\end{aligned}$ & 1 & S-51 & .082 & 100 & .082 & .. & .. & .. & -.74 \\
\hline 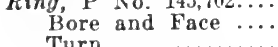 & OP No. 76,325 & $\begin{array}{l}1 \\
. .\end{array}$ & S-51 & -.039 & 100 & -.039 & $2.25 \mathrm{M} 1$. & .04 & -.090 & \\
\hline $\begin{aligned} & \text { Tur } \\
& \text { Enit, I }\end{aligned}$ & “" $76,698-3$ & $\ddot{i}$ & S.51 & .066 & 100 & .066 & .. & .. & .. & -.30 \\
\hline Face, 7 & OP No. 15,982 & .. & S-52 & -.038 & 100 & -.038 & .. & .. & .. & \\
\hline & “ $\quad 11,297$ & $\because$. & S. & $\begin{array}{l}.013 \\
.013\end{array}$ & $\begin{array}{l}100 \\
100\end{array}$ & $\begin{array}{l}.013 \\
.013\end{array}$ & .. & & ... & \\
\hline Forge ......... & & $\therefore$ & $\mathrm{H}$ & .010 & 2 & .0 & H.R.S. & .015 & -.026 & \\
\hline & $\ldots \ldots$ & .. & $\begin{array}{l}\mathrm{H}-21 \\
\mathrm{H}-21\end{array}$ & $\begin{array}{l}.001 \\
.001\end{array}$ & $\begin{array}{l}175 \\
175\end{array}$ & $\begin{array}{l}.002 \\
.002\end{array}$ & .. & .. & .. & -.195 \\
\hline Bars, Gauge No. 42,975 . & $315,452-6$ & 33 & & & & & $6.7 \quad>$ & & & \\
\hline Blanking......... & $\ldots \ldots$ & . & P-17 & -.060 & 150 & -.090 & $\begin{array}{l}1.1 \\
5.6\end{array}$ & .10 & i. 27 & 1.42 \\
\hline $\begin{array}{r}\text { Mica } \\
\text { Strips, }, \text { P No. }\end{array}$ & $241,375-2$ & 33 & & & & & & & & \\
\hline${ }_{\mathrm{C}}^{\mathrm{M}}$ & ...... & & E-21 & -.037 & 60 & -.023 & .382 & .99 & -.378 & \\
\hline & & & $E-21$ & 二.037 & $\begin{array}{l}60 \\
60\end{array}$ & .02 & $\ddot{. .}$ & $\because$. & $\cdots$ & -.56 \\
\hline $\begin{array}{l}\text { Build } \\
\text { Mica.V }\end{array}$ & JP No. 76,485 & 1 & S. 51 & -.095 & 100 & -.095 & .. & .. & -.020 & -.21 \\
\hline $\begin{array}{rl}r 1 & 145 \\
145 & -1\end{array}$ & $241,375-3$ & 2 & $\mathrm{E}-21$ & & & & & & & \\
\hline & & $\because$ & $\begin{array}{l}\text { E-21 } \\
\text { F-21 }\end{array}$ & -.080 & 60 & -.048 & .50 & .90 & -.460 & \\
\hline & $\ldots \ldots$ & .. & $\mathrm{E} \cdot 21$ & .018 & $\because$. & .01 & $\because$. & $\because$ & $\because$. & -.68 \\
\hline & $225,640-7$ & 1 & E-21 & .010 & .. & .006 & .. & .. & .. & \\
\hline$\& \dddot{\mathrm{A} s}$ & TP No. 76,446 & 1 & $\mathrm{~T}-32$ & -.186 & 50 & -.279 & & & .. & \\
\hline & “ & .. & & .1 & & & .. & .. & .. & \\
\hline & 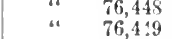 & .. & $\begin{array}{l}\mathrm{T}-32 \\
\mathrm{~T}-32\end{array}$ & $\begin{array}{l}.082 \\
.069\end{array}$ & $\begin{array}{l}150 \\
150\end{array}$ & $\begin{array}{l}.123 \\
.104\end{array}$ & $\because$ & .. & $\because$. & 1.536 \\
\hline Heat and l'ress ... & “ 99,675 & & $\mathrm{~T}-32$ & .150 & 150 & .225 & .. & $\cdots$ & .. & \\
\hline otals & & .. & ... & 1.460 & .. & 1.719 & $\because>>>>$ & .. & 2.464 & 5.61 \\
\hline
\end{tabular}

Fig. 31-Specimen of Cost I)ata Segregated by Operations

the situation and can now make a more detailed study of the cost of manufacture. The figures for factory costs used in the investigation up to this point are those eompiled in regular rontine by the various divisions of the cost department. Such figures are subject to more or less correction and revision, as inconsistencies and minor errors are unfortunately unavoiclable, so long as a large amount of work must be handled by clerks who are not expected to critically judge every figure turned in from feeder sections. For the general survey these figures 
were close enough, but for a detailed analysis it is necessary to review them critically.

To do this, it is not necessary to take the great mass of cost figures involved in a large line of apparatus, such as in the example above used for reference, where there may be over 1,000 ratings of directcurrent motors, all built along similar lines but differing in size and some of the details. It is better, in fact, to select only a comparatively few representative cases, say 10 or 20, and make the review of the figures that much more thorough. With reliable figures once established for a few sizes, selected over the entire range, it is a matter of duplication to correct the figures for the entire line on the same basis.

For these representative eases it has been found most desirable to record the cost of the corresponding component parts of all sizes together, rather than take all the incongruent parts of one unit of apparatus; that is, take the ten bearing sizes involved, then the ten frames, ten shafts and so on. The cost of each piece is then listed by operations, giving labor, material and overhead expense for each operation, noting at the same time the quantity of material, kind of material and base price used. For example, for a forging or casting the rough weight and finished weight should be given, the price per pound and the allowance for scrap material per pound. Further, there should be given the shop section where the operation is performed and the overhead percentage used. It is desirable to note all these details, so that in case of a change in material, price or overhead percentage, the figures can readily be revised and used for reference at a later date. In the table Fig. 31 a summary cost of a commutator is given as an example. It will be noted that labor rates (hourly, piece or premium work) and time per operation are not given. In many cases it will not be necessary to investigate the rates, but for those cases which appear unreasonable and capable of improvement further analysis as to time and labor rate will follow later.

The term "material" in the above is understood to mean actual raw material as purchased from outside concerns and without any feeder section labor performed on it. This is mentioned, because sometimes it is the practice to use the term material for semi-finished parts received by the assembling section from feeders. An addition is frequently made to the cost of raw materials, such as wire cables, pipe, etc., to cover the loss from short ends.

After corrected costs have been obtained along these lines, it is best to. show the results graphically before making any particular attempt at reducing the costs; that is, content ourselves for the time being with setting down the facts correctly and in detail. If the total costs of material, total labor and total overhead expense are determined for each of the ten sizes selected, it is possible to plot a series of curves as shown in Fig. 32. The first curve from the bottom shows the cost of material. Over this curve is plotted the amount of labor, so that the vertical distance between the two curves represents the labor cost. In the same manner is added the overhead expense in vertical lines, so that the resulting third eurve represents material plus labor plus overhead; that is, the factory cost. This curve is the true factory cost, as existing at the time and before any improvements are made other than 
the careful check of each item of cost. If we add to the factory cost the percentage of selling expense the fourth curve shows the commercial cost. On the same sheet is then plotted the regular listed selling price and the minimum selling price as granted under special contracts to large customers. The vertical distances between the last two curves show then the profits at minimum and regular sales price, as well as the rebates used.

The further steps in the analysis are largely a matter of correctly interpreting the various points brought out by the curves. Beginning with the first curve-material-we may ask ourselves, does this ma-

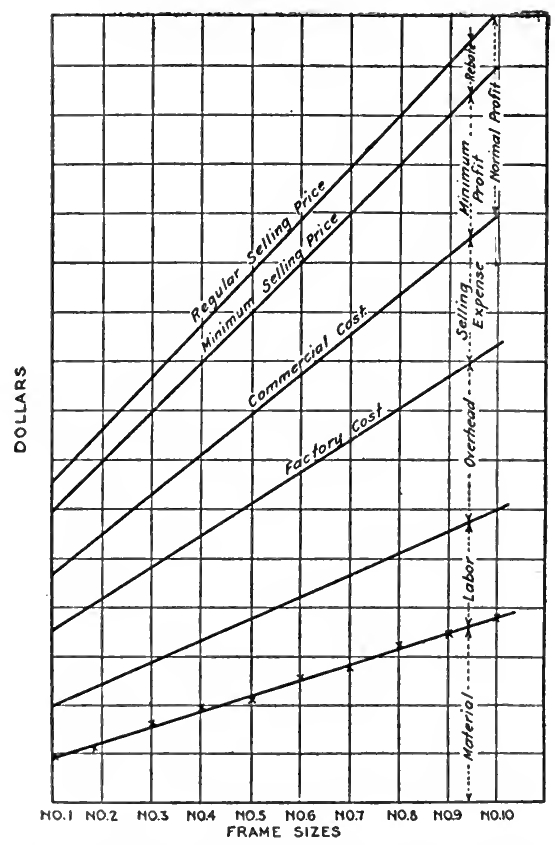

Fig. 32-Material, Lahor, Overhead, Factory Cost, Commercial Cost and Selling Prices

terial-eost bear the proper relation to the total factory costs? This may best be determined by comparison with other lines, perhaps older types, and a curve drawn showing the per pound cost of apparatus of various capacities. This per pound cost obviously cannot be a constant factor, as in large units the material cost will be found to constitute a larger part of the total, while the labor does not increase in direct proportion with increasing size of apparatus. The material cost may further be divided into kinds of matcrial, for instance, eopper, cast iron, forged steel, ete. This is best done for a given unit of completed apparatus and in graphice form as shown in Fig. 33. A segregation in active and inactive material is of particular interest. Such a division can be made with most classes of apparatus. To adhere to our example of electric motors, the active material would be that part which is actually 
used in producing or carrying current or magnetic flux, such as punchings, windings, ete., while under inactive material would be listed bearing brackets, bearings, bedplates, ete. There should, in a well proportioned design, be a certain relation between the two. This may readily be checked by reference to the curves or diagrams for similar apparatus. Further there should be a definite relation between the amount of copper and iron used in an electric machine of given size and characteristics. Their proportion may be influenced to an extent by the relative cost of the two materials, as with a radical change in their cost-relation a revision of the design may be called for to allow the machine to remain economical in production and be marketable.

Returning to Fig. 32 the curves for material and labor indicate whether or not there is a consistent gradual inerease in these items with increasing size of apparatus, that is, whether or not design and manu-

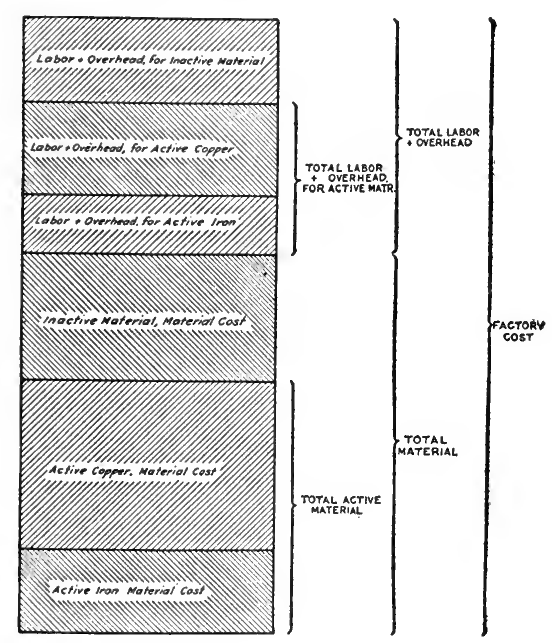

Fig. 33-Proportion of Active and Inactive Material

facturing methods are uniform and consistent or whether some sizes are out of line in one or the other respect. Further we may read from the curves whether the overhead percentage is distributed properly over the range of sizes, that is, in keeping with the actual conditions. Smaller sizes produced in large quantities may require less supervision, less expense for upkeep of tools, for handling facilities, etc. In other words, it may be that a graded percentage of overheads should be used, the higher percentage for the larger sizes, requiring greater tool investment, larger floor space and handling facilities, perhaps special attention from foremen and inspectors. In a similar manner the proportioning of selling expense or rebates for smaller and larger units is made clear by the curve. The question of a logical selling price, that is, a price in keeping with the cost of production may be raised and improvements taken up, in so far as market conditions will permit. As a rule uniformity of profits for all sizes of a line is not obtainable in practice, 
rather we must start out from a given market price and attempt to bring the cost of our product in line.

It is of course not possible in an article like this to discuss all these points in detail. Some of these questions represent in themselves quite formidable problems and they are here merely referred to, without further discussion. Cost analysis data furnish valuable pointers and helps to their solution.

The further steps in the cost analysis are of particular importance. They comprise a detailed study of the manufacturing operations listed on the summary cost sheet for each component part of each size. While it is not possible to state exactly what should be done in each individual ease, the analysis should in general first determine if the operations performed are necessary, if they are performed in the proper sequence without avoidable handling and delay to production, whether or not proper machine tools, fixtures and other equipment are used. Time studies on different machines, changes in designs or materials may be made involving building of experimental apparatus and tests to try out and prove the various suggestions. A study of new materials or new treatment of materials frequently yields good results. Purchasing methods may eome under consideration, improvements in preparing and transmitting of shop information.

These few items are enough to indicate that a thorough analysis reaches into practieally every activity of the different departments of a manufacturing organization. It cannot be expected that any one man possesses a thorongh knowledge of all thece specialized fields. It is necessary, therefore, for those making the investigation to take up the various points with those speeializing directly on the subject. The metallurgist or chemist is consulted on questions of material, the supervisor of rates will handle the time studies, and so on. Frequently this work of analyzing eosts is handled by a eommittee appointed for the purpose and consisting of representatives of the different departments interested, such as engineering, works and sales department. To secure results, however, it is necessary to have one man act as the leader, who must keep up interest in the work and be suffieiently broad to take the initiative or to judge suggestions made by others even in the more specializel fields not directly in his province. A certain amount of tact and diplomacy is required to insure willing and active co-öperation.

Ilaving then eompleted the investigation and before making any changes in manufacture or design a complete report should be drawn up statine the improvements recommended and containing a eonservative estimate of whatever lifferences in cost there may be. $A$ eareful estimate should be prepared of the expense involved in carrying out the rerommendations, including cost of tools, ete. All changes recommended should be carried out as much as possible at one time after proper anthorization has been given by the management so as to minimize the expense and upsetting of production.

To add to the value of the cost analysis it is desirable to keep a permanent record of the fluctuations of manufacturing costs. This is best done in the form of conves which extend over a long periogl of time and indicate at a crlance the lowering of cost, as the results of an analysis gradually become apparent in the cost returns from the works 
department. If there are increases in cost due to changes in material price, labor rates or overhead conditions this will be apparent from the curves, indicating perhaps the need of further steps for improvements under the changed conditions. Fig. 33B illustrates such curves, the cost status per unit being recorded in half-yearly intervals for each size. A curve of totals on the same sheet is intended to tell a more complete story by giving the total factory cost taking into account the

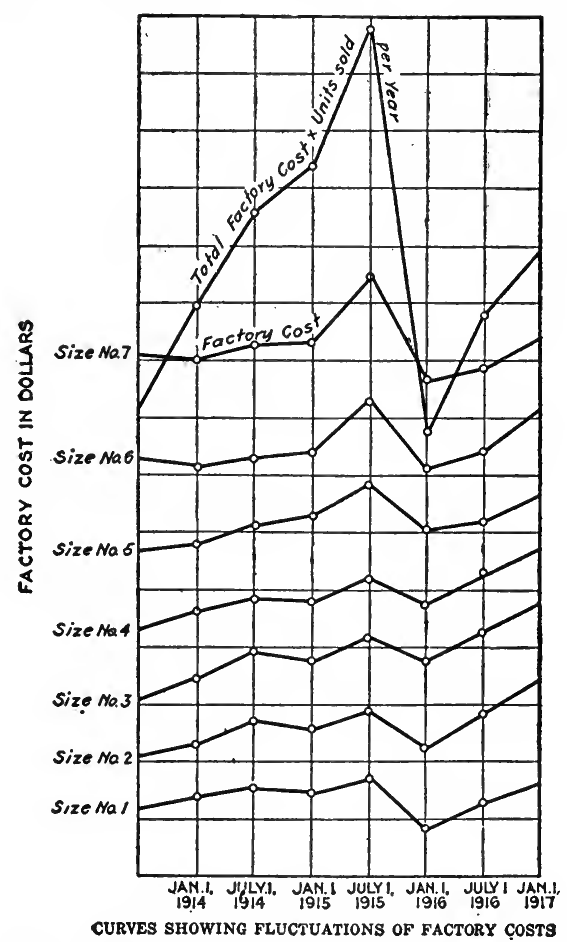

Fig. 33B-Curves Showing Fluctuations of Factory Costs

number of units sold of each size. The number of units sold is included in order to bring out the complete results, rather than the cost of units, some of which are more important than others from a standpoint of quantities sold. It is necessary and sufficiently accurate to assume a fixed number of units sold per year for each size, in order to have the totals at each time period expressed on the same basis for direct comparison.

Manufacturing Costs. In an article, "How to Find and Use Barometric Costs," published in Industrial Management, August, 1918, Mr. Starker describes a system for determining fluctuations in factory costs when materials, wages and overhead are changing. The following is quoted :

The basic idea is to provide a flexible, sensitive method of telling immediately the influence of market and manufacturing conditions upon 
selling price and profits, much as a barometer indieates the trend of the weather on the basis of changes in atmospheric conditions. So far as material is eoneerned our purpose is aceomplished by segregating first the various kinds of material used in the apparatus, giving the number of pounds gross weight for each and the base price and total value of each material at a certain date. Any corrections or revisions of these figures, from time to time, are entered in the sueceeding columns, giving the date when the eorreetion was made. Similarly we record in the table the different departments performing work on the apparatus and give the amount of flat labor cost in each department. The eorrections are made in suceeding columns at different dates as may beeome necessary by a change oecurring in the labor rates. Next we give the overhead pereentages used for each department at a given date and the total amount, making again corrections as they become neeessary. From these figures the total amount of ehange in material or labor eost and overhead can be calculated quickly and a percentage established for the total inerease or decrease relative to the previous factory cost of the individual unit. With the barometric cost system a number of typical sizes or ratings are selected, and percentages of inerease or decrease are ealculated for each of these and it has been found that, with a uniform design and eonstruction of all sizes, interpolation permits a very satisfactory and suffieiently aceurate ealculation of the new factory cost for all the intervening sizes. The main advantage and objeet of this barometrie cost system is that these figures are obtained at a moment's notice for any condition, existing or anticipated, of the labor or material market and for any of the many different sizes. It is not intended to do away with the thorough ealculation and revision of all the costs at reasonable intervals. From time to time a thorough going over of all the detail figures is, of course, required for all the typical cases selected as "barometer units." The intention is merely to lengthen these intervals and to provide a ready means for taking into aceount ehanging conditions of basis costs, so as to obtain a factory cost sufficiently aceurate for use as a basis for establishing selling prices and for billing to the general stores. In Fig. 34 is illustrated, as an example, assumed changes in the eost of raw material, labor and overhead, giving also the selling expense, profit and sales priee at assumed different periods of the year. From the foregoing the curves will be realily understood. The values are plotter in cumulative form, that is, the vertical distances between the curves give the amount of the partieular item and these values are added to one another. The rise in eost is indicated by the differenee in the leneth of the ordinates. Changes in priees of various materials may orecur at different times as will be noted in the eurves. The effect of a sulden increase in labor rates is also shown. The average overhead perentage may actually be lower due to inereased production, but it will be noted in the curves that the actual amount of overhead expense absorberl, that is, the produet of flat labor and the overhead percentage still shows an inerease. A similar eonsideration applies to the percentage and actual amount of selling expense, justified by the fact that these expenses, due to general conditions, increase more than proportionately to the increase in volume of business. 
As the sales price of necessity remains constant through a certain period of time, even though the cost of a product is creeping up continuously, it follows that a point is reached from time to time where the profit approaches zero and an increased price becomes a necessity. This is readily seen by reference to the curve. For the price, sales and executive departments it is very interesting to follow on such curves the changes in the cost of the product and the influence of such changes upon the margin of profit. The curves tell at a glance when an increase in the same price will become necessary to maintain reasonable profits. In these days of rapidly rising prices of practically all com-

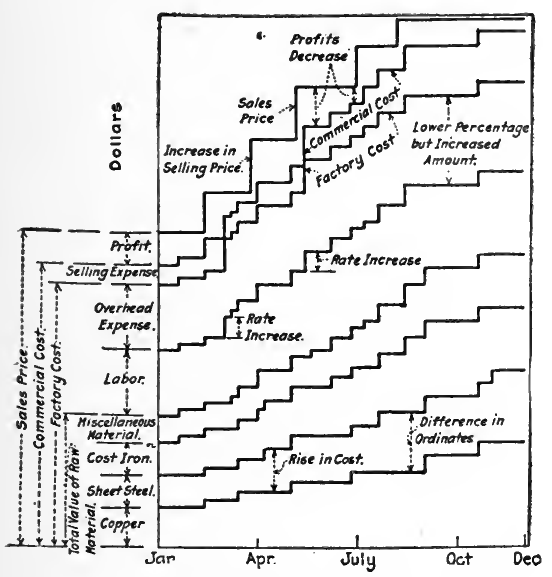

Fig. 34-Itemized Cost Changes Per Unit

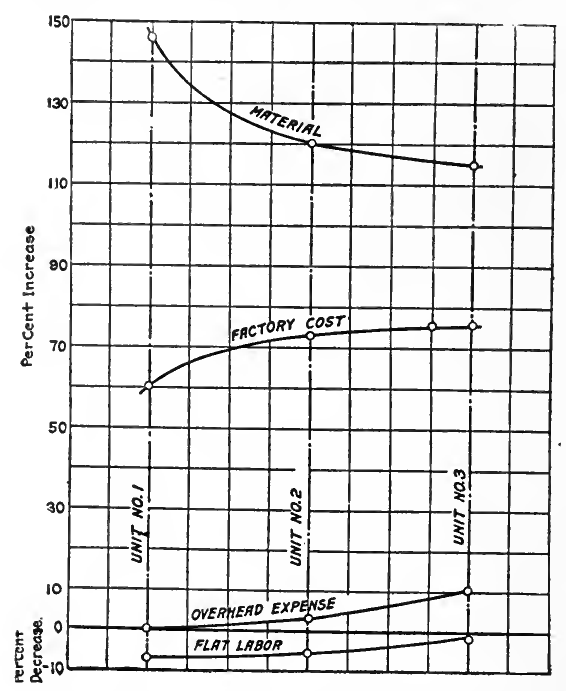

Fig. 35-Summary of Increases of Cost for the Different Items Involved

modities the purchaser at times feets that the increase is unwarranted and made arbitrarily. A look at the curve will be more convincing than many words of apology on the part of the salesman. He can point to the fact that the increase in sales price is established in a scientific manner and based on unrefutable facts. It may be shown, assuming this to be the case, that as a matter of fact the increase in price has not kept pace with the increased cost of raw material and labor, even if those two items were partly offset by more efficient production and increased output.

While in Fig. 34 the changes in cost are shown for one unit of apparatus and recorded as they occurred from time to time, in Fig. 35 is a sort of summary. These curves express the total change between the beginning and end of a certain period of time and give the change in cost for all the sizes of apparatus selected as barometric units, three for the case in hand. Such curves are particularly instructive inasmuch as they show to what extent labor, material or overhead have affected the total result-the factory cost-and also show how a change, for instance in the cost of material, affects different units. All values 
are plotted as pereentage of inerease or deerease. It will be noted that under the conditions assumed as basis for these curves, due to increased efficiency in manufacturing methods an aetual decrease in flat labor costs was accomplished in spite of repeated increases in labor rates and that this reduction affected the smaller sizes to a greater extent than the larger ones. The overhead expense, therefore, although greater in percentage, showed no increase or only a slight on. As for material it will be noted that there was a very great advance in cost and that this item affected the smaller units to a greater extent than the larger

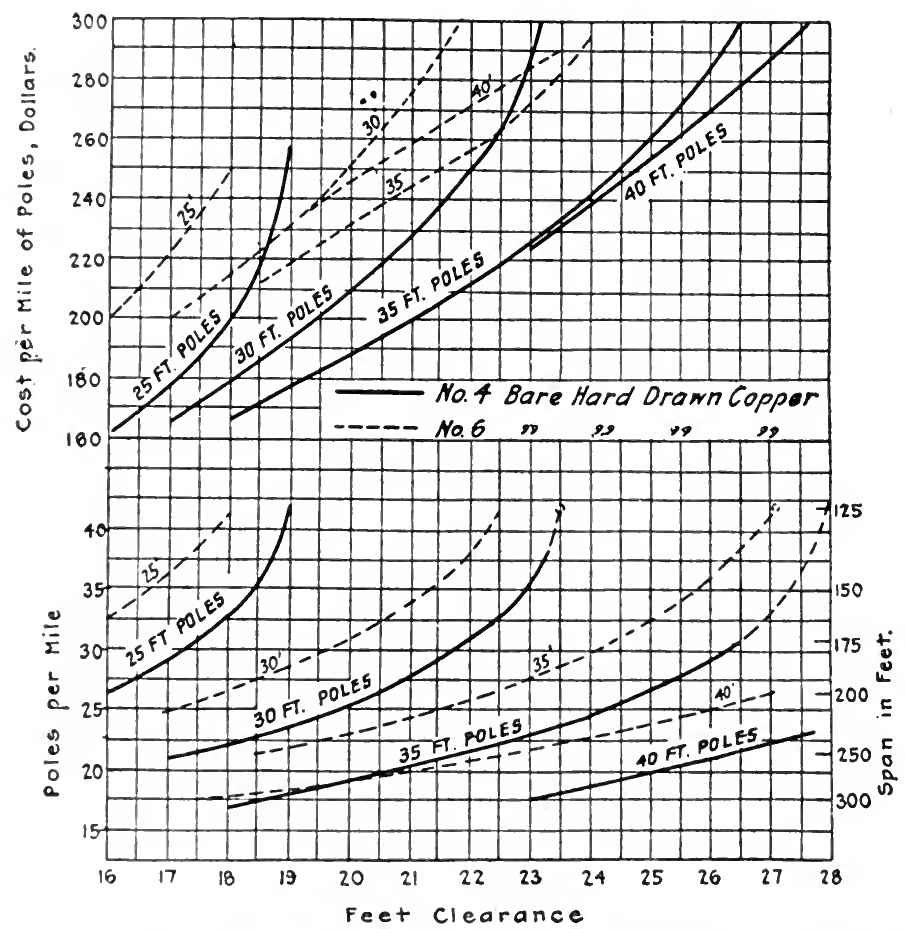

Fig. 36-Cost of Poles Per Mile with Two Sizes of Wire and Different Ground Clearances

sizes. This is readily explained by the fact that the larger units contain a greater bulk of the cruder materials. The curve for factory eost shows how the increase in material prices has to a certain extent been offset by the other factors mentioned, and shows how the percentage of rise in the factory eost is not uniform for all the sizes of a line, but that a lower factor should be applied to the smaller sizes. Intermediate sizes are rearlily interpolated, if the three sizes chosen are selected so as to fairly represent the lower, middle, and upper third of the entire range of sizes. For greater accuracy, or where variations in construction are to be considered, it is desirable to take more than three representative cases as barometer units.

Cost of Poles Per Mile. An excellent example of the value of charts. 
in cost analysis is given by the following from an article by L. P. Perry, "Iron-Wire Transmission for Rural Extensions," published October 13, 1917, in Electrical World.

The results of the study made by the Central Connecticut Power \& Light Company to ascertain the most economical pole spacings for ironwire line construction are given in Fig. 36. Selecting a given clearance of $18 \mathrm{ft}$. from the lowest point of the conductor to the ground, and using No. 4 bare conductors, it appears from the figure that 1735 -ft. poles are required per mile, whereas 2230 -ft. poles must be used in the same distance. From the upper set of curves it can be seen that the 35 -ft. poles cost $\$ 167$ per mile, while the $30-\mathrm{ft}$. poles would cost $\$ 178$, making the cost of poles for an $18-\mathrm{ft}$. clearance $6 \%$ cheaper with 35 -ft. poles than with $30-\mathrm{ft}$. poles.

With No. 6 bare conductors relatively larger sags would be required to keep the tension within safe limits. 2135 -ft. poles or 2730 -ft. poles would be required per mile with an 18 -ft. clearance from wire to ground. In this case the pole cost would be $\$ 208$ and $\$ 214$ per mile respectively, a difference of only $\$ 6$ per mile; thus it would hardly matter whether No. 6 bare conductors were supported on 35 -ft. or $30-\mathrm{ft}$. poles.

It was assumed when this study was first undertaken that the most inexpensive line would be that built with the shortest poles, but reference to the upper set of curves shows that for an 18 -ft. clearance the 25 -ft. pole line of 33 per mile costs considerably more than a 35 -ft. pole line with 17 per mile. At the then existing cost of materials it is, therefore, evident that it is no more expensive to use No. 4 conductors with long spans than to use a smaller conductor with shorter poles.

Analysis of Relation Between Electric Railway Investment and Traffic Density. D. J. McGrath in an article, "Investment per Revenue Passenger and Density of Traffic,' published in Electric Railway Journal, July 8, 1916, states:

During the past year, in connection with the investigation which is being made of the electric railway fare and traffic problem, the research division of the Massachusetts Institute of Technology has acquired a considerable amount of additional data along the lines of the actual investment in various street railway companies and has reduced these data to the revenue passenger unit.

As a result of this form of analysis it has been found that while there is a certain natural and proper variation in the costs of constructing and equipping different street railways, there appears to be, if the data available may be considered fairly representative, a reasonably consistent relation between the investment per revenue passenger and the density of traffic.

In considering the following discussion and statistics, it must be remembered that they are applicable only to ordinary street railways which are wholly, or almost wholly, engaged in passenger business at small unit fares such as 5 or 6 cents. The curves were derived from the data of thirty-five different street railways, more than half of which are in the State of Massachusetts, where the capitalization of the companies has always been limited to the actual investment of money in the property and has been under the supervision of a State commission. The remainder of the companies included in this study were ones for which 
reliable engineering appraisals were available in which the investment or the reproduction value had been determined. Systems serving various classes of territory were included, city suburban and rural lines all being treated alike, but the high-speed interurban type of electric railway, which corresponds more nearly to the steam railroad type of service, was excluded. No company was included which rented or leased any considerable part of the track or cars which it operated, unless the investment represented by such rented property could be determined and included. On the accompanying plots, the data are specially indicated for companies which purchase all or most of their power, and so have little or no investment in power plant. The figures are practically all from reports of the years 1914 or 1915.

The principal relation is shown in Fig. 37. The curve which has been drawn in is merely intended to guide the eye to the approximate average location of the points and not to represent any arbitrary standard. Granting that the data used are fairly typical of street railway conditions generally in this country, it may be concluded from this plot that the average investment per revenue passenger decreases as the density of traffic increases up to a certain point, after which higher traffic densities fail to cause any material decrease in the investment per passenger. This seems to indicate that in the larger cities, the traction companies have to put in so much more investment in heavier track, better cars, more power plants and the added track and equipment required for long hauls that any advantage which might be gained from increasing density of traffic is offset.

An example of this is shown by the two points which are considerably above the average curve, one near 400,000 and the other near 750,000 revenue passengers per mile of single main track. These points represent the investment in two very large cities where long hauls are given for the single fare and where there is a material amount of unusually expensive construction.

The points for companies which do not own their power plants are shown by the hollow circles as distinguished from the other solid dots. The former are generally somewhat lower on the curves than the others, as would naturally be expected.

It is plain that the investment per revenue passenger is subject to some considerable variation on different systems even at approximately the same densities of traffic, but this form of analysis seems to give somewhat more consistent results for comparative purposes than a mere seattering of investment data without reference to any of the limiting conditions.

Where the density of traffic is less than 100,000 revenue passengers per mile of single main track, the investment per revenue passenger becomes rapidly greater. In fact, these statisties indicate that operation is not so likely to be profitable at 5-ct. fares where the density of traffic is much less than 100,000 per mile of track.

When the investment per passenger gets to be 30 cts. or more, as it evidently does at these low densities, an average rate of return of, say, $7 \%$ on investment, recquires $7 / 100$ of 30 ets., or 2.1 cts. at least, out of each passengor's fare. Since a low density of traffic also means higher operating expenses per passenger, there is little likelihood of 2.1 cts. 
being available for return on investment after the nickel fare has been "split" for the other expenses. A 7\% average return is used here merely as an illustration; it is not intended to stand as an arbitrarily selected "fair" rate.

The general precept may be laid down that the more revenue passengers per mile of track, the better are the chances for profitable operation. However, a practical limit is reached along this line in the operation of street railways in very large cities where the length of passenger haul for the 5-cent fare becomes so great as materially to reduce the earnings per car-mile. Moreover, when any part of the trackage becomes so

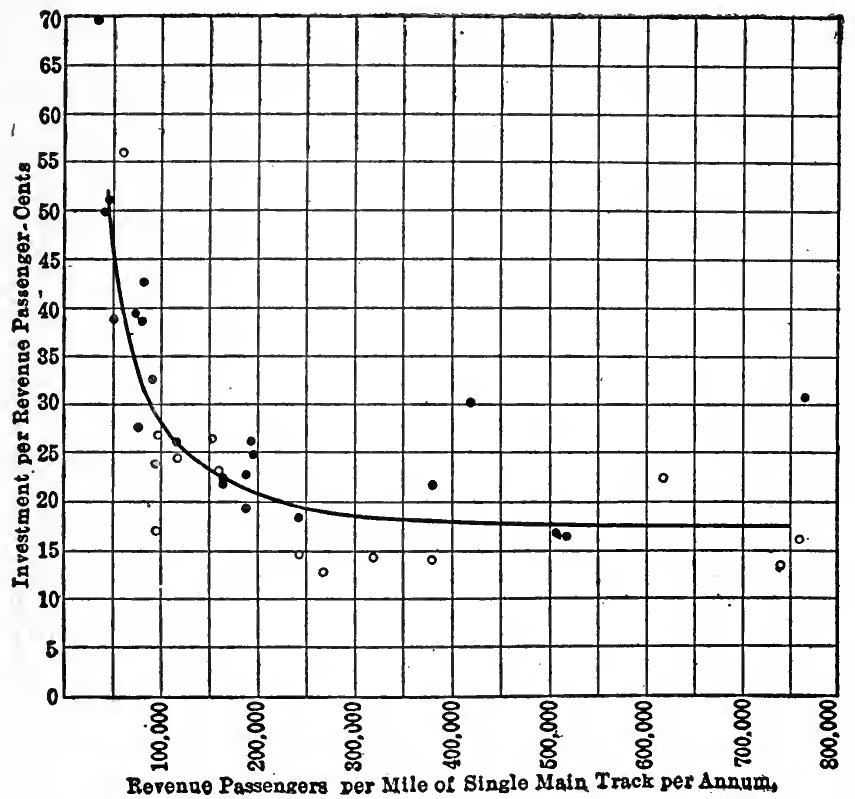

Fig. 37-Relation Between Total Annual Cost of Permanent Property Per Revenue Passenger Per Annum and Density of Traffic for Thirty-Five Typical Street Railways

congested that the company has to resort to the building of subway or elevated lines to handle the traffic. then the investment will be abnormally increased.

In Fig. 38 there is shown that part of the investment which is devoted to the construction of road and track, exclusive of power plant buildings, power equipment and rolling stock. The curve is similar in shape to the total investment curve shown in Fig. 37 but of course it is somewhat lower on the scale. All the companies included in making up Fig. 37 were not included in the construction of the following charts because the more specific data required were not available.

In Fig. 39 the other elements which go to make up the total investment are compared with density of traffic. These elements, however, seemed to be somewhat more closely related to the density of traffic 
expressed in terms of revenue passengers per car-mile, rather than per mile of track, although the same general form of relation naturally holds true in both eases.

The old unit, investment per mile of track, is also capable of analysis with respect to the density of traffic as is shown in the plot marked Fig. 40 which should prove generally useful when considering statistics in this form of comparison. The reasons for the increase in investment per mile of track as the density of traffic inereases are not difficult to explain. In the first place track built for dense traffic is generally, and should be, of heavier and more expensive construction. The electrie line for distributing the power along the track must be of greater current earrying capacity and consequently requires more copper. These railways with dense traffic are almost always in the large cities and towns where expensive paving is required which is not necessary in rural districts. Fully as important is the fact that the denser traffic

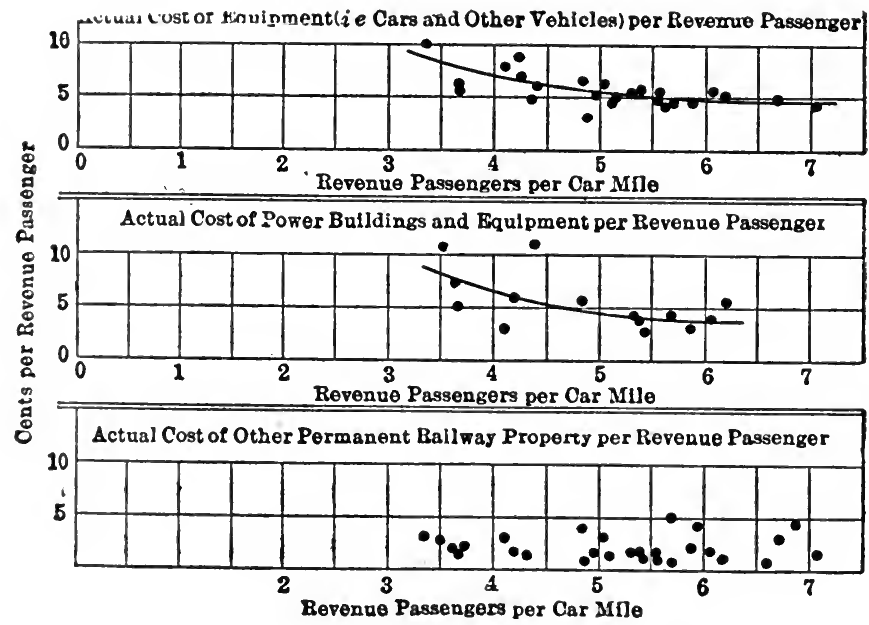

Fig. 39-Relations Between Actual Costs of Equipment, Etc., Per Revenue Passenger and Revenue Passengers Per Car Mile

requires more cars and power plant equipment for each mile of track operated.

It is not to be supposed that these general statistics can be used to make any definite or final deeision as to whether a given company's capitalization represents a fair investment value or not. Obviously there can be no absolute statistical standard for determining investment or fair value.

If in any given case the unit capitalization falls above the average curve it is by no means a proof of overcapitalization. All the conditions and local circumstances as well as the past history of the upbuilding of the system must be given due consideration. Indeed, the mere fact of the data falling exactly upon the average curve is not in itself a demonstration that a company is not over-capitalized for the actual amount, condition and kind of property it has on hand.

The statistics of any one particular year are not always a safe criterion 
to rely upon in these comparisons. A company may have been obliged to make a large investment in an extension or improvement, which investment is included in its capitalization for a certain year before the extension or improvement has been put into use. This would naturally raise the investment per revenue passenger somewhat above its normal level and it might take one or two years before the extension or addition began to receive its normal share of the traffic.

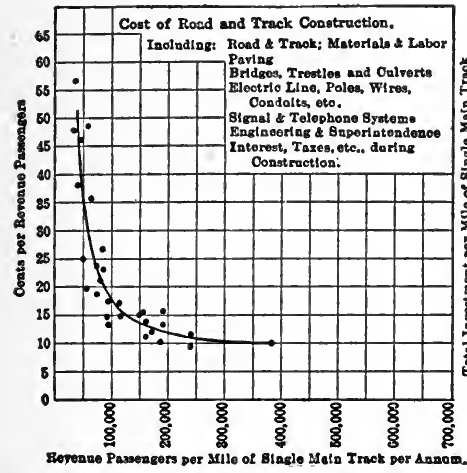

Fig. 38-Relation Between Cost of Road and Track Construction and Density of Traffic

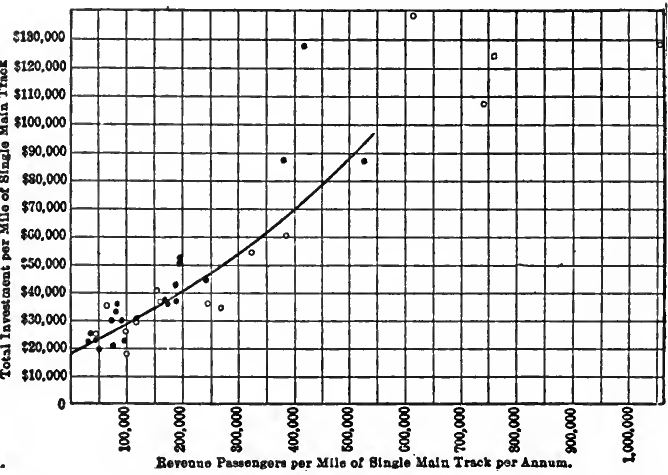

Fig. 40-Relation Between Total Investment Per Mile and Revenue Passengers Per Mile

In general, however, it would seem that these average curves should be of service in broad general considerations of the cost of street railway service and the determination of reasonable systems and rates of fare. They show clearly the general nature of the effect of density of traffic upon the investment in street railway property used for the transportation of passengers. These statisties may also prove useful in making rough comparisons of the capitalization of any particular street railway with others of a similar nature.

Cost of Combination Electric Service. From an article of this title published in Electrical World, May 8, 1915, is taken the following excellent example of the value of charts in an investigation of this kind.

One of the most flourishing communities in western Canada is the city of Calgary, Alberta. The city has at present two sources of electrical supply, one the Calgary Power Company's terminal station in East Calgary and the other its own steam plant at Victoria Park.

The municipality is bound by contract to take at least $5000 \mathrm{hp}$. from the Calgary Power Company for the next four years, and, in addition, the standby charges of the present steam station, which must be held in readiness in case of failure of the transmission system, must also be paid. There was therefore much speculation as to the most economical way of taking electricity from the two sources, the hydroelectric company having different summer and winter rates.

The accompanying curves and calculations are the result of an investigation made under the direction of City Electrician Robert Brown for determining with a fair degree of accuracy the cost of electrical energy to the city of Calgary up to the point of delivery to the distribu- 
tion system of the electric-light department under different conditions of load and methods of operation.

Fig. 44 is a typical load curve for a winter day, showing that there is quite a demand for energy between midnight and morning for the

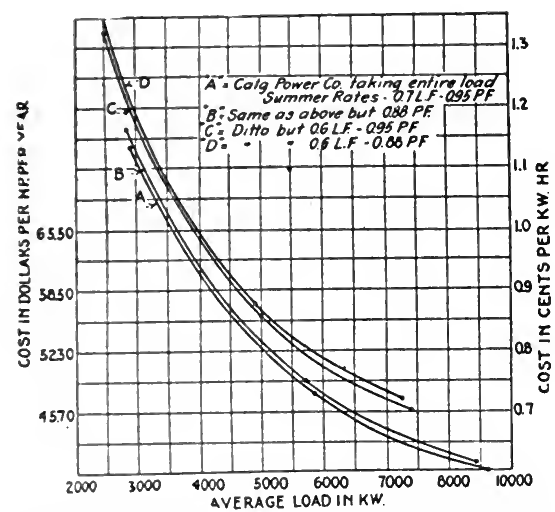

Fig. 41-Effect of Power-Factor and Load-Factor on Hydroelectric Rates

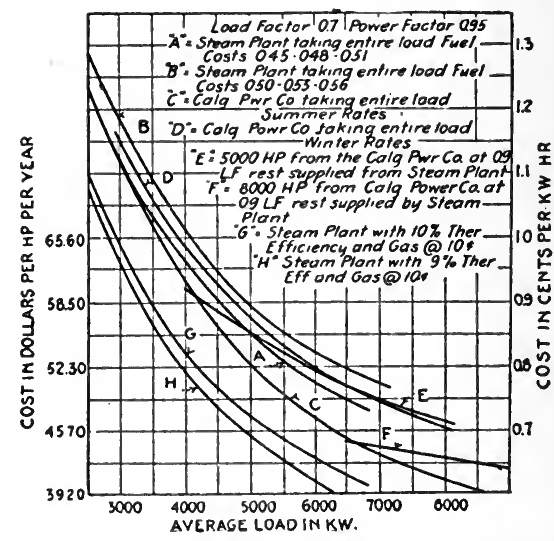

Fig. 43-Cost of Generating Electricity by Steam, Gas and Water-Power

following reasons: The street lighting demand is over $1000 \mathrm{kw}$. and is on an all-night schedule for the most of the year; water-pumping machinery in the eity is driven electrically and storage tanks are filled at off-peak hours; several large mills and factories operate all night,

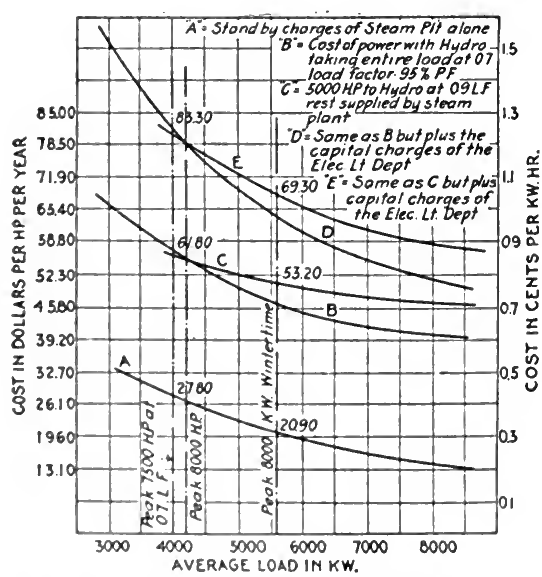

Fig. 42-Standlyy ('harges on Generating System

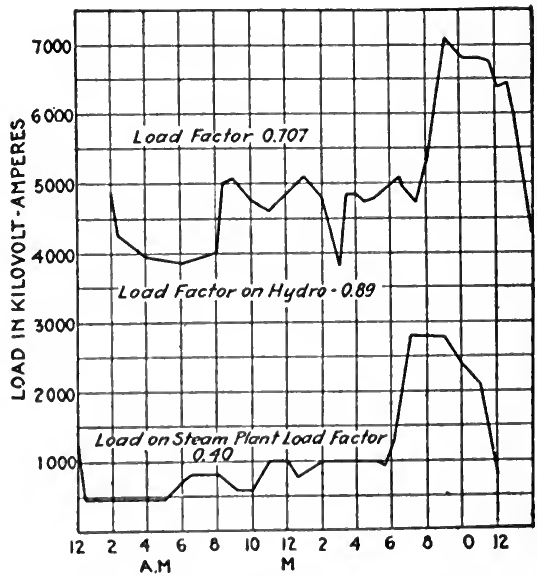

Fig. 44-Total Load Curve for Feb. 14, 1914

and the electric-vehicle-charging load is an all-night load and is beginning to become appreciable. The load-factor (ratio of average load to peak loarl) is therefore very high, being between 0.6 and 0.7 . The load-factor for the particular day the load curve of which is shown was 
0.707. That on the hydroelectric system was 0.89 , and that on the steam station was 0.40 . The yearly load-factor was probably a little less than this, and as the load increases from year to year there is no doubt that the load-factor will decrease.

The power-factor of the city's system is high, being between 0.9 and unity, because the street-railway system is supplied with direct current from synchronous motor-generator sets.

During the times in which the peak load of the Calgary system is reached there are three $1000-\mathrm{kw}$. sets and one $500-\mathrm{kw}$. set in operation.

By over exciting the synchronous motor the mattless kva. of the system can be entirely compensated for.

The contract with the Calgary Power Company will be enforced for the next four years. The following are some abstracts from it:

Rates :-winter rates, first $5000 \mathrm{hp}$. at $\$ 26$ per hp.-year; next $1000 \mathrm{hp}$. at $\$ 25$; next $1000 \mathrm{hp}$. at $\$ 24$; next $1000 \mathrm{hp}$. at $\$ 23$; next $1000 \mathrm{hp}$. at $\$ 22$; next $1000 \mathrm{hp}$. at $\$ 21$; all over $10,000 \mathrm{hp}$. at $\$ 20$.

Summer Rates (April-October)-First $5000 \mathrm{hp}$. at $\$ 26$ per hp.-year; all over this, $\$ 15$ per hp.-year.

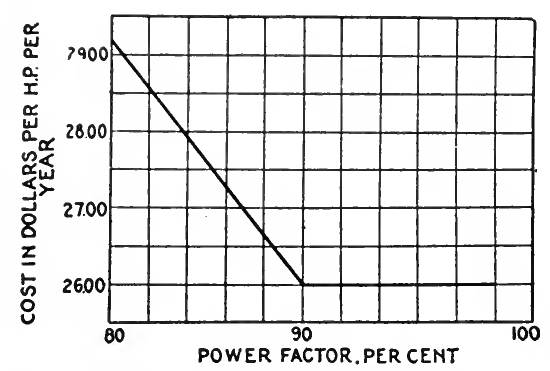

Fig. 45-Effect of Power-Factor on Hydroelectric Rates

The basis of charge per month is on the maximum thirty-minute peak during the month. In the Calgary Power Company's terminal station there is a curve-drawing ammeter connected to one phase of the feeder to the eity's lines. The power supplied is figured as the voltage multiplied by the maximum thirty-minute reading of the ammeter multiplied by the square root of three multiplied by the power-factor at this particular load. As long as the power-factor does not go below $90 \%$, the actual power-factor is used in figuring the power supplied. When the power-factor does drop below $90 \%$ the factor 0.9 is used, even though the power-factor be only $80 \%$. Fig. 45 shows how the rates vary with the power-factor of the city's lines. The minimum load to be paid for is $5000 \mathrm{hp}$, and $8000 \mathrm{hp}$ is the maximum load that the power company will supply.

At present the municipal steam plant contains the following: one 6250-kva steam generator turbo set, one 2800-kva steam generator turbo set, one 3125 -kva steam generator turbo set, one $600-\mathrm{kw}$ steam-driven street-railway generator, one 500-kva vertical steam driven alternator.

Half of the boiler equipment is fired by natural gas (1100 lb.-Fahr. heat units) at $15 \mathrm{cts}$. per $1000 \mathrm{cu}$. ft., and the other half by coal. In 
making up the curves actual figures were obtained from the power department as to labor, maintenance and the cost of supplies for three winter months, and for the month of April when the water-power company took the entire load and the steam plant was kept in readiness.

The fuel costs for these months were also calculated accurately. Some time ago an ideal full-load test was made on the 3125-kva steam turbo set, and the fuel cost of 0.47 ct. per kw-hr. was obtained. This shows what the plant can do under favorable conditions.

The possible methods of operating now are as follows:

(1) Allowing the Calgary Power Company to take the entire load and keeping the steam plant in readiness. This method is possible only in summer, as the water-power is not sufficient to supply more than the minimum contract load in winter.

(2) Allowing the steam plant to carry the entire load. If there were no contract with the hydroelectric company, this method would be possible.

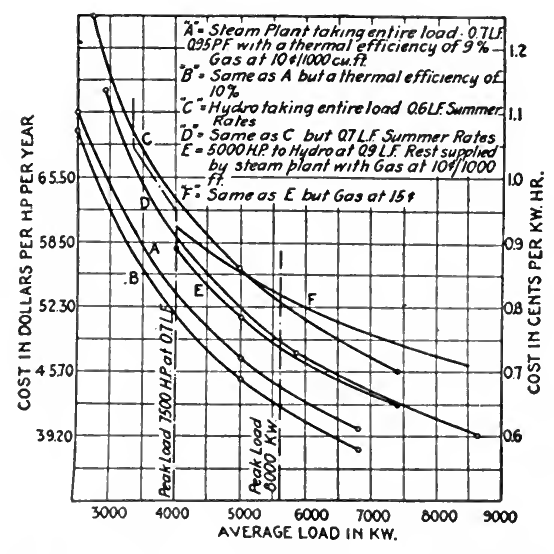

Fig. 46-Cost of Generating Electricity by Steam and Water-Power

(3) Allowing the Water Power Company to supply the minimum contract amount of power of $5000 \mathrm{hp}$., the rest being supplied by the steam plant. This is possible in summer and necessary in winter.

(4) Allowing the Water Power Company to carry the maximum contract amount of power $(8000 \mathrm{hp}$.) and supply the rest by steam plant. This is the method which will have to be used when the summer power demand exceeds 8000 hp., unless the Calgary Power Company raises its maximum.

The coal has a heat value of about $12,500 \mathrm{lb}$. Fahr. heat units per lb. and rosts about $\$ 3.75$ per ton of $2000 \mathrm{lb}$. With gas at 15 ets. per 1000 cu. ft. the enal is equal to about $\$ 3.40$ worth of gas.

In the winter the Calgary Power Company carries $5000 \mathrm{hp}$. and the rest is supplied by the steam plant. At times of low load the total power demand drops below $5000 \mathrm{hp}$. It is hard to keep the Calgary Power Company s load at exactly $5000 \mathrm{hp}$. so that the loarl-factor on the supply from the Calgary Power Company is about 0.9. The load on the Calgary 
Power Company is regulated by adjusting the governor on the turbines in the steam plant. During the summer the Calgary Power Company is allowed to take the entire load and the boilers in the steam plant are kept in readiness. The cost of labor, maintenance and supplies is, of course, reduced somewhat under these conditions.

The cost of energy from the steam plant is made up of several itemscapital charges, fuel costs, maintenance, supplies and labor. In making up the curves the capital charges, maintenance, supplies and labor were averaged from the actual figures from the power department. Fuel costs have to be assumed more or less, as they cannot be determined so accurately as the other costs. In the case where the Calgary Power Company is allowed to take the entire load the cost of energy is made up of charges to the water-power company and the standby charges of the steam plant. In figuring capital charges the capitalization of the power department was reduced by $\$ 70,000$ because it included the cost of some street-railway motor-generator sets, which are correctly classed as part of the distribution system.

A consulting engineer who was called in to report on power estimated the fuel costs to be 0.45 ct. for a $10,000-\mathrm{kw}$ gas-fired steam plant, working on $30 \%$ load-factor with gas at 15 cents per $1000 \mathrm{cu}$. ft. and 1100 lb.-Fahr. heat units per cu. ft. In an ideal test of the 3125-kva turbogenerator at full load, 0.455 ct. per kw-hr. and 0.47 et. per $\mathrm{kw}-\mathrm{hr}$. were obtained as fuel costs. A fuel cost of 0.45 ct. per $\mathrm{kw}$-hr. gives a thermal efficiency of $10 \%$ with gas at 15 cts. and $1100 \mathrm{lb}$-Fahr. heat units per cu. ft.

Fig. 48 shows thermal efficiencies for different prime movers. The efficiency of the boiler (about $75 \%$ ) and auxiliaries, such as exciters, vacuum pumps, hotwell pumps, etc., must also be taken into account. It can be seen from the above data that $10 \%$ thermal efficiency is not too high an estimate.

When the plant is operating at small load, however, the fuel cost will naturally be higher, so in making curves a fuel cost of 0.45 ct. at an average load of $7500 \mathrm{kw}$., 0.48 ct. at an average load of $5000 \mathrm{kw}$. and $0.51 \mathrm{ct}$. at an average load of $2500 \mathrm{kw}$. were used for the case when the load-factor equals 0.7 and the power-factor 0.95 . At 0.6 load-factor, costs of 0.46 ct., 0.49 ct. and 0.52 ct. were used. Some curves were drawn up assuming $9 \%$ thermal efficiency, with fuel costs of 0.50 ct., 0.53 ct. and 0.56 ct. These last costs represent the maximum, because in the figures supplied by the power department fuel costs of 0.53 ct. and 0.56 ct. have already been obtained with average loads of $1700 \mathrm{kw}$ and $40 \%$ load-factor. With gas at 10 ets. fuel costs of 30 ets., 32 ets. and 34 ets. were used.

Fig. 45 shows how the water-power company's rates increase with decrease in power-factor. Fig. 43 shows how the total costs vary with power-factor and load-factor.

As to the accuracy of the curves and calculations certain assumptions of course had to be made. The cost of energy is made up of the Calgary Power Company's power charge and the steam-plant charges, which consist of capital charges, labor, maintenance and supplies. The Calgary Power Company's charge amounted to $\$ 303,250$ in this case. The capital charges of the steam plant were taken from the auditors' figures and 
amounted to $\$ 98,000$ of $\$ 153,200$ assumed for the steam-plant charges; $\$ 33,000$ was for labor, and these charges were taken from actual figures supplied by the power department. The remaining $\$ 30,000$ is for maintenance, supplies and office charges. These charges were estimated from the power-department figures.

The costs with the steam plant taking the entire load are made up of the fuel costs plus the above steam-plant charges. Fuel costs cannot be estimated exactly, so that the maximum and minimum charges for fuel were assumed and curves drawn for the two figures.

The justification for the different fuel costs was given above. The variation in maximum and minimum fuel costs is not over $8 \%$ of the total cost of power. The minimum fuel costs assumed have already been proved by tests on the plant under favorable conditions, so that the curves using the minimum fuel costs are not in error more than $3 \%$ or $4 \%$.

The eurves show the necessity of keeping the power-factor and loadfactor high.

In the ease of the power-factor, as long as the street-railway load keeps pace with the total load there will always be enough synchronous motor load to give a leading current to compensate for the wattless kva of the system. If it does not keep pace, it might be possible during peak-load hours in the summer to run one of the turbo sets without

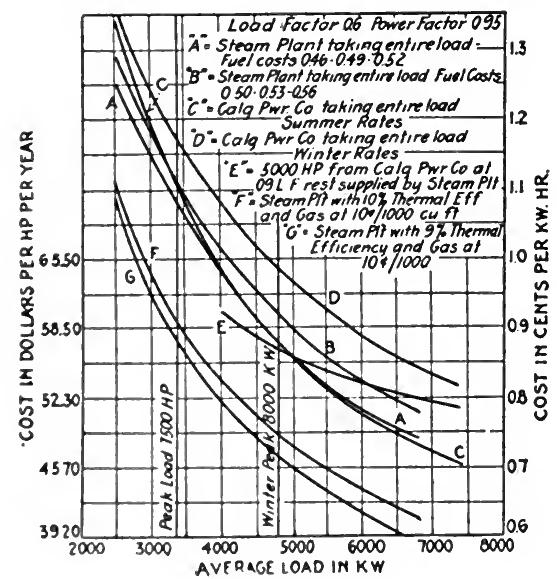

Fig. 47

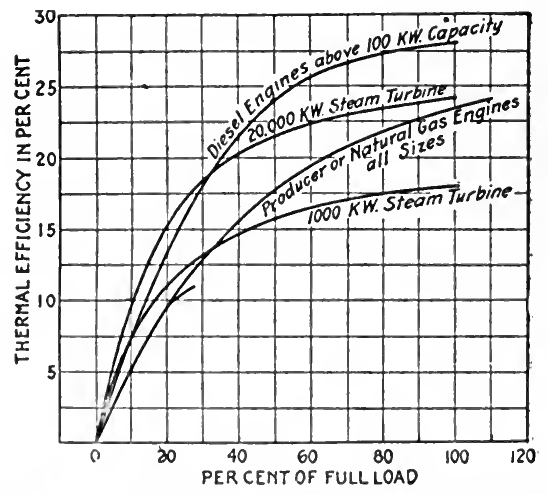

Fig. 48-Thermal Efficiency of Prime Novers not Including Auxiliaries

steam turned on and overexcite the generator, or the eonnecting rods. on the $7(0)$-kva. vertical-steam-engine-driven alternator might be takcn off and the alternator operated as a synchronous condenser.

Fig. 41 shows that in summer it is most economical to let the Calgary Power Company take the entire load if gas costs 15 cts. With gas at 10 cts. it wonli be cheaper to run the steam plant alone. This would be possible if the hydroelectrie contract did not exist. In winter the curve shows the economy of allowing the hydroelectric system to take $5000 \mathrm{hp}$. at 0.9 load-factor and supply the rest of the energy from the steam plant. 'This is the only method that ean be used on account of the hydroelectric eontract. 
Fig. 47 shows the cost of energy from the steam plant with 10-ct. gas. This shows that in the summer 10-ct. gas would not improve matters much as long as the load-factor is high.

Fig. 46 shows that at the usual summer peak loads of $7500 \mathrm{hp}$. the cost of the steam plant forms nearly half the cost of energy and the total cost of energy up to the point of delivery to the distribution system of the electric-light department is about 0.94 ct. per $\mathrm{kw}-\mathrm{hr}$., or $\$ 61$ per hp. per annum. Adding the capital charges of the electric-light department brings the total cost up to nearly 1.3 ets. per kw-hr., or $\$ 83.30$ per $\mathrm{hp}$. per annum.

Coal Cost and Selling Price Analysis. J. D. Skinner, in an article entitled "Graphic Determination of Prices," published in Coal Age, May 25, 1918, describes in detail the method he uses, thus making it valuable for similar analysis of other materials. The following is quoted:

When varying prices are received for run-of-mine, lump and slack coal and certain percentages of the two latter are made, it is somewhat difficult to determine quickly whether it will be advantageous to sell the mine output with or without preparation. And in whatever condition the coal is disposed of, it is advisable to know both "why" and "how much."

To provide a means and basis for quickly comparing the prices of these three grades of coal and determining which will yield the greatest return, I have constructed the accompanying charts. These are based on the following simple calculations:

Let

$P=$ Price of mine-run per ton;

$x=$ Corresponding price of lump per ton;

$y=$ Corresponding price of slack per ton;

$A=$ Percentage of lump made;

$B=$ Percentage of slack made.

Hence $A+B=100$.

Problem-Given $P, A$ and $B$ to find $x$ and $y$.

In one ton of mine-run there is an amount of lump represented by the decimal fraction $A$ and an amount of slack represented by the decimal fraction $B$. Hence, when the portion $A$ of a ton of lump is sold at $x$ dollars per ton, and the portion $B$ of a ton of slack is sold at $y$ dollars per ton, the total receipts will be $P$ dollars; and we have the relation

$$
A x+B y=P
$$

Since equation (1) is of the first degree, its graph is a straight line. Letting $x=o$, the intercept on the $\mathrm{Y}$-axis is $O b=\frac{P}{B}$

Letting $y=o$, the intercept on the $\mathrm{X}$-axis is $O a=\frac{P}{A}$

Hence, for given values of $A, B$ and $P$, the graph of (1) can be drawn by connecting the intercepts. If $A$ and $B$ are supposed to be constant. 
and $x$ and $y$ have different values, a series of graphs may be constructed for different values of $P$. That these lines will all be parallel may be shown as follows:

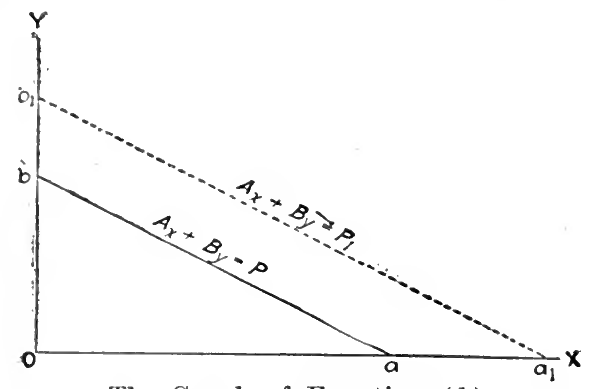

The Graph of Equation (1)

Transposing (1) and dividing both terms by $B$ to put it in the slopeintercept form:

$$
y=-\frac{A x}{B}+\frac{P}{B}
$$

$A$

Hence, the slope of (1) or $M=-\frac{A}{B}$

Now the equation of any other line having a different value of $P$, say $P_{1}$, is

$$
y=-\frac{A x}{B}+\frac{P_{1}}{B}
$$

Since the slope of $(2)$ is likewise $-\frac{A}{B},(1)$ and (2) make the same angle with the axis of $\mathrm{X}$ and hence are parallel.

The following illustrates the method of finding the intercepts:

$$
\begin{gathered}
\text { Sereen percentages }\left\{\begin{array}{l}
\text { Iump } 70 \% \\
\text { Slack } 30 \%
\end{array}\right. \\
\text { Hence } A=0.70 \text { and } B=0.30 \\
\text { Intercerpt } \\
\text { on } X-A x i s
\end{gathered}
$$

Price of

Mine-Run

$P$

$\$ 1.00$

1.15

1.30

Etc.

$$
\begin{aligned}
& \mathrm{Oa}=\frac{P}{A} \\
& \$ 1.43 \\
& 1.64 \\
& 1.86 \\
& \text { litc. }
\end{aligned}
$$

$$
\begin{gathered}
\text { Intercept } \\
\text { on } \mathbf{Y} \text {-Axis } \\
\mathrm{Ob}=\frac{\mathbf{P}}{\mathrm{B}} \\
\$ 3.33 \\
3.63 \\
4.33 \\
\text { Etc. }
\end{gathered}
$$

Erample 1. Given eost of mine-run fo.b. railroad ears at mine (including cost of sereening lump and slack $=\$ 2.30$ per ton. Profit $15 \mathrm{c}$ per ton. Selling jrice of lump f.o.b. mine $\$ 3.20$ per ton. Sereen product: $70 \%$ lump; $30 \%$ slack.

Find corresponding price, f.o.b. mine, that slack must be sold for in order that the combined profit on lump and slack will be 15 ets. per ton on a mine-run basis. 
Solution-On Fig. 49 for $70 \%$ lump and 30\% slack follow along the diagonal line marked $\$ 2.45$ (the selling price of mine-run) until it intersects the vertical line through $\$ 3.20$ (the selling price of lump to the scale marked on the axis $O Y$.) The distance this point of intersection is above $O X$, measured along the line through $\$ 3.20$ and perpendicular to $O X$, is the corresponding price of slack, which, by the scale on the axis $O Y$, is $\$ 0.70$. If the price of slack had been given instead, the corresponding price of lump could be found in a similar manner.

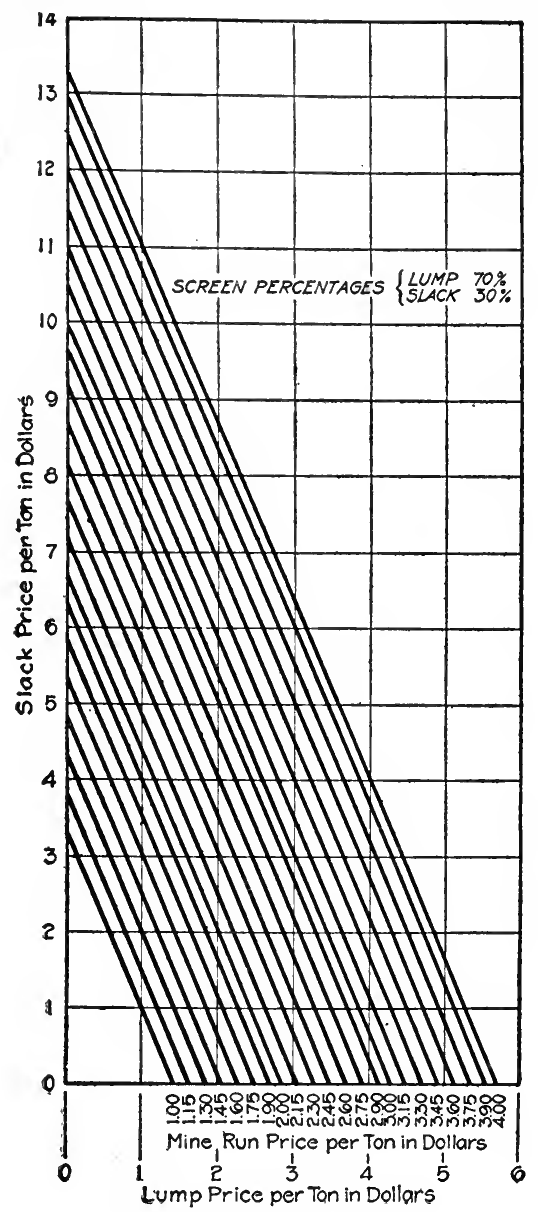

Fig. $49-70 \%$ Lump

Example 2. Given selling price of lump $=\$ 4.10$ f.o.b. mine. Selling price of slack $=\$ 1.20$ f.o.b. mine. Cost of mine-run (including screening, etc. $)=\$ 2.90$ f.o.b. mine. Sereen product $65 \%$ lump; $35 \%$ slack.

Find profit per ton of mine-run sold as lump and slack.

Solution-On a similar diagram for $65 \%$ lump and $35 \%$ slack find the 
intersection of the line perpendicular to $O X$ at $\$ 4.10$ with the line perpendicular to $O Y$ at $\$ 1.20$. This point of intersection lies a little over halfway from the diagonal marked $\$ 3$ to that marked $\$ 3.15$, which gives. $\$ 3.08$ for the corresponding selling price of mine-run. Hence the profit. per ton mine-run at the mine is $\$ 3.08-\$ 2.90=\$ 0.18$.

Example 3. Given selling price of mine-run, including operator's profit $=\$ 2.90$ per ton f.o.b. mine. No market for slack, which therefore has to be wasted. Screen product: $50 \%$ lump; $50 \%$ slack.

Find corresponding price, f.o.b. mine, at which lump must be sold to obtain the given price for mine-run.

Solution-On a diagram for $50 \%$ lump and $50 \%$ slack follow along. the diagonal marked $\$ 2.90$ until it interseets the axis $O . X$ (on which the selling price of slack is zero). This point of intersection is at $\$ 5.80$, which is the required price of lump.

Mine-run prices between those given may be seeured by laying a transparent ruler between and parallel to the proper diagonal lines and reading its intersections with the vertical and horizontal lines.

These diagrams also ean be used to compute costs as well as selling prices.

Analysis of Motor-Truck Transportation. The following is from an article by Francis W. Davis, "Truck Efficiency Graphically Shown," published in Automotive Industries, January 3, 1918.

The problem of analyzing motor-truck transportation is fundamentally different from other modes of transportation in that we have to do with a factor of far-reaching importance in determining the capacity or ability of motor trucks to perform a given amount of work. This factor is the loading and unloading time.

At first sight, it appears to be of little importance, as when considering horse team haulage very little attention is given to it. This is (!uite natural when we consider the fact that a team of horses can only cover a relatively small mileage in a day, and their average speed is of sufficient magnitude to cover this limiting daily mileage in a few hours, eonsequently the necessity for quick loading and unloading can be almost disregarded when dealing with team haulage.

With a motor truck we are face to face with entirely changed conditions. The eapacity of the truck is only limited by the number of hours it is in operation, and by operation we mean actual running time and not merely the time the truck is in eommission.

It is true that the faets as outlined above are instinctively understood by truck operators, and more and more time and effort is put into devising ways and means for reducing the so-called loading and unloading time of motor trucks. We hear of overhead hoppers, demountable borlic's, loarling eranes, winches, mechanical dump bodies, and innumerable other devices for saving time. This is all done for a very definite purpose, and it is strange that up till very recently little effort has been given to the important study of the absolute necessity for reducing this loading and nnloarling time and showing the relative amount of work that can be produced where efficient loading and unloading appliances are comsidered as compared with old out-of-date horse methods.

In approaching a problem to determine whether motor trucks can be used to advantage in a given transportation problem or to study 
means for increasing the efficiency of a motor-truck installation, it is customary to carefully investigate the loading and unloading conditions, nature, and distance of routes traveled, and other facts of more or less importance in order to draw up a report covering the capacity of a. given truck under certain conditions.

In addition to this, it is also customary to investigate the problem from a cost standpoint to determine the fixed charges per day and so-called operating expense per day, the latter usually worked down to a cost-per-mile basis.

Experience indicates that while a truck is in motion it averages approximately $75 \%$ of the full governed speed. The loss of $25 \%$ is occasioned through traffic dclays, varied road conditions, and other factors which are quite beyond control. With the 5-ton truck governed to 14 m.p.h., the $75 \%$ figure amounts to an average rate of $10 \frac{1 \%}{2}$ m.p.h.; therefore, while in operation we say the 5 -ton truck will cover a mile in 5.7 minutes' running time.

The following symbols will be of assistance in following this analysis :

D-Distance per round trip in miles.

L-Loading plus unloading time per trip in minutes.

$\mathrm{R}$-Running time per round trip in minutes.

$\mathrm{T}$-Total time per round trip in minutes.

M-Maximum speed in miles per hour.

$75 \%$ of $\mathrm{M}=$ running speed.

$$
\begin{aligned}
& \mathrm{T}=\mathrm{L}+\mathrm{R} \\
& \mathrm{R}=\frac{60 \mathrm{D}}{0.75 \mathrm{M}}
\end{aligned}
$$

$$
\begin{aligned}
& \mathrm{T}=\left(\mathrm{L}+\frac{60 \mathrm{D}}{0.75 \mathrm{M}}\right) \\
& \mathrm{M}=14 \text { m.p.h. (for Pierce-Arrow 5-ton truck) } \\
& \mathrm{T}=(\mathrm{L}+5.7 \mathrm{D}) \\
& \text { 10-hr. working day }=600 \text { minutes. }
\end{aligned}
$$$$
\text { Trips per day }=\frac{600}{\mathrm{~T}}=\left(\frac{600}{\mathrm{~L}+5.7 \mathrm{D}}\right)
$$

Truck carries out 5 tons; returns empty

$$
\begin{aligned}
& \text { Tons per day }=5 \times(\text { trips per day })=\left(\frac{3000}{\mathrm{~L}+5.7 \mathrm{D}}\right) \\
& \text { Miles per day }=\mathrm{D} \times(\text { trips per day })=\left(\frac{600 \mathrm{D}}{\mathrm{L}+5.7 \mathrm{D}}\right) \\
& \text { Ratio running time to total time }=\frac{\mathrm{R}}{\mathrm{T}}=\left(\frac{\mathrm{T}-\mathrm{L}}{\mathrm{T}}\right)
\end{aligned}
$$

The above method of figuring gives a rapid and accurate method of determining the capacity of a motor truck under any given conditions. However, very often in discussing the usage of motor trucks with a prospective owner the item of loading and unloading time is not only 
difficult to estimate but often is subject to considerable revision through the consideration of some simple auxiliary equipment. The prospective owner is usually quick to raise objections against this, due to the cost of the equipment. Then the figures showing the capacity of the truck must be corrected to show its increased capacity due to the addition of this extra equipment.

This process can be repeated a number of times, and if done rapidly and accurately will give the truck owner a good idea of the increased eapacity of his equipment due to quick loading and unloading.

With a view to showing this graphically, and in such a way as to admit of instantaneous comparisons, the accompanying charts, Figs. with the above facts incorporated, obviate the necessity for actually putting down figures, and permit of comparisons, one truck with another, under varying conditions. The charts also answer in a remarkable manner the old-time fallacy that motor trucks cannot compete with teams in short" hauls. The short haul is the "bonanza" of motor-truck haulage where the conditions are right.

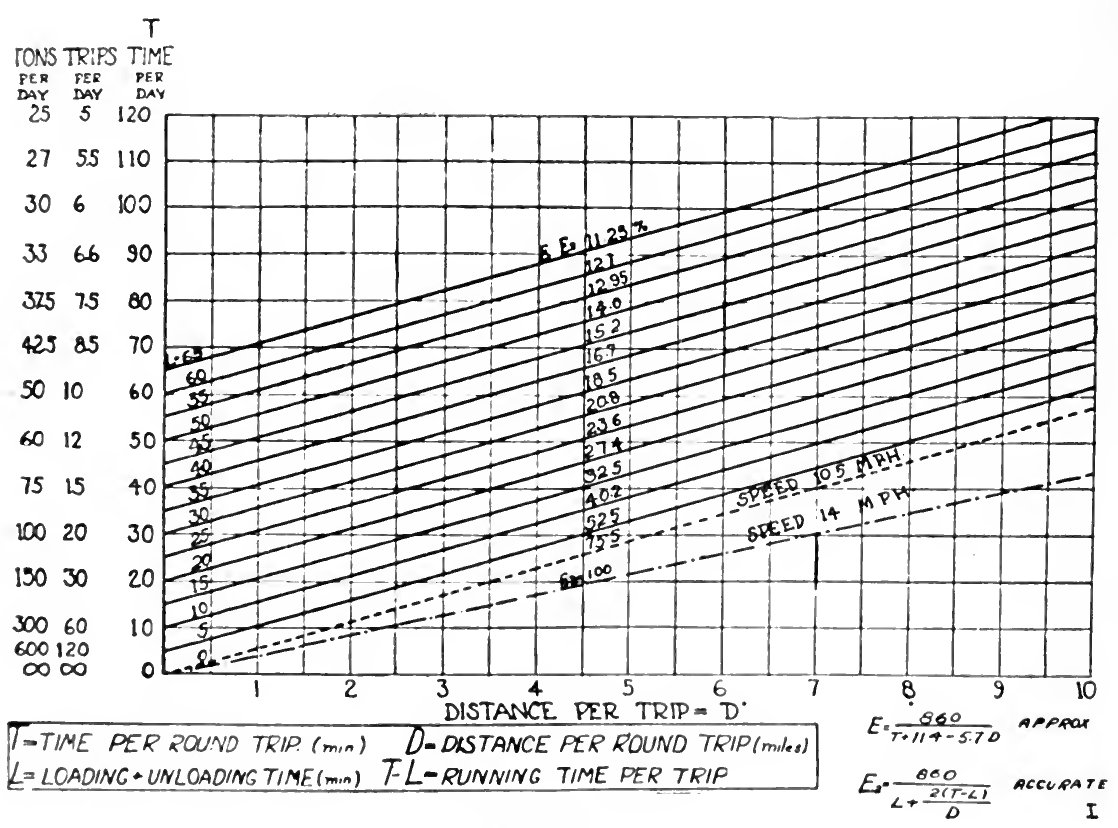

Fig. 50

Fig. 50 is drawn up for the Pierce-Arrow 5-ton truck, on the assumed ruming speed of 10.5 m.p.h. The distance per round trip $D$ is shown on the hare line up to 10 miles. The time per round trip $T$ is shown on the vertical line at the left of the chart, and runs up to 120 minutes. The loading and unloading times are indicated by $L$, and vary from zero up to 65 minutes per trip. Two other vertieal seales are ineluded, namely, the trips per day and tons per day, the tons per day bearing the relation of five times the number of trips per day, inasmuch as we 
are concerned with a 5-ton truck and assume a load of 5 tons carried out.

The chart is read by:

1. Running out the base line to the distance per round trip.

2. Then vertically to the intersection with the $L$ line.

3. Then follow horizontally to the left to the intersection with the three vertical scales, giving the time per round trip, trips per day; and the tons per day.

By using the same distance per round trip, and dropping down to a lower $L$ figure, we arrive at the time per round trip, trips per day, and tons per day with the new loading and unloading condition. This can be done rapidly, and gives surprising results when dealing with reasonably short hauls and small values for $L$.

The other figures of $E$ and $E 2$ represent efficiency figures which the writer has worked out at considerable length, starting from quite different grounds of reasoning, and yet the lines representing values of $L$ are constant efficiency lines, and the formulæ $E$ and $E 2$ give equal values for truck efficiency on these lines.

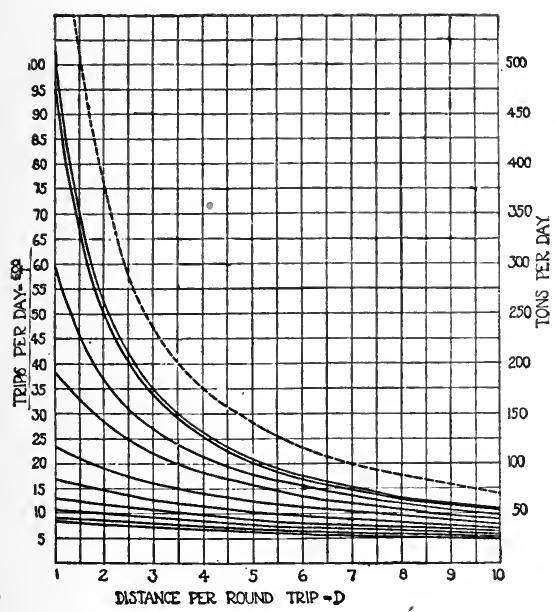

Fig. 51

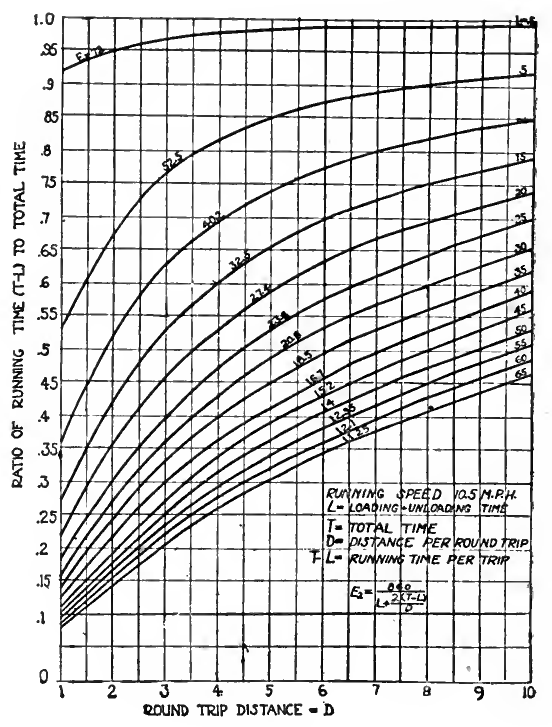

Fig. 52

Fig. 51 uses the same base line as 50, with altered scale of trips per day and tons per day, so as to show the enormous increase in capacity of the truck when operating on short hauls under efficient conditions of loading and unloading, and it furnishes a graphical proof of the often repeated assertion that quick loading and unloading time is much more essential in short hauls than in long hauls.

This statement is true, and with this chart it is brought out to an astonishing degree. For instance, it shows that in a round-trip haul of one mile, with loading and unloading time $L$ of 60 minutes, the 5 -ton 
truek ean make approximately nine trips per day, carrying a total of 45 tons. When redueing $L$ to five minutes per round trip, the truek will make approximately sixty trips per day earrying 300 tons. This represents an inerease in eapaeity of over $600 \%$. Further, if we consider this same haul with a loading and unloading time of $1 / 2$ minute, which has been reached in eertain installations, the truek has a capacity of 96 trips per day of 480 tons. This represents an inerease over the first condition of approximately $1000 \%$. These are the facts that must be brought home to the truck user in order for him to get the full benefit of his esmipment.

How often do we hear that a truek is on the road only one-fifth of the day? This is the eondition under which a great many trucks operate, and also a great many do not equal this figure. Fig. 52 gives graphically a picture which shows that when a truck operates under this condition the owner should be indicted for eriminal misuse of his equip-

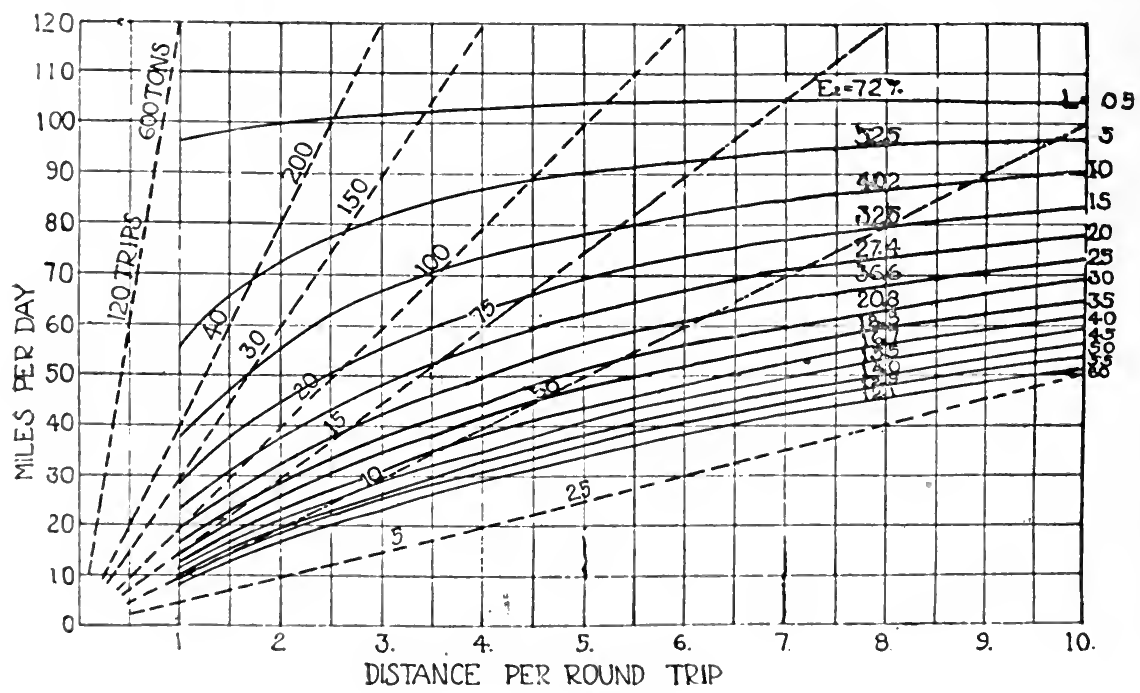

Fig. 53

ment. On Fig. 52 the point two-tenths of the way up on the left-hand seale-i.e., representing the eondition of one-fifth running time-shows that the operator in this ease is operating his truek only up to this level. Alove that is the region which he knows nothing about, and in which he denies the truck the opportunity of working. The limiting capacity of the truck, of course, is that it may be on the road the entire time. Figures of $\mathrm{F} 2$, given both on Figs. 51 and 52, represent constant efficiency lines the same as on Fig. 50.

There is another figure of importance, namely, the number of miles per day the truck will eover. Fig. 53 is drawn up with the same base line as the other charts, and indieates the miles per day as related to the loading and muloading time, and also the relation of the trips per day and tons carrierl, as related to the miles per day. This ehart again shows that when we reduce the loading and unloading time we increase 
the capacity of the truck, and on short hauls this increase goes up to an amazing figure.

Fig. 54 is a master chart of the others shown before, and includes all of the information contained on the foresoing charts. In addition, the base line is extended up to 22 miles and the time per round trip extended up to 300 minutes. The vertical scale for the total time per trip is marked T. A ratio table will be noted at the left end of the chart. This is useful where making comparisons so as to show the ratio of increase in the number of trips per day and tonnage hauled under

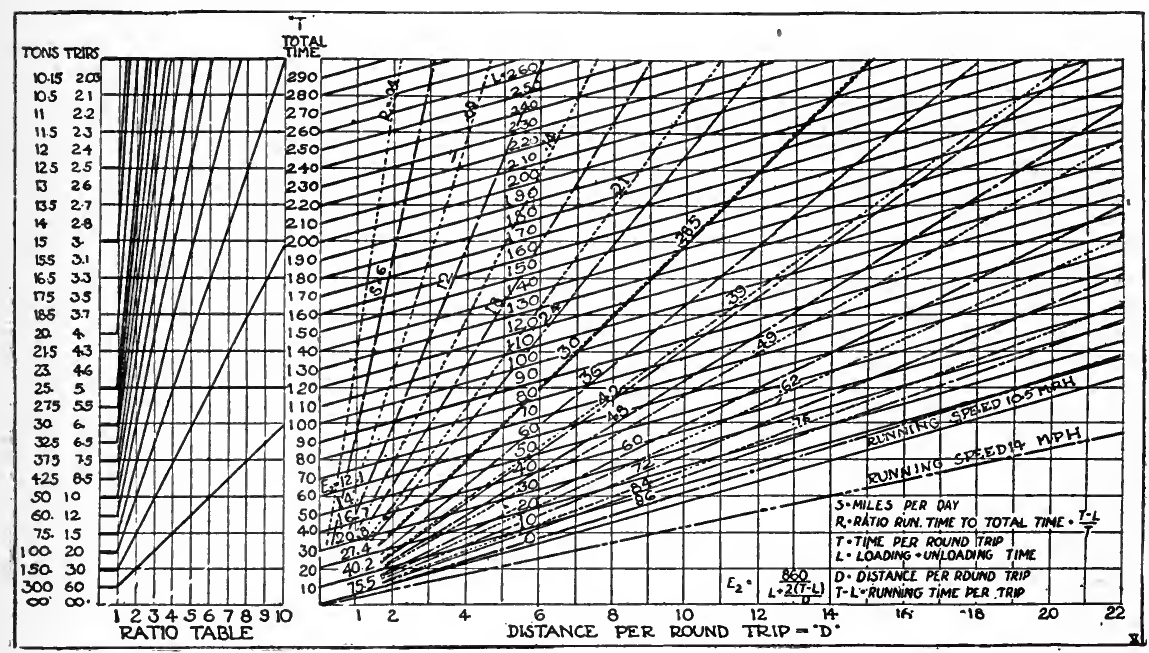

Fig. 54-Master Chart Showing the Relation Between All Factors Involved in Motor Truck Operation

conditions where quick loading and unloading are considered, as against one where slow loading and unloading are considered.

The procedure is to locate the number of trips per day on the vertical scale corresponding to the slow-loading figure, and, by tracing out to the diagonal line, reaching down to the greater number of trips, the vertical line from that point to the ratio table indicates the ratio of increase. This is somewhat difficult to explain in wording, yet a short study of the chart will show how easily and quickly it can be done.

The foregoing charts should prove not only of great value in analyzing new transportation problems, but should place in the hands of traffic managers means whereby trucks can be distributed for a given day's work so as to utilize the transportation equipment to the best possible advantage.

Cost of Plowing. The following is quoted from an article in Automotive Industries, by E. Goldberger, published December 6, 1917.

We have on the market practically any size of tractor ranging from $10 \mathrm{up}$ to $120 \mathrm{hp}$., and from their performance and prices and being acquainted with the requirement on the farm and the manufacturing problems, i.e., all the premises being known facts, it is possible not only to draw correct conclusions referring to the present conditions, but also, 
what from a business standpoint is more important, to anticipate the future development.

I shall consider in the following three sizes of traetor that would be built along exactly the same standards of quality, design and workmanship :

A-Capable of pulling two 14 -in. plows, 6 in. deep, in average soil, at a speed of $21 \frac{1}{2}$ m.p.h. This would require about $9 \mathrm{hp}$. on the drawbar.

B-Same quality tractor, of double size, pulling four 14-in. plows, 6 in. deep in the same soil, 21/2 m.p.h. speed. Drawbar of $18 \mathrm{hp}$.

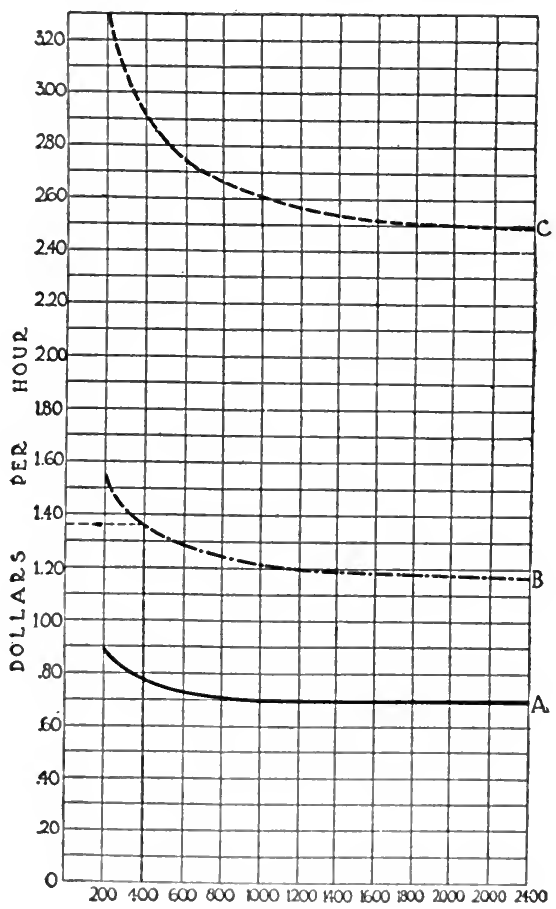

Fig. 55

$\mathrm{C}$ - Twice as strong as $\mathrm{B}$, pulling eight 14 -in. plows, $6 \mathrm{in}$. deep in the same soil, $21 / 2$ m.p.h. Drawbar 36 hp.

According to the standards of quality adopted by the truck manufacturers, I shall estimate the prices as follows:

Tractor $\Lambda, \$ 600$; Tractor $13, \$ 1,500$; Tractor C, $\$ 3,600$.

The price for tractor $\Lambda$, if the design, material and workmanship be first class, annot be reached unless quantity-production methods. are adopted. For $100 \%$ increase in size the price increases $150 \%$ in the above estimates, since I make the preliminary assumption that with the size of tractor inereasing, the demand might decrease and the manufacturing methods to be adopted beeome more expensive.

I shall not consider the cost of plows and other equipment, since for 
a comparative study of the cost of plowing with different sizes of tractors it matters very little.

A two and a four-bottom tractor and plow can easily be operated by one man, while for an eight-bottom tractor two men are necessary, not for lifting and lowering the bottoms, which can be done automatically, but for cleaning and unclogging the plows, which would mean stopping the machine often if it were expected to be done by the man steering the tractor.

The expense for operator would therefore come to $30 \mathrm{cts}$. per hr. for the two and four-plow outfit and 60 cts. for the eight-plow outfit.

Experience has shown that the fuel consumption of gasoline tractors per hp.-hour changes little or nothing with the size of motor, provided all are built according to the same standards and are run at the same percentage of load. In our case the cost for fuel (and oil, etc.) should be for tractor A 25 cts. per hr., for tractor B $50 \mathrm{cts}$. per hr. and for tractor C $\$ 1$ per $\mathrm{hr}$.

It is a conservative estimate to say that tractors of modern design of any size will depreciate after about $4500 \mathrm{hrs}$. of plowing, which would give a tractor, plowing $10 \mathrm{hrs}$. a day, for 90 days in a year, a life of 5 yrs: provided no other work is done but plowing. This estimate is safe, since should the tractor in the last case become obsolete after 8 years, $80 \%$ of its value has already been figured in the item, for wear and tear, having left only $20 \%$, part of which can easily be recovered from the sale of its high-grade materials as junk.

For our tractor A the amount for depreciation per plowing-hour 600

figures at $\frac{}{4500}=\$ 0.13$, for the tractor $B$ at $\frac{1500}{4500}=\$ 0.33$, for the tractor 3600

C at $\frac{}{4500}=\$ 0.80$.

4500

These two factors are considered together and estimated at $6 \%$ per year on the initial price of the tractor. Since the value of the tractor depreciates constantly, in time the interest on this value also decreases. Suppose the tractor is figured to depreciate in 6 years, the interest during the first year would be $6 \%$ of the initial price paid, the following year it would be $6 \%$ on the present value of the tractor, or $5 \%$ on the total initial price, and so on. The allowance for interest and repairs being $6 \%$ per year on the total initial price, it results that no repair allowance is made for the first year, $1 \%$ of total initial value for the second, $2 \%$ for the third, and so on, an estimate which corresponds with the actual conditions as they occur with tractors of modern design.

Suppose we figure every one of these items for the tractors chosen, for a variable number of actual plowing hours per year, Fig. 55 is drawn based upon these figures, marking on the horizontal co-ordinate the number of actual plowing hours per year, 200, 400, 600, etc., and on the vertical co-ordinate, the cost for plowing per hour; the curves marked $\mathrm{A}, \mathrm{B}$ and $\mathrm{C}$ correspond to the machines above mentioned.

Fig. 55 does not give a final indication as to the comparative cost of plowing with the different sizes of tractors, but it is used for making Figs. 56 and 57. Fig. 56 gives the cost in dollars per acre, varying 
with the number of hours of plowing. Tractors A, B, C are supposed to pull 2, 4 and 8 bottoms, 14 in. wide, 6 in. deep at a speed of 2.5 m.p.h. It is assumed that the tractors will plow only $85 \%$ of time they are in the field, $15 \%$ being the allowance for turning the headlands, oiling, trouble, ete. The area aetually plowed per hour figures then at .6, 1.2 , or 2.4 acres per hr. respectively. The small figures along the eurves give the number of acres per year the chosen tractor would actually plow.

From Fig. 56, as a basis, Fig. 57 has been drawn, and this gives now the final information we were after; we read the area to be plowed in a year on the horizontal co-ordinate, follow the vertical through this point

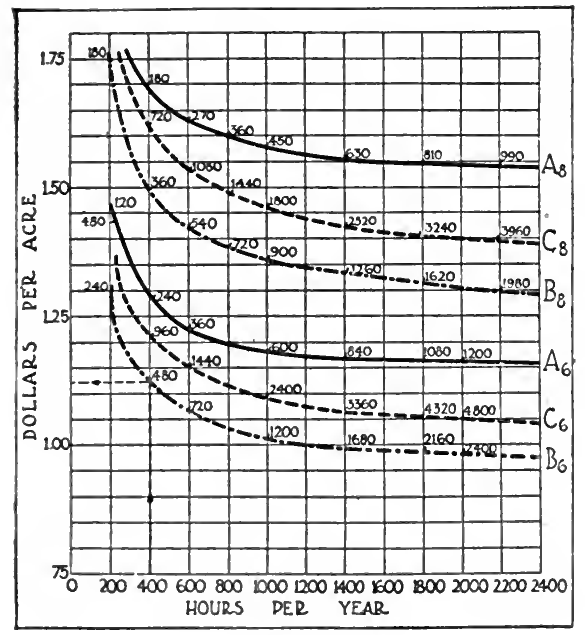

Fig. 56

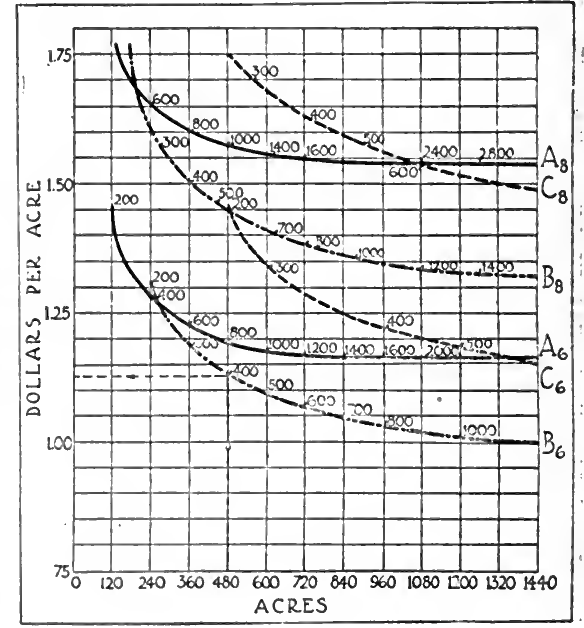

Fig. 57

until it intersects with the curve representing the $\mathrm{A}, \mathrm{B}$ or $\mathrm{C}$ tractor, and following to the left we read on the vertical co-ordinate the cost of plowing per acre. The figures along the eurves give the number of hours the tractor will have to work in order to perform the work expected. Recalling now that $A_{6}, B_{6}$ and $\mathrm{C}_{6}$ represent the conditions for a 2,4 or 8-plow tractor plowing $6 \mathrm{in}$. deep, while $\mathrm{A}_{8}, \mathrm{~B}_{\mathrm{s}}$ and $\mathrm{C}_{8}$ represent these tractors plowing 8 in. deep, let us now consider the signifieance of Fig. 57.

If we have to plow less than 250 acres per year and not deeper than 6 in., the tractor with 9IDB-IIP plows the cheapest, while for anything above this amount the 4-plow tractor with about 18 IBS-IIP is the eheapest in the end, i.e., it is cheaper than the 2-plow tractor for small farms, while even for farms so large that one 4-plow tractor be too small, it pays better to have two 4-plow tractors than one 8-plow tractor. The advantages of having two smaller power plant units instead of one large one from the point of flexibility and in emergeney cases (break down, e.te.) are not considered at all in the charts.

The chart shows much more than this. It shows how much eheaperthe plowing comes if there be much of it to be done. There are, never-- 
theless, two ways that will make the small farmer meet the competition of the big one, without having to buy his neighbor's land: by deeper plowing, and by plowing early and quick. To meet both of these conditions the 2-plow tractor will give way to the larger one; in fact, we find on the upper curves that represent the cost for plowing 8 in. deep, that for any land above 180 acres of plowing per year, the 4-plow tractor is the cheapest. While for 240 acres of plowing per year the 4plow tractor figures only a little cheaper in dollars and cents, it has the advantage that it performs the work in about 30 days instead of 60 , as is necessary with the smaller one.

Now, there are two ways of making a tractor, intended to pull 4 bottoms 6 in. deep, plow 8 in. deep. First, by hitching 3 plow bottoms instead of 4 ; second, by leaving the 4 on and plowing at the lower speed of about 1.9 m.p.h. instead of 2.5 m.p.h. (which every tractor should have); the area plowed per hour and the cost is nearly the same. In fact, there is a great advantage by applying the first method, since we can easier count on continually using the full power of the tractor where its efficiency is the highest. Hard spots on the land, or grades, will be overcome by shifting to the low-speed gear. On the other hand, with a two-plow tractor intended for 6-in. deep plowing we have no. other choice, if extra expense for specially narrow bottoms should be avoided, but to run the plows 8 in. deep on low speed, all the time.

Manufacturers have too long likened the tractor manufacturing problems to the truck problems. There is a good reason for building 4 or 5 sizes of trucks in the same plant: A 5-ton load cannot be handled sometimes by either one or two 3-ton trucks, nor is it economical to run a 3-ton truck with a 1-ton load, but in plowing the load can easily be adapted to the full power of the tractor.

It is important to note that the above conclusion has been reached, although the preliminary assumption was made that the 2-plow tractor will probably be the most popular type and therefore the cheapest to manufacture. But since we have reached the conclusion that the 4plow outfit is the most economical it can be expected that due to its. production in large quantities its price per horse-power will not be $25 \%$ higher than that of the 2-plow outfit as assumed at the start of this analysis but perhaps even lower.

Following, is a list of articles eontaining further illustrations of the use of graphic charts of the class discussed in this chapter and pertaining to kindred subjects.

Upon request, the Codex Book Co., Inc., 19 William Street, New York, will be glad to procure, where possible, copies of magazines containing the articles needed, only charging the "old magazine" price, plus postage.

"Making a Cost Profile," P. L. Mathews, Coal Age, November 4, 1916.

"Mastering Power Production," Series I-X, Walter N. Polakov, Industrial Management, January, 1918 to November, 1918.

"Power Plant Operating Costs," Walter N. Polakov, Power, February $15,1916$.

"Standardization of Power Plant Operating Costs," Walter N. Polakov, Electric Traction, February, 1916. 
“Expenditure Chart for Executives," F. J. Schlink, Industrial Management, March, 1918.

"Relation of Quantity Production to Cost," A. G. Popcke, Industrial Management, January, 1918.

"Ilow to Determine the Amount That Can Be Economically Spent for Remedying Plant Defects," Electrical World, April 8, 1916.

"Pieturing Prices by Charts," H. B. Twyford, Factory, June, 1916.

"Diagram Showing Increasing Cost of Rock Excavation as Cuts Become Shallow," L. N. Jenssen, Engineering and Contracting, October 30,1907 .

"Graphic Method of Estimating Core Costs," Edw. S. Dean, Iron Age, Nay $6,1915$.

"Choice of Electrification for a Concrete Case," Electric Railway Journal, December 27, 1913.

"Cost of Compressing Air by Electricity,", W. C. Lancaster, Power, February 3, 1914.

"Joint Use of Central Station Service and an Isolated Plant," Electrical World, January 13, 1917.

"Power Requirements of Grain Elevators," F. F. Espenschied, Electrical World, June 30, 1917.

"Power Generation for Electric Railways," Henry C. Stott, Electrical World, October 13, 1917.

"Some Difficulties with Iron-Wire Transmission," M. D. Leslie, Electrical World, October 13, 1917.

"A Graphic Chart for the Evaluation of Coal," A. F. Blake, Journal of Industrial and Engineering Chemistry, December, 1916 and August 1, 1918.

"Cost of Coal on the Heat Value Basis," Railway Review, December $9,1916$.

"Equivalent Cost of Coal and Oil as Fuel," R. L. Wales, Power, March 13, 1917.

"Belts, Their Selection and Care," C. J. Morrison, Engineering Magazine, July, 1916.

"The Life of a Truck," by W. F. S., Factory, May, 1918. 


\section{CHAPTER XII}

\section{Scheduling and Progress}

In this chapter are given examples of another important function of charts-that of graphic scheduling and the recording of the progress of work. There is small need, in these times, to dwell upon the absolute necessity of laying out a schedule in advance for all work which is to be efficiently and economically done. All of the many books upon the science of management emphasize the importance of this feature and the proof of the practical value of it lies in the fact that every concern which makes any pretense whatever of employing the principles of efficiency has some method of planning its work in advance.

A schedule is of little value unless it is known whether it is lived up to, and if there is any departure from it there must be a means of knowing it at once so that the causes may be investigated and remedied. It is these things which the chart accomplishes better than any other method as it furnishes a picture which cannot be misinterpreted.

Construction Work. An example of a schedule chart, Fig. 1 together with the explanation of how it operates, is given by A. G. Hillberg in Contracting, November, 1916.

It is necessary for a contractor to plan his job in such a way that work on one part of it will not interfere with work on another part, and so that when the work has progressed to any particular point the required machinery and materials are on the job, thus preventing unnecessary delays. In planning the construction, therefore, a schedule should be made showing the time allotted to each part of the work. The plant and supplies should be ordered in accordance with this schedule, and a wise contractor should see that they are on the ground and in advance of the time when they are actually required.

In planning the construction of a 25,000-hp. hydro-electric development in the Southwest, on which work is to begin soon, the writer made up the accompanying construction schedule. $15 \mathrm{mo}$. are allotted to complete the work, and it was therefore deemed wise to try to finish the job in 12 mo., thus being able to claim a substantial bonus.

The hollow dam is $400 \mathrm{ft}$. long and $145 \mathrm{ft}$. high, with large buttresess supporting a deck. The power house has a substructure of mass concrete and a superstructure of structural steel with brick walls. Concrete without reinforcement was specified because it was desired to make immediate use of the power house crane in the erection of the machinery. A structure of reinforced concrete would have to be at least three months old before it could safely carry the heavy crane, to say nothing about the form work, which would interfere with the work in the generator room.

The construction has been classified in three divisions: I, dam; II, 229 
power house, and III, transmission lines and substations, and arranged so that work can be carried on independently in every division.

Work is commenced on division I with the construction of cofferdams and the installing of the construction plant. About one mile of railroad must be built, but otherwise the work required is not extensive as an abumdant supply of gravel, sand, and boulders can be obtained in the river banks. Therefore no crushing plant is needed.

After the mattress and the cut-off have been built on the dry east bank, it will be necessary to cofferdam and divert the river. It is planned to do this during the latter half of the fourth month and first half of the fifth month. The work on the dam can then proceed without further interruptions, as it will be above high water level.

During the later half of the eighth month the penstocks will be placed and during the ninth month the intake will be built around their front parts. The penstocks cannot be delivered soon enough for any earlier installation. All orders to manufacturers are assumed to be given out during the first month of the work.

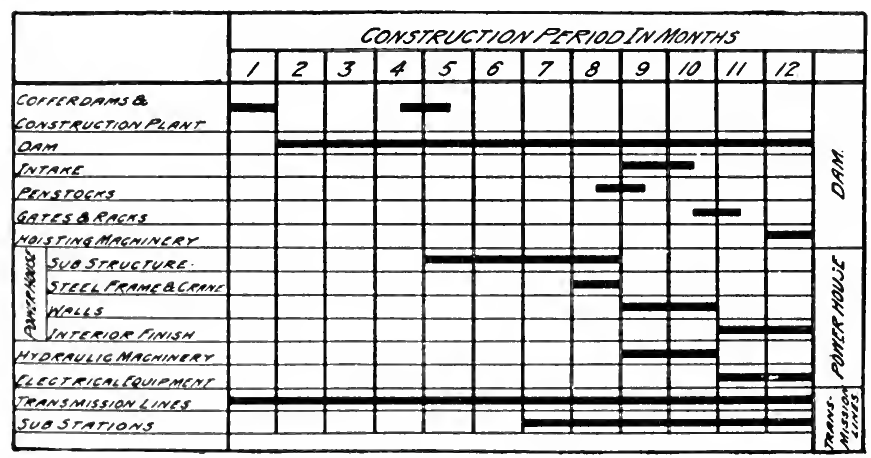

Fig. 1-Construction Chart for Dam, Power House, and Transmission Line

The gates, racks, and gate operating machinery ean be placed at leisure any time after the concrete work on the intake has been completed, and have been planned to take place as shown on the chart. This completes the work on the dam.

The work on division II, the power house substructure, cannot be commenced before the upstream cofferdam has been completed across the river, which will be about at the end of the fourth month.

The work on the substructure ean be stretched over four months, which means one and one-half units per month, the power house consisting of five 5,000-hp. units and one exciter bay.

At the beginning of the eighth month the steel will arrive and as it woighs only about 85 tons, it can easily be erected in one month so that the substructure and the framing for the superstructure will be finisher at the same time. The erane can then be installed and the ereetion of the machinery eommeneed.

Two months have heen allowed for the erection of the hydraulic machinery and two months for the electrical machinery. The work on the 
brick panels of the side walls and the interior decorating can be carried on independent of the installation of the machinery.

The final locations of the transmission lines, division III, will be determined during the first three months of the work; then the materials will be ordered for them and their erection begun. As the substations are few and small and can be built quickly, they will be erected during the last six months of the work, after their final locations have been decided upon in connection with the survey of the transmission system.

From this chart the size of the construction plant can be determined. Later on each group on this chart must be subdivided so as to show exactly how to carry on the work on each part. For instance, the dam
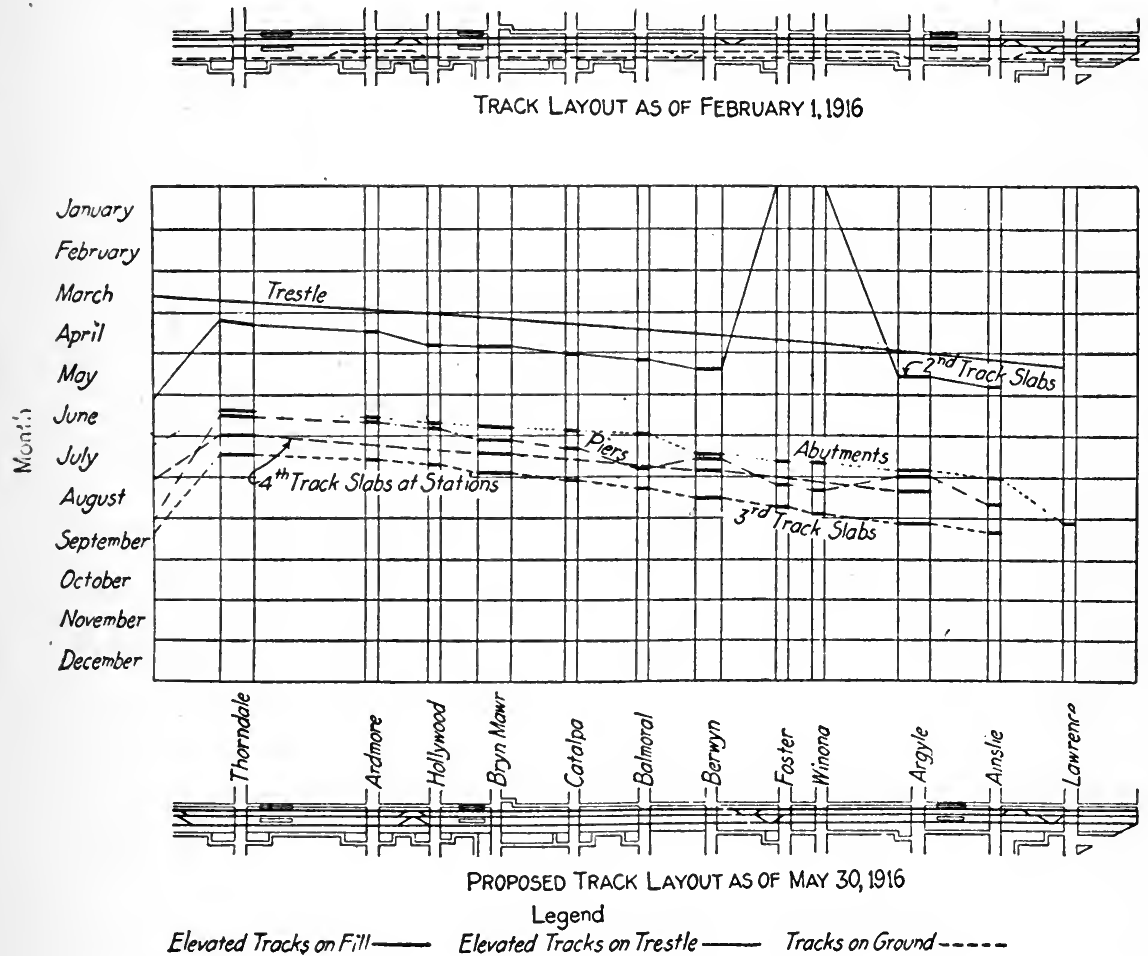

Fig. 2-Track-Elevation Program

consists of deep cutoff walls, a distributing mattress, buttresses, a concrete deck, bridge along the top, etc. A separate chart must be made showing the scheduled progress on these parts by weeks or even days, so as to enable the contractor to see at an early date whether he needs additional plant and men to complete the job on time.

Railroad Work. Fig. 2 and the following excerpt from Engineering Record, July 15, 1916, describe a schedule chart for track elevation work.

After considerable experimenting C. H. Buford, assistant engineer of the Chicago, Milwaukee \& St. Paul Railway, who is in charge of the 
North Side track elevation work of that road in Chicago, has devised a diagram for the desired progress of his work. It will be seen that at the top and bottom of the chart are diagrammatie plans showing the streets, tracks and stations as of Feb. 1, before the season's work was. started, and as proposed for May 30 . In the center section the abscissas correspond to the location, and the vertieal spaces are months.

This part of the diagram is self-explanatory. Each part of the work is represented by different conventions. For the trestles and the like, which are eontinuous, the line is also continuous. MIost of the items, however, have to do with the bridges, which are only at the streets, and for these items the heavy lines across the street are conneeted by lighter broken ones of the proper convention.

The various lines show elearly the desired sequence of operations and the time intervals planned between them.

Scheduling Labor Requirements. A somewhat different use for a schedule chart is elearly explained by R. von Huhn, in Industrial Management, February, 1919, in an article entitled "Graphical Analysis of an Overtime Problem." The following is quoted:

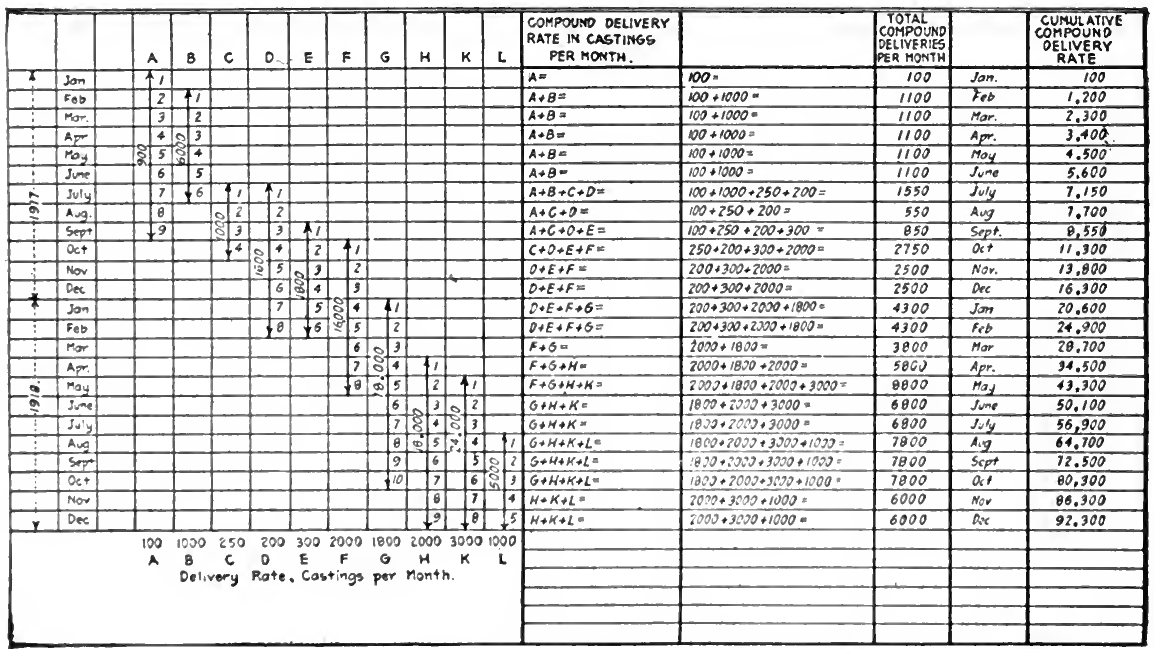

Fig. 3-Delivery Rates from Sub-Contractors and Compound Deliveries Per Month

A large manufacturing company was given a eontract requiring $92,3(0)$ rastings. At the time the company accepted the contraet its own fomdry was turning out maximum production. The management, thorefore, was forced to seek outside help and to issue sub-contracts with guaranteof delivery rates, the production of the 92,300 castings being distributed among 10 different concerns.

The delivery rates being quoted, the question immediately arose as to how much time womld be required to machine-finish the eastings ready for assembly. Naturally, the delivery promises of the $10 \mathrm{sub}$ contractors varief eneatly and were a fumetion of the magnitude of the conerrus involvecl, as well as their working capacity, other contracts being booked for other customers. 
The delivery promises were arranged in a table, according to the dates at which deliveries could begin, and each sub-contractor was given a letter. Fig. 3 shows this arrangement. It gives a complete record of the expeeted output, and of the delivery promises for the different time intervals. With the aid of this table, the "resultant" or "compound delivery rate" may be calculated at which the combined companies will furnish the eastings during each month; company A, for instance is booked for a total of 900 castings, which were contracted to be delivered during a period of nine months, starting· with January
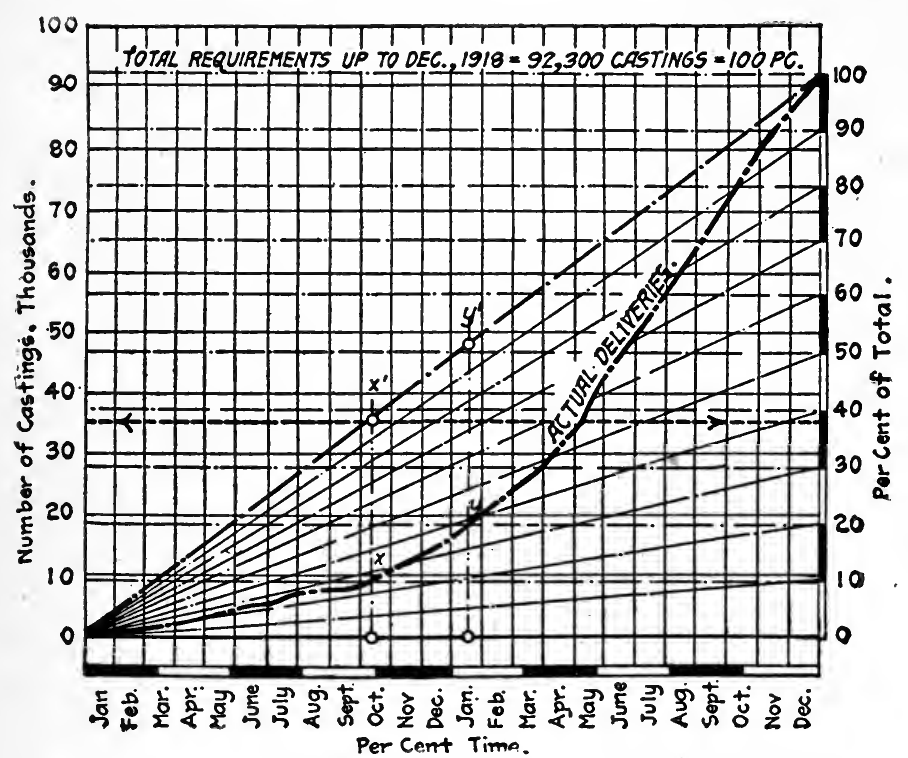

Fig. 4-Time Curve of Cumulative Compound Deliveries

1, 1917-which means that company $\mathrm{A}$ is working at a delivery rate 900

of $\frac{}{9}=100$ castings per month.

On February 1 company B, which agreed to deliver 6,000 eastings within a period of six months, starts delivery too, with a guaranteed delivery rate of $\frac{6000}{6}=1,000$ castings per month.

$$
1000+100=1100 \text { eastings per month. }
$$

In this way, the compound delivery rates may be ealculated for the entire period, and for all the companies involved, and the results are compiled in the column "Total Compound Deliveries Per Month"; Thus, the exact number of castings expected, and consequently to be machined ready for assembly, can be determined. If, furthermore, the monthly compound delivery rate is cumulated and plotted from the column "Cumulative Compound Delivery Rates," against time (24 
months in our case), the line, "Actual Deliveries" which will be found in Fig. 4 is obtained.

A glance at the diagram, shows that the delivery rate does not proceed at a uniform or average rate, as the dot-and-dash straight line indicates, but that the delivery rate is very fluctuating on account of the uneven deliveries during the different time intervals. The total deliveries require 24 months, or at December 31, 1918, the desired 92,300 castings are supposed to be on hand.

Let us eall this number, or the ordinate, on December $31,1918,100 \%$ and let us divide it into 10 parts; then each part of the total length of the ordinate, represents $10 \%$ of the total expected deliveries, or, in figures, 9,230 castings. It is obvious that the intersection of $X$ of the cumulative production eurve with the horizontal dotted $10 \%$ line (righthand scale) indicates that up to October 10,1917, only $10 \%$ of the total expected output will be delivered. The next intersection, $\boldsymbol{Y}$, shows that $20 \%$ of the total output will be delivered the middle of January, 1918.

In relation to time, this means: It took about $38 \%$ of the total time to produce the first $10 \%$ of the total number of castings; whereas, it

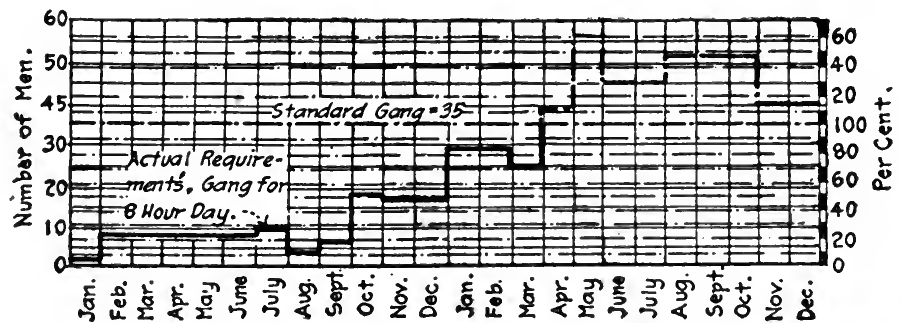

Fig. 5-Number of Men Required, Based on an Eight-Hour Day

took only about $14 \%$ of the total time to produce the next $10 \%$; which nicans that the delivery rate from October 10 to Jamuary 15 was, approximately, 2.7 times that of the first $10 \%$.

The ideal production line is, of course, the dot-and-dash line, or the line of uniform delivery rate. If production were uniform the point $x^{1}$ would hold good for October 10 . This point represents $100 \%$ of the total required up to that date, or, about $39 \%$ of the total required at the end of the expiration date of the contract December 31, 1918, or, reading left-hand seale (dot-and-dash line and arrow indicate the points in both eases), percentage as well as actual number.

Therefore the magnitude of the line $X X^{\mathrm{x}}$ represents the deficit of uniform production and, if plotted, will represent a new curve, which in some particular instances is of great interest to the executive.

Based on this production diagram,, the next phase of the analysis follows: The machine department of the company in question figures on a standard force of 35 men available for turning and finishing the eastings. It has been ascertained that five castings can be turned out by one man in cight hours per day, or 150 per month per man.

It also was eonsidered necessary to finish up each month the total number of castings received in order to keep pace with the output 
of the castings which means a considerable fluctuation of the working force requirements.

Let us assume that 35 men as a standard gang are available, and call that number $100 \%$. If, now, the number of men required is plotted for each month as indicated, the production office will have a means of analyzing the situation throughout the entire period. Fig. 5 gives a complete record of the working force which is necessary for the output

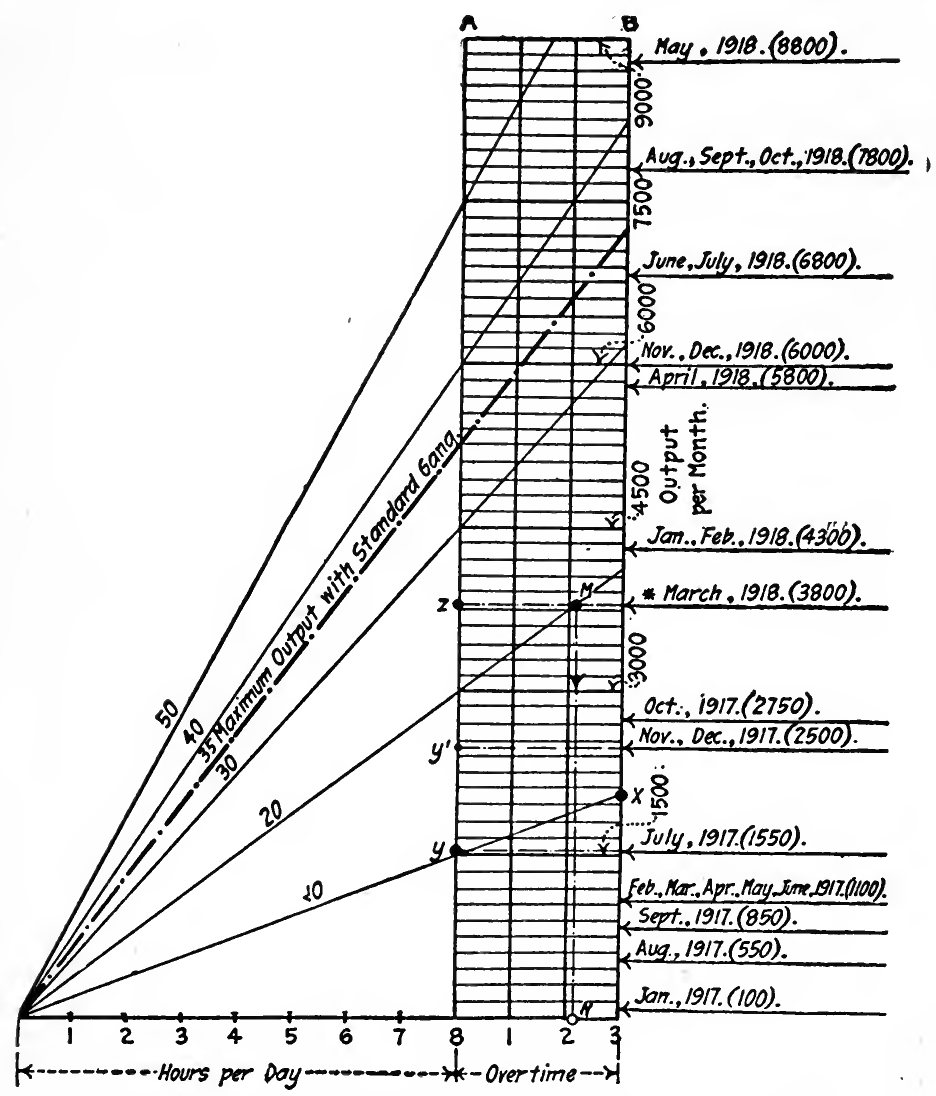

Fig. 6-Overtime Required, Based Upon Number of Men

if an 8-hour day is to be maintained. The line "Actual Required Gang for 8-Hour Day"' indicates that, up to April 1, 1918, production can be handled by the "Standard Gang." After that outside help must be hired if production shall keep up with the delivery rate. The hatched area of Fig. 5 shows the percentages of outside help necessary to machine-finish the delivered castings.

Suppose now, that for various obvious reasons the management is not inclined to increase the force but would rather introduce overtime work. A new diagram, Fig. 6, was devised to analyze what max- 
imum production could be covered with the standard force. The abscissa in this diagram is time, and the ordinate again represents production per month; or, as production depends directly on the number of men, two scales are possible; one scale in units of production, the other seale in units of men. Therefore, point 10 represents the number of castings turned out by ten men $=1,500$ castings per month based on an 8-hour day.

Let us assume now that a crew of ten men work 11 hours per day throughout the month instead of 8 hours. This would mean three hours overtime throughout this period, which would increase the production accordingly, as shown where the 10-line intersects with the three hours abscissa (point $X$ ). Each unit represents 150 castings, and the increased amount produced would be approximately, 550 castings, or the total for that period $525+1,500=2,025$ castings, or about $35 \%$ increase. This increase the diagram also shows at point $X$. In other words, point $X$ shows the actual increase as well as the percentage increase caused by overtime work.

The next step was to plot from Fig. 3 the column "Total Deliveries Per Month" as abscissa. The respective positions of the points are indicated by the horizontal lines, with the months during which these deliveries are expected, and the actual delivery number in parenthesis. The result obtained is a complete analysis of the required working force. During the month of July, for instance, 1,550 castings are the compound delivery rate. Reading horizontally across, (dot-and-dash line,

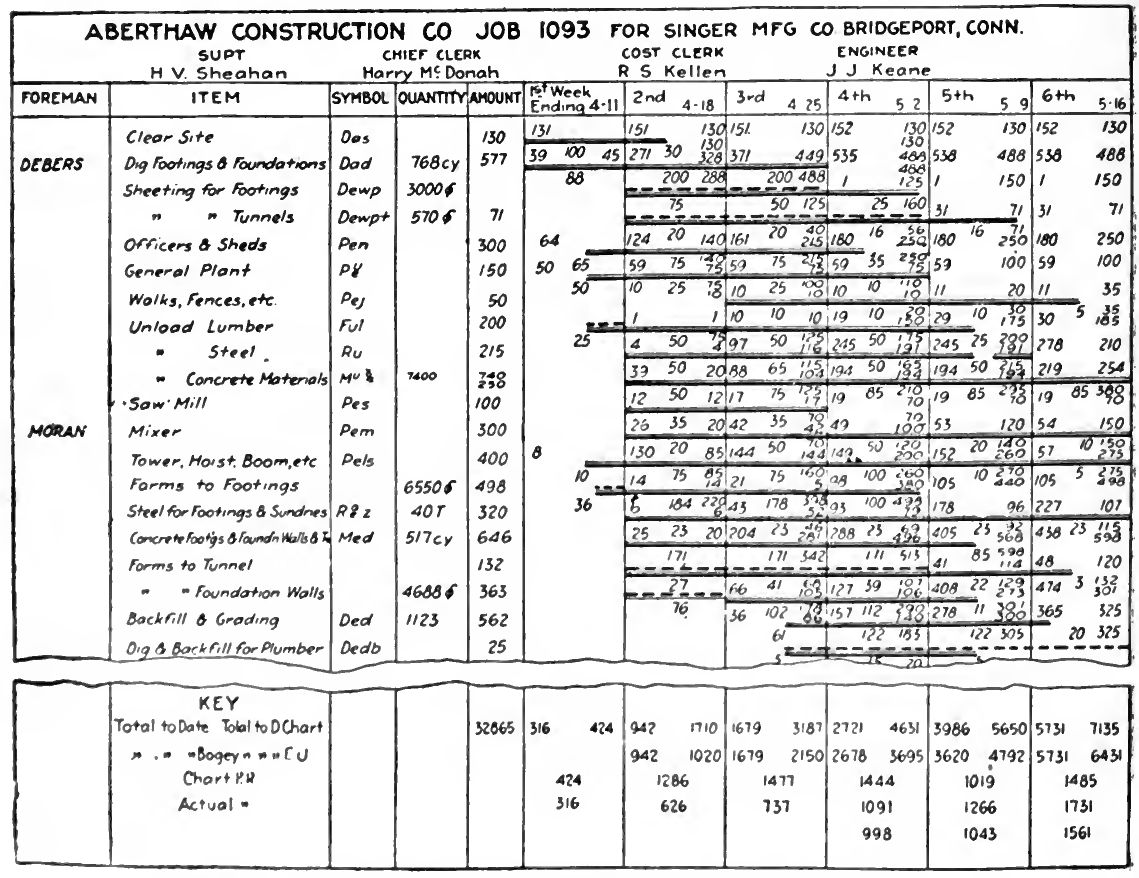

Fig. 7-Progress Chart Combined with "Bogey Score" 
point $Y$ ) we find that this number can be machined by ten men (based on an 8-hour day) during the month.

Considering overtime the management can make for the month of March (as marked by *) with 3,800 castings to be machined the following choice:

Reading straight across, the amount could be handled (based on an 8-hour day) with approximately 25 men (point $Z$ ) or, following the "20-men" line until it intersects with the horizontal 3,800 line in $\boldsymbol{M}$, and then reading vertically downward where the dot-and-dash line intersects with the "overtime" scale in $N$, we see that about $2 \mathrm{hrs}$. 10 min. overtime has to be worked to handle the work with a 20-man erew.

The maximum output is indicated by the 35 -men line, which readily shows that the May, August, September and October deliveries cannot be handled by the standard gang even with three hours overtime, without outside help.

The manager, therefore, is forced either to reduce output for these above-mentioned periods or to hire additional skilled labor to take care of the excessive production.

All phases of a contract can be analyzed in this way, and the information thus obtained is of supreme value and readily obtainable.

Combination Schedule and Progress Chart. An illustration of this type of chart is given by Dan Patch in his article "Predict Labor Demand Before Job is Started," published October 11, 1917, in Engineering News-Record, as follows:

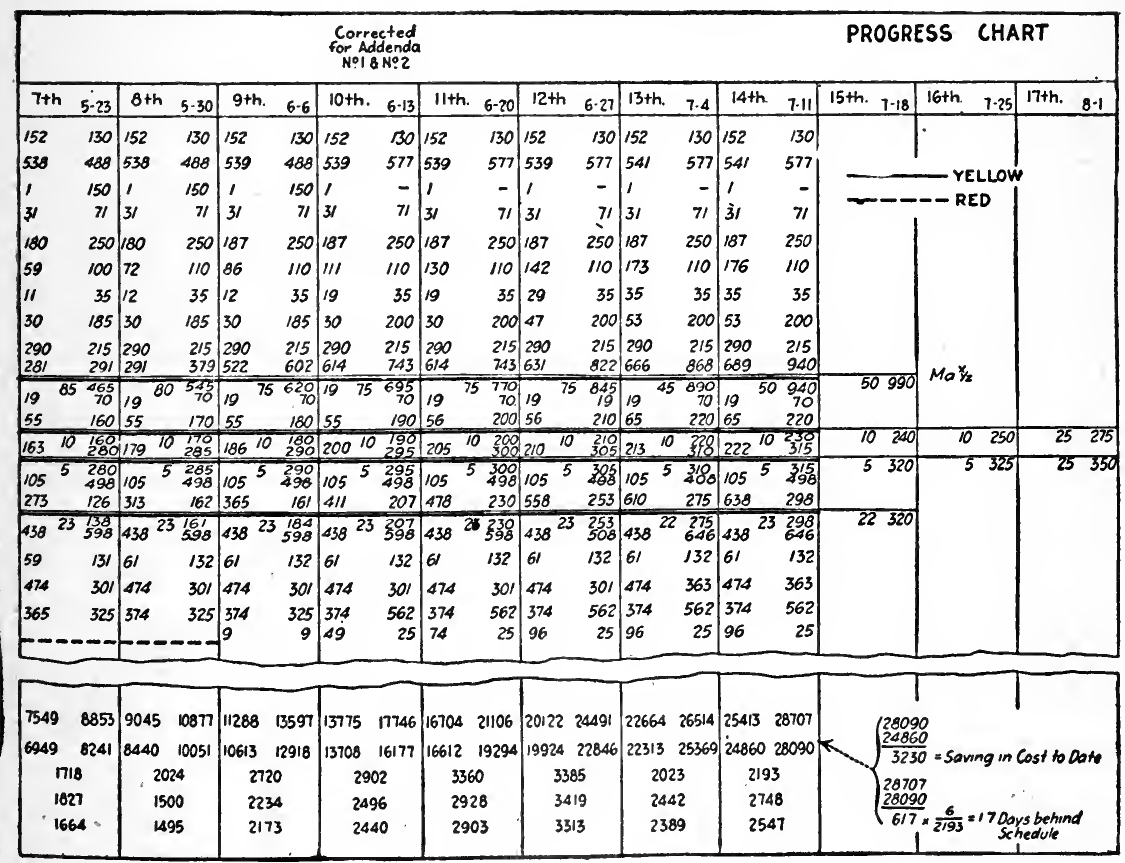


In seeking a record of a job's actual cost in time and money as compared with the progress schedule made for it in advance, the Aberthaw Construction Co. has modified its methods somewhat, so that it is possible to tell in advance how much weekly payrolls must amount to at each stage of the work; to tell, moreover, not only whether the material progress is up to this schedule on any date, but exactly how far behind or ahead it may be in terms of materials and labor spent; and to tell in addition, whether the work is ahead or behind in eost, and how the work actually done eompares, unit for unit, with the estimates. After predicting the weekly labor expenditure, made up of the cost of all sehedules to be under way in any week, these methods make it possible to tell definitely in terms of dollars and cents, and without elaborate peneil-sharpening or guesswork, whether the job is gaining or losing and exactly which features of the work are responsible for the variation from the estimate.

It is of importance to those financing the work to know not only how much money is to be spent but when, and of quite as much importance to the eontractor to know not only how large gangs he must have but when he will need them.

The construetion eompany for which the writer works has for some time plotted on a progress chart its schedule of dates for the operations to be undertaken on each of its important jobs, as an aid to the logical ordering and delivery of materials to the job and as a scheme for the job superintendent to follow in order to reach the ultimate date of delivery on time. In addition, however, the management desired a method of keeping in the central office a reeord of the job's effect on the schedule. As a result of this, the new method was devised.

The progress chart now has combined with it a "bogey score," or list of items of work with the estimated quantities and amounts of money allotted for their performance, Fig. 7. The money allotted for each item is divided up throughout the time scheduled in weekly amounts, which are placed below the sehedule line in the middle of the week space. At the right of the week space, below the line, is put the sum to date of these estimated weekly amounts. This, of course, should equal at the end of the line the amount allotted in the "bogey score." When all the money has been thus distributed, each column is added and the total weekly payroll for each determined. These in turn are summed to date, the total equaling the total of the "bogey score."

The prediction of the weekly roll and the total to date are plotted on a progress curve sheet, Fig. 8 , in fine lines, the horizontal lines representing the payroll and their connections making the figure resembling the cross-section of a mountain range. The form of this figure will vary according to the type of job and speed of eonstruetion. The eurve of "total to date" is the typical sinusoid of most construction costs.

With the construction of the chart and eurves understood, the methods of following the performance will be grasped readily. The cost of each item is kept by the field timekeepers against the mnemonic symbols set opposite cach item in the "bogey score." These costs to date are entered each week in the left-hand side, above the line for each item. But the enst of most items is made up of a quantity of work done at 
a certain cost. This quantity of work, at the unit cost allowed in the "bogey," is set down in the right side of the week, above the line. The total weekly roll is entered at the foot of the column under the predicted roll, and this roll, less extras, underneath this.

Now, if any one item is considered the rectangle for any week which has passed, it will look as follows:

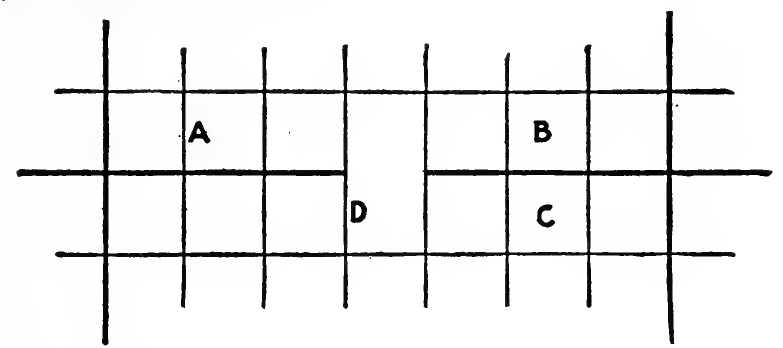

A-Actual cost of item to date.

B-What cost would be if done at the units allowed by "bogey score." C-What cost would be if the quantity scheduled had been done at the units allowed in the "bogey score."

D-Predicted weekly expenditure on item.

Then if $\mathrm{A}$ is less than $\mathrm{B}$, the job is saving money on the item; if $\mathbf{A}$ is greater than $\mathrm{B}$, money is being lost. If $\mathrm{B}$ is greater than $\mathrm{C}$, the job is pushing the item ahead of schedule; if $B$ is less than $C$, the item is behind.
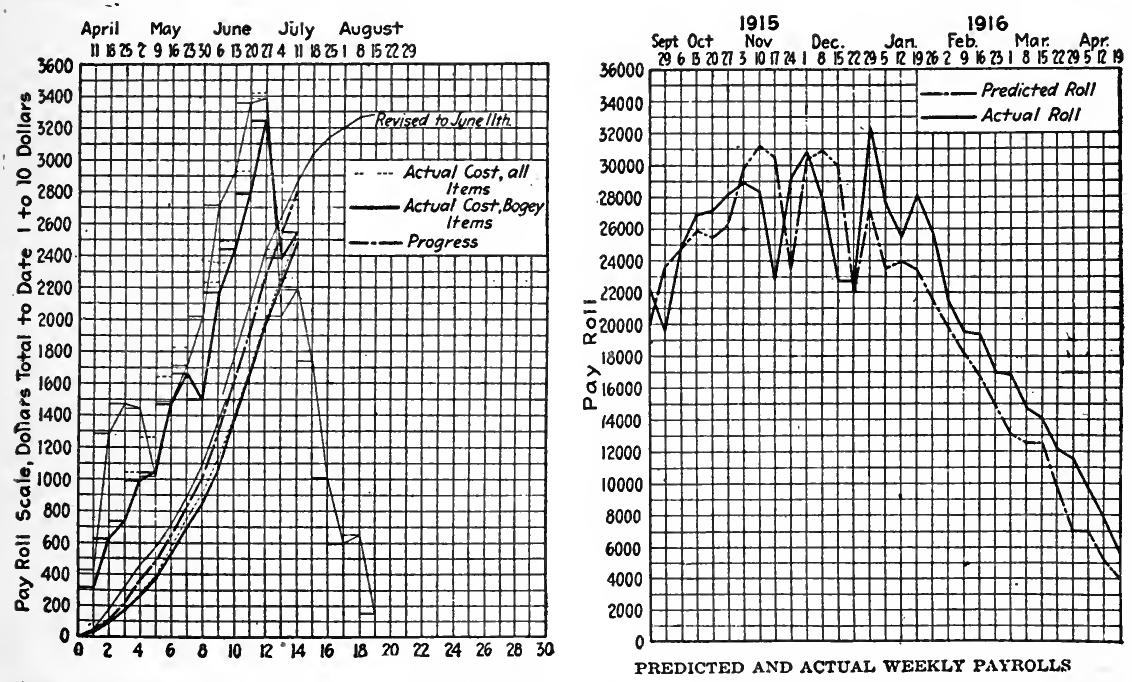

Fig. 8-Curve of Total Payroll to Date

Fig. 9-Predicted and Actual Weekly Payrolls

The blueprints which are posted on the central office board are not filled out each week so as to give these detail figures, but a graphic method is used to give the results. 
If any item does not start on the date set, a red line is started above and continued until the item starts or is eancelled. The traveling superintendent is notified of the red line, and he either notifies the office that the item has been found unnecessary, as is sometimes true of sheeting for footings, ete., in which case a white line is drawn underneath, or takes steps to get the item started. When the item starts, a yellow line is placed beneath and drawn up to the point where the money to date, under the line, is equal to the cost to date at "bogey" units; that is, where the value of $\mathrm{C}$ equals $\mathrm{B}$. This graphically represents the proportion of the item done, and at the completion of the item, will just reach the end of the item line. As each week is entered, a white ver-

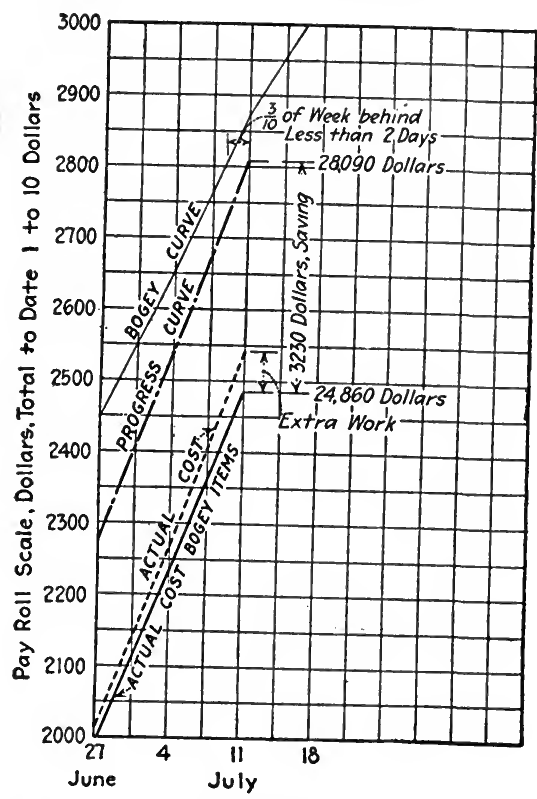

Fig. 10-Graphic Method of Showing Progress

tical difference line is drawn through the date, so that it is more easily seen how the job stands. The bottom of the chart has the standing of the whole job plotted. These results are also plotted for the total job on the progress eurves in the heavy black lines.

Now, it is to be noticed how closely the actual results follow the predictions; that is, how similar the heavy is to the light form. This would indicate that for any given job, a very elose approximation to the labor demands and expenditures can be predicted a considerable time ahearl. By reference to the ehart, the trend of the labor at any particular time during the job can be determined; that is, when masons will be demanderl and how many, ete.

In ease of large operations, eovering groups of buildings, the superposing of these individual job eurves, showing the predicted and actual weekly rolls, of which Fig. 9 for one of the company's large contracts 
is an example, will give a resultant curve of the total operation to be financed. Labor is an active liability and has to be met with cash. Merely dividing the total estimated cost by the length of the job gives no idea of the peak load, which may be of importance.

It may be of further interest to note the graphic method of showing the progress curve sheet. To do this, consider the curves, Fig. 10, for the job for which the progress chart is shown. Enlarge the section near the terminal of the lines to date of July 11, and the diagram will indicate the conditions of the job. Should the progress line be on the opposite side of the "bogey" curve, then the job would be ahead of the schedule; if the curve of the actual cost of "bogey" items should be above the progress curve, there would be indicated an overrun in cost.

Another Form of Progress Chart is illustrated and described by E. W. Robinson in Engineering and Contracting, December 17, 1913. Part of this article follows:

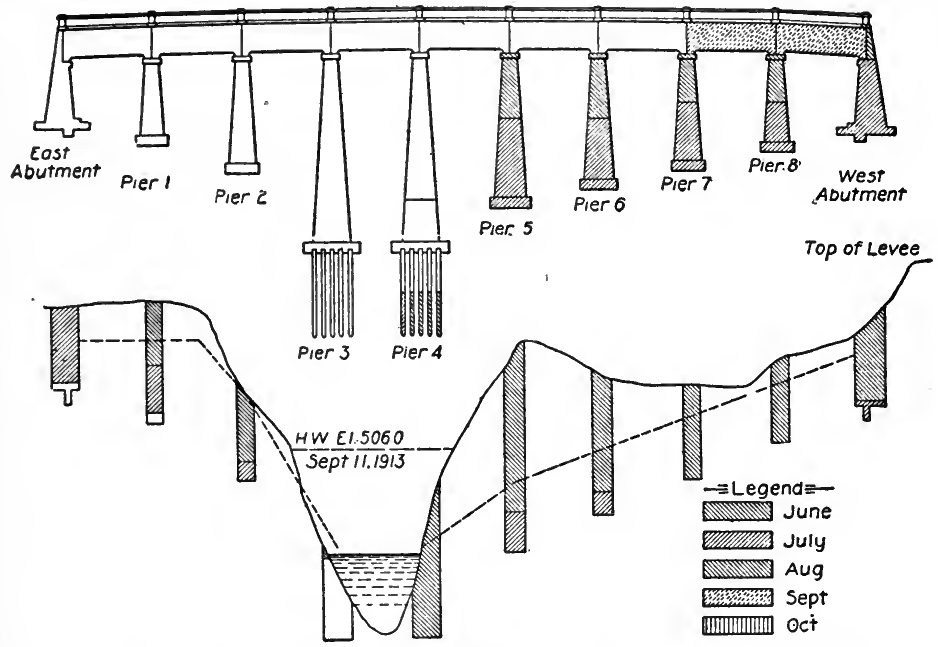

Fig. 11-Specimen Progress Elevation and Profile of a Bridge

The following is a general outline of an office system that has been worked up and tried, with more or less modification, on five reinforced concrete jobs varying in size from $\$ 20,000$ to $\$ 115,000$. The cost-keeping and progress charts were taken, with very few changes, from a system worked out by another, but the rest of the system described herein was worked out by trial from a knowledge of the results desired, aided by suggestions obtained from reading many articles in the various engineering and contracting magazines and treatises.

A more or less permanent record of the progress of the work is obtained by means of diaries, notes on the timekeeper's daily reports and photographs taken at regular intervals.

The weather report of the U. S. Department of Agriculture is received each day from the nearest weather bureau and filed as a permanent record of the weather to supplement the records of the daily 


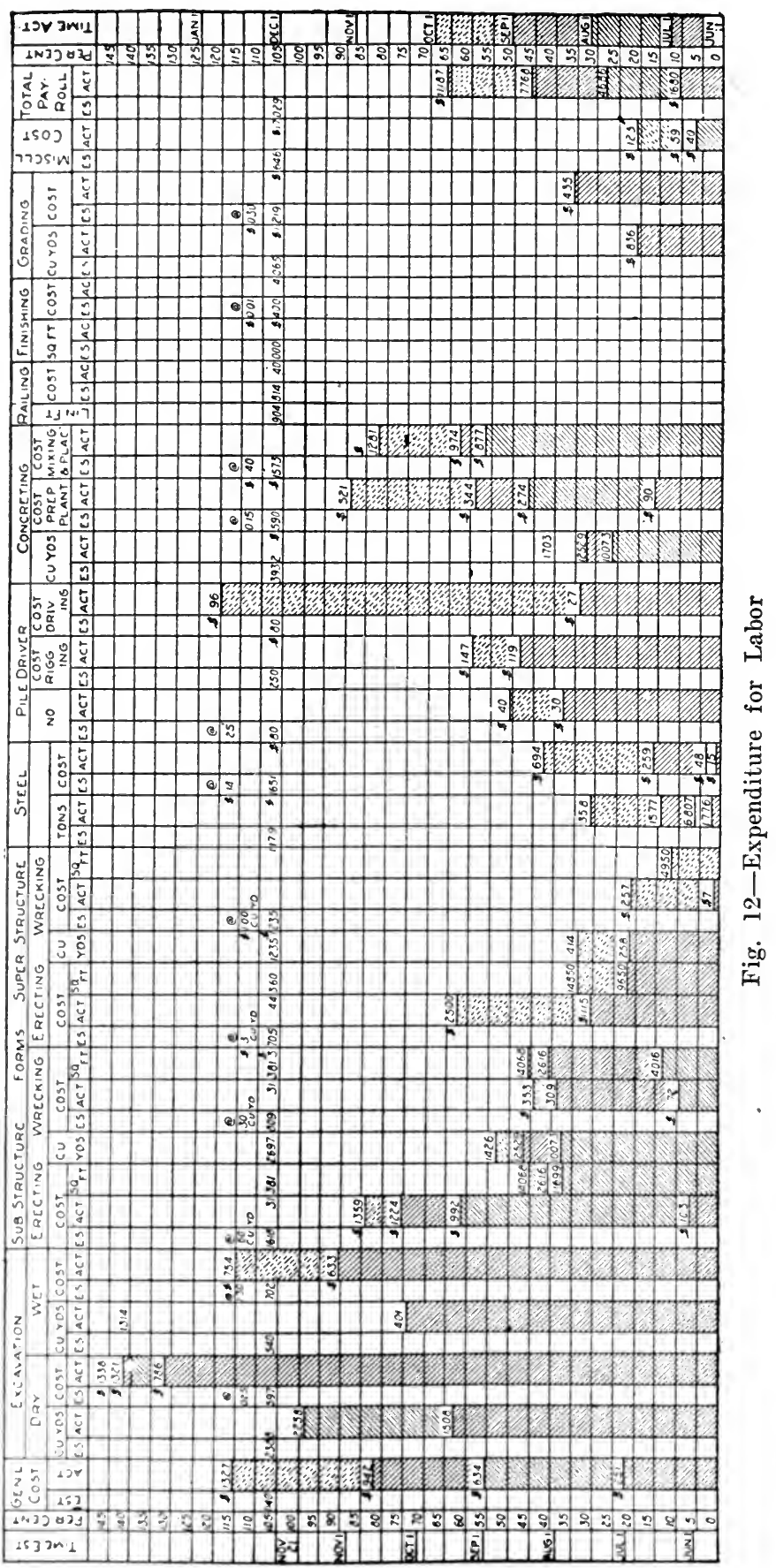




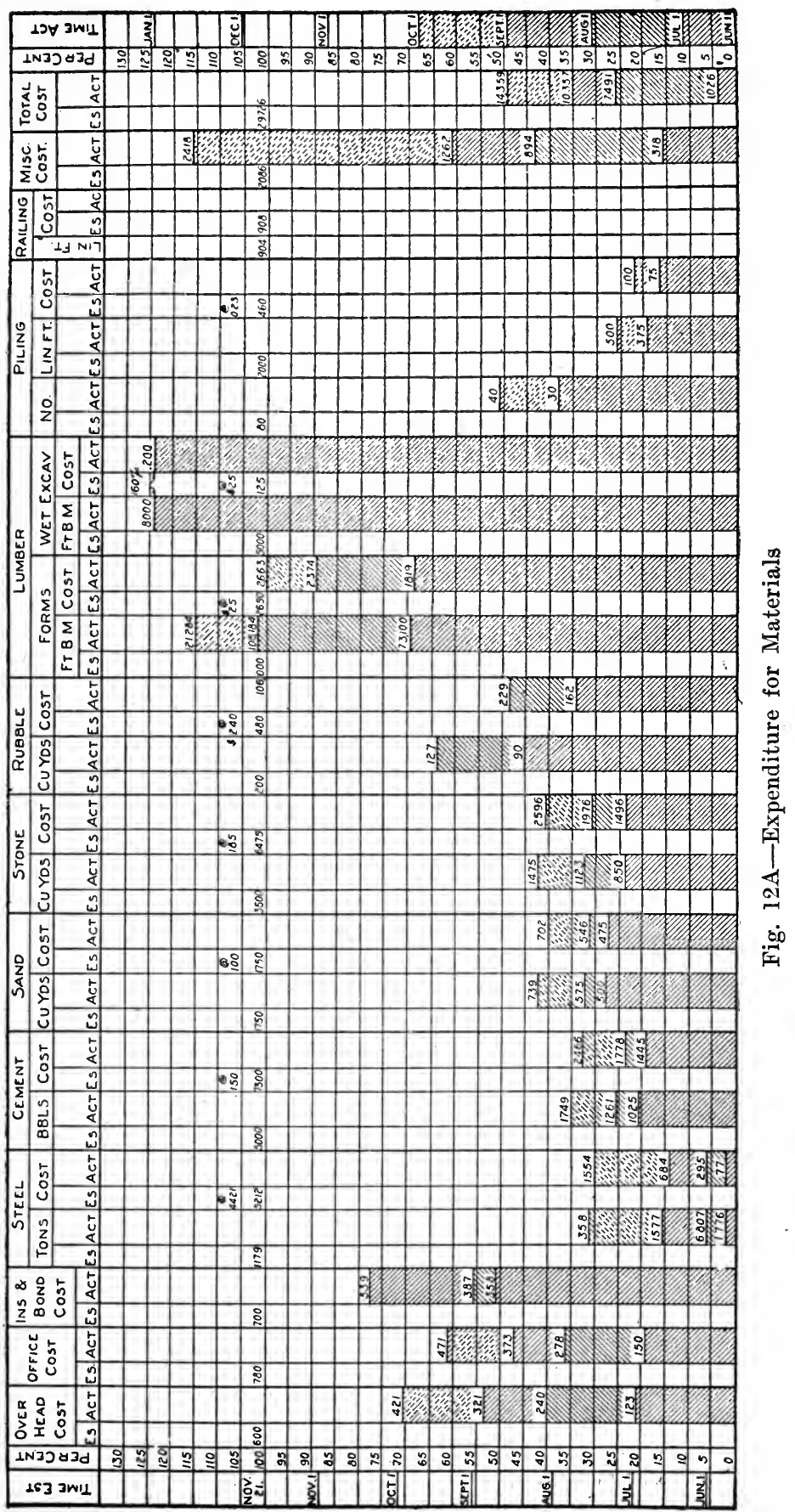


reports. On bridge work a water gauge is set near each job and a record made and plotted on cross-section paper of each" and every change in the water level of the river. All unustial rises in the river are shown on the progress profile which is described later. This shows at a glance the effect of the high water upon the progress of the work.

An accurate record of the amount, kind, location, etc., of the various items of work done each month is obtained at the time the monthly estimate is made up and is shown graphically on a profile and on the cost charts. In the case of bridge work a profile on the longitudinal center line of the structure is plotted to some convenient scale, and directly above this is drawn to the same scale an elevation of the completed structure in outline only (Fig. 11). Different kinds of crosshatching are used to show the location of work done during different months, and the same convention for a certain month is used throughout on all charts. This profile shows the method used in prosecuting the work, and is valuable in connection with the cost data, as it explains many things regarding unusual unit costs for any particular month. On a concrete bridge this profile will show the progress of the excavation, concreting, piling driven, together with any unusual stages of the river as stated in the preceding paragraph.

The progress charts (Figs. 12, 12A, 13) serve two purposes, to show the actual amounts of materials used and work completed and to give the costs of the same. They consist of parallel vertical columns which are filled with the particular kind of cross-hatching for the month in which the work was done. About two-thirds the way up from the bottom a heavy horizontal line is drawn through the columns to represent the estimated quantities and costs or $100 \%$. This line also represents the time for completion as shown in the contract. Then if there is estimated to be 1,000 cu. yds. of conerete to be poured, and the total time for completing the job is four months, if at the end of the second month there are only $250 \mathrm{cu}$. yds. in place, the cross-hatching in the quantity column under the heading of "concrete" will be advanced from where it was the previous month to a point $25 \%$ from the bottom line of the chart, while in the time column the same style of cross-hatching will be advanced to a point $50 \%$ from the bottom. This will show for that particular item we are only $25 \%$ completed, when to finish according to schedule time we should be $50 \%$ completed. On a large job with many items of nearly equal importance it requires judgment to state the stage of the work from these charts, for the reason that when our time is $50 \%$ gone several items may be $99 \%$ completed and others only $5 \%$, and the relative importance of the different items must be taken into areount. The principal value of the charts, however, is in showing the unit eosts from month to month, and their variation according to quantities, locations, weather, etc. The same variation in regard to eost may exist as in regard to time. That is several items may run $200 \%$ or even more above the estimated cost for that item, and yet the job as whole be completed below the estimate. If the unit costs as a whole are rumning close to the estimated costs, then the total actual cost column, when compared with the time column, shows a fairly accurate representation of the rate of progress.

Three separate charts are made for each job. The first, Fig. 12, is 
called the Labor Chart and contains only the items shown on the pay-roll sheets. While there are some labor items that never get on the pay-roll sheets, the reason this separation is made is because of the ease with which a check is kept on the costs. The timekeeper makes out a daily report showing the distribution of labor to each item listed on the chart. This is made to check the actual pay roll each week. It is easy for the timekeeper to keep an accurate record down to the nearest half hour for each man on each class of work.

\begin{tabular}{|c|c|c|c|c|c|c|c|c|c|c|c|c|c|}
\hline \multirow[t]{2}{*}{$\begin{array}{l}\text { TIME } \\
\text { EST }\end{array}$} & \multirow[t]{2}{*}{$\begin{array}{l}\text { PER } \\
\text { CENT }\end{array}$} & \multicolumn{2}{|c|}{$\begin{array}{l}\text { TOTAL } \\
\text { PAYROLL } \\
\text { SHEET } 2\end{array}$} & \multicolumn{2}{|c|}{$\begin{array}{c}\text { TOTAL } \\
\text { MATERIAL } \\
\text { SHEET } 3\end{array}$} & \multicolumn{2}{|c|}{$\begin{array}{l}\text { TOTAL COST } \\
\text { SUM OF } \\
\text { SHEETS 2.43 }\end{array}$} & \multicolumn{2}{|c|}{$\begin{array}{l}\text { PLANT } \\
\text { COST }\end{array}$} & \multicolumn{2}{|c|}{$\begin{array}{c}\text { GRANO } \\
\text { TOTAL } \\
\text { COST }\end{array}$} & \multirow[t]{2}{*}{$\begin{array}{l}\text { PER } \\
\text { CENT }\end{array}$} & \multirow[t]{2}{*}{$\begin{array}{l}\text { TIME } \\
\text { ACT. }\end{array}$} \\
\hline & & EST & ACT & EST & ACT. & $E S T$ & $A C T$ & EST & ACT & EST & ACT & & \\
\hline & 145 & . & & & & & & & & & & 145 & \\
\hline & 140 & & & & & & & & & & & 140 & \\
\hline & 135 & & & & & & & & 4082 & & & 135 & \\
\hline & 130 & & & & & & & & 3092 & & & 130 & \\
\hline & 125 & & & & & & & & & & & 125 & Jan. 1 \\
\hline & 120 & & & & & & & & 3.613 & & & 120 & \\
\hline & 115 & & & & & & & & \multirow{3}{*}{3286} & & & 115 & \\
\hline & 110 & & & & & & & & & & & 110 & \\
\hline & 105 & & & & & & & & & & & 105 & Dec 1 \\
\hline \multirow[t]{3}{*}{ Nov 21 } & 100 & 317,029 & & 329.726 & & $\$ 46,755$ & & 83.000 & & $\$ 49755$ & & 100 & \\
\hline & 95 & & & & & & & & & & & 95 & \\
\hline & 90 & & & & & & & & & & & 90 & \\
\hline \multirow[t]{2}{*}{ NovU } & 85 & & & & & & & & & & & 85 & Nov \\
\hline & 80 & & & & & & & & & & & 80 & \\
\hline \multirow{4}{*}{ Oct 1} & 75 & & & & & & & & & & & 75 & \\
\hline & 70 & & & & & & & & & & & 70 & \\
\hline & 65 & & 11187 & & & & & & & & & 65 & 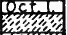 \\
\hline & 60 & & 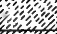 & & & & & & & & 29628 & 60 & 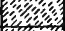 \\
\hline \multirow[t]{3}{*}{ Sept 1} & 55 & & 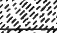 & & & & 25,546 & & & & 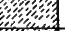 & 55 & 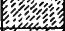 \\
\hline & 50 & & 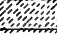 & & 14359 & & 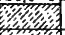 & & & & 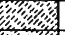 & 50 & 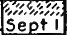 \\
\hline & 45 & & 7,768 & & 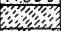 & & 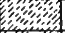 & & & & 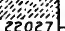 & 45 & \\
\hline \multirow{4}{*}{ Aug 1} & 40 & & & & 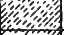 & & $\begin{array}{l}0 \\
18125\end{array}$ & & & & $\frac{20}{m}$ & 40 & \\
\hline & 35 & & & & $\begin{array}{l}0.3 \% \\
10.357\end{array}$ & & & & & & & 35 & \\
\hline & 30 & & & & & & & & & & 15790 & 30 & Aug I. \\
\hline & 25 & & 4686 & & 7491 & & 12177 & & & & & 25 & \\
\hline \multirow[t]{3}{*}{ July!. } & 20 & & & & (II & & & & & & & 20 & \\
\hline & 15 & & & & & & & & & & & 15 & \\
\hline & 10 & & 1680 & & & & & & & & 5992 & 10 & July I \\
\hline June 1 & 5 & & & & $\int_{1026}$ & & 2706 & & & & $m$ & 5 & $\mathbb{E}$ \\
\hline May 21. & 0 & & & & & & & & & & 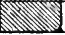 & 0 & $\begin{array}{l}\text { June I } \\
\text { Juln }\end{array}$ \\
\hline
\end{tabular}

Fig. 13-Summary of Labor and Material Expenditure and Plant Cost

The unit costs for each month are written in small figures in the total cost column. These vary considerably from month to month, and would occasionally be cause for alarm were it not for the explanation given by the progress profile. For instance, one month the cost per square foot, or per cubic yard, too, for erecting forms for sub-structure was three times as high as it was the month previous. Turning to the progress profile we see that on this particular month the tops of four piers only were finished which had small yardage and superficial area, but were so cut up with offsets, curved surfaces, coping, etc., as to 
be very costly as compared with the massive work of the month before.

While the second ehart, Fing. $12 \Lambda$, is called the Material Chart it really shows all other items of expense not shown on the Labor Chart except that of plant or equipment. As shown, there are three items that are not material; namely, general overhead expense, office expense and cost of insurance and bond. All other items are material items and are taken directly from the books for that job. All hauling, loading and unloading, etc., of material is charged to material directly and not as a labor item. The unit costs of the different items on the Material Chart give the costs placed on the job ready to be incorporated into the completed strueture.

The third ehart, Fig. 13, is merely a summary of the first two, together with the plant expense, chargeable to that job. The first cost, in the ease of new equipment, and the invoiced value in the case of second-hand equipment, is charged directly to each job as it is placed on the work. Then the sale price, if sold, or the invoiced value if moved to another job, is given as a credit, leaving the balance as the depreciation or cost of plant for that job.

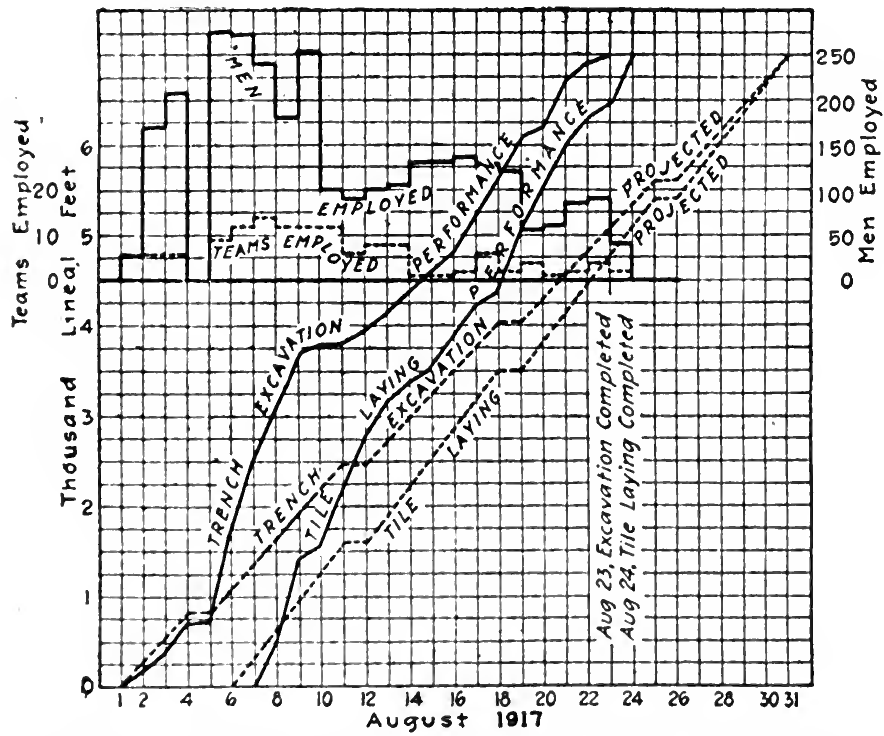

Fig. 14-Progress Chart, South Trunk Sewer, Camp Jewis

Sewer Work. Still another form of progress chart is shown in Figs. 14 and 15 . These, with the following description, are taken from Engineering Veus-lecord, June 13, 1918.

Progress charts on sewer construction at Camp Lewis, American Lake, Wash., were used with great success by the superintendent to keep the various divisions of the work balanced as to men and material, so that all the work would be eompleted at the same time. They were also used to gage the probable date of eompletion and in making 
reports to the contracting quartermaster and the contractors' advisory committee.

When the plans had been made, and as each division was released by the engineering department, a progress chart was immediately made up, a projected progress charted, and an estimate made of the men, teams and materials required. These were immediately assembled and the progress charted daily. September 1 was the projected date of completion in every case.

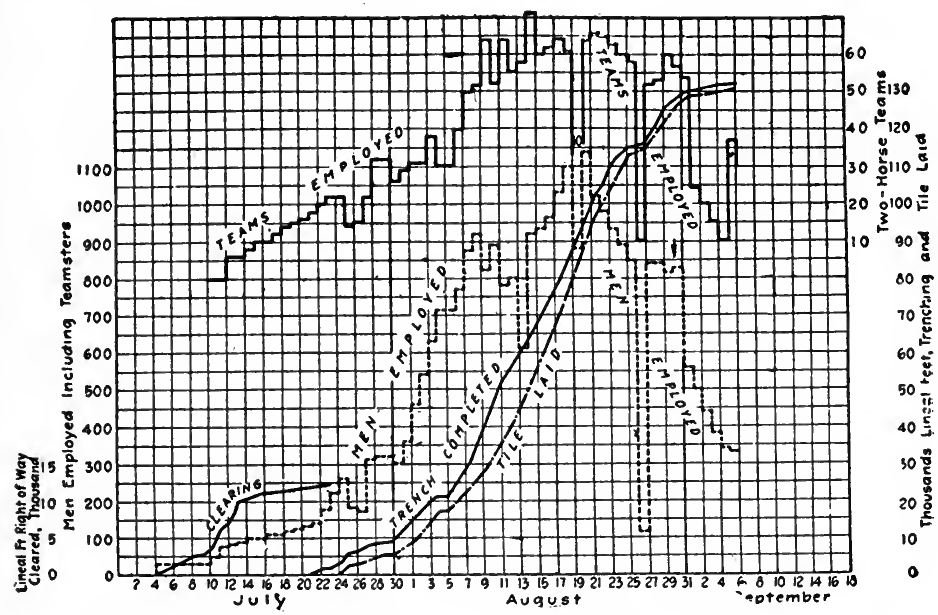

Fig. 15-Combined Progress Chart for Entire Sewer System, Camp Lewis

It was found as the work progressed that the line of progress on the chart indicated an earlier date of completion than that projected. After careful study of the charts a new date was set as Saturday night, $\mathrm{Au}$ gust 25, and the distribution of labor and material was made with this date in view. All work embraced in the original plans was completed on that date, the last piece of tile to make the connecting link on each division being laid on the same day.

Figs. 14 and 15 show the chart for the south trunk sewer and the combined progress chart for the whole system with the projected and actual lines of progress plotted. The distribution of men and teams was plotted on the same charts.

Transmission Line Progress Chart. Fig. 16 and the accompanying description are from Electrical Review, September 7, 1918.

In the erection and construction of transmission lines, traversing as they do in many cases long distances, the need for definite and yet concise progress reports is pronounced. Gangs of men often move from camp to camp with the progress of the line, only coming into town once a month or so. Materials have to be delivered where wanted, otherwise the cost of doing the work, delays and disorganization are bound to follow.

By drawing up a simple yet complete form of progress chart, which may be mailed or in some other way gotten into headquarters or office 


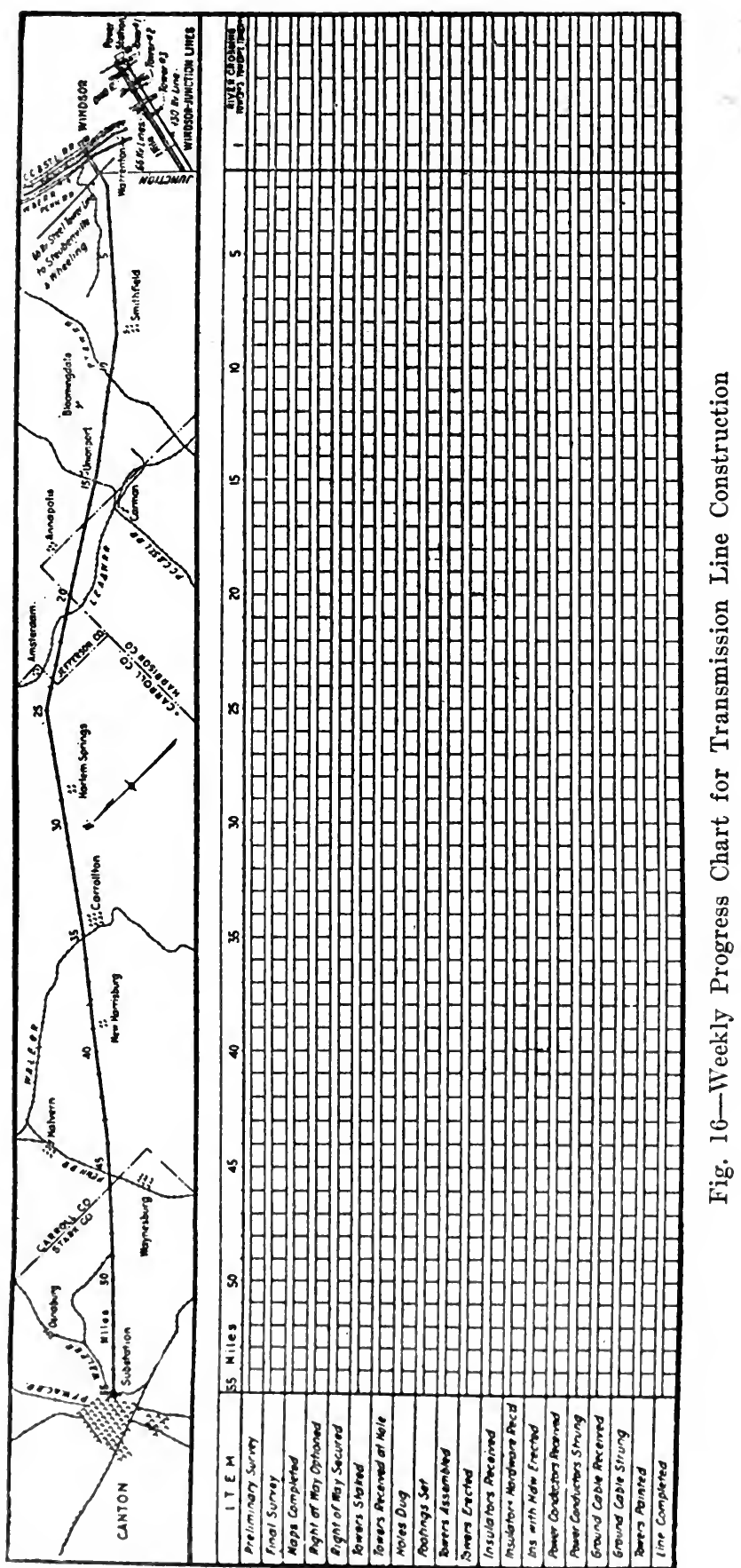


of the engineer, delays and loss of material can be avoided, and the work may be prosecuted with despatch at minimum cost. Fig. 16 shows part of one form of progress chart that is at once convenient, accurate and simple. To make it complete requires a minimum amount of effort on the part of the foreman and little actual' writing at that. Yet it tells the tale.

The chart covers one week's work. Progress obtained during the week is indicated by filling in the squares in blue, while the preceding progress is shown in red. This form of chart is about as fool-proof as it is possible to make such a report. A profile in addition to the map shown is also included in the progress chart, but this is not here shown.

Railway Scheduling. An excellent example of how clear a chart makes a comparison of schedules is given by C. W. Squier in Electric Railway Journal, March 21, 1914. In part, this is as follows:

The following comparison of operating conditions on three elevated lines which have the same equipment and service requirements is made from actual tests. It will serve as an example of the relation between running time and distance between stops:

$\begin{array}{cc}\text { Average Distance } & \text { Schedule Speed, } \\ \text { Between Stops, Ft. } & \text { M.p.h. } \\ 1859 & 13.5 \\ 1926 & 13.7 \\ 1972 & 13.8\end{array}$

A comparison of these figures shows how the schedule speed drops off as the distance between stops decreases. The line with the greatest distance between stops, $1,972 \mathrm{ft}$., has a schedule speed of $0.3 \mathrm{~m} . \mathrm{p} . \mathrm{h}$. greater than the line with 1,859 ft., or there is an increase of 0.3 m.p.h. for an increase of but $113 \mathrm{ft}$. between stops.

To follow the comparison further, I have drawn the four distancetime curves shown in Fig. 17. These are plotted with the same operat-

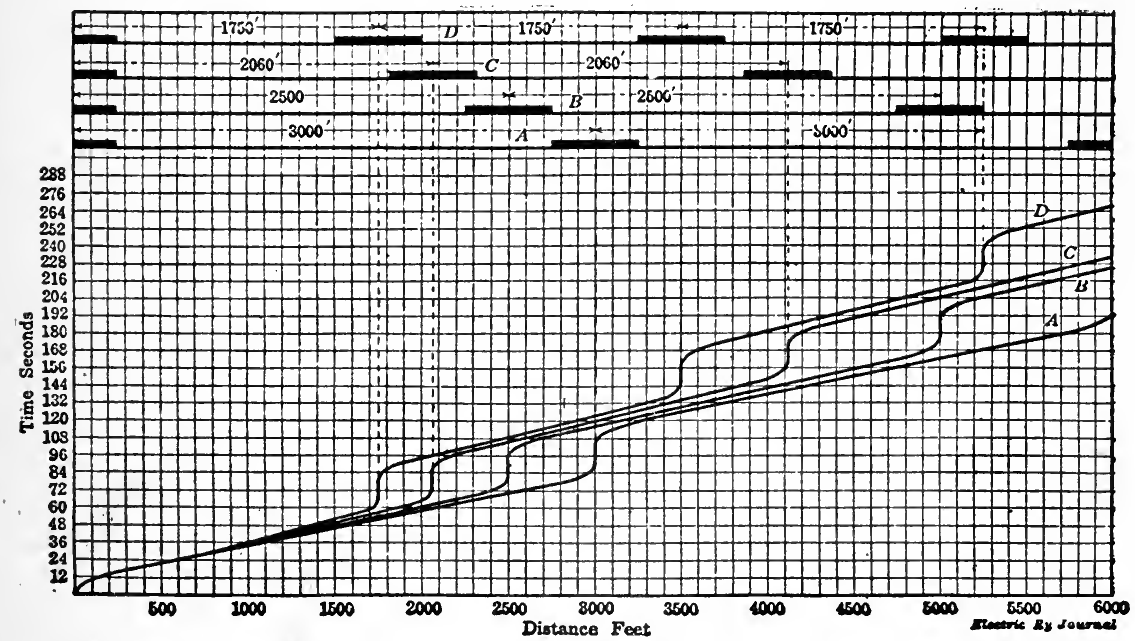

Fig. 17-Effect of Spacing of Stations 
ing characteristics so that the resulting running time is comparable with the distances between stops, which have been taken as 1,750 ft., $2,060 \mathrm{ft}$., $2,500 \mathrm{ft}$. and $3,000 \mathrm{ft}$. respectively.

If we assume that with any increase in the distance between stops a passenger will have to walk one-half this increased distance, and if we add this time to the time taken by the train, we find that a person traveling 7 miles will save $1 \mathrm{~min}$. 37 sees. when the distance between stops is increased from 1,750 ft. to $2,060 \mathrm{ft}$., $3 \mathrm{~min}$. 9 sees. when the distance between stops is 2,500 ft., and $4 \mathrm{~min}$. 5 secs. when the distance between stops is $3,000 \mathrm{ft}$.

A further betterment of the service will be brought about by the

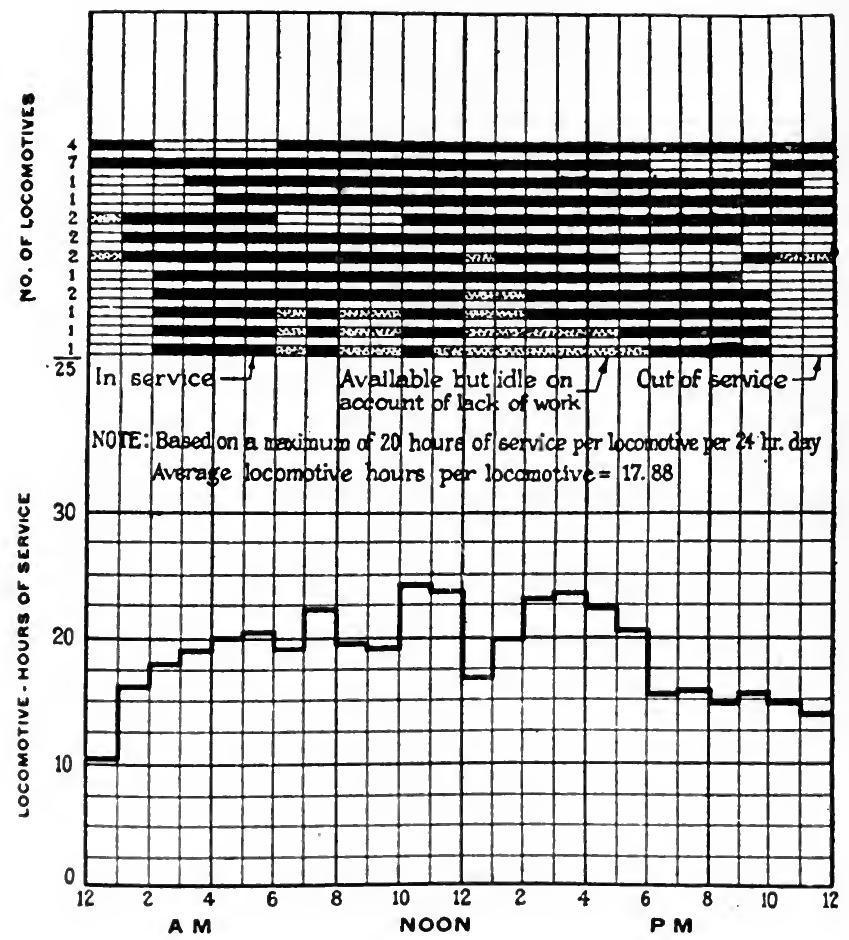

Fig. 18-Electric Switch Engine Requirements

decreased headway and increased number of passenger seats which would result from this greater distance between stops. With a line 10 miles long and stations spaced 1,750 ft. apart, seventeen trains would be required to maintain a $5 \mathrm{~min}$. headway. With the distance between stops increased to $3,000 \mathrm{ft}$. the same number of trains would be able to maintain a 4-min. headway. Thus the time between trains and the consequent delay occasioned by waiting for them is reduced one minute. This saving will offset some of the extra time taken to walk the increased distanee.

This decreased time between trains will give three more trains per 
hour passing any point on the line. If we assume that each train is capable of seating 500 passengers, then by increasing the distance between stops we would provide 1,500 more passenger seats per hour and thus add to the comfort of travel.

So far I have referred only to such points as might be of advantage to the traveling public. The operating company would also be benefitted by an increased distance between stops. Energy consumption would decrease as the total number of stops would be less, and the rheostatic losses during acceleration would be proportional to the number of stops. Finally, for the same service the same headway could be maintained with fewer cars. This would meàn less capital invested and lower maintenance costs.

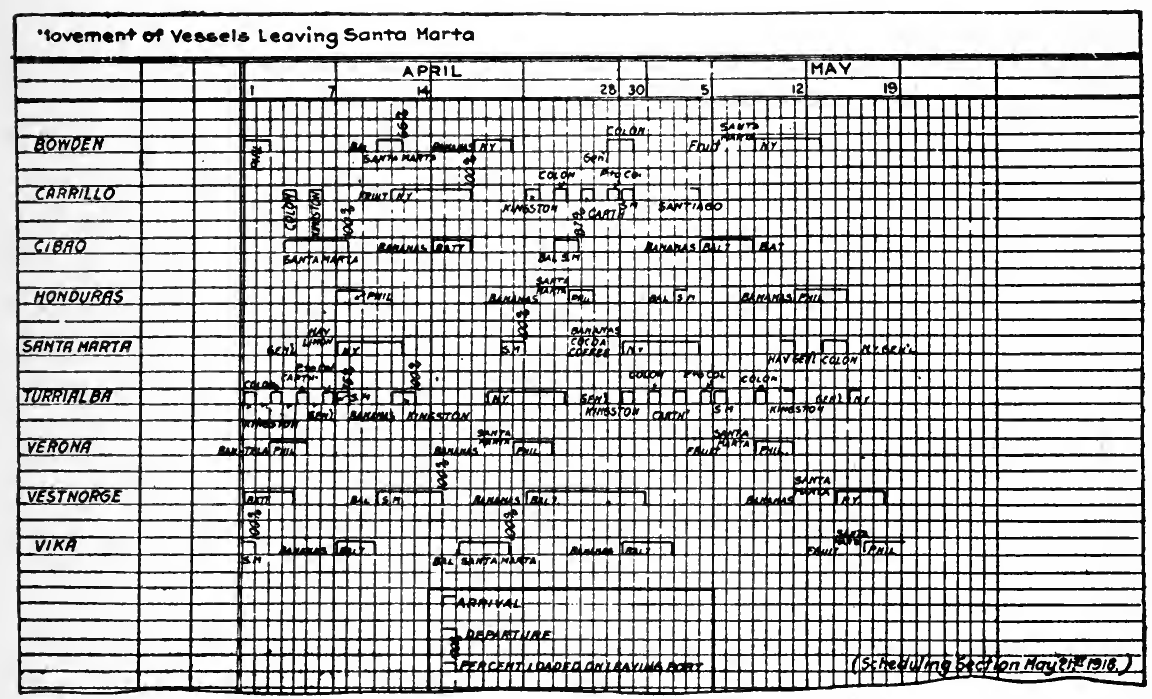

Fig. 19-Specimen Movement Sheet for Ships

Scheduling Equipment Requirements. The following is from Electrical Railway Journal, December 11, 1915 :

Locomotive-hour diagrams (Fig. 18) have been worked out for eąch road, and the number of electric locomotives required to handle the yard and transfer service of the road in question has been determined from these.

Scheduling Merchant Ships. L. P. Alford, in Industrial Management, September, 1918, very interestingly describes how the U. S. Shipping Board employed the H. L. Gantt progress charts to control the ship movements and the handling of their cargoes during the war. The methods described not only are just as valuable now that peace has come but also they are applicable, with the necessary variations, of course, to other industries where the control of outside moving units is essential. A portion of the article follows:

Simplicity is the keynote of these straight line charts and the feature 


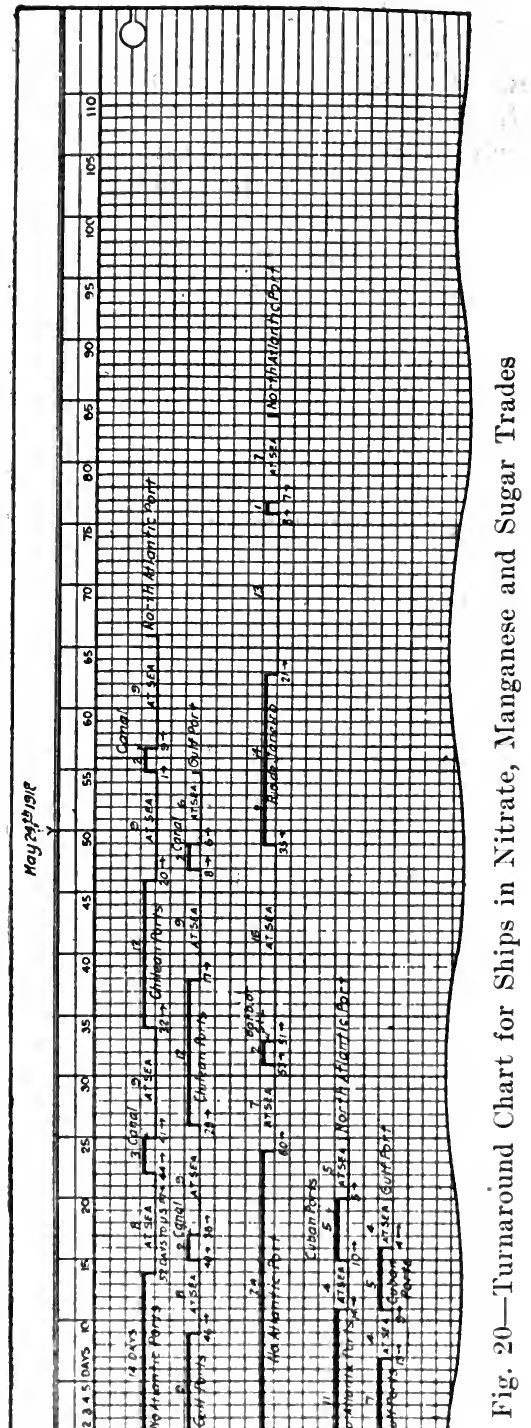


which distinguishes them from other charts is the fact that they indicate progress both as to time and amount. They show:

What the job is.

When it is to be begun.

When it is to be finished.

What progress has been made in the past.

What progress is likely to be made in the future.

These are not the methods of men who have spent their lives in shipping circles, but of an engineer who tackles the problem of world-wide shipping just as he would plan putting an order through a machine shop.

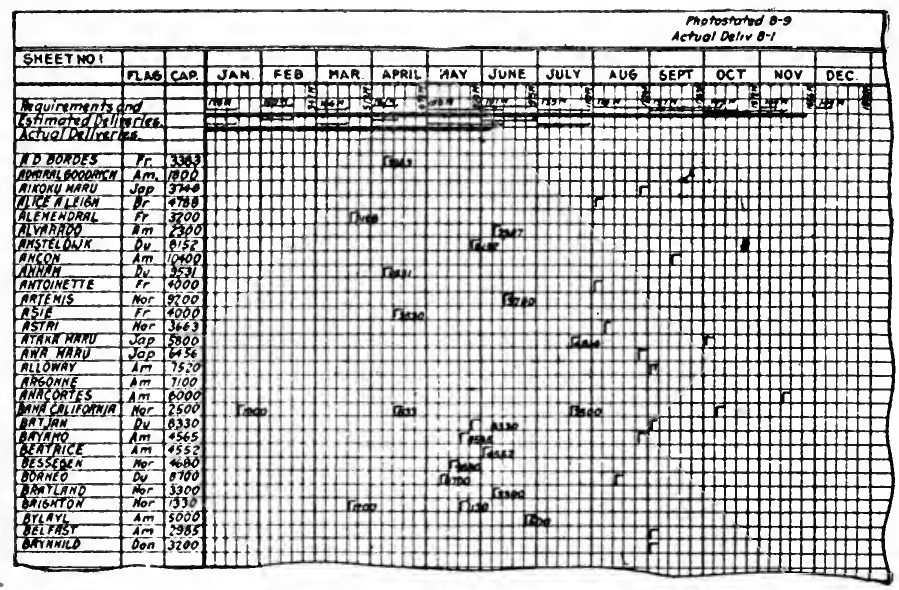

Fig. 21-Specimen Ship Chart of Commodities

Ships are of no use whatever except for the cargoes they deliver, and no two ships ever do a thing in the same way. They must be handled individually - not in bunches. In the Shipping Board, records are kept on ship movement charts of more than 11,000 vessels. Ships of every nationality are shown moving in and out of the nearest and most remote ports. They move up and down the Atlantic from Reykjavik to Tierra del Fuego, and from Antofagasta on one side of the Pacific to Vladivostock on the other. The chart illustrated in Fig. 19 traces the movements of a few vessels in the fruit trade between Santa Marta, Colombia, and the United States. The key in the lower right corner shows the meaning of the plotted symbols. The ports at which the vessels touch, the time they spend in those ports, the time at sea, and the cargoes they carry, are all shown clearly and compactly.

The purpose of these movement charts is to keep in one place all information in regard to locations and movements of vessels-the cargoes delivered depend on the movements of the vessels. The information entered on these records comes from various sources and in various forms: the daily report of the Naval Communication Service gives movements in all ports of the United States and its possessions; cables, some daily and some weekly, give locations of vessels in foreign ports. Every available source is drawn upon for this information. 
Other government departments have cordially co-operated with the Shipping Board in securing information as to cargoes carried by the ships. The Shipping Board was experiencing considerable difficulty in securing the necessary, prompt information as to movements of commodities when the Department of Commerce and the Treasury Department came to its aid and offered the services of their seasoned and world-wide organizations. Hundreds of thousands of cargoes have been checked in and recorded by them and the information forwarded to the Shipping Board daily. They have even gone further and have digested the information and at ten-day and monthly intervals they furnish summaries to assist in planning the operation of our shipping.

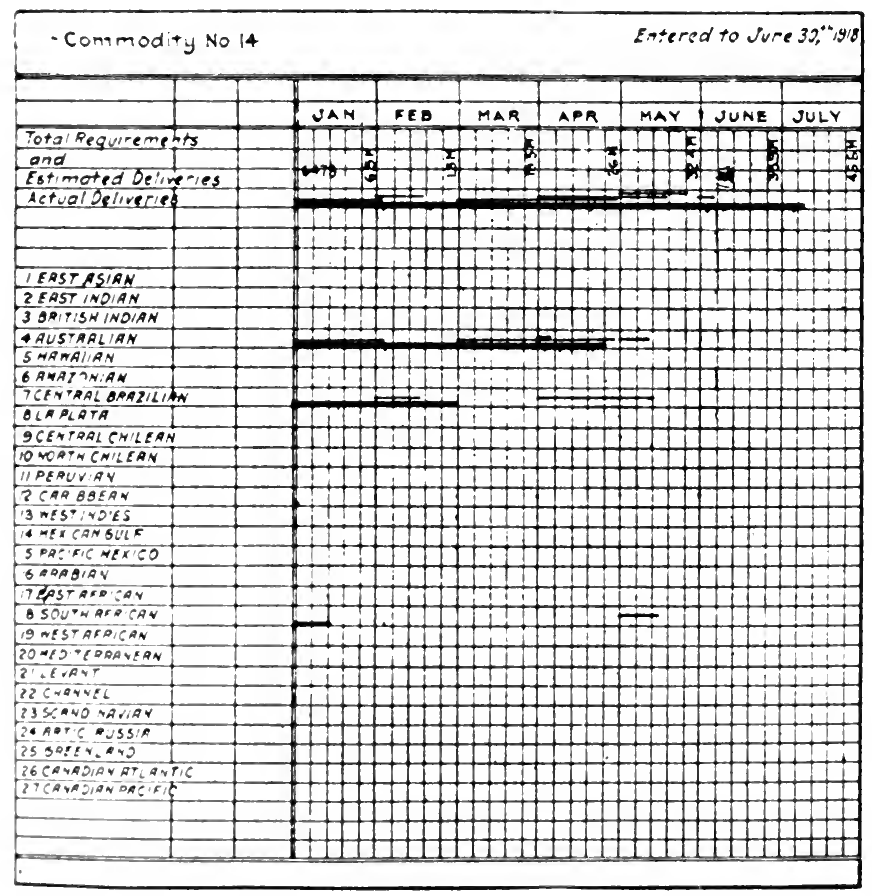

Fig. 22-An Individual Commodity Chart

The round trip, or "turnaround" as it is called in shipping circles, is the time it takes a vessel to do a task set for it-i.e., the interval between the delivery of one eargo and the delivery of the next. Fig. 20 illustrates the turnaround of vessels in the nitrates, manganese and $\mathrm{Cu}$ ban sugar trades. This ehart is, of course, based on the actual performance of vessels in these trades and its purpose is to furnish a basis for forecasting the dates on which vessels will deliver their eargoes. The figures above the lines indicate the time consumed in the various parts of the voyage (the delays in ports become immediately apparent) and the figures below the lines tell how many days are likely to elapse before the vessel delivers her eargo. 
The chart illustrated in Fig. 21 connects the individual vessels and the cargoes they deliver with the task that has been set. The angles opposite the various vessels indicate the time of delivery of cargoes; the figures in the angles indicate the tons of cargo delivered. The angles which contain no figures indicate the time of future arrivals, based on turnaround charts. In the absence of information as to the amount of cargo to be lifted, the capacity of each vessel, as shown at the left of the chart, is taken as the amount she will deliver at the time indicated by the angle.

At the top of the sheet, the horizontal figures show monthly requirements; vertical figures show cumulative requirements; light lines indicate monthly deliveries (the ratio of the length of the line to the

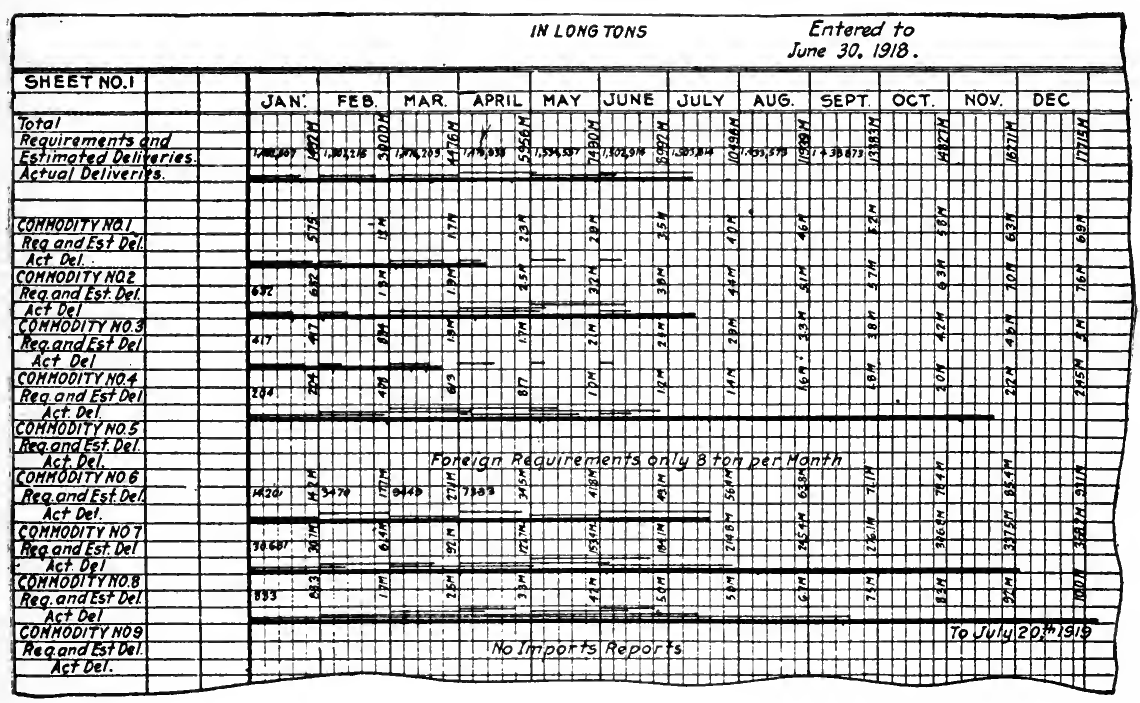

Fig. 23-Summary of Commodities Showing State of Deliveries

monthly space is the same as the ratio of the delivery to the requirement); heavy lines show to what extent our present estimated or actual imports will meet our requirements. The purpose of this chart is to show the progress made toward meeting the requirements and to show the effect of allocating vessels to any particular trade or taking them off that trade.

The number of commodities which it is considered necessary to import in view of the shortage of ships, is less than 100. For each one of these commodities there is a chart similar to the one shown in Fig. 22 on which are plotted the amounts received from the 27 trade regions into which the world has been arbitrarily divided, all plotted on the same scale as the total. This chart was developed for the use of the experts who had determined the requirements so that they might be kept informed as to the import situation.

Fig. 23 illustrates a few of the hundred necessary commodities placed one above the other. This shows shortages of some commodities and 
of others, over-deliveries equalling many months' requirements. This chart makes it easy to eompare the progress made on a large number of imports and presents the facts in such a way as to induce action.

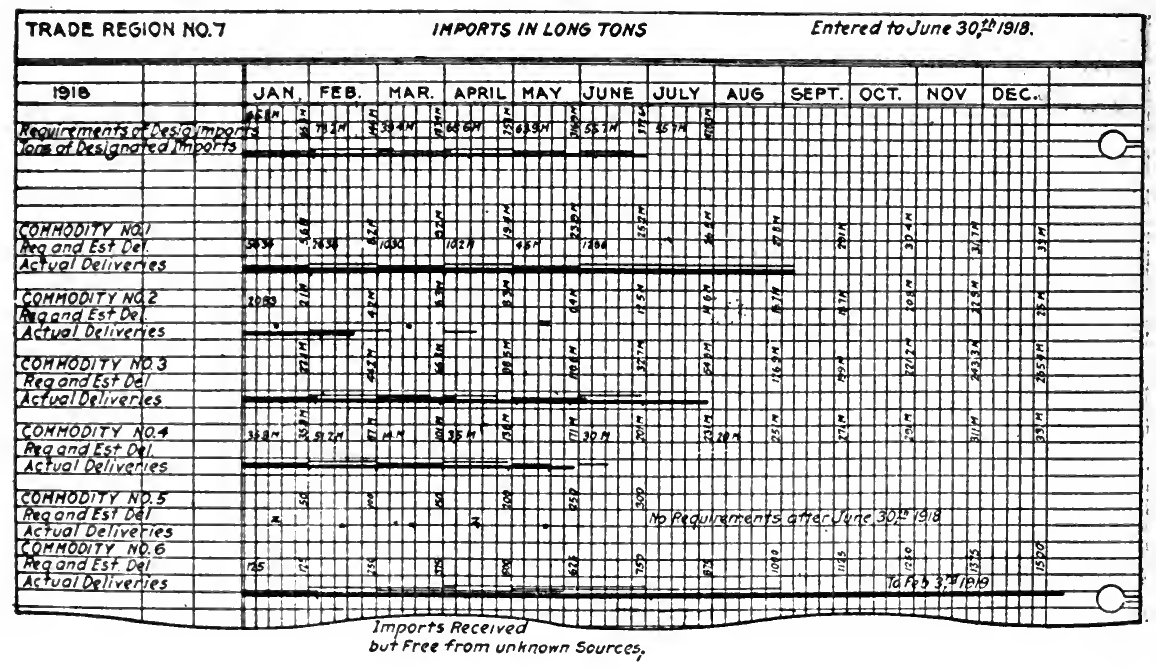

Fig. 24-Summary of Imports for a Trade Region

At the top of the sheet the import situation as a whole is shown in a single line.

From trade region No. 7 (see Fig. 24) there are only six commodities which it is considered necessary to import. The progress made in im-

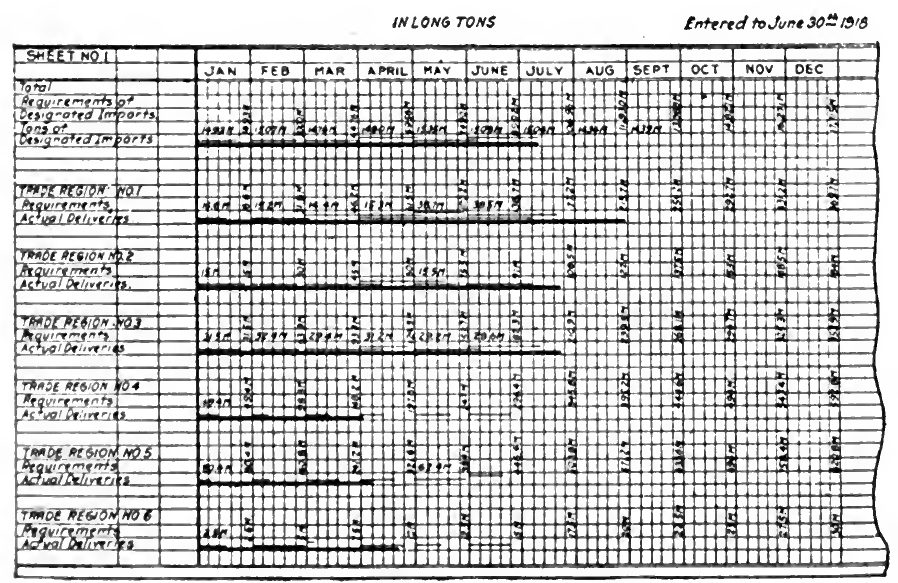

Fig. 25-Summary Chart for Essential Commodities

porting these commodities from this particular trade region is plotted here. Inerualities berome very apparent in this arrangement. The individual commodity charts showing the amounts of each commodity 
received from all parts of the world are studied with these trade region charts in order to get comparisons two ways. At the top of the sheet the situation in this trade region as a whole is plotted.

Fig. 25 shows the situation in a few of the 27 trade regions. The

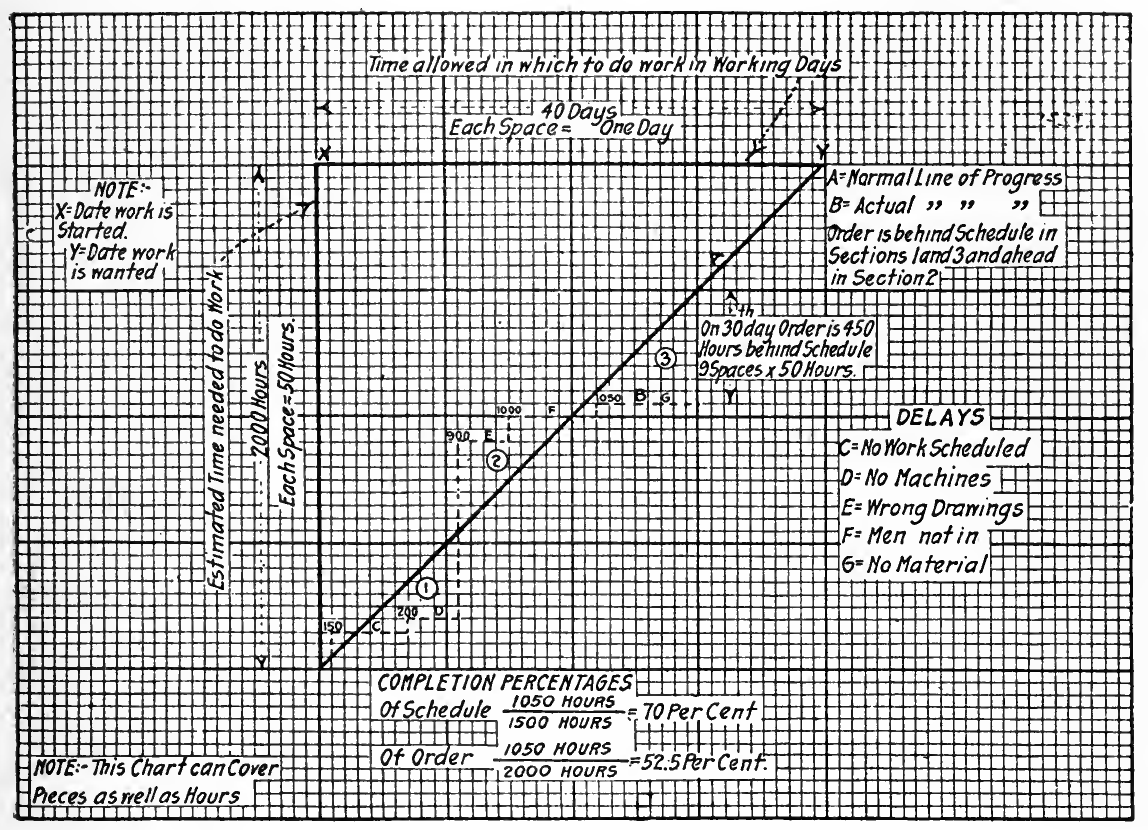

Fig. 26-Angle Graph Showing How Progress is Watched

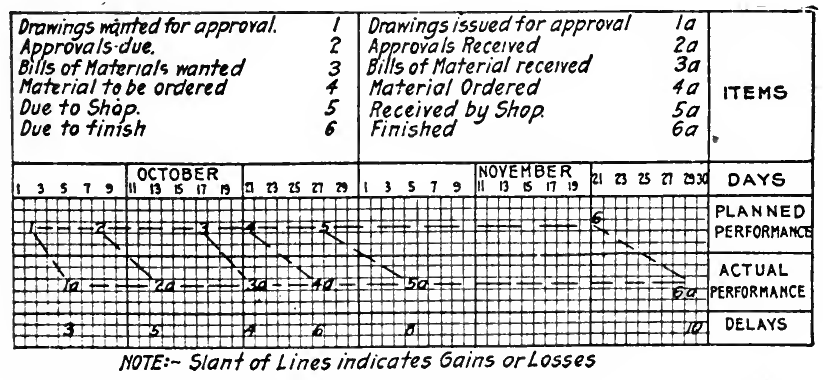

Fig. 27-Showing Method of Graphically Following Up Important Features

NotE-This method is especially useful in plants where orders, from receipt to completion, pass through a larger number of departments.

first three show considerable over-deliveries while each of the second three is, however, from two to three months behind its requirements. It is evident that the next vessels arriving from the first three regions should be transferred to those regions in which the shortage is the most 
acute. The line at the top of the sheet shows the progress made on the trade regions as a whole and is the same as the line on the "Summary of Imports." It shows whether or not the vessels at the disposal of the Shipping Board are sufficient to bring in the necessary imports.

This export problem differs only in detail from the import problem and similar charts set forth the facts.

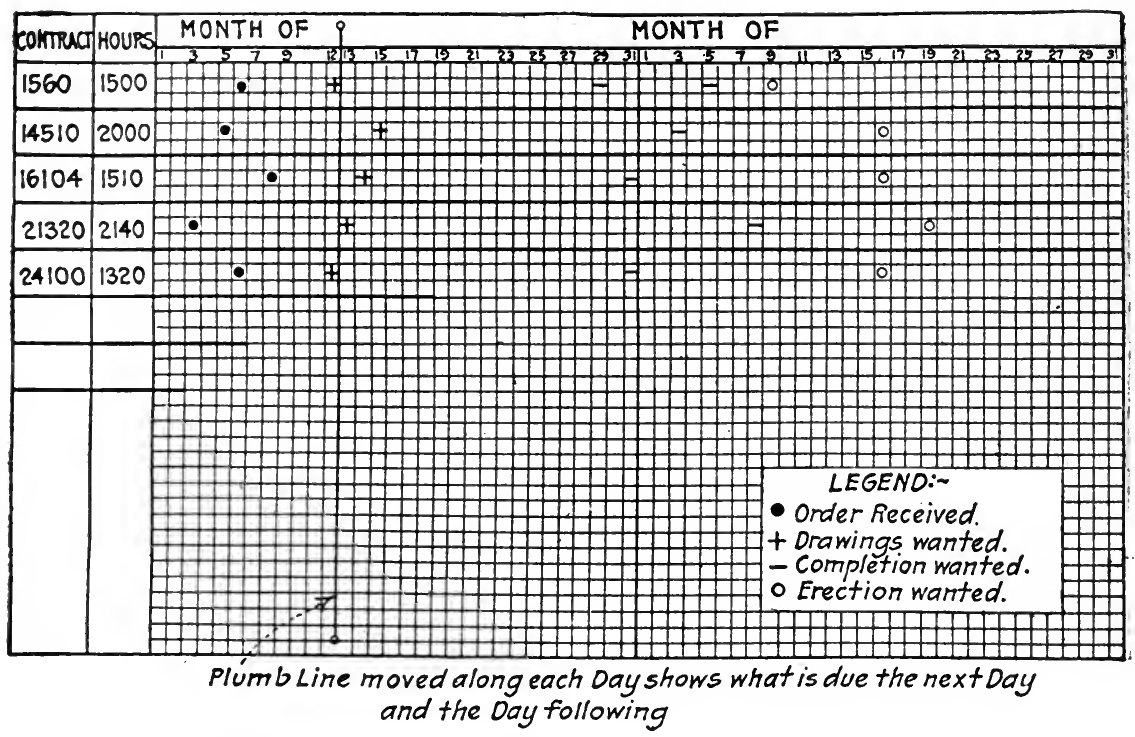

Fig. 28-Control Showing Important Things to Follow Up

Graphic Production Control. Under this head C. E. Knoeppel wrote a series of six papers which were published during consecutive months. in Industrial Management, beginning September, 1918. Space does not permit quoting them but they present an exceptionally complete and instructive exposition of the use of charts in connection with scheduling and progress, and it is suggested that the reader go over the articles carefully if they are available. Figs. 26 to 34, which offer many suggestions for the profitable use of such charts, have been reproduced.

Following, is a list of articles eontaining further illustrations of the use of graphic charts of the elass discussed in this chapter and pertaining to kindred subjects.

Cpon refuest, the Codex Book Co., Ine., 19 William Street, New York, will be glad to procure, where possible, copies of magazines containing the articles needed, only charging the "old magazine" price, plus postage.

Schedules-"How to Make Them Work," Arthur Van Vlissingen, Jr., Siystem, September, 1918.

"How Construction Work is Scheduled," Fred R. Lufkin, 'Building Age, November, ${ }^{1917 .}$ 

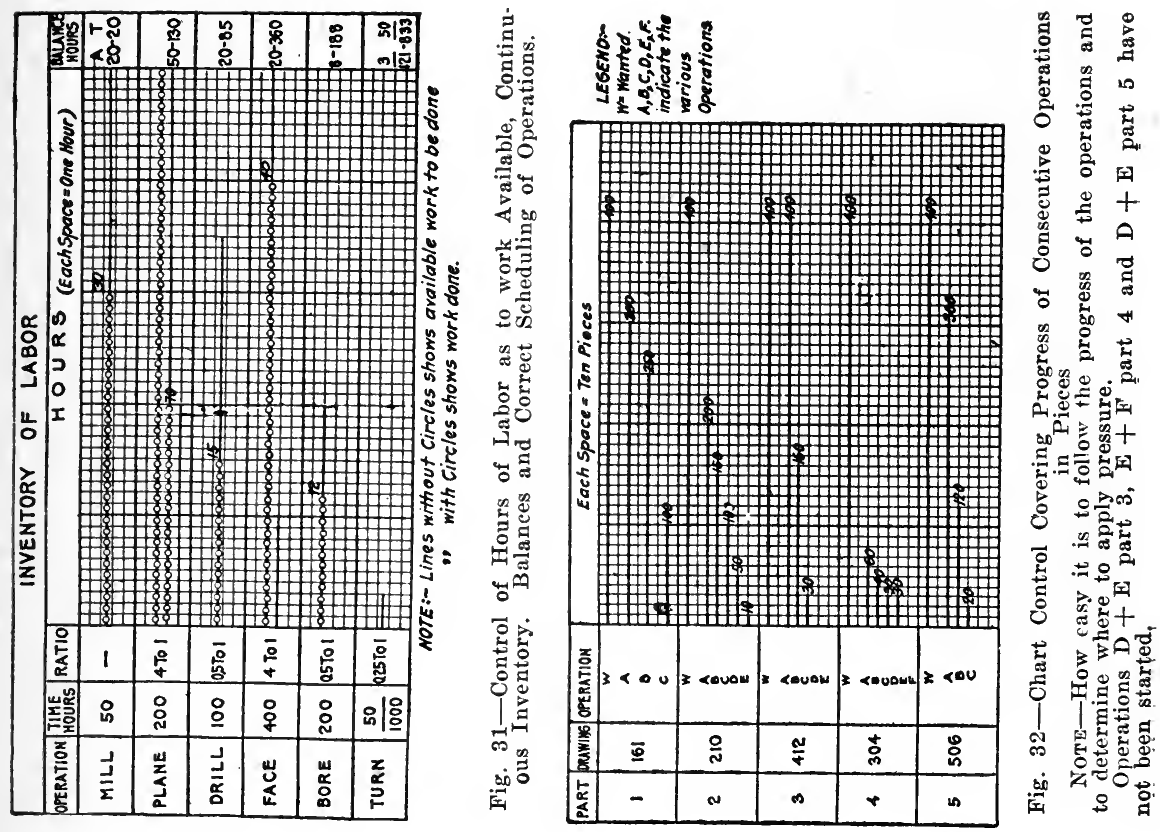

In!

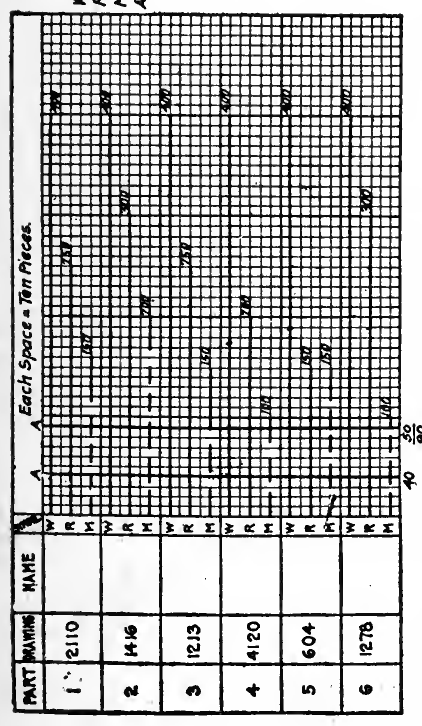

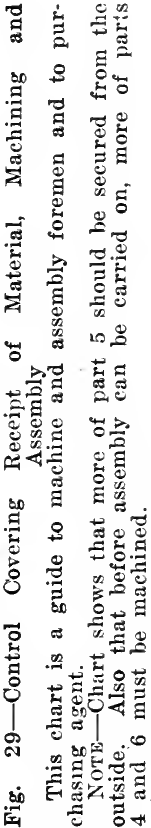

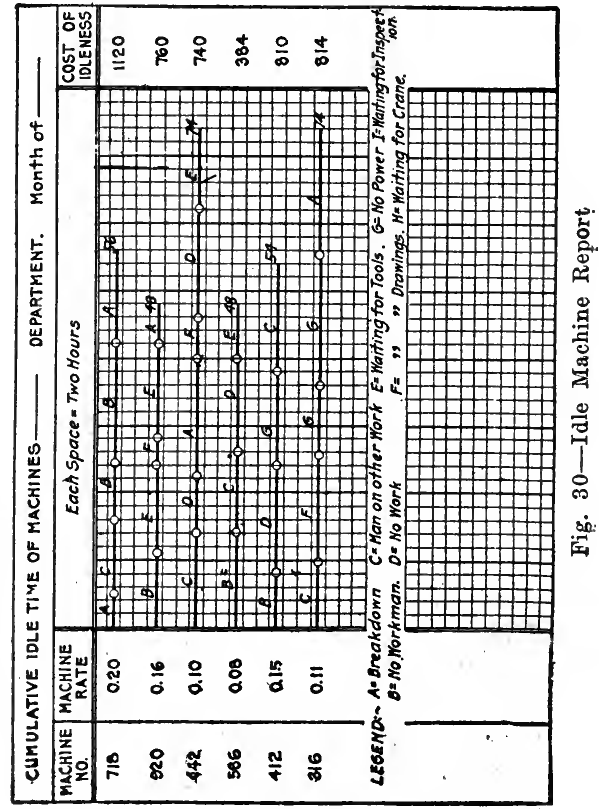




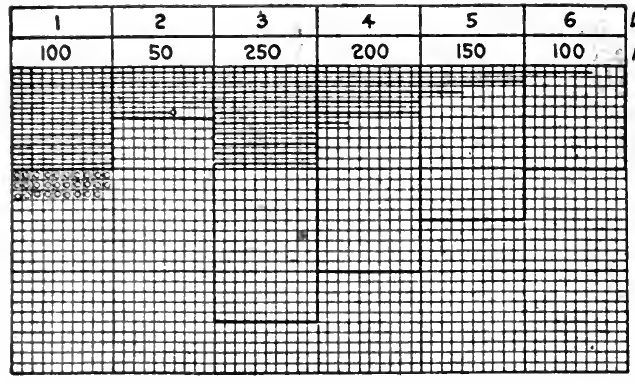

OEPARTMEAT OROPERATION HOURS ESTIMATED

\section{IMPOATANT:- \\ Costs can be inserfed on \\ Chart by Multiplying Hours by Proper Rates. \\ ESTIMATE: iAsshomb by Heary Lines. \\ PAOARESS. \\ Department I Finished \\ " 20 \\ " 3 100 Hours \\ 04530 \\ (1) 5250}

Fig. 33-Ccntrol Showing Estimated Times, Actual Times, and Progress

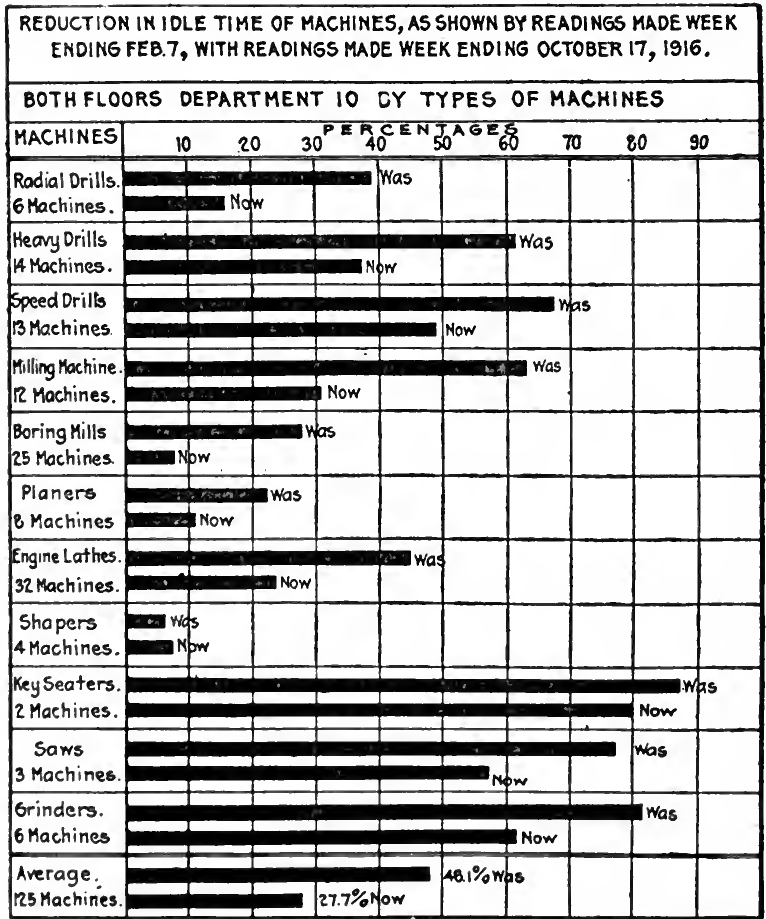

Fig. 34-Department 10-Idle Time Report, Showing Compari. sons

"The Organization of Manufacturing Plants, A. D. C. Parsons, Iron Age, Decembrer 16, 1916.

"The Practical Introduction of Efficiency Principles," (Chapter VIII), Series I-XII, C. E. Knoeppel, Engineering Magazine, January, 1914, December, 1914.

"Graphic Production Control," Series I-VI, C. E. Knoeppel, Industrial Management, September, 1918, to February, 1919. 
"An Industrial Achievement of the War," L. P. Alford, Industrial Management, February, 1918.

"Being Sure of Your Delivery Dates," D. G. Robinson, Factory, September, 1918.

"Charts Show Monthly Progress and Expenditure on Road Work," H. S. van Scoyoc, Engineering Record, April 15, 1916.

"Field Progress Diagram," Railway Engineering, July, 1913, P. 337.

"Making and Filing Valuation Maps," James G. Wishart, Engineering Record, February 24, 1917.

"Efficiency Chart of Car-Loading Gang," Engineering News, September 10,1914 . 


\section{CHAPTER XIII}

\section{Operating Characteristics}

In the first part of this chapter are presented examples of charts which show how equipment operates, that is, they give a graphical picture of performance. Such charts are of great value not only as showing what the equipment will do under the conditions examined, but also as furnishing a method for determining what it will do under different conditions. They also frequently show up opportunities for improving the operating efficiency of the apparatus in question.

The latter portion of the chapter deals with the operating characteristics of plants as a whole.

Operating Characteristics of Distributing Transformers and Squirrel-cage Motors. In an article, "Capacities of Transformers for Induction Motors," published in Power, July 24, 1917, G. P. Roux describes the operating characteristies of these two kinds of equipment and an excerpt follows:

In industrial power installations supplied from transformers connected to distribution lines, the question of capacity to be provided arises almost daily and no fixed rules, other than mere speculative estimates, are available for the solution of these practical problems. It is therefore of interest to study and discuss this question in order to more conveniently meet the requirements of each application. Inasmuch as nearly $90 \%$ of the induction motors in use are of the squirrel-cage type, its performance and also that of the standard distribution transformer will be analyzed, taking as a typical example an installation with a 30 -hp. 220-volt three-phase 60-cycle 900-r.p.m. constant-speed induction motor with a starting compensator.

To supply power to moderate-sized industrial installations, ordinary standard distribution transformers are generally used, the power ratings of which are given in full-load kilovolt-amperes. The voltage rating of the primary is adlapted to some standard distribution-line voltage, commonly 2,200 volts, and the ratio of voltage transformation is 20 to 1,10 to 1 or 5 to 1 according to the requirements.

The actual line voltage is, as a rule, somewhat different from the standard line voltage and varies according to the line losses, which are in most cases partly compensated for at the power house or substation by boosting the voltage of the outgoing lines. The secondary voltage if the transformers connected to distribution lines therefore varies from place to place and throughout the day aceording to the uncompensated line drop. Sometimes it is possible to give the secondary voltage a further arljustment when the transformers are provided with regulation taps, a practice that is gradually being abandoned by many manufacturer's because of complieations and difficulties in the manu- 
facture of the apparatus which increase its cost and decrease its reliability.

The kilovolt-ampere rating of a transformer does not necessarily present its effective rating in kilowatts. The latter will vary according to the power factor at which the apparatus operates, but the kilovoltampere load supplied by the transformer will govern its operating characteristics such as heating, efficiency and regulation, all of which is shown in Fig. 1, for a standard 10-kv.-a. 60-cycle distributing transformer, having a 2,200-volt primary and 110 and 220-volt secondary.

At constant kilovolt-ampere output, normal primary voltage and frequency, the available kilowatt output decreases in proportion to the power factor, the efficiency slightly decreases owing to the reduction in effective capacity; hence, the input slightly increases. The regula-

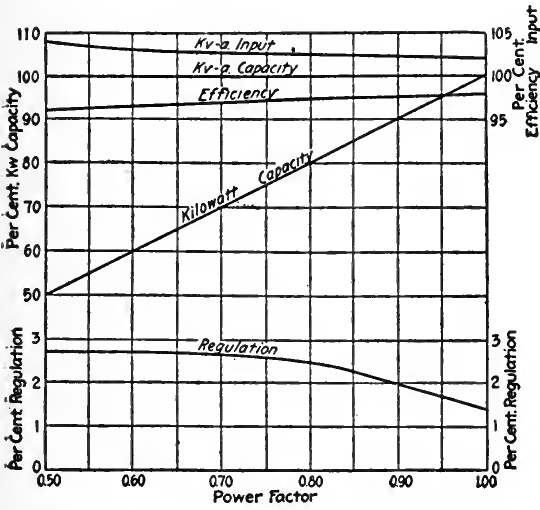

Fig. 1-Performance of 10-Kv.-a. Transformer at Constant Kv.-a. and Different Power Factors

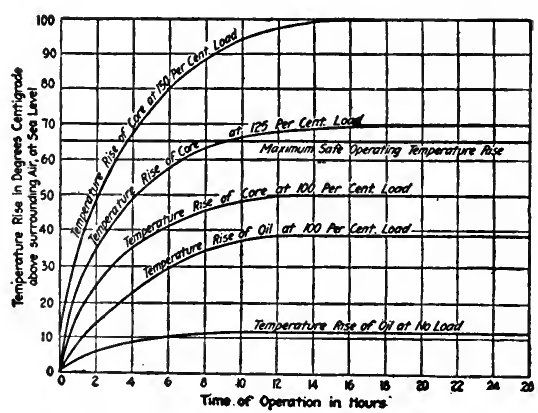

Fig. 2-Temperature Rise of Oil and Core of $10-\mathrm{KV}$.-A. Transformer

tion, or percentage reduction of secondary voltage, increases as the power factor decreases.

The full-load kilovolt-ampere rating of a transformer is not the maximum load that the apparatus can safely carry, which depends on its internal losses' and details of construction affecting its cooling capacity. Most of the standard transformers are designed and built to carry $125 \%$ full load for two hours and $150 \%$ full load for one hour without injurious heating. However, if the overload is applied after a long period of operation at full load, the heat developed may become injurious to the insulation and to the oil, if maintained for a sufficient length of time and repeatedly. The overload limitations of a transformer are merely a matter of heating and heat dissipation. In the absence of means to determine the exact temperature of the windings, it follows that for safety of operation the amount of overhead and the length of time a transformer can be operated above its normal rating must be limited to the periods and overload values already given, in order not to exceed a temperature rise in the copper and iron of more than $65^{\circ}$ C. above that of the surrounding air, taken for reference at $40^{\circ}$ C., as recommended in the Standardization Rules of the A. I. E. E. 
A typical diagram of the heating performance of the standard 60-cycle distribution transformer is shown in Fig. 2. The oil never reaches the temperature attained by the copper windings or core, therefore thermometer readings of the temperature of the oil do not give the temperature of the windings. It will be noticed from Fig. 2 that after a certain lapse of time the temperatures become constant for a constant load.

While the eurves of Fig. 2 represent average conditions for indoor transformers operating at altitudes up to $3000 \mathrm{ft}$., and with a ratio by weight of oil to eore of 1 to 5 , the results vary according to the characteristies of each make of transformer, the quality and viscosity of the oil and atmospheric conditions. The outdoor transformer has greater cooling facilities than the indoor type. The limiting temperature rise being based on a surrounding temperature of reference of $40^{\circ} \mathrm{C}$., this allows a final maximum temperature of $105^{\circ} \mathrm{C}$., but if the ambient temperature is less than $40^{\circ} \mathrm{C}$. $\left(104^{\circ} \mathrm{F}\right.$.) -as for instance, in the winter-then the transformer ean be subjected to a greater overload, as long as the final temperature does not exceed the maximum of $105^{\circ} \mathrm{C}$. ( $221^{\circ} \mathrm{F}$.).

With a diagram similar to the one shown in Fig. 2 and referring to a particular type of transformer, it is possible to determine its heating characteristic and the amount of overload that it can safely carry for any period of time.

Variation in the voltage and frequeney of the supply line affects the operation of the transformer as summarized here:

\begin{tabular}{|c|c|c|c|}
\hline & Exciting Current & Regulation & Power Factor \\
\hline Iov & Decreased & Worse & Higher \\
\hline ge $\ldots$ & Increased & Improved & Lower \\
\hline ency $\ldots \ldots \ldots$ & Increased & Improved & Lower \\
\hline High frequency $\ldots \ldots \ldots$ & Decreased & Worse & Higher \\
\hline
\end{tabular}

The eonstant-speed squirrel-cage polyphase induction motor represents the type most generally used in alternating-current power installations. An induetion motor is in many ways similar in its operation to a transformer. It consists of a system of polyphase primary windings produeing a rotating magnetic field which is completed through the rotor, or seeondary, and provided with short-cireuited windings. The eonductors, being cut by the revolving magnetic flux, are eaused to move and rotate at the same synchronous speed (less the slip) as the primary system.

While the mechanical characteristies of an induction motor eompare very closely to those of a shunt-wound direct-current motor, its electrie and magnetic performances resemble those of a transformer with an air gap between the primary and seeondary. This eauses a eonsiderably greater magnetizing current and leakage reactance, which in turn decreases the power factor.

An induction motor, like a transformer, ean be designed with its characteristics so adjusted as to meet a great variety of requirements. The improvement of one characteristic is, however, obtained to the detriment of another, and unless intended for special duties, the polyphase induction motors manufactured in this country have the average performances shown in Fig. 3. These performances are naturally sub- 
ject to slight departures according to the make, type, speed and voltage of the motor.

When standing still the induction motor is like a transformer with its secondary short-circuited; therefore it takes a considerable current when connected to a source of voltage. The value of this current depends on the applied voltage and impedance of the motor. Reducing the voltage or increasing the impedance reduces the value of the starting current. This is a very important item, as it affects the source of supply of power and the operation of other apparatus connected thereto.

The starting current taken by an induction motor is equal to $\frac{E}{Z}, E$ being the applied voltage and $Z$ the total impedance of the motor. The current rises rapidly upon closing the circuit to start the motor and falls gradually as the speed increases and becomes finally steady

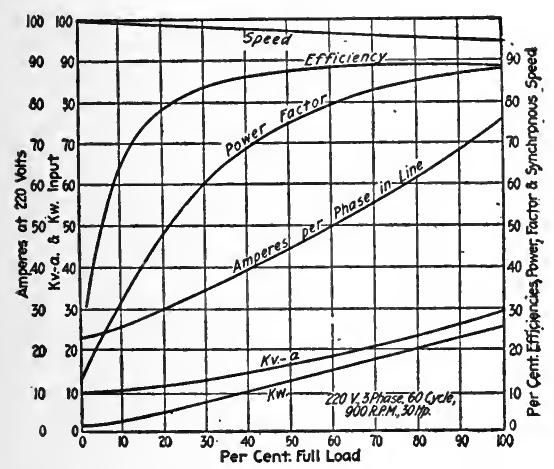

Fig. 3-Induction-Motor Characteristic - Curves

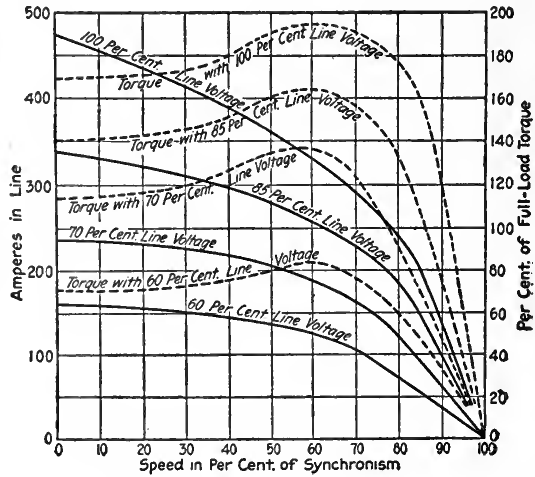

Fig. 4-Starting-Speed Torque Curve of 30-Hp. Three-Phase Motor on Four Different Voltages

when approximate synchronous speed is reached, rising subsequently as the mechanical load is increased. The starting torque is directly proportional to the square of the impressed voltage and the secondary or rotor resistance, and inversely to the impedance.

In Fig. 4 are shown graphically the starting performances of a 30-hp. 220-volt three-phase motor, from standstill to full synchronous speed, when started by means of a starting compensator connected on different voltage taps. The dotted curves indicate the values of the torque for the different percentages of line voltage. Both the starting current and the starting torque depend on the connections made at the auto-starter, the rotor resistance remaining unvaried. The object of the auto-starter is to reduce the line voltage and increase the current by auto-transformation. This apparatus generally consists of a number of single phase windings connected either open-delta or star and provided with taps giving different voltages below that of the line. The coils are designed for intermittent operation only and made to carry a current density considerably greater than in an ordinary auto-transformer in 
order to reduce their size to a minimum, which is permissible, as they do not remain in the circuit after the motor is started.

It is general practice to start a squirrel-cage motor on one low-voltage tap on the compensator; after the motor reaches near synchronous speed, it is thrown on full-line voltage. The ratio of starting voltage to line eurrent is given in the following table:

$\begin{array}{llrrrrrrr}\text { Line voltage } \ldots \ldots \ldots \ldots \ldots \ldots \ldots \ldots & 100 & 100 & 100 & 100 & 100 & 100 & 100 \\ \text { Per cent. tap and voltage at motor } \ldots & 40 & 50 & 58 & 65 & 70 & 80 & 85 \\ \text { Per cent. full-voltage starting current.. } & 16 & 25 & 34 & 42 & 50 & 65 & 72\end{array}$

The performance of a $30-\mathrm{hp}$. motor from standstill to full synchronous speed, against a starting torque equivalent to two-third full-load torque, is shown in Fig. 5. It is seen that at the moment of starting $61 \mathrm{kv}$.-a. is taken by the motor. The kilovolt-ampere input, however, gradually decreases and then rapidly falls as the speed increases, to a value for full speed and no load represented by the exciting current plus the friction and windage losses, or about $9 \mathrm{kv}$.-a., but with a very low power factor. The power factor improves as the mechanical load is applied and reaches about 0.87 at full load, as shown in Fig. 3 .

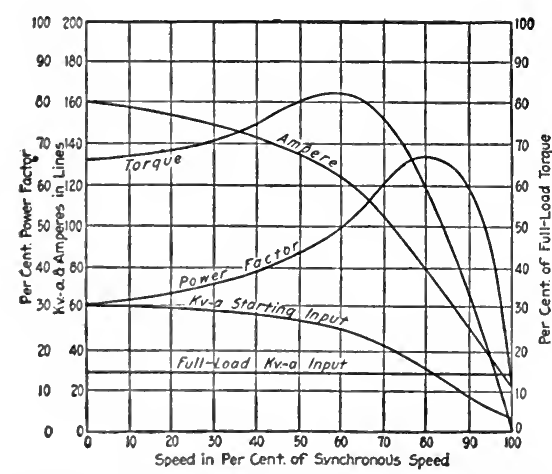

Ficr. 5-Starting Characteristics of 30 IIp. Induction Notor at $58 \%$ Normal Voltage

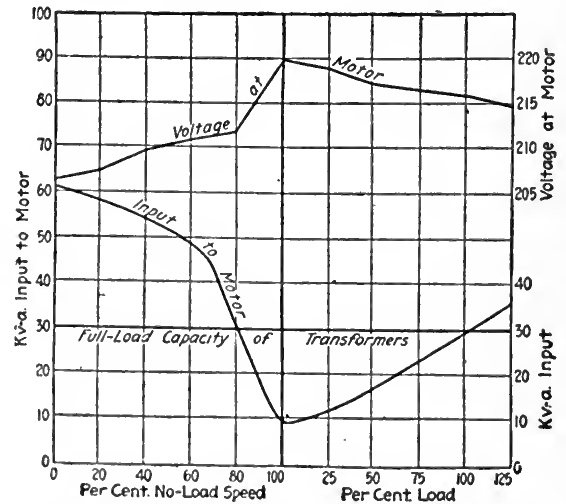

Fig. 6-Starting and Operating Kv.-a. Requirements of $30-\mathrm{Hp}$. Notor

Assuming that three $10-\mathrm{kv}$.-a. transformers have been provided to supply power to the motor in question, it will be seen from Fig. 5, that for starting duty they will be subjected to a load of $203 \%$ their full rated callacity. However, this overload will not remain for more than from one-fourth to one-half a minute each time the motor is started, the load on the transformers thereafter increasing from $9 \mathrm{kv}$-a. at no load to $29 \mathrm{kv}$-a a at full-rated load.

Inder normal starting and operating conditions the kilovolt-ampere load drawn from the transformers in question and the secondary voltage are shown in Fig. 6. Both eurves indicate that it is safe to provide in such ases transformer eapacity at the rate of one kilovolt-ampere for each horsepower of rated capacity of the motor, even if the motor is to operate oceasionally with overload, provided the overloading periods do not exceed the heating guarantees of the transformers, as shown in Fig. 2. 
In installations where more than one motor is supplied from the same bank of transformers, an investigation of the duties of the motors must be made to determine the demand factor, or ratio of maximum combined demand to transformer capacity, together with the length of time of maximum demand, and the transformer capacity provided accordingly.

Taking, for instance, an installation consisting of one 30-hp., one 10- and two 5-hp. motors, not to be started at the same time, and normally operating with an average combined demand of $50 \%$; assuming the 30 - and 5-hp. motors to operate at $125 \%$ load two hours a day and the 10-hp. motor to start and stop frequently, and a fairly constant supply voltage and frequency, the combined operating conditions can be analyzed and tabulated as follows:

\section{KILOVOLT-AMPERE REQUIREMENTS}

\begin{tabular}{|c|c|c|c|c|c|c|c|c|}
\hline \multirow[b]{2}{*}{ Motor } & \multirow{2}{*}{$\begin{array}{c}\text { Full } \\
\text { Load } \\
\text { Rating }\end{array}$} & \multicolumn{4}{|c|}{$\multimap$ Starting Periods $\neg$} & \multicolumn{3}{|c|}{ Operating $\mathrm{Pe}$} \\
\hline & & 1 & 2 & 3 & 4 & Normal & Overload & $\begin{array}{l}\text { and } \\
\text { Starting }\end{array}$ \\
\hline $1-30$ hp.. & . 30 & 60 & 9 & 9 & 9 & 15.0 & 37.5 & 37.5 \\
\hline $1-10$ hp............ & 10 & 0 & 20 & 3 & 3 & 5.0 & 5.0 & 20.0 \\
\hline $1-5$ һp......... & 5 & 0 & 0 & 15 & 1.5 & 2.5 & 6.25 & 6.25 \\
\hline $1-5$ hр......... & 5 & 0 & 0 & 0 & 15 & 2.5 & 6.25 & 6.25 \\
\hline Cotal kv.-a..... & 50 & 60 & 29 & 27 & 28.5 & 25.0 & 55.00 & 70.00 \\
\hline
\end{tabular}

The maximum instantaneous load is $70 \mathrm{kv}$.-a., the maximum twohour load is $55 \mathrm{kv}$.-a. and the normal load is $25 \mathrm{kv}$.-a. only. The maximum starting load is $60 \mathrm{kv}$.-a.

Three 15-kv.-a. transformers, or a total of 45-kv.-a. transformer ca$25 \times 100$

pacity, would supply this installation, earrying a load of - or 45

$$
55 \times 100
$$

$55 \%$ under average conditions, $\frac{}{45}$ or $121 \%$ for two hours at 45

$70 \times 100$ time of overload and $\frac{X 1}{45}$ or $155 \%$ at times of overload during

the brief but frequent periods of starting the 10-hp. motor. The temperature rise of the transformers under the worst condition would not exceed $65^{\circ} \mathrm{C}$. The demand ratio or capacity of transformer to be pro$45 \times 100$

vided in the case eited is therefore or $90 \%$ of the horsepower 50

rating of the installation.

Knowing the torque $t$ required to start a certain load and the fullvoltage starting torque of the motor $T$, and also the line voltage $E$, the voltage $E^{\prime}$ necessary to start the motor is

$$
E^{\prime}=E \sqrt{\frac{t}{\iota^{\prime}}}
$$

For instance, if the starting torque $T$ of a 30 -hp. motor at full voltage 
is twice its full-load torque, the line voltage, $E, 220$ and the torque $t$ required to start the motor with its connected load equal to only $66 \%$ of the full-load torque, the starting voltage necessary to develop the; required torque is

$$
E^{\prime}=220 \sqrt{\frac{0.66}{2}}=220 \times 0.575=126 \text { volts }
$$

that is, sufficient torque will be developed by connecting the motor to $60 \%$ taps of the auto-starter, and the line current is reduced to about. $36 \%$ the full line-voltage starting current.

Where the starting torque required by the motor to start its connected load (which should be reduced to the minimum consistent with the circumstances) cannot be conveniently ascertained, the motor can be connected first to the lowest tap of the starting compensator. If insufficient torque is developed on that tap, the next higher one can be tried until the right tap is found.

In installations requiring motors to start with an appreciable load, it is preferable to use the slip-ring motor, which is provided with a wound rotor having its winding connected to a set of collector rings, thus permitting increasing the resistance of the rotor for starting duties. The effect is to decrease the starting current while increasing the starting torque and to bring the motor under load to speed without excessive. strain on the transformers and supply line.

Variations of voltage and frequency affect all induction motors; their performances vary as follows:

\begin{tabular}{|c|c|c|c|c|}
\hline & Torque & Slip & Power factor & $\begin{array}{l}\text { Efficiency } \\
\text { Gay slightly }\end{array}$ \\
\hline & Dec & Increased & & May sl \\
\hline & Increased & Decreased & Decr & Increase \\
\hline$\ldots$ & Increased & No change & Decreased & or \\
\hline uency & Decreased & No change & Increased & Decrease \\
\hline
\end{tabular}

In general, slight variations of either voltage or frequency are in most eases not objectionable, provided, however, these variations do not exceed $10 \%$ and occur in opposite directions; that is to say, a lower voltage should coincide with a lower and not with a higher frequency and vice versa, otherwise the effects mentioned in the table would be cumulative instead of practically compensating each other. However, a variation in voltage of $10 \%$, or in frequency of $5 \%$, is not desirable for the successful operation of either transformers or motors in any installation.

Operating Characteristics of Centrifugal Pumps. There follows an article under this title presented in Power. February 3, 1914, by A. B. Morrison. Jr., which is a good example of how valuable the operating characteristics curves may be for figuring out the effects of various factors upon output, ete.

While e'sitrifugal pumps are in general use in nearly all lines of work there is still, among many engineers, a laek of knowledge as to what their performance will be under anything but the conditions for which they happen to be installed. The man who has to operate the pump or is responsible for its operation is not particularly concerned with the reasons which prompted the designer to use an impeller with 
certain dimensions or with a certain blade angle, but he is interested in the effect which certain variables, such as speed and head against which the pump is to work, will have on the eapacity if he wants to use the pump in some other location.

He knows the centrifugal is not a positive pump but he is not able, usually, to forecast with any degree of accuracy how fast he ought to run the pump under new conditions to do certain work. It is generally stated that with a centrifugal pump the capacity varies as the speed, the head genterated by the pump as the square of the speed, and the power required to drive the pump as the cube of the speed. While this is true theoretically of the pump and also under certain operating conditions, it does not mean, as is sometimes supposed, that to get an increase, for example, of $5 \%$ the pump should be speeded up $5 \%$, since the eapacity discharged, as will be shown later, depends on certain characteristics of the system to which the pump is connected.

It is also desirable to know what effect throttling the discharge will have on the efficiency of the pump and whether it is better to use, where feasible, some form of speed control in preference to throttling.

It is absolutely essential, in investigating the behavior of a centrifugal pump in operation, to know the head curve of the system to which the pump is to be connected and for that reason the head curve of the system will be considered before attempting to do anything. with the centrifugal-pump curves proper. The head, which includes both suction and discharge, against which the pump works may be any one of three distinct classes: (1) Head entirely static; (2) head entirely friction; (3) head part static and part friction.

The first case seldom occurs in practice. If the pump were under a constant suction head, without any connecting piping, and the outlet of the pump were connected, without any piping, direct to a large standpipe of such size that practically no friction existed in it and in which the water, due to an overflow pipe, were at constant level, we would have the first case. In this instance, the head curve would be a straight horizontal line. Under similar conditions, no piping on suction or discharge, but with the standpipe gradually filling, the head curves would be represented by a series of horizontal lines taken at whatever distances apart were felt advisable to get the data required as to speed, capacity, etc., on the pump.

The second case mentioned is not common, either, but occurs more frequently than the first. It would be represented in practice by a level pipe with the suction and the discharge at the same level. The head curve under such conditions is shown in curve $E$, Fig. 7 . In laying out such a curve the friction head at the rating of the pump, which we will call $100 \%$, is obtained from friction tables, showing the loss in head at the rated capacity of the pump through the size of pipe used. This gives one point on the curve, the capacity being represented always by abscissas (horizontal scale), and the head by ordinates (vertical scale). The friction head varies as the square of the velocity which, with a given pipe, is equivalent to the square of the quantity discharged. The following table shows the friction heads at less than rating, all quantities being in percentage of those at rating: 


$\begin{array}{llllllllrrr}\text { Capacity, } \% \ldots \ldots \ldots & 90 & 80 & 70 & 60 & 50 & 40 & 30 & 20 & 10 \\ \text { Head, } \% \ldots \ldots \ldots \ldots & 81 & 64 & 49 & 36 & 25 & 16 & 9 & 4 & 1\end{array}$

The third case is the one which almost invariably occurs. In laying out this eurve the ordinate at zero discharge is the actual elevation through which the liquid is to be raised. At any other rate of discharge the friction in the pipe, based on the quantity of water flowing, is computed and the ordinate at this discharge is the static head plus the friction head. The general form of this curve is shown in eurve $A$, Fig. 7 , where it is assumed the head is based on $20 \mathrm{ft}$. of friction at the normal discharge (1000 gal.) of the pump. At any other point the friction head is computed from tables or can be readily aseertained in terms of the friction head at rating by using the table just given. At $125 \%$ of rating the friction head is $156 \% \%$ greater than at normal and at $150 \%$ of rating is $225 \%$ more than at normal, or based on $20 \mathrm{ft}$. of friction head at normal eapacity these quantities are $31.25 \mathrm{ft}$. and $45 \mathrm{ft}$., respectively, to be added to the static head to secure the total head.

The characteristic curves of a centrifugal pump, as shown in Fig. 8, are more or less familiar to all engineers. While these eurves differ for various makes and types, their general form is eharacteristic of all centrifugals. They are plotted for constant speed. One important fact to be noticed is that the head given by the pump depends entirely on the amount of liquid being pumped; or, to state it the other way around, the pump will discharge against a given head only a certain amount of water. The only way in which the head developed by the pump for a given discharge can be increased is by speeding it up. The discharge, as will be noticed, ean be entirely closed without harming the pump.

In Fig. 7 the quantity-head curve of the given pump is shown together with the head curve of the system, both in heavy lines. For ease of eomputation the capacity at normal rating is taken as 1000 gal. per min. and the total head as $100 \mathrm{ft}$., eomposed of $80 \mathrm{ft}$. static and 20 $\mathrm{ft}$. friction. Suppose, however, that it is desired to move the pump to a new location in which the static head is increased to $100 \mathrm{ft}$., the friction head at rating remaining unchanged, giving a total head of $120 \mathrm{ft}$., and it is desired to have the pump discharge the same amount as formerly, the new head curve $B$ is obtained. Since the interception of the quantityhead curve of the pump with the head curve of the system gives the quantity of water which the pumo will discharge it is evident that at the original pump speed only $76 \%$ of normal discharge will take place. To determine the new speed at which the pump must operate to give the head of $120 \mathrm{ft}$. necessary to discharge 1000 gal. per min., the pressure given by the pump is taken as proportional to the speed at which it operates. 'This is approximately true and close enough for all practieal purposes. The relation then holds

\section{0) (head at normal speed) : 120 (head required) : : given rovolutions squared : required revolutions squared}

IIence the refuired r.p.m. efuluals $109.5 \%$ of the original r.p.m., or the pump should be speeded up practically $10 \%$, to meet the new eonditions and discharge practically the same amount of water as at first. The new quantity-hearl curve of the pump at approximately $10 \%$ increase in speed 
is shown by the dotted curve $B_{1}$ in Fig. 7 . It has the same general form as the original.

If it is assumed that the static head on the pump is decreased to $60 \mathrm{ft}$., the friction head remaining the same, and the total head being $80 \mathrm{ft}$. in place of $100 \mathrm{ft}$., the new condition can be met in two ways. To meet the new head conditions by changing the speed of the pump, the former

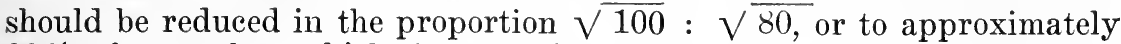
$90 \%$ of normal at which the quantity-head curve at rating will exactly balance the computed head curve of the system and the pump will discharge its normal quantity of liquid. Curve $C$, Fig. 7, shows this curve of the pump at the reduced speed.

It is apparent, however, that this new condition can also be met by allowing the pump to operate at normal speed and by throttling the discharge so that only the head necessary to force 1000 gal. per min. through the system will be available, the rest being wasted in friction in the throttle valve. A comparison of these two methods will be taken up in detail later.

There is a further interesting fact brought out by these curves. It has been mentioned that the quantity discharged by a centrifugal pump is proportional to the speed, and that this is sometimes thought to mean that for a certain increase in speed the amount discharged is proportional to such increase. In Fig. 7, however, curve $E$ is a head curve corresponding to the first case, viz., entirely static ; curve $D$ corresponds to the second case, entirely friction; and curve $A$ is the third case. Taking curve $E$ where the head is constant for all capacities, an increase in speed of approximately $10 \%$ produces an increase of $21 \%$ in the quantity discharged. For curve $D$ the quantity discharged at $10 \%$ above normal speed is $10 \%$ more than normal or the increase is exactly proportional to the speed, and this is the only case where the relation holds.

In curve $A$, where the head is partly static and partly friction, an increase in speed of approximately $10 \%$ produces an increase in the volume discharged of $15 \%$. Furthermore, the only case where the head on a pump for a given discharge is exactly proportional to the square of the speed and the discharge is proportional to the speed is for the second case where the head is entirely friction. Hence, as stated earlier, the capacity discharged by the pump and consequently the head depend entirely on the head of the system against which the pump works.

So far, only the speed necessary to obtain a certain discharge under new conditions has been considered and no investigation made of the efficiency of the pump nor the power required to operate it. Unless this be known, there is the possibility of the motor or shafting being overloaded.

An approximate method for obtaining this horsepower, and one which is accurate enough for ordinary calculations, is as follows: The quantity in gallons per minute discharged at the new speed is divided by the new speed taken in percentage of normal. From the brake horsepower curve in Fig. 8 the horsepower corresponding to this quantity just obtained is found and this brake horsepower is multiplied by the cube of the new speed expressed in percentage of normal speed. The result is the brake horsepower required at the new speed. Applying this rule to 
the results shown by the curves in Fig. 7, the following results are obtained:

For curve $B, 10 \%$ speed increase: Gallons discharged per minute, 1000 ; new speed in per cent of normal, $110 ; 1000 \div 1.10=909$; b.hp. for 910 gal. per min. (Fig. 8), $37 ; 1.10^{3}=1.331 ; 37 \times 1.331=49.25$ b.hp. required at 1000 gal. per min. and $120-\mathrm{ft}$. head.

B.hp. at 1000 gal. per min. and 100 -ft. head (normal rating) $=38.5$.

Increase $128 \%$ which is slightly less than the eube of the speed.

Applying the same methods to curve $C$, showing $10 \%$ decrease in speed, the b.hp. required is 29.2 , about $76 \%$ of that required at normal, slightly

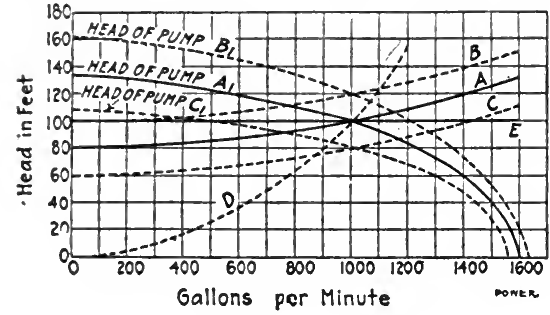

Fig. 7-Quantity-Head Curves of Centrifugal Pump

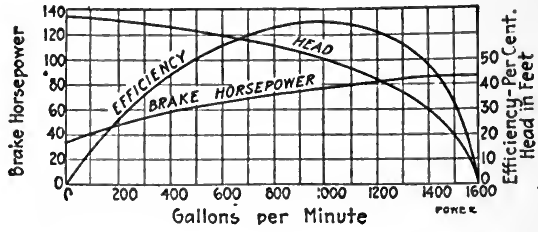

Fig. 8-Characteristic Curves of a Centrifugal Pump

more than if the power required were proportional to the eube of the reduced speed eompared to the cube of the normal speed.

It has been seen that at the reduced head the pump can be run at normal speed by throttling the discharge until just the proper amount of water flows. In this case, though, the brake horsepower required is the same as when discharging the same quantity at the higher head, since the pump generates this higher head at the given speed and capacity. The net efficieney when throttled is the efficiency at the normal rating multiplied by the ratio between the head actually required and the normal pump head generated. Referring to Fig. 8, when pumping $1000 \mathrm{gal}$. per min. the pump generates a head of $100 \mathrm{ft}$. and the efficiency is $65 \%$. The head actually required by the system, as shown in curve $C$, Fig. 7, is $80 \mathrm{ft}$. Hence, the net efficieney of the pump at normal speed when throttled to 80 -ft. head is

$$
0.65 \times \frac{80}{100}=52 \%
$$

As a check on this result the brake horsepower required at normal speed pumping 1000 gal. per min., as given by Fig. 8, is 38.5. The water horsepower recpuired at 1000 gal. per min. and $80-\mathrm{ft}$. head is

$$
\frac{1000 \times 80 \times 8.33}{33,000}=20.2
$$

Hence the efficieney

$$
\frac{\text { water horsepower }}{\text { brake horsepower }}=\frac{20.2}{38.5}=52 \%
$$

as just given. 
With a variable-speed direct-current motor the proposition of securing the correct speed for any given set of conditions is easy and the motor efficiency and the overall efficiency of the equipment are unimpaired. Where it is necessary, however, in order to get any change in speed, to use resistance in series with the rotor, it is interesting to compare the combined efficiency of the pump and the motor obtained by this method with that obtained by throttling alone. Referring again to Fig. 7, the speed required at the reduced head, curve $C$, at 1000 gal. per min. is approximately $90 \%$ of normal. The horsepower calculated above for this discharge at $90 \%$ of normal revolutions is 29.2 . In the case of a motor with resistance in series with the rotor, the efficiency at any reduced speed compared to that at normal speed with no resistance in the circuit is closely proportional to the revolutions at reduced speed compared with normal revolutions, so that reducing the speed to $90 \%$ of normal the efficiency is reduced correspondingly. In other words, at the reduced head

$$
\frac{29.2}{0.90}=32.4 \text { b.hp. }
$$

is required when resistance in series with the rotor is used.

Tabulating the results, the following comparison based on reducing head to $80 \mathrm{ft}$., as shown by curve $C$ in Fig. 7, is obtained. Each percentage gives the per cent. of brake horsepower required at rating:

B.hp. required by rotor control $\ldots \ldots \ldots \ldots \ldots . . .32 .4=84.4 \%$

B.hp. required by field control on motor.........29.2 $=75.8 \%$

B.hp. required by throttling discharge $\ldots \ldots \ldots \ldots . .38 .5=100 \%$

In other words, by using resistance in series with the rotor there is a saving of 6.1 b.hp., or $15.8 \%$, over throttling, and by using some form of variable-speed direct-current motor the saving is 9.3 b.hp., or $24.2 \%$. It is evident that where the head is likely to be less than normal for considerable periods and where the cost of power is important, some form of speed control is necessary. The variable-speed direct-current motor, as shown, is by far the best proposition. Armature control is sometimes used, but not often, as usually a variation of $10 \%$ or, at the most, 15 , will take care of most installations and that can usually be obtained from any standard shunt-wound motor. With alternating-current motors, throttling is generally resorted to, owing to the special character of alternating-current variable-speed motors unless the installation is of fairly large size.

In working out curves for any given pump, the results obtained at various heads and rates of discharge will depend entirely on the characteristics of the particular pump and these may differ decidedly from the one used here as an illustration. If the pump has a steeper characteristic-i.e., if the head falls off more rapidly as the discharge increasesthan the one shown, the difference in volume for any given change in speed will be less than for the curve given, whereas, with a flatter characteristic the change in volume for a given change in speed will be greater. With a flat characteristic, the head against which the pump 
works must be closely calculated in order to run the pump at the proper speed.

Wherever feasible, and especially if the pump is of large size, the writer wishes to emphasize the fact again, that some form of speed control should be used. It is almost impossible to determine accurately beforehand the head against which the pump will work, and with a fixed speed any change in piping or operating conditions may make a great difference in the amount discharged and in the power required.

Belt Conveyors. The following article by J. D. Morgan, "Belt-Conveyor Operating Data," published in Power, October 3, 1916, shows the value of charts for giving a eomplete exposition of just what equipment may be expected to do under given conditions.

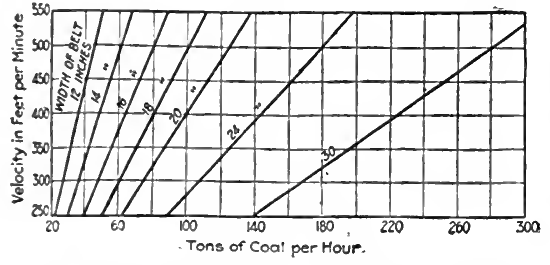

Fig. 9-Variation of Capacity with Velocity of Belt

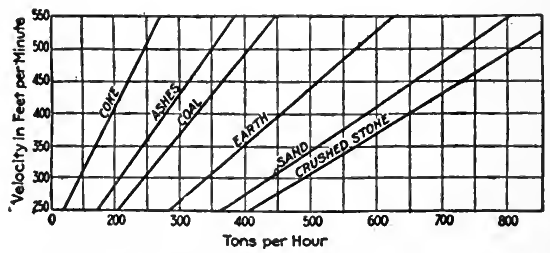

Fig. 10-Capacity of 36-In. Belt to Handle Different Materials

In view of the fact that few operating engineers are familiar with the operating characteristies of belt conveyors, this article was made up in eurve form so that the operating data would be available at a glance.

Belt conveyors are divided into two groups, horizontal and incline, or a combination of both, and each group has its own calculations.

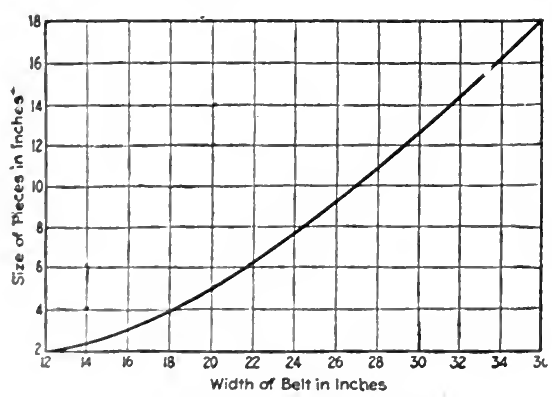

Fig. 11-Naximum Size of Pieces to Be Handled by Belt Conveyors

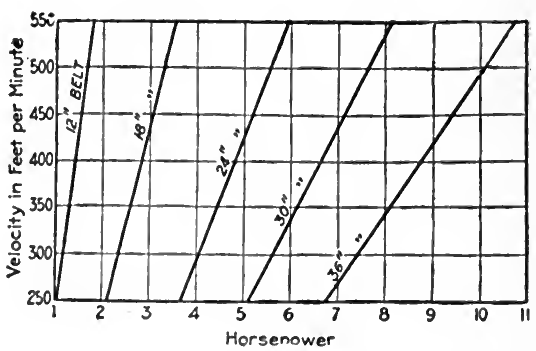

Fig. 12-Power Requirements of 100-Ft. C. to C. Level Conveyors

Fig. 9 shows the quantities of coal that ean be handled by different sizes of hell at varions speeds. In Fig. 10 the amounts of different materials that ean be handled by a $36-\mathrm{in}$. belt conveyor at different velocities are given. The maximum sizes of material for different widths of belt, consistent with good operation, are plotted in Fig. 11.

As will be noticed in these curves, the eapacity of a belt conveyor varies with the veloeity and the width of the belt, and as the eapacity changes so do the power requirements of the conveyor. Fig 12 
shows the power requirements of a 100-ft. center to center level conveyor with one movable tripper, for various belt sizes and velocities. Only by actual test can the power requirements of any particular conveyor be found, yet if the formula given is used, a good approximation is obtained. When using this formula it must be remembered that allowances for gear and friction losses must be made. For a level conveyor

$$
\text { Horsepower }=\frac{K L D}{1,000}
$$

For values of $K$ see Fig. 13. Throughout the article $K$ is a constant varying with different sizes of belt and the specific gravity of the material handled, $L$ is the load in tons of $2,000 \mathrm{lb}$. per hr., $D$ the distance from center to center of the conveyor in feet, and $H$ the vertical height in feet that the material is to be lifted.

Figs. 14 and 15 show the power requirements of a 200 -ft. center to center incline conveyor with one movable tripper, for various widths of belts, velocities and vertical heights. If the same allowances are made as were assumed in the calculation of level conveyors, the following formula will give good results for an incline conveyor:

$$
\text { Horsepower }=\frac{K L D+L H}{1,000}
$$

Often it will be found that it is desirable to change the size of the driving pulley. In Fig. 16 a curve is given showing the belt sizes of rubber-surfaced pulleys for different sizes of belt.

An important operating question is belt speed, which depends upon belt size and stress, for it has been found that $20 \mathrm{lb}$. per ply is the maximum allowable stress consistent with good belt service. Fig. 17 shows the maximum advisable speeds as obtained by a series of tests.

The matter of cost now enters in, and as a rule the first question is, "What is the right kind of belt to use?" This question has to be decided by the material to be conveyed. The writer is of the opinion that a balata belt of the first quality will outlast any other for use on soft coal and under various weather conditions. It is true that the price is higher, yet the wearing quality will more than make up for the difference. Fig. 18 shows the cost of varying sizes of balata belt of the first quality.

The final consideration is the maintenance cost per ton of coal conveyed. This item will vary from year to year, but a good average cost will be found to be about $0.247 \mathrm{c}$.

Comparison of Stokers. Fig. 19 and the following description were taken from Electrical World, October 31, 1914, to show how quickly and easily a comprehensive idea of the relative efficiencies of similar equipment may be obtained by plotting their operating characteristies on the same chart.

The efficiencies of modern stoker installation are shown by Fig. 19. These curves indicate boiler efficiency alone and do not allow for steam consumed by auxiliaries such as blowers, stokers and boiler-feed 
pumps. In general the best efficiency is shown when the boiler is operated at less than $100 \%$ rating. Curves $A$ and $B$ indicate an exception to this statement. As the net output of steam for a given coal input is affected relatively more at low loads than at high loads by the steam consumed by the auxiliaries, it is advisable to operate the

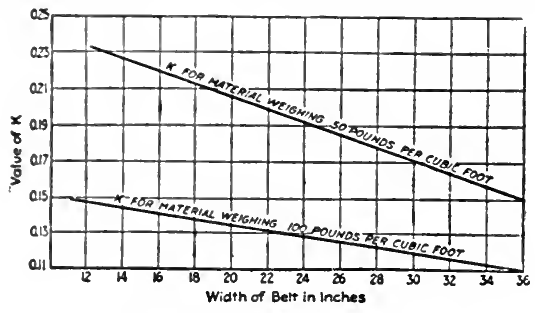

Fig. 13-Values of $\mathrm{K}$ in Horsepower Formula

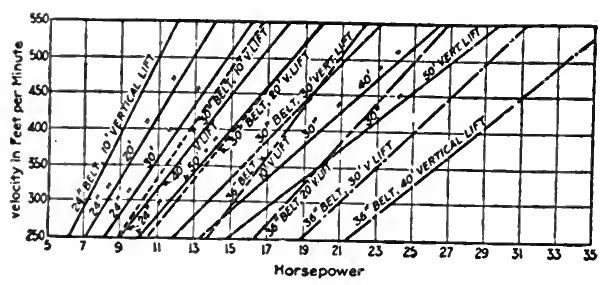

Fig. 15-Power Requirements of 200-Ft. C. to C. Incline Conveyor-One Movable Tripper

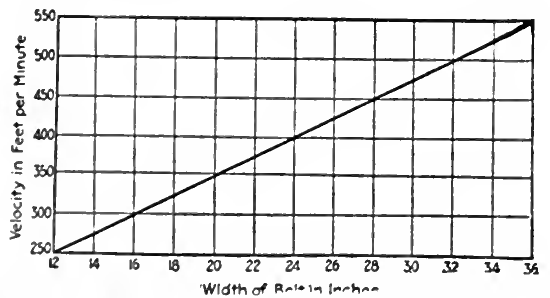

Fig. 17-Maximum Advisable Belt Speed

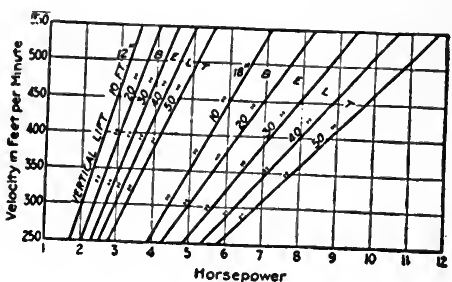

Fig. 14-Power Requirements of 200-Ft. C. to C. Incline Conveyor-One Movable Tripper

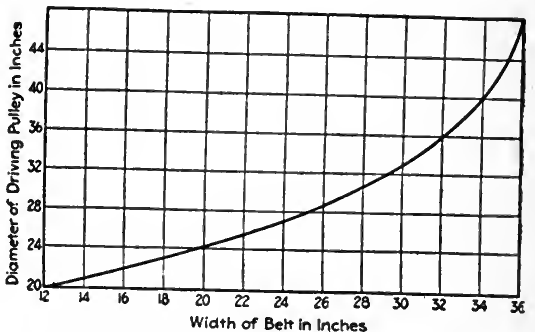

Fig. 16-Advisable Size of Driving Pulley

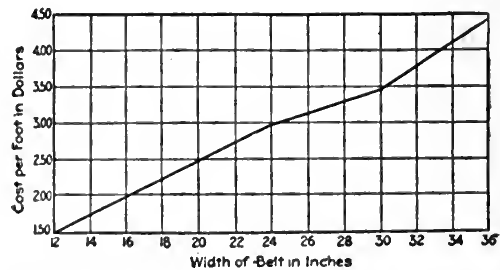

Fig. 18-Cost of Six-Ply First-Quality Balata Conveyor Belting

boilers at about $25 \%$ higher than their most efficient rating as shown by the curves to obtain the best plant efficiency. The principal factor influencing the load to be carried by a boiler is the relation between fixed and operating eosts. Foreing a boiler, however, will tend to deteriorate the furnace walls faster.

Operating Characteristics of Plants. There follow, to the end of this chapter, several examples of what may be termed-"Operating Characteristices of Plants as a Whole." These might have been included under the chapter on Management, as the function of the charts is invariably to permit a more comprehensive study of the operating 
characteristics of the plant with a view to increasing its efficiency. Under the Management chapter, major consideration was given to business management rather than management of operation or production. But just as we study the operating characteristics of a single piece of apparatus in order to become familiar with its probable behavior under all conditions so we study similarly the plant as a whole, and the great advantage of the chart method is that a bird's-eye view may be obtained of a situation better than in any other way.

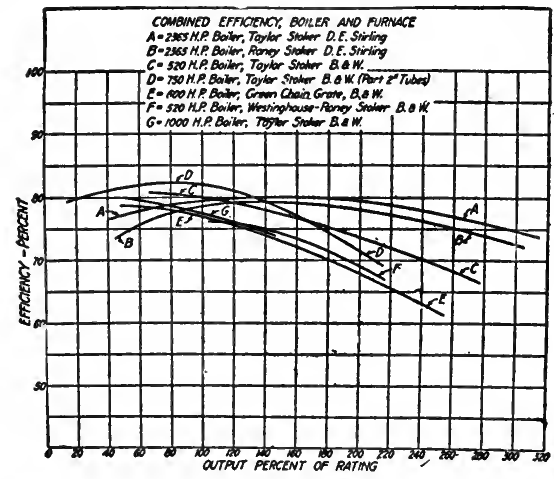

Fig. 19-Efficiency Curves for Various Stokers

Refrigerating Plant. The first article cited as an illustration is entitled "Refrigerating Plant Efficiency," published in Mechanical Engineering, April, 1919, and written by V. J. Azbe. The portions not dealing with charts have been omitted and if the reader is interested in this type of plant he is urged to read the complete article which takes up many points not mentioned here.

The cost of ice and of refrigeration is a composite figure of many different expenses coming under the heads of manufacturing, selling and general expenses. The cost of fuel is usually the largest item, and it may easily represent the difference between profit and loss.

During the last two years the cost of fuel per ton of ice has doubled, and many plants $a_{2}$ e now paying $\$ 1.50$ to $\$ 2.00$ per ton of ice for fuel alone. In spite of these conditions the majority of refrigerating plants are very wasteful of fuel as a result of improper design, run-down equipment or poor operation. While the possible savings are enormous and can usually be secured with little effort and slight expenditure, the necessity for improving equipment and operating conditions is not realized in the majority of plants.

In this paper the writer expresses himself frequently in terms of tons' of ice per ton of 10,000 B.t.u. fuel, to equalize the value of the various fuels (semi-bituminous, bituminous, lignite, oil, etc.), since by a simple recalculation it places them on a common basis.

The prime mover most generally used in refrigerating plants is the Corliss non-condensing steam engine. Next in order are the compound Corliss, electric motor, oil engine, and uniflow steam engine. If this 
order were reversed, however, enormous sums of money would be saved to ice manufacturers, for the following reasons:

In selecting the prime mover for an ice plant, the two most important items to consider are efficiency at rated load and efficiency at half load. While the average Corliss non-condensing engine consumes $20 \%$ more steam at half load than at full load, the uniflow engine uses only about $8 \%$ more. This is of great importance because of the great variations of load factor. Many engines can be found operating at one-eighth eut-off. This is the reason, also, why a steam cylinder should be adapted to the back pressure at which the compresser operates. It is most unwise to have a steam cylinder large enough for economical cut-off

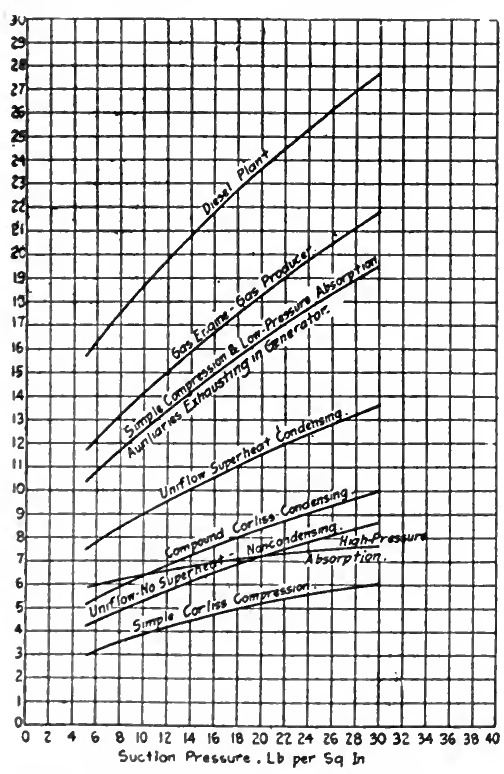

Fig. 20-Relative Efficiency of Various Ice-Plant Installations

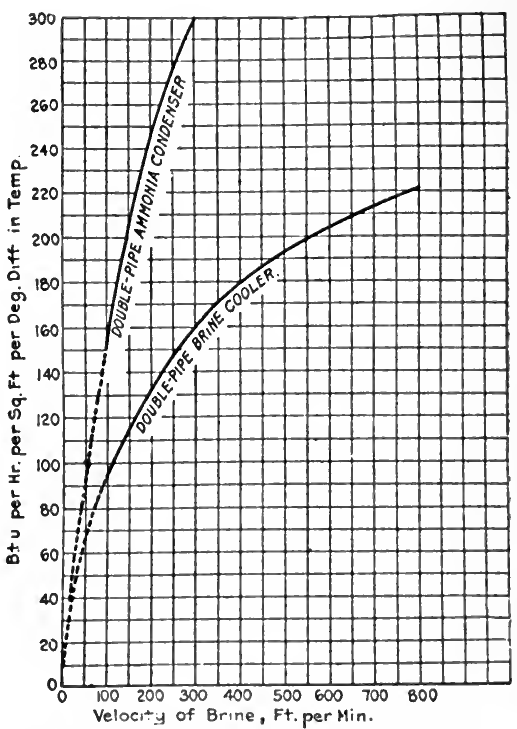

Fig. 22-Heat Transmission with Double- Pipe lirine Coolers and Ammonia Condensers

at $25 \mathrm{lb}$. suction pressure when the pressure to be maintained is $15 \mathrm{lb}$. or less.

Fig. 20 shows the results to be expected from various types of installations. The allowance made for auxiliaries ranges from one-half to one horsepower per ton of ice, depending upon conditions. The condenser pressure was taken at $185 \mathrm{lb}$. gage, and it was assumed that at the suction pressure given the machine would operate at about full Ioarl.

In small plants preference should ordinarily be given to the use of superheated steam, since as high an economy may be obtained from a uniflow non-condensing engine operated with superheated steam as from a compound Corliss condensing engine using saturated steam, and the former equipment is a great deal simpler and necessitates less auxiliary power. Furthermore, the steam-consumption curve is 
flat and the efficiency of the plant will be maintained during the winter time.

Superheaters can be installed with facility even in existing installations, and since the gain is greater with simple non-condensing engines, uneconomical ice plants will derive benefit from this procedure.

In order to gain in economy, a simple engine is often made to operate as a condensing engine. As a result, the temperature difference in the cylinder is increased and the cut-off is shortened, which increases the wall area at cut-off as compared to volume. This greatly increases cylinder condensation, which is directly proportional to the temperature difference and the area exposed. It therefore seldom pays to operate such a machine at more than 20 in. of vacuum, and if the plant is to be changed over to condensing operation, it is best to replace the simple Corliss by compound cylinders or by a uniflow engine and leave the rest of the machine intact.

Under proper conditions the ideal and most efficient prime mover for the ice manufacturer is the Diesel oil engine. By "proper condi-

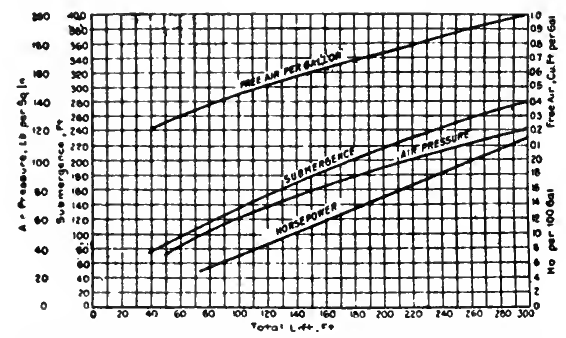

Fig. 21-Standard Air-Lift Performance

tions" is meant conditions of oil supply and cost, and kind of operating force to be employed. It cannot be over-emphasized that with the Diesel engine a high-grade engine-room force must be maintained, and especially during the overhaul period.

The curse of most ice plants is the auxiliaries. In many cases the steam consumption of the auxiliaries is as great as that of the engine running the compressor. In a certain ice plant of 100 tons capacity the following auxiliaries were found in operation: Electric-light engine; duplex circulating pump of ammonia condenser; duplex circulating pump for steam condenser; duplex brine pump; duplex boiler-feed pump ; ice-hoisting compressor; single-stage single-steam-cylinder deepwell compressor; agitator engine and cooling-tower fan engine.

One of the most uneconomical auxiliaries as usually operated is the air lift. This statement is meant as no reflection upon the air-lift pump as such, but on the way in which it is usually operated. Fig. 21 shows the performance of properly designed air lifts; the efficiency decreases somewhat as the lift increases, but should not be less than 60 to $80 \%$, whereas, actually in some cases it will be found as low as $20 \%$; the trouble is usually too great or too little submergence.

It is seldom advisable to use an air-lift pumping system for the circulation of water over the steam condenser because of the increased head required. The use of a cooling tower is ordinarily to be preferred, 
and any water needed for make-up may be taken from the air-lift system and passed over the distilled-water and liquid-ammonia coils, thus cooling the latter below the temperature of the circulating water proper.

For driving auxiliaries, electric current from generators driven by uniflow engines is to be preferred in the majority of installations, and the various units must be selected with a view to obtaining a flat efficiency eurve between half and full loads; all motors on the larger units should have variable speed control. Duplex steam pumps or steamdriven deep-well pumps should not be used except possibly as a reserve.

All the details incidental to the process of ice making should be so regulated as to secure a high rate of heat transmission, coupled with a low temperature difference. The ice tank is a most important member of the ice plant.

Brine velocity is helpful in two ways; it increases heat transmission between the brine and the can, and between the evaporating coil and the brine.

The average transmission of heat from the ice in the can to the brine outside is very poor, only about 2.5 B.t.u. per deg. per hr. per sq. ft., due to the insulating effect of the ice. The thicker the ice, the slower the heat exchange, consequently tank brine velocity has not nearly so great an effect upon heat transmission on the can side as it has on the coil side. The heat transmission between ammonia and brine through metal is between 10 to 20 B.t.u. per sq. ft. per hr. per deg. This comparatively low figure is primarily due to the superheating of the gas in the coil, which causes one side of the surface to be dry, and secondarily, to the low brine velocity.

Fig. 22 gives York Mfg. Co. curves showing heat transmission with double-pipe brine coolers and ammonia condensers. The curves were extended by dotted lines to show probable transmission at low velocity. These curves purely from their characteristics show the importance of velocity, an item which from the economy standpoint is certainly worthy of very careful investigation.

Ice storage is profitable in most cases if the storehouse is filled to eapacity during the winter and emptied entirely in the summer. The cost of storehouse will be from $\$ 6$ to $\$ 12$ per ton capacity and the investment will net a substantial return in most cases. The objections to it are the cost of refrigeration for the storage and the increased cost of handling the ice.

As far as plant economy goes, the value of ice storage is due to the equalization of the load factor. That is, the plant can be operated at higher rate during slack seasons and there may not be the neecssity for forcing the plant beyond its economical limit in summer. The ice plant and ice storage can be so proportioned that the plant will have a loarl faretor of 80 to $90 \%$, while without ice storage it would be only about $40 \%$, taking the whole year into consideration.

The small ice vault, or daily ice storage, exerts also quite an influence upon plant eronomy and it cannot be overemphasized that it is advantageous to make it amply large. The idea is to remove ice from tanks as quickly as possible-to use icc tanks for storing ice is a very poor practice. 
In Fig. 23 the full line represents the output of ice; the broken line indicates the sale of ice, and the dotted line the proper rate of manufacturing ice. These curves were plotted from the figures actually obtained in a plant. An exact determination of the proper rate of manufacturing ice will permit stopping the plant altogether for a certain time and attending to repairs.

Fig. 24 presents the effect on the transmission of heat of the velocity of the water in the pipes of a condenser. The points in the graphs were plotted from values obtained with a 12-pipe condenser of the drip-pipe type.

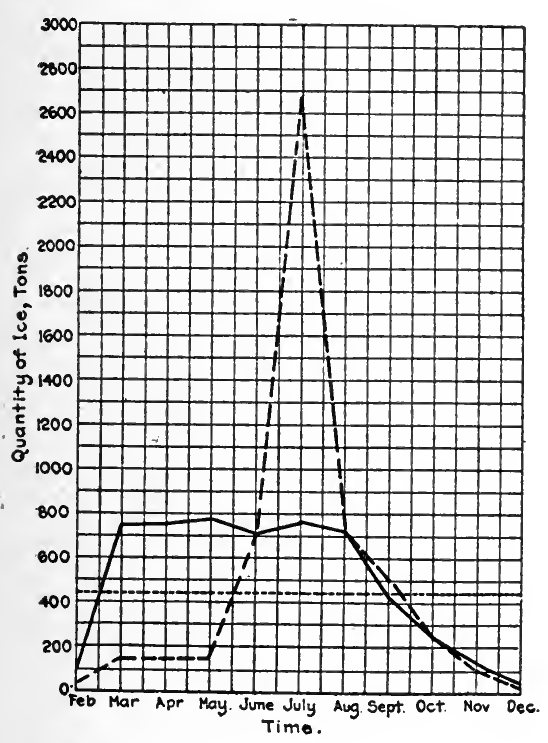

Fig. 23-Effect of Ice Storage on Extreme Load Factor Variations

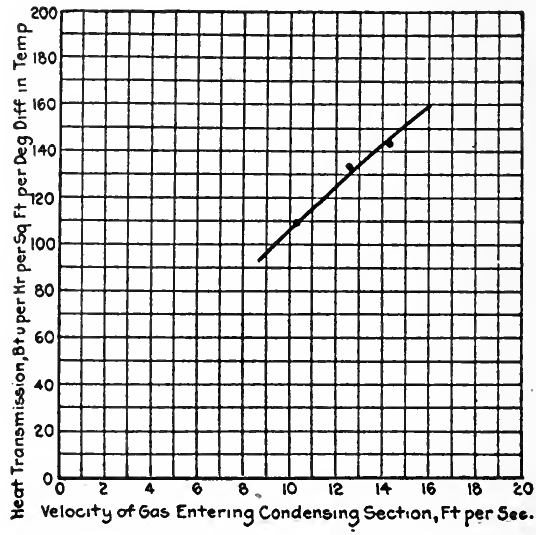

Fig. 24-Effect of Velocity Upon Heat Transmission

The velocity of the water in the pipes of a double-pipe condenser may be increased by allowing a greater amount of water to flow through them. The gas velocity increases when the height of the stand is increased by the addition of more pipes. In selecting between high and low condensers, however, other factors must be considered in addition to the water or gas velocity. Generally speaking, high condensers are preferable when the available water is cold.

The presence of air in the condenser often causes the cessation of the flow of the liquid through the pipes and thus seriously impairs the efficient performance of the condenser. The author has had occasion to observe in certain plants he inspected that the condenser pressure dropped 20 to $30 \mathrm{lb}$. after the air was removed from the system.

Fig. 25 shows distinctly how the compressor horsepower per ton of refrigeration increases with rising condenser pressure, and decreases with rising suction pressure.

Fig. 26 shows how the consumption of condenser water per minute per ton of refrigeration increases as its temperature range over the 
condenser is decreased, it being assumed that for each ton 350,000 B.t.u. must be removed per 24 hours, inclusive of the heat equivalent of the compressor horsepower.

The spray system has reeently been introduced in eonnection with ammonia condensing systems and very good results are being obtained. Its eooling efficiency varies from 45 to $70 \%$, depending upon humidity,

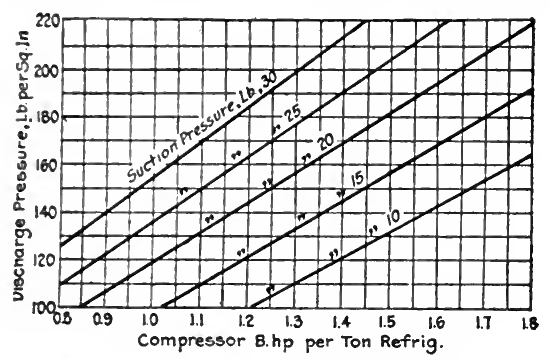

Fig. 25-Compressor Horsepower per Ton Refrigeration

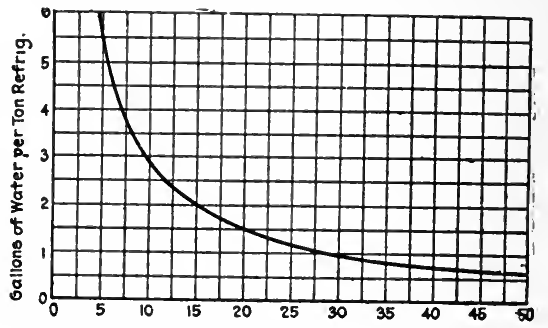

Fig. 26-Temperature Rise of Water Over Condenser with Various Amounts of Water and No Atmospheric Cooling

wind velocity, fineness of spray and pressure at the nozzles. In the calculations which must be made for designing a spray system, however, it is advisable to figure its operating effieieney at $50 \%$. This value has been assumed in the preparation of the eurves in Fig. 27, which show the value of the temperature of the water before spraying and the corresponding values after spraying, for various wet-bulb temperatures.

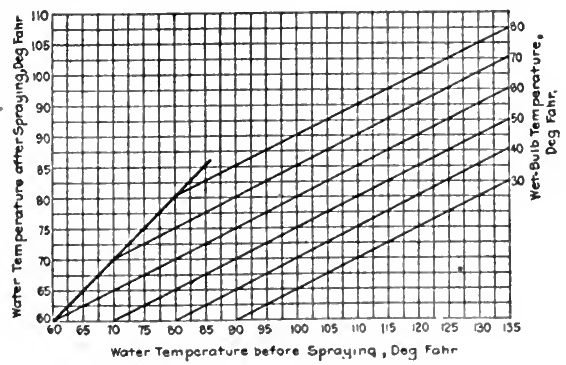

Fig. 27-Performance of Spray System

The advantage resulting from the use of a spray system in an ammonia condensing installation is that it reduces the power required, because a spray system does not involve the action of a fan, and a pressure at the nozzle of only six or seven pounds, that is, the equivalent of a tower $1+\mathrm{ft}$. high is sufficient. Also, the eost of eonstruction and maintenance is less for a spray system than for a cooling tower.

Electric Railway. The following article and eharts were printed in Electric Railwa!y Journal, November 6, 1915, under the title "Graphical Maintenance Work:"

For the purpose of furnishing an instructive exhibit of the kinds of records that are being found useful, a number of typical examples were selected as represniting good praetice and possessing commendable fea- 
tures, but no attempt has been made to draw general conclusions from them. They will suggest to some companies how greater use might be made of this method of presenting data, while others may see how their practice can be simplified.

To be effective the information to be put in graphical form must be such as can be used in impressing some lesson in the direction of improvement, or such as it is desirable for employees to keep before them, in a comprehensive way. For example, a graphical record of trolley breaks by months and years cannot but lead to study of causes and

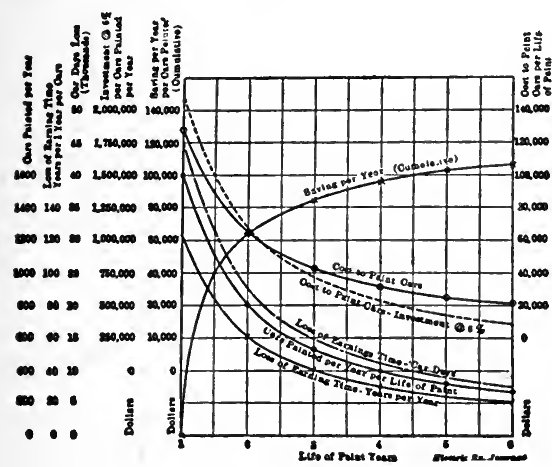

Fig. 28-Life-of-Paint Curves

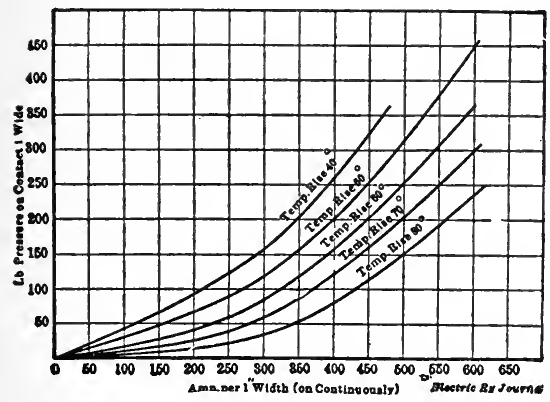

Fig. 29-Spring Contact

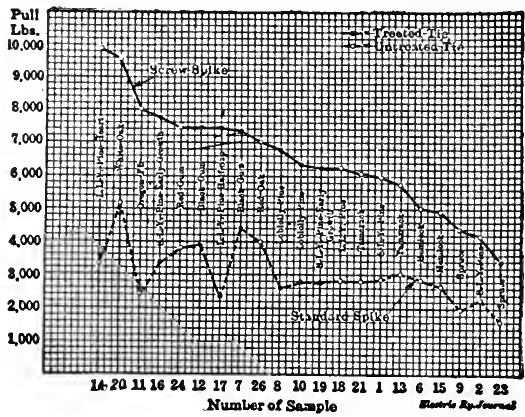

Fig. 30-Holding Power of Spikes

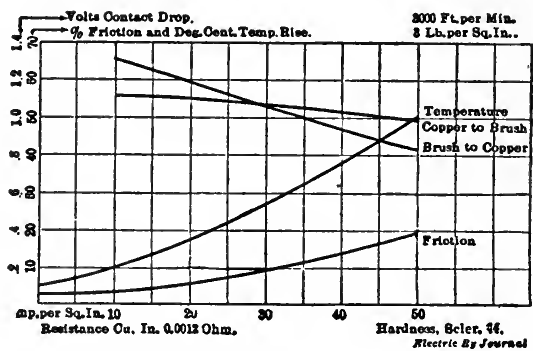

Fig. 31-Carbon Brush

remedies. Again, an organization chart of a shop maintenance force shows at a glance "who's who" in the company and how the responsibility is subdivided.

The present practice in graphical records appears to comprehend the following varieties for the purposes indicated:

(1) Curves plotted between variable quantities affecting equipment details, such as the relation between current-carrying capacity of spring contacts and the pressure at the point of contact, or the relation of the life of paint and total cost. Such curves are of great value in determining details of practice on the basis of real information. Manufacturers are in a position to furnish data in this form.

(2) Records of costs plotted, usually, against time for maintenance 
of detail parts and totals. Such are valuable for the determination of the durability of the individual parts of the equipment and especially as a basis for conference and discussion.

(3) Records of performance, usually on a time basis, such as numbers of cars pulled in by months, car-miles per defective car removed from line, hot-bearing records, etc. These are useful for the same purposes as (2).

(4) Construction progress records, partly for the purpose of insuring the delivery of parts at the times they are needed.

(5) Organization and routeing diagrams.

As examples of special studies, Figs. 28-31 have been selected.

Fig. 28 is a study made by the Detroit United Railway in the process of reducing the cost of car painting by improving washing methods. It shows at a glance the results of increasing the life of ear finish and could be used in selecting paint on an economical basis or, as it was, for determining the best methods of washing. Fig. 29 was used by C. W. Squier in his "Equipment Defects" series of articles in indicating how circuit-breaker contact pressures affect carrying capacity. Such data for every element of equipment should be in the hands of all equipment men.

Fig. 30 is from a report of the Board of Supervising Engineers, Chicago Traction. It is typical of the kind of information needed in track work in selecting ties and spikes. By the ingenious plan shown a great deal of information is condensed in a small space. This board makes extensive use of graphical presentation of data. Fig. 31 shows curves prepared by a brush manufacturer in the course of improving his product, but they supply exactly the information needed in the shop. Similar curves between brush pressure and voltage drop, pressure and life of brushes, etc., would also be convenient.

Continuous records of maintenance cost data are easily kept graphically and the advantages of so doing are obvious from an inspection of Figs. 32, 33 and 34. Fig. 32 is from the New York, Westchester \& Boston Railway. It serves to show elearly how the cost of repairs was brought down to a reasonable value until it is nearly down to inspection cost. Fig. 33 gives a more elaborate analysis of the United Railroads of San Franciseo records prepared by B. J. Arnold. It contains more records in a single sheet than are usually necessary but has the advantage of condensation. In reading diagrams like Figs. 32 and 33 it is necessary to remember that the points represent average values for the period corresponding to the horizontal units and not instantaneous values. Fig. 34 is a sample diagram from the equipment department of the New York Railways, which employs graphical records on a large scale. This particular chart was prepared for the purpose of showing the change in maintenance costs over a long period, but similar charts, with the month as the time unit, are plotted for all elements of maintenance cost. These are sent monthly to heads of departments and are made the basis of discussion at staff ennferences. Obviously diseussion with the record of operating results at hand in such tangible form eannot but result in more intelligent shop practice. The advantage of keeping such records in some standarl form ean be seen from Figs. 35 and 36 from different roads, for if it is profitable to compare performance month 


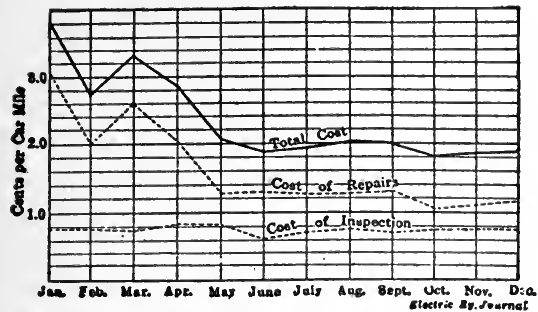

Fig. 32-Car Maintenance Cost, New York, Westchester \& Boston Railway

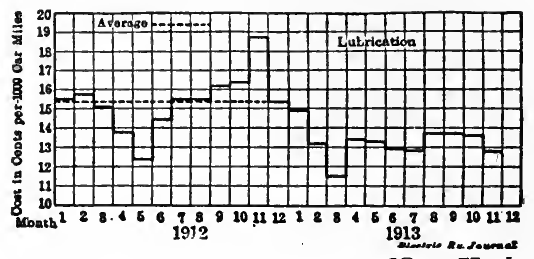

Fig. 36-Lubrication Cost, New York Railways

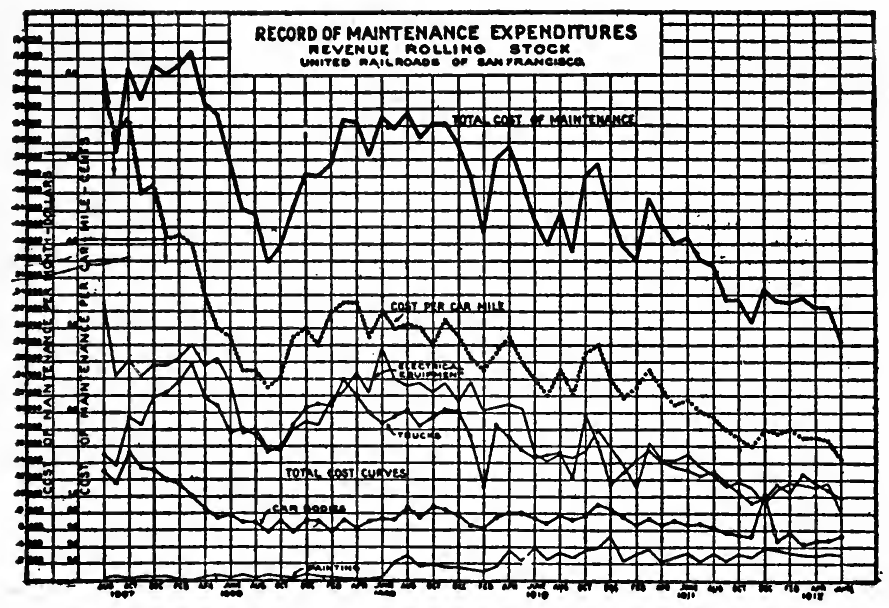

Fig. 33-Car Maintenance Cost, United Railroads of San Francisco

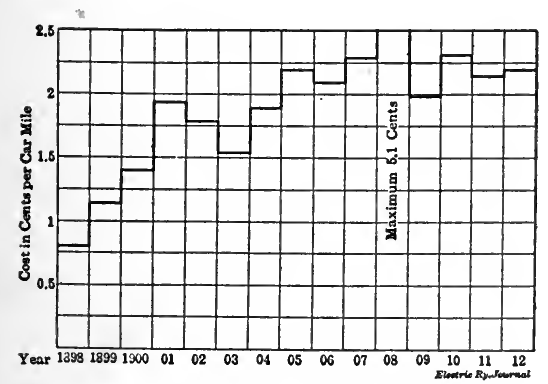

Fig. 34-Car Maintenance Cost, New York Railways
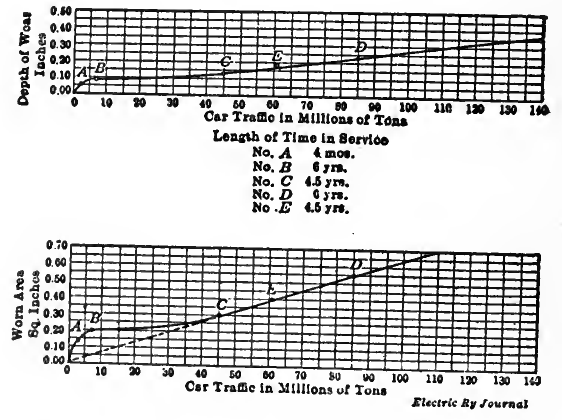

Figs. 38 and 39-Rail-Wear Curves, United Railroads of San Francisco 


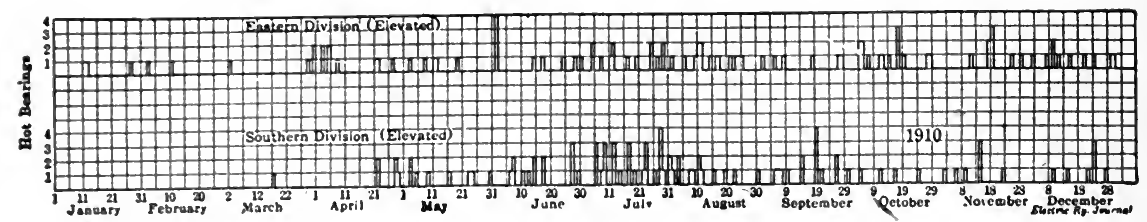

Fig. 35

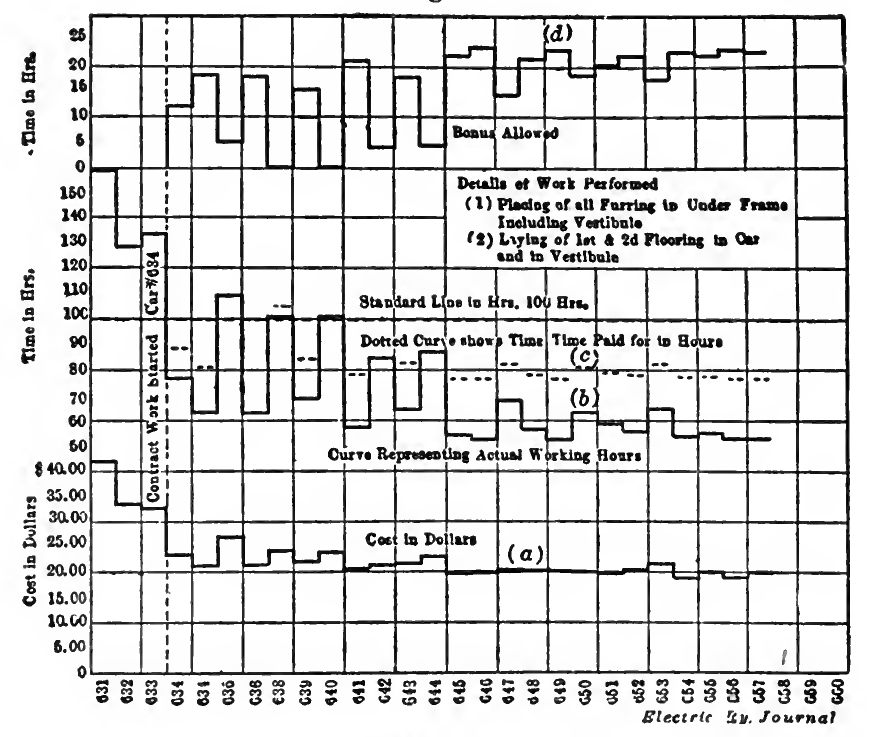

Fig. 37-Car Flooring Cost, Milwaukee Electric Railway \& Light Company

by month on one system it is equally so to make intercompany eomparisons.

Cost diagrams can often eontain other information as to factors affecting cost as, for example, in Fig. 37, which was prepared by the efficiency engineering department of The Nilwankee Railway \& Light Company. It shows the effect of introducing a premium system in the flooring of ears. Cars 631 to 633 were framed and floored by day work, (ars 6.34 to 657 under the premium system. Line $d$ is the bonus time allowed above a standard of 100 hours set from observation on the first three cars. Line $b$ is the actual time consumed. in man-hours. Line $c$ is the time paid for, and line $a$ is the cost to the eompany for labor on underframe and floor eonstruction.

Another class of diagram, typified in Figs. 38 and 39, is of value in keeping track of performanee in terms of traffic or time. These diagrams were made by B. F. Legare, engineer maintenance of way United Railroads of San Franciseo. They depict rail-head wear, in terms of both traffic and time, by the simplest expedient of indicating timenoints on the wear corves by letters. Fig. 40 shows a Brooklyn Rapid Transit daily hot-bearing record, in which a small rectangle represents ('ne hot bearing. 
Fig. 41 is a hot-bearing record from the New York Subway showing averages by months. The record for the succeeding year is placed on the same sheet for comparison of similar months. Generally this would answer the same purpose as the preceding and is much cheaper to produce. In Fig. 42 is given a "cars-pulled-in" record of the Connecticut Company, Hartford shops. This company uses similar diagrams for different car troubles, arranging data for comparison of carhouses with a view to stimulating competition. The lines for the several years are distinguished by their character.

In addition to the examples already cited, there are many special

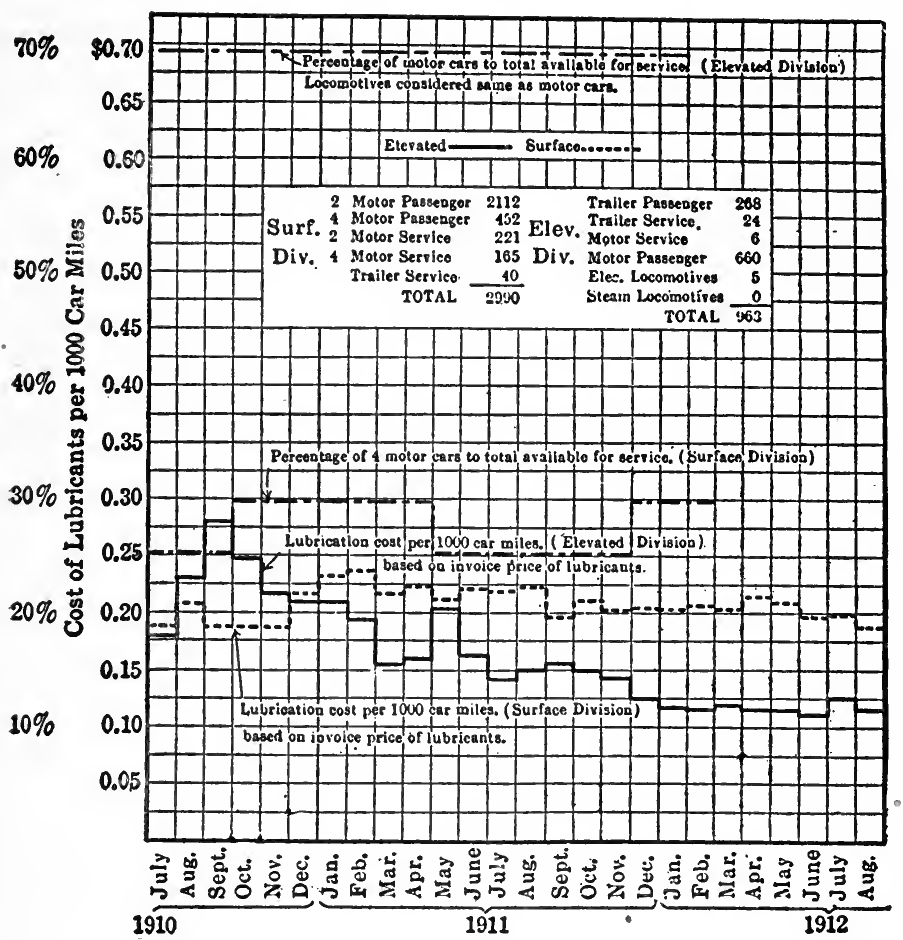

Fig. 40.-Lubrication Record

uses of graphics in maintenance work. For instance, autographic records of wheel press pressure, such as shown in Fig. 43 which is from a car-shop hydraulograph operated by the Boston Elevated Railway. Construction progress diagrams are also very useful. The style shown in Fig. 44, as used by the Connecticut Company, is good for general summaries, but for daily records that shown in Fig. 45 is better. The latter is used by the British Columbia Electric Railway of Vancouver.

A wheel record form that combines simplicity and effectiveness is the card used by the Massachusetts Electric Companies and shown in Fig. 46. A history of each pair of wheels is entered on the back of the card. In the January 5, 1918, number of Electric Railway Journal were 
printed a large number of additional charts pertaining to electric railway operating characteristics. These with certain abstracts from the articles which accompanied them, have been reproduced to show how completely the various phases of operation may be recorded by charts. Several portions of the article not having particular reference to the charts and all of the tables have been omitted, so that the following does not present a complete resumé, and those interested in the subject. should read the original reference noted above.

In the growing community a railway manager can get a great deal of helpful information from the following:

First-Graphs of the increase in population according to the shortest.

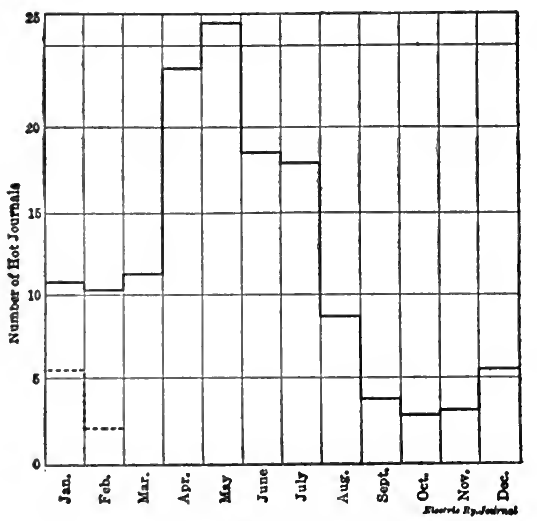

Fig. 41-Hot Journal Record

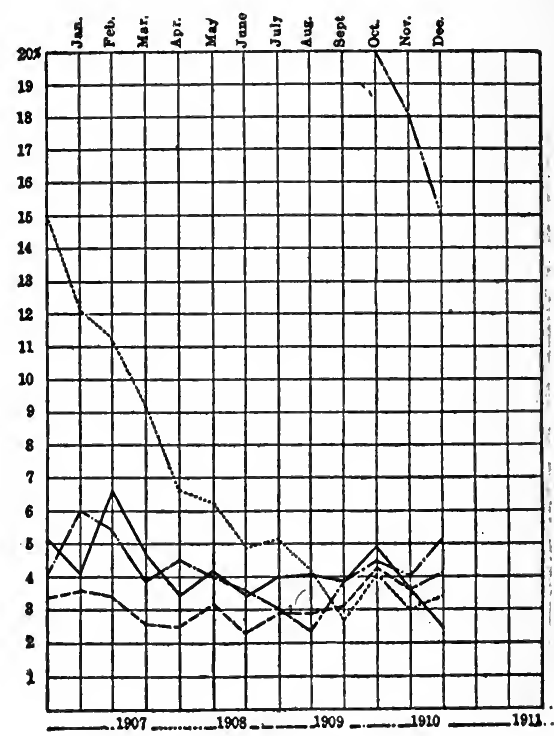

Fig. 42-Record of Cars Pulled In

intervals available. Figs. 47 and 48 present such graphs for two Amer-ican cities, both being reproduced to show how the ratios of increase may differ. Nor should we stop here. If at all possible similar graphs: should be made by districts, to determine where track should be put. down and where track should be pulled up.

s'ecoud-Giraphs of growth of employment establishments and wage earners. Fig. 49 shows that these two elements in eity growth are by no means parallel. $\Lambda$ slight increase in shops against a big increase in men discloses a greater likelihood of congestion than if the shops also. had increased in the same ratio. Factory eapacity is usually increased vertically or by annexes; but factory number is usually increased by new construction in another district. Some railways, in the absence of these graphs, have overlooked developments like these; or, contrariwise, have continued excess service to a rundown factory district.

Third-Graphs which indicate the financial progress of the eommunity, such as value of products, capitalization, cost of material and wages. 


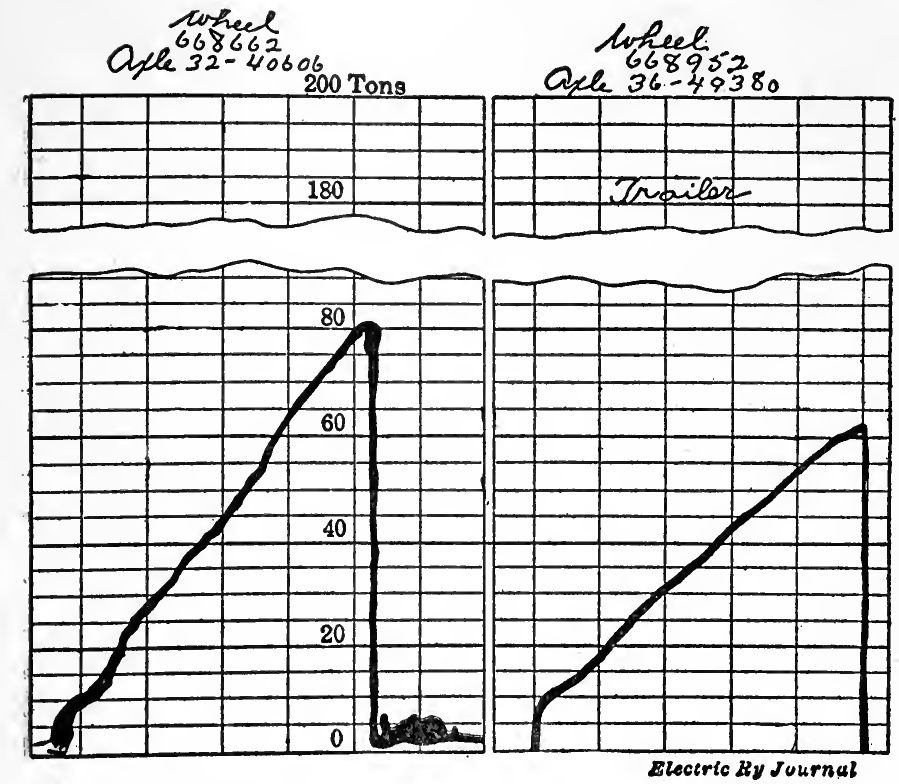

Fig. 43-Graphical Wheel-Press Records

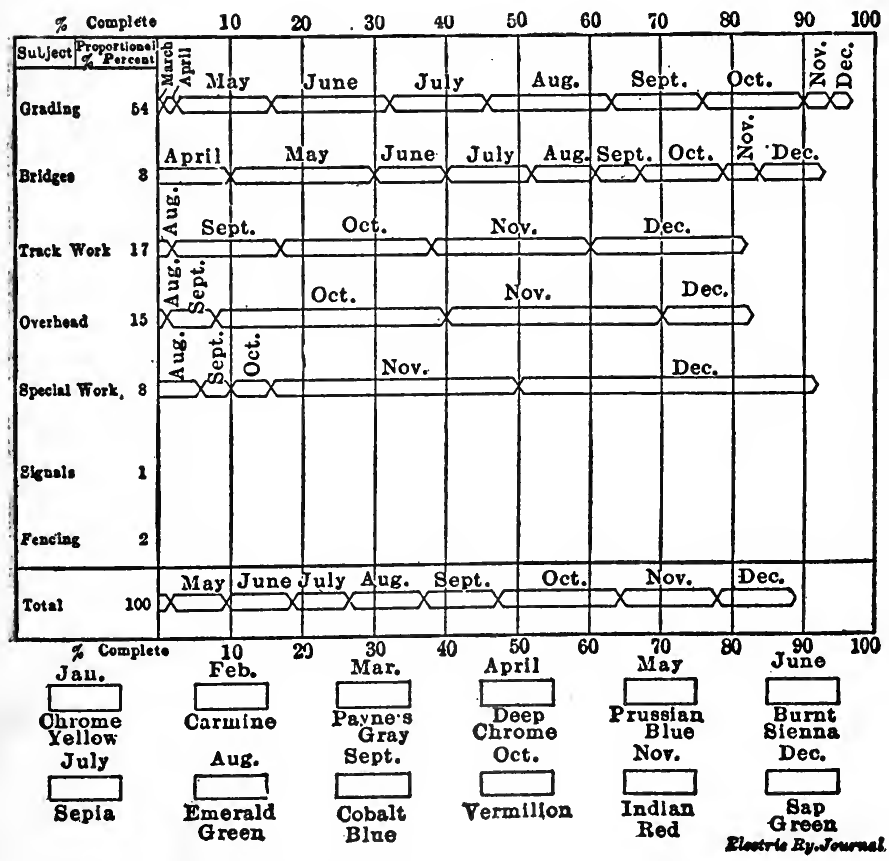

Fig. 44-Way Construction Diagram, Connecticut Company 


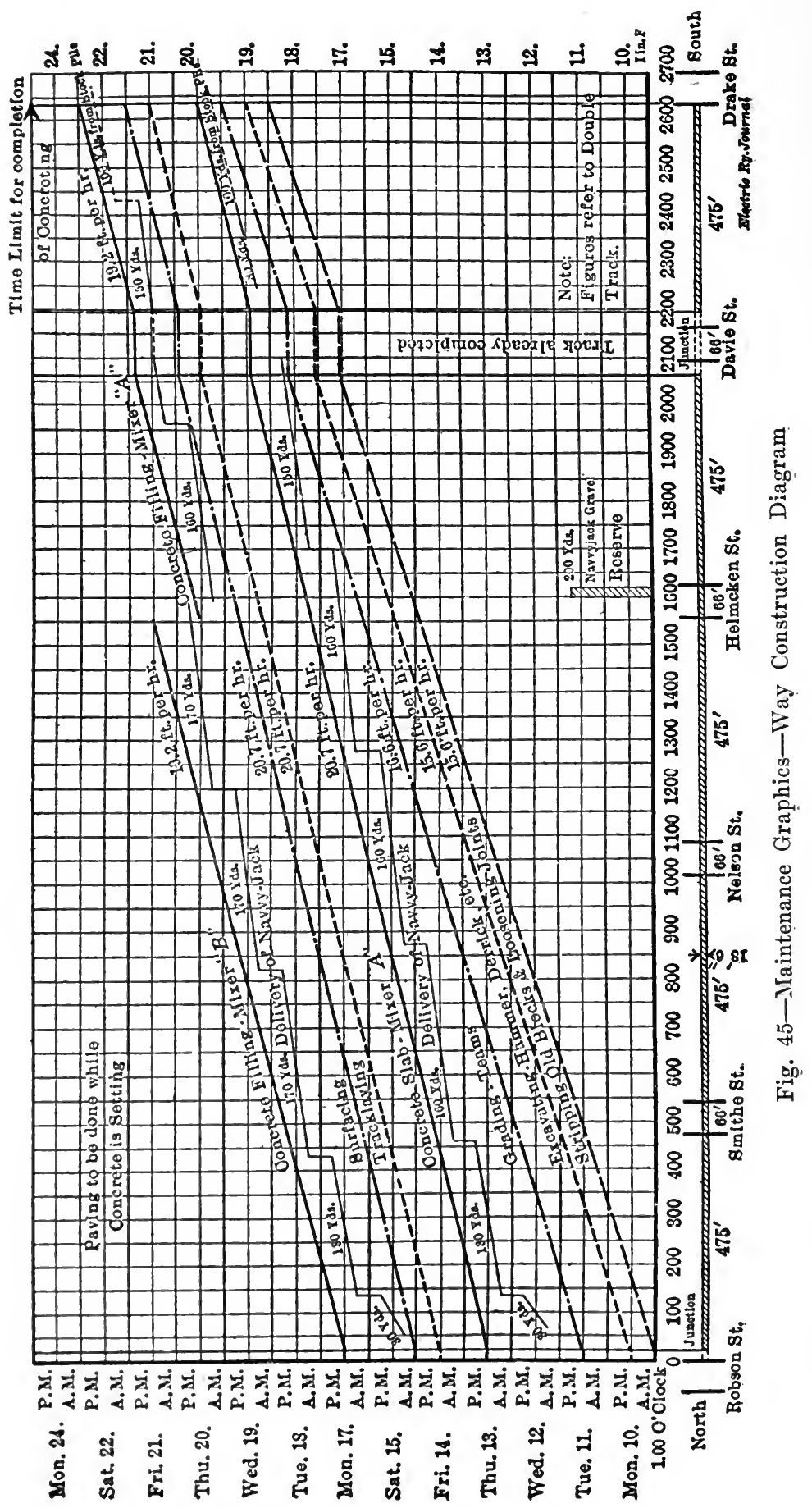


(Fig. 50) and the bank clearings (Fig. 51). Bank deposits should also be plotted. Such graphs as these will speak in mighty convincing tones alike to the banker, the board of directors, the management and the community.

To know how the general finances are tending, keep graphs of the gross earnings, operating expenses, interest charges, net earnings, taxes and the remainder necessary for paying the interest on stocks, bonds, mortgages and reserve funds. They are usually made on what is commonly known as a twelve-months' ending curve, namely, each point on the graph represents the business of the preceding lunar year, like January to December, February to January, inclusive, etc. When we plot the graph on this basis, the influence of fluctuations is decreased so that it is easier to form a direct idea of general tendencies in either the increase or decrease of the different items. This is illustrated by Fig. 52.

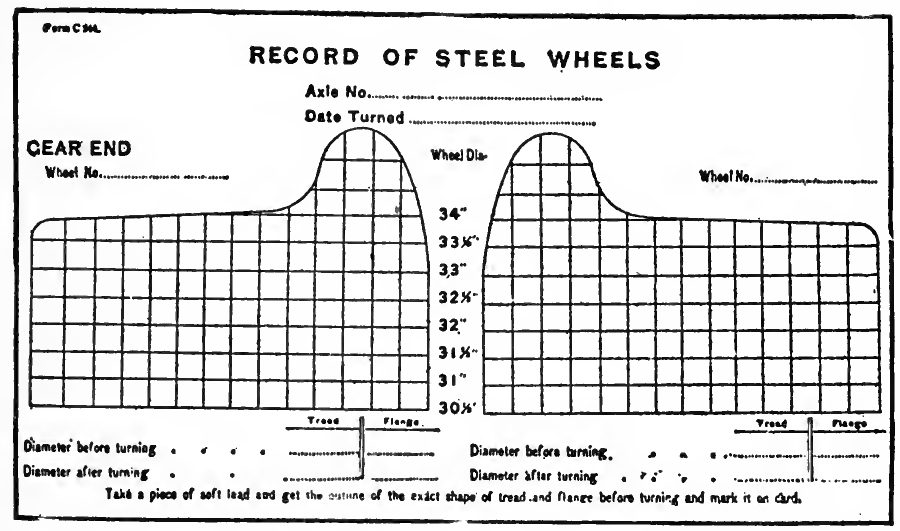

Fig. 46-Wheel-Wear Record Form, Massachusetts Electric Companies

Other important studies are the preparation of graphs to show the gross receipts, increase in population and ear-miles. On the same chart are plotted receipts per capita, car-miles per capita, etc., as in Fig. 53.

To find if the expenditure of the various departments varies in the same ratio as the gross income of the company, graphs are prepared of the gross receipts, total operating expenses and car-miles, as in Fig. 54. It is good practice to show the gross and operating expenses in total dollars, while plotting directly underneath it the receipts, operating expenses, cost of conducting transportation, power, maintenance of way and structure, maintenance of equipment and general and miscellaneous expenses in cents per car-mile. With the car-mile basis, the manager can see at once if departmental expenses are increasing or deereasing in proportion to the railway's gross earnings. An opportunity to compare relative total operating expenses and receipts on a cents per car-mile basis is presented in Fig. 55 .

The same method of analyzing accounts applied to the system as a whole should be extended to the individual lines. It is absolutely vital to know the earning value of each line by itself; and not the least im- 


\section{HOW TO MAKE AND USE GRAPHIC CHARTS}
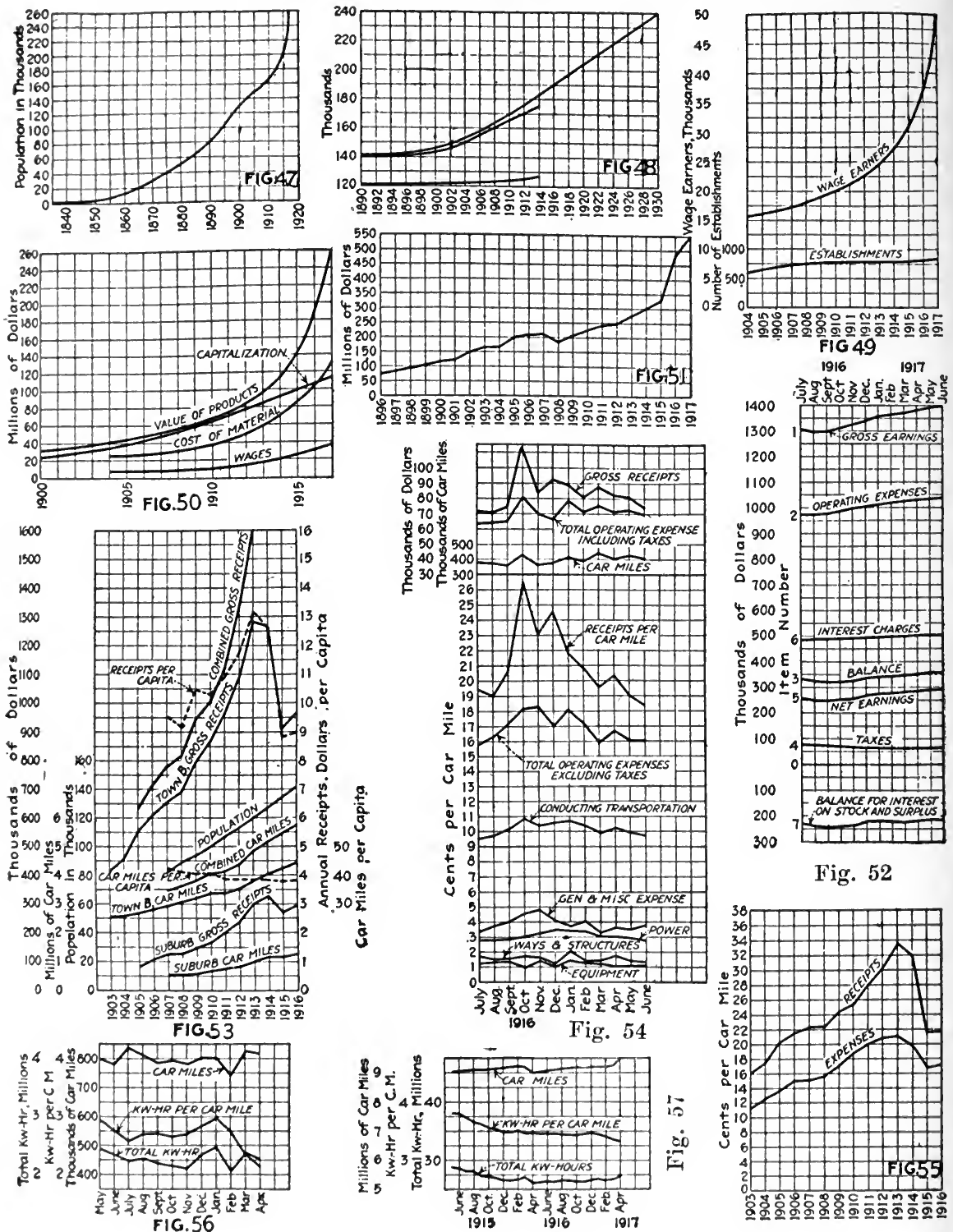

Fig. 52

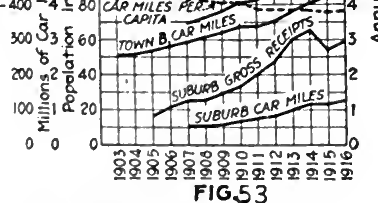

Fig. 52-Company Records, Town B,

Fig. 47-Growth in Population, Town A Fig. 48-Growth in Population, Town B, from Top Down, Combined, City and Suburbs

Fig. 4!)-Relation Between Growth in Numbers of Wage Earners and Establishments

Fig. 5)-Relative Increases in Value of Products, ('apitalization. Cost of Material and Wages, Town A

Fig. 5l-Record of Bank Clearings, Town A
Suhurbs Included

Fig. 53-Company Records, Per Capita Data, Town B, Sulourlss Included

Fig. 54-Total Departmental Data, Town 13, Suburls Ineluded

Fig. 5.-Ciross Receipts and Operating

Expenses Per Car-Mile, Town B Alone

Fig. 56-Car-Miles and Energy for One Year by Months

Fig. 57-Car-Miles and Energy for Two Years by Months 
portant reason is the ability which it lends to a manager to prove his case when he is asked to make an unreasonable increase in service.

He knows, for example, that an extra stop slows down schedules and costs money but he must prove this to the satisfaction of the complainants in figures, for his say-so is no better than that of any other man.

Figs. 56 and 57 contrast the average car-mile earnings, car-hours and car-mileage of a certain city with those of its best-paying line. Going still further, Fig. 58, we get a contrast of all the lines individually (on another system) revealing the perceptible disproportion between carmiles and earnings per mile. The operating expenses of a line may seem to be reasonable when measured on a car-mile basis but be found unduly high when measured on a car-hour basis. For this reason, if a railway has the information figured on a basis of both car-miles and car-hours, it comes pretty close to knowing what parts of the system are bringing in the net income, where service should be added and where it should be taken off.

By further segregation of the standard classification of accounts, the cost of operation of individual lines can be obtained. Thus where several lines operate over one track, the expense of maintenance and operation can be distributed in proportion to the car-mileage of the individual lines.

To get these data, the superintendent or manager instructs the conductors to count the number of passengers at each point selected, and each conductor also receives a printed card, Fig. 59, for writing down his figures.

When the service of the individual lines is analyzed, it is well to consider the cost or appraisal value of each of the lines which we are comparing. Frequently, a large amount of money necessarily has to be invested to construct over a bridge or some costly fill-items which are not taken into consideration when operating costs ony are borne in mind.

Still another subject of profitable study for the average road is a careful analysis of the turnback situation. To determine the turnback points for any particular line, it is necessary to analyze the passenger loads on all cars at all hours of the day.

Of course, in making comparisons of total and departmental expenditures we should be sure that apparent savings in way or equipment maintenance, power or transportation expenses are really savings. They may be merely transfer charges, as when a saving in power possibly is transferred to the cost of transportation. Therefore, each saving must be considered in the light of what effect it will have on other costs.

The importance of departmental studies like Fig. 54 is emphasized by comparisons with the statistics of the United States Census Bureau. Its 1912 report shows that of the gross income of all the electric railways of the United States. the $58.18 \%$ operating ratio was divided as follows:

Maintenance of way and structures ....................... $8.17 \%$

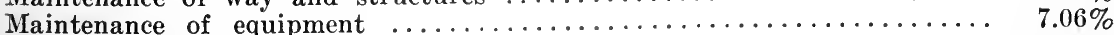

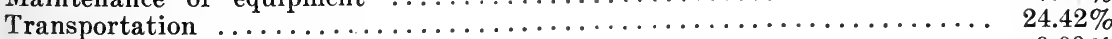

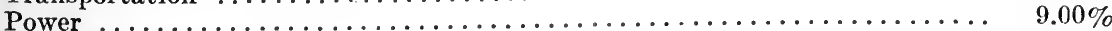

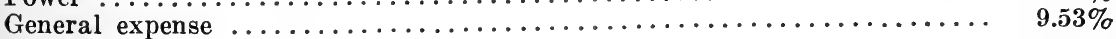

Total

$58.18 \%$ 
We all know that the recent exceptional increases in the price of labor and material will raise this percentage. Thus the figure for transportation expenses is nearer 35 and $40 \%$ than $24.42 \%$.

It would seem advisable also to include the depreciation, contingency funds, taxes and terminal charges in the operating ratio to obtain a true idea of what it really costs to conduet electric railway business.

The data from the turn-back studies may be used for chief aid in determining another important matter: The best size of car.

To do this we must develop loading graphs which will show at each cheeking point the maximum and minimum number of passengers on all cars at hourly intervals throughout the day, the two elasses of loading graph being contrasted with the straight line which represents the seating capaeity and with the derived graph of average loading. It is this "average loading" which should determine the size of the car best suited for the line.

For instance, an examination of the upper graphs in Fig. 60 shows that the car should be a little larger to care for the worst condition or that more of the present type should be operated. By making similar studies for say a dozen points (as indicated in Figs. 61 and 62) we can deeide definitely what is the best car for the line as a whole, while for the congested sections we can arrange shorter headways through turn-backs or trippers.

Again, we may look at the question in another way: Will the combined effects of increased rates of acceleration and braking, shorter length of stops, fewer stops per mile, etc., be of sufficient importance to give a shorter headway with the same number and size of ears now in use? We must not ignore the probability that shorter headways will bring more gross revenue.

Such studies as these should be considered in connection with the analysis of line travel by zones as detailed in the following paragraphs.

Both in securing general data on a line and the making of schedules it is best to divide the line into zones. In the downtown districts the travel is congested and stops frequent, so the running time must necessarily be relatively slow. As we leave the center of the eity, stops are less frequent and vehicle and pedestrian interference greatly reduced; therefore the schedule speed is much higher. When we also eonsider the possible turn-baek points, we are ready to lay out the following data:

\begin{tabular}{|c|c|c|c|c|c|}
\hline \multicolumn{6}{|l|}{ I-I)istance } \\
\hline $2-$ & $\begin{array}{c}\text { Running } \\
\text { Time }\end{array}$ & Stops & $\begin{array}{l}\text { Length } \\
\text { of Stop }\end{array}$ & $\begin{array}{l}\text { Schedule } \\
\text { Speed }\end{array}$ & $\begin{array}{l}\text { Average } \\
\text { Voltage }\end{array}$ \\
\hline A.M. rush $\ldots \ldots \ldots \ldots$ & $\ldots \ldots$ & $\cdots$ & $\ldots \ldots$ & $\cdots \cdots$ & $\ldots \ldots$ \\
\hline A.Ml. non-rush $\ldots \ldots \ldots$ & $\ldots \ldots$ & $\ldots$ & $\ldots \ldots$ & $\ldots \ldots$ & $\ldots \ldots$ \\
\hline Noon rush $\ldots \ldots \ldots \ldots$ & $\ldots \ldots$ & $\ldots$ & $\ldots \ldots$ & $\ldots \ldots$ & $\ldots \ldots$ \\
\hline P.Xl, rush $\ldots \ldots \ldots \ldots$ & $\ldots \ldots$ & $\ldots$ & $\ldots \ldots$ & $\ldots \ldots$ & $\ldots \ldots$ \\
\hline P.M. non-rush . . . . & $\ldots \ldots$ & $\ldots$ & $\ldots \ldots$ & $\ldots \ldots$ & $\ldots \ldots$ \\
\hline Evening .......... & $\ldots \ldots$ & $\ldots$ & $\ldots \ldots$ & $\ldots \ldots$ & $\ldots \ldots$ \\
\hline Night $\ldots \ldots \ldots \ldots \ldots$ & $\ldots \ldots$ & $\ldots$ & $\ldots \ldots$ & $\ldots \ldots$ & $\ldots \ldots$ \\
\hline
\end{tabular}

3-curves and grade (ondition

4-Sipecial traflio condicions 
When we have these data (see. Fig. 63) plus the schedule possibility of each car, we know if we are getting all out of our equipment that is advisable, making due allowance against a schedule that is "too tight" to keep the men ambitious to hold the cars to time. The stops given in Fig. 63 are equivalent stops, and include slowdowns.

At any rate, we are now armed with definite information and can take up intelligently such problems as municipal traffic rules to secure the street car a right-of-way that will increase the schedule speeds so that it will not be necessary for the crew to take chances in meeting tight schedules.

Or to take a less obvious condition: Exact knowledge of the voltage in each of the zones will show if the bonding is right or if there is enough feeder in the section observed. For example, Fig. 64 shows how radically the schedule speed or mileage output of a car is affected over a range of 400 to 600 volts. What this low voltage means can therefore be calculated directly in dollars and cents so that it is easy to determine whether it will pay to buy better bonds, use automatic substations, install more feeder copper or to take any other measures that will improve the quality of service while lowering the cost of service.

TABLE I-SCHEDULE SPEED EFFICIENCY AS AFFECTED BY VOLTAGE

\begin{tabular}{|c|c|c|c|c|c|c|}
\hline \multirow[b]{2}{*}{$\begin{array}{l}\text { Stops per } \\
\text { Mile }\end{array}$} & \multicolumn{2}{|c|}{$\begin{array}{l}400-\text { Volt } \\
\text { Schedule }\end{array}$} & \multicolumn{2}{|c|}{$\begin{array}{l}500 \text {-Volt } \\
\text { Schedule }\end{array}$} & \multicolumn{2}{|c|}{$\begin{array}{l}600-\text { Volt } \\
\text { Schedule }\end{array}$} \\
\hline & M.P.H. & $\begin{array}{l}\text { Per } \\
\text { Cent }\end{array}$ & M.P.H. & $\begin{array}{c}\text { Per } \\
\text { Cent }\end{array}$ & M.P.H. & $\begin{array}{l}\text { Per } \\
\text { Cent }\end{array}$ \\
\hline 3 & 12.4 & 79 & 14.2 & 90.5 & 15.7 & 100 \\
\hline 5 & 11.0 & 84 & 12.2 & 93.2 & 13.1 & 100 \\
\hline 7 & 9.9 & 86.6 & 10.75 & 94.3 & 11.4 & 100 \\
\hline 9 & 9.1 & 91 & 9.6 & 96.0 & 10.0 & 100 \\
\hline
\end{tabular}

Calculated on basis of tangent level track and without leeway.

From Fig. 64, just mentioned, Table I has been prepared to show the schedule possibilities with a 20 -ton ear at 400,500 and 600 volts.

What has already been said about the essential records required by an electric railway indicates the need for a statistical department. Preferably this bureau should be combined with the schedule department because most of its work bears so directly upon transportation problems.

The work should be guided by an experienced transportation engineer who would have the tact to co-operate with all of the other departments and who would also have the ability to analyze every function of operation. To make a success of this department, it is also necessary to have its findings presented to the chief executive in such a way as to make its studies of practical instead of theoretical value.

This would be the department to prepare all the graphs mentioned in the different portions of this study. The chief executive of a railway may lack the time for details, but it is his duty thoroughly to review all of the phases of the work of this department, to keep in touch with the transportation engineer and to transmit to him such directions as are necessary to develop the usefulness of the statistical and schedule studies.

A truly scientific schedule department will solve many problems that cannot well be handled directly by the operating department. For ex- 

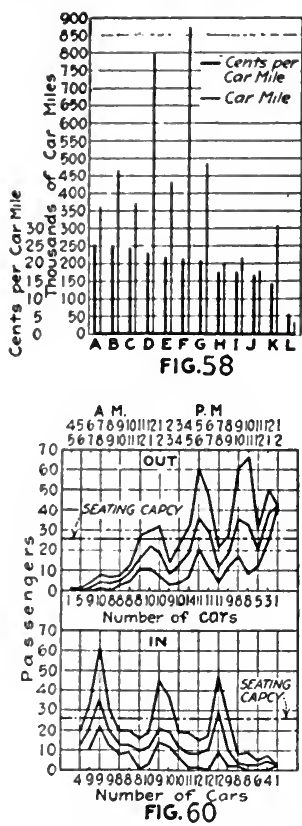

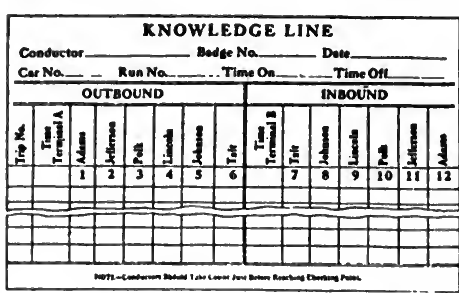

FIG.59

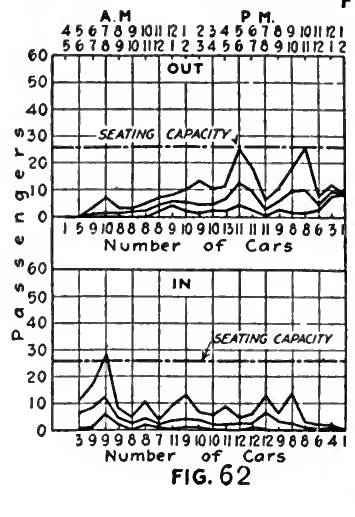

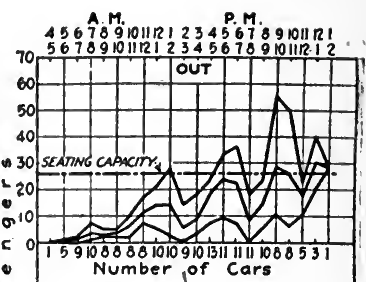

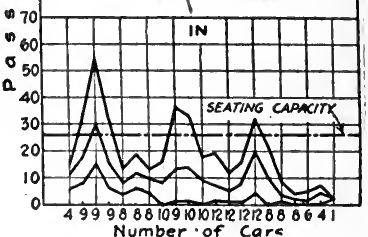

.4 FIG.6I,

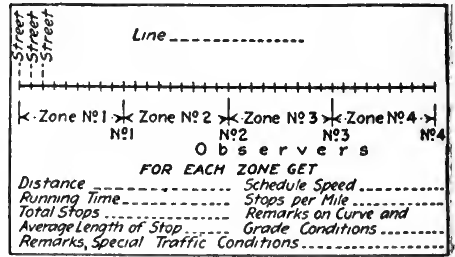

'FIG.63
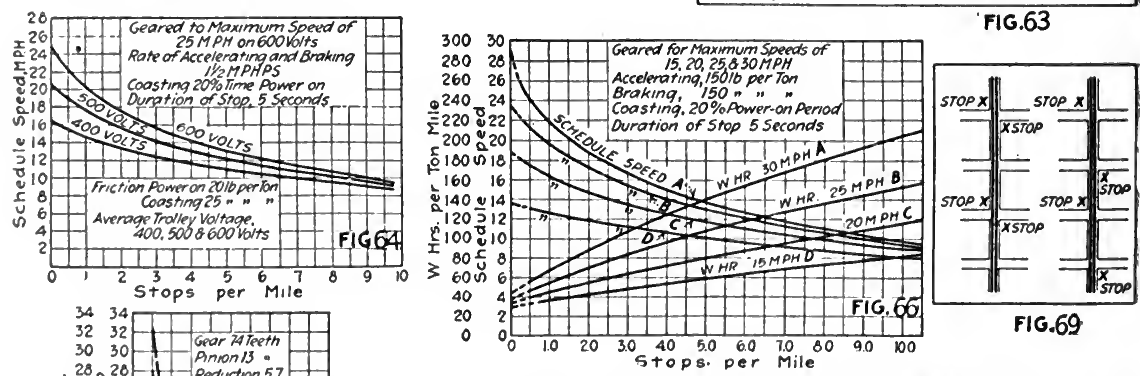

FIG.69
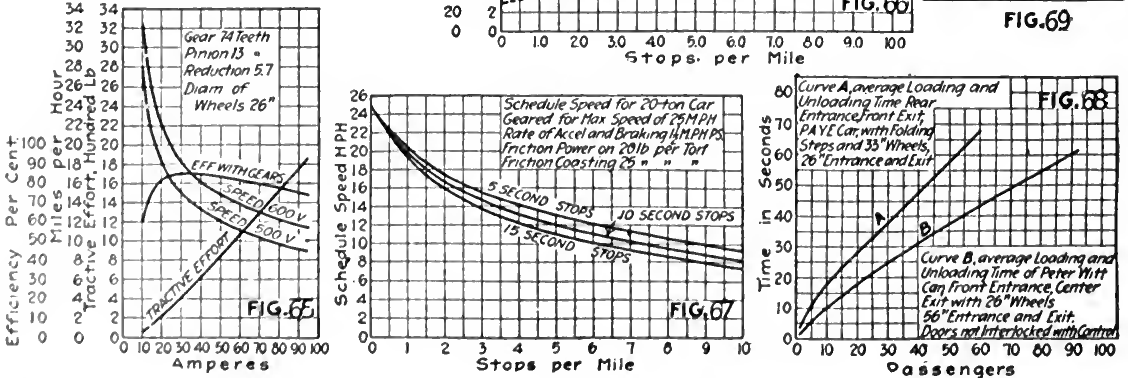

Fig. 5s-Ciross Farnings Per Car-Mile

Fig. 5) - liank Form for Traffic Count Fig. 6(0-I)etermining Car Loading Fig. fil-loncrmining Car Loading Fig. fiz-loctrormining Car Trading Fig. (i.3-Isavout for Traffic Situdy by \%ollos

Fig. 64-Sichedule speed Curves for 20.Ton Car
Fig. 65-Characteristic Curves GE-258 Motor, (icar Ratio $13: 74$

Fig. 66-Sicherlule Sipeed and Energy Curves for 20-Ton Car

Fig. 67-Influence of Length of Stop on Sichedule Sipeed

Fig. 68-Loading Time Curves for Two Types of Car

Fig. 69-Comparison of Skip Stop (Left) and Stagger Stop (Right) 
ample, there is the problem of determining whether it is desirable to have four different running speeds a day; and if so, what are the most economical figures, the question of balancing platform cost against power voltage, and others.

Or if it is a problem of removing an obstacle not within the control of the railway, such as vehicular and automobile congestion, better results will be obtained by publishing charts of surveys and detail reports of the delays so caused to the riding public than any amount of recrimination without evidence. If parking and safety zone ordinances are wanted, let this department prove the necessity for them.

In the past it has been too often the practice to lay out schedules on what might be termed the "cut and try" method. By this is meant that a trial car is operated over the line, usually under the supervision of some competent man, and the running time so made generally determines the future schedule.

On the other hand, every individual type of car has a certain schedule possibility which is almost entirely determined by the free running speed of the equipment, the arrangement of passenger interchange and fare collection facilities. The free running speed of each. car being known and the other conditions outlined in the present study being reviewed, schedules can be calculated, based on what each type of car is actually capable of performing. Schedules prepared on such a basis should prove more satisfactory than those established by guess, although they should, of course, be carefully checked by actual trial as formerly.

To facilitate the making up of schedules on this basis, two graphs, Figs. 71 and 72 , will be discussed later in this study; one for a 20-ton car and one for a 71/2-ton car. These graphs give the schedule speeds which are possible with an equipment geared for any free running speed within certain limits. It is appreciated that the voltage will change the free running speed. The average voltage can be obtained by observation and due allowance made in the free running speed. With but a very small commercial error, free running speeds of all cars decrease in proportion to the voltage, as outlined later.

This speed can be raised or lowered by changing the gear ratio. This change affects directly the amount of energy used and so has certain practical limitations set by the horse-power capacity of the equipment. It is always safe to lower the free running speed of the car, assuming this to be advisable, but when the speed is raised it is well to consult the equipment manufacturer and give him all the operating conditions before making a change.

One of the most important things is to know the schedule possibility of every car which the company owns.

This is directly affected by the free running speed of the car, namely, the speed which a car will ultimately attain if the controller is at full "on" position and the car is running on tangent level track.

One of the basic factors in the railway business is the number of stops the car has to make. This factor directly affects the line capacity, numbers of cars for a given service, car-hours, power, maintenance of equipment, size of motors, etc.

The length of stop can be decreased if passengers are urged both by 
posters and by word of mouth to board and leave the ear as expeditiously as possible, while the conductor should be urged to be prompt in giving signals to the motorman through push-buttons or otherwise. The value of decreasing the length of stop is strongly brought out by Fig. 67 .

One way of operating fewer-stop service is to stop on alternate corners; the other is to have outbound cars stop at one series of corners while inbound cars stop at the other series of corners. The latter method, or "stagger" stop, illustrated in Fig. 69, has the advantage of equalizing the walking done by all passengers, since the passenger who walks an extra block in the morning does not have to do so at nightand vice versa. The stagger stop has been successfully introduced at Cleveland, Detroit, Buffalo, Kansas City and Toledo, and is now being tried at Baltimore, Dallas, Cincinnati and Columbus.

To show that the stagger stop plus walking is quicker for the patron than haphazard stopping, Fig. 70 has been prepared. This shows the time required to walk distances up to $1,000 \mathrm{ft}$. Of course, the distance between stops does not have to be traversed by every individual car user. The greatest distance which he will have to walk will be one-half of the distance between the possible ear stops.

The great importance of the system of fare eollection on loading time is shown in Fig. 68, which brings out the greater passenger-interchange speed of the pay-as-you-pass car over a prepayment car in exactly the same service. Thus the pay-as-you-pass car interchanged sixty on or off passengers in 43 sees. while the other car required 68 sees. or 25 sees. more!

To show definitely what can be secured from schedules with different rates of acceleration and braking, three sets of graphs have been made up. Fig. 71 shows a 40,000-lb. ear complete with load and geared for a free running speed of 25 m.p.h., assuming a definite length of stop of five seconds. The calculations are made on the basis of accelerating and braking at 1, 11/2,2 and $2 \frac{1}{2}$ m.p.h.p.s. With an equipment on cars of this weight the best rate of acceleration which we have been able to obtain commercially is 2 m.p.h.p.s. However, since the future may give us an equipment and car construction which will enable us to accelerate at $2 \frac{1}{2}$ m.p.h.p.s. we have included this figure.

On the automatie safety car, Fig. 72, data have been prepared for a car which will weigh complete with live load $7 \frac{1}{2}$ tons or $15,000 \mathrm{lb}$. The rates of acceleration and braking specified for this car are 11/2, 2 and $2^{1 / 2}$ m.p.h.p.s. This ear is also geared for 25 m.p.h. free running speed, and we are assuming that the stops are of the same length as for the larger car, that is five seconds. On these two graphs we have taken the example of a car accelerating and braking at different rates and have taken full arlvantage of all the schedule possibilities of the equipment when accelerating and braking at $1 \frac{1}{2}$ m.p.h.p.s. It will be noticed that the power increases with the more rapid rate of acceleration, but that we secure a greater sehedule speed.

Another (ondition which we ean consider is that illustrated in Fig. 73. This graph shows the maximum speed which ean be obtained by accelerating and braking at $1 \frac{1}{2}$ m.p.h.p.s. The power when this acceleration is in use is shown in the upper graph.

We all know that by accelerating and braking at 2 m.p.h.p.s. rather 
than $1 \frac{1}{2}$ m.p.h.p.s. there is a considerable saving in energy consumption, providing the same schedule is maintained in both cases. This fact is brought out by Figs. 74 and 75, which show the power saving which ean be made by accelerating and braking at different rates, while maintaining the same schedule. These two graphs are made for cars operating in runs of $1,000 \mathrm{ft}$. each.

Fig. 73 shows the saving which ean be made in energy by operating a maximum schedule with $1 \frac{1 / 2}{2}$ m.p.h.p.s. acceleration and braking; and also the energy which will be used providing this acceleration and braking are changed to 2 m.p.h.p.s. With this latter condition we will still maintain the maximum schedule possible with the lower rate of acceleration and braking.

An idea of the schedule speeds which can be made with cars geared for different free running speeds may be obtained from Fig. 66. We have assumed that the car weight complete with all equipment and live load will total $40,000 \mathrm{lb}$. and that by substituting different gearing we can arrange the car to have free running speeds of 15, 20, 25 or 30 m.p.h.

The service fundamental of weight embraces the size of car or seating capacity and the design and structure of a comfortable vehicle capable of operating at a speed which will satisfactorily meet the conditions of traffic.

To help us review the influence of weight propelled on a particular motor on which the same gearing is used, graphs have been prepared to show the free running speed which determines the schedule possibilities with ears having a total weight of 20,28 and 36 tons respectively.

Fig. 76 shows that the free running speed with the three different classes of equipment will be

20-ton car-25.6 m.p.h. free running speed
28 -ton car-23.4 m.p.h. free running speed
36 -ton car-21.4 m.p.h. free running speed

It will be noted that this graph has been made up to show three different runs which are of the same distance. However, the time of making the run varies with the different weights of cars.

By referring to Fig. 77 we see that the highest speeds which are attained when the car is making six stops per mile are as follows:

20-ton car-21.0 m.p.h. max. running speed
28-ton car-19.4 m.p.h. max. running speed
36-ton car-18.1 m.p.h. max. running speed

The time required for each cycle of operation will be as follows:

$$
\begin{aligned}
& 20 \text {-ton-41.00 seconds } \\
& 28 \text {-ton-44.00 seconds } \\
& 36 \text {-ton-47.25 seconds }
\end{aligned}
$$

By referring to Fig. 78 we find that the schedule speeds which ean be operated without leeway are as follows:

$\begin{array}{lcccc}\text { Stops per mile } \ldots \ldots & 3 & 5 & 7 & 9 \\ 20 \text {-ton car } \ldots \ldots \ldots & 16.5 & 13.9 & 12.25 & 10.9 \\ 28 \text {-ton car } \ldots \ldots \ldots & 15.4 & 13.2 & 11.65 & 10.4 \\ \text { 36-ton car } \ldots \ldots \ldots & 14.6 & 12.2 & 10.8 & 9.65\end{array}$



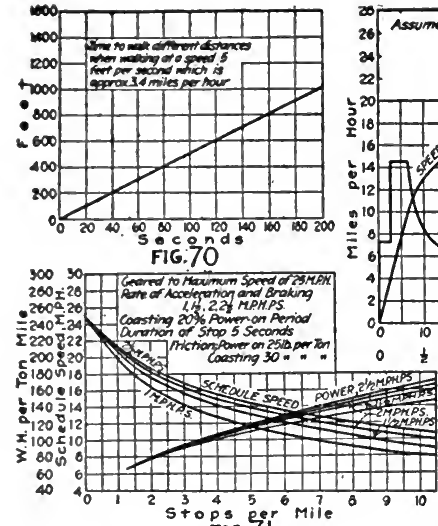
FiG. 7 il

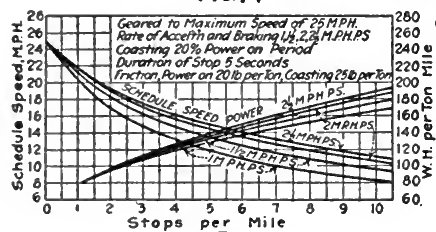

FIG.72

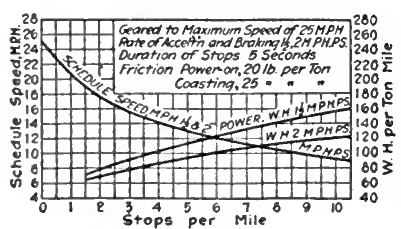

FIG.73

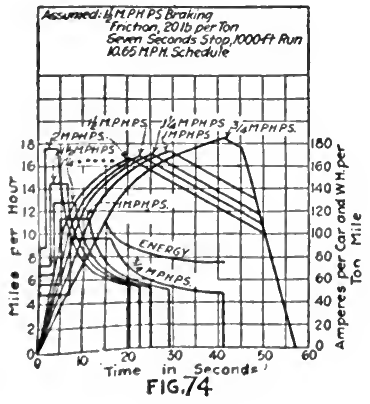

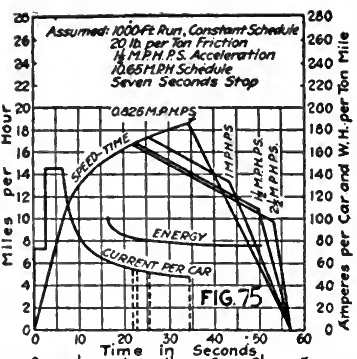

M.P. H.P. S. Braking

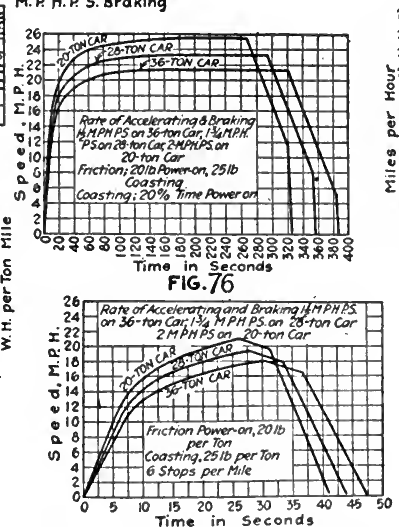

FIG. 77

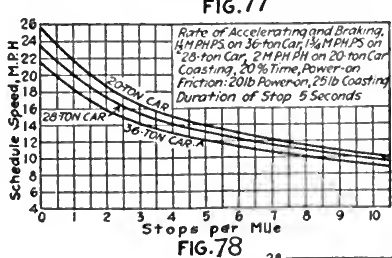

FiG.78
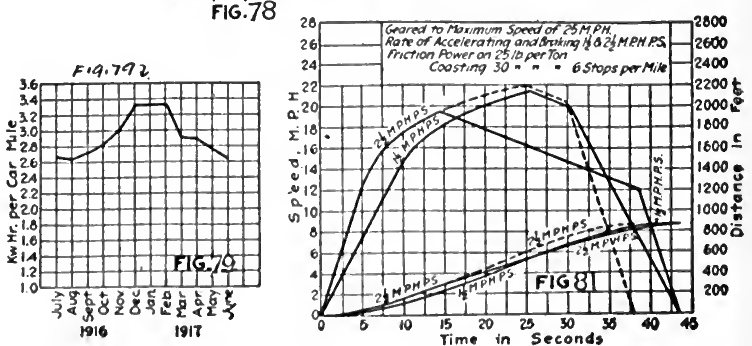
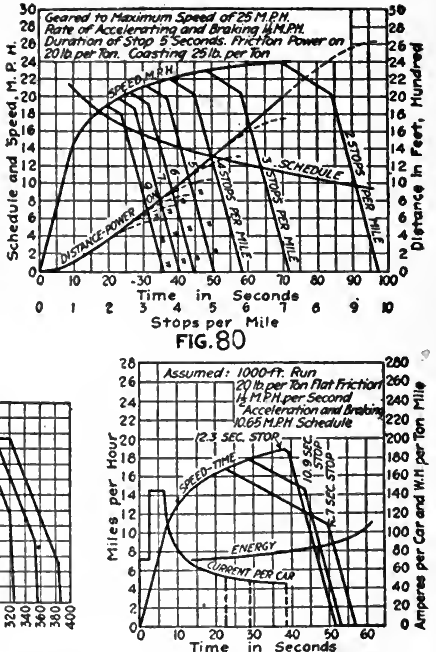

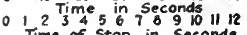

FIG 82

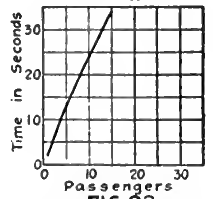

FIG.83

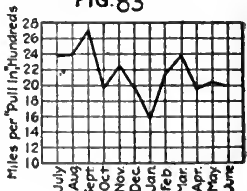

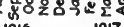
FIG.84
Fig. 70-Relation of Walking Distance and

Fig. 71-Schedule Sime Sined and Energy ConFig. sumption Curves for 20-Ton Cur

Fig. T2-Schedule speed and Fnergy Consumpion Curves for $7 \frac{1}{2}$. Ton Car

Fig 73-schedule sueed and Energy Con. sumption Curves for 20-Ton Car

Fig. 74-Chart Showing Decrease in Energy as Rate of Acreleration Is Increased

Fig. 75-Chart Showing Defrease in Fnergy

as Rate of Braking Is Increased
Fig. 76 -Siper-Time Graphs for 20-Ton, 28 .

Th. Ton and 36.Ton Cars

Fig. 77-Speed Time Gruphs for 20-Ton, 28 Ton and 36-Ton Cars

Fig. 78-Schedule Speed Curves for 20-Ton, 28.'Ton and 36-Ton Cars

Fig. 79-Graph Slowing Effect of Heating on Energy Consumption

Fit. 80-Speed, Schedule Speed and Distance Graphs for 20-Ton Car

Fig. 81-Speed and Distance Graphs for $7 \frac{1}{2}$ Ton Car

Fig. 82 -Chart Showing Increase in Energy Consumption as Duration of Drop Is Increased

Fig. 83-Loading Time of Fort Worth Safety

Fig. 84-Graph of Miles per "Pull In" (Total "Pull Ins") 
This is but one phase of the study of what weight means. Another phase is that every time excess weight is moved it is paid for in power. So we should keep weights down to the possible minimum and still retain safety and reasonable maintenance. If we desire to know the influence of weights of all different cars on the system which are in service, we can ascertain from characteristic curves what is the free running speed of each car which is in service. We can also follow up the service conditions as they actually are and obtain the number of stops per mile with which the cars are operating. From these data, and also from the data which are given on the graphs of power consumption and schedule speed possibilities, we can determine to a surprising degree of accuracy the amount of power taken by the different cars on the system. Due allowance can be made for transmission, substations and line losses, and so we can figure out the total amount of power used by any combination of equipments.

Where the mileage operated by each car and also the service in which it is operated are given, relative comparisons can be made as to what different cars cost for power consumption. Our estimates for wear and tear on track by equipments of different weight are not definite, but approximate figures can be taken to enable the operator practically to determine just what heavy cars cost. By making a comparison of this kind, the operating department will learn what cars should be run for the maximum mileage and what cars should only be used as trippers.

A number of factors which affect cost of power, such as number and time of stops, gear ratios, weights, etc., have already been discussed. The time elements, of course, also have considerable effect on platform costs.

Undoubtedly there is a great deal of loss through wasteful operation of electric heaters and motor-driven hot-air heaters. If thermostats and automatic switches were used, the ear-heating peak shown in Fig. 79 would be reduced 25 to $33 \frac{1}{3} \%$. At the same time, the riders would be more comfortable, because of the more uniform temperature attained. $30 \%$ of the electric heaters ordered last year from one maker included thermostats.

Among the minor power losses are those due to dragging brakeshoes - a condition that can be eliminated partly by the use of air brakes, automatic slack adjusters and more coasting. The installation of these devices will require new capital, which the operating company may or may not be able to secure at a reasonable rate of interest.

In addition to these factors is the overwhelmingly important one of the motorman's handling of the car operating cycle, namely acceleration, coasting; braking. Here are important opportunities if we first learn how to analyze the possibilities for ourselves and then instruct the motormen with the aid of car checking instruments to operate exactly as they should.

Therefore, it will be of decided advantage to know just how many seconds are required for each particular part of the cycle of car operation. In order to facilitate our study Figs. 80 and 81 have been made up for the big and small cars respectively. Fig. 80 illustrates a car weighing 20 tons, which is geared for a maximum free running speed of 25 m.p.h., when accelerating and braking at 11/2 m.p.h.p.s. using $20 \mathrm{lb}$. 
per ton friction during the power-on period and $25 \mathrm{lb}$. per ton friction during the coasting period. Let us assume that this car is operating in a service with six stops per mile. Then the car will require 26.3 secs. to reach the point where the current is cut off and will have to traverse a distance of $580 \mathrm{ft}$. The time required to the end of the coasting will be 31.7 secs. and the distance will be $700 \mathrm{ft}$. The time required to the end of the braking, namely, when the car comes to a complete stop, will be 44.5 sees. and the distance traveled will be $880 \mathrm{ft}$. On the lower ordinate it will be noted that time and stops per mile are specified. The stops per mile refer to the schedule speed graph which is shown above and should not be confused with the distance graph or with the graphs which illustrate the actual car speed, coasting and braking.

Since the energy that can be saved during the run cycle depends largely upon the time available for coasting, it is not only desirable to use high rates of acceleration and braking, but also to shorten the length of stop. By referring to the graphs, Fig. 82, we see that by making a seven-second stop the coasting will amount to $120 \%$ of the power-on period, while by extending the length of stop to 10.9 secs. this figure is further reduced to $47.8 \%$; by further increasing the length of stop to 12.3 secs. the coasting period is reduced to $2.63 \%$. It will therefore be readily appreciated that if we are operating a car at a given schedule, the length of stop is a big factor in the amount of coasting possible. We should, therefore, help the crews all we possibly can to take advantage of this phase of operation in addition to obtaining the permissible maximum rates of acceleration and braking.

The use of anti-friction bearings, particularly for the journals, naturally will extend the coasting period.

It may be added here that even on lines of three or four cars, where the larger economies (like saving a car) are impracticable it is at least possible to get more coasting, which means power savings.

The number of cars taken from service every day because of equipment defects directly pictures the physical condition of the apparatus and, broadly speaking, usually represents the amount of money which is spent for equipment maintenance. Where there are a large number of failures the cost of maintenance is in proportion.

Records should be made of all crippled cars turned in by the transportation department; and it is advisable to transeribe this information into classified graphs which will show on the basis of car-miles per failure, the failures to motors, electrical efjuipment, car trucks, bodies and hrakes respectively. On large, well-operated systems mileage per failwre varies from 2,800 to 4,400 car-miles. By the proper segregation of these failures the mechanieal department knows exactly what portion of the efuipment is causing the largest number of troubles and can use its energies intelligently to eare for each trouble as it comes along.

It is even more important to know just how many minutes delay certain defects caused in service on the line. With such a comparison, added to the ineehanical analysis, it will not take long to learn what erguipments are umprofitable and unsatisfactory.

There should always be graphs showing the effect of the total pull- 
ins. A glance at these charts, such as Figs. 84, 85 and 86, will show whether or not the mechanical department is maintaining the proper relations betwen failures and pull-ins.

The influence of rates of pay and considerate treatment of platform employees is strongly brought out by a study of the Dallas Railway under Richard Meirwether, now general manager. The attached graphs, Figs. 87 and 88, show how a voluntary increase in the average rate of pay from 21 cents to 26 cents an hour over a period of six years held the men.

In 1911 the average length of service of a trainman was 26 mos. while in the first half of 1917 the average length of service had increased to 49 mos.

The influence which the retention of the men in the service has to accidents is very striking. It will be noted that the total expense in settling claims for this property was reduced from approximately $\$ 97$,000 in 1911 down to approximately $\$ 50,000$ in 1916 . The costs in each case are exclusive of the shop repairs and mileage losses due to shopping time.

Aside from the decreased cost of accidents we must consider the saving in instruction cost and apprenticeship periods.

Fig. 89, derived from"Blake \& Jackson's "Electric Railway Transportation," presents these figures on a year-by-year basis.

One example of the effect of jitney competition will be quoted to show what records were kept as a basis for discovering the habits of the intruder, for determining the extent of the losses and for finding a remedy.

Although the jitney appeared in this particular city in 1914, there were still 268 jitneys in July, 1916, and by July, 1917, the number actually rose to 282 . In the intervening months, however, as Fig. 90 shows, the number declined with the severity of the weather.

The small initial investment in a jitney and the usual absence of all service obligations make it easy to withdraw individual machines from service; the large collective investment and fixed charges in an electric railway and the franchise requirements compel a large measure of service. Interest necessarily has to be paid on the investment, and every intelligent effort possible must be made to conserve that investment so that new capital may be obtained for new work.

Of course, those companies which tried to answer jitney competition by cutting down their own service made a tactical error. It was not necessary, however, to go to the other extreme of increasing the service at all hours of the day. If the jitney traffic had been plotted by hours, as in Figs. 91, 92 and 93, the extra service could have been put on only when it served the purpose of making competition unprofitable.

That the electric railway ean win on the basis by the use of small, fast, one-man ears on short headways is now certain. The safety car in particular has proved that fact in a host of places.

Hotel Operating Characteristics. From an article, "Records at the Blackstone," by W. A. Eberman, published April 18, 1916, in Power, the following is quoted to show the use of charts in obtaining efficient supervision.

To make a comparison of one period with another, monthly results 

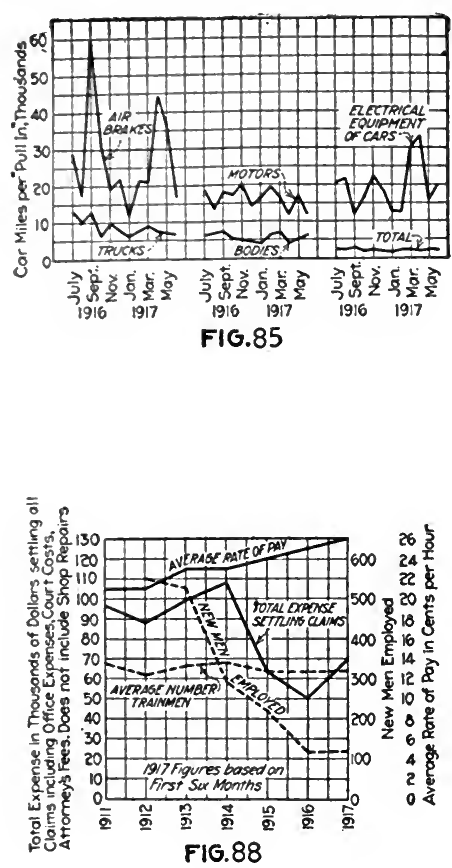
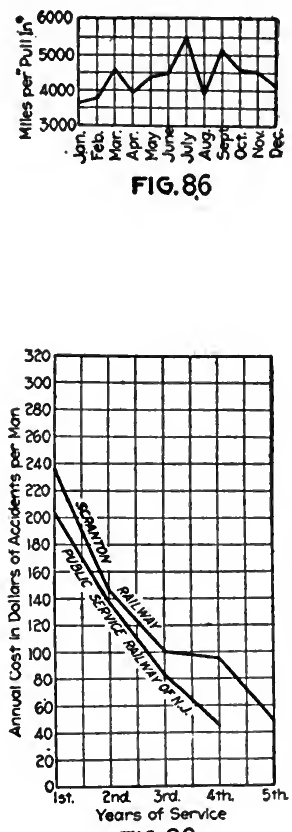

FIG,89
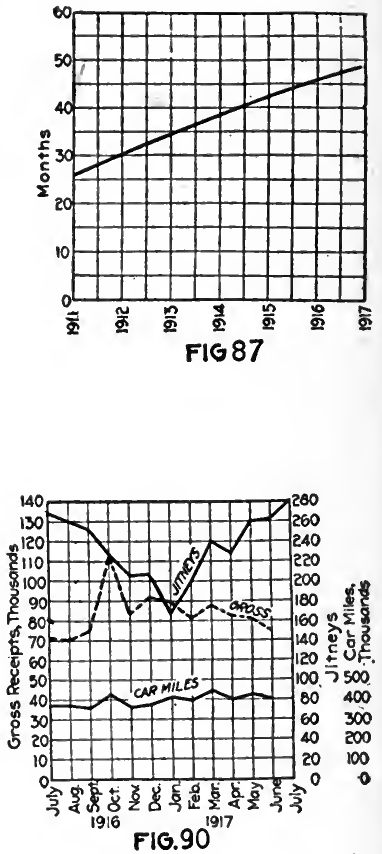

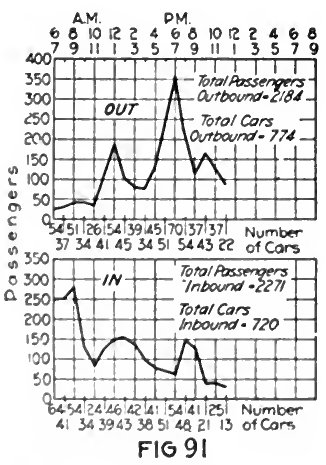

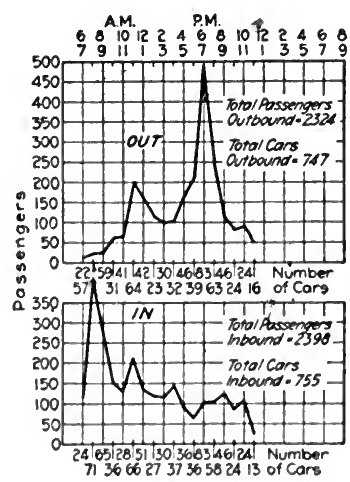

FIG 92

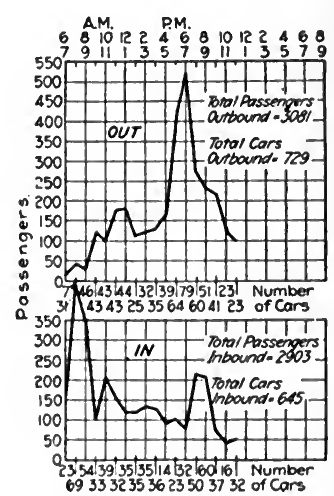

FIG.93
Fig. 8.j-C"ar-Miles per "Pull In," i.e., Cars Removed from Service Due to Car Defects

Fig. 86-Car-Miles per "Pull In"

Fig. 87-Average Duration of Service of Trainmen, Dallas

Fig. 8. - Relation of Now Mon Employed and Cost of Settling Claims

Fig. 8!-Ciraphs Showing How Platform Experience ('uts Platform Cost
Fig. 90-Craphs Showing Irregularity of Jitney Service and Regularity of Street Car Service, Town B, Excluding Suburbs

Fig. 9l-Jitney Cheek in Town B, on C Street at Corner of First Street

Fig. 92-Jitney Check in Town B, on $\mathbf{E}$ Street at Corner of Tenth Street

Fig. 93-Jitney Check in Town B, F Street, Corner Fifth Street 
most often wanted for reference are plotted on cross-section paper in a series of curves that show at a glance whether the consumption, output or expense at one period is higher or lower than normal. The curves make plain the connections between changing conditions and varying output. After plotting them for a few years, a lot of information is secured as to what may be expected in certain seasons of the year, and any great departure from previous seasons stands out prominently. In this plant curves are plotted not only of engine-

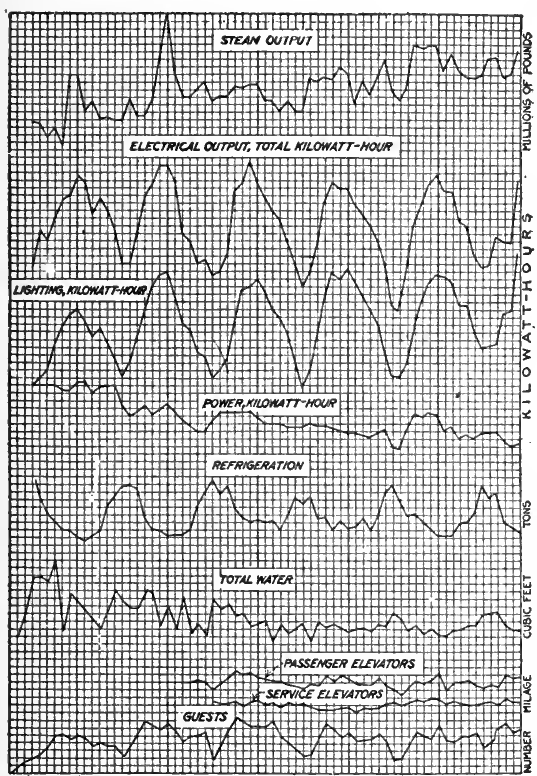

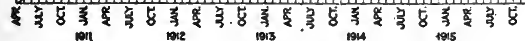

Fig. 94-Some of the More Important Curves, Including Steam and Kilowatt Output

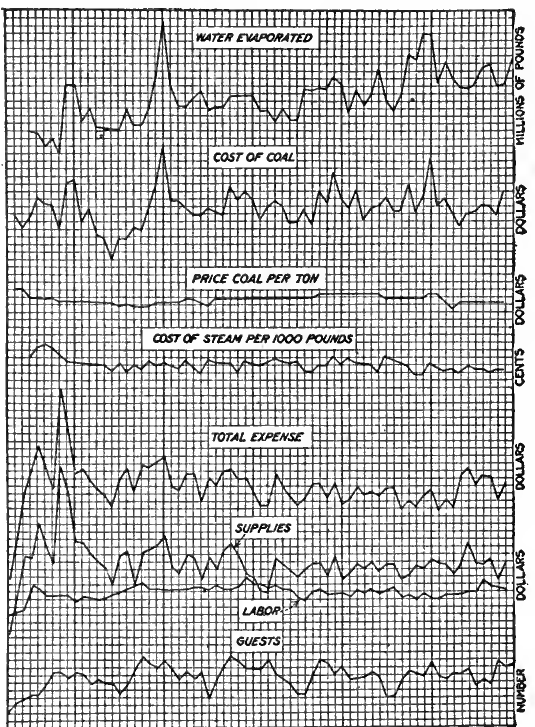

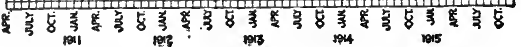

Fig. 95-Cost Curves and Total Operating Expenses

These curves show the cost of water evaporated, cost of coal and the price per ton, also the cost of steam per 1,000 lb. The total expense curve is shown, also those for supplies, labor and the number of guests.

room performances, but of costs in the various subdepartments, these costs being subdivided between labor and material. Some of the more important curves are reproduced in Figs. 94 and 95. The curves kept on file are of steam output, total electric load, current for lighting and for power, refrigeration load, water handled, temperature averages and elevator mileage. Other charts take care of the expense incurred and show the total expense, total labor and supplies. The expenses of subdepartments in which labor and supplies are large and subject to considerable variation are charted, and another chart divides the cost of labor and material between the mechanical division and the engine room. 
To the mechanical division are applied expenses other than for the actual operation of the engine-room machinery.

To show the convenience of these charts, suppose the engineer was asked why the expense for a given month was higher than the corresponding month of a previous year. Reference to the charts will show

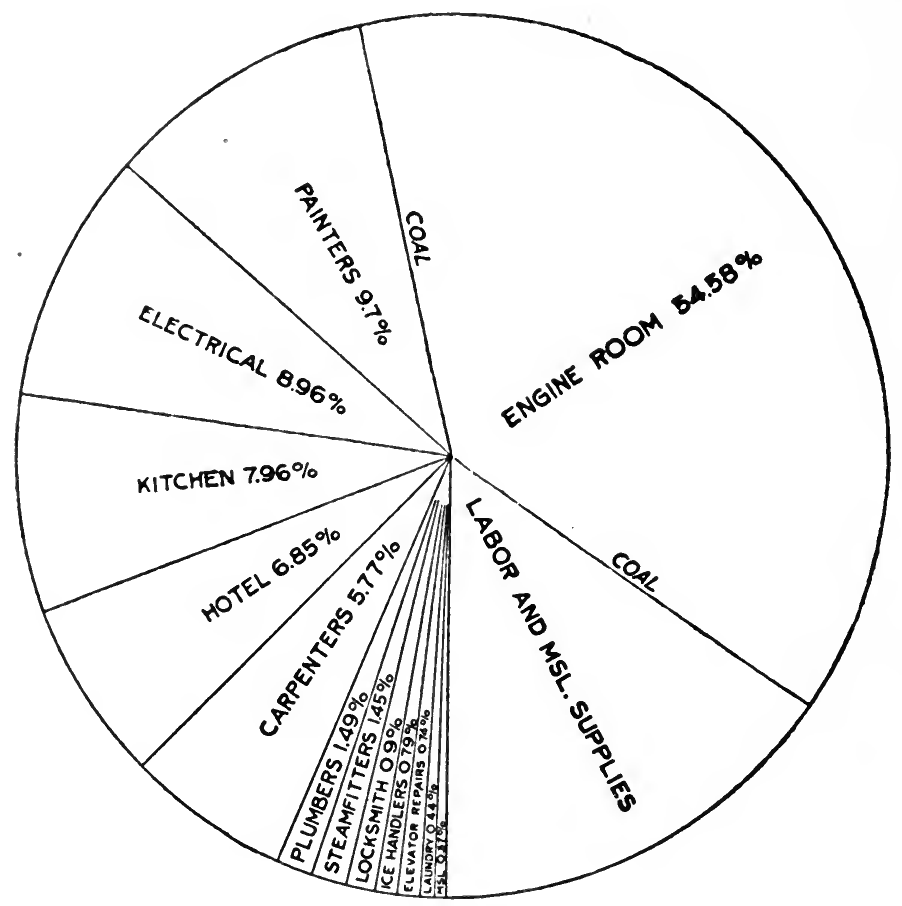

Fig. 96-Percentage Each Subdivision Bears of Total Cost of Mechanical Department

that the total expense is higher and that the increase occurs in both the mechanical division and in the engine room. It is shown that the mechanical-expense increase is due to more work by the painting department, which has a larger force working, and reference to the card index shows that a large stock of material was bought during that month. The cngine-room expense is higher, but no increase in labor is shown, so it must be due to output in excess of that of the previous month in (puestion. The steam curve shows an increase, and as the coal is up in the same ratio, it is evident that the efficiency has not been lowered. The clecetrical load is higher, and the eurves show whether or not it is due to extra lighting or power. Assume that they are both high. Part of the power consumption can be accounted for by referring to the elevator mileage "urve, which shows a deeided rise. The increase of lighting is due to more people being served, which is indicated both by the elevator mileare-a good index-and the curve giving the number 
of people served. A greater water consumption is also evident. This is to be expected with more people using it in their rooms, more linen to be washed, more food to be prepared and cooked and more dishes to be washed, all of which take water.

The question is raised, "Why is it that in the summer months, with current consumption at its lowest, the steam does not come down in proportion?" The answer is found by looking at the curve of refrigeration, where it is seen that the output was at its greatest. The reason for this is found by looking up the weather curve showing the average mean temperature for the month, which in this case is high, and the necessity for more ice and box cooling and more and cooler air for the restaurant, lobby, etc. In fact, the whole story is plotted out so that anyone can easily read it. It is worth the trouble.

The curve charts are fastened to thin boards, $18 \times 24$ in., which slide in grooves in a cabinet made for them. Both sides of these boards can be used, so that 22 sheets can be handled. Fig. 96 is a chart prepared to show at a glance the percentage each subdivision of the mechanical department bears to the total expense for labor and supplies.

Following, is a list of articles containing further illustrations of the use of graphic charts of the class discussed in this chapter and pertaining to kindred subjects.

Upon request, the Codex Book Co.. Inc., 19 William Street, New York, will be glad to procure, where possible, copies of magazines containing the articles needed, only charging the "old magazine" price, plus postage.

"The Individuality of Water Works System," Engineering and Contracting, June 3, 1919.

"Coasting Records of Northern Texas Traction Company," Electric Railway Journal, June 26, 1915.

"Electric Car Maintenance, J. F. Layng, Paper presented at meeting of Pennsylvania Street Railway Association, Scranton, Pa., December 14, 1915.

"Power Plant Efficiency" Victor J. Azbe, Journal of American Society of Mechanical Engineers, December, 1916.

"Value of Fuel Economy in the Power Plant," Reginald Trautschold, Industrial Management, August, 1917.

"Cost of Manufacture in Distilled-Water Ice Plants," Peter Neff, November 25, 1913.

"Relative Resistance of Various Hard Woods to Injection of Creosote," Clyde H. Teesdale and J. P. MacLean, Railway Review, July 20, 1918.

“"High Vacuum Condenser," Electrical World, February 22, 1913.

"Diversity and Diversity Factors," Terrell Croft, Power, February 4, 1917.

"Coal Consumption Required for Certain Pump Duty," Electrical World, December 2, 1916.

"To Indicate the Amount of Feed-water Pumped into Boilers," F. Sawford, Power, October, 1907.

"Centrifugal Versus Reciprocating Boiler Feed Pumps," M. William Ehrlich, Electrical World, November 11, 1916. 
"The War-Time Operation of Motor-Driven Deep-Well Pumps," P. S. Biegler and I. W. Fisk, Electrical World, August 17, 1918.

"Stray Losses in Direct-Current Machines," W. C. King, M. B. McDermott, and F. W. Maxstadt, in Electrical World, August 12, 1916. "Capacity of Beit Magnetic Separätors," Geo. J. Young, Engineering and Mining Journal, November 16, 1918.

"Graphs for Finding Safe Current-Carrying Capacity of Insulated Copper Wire," M. C. Mason, Electrical Review, December 28, 1918.

"Effect of Grades and Curves in Plotting Speed-Time Graphs," C.

W. Squier, Electrical Railway Journal, July 20, 1918.

"Current and Power Curves Show Results to be Fxpected of Motors."

C. W. Squier, Electrical Railway Journal, July 15, 1918.

"Solution of Grinding Problems," Sergio Bagnaro, Engineering and Mining Journal, July 14, 1917.

"Orifice Bucket for Measuring Water," Engineering and Mining Journal, Nareh 29, 1919.

"Flow of Water in Pipes," E. W. Greene, Power, December 4, 1917.

"Developing Efficiency in Central Stations," C. M. Rogers, Power, July 4, 1916.

"Storage Batteries for Handling Peak Loads," Everard Brown, Power, April 6, 1915.

"The Significance of Load Graphs," Terrell Croft, in Power, October 2,1917 .

"Thermal Value of Coal," W. C. Stripe, Power, January 11, 1916.

"Chart for Determining Heat Loss in Flue Gases," II. F. Hutzel, Power, December 12, 1916.

"Characteristies of Uniflow Engine Generating Units," E. Hagenlocher, Electrical World, February 10, 1917.

"An American Locomobile," Power, Décember 16, 1913.

"Cutting Power of Lathe Turning Tools," Wm. Ripper and G. W. Burley, Iron Age, December 18, 1913. 


\section{CHAPTER XIV}

\section{Showing the Results of Tests and Experiments}

As an aid to research charts are invaluable. Almost every large industrial concern now has its own testing and experimental departments and in all big cities there are laboratories devoted entirely to investigation along untraveled paths. The scope which such tests cover is endless and the purpose here is to show by a few illustrations the methods, in the hope that they will offer valuable suggestions that may be applied in many other directions.

Strength of Materials. E. R. Hedrick is authority for the following, taken from his article, "A Generalized Form of Hooke's Law," in Engineering News, September 16, 1915:

It has been known for a long time that many materials fail to satisfy Hooke's law exactly, even within the range usually described as below the elastic limit. Even such substances as steel and wool have measurable variations from this law that have long been recognized by physicists and engineers. Other substances, such as cast iron, concrete, bronze, copper, stone, rope and so on, depart from the law very widely indeed.

The writer discovered originally for a set of data on the extensions of rubber that the measured values of forces and extensions gave a very much better straight line on logarithmic paper than on squared paper. This means, of course, that the relation between the force $p$ and the extension $e$ is in that example much better expressed by a relation of the form

$$
p=k e^{\mathrm{n}} \quad \text { or } \quad e=c p^{1 / \mathrm{n}}
$$

if $n$ is taken different from unity. In the example just mentioned the value of $n$ was found to be approximately 0.625 .

I have been working over the data contained in the Reports of the Watertown Arsenal, in which the numerical results of the actual tests of a large number of substances are given. Contrary to my original expectation, I soon began to suspect that the effect noticed in the example mentioned is not peculiar to rubber, but occurs also with many other substances.

I have now worked over a substantial part of the tests in several of the volumes of the Watertown Reports, and I think it is safe to announce that these results follow a straight line on logarithmic paper better than they follow a straight line on squared paper, in a very large number of cases. Fig. 1.

For steel, of which the tests are most numerous, the value of $n$ is quite close to unity, but it is in most instances very definitely different from unity, and there are specimens that give values as low as 0.69. 
The majority of specimens of steel give values of $n$ over 0.85 , but many specimens give values less than 0.9 .

Some other substances give values of $n$ near to unity. Thus, an excellent speeimen of long-leaf yellow pine, under compression, follows a straight line on logarithmic paper with remarkable fidelity, and gives a value not far from 0.95 . Other specimens of wood show greater divergence; thus, a piece of fir, across the grain, gave $n=0.85$, approximately.

Uther substances show still great divergences. Thus, a specimen of

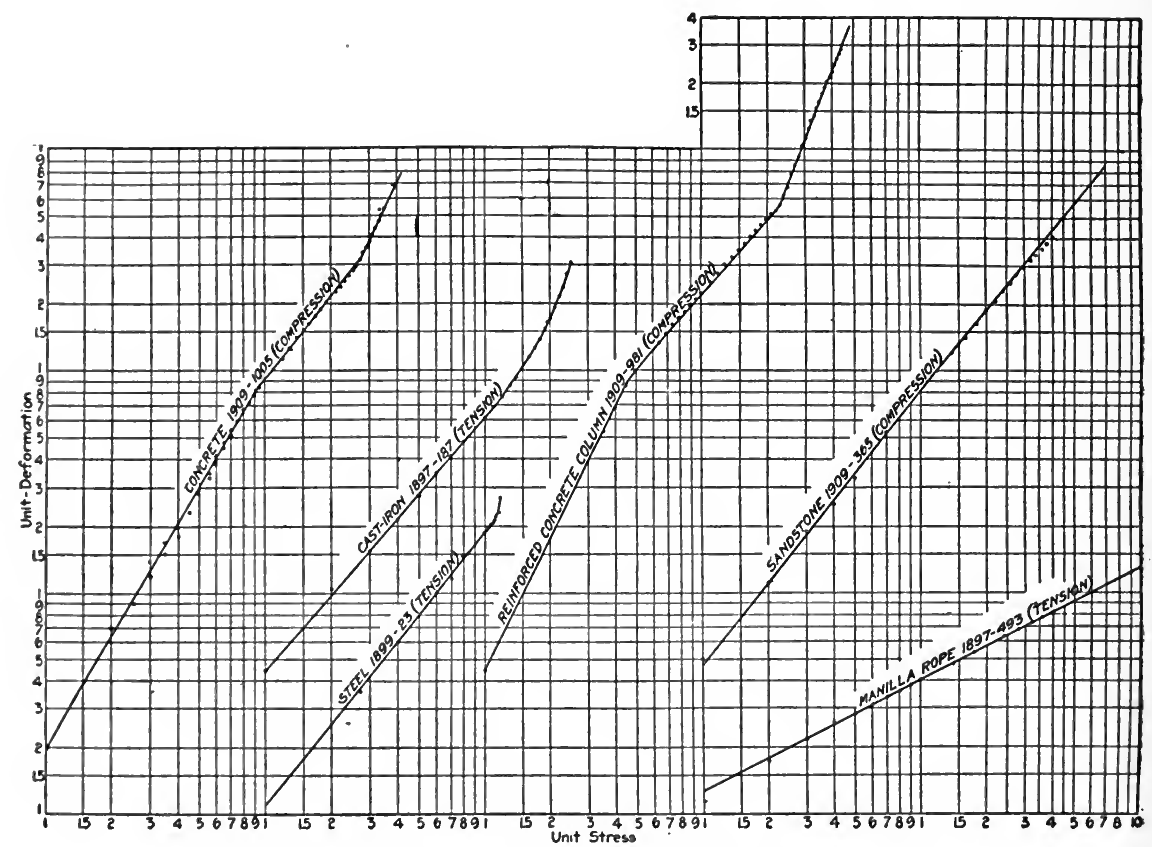

Fig. 1-Strength Tests of Various Materials

east steel gives $n=0.74$. Two specimens of east iron give 0.69. Several specimens of copper give a set of remarkably parallel lines that correspond to 0.56 . A specimen of sandstone gives 0.80 .

$A$ case that is worthy of mention is that of rope. A piece of manila rope gives a very good line for $n=1.94$.

The behavior of concrete, both plain and reinforeed, is very remarkable. Evidently, a further study of this important substance is desirable as will be seen if an attempt is made to draw the corresponding lines. Throughout long ranges of values the pressures and compressions follow a straight line on logarithmic paper. These lines take suddenly a different direction, as will be seen in a most pronouneed fashion if even a hasty sketch is made of the test in Watertown Report 1909, pp. 981-982, in the range from $1,000 \mathrm{lb}$. to $4,400 \mathrm{lb}$. per sq. in.

Soil Pressures. From an artiele by J.'A. Moyer in Engineering Record, March 13, 1915, is taken the following: 
To develop mathematical formulæ useful to the practical designer of culverts, arches and subways, investigations of the vertical pressures due to the loading of sand and other soils on such structures are being conducted by the Engineering Experiment Station of Pennsylvania State College. The results of the tests thus far indicate that the per cent of transmission increases with the increase of load, that for depths of earth greater than $2 \mathrm{ft}$. this per cent of transmission is always less than 20, and that for eccentric loads it is always less than 20 when the loading strip is not over any part of the weighing strip. The loading was carried as high as 6 tons.

The apparatus used consisted of a large wooden box, $6 \times 12 \mathrm{ft}$. supported on concrete piers by means of I-beams and joists. In the center of the floor of the box there was an opening $12^{1 / 4}$ in. square, into which the weighing strip or "block," measuring $12 \times 12$ in., was placed. The load on this weighing strip was supported on a large Standard platform scales. A "restricting" box, containing the soil tested surrounded the weighing strip. The size of this box was $4 \frac{1}{2} \times 9 \frac{1}{3} \times 5 \mathrm{ft}$. deep. The loads were applied by a calibrated hydraulic jack, exerting its load on a wooden strip $12 \times 12 \times 3^{1 / 2}$ in., which rested on the surface of the soil. The jack was blocked up so that it acted against an overhead I-beam. The tension rods were threaded to permit ready adjustment of the height of the beam above the surface of the soil as required for the various depths.

A piece of canvas was spread lightly over the weighing strip to prevent the soil from running through the $1 / 8$-in. spaces between the weighing strip and the box. The soil was shoveled in and leveled off at a certain depth. The loading strip was firmly embedded by hand pressure so as to have a good bearing area. The jack was placed in position and blocked up against the I-beam. A dead-load reading of the platform scale was taken and loads were applied and readings of the scale beam noted for various loads as indicated by the gage up to the capacity of the apparatus, or to a point when the loading block sank into the soil as fast as the load was applied. The difference between the two scale readings-before and after applying the load-gave the amount of pressure transmitted through the soil. One hundred times this difference divided by the known weight of the load determined from the calibration curve gave the percentage of the load transmitted vertically through the soil. The distribution of this transmitted pressure was readily determined by varying the position of the loading strip. To make sure that the loading strip would sink evenly into the soil great care was exercised in preventing the jack from getting out of plumb. Whenever uneven sinking occurred the test was discontinued and run over again. Throughout all the tests the method of applying the load was precisely the same as that employed in calibrating the jack and gage.

A series of tests was run on dry sand for the purpose of determining the effect of the size of the "restricting box." The size of the box used for this purpose in previous tests was $3 \times 4 \times 4 \mathrm{ft}$. deep. In the present series this size was increased to the larger size above mentioned. Suitable bracing was provided in order to prevent the sides of the box from bulging when large pressures were used. The sand used in these 
experiments was the same as that used in the former experiments, namely, clean, dry river sand, of medium sharpness-a typical brown building sand. The following depths of this sand were tested: $3,6,12,18,24$, 36,48 and $59 \mathrm{in}$. For each depth the eccentricity of the load was varied from 0 to $42 \mathrm{in}$. both right and left. The loads were applied in increments of 1,000 lb., as indicated by the gage on the hydraulic jack, up to the point where the loading strip sank into the sand as fast as the load was applied. As determined from the calibration curve these loads varied from about $600 \mathrm{lb}$. to between 2,200 and 6,000 $\mathrm{lb}$. according to the depth of the sand.

The curves in Fig. 2 show the distribution of the vertical component of pressure due to a concentrated load. The points plotted are the averages of all the results obtained for a given depth of sand and a

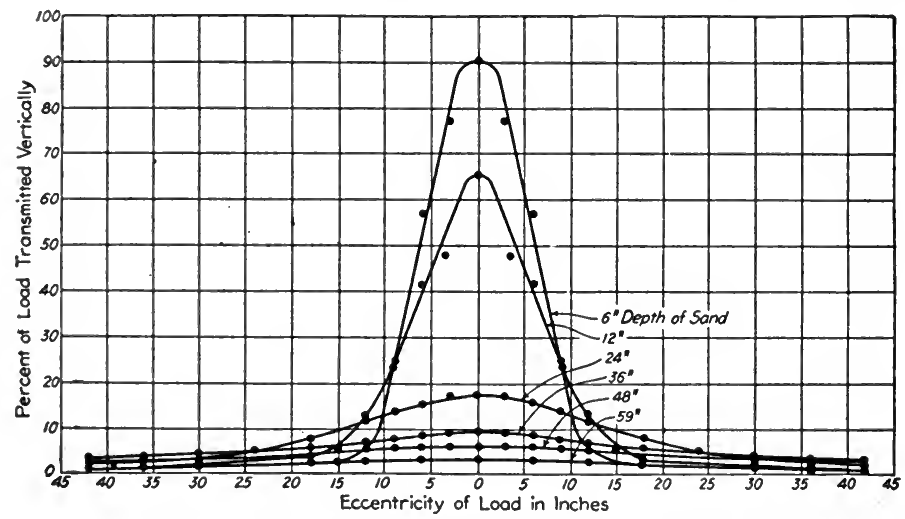

Fig. 2-Per Cent of Load Transmitted for Different Depths of Sand

Loads, varying from 1,400 to $5,500 \mathrm{lb}$., applied on $12 \times 12$-in. area; weighing area $12 \times 12 \mathrm{in.}$

given ecentricity of load. On the average about eight observations are represented by each of these points. The calculated percentages of transmission lie for the most part well within $10 \%$ of the mean value, as in the previous tests. It will be noted that as before there was a very marked change in the per cent of transmission when the eccentricity of the load was equal to the width of the weighing strip, and at this point the maximum value was $13 \%$. For greater eccentricities this value was never exceeded. By comparing the results of the present tests with those of the previous ones it will be readily seen that the size of the restricting box does not make any appreciable difference.

The maximum bearing power of the sand decreased with the depth of sand, as shown in Fig. 3, the plotted points in the curve being the averages of the maximum loads that could be applied at zero eccentricity. This was possibly due to the fact that the sand had more opportunity to flow at the greater depths. Furthermore there was a distinct tendency for the percentage of transmission to increase as greater loads were applied. The point is well illustrated in Fig. 4. Curve A shows the maximum values for sand obtained by averaging the results for the 
two highest loads that were applied. These loads averaged from 3,000 to 5,000 lb. per sq. ft. Curve B shows the results obtained in previous tests under the same conditions, but with loads up to $300 \mathrm{lb}$. per square foot only. Similar data and curves are given for a clay mixture and for loam, together with the conclusions resulting therefrom.

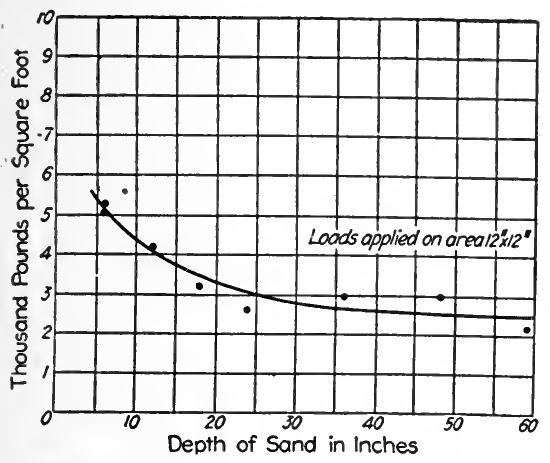

Fig. 3-Relation Between Maximum Bearing Power and Depth of Sand

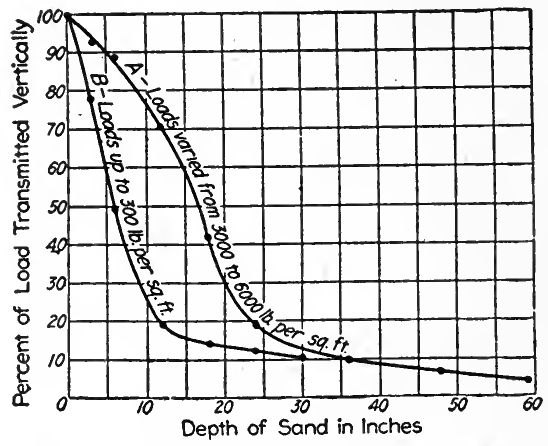

Fig. 4-Per Cent Transmission in Sand for High and Low Pressures

Test Concrete Under Freezing Conditions. Under this title Engineering Record, July 15, 1916, published an article from which is quoted the following:

Pouring of the floor slabs between steel beams in the Philadelphia Electric Power House, had to be continued during the cold winter weather. While no concrete was poured during freezing weather, the temperature was frequently much below freezing for a long period after pouring. In fact there were times when the water froze in icicles below the slabs on the day after pouring.

Although the materials were all heated before pouring (steam coils being used for the sand and gravel), so that the concrete probably had some time to set before reaching the freezing temperature, it was desired to make certain that the strength was sufficient before removing the forms. For this purpose a schedule was prepared for the various parts of the work, with greater time requirements as the length of span increased. This schedule was made by counting only the hours that the concrete had cured above a temperaure of $32 \mathrm{deg}$.

In addition 6-in. test cubes were poured from the concrete going into the floor and exposed to the same temperature conditions as the slab they represented. These cubes were tested in the laboratory, and if the strength exceeded 800 to 1,000 lb. per square inch the forms could be removed, provided there was not too much evidence of frost in the structure, and provided the floor was not loaded with material or by shores from above.

Fig. 5 shows the results of tests of both laboratory and field specimens. For the latter the results are plotted in terms of number of hours above 32 deg., the actual age being noted only in a few special cases. For example, one cube 14 days old, but with only 60 hrs. above 32 deg., failed at 250 lbs. per sq. in., and one cube 20 days old, almost half of 
that time at a temperature below freezing, failed at a unit load less: than $600 \mathrm{lbs}$. In general the results plotted on this basis of time above 32 deg. fall within the approximate curves drawn on the diagram for limits found by testing the standard laboratory eylinders kept at a temperature of $70 \mathrm{deg}$. In comparing results, however, it should be noted that eubes should give higher. results than the standard cylinders. Furthermore the cubes east on the work had the advantage of the beneficial effect of the heating of the materials and of machine mixing.

The comparison between the strength of $1: 2: 4$ conerete made of pebbles and similar conerete made of erushed stone shows no consistent. advantage either way, although the cylinders made with pebbles gave' slightly better results. This was probably due to the fact that these

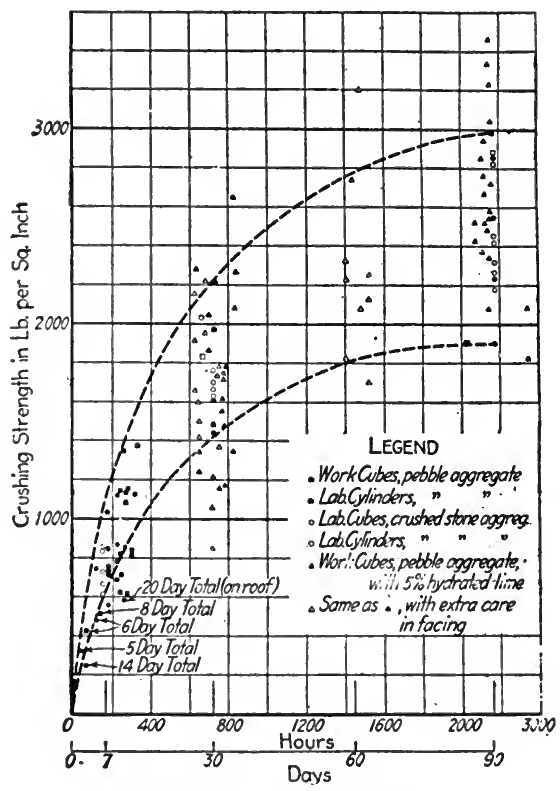

Fig. 5-Concrete Tests

pebbles were almost pure quartz, with some of granular quartz formation and some of dense sandstone.

The test results for the specimens east on the work in which $5 \%$ of hydrated lime had been used are seen to be variable, no noticeable effects on strength being obtained. It was thought that a eareful bedding of the ends of the test eylinders in the testing machine might produce better results, but, as the plotted points show, there seems to be no definite advantage.

It may be inferred from these test results that a satisfactory and reliable method of estimating the strength of concrete eured under freezing conditions is to consider the age to be the same as the number of hours of temperature above 32 deg., or preferably above $35 \mathrm{deg}$. Thus, knowing the approximate strength, it is possible to determine when it is safe to remove the forms. 
Artificial Light for Hens. The following is a very short abstract from an article by Prof. J. E. Rice, published in The Country Gentleman, June 7, 1919, telling about the results of tests of the use of artificial light to control egg production. Fig. 6 shows the comparative yield under ordinary conditions and by the use of electric lights.

Our tests at Cornell, as well as surveys of many flocks scattered through New York State and elsewhere, have proved beyond all doubt that not only egg yield but net profit has been greatly increased. Accurate records on $100 \mathrm{~S}$. C. White Leghorn hens and pullets in equal numbers showed that for a period of forty-eight weeks the net gain in production was $135.37 \mathrm{doz}$. eggs, which represented a gain in value of $\$ 71.88$. With these birds lights were used from twilight to nine P. M. The gain in production lasted from November 28, through the

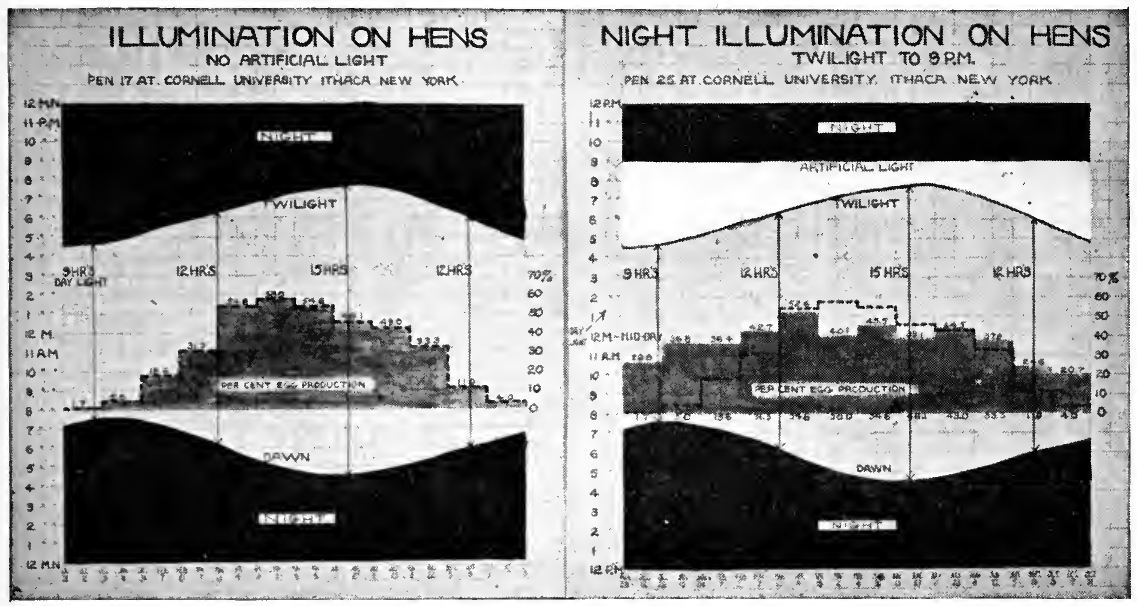

Fig. 6

winter until March 19. There was a decrease in production from March 20 to August 6. From August to November there was a gain by the flocks under lights. The total gain of the birds under artificial light as compared with the birds without lights was 212.90 doz. against a loss of $77.53 \mathrm{doz}$., leaving the net gain of $135.37 \mathrm{doz}$. Very much larger gains have been secured for several months in succession on private flocks. The left hand portion of Fig. 6 shows the average length of Daylight for a Year in New York State with the average egg production for various months. Note how yield increases with the length of day.

The right-hand part shows how by the use of lights the light period is extended to $9 \mathrm{P}$. M. Note how the egg yield is spread through the year and increased in the winter months, when the yield is very low without lights.

Light Distribution. For plotting the results of luminosity tests polar paper is best adapted, as the intensity at different angles may be readily shown. Fig. 7 illustrates the maximum and minimum distribution 
curves for reflector for lighting units placed at approach to apex of hump of a railroad classification yard, candle power values for $35 \mathrm{ft}$. mounting height. This is from Railway Review, November 25, 1916.

Tests of Efficiency of Train and Team Drawn Dump Wagons. As a result of an investigation made by $R$. T. Dana for the Troy Wagon Works Company to determine the relative eosts of operation of reversible dump wagons handled in trains and team dump wagons, Fig. 8 was plotted.

The operation of the Troy reversible dump wagons of $3 \mathrm{cu}$. yds. capacity was compared with that of team dump wagons of $1 / 2 \mathrm{eu}$. yds. capacity on four road construction jobs, under various conditions. The

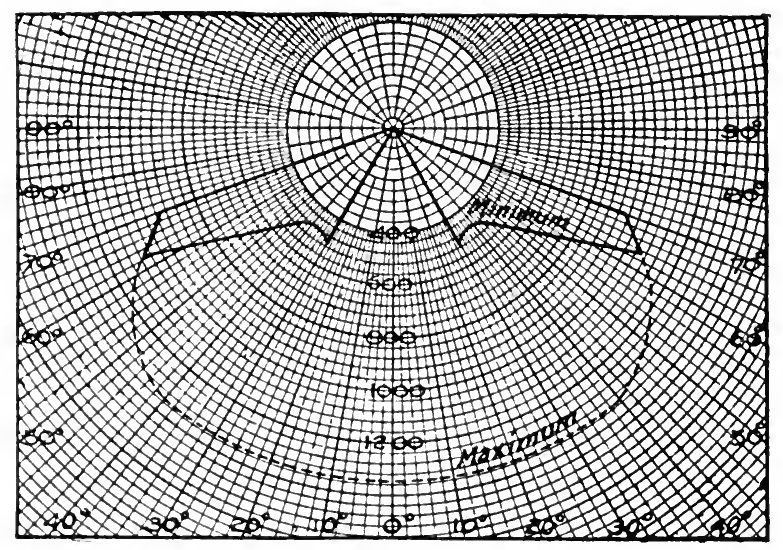

Fig. 7

haulage costs per ton in cents up to $3 \%$ miles haul were deduced from the following formulas in which $C=$ total expense per day; $t=$ the rated eapacity; $S=$ the average speed loaded; $K=$ ratio of the speed light to the speed loaded; $K S=$ the average speed returning; $L=$ the time lost in loading, unloading, turning, resting and wasted for an average round trip; $R=$ the total cost per ton for transportation, and $W=$ the number of minutes or hours in the working day.

(1) $D / S=$ time for loading trip.

(2) $D / K S=$ time returning.

(3) Combining with $L$ (above) and factoring:

$T=D / S(1+1 / K)+L=$ average time for round trip.

(4) $N=W / T=$ average number of round trips per day. The value must be a whole number for average work for any one day.

(5) $N t=$ average total amount transported per day.

(6) $R=C / N t=$ cost of transportation per pound or per ton.

$\mathrm{C}$ depends so largely on the prevailing price of teams that it will vary aceording to locality.

Studying the curves it will be noted that wide savings are effected by the train (indicated by the solid line) over the teams (indicated by the dotted line) in the longer hauls, as, for instance, assuming a haulage of erushed stone in a six-wagon train (or 40,000 lbs. per load), and in 
evidenced by the fact that several times, when, governed by the idea that the more scrapers he had working the more material he could handle, it was found by test that it actually cost less in cents per cubic yard and more material was moved, by taking off one or more scrapers. By tests it was determined what were the economical hauls for each type of scraper and the economieal number of scrapers for each haul.

Traffic Census. In an article published in Engineering News, November 2, 1916, Wm. Holden describes the methods, forms, etc., used by the City of St. Louis in making a traffic census. With respect to charts he says: In order to present the traffic data a little more clearly, traffic charts were plotted as follows: (1) Average traffic for each

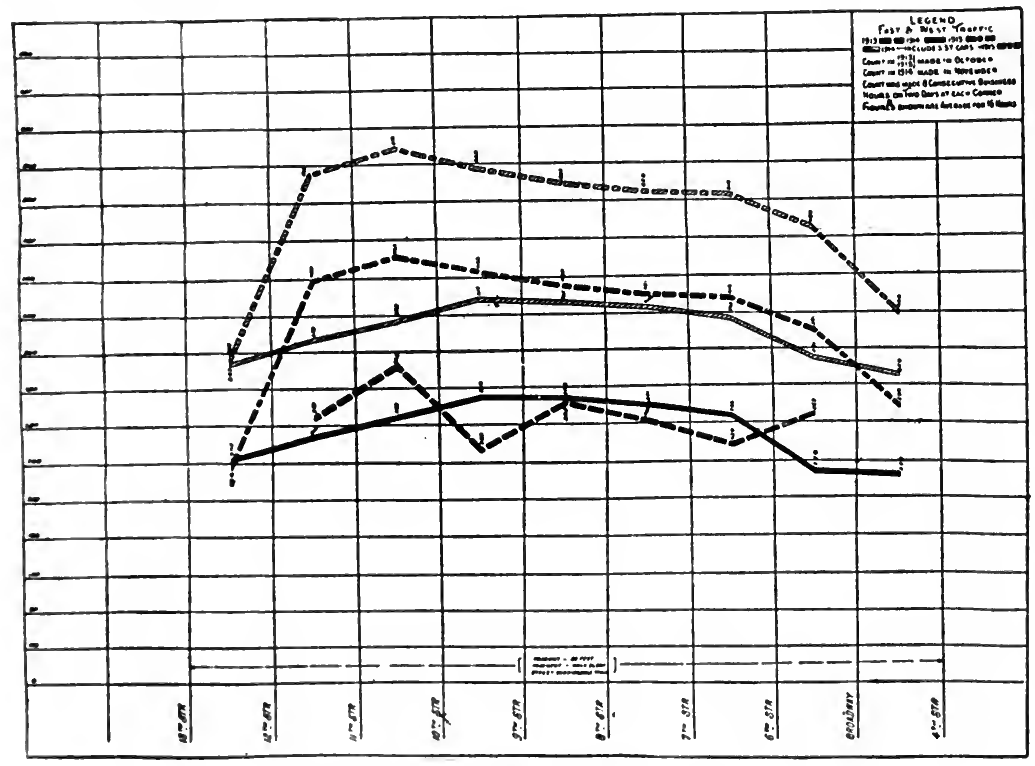

Fig. 9-Average Traffic Chart

street in the downtown distriet: (2) maximum traffic for intersections in the downtown district; and (3) traffic of the enngested district.

The Average Traffie charts show the average number of vehicles per hour (sum of both directions) and vehicles plus strect ears in each block observed on the street nuder ennsideration. On these charts, as on Maximum Traffic charts, black lines represent total vehieles and the shaded lines represent total vehicles and st peet cars combined. On the Average Traffic (harts (Fig. 9) the horizontal base represents the street, divided into blocks by the vertical lines representing the interseeting streets. The vertical dimension is ruled off into 1-in. spaces which represent 40, 60 or 100 vehicles (or vohicles and street cars), depending on the seale used, which varied in aceordance with the amount of traffic carried. The average number of vehicles per hour (total of all classes) for each block was plotted in the midlle of the seetion $r$ presenting that block on the chart. These points were eonnected by lines. The com- 
bined number of vehicles and street cars was platted for each block, and the points were connected with a shaded line. From the Average Traffic charts the fluctuation of traffic per hour in different blocks can readily be seen. On account of their effect on the volume of traffic there are also shown width of roadway, kind of pavement and number of car tracks on the Average Traffic chart of each street.

The Maximum Traffic chart, Fig. 10, for each street shows the number of vehicles and vehicles plus street cars crossing each intersection

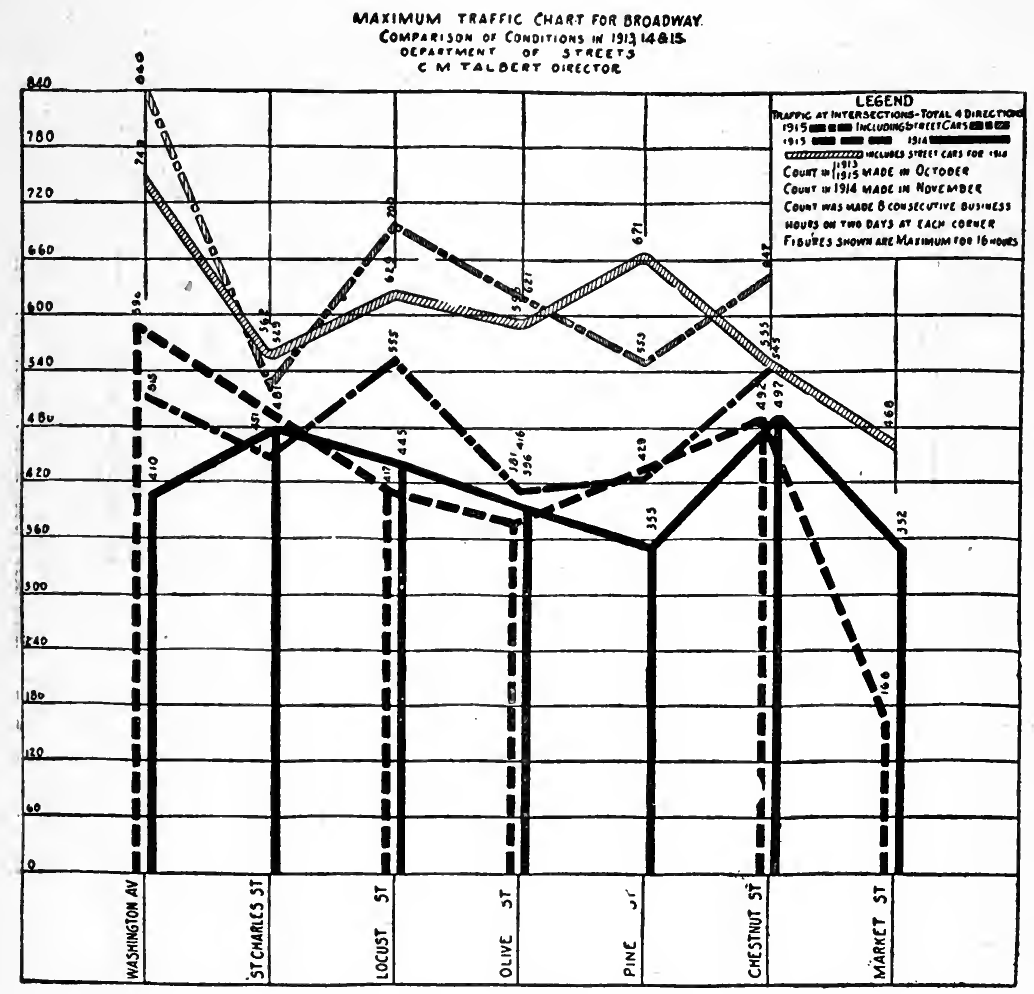

Fig. 10-Maximum Hour-Traffic Chart

on the street during the maximum hour from all four directions. The horizontal base line represents the street under consideration, and the vertical lines represent the cross-streets. The total number of vehicles during the maximum hour for each intersection is platted on the proper vertical line representing the cross-street. The relative volumes of traffic at the different intersections, as well as that of the 1914 census, are readily compared from these charts.

A Traffic Chart of the Congested District (1915) shows the average number of vehicles per hour (totaling both directions) for each block of every street observed in the 1915 census. The vehicles are platted to a scale of $1,000=1 \mathrm{in}$. in width on a map of the territory drawn to a scale of $200 \mathrm{ft} .=1 \mathrm{in}$. Thus by comparing the relative widths of the 
black lines representing traffic we see at a glance the eomparative volumes of traffic on different streets and different parts of the same street.

Rail Wear. Electric Railway Journal, June 26, 1915, describes tests made in Chicago, as follows:

To determine the extent to which vehicle traffic is responsible for the wear of rails, a large number of observations were made at different points of the system. The results are thoroughly eonsistent and are shown in Fig. 11, in which the relative portions of wear from cars and.

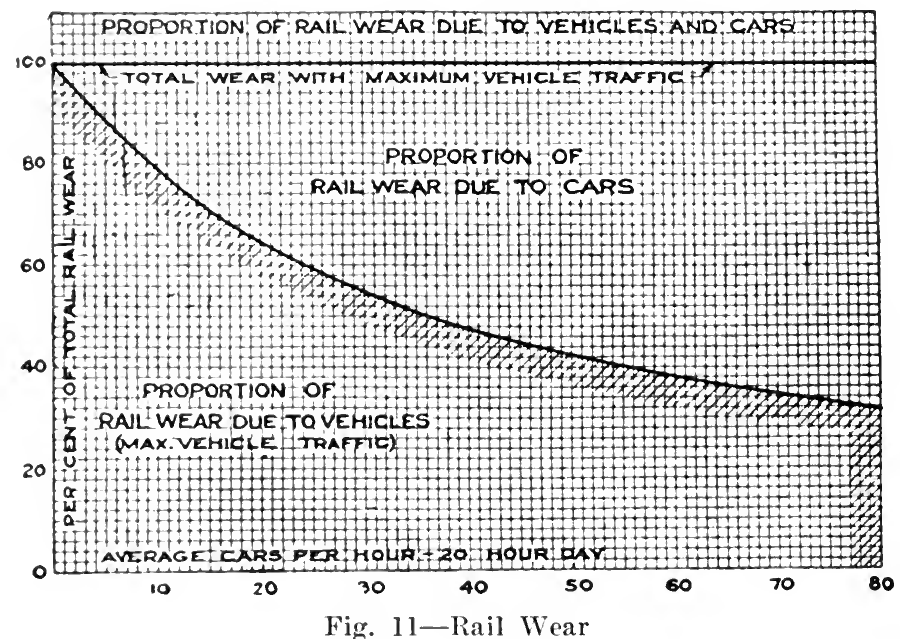

vehicles are shown for varying average headway of cars along the street. This diagram shows that even on a line with a great deal of railway traffic, such as 40 cars per hr. for a $24 \mathrm{hr}$. day, vehicle wear may amount to as much as half of the total wear on the rail head, thus reducing the life of the rail by a corresponding amount. Other measurements, taken on short sections of track where the rail was not subject to any vehicle wear but carried a large car traffic, showed a wear no greater than that on streets, with only half the car traffic but with heavy team traffic.

Gasoline Engine Test. A letter from R. S. IIawley to Power, printed A pril 6. 1915. describes a brake test on a gasoline engine.

Fig. 12 shows the results of a brake test on a 10-hp. gasoline engine, made to determine the cost ner brake horsepower per hour under different loads and, incidentally, the regulation under these loads. The gasoline pump was diseonnected and the gasoline was fed to the vaporizer ly gravity from a 5-gal. can provided with a nipple and cock; the flow being regulated so that only a small amount appeared at the overflow. This was collected and poured back into the supply ean, which was weighed at the beginning and at the end of each run. The revolutions per minute were taken almost continuously by speed indicators and the: average readings were used in the ealeulations. Each run was of $30^{n}$ min. duration, which, although not long enough to obtain extremely 
accurate results, was sufficiently accurate for the purpose. The cost is based on gasoline at 15 cts. per gal.

Friction of Lineshaft Bearings. Power, March 10, 1914, printed the following:

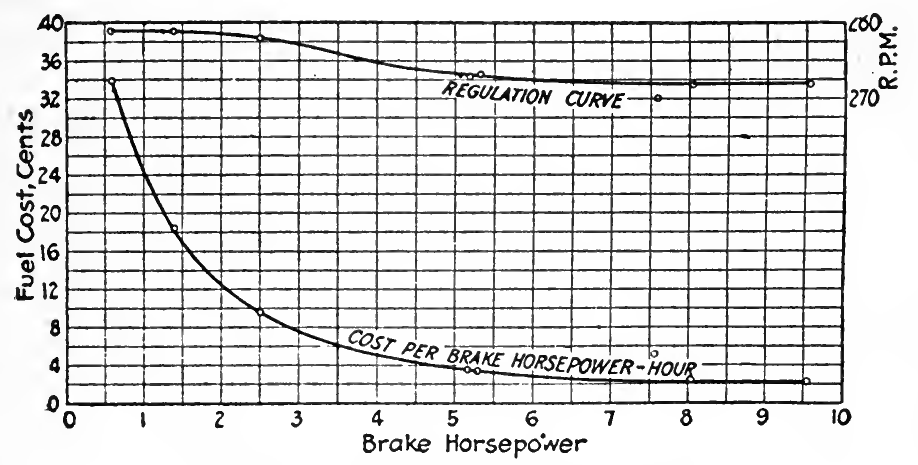

Fig. 12-Regulation and Cost Curves

In a paper read before the A. S. M. E., by Carl C. Thomas, E. R. Maurer and L. E. A. Kelso, some interesting data were given on the absolute and relative power required to drive a specially constructed series of shafts, flexibly connected and carrying loads uniformly dis-

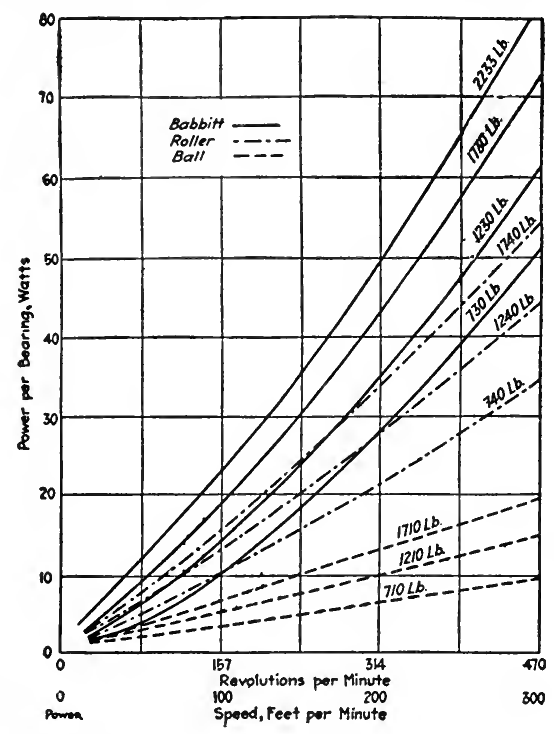

Fig. 13-Power Consumed by Friction; Bearings at 100 Deg. F.

tributed, when the shafts were supported successively by 20 ring-oiled babbitt bearings, then by 20 roller bearings, and finally by 20 ball bearings. Wide ranges of loads and speeds were covered and the coefficients of friction were worked out for each type of bearing. 
The apparatus, which was designed and built in the department of steam and gas engineering at the University of Wisconsin, consisted of $25 \mathrm{ft} .10 \mathrm{in}$. of line-shafting in five equal sections, mounted in hangers. which were inverted and used as floor stands. The hangers were bolted to two 8-in. I-beams which were leveled on the floor, and the shafts. were of cold-rolled steel, $2^{7 / 16}$ in. in diameter. Each section was $5 \mathrm{ft}$. $2 \mathrm{in}$. long; the adjacent sections were coupled together by means of a flexible leather disk or two straps connecting the two flange couplings. The flexible couplings prevented transmitting any part of the load applied on one shaft to either adjoining section and binding between shafts and bearings due to possible lack of alignment. A direct-current Fort Wayne motor was directly connected to one end of the shafting. by means of a flexible coupling.

The three kinds of bearings tested were the Hess-Bright ball bearing,

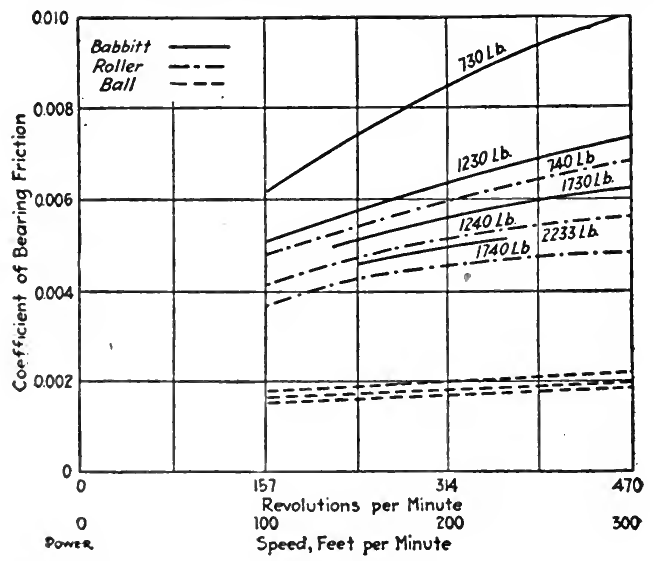

Fig. 14-Coefficients of Friction; Bearings at 100 Deg. F.

the ring-oiled babbitt bearing made by the Dodge Manufacturing Co. and the Hyatt roller bearing. All bearings were for the same size of shaft, and the same pieces of shafting were used for all the tests. The babbitt bearing was $9^{21} / 32 \mathrm{in}$. long and its projected area 22.36 sq.in. Each roller bearing contained six right-hand and six left-hand rollers $0.78 \mathrm{in}$. in diameter; six were $9 \% 16 \mathrm{in}$. and six were $93 / 16 \mathrm{in}$. long. The bearings were of the type in which a cage is used for holding one-half the rollors. Each ball bearing contained a single set of balls $\% 16$ in. in diameter. The diameter in the inner race through the ball groove was $3.4729 \mathrm{in}$.

In the tests the speeds were between 150 and 450 r.p.m., eorresponding respectively to about 100 and $300 \mathrm{ft}$. per min. peripheral speed. Most of the loads were between 700 and $1800 \mathrm{lb}$. per bearing, corresponding. to about 30 and $80 \mathrm{lb}$. per sq. in. for the babbitt bearing.

Fig. 13) shows a comparison of the babbitt, roller and ball bearings. for $100^{\circ}$. Fach curve gives the power required per bearing to run theshaft under the load and speed indicated. The power for the babbitt is seen to be higher than for the other bearings except perhaps at low 
loads and speed, and that the power for rollers is higher than that for balls. The excess of power for babbitt over rollers and rollers over balls increases with the increase of speed for all loads.

Fig. 14 presents the comparison in another way; the ordinates are coefficients of friction instead of power as in the preceding figure.

A series of curves were presented to show how the coefficients for babbitt, roller and ball bearings change with the time after starting. In each case the coefficient, and hence the power, decreased rapidly at first. For the babbitt bearing there is a marked difference between the average coefficient for the first and for the second hour; this difference is less marked with the roller bearing and still less with the ball bearing.

Fig. 15 shows the rise in temperature for the different kinds of bearing under practically identical conditions. Curve $A$ is for babbitt bearings under a load of $1230 \mathrm{lb}$. per bearing, curve $B$ is for roller bearings under $1240 \mathrm{lb}$, eurve $C$ for roller bearings under $1740 \mathrm{lb}$.

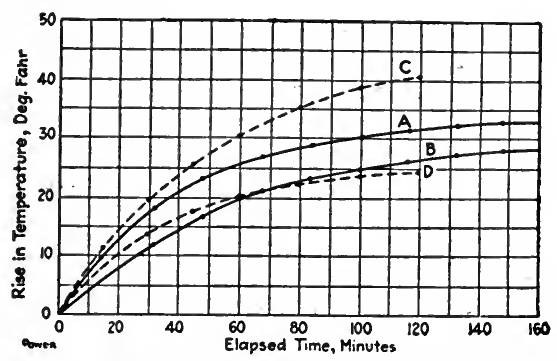

Fig. 15-Rise in Temperature with Bearings Under Practically Identical Conditions

and curve $D$ for ball bearings under $1710 \mathrm{lb}$. In the tests represented by curves $A$ and $B$, the speed was 350 r.p.m., and the room temperature 80 to 81 deg.; in those resulting in curves $C$ and $D$ the speed was 450 r.p.m. and the room temperature 78 to $80 \mathrm{deg}$.

Breakdown tests were run on each type of bearing with only one section of shafting on which were four bearings. The maximum load was about $5000 \mathrm{lb}$. per bearing. A speed of 200 r.p.m. was chosen because it represents about the average lineshaft speed in practice. The tests began at about $3200 \mathrm{lb}$. per bearing. Failure occurred at about $4250 \mathrm{lb}$. per bearing in the case of the babbitt, $4650 \mathrm{lb}$. of the ball bearing and about $5100 \mathrm{lb}$. of the roller bearing. The bearings did not fail structurally as the power was cut off soon after distress was manifested, but the failure was simply that of the lubricant. The authors also told how the methods of testing were developed and in an appendix gave diagrams of motor losses and graphical logs of tests.

Following is a list of articles containing further illustrations of the use of graphic charts of the class discussed in this chapter and pertaining to kindred subjects.

Upon request, the Codex Book Company, Inc., 19 William Street, New York, will be glad to procure, where possible, copies of magazines 
containing the articles needed, only charging the "old magazine" price, plus postage.

"The Heat Insulating Properties of Commercial Steam Pipe Coverings," L. M. MeMillan, Heating and Ventilating, Jan., 1916.

"Direct-Current vs. Alternating-Current for Cable Testing," Electrical World, August 12, 1916.

"Characteristies of Iron Wire for Transmission Purposes," L. W. W. Morrison, Electrical World, July 14, 1917.

"Factors That Determine Economical Life of Transformers," Theodore B. Morgan, Electrical World, Jan. 13, 1917.

"Traction is a Straight-Line Function of Tire Width," Engineering Record, Narch 24, 1917.

"Results of Tests of Bearing Power of Moist Blue Clay Underlying Chicago," Engineering and Contracting, April 17, 1912.

"Slippage of Reciprocating Pumps," T. B. Hyde, Power, April 6. 1915.

"Chart for Determining Economy of Insulation," Austen Bolam. Electrical World, June 15, 1918.

"Reaming Charts and Data," Karl G. Keck, American Machinist, Nov. 22, 1917.

"The Moisture Content of Textiles and Some of Its Effects," William

D. Hartshorne, Journal American Soc. Mechanical Engineers, Nov., 1917.

"Flow Measurement in a Water-Collecting Gallery by the Chemical Method," Engineering and Contracting, April 11, 1917.

"Soot Removal," H. R. Blessing, Power, May 4, 1915.

"An Investigation of Stenches and Odors for Industrial Purposes,"

V. C. Allison and S. H. Katz, Journal of Industrial Engineering \& Chemistry, April, 1919.

"The Economical Loading of Transformer Banks," Electrical World, October 19, 1918.

"Plotting Blower-Test Curves," A. II. Anderson, Journal Amer. Soc. Mechanical Engineers, Nov., 1917.

“'Traffic Study Data," D. D. Ewing, Electrical Railway Journal, Feb. $19,1916$.

"Effect of Gear Ratio on Operating Economy," Electric Railway Journal, .July 10, 1915.

"Rail Bond Testing-Determining and Interpreting Bond Resistance,"

II. H. Febrey, Electrical Railuay Journal, Dec. 11, 1915.

"'Brush Tests,"' Keith MacLeod, Electrical Railway Journal, Dec. 11, 1915

"Characteristies of Iron and Steel Conductors," Charles E. Oakes and Winfield Eckley, Electrical World, Oct. 14, 1916.

"Iron Wire for Distribution and Transmission Lines," Electrical World, April 8, 1916.

"Comparative Test of Water Heaters," Ileating and Ventilating Magnzine, .Jan., 1916.

"T'esting Drain Tile and Sewer Pipe," Mont Schuyler, Engineering Record, Dece. 20, 1913.

"A Logarithmic Aggregate Card and Its Application to Asphaltic 
SHOWING RESULTS OF TESTS AND EXPERIMENTS 325

Paving Mixtures,' C. C. Kennedy, Engineering and Contracting, March

7, 1917.

"Determining Efficiency of Gas-Fired Boilers," The Iron Age, March $15,1917$. 


\section{CHAPTER XV}

\section{Trends, Tendencies and Statistical Prediction}

In this chapter are presented several examples of how to use charts to show trends and tendencies such as prices, design, etc. The value of such data invariably lies in the means which they afford for the prediction of future events. At best such methods are bound to be approximations and their value is enhanced in just the degree that good judgment and experience are employed in connection with them. By the use of charts, however, a method of looking into the future is afforded which is of the greatest help and value.

Trend of Bond and Stock Markets. Figs. 1 and 2 are examples of charts published from time to time by the financial department of the New York Tribune to show the course of the bond and stock markets in New York over a considerable period. Such charts are particularly

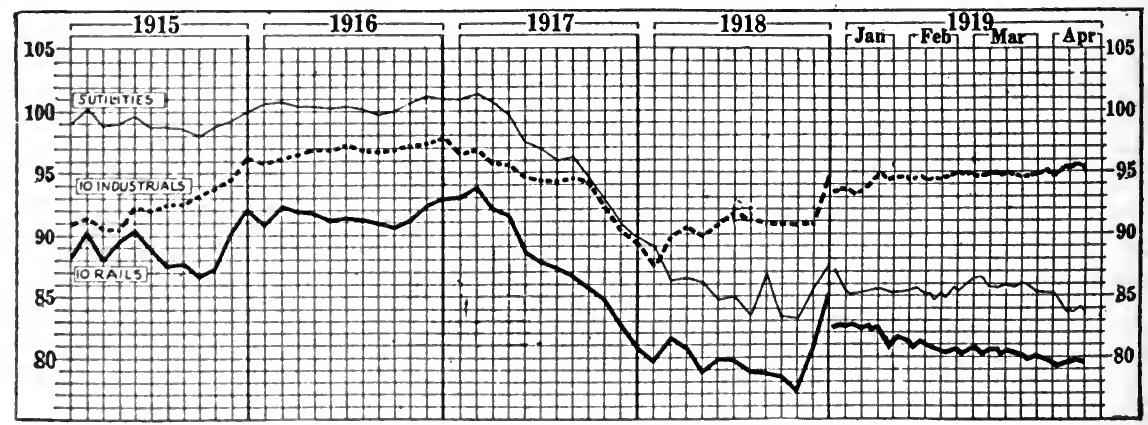

Fig. 1-Price Trend of New York Bond Market

interesting to those holders of securities who, owing to their isolated locations, are not in close touch with the New York market and daily quotations. The points plotted are for the first business day of each month. For such eharts the 12 by 20 divisions per inch paper is designer.

Prices of Commodities. An illustration of what an excellent picture one may obtain of the fluctuations and trends of prices of commodities is given by Fig. 3. This is reproduced from the Iron Trade Review, January 4, 1917.

Trends in Design and Manufacture. Fig. 4 is a good example of trends in design and manufacture. These curves are especially interesting to a prospective purchaser, and would also be of inestimable value to one about to start business along that line. This chart is from Automotive Industries, January 3, 1918, and they explain that considering all the chassis listed without regard to the output of each 326 
maker the averages deduced are averages of engineering opinion; and it is this which such analyses seek to discover. An analysis by production would throw into absurd prominence the features of a very few cars which happen to be very cheap to be made in huge amounts.

Membership Trends. Fig. 5, taken from a report of the American Institute of Mining Engineers, shows the tendency of membership increase for the four large engineering societies.

Steel Prices Reflect Demand. A letter from Dan Patch to Engineering Record, February 24, 1917, states that he has just completed an investigation of steel prices for the information of the buying staff of the company with which he is connected. Fig. 6 indicates that steel prices show a suspicious tendency to follow closely the old law of supply

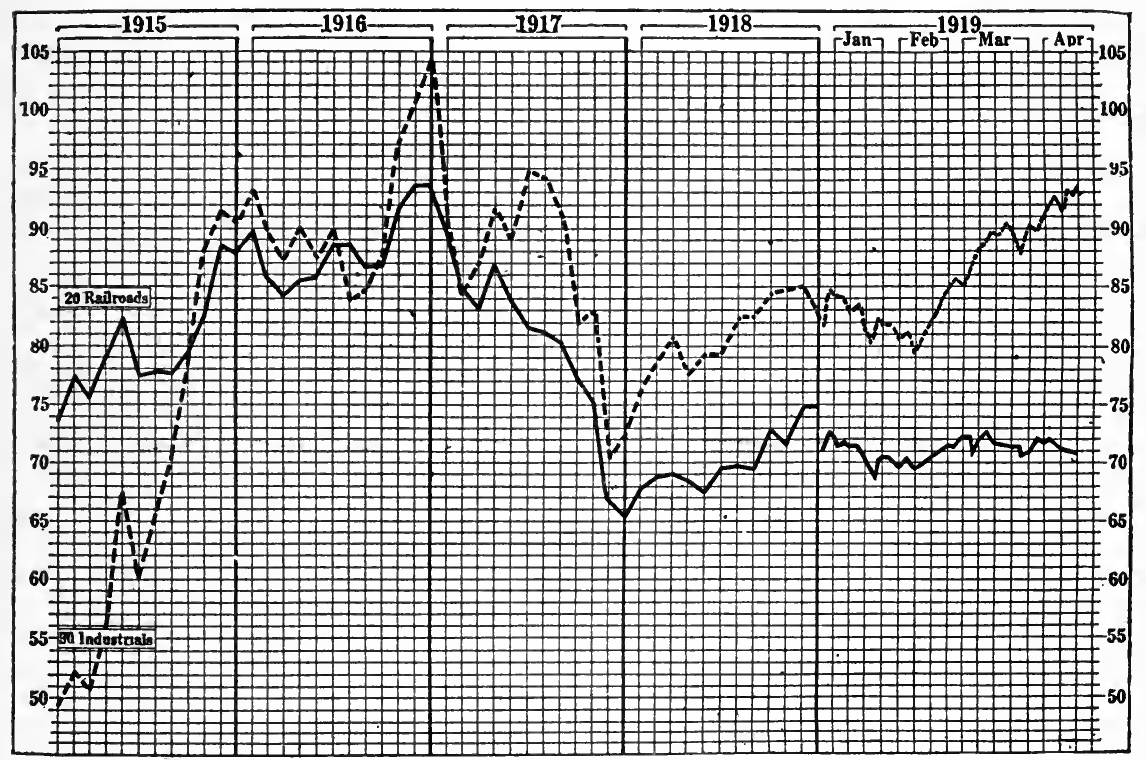

Fig. 2-Price Trend of New York Stock Market

and demand. The dashed line was made by plotting the monthly balance of unfilled orders published by the United States Steel Corporation, which is taken as a fair barometer of the demand for steel. The solid line is a curve showing the prices per $100 \mathrm{lbs}$. quoted on corresponding dates for steel bars, f.o.b. Pittsburgh.

Business Death Rate. A short portion of an article by S. A. Dennis, "When Will My Business Die?" published in System, October, 1917, shows a method of gathering and arranging statistics to give tendencies -in this instance whether the death rate of business concerns in the locality investigated is increasing or decreasing. This is cited merely as an illustration of a method of analysis applicable to an almost endless variety of cases.

Insurance men know a great deal about how fast human beings die. Business men, however, know next to nothing about the business death 


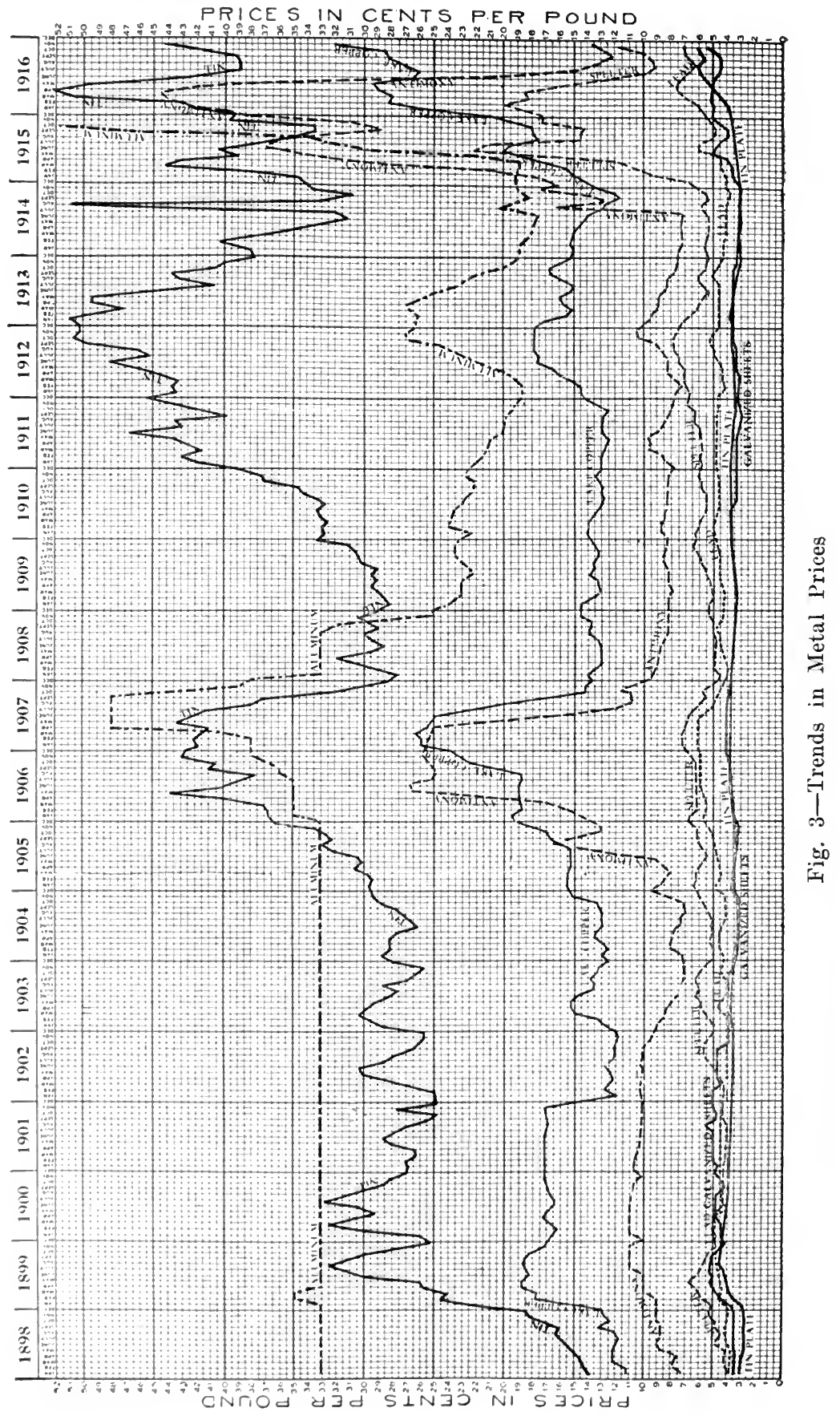


rate. Yet the two subjects are analogous-and for business men the business death rate is surely as important as the human death rate is for insurance men.

The human death rate tells how many persons for each 100 of population will on the average die each year. It shows more or less accurately how many people will die of each principal disease. The causes behind these diseases are of course well understood in many instances. Records being compiled each day indicate whether the human death rate is increasing or decreasing. If it is increasing, the causes are often easily determined-and once the causes are determined, it is usually not difficult to apply a remedy. Certain diseases that used to cause epidemics are today almost never heard of, simply because

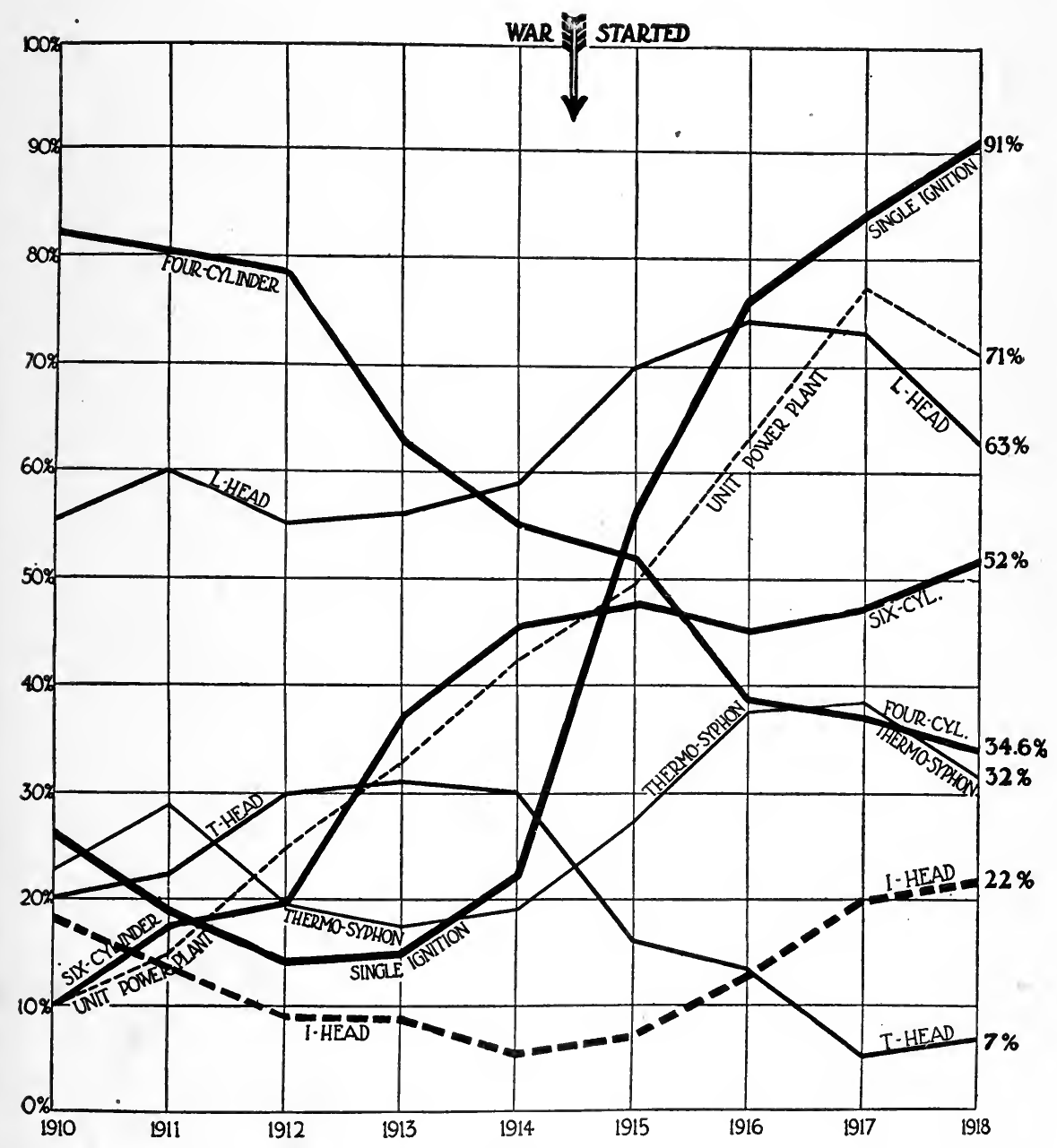

Fig. 4-Trends of the American Automobile for 8 Years, Showing Variations of Most Important Factors in Design and Manufacture 
medical men have learned the causes and the remedies; and that has made the world a much healthier place.

Does it not seem logical to suppose that in almost the same manner a great work can be done for business? In other words, if we knew more or less accurately how fast concerns die, the common causes behind such failures, and the methods most effective in fighting them, we would surely be forearmed in an important way.

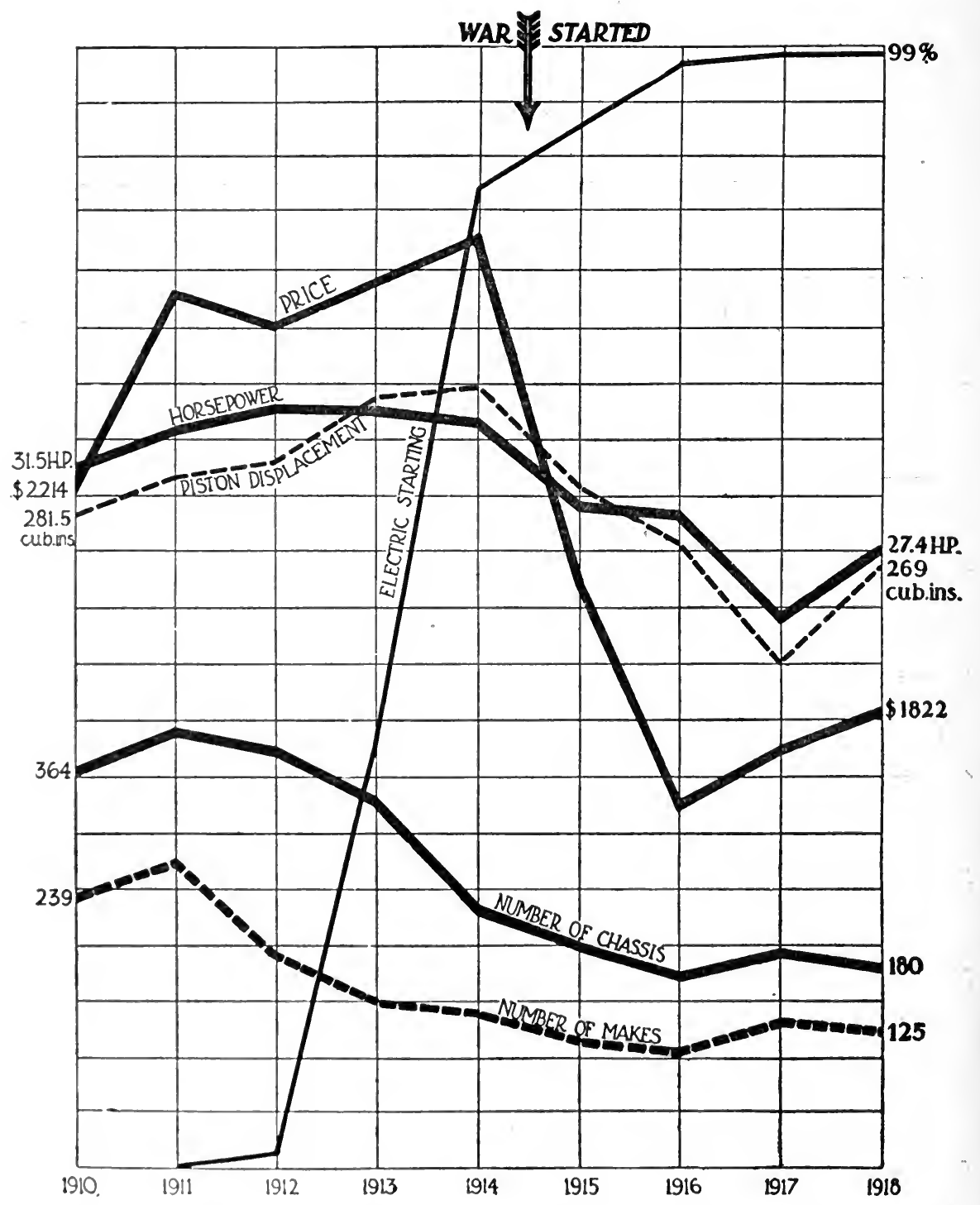

Fig. 4-Trends of the American Automobile for 8 Years, Showing Variations of Most Important Factors in Design and Manufacture 
TRENDS, TENDENCIES AND STATISTICAL PREDICTION 331

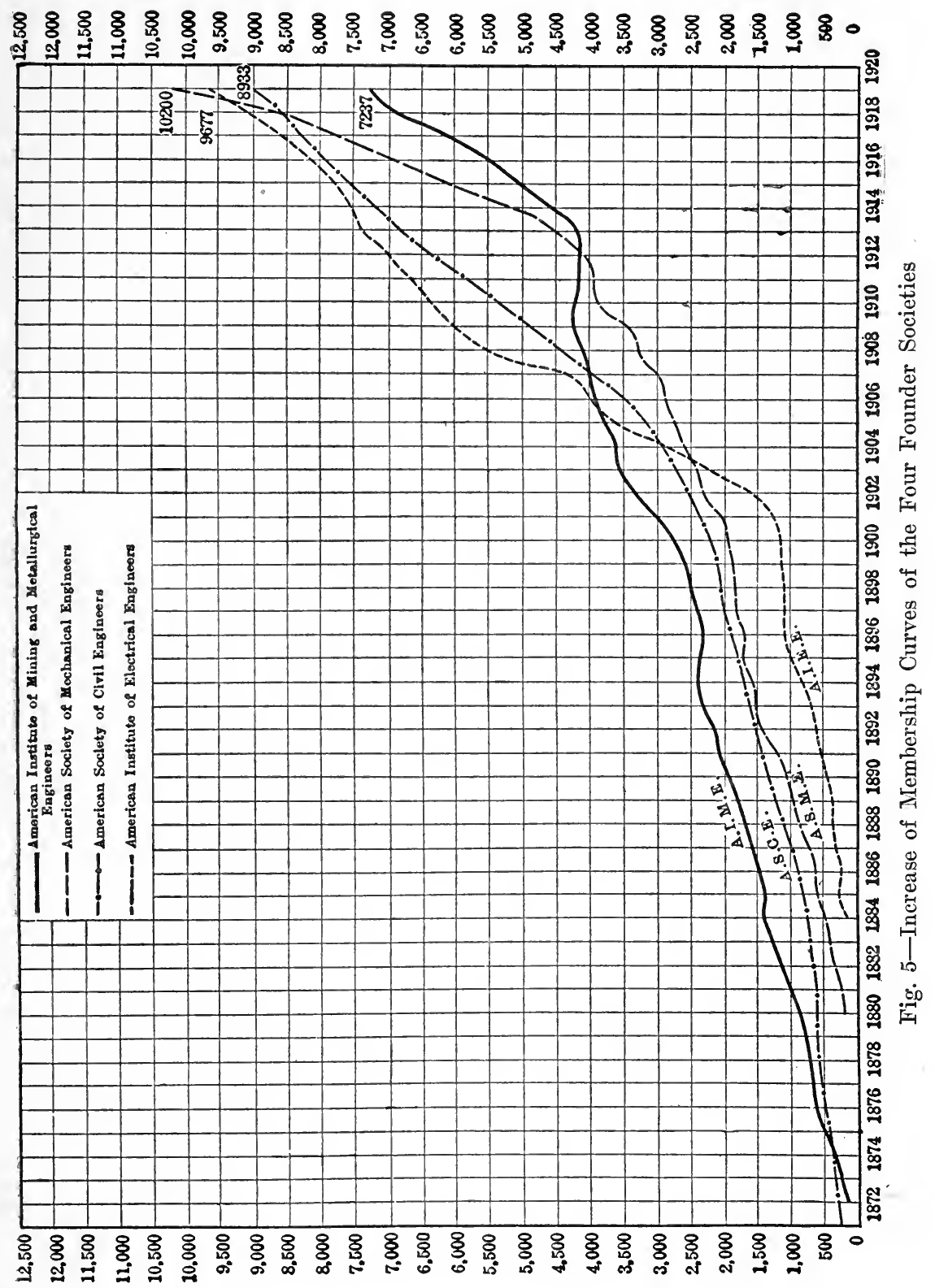


Naturally, the first facts to know are: (1) how fast do businesses die? and (2) what are the diseases to which they are most eommonly subject?

In an investigation made by System's Bureau of Business Standards a typieal eity was seleeted-a eity that possesses what it does largely because of a good fight and not beeause of an unusually favorable location or some other peeuliar gift of nature.

The death rate figures obtained from this survey into 30 years of the business history of this eity could doubtless be duplieated in almost any Ameriean community of equal size. Many similar eommunities. could be even found, indeed, in which the business death rate would probably be much higher. And, on the other hand, it is quite probable that communities could be found in which it would be lower.

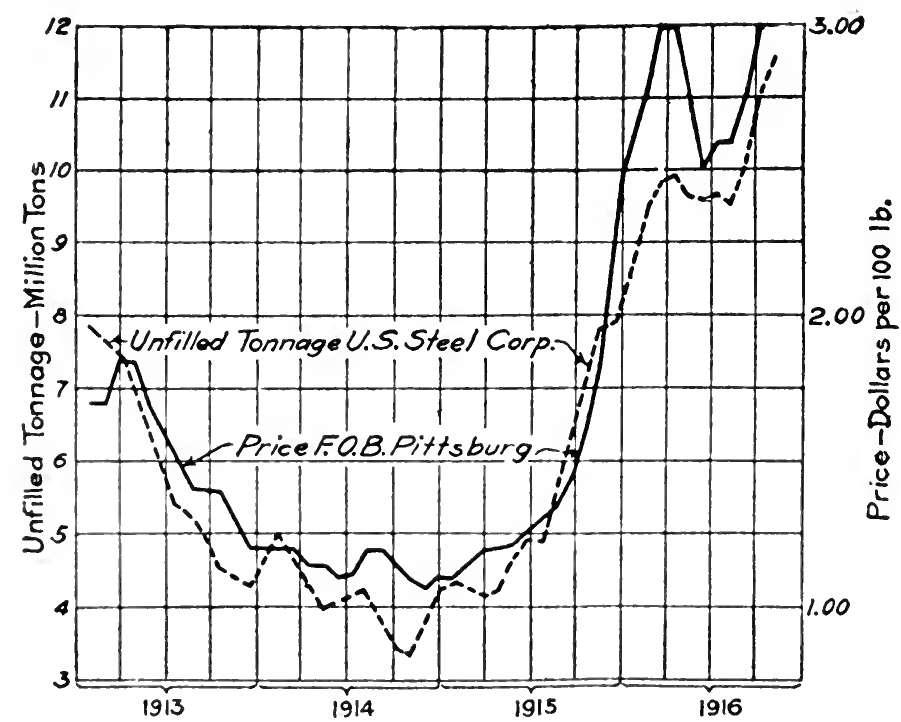

Fig. 6-Price of Bars Fluctuates with Steel Corporation's Unfilled Tonnage

The figures are not deeisive or absolute. They are indieative only, and no further elaim is made for them. They have been established with sufficient accuracy, however, to give them a laboratory value in the study of the business death rate.

What these figures show is how many and how fast eoneerns are dying today in a typical American community. Also, they indieate whether or not concerns are dying faster today than they did 25 or 30 years ago. Fig. 7 is but one illustration from the many eited in the article.

Predicting Yearly Earnings. From a letter, by Henry Hess to the editor of Mechanical Engineering, February, 1919, is taken the following:-

The executive whether he deal with materials or dollars, is not satisfied with a mere record of what has occurred in the past, but he wants. 
to use the past experience as a basis for consideration or production of the future. It is possible to extend a curve of monthly fluctuations to indicate a future probability, but this is neither safe nor simple.

Plotting the data as in Fig. 8 answers every question as to the past and also readily predicts the probabilities of the future.

To deal with something concrete, let us say that it is the profits of the business that are being examined, that these are being ascertained monthly, that the corresponding result for the year is desired and that the prediction is to be brought up to date or corrected each month.

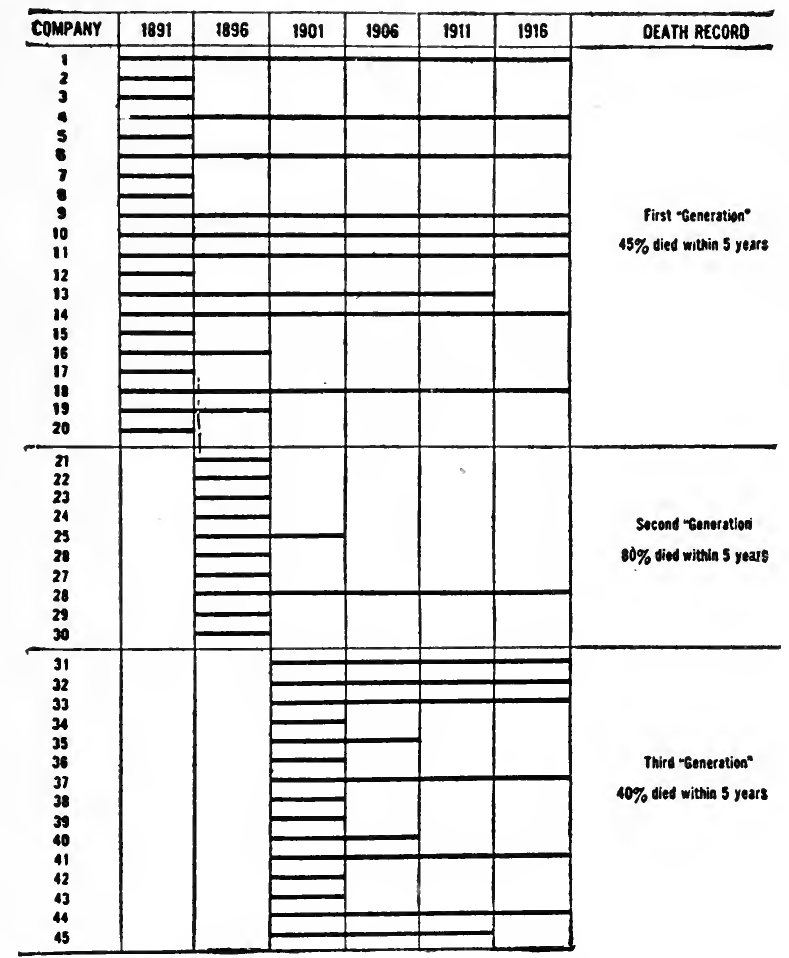

Fig. 7-How Fast Three "Generations" of Retail Clothing Stores Died

In Fig. 8 each month's earnings are plotted to a curve, the lower one shown.

Over each month there is plotted the total profit to date. For instance, January shows $\$ 250$, February, $\$ 300$, and the total therefore to date is $\$ 550$. These are shown by the upper solid curve.

To predict the probable profit for the year it is only necessary to draw a line from zero through the total earnings to date and to continue that line to the twelfth month; for example, the total profit by the end of the fourth month was $\$ 1150$; the projection shows $\$ 3450$ as the probable rate of profit for the year resulting from the first four months. An examination of the diagram shows that there is an improvement in 
the second and third months over the first month and an improvement in rate by the end of the fourth month over the previous months. By the eighth month, however, the rate had fallen off from that of the fifth, sixth and seventh months, as is clear from the production line for the eighth month falling below that of these previous three months. The ninth month, however, showed clearly an inprovement in the rate to date.

The inclination of the lines giving the totals of each month also furnishes useful data. It is clear that if these lines all have the same slope, the increase from month to month is then equal. A lesser angle shows a deerease in monthly earnings and a greater angle shows an inerease in monthly earnings.

The most useful element of this form of plot, however, is the projection into the future and the ability that it gives to instantaneously correct the indications of previous periods by the result of the last period.

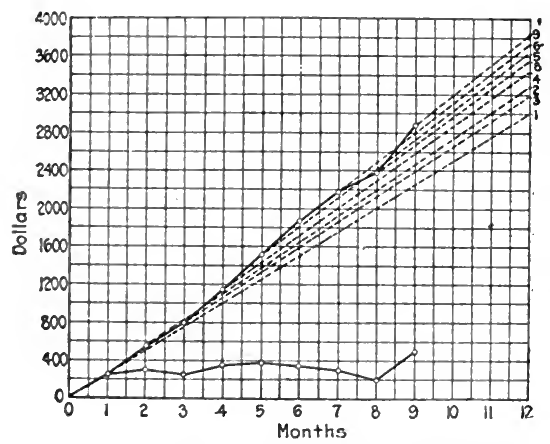

Fig. 8-Diagram for Predicting Yearly Earnings from Earnings for Part of Year

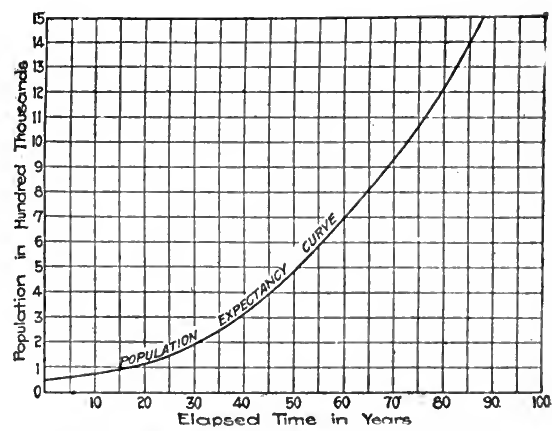

Fig. 9-Moore Expectancy Population Curve

Probable Population. In Engineering News; November 2, 1916, Geo. II. Noore had the following:-

What the author has named the Moore expectancy eurve is a graphical representation of the population a eity may be expected to have after any given clapsed time in years. The eurve is derived by plotting on cross-section paper the actual populations of selected cities after equal periods of elapsed years, ignoring exact dates and choosing such values for "elapsed time in years" as may enable the respective curves to conform most nearly to a final mean value for the group. After the several curves have been plotted, the mean or composite eurve is sketehed in. This alone is traced off on transparent paper, as shown in Fig. 9, for use by itself or for superimposing upon other eurves for various comparative purposes.

Tr) illustrate a simple use of the curve: Suppose one desires to obtain the most probable population that a city of 500,000 will possess after a lapse of 20 years. It is necessary only to mark the 500,000 point on the expectancy curve, add 20 years to the elapsed time, which in this case will grive a final of 71 years, and read off 960,000 . 
The method can be used in almost every problem that involves either population estimate or population analysis. It can be used to predict the most probable population of a city of any size whatever at any given future time. Or, on the other hand, by comparing the plotted curve for any city with the expectancy curve it becomes evident at a glance whether the city in question is growing, or has grown, faster or slower than the average of its class.

Finally, it will be evident after a little reflection that analyses based on the expectancy-curve method are exactly as reliable as are insurance risks written on the basis of mortality tables. And since by its use the three quantities of maximum, minimum and mean populations to be provided for are readily obtainable, it is claimed that the expectancy curve provides a thoroughly defensible method-possibly the first-for the exact analysis of population problems.

Probable Future Traffic. The following is from a report on "Con-

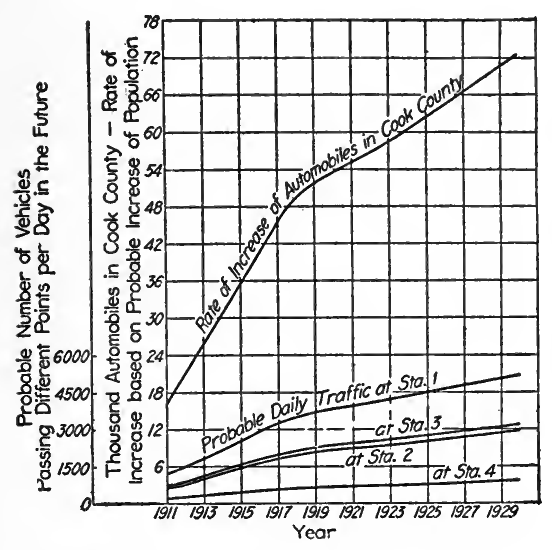

Fig. 10-Probable Future Traffic

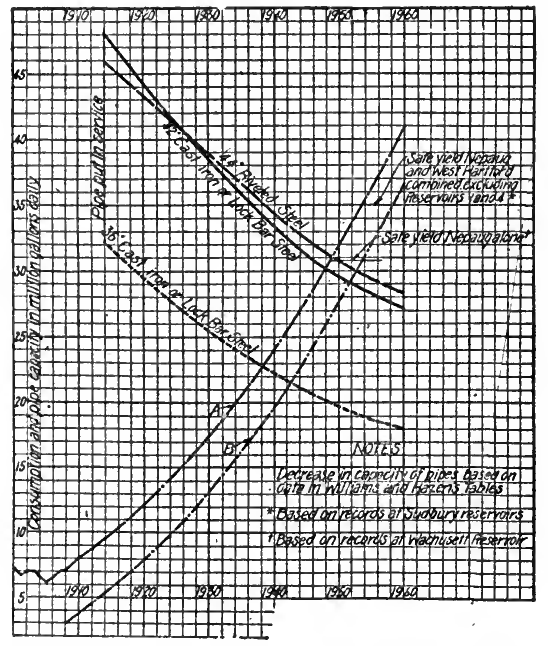

Fig. 11-Estimated Increase in Water Consumption at Hartford, Conn., and Decrease in Capacity of Pipes with Age

dition of Famous Chicago Road," published in Engineering Record, September 12, 1914.

Traffic increase curves (Fig. 10) were prepared for the purpose of estimating the future number of automobiles using the road in 1930 . The number of machines in Cook County from 1900 to 1911 was used as the basis because the automobile traffic is $80 \%$ of the total, and probably more than $50 \%$,were from Cook County rather than the local towns. It was assumed that the traffic increase curve would parallel the curve of the automobile increase. The rate of increase of the number of cars was assumed to decrease regularly until 1920, after which the curve would parallel the population curve. 
Predicted Water Consumption. From Engineering and Contracting, December 17, 1913, is taken the following:

Works for the additional water supply of Hartford, Conn., are now under construction or will soon be contracted for. The plan contemplates a collecting and storage reservoir, known as the Nepaug Reservoir, a compensating reservoir, a pipe line connecting the proposed storage reservoirs with the present reservoir system in West Hartford, a slow sand filtration plant to treat all the water passing to the city and a large size main pipe line running from the filter plant and entering Hartford in the northern part of the city.

Fig. 11 is a diagram showing the estimated increase in water consumption and the decrease in carrying capacity with age of steel pipe from 36 to 44 ins. in diameter and of cast iron from 36 to 42 ins. in diameter.

Curve $A$ shows the demand on the pipe when no supply is furnished by West Hartford. Curve B shows the demand on the pipe when West Hartford is supplying 4,250,000 gals. per day safe capacity, excluding present reservoirs Nos. 1 and 4. The curves are based on the following assumptions : 8,600,000 gals. per day present consumption; 400,000 gals. per day present yearly increase; 50,000 gals. per day added every four years to rate of increase.

Going Value. From a paper by John W. Alvord, reprinted in part in Engineering and Contracting, August 5, 1909, is taken only that portion describing the method of computing Going Value by means of a chart, omitting the development of the theory, etc. It is a form of prediction.

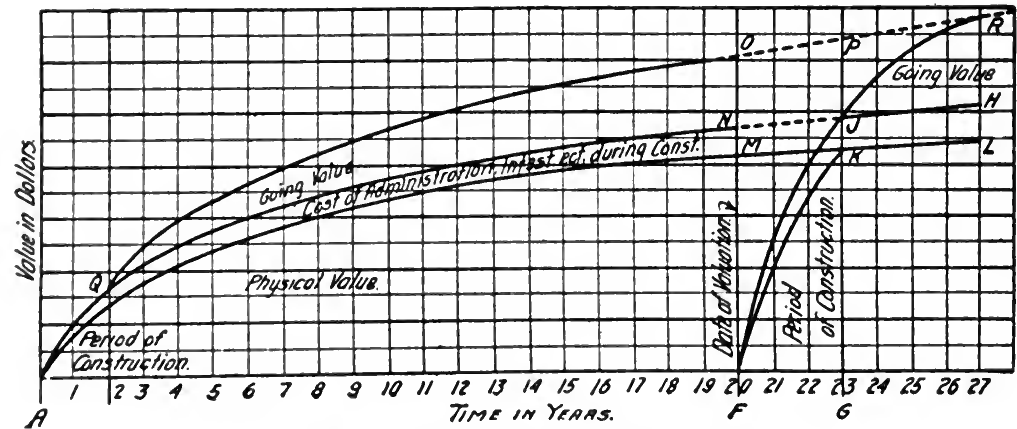

Fig. 12-Computing Going Value

To those who are accustomed to thinking by the aid of diagrams, the subject of going value may perhaps be made more clear by the one here shown depieting the typical growth in value of a water works plant, and the time element involved in reproducing the same. This diagram, Fig. 12, is not illustrative of any particular plant, but is rather a eomposite of many plants which have eome under the writer's notice.

The spaces along the bottom of the diagram give the time in years which have clapsed since the original construction, and it is assumed that about the twentieth year it is desired to value the property for purposes of purchase or fixing rates. 
The vertical spaces upon the diagram represent increments of value due to the cost of its construction and its going value. In the beginning of its history two years are assumed to have been necessary for construction, after which time the plant begins to acquire its going value. In the earlier years of operation the acquisition of going value is necessarily quite slow. An additional curve of growth shows, roughly, the proportionate costs of administration, interest, etc., during construction, which are added to and are a part of the physical value.

For purposes of illustration, it is assumed that in its twentieth year this plant is to be valued. The estimator thereupon proceeds to imagine the construction of a conceptual duplicate plant and, as the original plant has grown in value year by year through minor additions and extensions, he is now facing the contingency that it will require perhaps three years instead of two for the actual physical replacement. This, as shown by the area $F K G$, is in advance of the date of the estimate, as must be the case if the estimator is limiting himself to what is humanly possible.

During the construction the estimator computes the necessary expenses for administration, cost of interest on capital being invested, engineering expenses, etc., which may be graphically represented as added to the other construction by the area $F J K$, reproducing at the end of the proper assumed construction period the value of such items as they already exist in the going plant.

The estimator then arrives at the value $G J$ for the physical property, and it remains for him to reproduce the going value $O M$, existing at the date of his valuation, but in order to recover this going value we must again be cautious enough to proceed in a manner which is humanly possible, that is to say, the conceptual plant will not only have to recover this cost of reproducing the income, but at the same time, it will be hampered by the necessity in common with the going plant of recovering such growth of revenue as the near future may reasonably yield. This, of course, is not an element of its credit except as it embarrasses and delays it in acquiring the full going value $O M$.

If the estimator further proceeds in a manner which is humanly possible, he must consider this recovery of going value as taking place in the period following the periods of construction, which period is assumed for illustrative purposes in this case to be four years, and after having made the assumption and computations for credits to the going plant must of necessity refer them back to their present worth. This reduces his values back to the twentieth year, and completes his valuation of $F O$ upon the diagram.

This total is a "total value" diagram and must not be confused with a "revenue diagram" which is frequently used in somewhat the same manner.

Determination of Unit Prices of Material for Purposes of Valuation of Plant. Under this title Engineering and Contracting, May 13, 1914 , published an article by $R$. V. Achatz, from which the following is quoted:

One of the first questions which must be decided in making a valuation of a physical plant is what unit costs of material and labor are to be used. In making a valuation on the cost of reproduction basis, 
it is agreed that these costs should be present costs but there is a question of just what is present cost. In the ease of labor present cost is a cost based on the current wage scale but in the case of material it is quite generally agreed that the market price on a given date, particularly for those materials which are subject to constant fluctuations in price, eannot always be used with justice. Many writers in discussing this question have said that, in ease of materials subject to price fluctuations, the market price on a given date should not be used but an average price over a number of years past, usually five or ten, should be adopted. There immediately arises a question as to the propriety of using an average of past prices in a valuation on the eost of reproduction basis, and there is also a large question as to whether such an average actually represents a fair present price. It has been proposed that the present normal price ean be determined by plotting the prices for a number of years past and drawing a smooth curve representing an average of the prices as shown by the yearly price curve.

In order to make a study of the different methods of determining unit costs of materials the prices on three metals, copper, tin and lead,

- were used. These metals were adopted beeause prices were available for many years past, because of their importance in telephone and other electrical properties, and because the prices are representative of three types of price variation. Copper prices have fluctuated continuously and sometimes violently with a general tendeney toward inerease. Tin prices have also fluctuated considerably and also have shown a marked increase in the past fifteen years. Lead prices have in general been stable and show little if any tendency toward ehange.

In making the study curves were plotted for the monthly average prices of the metal by plotting at the abseissa representing each month, the average price for that month and eonneeting the points by straight lines. In a similar way curves were plotted for the average prices for 5,10 , and 15-year periods by plotting at the elose of each year the average of the prices for the period preeeding, and, as before, eorrecting the points by straight lines. A smooth curve was also drawn representing a mean between the higher and lower changes in the monthly average prices. Theoretically there should be equal areas, above and below, between this curve and the monthly average eurve but in practice it can be drawn by eye with sufficient aceuracy. This has been designated a "normal trend price" eurve.

In Fig. 13) are shown the eurves of eopper prices. These prices extend from 1884, the earliest period that prices were available, to the present. It will be noted that the prices have had peaks at periods of from seven to ten years apart, the highest peaks oecurring in 1889. 1899, and 1907, followed by periods of comparatively low prices. The dotted curve represents suceessive five year averages. This eurve also has peaks coming at the frequency of the peaks in the monthly average curve but displaced so that the maximum points in the five year average curve come later than those of the monthly average and oecur during times of low market prices. For example, at the close of 1909 the market price of eopper was 13.75 ets per 11 ., while the five year average was 16.808 cts. per lb. At the elose of 1912 the market price was 17.75 ets. per lb., while the five year average was 13.91 cts. The 
high average for the five years ending with 1909 was due to the influence of the very high peak in the market in 1906-7 and the low average for the five years ending in 1912 was due to the abnormally low prices following the 1907 peak, the influence of that peak on the five year average having passed. It is at once seen that a five year average price at either of these times would be unjust, the price in 1909 being too high to be just to the public and the price in 1912 too low to be just to the utility.

The ten year average prices of copper are shown in Fig. 13 by the broken line. This curve has peaks in much the same way as the five year average curve but the differences from time to time are not so great. The ten year average at the close of 1911 is 1.1 cts. less than at the close of 1908 . The use of this average would be less open to objection than the five year average on account of smaller variation from time to time.

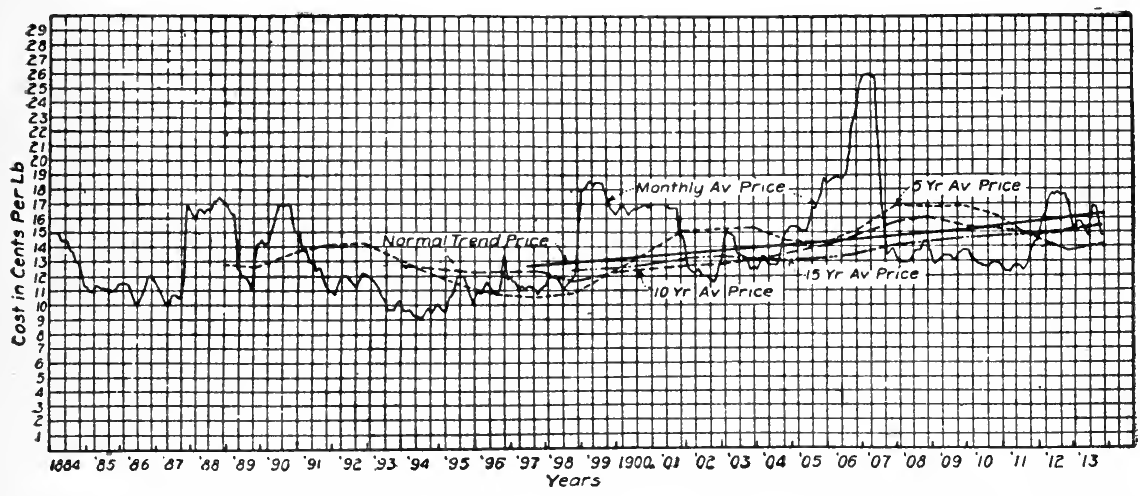

Fig. 13-Variation and Trend of Prices for Copper for Period 1884 to 1913, Inclusive

The 15 year average is shown on Fig. 13 by the dot and dash line. It is entirely free from variation due to the influence of peaks in the market price and shows a general tendency upward. Its use might be objectionable on account of the influence of a period of low prices a long time ago.

The smooth curve which has been called the normal trend price has been drawn to represent an average of the market prices. At any given time the price read from this curve may be considered to be the price of copper under normal conditions. A buyer of copper in the future would expect to find prices following this curve more or less closely. The price as indicated by this curve was $15.2 \mathrm{cts}$. at the close of 1.909 , 15.8 cts. at close of 1911 and 16.3 cts. at close of 1913 . The discussion pertaining to the tin and lead curves has been omitted.

From the study of the curves and the above discussion the following conclusions are drawn regarding the use of the different methods of determining unit costs of material for valuation purposes:

(1) The use of the market price of materials, especially those which are subject to price fluctuations is likely to be unfair.

(2) Average prices for a period of five years previous to the valua- 
tion are unreliable on account of the influence of periods of high market prices which tend to raise the five year average during the period of low prices that usually follows a period of high prices and the corresponding decrease in the five year average following a period of low prices even after prices have again increased.

(3) An average of the prices for a period of ten years previous to the valuation fluctuates in a less degree than the average for five years, but may be lower than a fair price or higher than a fair price due to the influence of a period of low or high prices many years before.

(4) An average of the prices for 15 years may be unfair for the same reason as the second given under the ter year average, viz.: the effect of prices of many years previous.

(5) An average smooth curve can be drawn, taking into consideration market prices for a number of years past and also successive average prices for periods of 5,10 and 15 yrs. which will represent the normal present price of the material. In general the use of this curve as a basis of unit price of material will be more fair and less open to objection than any average price.

(6) Before unit prices are adopted it is necessary to make a study of the past and present prices of materials, particularly the more important ones which may represent a large portion of the total cost of material in the plant. Such materials would be poles, copper wire, lead covered eable, duct materials and Portland cement in the case of telephone, street railway and other electrical properties, and cast iron pipe in case of gas and water plants.

There may be some objection to a price based on the trend curve on account of the fact that the judgment of the appraiser is brought into its determination, but it must be remembered that a valuation is an estimate of cost to reproduce a given plant and the entire result is based on engineering judgment. Furthermore a result arrived at by the use of well trained judgment after considering all the facts is more likely to be fair than an average over an arbitrary period without further consideration.

Following is a list of articles containing further illustrations of the use of graphic charts of the class discussed in this chapter and pertaining to kindred subjects.

Upon request, the Codex Book Company, Inc., 19 William Street, New York, will be glad to procure, where possible, copies of magazines containing the articles needed, only charging the "old magazine" price, plus postage.

"'The Estimation of Petroleum Reserves," Robert W. Pack, Metallurgical and Chemical Engineering, September 1, 1917.

"A Manufacturer's Reflections on Textiles, Rubber and Finance," Archer Wall Douglas, Factory, April, 1919.

"The Anthracite Situation," William Griffith, Coal Age, May 19, 1917.

"Fngine Design for 1918, (Ignition and Lubrication)," Automotive Industries, January 3, 1918.

"1918 Engineering Trends," A. Ludlow Clayden, Automotive Industries, January 3, 1918. 
"1918 Chassis Design Features," Automotive Industries, January 3, 1918.

"Charts showing prices fúrnace coke, pig iron, unflled tonnage, net earnings, etc." Iron Trade Review, January 4, 1917.

"Some Practical Applications of the Principles of Statistics," Journal of the Association of Engineering Societies, December, 1914.

"Method of Estimating the Probable Volume of Railway Traffic," Journal of the Association of Engineering Societies, March, 1911.

"Analyses of P. R. T. Proposals," (Railway Data), Electric Railway Journal, April 7, 1917. 


\section{CHAPTER XVI}

\section{Computation, Arithmetical and Geometrical}

Generally speaking, the plotting of a curve consists of graphically representing numbers and equations by the relation of points and lines. with reference to other given lines or to a given point.

In Chapter II, the theory of rectangular co-ordinates was explained briefly, showing how it is possible to definitely locate any point by the algebraic values of its co-ordinates, $x$ and $y$. The lines from which these distances $\mathrm{x}$ and $\mathrm{y}$ are measured are the reference lines-the $\mathrm{x}$-axis and $y$-axis, and these cross at right angles at the origin, 0 . Reference to Chapter II will make this clear.

Plotting an Equation. The next step is to show how to locate on a. chart the locus of points which shall satisfy a given equation containing variables. This has been so clearly and simply explained in a series. of articles on "Curve Plotting," published in Power that the author feels he eannot do better than quote these directly. The following was published in the Narch 10 and 17, 1914, numbers. The proper understanding of the principles outlined requires a comprehension of the fundamentals of algebra, but it is assumed that any reader interested in the use of charts for computing would have this.

It is possible to represent any equation in $x$ and $y$ by a curve or group of curves. but not all curves are capable of being represented by equations. although the simple ones can be. The two unknown quantities: (or variables, as they are called) in the equation need not be represented by $x$ and $y$; an equation in $u$ and $v$ or any other letters would do just as well but then instead of calling the axes those of $x$ and $y$, we simply call them those of $u$ and $v$.

To plot a curve from its equation, we assume various values for one variable, and find what the other variable will equal under those conditions. For example: To plot the equation, $y=3 x+4$, we make a. table as follows:

$$
\text { If } \begin{array}{rl}
x=-3 & y=-9+4=-5 \\
x=-2 & y=-6+4=-2 \\
x=-1 & y=-3+4=+1 \\
x=0 & y=0+4=+4
\end{array}
$$$$
\begin{array}{ll}
x=1 & y=3+4=+7 \\
x=2 & y=6+4=+10 \\
x=3 & y=9+4=+13
\end{array}
$$

If we now lay off on cross-section paper the points $(-3,-5),(-2$, $-2),(-1,+1),(0,+4),(1,7),(2,10)$ and $(3,13)$ and connect. them we have the curve represented by this equation, in this case a straight line, Fig. 1. That is, to plot an equation, we assume values for one of our unknown quantities, find the values of the other unknown quantity corresponding to the first, mark off the points corresponding to them and connect them.

There are one or two special eases. The equation $x=4$, means $x=4$ 
for any and all values of $y$. That is, this is a line parallel to the axis of $y$, and 4 units from it. Similarly, $y=3$, represents a condition that $y=3$ for any and all values of $x$, which is fulfilled by a line parallel to the axis of $x$, and 3 units from it.

However, these are, as said, special cases, and in general any equation of the first degree can be reduced to the form

$$
y=m x+b
$$

$m$ and $b$ representing any two constant numbers. This is the usual form in which the equation of a straight line is written, and has this convenience, that $m$, whatever number it is, is the tangent of the angle

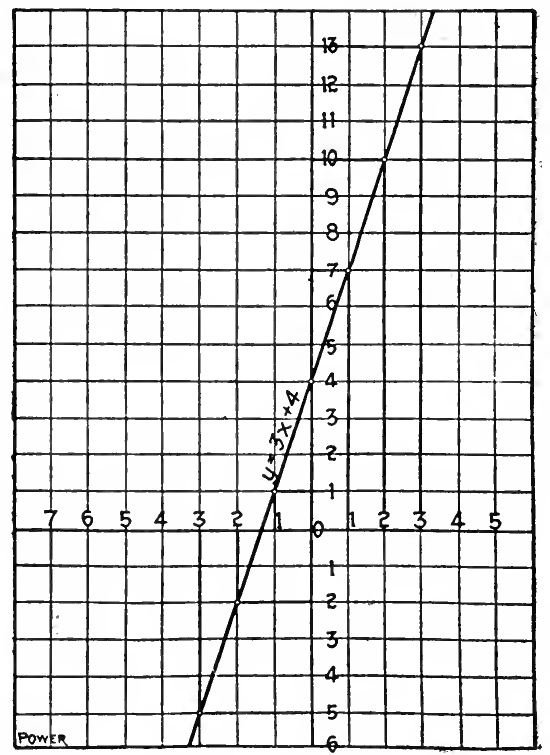

Fig. 1-Straight Line

the line makes with the axis of $x$, and $b$, whatever number it is, is the distance from the center $O$, that the line cuts the axis of $y$. For instance, the equation

$$
4 y=3 x+7
$$

can be written

$$
y=\frac{3 x}{4}+\frac{7}{4}
$$

and this particular equation could be immediately plotted by taking a distance $7 / 4$ out on the axis of $y$, and drawing a line through this point making an angle with the axis of $x$ whose tangent is $3 / 4$.

Another way in which equations of the first degree can always be written is: 


$$
\frac{x}{a}+\frac{y}{b}=1
$$

The same equation

$$
4 y=3 x+7
$$

reduces to

$$
\frac{y}{7 / 4}-\frac{x}{7 / 3}=1
$$

or in the above formula $a=-7 / 3, b=7 / 4$.

In this form of writing the equation, $a$ and $b$ are the distances from the center on the axes of $x$ and $y$, where the straight line cuts these axes. These distances are known as the intercepts on the axes of $x$ and $y$.

Knowing this much about the straight line, we can find the equation where we have the line. Suppose a straight line passes through the points $(4,2)$ and $(-5,3)$. Let us find the equation in the form

$$
y=m x+b
$$

When $x=4, y=2$; when $x=-5, y=3$ therefore,

$$
\begin{aligned}
& 2=4 m+b \\
& 3=-5 m+b
\end{aligned}
$$

Substract (2) from (1) $-1=9 m$, or $m=-1 / 6$;

Substituting in (2)

$$
3=\frac{5}{9}+b, \text { or } b=\frac{22}{9}
$$

Then the equation of a line passing through points $(4,2)$ and $(-5,3)$ will be

$$
y=-\frac{1}{9} x+\frac{22}{9}, \text { or } 9 y=-x+22
$$

The matter becomes a little more eomplicated when the equation is quadratic, i.e., contains squares of $x$ and $y$.

Suppose $x^{2}+y^{2}=25$, then

$$
\text { If } \begin{aligned}
x=0, & y=\sqrt{25}=5 \\
& x=1, \quad y=\sqrt{24}=4.9 \\
& x=2, \quad y=\sqrt{21}=4.6 \\
& x=3, \quad y=\sqrt{16}=4 \\
& x=4, \quad y=\sqrt{9}=3
\end{aligned}
$$$$
\begin{array}{lll}
x=5, & y=\sqrt{0}=0 \\
x=-1, & y & =4.9 \\
x=-2, & y & =4.6 \\
x=-3, & y & =4 \\
x=-4, & y & =3 \\
x=-5, & y & =0
\end{array}
$$ 
If we plot the points corresponding to these values, and connect the points, we find we have a circle, as in Fig. 2. In general, any equation of the form $x^{2}+y^{2}=r^{2}$ is a circle of radius $r$. (In the above example the radius is $\sqrt{25}=5$.

If we take the curve $p v=25$, then when

$$
\begin{array}{llll}
p=10 & v=2.5 ; & p=5 \quad v=5 \\
p=9 & v=2.78 ; & p=4 \quad v=6.25 \\
p=8 & v=3.13 ; & p=3 \quad v=8.33 \\
p=7 & v=3.57 ; & p=2 \quad v=12.50 \\
p=6 & v=4.17 ; & p=1 \quad v=25
\end{array}
$$

If we plot this curve, measuring the " $p$ 's" on the axis of $y$, and the " $v$ ' $s$ " on the axis of $x$, we obtain a curve which represents the behavior of steam as it expands, or air as it is compressed, without change of

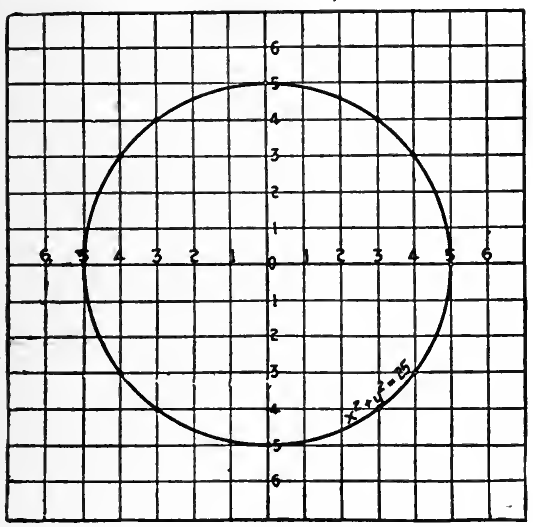

Fig. 2-Circle

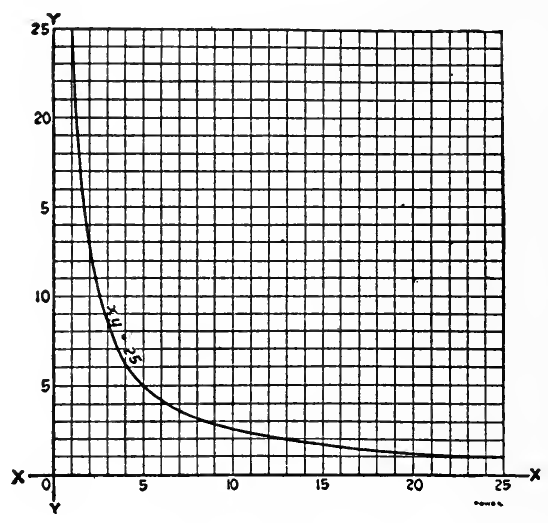

Fig. 3-Hyperbola

temperature. (The engineer will immediately recognize this as a part of his indicator diagram.) It is a part of a curve known as an equilateral hyperbola, and the rest of the curve may be obtained by plotting:

$$
\begin{array}{ll}
p=-10 & v=-2.5 \\
p=-9 & v=-2.78 \text { etc. }
\end{array}
$$

(The minus branch has no physical meaning).

Fig. 3 is an hyperbola, a plot of an equation of the form $x y=25$.

In like manner we may plot any equation, by making a table of values of $y$ corresponding to various assumed values of $x$, finding the points corresponding to each pair of values, and passing a straight or curved line through these points.

We can summarize much information concerning the simpler equations. Every equation of the first degree is a straight line. Every equation of the second degree is either a circle, an ellipse, a parabola, a hyperbola, or a pair of straight lines.

Any equation of the form $y=m x+b$ represents a straight line in 
which $m$ is the tangent of the angle between the line and the axis of $x$, and $b$ the intercept on the axis of $y$.

$$
\frac{x}{a}+\frac{y}{b}=1
$$

is a straight line where $a$ and $b$ are the intercepts on the axis of $x$ and $y$ respectively.

$$
x^{2}+y^{2}=r^{2}
$$

is a circle, whose center is the point, $O$, and of radius $r$.

$$
(x-a)^{2}+(y-b)^{2}=r^{2}
$$

is a circle, whose center is the point $a, b$, and of the radius $r$. (The simpler equation may be derived from this by putting $a=0, b=0$ )

$$
\frac{x^{2}}{a^{2}}+\frac{y^{2}}{b^{2}}=1
$$

is an ellipse, with $O$ as the center, and $a$ and $b$ as semi-diameters.

$$
\frac{x^{2}}{a^{2}}-\frac{y^{2}}{b^{2}}=1
$$

is a hyperbola, with the axes of $x$ and $y$ as its axes.

$x y=a$ is an equilateral hyperbola, with its axes at $45 \mathrm{deg}$. to $X^{r}$ $O X$ and $Y^{\prime} O Y$.

$y^{2}=k x$ is a parabola, whose axis is $X^{\prime} O X$.

Now, what is the good of all this? Can any problems be solved by it which are not easily solved otherwise? Here is an elementary one which is more easily solved by plotting equations than in any other way.

Suppose, as was an actual case, a flywheel burst at $B$, Fig. 4, a piece:

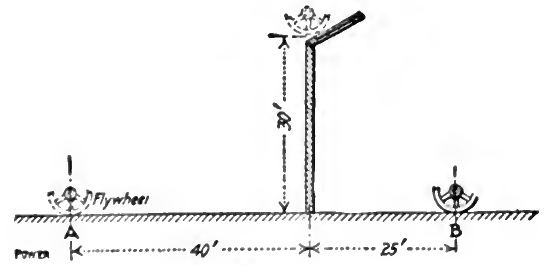

Fig. 4-Problem of Burst Flywheel

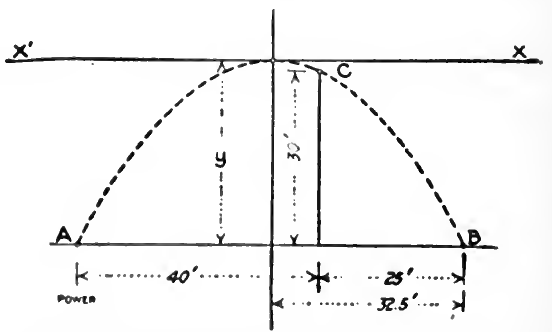

Fig. 5-Plot of the Path Taken by Flywheel Piece (Parabola)

just cleared the wall $25 \mathrm{ft}$. away, $30 \mathrm{ft}$. high (it actually went through a skylight) and alighted $40 \mathrm{ft}$. farther off. How high did it go?

Bodies thrown into the air travel in parabolas, and the highest point is midway from $A$ to $B$. We draw our figure then, as in Fig. 5 .

The parabola is of the form $x^{2}=-$ ky. By the conditions of the problem, the parabola goes through point $B(32.5,-y)$ and through 
$C(7.5,-y+30)$. (Try to see how those co-ordinates are obtained). Then

$$
\begin{gathered}
32.5^{2}=+k y \\
7.5^{2}=k(y 30)
\end{gathered}
$$

Solving by dividing (2) by (1)

which gives

$$
\frac{7.5^{2}}{32.5^{2}}=1-\frac{30}{y}
$$

$$
y=\frac{507}{16}, \text { or }-y=-\frac{507}{16}
$$

That is: the wheel at its highest point was $31^{11 / 16} \mathrm{ft}$. off the ground.

Other applications are: If two simultaneous equations are given, as

$$
\begin{aligned}
& x+3 y=16 \\
& x^{2}+y^{2}=36
\end{aligned}
$$

an approximate idea of the solution may be had by plotting these equations. The intersections of their graphs are the solutions of the simultaneous equations.

Or, in the equation

if we put

$$
x^{3}-2 x^{2}+3 x-4=0
$$

$$
y=x^{3}-2 x^{2}+3 x-4,
$$

and plot the graph, we can get an idea of the approximate roots of the equation, as these will be where the graph crosses the $x$ axis.

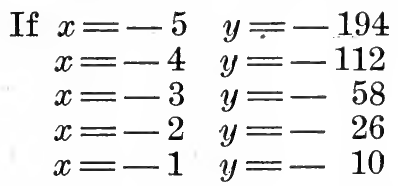$$
\begin{array}{ll}
x=0 & y=-4 \\
x=1 & y=-2 \\
x=2 & y=+2 \\
x=3 & y=+14
\end{array}
$$

There is a root between +1 and +2 , and by computing betwen these points we can approximate it as closely as we please.

From the preceding we can conclude that all quantities may be divided into two classes: constants and variables. A constant quantity is one that maintains a fixed value, for example, the length of the stroke of an engine does not change. The speed and the load may change but the stroke remains the same under all conditions. A variable is a quantity whose value is changing. Variables may be subdivided into dependent and independent variables. Thus the mean effective steam pressure in an engine cylinder is a dependent variable, for its value is determined by the load on the engine. The load on the engine is an independent variable, for it can be changed at will. (In a sense the load is' a dependent variable, as it is limited by the power of the engine.)

As explained in the pages above, a physical law may be stated in the form of an algebraic equation, or it may be represented graphically by 
the aid of a curve or graph, and in most eases the latter method will give a clearer idea of the law of variation than will a mere setting down of the equation. Take, for example, the law of the expansion of gases which states that the product of the absolute pressure times the volume is equal to the weight of the gas times the absolute temperature of the gas, times a constant which is dependent upon the gas. Stated in the form of an equation there results,

$$
P V=W R T
$$

Assume $W$ as unity and let $T$ remain eonstant, and we have the well known isothermal law that if the temperature of a gas remain constant.

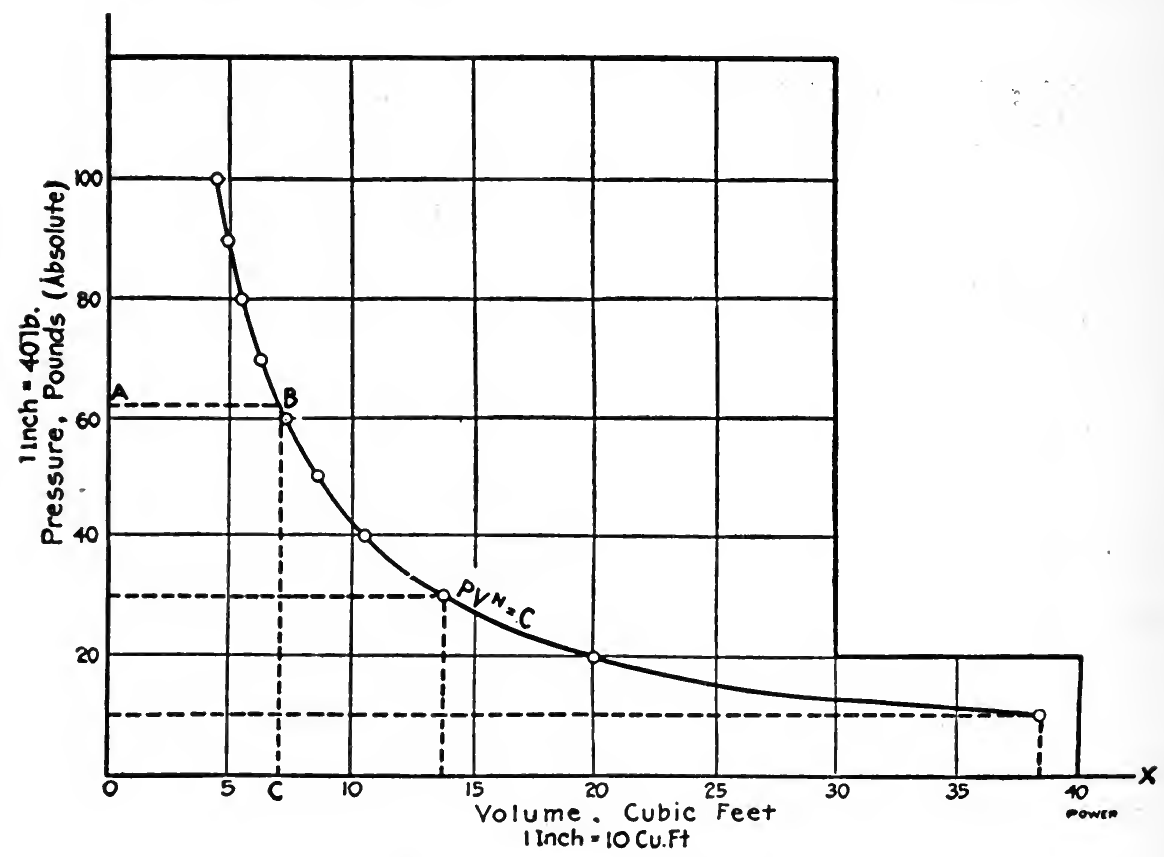

Fig. 6-Pressure-Volume Curve

then the product of the absolute pressure and volume of the gas will also remain constant. This is the approximate law for the expansion of steam in the engine cylinder.

For every pressure, saturated steam has a definite volume, and a fixed temperature. These values have been determined largely from experiment. The following table gives the values of the volume for certain pressures:

Absolute

$$
\begin{aligned}
& \text { Pressur } \\
& 20 \quad 30 \\
& 40 \quad 50 \\
& \begin{array}{lll}
60 & 70 & 80
\end{array}
\end{aligned}
$$

Volume, cubic 
Frequently the engineer desires values not given in the table and is at a loss to know how to determine them. This gives rise to another useful application of curves, namely, the determining of intermediate values not given in the table. Let us now plot the curve showing the relation between the pressure and the volume of saturated steam. Let distances (abscissas) measured along the line $O X$, Fig. 6, represent the volume in cubic feet to a definite scale; let distances measured along the vertical $O Y$ represent pressure, to another scale. Lay off an ordinate equal to $10 \mathrm{lb}$. From the table the volume for this pressure is $38.38 \mathrm{cu}$. ft. These two values give us the first point on our curve and similarly other points can be plotted. Through the points thus plotted draw a smooth curve and we have a graphical relation between the volume and pressure of saturated steam. Suppose the volume is desired at $62 \mathrm{lb}$. pressure. This can be found from the curve as follows :

Lay off the distance $O A$ (to scale) to represent $62 \mathrm{lb}$., draw the horizontal line $A B$; at the point of intersection $B$ with the curve drop a perpendicular $B C$, cutting the volume axis at $C$. Then the line $O C$ multiplied by the volume scale gives the desired volume which in this case is $6.95 \mathrm{cu}$. ft.

Another instance where a knowledge of curve plotting is essential is in the testing of boilers, engines, generators, motors, etc. A mere tabulation of the data taken during the test is of little value in giving a general conception of the performance of the given unit. The following table gives the results of a series of tests on a four-cylinder fourcycle Diesel oil engine.

\begin{tabular}{|c|c|c|c|c|c|c|}
\hline est No. & 1 & 2 & 3 & 4 & 5 & 6 \\
\hline & & 31 & 71 & 109 & 150 & 169 \\
\hline b.hp.............. & 2 & 50 & 111 & 163 & 220 & 246 \\
\hline of fuel per b.hp. & 171 & 169 & 167 & 164 & 165 & 162 \\
\hline hermal efficiency ... & & $\begin{array}{r}0.77 \\
17.4 \%\end{array}$ & $\begin{array}{r}0.48 \\
27.8 \%\end{array}$ & $\begin{array}{r}0.45 \\
29.5 \%\end{array}$ & $\begin{array}{r}0.44 \\
30.3 \%\end{array}$ & $\begin{array}{r}0.444 \\
30.2 \%\end{array}$ \\
\hline
\end{tabular}

In Fig. let distances along the axis $O X$ represent the net brake horsepower (b.hp.) to an assumed scale. Let the ordinates along $O Y$ equal the revolutions per minute to a definite scale. From the values given in the table locate the necessary points and plot the curve $A B$ which shows at a glance the variation in speed of the engine as the load is increased or decreased.

It is often desirable to have on the same chart a series of curves showing the relation between the dependent and the independent variables. In a commercial test the load is taken as the independent variable. In this case we will take speed, thermal efficiency and fuel consumption as the dependent variables. To plot these new curvas the same abscissas (load) can be used, but new vertical scales must be assumed. At the right of Fig. 7 let distances along $X Y^{\prime}$ represent thermal efficiencies in per cent. to an assumed scale-one unit on this line will necessarily mean something entirely different from one unit on the line $O Y$. Next take the values of the efficiencies from the table corresponding to the brake loads; locate a new set of points and through 
them draw the eurve $C D$. From this eurve we find that the maximum efficieney oceurs when the load is 220 b.hp. The curve also shows how the efficieney deereases under light loads and also on the overloads.

There remains the fuel-consumption curve. Here, again, a new vertieal seale must be assumed and will represent pounds of fuel per brake horsepower per hour. To avoid confusion it is well to mark the values for fuel inside the line $O Y$ and keep the values of speed outside the line $O Y$. Taking the necessary values from the table and plotting the points there results the eurve GH. Still further eurves might be plotted on this same chart. The student is urged to plot these same curves to different seales and compare the results.

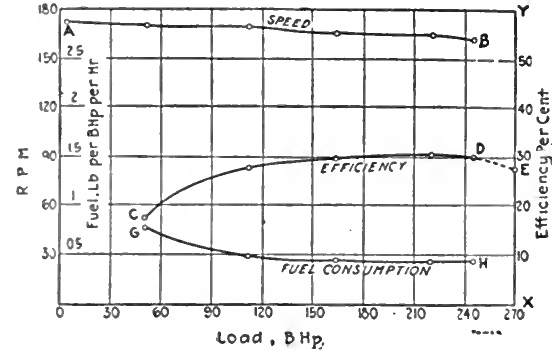

Fig. 7

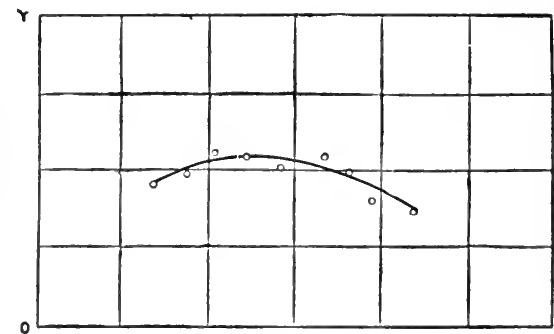

Fig. 8

By a careful study of the curves shown in Fig. 8 predictions can be made as to the probable values under other conditions of load. Thus if the net brake horsepower were run up to 270 (provided the engine would carry such a load) the efficieney would likely drop to the point $E$ or about $27 \%$. This value is found by extending the eurve to the point $E$ by following the general outline of the eurve $C D$.

In plotting curves of this type great eare must be taken to select suitable seales or false deduetions may be drawn from the results. No definite rules can be given as to what units should be used but it is hardly probable that we would take, say, seven or nine units per ineh but rather four or eight, or still better multiples of ten. In choosing the seales two things should be borne in mind: (1) That the seales should be large enough to give the reculuired degree of aceuraey and (2) that they should not be so large as to separate the points of observation hy great distances or to represent a degree of aceuracy greater than the original figures. In all sueh eases the seales should be elearly marked upon the axes and both seales should start with 0 value at the origin or the intersection of the axes of $X$ and $Y$.

Af times when an attempt is made to plot a curve between two variables it. will be found that a smooth eurve caunot be drawn that will tronch or pass through all the points of observation, due, perhaps, to mavoidable inacouracoses in the readings. In such rases (see Fig. 8) locate all the points and mark them by a ${ }^{\circ}$ or + , and then draw a smooth curve, as shown, to touch on the average values or pass as near to them as possible.

As a final application let us eonsider the ease of a gas engine. It is 
desired to find the probable pressure at the end of the compression stroke. The approximate law of compression is

\section{$P V^{\mathbf{1 . 4}}=$ constant}

Let the clearance volume equal $V_{1}$ and the total cylinder volume (piston displacement plus clearance) equal $V_{2}$. Let $\mathrm{P}_{1}$ equal the pressure at the end of compression and $P_{2}$ equal 14.7 equal the pressure at the beginning of compression. Then

$$
\text { or } \frac{P_{1} V_{1}{ }_{1.4}=P_{2} V_{2}{ }^{1.4}}{P_{2}}=\left(\frac{V_{2}}{V_{1}}\right)^{1.4}
$$

This makes a hard equation to solve and to facilitate computations of this character curves are plotted giving the ratio between pressures for given volume ratios. If the clearance is $33 \frac{1}{3} \%$ the volume ratio is then 4 and from the curve, Fig. 9 (plotted from computed data) the pressure

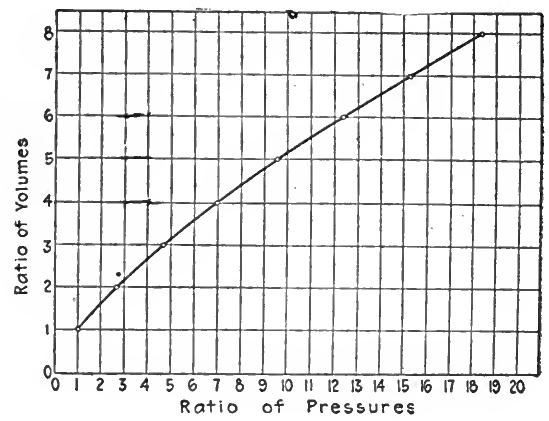

Fig. 9

ratio is approximately seven. Therefore, the pressure at the end of compression equals

$$
7 \times 14.7=102.9 \text { lb. abs. }=88.2 \text { lb. gage. }
$$

From these illustrations we see that the plotting of curves is very useful to the engineer in helping him to interpret data from tests, to predict results under other conditions, to find intermediate values not given in tables and to aid in the solution of complicated ratios

Diagrams for Safe Loads on Piles. The following published in Engineering and Contracting, January 24, 1912, gives a good illustration of the method of procedure.

Figs. 10 and 11 are for determining safe loads for piles. The first is for piles driven with a drop hammer and the second for piles driven with a steam hammer. The diagrams are constructed with the use of the Engineering News formula as given by the late A. M. Wellington as follows:

$$
P=\frac{2 w h}{1+S}
$$


Where $P=$ safe load in tons, $w=$ weight of hammer in tons, $h=$ fall of hammer in feet, and $S=$ penetration of pile at last blow.

The diagrams given here are by Prof. Edward R. Cary, as published in the Polytechnic of the Stevens Institute of Technology.

For the construction of the diagrams the formula may be expressed. as follows:

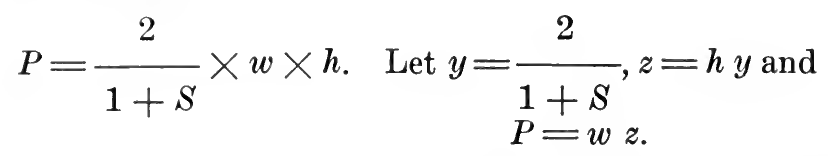

Values of $S$ are taken on the $X$ axis of the diagram and the corresponding values of $Y$ are plotted as ordinates. A curve is drawn through the plotted points, and lines parallel to the $X$ axis are also drawn through these points. The equation $z=h y$ is that of a straight line making with the $Y$ axis an angle whose tangent is $h$. A point is. taken on the $X$ axis, at such a distance from the origin, used for plotting points of above curve, as will make a convenient size for the dia-

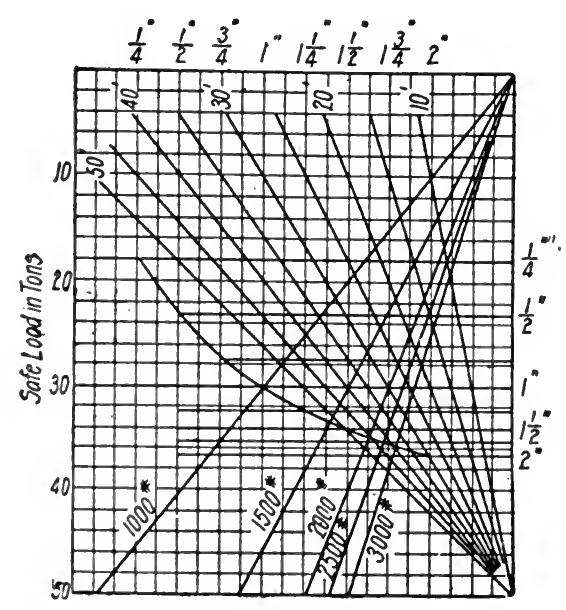

Fig. 10-Diagram for Safe Load on piles Driven with Drop Hammer

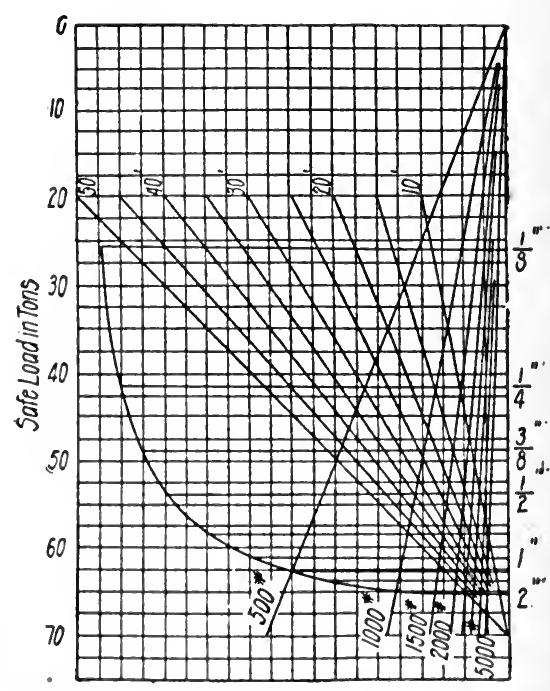

Fig. 11-Diagram for Safe Load on: Piles Driven with Steam Hammer

gram. This point is used as the origin for plotting the line $z=h y$ : (I) the line of $y$ for a value of $S=1 \mathrm{in}$., two divisions on the cross section paper are taken for the value of $z$ when $h=10 \mathrm{ft}$., four divisions when $h=20 \mathrm{ft}$., six divisions when $h=30 \mathrm{ft}$., etc. From these points: lines are drawn to the origin for $z$. On a line parallel to the $Y$ axis: and drawn through the origin for $z$, a point is taken for the origin for $I^{\prime}=u z$. On a line parallel to the $Y$ axis and drawn through the intersection of the $40 \mathrm{ft}$. line for the fall of the hammer and the line marked $1 \mathrm{in}$. parallel to the $Y$ axis, are laid off, to an adopted scale, 
values of $P$ for $w=1,000$ lbs., $1,500 \mathrm{lbs}$., etc., and for $S=1 \mathrm{in}$. and $h=40 \mathrm{ft}$. The lines above described and the $Y$ axis are properly numbered to make the completed diagram.

Knowing the penetration in inches for the last blow, find the intersection of the line parallel to the $X$ axis that is marked with this amount and the line which gives the fall of the hammer, then go parallel to the $Y$ axis until the line which gives the weight of the hammer is reached, then go parallel to the $X$ axis to the left edge of the diagram and read the safe load in tons; $e$. g., for a penetration of $3 / 4$ in., fall of hammer of $20 \mathrm{ft}$. and weight of hammer of $2,000 \mathrm{lbs}$., find intersection of $3 / 4 \mathrm{in}$. and $20 \mathrm{ft}$. lines, then go parallel to $Y$ axis until 2,000 lb. line is reached, then go parallel to $X$ axis and read $22+$ tons.

For steam hammer driven piles the formula $P=\frac{2 w h}{0.1+S}$ is used,

where terms represent the same quantities as above. The methods of construction and use are the same as for the above. The diagram is seen to be very similar in form to the one for drop hammer driven piles.

This formula is intended for piles not driven to rock, as such piles should be figured as columns. The quantity $S$ is the average penetration for the last few blows, which under ordinary circumstances becomes fairly constant. This condition in the driving shows that the pile is in such material that the formula may safely. be used for figuring its loading.

Resistances in Parallel. A. S. L. Barnes gives the following in Electrical World, June 22, 1918.

Calculation of resistances in parallel entails a considerable amount of routine work which can be eliminated by means of the graphical system shown here. This gives a simple method whose accuracy is directly proportional to the size of chart used.

As shown in Fig. 12, ordinate and abscissa scales are marked identically on cross-section paper, and a line is drawn from the common zero point at an angle of 45 deg.

If it be assumed that three resistances in parallel are 62, 43.5 and $13 \mathrm{ohms}$ respectively, the ease of determining their total resistance is seen by reference to the chart. The resistance $R_{1}$ is measured on the ordinate scale and resistance $R_{2}$ on the abscissa scale, or vice versa. These lines joining the scale points representing these values will cut the line $O X$ at a point which, projected on either scale, will give their joint resistance in parallel. This new value, $25.56 \mathrm{ohms}$, if joined to a point on the opposite scale representing $R_{3}$, will give a new value, $8.62 \mathrm{ohms}$, which represents the combined resistance of the three circuits in parallel. This scheme can be extended to include any number of resistances in parallel.

Logarithmic Paper for Computation. The following is an exceptionally good description of the use of logarithmic paper for various kinds of computations. It is taken from an article by W. F. Durand published in Engineering News, September 28, 1893.

It is the peculiar property of logarithmic section paper that for all relationships which involve multiplication, division, raising to powers, 
or extraction of roots, the lines representing them are straight. This may be readily proved as follows:

Any such relationship may be represented by an equation of the form: $y=13 x^{n}$. Taking logarithms we have: $\log . y=\log . B+n \log$. $x$. Suppose we proceed to lay off this equation on ordinary cross-seetion paper. We way off $\log x$ horizontally on the $x$-axis and at the point so determined we lay up parallel to the axis of $\mathrm{y}$ a distance $\log . \mathrm{B}+\log$. $\mathrm{x}$ and through this point draw a line inclined to the axis of $\mathrm{x}$ at an

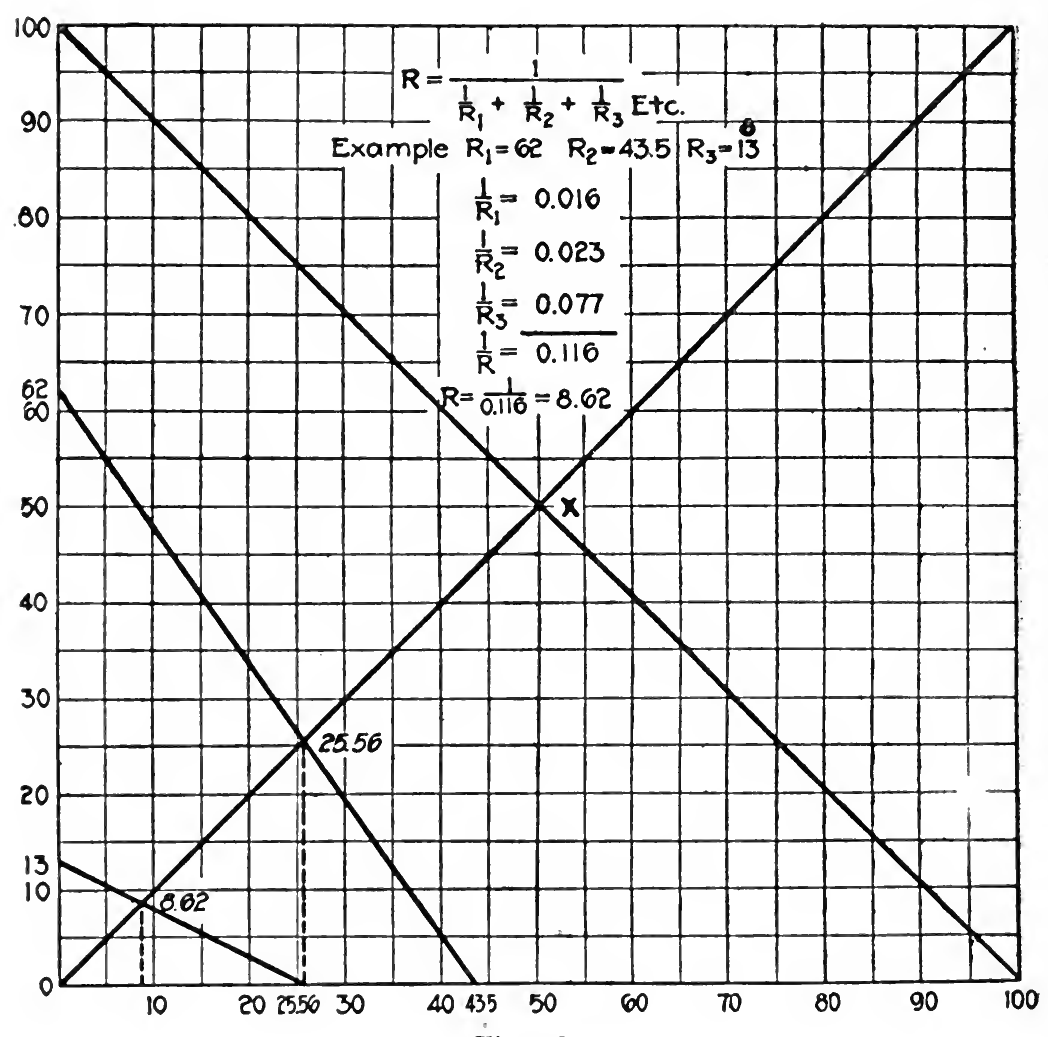

Fig. 12

angle whose tangent is $n$. This gives at each point the value of $\log y$, and the result will be a straight line. This may be the more readily secn by noting that it is exactly similar in operation to the plotting of $y=\mathrm{I}+11 \mathrm{x}$, a form of equation which gives a straight line eutting the axis of $y$ at a distance $I 3$ above the origin and inclined to the axis. of $x$ at an angle whose tangent is $n$.

Now, locrarithmic section paper is simply a short and ready means of blotting such logarithmic equations. The scales on each side are logarithmic instead of miform, as in ordinary cross-section paper. The numbers and divisions marked are placed at such points that their distances from the origin are proportional to the logarithms of such 
numbers instead of to the numbers themselves. It follows that if we take any point, as 3, for example, on such a scale, the real distance we are dealing with is log. 3 to some particular base, and not 3 itself. With such scales, then, we may proceed to plot numbers in the usual way, knowing that in so doing we deal with the logarithms of the numbers instead of with the numbers themselves. In general we may remember that any scale involves two distinct parts :

(1) The divisions marking off certain distances from the origin.

(2) The numbers marking such divisions.

If both of these correspond, we have the ordinary plain scale or scale of equal parts. We may, however, have the numbers mean one thing and the distances another, in any desired relationship one to the other. Thus, we might have the distances uniformly spaced, while the numbers might give the squares of such distances from the origin. Or, vice versa, we might have the distances give the squares of the numbers. In a similar manner on the logarithmic scale the distances give the logarithms of the numbers. It should be noted that the number at the origin of such a scale is always 1 and not 0 . This is, of course, because 1 is the number whose logarithm is 0 . This 1 may, however, represent a unit of any order, so that quantities of any size whatever may be dealt with. Those familiar with the slide rule will note the identity between the foregoing considerations and those which underlie the use of that instrument.

It appears, therefore, if we have a series of values of $\mathrm{x}$ and of $\mathrm{Bx}^{\mathrm{n}}$, and plot on logarithmic section paper $\mathrm{x}$ horizontally and $\mathrm{Bx}^{\mathrm{n}}$ vertically, that the actual distances involved will be $\log . \mathrm{x}$ and $\log$. $\left(\mathrm{Bx}^{\mathrm{n}}\right)$ or $\log$. $\mathrm{B}+\mathrm{n} \log . \mathrm{x}$. But these distances, as we have seen, will give a straight line as the locus. Hence all relationships expressible in this form are represented on logarithmic section paper by straight lines. It follows that the entire locus may be determined from any two points; that is, from any two values of $\mathrm{Bx}^{\mathrm{n}}$; or, again, by any point and the angle of inclination; that is, by one value of $B x^{n}$ and the value of $n$, remembering that $\mathrm{n}$ is the tangent of the angle of inclination to the horizontal.

We will next proceed to some details of the considerations involved, and show how a single square plotted on each edge with a logarithmic scale from 1 to 10 may be made to serve for any number whatever from 0 to $\propto$. To make the illustration simple we will take the case of a table of $3 / 2$ powers. That is, we wish to express graphically the locus. of the equation: $\mathrm{y}=\mathrm{x}^{3 / 2}$. Let Fig. 13 denote a square cross sectioned with logarithmic scales, as described. Suppose that there were joined to it and to each other on the right and above, an indefinite series of such squares similarly divided. Then, considering, in passing from one square to an adjacent one to the right or above, that the unit becomes of the next higher order, it is evident that such a series of squares would, with the proper variation of the unit, represent all values of either $\mathrm{x}$ or $\mathrm{y}$ between 0 or a quantity indefinitely small, and $\propto$ or a quantity indefinitely large.

Suppose next the original square divided on the horizontal edge into 3 parts, and on the vertical edge into 2 parts, the points of division being given at A, B, D, F, G, I. Then lines joining these points, as. 
shown in the diagram, will be at an inclination to the horizontal whose tangent is $3 / 2$, and will be, therefore, in the right direction. Now, beginning at 0 , it is evident that $0 \mathrm{~F}$ will give the value of $\mathrm{x}^{3 / 2}$ for values of $\mathrm{x}$ from 1 to that denoted by HF, or $0 \mathrm{~B}$, or about 4.6. For greater values of $x$ the line would run into the next adjacent square above. Now, the location of this line if continued, may be seen to be exactly similar to that of $\mathrm{BD}$ in the square before us. It follows, therefore,

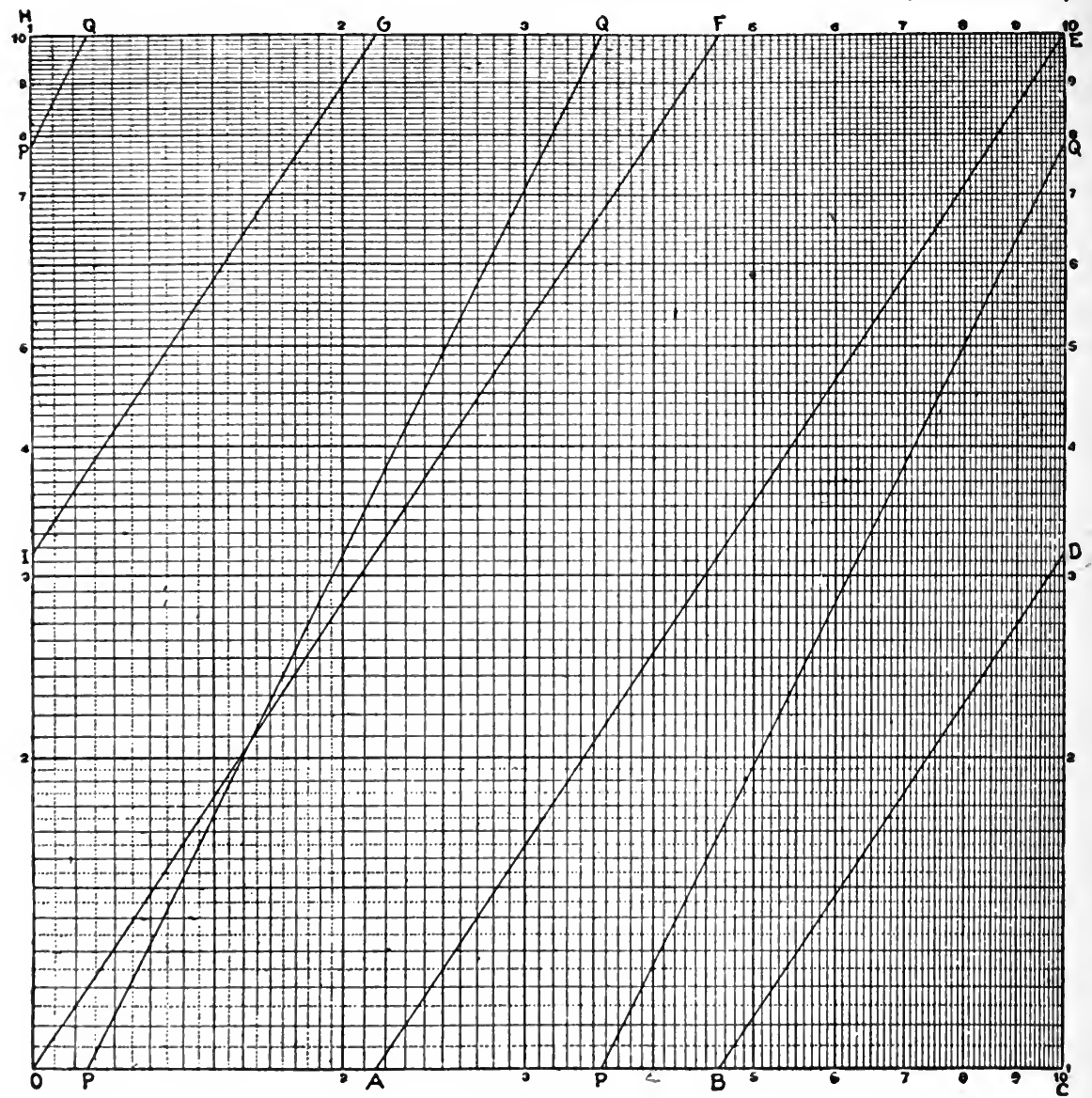

Fig. 13

that considering the units as of next higher order, the line $\mathrm{BD}$ will give values of $x^{3 / 2}$ for $x$ between $B$ and $C$, or 4.6 and 10 . For larger values of $x$ we consider the unit on $x$ as of next higher order, and note that we should again run into an adjacent square to the right without change of unit for $y$. In this square we should traverse a line similar to IG. Therefore, by a proper choice of units we may make use of IG for the determination of values of $x^{3 / 2}$, where $x$ lies between $I(x$ and the value at $r_{r}$, or abont 21.5. We should then run into an adjacent square above, reculuiring the unit on $\mathrm{y}$ to be of the next higher order, and 
traverse a line similar to $\mathrm{AE}$, which takes us finally to the opposite corner and completes the cycle. Following this, the same series of lines would result for numbers of succeeding orders.

A little consideration of the subject will show that the value of $\mathrm{x}^{3 / 2}$ for any value of $\mathrm{x}$ between 1 and $\propto$, may thus be read from one or another of these lines; and if for any value between 1 and $\propto$, then likewise for any value between 0 and 1 . The location of the decimal point is readily found by a little attention to the numbers involved. A rule for its location might be derived, but is of little additional value in practice. The limiting values of $\mathrm{x}$ for any given line may be marked on it, thus enabling proper choice to be readily made. Thus, in Fig. 13 we mark $\mathrm{OF}$ as $0-4.6$; $\mathrm{BD}$ as $4.6-10$; $\mathrm{IG}$ as $10-21.5$, and $\mathrm{AE}$ as 21.5-100. If values of $x$ less than 1 are to be dealt with, we readily see that $\mathrm{AE}$ will serve for values of $\mathrm{x}$ between 1 and .215 ; IG for values between .215 and .1; BD for values between .1 and .046, and $\mathrm{OF}$ for values between .046 and .001 .

The principles involved in this case may be readily extended to any other, and it will be found in general that if the exponent be $\mathrm{m}$

represented by - the complete set of lines may be drawn by dividing $n$

one side of the square into $\mathrm{m}$ and the other into $\mathrm{n}$ parts, and joining the points of division as in Fig. 13. In all there will be $(m+n-1)$ lines, and opposite to any point on $\mathrm{X}$ there will be $\mathrm{n}$ lines corresponding to the $n$ different beginnings of the $n$th root of the mth power, while opposite to any point on $\mathrm{Y}$ will be $\mathrm{m}$ lines corresponding to the different beginnings of the $\mathrm{mth}$ root of the $\mathrm{mth}$ power. Where the complete number of lines would be quite large, it is usually unnecessary to draw them all, and the number may be limited to those necessary to cover the needed range in the values of $x$.

If, instead of the equation $\mathrm{y}=\mathrm{x}^{\mathrm{n}}$, we have a constant term as a multiplier, giving an equation in the more general form $\mathrm{y}=\mathrm{Bx}^{\mathrm{n}}$, there will be the same number of lines and at the same inclination, but all shifted vertically through a distance equal to log. B. If, therefore, we start on the axis of $Y$ at the point $B$, we may draw in the same series of lines and in a similar manner. In this way $\mathrm{PQ}$, Fig. 13, represents the locus giving the values of the areas of circles in terms of their diameters, being the locus of the equation $\mathrm{A}=1 / 4 \pi \mathrm{d}^{2}$ or $\mathrm{y}=1 / 4 \pi \mathrm{X}^{2}$.

If in any case we have $\mathrm{x}$ in the denominator such that the equation B

is in the form $y=\frac{-}{\mathbf{x}^{\mathbf{n}}}$, we note that this is equal to $\mathrm{y}=\mathrm{Bx}^{-\mathrm{n}}$ and that the same general rules hold. The lines in such case simply slant downward to the right instead of upward. As an instance of this we may take the equation $\mathrm{pv}^{10 / 9}=\mathrm{C}$, a formula well known to the steam engineer. This in another form is $\mathrm{p}=\mathrm{Cv}^{-10 / 9}$. In Fig. 14 we have a line $\mathrm{PQ}$ 10

drawn at an inclination whose tangent is $-\frac{1}{9}$, and which therefore readily gives the value of $\mathrm{v}^{-10 / 9}$. We thus find the resulting value of 
$\mathrm{p}$, and by this means a eurve for this law may be laid down with almost the same ease as that for the more commonly assumed law $\mathrm{pv}=\mathrm{C}$.

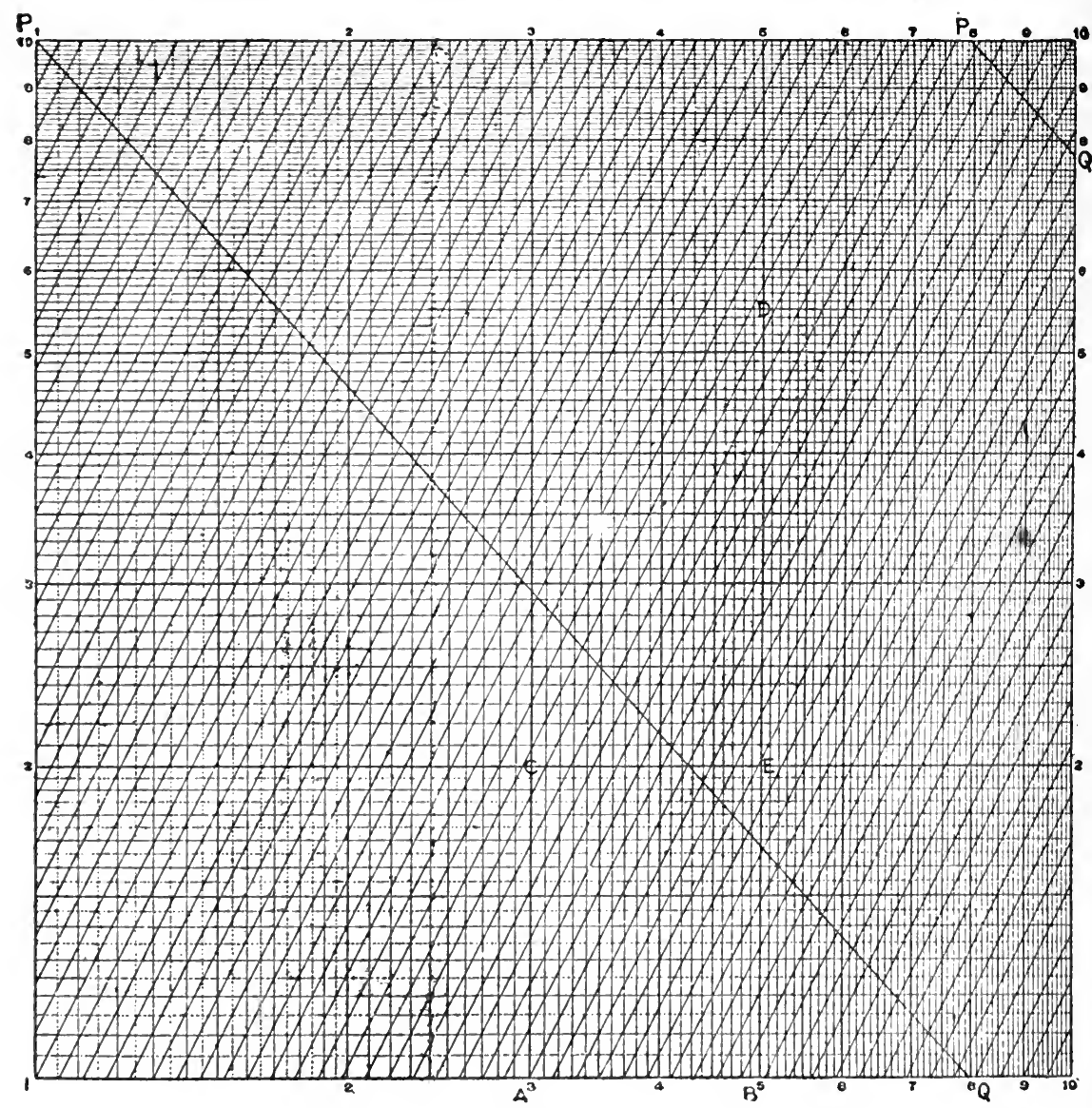

Fig. 14

The same problem may be differently treated thus: We have $\mathrm{pv}^{10 / 9}=$ $\mathrm{p}_{1} \mathrm{v}_{1}{ }^{10 / 9}$ or $\mathrm{p}=\mathrm{p}_{1}\left(\frac{\mathrm{v}_{1}}{\mathrm{v}}\right)^{10 / 9}$. Now, if the operation under consideration is an expansion, we have $v>v_{1}$ and $-=r$, the expansion ratio. Hence $p=p_{1} \mathrm{r}^{-10 / 9}=\mathrm{p}_{1} \div \mathrm{r}^{10 / 9}$. If the operation is a. compression then $\mathrm{v}<\mathrm{v}_{1}$ and $\frac{\mathrm{v}_{1}}{-}=\mathrm{r}$, the compression ratio. This gives $\mathrm{p}=\mathrm{p}_{\mathbf{1}} \mathrm{r}^{10 / 9}$ 
$=p_{1} \div r^{-10 / 9}$. Either of these values may be treated by means of a line inclined at an angle whose tangent is either $-\frac{10}{9}$ or $+\frac{10}{9}$.

The applications of this paper thus far mentioned have been concerned chiefly with quantities involving powers and roots. It must not be supposed, however, that it cannot be as readily used to express relations involving only the first power. Here, however, it has no especial advantage over ordinary paper, for with each the locus in such ease is a straight line. With logarithmic paper we may note that the direction of the line in such case is always at an angle of $45^{\circ}$, cutting, of course, the axis of $\mathrm{Y}$ at a distance above the origin equal to the logarithm of the constant term.

Among the various relationships which may be represented by the means above discussed, the following may be mentioned as those more frequently occurring: Circumferences and diameters of circles in terms of their radii or diameters, or the inverse; moments of inertia and radii of gyration in terms of a linear dimension, or the inverse; length of pendulum and time of oscillation; powers and roots of any and all indices; weights of a series of bodies of the same substance and form but of varying size, in terms of a linear dimension, or the inverse; sizes of shafts, struts, tiebars, etc., in terms of varying load, or the inverse; shearing stress, bending moment, or deflection of beams in terms of load, or the inverse.

It will be noticed in the above that we have mentioned both the direct and the inverse relationships, and this is one of the advantages which all such modes of representation possess, rendering estimation and interpolation equally easy in both directions.

iIany other applications might be mentioned, and each branch of engineering would furnish a large variety of uses adapted to the peculiar problems arising in such special field. In this way numerous applications will be found in the various laws of thermodynamics, in the laws relating to electricity and magnetism and electrical engineering in general, in hydraulies, in naval architecture, etc. In engineering work of an experimental character, and in various fields of physical research, special advantage will be found in the use of this form of section paper for the plotting of results.

Where, for example, it is desired to plot a result $\mathrm{y}$ which varies as some unknown power of a variable $x$, this method will be found very useful. The equation assumed to be fulfilled will be of the form $\mathrm{y}=$ $B x^{n}$, where $B$ is a constant and unknown, and $n$ is unknown and may be either constant or variable. If it is constant the line will be straight, and the tangent of its inclination to $\mathrm{X}$ will equal $\mathrm{n}$, while the distance from the origin to where it cuts $\mathrm{Y}$ will be equal to log. $\mathrm{B}$, and will therefore determine $B$ directly by the reading at that point. If $n$ is not constant then the locus will be curved, and the instantaneous exponent of the variation of $y$ with $x$ is given by the value of the tangent of the inclination of the tangent line to the curve at such point. It may be remarked in such case that we have really to deal with two exponents, one giving the instantaneous exponent as noted above, and the other an exponent such that it will satisfy the equation $\mathrm{y}=\mathrm{Bx}^{\mathrm{n}}$. Unexpected 
though it may seem, these two exponents are not the same, but both may be readily found by a proper examination of the resulting locus. For further information on this point the reader is referred to a paper published in the Journal of the Society of Naval Engineers, on "The: Analysis of Certain Curves Arising in Engineering Investigation.'

We will next examine the use of logarithmic section paper as an actual instrument of computation, rather than as a means of graphically representing various algebraic relationships.

Proportions of the form $\mathrm{y}_{2}: \mathrm{y}_{1}:: \mathrm{x}^{\mathrm{n}}{ }_{2}: \mathrm{x}^{\mathrm{n}}{ }_{1}$, frequently arise in engineering investigations. In the form of an equation this becomes $y_{2}=$ $\mathrm{y}_{1}\left(\frac{\mathrm{x}_{2}}{\mathrm{x}_{1}}\right)^{11}$ whence log. $\mathrm{y}_{2}=\log \cdot \mathrm{y}_{1}+\mathrm{n}\left(\log \cdot \mathrm{x}_{2}-\log . \mathrm{x}_{1}\right)$. Now in Fig. 14 let $\mathrm{A}$ and $\mathrm{B}$ denote the points $\mathrm{x}_{1}$ and $\mathrm{x}_{2}$, and $\mathrm{C}$ and $\mathrm{D}$ be at the heights denoted by $y_{1}$ and $y_{2}$. Then, remembering that the actual distances involved are the logarithms of the corresponding quantities, we readily see that $\mathrm{BD}=\mathrm{AC}+\mathrm{nAB}$ or $\mathrm{AC}+\mathrm{nCE}$. Therefore, a line from $\mathrm{C}$ to $\mathrm{D}$ will be at an inclination to $\mathrm{X}$ whose tangent is $\mathrm{n}$. If, therefore, we have any ready means of passing from $C$, a point determined by the co-ordinates $\mathrm{x}_{1} \mathrm{y}_{1}$, along an oblique line inclined to the axis of $\mathrm{X}$ at an angle whose tangent is $n$, it is evident that we shall pass along a continuous series of points $x_{2} y_{2}$ so related to $x_{1} y_{1}$ that the proportion above mentioned will be fulfilled. To solve any such proportion, therefore, we have simply to start at the given point, $x_{1} y_{1}$, and pass along the oblique line until we reach a point whose abscissa is $x_{2}$. The corresponding ordinate will be the desired value of $y_{2}$, or, vice versa, if we stop at any given value of the ordinate $y_{2}$, the eorresponding abscissa will be $\mathrm{x}_{2}$, so related to the other quantities that the proportion is fulfilled. To provide the necessary means for moving in the right direction from any point whatever as $x_{1} y_{1}$, a series of equidistant lines at the proper angle may be ruled. With this aid a very close approximation to the proper values of $x_{2} y_{2}$ may be made. As an instance of the use of this proportion, suppose that we wish to compare the areas of similar figures of different size. Such areas are in the ratio of the srfuares of their linear dimensions, and a corresponding series of oblique lines will be at an inclination whose tangent is 2 . Now, suppose we have given any one area which we denote by $\mathrm{y}_{1}$, and the linear dimension $x_{1}$. Then, using any convenient units we note the point so determined, and passing thence along the sheet parallel to the oblique lines we shall find corresponding to any linear dimension $\mathrm{x}_{2}$ the corresponding area $y_{2}$, or, vice versa, corresponding to any given area $y_{2}$, the corresponding linear dimension $\mathrm{x}_{2}$.

Reference has been already made to the similarity in fundamental principle between logarithmic section paper and the slide rule. This similarity is only to be expected from the fact that the fundamental scale is the same in both. We may, therefore, expect to find in the section paper, all the inherent properties of the slide rule, and in fact it may he used as such, provided only that we may be able to take the necessary mechanical advantage of them. Having in this ease no slide to, serve as a carrier of logarithms from one part of the seale to another, we may substitute for it a pair of dividers. By this means we find 
that we can perform all ordinary operations quite as readily as with the slide rule. We note that with one point of the dividers on the axis $\mathrm{X}$ we may reach vertically upward to any number $\mathrm{y}$. The distance between the points will be log. $y$. If we then swing the dividers down to the right we shall add $\log$. $y$ to $\log$. $x$, and the number at the right hand point will be the product xy. If instead, we swing to the left, we shall subtract log. $y$ from log. $x$, and the number at the left hand point will be the quotient $\mathrm{x} \div \mathrm{y}$. This may be kept up indefinitely, and the result of any continued multiplication and division thus found. Again, if on either of the scales the two points are opened to two numbers, $x$ and $y$, the distance between them will be the log. of their quotient, and placing one point at the origin we read off the quotient at the other.

We may also remember with advantage that so far as the digits in a result are concerned. the addition or subtraction of a logarithm is the same as the subtraction or addition of the complement of the logarithm. If, therefore, in any of the above operations either point of the dividers should go off the scale, the result may be found by taking advantage of this principle. To illustrate this point suppose that we wish to multiply 82 by 36 . With one point of the dividers at 82 we reach up to 36 and then find on swinging to the right that the point comes off the scale. We obtain the correct result, however, by subtracting from 82 the distance from 36 to the right hand end of the scale, this distance being the complement of the log. of 36 . A little familiarity will enable such occasions to be foreseen, and the complementary log. would be taken at the start. The same principle may be utilized to avoid opening the dividers to more than half the length of the scale, and a little attention to this matter will keep the distances involved down to a minimum. In the preceding example the preferable course would be to step from 82 to the right hand end of the scale and then subtract this distance from 36 .

Rules for the location of the decimal point are, of course, the same as for the ordinary slide rule. For powers and roots it is preferable to have sheets ruled with a line giving the desired power or root at a glance. If this is not at hand, however, a power is readily found by simply stepping off the log. to the right or its complement to the left, as many times as there are units in the index. Thus, to cube 13 we open from 10 to 13 and step this distance three times along the scale, reading off at the end the cube desired. To cube 8 we would preferably open from the right hand end to 8, and step this distance three times to the left. To find a root, an inverse operation is necessary, remembering that there are as many different beginnings to a root as there are units in its index, and that the successive ratio between these is the nth root of 10 .

With regard to accuracy, it is found that section paper can be readily printed so that the error is well within $1 / 3$ of $1 \%$, and, therefore, that the accuracy is abundantly sufficient for a very large class of engineering work. On this point it may be well to note that from the peculiar properties of logarithmic scales, an error of a certain amount in the location of a line results in the same proportional error at all parts of the scale. This is readily seen to be due to the fact that in such case 
the true result is multiplied or divided by a number whose logarithm is the amount of error in question. The peculiar value of this may be seen from an illustration. Suppose, on the scales used, the true distance between 40 and 41 to be 0.1 in., while actually it is in error .01. This would be an error of $10 \%$ in itself, but the error resulting from the use of this distance in finding the quotient of 41 by 40 would be simply the ratio whose log., on the base used, is .01, which in such case would be about 1.002. Hence, the error in the result is $1 / 2$ of $1 \%$, while the error in the logarithm was relatively 50 times greater.

Locomotive Horsepower. Figs. 15 and 16 and the following are from an article by T. Price, Railway Age Gazette, March, 1914.

Suppose that we wish to prepare a chart for quickly finding the ap-

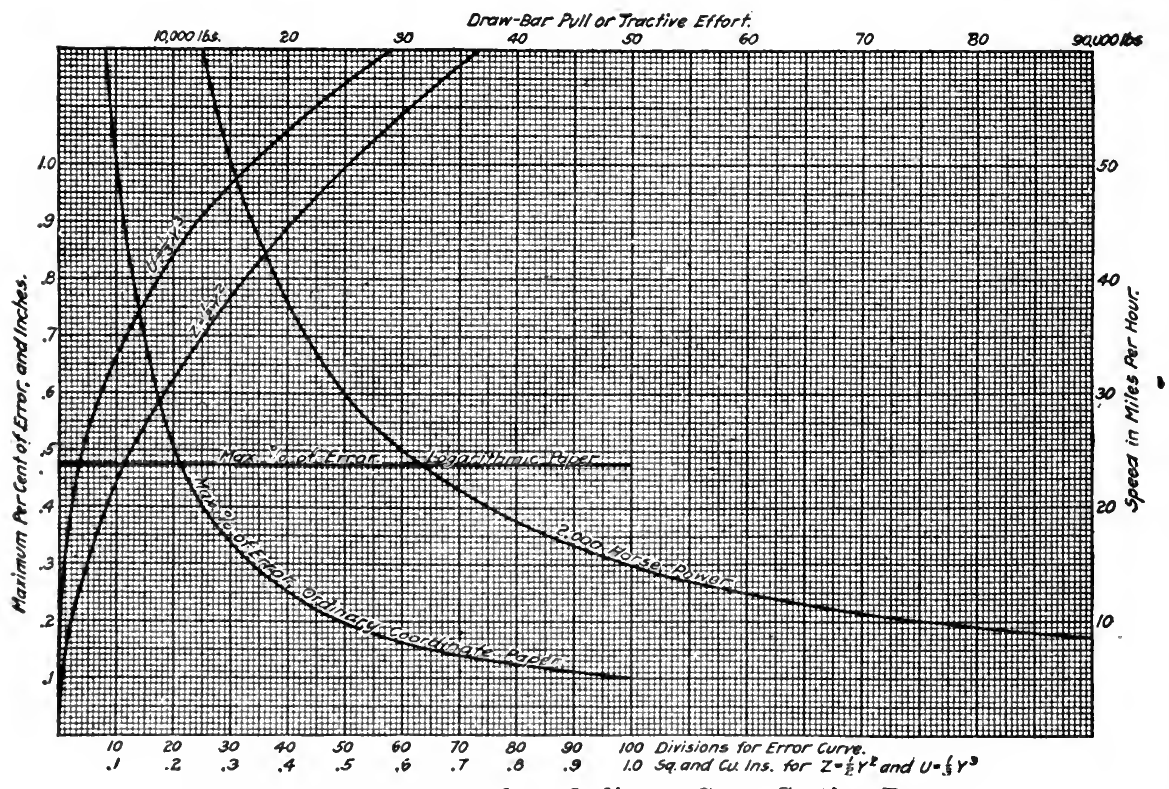

Fig. 15-Curves Plotted on Ordinary Cross Section Paper

proximate horsepower a locomotive is developing for the known draw bar pulls and speeds on a dynamometer test.

Let IIP = horsepower, $\mathrm{F}=$ tractive effort or draw bar pull in pounds, and $\mathrm{V}=$ speed in miles per hour. Then $\mathrm{IIP}=\mathrm{FV} \div 375 . \Lambda$ series of horsepower lines on ordinary rectangular cross section paper would he a series of hyperbolas, Fig. 15, as this erpuation is of the form $x y=c$, $\mathrm{x}=\mathrm{F}, \mathrm{y}=\mathrm{V}$, and $\mathrm{c}=375 \mathrm{HP}$. It would be a laborious task to construct a large number of these; also they would not be very accurate unless a eonsiderable number of points were plotted for each. On logarithmic paper, on the contrary, these horsepower lines are all straight and parallel to each other, making angles of 45 deg. with the axes. This makes it necessary to find only one point for each line to make the chart as shown, Fig. 16 .

It is only necessary to plot two points for each of the equations $Z=$ 
$1 / 2 \mathrm{Y}^{2}$ and $\mathrm{U}=1 / 3 \mathrm{Y}^{2}$. Thus, draw a straight line through the points .2 , .02 and $.4, .08$ for the first and one through the points $.3, .009$ and $.6, .072$ for the second.

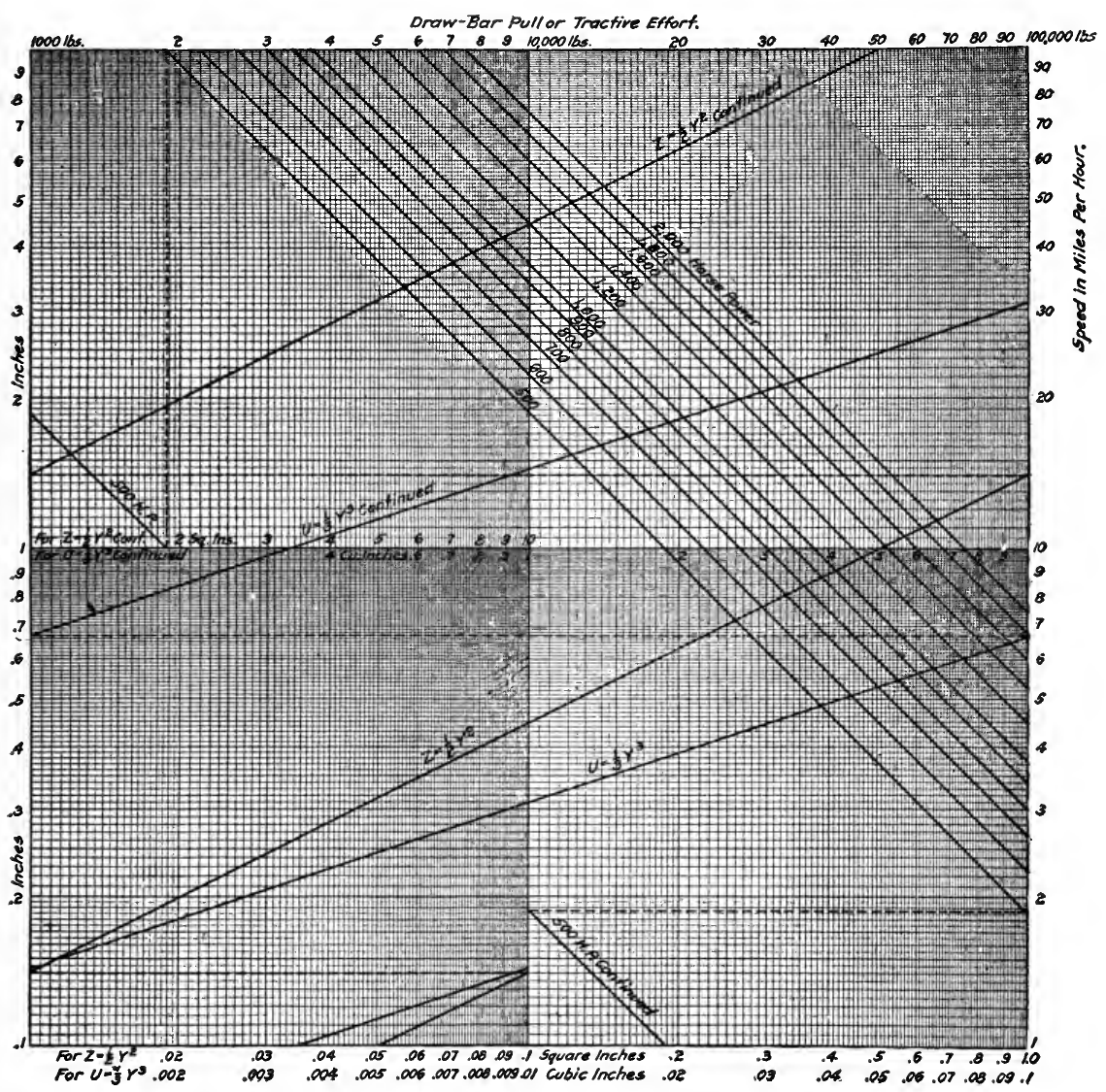

Fig. 16-Illustrating the Uses of Logarithmic Paper

Logarithmic Paper for Finding Formulas to Fit Given Curves. The following is from an article by E. W. Lane, published in Engineering News-Record, September 20, 1917.

Following the universal adoption of the slide rule for making ordinary computations, the use of logarithmic co-ördinate paper is becoming increasingly common in engineering studies. Some of the properties of such paper are obscure and complex and this article aims to set forth in plain directions a systematic procedure to be followed in one of the most valuable applications of this useful modern device-the determination of empirical exponential formulas to fit given curves.

The use of logarithmic paper in finding equations to express the laws governing phenomena in certain simple classes of cases is already familiar to most engineers. It has been found especially valuable in the 
analysis of experimental results on account of the great variety of cases in which it can be applied and the ease with which equations may be derived by its aid. An important property of this paper is that any straight line drawn upon it has a simple and easily determined exponential formula, and its use has been largely confined to cases in which the data fall on such a line. Simple formulas may in many cases be found, however, where the experimental data plot on a curve, sometimes even on reverse curves.

The following discussion was prepared by the writer under the direc-

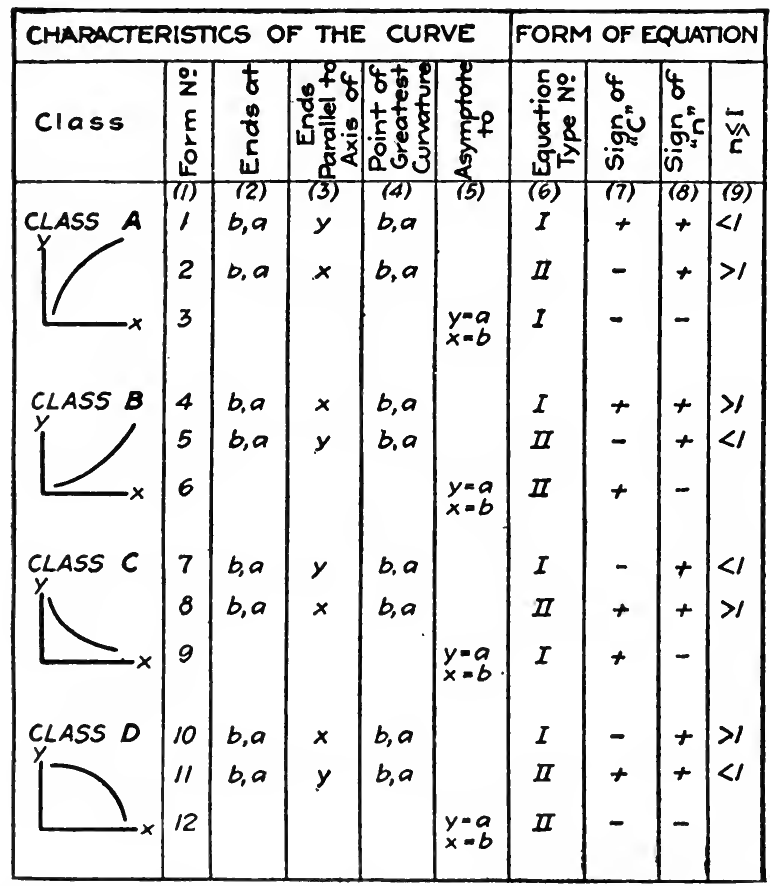

Fig. 17-Tabulated Curve Characteristics and Equation

Forms for Determining Empirical Exponential Formulas

tion of S. M. Woodward, in connection with the design of the floodprevention works of the Miami Conservancy District. Its purpose is to point out some of the possibilities of the determination of equations by the aid of logarithmic paper and the characteristics of the curves to which the process may be applied. In this article the mathematical principles are not discussed, but an effort is made to present the subject in the form in which it will be of greatest practical use. It is assumed that the reader is already familiar with the theory and principal properties of logarithmie paper, and the ease of the straight line will therefore not be considered.

Simple exponential equations can be found by the aid of logarithmic paper for a great variety of curves. The formulas are of two general types: 


$$
\begin{aligned}
& \text { Type I, } y-a=c(x-b)^{\mathrm{n}} \\
& \text { Type II, } y-a=c(b-x)^{\mathrm{n}}
\end{aligned}
$$

where $a, b, c$ and $n$ are any real numbers. Most graphs, in which one variable is a single-valued increasing or decreasing function of the other, can be closely approximated by one of these two equations.

In this discussion only the cases where $y-a$ and $x-b$ or $b-x$ are positive will be considered, since when they are negative, complications are introduced, a consideration of which will add little to the value of the discussion. Depending on the sign and magnitude of the constants $c$ and $n$, these two type formulas cover 12 distinct forms of curve, each having different eharacteristics.

To aid in selecting the equation which will fit any desired curve, the
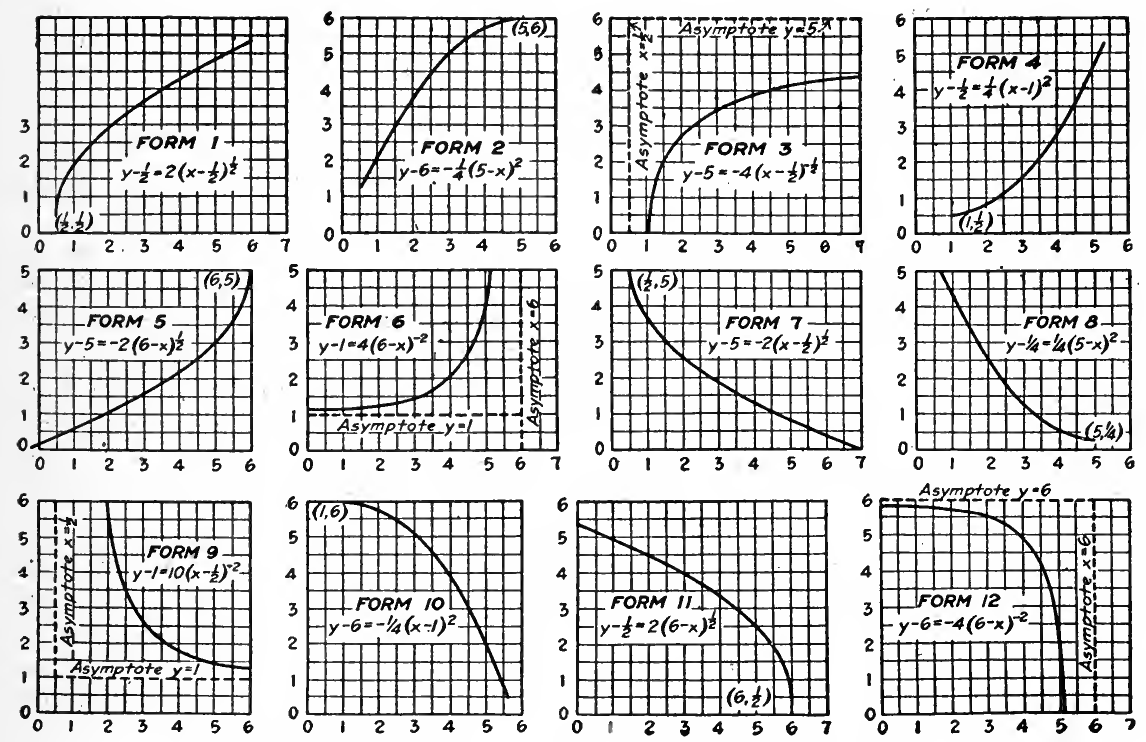

Fig. 18-Twelve Typical Exponential Curves of the Simpler Forms

forms here have been classified according to their shape when plotted on rectangular co-ordinates. In Fig. 17 these classes are tabulated, together with the characteristics of the three forms in each class, and the magnitude and sign of the constants which distinguish them. Typical curves of each case are shown in the 12 sketches, Fig. 18.

The basis of classification used is the well-known one of differential calculus, depending on the signs of the first and second derivative. In other words, all curves whose slopes are of the same sign, and which are concave in the same direction, are in the same class. For example, class A contains all those forms which slope upward to the right and are concave downward, or for which the first derivative is positive, while the second is negative. There are three of these forms, which, for the purpose of identification, have been numbered 1 to 3 inclusive. Ex- 
amples of each form are shown and in the table are listed their characteristie features.

Consider a curve of form 1: The second column of the table shows that it ends at the point $(b, a)$. The third column shows that at this point the curve is parallel to the axis of $y$, while the fourth column shows that it is also the point of greatest curvature. Column 5 indicates that the curve is not asymptotic to any line. In the last four columns are given determining features of the formula for the curve. In the first of these, column 6 , is given the type of equation for this form-in this case type $\mathrm{I}$, or $y-a=c(x-b)^{\mathrm{n}}$. The next two columns show that the signs of both $c$ and $n$ are plus, while column 9 shows that $n$ must be less than unity. In the sketches is a typical curve of this form, the equation being $y-1 / 2=2(n-1 / 2)^{1 / 2}$.

Consider a curve of form 3 . Columns 2 and 3 of the table indicate that this curve has no finite end. Since the expression for the point of greatest curvature is complicated, it is not included in column 4. Column 5 shows that the curve has two asymptotes, $y=a$ and $x=b$. Columns 6 to 9 inclusive give the characteristics of the formula. It belongs to type $\mathrm{I}$; both $c$ and $n$ are negative, and $n$ may be greater or less than unity. A typical example of form 3 is drawn for which the equation is $y-5=-4(x-1 / 2)^{1 / 2}$.

The first step in the determination of the formula of a curve is to find to which class and form it belongs. For example, consider a curve which slopes upward toward the right, is concave upward, is asymptotic to a line parallel to the axis of $x$ and possibly also to one parallel to the $y$ axis. The first two characteristics, a plus slope and concavity upward, place this curve in class B of the table, while having asymptotes, places it in form 6 . The equation whose graph will most nearly fit the given curve is therefore $y-a=c(b-x)^{n}$, where $c$ is positive and $n$ is negative.

It sometimes happens that the curve is not so easily classified as this example and it is necessary to try out several forms in order to arrive at the best one.

Where a high degree of precision is not required, the determination of the values of the constants can best be made by a cut-and-try process. In order better to understand the method, consider the two type forms:

$$
\begin{aligned}
& y-a=c(x-b)^{n} \\
& y-a=c(b-x)^{n}
\end{aligned}
$$

If we replace $y-a$ by another variable, $z$, and $x-b$ or $b-x$ by $w$, we have the equation

$$
z=c w^{n}
$$

This will be recognized as the equation of a straight line on logarithmic paper. The method of determination of the proper values of $a$ and $b$ is therefore to find those values which will eause $z=y-a$ when plotted against $w=x-b$ or $b-x$, to form as nearly a straight line as possible. The shape of the graph when plotted on rectangular co- 
ördinates often indicates approximately the values of $a$ and $b$. If the curve belongs to one of the forms where $n$ is negative, the asymptotes will be at the values of $b$ and $a$. If $n$ is positive, the curve will start from the point $(b, a)$ parallel to the $x$ or $y$ axis, depending on whether $n$ is greater or less, respectively, than unity.

Having determined the form of the equation and the approximate values of $a$ and $b$, plot on logarithmic paper the values of $y-a$ against $x-b$, using these approximations of $a$ and $b, x$ and $y$ being any of the simultaneous values of the two variables the relation between which it is desired to express by a formula. If $c$ is negative, the form of the type equations must be changed to

or

$$
a-y=-c(x-b)^{n}
$$

$$
a-y=-c(b-x)^{n}
$$

and the values of $a-y$ plotted against $x-b$ or $b-x$. The plotted points, using the approximate values, will probably lie on a line which is nearly straight. To transform this into a straight line it will be necessary to use somewhat different values of $a$ and $b$.

The following rules will aid in determining the nature of the required changes. To alter the values of these constants in such a way that the curve is moved in the direction in which it is concave, increases the concavity, while to move it in the opposite direction decreases the concavity. Thus, if the graph obtained by plotting $y-a$ and $x-b$ or $b-x$ slopes upward to the right, and is concave upward, to make it more nearly straight change $a$ and $b$ in such a way as to move the graph downward or to the right or both. In any such movement those parts of the graph nearer the lower and the left-hand parts of the sheet tend to move faster than the other parts. With the above rules in mind, a little practice will enable one quickly to select $a$ and $b$ of such magnitude that the points will fall as nearly on a straight line as it is possible to make them.

From this straight line the values of $c$ and $n$ of the formula $y-a=$ $c(x-b)^{n}$ or $y-a=c(b-x)^{n}$ are easily determined, since, on account of the properties of logarithmic paper, the value of $c$ with its sign is the ordinate as read on the logarithmic paper of the point at which this line crosses the axis along which the values of $y-a$ were plotted; while the value of $n$, with its sign, is the tangent of the angle which the line makes with axis along which the values of $x-b$ or $b-x$ were plotted.

In case greater precision is required than can be obtained by the use of ordinary logarithmic paper, the logarithms of the values of $y-a$ and $x-b$ or $b-x$ may oe plotted to any desired scale on rectangular co-ördinates and the values of $a$ and $b$ adjusted until a straight line is obtained. In this case the value of $c$, with its sign, is the number whose logarithm is the magnitude of the ordinate where the straight line crosses the axis along which the logarithms of $y-a$ were plotted, while the value of $n$ is the slope of the line with respect to the axis of the logarithm of $x-b$ as was the case with the logarithmic paper.

Logarithmic Charts for Hydraulic Formulas. The following is from 
a eontribution by L. G. Hall to Engineering and Contracting, July 14, 1915 .

Williams' formula, which reduces to $Q=1.29 D^{2.668} H^{0.535}$, is plotted in Fig. 19. In this case $Q$ is the discharge in second-feet, $D$ is the diameter of the pipe in feet, $H$ is the loss of head in $1,000 \mathrm{ft}$. of pipe, expressed in feet.

Compute and plot.curves for the desired size of pipe. These curves are all parallel straight lines. Two points only need be computed, when the line ean be drawn. After the first curve has been drawn, the others can be drawn from it with a parallel ruler, one point on each, only, being located.

In locating these points, it is well to choose points on the line, $H=1$. This does away with the handling of one fractional exponent in each equation, as any power of one, is one,

Handling the fractional exponents ean be simply done by the use of logarithms. For instance, to find the value of $2^{2.668}$, look up the log. of 2 , which is .30103. Multiply this by the exponent, and the product is .803148. Look up the number which corresponds to this logarithm, and you have 6.355 , the required result. The multiplication may be done on the slide rule accurately enough for practical purposes.

When the 12 -in. and 24 -in. curves have been located, the 6 -in. and 48-in. curves may be immediately located without solving the equation. This is by virtue of the fact that the distanees of these eurves apart form another logarithmic scale similar to the horizontal and vertical scales. For instance, the distance from the 12-in. curve to the 24-in. curve is equal to the distances between the 24-in. and 48-in curves, or between the 12 -in. and the 6 -in. curves, measured along any ordinate. And when the 5-in. curve has been computed, the distances between the 5-in., 10-in., 20-in. and 40-in. curves will be the same as that from the 12-in. to the 24-in. curves. From this, after the 5, 7, 8, 12 and 24in. enrves are drawn, the entire series can be laid out graphically, without further solutions of the equation.

The velocity curves are next drawn. These, too, are parallel straight lines, and ean be drawn in the same manner as the first set from the $V \pi D$

formula, $Q=\frac{\pi}{4}$, using the diameter curves, and the discharge or4

dinates as reference lines. Points on the lines for constant velocity can be locater by taking the velocity and assuming a eonstant value for $D$, then solving for $Q$.

When completed, the chart presents four series of ordinates When values are given for any two of the ordinates, the point thus located immediately shows the values of the other two. When logarithmic paper is at hand, the entire chart ean be drawn in about an hour's time.

Logarithmic Diagram for Determining Cutting Time. This is taken from an article by I. Schelbeck, in Machinery, February, 1915.

The shop manager frequently finds it necessary to obtain a quick and more or less exact determination of the cutting time for a given piece of work that is to be handled on the lathe, milling machine, drill press, etr. For a piece of work handled on the lathe, the expression for the entting time is: 
COMPUTATION, ARITHMETICAL AND GEOMETRICAL 369

$$
Q=1.29 D^{2.660} \mathcal{H}^{0.535}
$$

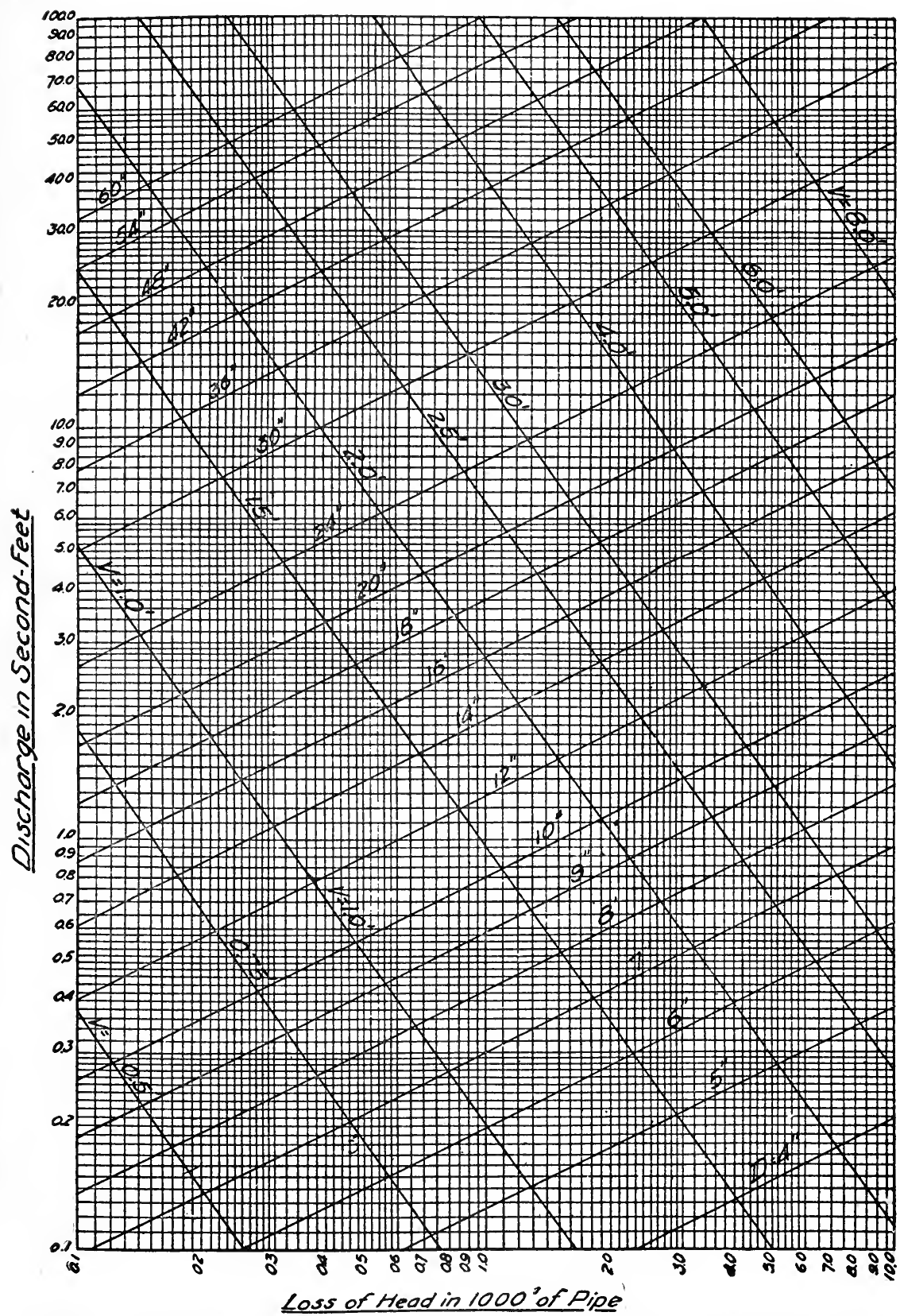

Fig. 19-Chart of Hydraulic Properties of Wooden Stave Pipe of 4 to 60 Ins. in Diameter 


$$
\text { Cutting time }=\frac{\pi D L}{S F}
$$

where $D=$ diameter of work in inches;

$L=$ length of work in feet;

$S=$ cutting speed in feet per minute;

$F=$ feed in inches per revolution.

The diameter of the work and the length to be machined will be specified on the drawing, and the choice of a suitable speed and feed will be governed by the size of the work, the material, the type of tool used, etc. In addition to finding the cutting time, it will be required to know the number of revolutions per minute at which the work can be rotated while machining. When the diameter and the cutting speed are known, the number of revolutions per minute is:

$$
\text { Revolutions per minute }=\frac{S}{\pi D}
$$

where $S$ and $D$ are the same as before.

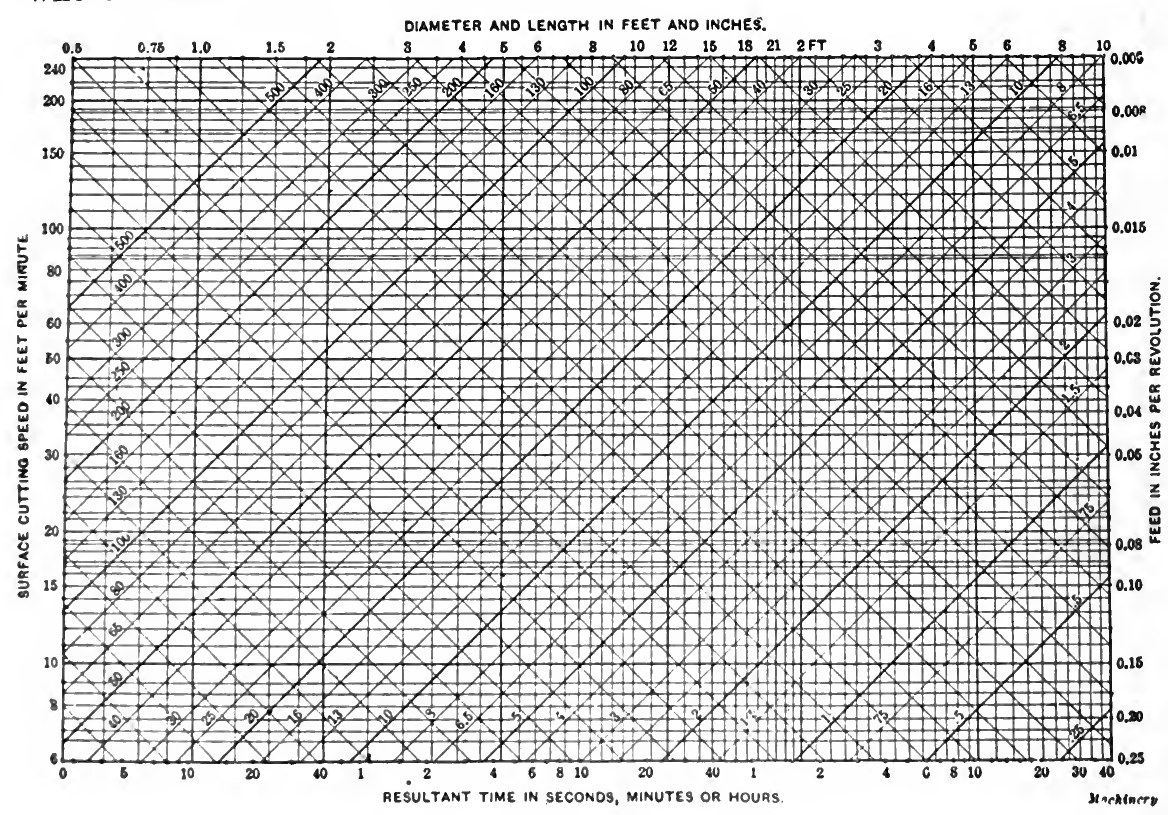

Fig. 20-Rapid Determination of Cutting Time

Fig. 20 gives a complete solution of both problems, and its use is as follows:

Consider a piece of work 3 inches in diameter by $8 \mathrm{ft}$. in length, which is to be machined with a tool that will permit a cutting speed of 50 feet per minute to be employed. Operating at this speed, the lathe on which the work is to be done is capable of taking the cut at a 
feed of 0.040 in. per revolution. With this data at hand and making use of Formula (1), the cutting time is found to be:

$$
\text { Cutting time }=\frac{\pi \times 3 \times 8}{50 \times 0.040}=37.7 \text { minutes. }
$$

Similarly we find from Formula (2) that the number of revolutions per minute at which the work will run is :

$$
\text { Revolutions per minute }=\frac{50 \times 12}{\pi \times 3}=64 \text {. }
$$

With the preceding results as a guide we will now proceed to solve the same problems by means of the chart. Entering the diagram from the left-hand side along the horizontal line through a cutting speed of $50 \mathrm{ft}$. per min. and following it to the vertical line coming down from the top of the chart through a diameter of 3 ins., a diagonal line showing the number of revolutions per minute to be 65 is located. Then by entering the diagram along the horizontal line from the right-hand side marking a feed of 0.040 in. per revolution, and following it to the vertical line coming down from the top of the diagram indicating a length of $8 \mathrm{ft}$., a second diagonal line is located. At the point where the two diagonal lines intersect, the vertical line is followed to the base of the diagram and shows the resultant cutting time to be a little less than 40 mins. The same system may also be used in determining the cutting time for milling and drilling machine operations.

Isometric Plotting. From an article by G. H. Marx, published in American Machinist, November 12, 1908, the following is quoted:

Rectangular co-ördinate paper, whether laid off on either a plain or a logarithmic scale, can present directly a picture concerning the relations of two variables only. In mathematical terms such a chart expresses the relation between two terms one of which is a "function" of the other; that is, the values of the first term vary according as different values are assigned to the second term (or vice versa).

But very frequently our problem involves three terms and it is quite as desirable to represent their relations graphically. To give a single concrete illustration: We may wish to show the gallons of water discharged per minute by a centrifugal pump, throughout a range of varying peripheral speeds, and against a series of varying heads. If such a chart were constructed it would show at a glance the proper combination of speed and head for maximum performance, and much else besides into which it is needless to enter here.

In all such cases we have three variable factors to deal with which we may term $x, y$ and $z$; to represent them graphically requires the use of three corresponding axes which we may term $X, Y$ and $Z$. To represent a set of three such axes on a plane surface we may avail ourselves of the well known system of isometric projection, which is a form of perspective drawing in which there is no foreshortening but each dimension of length, breadth or height is given its actual full value. The picture thus presented is much the same as if an object were set 
in the eorner of a room and the eye of the observer was at an equal distance from the floor and each wall. The lines of intersection of the two walls, and of each wall and the floor would form the three axes. The angles between the pairs of axes become 120 degrees in this ease. If, now, we wish to show the point whose three eo-ördinates are $x, y$ and $z$, we may measure off from the corner the distanee $y$ along the $Y$ axis; from the point thus located measure the distance $x$ in a direction parallel to the $X$ axis, thus locating a point $x, y$; at $x, y$ erect a perpendicular whose length is equal to $z$, and the extremity of the perpendicular will be the point whose position expresses the given relation

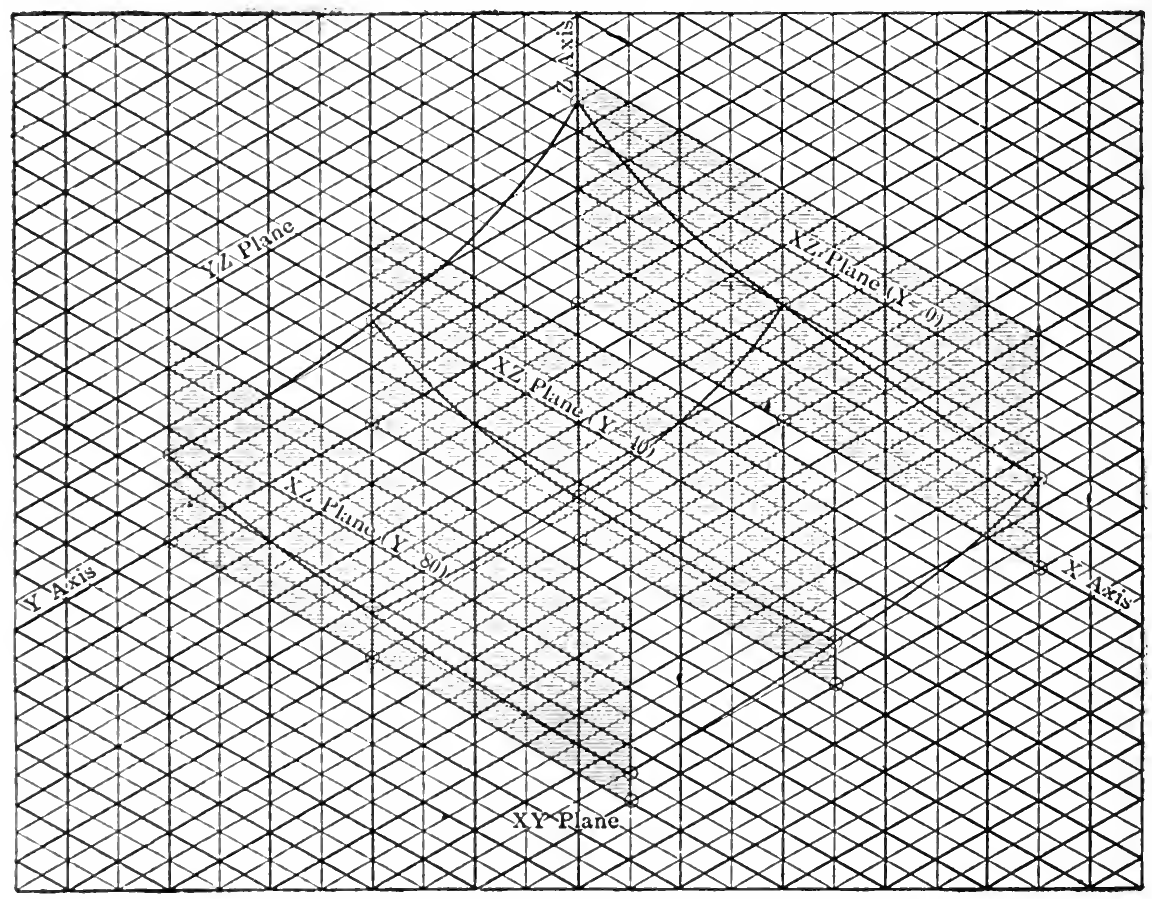

Fig. 21-A Typical Example of Isometric Plotting

of $x, y$ and $z$. It is seen that if we take a series of different $x y$ 's and at each erect the corresponding $z$, the upper extremities of the $z$ 's will locate a surface whose form and position will give a very elear pieture of the relationships of the three variables.

The typical use of isometrie paper is seen in Fig. 21, where points are located and curves drawn which show the surface determined by the following set of (co-ïrdinates:

$$
\begin{array}{cl}
y=0 & \multicolumn{1}{c}{y=40} \\
x=0, z=35 & x=0, z=17.5 \\
x=40, z=20 & x=40, z=7 \\
x=90, z=15 & x=90, z=7
\end{array}
$$




$$
\begin{gathered}
y=80 \\
x=0, z=15 \\
x=40, z=7 \\
x=90, z=5
\end{gathered}
$$

It is to be borne in mind that $x, y$ and $z$ are used here in their algebraic sense, and may represent actual factors of velocity, resistance, pressure, temperature, horsepower, efficiency, or any other terms whose relations it is desired to express graphically and which are interrelated in groups of three.

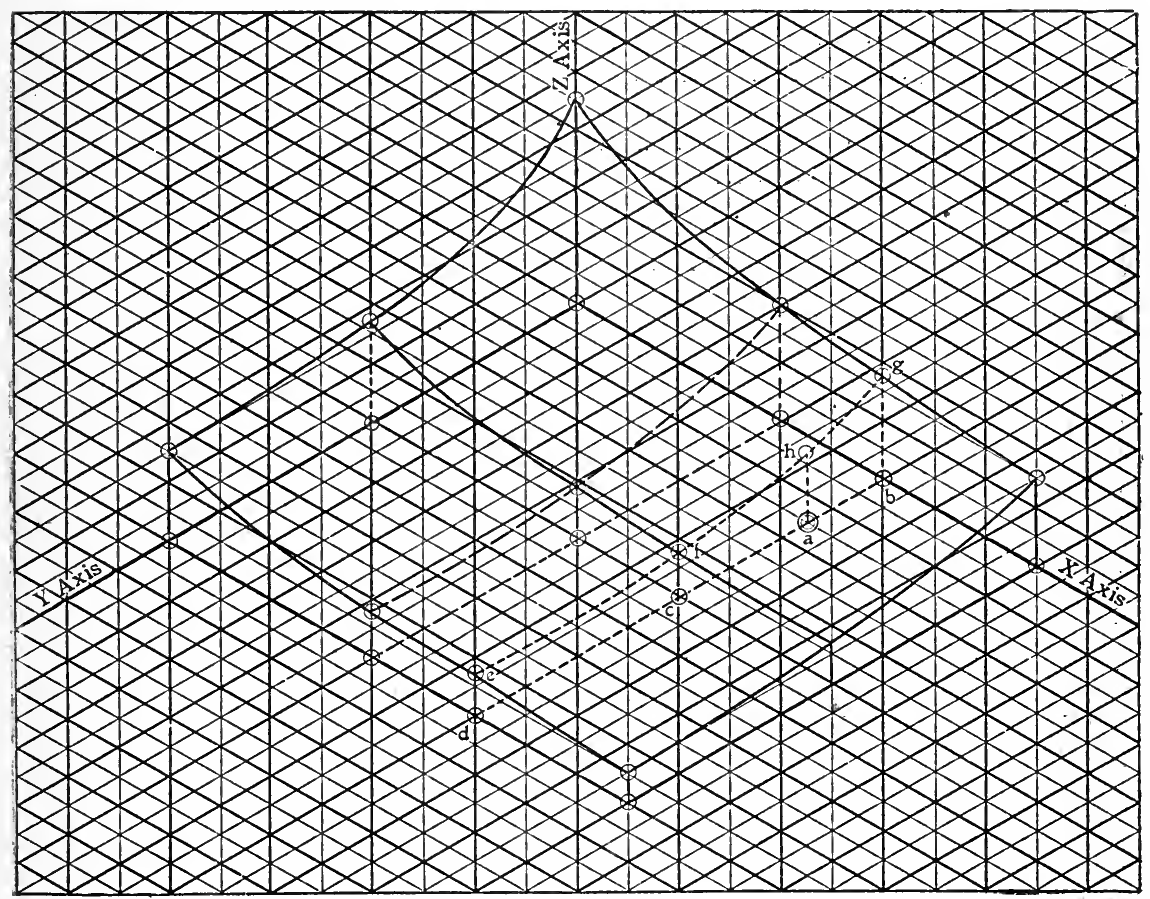

Fig. 22-An Illustration of Interpolation in Isometric Plotting

The use of such isometric co-ördinate paper by no means ends when one has plotted isolated observed or computed relations. Fig. 22 shows one means for ready interpolation. The surface is similar to that shown in Fig. 21, and it is desired to find the value of $z$ corresponding to $x=$ $60, y=15$.

The point $a$ is located for these values of $x$ and $y$, and through $a$ the line $b \quad c d$ is drawn parallel to the $Y$-axis. Where this line cuts the base lines at $b, c$ and $d$, respectively, erect perpendiculars $b g, c f$ and $d e$. Draw proper curve through $g, f$ and $e$. At $a$ erect a perpendicular until it cuts this curve at $h$. The ordinate $a h$ is the required value of $z$; in this case $z=17$. The foregoing example illustrates also the method 
of use of the paper for charts for the graphical solution of problems involving three quantities.

Another valuable characteristic of the paper is the ease with which "contour" lines may be shown, by passing planes parallel to any one of the three co-ordinate planes. Thus, in Fig. 23, if the plane $a b c d$ is passed parallel to the original $X Y$-plane, at a height of 15 divisions above it, the curve $a$ e $f c$ will be established ( $p a, g e, h f$ and $q c$, each being equal to 15), which will give all the possible relations of $x$ and $y$ corresponding to a value of 15 for $z$. Similarly, the curve $j f k$ gives all the possible relations of $y$ and $z$ for $x=40$; and the curve $l e m$ gives all relations of $x$ and $z$ for $y=40$. This, to give but a single illustration, is of extreme value in showing at a glance all of the possible combinations of two variables for a given value of the third factor; as, for instance, the series of peripheral speeds and corresponding discharge heads coresponding to a given efficiency of a centrifugal pump.

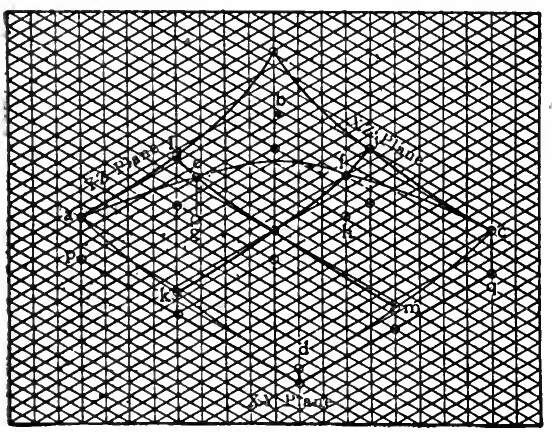

Fig. 23-Isometric Plotting of Contour Lines

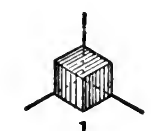

1
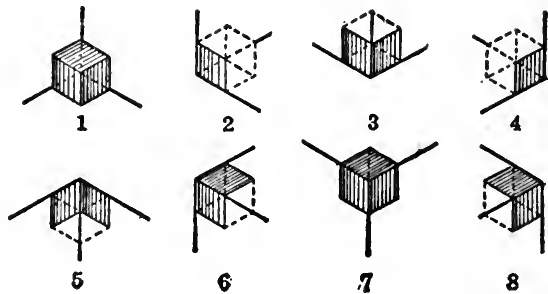

Fig. 24-The Eight Sets of Isometric Axes

The values of the variables corresponding to maximum or minimum conditions are equally evident from a glance at an isometric diagram.

It may be borne in mind that at the intersection of the three co-ordinate planes eight corners or angles are formed and that in constructing a diagram one is not limited to the ordinary set of isometric axes (as used in Figs. 21-23), but has a choice of eight sets, as shown in Fig. 24. Here, to make matters clear, a cube has been placed in the "corner" in each case, with those faces shaded which lie in or in front of co-ordinate planes. While the set of axis No. 1 will be found to answer ordinarily, one of the others occasionally may be found to suit some particular data with less confusion of lines.

Just as, with ordinary rectangular co-ordinate paper, familiarity has led to an increasingly wide field of usefulness, so it will be found with this. Where three interdependent quantities are to be dealt with the isometric paper is quite as valuable as is the rectangular paper for expressing the relations between two variable terms.

Nomographic, or Alignment Chart, Computations. Mr. R. C. Strachan presented a paper before the A. S. C. E. on Feb. 3, 1915, entitled "Nomographic Solutions of Formulas of Various Types," which was published in Transactions A. S. C. E. Vol. IXXVII (1915), Page 
1359, and which gives the following analysis of charts constructed in accordance with his method described in Chapter VIII.

Chart for Pin Moments. Tables for bending moments on pins are found in all engineers' pocket books, but interpolations are frequently necessary, particularly in reviewing a completed design. For this purpose a nomograph is especially useful, and a very neat one may be constructed with scales of natural numbers for moments and stresses. (Fig. 25.)

Let $\mathrm{M}=$ moment, in inch-pounds;

$\mathrm{D}=$ diameter of the pin, in inches;

$A=$ area of the pin, in square inches;

$\mathrm{F}=$ extreme fiber stress, in pounds per square inch.

$$
\text { Then } M=f \frac{D A}{8} \text {. }
$$
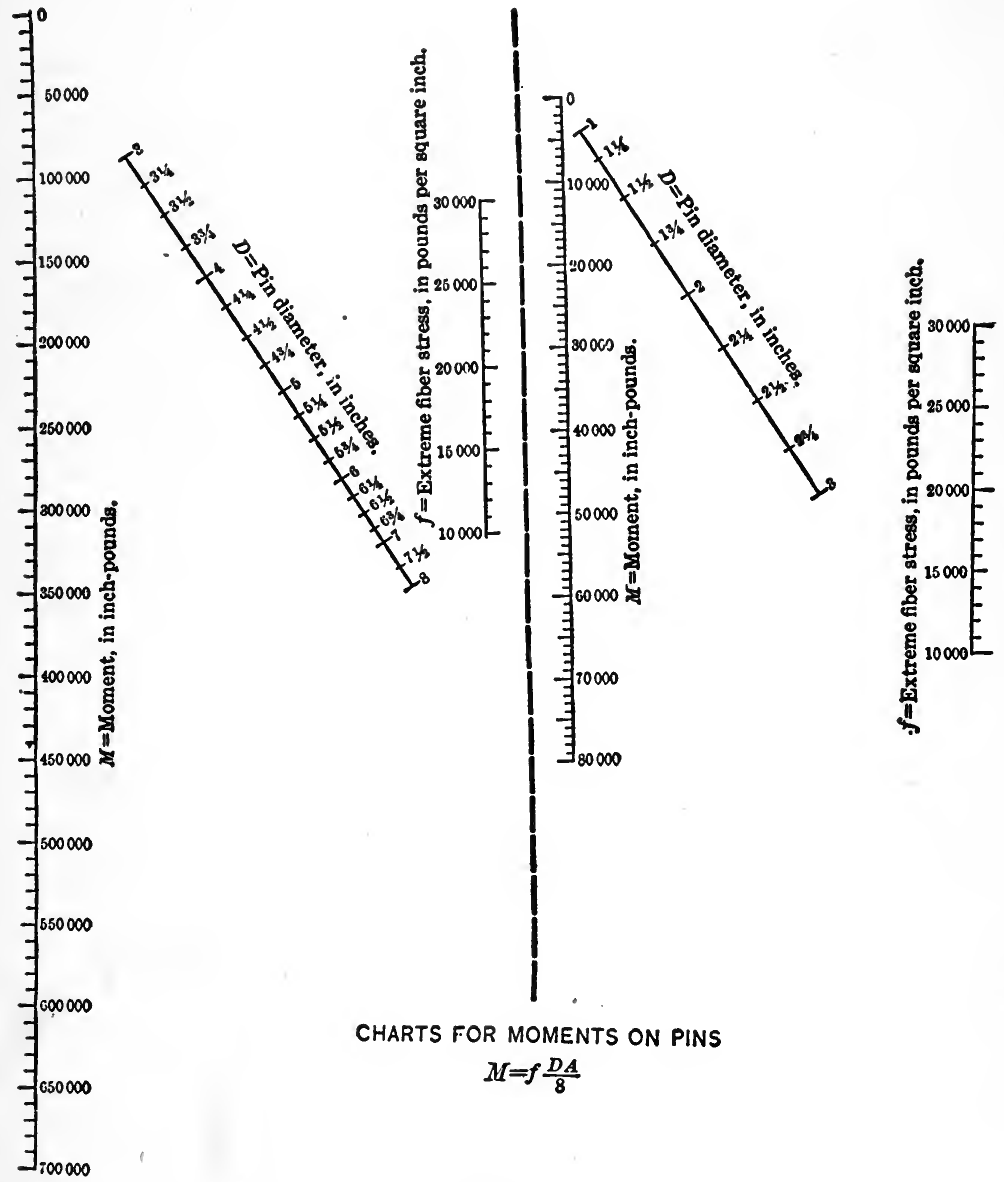

CHARTS FOR MOMENTS ON PINS

$M=f \frac{D A}{8}$ 
Or, by substituting $0.7854 D^{2}$ for $A$,

$$
M=0.098 \mathrm{f} D^{3} \text {. }
$$

In Fig. 26, let the scales of equal parts for $f$ and $M$ be parallel, with the zeros at $O$ and $O_{1}$, as shown, and plotted at a convenient distance apart. Connect $O_{1}$ and $O$, and let any line, $a b$, intersect the three lines.

Then

therefore,

$$
\frac{O l}{O_{1} k}=\frac{c}{e}
$$

$$
\frac{o l}{O l+O_{1} k}=\frac{c}{L} \text {. }
$$

Also,

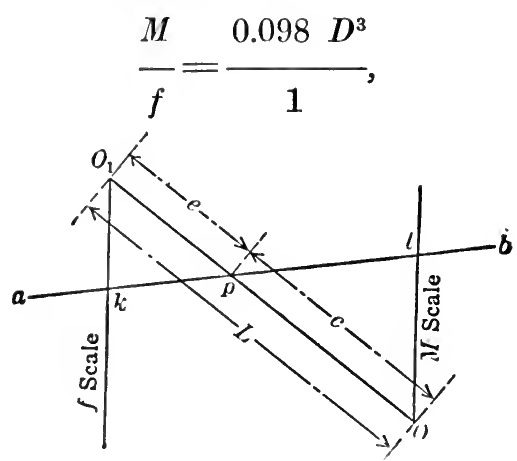

Fig. 26

and, if the seales for $M$ and $f$ were plotted on $O l$ and $O_{1} k$ with units of the same magnitude, we should have, for any position of $a b$,

$$
\frac{M}{M+f}=\frac{0.098 D^{3}}{0.098 D^{3}+1}=\frac{c}{L} .
$$

It will be found more satisfactory if the $M$ units are one-tenth as great as the $f$ units, for diameters from 3 to 8 in., the limits of one chart (that on the left), and one-half as great as the $f$ units for diameters from 1 to 3 in., as in the ehart on the right of Fig. 25 .

Then for the left-hand chart,

$$
\begin{aligned}
& \frac{M}{M+10 f}=\frac{0.098 D^{3}}{0.098 I^{3}+10}=\frac{c}{L} . \\
& \text { And } c=L \frac{0.098 I^{3}}{0.098 I^{3}+10} . \\
& 2.65
\end{aligned}
$$

When $D=3, c=L \frac{2.65}{1205}=0.209 L$; 


$$
\text { when } D=4, c=L \frac{6.27}{16.27}=0.385 L \text {; }
$$

and similarly for any other diameter.

In the right-hand chart, for small pins, 2 will be used in the expression for $c$ in place of 10 . If we then note the diameters at the points $p$, thus found, the line, $a b$, is an isopleth giving scale readings for $f, D$, and $M$ which satisfy the equation, $M=0.098 f D^{3}$.

The length and inclination of the line, $O_{1} O$, should be chosen so as to give good intersections within the limits of the chart. A unit of 1 in. to 5,000 lb. for the $f$ scale will be suitable for practical purposes.

Chart for Column Formula. The Gordon column formula, when suitably transformed, may also be adapted to nomographic representation obtained by the relation of similar triangles.

The constants used in the chart, Fig. 27 , are 20,000 and 8,000, or,

$$
P=\frac{20000}{1+\frac{l^{2}}{8000 r^{2}}}
$$

in which $\quad l=$ length of columns, in inches; and $r=$ radius of gyration, in inches.

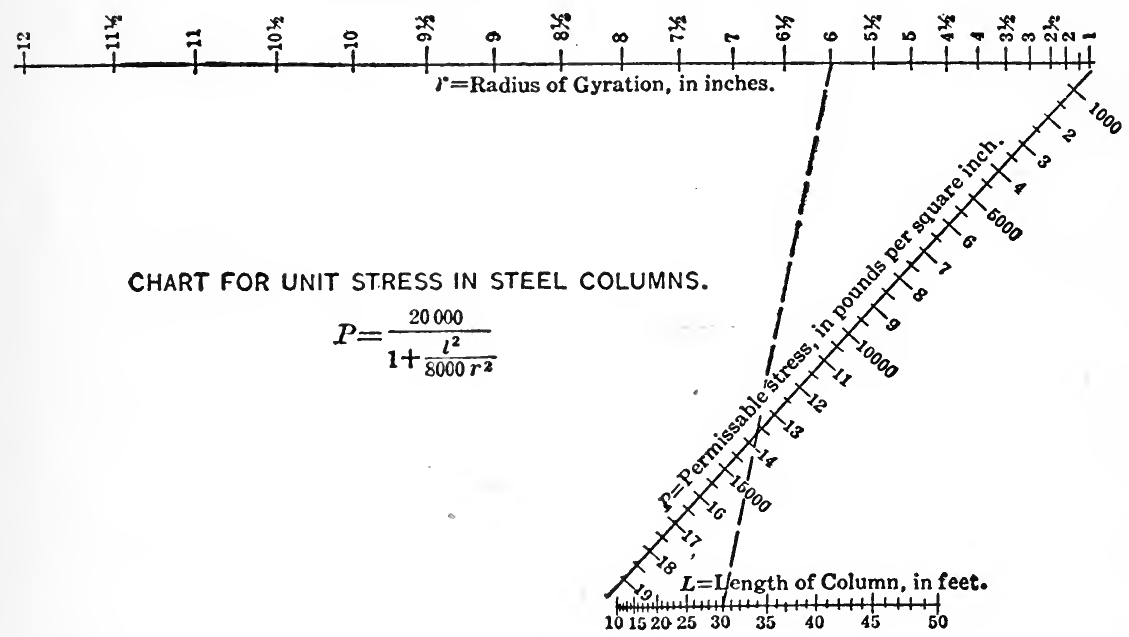

Fig. 27

The length is usually given in feet, and therefore, inserting $12 L$ for $l$, we have

$$
P=\frac{20000}{1+\frac{144 L^{2}}{8000 r^{2}}}
$$


This may be written

$$
\frac{P}{20000}=\frac{8000 r^{2}}{8000 r^{2}+144 L^{2}}=\frac{r^{2}}{r^{2}+0.018 L^{2}}
$$

Referring to Fig. 28,

$$
\frac{O_{1} k}{O l}=\frac{c}{d}
$$

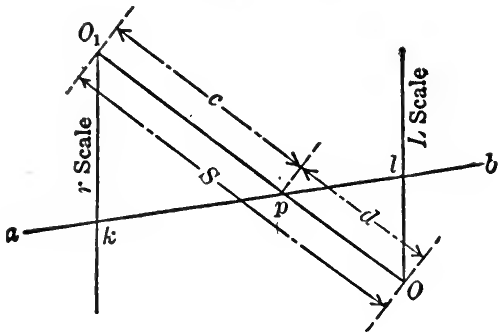

Fig. 28

Therefore,

$$
\frac{O_{1} k}{O_{1} k+O l}=\frac{c}{s} \text {. }
$$

If now we lay off from $O_{1} 1,4,9,16$, ete., units, placing the numbers $1,2.3,4$, at these points, and similarly from $O 0.018 \times 1,0.018 \times 4$, $0.018 \times 9,0.018 \times 16$ units, in other words, making the unit for the $L$ scale 0.018 times the unit for the $r$ seale, we shall have

therefore,

$$
\frac{r^{2}}{0.018 L^{2}}=\frac{c}{d}
$$

and as

$$
\frac{r^{2}}{r^{2}+0.018 L^{2}}=\frac{c}{S}
$$

$$
\frac{P}{20000}=\frac{r^{2}}{r^{2}+0.018 L^{2}},
$$

if $O_{1} O$ were graduated decimally from $O_{1}$ as the zero point, the reading at $p$ would be the value of $c$ as a fraetion of $S$, or $P$ as a fraction of 20,000 .

If we place 1,000 at the distance $0.05,2,000$ at the distance 0.1 , and so on, we may read values of $P$ directly.

The line, $a b$, is then an isopleth giving scale readings for $r, L$, and $P$ which satisfy the equation,

$$
P=\frac{20000}{1+\frac{144 L^{2}}{8000 r^{2}}} .
$$


The unit used for the chart, of which Fig. 27 is a reproduction, is $1 / 10$ 'in. for the $r$ scale, and the distance, $O_{1} O$, is $10 \mathrm{in}$. These dimensions, of course, must be chosen with regard to the degree of precision desired in the scale readings. When constructed as stated, the value of $P$ may be read to $100 \mathrm{lb}$.

Good intersections may always be obtained, since $P$ is the same for $L \quad n L$

$\frac{-}{r}$ and $\frac{}{n r}$.

The broken line on the chart shows the solution for the case $L=30 \mathrm{ft}$. and $r=6$ in.

Chart for Impact Formula. Fig. 29.--The percentage of live-load stress to be added for the effect of impact in designing bridge trusses is specified by the Public Service Commission of the First District, New York, as

$$
S=125-\frac{1}{8} \sqrt{2000 L-L^{2}}
$$

in which $S=$ percentage, and $L=$ length of load producing the stress to which the percentage, $S$, is to be added.

If we represent $2000 L-L^{2}$ by $q$, this may be written

$$
125-S=\frac{1}{8} \sqrt{q}
$$

Also,

$\log .(125-S)=\frac{1}{2} \log . q-\log .8$.
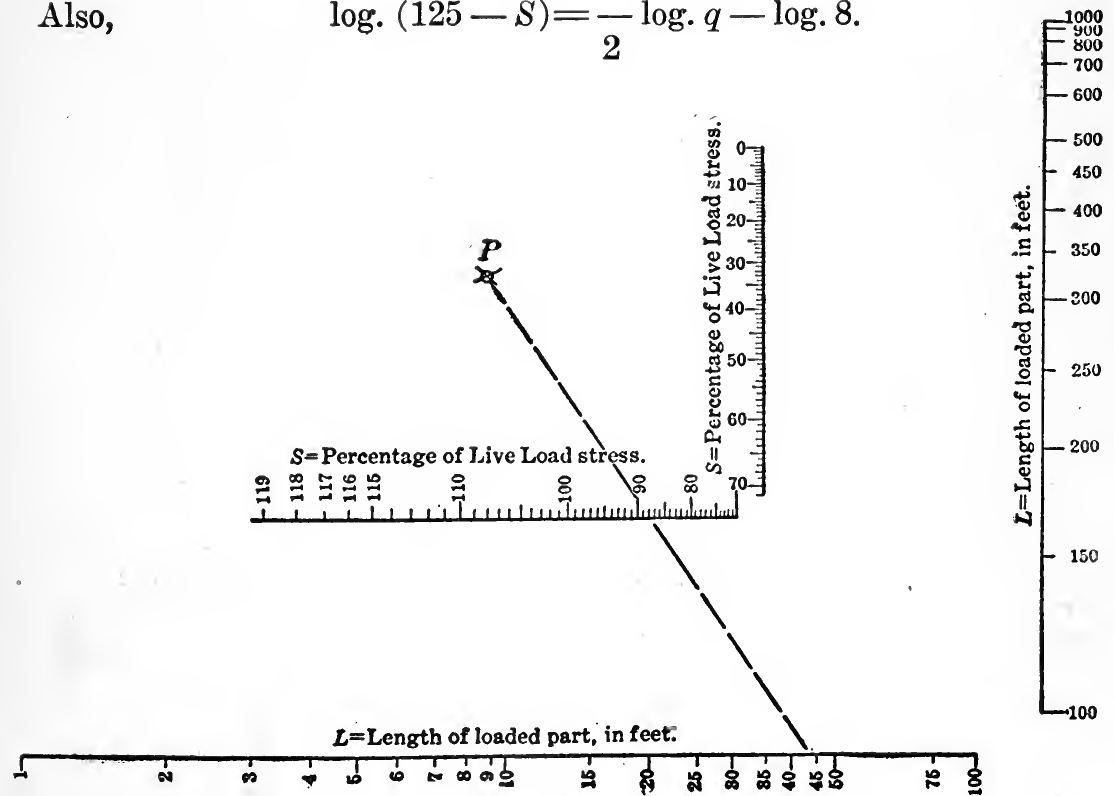

Fig. 29 
The series of values of $q$, taken with suitable intervals in the value of $L$, may be easily dedueed, beeause, for consecutive values of $L$ differing by unity, the consecutive values of $q$ differ by $(2,000-3),(2,000-$ $5),(2,000-7)$, ete.

$$
\begin{aligned}
& \text { When } L=1, q=1999 \text { log. } q=3.301 \\
& 2 \quad 3996 \quad 3.601 \\
& \begin{array}{lll}
3 & 5991 & 3.777
\end{array} \\
& \begin{array}{lll}
4 & 7.984 & 3.902
\end{array} \\
& 5 \quad 9975 \quad 3.999
\end{aligned}
$$

Referring to Fig. 30, lay off the logarithms of $q$ from the point, $f$, marking 1 opposite the log. 1999,2 opposite log. 3996 , ete.

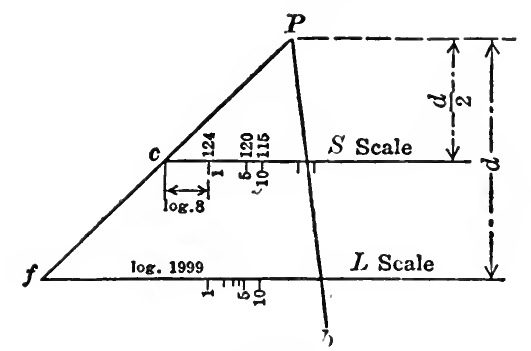

Fig. 30

From the extremities of this scale, when completed, draw lines to a point, $P$, and place the line, $S$, parallel to the line, $L$, at a distance of 1

$-d$ from it. If a logarithmie scale of the same unit magnitude as 2

the scale, $L$, were placed on the line, $S$, beginning at $c$, any straight line from $P$ through the $L$ seale would give the number corresponding to 1

- log. $q$ at the point of crossing the line, $S$; but, by beginning at a 2

distance of $0.908=\log .8$ from $c$, the point of erossing oceurs at $\frac{1}{2}$ log. $q-\log .8$.

\section{1}

The reading on this seale would then be $-\sqrt{q}$, or $125-S$.

8

If we now number the $S$ scale by putting 124 for 1,123 for 2 , etc., any line, $I^{\prime} b$, is an isopleth giving scale readings for $S$ and $L$ which satisfy the equation.

$$
S=125-\frac{1}{8} \sqrt{2,000 L-L^{2}}
$$

In the chart, Fig. 29, the seales, for the sake of compactness, are turned through $90^{\circ}$ at the point, $L=100$. The logarithmic unit also 
changes, so as to keep the graduations of the $S$ scale large enough to be easily read.

The broken line in Fig. 29 shows that when $L=44$ ft., $S=88 \%$.

Chart for Reinforced Concrete Beam Formulas. Fig. 31.-On the theory commonly applied in the design of concrete beams of rectangular cross-section reinforced on the tension side, the tensile strength of the concrete is neglected and a straight-line variation in compressive stress is assumed.

Following the usual notation,

$b=$ breadth ;

$d=$ depth, measured to center of reinforcement;

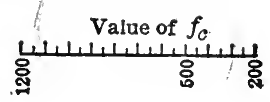

DIAGRAM $\mathrm{B}_{2}$
$n=12$
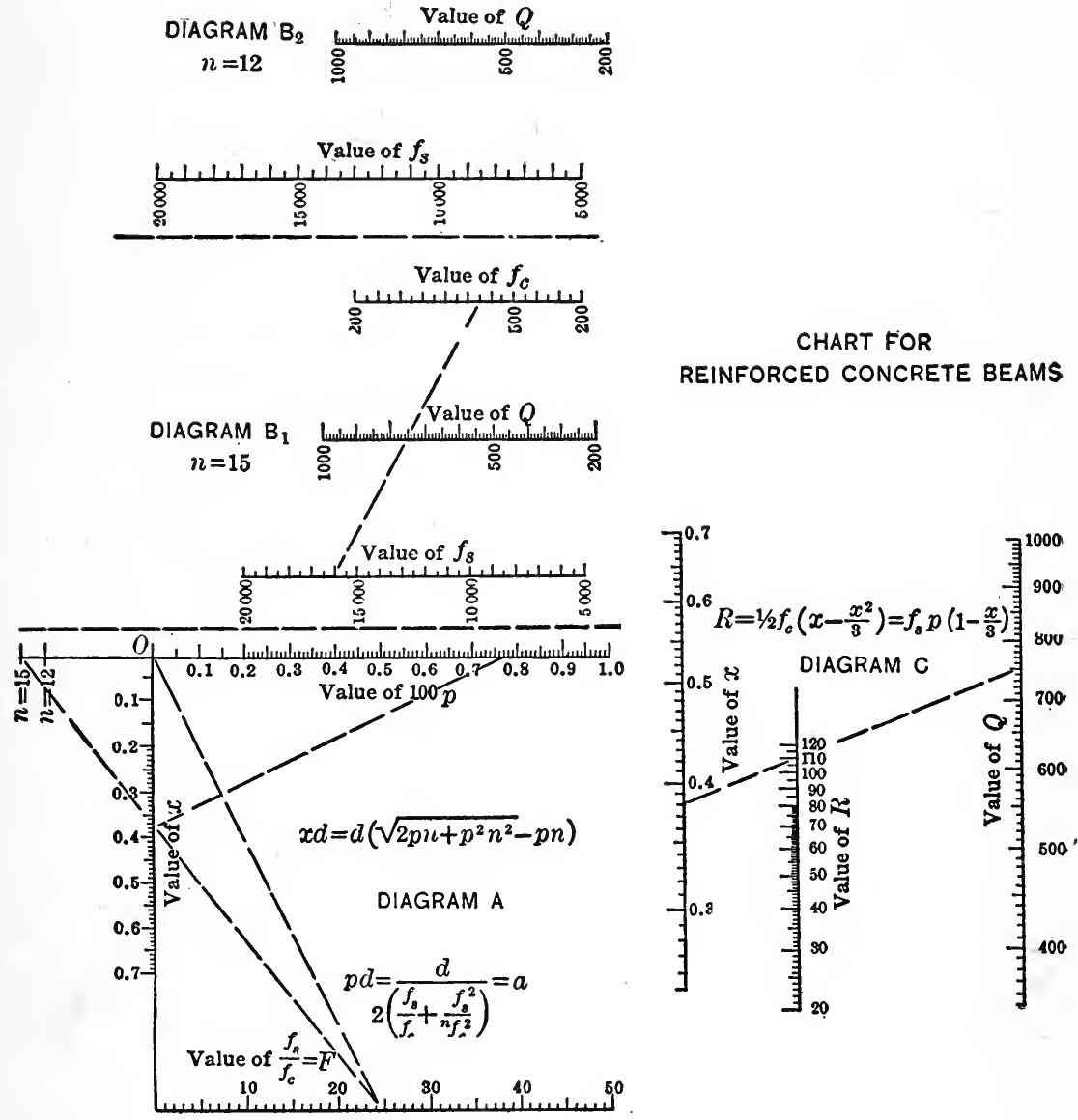

Fig. 31 
$X=x d=$ distance from compression side to neutral axis;

$f_{\mathrm{s}}=$ unit stress in steel;

$f_{\mathrm{c}}=$ unit stress in concrete;

$n=E_{\mathrm{s}} \div E_{\mathrm{c}}$

$M=$ moment of resistance of beam;

$p=$ pereentage of reinforcement;

$a=$ area of reinforcement per unit width $=p d$.

Then

$$
\begin{array}{r}
X=x d=d\left(\sqrt{2 p n+p^{2} n^{2}}-p n\right), \\
a=p d=\frac{d}{2\left(\frac{f_{\mathrm{s}}}{f_{\mathrm{c}}}+\frac{f_{\mathrm{s}}{ }^{2}}{n f_{\mathrm{c}}{ }^{2}}\right)}, \\
M_{\mathrm{s}}=f_{\mathrm{s}} p d^{2}\left(\begin{array}{c}
x \\
1-\frac{3}{3}
\end{array}\right), \\
M_{\mathrm{c}}=\frac{1}{2} f_{\mathrm{c}} d^{2}\left(\begin{array}{r}
x^{2} \\
3
\end{array}\right) .
\end{array}
$$

And, for balanced reinforcement, $M_{\mathrm{s}}=M_{\mathrm{c}}$.

These expressions may be transformed so as to adapt them to nomographic representation.

If

$$
\frac{f_{\mathrm{s}}}{f_{\mathrm{c}}}=F,
$$

and the moment of resistance $R b d^{2}$, or $R d^{2}$ per unit width,

then

$$
\begin{aligned}
x & =\frac{n}{n+F} \ldots \ldots \ldots \ldots \ldots \ldots \ldots \ldots \ldots \ldots \ldots \ldots \ldots \ldots \ldots \ldots \ldots \ldots \ldots \\
p & =\frac{x}{2 F} \ldots \ldots \ldots \ldots \ldots \ldots \ldots \ldots
\end{aligned}
$$

Four diagrams will be required, one for $x$, one for $p$, one for the quantity, $\frac{f_{\mathrm{s}}}{2 n}+\frac{f_{\mathrm{c}}}{3}$, and one for $R$.

Referring to Fig. 32,

$$
\frac{O t}{u v}=\frac{O w}{w u}
$$




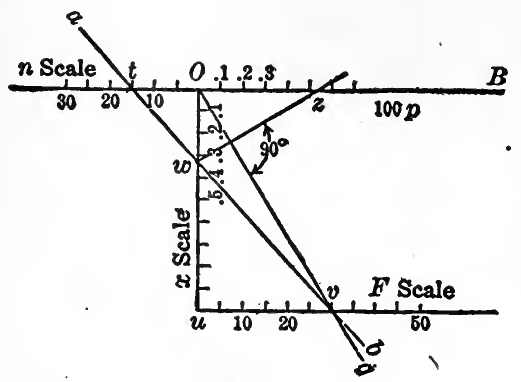

Fig. 32

therefore,

$$
\frac{O t}{O t+u v}=\frac{O w}{O u}
$$

If we lay off scales of equal parts on $O t$ and $u v$, the former for values of $n$ and the latter for values of $F$, using units of the same magnitude, then, for any position of $a b$,

$$
\frac{n}{n+F}=\frac{O w}{O u},
$$

and if $O u$ be graduated decimally, as on the chart, Fig. 31, $a b$ (of Fig. 32) is an isopleth giving values of $n, F$, and $x$ satisfying Equation 1.

Only those values of $n$ which are commonly used appear on the $n$ scale of the completed chart, Fig. 31.

Draw $O v$, and from $w$ draw $w z$ perpendicular to $O v$. Then

$$
\frac{u v}{O u}=\frac{O w}{O z} .
$$

If the unit for the $F$ scale is one-fiftieth of $O u$, the line from 0 to 50 is at an angle of $45^{\circ}$ to $O u$.

From Equation 2, when $F=50, x=100 p$, therefore, if lines be drawn from the $x$ scale sloping upward to the right at $45^{\circ}$, and if the intersections with $O B$ are marked with the same figures as the points on the $x$ scale from which they start, we shall have the scale of $100 p$ on $O B$.

A line, $O g$, through the $F$ seale and a line perpendicular to $O g$ through $w$ and $O B$ will then give values of $F, x$, and $p$, satisfying Equation 2. This completes Diagram A of the chart, Fig. 31.

To obtain $R$ it is necessary first to obtain $\frac{f_{\mathrm{s}}}{2 n}+\frac{f_{\mathrm{c}}}{3}$. Denoting this quantity by $Q$, when $n=15$,

$$
Q=\frac{f_{\mathrm{s}}}{30}+\frac{f_{\mathrm{c}}}{3} \text {. }
$$


In Fig. 33, if $k g=k h, k m=\frac{1}{2} g n+\frac{1}{2} h p$.

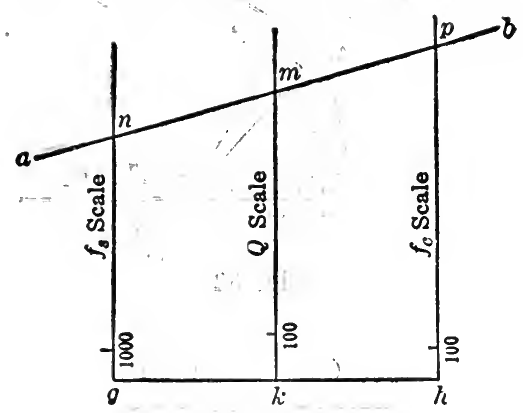

Fig. 33

Lay off scales of equal parts for $f_{\mathrm{s}}$ and $f_{\mathrm{c}}$, using natural numbers, and making the unit for $f_{\mathrm{c}}$ ten times that for $f_{\mathrm{s}}$.

If the $Q$ seale be laid off with unit one and one-half times the $f_{\mathrm{c}}$ unit. 30

or $\frac{-}{2}$ of the $f_{\mathrm{s}}$ unit, the reading at $\mathrm{m}$ will be

$$
\frac{2}{30} \times \frac{1}{2} f_{\mathrm{s}}+\frac{2}{3} \times \frac{1}{2} f_{\mathrm{c}}
$$

Diagram $B_{1}$ of the chart, Fig. 31, is thus constructed.

In Diagram $B_{2}$, of Fig. 31 , the unit for $f_{c}$ is elght times that for $f_{s}$, and the $Q$ unit is $\frac{3}{2}$ of the $f_{\mathrm{c}}$ unit or $\frac{24}{2}$ of the $f_{\mathrm{s}}$ unit. The reading at $m$ in this case, therefore, is

$$
\frac{2}{24} \times \frac{1}{2} f_{\mathrm{s}}+\frac{2}{3} \times \frac{1}{2} f_{\mathrm{c}}
$$

The line, $a b$, is an isopleth giving, in Diagram $\mathrm{B}_{1}$,

and, in Diagram $\mathrm{B}_{2}$,

$$
\mathrm{Q}=\frac{f_{\mathrm{s}}}{30}+\frac{f_{\mathrm{c}}}{3},
$$

$$
Q=\frac{f_{\mathrm{s}}}{24}+\frac{f_{\mathrm{c}}}{3} .
$$

Diagram C, of Fig. 31, is constructed to give the product $x^{2} Q=R$, as follows:

Place the $R$ line as shown in Fig. 34, making $k g=\frac{1}{3} g h$. Lay off 
the logarithms of $x$ and $Q$ on their respective lines, using the same unit. Then, for any position of $a b$,

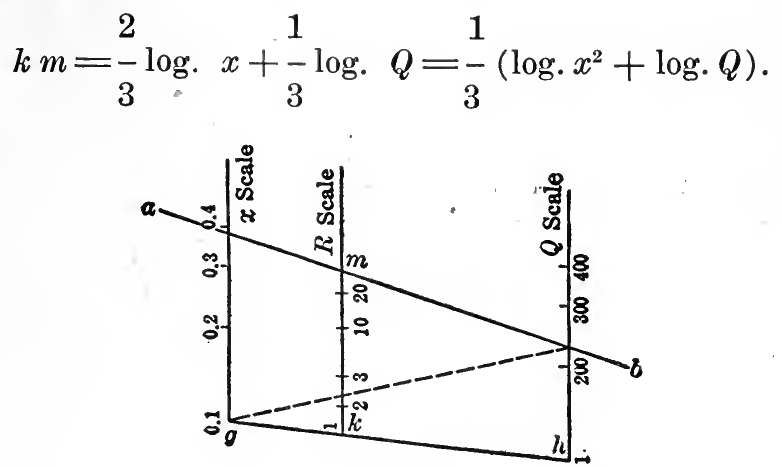

Fig. 34

The $R$ scale is laid off with unit one-third of that used for $x$ and $Q$, making the reading at $m$ the number corresponding to $\left(\log . x^{2}+\log . Q\right)$.

If the points, $2,3,4$, etc., on the $x$ scale are noted as $0.2,0.3,0.4$, and the points, 2, 3, 4 , etc., on the $Q$ scale are noted as 200,300 , etc., their product is

$$
\left(\begin{array}{c}
x \\
10
\end{array}\right)^{2} \times Q \text { hundreds }=x^{2} Q \text { units. }
$$

Diagram $C$ is then a nomograph for the expression, $R=x^{2} Q, Q$ being taken from Diagram $\mathrm{B}_{1}$ when $n=15$, and from Diagram $\mathrm{B}_{2}$ when $n=$ $12, f_{\mathrm{c}}$ and $f_{\mathrm{s}}$ being in pounds per square inch, $b$ and $d$ in inches, and $R b d^{2}$ in inch-pounds.

To illustrate the use of the chart, Fig. 31 , assume $f_{\mathrm{s}}=16,000, f_{\mathrm{c}}=$ $650, n=15$, and $b=12$ in.

Moment of external forces $=517000$ in-lb.

On Diagram A connect the point $n 15$ with $F 24.6$ and read $x=0.38$.

Connect the origin, $O$, with $F 24.6$, and, on a perpendicular to this line, drawn from 0.38 on the $x$ scale, read $100 p=0.77$, or $p=\frac{77}{100}$ of $1 \%$.

- On Diagram $\mathrm{B}_{1}$ connect $f_{\mathrm{s}} 16000$ with $f_{\mathrm{c}} 650$, and read $Q=750$.

On Diagram C connect $x 0.38$ with $Q 750$, and read $R=109$.

$$
\begin{aligned}
\text { Then } 517000 & =109 \times 12 d^{2}, \\
\text { therefore, } \mathrm{d} & =20 \mathrm{in}
\end{aligned}
$$

Allowing 2 in. of concrete below the center of reinforcement, the required beam would then be 12 by 22 in., and the steel area would be $0.77 \%$ of 240 sq. in. $=1.85$ sq. in.

Charts for Williams-Hazen Formula. Many hydraulic formulas contain fractional exponents, and are cumbersome on account of the time required for their solution. This difficulty is partly overcome by tabulating the results for a series of eases. Interpolations in such tables, how- 
ever, are not invariably simple, and resort is had to many expedients: to simplify the work. Special "hydraulic slide-rules" have been devised, which give, with sufficient precision, the results of the formula. for which they are designed, but naturally such special rules can be of no general utility.

The charts for the Williams-Hazen formula, Figs. 35 and 36, are. given as examples of the beauty of the nomograph in its application to. the solution of an exponential equation.

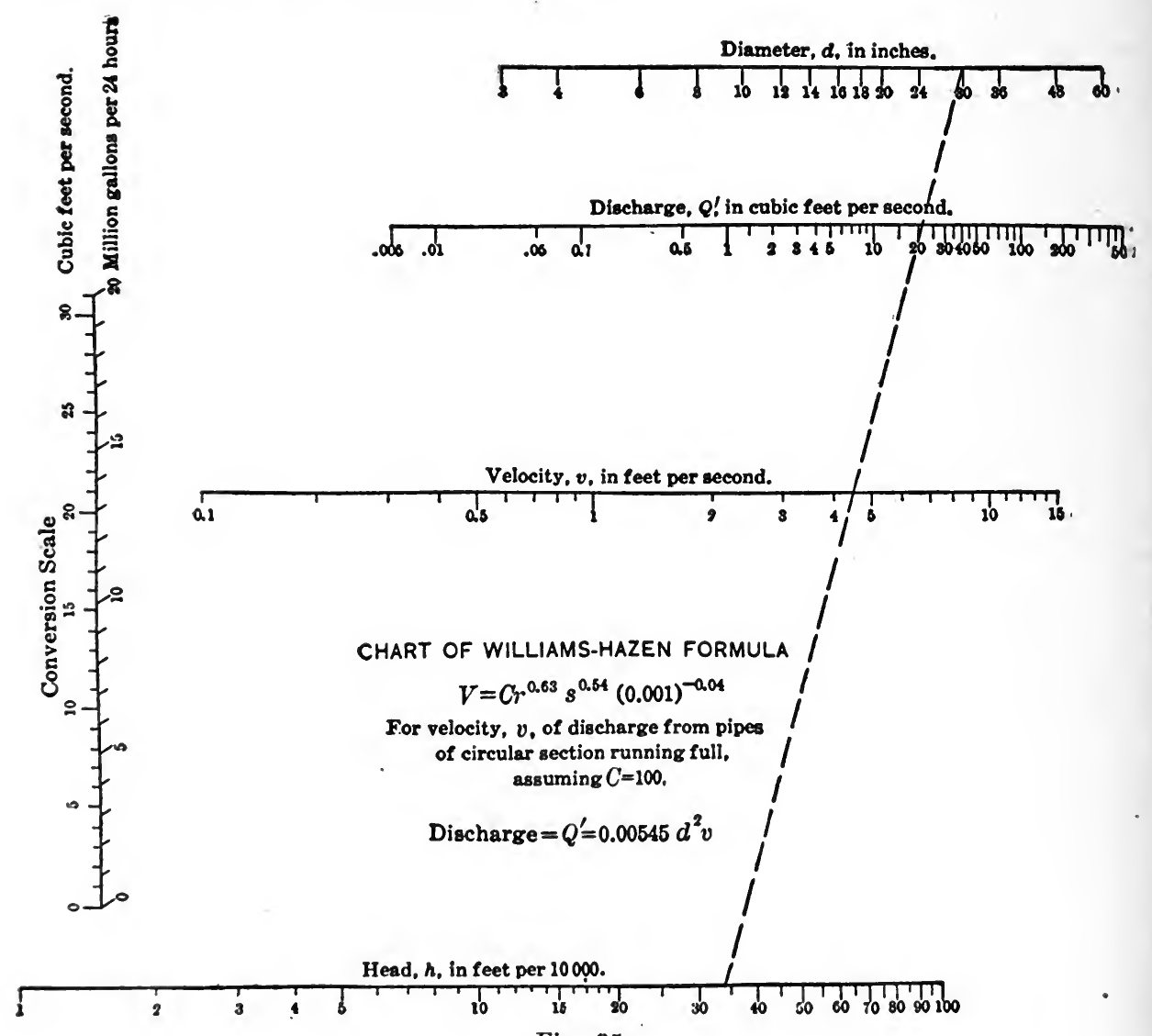

Fig. 35

As usually stated, this formula is

$$
V=C r^{0.83} S^{0.54} 0.001^{-0.04} \text {, }
$$

in which $V=$ velocity, in feet per second;

$r=$ mean hydraulic radius;

$S^{\prime}=$ slope or ratio of rise to length;

$C=$ a coefficient depending on the condition of the inner surface of the pipe. 
Diameter, $d$, in inches.

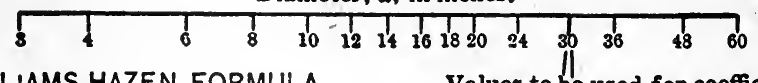

CHART OF WILLIAMS-HAZEN FORMULA

$$
V=C r^{0.63} s^{0.54}(0.001)^{-0.04}
$$

For velocity, $V$, of discharge from pipes of circular section running full.

$$
\text { Discharge }=Q=0.00545 d^{2} V^{V}
$$

Discharge, $Q$, in cubic feet per secón

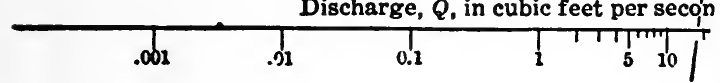
for pipes 3 "to 60 "diam.

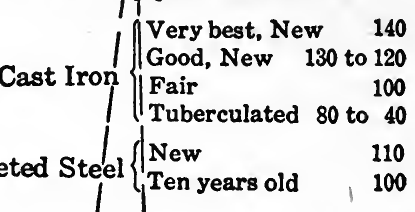

Velocity, $V$, in feet per second.

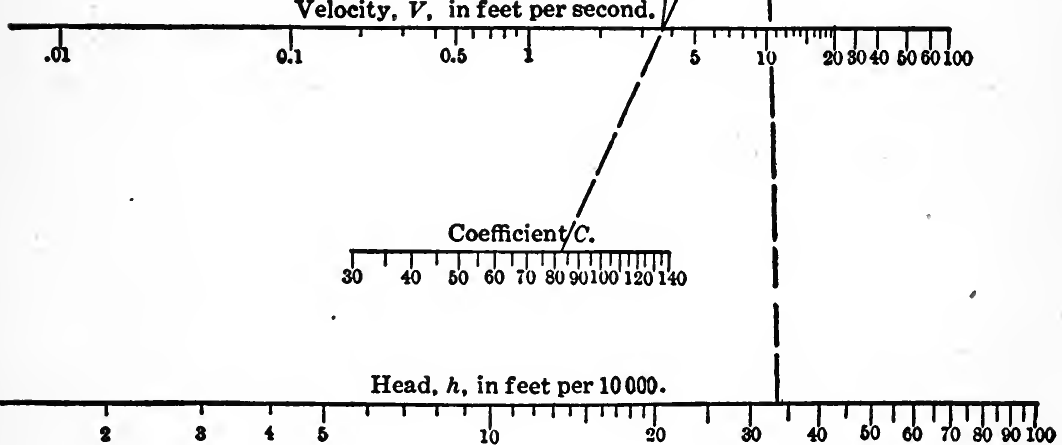

Fig. 36

In constructing a nomograph of this formula, applicable to pipes of circular section running full, it will be convenient first to transform it so as to express $r$ in terms of the diameter, and $s$ as feet of head in $10000 \mathrm{ft}$.

Letting $d=$ diameter of pipe, in inches;

and $h=$ feet of head in $10000 \mathrm{ft}$.,

then

$$
r=\frac{D \text { (feet) }}{4}=\frac{d \text { (inches) }}{48}
$$

and $S=\frac{h}{10000}$. 
Making these substitutions, we obtain

$$
V=0.000796 C d^{0.63} h^{0.54} \text {. }
$$

$C$ has values from 30 to 140 , and we will limit the charts, Figs. 35 and 36 , to values of $d$ from 3 to $60 \mathrm{in}$. and to values of $h$ from 1 to 100 .

$$
\text { If } \begin{aligned}
v & =\text { velocity when } C=100, \\
v & =0.0796 d^{0.63} h^{0.54},
\end{aligned}
$$

and $V$ will be obtained by multiplying $v$ by a factor, 0.3 to 1.4. The process of constructing the chart so as to give the product, $0.0796 d^{0.63}$ $h^{0.54}$, will be the first step.

Using the decimal seale, and taking $1 / 2$ in., or 1 on the 20 th scale, to represent 0.1 , lay off two parallel scales, one being the logarithms of all diameters from 1 to $60 \mathrm{in}$. and the other the logarithms of all the $h$ 's from 1 to 100 . Draw the $v$ line parallel to the $d$ and $h$ lines, dividing the space between in the ratio of 63 to 54, as shown in Fig. 37.

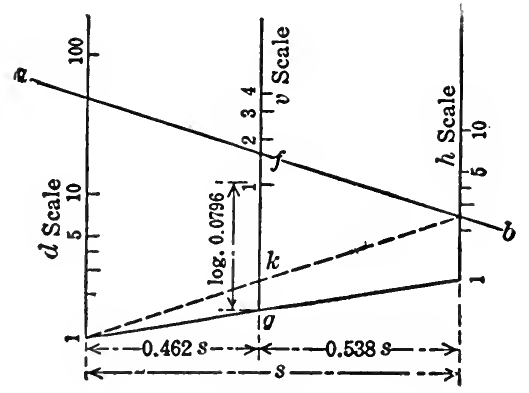

Fig. 37

If the scales are intersected by any straight line, $a b$, the distance, 6354

$f g=f k+k g=\frac{1}{117} \log . d+\frac{}{117} \log . h$. If the $v$ 's were laid off by the scale of 20ths beginning at the point, $g$, the reading at $f$ would give the product, $d^{\frac{53}{117}} h^{\frac{54}{117}}$

63

117 $=0.855 \times \frac{63}{100}$, hence if the logarithms of the $v$ 's are laid off with a unit of $(1 / 2$ in. $\times 0.855)$ to 0.1 , the reading at $f$ would give the product, $d^{0.63} h^{0.54}$. This may be accomplished by using for the $v^{\prime} s$ the seale of 20 ths after multiplying the logarithms by 0.855 , thus:

$$
\begin{aligned}
& \log .2=0.301 \times 0.855=0.257 \\
& \log .3=0.477 \times 0.855=0.408 \\
& \log .4=0.602 \times 0.855=0.515,
\end{aligned}
$$

or by any other convenient method of reducing the unit magnitude.

The logarithm of $0.0796=\overline{2} .901=-1.099$, and by placing the starting point of the $v$ 's above $g$ a distance $1.099 \times 0.855=0.940$ on 
the scale of 20ths, thus adding -1.099 , the logarithm of 0.0796 , the reading on the $v$ line is $0.0796 d^{0.63} h^{0.54}$.

To introduce the factor, $C$, draw the $C$ line parallel to the others at any suitable place between the $v$ line and the $h$ line. Draw the $V$ line midway between the $v$ line and the $C$ line, as in Fig. 38 .

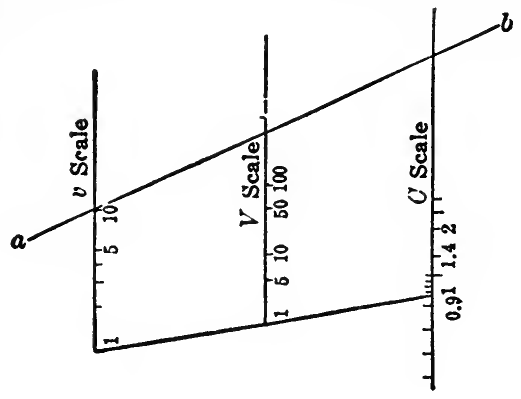

Fig. 38

From the point, 1 , on the $v$ scale, draw a line across the $v, V$, and $C$ lines at any convenient angle, in order to locate the " 1 " points of the scales about to be plotted. Using the same unit as for $v$, lay off on the $C$ line the logarithms of $1.1,1.2,1.3$, and 1.4 above 1 and the logarithms of $0.9,0.8$, etc., below it. If the logarithms on the $V$ line were now laid off with the same unit as those on the $v$ and $C$ lines, the reading at the crossing of any line, $a b$, would be the number corresponding to 1 $-\log v+-\log . C$.

Therefore we make the $V$ scale one-half as large as the other two, that is, with a unit of $(1 / 4$ in. $\times 0.855)$, which will give the product, $v C$, as required.

It is apparent that no divisions are required on the $v$ line after the starting points for the $V$ and $C$ scales are drawn. Therefore it is left blank in the completed chart, Fig. 36, and marked "Line $P$ " for reference.

In order to make the chart of greater use, it will be well to introduce another scale giving $Q$, the quantity discharged.

If $Q=$ discharge, in cubic feet per second;

$d=$ diameter, in inches;

$V=$ velocity, in feet per second;

$Q=0.00545 d^{2} V$.

The $d$ scale was plotted with a unit of $1 / 2$ in. to 0.1 . Therefore it will serve for log. $\left(d^{2}\right)$ with a unit of $1 / 4$ in. to 0.1 . The $V$ scale is made with a unit of $(1 / 4$ in. $\times 0.855)$, as shown on Fig. 39.

If we place the $Q$ line between the $d$ and $V$ lines, as shown, the distance, $\quad f g=n \times \log$. $\left(d^{2}\right)+(1-n) \times 0.855$ log. $V$, and if $n=(1-n) \times 0.855$,

then

$$
\begin{aligned}
n & =0.462 \\
f g & =0.462 \log .\left(d^{2}\right)+0.462 \log . V .
\end{aligned}
$$


If the $Q$ scale were now laid off from $g$ with a unit of $(1 / 4$ in. $\times 0.462)$, the reading at $f$ would be the number corresponding to log. $d^{2}+\log$. $V$.

Log. $0.00545=\overline{3} .7364=-2.2636$, and if we place the " 1 " of the $Q$ scale above $g$ a distance, $2.2636 \times 0.462=1.045$ on the 40 th scale $(1 / 4$ in. representing 0.1 ), thus adding log. 0.00545 , the reading at the intersection of $a b$ will give $0.00545 d^{2} V$, as required.

Assuming $d$ and $h$ to be known, and a value of $C$ selected according to the condition of the pipe, place a straight-edge so as to connect $d$ and $h$, and mark its interseetion on "Line $P$ ". Similarly, connect the point just marked with the given $C$ and at the intersection on the $V$ line read the value of $V$. Then connect this $V$ with the given $d$, and at the intersection on the $Q$ line read $Q$ in cubic feet per second.

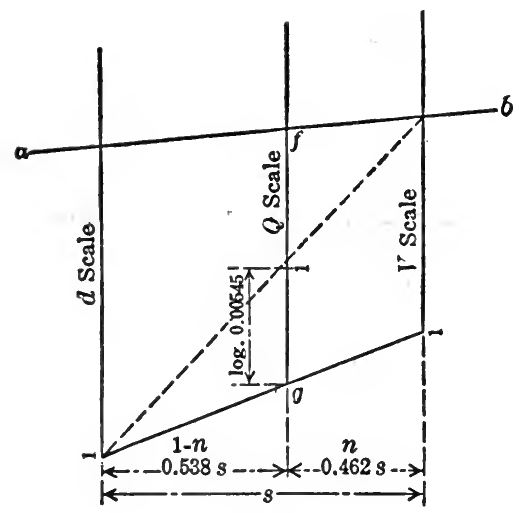

Fig. 39

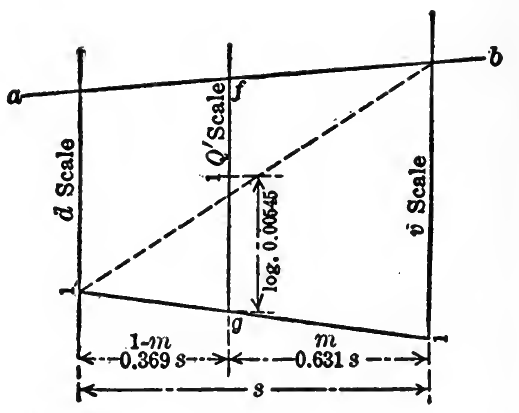

Fig. 40

It is evident that, by a suitable modification of the process described, either of the four quantities, $d, h, V$, or $C$, may be obtained when the other three are given.

As $C$ is usually assumed at a round figure, multiplication or division by it is so simple that some might prefer a chart based on the value 100 for this coefficient. The chart, Fig. 35, is constructed in this way, as follows:

Lay off the $d, h$, and $v$ seales as described. The $v$ scale will have its subdivisions eompleted and will occupy the position of "Line $P$ " in the chart, Fig. 36.

The subdivisions on the $d$ line represent $\log$. $\left(d^{2}\right)$ with a unit of $1 / 4 \mathrm{in}$, and those on the $v$ line represent log. $v$ with a unit of $(1 / 2 \mathrm{in} . \times 0.855)$, equivalent to $1 / 4$ in. $\times 1.71$.

If we place the $Q^{\prime}$ line as shown in Fig. 40, the distance,

$$
\begin{gathered}
f g=m \log .\left(d^{2}\right)+(1-m) \times 1.71 \log . v ; \\
\text { and if } m=(1-m) \times 1.71, m=0.631 .
\end{gathered}
$$

Then

If the $Q^{\prime}$ seale were lail off from $g$ with a unit of $(1 / 4$ in. $\times 0.631)$, the rearling at $f$ would be the number corresponding to $\log$. $\left(d^{2}\right)+\log . v$.

Log. $0.00545=-2.2636$, and if we place the " 1 " of the $Q^{\prime}$ scale 
above $g$ a distance of $2.2636 \times 0.631=1.428$ on the scale of 40 ths $(1 / 4$ in. to 0.1 ), the reading at the intersection of $a b$ will give $0.00545 d^{2} v$, as required.

It is evident that any straight line intersecting the four scales gives values of $d, h, v$, and $Q^{\prime}$ satisfying the equations,

$$
\begin{aligned}
v & =100 \times 0.000796 d^{0.63} h^{0.54}, \\
\text { and } Q^{\prime} & =0.00545 d^{2} v .
\end{aligned}
$$

By means of the "conversion scale," cubic feet per second may be read off at once, in millions of gallons per day.

The broken lines drawn on the charts, Figs. 35 and 36 , show that when $d=30$ in., $h=33 \mathrm{ft}$, and $C=82$, the formula gives $V=3.7$ and $Q=18 \mathrm{cu}$. ft. per sec. Also when $d=30 \mathrm{in}$. and $h=33 \mathrm{ft}$., $v=$ 4.5 and $Q^{\prime}=22 \mathrm{cu}$. ft. per sec.

In the discussion of the above paper, Messrs. P. C. Nugent, W. M. Eliot, and F. W. Green sent interesting letters, which have also been reproduced.

Paul C. Nugent.-Mr. Strachan has covered admirably the theory of the nomographic diagram. His paper is of especial value in calling attention to some of the less familiar forms. Particularly to be noted are the diagrams in which the natural scales are. used. As a somewhat different method of presentation is often of interest, the following treatment of the theory and construction of a logarithmic nomograph, similar to the one representing the Hazen-Williams formula, is here submitted:

The typical form of an equation of this. kind is

$$
K_{\mathrm{q}} Q^{\mathrm{a}}=K_{\mathrm{d}} D^{\mathrm{b}} \times K_{\mathrm{h}} H^{\mathrm{c}}
$$

in which $Q, D$, and $H$ are variables, and $K_{\mathrm{q}}, K_{\mathrm{d}}, K_{\mathrm{h}}, a, b$, and $c$, are constants. A representative formula is that of Flamant, which may be written

$$
Q=0.001361 D^{\frac{19}{7}} H^{\frac{4}{7}}
$$

In Equation (2) $Q$ is in cubic feet per second; $D$ is the diameter of the pipe, in inches; and $H$ is the drop, in feet, of the hydraulic gradient per $1,000 \mathrm{ft}$. of pipe line.

Referring to Fig. 41, on the vertical line, $H$, are supposed to be laid off, from the point $H$ downward, the logarithmic values of $K_{\mathrm{h}} H^{\mathrm{c}}$. It is next necessary to assume the distances, $x$ and $y$. These, for convenience, may be equal. Having assumed them, draw two vertical lines, $D$ and $Q$, as shown. Assuming any convenient point, $Q$, on the $Q$ line, draw a straight line, $R S$, through this point and $H$. $Q$ is the beginning or zero point of the $Q$ line, and $D$, the intersection of $R S$ with the intermediate vertical previously drawn, is the corresponding point on the $D$ line

To discuss first the case where the $Q, D$, and $H$ factors, including their constant exponents and coefficients, are each not less than unity, suppose any straight-edge, $L_{1} M_{1}$, laid across the diagram as shown in Fig: 41. It is evident that the $Q$ and $D$ quantities must increase upward, and the $H$ quantities downward, from what may be termed the "zero line," $R S$. Consequently, in the assumed case (all factors not less 
than unity and therefore all logarithms plus) $L_{1} M_{1}$ will intersect $R S$ to the right of the $D$ line, and will eut the three verticals in the points, $Q_{1}, D_{1}$, and $H_{1}$.

From the figure,

$$
\begin{aligned}
& \frac{H H_{1}+Q Q_{1}}{x+y}=\frac{H H_{1}+D D_{1}}{y}, \text { from which } \\
& Q Q_{1}=-H H_{1}+\frac{(x+y)}{y} D D_{1} \ldots \ldots \ldots
\end{aligned}
$$

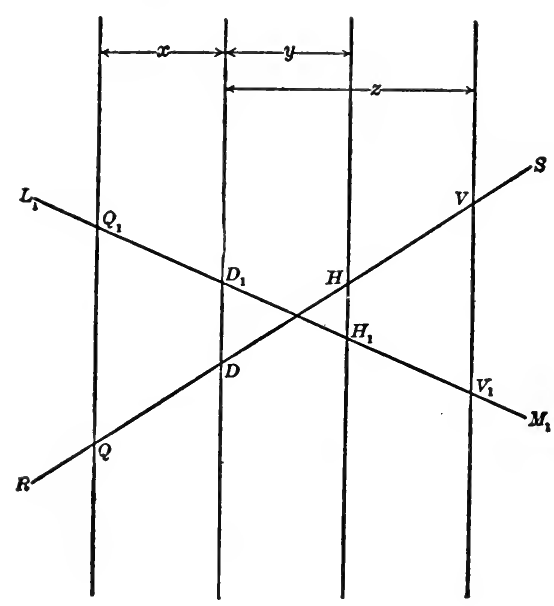

Fig. 41

Now, suppose that the scale to which the $Q$ quantitics are laid off is $n_{\mathrm{b}}$ and $n_{\mathrm{d}}$ times the scales for the $I$ and $D$ quantities. That is, the actual space, say in inches, on the $Q$ scale which corresponds to the logarithm of any number as 5 , will be $n_{\mathrm{h}}$ times the actual corresponding space on the $I I$ seale. From an inspection of Equations (1) and (3) it is evident that, if the nomograph is to represent the typical Equation (1), $n_{\mathrm{b}}$ and $n_{\mathrm{d}}$, respectively, must equal - and $\frac{x+y}{y}$. Any convenient scale having been selected for the $I I$ line, the $Q$ and $D$ scales may now be determined.

The distanees along any vertical, as the $I$ line, from a point marked with a certain value of $I)$ to a value ten times as great, will be eonstant. These distances may be called the "secondary unit lengths" and denoted by $L^{\prime}{ }_{\text {(1, }} L_{\text {d }}^{\prime}, \dot{L}_{\mathrm{h}}^{\prime}$. The "unit lengths," that is, the lengths eorresponding to the logarithm of 10 (unity) are symbolized by $L_{\mathrm{q}}, L_{\mathrm{d}}$, and $L_{\mathrm{h}}$ 。

The additional details of the construction may perhaps be best illustrated by the applieation of the theory to Equation (2) and its nomo- 
graph, Fig. 42. As shown in this figure, $x$ and $y$ are each assumed as 1.25 in. Consequently, $n_{\mathrm{h}}=1$ and $n_{\mathrm{d}}=2$.

In the formula, $D$ occurs as $0.001361 D^{\frac{19}{7}}$ and $H$ as $H^{\frac{4}{7}} . Q$ is found simply as $Q$. The scale assumed for the $H$ line is 3 in.= unity. There4

fore, $L_{\mathrm{h}}^{\prime}=\frac{4}{7} \times 3=1.71$ in. Similarly, $L_{\mathrm{q}}^{\prime}=3.00$ in. and $L_{\mathrm{d}}^{\prime}=4.07$ in. The spaces, $L^{\prime}{ }_{\mathrm{q}}$ and $L^{\prime}{ }_{\mathrm{h}}$, are laid off up and down the $Q$ and $H$ lines from the zero line, $R S$.

Turning to the $D$ line, we note that where it is intersected by $R S$, the value written for $D,\left(D_{0}\right)$ must be such that

$$
\log .\left(0.001361 D_{0}^{\frac{19}{7}}\right)=0
$$

from which, $D_{0}=11.38$.

As shown above, the $D$ scale is one-half the $H$ scale, and consequently 1

$L_{\mathrm{d}}=\frac{1}{2} \times 3=1.50 \mathrm{in}$. To get back to the point marked 10 on the $D$ line, we have

[log. $\left.\left(0.001361 \times 11.38^{\frac{19}{9}}\right)-\log .\left(0.001361 \times 10^{\frac{19}{7}}\right)\right] \times 1.50 \mathrm{in}$. $=0.23 \mathrm{in}$.

This locates the 10 point on the $D$ line from which the spaces, $L_{\mathrm{d}}^{\prime}=4.07$ in., are laid off up and down the line.

If a $V$ line is desired, we may transform Equation (2) by writing $A V$ for $Q$, obtaining finally

$$
V=0.2495 D^{\frac{5}{7}} H^{\frac{4}{7}}
$$

and, proceeding as above, construct a diagram based on the $D, H$, and $V$ lines. More simply, however, we may write

$$
Q=A V \frac{\pi D^{2}}{4 \times 144} V=0.005454 D^{2} V \ldots \ldots \ldots \ldots
$$

Assuming the $\mathrm{V}$ line $z$ inches to the right of the $\mathrm{D}$ line, just as above, and considering the $Q, D$, and $V$ lines, $n_{\mathrm{v}}=\frac{x}{z}$ and $n_{\mathrm{d}}=\frac{(x+z)}{z}$. $Q$ is involved in Equation (4) just as in Equation (2), and we may, therefore, assume the plotted $Q$ line as a basis for the $V$ construction. The coefficients, $K_{\mathrm{d}}$, etc., in the general Equation (1) do not enter into the determination of the secondary unit lengths. These are matters of the exponents, and, having determined $L_{\mathrm{d}}, L_{\mathrm{d}}^{\prime}=b L_{\mathrm{d}}$. Thus, considering the $Q, D$, and $V$ lines,

$$
L_{\mathrm{d}}^{\prime}=b L_{\mathrm{d}}=b \frac{L_{\mathrm{q}}}{(x+z)}, \text { from which }
$$




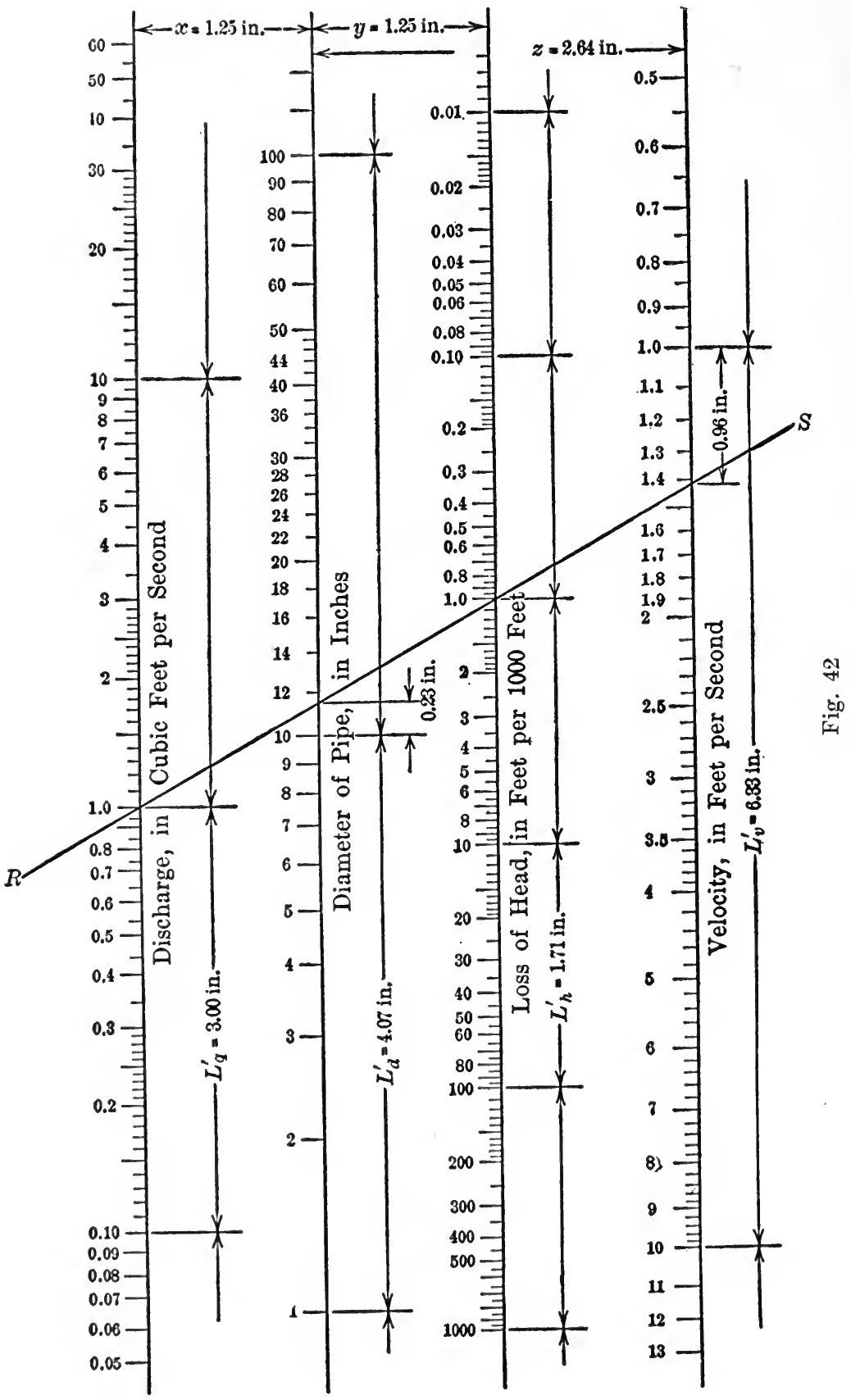




$$
z=\frac{L_{\mathrm{d}}^{\prime} x}{b L_{\mathrm{q}}-L_{\mathrm{d}}^{\prime}}
$$

Using the values above given, from Equation (5), $z=2.64$ in. Note that what has been done involves the assumption of a new $L_{\mathrm{d}}$ for the $D$ line. Since the unit lengths have not been shown on the diagram, no confusion will be caused thereby.

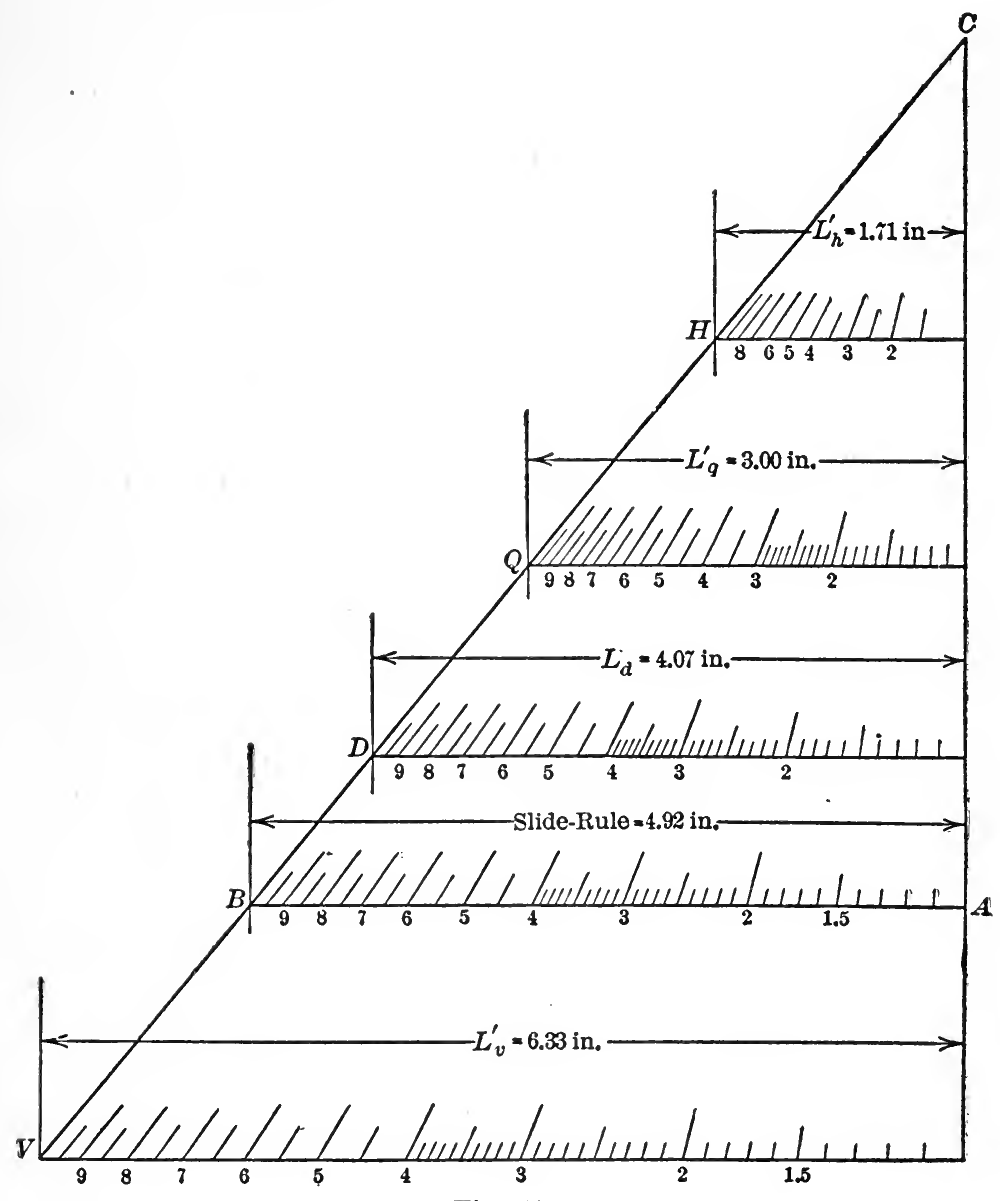

Fig. 43

The determination of the value, $D_{0}$, to be written at the zero point of the $D$ line, has involved both the coefficient and exponent of the $D$ factor in Equation (2). The general condition to be satisfied here is $\log . K_{\mathrm{d}}+b\left(\log . D_{0}\right)=0 \ldots \ldots \ldots \ldots \ldots$ (6)

From Equation (4), $b=2$, and we have already found that $D_{0}=11.38$. Making these substitutions in Equation (6), we obtain $K_{\mathrm{d}}=0.007729$. 
It now becomes necessary to write Equation (4) in such a way that this speeial value of $K_{\mathrm{d}}$ appears as the eoefficient of $D^{2}$. Equation (4), therefore, is transformed to

$$
Q=0.007729 D^{2} \times 0.7057 \mathrm{~V}
$$

We next have $L_{\mathrm{v}}\left(=L^{\prime}{ }_{\mathrm{v}}\right)=\frac{L_{\mathrm{q}}}{n_{\mathrm{v}}}$, from which, with the foregoing values, $L^{\prime}{ }_{\mathrm{v}}=6.33 \mathrm{in}$. The value to be written at the intersection of the $R S$ and $V$ lines and the distance from this intersection to the end of the nearest $L^{\prime}$ v space are determined as with the $D$ line. $V_{0}$ is found to

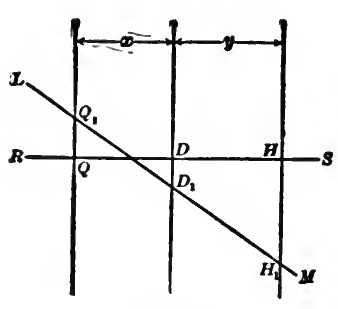

Fig. 44

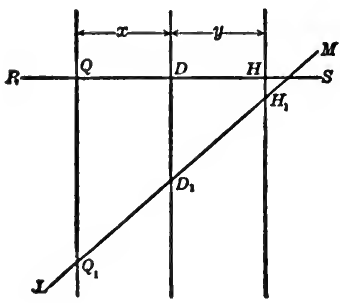

Fig. 46

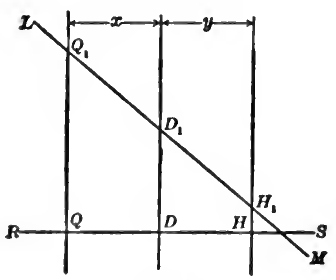

Fig. 48

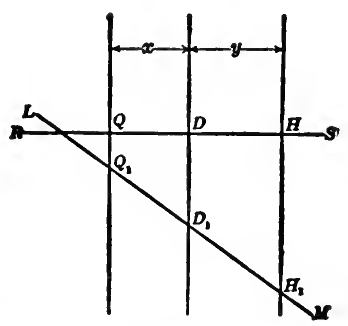

Fig. 45

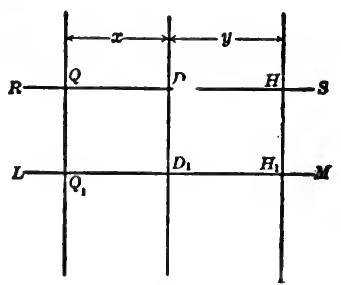

Fig. 47

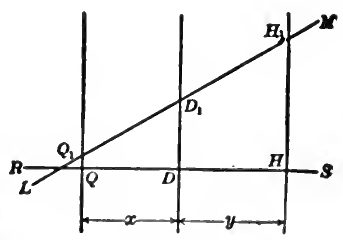

Fig. 49

equal 1.417, and the distance up to the point marked $l$ is 0.96 in. From this point the $L^{\prime}$ v spaces are laid off upward and downward. Less than two such spaces are needed in the present diagram.

For subdividing the spaces, $L_{\mathrm{q}}^{\prime}, L_{\mathrm{d}}^{\prime}$, ete., the construction shown in Fig. 43 may conveniently be used. A right triangle is drawn with a base of any convenient length and an altitude equal to the longest of these spaces, in the present case $L^{\prime}{ }_{\mathrm{v}}$. On the hypotenuse are chosen 
points at distances from the base equal to the various lengths to be subdivided and also to the unit length of an ordinary slide-rule. Lines are drawn from these points perpendicular to the base, and the line corresponding to the slide-rule is divided as on that instrument. A series of lines through these points of division and the vertex of the triangle will subdivide the other lines proportionately. The subdivisions are finally transferred to the nomograph.

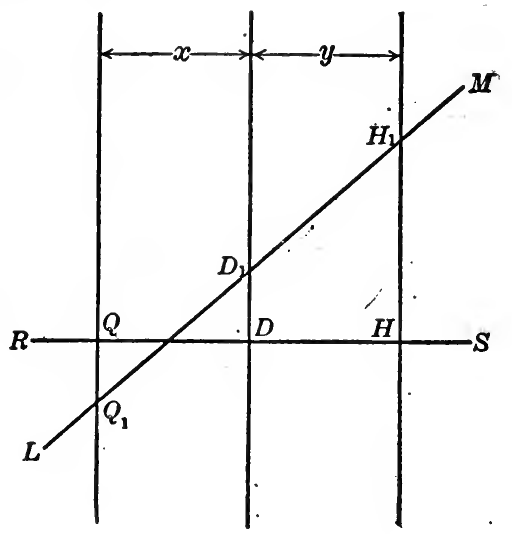

Fig. 50

To consider the case where one or more of the various factors are less than unity, Figs. 44 to 52 show the nine possible positions of the straightedge, or isopleth, $L M$. With each scale $(Q, D, H$, or $V)$ increasing in the same direction both below and above the line, $R S$, the numerical value of the logarithm of any quantity read off on the diagram is always proportional to the distance between the point read and the $R S$ line. In the general Equation (1), in solving for the $Q$ factor, if its

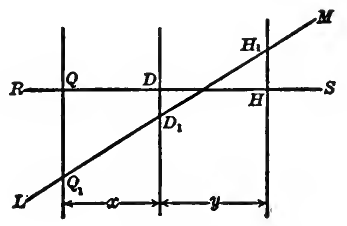

Fig. 51

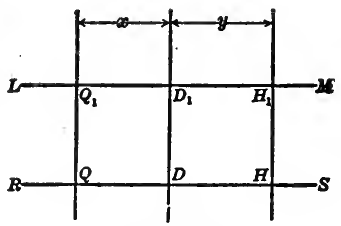

Fig. 52

logarithm is plus and the logarithms of the other two factors are one plus and the other minus, the logarithm of the $Q$ factor will be numerically equal to the difference between the other two. This corresponds to Figs. 44, 48, 49, and 52. The same thing is true if the logarithm oi the $Q$ factor is minus and the other two logarithms are one plus and the other minus. This corresponds to Figs. 45, 46, 47, and 50. If all the logarithms are negative, the logarithm of the $Q$ factor is numerically equal to the sum of the other two; this corresponds to Fig. 51. This last case is entirely similar to that already discussed, where all the 
logarithms are positive. Cases where one or more of the logarithmic factors become equal to zero, may be treated as special cases under some of those previously mentioned.

To justify the diagram for the cases shown in Figs. 44, 50, and 52, it is necessary to show for each only that

$$
Q Q_{1}= \pm\left(n_{\mathrm{r}} H H_{1}-n_{\mathrm{d}} D D_{1}\right)
$$

With Figs. 44 and 50 we may write

$$
\frac{Q Q_{1}+H H_{1}}{x+y}=\frac{Q Q_{1}+D D_{1}}{x},
$$

from which, $Q Q_{1}=\frac{x}{y} H H_{1}-\frac{(x+y)}{y} D D_{1}=n_{\mathrm{h}} H H_{1}-n_{\mathrm{d}} D D_{1}$, and with Figs. 45 to 49 and 52,

$$
\frac{Q Q_{1}+H H_{1}}{2}(x+y)=\frac{\left(Q Q_{1}+D D_{1}\right)}{2} x+\frac{\left(D D_{1}+H H_{1}\right)}{2} y,
$$

from which, $Q Q_{1}=\frac{(x+y)}{y} D D_{1}-\frac{x}{y} H H_{1}=n_{\mathrm{d}} D D_{1}-n_{\mathrm{h}} H H_{1}$.

W. MI. ELIot.-The author has opened a new field to those engineers who hitherto have been unacquainted with this method of platting. As a general rule, any formula can be solved with nomographs by the

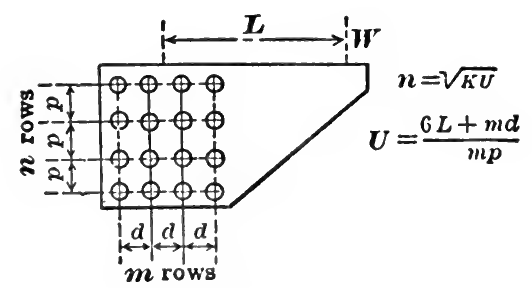

Fig. 53

elimination of one variable at a time. In an equation with several independent variables, however, it happens, frequently, that results are wanted for a few values only of one or more of them. The solution of the formula by using a nomograph can be made much simpler in this case by platting the few values required on the same graph.

As an illustration, in a recent issue of a technical publication there appeared the following formula for the approximate number of rivets recpuired in an eccentric connection ( Fig. 5.3):

$$
n=\sqrt{\frac{K(6 L+m d)}{m p}}
$$


$n=$ number of horizontal rows of rivets;

$m=$ number of vertical rows of rivets;

$p=$ vertical rivet spacing;

$d=$ horizontal rivet spacing;

$L=$ eccentricity of load, $W$;

$K=$ ratio of load to the allowable rivet stress $=\frac{W}{S_{\mathrm{R}}}$.

For ordinary detailing, values of $m=1,2,3$, or 4 , and $p=2 \frac{1}{2}$ or 3 in., will be sufficient to cover most cases.

The formula will be divided into two parts :

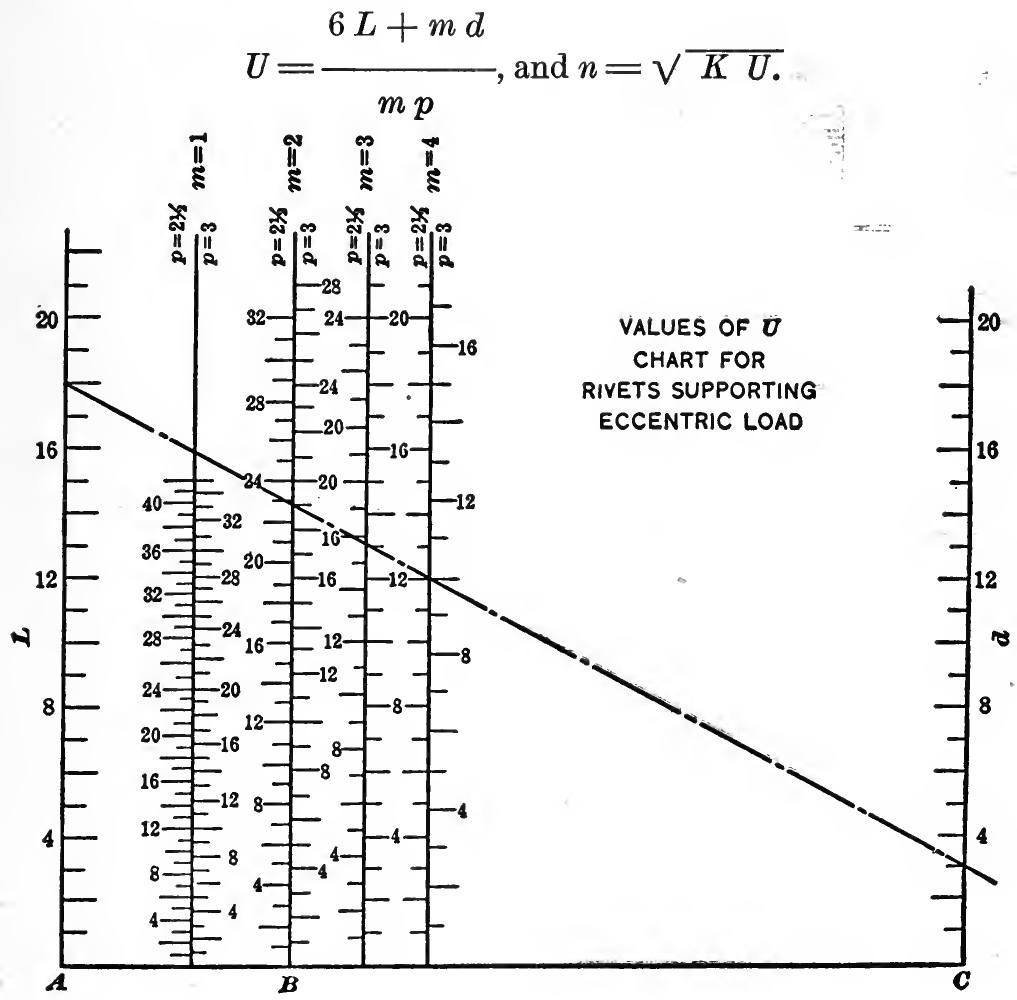

Fig. 54

A nomograph will be made first for the solution of $U$, and then one for $n$.

$$
\begin{gathered}
U=\frac{6 L+2 d}{2 \times 2 \frac{1 / 2}{5}}, \text { when } m=2, \text { and } p=2 \frac{1}{2} \\
\frac{5}{8}=\frac{3}{4} L+\frac{1}{4} d .
\end{gathered}
$$


On the base line (Fig. 54), $A B=1 / 4 A C$. The scale for $m=2, p=$ $2 \frac{1}{2}$, is five-eighths of that used for $L$ and $d$. Other values of $U$ are similarly platted.

Scales $U, K$, and $n$, are platted logarithmically (Fig. 55), lines for $U$ and $K$ being equidistant from $n$.

Given $K=9 \frac{1}{2}, L=18$ in., $m=4, p=3$, and $d=3$, to find $n$. On Fig. 37, place a straight-edge on $18 \mathrm{in.}(L)$ at the left and on 3 in. $(d)$ at the right; read $10.0(U)$ on the line, $m=4$, right side $p=3 \mathrm{in.} \mathrm{On}$ Fig. 38, place a straight-edge on $10.0(U)$ at the left, and on $91 / 2(K)$ at the right; read $9.7(n)$. Use four vertical rows of rivets, ten in each row. By this method, an expression containing six variables is solved for certain values with two nomographs.

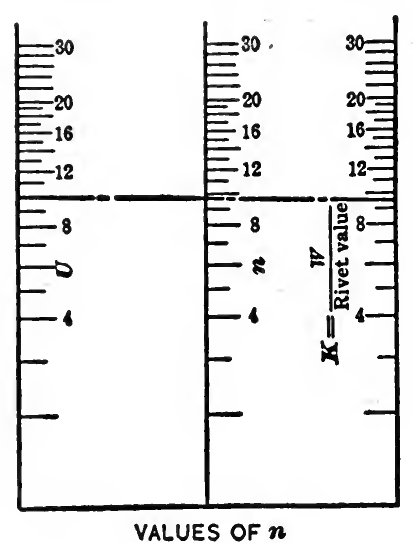

Fig. 55

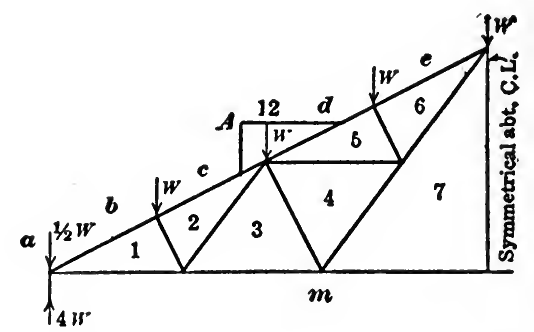

Fig. 56

One of the main advantages of the nomograph is the few lines required to represent a formula. On this account, several results may often be shown on the same plat. The stresses in a roof truss (Fig. 56) of any slope and panel loading may be ascertained by the same general method as that used by the author for stresses in pins. As it is impracticable to use a separate graph for each member, they have been combined as shown in Fig. 57, two scales being used to give greater accuracy in the results. The stress in $m 1$ for a panel load of 2,000 lb., bevel of top chord 6 in 12, is $14,000 \mathrm{lb}$.

Other simple types of trusses have been similarly platted by the writer, but are not reproduced here, as the method is the same for all. By the use of these graphs, and diagrams of angles platted for strength in compression for different lengths, and for tension values with the proper size and number of holes deducted, it takes only a few minutes to ascertain the makc-up of a roof truss for an ordinary mill building. An excess vertical load is assumed to take care of wind stresses, but this need not be the same for all members.

The two nomographs which follow, though only for stresses of 650 and $16,000 \mathrm{~h}$. per sq. in. on the conerete and steel, respectively, give more direct results than the one proposed by the author. The diagram, 
Fig. 58, gives the size of beam and area of steel required to resist a given moment, when the concrete and steel are each stressed to the working limit. For the case shown, a moment of 428,000 in-lb. requires

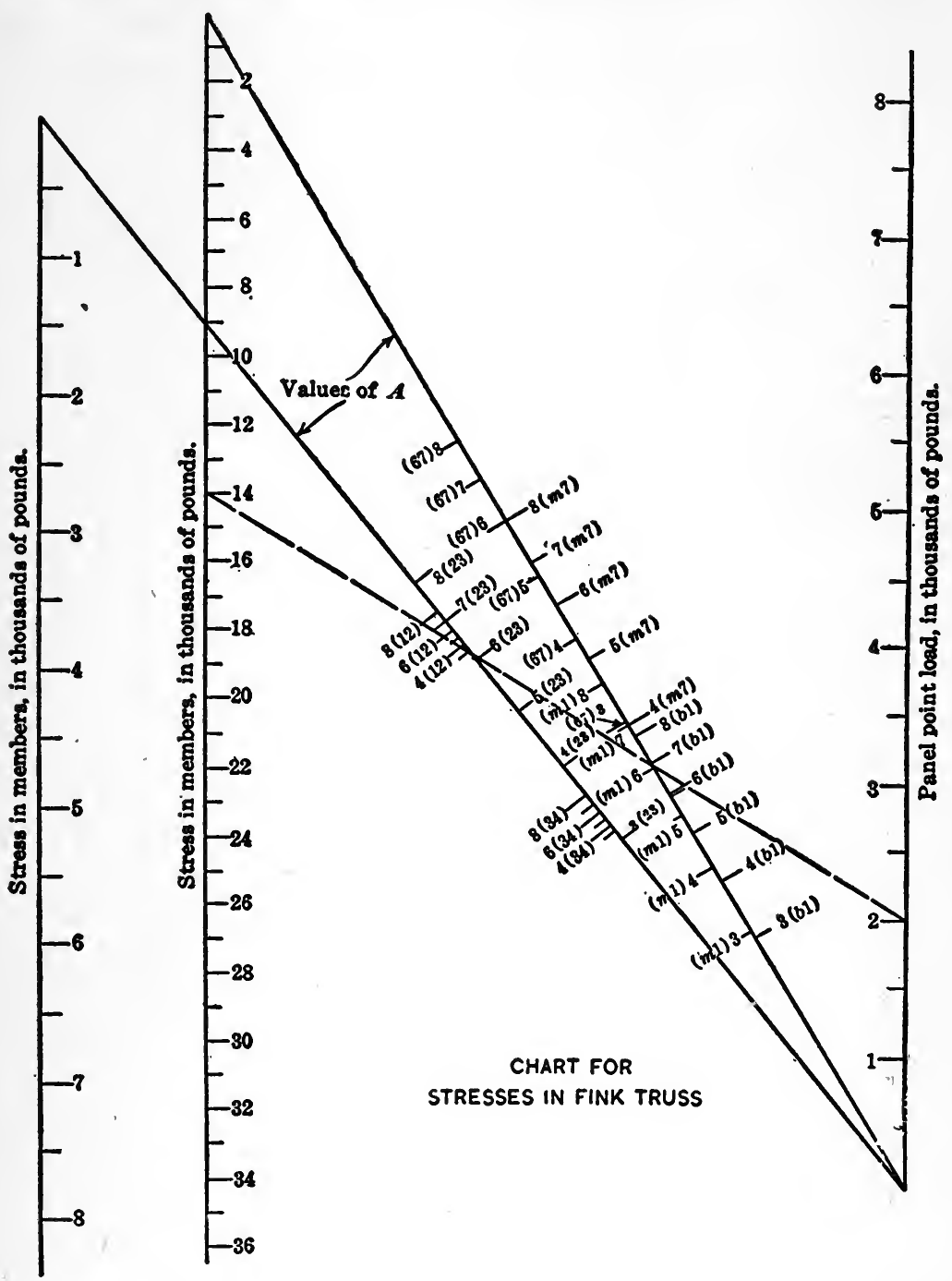

Fig. 57

a beam 10 by $20 \mathrm{in}$. (effective depth), and an area or steel $=1.54$ sq. in. Often the size of beam is fixed, and it is desired to know the quantity of steel required for a given resisting moment. This is given by the nomograph, Fig. 59; the resisting moment is determined by the steel being stressed to the working limit for values of $p$ less than 0.0077 , and 
by the concrete for values of $p$ greater than 0.0077 . Given a beam, 10 by $20 \mathrm{in}$. (effective depth) to resist a moment of 30,000 in-lb.: Place a straight-edge on the moment $35,000 \mathrm{lb}$. (for $1 \mathrm{in}$. in width) and on $d=20$; read $p=0.0062$. Area of steel required $=p b d=1.24$ sq. in. One designing many beams can well afford to make similar nomographs for the stresses he uses, as little time is required.

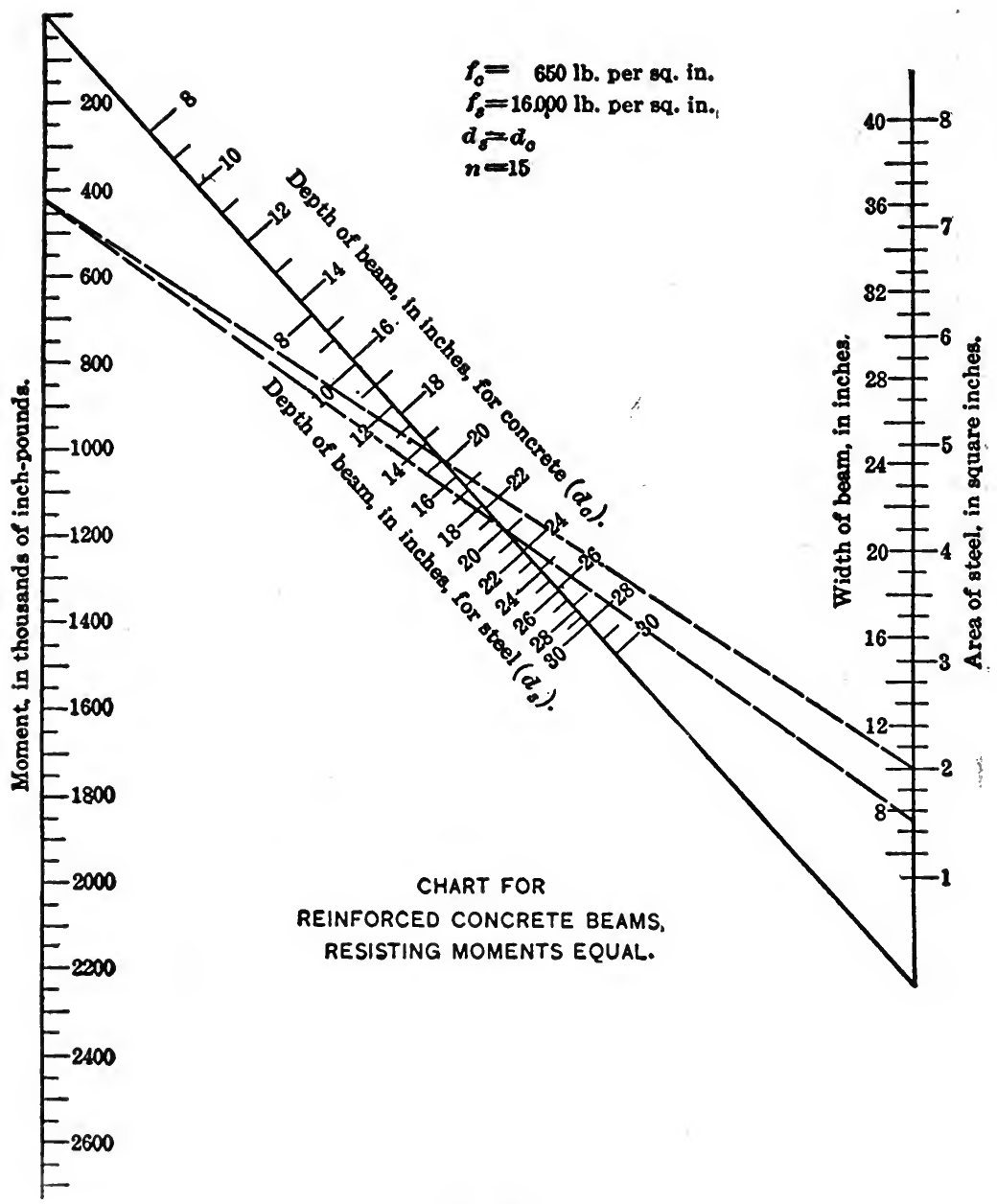

Fig. 58

The diagram, Fig. 60, gives the dimensions of a yellow pine beam directly, when the moment is known. If the total uniform load and span are given, two isopleths are required to ascertain the size of beam. In the example shown, a load of $19,000 \mathrm{lb}$. on a span of $20 \mathrm{ft}$. requires a beam 10 by $16 \mathrm{in}$. (actual size $9^{1 / 2}$ by $15^{1 / 2} \mathrm{in}$.). The moment itself need not be read. 
The pitch of rivets connecting the web and flange of a plate girder (see Fig. 61) is given approximately by the formula:

$$
\begin{gathered}
p=\frac{R h}{V}, \\
\text { or, } \frac{10 p}{h}=\frac{10 R}{V}=Q .
\end{gathered}
$$

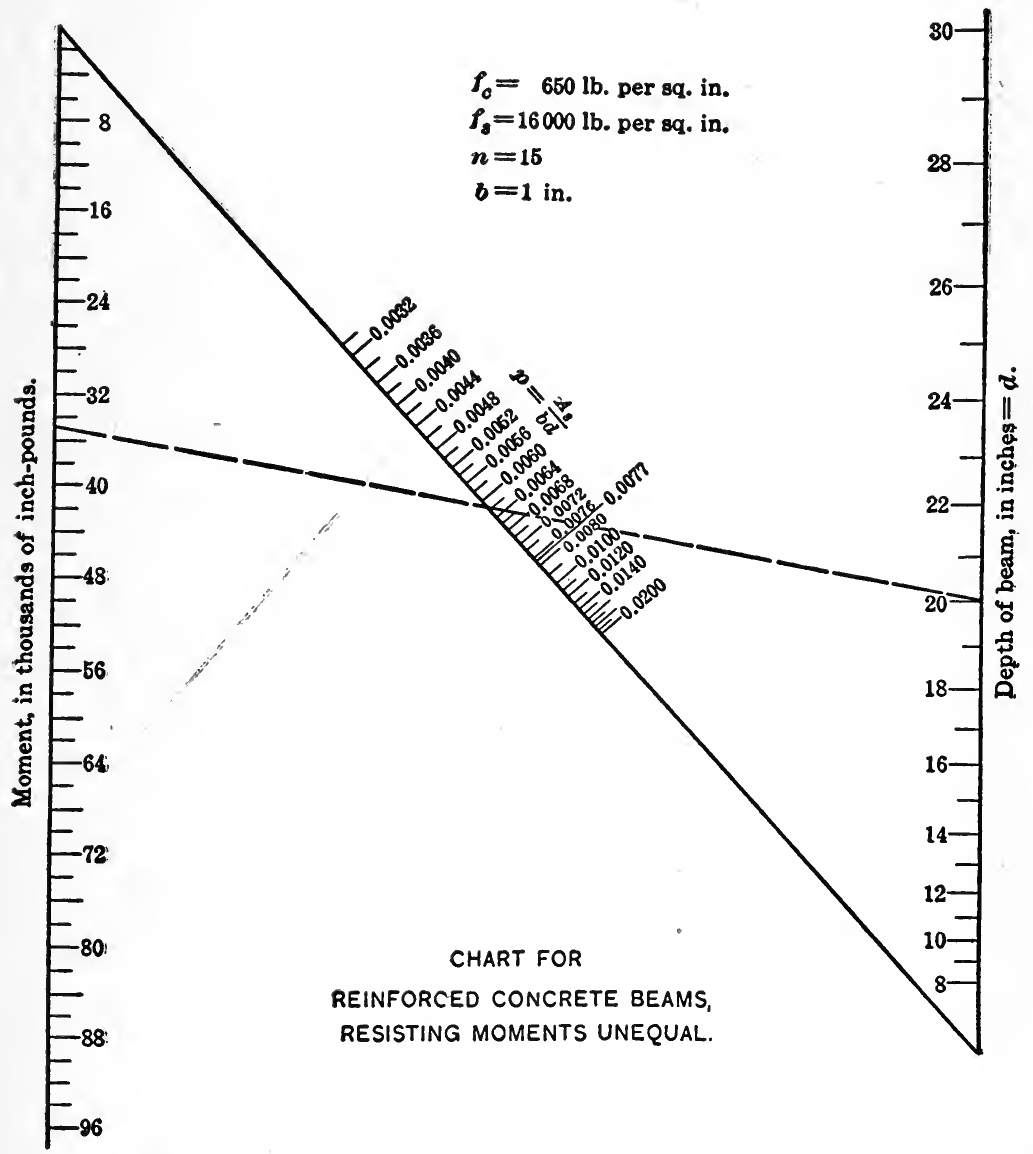

Fig. 59

The isopleths joining $p$ and $h$ and $R$ and $V$, intersect on the diagonal line for $Q$ connecting the ends of the scales. Thus the pitch is $2.8 \mathrm{in}$. for a vertical shear of $200,000 \mathrm{lb}$. on a girder having an effective depth of 70 in., with a rivet value of $8,000 \mathrm{lb}$. As this formula gives a pitch that is a little low, correction can be made to some extent by using the 
total for the effective depth of the girder. As the values of $Q$ are not: platted, nomographs for equations of this type are easily made.

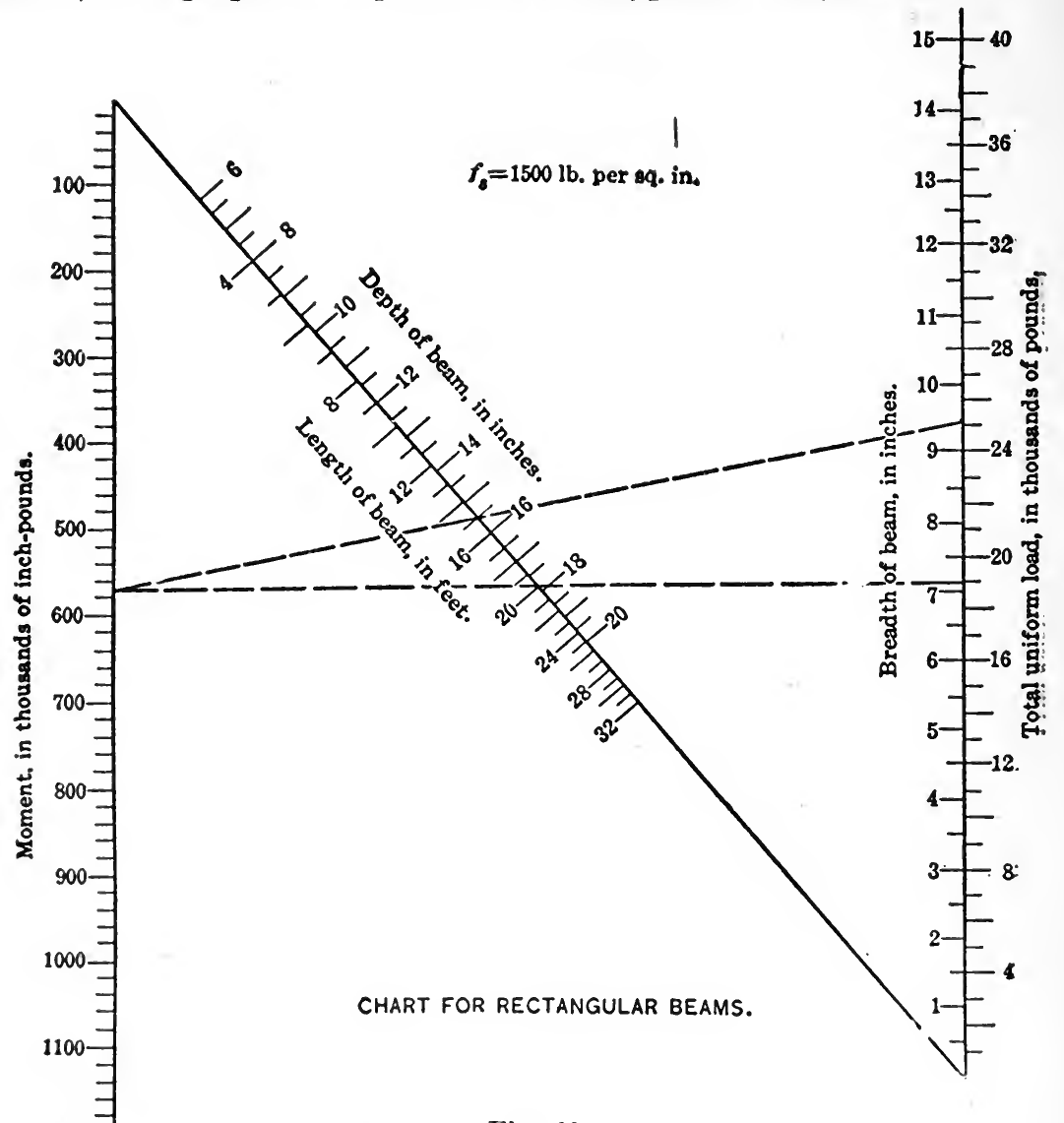

Fig. 60

This method can be applied in the same way to more complex formulas, such as that for the deflection of beams (Fig. 62).

$$
\begin{aligned}
& \Delta=\frac{5 W L^{3}}{384 E I}, \\
& \text { or, } \frac{384 \times 58,000 \Delta}{L^{3}}=\frac{W}{100 I}=Q, \\
& \text { when } E=29,000,000 \text { for steel; } \\
& \Delta=\text { deflection; } \\
& L=\text { span; } \\
& W=\text { total uniform load; } \\
& I=\text { moment of inertia. }
\end{aligned}
$$


The cubes of $L$ are used, and the beams are written at their respective moments of inertia when the members are platted. The deflections for a concentrated center load are similarly shown. A 20-in. I-beam of $65 \mathrm{lb}$. per $\mathrm{ft}$., with a total uniform load of $50,000 \mathrm{lb}$. on a span of $25 \mathrm{ft}$., has a deflection of $0.52 \mathrm{in}$.

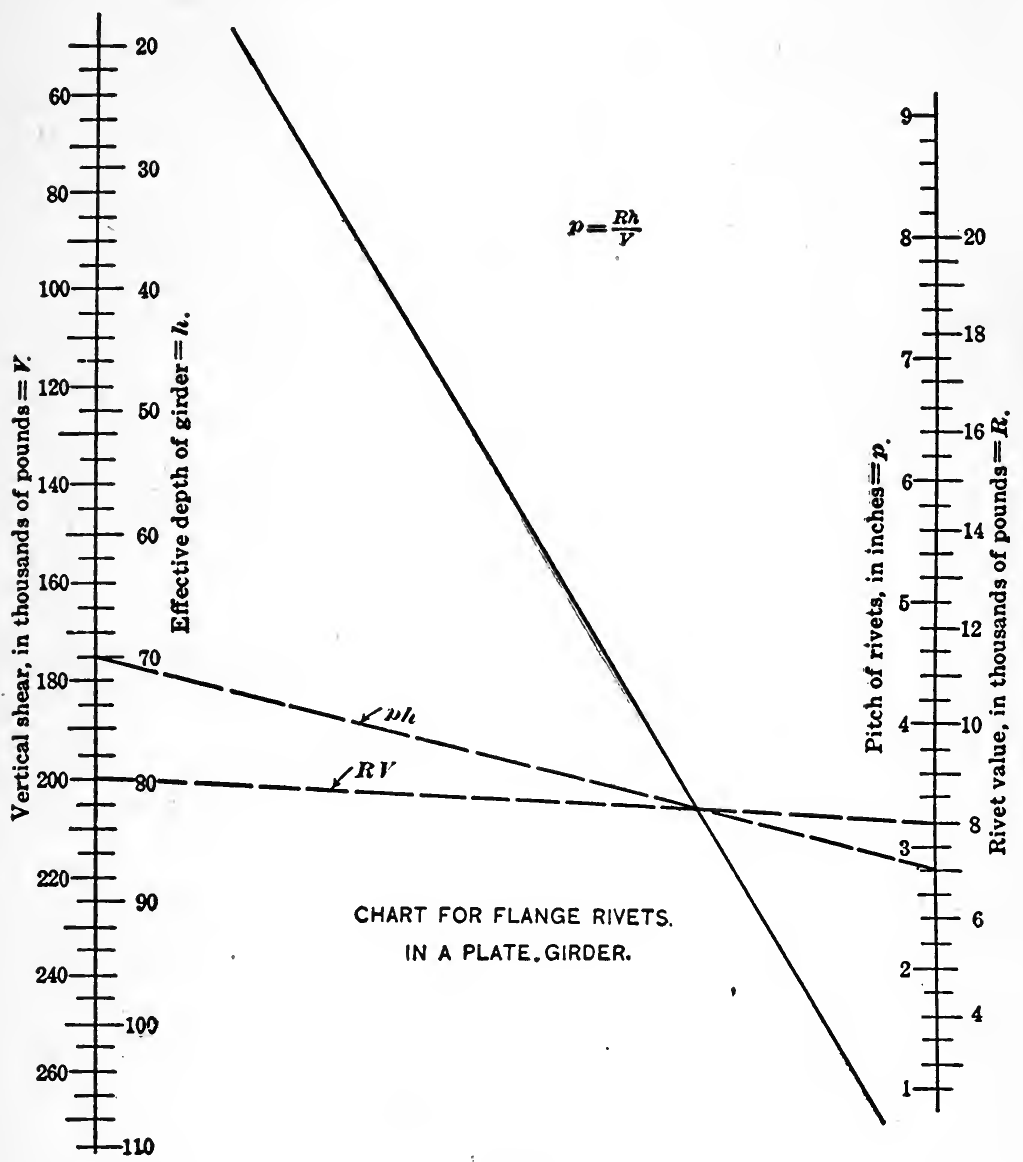

Fig. 61

The writer, in presenting these nomographic solutions for formulas used in structural engineering, has not given the details of construction in each case, as the method will be apparent to any one who has read the paper. The alignment diagram is very useful in this field, even though many of the formulas are readily solved by direct computation. It would seem that this method would be of greatest value, however, in hydraulic and experimental work, where fractional exponents frequently occur.

The author has ably presented this method of solving formulas. The 
system has heretofore been used to some extent, but its principles have not been generally understood.

F. W. GREen.-As the author's presentation of this subject seems to the writer to leave rather too much for the reader to demonstrate for

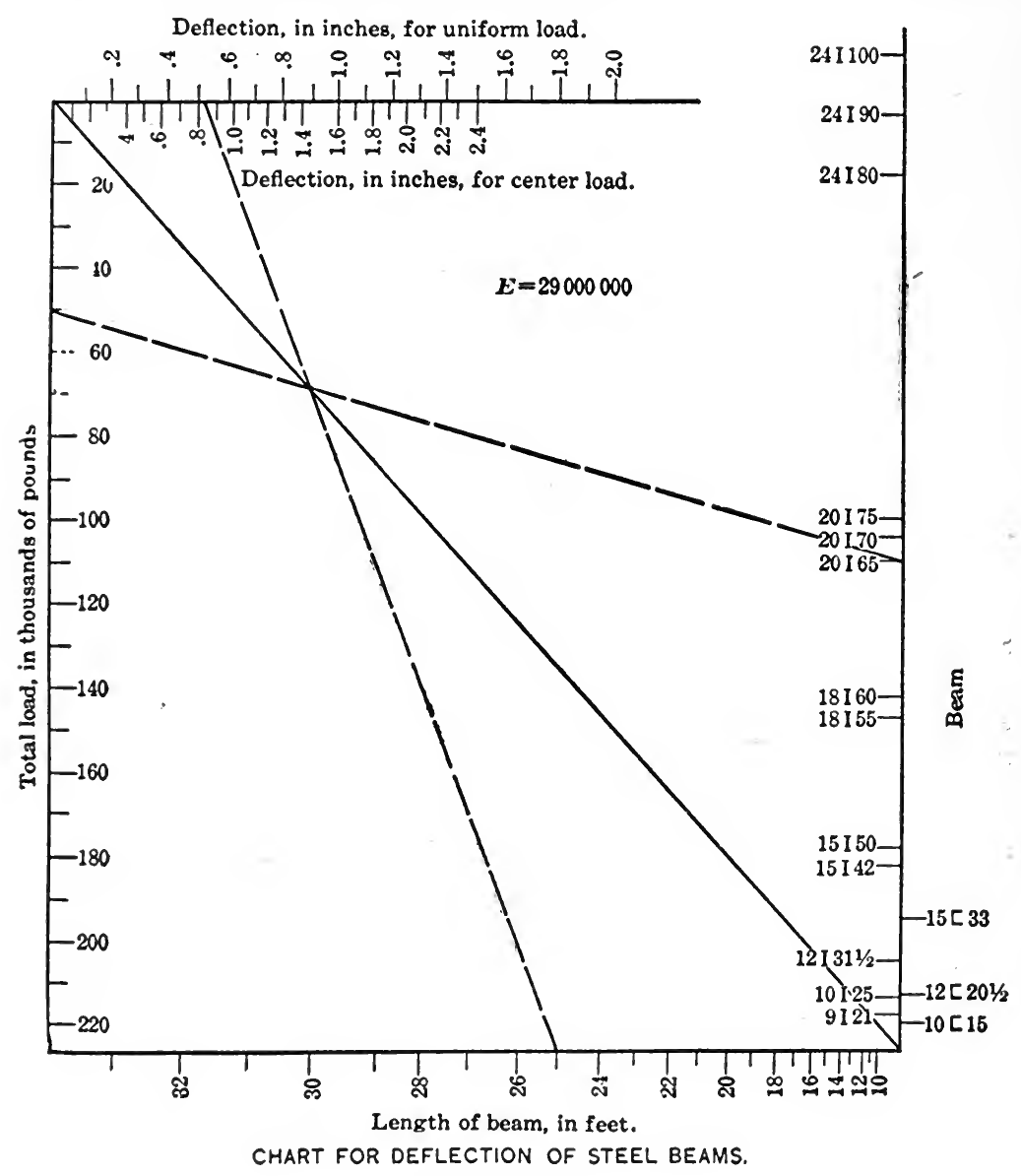

Fig. 62

himself, the following discussion will be addressed to clearness of presentation rather than to the addition of anything new.

Assume an expression of the form,

$$
A x^{\mathrm{D}}=B y^{\mathrm{q}} C z^{\mathrm{r}} \text {. }
$$

Using logarithms,

$$
\text { log. } A+p \log . x=\log . B+q \log . y+r \log . z+\log . C,
$$

in which $A, B$, and $C$ are constants, $x, y$, and $z$ are variables, and $p, q$, and $r$ are exponents. 
Dropping the constants for the time being,

$$
p \log . x=q \log . y+r \log . z
$$

which will be considered as the equation for which a nomograph is to be constructed.

In Fig. $63, y, x$, and $z$ are three parallel lines the scales of which are arranged so as to satisfy the required equation. The line, $A B$, is an isopleth. The arrangement of the three parallel scales in such a way as to solve such an equation is called a nomograph.

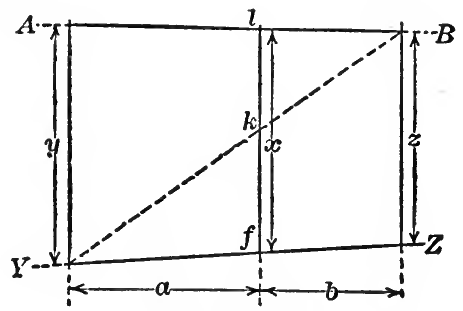

Fig. 63

From geometry, $f k: a:: x: a+b$,

whence

and, similarly, $\quad k l: y:: b: a+b$,

whence,

$$
f k=\frac{a}{a+b} z,
$$

$$
k l=\frac{b}{a+b} y .
$$

therefore,

$$
x=\frac{a}{a+b} z+\frac{b}{a+b} y \text {. }
$$

Equation (2) shows the relation of the scales of values for the variables, which is required in order to satisfy the given equation.

The expression assumed was,

$$
A x^{\mathrm{p}}=B y^{\mathrm{q}} C z^{\mathrm{r}},
$$

whereas Equation (2), in the right-hand member, involves addition instead of multiplication. Therefore logarithms must be used in order to make Equation (2) applicable, thus,

$$
\text { log. } A+p \log . x=\log . B+q \log . y+\log . C+r \log . z,
$$

which, after dropping the constants, becomes,

$$
p \log . x=q \log . y+r \log . z
$$

which is Equation (1).

Comparing Equations (1) and (2) shows that it is necessary to de- 
vise a method of converting the coefficients of $z$ and $y$, respectively, in Equation (2) to those in Equation (1). Therefore, a modulus is used, thus :

whence,

$$
\begin{array}{r}
\frac{a}{a+b} \log . z m_{1}=r \log . z, \\
m_{1}=r \frac{a+b}{a} \ldots \\
\frac{b}{a+b} \log . y m_{2}=q \log . y \\
m_{2}=q \frac{a+b}{b} \ldots
\end{array}
$$

whence,

Dividing Equation (3) by Equation (4)

$$
\frac{m_{1}}{m_{2}}=\frac{r(a+b)}{a} \div \frac{q(a+b)}{b}=\frac{b r}{a q} .
$$

By inspection of Equation (5) it is seen that $m_{1}$ and $m_{2}$ vary as $a$ and $b$, respectively, or

$$
a q m_{1}=b r m_{2} \text {. }
$$

Particular attention is called to this, which is the fundamental principle of the nomograph. The condition, $a q m_{1}=b r m_{2}$, must be satisfied in every case, or any assumed isopleth fails to solve the given equation. If any changes occur, either in $a$ or $b$, the corresponding changes must be made in the other factors. Until the reader has a clear conception of this he cannot make further satisfactory progress.

To illustrate this principle, one example will be worked out in detail. The author refers to a modification of the Williams-Hazen formula as

$$
v=0.0796 d^{0.63} h^{0.54}
$$

For convenience in applying the formula, this will be written,

$$
x=0.0796 y^{0.63} z^{0.54} \text {. }
$$

Using logarithms,

$$
\begin{aligned}
& \log . x=\log .0 .0796+0.63 \log . y+0.54 \log . z \\
& \log . x-\log .0 .0796=0.63 \log . y+0.54 \log . z .
\end{aligned}
$$

$a$ and $b$ may be assumed as having any arbitrary value whatever. In this case, the exponents will be used for such arbitrary values, and it will be assumed that $a=63$ and $b=54$.

Then, from Equation (2),

$$
\log . x-\log .0 .0796=\frac{63}{117} \log . z+\frac{54}{117} \log . y .
$$


Substituting in Equation (5) and solving for $\frac{m_{1}}{m_{2}}$

$$
\frac{m_{1}}{m_{2}}=\frac{b r}{a q}=\frac{0.54 \times 54}{0.63 \times 63}=\frac{29.16}{39.69}=\frac{1}{1.361} .
$$

Draw three parallel lines for the $x, y$, and $z$ scales, respectively, with 63

the distance from $y$ to $x=\frac{}{117}$ of that from $y$ to $z$. If the highest 117

values desired are for $y=60$, and $z=100$, an isopleth may be drawn across these scales near the top (as the line, $A B$, in Fig. 46), and the intersection with $y$ marked 60 and that with $z$ marked 100 . Substituting these values in Equation (6) and solving for $x$, gives $x=12.62$, which value is marked on the $x$ scale, similarly.

Next make a table (Table 1) in order to obtain values for plotting the various scales.

\begin{tabular}{|c|c|c|c|c|c|c|c|}
\hline $\begin{array}{c}y \\
(1)\end{array}$ & $\begin{array}{l}\log . y \\
(2)\end{array}$ & $\begin{aligned} & m_{2} \log \cdot y \\
= & 1.361 \log \cdot y \\
& (3)\end{aligned}$ & $\begin{array}{c}z \\
(4)\end{array}$ & $\begin{array}{c}\log . z \\
(5)\end{array}$ & $\begin{array}{l}m_{1} \log . z \\
=1 \log . z \\
\quad(6)\end{array}$ & $\begin{array}{c}x \\
(7)\end{array}$ & $\begin{array}{c}\log . x \\
(8)\end{array}$ \\
\hline 60 & 1.778 & 2.420 & 100 & 2.000 & 2.000 & 12.62 & 1.101 \\
\hline 50 & 1.699 & 2.312 & 90 & 1.954 & 1.954 & 12.00 & 1.079 \\
\hline 40 & 1.602 & 2.180 & 80 & 1.903 & 1.903 & 11.00 & 1.041 \\
\hline 30 & 1.477 & 2.008 & 70 & 1.845 & 1.845 & 10.00 & 1.000 \\
\hline 20 & 1.301 & 1.770 & 60 & 1.778 & 1.778 & 9.00 & 0.954 \\
\hline 10 & 1.000 & 1.361 & 50 & 1.699 & 1.699 & etc. & etc. \\
\hline 9 & 0.954 & 1.298 & 40 & 1.602 & 1.602 & $\ldots$ & $\ldots$ \\
\hline 8 & 0.903 & 1.228 & 30 & 1.477 & 1.477 & $\ldots$ & $\ldots$ \\
\hline 7 & 0.845 & 1.150 & 20 & 1.301 & 1.301 & $\ldots$ & $\ldots$ \\
\hline 6 & 0.778 & 1.058 & 10 & 1.000 & 1.000 & $\ldots$ & $\ldots$ \\
\hline 5 & 0.699 & 0.952 & 9 & 0.954 & 0.954 & $\ldots$ & $\ldots$ \\
\hline 4 & 0.602 & 0.820 & etc. & etc. & etc. & $\ldots$ & $\ldots$ \\
\hline 3 & 0.477 & 0.649 & $\cdots$ & $\cdots$ & $\ldots$ & $\cdots$ & $\cdots$ \\
\hline 2 & 0.301 & 0.410 & & & & $\because \cdots$ & \\
\hline 1 & 0.000 & 0.000 & 1 & 0.000 & 0.000 & 1.00 & 0.000 \\
\hline
\end{tabular}

Using any convenient scale (as the 20th) plot the values of $y$ from the items in Column (3), and, similarly, with the same scale, plot the values of $z$ from the items in Column (6).

Using the same scale, the values of log. $x-\log .0 .0796$ are plotted as follows:

$$
\begin{aligned}
& \text { log. } 12.62=1.101 \\
& \text { log. } 0.0796=\overline{2} .901=-2+0.901=-1.099 \\
& \text { log. } 12.62-\log .0 .0796=1.101-(-1.099)=2.200
\end{aligned}
$$

The point on the $x$ scale where log. $x-\log .0 .0796=1$ is, therefore, 2200 units beluw the isopleth intersecting the $x$ scale at a value for $x=12.62$; but, as it is necessary to plot values of $x$, and not values of

$x$ the value of log. 0.0796 is subtracted by adding 1.099 to the point $(2,200$ units below the value of $x=12.62)$ where log. $x-\log .0 .0796$ 
$=1$. Marking this latter point 1 , the values are sealed from the items: in Column (8).

In any case, the numerical coefficients of any of the variables may be treated in a similar way. The method of working with exponents has. already been developed quite fully. Fig. 64 is the chart made from the foregoing description.

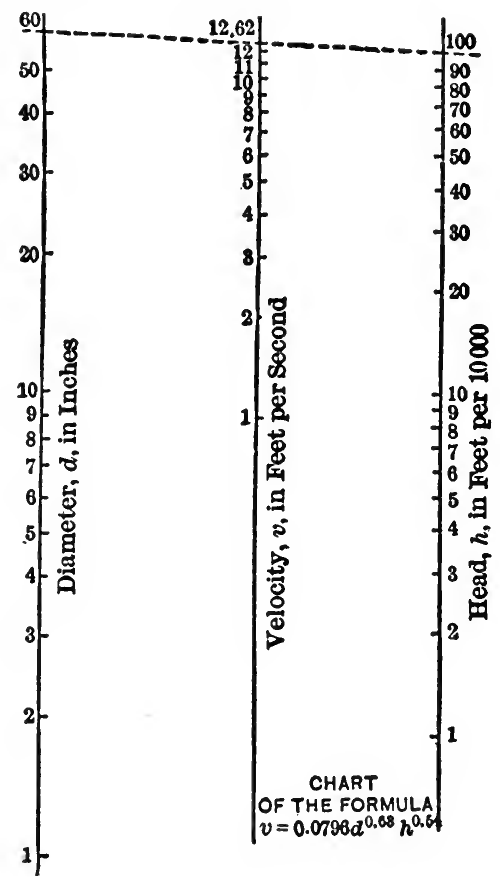

Fig. 64

What Chart to Use. It is not possible to lay down any hard and fast rules for determining what chart is the best for any given problem. Ordinarily that one is the best which will produce the quickest and clearest results. but unfortunately it is not always possible to construct the clearest one in the least time. Experience is the best guide. Generally speaking, a rectilinear chart is best adapted for equations of the first degree, logarithmie for those other than the first degree and not containing over two variables, and alignment charts where there are three or more variables. IIowever, nearly every person becomes more or less familiar with one type of chart and prefers to adhere to the use of that type because he does not care to take the time and trouble to find ont how to nse the others. It is best to know what the possibilities of all types are and to be governed accordingly when selecting one or the other for presenting or working out certain data.

Fins. 65, 66, and 67 are examples of three methods of determining the number of feet, board measure. in timbers. On Fig. 65 it will be noted that each of the curves has a different curvature, necessitating the plotting of several points to determine each one, a laborious task. It re- 
quired 25 mins. to make Fig. 66 on logarithmic paper, the lines all being parallel and at $45^{\circ}$. The alignment chart, Fig. 67, once constructed, is the simplest to use and the time to construct it would depend largely upon one's familiarity with the method. There is considerable computation necessary to determine the proper spacing of the

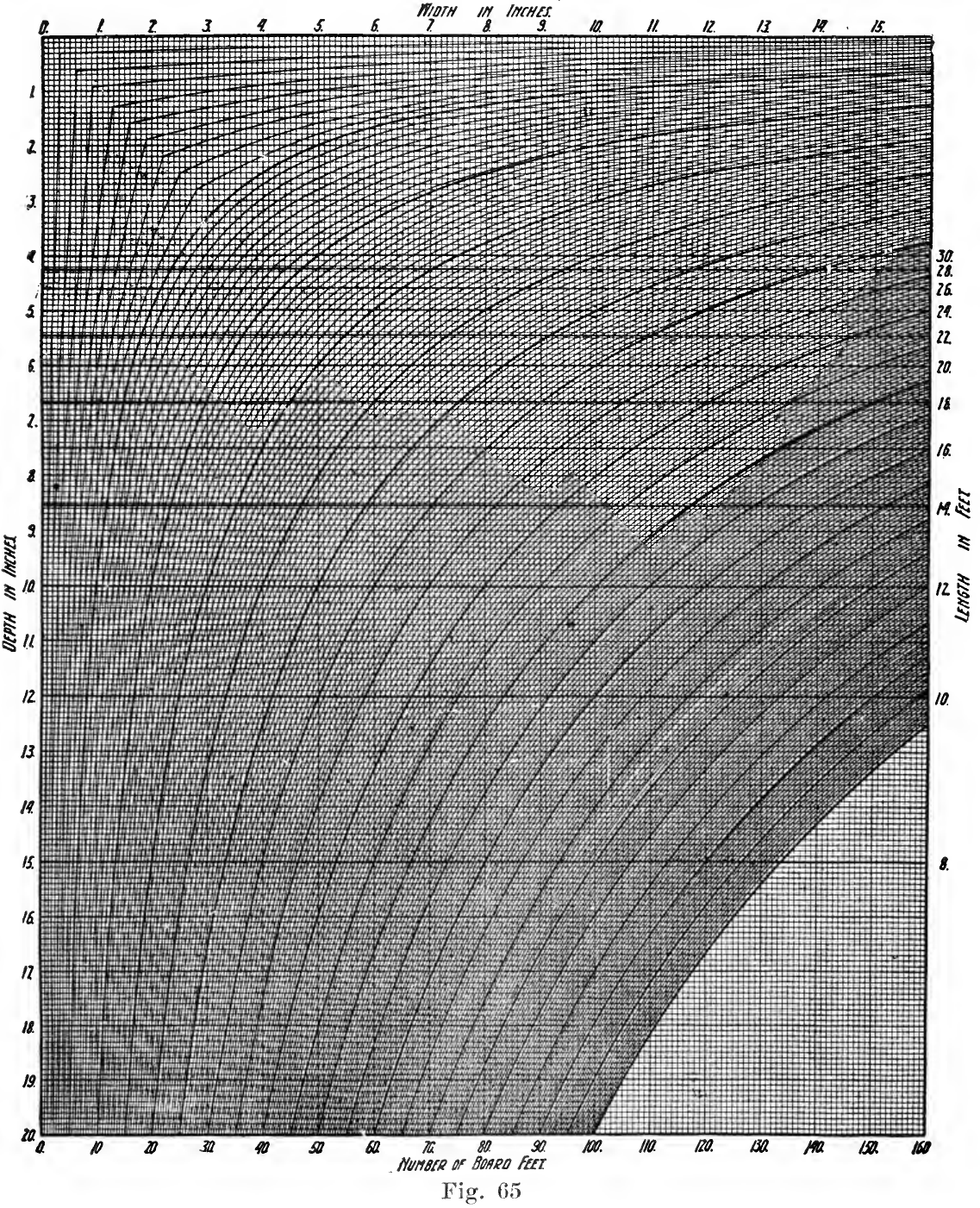

axes, the best selection of the scales and the marking off of the scale divisions after they have been decided upon.

Diagrams for Obtaining Number Feet B.M. in Various Timbers. Fig. 65 and the following explanation of its use are from Engineering and Contracting, November 22, 1916. W. R. Roof is the author. 
Find the number of board feet in a piece of timber 8 in. $x 10$ in. $x 18$ ft. : First, read down from top of diagram on line corresponding to 8 -in. width. Second, read to right from left hand side of diagram on line corresponding to 10-in. depth. Third, from intersection of these two

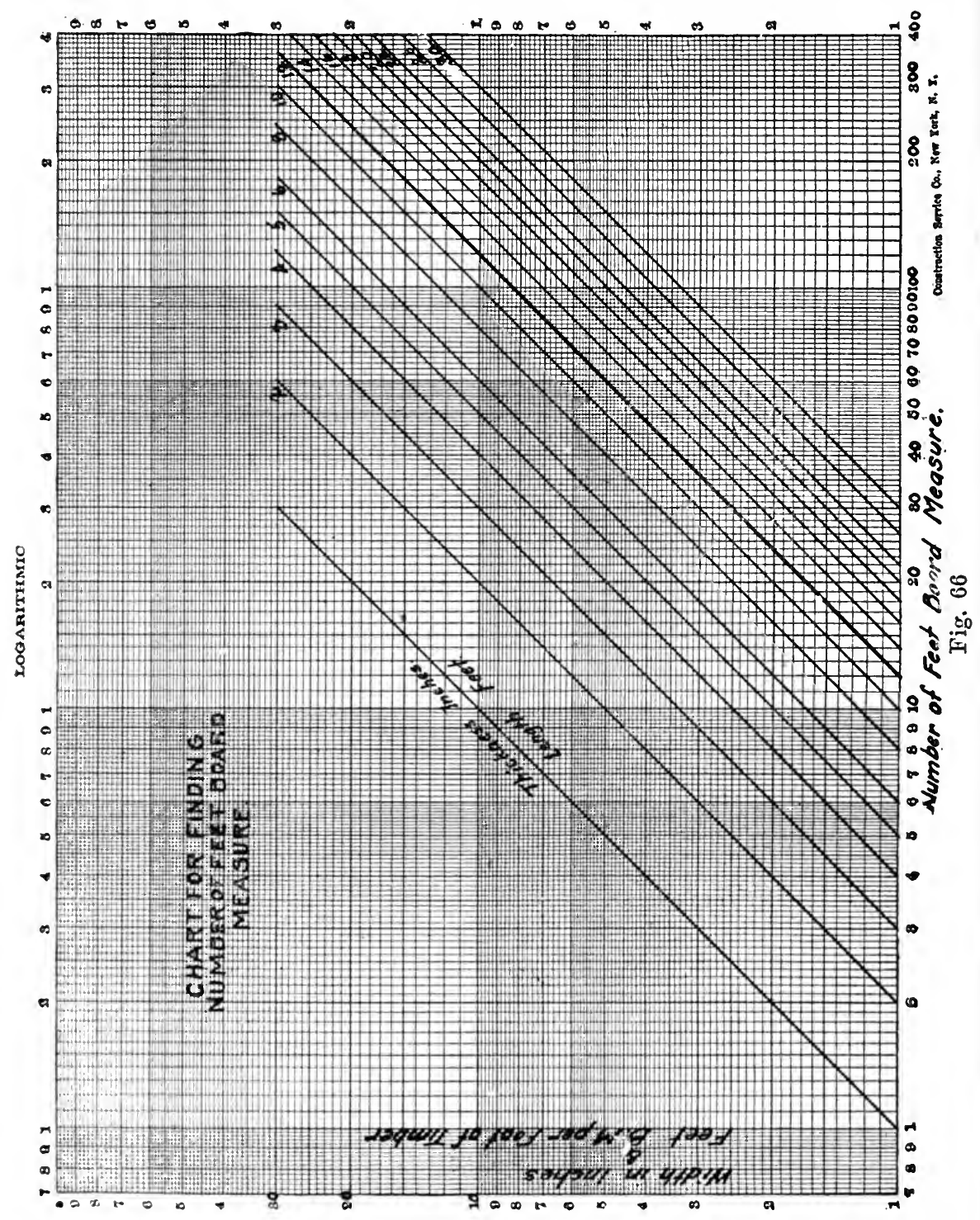

lines, follow curve until it intersects horizontal line eorresponding to 18-ft. Iength. Fourth, from this intersection read downward to bottom of diagram, at which place the number of board feet is found to be 120 . 
Fig. 66 and the description of its use were prepared by James MI. Kingsley. The following formula indicates the steps taken in calculating the number of feet board measure in a timber.

$$
\begin{aligned}
\text { WT } \times \frac{1}{12} \times \mathrm{L} & =\text { Feet B.M. } \\
\text { In which } \quad \mathrm{W} & =\text { width in inches } \\
\mathrm{T} & =\text { thickness in inches } \\
\mathrm{L} & =\text { length in feet. }
\end{aligned}
$$

This is a simple process of multiplication and division and is therefore easily reduced to a straight line chart on logarithmic cross-section paper.

In order to show the manner in which the chart is used, consider a typical case.

Find the number of feet board measure in a timber 8 in. $x 10$ in. $x 18$ ft. long.

Enter chart on left, at point indicating 8 in. width and follow horizontal line to the right to the inclined line for $10 \mathrm{in}$. thickness. From the intersection of these lines follow the vertical line down to the inclined line marked 12 (to divide the product $\mathrm{W} \times \mathrm{T}$ by 12 - read $6.7 \mathrm{ft}$. B.M. on left hand margin). From this intersection follow the horizontal line to the inclined line marked 18 and from this intersection follow the vertical line down to bottom of the chart to find the result, 120 ft. B.M.

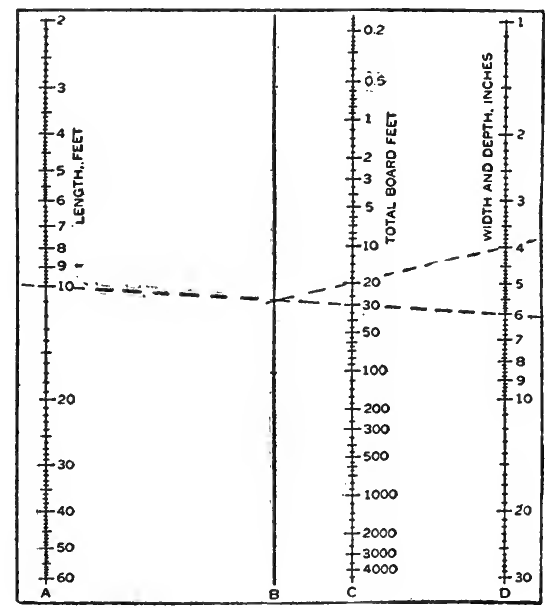

Fig. 67

Fig. 67 with the following are taken from the Woodworker, September, 1917. Lay a straight-edge across the chart twice, as indicated by the dotted lines. and the board feet are found without any multiplying, dividing or bothering with converting inches into feet, etc.

For example, how many board feet in a timber 10 -ft. long, 6-in. wide 
and 4-in. thick? Run a straight line from the 10 (column $A$ ) to the 6 (column $D$ ) and locate the intersection with column $B$. Then from. that point of intersection run over to the 4 (column $D$ again), and the answer (20 board feet) is found in the intersection with column $C$.

If the timber is square in section one operation is sufficient, and the answer is immediately found in column $C$. For example, take a timber 10 -ft. long and 6-in. square. The dotted line drawn from the 10 (column $A$ ) to the 6 (column $D$ ) solves the problem alone, and the answer in column $C$ is, 30 board feet.

Some Uses for the Polar Chart in Computing. The following is from a letter by S. G. Haverstick to Coal Age, July 7, 1917.

I noticed the inquiry of "Surveyor," Coal Age, June 2, p. 970, in regard to the ealculation of room centers when the rooms are turned at an angle with the entry or gangway, and thought it would be of interest to the readers of Coal Age to submit a diagram or chart that $\cdot \mathrm{I}$ designed some years ago for a similar purpose. Since that time it has. been in constant use by the engineers of many coal companies.

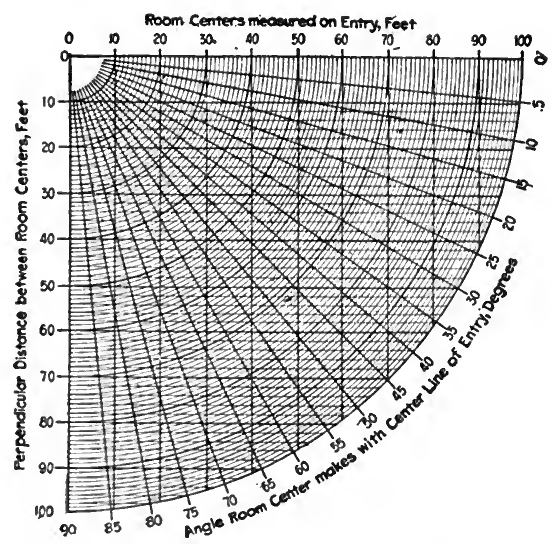

Fig. 68-Handy Chart for Mining Engineers

As shown in Fig. 68, this chart consists of horizontal and vertical lines spaced at regular distances apart and erossed by ares of concentric eircles and diagonal lines, the latter radiating from the common center of the ares and spaced 5 deg. apart, marking angles from 0 to 90 degrees.

This chart can be used in a number of ways, as for instance, where it is necessary to find the projection of a given line on another when the angle between the two is known. Thus, it is useful in finding the latitude and departure for any given course in surveying. It has, besides, a convenient application in ascertaining quickly the distance apart of room centers, measured on the entry, when the rooms make a given angle with the entry, to provide for a certain width of room and pillar.

For example, in the inquiry to which I have referred the distance between.room centers measured on the entry is given as $55 \mathrm{ft}$., and the rooms make an angle of $50 \mathrm{deg}$. with the entry. Hence, to find the 
perpendicular distance between room centers by means of the chart, in this case. follow the radius marked 50 deg. to its intersection with the circle whose radius is $55 \mathrm{ft}$. From this intersection follow the horizontal line to the scale on the left, which shows the perpendicular distance between room centers to be $42 \mathrm{ft}$.

When the desired width of room and pillar and the angle the rooms make with the entry are known, and it is required to find the distance between centers measured on the entry, it is clear that the order just given must be reversed. For example, in this case, first follow the horizontal line corresponding to the given width of room and pillar (42 ft.), as indicated by the scale on the left of the diagram, to its intersection with the radial line marked $50 \mathrm{deg}$. Then, follow the arc of the circle that would pass through this intersection to either scale or the margin of the diagram, and the reading of the scale will be the required distance between room centers measured on the entry.

When surveying a mine the same chart can be used to find the latitude and departure of any course. In that case, follow the radial line marked with the bearing of the course to its intersection with the are indicating the length of the course, and from this intersection follow the vertical and horizontal lines respectively to the scales at the top and the left side of the diagram. The seale at the top of the diagram will show the latitude and that at the left the departure of the given course.

In the solution of any right triangle the diagonal line corresponding to the given angle is the hypotenuse of the triangle, its length being marked by the arc corresponding to the length of the hypotenuse. The two scales at the top and side of the diagram respectively will then give the corresponding lengths of the two sides of the triangle.

The Use of Diagrams in Chemical Calculations. The following article by H. S. Deming, published March, 1916, in the Journal of Industrial and Engineering Chemistry is given as an excellent demonstration of how chart calculations may be utilized.

The use of charts or diagrams for the solution of arithmetical problems is well known to the engineering profession, and several books have been written on the subject. Thus we have the graphical representation of forces and moments, Kutter's formula for the flow of water, indicator diagrams for steam engines, and vector diagrams for the diagrammatic representation or graphical solution of problems in alternating current theory. In metallurgy we have diagrams for the representation of the composition of slags; in chemistry the familiar rectangular and triangular diagrams for the representation of the phase relations between the members of two-component and three-component systems; and, in chemical technology, diagrams for the calculation of mixtures for the manufacture of cement.

In spite of such scattering instances of the use of graphical methods in industrial chemistry, it appears that chemists do not in general take advantage of the really remarkable opportunities that the use of diagrams presents for the quick solution of chemical problems met in everyday work; and but little systematic study of the possibilities of the graphical method in chemistry has ever been published. The diagrams that are presented are selected from among a large number devised by the writer with a view to illustrating some of the principal computations 
that may be solved by graphical means; they indicate at the same time what a great variety of problems are susceptible to such treatment, and what diverse types of diagrams may be used. It is hoped that those presented may suggest others better adapted to the individual needs of the readers of this .Journal ; for this reason it will be necessary to mention the mathematical principles on which the construction of the different types of charts is lased; but, since we are concerned rather with general principles than with details of execution, we can do no more than refer to many interesting charts that differ from those here given in but a few particulars.

It is evident that any relation capable of exact mathematical expression may be given in graphical form. There are, in fact, many methods that might be used in any particular case. Considerations of ease of construction and accuracy of reading must determine which of the different possible charts is the one to be employed; or whether the desired result may not be most easily obtained by calculation with a slide-rule or by direct reading from a table.

The ordinary slide-rule is accurate to within about 1 part in 500, and the ordinary chart to within about 1 part in 250 . The graphical method has the advantage that it ean cover many complicated formulas involving operations impossible with a slide-rule; and a combination of slide-rule and chart will in many cases effect the greatest saving of time. For problems that demand a degree of precision not to be reached by the use of graphical methods, the latter often still afford a quick and convenient means of checking results, a fact which will lead us to consider one or two charts that we should otherwise regard as of little practical use.

The familiar type of diagram, in which two variables are laid off along perpendicular axes on cross-section paper and the relation between them expressed by means of a curve, serves principally for the graphical representation of experimental results, presenting to the eye in an easily comprehensible form a collection of data that could not be so easily interpreted if given in a table. But, as a means of computation, charts of this form are of rather limited use, for the reason that the relation between two variables may generally be expressed most conveniently and accurately in the form of a table. It is only where the precise mathematical relation between two variables is unknown or very complicated, or where more than two variables enter that it may be worth while to construet a diagram to save a part of the labor involved in drawing up a table.

As an example of the simplest chart of this description let us consider the conversion of a temperature reading in Centrigrade degrees into degrees of the Fahrenheit scale, according to the equation

$$
F=9 / 5 C+32 \text {. }
$$

This relation may be expressed as a straight line inclined to the horizontal axis at an angle whose tangent is $9 / 5$, and cutting the vertical axis 32 units above the origin. This chart is shown in Fig. 69, its use being self-explanatory. It is evident that any other relation between two variables that ean be put into the form

$$
y=a x+b
$$


can be expressed in a diagram of this type. In practice such a chart is best constructed by locating two points by calculation, then passing through them a straight line of indefinite length. - The best sort of crosssection paper to use is that having heavily ruled square-centimeter divisions, with square-millimeter subdivisions (the so-called standard millimeter cross-section paper).

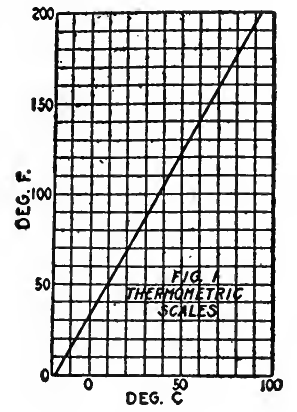

Fig. 69

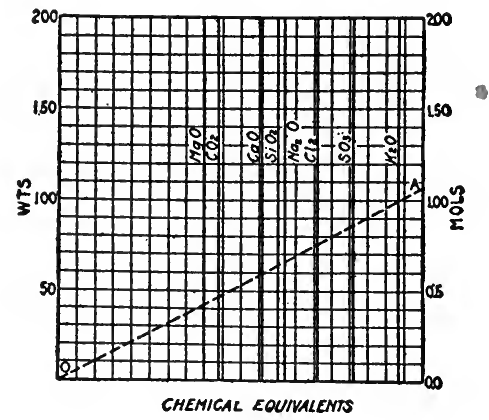

Fig. 70

When the constant $b$ in the above equation becomes zero we have a simple proportionality between the two variables. Problems of this sort are best solved with a slide-rule, where the quantity $a$ is a real constant, invariable from problem to problem. But where $a$ may have different values we make use of one of the types of charts illustrated in Figs. 70 and 71 , which will be called radial charts, because they are characterized by a series of axes and construction lines that radiate from the lower left-hand corner.

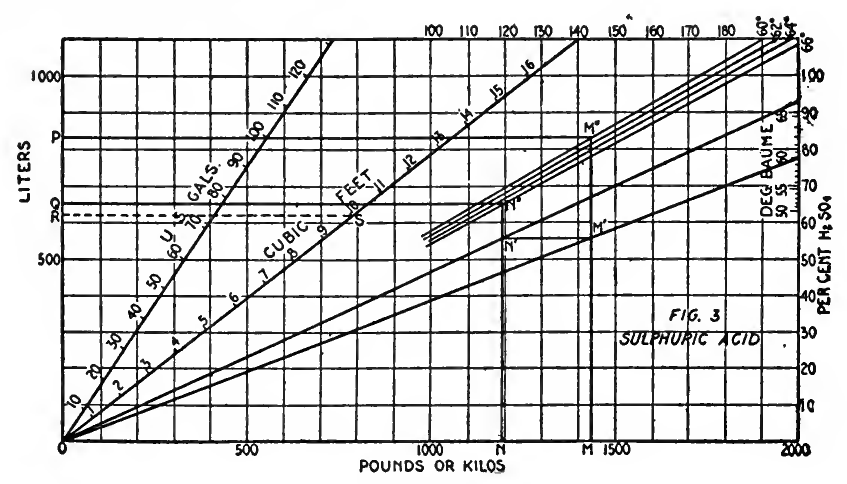

Fig. 71

In Fig. 70 we have a chart for the conversion of a given weight of one compound into the equivalent weight of another. In constructing this chart distances were first measured to the right along the horizontal axis, each equal (in centimeters) to one-fifth the molecular weight of some definite compound. From the points thus located vertical axes were erected and labeled with the formulas of the compound or elements to which they correspond. Thus the axis for magnesium oxide 
is placed $1 / 5 \times 40.32=8.06 \mathrm{~cm}$. to the right of the vertical axis; along the left side of the ehart is laid down a scale of weights, each $\mathrm{cm}$. being: equivalent to $10 \mathrm{mg}$.

If it be required to eonvert $100 \mathrm{mg}$. of potash into its equivalent weight of carbon dioxide, locate a point $A$ on the $K_{2} O$ axis, corresponding to $100 \mathrm{mg}$. wt. Connect this point with the origin of $O$. Notice that the $\mathrm{CO}_{2}$ axis is crossed at an elevation corresponding to $47 \mathrm{mg}$., the equivalent of $100 \mathrm{mg}$. of potash.

An axis at the right-hand margin of the chart, 20 centimeters from. the origin, gives the number of millimols of the substances concerned. Thus, in the problem we have just been eonsidering, the dotted eonstruction line cuts this axis at 1.06 , the molecular equivalent of $100 \mathrm{mg}$. of potash or $47 \mathrm{mg}$. of carbon dioxide. It is apparent that the construction line $O A$ need not actually be drawn, it being sufficient to lay a transparent straight-edge on the ehart to connect the points $A$ and $O$.

A ehart of this kind might be used for calculating the weight of oxygen needed to burn a fuel of a given composition, the weight of silica necessary for combination with a mixture of basic oxides, or in general the weight of any one substance needed to react with definite weights of one or several others. Since the volume occupied by a given weight of a gas is inversely proportional to its molecular weight, we may use the same ehart in ealculating the volumes corresponding to definite weights of different gases, an additional axis in this case being erected $22.38 \mathrm{~cm}$. to the right of the origin, since the gm.-mol. volume of gases is $22.38 \mathrm{l}$., and the oz.-mol. volume (by a happy accident) is very closely $22.38 \mathrm{cu}$. ft. If the weight of the gases be expressed in grams, their volume, read from the volume-axis, will be given in liters; if their weight be given in ounces their volume will be in cubic feet. Each centimeter of distance along the volume-axis will represent 2 units of volume, this being dependent on the scale-units used in graduating the horizontal and vertical axes. A chart derived from that just discussed may be used for converting solutions of one strength of specific gravity into equivalent weights or volumes of another strength. In Fig. 71 weights in lbs. or $\mathrm{kg}$. are plotted along a horizontal axis, and percentages of sulfuric acid along a vertical axis at the right-hand edge of the diagram. Specific gravities or degrees Baumé may conveniently be laid off along the vertical seale as well.

Let it be required to find what weight of acid of $66^{\circ} \mathrm{B}$., corresponds. to $1430 \mathrm{lbs}$. of acid of $60^{\circ} \mathrm{B}$. Draw lines connecting the origin at $O$ with the points corresponding to $60^{\circ}$ and $66^{\circ} \mathrm{B}$. From the point $M$ corresponding to $1430 \mathrm{lbs}$. pass vertically to the $60^{\circ}$ line at $\boldsymbol{I}^{\prime}$, thence horizontally to intersect the $66^{\circ}$ line at $N^{\prime \prime}$, thenee downward to $N$, where we read $1190 \mathrm{lbs}$. of $66^{\circ}$ acid.

A slight modification of this chart will enable us to calculate volumes. $\Lambda t$ the left elge of the diagram lay down a scale of liters, and from the tenth main division of this scale lay off to the right a scale of specific gravities, in such a way that the slopes of straight lines drawn to connect points on the latter seale with the origin will in all cases be equal to the reciprocal of the specific gravity. In the figure, four such lines have been drawn, namely those passing through points on the specific gravity scale corresponding to $60^{\circ}, 62^{\circ}, 64^{\circ}$ and $66^{\circ} \mathrm{B}$. 
Let it now be required to convert $1190 \mathrm{~kg}$. of acid of $66^{\circ} \mathrm{B}$. into liters. From 1190 on the horizontal scale (which in this case must be read in kg. instead of in lbs.) we pass upward to $\mathrm{N}^{\prime \prime}$ on the line drawn through the gravity corresponding to $66^{\circ}$ B., thence horizontally to $Q$, where we read 650 liters, Ans.

Let us consider one more problem, in which 650 liters of acid of $66^{\circ}$ B., are to be converted into liters of acid of $60^{\circ} \mathrm{B}$. From the point corresponding to 650 liters, at $Q$, on the vertical scale, pass horizontally to intersect the $66^{\circ}$ gravity line at $N^{\prime \prime}$, thence vertically downward to intersect the $66^{\circ}$ weight line at $N^{\prime}$, thence horizontally to the $60^{\circ}$ weight line at $M^{\prime}$, thence upward to the corresponding gravity line at $M^{\prime \prime}$, thence horizontally to $P$, where we read 830 liters, $A$ ins.

If weights of acid are not to be considered, but volumes alone, as in the last example, the chart as just described may be made to read in other units than those of the metric system, the vertical scale at the left being simply relabeled gallons or cubic feet. But if weights of acid are to be used in the calculation, it will be necessary to graduate new axes for the U. S. gallons or cubic feet. If the horizontal axis be read in lbs. the vertical axis will give the number of lbs. of water occuying the same volume at $60^{\circ} \mathrm{F}$. Since $623 \mathrm{lbs}$. of water $=10 \mathrm{cu}$. ft., an axis for cu. ft. may be located by drawing through the point $R$, at 623 , a horizontal line to intersect a $10 \mathrm{~cm}$. radius from the origin at a point $S$, which is marked $10 \mathrm{cu}$. ft. The line $O S$ is then drawn and graduated to that scale. An axis for U. S. gals. may be located in a similar way, having given that 833.7 lbs. of water at $60^{\circ} \mathrm{F} .=100 \mathrm{U}$. S. gals.

In certain problems we have to deal with two constituents. For example, we may need to find the $\mathrm{SO}_{3}$ content of sulfuric acid of a given percentage of $\mathrm{H}_{2} \mathrm{SO}_{4}$. This is most conveniently done by graduating the scale at the right of the diagram, on one side with percentages of $\mathrm{H}_{2} \mathrm{SO}_{4}$, and on the other with percentages of $\mathrm{SO}_{3}$. The vertical axis of Baumé degrees is in this case displaced to the right, and graduated by locating the points of intersections of radial lines passing through definite percentages. With such a chart we can calculate the amount of water to be added by fuming acid in order to convert it into commercial acid of any desired strength.

This type of chart might be used to calculate the amount of water to be added to a solution of definite strength to produce a solution of another strength, for the difference between equivalent weights of two solutions must represent the weight of water to be added to the stronger one. Charts of this type have also been devised for calculating the amount of filler to be added to a fertilizer to reduce it to a desired percentage of a single element; for determining the amount of water to be removed by evaporation during the concentration of a solution from a lower to a higher density; and for calculating the proportions in which two raw materials need to be mixed in order to produce a mixture containing a given percentage of some one constituent. But, since problems of this sort may be solved somewhat more conveniently by means of other charts to be described later, no description of these diagrams need be given.

It is worth while noting that radial charts, such as those described 
above and illustrated in Figs. 70 and 71, may be used for the solution of any problem, in which the relation

$$
x / a=x^{\prime} / a^{\prime}
$$

obtains, $x$ and $x^{\prime}$ being distances along the horizontal axis, and $1 / a$ and $1 / a^{\prime}$ being constants proportional to the slopes of the radiating lines. The eonstants $a$ and $a^{\prime}$ should have unchanging values in all the problems that are to be solved by the use of a given ehart, since in this case we may draw the radiating lines once for all when the chart is first constructed. If $a$ and $a^{\prime}$ are variables, we are foreed to draw new lines with slopes respectively proportional to $1 / a$ and $1 / a^{\prime}$ for each problem to be solved, or to draw in the beginning a great number of radiating lines, and afterward resort to interpolation. For this reason a set of computations in which the constants $a$ and $a^{\prime}$ vary from problem to problem is best earried out by a modification of the "figure-four" chart to be described later.

The principles involved in the preceding diagrams may frequently be applied in the solution of rather complex formulas. As an example, let us eonsider that for the constant of dissociation of a gas yielding two gaseous products of dissociation:

$$
k=\frac{x^{2}}{(1-x) V} .
$$

In Fig. 72 the values of $x$ are laid down along the horizontal axis and the curve $O A B$ drawn through the calculated values of $x^{2}$. The values

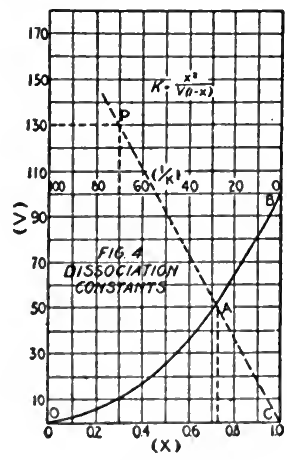

Fig. 72

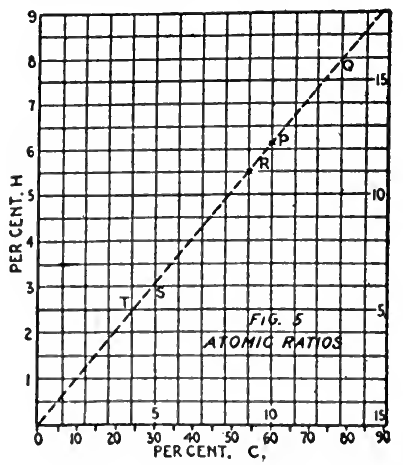

Fig. 73

of $V$ are then laid down along the vertical axes and the values of the reciprocal of $k$ from right to left along a horizontal axis. Given $72 \%$ dissociation and a volume of 130 calculate the constant of dissociation. Enter the diagram at 72 on the base-line, pass upward to cross the curve at the point $A$. Connect $A$ with $C$ and produce the line thus obtained until it euts the horizontal line passing through 130 units of volume, at $P$. Passing downward we read the value of $1 / k$, viz., 70 , from which $k=0.143$. Viee versa, if we have a volume of 130 units and know that the value of $1 / k$ is 70 , we locate the point $P$ correspond- 
ing to these data and draw the line $P C$; from the point $A$ where this line cuts the curve, we pass vertically to the base line, where we read $72 \%$ dissociation.

Of course in practice it is unnecessary to draw the construction lines in this and similar diagrams. If the edge of a transparent triangle is passed through the desired points or, better yet, a straight line scratched on the other side of a sheet of celluloid, it will be possible to make readings directly. The horizontal scale through the middle of the diagram might also have been graduated directly in values of $k$, instead of in reciprocal values, by laying down graduations proportional to the reciprocal of the numbers that appear in Fig. 72 ; but scales of reciprocals have the disadvantage that their divisions are not equal, hence there is a loss of accuracy in interpolation. For this reason it is better to read the values of $1 / k$ directly, and convert them into values of $k$ by means of a slide-rule or table of reciprocals.

Closely related to the preceding type of chart is one in which the composition of a compound or mixture is expressed by the rectangular co-ordinates of a point in a plane or in space. As an example, let us take a chart for the determination of the ratio of the number of atoms of carbon and hydrogen in an organic compound, having given the percentage of each of these elements (Fig. 73).

Along the vertical axis of the diagram is laid down a scale of hydrogen, in such a way that the percentages of that element in the compounds to be considered fall within the limits of the chart. Along the horizontal axis is laid down a scale of percentages of carbon, in such a way that $11.9 \%$ of earbon is the same linear distance as $1 \%$ of hydrogen, since the atomic weight of carbon is 11.9 (on a basis of $\mathrm{H}=1$ ). The horizontal and vertical divisions of the chart are also marked 5, 10, 15, etc., to indicate the relative number of atoms of each element.

Let us suppose that a given compound contains $60.0 \%$ of carbon and $6.1 \%$ of hydrogen. Locate the point $P$ corresponding to this composition and lay a straight edge through $O P$. Where this line happens to pass through the corner of a square may be read the atomic ratio. It will be seen that there are four corners, $Q, R, S$, and $T$, which very nearly concide with the given line. $Q$ corresponds with the ratio $\mathrm{C}_{13} \mathrm{H}_{16}, R$ with $\mathrm{C}_{9} \mathrm{H}_{11}, S$ with $\mathrm{C}_{5} \mathrm{H}_{6}$, and $T$ with $\mathrm{C}_{4} \mathrm{H}_{5}$. Which of these formulas is the correct one will of course need to be determined from other considerations; for the errors of organic combustion methods are of such a magnitude as to leave the matter of a formula more or less in doubt, unless the percentages of the elements other than carbon and hydrogen be taken into consideration.

Another method of working this same problem is to convert the percentage of hydrogen found into its equivalent percentage of carbon by means of the radial diagram of Fig. 70. The ratio between this number and that giving the percentage of carbon actually found is then taken by means of a slide-rule. The chart just described may of course be modified for use with any other pair of elements.

In Fig. 74 we have a diagram for the adjustment of various raw materials to give a mixed fertilizer containing desired percentages of nitrogen and phosphorus. Percentages of nitrogen are laid off along the horizontal axis, and percentages of phosphoric acid $\left(\mathrm{P}_{2} \mathrm{O}_{5}\right)$ along a 
vertical axis. Suppose that we have to prepare a mixed fertilizer containing $10 \%$ nitrogen and $3 \% \mathrm{P}_{2} \mathrm{O}_{5}$ (point $\boldsymbol{M}$ ) and that there are available for the purpose the four fertilizers whose compositions are expressed by the four points, $A, B, C$ and $D$, together with filler, which, since it contains zero per cent of the elements of plant food, is of course represented by the point $O$. It will first be noticed that the point $M$, representing the required composition of the mixed fertilizer, lies within a triangle formed by comnecting up the points $A, B$ and $C$; this means that it is possible to secure the desired mixture by a combination of these three raw materials. On the other hand, a triangle obtained by comnecting the points $A, C$ and $D$ does not include the point $M$; it is therefore impossible to produce the desired mixture by any combination of these three raw materials alone, in spite of the fact that some of the raw materials contain more and others less nitrogen or phosphoric acid

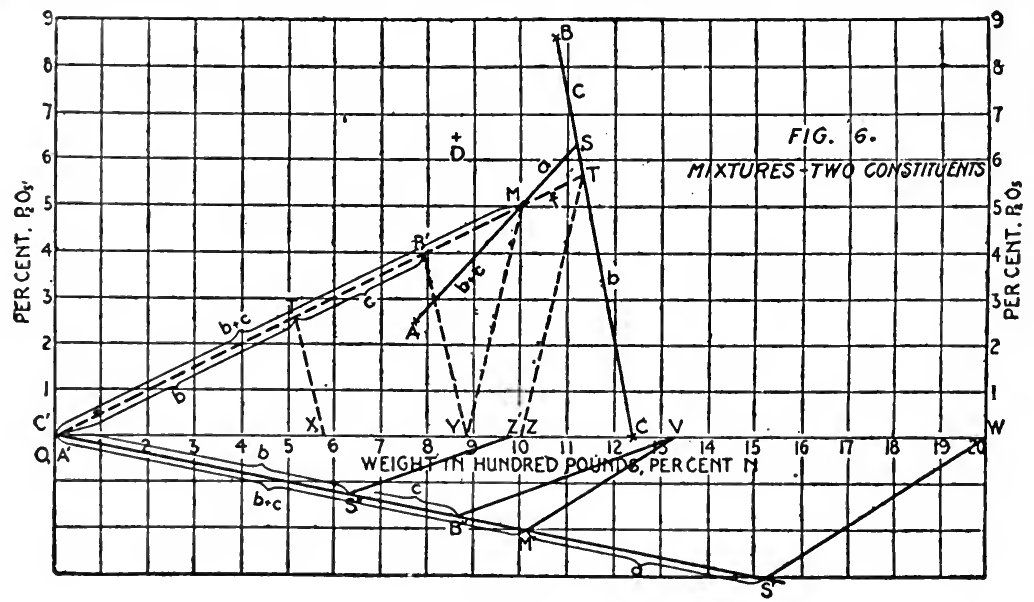

Fig. 74

than the mixture. The first use of the chart we have constructed is therefore to tell us which of the various raw materials placed at our risposal may be used in making up a mixture of the desired composition.

Now suppose that it is desired to make up $1,000 \mathrm{lbs}$. of the mixture having the composition denoted by the point $M$, using for the purpose the raw materials $B$ and $C$ and filler. Connect $B$ and $C$, and draw from the point $O$, representing filler, a line passing through $M$, which is produced until it euts the line $B C$ at $T$. From $T$ draw the line $T Z$ to the point representing $1.000 \mathrm{lbs}$, the weight of mixture to be made. Draw the line $M Y$ parallel to $T Z$ to intersect the base line at $Y$.

That portion of the line $B C$ most distant from $B$ is marked $b$, and that most distant from $C$ is marked $c$; in the same way that portion of () $T$ most distant from $O$ (filler) is marked $f$, while that most distant from $B$ and $C$ is marked $b+c$. The distances $O B^{\prime}$ and $O T^{\prime}$ are laid off equual to $C B$ and ('T', respectively and the segments thus found are markerl $b$ and $c$ according to the portion of $B C$ to which they corresnomd. Noxt connect $B$ ' with the point $Y$, previously located, and draw $T^{\prime} X$ parallel to $B^{\prime} Y$. The distance $O X$, lying under the segment $b$ of 
the line $O T$, is the amount of raw material $B$ needed to make up the fertilizer; the distance $X Y$, lying under the segment $c$, is the amount of $C$ to be taken; while the distance $Y Z$, lying under the segment $f$, is the amount of filler needed.

If for any reason it had been decided to make up the mixed fertilizer from $A, B$ and $C$, without using any filler, the construction lines needed would have been those shown full length in the diagram to distinguish them from the broken construction lines of the preceding problem. Draw the line $A M$ through $M$, the point representing the composition of the desired mixture, and produce it to intersect $B C$ at $S$. Since the line $A S$ does not pass through $O$, it will be necessary in this case to draw an auxiliary line $A^{\prime} S^{\prime}$ making any convenient angle with the base

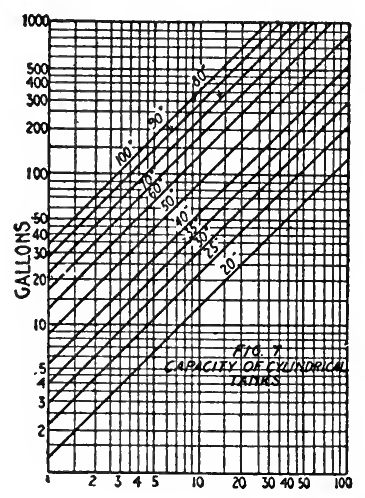

Fig. 75

line. Along this line take $A^{\prime} M^{\prime}$ any convenient multiple of $A M$ (say three times $A M$ ), and $A^{\prime} S^{\prime}$ that same multiple of $A S$. If $2,000 \mathrm{lbs}$. are to be made, connect $S^{\prime}$ with the point $W$ representing that weight, and draw $M^{\prime} V$ parallel to $S^{\prime} W$. The segments $M S$ and $M^{\prime} S^{\prime}$ are labelled $a$, and the segments $A M$ and $A^{\prime} M^{\prime}$ are labelled $b+c$. The distances $C S$ and $C B$ are transferred to $O S^{\prime \prime}$ and $O B^{\prime \prime}$, and the construction finished by drawing the parallel lines $B^{\prime \prime} V$ and $S^{\prime \prime} Z^{\prime}$. The distance $O Z^{\prime}$, under the segment $b$ of the line $C^{\prime} S^{\prime}$, represents the amount of raw material $B$ to be taken; the distance $Z^{\prime} V$ represents the amount of $C$; and the distance $V W$ represents the amount of $A$ that will be needed.

The preceding chart may be adapted very readily to the preparation of mixtures of any sort in which definite percentages of two different components are to be secured. If three different components are to be considered, the same chart may be used provided that the sum of the three adds up to $100 \%$, or a slight modification of the chart, which cannot be presented here, will serve for more complex cases. If each of the raw materials contains but a single one of the constituents to be considered in the final mixture, the problem becomes simply one in inverse proportion, and may be most simply solved by means of a slide-rule. It is worth mentioning that another modification of the chart last described will permit one to calculate the proportions in which feeding- 
stuffs need to be mixed in oräer to form a standard ration according to any one of the several systems in use.

Our next elass of charts includes those plotted on logarithmic erosssection paper, namely, on that in which the graduations along the perpendicular axes are proportional to the logarithms of the variables, instead of to the variables themselves. This paper may be obtained from dealers in draftman's supplies.

Since the equations

$$
y=a x \text { and } x / a=x^{\prime} / a^{\prime}
$$

may be put in the logarithmic form

$$
\log y=\log a+\log x \text {, and, } \log x-\log a=\log x^{\prime}-\log a^{\prime},
$$

it is possible to construct logarithmic cross-section eharts to solve the same problems as the radial charts of Figs. 70 and 71 . There is, however, nothing important to be gained by such a procedure, and there is even some loss of aecuracy in so doing, since interpolations are less easily made on logarithmic cross-section paper.

The most important use of the logarithmic charts is in the solution of equations of the general form

$$
y=a^{\mathrm{m}} x^{\mathrm{n}} ;
$$

but in equations in which several factors are to be multiplied together such charts are very convenient, providing that a reading to the nearest 2 or $3 \%$ is accurate enough for the purpose in hand. In Fig. 75 is a chart for the eapacity of eylindrical tanks in U. S. gallons, the diameter of the tanks and the depth of the liquid being given in inches. In this case the formula used is

$$
\mathrm{C}=\frac{\pi d^{2} h}{4 \times 231} .
$$

where $d$ is the diameter of the tank in inches, and $h$ is the depth of the liquid it contains. Putting this into the logarithmic form we have

$$
\log C=\log \frac{\pi d^{2}}{924}+\log h .
$$

Note that all the constants in the equation are combined with a single variable $d$, the variable $h$, representing the depth of liquid in the tank, being kept free of constants.

To construet the chart work out the values of $\frac{\pi d^{2}}{924}$ by means of a sliderule for all the values of $d$ that are likely to be used, and lay off the results along the vertical logarithmic seale. From the points thus located draw a series of lines sloping upward toward the right at an angle of $45^{\circ}$ with the horizontal, and label each with that value of $d$ to which it corresponds. Alome the horizontal axis lay down a logarithmic seale of depths (values of $h$, in inches) and graduate the vertical axis in gallons. 
Logarithmic cross-section paper is very useful in recording results that would extend over too great a range if plotted on ordinary crosssection paper; for example, in the plotting of absorption spectra, the co-ördinates representing respectively logarithms of vibration frequencies and millimeters length of the absorbing layer of solution. In certain work experimental results plotted on logarithmic cross-section paper fall very nearly on a straight line, where ordinary cross-section paper would give a curve; this happens, for example, in a study of the volumes of filtrate delivered by a filter-press in varying periods of time. If an empirical equation exists connecting the variables in such a set of experimental data, a logarithmic chart will frequently reveal its form and the values of some of the constants that appear in it much more readily than they may be obtained in any other way.

An equation of the general form

$$
a x+b y=u / v
$$

may be treated by means of a chart we are about to discuss, which has two parallel axes intersected by a third in such a way as to remind one of a "figure-four."

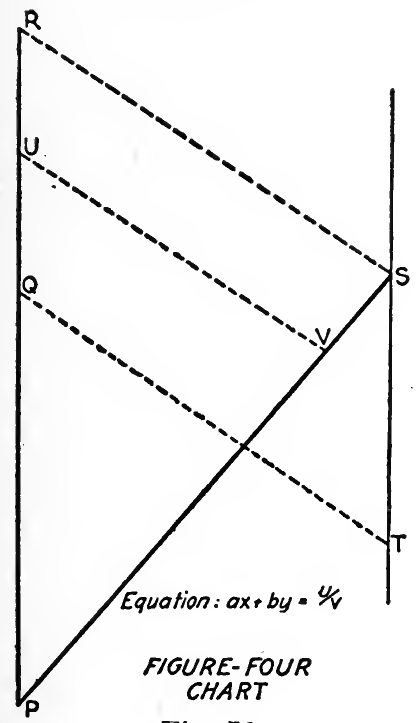

Fig. 76

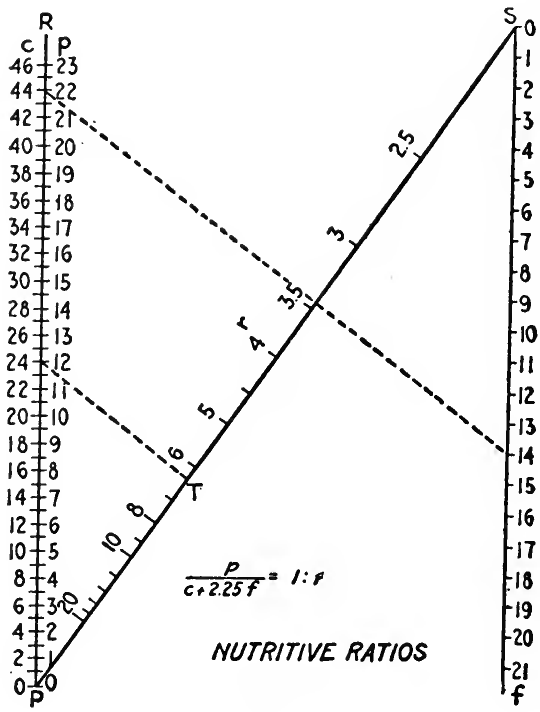

Fig. 77

In Fig. 76 the axes of the chart are $P R, P S$ and $S T$. Let the distance $P Q=a x$; let $T S=b y$; let $P U=u$; and let $P V=v, U V$ and $R S$ being drawn through $U$ and $S$ parallel to a line connecting $Q$ and $T$. We have then $Q R=T S$, whence $P R=a x+b y$. From similar triangles we have then

that is,

$$
P R: P S=P U: P V ;
$$

$$
a x+b y=u / v,
$$

if the distance $P S$ be taken as unity. 
As a first example of the application of this chart let us consider the equation for finding the nutritive ratio of a foodstuff, e.g.,

$$
\frac{p}{c+2.25 f}=\frac{1}{r}
$$

Putting this in the standard form for the type of chart we are discussing, we have

$$
c+2.25 f=\frac{p}{1 / r} .
$$

To construct this chart (Fig. 77) lay down a scale of percentages of digestible earbohydrates along the axis $P R$, in such a way that the highest percentage met will fall within the limits of the chart. Draw an inclined axis making any convenient angle with the axis just drawn, and take $P S$ a measured distance equal to, say, 30 centimeters. From $S$ lay down a scale of percentages of digestible fat, and graduate it downward, using a scale unit $\frac{1}{2.25}$ times that used in graduating the scale of digestible carbohydrates.

On the opposite side of the axis $P R$ from the graduations representing pereentages of digestible earbohydrates, lay down a scale of digestible protein. If the unit of this scale be taken as $n$ times the unit of the carbohydrate scale, then unit distance along the inclined axis will be $n$ times the distance PS. In this particular ease it will be convenient to have the divisions of the protein scale twice as long as those of the carbohydrate scale; the unit distance along $P S$ will then be $2 \times 30=60$ $\mathrm{cm}$. Now since the quantity $1 / r$ appears in the equation above in place of the variable $v$ in the general equation of this type of chart, we must take the reeiprocals of the various values of $r$, thus getting a series of fractions $1 / 3,1 / 4,1 / 2$, etc. Since the unit distance along $P S$ is $60 \mathrm{~cm}$. the point corresponding to the fraction $1 / 3$ will be $20 \mathrm{~cm}$. from the left end of the inclined axis. This point is marked with the value of $r$, which is of course 3 , since $1 / r$ is $1 / 3$. In a similar way locate other points corresponding to other values of $1 / r$ and mark them with the values of $r$. A skillful combination of slide-rule and engincer's scale will enable any one to graduate this part of the chart in a few minutes.

To use the chart lay down the edge of a draftsman's triangle to connect the percentages of digestible earbohydrates and fat (say 44 and $14 \%$, respectively). Slide the triangle along the edge of another triangle until its ruling edge passes through the percentage of digestible protein $(\operatorname{say} 12 \%)$. The place where this edge of the triangle cuts the inclined axis (at $T$ ) gives the second term of the nutritive ratio, which in this case is read $1: 6.3$.

Another way to use this chart is to construct it on paper thin enough to be transparent to a series of heavily ruled narallel lines on a sheet placed beneath it. The chart is moved until one of these parallel lines, seen through the transparent paper of the chart, connects the percentages of carbohydrate and fat; then another parallel line, passing through 
the percentage of protein, is followed up until it intersects the inclined axis, where the nutritive ratio is read.

A chart very similar to that just described might be constructed for calculating the rate of transfer of heat from circulating hot water to iron, according to the equation

$$
\frac{H}{t}=\frac{1.67+\sqrt{v}}{0.02},
$$

where $H$ is the number of calories of heat transferred in one second to a square meter of surface, $t$ is the temperature difference between water and metal (degrees C.), and $v$ is the velocity of the water in centimeters per second.

In Fig. 78 we have a chart for converting specific gravities greater than unity into degrees of the Baumé scale. In this case the inclined

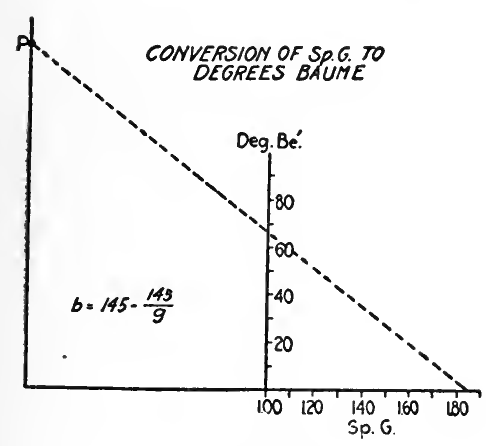

Fig. 78

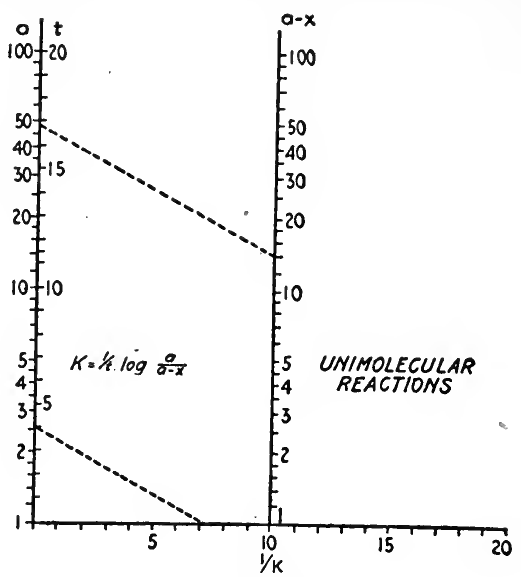

Fig. 79

axis of the chart has been made horizontal, though this need not necessarily be done. A point $P$ is located on the left axis 145 units above the horizontal axis; the right-hand axis is graduated in the same scale units as those used in locating $P$, while the horizontal axis is graduated in specific gravities, the distance between the two vertical axes being taken as unity. A line connecting the point $P$ with the observed specific gravity will intersect the vertical axis in the corresponding number of the Baumé scale. The equation for this chart is

$$
145-b=145 / g,
$$

the values of $b$ being laid off upward along the right-hand axis, because of the negative sign of $b$ in the equation.

The equation for the constant of a unimolecular reaction may be put into the form

$$
\log a-\log (a-x)=\frac{t}{1 / k}
$$


which is evidently one eapable of being solved with the help of a chart of the kind we have been considering. In Fig. 79 the scales for $a$ and $a-x$ are logarithmie, while those for $t$ and $1 / k$ are not. Of course the values of $k$ corresponding to the different values of $1 / k$ might have been calculated and laid down on the chart, but in practice it will be found more eonvenient to convert values $1 / k$ read from the ehart into values of $k$ by the use of a slide-rule or table of reciprocals. The equation for the constant of a bimolecular reaction may be worked out with this same chart, the values of $a(b-x)$ and $b(a-x)$ being read from a slide-rule before the chart is entered. A diagram of this sort is probably the quickest means of determining the order of a reaction being investigated for the first time.

As a final example of a chart of this class, consider that in Fig. 80 for the representation of the composition of furnace slags. This chart

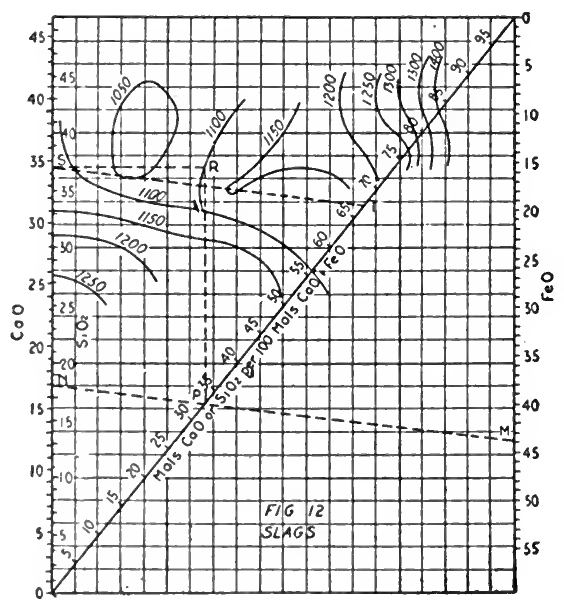

Fig. 80

is construeted on cross-section paper, each main (centimeter) division of the cross-sectioning representing $2 \%$ silica in the slag, or some equally convenient figure. A scale of percentages of silica is plotted just within the left-hand border of the chart.

.Just outside the scale of silica lay down a scale of lime, such that the point corresponding to $30.15 \%$ silica $\left(\mathrm{SiO}_{2} \div 2=30.15\right.$ ) coincides with the point corresponding to $28.04 \%$ lime $(\mathrm{CaO} \div 2=28.04)$. At the right-hand side of the chart construct a scale of ferrous oxide, letting $35.92 \% \mathrm{FeO}$ occupy the same linear distance as $30.15 \% \mathrm{SiO}_{2}$.

As an example of the use of the chart let us suppose that a slag is found to have the percentage composition $\mathrm{FeO}, 44: \mathrm{CaO}, 17 ; \mathrm{SiO}_{2} .37$; other substances, 2 . Required its ehemical formula and approximate melting point. Connect the point $\boldsymbol{M}$, representing the ferrous oxide, with the point $N$, representing the lime; at $P$, where the inclined axis is crossed, we read "33) molecules of $\mathrm{CaO}$ in the slag for 100 molecules of $(\mathrm{FeO}+\mathrm{CaO}) . "$ From a point, $S$, representing the silica in the slag, draw a line, $S T$, parallel to $M N$. The point $T$, where the inclined 
axis is crossed, will give the number of molecules of $\mathrm{SiO}_{2}$ for 100 molecules of $(\mathrm{CaO}+\mathrm{FeO})$. In the present case there are 33 molecules of lime, 67 molecules of ferrous oxide, and 67 molecules of silica for 100 molecules of basic oxides, hence the formula of the slag is very closely

$$
\mathrm{CaO} .2 \mathrm{FeO} .2 \mathrm{SiO}_{2} \text {. }
$$

This chart gives also the melting points of slags of various compositions. Thus, if we pass vertically upward from $P$ to meet the horizontal from $S$ at the point $R$, we find ourselves on the contour line marked $1100^{\circ}$, the melting point of the slag in question. The diagram shows, moreover, that a slight increase in the percentages of ferrous oxide and silica at the expense of the lime would result in a slag of lower melting point.

In the case of highly acid slags the point $T$ will lie so far to the right as to fall entirely without the limits of the diagram. In this case it is best to enter the chart with half or one-fifth of the actual percentage of silica, the molecules of silica per 100 molecules of basic oxides read from the chart being multiplied by 2 or 5 , respectively. Melting points are in this case best gotten by consulting a separate set of melting-point curves, which are always more accurately read than contour lines. The latter have, moreover, the disadvantage of being too much dependent on the judgment of the person who first plots them from experimental data; yet for rough approximations within a limited range, the melting point contour lines shown in the diagram will be found very useful.

Another class of charts closely related to the one just described is that for dealing with mixtures. Many special cases occur, according to whether the composition of the final mixture or either of its constituents is constant or variable in composition; according to whether results are to be calculated to a basis of a fixed weight of mixture or of one of its constituents; and according to whether or not different ingredients are to be reduced to a basis of chemically equivalent quantities, etc. Lack of space prevents us from giving more than three of the many interesting charts that might be presented here, each with its own special advantages.

In Fig. 81 is a chart for preparing mixtures for the manufacture of cement from clay and limestone, assuming the content of alumina and silica in the raw materials to be so nearly constant as to permit the mixture being controlled by determinations of calcium carbonate alone. To construct this chart draw two vertical axes, any convenient distance apart, and graduate them, both downward, in percentages of calcium carbonate, choosing such a scale unit that the percentages of calcium carbonate likely to be met in the clay or cement rock (left axis) and in the limestone (right axis) fall within the limits of the chart. It is necessary to use the same scale unit in graduating both axes, but neither scale needs to be carried upward to zero, nor is the relative position of the lower limits of the two scales a matter of any importance. If the final mixture is to be adjusted to $75 \% \mathrm{CaCO}_{3}$, connect the points marked $75 \%$ on the two seales, and divide the inclined axis thus gotten into twenty equal parts, each representing $100 \mathrm{lbs}$. of limestone in one ton of final mixture.

As an example of the use of this chart, suppose that we have a cement 
rock containing $58 \% \mathrm{CaCO}_{3}$, and a limestone containing $97 \% \quad \mathrm{CaCO}_{3}$. Connect these two points, best by means of a straight line scratched on the lower surface of a sheet of transparent celluloid. Where the inclined scale is crossed is read $875 \mathrm{lbs}$., the weight of limestone to be contained in one ton of the mixture.

Not only may diagrams similar to that just described be used to ealculate the proportions in which two raw materials of variable composition need to be mixed in order to give a mixture of constant composition, but the converse problem may be solved: if the ingredients of a,mixture are of constant composition the proportions in which they are present in a given mixture may be readily determined. Thus, if a sample of red lead is assumed to consist of a mixture of litharge and lead dioxide (disregarding the possible presence of other oxides of lead) the percentage of each may be gotten from a determination of the per-

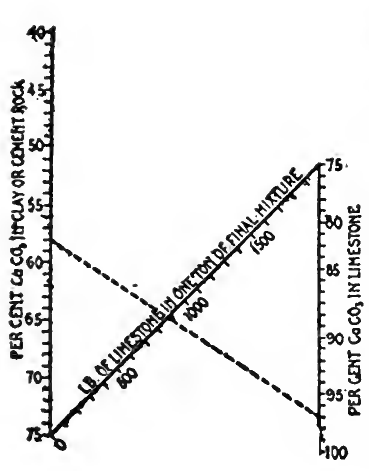

Fig. 81

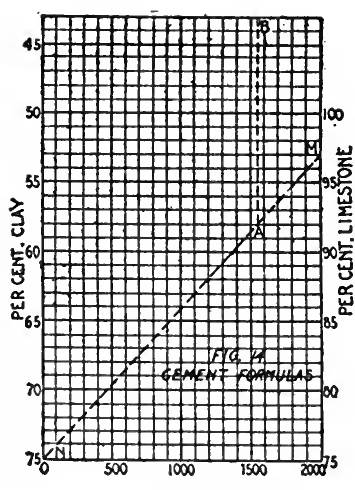

Fig. 82

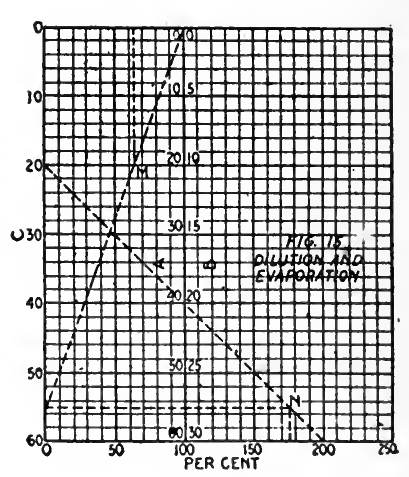

Fig. 83

centage of lead in the mixture, points representing the perentages of lead in litharge and in lead dinxide, respectively, being located on separate axes and connected by a line, which is graduated from left to right in pereentages of that oxide whose composition has been located on the right-hand axis. By connecting points on the two vertical axes representing the percentage of lead found in a given sample of red lead, the percentage of litharge and of lead dioxide may be read directly from the chart. The reader will have little difficulty in adapting this chart to other cases of indirect analysis.

Returning now to a consideration of the preparation of mixtures of limestone and clay for the manufacture of cement, if we wish to calculate the weight of limestone to be taken, not for a ton of mixture as hefore, but for a ton of clay, we have the diagram shown in Fig. 82 . Notice that here the percentages of ealcium carbonate are laid down along vertical axes, starting from a horizontal base-line, the graduations of one seale rumning downwarl, and those of the other upward. To use this chart pass a straight line through the point denoting the percentage of calcium carbonate in the limestone $(M)$ and that denoting the percentage of calcium carbonate in the final mixture $(N)$. This line intersects a horizontal line (of the cross-sectioning) passing through the 
percentage of calcium earbonate in the clay at some point $A$. From this point pass upward to the point $B$, where we read the number of pounds of limestone that will be needed for one ton of clay. Thus, if the clay contains $58 \% \mathrm{CaCO}_{3}$ and the limestone $97 \% \mathrm{CaCO}_{3}$, there will be needed $1,535 \mathrm{lbs}$. of limestone for every ton of clay. If the percentage of calcium carbonate in the clay is so low that there will be needed more limestone than clay, the cross-sectioning of the chart may be carried further to the right; or the problem may be so changed as to calculate the number of pounds of clay to be used for one ton of limestone.

In Fig. 83 we have a chart for determining the number of pounds of water that must be added to a given weight of solution of definite specific gravity or percentage strength in order to produce a definite diluted solution; or the number of pounds of water that must be evaporated from a given solution in order to produce a more concentrated one.

The chart is constructed with three vertical axes, $A, B$, and $C$, the unit of scale $B$ being twice that of scales $A$ and $C$. The horizontal distance between the axes $A$ and $C$ is divided into 100 equal parts representing percentages of evaporation or dilution. Since dilution may amount to more than $100 \%$ the graduations are carried out to the right of scales $A$ and $B$, say to $250 \%$.

As an example of the use of this chart, let us suppose that a sugar solution of $20^{\circ}$ Brix is to be evaporated to a syrup of $55^{\circ}$ Brix. Connect this point on scale $C$ representing $55^{\circ}$ Brix with the zero of scale $A$ (representing water). At the point $M$, where the horizontal through $20^{\circ}$ Brix is crossed, we read $64 \%$ evaporation.

Conversely, if $64 \%$ of water were to be evaporated from a solution of $20^{\circ}$ Brix, locate the point $M$ where the vertical through $64 \%$ intersects the horizontal through $20^{\circ}$ Brix (scales $A$ and $C$ ). Connect the point $M$ with the zero of scale $A$, and produce the line thus gotten until it cuts the $C$-scale at $55^{\circ}$, the required Brix of the syrup.

In a similar way we might calculate the amount of water that would need to be added to a syrup of $55^{\circ}$ Brix to dilute it to $20^{\circ}$ Brix. Note that in this case the result is the number of pounds of water to be added for every 100 pounds of diluted solution.

If it is desired to calculate the dilution for 100 parts of syrup as is generally the case, instead of for 100 parts of diluted solution, a somewhat different method is used. Thus, if we wish to determine the water that would have to be added to a solution of $55^{\circ}$ Brix in order to produce one of $20^{\circ}$ Brix, connect the points representing $20^{\circ}$ Brix on scales $B$ and $C$. Where this line cuts the horizontal through $55^{\circ}$ Brix (scale $A$ ) we read 175 , the number of parts of water to be added to one hundred parts of syrup by weight).

Since scale $A$ is used only in problems dealing with dilution, it may be omitted from the chart if only evaporation is to be considered. In any case it is better to letter scale $A$ in red ink, since there is otherwise some danger of interchanging seales $A$ and $B$ in reading the chart. The reader will perceive that a chart of this sort may be used for diluting concentrated solutions of any kind from one percentage strength to another. If specific gravities or degrees Baumé are to be used the scales 
are graduated, first in percentages, the percentage graduations being erased after a scale of specific gravities or degrees Baumé has been laid down. It is only with sugar solutions and the Brix scale that the percentage strength of the solution will be directly given by the areometer realing. If volumes of solutions are to be brought into consideration in dilution problems, a modification of the diagram given in Fig. 71 will be found to offer the most convenient solution.

The next type of chart to be considered is that in which three variables are comnected by the equation

$$
a x+b y=c z .
$$

This chart consists of three parallel axes (Fig. 84), the left-hand one being for the values of $x$, the right-hand one for $y$, and the middle one for $z$. To construct this chart draw the left and right axes exactly parallel, at any convenient distance apart. Graduate the left axis in values of $x$ at the rate of $A$ units per centimeter beginning the graduations at any convenient point on the axis, $A$ being so chosen that the scale for $x$ and the scale for $z$ (to be graduated afterward) will not be inconveniently long or short. In a similar way graduate the right axis at the rate of $B$ units per centimeter. Now it may be shown by geometry that if each value of $z$ is to lie in the same straight line with corresponding values of $x$ and $y$ (on the other two axes), then the distance $m$ from the left axis to the middle axis must be such that

$$
m=\frac{b B n}{a A+b B},
$$

where $n$ is the distance between the two outside axes; and that the middle axis must be graduated with values of $z$ at the rate of $C$ units per centimeter, where

$$
C=\frac{a A+b B}{c} .
$$

The chart is used by lining up corresponding values of $x, y$ and $z$ by means of a straight edge, whence the name alinement chart.

In Fig. 85 we have a chart for determining the milligrams of sodium oxide and of potassium oxide, respectively, shown by an analysis in which the two metals are first weighed as chlorides, the potassium being afterward collected and weighed as potassium chloroplatinate. Since the factor for converting potassium chloroplatinate into potassium chloride is 0.3068 , and that for converting sodium oxide into sodium chloride is 1.583 , we have

$$
0.3068 x+1.583 y=z,
$$

where $x$ is the number of milligrams of potassium chloroplatinate obtained, $y$ is the number of milligrams of sodium oxide in the sample, and $z$ is the combined weight of sodium and potassium chloride.

Tet the ontside axes be taken $20 \mathrm{~cm}$. apart. (iraduate the left axis at the rate of $5 \mathrm{mg}$. of potassium chloroplatinate per centimeter. The right axis is to be graduated in milligrams of sodium oxide at the rate 
of $2 \mathrm{mg}$. per centimeter, beginning with $20 \mathrm{mg}$. (if that is the least weight of sodium oxide that is likely to be met in any of the analyses). The distance from the left axis to the middle axis is then

$$
m=\frac{2 \times 1.583 \times 20}{5 \times 0.3068+2 \times 1.583}=13.47 \mathrm{~cm} .
$$

Locate the midle axis at this distance to the right of the left axis. Now $40 \mathrm{mg}$. of sodium chloride are equivalent to $40 \div 1.583=25.25 \mathrm{mg}$. of sodium oxide. Therefore lay a straight edge to connect the zero of the

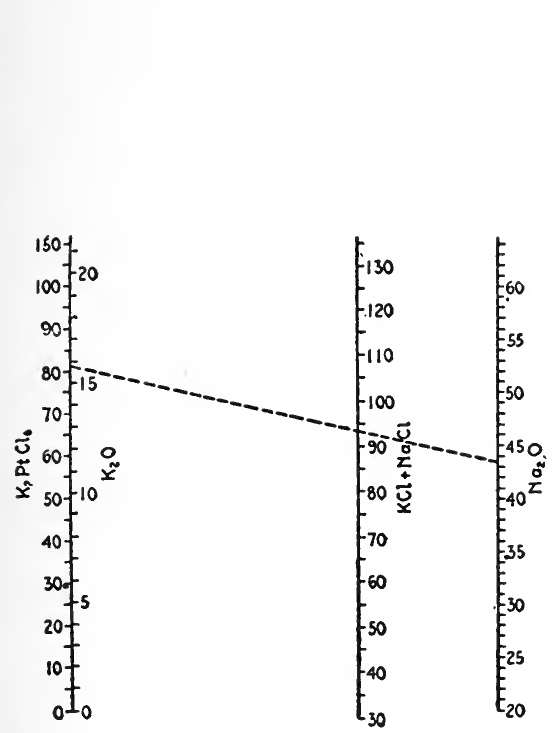

Fig. 85

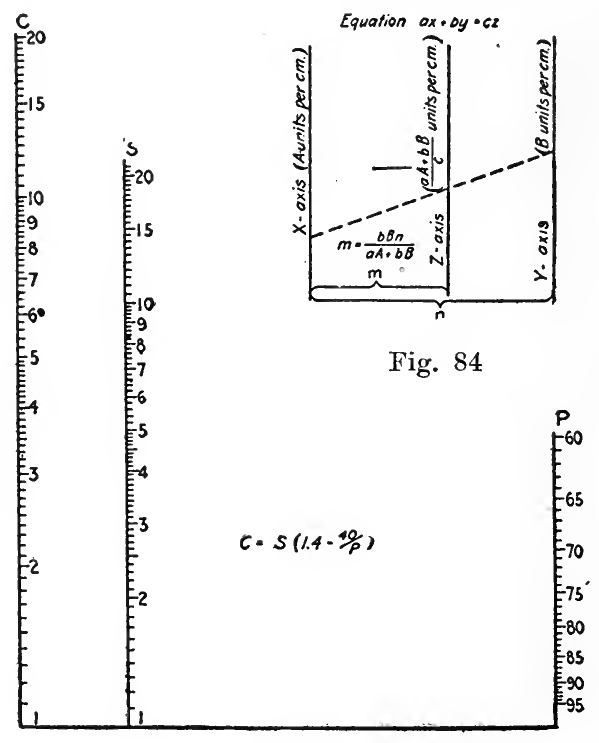

Fig. 86

potassium chloroplatinate scale with 25.25 of the sodium oxide scale. The point where the middle axis is crossed is marked 40. In the same way $100 \mathrm{mg}$. of sodium chloride correspond to 63.16 parts of sodium oxide; therefore connect the zero of the potassium chloroplatinate scale with 63.16 on the sodium oxide scale, and mark the point where the middle axis is crossed 100. The distance between the points 40 and 100 on the middle axis is then divided into sixty equal parts and the graduations continued upward and downward to the limits of the chart.

As an example of the use of the chart, let us suppose that a mixture of the chlorides of sodium and potassium weighs $94 \mathrm{mg}$. and that 81.8 $\mathrm{mg}$. of potassium chloroplatinate are obtained. Connect these points on the middle and left axes, respectively, by a straight edge; where the latter intersects the right axis is read $43.5 \mathrm{mg}$. of sodium oxide, while just within the left margin of the chart we read $15.9 \mathrm{mg}$. of potassium oxide. The scale of potassium oxide here referred to is constructed by dividing the space between 0 and 100 of the potassium chloroplatinat ${ }^{\text {n }}$ 
scale into 19.38 equal parts, sinee the factor for converting potassium chloroplatinate into potassium oxide is 0.1938 .

Of course the preceding chart might have been constructed with any other rate of graduation for the outside axes, the middle axis being placed accordingly, and either of the outside axes might have been begun at any convenient point. When one point of the middle axis has been located by calculation, the other graduations may be laid down by the method just described; or, if the caleulated value of $C$ in the equation above happens to be a round number all the divisions of the middle seale may be laid off directly with an engineer's rule. This type of ehart may be used in a great many indirect analyses; for example, those in which the percentage of each of two metals in a mixture of their carbonates is determined from the volume of carbon dioxide that the mixture evolves on being treated with an acid; or those in which the amount of each of several gases in a mixture is determined from the decrease in volume observed and carbon dioxide formed when the mixture is burned. A modification of the ehart in Fig. 81 will frequently serve the same ends. Other uses for the alinement chart are in caleulating the formulas of isomorphous mixed minerals from analytical data, in caleulating heat exchange and loss by radiation in evaporation problems, and in finding the amount of a given substance necessary to react with a mixture of two others.

Fig. 86 is an alinement chart for determining the crystallizable sugar in sugar-cane juice, according to the formula

$$
C=S(1.4-40 / P),
$$

where $C=$ per eent crystallizable sugar; $S=$ per cent sucrose; and $P$ $=$ per cent purity of the juice. Putting this in logarithmic form we have

$$
\log S=\log C-\log (1.4-40 / P),
$$

Plot values of log. $C$ along the left axis, letting unit logarithmic distance equal 25 centimeters $(A=1 / 25)$. Plot values of $-\log (1.4-40 / P)$ along the right axis, letting unit logarithmic distance equal 100 centimeters $(B=1 / 100)$. Since the quantity $(1.4-40 / P)$ is less than unity, its negative logarithm will be positive, and is hence measured upward along the right axis. If the total distance between the outside axes is $20 \mathrm{~cm}$., the middle axis will need to be $4 \mathrm{~cm}$. from the left axis. The micldle axis is graduated at a rate which may be calculated to be $1 / 20$ unit per centimeter (from the general equation for the value of $C$ in the discussion above of the principle of the alinement chart).

This chart is given, not so much because of its practical importance, as because it illustrates as well the way in which a very complicated equation may often be solved by the alinement chart method. In practice it would be easier to calculate erystallizable sugar from another formula, in which the Brix of the solution is used instead of its purity, e. g.,

$$
C+0.4 B=1.4 S \text {. }
$$

This may be eharted at once by the alinement method, without recourse to logarithms, or even as a figure-4 chart. 
Other types of charts might be described, but it will be found that in practice the ones here given will serve for the solution of almost any problem that does not demand greater accuracy than that of which the graphical method is capable. The different types of charts are all interrelated, and there are so many intermediate types that the classification here adopted is but a very arbitrary one. A single chart may

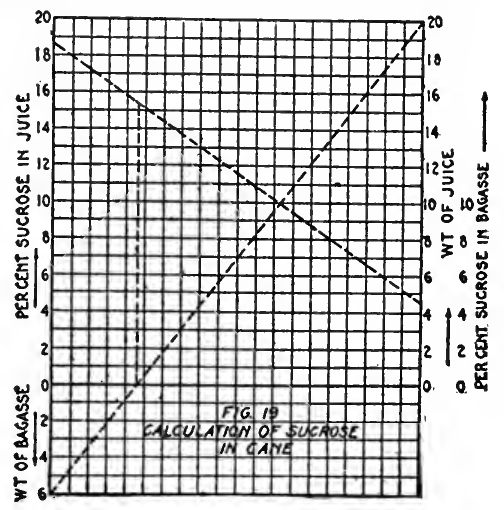

Fig. 87

ilustrate at once the principles of several different types, so that we have what may be termed combination charts, illustrated in Figs. 87 to 89 .

Fig. 87 is a chart that is a combination of an alinement chart with a mixture chart, for calculating the percentage of sucrose in sugar cane, having given the weight and composition of the bagasse and expressed juice (dry grinding). It is worth noting that this chart may be used

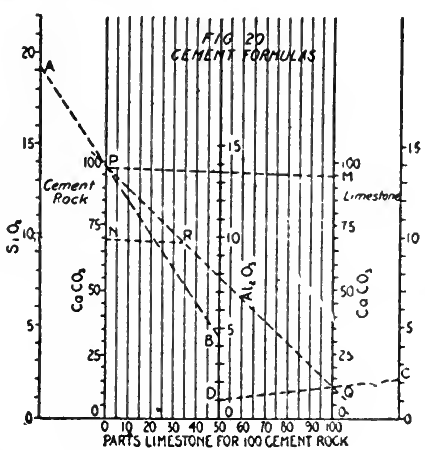

Fig. 88

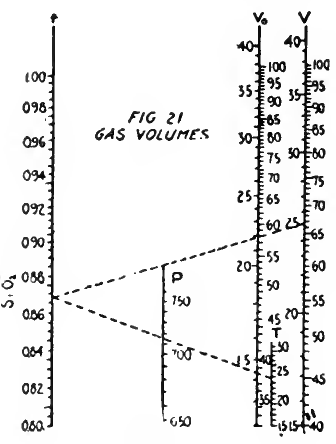

Fig. 89 .

to solve the most general case of mixtures, namely, that in which two constituents of varying composition are mixed in any proportion to give a final mixture of varying weight whose composition is to be calculated.

In Fig. 88 is a combination of two alinement charts with a mixture chart for calculating the number of pounds of limestone to be added 
to one ton of cement rock to prepare a mixture for the manufacture of eement, aceording to the formula of Meade, which demands $90 \%$ of the calcium oxide that would be needed to form tricalcium. silicate and triealeium aluminate with the silica and alumina in the raw materials.

In Fig. 89 is a double logarithmic alinement chart for reducing the volumes of gases from one temperature and pressure to another. It might easily be constructed for problems in which the temperature is expressed in degrees Fahrenheit, and the pressure in inches of mercury.

Solution of Pulp Problems by Graphic Methods. W. J. MeCauley, in Engineering and Mining Journal, July 17, 1915, gives the following:

In many hydrometallurgical plants the importance of making accurate pulp measurements and keeping a systematic record of them is too often overlooked. The importance of such observations can hardly be underestimated, as they are essential to the proper working of any mill. The excuse for their absence usually is that no one on the job has time to look after the work, and as the mill is apparently running pretty well as it is, there seems to be no real need of keeping such records. Most mill men will admit, however, that if such information is systematically kept through several years, it is valuable and often a money maker. The trouble encountered in the taking of such measurements and the difficult nature of the subsequent calculations are sometimes causes for inattention to such details.

It is the custom in most mills where such observations and measurements are taken to first determine the constants of the ore treatedthe density and such others as the practice of the mill and the systems of measurement make possible. These eonstants are substituted in the various formulas as one of the three factors of pulp problems. The second quantity is found by measurement and the formula is solved for the third.

It is not the purpose of this article to give methods for making pulp measurements or to derive the formulas neessary for the solution of the problems. This subject has already been thoroughly diseussed.

The drudgery of solving each problem every day is eliminated by solving onee all the problems concerning a eertain measurement which are likely to occur in practice. Tables or curves from which the desired results of any observation may be read directly are then made. Such conveniences are somewhat diffieult to formulate becanse to have them aceurate it is necessary to solve substantially every problem within the range of the table or enrve. This is usually a task of no small proportions, and should one of the previously determined eonstant quantities become changed at any time, the labor must all be repeated.

With the idea in view of rendering the making of such eharts simpler I have undertaken to produce a method of graphic analysis for the solution of pulp problems, and to make a system of straight-line charts which may be drawn in a few minutes and with little or no calculation.

The basie principles of this system were sugogested in an artiele published by (ieorge B. MeLain in the Mining and Scientific Press, Ang. 3, 1912. In this article the method of making and using a straightline chart for the determination of the pereentage of solution and solids in mill pulps is explained: the density of the ore being a constant and the gravity of the pulp being known. 
The equations may be divided into two general classes: First, those in which it is possible to assume one quantity as constant so that when a second is made to vary by equal increments the third will also be found to vary by equal increments. Equation (1) is of this character. If in this equation either $s$ or $x$ is held constant it will be found that if $y$ is varied by equal amounts, then will $x$ or $s$ also vary by equal amounts.

$$
y=s x
$$

The second class consists of those equations in which the above relationship does not exist. In this case it is impossible to select any one value as constant so that if a second be varied by equal amounts the third will also vary by equal amounts. Equation (2) is of this nature.

$$
y^{2}=s x^{2}
$$

'The distinction between these two cases is really that of degree. The equations of the second class are formed usually not by the occurrence

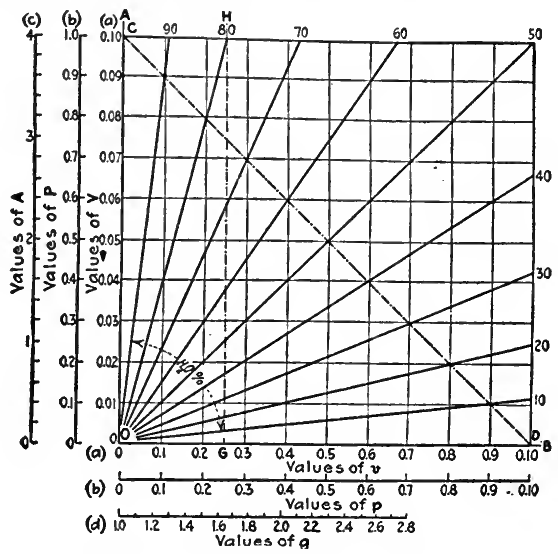

Fig. 90-Solving Pulp Problems

of the higher powers of the three variables, but by the way in which these quantities are related to one another and to the constants of the equation.

Let us now consider the nature of such equations and methods for their graphical solution, following up by some practical examples of their applications.

Formula (1) is the equation of a straight line passing through the origin, in which $s$ is the tangent of the angle that this line makes with the axis of $x$. Such a problem may be solved for all varying values of $x$ and $y$, when $s$ is a constant, by simply drawing on cross-section paper a straight line through the origin of points, which makes an angle with the axis of $x$ whose tangent is $s$. A series of such lines radiating from the origin may be drawn to represent varying values of $s$, as in Fig. 90 . All problems of this nature may now be solved by inspection of the chart.

One or two such practical pulp problems may be worked out. In 
explaining these charts there will be no attempt to make mathematical proofs of their eorreetness or the formulas used in making them.

The following symbols will be used throughout:

$a=$ percentage of water by weight, in any pulp, expressed as a. decimal.

$c=$ a constant.

$d=$ density of the dry pulp or ore.

$g=$ gravity of the wet pulp.

$p=$ number of pounds of eyanide ( $\mathrm{KCN}$ or $\mathrm{NaCN}$ ) per ton of solution.

$P=$ number of pounds of cyanide per ton of solids.

$R=$ water ratio.

$t=$ a length of time measured in seconds.

$T=$ tons of dry pulp discharged in $24 \mathrm{hr}$. by a pulp stream.

$v=$ dissolved metal ( $\mathrm{Au}$ or $\mathrm{Ag}$ ) per ton of solution in any wet pulp.

$V=$ Dissolved metal per ton of solids in any wet pulp.

The dissolved metal in discharged tailings is usually expressed as a. value in cents per dry ton of pulp discharged, the value per ton of solution and the pereentage of solution being known. This is aecomplished by taking a sample of the tailings and determining the percentage of moisture by the gravity bottle, or other eonvenient method. The slime is allowed to settle from the solution and a known amount. is decanted and assayed. The following formula is then employed:

$$
V=\frac{a v}{1-a}
$$

This is of the form of equation (1). Here $s=\frac{a}{1-a}$, and a chart, similar to the one above described, may be used.

To make this chart lay off along $O B$, Fig. 90, to any convenient scale, values of $v$, and to the same scale lay off along $O A$ values of $V$. Now draw a $45^{\circ}$ line $C D$ and divide it into 100 equal parts. Through each of these points of division and a draw diagonal lines as shown. Number these lines from 0 to 100 starting with $O B$ as 0 and ending with $O A$ as 100 . These numbers and the lines to which they are attached represent the varying precentages of water or solution in a pulp.

This method of drawing in the diagonal lines is evidently eorreet. In formula (3) let $V$ and $v$ be made to vary in such a manner that their sum is always a constant. The equation representing these changing values is $V+v=c$. This is the equation of a $45^{\circ}$ line intersecting $O A$ and $O B$ in points other than $O$, such as $C I$. It will now be found that $a$ varies by equal increments.

To use the chart, follow vertically up the line representing the value of $v$ found by assay until it intersects the line representing the percentage of water previously determined. Thence follow horizontally to. the left. On $O A$ will be found the value of $V$ sought.

In practice it is desirable to assay more than one assay ton of the decanted solution from the pulp, and it is usually desired to express $V$ 
in terms of cents per ton. In this case it is better to lay off values of the actual weights of gold or silver found in milligrams along $O B$, and to lay off values in cents per ton along $O A$. Here $C D$ will probably not be a $45^{\circ}$ line, but will be one connecting two points, $C$ and $D$, which have values such that

$$
\frac{O D}{x}=\frac{O C}{y}
$$

where $x$ is the number of assay tons of the solution taken for assay, and $y$ is the value in cents per ounce of gold or silver.

It sometimes happens that the pulp is too thick to make practicable the method just described of direct decantation and assaying of solution for the value of $v$. For notes on a method recommended in such cases, and the explanation of a chart for the calculations of same, the reader is referred to the Engineering and Mining Journal of March 27, 1915.

The cyanide loss in tailings is usually expressed as a number of pounds per ton of dry pulp discharged. The number of pounds per ton of solution is found by titration and the following formula applied:

$$
\frac{a p}{1-a}=P
$$

Formula (4) is of exactly the same nature as formula (3) and the same chart, Fig. 90, may be used for both.

It is often desired to know the water ratio of a pulp; that is, the number of tons of solution per ton of solids. The water ratio of any pulp is expressed by the formula:

$$
R=\frac{a}{1-a}
$$

Now, as has been shown, the quantity $\frac{a}{1-a}$ has the value of $s$ in the equation of a straight line passing through the origin. It is the tangent of the angle which the diagonal line makes with the axis of $x$, or in Fig. 90 with the line $O B$. Hence the water ratio is the tangent of the angle which the percentage water line makes with the line of zero percentage water, or $O B$ of Fig. 90. This being the case we may lay off on $O A$ values of $R$, and to the same scale lay off $O G$, on $O B$, equal to unity, and draw $G H$ parallel to $O A$. Now the percentage water lines will cross $G H$ on that horizontal line corresponding to the desired value of $R$.

A chart similar to the water ratio chart may be used to determine the number of cubic feet of solution per ton of dry pulp and many other similar problems.

The tonnage measurement of pulp streams of nearly constant volume, such as tube-mill discharges, offers a considerable problem to the metallurgist. Such measurements are usually made by taking the time 
required for the stream to fill a given volume, such as one cubic foot. The following formula may then be applied:

$$
T=\frac{2700 d(g-1)}{t(d-1)}
$$

in which $t$ is the time in seconds required to fill one cubic foot of volume. $2700 d$

For any ore $d$ is a constant and hence the quantity $\frac{2700 d}{d-1}$ is a constant. Onee $d$ is known for the given ore the formula becomes:

$$
T=\frac{c(g-1)}{t}
$$

This equation is a rather special ease of equation (1), but it can be solved by the same general methods.

Referring to Fig. 91, on $O B$ lay off values of $T$ to some convenient. scale, starting at $O$ as zero, and on $O A$ lay off values of $g$, starting with.

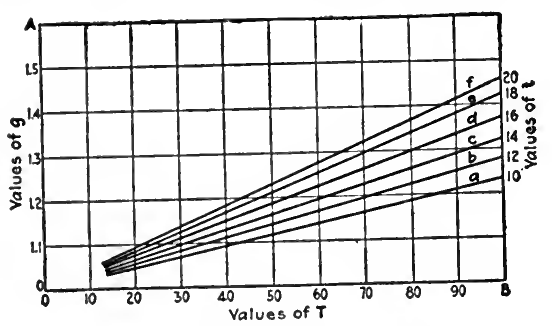

Fig. 91-Chart for Pulp-Stream Measurements

$O$ as the point where $g=1$. Or, which is the same thing and practically more handy, OA may read the actual weights of the eubic-foot volume taken, starting at $O$ with the weight of the container when full of water.

Diagonal lines, similar to those of Fig. 90, may now be drawn representing values of $t$, so that the horizontal line representing the value of g, or weight found, will intersect the diagonal representing the observed time of filling $(t)$ vertically above the desired value of $T$.

Now if $g=1$, $\mathrm{T}$ will equal zero and it follows that all the $t$ lines intersect at $O$. If $t$ is equal to zero then will $T$ equal infinity except for the case where $g$ is equal to unity ; here $T$ is equal to zero divided by zero which is an indeterminate and may have all values from zero to infinity. Hence the line representing $t$ equals zero and coincides with $O H$. If $t$ equals infinity, then will $T$ equal zero, and the line representing $t$ equals infinity and coincides with $O A$. All other values of $t$ are diagonals lying between $O A$ and $O B$.

The $t$ lines are all straight lines; becanse if $t$ be held constant and $g$ varied by equal increments, then will $T$ vary by increments. If $g$ is held constant and $t$ is made to vary by equal amounts, then will $T$ vary along a curve. Practically the best way to draw in the $t$ lines is to solve the equation for each value of $t$ liable to oceur in the practice for which 
the chart is made, holding $g$ constant. By this method a series of other points besides $O$, such as $a, b, c, d$, etc., on each desired $t$ line, is found. It now only remains to draw in the $t$ lines and number them.

In the second case the three quantities going to make up the equation bear such a relation to one another that if any one of the three quantities be held constant and a second varied by equal amounts, the third would be found to vary by unequal amounts, or along a curve. In problems of this kind it is impossible to construct a simple chart on the lines previously outlined, for we have no longer the simple equation of a straight line, but that of a curve. It may, however, be solved by a straight-line graphic method by drawing in two sets of diagonal lines, radiating from different points, so as to cross each other. These sets of lines represent two of the three values of the equation, while ordinates or abscissas represent the third. The lines representing any two of these factors meet on that point where the proper value of the third factor, necessary to balance the equation, meets them both.

There is at least one pulp problem of prime importance which falls in this class. By referring again to Fig. 90 it may be discussed.

This problem was solved graphically in a very ingenious way by Mr. McLain. My method is similar, but in many respects the reverse of that given by McLain. My three quantities entering into this equation are $a, b$ and $g$. The relationship between them is expressed by the formula:

$$
a=\frac{d-g}{g(d-1)}
$$

which is evidently of the form of the second case.

On a chart constructed in accordance with Fig. 90, lay off on $O B$ to some convenient scale, the density, $d$, of the ore. Let this be $O F$. Now on $O A$ lay off, to the same scale, $O E$ equal to the gravity of the solution; that is, unity as a rule. Divide $O F$ into equal parts varying from unity at $O$ to $d$ at $F$. These divisions represent the gravities, $g$ of the pulps which are possible from the ore whose density is $d$. Draw $E F$. Now when $g$ is determined by the gravity bottle or other method, the vertical line representing this value of $g$ intersects $E F$ at the same point as does the diagonal line representing the percentage of solution, $a$, sought.

This last described chart, giving the relation between $a, d$ and $g$, may be made according to the method outlined only when $C D$ is a $45^{\circ}$ line. If the dissolved value chart be made along the line previously suggested; that is wherein $C D$ is not a $45^{\circ}$ line, it is necessary that $O F$ and $O E$ bear a relationship different to one another than that of $O E: O F:: 1: d$. If the chart is constructed in this manner the relationship between $O F^{\prime}$ and $O E$ will be such that

$$
\frac{v}{x}=d \frac{V}{y}
$$

where $x$ and $y$ have the same values as that assigned to them in the discussion of the dissolved value chart. 
In discussing the various charts a single figure has, for the most part, been used. In the actual use of this method of analysis it is better to make individual eharts for each problem to be solved; thus avoiding unnecessary lines and figures.

In what has gone before I have attempted to give the outline of a method of graphic solution of pulp problems, and explain the same by giving methods for the analysis of a few of the more eommonly oceurring ones. The seope of this field has only been touched; but the underlying principles of the method may be applied to nearly every pulp problem, and little difficulty should be encountered in extending them beyond the scope of this paper.

By drawing such charts to large seale and exercising eare in their construetion, they may be made as accurate as, or even more so than, the measurements can be taken, and fully aceurate enough for all practical purposes.

Following is a list of articles containing further illustrations of the use of graphic charts of the class discussed in this chapter and pertaining to kindred subjects.

Upon request, the Codex Book Company, Inc., 19 William Street, New York, will be glad to procure, where possible, copies of magazines containing the articles needed, only charging the "old magazine" price, plus postage.

"I)iagram for Computing Rock in Sewer Trenches,"' C. A. Bryan, Engineering News, June 3, 1915.

"Nomography in Engine Design," F. Leigh Martineau and A. Marshall Arter, Automotive Industries, Series, May 16, 23, 30, 1918.

"Flow of Water in Pipes," Hazen \& Williams formula, Journal of Electricity, Power and Gas, October 2, 1915.

"Darey's Formula (Flow of Water)," Albert E. Guy, Catalogue American Spiral I'ipe Works, Page 7.

"Chart Determining Flow of Water Through Pipes and Friction Loss," Electrical World, November 4, 1916.

"Diagrams for Determining Friction Loss in New Cast Iron Pipe," Engineering and Contracting, January 31, 1912.

"To Find the Length of a Leather Belt by Weighing," W. F. Schaphorst, Engineering and Mining Journal, .July 14, 1917.

"A Logarithmic Diagram for Finding Brake Horse Power," Engineering and Contracting, July 11, 1917.

"Solar Declinations Computed by Graphic Method," Robert R. V. Reynolds, Engineering Record, August 7, 1915.

"Graphical Presentation of Conductor Properties," Andres Ham, Electrical World, February 10, 1917.

"Electrophysies and Magnetism, (Hysteresis Loss)," A. Castex, Electrical World, Sept. 30, 1916.

"('leveland Municipal Electric Plants," A. I). Williams, Power, Nov. $25,1913$.

"('harts for Eceentric Loading on Rectangular Areas," S. MI. Cotten, Engineering News, Dec. 7, 1916.

"Chart for Equivalent 'T'wisting Moments," Brent C. Jacob, American Machinist, Aug. 2, 1917. 
"Chart for Obtaining the Velocity of Air," Francis J. G. Reuter, American Machinist, Feb. 1, 1917.

"Graphic Solution of Compressed Air Transmission Formula," Engineering and Mining Journal, Jan. 25, 1913.

"Graphic Solutions of Some Compressed-Air Calculations," C. W. Crispell, Transactions of the Amer. Inst. of Mining Engineers, June, 1917.

"To Determine Income at Known Load Factor," Electrical World, Aug. 17, 1918.

"Dimensions of Dished Heads," E. W. Ashenden, Power, March 6, 1917.

"Diagram for Computing Earthwork," Engineering and Contracting, July 19, 1916.

"Ice and Salt Mixtures," Gilbert H. Crawford, Jr., Power, June 10, 1913.

"Graphical Determination of Trigonometric Functions,"' Power,

February 3, 1914.

"Diagram for Estimating Earthwork in Canals," Charles K. Fox and Charles M. Stokes, Western Engineering, May, 1917.

"A Method of Plotting River Stage-Discharge Data," Ernest W. Schoder, Engineering Records, August 3, 1912.

"Diagrams for Thermal Corrections for Road Oil and for Contents of Tanks," E. Earl Glass, Engineering and Contracting, December 6, 1916.

"Charts Give Contents of Cylindrical Tanks Without Computation,"

E. Earl Glass, Engineering News-Record, October 3, 1918.

"Diagram Giving Excess Loss of Head in $90^{\circ}$ Bends," Frank S. Bailey, Engineering News, March 2, 1916.

"Curves for Irrigation-Ditch Velocity and Discharge," Julian Hinds, Engineering News, August 24, 1916.

"Alignment Chart for Feeds, Speeds, etc., for Lathe Tools," A. Lewis Jenkins, American Machinist, March 14, 1918.

"Chart for Milling Cutters," A. Lewis Jenkins, American Machinist, July 19, 1917.

"Chart for Determining Planing Time," A. Lewis Jenkins, American Machinist, October 24, 1918.

"Chart for Quickly Determining Cutting Speeds and Feeds, J. B.

Peddle, American Machinist, March 15, 1917.

"Diagram for Overturning Moments on Retaining Walls for Earth or Water," C. H. Hoyt, Engineering News, April 25, 1907.

"Power Transmitted by Belts," W. F. Schaphorst, Engineering and Mining Journal, August 18, 1917.

"Charts Tell Weight of Sheeting for Round or Box Cofferdams," N.

G. Near, Engineering Record, October 7, 1916.

"Determining Pipe Main Sizes," Alfred Iddles, Power, June 19, 1917.

"Chart for Pipe Areas," W. F. Schaphorst, Engineering and Mining Journal, November 16, 1918.

"A Handy Excavation Chart, Field Note Dept.," Excavating Engineer, P. O. Box 607, Milwaukee, Wis.

"A Handy Chart for Figuring Power Capacity and Efficiency of 
Pumps,' W. F. Schaphorst, Engineering and Contracting, August 9, 1916

"Boiler-Efficiency Chart," John C. Parker, Power, June 10, 1913.

"Nomographic Chart for Computing Flow in Wood-Stave Pipe," Carl Naughmer, Western Engineering, May, 1917.

"The Weight of Pipe," W. F. Schaphorst, The Gas Age, January $15,1917$.

"Chart for Finding Factor of Evaporation," Franz Szabo, Power, November 12, 1918.

"The Comparative Capacity of Elevators and Escalators for Handling Crowds," Chas. D. Seeberger, Engineering News, May 2, 1917.

"The Maximum Weights of Slow Freight Trains," The Engineering Record, MIarch 6, 1909.

“Determining Mine-Car Friction," N. G. Near, Coal Age, August 11, 1917.

"Calculating Illumination Density, Neville S. Dickinson, Electrical World, September 20, 1913.

"'The Ellenwood Steam Charts," C. Harold Berry, The Sibley Journal of Engineering, May, 1916.

"Calcr:lation of Plant Efficiencies and Fuel Costs," J. T. Foster, Power, March 4, 1919.

"Chart for Estimating Change in Declination of Sun," Coal Age, May 18, 1918.

“"The Ross Mediograph," J. T. Beard, Coal Age, April 20, 1918.

"Graphical Determination of Line Constants," T. A. Wilkinson, Electrical World, March 16, 1918.

"Method for the Graphical Construction of a Direct Reading Scale for Wheatstone Bridge," J. Carl Fisher, Scientific American Supplement, Nay 16, 1914.

"Sars and Tensions of Overhead Conductors," Alfred Still, Electrical World, May 11, 1912.

"Simplified Sag Formulas," II. V. Carpenter, Electrical World, July 13, 1912.

"Graphical Solution of Transmission Line Problem," T. A. Wilkinson, Electrical World, August 12, 1916.

"Pressure Drop in Alternating-Current Lines," M. J. Eichhorn, Electrical Review and Western Electrician, .January 11, 1913.

"Visits of Inspector Brown (diagraming formula for stay-bolt)," J.

E. Terman, Power, April 10, 1917.

"Rapicl Reckoning Chart," Electrical World, April 8, 1916.

"'The Flow of Steam Through Pipes," II. V. Carpenter, Power, June $10,1913$.

"Coal to IIeat Factory Buildings," James D. White, December 16, 1913. 


\section{CHAPTER XVII}

\section{Designing and Estimating}

There is almost no limit to the use of charts for designing and estimating purposes. In the former capacity they frequently eliminate the necessity of long and tedious calculations. Further, they furnish a means for determining economical designs much more easily and readily than by the comparison of the results of long computations.

For estimating purposes they present a rapid and accurate method and one which is highly recommended for wider use.

Out of the multitude of examples naturally it has been possible to quote but a few, but these few have been selected so that they cover a wide field and it is hoped that they will offer many valuable suggestions for the adoption of similar methods in other directions.

Concrete Bridge Floors. Wm. Snaith shows the application of graphical charts to design in his article, "Standard Bridge Floors of Concrete Slabs on Steel Beams," published July 12, 1917, in Engineering News-Record.

Concrete floors on highway bridges are a sufficiently recent development to make of value any data on the design of the steel floor system to support them. Even the latest works on the subject discuss wooden floors exhaustively, but contain little information as to the stringers and floor-beams for concrete floors. It is the intention of the writer to supply this deficiency by the diagrams here given for standard clear widths of roadway varying from 14 to $20 \mathrm{ft}$. and for panel lengths from 6 to $35 \mathrm{ft}$. The economical panel length for concrete floors, when the floor only is considered apart from the trusses, will appear from inspection of a cost chart presented herewith.

All the floor systems investigated have been designed to meet the usual standard specifications as to construction. The dead-load $D$ is taken to include the weight of the concrete slab, the supporting steel and two handrails each weighing $200 \mathrm{lb}$. per lin. ft. The live-load $L$ is either a 15-ton road roller or a uniform load of $100 \mathrm{lb}$. per sq. ft., whichever gives the greatest stresses. The roller is assumed to consist of two back wheels with 20-in. face and 5-ft. center to center and of one front wheel with 40-in. face, the axles being $10 \mathrm{ft}$. center to center and the load on each of the three wheels being 10,000 pounds.

The effect of impact is calculated from the formula:

$$
\text { Impact }=\frac{L_{2}}{2(D+L)}
$$

This impact formula will give uniformly better results than a straight percentage of the live-load or a formula based only on live-load and panel length. 
The ealeulations have been simplified by several assumptions that err on the side of safety and are only slightly in error.

The maximum bending moment has been assumed to equal the sum of the maximum live-load and derd-load moments. The dead-load per lineal foot per stringer has been raken in round figures at the nearest multiple of 25 above the actual. The live-load from the roller loading on the side stringers is taken in even thousands of pounds.

The weight of each wheel is assumed to act at a point in stringer and floor-beam ealculations. In stringer calculations the slab is assumed not to be continuous.

The theoretical length of floor-beam or distance between center lines of trusses is assumed to be $2 \mathrm{ft}$. greater than the clear width of roadway.

The depth of concrete floor slabs is 1/12 the maximum span between stringers, with a wearing surface of $3 \mathrm{in}$. of concrete. An asphalt wearing surface or ereosoted wood block on sand will give approximately the same loading. Earth fill to a depth of $4 \mathrm{in}$. would do so also, but this depth is insufficient. To increase the earth fill to about 9 in. average depth will increase the total stresses in stringers and floor-beams by about $15 \%$.

The total width of slab has been taken to be $1 \mathrm{ft}$. wider than the clear width of roadway, to allow for a wagon guard on the eurb 6 in. high on each side.

The dead-load on the middle stringer of course includes the weight of the slab and stringer. For maximum live-load stresses the 15-ton roller only need be considered. For 35-ft. span and 5-ft. spacing this gives greater stresses than a uniform live-load of $165 \mathrm{lb}$. per sq. ft.

For a roller of the dimensions and weight assumed, and for spans up to $17.1 \mathrm{ft}$., the maximum bending moment will oceur under one wheel when it is at the center of the span. For spans beyond $17.1 \mathrm{ft}$. the roller is assumed to be slewed slightly on the bridge, so that one back wheel and the front wheel come directly over the stringer. The maximum bending moment then oceurs under the wheel, which has traveled $2 \mathrm{ft} .6 \mathrm{in}$. beyond the center of the span. The value of the maximum hending moment in foot-pounds when one stringer is assumed to sustain the whole load is given by the formula:

$$
M_{\max }=W\left(\frac{l}{2}-5+\frac{12.5}{l}\right)
$$

where $W$ is weight of one wheel and $l$ is the panel length, or span, of the stringer.

The maximum end reaction $R$ is

$$
R_{\max }=W+W\left(\frac{l-10}{l}\right)
$$

the last term vanishing for spans less than $10 \mathrm{ft}$.

The sicle stringers are designed for a dead-load consisting of one-half the slab between the side and nearest middle stringer, all the slab and curb overhanging, the stringer itself and a handrail assumed to weigh $200 \mathrm{lb}$. por linear foot. 
The roller will produce the maximum live-load stresses in the side stringers when the front wheel and one back wheel are just touching the curb. The portion of each wheel to be supported by the side stringers will be different for different distances of the stringers from the curb. Values for these loadings were carefully calculated for each of the floor systems considered.

The dead-load on the floor-beam is conveniently calculated from the stringer reactions, the amount of excess due to increasing the actual figures into round numbers being more than sufficient to account for the weight of the floor-beam itself.

The maximum bending moment due to the live-load occurs with the

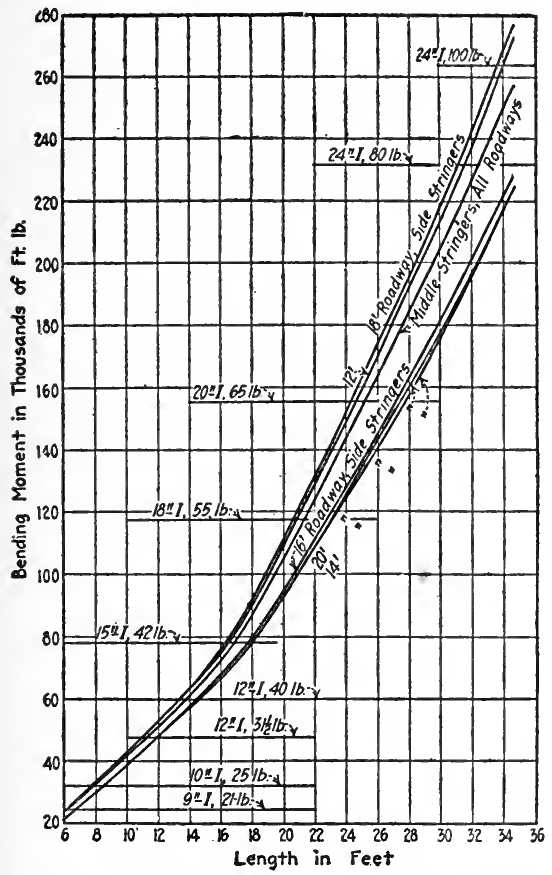

Fig. 1-Bending Moments for Stringers

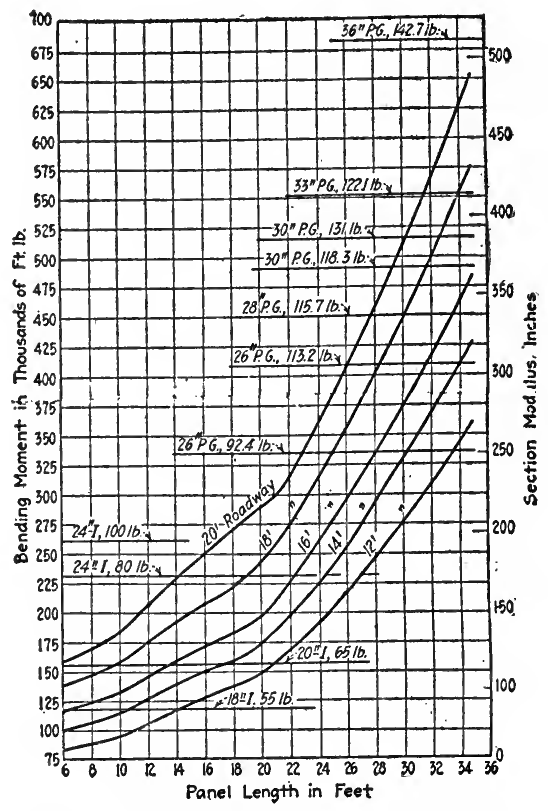

Fig. 2-Bending Moments for FloorBeams

two back wheels on the floor-beam under the wheel which is $1 \mathrm{ft}$. 3 in. from the center of the span. The front wheel at the same time is on the center line of the bridge. For panel lengths less than $10 \mathrm{ft}$. the front wheel is not considered, and for those over $20 \mathrm{ft}$. the uniform load of $100 \mathrm{lb}$. per sq. ft. will give greater values, in general, but in the latter case it is necessary to make the calculations for both conditions.

For the maximum end reaction the roller is placed with the front wheel and one back wheel near the curb and the two back wheels directly over the floor-beam. When the panel length exceds $20 \mathrm{ft}$., it is advisable also to calculate the results for uniform loading.

The particulars of the floor systems considered are given in Table 1. The values given for the dead-load per lineal foot on the stringers are 
based on actual calculations made for panel lengths of 11, 19, 30 and 35 feet.

The maximum bending moments on the stringers are shown by the curves in Fig. 1. The maximum bending moments for the usual stock sizes of I-beams have also been indicated, and these sizes were adopted in calculating the total weight of the steel in each system. From these eurves the stringers used for any span of the standard floor systems can be read directly. Where heavier than standard rolled sections are available, the necessary interpolation is a simple matter.

The maximum bending moments and end reactions of the floor-beams are given by the curves in Figs. 2 and 3 . The section moduli required for a unit stress of 16,000 lb. per sq. in. are shown in Fig. 2, and the

\section{TABLE I. PARTICULARS OF FLOOR SYSTEMS CONSIDERED IN ANALYSIS OF}

\begin{tabular}{|c|c|c|c|c|c|}
\hline \multirow{6}{*}{ 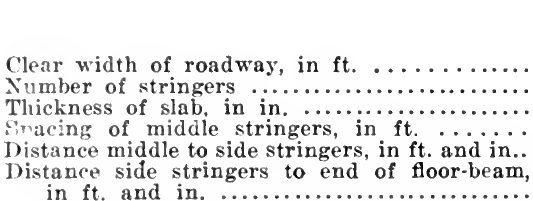 } & \multicolumn{5}{|c|}{$\begin{array}{c}\text { Dimensions of } \\
\text { Standard Floor Systems }\end{array}$} \\
\hline & . 12 & 14 & 16 & 18 & 29 \\
\hline & 3 & 4 & & & \\
\hline & 8 & & & & \\
\hline & & & & & $\begin{array}{r}5 \\
4-6\end{array}$ \\
\hline & $2-0$ & $1-9$ & $1-6$ & $2-6$ & $1-6$ \\
\hline Dead-load in lb. per lin. $\mathrm{ft}$. on middle stringers: & & & & & \\
\hline is $6 \mathrm{ft}$. to $19 \mathrm{ft}$. & .550 & 550 & 550 & 550 & 550 \\
\hline Panels $20 \mathrm{ft}$. & .575 & 575 & 575 & 575 & 575 \\
\hline Dead-load in lb & & & & & \\
\hline Panels $6 \mathrm{ft}$. to 19 & & 600 & 625 & 725 & 600 \\
\hline Panels $20 \mathrm{ft}$. to $35 \mathrm{ft}$. & 700 & 625 & 650 & 750 & 625 \\
\hline
\end{tabular}

\begin{tabular}{|c|c|c|c|c|c|}
\hline \multicolumn{6}{|c|}{ Dimensions of } \\
\hline $\begin{array}{r}12 \\
4 \\
8 \\
5 \\
3-9\end{array}$ & $\begin{array}{r}16 \\
5 \\
8 \\
5 \\
3-3\end{array}$ & $\begin{array}{r}16 \\
6 \\
7 \\
4 \\
2-3\end{array}$ & $\begin{array}{r}16 \\
7 \\
6 \\
3 \\
2-3\end{array}$ & $\begin{array}{r}16 \\
9 \\
5 \\
2 \\
2-3\end{array}$ & $\begin{array}{r}18 \\
5\end{array}$ \\
\hline $0-9$ & $0-9$ & $0-9$ & $0-9$ & $0-9$ & \\
\hline $\begin{array}{l}550 \\
525\end{array}$ & $\begin{array}{c}\mathbf{5 5 0} \\
\mathbf{5 0 0}\end{array}$ & $\begin{array}{r}400 \\
400\end{array}$ & $\begin{array}{l}270 \\
350\end{array}$ & $\begin{array}{r}160 \\
375\end{array}$ & $\begin{array}{l}550 \\
57\end{array}$ \\
\hline $\begin{array}{l}500 \\
575\end{array}$ & $\begin{array}{c}475 \\
575\end{array}$ & $\begin{array}{c}400 \\
400\end{array}$ & $\begin{array}{c}350 \\
270\end{array}$ & $\begin{array}{c}375 \\
160\end{array}$ & $\begin{array}{r}550 \\
575\end{array}$ \\
\hline
\end{tabular}

maximum bending moments on the usual stock I-beams and a selected line of plate girders are also indicated. From the curves in Figs. 2 and 3 the floor-beams for any span up to $35 \mathrm{ft}$. can be obtained. Fig. 3 can also be used to obtain maximum panel loads for truss bridges.

Average prices for steel beams in place in bridge floors and for concrete in place were assumed, and the total cost of one bay (slab, curb, stringers and floor beam) was calculated. These values were divided by the area of floor supported (nominal width multiplied by panel length), and the results were expressed in curves, Fig. 4.

Owing to the use of eommercial sizes and abrupt changes in loading, when, for example, the whole roller is taken into account instead of only two back wheels, or when the uniform live-load replaces the roller load in the calculations, these curves are not smooth and actually cross over one another. It is not to be understood that the figures represent actual probable eosts; they are approximate only and will be affected by changes in cost of materials and locality. However, they are of value for purposes of comparison and clearly indicate the economic panel length when the floor systems only are eonsidered.

Alternative floor systems were carefully calculated by the same methodts as those adopted and proved to be more expensive in every case. An interesting comparison was made in the ease of the 16 -ft. roadway system. Five systems were investigated out with various thicknesses of slah and were plotted similarly to Fig. 4. The four-stringer system shows a notable economy at all spans. The seven-stringer system is almost as ceonomical at 10 -ft. panel length as the four-stringer system and least so at 35-ft. panel length. Exactly the reverse is true of the six- 
stringer system. The way in which the curves crossed one another would show that no general statement would be warranted that the fewer the stringers the greater the economy. Each case must be settled on its merits.

The amounts of the errors due to the various assumptions were carefully investigated. They are inconsiderable and invariably on the side of safety. The expression of the dead-load figures in multiples of $25 \mathrm{lb}$. will not in any case involve an error of more than $1 \%$ in the total, and

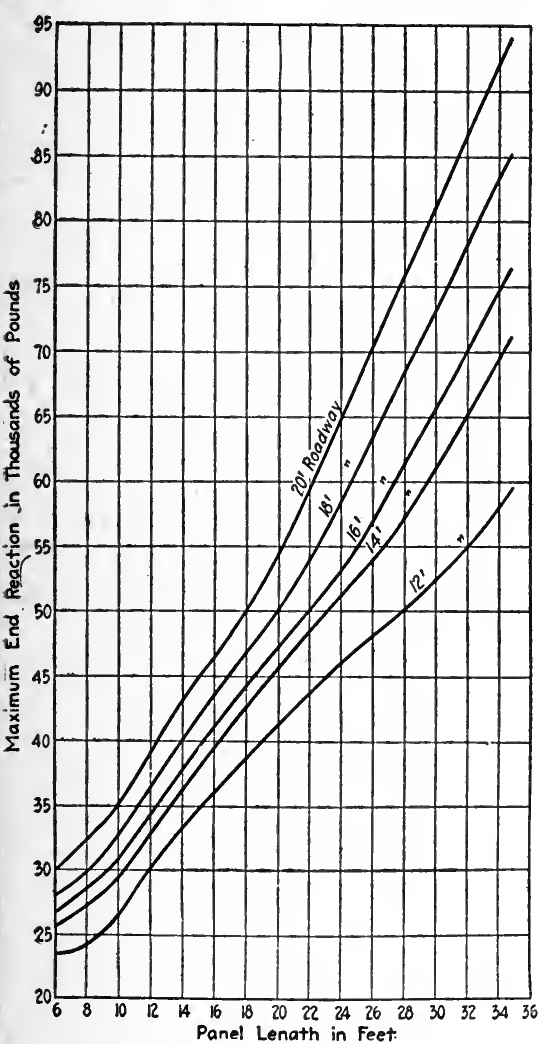

Fig. 3-End Reactions for Floor-Beams

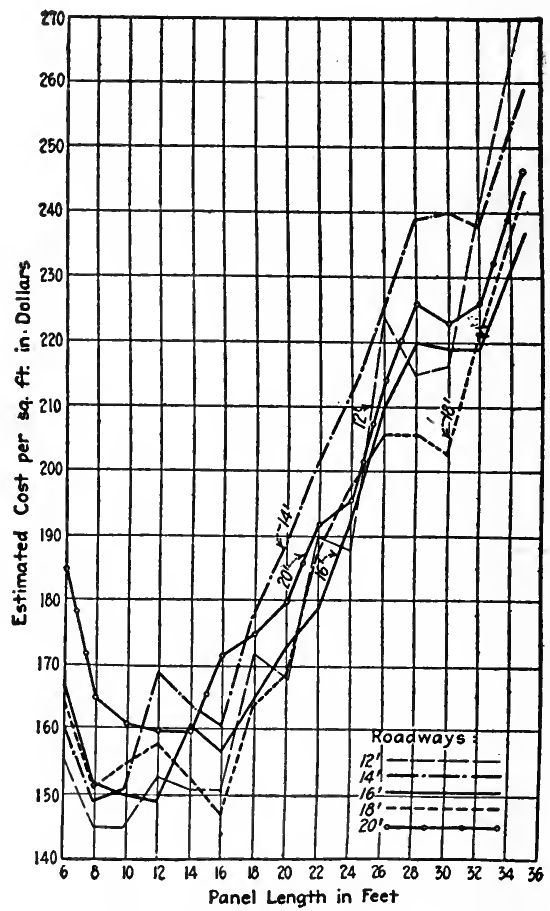

Fig. 4-Estimated Costs of Floors per Square Foot

Includes floor-beams, stringers, slab and curb.

the effect of the round figures adopted for the roller loadings on the side stringers does not exceed the actual loadings by more than $4 \%$ of the total results at 35-ft. panel lengths. The sum of the three errors above will hardly amount to $4 \%$ for panel lengths less than $20 \mathrm{ft}$. and not more than $6 \%$ at $35 \mathrm{ft}$. in any instance.

Column Design. From Engineering and Contracting March 4, 1914, is taken the following article, "Graphical Designs of Eccentrically Loaded Columns,' by H. B. Cooley.

The design of columns subject to cross-bending stresses in addition to axial loads involves a preliminary approximation, either as to size of section or unit stress, followed by investigation using a rather compli- 


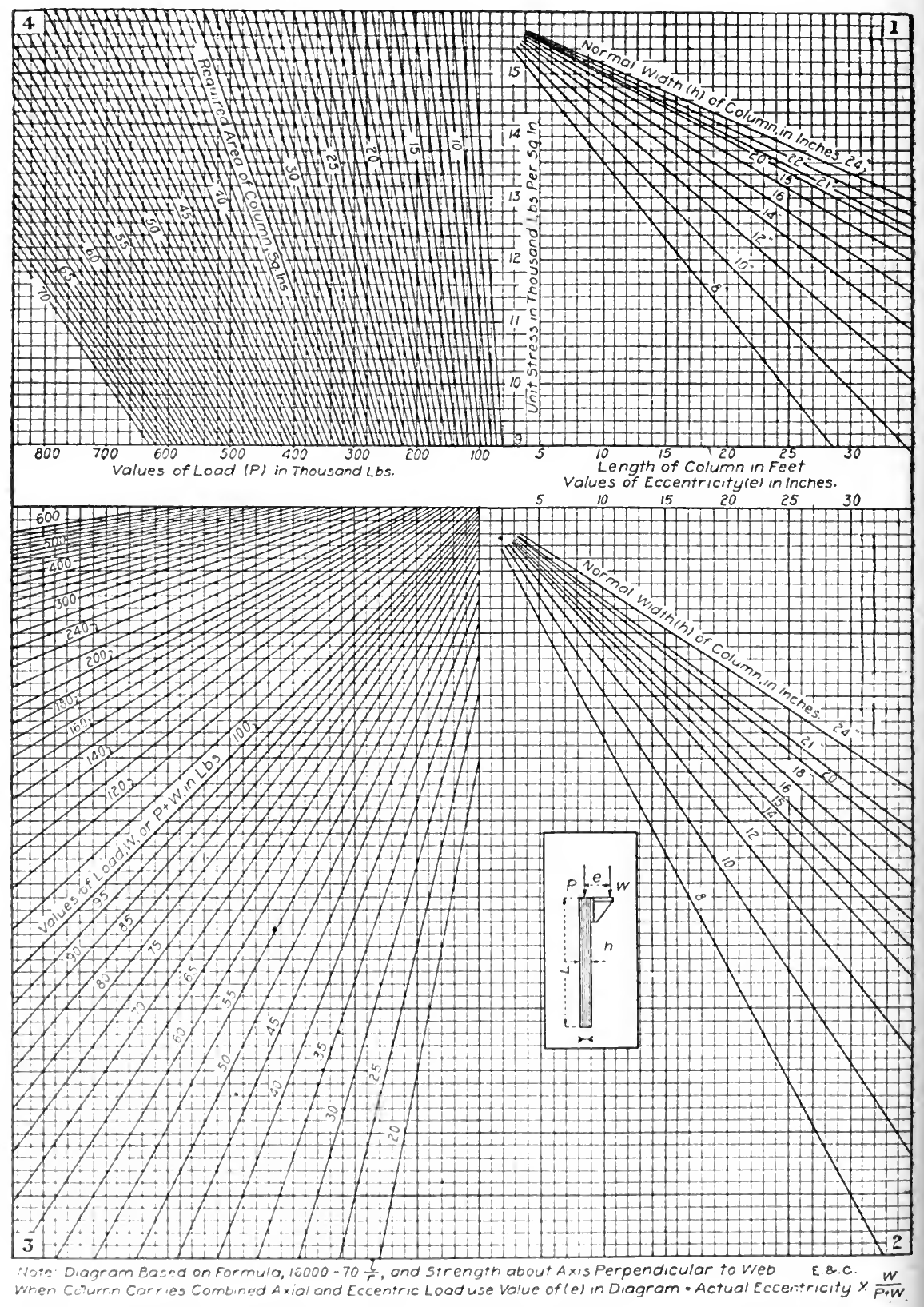

Fig. 5-Diagram for Dotermining the Required Area for Plate-and-Angle Columns Eccentrically Ioaded-Diagram also Applicable to Axially Loaded Columns 
cated formula. In some cases this process must be repeated several times before a suitable section is obtained, and it is to avoid this labor that the diagram of Fig. 5 was plotted.

Although the method used in constructing the diagram is general, the following limitations are involved for the particular graph, shown in Fig. 5.

(a) The diagram as plotted considers only the I-section type of column, i. e., a column composed of a web plate and four angles, either with or without cover plates. The nominal width shown in Fig. 5 is that of the web, but the width used in plotting was the width of the web plate plus $1 / 2$-in.

(b) The safe loads as given are for the strength of a column about an axis perpendicular to the web.

(c) The allowable unit stress is based upon the formula $16,000-70-\frac{l}{r}$, where $l=$ length of column, in inches, and $r=$ radius of gyration about an axis perpendicular to the web.

The method of deriving the equations used in plotting the various lines of the diagram is herewith given, the nomenclature being as shown below :

$S=$ unit stress in pounds per square inch,

$l=$ length of column in inches,

$L=$ length of column in feet,

$r=$ radius of gyration about an axis perpendicular to the web,

$P=$ axial load on column in thousands of pounds,

$W=$ eccentric load on column in thousands of pounds,

$h=$ width of column web plus $1 / 2$ in.,

$c=$ distance from neutral axis to extreme fiber,

$a=$ area of column in square inches,

$i=$ moment of inertia about an axis perpendicular to the web, $e=$ eccentricity of load, $W$, in inches.

Then

$$
S=16000-70 \frac{l}{r}
$$

also $\quad S=\frac{P}{A}+\frac{M c}{I}$.

But $I=a r^{2}$.

Substituting $r=0.4 h$ in equation (3) (this value of $r$ is approximate, but for sections in common use gives close results), we have

$I=0.16 a h^{2}$

Substituting in equation (1) for $r$ its value $0.4 h$, and for $l$ its value in feet; also substituting in equation (2) the value of $I$ given in equation (4), we have 


$$
\begin{aligned}
& S=16000-2100 \frac{L}{h} . \\
& S=\frac{P}{a}+\frac{M}{0.32 a \cdot h} .
\end{aligned}
$$

Equating values of $S$ given in equations $(1, a)$ and $(2, a)$, we have:

$$
16000-\frac{2,100 L}{h}=\frac{P}{a}+\frac{M}{0.32 a h} \text {. }
$$

The above equation now contains five variables, and can be plotted in four quadrants, as shown in Fig. 5.

There are three general cases of column loading that may arise, any one of which ean be solved by use of the diagram, as will now be shown by the solution of particular examples:

Case I: Axial Load Only.-In this case the last term on the righthand side of equation (5) is zero; therefore only two quadrants are required for the solution.

Example: Column $15 \mathrm{ft}$. long, with 10 -in. web plate; load $=300,000$ lbs.; find required area of the column, for strength about axis perpendicular to the web.

Under the line marked "Length of Column in Feet" proceed vertically upward to the diagonal line marked 10 ins.; thence horizontally to the left, noting where the horizontal line intersects a vertical line passing through the given load 300 (thousand), which is marked on line "Axial Load $P$ in 1,000 Lbs." ; the required area is found to be 23 sq. ins. From the handbooks or from specially prepared tables this area can be made up to suit conditions. For example, four $6 \times 4 \times 1 / 2$-in. angles and one $10 \times 3 / 8$-in. web plate give an area of 22.75 sq. ins., and $r=4.20$ ins. The efficiency of this section is about $99.5 \%$.

Case II: Eccentric Load Only.-Replacing the eccentric load by an axial load and a couple equal to $W e$, we have:

$$
\begin{aligned}
& 16000-2100 \frac{L}{h}=\frac{W}{a}+\frac{W e}{0.32 a h}= \\
& \frac{W}{a}\left[1+\frac{e}{0.32 h}\right]
\end{aligned}
$$

Knowing any four of the above quantities, the fifth can be found directly from the diagram.

Example: Column $20 \mathrm{ft}$. long, with 12 -in. web; load $=75,000$ lbs.; eccentricity $=20$ ins.; find required area of column.

Starting at line marked "Length of Columns in Feet," read around the diagram in a clockwise direction, considering the width of column and the eccentric load, in the second and third quadrants; then starting with line marked "Values of Eccentricity $e$, in Inches," read around the diagram in a counter-clock-wise direction, considering the width of 
column; from the intersections in the third and first quadrants, project lines to meet in the fourth quadrant; their intersection gives the required area $35.6 \mathrm{sq}$. ins. A section can now be chosen which will give this area.

Case III : Combined Axial and Eccentric Load.-The allowable stress,

$$
S=16000-2100 \frac{L}{h}=\frac{P}{a}+\frac{W}{a}+\frac{W e}{0.32 a h}
$$

Let $W e=(P+W) e^{1}$;

$$
\text { then } e=\frac{(P+W) e^{1}}{W} \text {. }
$$

Substituting this value of $e$ in equation (7), we have:

$$
16000-2100 \frac{L}{h}=\frac{P+W}{a}\left[1+\frac{e^{1}}{0.32 h}\right]
$$

It is seen from equation (8) that, for an application of Case III to the diagram, it is only necessary to use a value for the eccentricity, $e$, (shown on the diagram) equal to the actual eccentricity multiplied by

$W$

$$
\overline{P+W} \text {. }
$$

Example: Column $18 \mathrm{ft}$. long, with 18 -in. web; axial load $P=120000$ lbs., eccentric load $W=80000$ lbs., eccentricity, $e$, of load $W=35$-ins., find required area of column.

First compute the value of $e^{1}$

$$
e^{1}=\frac{80000 \times 35}{200000}=14 \text { ins. }
$$

Starting with this value of $e^{1}$ at the line marked "Values of Eccentricity, $e$, in Inches," and proceeding as in case II, we find the required area to be 49 sq. ins. A section can now be chosen which will give this area.

In all cases care must be taken to see that the column selected is sufficiently strong about an axis parallel to the web. This can be done, either by making an investigation in the usual manner, or by plotting additional diagrams similar to those shown in Fig. 5 using the approximate values for the radius of gyration about an axis parallel to the web.

C. D. Conklin, in Engineering Record, March 17, 1917, gives a nomographic chart for column design. A part of his article follows:

Fig. 6 will aid the structural designer in selecting sections for columns required to carry a given load, or to find the safe load of a given column, according to the well-known straight-line formula $S=16000-70 L / R$. The chart is particularly useful in cases where numerous column sizes sre required, the load and length of each being known. 
Given the load, length and any assumed radius of gyration, the slenderness ratio $L / R$, the average allowable unit stress $S$, and the required area $A$ ean be determined by two moves of a triangle or other straight edge. An example will indicate the procedure, as illustrated by the dotted lines on the diagram.

For a column to carry 140,000 lb., $18 \mathrm{ft}$. long, least radius of gyration 2.1, place a straight edge at 18 on the inner side of the left-vertical scale and at 2.1 on the inclined scale for $R$. The slenderness ratio is

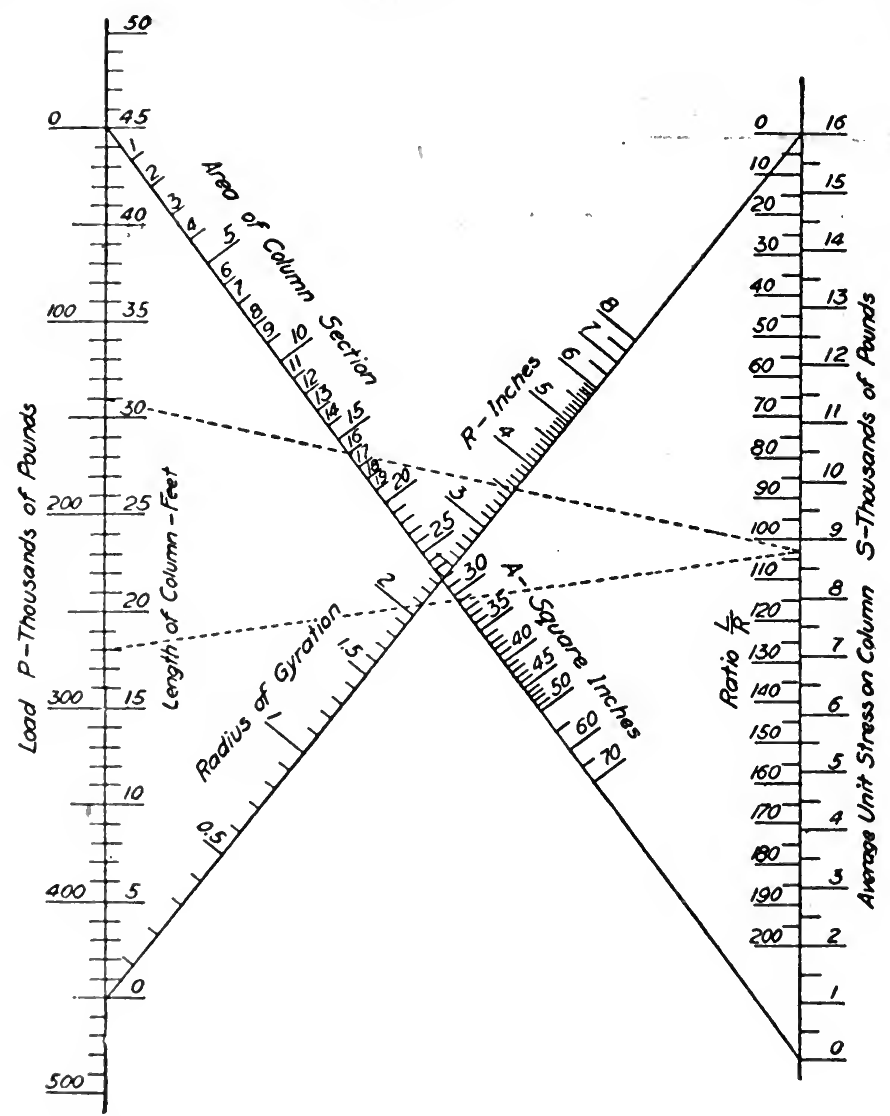

Fig. 6-Simplifies Solution of A. R. E. A. Column Formula

then read as 103 on the inside of the right vertical seale, the straight edge is swung around this point to an intersection with 140000 on the outside of the left vertical scale, and the desired value of $16 \mathrm{sq}$. in. for the area $A$ is read on the other inclined scale. If desired, the average unit stress is easily read as $8,800 \mathrm{lb}$. per sq. in. on the outside of the right vertical scale.

If it be desired to investigate a given eolumn of known area and properties, the procedure is the same except that the seeond line con- 
nects with the known area $A$ and gives the desired allowable total load $P$ on the outside of the left vertical scale. Any other straight-line column formula can readily be charted in a similar manner.

Boiler Design. "Calculation for Boiler Stresses," an article by G. E. Parks, published in Railway Mechanical Engineer, March, 1918, contains a good demonstration of the use of charts for design.

In boiler design and the calculation of boiler stresses, systems of curves like those illustrated below have proved of material value in Michigan Central practice. They are not only a great labor saver and aid in checking boiler specification cards, but they will often show at a glance the best possible arrangement of detail or size of part. Their accuracy will depend upon the scale employed.

Fig. 7 shows the relation between the efficiency of the boiler seam and the tensile strength of the plate for various thicknesses of plate and as two systems of curves are represented on a single co-ordinate field, care should be exercised in using the respective scales. The curved lines on the co-ordinate field represent the efficiency of the American Locomotive Company's seam, drawings 142-S-30,080-81-90 (known as seam No. 1), for different tensile strengths of plates. The dimensions of this seam are given in Fig. 8 and the curves are plotted from the equation

$$
\mathrm{T}=\frac{\mathrm{SA}}{\mathrm{t}\left(\mathrm{p} \frac{\mathrm{E}}{100}-\mathrm{c}\right)}
$$

where:

$\mathrm{T}=$ Tensile strength of plate in pounds per square inch.

$\mathrm{S}=$ Shearing strength of rivets in pounds per square inch; taken as $44,000 \mathrm{lb}$.

$\mathrm{A}=$ Area of rivet hole in square inches.

$\mathrm{t}=$ Thickness of sheet in inches.

$p=$ Pitch of outside row of rivets in inches.

$\mathrm{E}=$ Efficiency of seam in per cent.

$\mathrm{C}=$ The pitch, minus twice the diameter of rivet hole $(p-2 d)$, or the length of metal between alternate rivets in the second row.

The straight lines on the co-ordinate field represent the relation between the efficiency of seam No. 1 and the efficiency of the various other seams as plotted. If we assume that the tensile strengths and thickness of plate is the same in each case, the equation for the straight lines becomes

$$
\mathrm{E}_{2}=100\left[\frac{\mathrm{A}_{2} \mathrm{p}_{1}}{\mathrm{~A}_{1} \mathrm{p}_{2}} \mathrm{E}_{1}+\frac{\mathrm{A}_{1} \mathrm{C}_{2}-\mathrm{A}_{2} \mathrm{C}_{1}}{\mathrm{~A}_{1} \mathrm{p}_{2}}\right]
$$

In this equation the letters represent the values as given above, the subscript figure " 1 " representing values in seam No. 1 and the subscript figure " 2 " representing values in the seam under investigation.

The points marked "X" at the lower end of the straight lines representing seams 15 and 57 , also seam 64 , indicate the position of the 
curve at which the efficiency of the seam for shearing all of the rivets and the efficiency of the seam when tearing through the second row of rivets and shearing the rivets in the outside row are equal. Therefore, if the thickness and tensile strength of plate in seams 15, 57 and 64 are such that the point of intersection between the curved line representing the thickness of plate, and the horizontal line representing the tensile strength of plate falls at the left of the point " $X$," the seam will fail by the shearing of all of the rivets. The efficiency is determined as follows:

From the point of intersection between the eurved line representing the thickness of the sheet and the horizontal line representing the tensile strength of the sheet, project a line in a vertical direction until it comes in contact with the straight line, marked "Actual Shearing Efficiency." Opposite this point of intersection, read on the vertical scale the shearing efficiency of the seam. The co-ordinate field as plotted does not show the points where the shear of all of the rivets is equal to the efficiency of seam through the second row of rivets, for seams Nos. 2, 3, 14 and 55, therefore, these seams would not fail due to shear of all of the rivets for any thickness of plate or tensile strength of plate, which is given on the drawing.

The equation of the straight line marked "Actual Shearing Efficiency" is :

$$
\mathrm{Es}_{2}=\frac{\mathrm{A}_{2} \mathrm{~N}_{2}}{\mathrm{~A}_{1} \mathrm{P}_{2}}\left(\mathrm{P}_{1} \mathrm{E}_{1}-\mathrm{C}_{1}\right)
$$

where:

$\mathrm{Es}_{2}=$ Efficiency due to shear of all of the rivets in seam under investigation represented by the straight line.

$\mathrm{A}_{2}=$ Area of rivet hole in seam under investigation.

$A_{1}=$ A rea of rivet hole in seam No. 1, represented by the eurved lines.

$\mathrm{N}_{2}=$ Number of rivet shearing planes of seam under investigation. In all seams plotted on this sheet, $\mathrm{N}_{2}$ equals 9 .

$P_{2}=$ Pitch of rivets in outside row of seam under investigation.

$\mathrm{P}_{1}=$ Pitch of rivets in outside row of seam, No. 1.

$\mathrm{E}_{1}=$ Efficiency of seam, No. 1.

$\mathrm{C}_{1}=$ Pitch minus twice diameter of rivet hole in the seam, No. 1.

At a point representing $85.52 \%$ efficiency for seam No. 1 is a vertical dotted line which is the efficiency of the seam through the first row of rivets. If the thickness and tensile strength of plate are such that the effieiency for seam No. 1 is higher than $85.52 \%$ and is shown on the right of the dotted line, the higher efficiency should not be considered as the seam would fail through the first row of rivets which has an efficiency of $85.52 \%$. At the right end of the straight lines representing seams No. 2, 3, and 14 are the points where failure will occur in the first row of rivets and these points represent the maximum efficieney of the seams. The co-ordinate field is not large enough to show similar points for seams $N o, 15,57$ and 64 .

I'roblem 1.- To find the effisiency of boiler seam No. 1 having 11/8-in. plate, the tensile strength of which is $58,000 \mathrm{lb}$. 


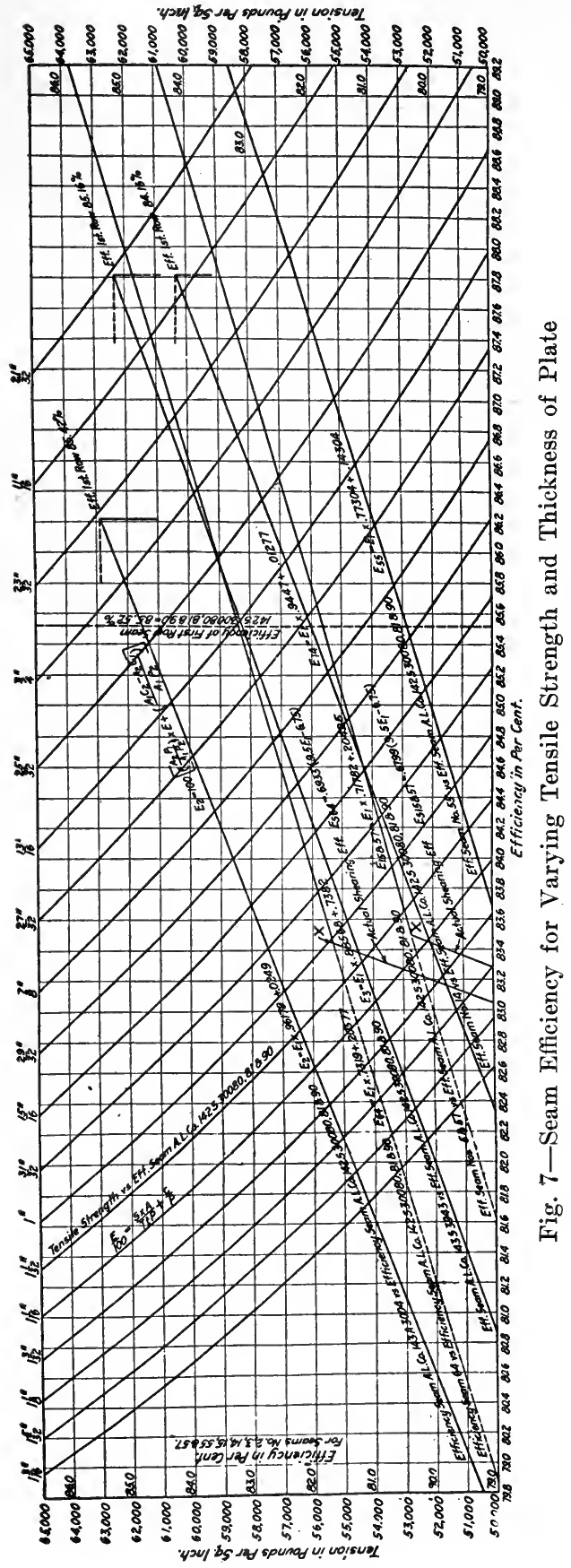


From the point of intersection of the $58,000 \mathrm{lb}$. line on the horizontal scale and the curve representing a $1 \frac{118-i n}{}$. plate, project a line vertically either to the top or bottom axis and read off the efficiency, which is $81.6 \%$.

Problem 2.-To find the efficiency of boiler seam No. 2 having 118-in. plate, the tensile strength of which is $58,000 \mathrm{lb}$.

First find the efficiency that seam No. 1 would have under the same conditions, which is $81.6 \%$. Then from the point where the vertical line representing $81.6 \%$ intersects the straight line representing seam No. 2 find the efficiency corresponding to this point on the vertical scale, which is $81 \%$.

This system of curves may be used to determine the value of any of the factors in the general equation when the other factors are known.

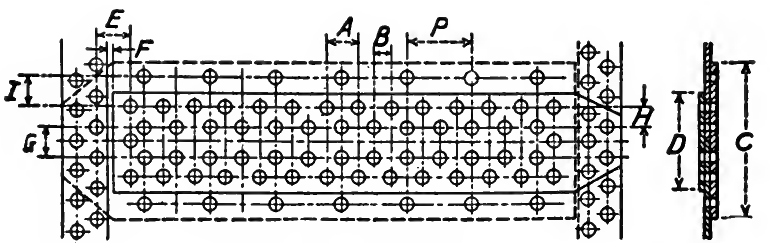

Longitudinal Seam

\begin{tabular}{|c|c|c|c|c|c|c|c|c|c|c|c|c|}
\hline \multicolumn{13}{|c|}{ Dimensions of Seams } \\
\hline Seam & \begin{tabular}{|l|} 
Diam. \\
Rivet
\end{tabular} & $\begin{array}{l}\text { piam } \\
\text { River }\end{array}$ & $P$ & $A$ & $B$ & $c$ & 0 & $E$ & $F$ & $G$ & $H$ & $I$ \\
\hline $\begin{array}{l}12530080,81,90 \\
\text { Seam No.1 }\end{array}$ & $1 \frac{5^{n}}{16}$ & $1 \frac{31}{8}$ & $9 \frac{1 "}{2}$ & $4 \frac{3}{4}$ & $2 \frac{3}{8}^{3 \prime \prime}$ & $19 \frac{3}{4}$ & $12 \frac{1}{4}$ & $4^{\frac{3}{4}}$ & $\frac{3}{4}$ & $3^{3}{ }^{\prime \prime}$ & $23^{3 \prime \prime}$ & $\overline{3 \frac{3}{4}}$ \\
\hline $\begin{array}{l}143 A 3004 \\
\text { Seam No.2 }\end{array}$ & $1 \frac{1}{4}$ & $1 \frac{5}{16}$ & 9 & $4 \frac{1}{2}$ & $2 \frac{1}{4}$ & $18 \frac{1}{2}$ & $\| \frac{1}{2}$ & $4 \frac{1}{2}$ & $\frac{3}{4}$ & $3 \frac{1}{2}$ & $2 \frac{1}{4}$ & $3 \frac{1}{2}$ \\
\hline $\begin{array}{r}14353943 \\
\text { Seam No.3 }\end{array}$ & $1 \frac{1}{8}$ & 1/3 & 8 & 4 & 2 & 17 & $10_{\frac{1}{2}}^{\frac{1}{2}}$ & 4 & $\frac{3}{4}$ & $3 \frac{1}{4}$ & 2 & $3 \frac{1}{4}$ \\
\hline 14 & $1 \frac{1}{8}$ & $1 / \frac{3}{6}$ & $7 \frac{1}{2}$ & $3 \frac{3}{4}$ & $1 \frac{7}{8}$ & $16 \frac{1}{4}$ & 10 & 3.7 & $\frac{3}{4}$ & $2 \frac{3}{4}$ & 2 & $3 \frac{1}{8}$ \\
\hline $15 \& 57$ & $\frac{15}{16}$ & I & 7 & $3 \frac{1}{2}$ & $1 \frac{3}{4}$ & $14^{\prime}$ & $8 \frac{3}{4}$ & $3 \frac{1}{2}$ & $\frac{3}{4}$ & $2 \frac{1}{2}$ & $1 \frac{3}{4}$ & $2 \frac{3}{4}$ \\
\hline 55 & $\frac{15}{16}$ & 1 & $6 \frac{1}{2}$ & $3 \frac{1}{4}$ & $1 \frac{5}{8}$ & $14 \frac{1}{4}$ & $8 \frac{3}{4}$ & $3 \frac{1}{4}$ & $\frac{3}{4}$ & $2 \frac{1}{2}$ & $1 \frac{3}{4}$ & $2 \frac{3}{4}$ \\
\hline 64 & 1 & $1 / 15$ & $7 \frac{3}{4}$ & $3 \frac{7}{8}$ & $1 \frac{15}{15}$ & $16 \frac{3}{4}$ & $10 \frac{5}{8}$ & 37 & $\frac{3}{4}$ & $3 \frac{1}{2}$ & $2 \frac{6}{4}$ & $3 \frac{1}{2}$ \\
\hline
\end{tabular}

Fig. 8-Dimensions of Standard Boiler Seams

Problem 3.-To find the tensile strength required for 15/16-in. plate to give an efficiency of $80.5 \%$, using sam No. 14 as an example.

On the vertical scale of efficiency find $80.5 \%$, and project a line in a horizontal direction until it intersects the line representing seam 14. From this point project vertically to the $15 / 16-i n$. line, and this intersection projected to the vertical axis grives a tensile strength of 57,000 lb. per sce. in.

I'roblem 4.-If seam No. 14 has an efficiency of $80.0 \%$ what will be the efficiency of seam No. 2, providing that the thickness of plate, tensile strength of plate and shearing stress of rivets are alike for both seams?

From the point where the $80.0 \%$ line from the vertical scale intersects the line of seam No. 14, project a line vertically to the line of seam No. 2 and from this point project a line horizontally and find the efficiency, which is $82.7 \%$. 


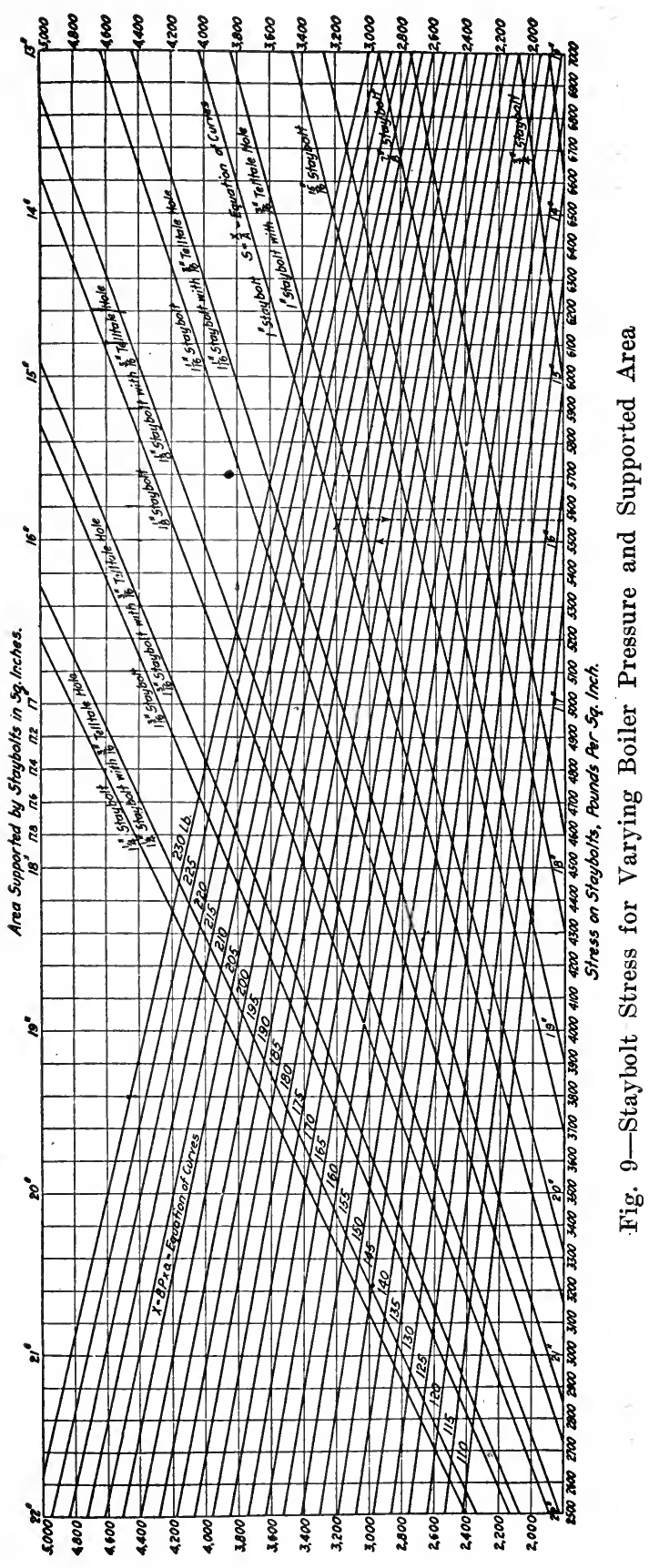




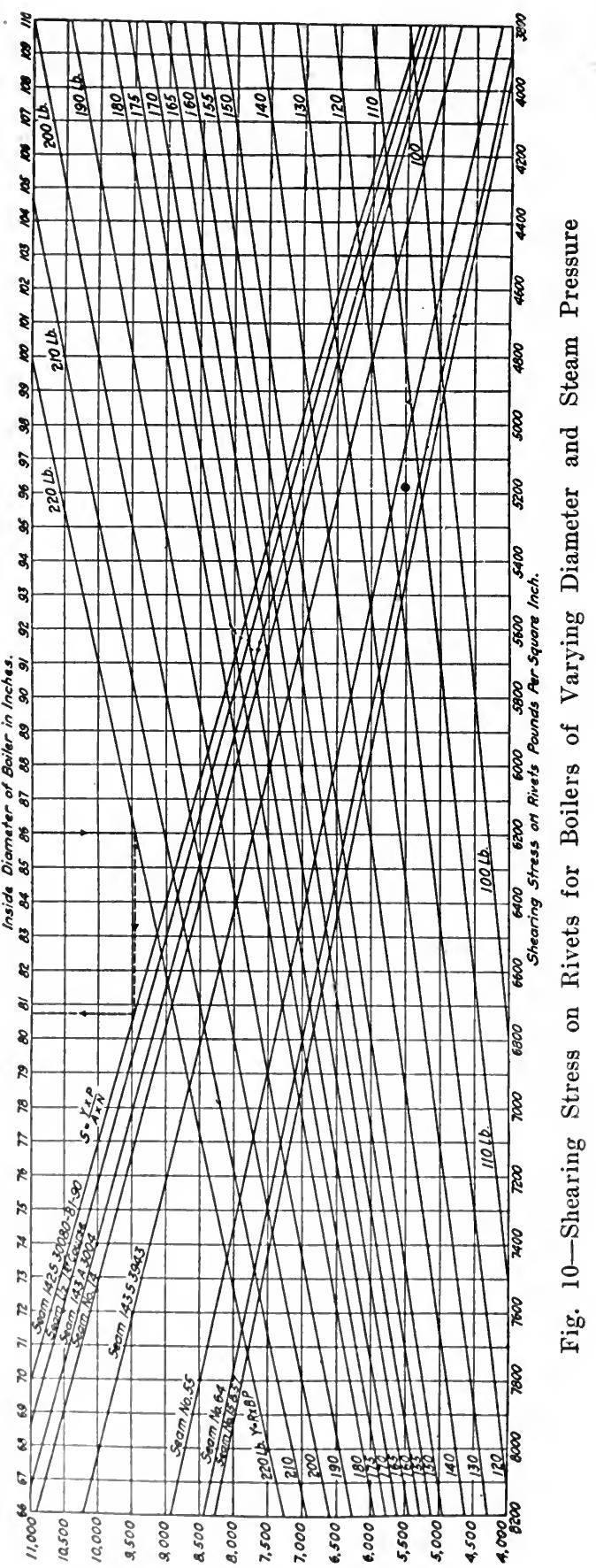


Fig. 9 shows the stress on several sizes of stay bolts, under conditions varying from supporting $13 \mathrm{sq}$. in. to $22 \mathrm{sq}$. in. of area with boiler pressures varying from $110 \mathrm{lb}$. to $230 \mathrm{lb}$. gage, advancing by five pounds each.

The general equation is

$$
\mathrm{S}=\frac{\mathrm{BP} \times \mathrm{a}}{\mathrm{A}}
$$

where:

$\mathrm{S}=$ Stress on stay bolt in pounds per square inch.

$\mathrm{BP}=$ Boiler pressure in pounds per square inch as shown by gage.

$\mathrm{a}=$ Area in square inches supported by stay bolt.

$\mathrm{A}=$ Area of stay bolt in square inches.

There are two systems of curves on the co-ordinate field, and care should be exercised in using the respective scales. The lines marked with the boiler pressure show the relation between the boiler pressure and the area supported by the stay bolts and the lines marked with the various sizes of stay bolts show the relation between the product of boiler pressure times area supported and the stress on stay bolts as illustrated. The area for the stay bolts is taken at the root of the thread, for a $V$ thread, 12 threads per in., and there are also lines for stay-bolts which have $3 / 16$-in. telltale holes.

To use the curve it is first necessary to determine the number of square inches of flat surface that is to be supported.

Problem 5.-What is the stress on a 1-in. solid stay bolt with $V$ thread, 12 threads per inch when supporting 16 sq. in. at $200 \mathrm{lb}$. pressure?

On the horizontal axis, either at the top or bottom, find the area supported, in this case 16 sq. in., and project a line vertically until it intersects the $200 \mathrm{lb}$. line. From this point project a line horizontally to the right until it intersects the 1-in. stay bolt line. This intersection projected vertically either to the top or bottom axis gives the stress on the stay bolt, which in this case is $5,560 \mathrm{lb}$. per sq. in.

Fig. 10 shows the shearing stress on rivets for such seams as outlined for boilers varying from 66 in. to 110 in. in diameter, and for steam pressures between the limits of $100 \mathrm{lb}$. and $220 \mathrm{lb}$. gage pressure.

The general equation for shearing stress on rivets is

$$
\mathrm{S}=\frac{\mathrm{R} \times \mathrm{P} \times \mathrm{BP}}{\mathrm{A} \times \mathrm{N}}
$$

where :

$\mathrm{S}=$ Shearing stress on rivets in pounds per square inch.

$\mathrm{R}=$ Radius in inches of inside of boiler shell.

$\mathrm{P}=$ Pitch in inches of rivets in the outside row of seam.

$\mathrm{BP}=$ Boiler pressure in pounds per square inch as shown by gage.

$\mathrm{A}=$ Area in square inches of rivet hole.

$\mathrm{N}=$ Number of rivet shearing planes in pitch length taken.

The system of straight lines marked with the boiler pressure, repre- 
sent the relation between the radius of the boiler and the boiler pressure. These lines are expressed by the equation $\mathrm{R} \times \mathrm{BP}=\mathrm{Y}, \mathrm{Y}$ representing the figures given on the $Y$-axis. The second system of straight lines represents the relation of the characteristics of each individual seam, so far as shearing stress on the rivets is concerned, to the radius. of inside of boiler and boiler pressure.

Problem 6.- What is the shearing stress on the rivets of seam No. 1 when used on a boiler 86 -in. inside diameter and carrying $220 \mathrm{lb}$. pressure?

On the top horizontal axis find the diameter of the inside of the boiler, in this case 86 in., and project a line vertically until it intersects the line marked $220 \mathrm{lb}$. From this point projeet a line in a horizontal direction until it intersects the line marked with the seam number, in this case seam 142-S-30,080. This intersection projected vertically to the bottom horizontal axis gives the shearing stress on rivets which is $6,730 \mathrm{lb}$. per sq. in.

Fig. 11 shows the tension on net section for various thicknesses of plate, boiler pressures, efficiency of seams and diameters of boilers.

The general equation is

where:

$$
\mathrm{T}=\frac{\frac{\mathrm{D}}{2} \times \mathrm{BP}}{\mathrm{t} \times \mathrm{E}}
$$

$\mathrm{T}=$ Tension on net section in pounds per square inch.

D

$\longrightarrow=$ Radius in inches of inside of boiler.

2

$\mathrm{BP}^{\mathrm{P}}=$ Boiler pressure in pounds per square inch as shown on the gage.

$\mathrm{t}=$ Thickness of plate in inches.

$\mathrm{E}=$ Effieiency of the boiler seam in per cent.

There are two co-ordinate fields shown on the sheet, both of which are used in determining the tension on a net seetion. One field consists of a series of straight lines showing the relation between boiler pressure and the inside diameter of the boiler. The equation for these lines is

$$
\mathrm{X}=\frac{\mathrm{D}}{2} \times \mathrm{BP}
$$

where $\mathrm{X}$ is equal to the product of boiler pressure and the radius of the inside of the boiler. 'This is transferred to the second field in obtaining the tension on a net section.

The second field consists of two series of straight lines crossing each other. The series of lines upon which are marked the thicknesses of plate are plotted from the equation

$$
Y=\frac{X}{t}
$$




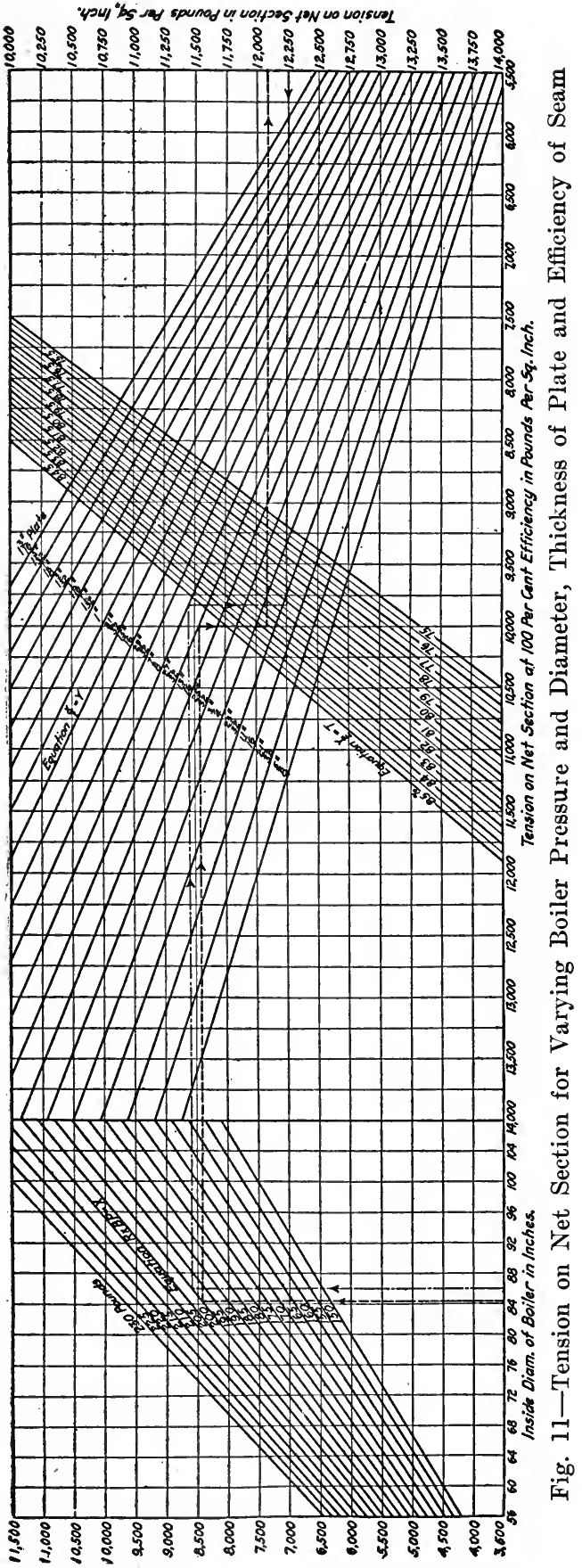


where:

$\mathrm{Y}=$ Tension on net section at 100 per cent efficiency

$\mathrm{t}=$ Thiekness of plate in inches.

D

$\mathrm{X}=$ Value of $\frac{-}{2} \times \mathrm{BP}$ as explained above.

The other series of straight lines upon which are marked the different efficiencies, show the relation between the tension on a net section at $100 \%$ efficieney and on a net section for the various other efficiencies. of seams as' noted.

These lines have been plotted from the following equation:

$$
\mathrm{T}=\frac{\mathrm{Y}}{\mathrm{E}}
$$

where:

$\mathrm{T}=$ Tension on net section at the desired efficiency.

$\mathrm{E}=$ Efficiency in per eent.

$\mathrm{Y}=$ Value of $\frac{\mathrm{X}}{\mathrm{t}}$ as explained above.

Problem 7.- To find the tension on a net section of a boiler of $84 \% 16$ in. diameter, ${ }^{2} \pi / 32-\mathrm{in}$. thickness of plate, $200 \mathrm{lb}$. boiler pressure and $82.75 \%$ efficiency of seam.

From the point representing $84^{5 / 16}$-in. diameter on the horizontal axis of field No. 1, project a line vertically until it intersects the $200 \mathrm{lb}$. line and from this point project a line horizontally into field No. 2 until it intersects the $27 / 32-i n$. line. Project this point vertically until it intersects the line representing $82.5 \%$, where it will be necessary to interpolate to reach an imaginary line representing $82.75 \%$. This point projected horizontally to the vertical axis gives the desired tension on net section as $12.100^{\circ} \mathrm{lb}$ per sq. in.

I'rob'em 8.- What efficiency must a boiler seam have when the tension on a net section is $12,250 \mathrm{lb}$. per sq. in. with inside diameter of. the boiler $86 \mathrm{in}$., boiler pressure $200 \mathrm{lb}$. and thickness of plate $7 / 8 \mathrm{in}$.

From the point representing 86 in. on the horizontal axis of field No. 1, project a line vertically until it intersects the $200 \mathrm{lb}$. line, and from this point projocet a line horizontally into field No. 2, until it in-

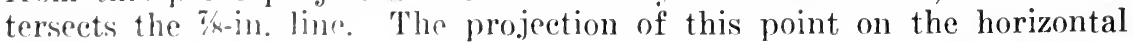
line showing 12.250 16 . tension on a net section gives the required efficiency which is hy interpolation $80.2 \%$.

Engine Dcsign. Many charts have been devised to assist in engine design, such as obtaining mint force due to inertia of reciprocating parts, determining the sizes that will meet particular requirements, cte. The example given here is from an article, "Chart for Determining Engine Frame Loads," by E. A. Andrews, published in Ma chinery, August, 1916.

It is the purpose of this article to explain the use of a chart, Fig. 12, for finding the loads on the following members of an engine: (1) main 
bearings ; (2) crankpins and crosshead pins; (3) crosshead guides. The horizontal line $A B$ represents various values of the total load on the piston in pounds; the vertical line $C D$ indicates the projected area of the main bearings in square inches; vertical line $A E$ represents the projected area of the crankpins and crosshead pins in square inches; and the horizontal line $E F$ shows the projected area of the crosshead bearing surface in square inches.

To illustrate the use of this chart, let us assume a case in which the

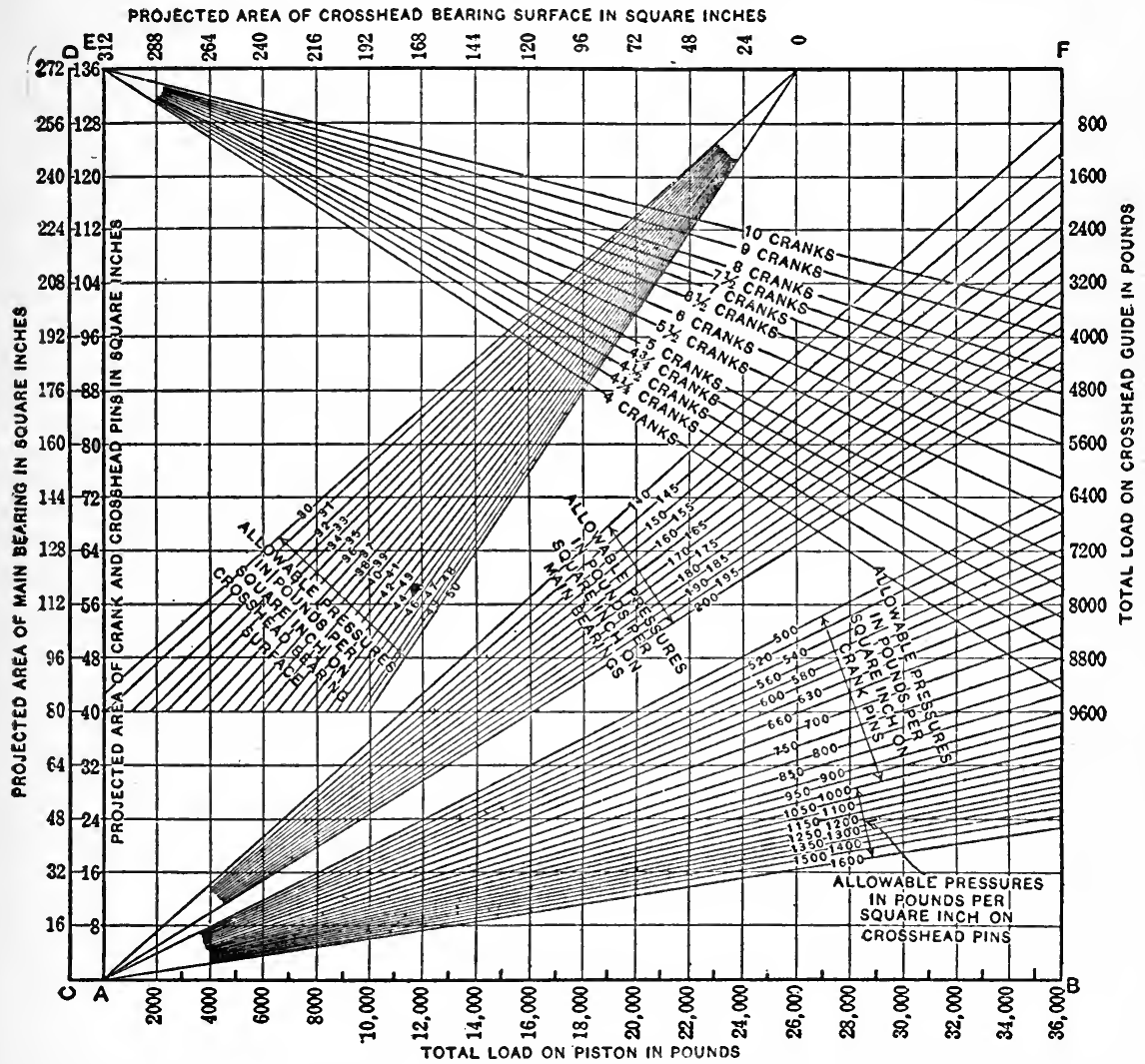

Fig. 12-Chart for Use in Rapidly Determining Loads on Important Engine Frame Members

total load on the piston is $24,000 \mathrm{lbs}$. This value is located on line $A B$, and assuming that it is required to find the size of the main bearings, we follow the $24,000 \mathrm{lb}$. line vertically to the radial lines indicating allowable main bearing pressures. It will be seen that these lines cover a range of from 140 to $200 \mathrm{lbs}$. per sq. in. and assuming that we decide to use a value of $150 \mathrm{lbs}$. per sq. in., the radial line corresponding to this value is located, after which the horizontal line is followed across

Copyright 1916 by the Industrial Press, publishers of Machinery, 148 Lafayette St., New York City. 
the chart to the vertical line $C D$. Here we find that the required area for the main bearings is $159 \mathrm{sq}$. ins. Following the same method in determining the areas for the crankpins and crosshead pins, the required values may be quickly determined from the chart, taking care to use the proper section of the chart in each case.

To determine the proper area for the crosshead bearing surface, it is necessary to know the ratio of the length of the connecting-rod to the radius of the crank circle, and assuming that for the case under consideration the value of this ratio is 5 to 1 , or "five cranks," we follow the $24,000 \mathrm{lb}$. piston load line until it crosses the radial line marked 5 cranks; from this point of intersection, move across the chart to the left to the radial line of allowable crosshead bearing surface pressure. Assuming that the value is $40 \mathrm{lbs}$. per sq. in., we follow up the vertical line to $E F^{\prime}$ at the top of the chart, and find that $123 \mathrm{sq}$ ins. is the proper area for the crosshead bearing surface.

The total load on the crosshead guide is represented on line $B F$ at the right-hand margin of the chart, and is found by moving across the chart to the right from the point of intersection of the piston load line and the radial line representing the ratio of connecting length to crank radius.

Sizes of Pipes. Charts are a great help in determining the sizes of steam pipes, the flow of steam in pipes, the areas of gas passages in steam boilers or of the flues and breechings connecting boiler and chimney, the proportioning of trunk mains for the most economical supply to the branch leaders, and in selecting a stack of proper diameter and height for natural draft, etc. "Two examples are given, the first from an article by W. L. Durand, "Flow of Steam in Pipes," published in Power, March 7, 1916, and the second from a paper by Wm. Wipperman, published in P'ower, February 29, 1916, entitled "Selection of Stacks and Breeching."

The commonest method for determining the size of steam pipes is to assume a velocity of $6,000 \mathrm{ft}$. per min. for high-pressure pipe and 4,000 ft. for low-pressure pipe. That this method will not give satisfactory results, except over a very limited range, may easily be seen if the drop in pressure at these velocities is computed for different sizes of pipe. The accompanying table is based on a length of pipe of $100 \mathrm{ft}$. and an initial pressure of $100 \mathrm{lb}$. at 6,000 ft. per min.

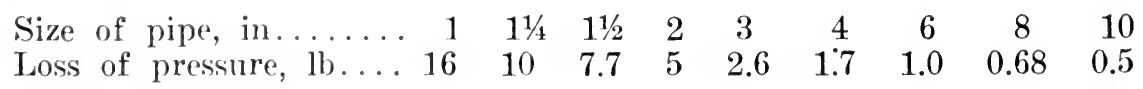

From the foregoing it may be seen that in small pipes the loss in friction is so great as to affect the economy of operation; on the other hand, in the large sizes of steam lines bigger pipes are speeified than are necessary. This increases the eost of installation to an appreciable extent since the eost of pipe and fittings increases rapidly with the size.

The factors that enter into any problem of this character are weight or quantity of steam, length of pipe, density of the steam, drop in pressure and the diameter of the pipe. To obtain accurate results, it is neessary to take into consileration all of the foregoing factors, and any formula used in this comnection should contain all of them. 
The most commonly accepted formula for the flow of steam is Unwin's, which is as follows:

$$
W=87.5 \sqrt{\frac{D p d^{5}}{L\left(1+\frac{3.6}{d}\right)}}
$$

where

$W=$ Weight of steam flowing in pounds per minute;

$L=$ Length of pipe in feet;

$d=$ Internal diameter of pipe;

$D=$ Density, pounds per cubic foot;

$p=$ Drop in pressure, pounds per square inch.

The reason that this formula is not more generally used is presumably because of the excessive time required in solving it. Recently the writer published a table based on dividing Unwin's formula into three parts. The length of pipe and friction loss may be reduced to one variable "friction loss per unit length," such as "per hundred feet."

$$
W=87.5 \sqrt{\frac{p}{100}} \times \sqrt{D} \times \sqrt{\frac{d^{\circ}}{1+\frac{3.6}{d}}}
$$

But even the use of this may require more time than many engineers desire to spend in sizing pipes. Fig. 13 is based on the table and gives

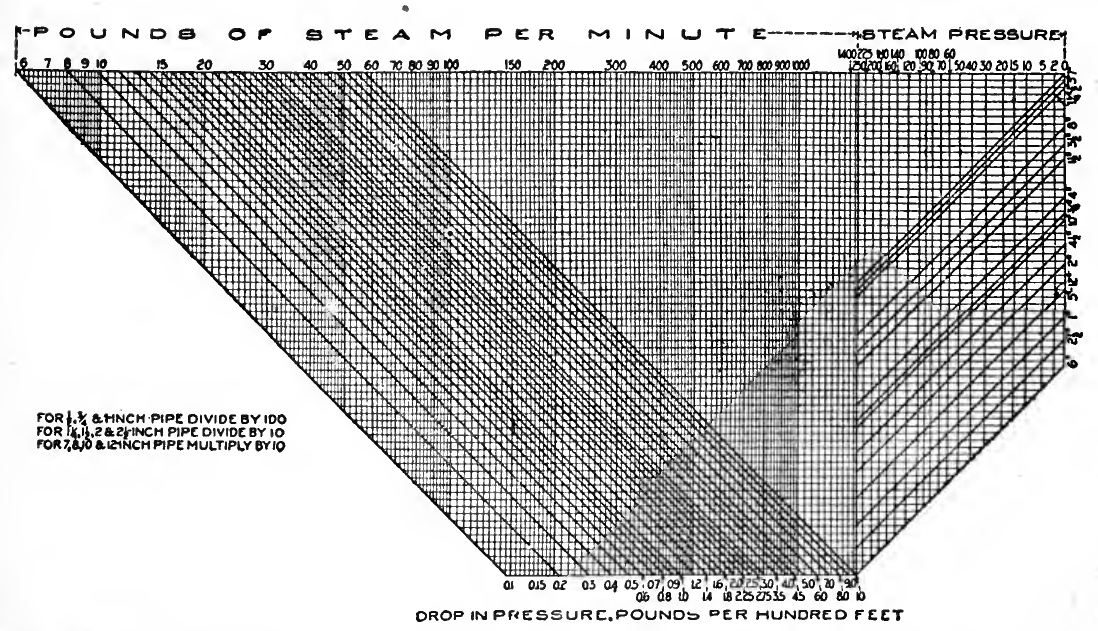

Fig. 13-Curves by Which if Any Three Variables Are Known the Fourth Can Be Found

quickly and accurately the result in any problem relating to the flow of steam in pipes if three of the four variables are known.

These curves are really a slide-rule and mechanically add the log 
of the factors in the table. The $\log$. of $\vee D$ is laid off corresponding to the steam pressure, the log. of $\sqrt{\frac{d^{5}}{1+\frac{3.6}{d}}}$ is laid off for the diameter of the pipe, the log. of $87.5 \sqrt{\frac{p}{100}}$ is laid off for the drop in pressure, and the log. of the quantity of steam is laid off as indicated. This is accomplished by plotting these curves on logarithmic paper.

Starting at the pressure line and moving down to the steam pressure desired, the length is proportional to the log. of the square root of the density, and by projecting that to the first 45-deg. line, an equal distance at right angles is obtained. Continuing this line to the required pipe size adds the log. of the pipe factor. Projecting that distance to the bottom of the 45-deg. lines representing the drop in pressure, a distance equal to the sum of the pressure and diameter is obtained on the vertical scale. By moving up to the required drop the log. of the factor for the pressure drop is added, and projecting the sum of the logs. of the three factors, the answer is read directly on the quantity scale in pounds of steam per minute.

In order to show the use of this chart, take a hypothetical case of a steam main $800 \mathrm{ft}$. long and $4 \mathrm{in}$. diameter with steam at $100 \mathrm{lb}$. pressure. What will be the flow of steam if the drop is limited to $4 \mathrm{lb}$.? The drop per $100 \mathrm{ft}$. is $1 / 2 \mathrm{lb}$. Starting at $100 \mathrm{lb}$. and running down to the 4 -in. diagonal line and over to $1 / 2 \mathrm{lb}$., $73 \mathrm{lb}$. of steam per min. is read on the "pounds of steam" scale. Suppose the problem to be the same except the pounds of steam given and the drop was to be found. Starting at $100 \mathrm{lb}$. and running down to the 4-in. diagonal line and over $73 \mathrm{lb}$., $1 / 2 \mathrm{lb}$. is found at the intersection. Given any three of the four variables, the fourth ean be found from this chart.

The Determination of the Height and Diameter of a Chimney from tables or formulas connecting height and diameter with horsepower often results in an impossible stack. As a rule there are so many factors involved that no one table or formula meets the requirements of the individual problems. But it is possible to present the data on a chart in such a way that chimney and breeching dimensions ean be determined with ease and certainty. That was the aim in designing Fig. 14.

The function of a chimney is to carry off the gases and to produce draft. Its height is fixed by the draft necessary to overeome the resistance of bends, damper, connections, breeching, boiler and grate. This resistance is not a function of the horsepower, but varies with every installation. The cross-sectional area must be so large that the stack will handle the volume of gas without excessive loss in friction. By increasing the height, the diameter can be reduced, and conversely, but among the various chimneys that are large enough, there is one that is cheaper to build.

The draft available for producing flow is usually measured in inches of water column. Its intensity depends upon the difference in weight of the outside and inside air columns, redueed under flow eonditions 


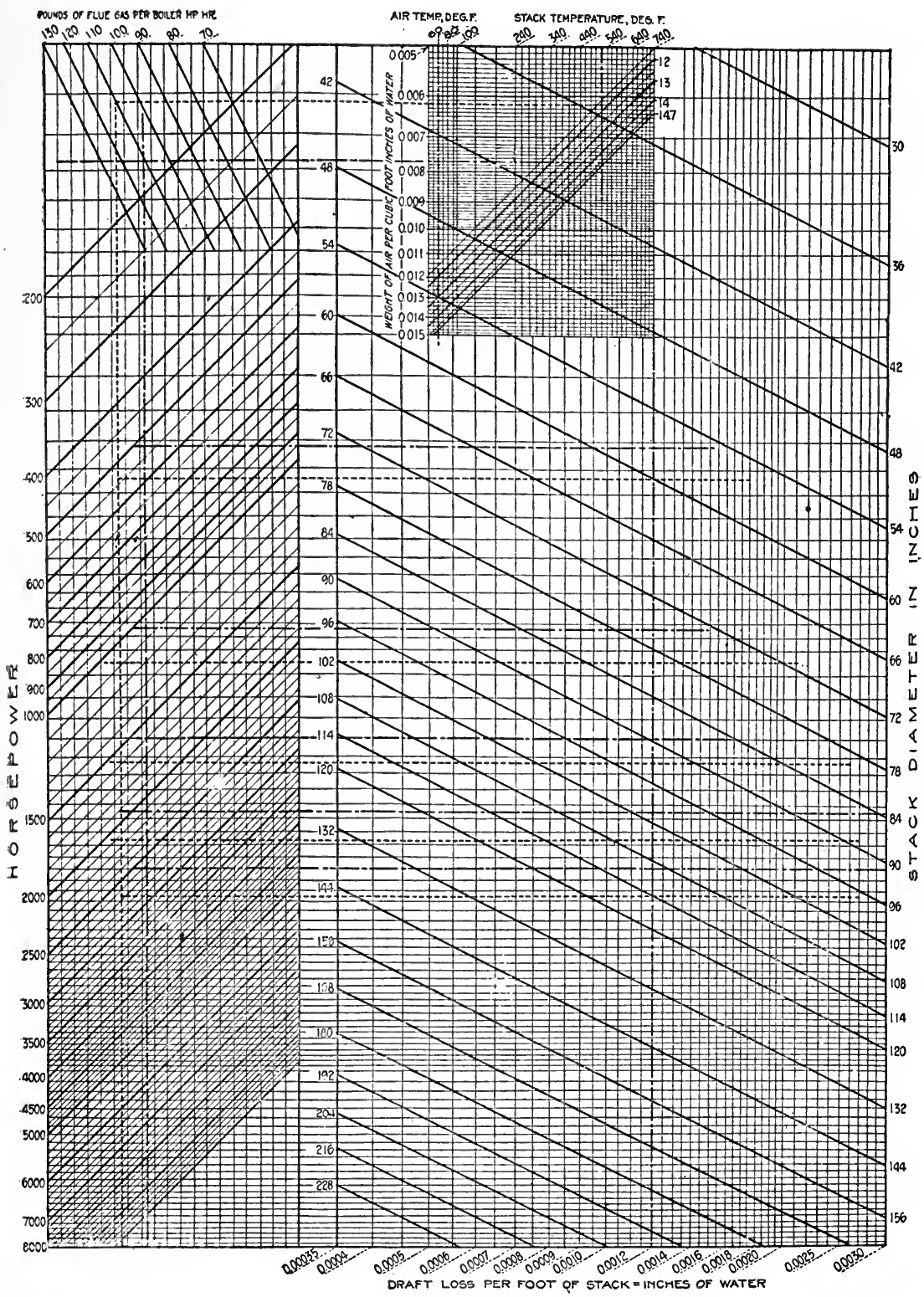

Fig. 14

by the friction loss in the stack. For a given temperature and pressure it is a maximum when no gases are flowing. The weight of a cubic foot of gas depends upon the temperature, pressure and nature of the gas. For chimney calculations flue gas is always considered to be the same as air. Inasmuch as draft is always measured in inches of water 
column, the scale on the chart shows the weight of air in inches of water columm.

The use of this chart will be illustrated by determining the dimensions of a chimney and breeching for a boiler house containing five 350 -hp. water-tube boilers vertically baffled and equipped with chain grates burning Illinois, or equal, coal. The overload is not to exceed $50 \%$ of rating. The stack temperature will average 520 deg. F. at maximum rating and about 440 deg. F. at normal rating. The outdoor temperature will be taken at $60 \mathrm{deg}$. F. and the atmospheric pressure: at $14.5 \mathrm{lb}$. per sq. in.

Referring to the chart, the intersection of a vertical representing 520 deg. F. with a diagonal representing $14.5 \mathrm{lb}$. per sq. in. determines a horizontal through 0.0077 in., the height of a water column $1 \mathrm{ft}$. square. that is of the same weight as a cubie foot of air at the given pressure: and temperature. The same method shows that 0.0145 in. of water weighs the same as a cubic foot of air at a temperature of $60 \mathrm{deg}$. F. and a pressure of $14.5 \mathrm{lb}$. per sq. in. The difference between 0.0145 and 0.0077 is $0.0068 \mathrm{in}$. This is the potental draft per foot of stack: when no gases are flowing. Under conditions of flow it must be reduced by an amount equal to the friction per foot of stack.

The head lost in friction is a function of the square of the velocity. It depends upon the roughness of the surface, on the density of the gas: and on the surface in contact with the gas. For a brick stack,

$$
D=54 \frac{W^{2} T H}{d^{5} P}
$$

in which

$$
\begin{aligned}
D & =\text { Draft loss in inches of water column; } \\
W & =\text { Pounds of flue gas flowing per second; } \\
T & =\text { Aboslute temperature, dcgrecs F.; } \\
P & =\text { Absolute pressure, pounds per square inch; } \\
H & =\text { Ileight in feet above center of breeching; } \\
d & =\text { Diameter in inches; } \\
54 & =\text { Factor depending on the roughness of a brick stack. }
\end{aligned}
$$

The atmospheric pressure is fixed by the altitude. The temperature of the flue gas depends upon the heat-absorbing qualities of the equipment. The quantity of flue gas $W$ depends upon the quantity and quality of eoal burned. It is represented on the chart by diagonal lines under the caption "Pounds of Flue Gas per Boiler Hp.-IIr.," which ean be estimated from the coal and $\mathrm{CO}_{2}$ guarantees made by the stoker and boiler manufacturers, or it can be estimated from experience. It varies: from 70 to $130 \mathrm{lb}$. per hp.-hr. In this problem it will be taken as 110 .

The chart is a graphical solution of the formula previously given. The draft loss per foot of stack $I$ ) is determined as follows: Through the intersection of the vertical through 520 deg. F. and the diagonal 14.5 lb. per sq. in. (lraw a horizontal line intersecting the diagonal marked 110 lb. of flue gas por hp.-hr. Through this intersection draw a vertical cutting all of the horsepower diagonals. Inasmuch as this line is referred to several times, it will be called the " $T, P$ and $W$ " line. Continuing, 
draw a horizontal line through the intersection of the " $T, P$ and $W$ " line with the horsepower line 2,625 (150\% of the rating of the five boilers). This line intersects some of the stack diameters, and the reading of the intersection on the "Draft Loss per Foot of Stack" scale is $D$ in "Inches of Water" column. The values of $D$ for different diameters are tabulated here:

$\begin{array}{cccc}\text { Stack } & \text { Draft } & \text { Stack } & \text { Draft } \\ \text { Diameter, in. } & \text { Loss } & \text { Diameter, in. } & \text { Loss } \\ 132 & 0.0005 & 108 & 0.0013 \\ 120 & 0.00075 & 102 & 0.0017 \\ 114 & 0.0010 & 96 & 0.0023\end{array}$

Under conditions of maximum flow the draft available for producing flow is the difference between the potential draft and the friction loss. If the total draft required is known, the height of stack necessary can be computed by dividing the draft required by the available draft per foot of stack.

Before the height of the stack can be computed, an estimate must be made of the drop through the furnace, boiler, breeching, etc. The furnace draft, the drop through the boiler and the drop through the economizer, if one is used, can be furnished by the manufacturers. It is best to get these data from them because there is considerable variation in the different types of apparatus. It is customary to figure on a drop of $0.1 \mathrm{in}$. per $100 \mathrm{ft}$. of breeching of the proper size, on a loss of $0.05 \mathrm{in}$. for every right-angled bend; $0.05 \mathrm{in}$. for every boiler connection or entrance to breeching and breeching entrance to stack and 0.05 in. for a damper of proper design-that is, one that offers resistance at the lower capacities and a minimum of resistance at high ratings when maximum draft is required. Additional obstructions must be allowed for.

For the given problem the furnace draft at $150 \%$ of rating is $0.40 \mathrm{in}$. the drop through the boiler and damper is $0.47 \mathrm{in}$., the drop in $80 \mathrm{ft}$. of breeching is $0.08 \mathrm{in}$.; and the draft loss in entrance is $0.30 \mathrm{in}$., making a total draft required at the stack of 1.25 in. The heights of stack that will produce this draft are given in the following table. As previously figured, the potential draft per foot of stack is 0.0068 in. Deducting from this the draft loss per foot of stack, as itemized in the foregoing table, gives the available draft per foot of stack. Dividing the total draft required by the draft per foot of stack gives the height of stack.

$\begin{array}{cccccc}\begin{array}{c}\text { Stack } \\ \text { Diameter, In. }\end{array} & \begin{array}{c}\text { Available } \\ \text { Draft, In. }\end{array} & \begin{array}{c}\text { Stack } \\ \text { Height, Ft. }\end{array} & \begin{array}{c}\text { Stack } \\ \text { Diameter, In. }\end{array} & \begin{array}{c}\text { Available } \\ \text { Draft, In. }\end{array} & \begin{array}{c}\text { Stack } \\ \text { Height, Ft. }\end{array} \\ 132 & 0.0063 & 198 & 108 & 0.0055 & 228 \\ 120 & 0.0060 & 208 & 102 & 0.0051 & 245 \\ 114 & 0.0058 & 216 & 96 & 0.0045 & 278\end{array}$

The total height of the stack is the sum of the foregoing and the disstance from the center of the breeching to the base. In the second paragraph it was stated that the cross-section of a stack must be large enough to handle the gas without excessive friction loss, and by increasing the diameter and conversely a size of stack could be found that was cheapest. With the exception of the last two stacks all are equally good and wellproportioned. One of them, however, is cheaper than the rest. 
But for the following exception, the method of figuring the sizes of steel stacks is the same as for brick. The draft lost in friction in a steel stack is less than that lost in a brick stack because the resistance is less. It is 0.8 of the draft lost in brick or brick-lined chimneys. For a steel stack for the boiler house in question the friction head is tabulated. here :

Stack

Diameter, in. $132 \ldots .0 .0005 \times 0.8 \ldots 0.0004$ $120 \ldots 0.00075 \times 0.8 \ldots 0.0006$ $114 \ldots \ldots 0.0010 \times 0.8 \ldots 0.0008$
Friction Head, in.
Friction Head, in. $108 \ldots \ldots 0.0013 \times 0.8 \ldots .0 .0010$ $96 \ldots .0 .0023 \times 0.8 \ldots 0.0018$

Quite frequently it is necessary to estimate the capacity of a stackalready built. Take for example a stack $120 \mathrm{in}$. diameter and $208 \mathrm{ft}$. high. Using the data of the previous problem, the draft required at the entrance to the stack is $1.25 \mathrm{in}$. of water column. The available draft perfoot of stack is $1.25 \div 208=0.0060$. The difference between the potential draft (0.0068 in.) and the available draft (0.0060) is 0.0008 , the loss. per foot of stack. The intersection of the 120-in. diameter line with a. vertical through 0.0008 in. locates a horizontal line. The intersection of this horizontal with the " $T, P$ and $W$ "' line fixes the capacity of the stack.. It happens to be $2,625 \mathrm{hp}$. in this problem.

In tall buildings the height of the stack is fixed by the height of the building. If the boiler house in question is in the basement of a tall building such that the stack rises $300 \mathrm{ft}$. above the breeching connection, the solution for the diameter is as follows:

The estimated draft required at the stack is $1.25 \mathrm{in}$. The available: draft per foot of stack is $1.25 \div 300=0.0042$. The difference between the potential and available draft is $0.0068-0.0042=0.0026$, the friction head in inches of water. The intersection of a vertical through $0.0026 \mathrm{in}$. and a horizontal through $2,625 \mathrm{hp}$. fixes the diameter. In this case the nearest diameter is 96 in.

In making up the estimate of the draft drop in the breeching, the figure $0.1 \mathrm{in}$. per $100 \mathrm{ft}$. of flue was used. This is safe for round steel flues of the proper size, but for square, rectangular and brick flucs the retarding effect is greater, consequently the area of such flues should be larger than that of a round steel flue. It is not necessary that the breeching be of the same area throughout. It is best to proportion the cross-section according to the volume of gases passing.

The chart, which was designed for brick flues and stacks, ean be used to advantage in eomputing the cross-section of the breeching. $\Lambda$ s mentioned before, the draft lost in a steel flue is 0.8 of that lost in a brick flue. Consequently the figure 0.0012 in. on the "Draft loss per Foot of Stack". scale represents (0.0)(0) in. for steel flues, beeause $0.0012 \times 0.8$ is practically $0.0010 \mathrm{in}$, the draft drop per foot of flue used in making up the total draft estimate.

The cross-sections are determined in the following manner: Through $0.0012 \mathrm{in.} \mathrm{draw} \mathrm{a} \mathrm{vertical} \mathrm{line} \mathrm{and} \mathrm{through} \mathrm{the} \mathrm{intersection} \mathrm{of} \mathrm{the} \mathrm{horse-}$ power diagonals 525, 1,(5)(), 1,575, 2,100 and 2,625 with the " $T, P$ and W," line draw horizontal lines. The intersection of the $0.0012 \mathrm{in}$. ver- 
tical with the horizontal lines fixes the diameter of the flue. Inasmuch as a round breeching is seldom used, it is better to make it square than rectangular, making the side of the square equal to the diameter determined from the chart. Then the cross-section will be a trifle larger than necessary. The dimensions appear in the following table:

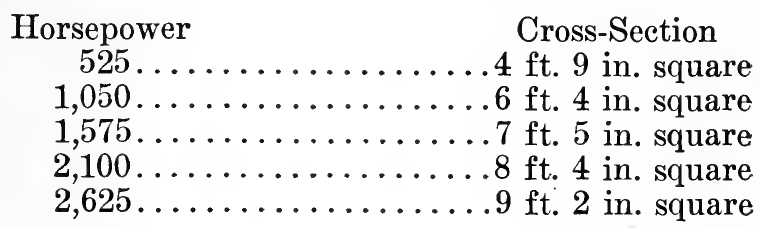

If rectangular cross-sections are used, the area must be increased because a rectangular section offers more resistance. General practice is to make them up to $120 \%$ of the area of the stack. If the flue is lined with tile or is of brick, the vertical line is drawn through $0.0010 \mathrm{in.} \mathrm{This} \mathrm{results}$ in proportionately larger flues, because the resistance is greater.

This method of chimney analysis is simple and easily learned. It cannot very well result in impossible stacks. Many of the chimney men use velocities in figuring areas. A proper analysis shows that high velocities are used in large-diameter stacks and low velocities in the smalldiameter stacks. The velocity may vary from 15 to $40 \mathrm{ft}$. per sec., depending on the size of the stack. Judgment of course is necessary in estimating the temperature of the flue gases, pounds of flue gas per boiler horsepower and the draft required at the stack. In making up the item last named, due consideration must be given to the fact that more draft. is required for higher altitudes than for the sea level. The items to be considered at the higher altitudes are the drop through the boiler and the furnace draft, and they can be estimated with ease by remembering that altitude has the same effect as increasing the capacity - more draft is needed. The cross-section of the breeching and the increased height necessary to overcome the increased friction loss in the stack are taken care of in the method of computing the respective dimensions.

If the atmospheric pressure had been $11.7 \mathrm{lb}$. per sq. in. instead of 14.5, the furnace and boiler drop for the specific problem under consideration would increase from 0.87 to 0.97 in., making a total of $1.35 \mathrm{in}$. of draft required at the stack. The solution of the problem may be traced by the light dotted lines on the chart. The results obtained follow :

\section{CHIMNEY ANALYSIS}

Potential draft, in. of water column........0.0117-0.0062 $=0.0055$ Draft loss (120 in. diam.), in. of water column............. 0.00095 . Draft loss (132 in. diam.), in. of water column...............0.0006 Available draft (120 in. diam.), in. of water column............ 0.0045 Available draft (132 in. diam.), in. of water column............ 0.0049 Height of 120 -in. stack above breeching, ft............ 300 Height of 132 -in. stack above breeching, ft............. 275 


\title{
BREECHING ANALYSIS
}

\author{
Horsepower (150\% rating) Cross-Section
}

$525 \ldots \ldots \ldots \ldots \ldots \ldots \ldots$ ft. 0 in. square

$1,050 \ldots \ldots \ldots \ldots \ldots \ldots 6 \mathrm{ft} .8 \mathrm{in}$. square

$1,575 \ldots \ldots \ldots \ldots \ldots \ldots . \ldots \ldots$ ft. 9 in. square

$2,100 \ldots \ldots \ldots \ldots \ldots \ldots$ ft. 9 in. square

$2,625 \ldots \ldots \ldots \ldots \ldots \ldots$ ft. 6 in. square

These results check very well with dimensions obtained by using the well-known methods of correcting for altitude. Chimney and breeching dimensions can be figured with ease and sufficient accuracy by drawing a few lines on charts ruled as shown. Such a chart is a permanent record of the analysis of the chimney and breeching.

Relation Between the Number of Trains and Passing Points. This article by Paul M. LaBach, was published in Railway Age Gazette, July 30, 1915. In discussions concerning the economies of railway operation the statement is frequently made that the number of meeting and passing points on a single line is in proportion to the square of the number of the (round trip) daily trains. That is to say, if the number of trains is doubled, the number of meeting and passing points will be multiplied by four; if the number is tripled these points will be multiplied
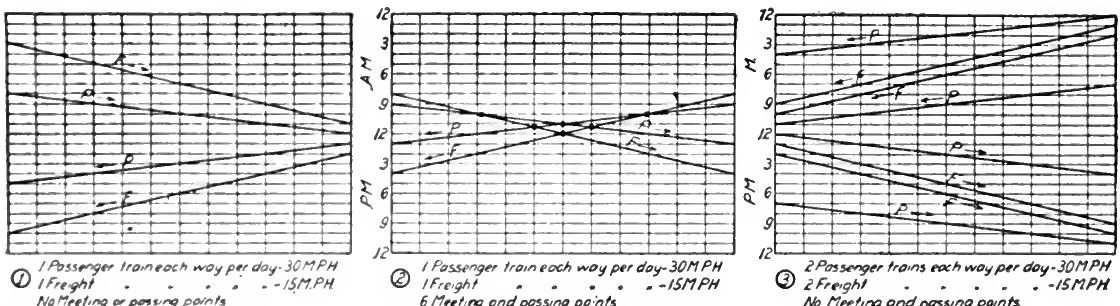

6 Meeting ond possing points
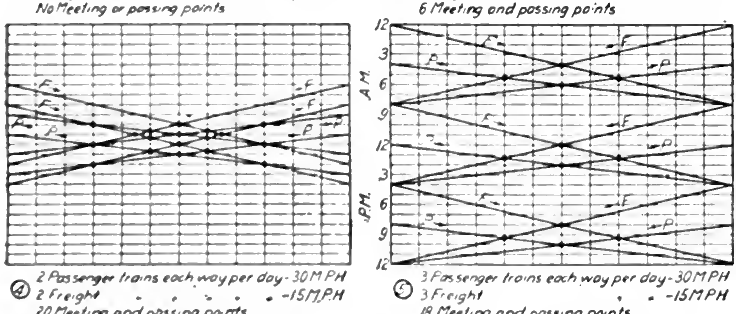

No Meeting and possing paints

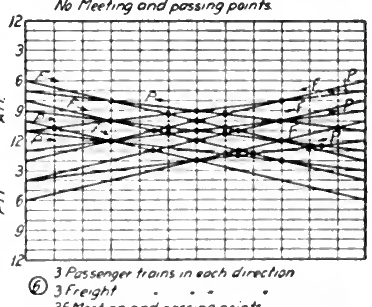

19 Meeting and poseing paints

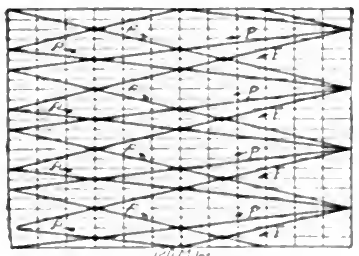

A Assienger trons each way per doy- $30 \mathrm{MPH}$ 30 meeting ond possing points"
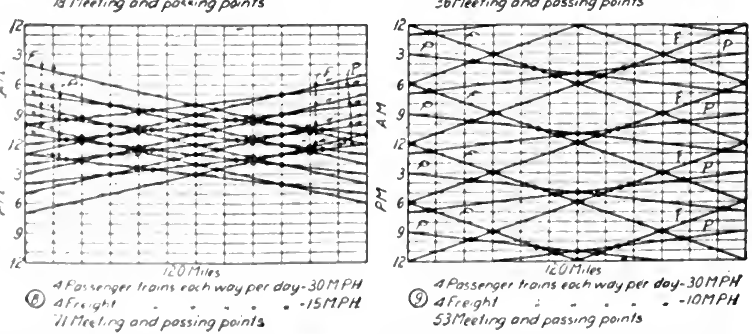

s3meeting ond passing points

Fig. 15-Maximum and Minimum Number of Meeting Points With Different Nimbers of Trains 
by nine, etc. At first glance this appears to be correct. Two trains in opposite directions will meet once; four trains may meet four times, etc. The error lies in the fact that in changing from one number of trains to another the operating conditions are not duplicated. If one should have a certain number of meeting and passing points under present conditions and should double the number of trains he might have four times as many meeting and passing points but the chances are that he would have considerably more. It makes a great deal of difference whether the trains are seattered at intervals throughout the 24 hours or are concentrated in a portion of that time.

To illustrate the point the accompanying diagrams, Fig. 15, have been made on the same principle as density diagrams; the vertical dimensions being divided into space for each hour and the horizontal distance being the length of an engine district. Passenger train speeds are assumed as being $30 \mathrm{mi}$. an $\mathrm{hr}$. and freight $15 \mathrm{mi}$.

The only general rule that ean be formulated is that the slow trains should follow immediately after the fast and be scattered at nearly equal intervals through the day. It is also apparent that increasing the speed will decrease the number of meeting and passing points. To make the rule general, the interval of time occupied by a train passing over the district, divided by 24 , should be as small as possible. Under the assumed condition, the following interesting results are found:

$\begin{array}{ccccc}\begin{array}{c}\text { Diagram } \\ \text { Nos. }\end{array} & \begin{array}{c}\text { No. of } \\ \text { round trip } \\ \text { trains } \\ \text { per day }\end{array} & \begin{array}{c}\text { Square } \\ \text { of the } \\ \text { number } \\ \text { of trains }\end{array} & \begin{array}{c}\text { No. of meeting and } \\ \text { passing points }\end{array} \\ \ldots \ldots . . & 1 & 1 & 0 & 1 \\ 1 \text { and } 2 & 2 & 4 & 0 & 6 \\ 3 \text { and } 4 & 4 & 16 & 0 & 20 \\ 5 \text { and } 6 & 6 & 36 & 18 & 36 \\ 7 \text { and } 8 & 8 & 64 & 30 & 71\end{array}$

In counting the meeting points one half value has been given to points on the lines dividing engine districts and days. As the number of trains increases it is apparent that the tonnage of freight trains will decrease unless there is considerable excess power in the locomotives for the rating, say in Diagram 1. This feature is of considerable importance in making calculations for tonnage ratings in problems of the economics of railway location. According to the older method laid down by Wellington and others, a rating was assumed for one daily train and this was multiplied by ten or twelve as the case might be, but the time spent in meeting and passing will be increased very rapidly and the available running time decreased after a traffic of about eight trains per day is passed, as is shown in Diagram 9, which can be compared with Diagram 7, as both are for the most favorable circumstances. The graphical method of handling such problems is not new, but new applications can be found for it, as it has as many variations as the kaleidoscope.

The proper location of passing sidings on new work is not always an easy matter. By turning the horizontal distance into time and finding the fraction of the whole run between each passing point this may be arrived at. The next step would be to develop a table of accelerations 
and retardations and plot a time curve. The location of the train when $1 / 6$ of its run (in time) has elapsed could then be fixed or any other fraction that might be eaused by the ordinary operation of trains.

A Graphical Method of String-lining Curves is given by H. M. Church in Railway Age Gazette, July 16, 1915. Some discussion has appeared regarding the advantages and results to be obtained by the use of a string for lining track, and the extensive use of various methods which have been tried where curves have not been staked and spiraled previously by an engineering corps. The following method of string lining has been found to be specially practicable and adaptable to use on all curves, simple or compound.

\section{TABLE OF MEASUREMENTS}

\begin{tabular}{|c|c|c|c|c|}
\hline & $\begin{array}{l}\text { Measu } \\
\text { taken on ex }\end{array}$ & $\begin{array}{l}\text { rements } \\
\text { isting curve }\end{array}$ & $\begin{array}{l}\text { Measur } \\
\text { re-establ }\end{array}$ & $\begin{array}{l}\text { ments for } \\
\text { shed curve }\end{array}$ \\
\hline $\begin{array}{c}31-\mathrm{ft} . \\
\text { stations }\end{array}$ & $\begin{array}{c}\text { Middle } \\
\text { ordinates of } \\
62 \text {-ft. chords } \\
\text { in inches }\end{array}$ & $\begin{array}{l}\text { Elevation } \\
\text { in inches }\end{array}$ & $\begin{array}{l}\text { Middle } \\
\text { ordinates of } \\
62-f t . c h o r d s \\
\text { in inches }\end{array}$ & $\begin{array}{l}\text { Elevations } \\
\text { in inches for } \\
40 \mathrm{mi} \text {. per hr. }\end{array}$ \\
\hline $1 \ldots \ldots \ldots \ldots$ & . 0 & 0 & 0 & 0 \\
\hline $0 \ldots \ldots \ldots \ldots$ & . & $1 / 2$ & $7 / 8$ & 1 \\
\hline $2 \ldots \ldots \ldots \ldots$ & $11 / 8$ & $3 / 4$ & $13 / 4$ & 2 \\
\hline $2 \ldots \ldots \ldots \ldots$ & $23 / 4$ & $13 / 4$ & $23 / 4$ & 3 \\
\hline $3 \ldots \ldots \ldots$ & $51 / 4$ & $23 / 4$ & $31 / 2$ & 4 \\
\hline $4 \ldots \ldots \ldots \ldots$ & $51 / 4$ & $21 / 2$ & $4 \mathrm{I} / 2$ & 5 \\
\hline $5 \ldots \ldots \ldots \ldots$ & 6 & 3 & $41 / 2$ & 5 \\
\hline $6 \ldots \ldots \ldots \ldots$ & $3 \mathrm{I} / 2$ & $31 / 4$ & $4 \pi / 2$ & 5 \\
\hline $7 \ldots \ldots \ldots \ldots$ & .. $61 / 2$ & $21 / 4$ & $41 / 2$ & 5 \\
\hline $8 \ldots \ldots \ldots \ldots$ & $7 \mathrm{I} / 2$ & 2 & $41 / 2$ & 5 \\
\hline $9 \ldots \ldots \ldots$ & 5 & 3 & $41 / 2$ & 5 \\
\hline $10 \ldots \ldots \ldots \ldots$ & .. $3 \mathrm{I} / 4$ & $23 / 4$ & $41 / 2$ & 5 \\
\hline $11 \ldots \ldots \ldots \ldots$ & . 4 & $21 / 2$ & $41 / 2$ & 5 \\
\hline $12 \ldots \ldots \ldots \ldots$ & . & $21 / 2$ & $41 / 2$ & 5 \\
\hline $13 \ldots \ldots \ldots \ldots$ & $31 / 2$ & $23 / 4$ & $35 / 8$ & 4 \\
\hline $14 \ldots \ldots \ldots \ldots$ & 3 & $25 / 8$ & $27 / 8$ & $31 / 8$ \\
\hline $15 \ldots \ldots \ldots \ldots$ & $11 / 2$ & $23 / 4$ & 2 & $21 / 4$ \\
\hline $16 \ldots \ldots \ldots \ldots$ & $11 / 2$ & $23 / 4$ & 2 & $21 / 4$ \\
\hline $17 \ldots \ldots \ldots \ldots$ & $23 / 4$ & 2 & 2 & $2 \mathrm{I} / 4$ \\
\hline $18 \ldots \ldots \ldots \ldots$ & $13 / 4$ & $23 / 4$ & 2 & $21 / 4$ \\
\hline $19 \ldots \ldots \ldots \ldots$ & $11 / 4$ & $21 / 4$ & 2 & $2 \% / 4$ \\
\hline $20 \ldots \ldots \ldots \ldots$ & $13 / 4$ & $21 / 8$ & 2 & $21 / 4$ \\
\hline $21 \ldots \ldots \ldots \ldots$ & . . $21 / 8$ & $21 / 8$ & 2 & $2 \pi / 4$ \\
\hline $22 \ldots \ldots \ldots \ldots \ldots$ & . $13 / 8$ & $21 / 2$ & 2 & $21 / 4$ \\
\hline $23 \ldots \ldots \ldots \ldots$ & $3 / 4$ & $21 / 4$ & 2 & $21 / 4$ \\
\hline $2+\ldots \ldots \ldots \ldots$ & 1 & $21 / 4$ & 2 & $21 / 4$ \\
\hline $25 \ldots \ldots \ldots \ldots$ & $7 / 8$ & $13 / 4$ & $13 / 8$ & $11 / 2$ \\
\hline $26 \ldots \ldots \ldots \ldots$ & .. $5 / 8$ & 2 & $5 / 8$ & $7 / 8$ \\
\hline $27 \ldots \ldots \ldots \ldots$ & . 0 & $11 / 2$ & 0 & 0 \\
\hline Total........ & . . $77 \% / \mathrm{s}$ & & $777 / 8$ & $\cdots$ \\
\hline
\end{tabular}


One takes the usual preliminary notes with a 62 -ft. chord, noting the middle ordinate which gives the degree of the curve at each station, observing the curvature and elevation at each station and recording this data in the usual engineers field book. On the opposite side of the book, usually ruled like cross section paper, the ordinates of the degrees of curve at each station can be plotted quickly so that the curve will show clearly the points of maximum and minimum variation. Fig. 16.

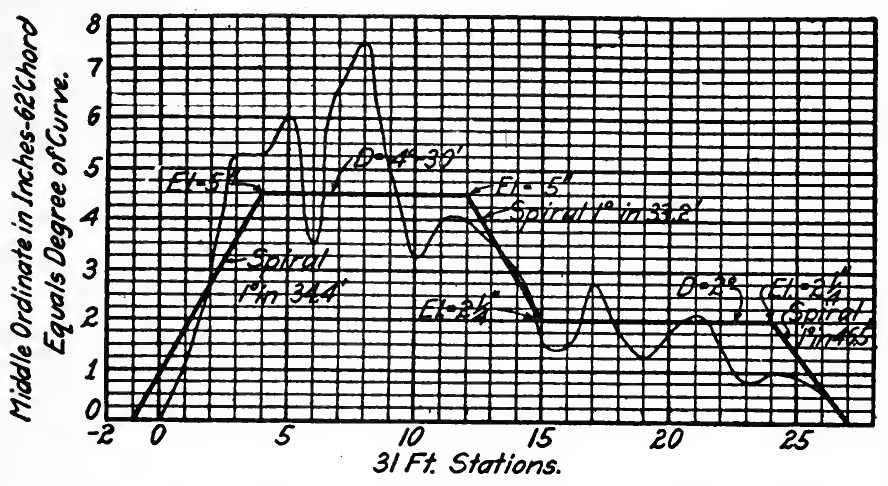

Fig. 16-An Example of the Graphical Determination of the Curve Offsets

If all the degrees of curvature in inches as originally taken are added, the sum for each particular curve will remain constant. After reading from the diagram the proposed degree of curve for each station and again adding, the totals should equal the previous sum, and if not, adjustments can be made.

The diagram of the curve will locate quickly the points of the maximum and minimum variation and then an average line for the spiral and curve can be plotted. It has been found in plotting the "Talbot Spiral" in this manner that it becomes a straight line inclining in proportion to the rate of runoff in feet per degree of curve. A similar diagram for elevation using inches as ordinates at each station can be correspondingly figured out to fit the new curve and spiral. This will give data that will permit a track man to line the track for the spiral and curve throughout readily.

Economical Size of Electrical Conductors. A good example of the use of charts in economical design is given by B. B. Hood in his article, "Proper Current Densities," published in Metallurgical and Chemical Engineering, November 15, 1916. There is a broad field for a similar use of this method and it is hoped that the illustration given here will suggest many possibilities to the reader.

When it is necessary to install large amounts of copper to transmit electrical power the question of interest on that copper should be taken into consideration when figuring the size of conductors. The cost of operating such transmission lines may be taken as the interest on the copper tied up plus a certain per cent for amortization plus the cost of the power lost. For direct-current work this will be a minimum when the cost of interest, etc., equals the cost of power lost, since interest varies 


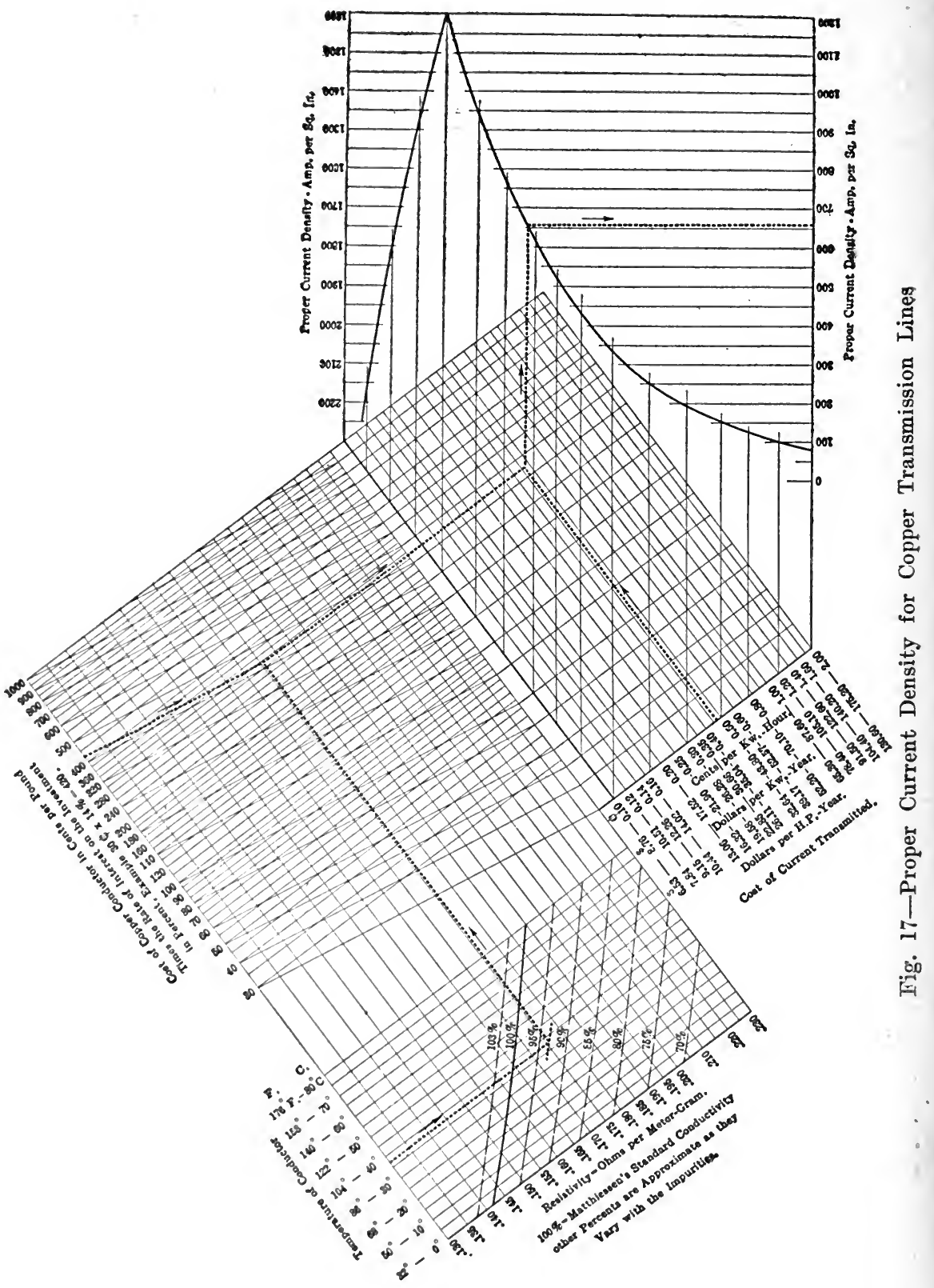


directly as the copper involved, while the power lost varies inversely. Having a certain amount of power to transmit, in order to figure the size of conductors, the proper current density should be determined. Into this problem will enter the resistivity or conductivity of the copper to be used, the temperature at which it is to be operated, the cost of copper installed, the rate of interest on the investment and amortization, and the cost of power to be transmitted.

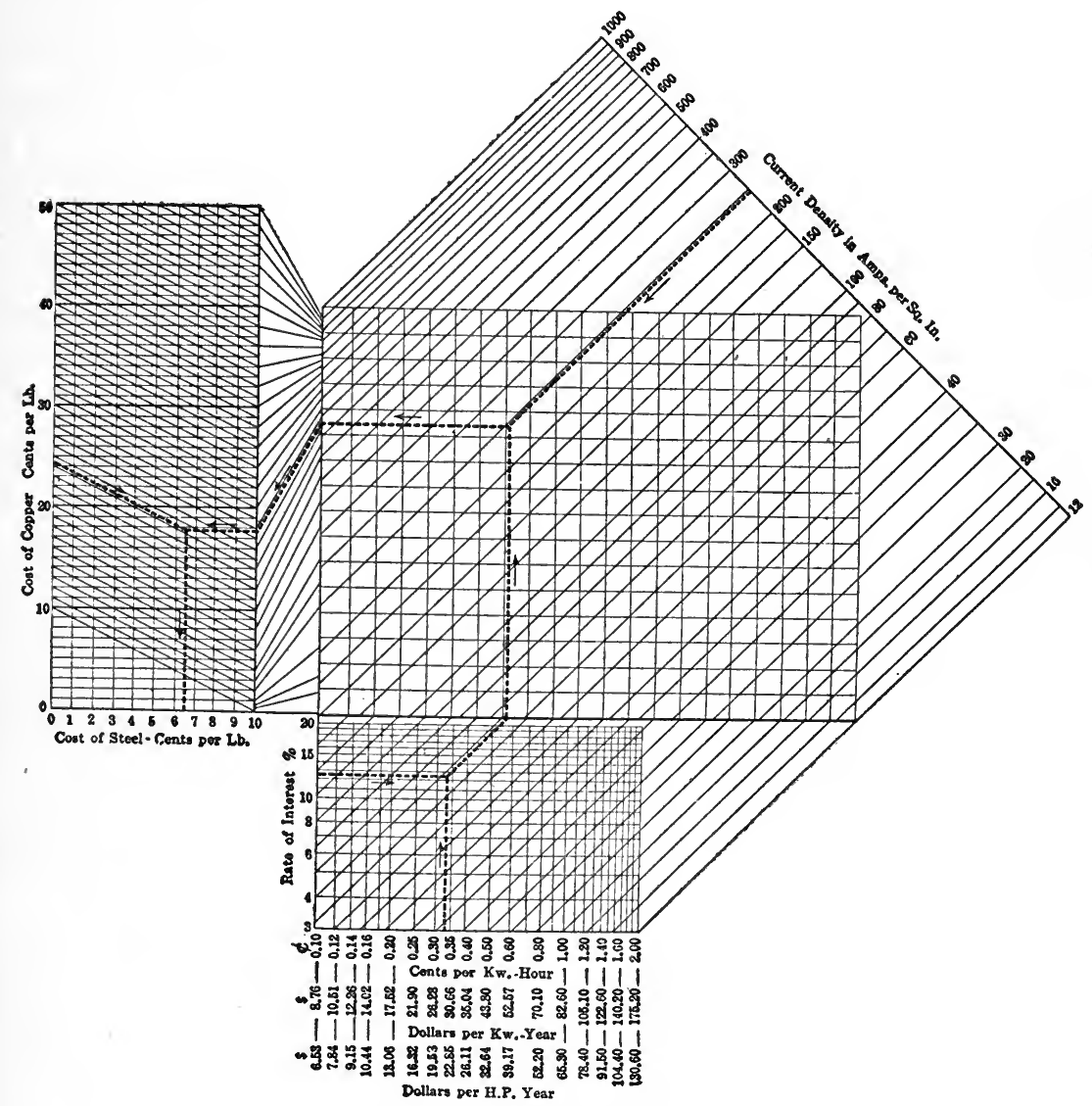

Fig. 18-Copper vs Steel Conductors

Fig. 17 shows the proper current density for copper transmission lines.

Example: Copper bus-bar having a conductivity of 93\% (Matthiessen's Standard) to be operated at an average temperature of $33^{\circ} \mathrm{C} . ;$ cost of copper,$=30 \mathrm{c}$ per lb.; interest plus amortization of investment $=14 \%$; cost of current $=\$ 40$ per $\mathrm{kw} . \mathrm{yr}$.; proper current density $=655$ amp. per sq. in.

Fig. 18 shows the relations between, cost of copper, cost of steel, rate of interest and amortization, cost of power transmitted, and current density, when copper conductors $=$ steel conductors in cost of operation. Cost of operation being equal to interest on and amortization of the investment plus the value of the power lost. Knowing four of the five factors the other one can be determined from the Chart.

Example: Current density $=248$; interest, etc. $=12.5 \%$; cost of power $=0.33 \phi \mathrm{kwh}$. Cost of conper $=24.5 c$. Ans. Cost of steel $=6.5 c$ per lb. If Steel costs less than $6.5 c$ it would be cheaper than Copper in Cost of Interest, etc. + Current Cost.

This chart is based on a copper resistivity of 0.155 ohms per meter gram, which is equivalent to approximately $99 \%$. 
From the chart Fig. 17 the proper current density in amperes per square inch may be determined, and from it the size of conductors readily figured. Taking the example given find the point where the $93 \%$ line crosses the $38^{\circ} \mathrm{C}$. line. Multiply the interest of $14 \%$ by the cost of copper, 30 ets., getting a factor of 420 . From these points follow the dotted lines until the line representing the cost of power at $\$ 40.00$ per $\mathrm{kw}$. year is reached, from thence over to the eurve and down to the proper current density 655 amperes per sq. in.

Suppose we consider an electrolytic refining plant using copper conductors under the above conditions. If instead of using the proper current density, $150,000 \mathrm{lbs}$. of conductors were installed, using a density of 900 amperes, what would be the yearly loss?

The interest charge on the investment would be $\$ 6,300$ per yr. If the density of 655 amp. were used, the power loss would amount to $\$ 6,300$ per year. With 900 amp. the power loss will be $\left(\frac{900}{655}\right)^{2} \times 6300=$ $\$ 11,890$, or a total of $\$ 18,190$ per yr. for operating. This makes no allowance for the increased temperature of the eonductors above 38 deg. $\mathrm{C}$., which would take place and increase the loss. If the installation were 900

made so that 655 amp. per sq. in. was obtained, there would be $-X$

$150,000=206,200 \mathrm{lb}$. of copper conductors installed which would have an interest and power cost of $\$ 8,660$ per yr. each, or a total of $\$ 17,320$. This would make a saving of $\$ 18,190-\$ 17,320=\$ 870$ per year over and above all allowances for amortization, etc.

Some times a conductor must be large enough to support a weight such as a cathode. In that case it may be possible to substitute steel with a saving, providing, of course, there are no other conditions which do not prohibit the use of steel. From the chart in Fig. 18 it is possible to determine whether steel will compete with copper in cost of operating. If in the example given steel costs less than $6 \frac{1}{2} \mathrm{c}$. per lb. installed it would be cheaper to use than copper. This situation exists largely because a lower current density is used than would be proper if the conditions, upon which the chart in Fig. 17 is figured, were all that were considered, instead of bringing in the question of strength.

Chart for Pulley Sizes. This article, by H. J. Fuller, was published in American Machinist. September 27, 1917.

The pulley user will find this chart a great time saver. When you have a lineshaft, the speed of which you know, and buy a machine that shruld run at a speed given by the maker, the question arises as to what pulleys you need to order.

There are a great many things that affect your ehoice besides the diameters that will grive the speed ratio: The drop of the shaft hangers on the lineshaft or comntershaft; the nearness of some pipe or beam; on the machine itself somo parts limit the size of the driven pulley; or if the drive is close, the are of contact may prohibit a smaller pulley.

'The right-hand fignese in Fig. 19 are for shaft speeds in revolutions per minute, as are the lower set at the bottom. The other two sets are pulley diameters in inches. 
For example, you have a driving shaft running at 150 r.p.m. and a machine or countershaft running at 250 r.p.m. Find 150 at the right. Follow the cross section horizontal line until it meets the cross-section vertical line that starts from 250 at the bottom of the page. The diagonal line or curve that crosses this intersection is the key to all the pulley combinations that will give these speeds.

Notice that the vertical from 33 and the horizontal from 20 meet on this line; also, 40 and 24, 50 and 30,67 and 40, 80 and 48, 90 and 54,5 and 3,15 and 9 , etc. It works both ways. If you have in stock a number of pulleys, you can soon tell how near any two of them will come to giving you the speeds you wish.

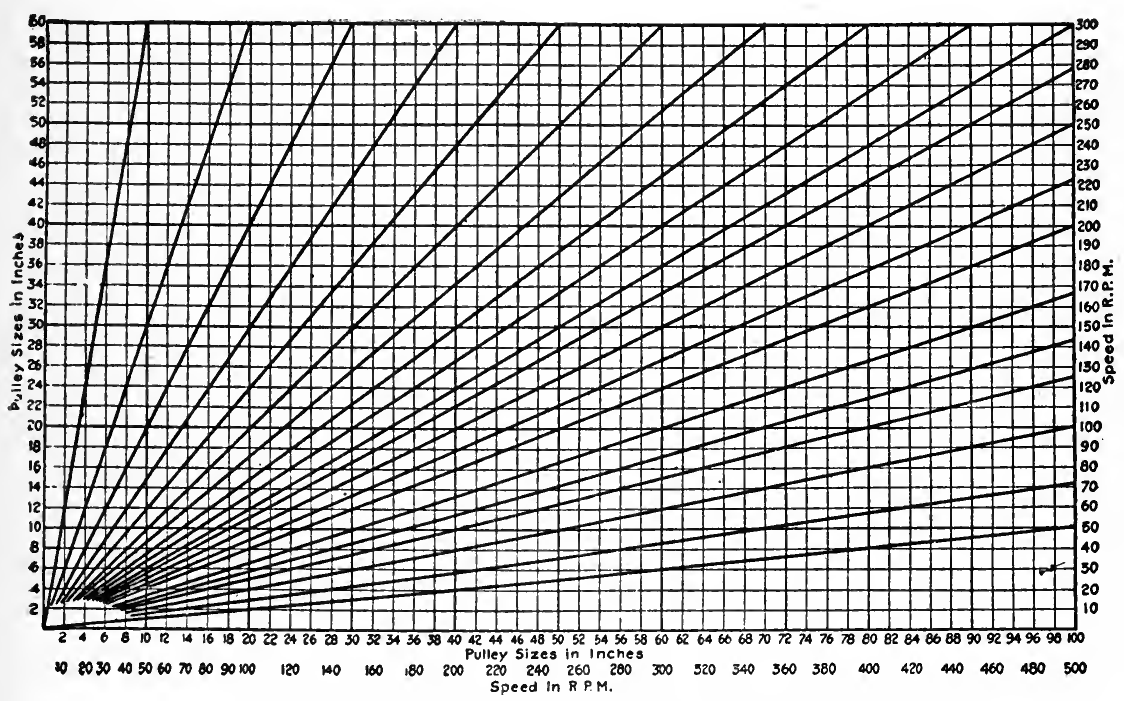

Fig. 19-Chart for Quickly Determining the Sizes for Pulleys

If the speed horizontal and vertical do not meet on any of the diagonals that are drawn to give the usual speed changes, you can easily place a ruler so that the edge passes through the intersection and also through the lower left-hand corner of the page and draw your own key line.

Then also, if you are limited to pulleys where horizontal and vertical do not quite meet at the diagonal of your chosen speed, a glance will show you how much your speed will be affected: for instance, pulleys 49 and 30 in. would make the speed 245 instead of 250.

In reading such a curve or chart, a celluloid rule or right triangle is an aid; and when the lines are close together, it is sometimes better to read a multiple, as in the last example 50 to 30 is the same as 5 to 3 . Likewise, for numbers larger than those shown, a smaller can be used, as 15 for 150 on the left-hand side; only it must be remembered to add the zero or point off in the answer, as the case may need.

Required Number of Plies of Conveyor Belt. Engineers who have occasion to ascertain the correct number of plies required for conveyor belt will find Fig. 20 useful. The calculations are based on the average 
safe strength, factor of safety of 15 , of the various standard rubber conveyor belts. The ealeulations assume maximum loading conditions; that is the belt is eonsidered as earrying the greatest load that it will handle without spillage at ordinary belt speeds. This not only produces the most economical operating conditions but also the maximum tension in the belts. The chart is drawn for simple drive with base pulling and for other drives the number of plies should be multiplied by the factors: 0.83 for simple lugged drive; 0.67 for tandem base drive, and 0.55 for tandem lugged drive.

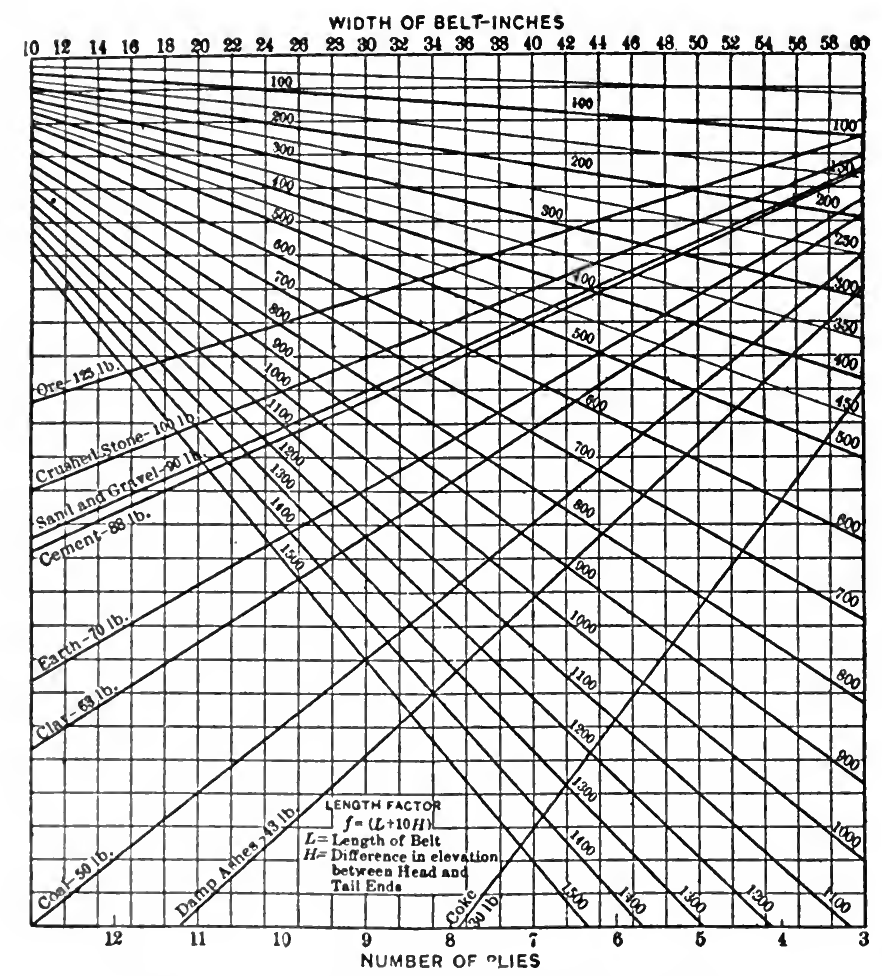

Fig. 20-Chart for Calculating Conveyor Belts

The length factor, $f=(L+10 H)$, represented on the chart by the lines 500, 600, 700, rte., is a devcloped factor equal to the sum of the length of the belt and ten times the difference in elevation between the head and tail pulleys.

To find the correct number of plies for a eonveyor belt, knowing the width, the length, the difference in elevation between the head and tail ends, and the kind of material to be handled:

Start from the width given at the top of the chart and move down until this line intersects the line corresponding to the proper length factor; then move either right or loft until the line corresponding to the given material is met; then move down again to the scale of plies, where the next largest figure will give the correct number of plies. 
For example: To find the correct number of plies for a conveyor belt $36 \mathrm{in}$. wide and $300 \mathrm{ft}$. long, with $20 \mathrm{ft}$. difference in elevation; handling sand and gravel: Follow the line from the 36-in. width downward until it intersects the 500 length factor line; then follow to the right until the sand and gravel line is intersected; then down to the ply scale, where the ply will be found to be 7 .

This chart is reproduced from a paper in Transactions, American Institute of Mining Engineers, September, 1915, by J. D. Mooney and D. L. Darnell with the B. F. Goodrich Co.

The above is from Engineering and Contracting, Dec. 22, 1915.

Size of Rope Required. W. B. Crowl, in Coal Age, August 11, 1917, gives the following:

A great number of the new coal-mine operations are so situated that

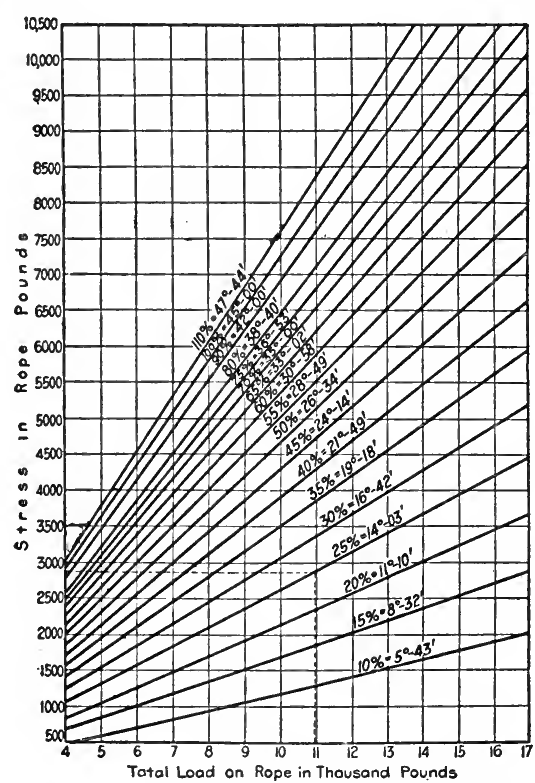

Fig. 21-Showing Stresses Produced in Rope by Various Loads and Grades

an inclined-plane machine is required to lower the loaded cars to the tipple. With such an installation one of the problems that confront the manager is the size of and the material to be employed in the haulage rope, in order to make the lowering operation safe without using an excessively large rope and thus incurring unnecessary expense. By using the accompanying charts this problem can be readily solved.

Example-To find the size of rope necessary to use on an inclined plane $400 \mathrm{ft}$. long, 25\% slope, with two loaded cars in the trip ; capacity of cars, $1 \frac{1}{2}$ tons each; weight of empty car, $2,000 \mathrm{lb}$; ; weight of coal, 11/2 tons $\left(1 \frac{1 / 2}{2} \times 2,240=3,360 \mathrm{lb}\right.$. $)$, making total weight of loaded car 5,360 lb. The weight of two loaded cars will thus be $2 \times 5,360=10,720 \mathrm{lbs}$.

Assume that it will take a $3 / 4$-in. rope weighing 0.89 lb. per ft.; $400 \times$ 
$0.89 \mathrm{lb} .=356 \mathrm{lb} .=$ weight of rope. The total load on the rope will then be $10,720+356=11,076$ lbs.

From Fig. 21, starting with a total load of $11,076 \mathrm{lb}$. and following the vertical load line to the $25 \%$ grade curve, the stress in the rope is found to be $2,875 \mathrm{lbs}$.

Using this stress in the rope with Fig. 22 as shown by the dotted line, a $\%$-in. extra-strong cast-steel haulage rope will be found to give a factor of safety of 9 , which is sufficient. A rope having a factor of safety between 7 and 10 will give satisfactory service, but any larger factor will make it stronger than is necessary.

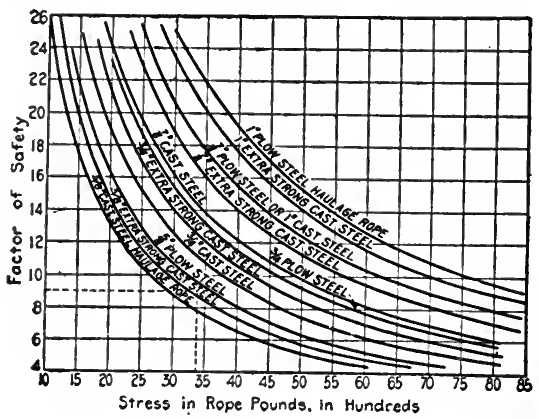

Fig. 22-Showing Stresses in Rope and Corresponding Safety Factors

Approximate Cost of Mill Buildings. Engineering Record, Jan. 29, 1910, says:

It is sometimes convenient to be able to tell offhand the approximate cost of proposed buildings, or the cost if new, of existing buildings without going through an estimate of all the quantities of materials and labor.

Some time ago Mr. Charles T. Main placed a valuation upon a portion of the property of a corporation, including some 400 or 500 buildings. In order to have a standard of cost from which to start in each case, he prepared a series of diagrams, Fig. 23, showing the approximate costs of buildings varying in length and width and from one story to six stories in lieight. The height of stories also was varied for different widths, as given below.

The costs used in making up the diagrams are based largely upon the actual cost of work done under average eonditions of cost of materials and lahor. and with average soil for foundations.

The costs given include plumbing, but no heating, sprinklers or lighting. These three latter items would add roughly 10 cents per square foot of floor area.

I'se of Inicgrams.-1. The diagrams ean be used to determine the probable approximate eost of proposed brick buildings of the type known as "slow-burning " to be userl for manufacturing purposes with total floor load of about 75 1h. per scfuare foot, and these can be taken from the diagrams readily. For example, if it is desired to know the probable cost of a mill $400 \mathrm{ft}$. long by $100 \mathrm{ft}$. wide by three stories high, refer to the diagram showing the cost of three-story buildings. On the curve for 
buildings $100 \mathrm{ft}$. wide, find the point where the vertical line of $400 \mathrm{ft}$. in length cuts the curve, then move horizontally along this line to the left-hand vertical line, on which will be found the cost of 81 cents.

The cost given is for brick manufacturing buildings under average conditions, and can be modified, if necessary, for the following conditions :

(a) If the soil is poor or the conditions of the site are such as to require more than the ordinary amount of foundations, the cost will be increased.

(b) If the end or a side of the building is formed by another building, the cost of one or the other will be reduced slightly.

(c) If the building is to be used for ordinary storage purposes, with low stories and no top floors, the cost will be decreased about $10 \%$ for large low buildings, to $25 \%$ for small high ones, about $20 \%$ usually being fair.

(d) If the buildings are to be used for manufacturing purposes and are to be substantially built of wood, the cost will be decreased about $6 \%$ for large one-story buildings, to $33 \%$ for high small buildings, and $15 \%$ would usually be fair.

(e) If the buildings are to be used for storage with low stories and built substantially of wood, the cost will be decreased from $13 \%$ for large one-story buildings to $50 \%$ for small high buildings, and $30 \%$ would usually be fair.

(f) If the total floor loads are more than $75 \mathrm{lb}$. per square foot the cost is increased.

(g) For office buildings, the cost must be increased to cover architectural features on the outside and interior finish.

The cost of very light wooden structures is much less than the above figures would give.

2. The diagrams can also be used as a basis of valuation of different buildings.

A building, no matter how built nor how expensive it was to build, cannot be of any more value for the purpose to which it is put than a modern building properly designed for that particular purpose.

3. The diagrams can be used as a basis for insurance valuations after deducting abuut $5 \%$ for large buildings to $15 \%$ for small ones, for the cost of foundations, as it is not customary to include the foundations in the insurable value.

Basis of Estimates. - Table 1 shows the costs which form the basis of the estimates, and these unit prices can be used to compute the cost of any building not covered by the diagrams.

The cost of brickwalls is based on 22 bricks per cubic foot, costing $\$ 18$ per thousand laid. Openings are estimated at 40 cents per square foot, including windows, doors and sills. Ordinary mill floors, including timbers, planking and top floor with Southern pine timber at $\$ 40$ per thousand feet board measure and spruce planking at $\$ 30$ per thousand, costs about 32 cents per square foot, which has been used as a unit price. Ordinary mill roofs covered with tar and gravel, with lumber at the above prices, cost about 25 cents per square foot, as used in the estimates.

Add for stairways, elevator wells, plumbing, partitions and special work. 

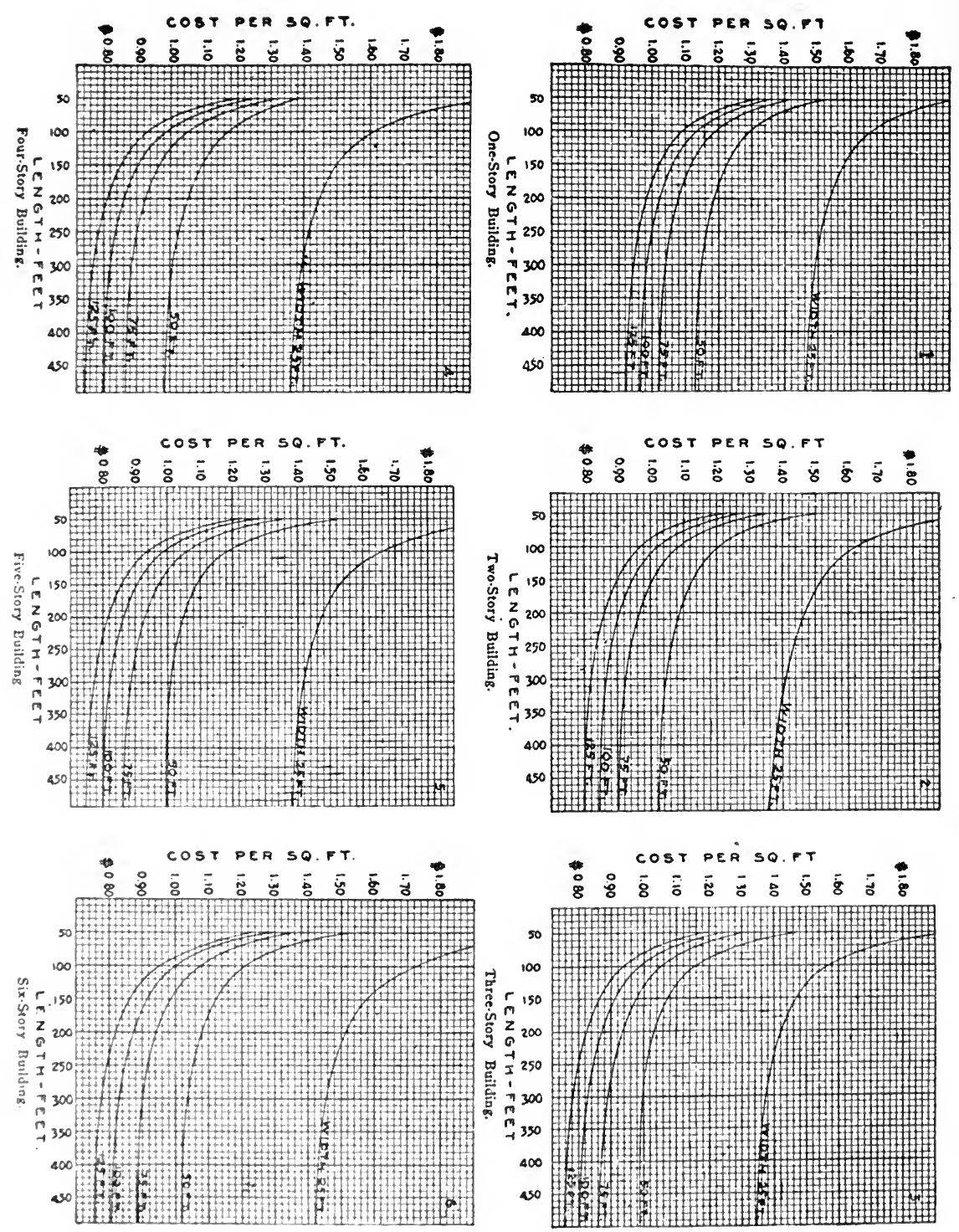

Fig. 23-Fstimaterl ('ost per Siquare Foot of Gross Flowr Area of Brick Buildings for 'Textile Manufacturing, Including Foundations and Plumbing 
Deductions from Diagrams.-1. An examination of the diagrams shows the decrease in cost as the width is increased. This is due to the fact that the cost of the walls and outside foundations, which is an important item of cost, relative to the total cost, is decreased as the width increases. For example, supposing a three-story building is desired with 30,000 sq. ft. on each floor. If the building were $600 \mathrm{x} 50 \mathrm{ft}$., its cost would be about 99 cents a square foot. If the building were $400 \times 75 \mathrm{ft}$., its cost would be about 87 cents a square foot. If the building were $300 \times 100 \mathrm{ft}$., its cost would be about 83 cents a square foot. If the building were $240 \times 125 \mathrm{ft}$., its cost would be about 80 cents a square foot.

2. The diagrams show that the minimum cost per square foot is reached with a four-story building. A three-story building costs a trifle more than a four-story. A one-story building is the most expensive. This is due to a combination of several features:

(a) The cost of ordinary foundations does not increase in proportion to the number of stories, and therefore their cost is less per square foot as the number of stories is increased, at least up to the limit of the diagram.

(b) The roof is the same for a one-story building as for one of any other number of stories, and therefore its cost relative to the total cost grows less as the number of stories increases.

(c) The cost of columns, including the supporting piers and castings, does not vary much per story as the stories are added.

(d) As the number of stories increases, the cost of the walls, owing to increased thickness, increases in a greater ratio than the number of stories, and this item is the one which in the four-story building offsets the saving in foundations and roof.

3. The saving by the use of frame construction for walls instead of brick is not as great as many persons think. The only saving is in somewhat lighter foundations and in the outside surfaces of the building. The floor, columns and roof must be of the same strength and construction in any case.

Re-inforced Concrete Buildings.-From such estimates and proposals as Mr. Main has been able to get, and from work done, it appears that the cost of re-inforced concrete buildings designed to carry floor loads of $100 \mathrm{lb}$. per square foot or less would cost about $25 \%$ more than the slowburning type of mill construction.

\section{Table 1.-Prices Used for Estimating.}

\begin{tabular}{cccccc} 
& \multicolumn{2}{c}{$\begin{array}{c}\text { Foundations, } \\
\text { including excavations, } \\
\text { per lin. ft. }\end{array}$} & \multicolumn{2}{c}{$\begin{array}{c}\text { Brick walls, } \\
\text { per sq. ft. of }\end{array}$} & $\begin{array}{c}\text { Columns } \\
\text { surface. } \\
\text { including } \\
\text { piers and }\end{array}$ \\
$\begin{array}{c}\text { castings. } \\
\text { Height in } \\
\text { stories. }\end{array}$ & $\begin{array}{c}\text { Outside } \\
\text { walls. }\end{array}$ & $\begin{array}{c}\text { Inside } \\
\text { walls. }\end{array}$ & $\begin{array}{c}\text { Outside } \\
\text { walls. }\end{array}$ & $\begin{array}{c}\text { Inside } \\
\text { walls. }\end{array}$ & $\begin{array}{c}\text { Cost of one. } \\
1\end{array}$ \\
$\$ 2.00$ & $\$ 1.75$ & $\$ 0.40$ & $\$ 0.40$ & $\$ 15$ \\
2 & 2.90 & 2.25 & .44 & .40 & 15 \\
3 & 3.80 & 2.80 & .47 & .40 & 15 \\
4 & 4.70 & 3.40 & .50 & .43 & 15 \\
5 & 5.60 & 3.90 & .53 & .45 & 15 \\
6 & 6.50 & 4.50 & .57 & .47 & 15
\end{tabular}


Data Used in Estimating.-Assumed height of stories : From ground to first floor, $3 \mathrm{ft}$; buildings $25 \mathrm{ft}$. wide, stories $13 \mathrm{ft}$. high; buildings $50 \mathrm{ft}$. wide, stories $14 \mathrm{ft}$. high ; buildings $75 \mathrm{ft}$. wide, stories $15 \mathrm{ft}$. high; buildings $100 \mathrm{ft}$. wide, stories $16 \mathrm{ft}$. high; buildings $125 \mathrm{ft}$. wide, stories $16 \mathrm{ft}$. high.

Prices for foundations, brick walls and columns are given in Table 1. Floors 32 cents per square foot of gross floor space, not including columns. If columns are included, 38 cents.

Roof 25 cents per square foot, not including eolumns. If columns are included, 30 cents. Roof to project 18 inches all around buildings.

Stairways, including partitions, $\$ 100$ each flight. Allow two stairways and one elevator tower for buildings up to $150 \mathrm{ft}$. long. Allow two stairways and two elevator towers for buildings up to $300 \mathrm{ft}$. long. In buildings over two stories, allow three stairways and three elevator towers for buildings over $300 \mathrm{ft}$. long.

In buildings over two stories, plumbing $\$ 75$ for each fixture, including piping and partitions.

Allow two fixtures on each floor up to $5,000 \mathrm{sq}$. ft. of floor space and add one fixture for each additional 5,000 sq. ft. of floor space or fraction thereof.

Note-From the above data the approximate eost of any size and shape of building can be estimated in a few minutes. After the eost of the items given is determined, about $10 \%$ should be added for incidentals.

Cost of Retaining Walls, by E. F. Kriegsman, published in Engineering Record, August 9, 1913. Fig. 24 is useful in determining approximate cost of walls in connection with regrade work, viaduct and tunnel

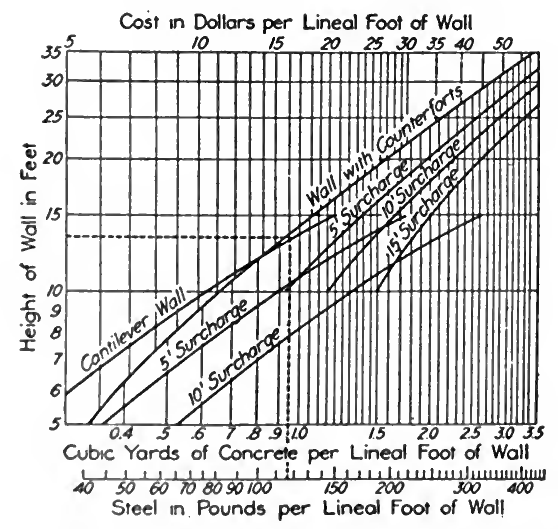

Fig. 24-Approximate Cost of Retaining Walls

approaches, etc. I'sually one has to wait for the designer to ascertain the quantities before a preliminary eost report ean be made. This manifestly has its disalvantages.

In constructing the diagram about forty wall designs were investigated and the quantities computed. The results were next plotted on logarithmie cross-section paper and average curves drawn representing the amounts of concrete and steel reinforcement for various heights of wall 
having a 1-ft. length. An average price of $\$ 17$ per cu. yd. for concrete, steel, forms and finish was applied to these yardages. All that one needs is the grades of the top and bottom of the proposed wall, when one can readily approximate the cost of the finished wall with the use of the diagram.

The price of $\$ 17$ per cu. yd. may not meet the requirements of many engineers, as local conditions vary considerably. If another unit price is desired all that is necessary is to trace the cost scale on tracing cloth and move it horizontally until the desired cost is in the same vertical with the 1-cu. yd. line in the diagram now occupied by $\$ 17$ at the top. The new location of the cost scale will give the desired cost for the given local conditions.

For illustration the dotted line shows that a cantilever wall $14 \mathrm{ft}$. high, without surcharge load, would contain $0.95 \mathrm{cu}$. yd. of concrete and $116 \mathrm{lb}$. of steel reinforcement and would cost approximately $\$ 16$ per linear foot. Excavation must be figured separately.

Printing Estimates. Rectilinear charts, 6 divisions per inch and 12 divisions per inch are very useful and time-saving in making layouts. On the former, each small divisions is a 12-point, or pica, space and on the latter each small division is $1 / 12$ of an inch or 6 points. The following
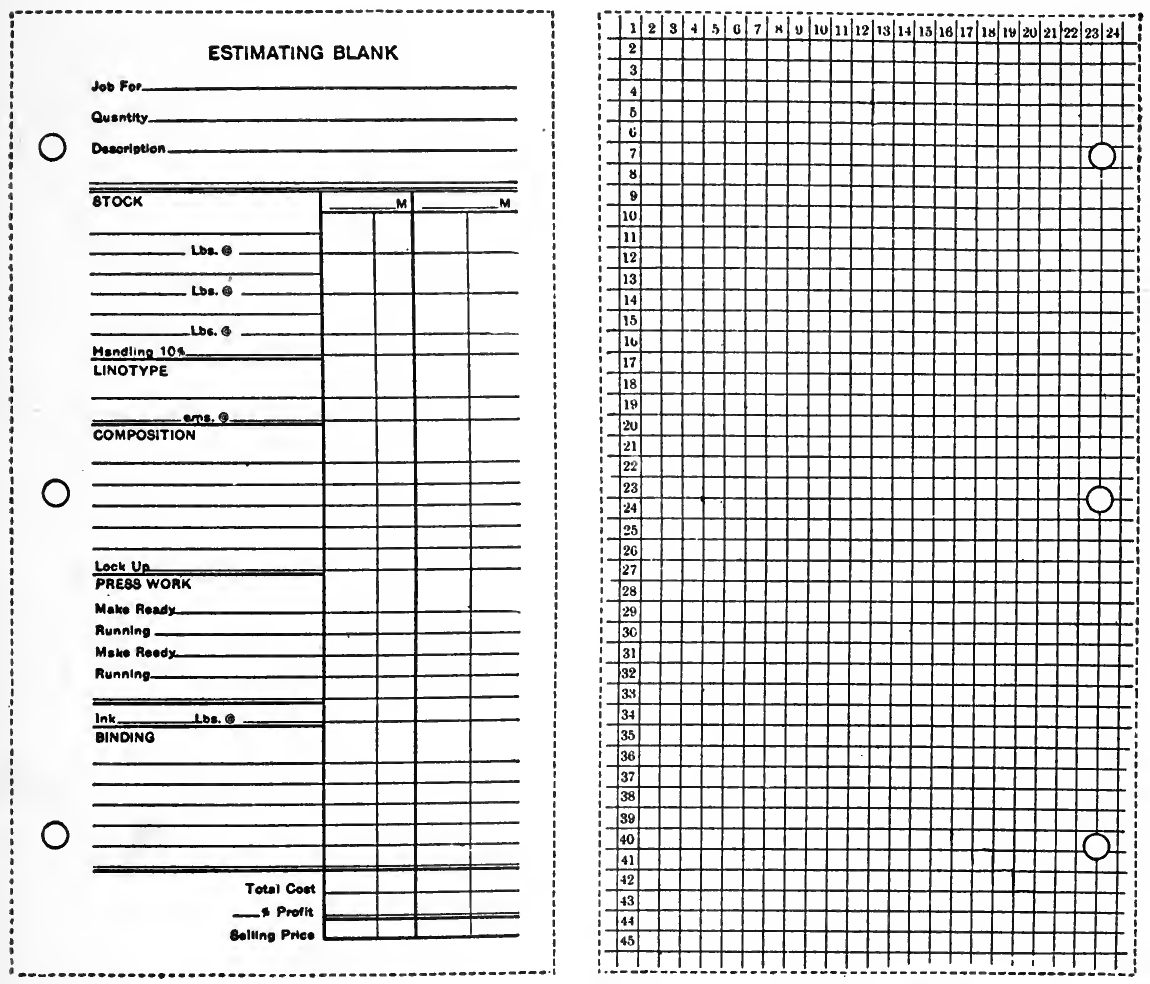

Fig. 25 
abstract and Fig. are from an article by R. T. Porte, "The Costs of Job Printing," in Inland Printer, March, 1919.

There are many and varied estimate blanks in use by printers, and some are decidediy elaborate.

For my own use I have prepared a very simple blank, punched to fit the "recipe book," with spaces for the various classes of work. Fig. 25, in reduced size, (the original is on a sheet $4 \frac{1}{8}$ by $7 \frac{1}{2}$ inches), will give a good idea of this. On examination it will be found simple yet complete.

On the back of the estimate blank I have quadruple-ruled twelvepoint (or pica) spaces, numbered. On this side I make necessary calculations for which I do not have room on the front, or sketches as to paper and the number that will cut out of a sheet, using the numbers for inches or half inches. Frequently I lay out a job on them. In many ways I have found these ruled lines of great value and help in preventing errors that might prove costly.

Estimates of Cost of Transportation. While the following charts pertain to moving earth with scrapers, the formula is applicable to the transportation of any material with any conveyance. Therefore, by following the method indicated, similar charts may be plotted for use in estimating by any contractor or concern interested in moving materials. As will be noted, this formula presents a means for accurately determining what method of transportation is most economical for a given job and the charts (Fig. 30, for example) indicate the economical haul for each type of equipment tested.

The cost of earth moving is a substantial factor in the total expense of nearly all construction work, and a considerable part of all earth moving operations is for work where it is not economical to use locomotives and ears, either because of the length of haul or because the magnitude of the work is not sufficient to justify the preparatory costs of a large plant. Under such conditions a choice must be made between wagons or carts and one or more of the various types of scraper.

It has been the experienee of the writer that a majority of contractors east of the Alleghenies are unfamiliar with the relative advantages of the different kinds of scraper and do not possess the data necessary to determine for the particular conditions of their work which style and size should be most economic. A contractor who has found wheel scrapers very successful in a eertain kind of earth is likely to be biased in favor of the wheel scraper for that kind of earth more or less regardless of the length of haul.

The general economic formula for transportation is as follows:

Symbol. Factor.

C .........The total expenses per day in ents.

$w \ldots \ldots \ldots$ The net load, for the average trip, in pounds, or other convenient unit.

$S$..........The speed (average) when loaded, in feet per minute. KS ..........The speed (average) when returning, in feet per minute.

D ..........The length of haul in feet.

$l \ldots \ldots \ldots \ldots$ The time lost in turning, resting, and wasted for an average round trip, in minutes. 
$R \ldots \ldots \ldots \ldots$ The total cost in cents per ton for transportation.

$W \ldots \ldots \ldots \ldots$ The number of minutes in the working day.

$D$

The following facts are deducible algebraically:

$\bar{S} \cdots \cdots \cdots$. Time for a loaded trip, in minutes.

$D$

$\bar{K} S$......... Time for the empty haul.

$D$

$l+\frac{1}{K s} \ldots \ldots$. Actual time not occupied in transporting material, in $\frac{D}{S}\left(1+\frac{1}{K}\right)+l$. Average time for one round trip, in minutes.

$W$

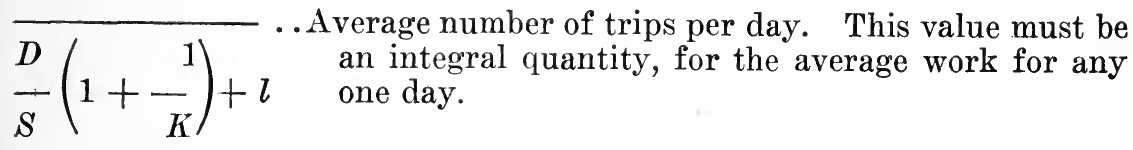
$W w$

$\frac{D}{S}\left(1+\frac{1}{K}\right)+l$

Average total amount transported per day.

$$
\frac{D}{S}\left(1+\frac{1}{K}\right)+l
$$

$\mathrm{R}=\mathrm{C}-$ Cost of transportation per pound, or other $W w$ convenient unit.

The value for $C$ will depend upon the number of horses used in a team, whether extra teams are employed in loading, the general organization of the gang, and to a lesser extent on the cost of repairs and depreciation of the scrapers themselves. The value $w$ of the net load will depend entirely upon the type and size of equipment, and the care with which loading is done. It is likely to vary much more with wheel scrapers than with Fresnos. In heavy ground which has not been well loosened, particularly where there are many roots of trees and occasional boulders, the wheel scrapers often fail to get more than a $60 \%$ or $70 \%$. load. The value for $w$ was determined by taking the average of several hundred trips, the amount of each load being estimated by the inspector on the basis of the space occupied loose, multiplied by a density factor and afterward checked by the place measure computations.

In a well organized scraper gang the speed loaded and that light are fairly constant for constant conditions, changing as soon as the local conditions vary. These conditions are the wetness of the ground hauled over, the "sea room" available for each team and the grades and curva-. 
tures along the line of haul. The length of haul is nearly always a constantly varying quantity. Its average value was determined by measuring it at regular intervals, and averaging the figures thus obtained. The time lost in turning, loading, etc., varies somewhat but for a given set of conditions was taken as an average of a large number of observations. The data obtained in this way by an inspector who timed serapers for two hours or so each day were afterward compared with a eount kept by a boy with a tally machine at the dump, resulting in very close agreement, except that the average costs obtained by the time study method were nearly always a few per cent less than the probable actual costs kept by the tally boy over the whole day's work. The main reason for this seems to be that when under the eye of an inspector with a watch in one hand and a note book on his knee the drivers keep their teams going a little faster when not loaded and are a little more prompt in dumping and turning than when observed by a boy.

\begin{tabular}{|c|c|c|c|c|c|}
\hline $\begin{array}{l}\text { Waiting. } \\
13: 30-15: 25 \\
14: 15-70: 20 \\
24: 20-25: 13 \\
24: 40-2.5: 50 \\
33: 18-34: 04 \\
33: 25-34: 58 \\
42-25-43: 12 \\
43: 20-44: 04 \\
50: 48-53: 20 \\
51: 07-53: 48 \\
\text { Footnote- } \\
\text { at which the } \\
\text { No. 1 waiting } \\
\text { to } 15: 25 ; \text { loar } \\
\text { ing from } 15: 3 \\
\text { Ohservation } \\
\text { lo or } 20 \text { to } \\
\text { Times were } \\
\text { to get a fair }\end{array}$ & $\begin{array}{c}\text { Load- } \\
\text { ing. } \\
15: 37 \\
16: 50 \\
25: 27 \\
26: 20 \\
34: 17 \\
35: 10 \\
43: 32 \\
44: 18 \\
53: 30 \\
54: 05 \\
\text { he time } \\
\text { rforman } \\
\text { t the lo } \\
\text { ng from } \\
\text { to } 18: 2 \\
\text { were gre } \\
\text { cilitate } \\
\text { alen up } \\
\text { cerage. }\end{array}$ & $\begin{array}{c}\text { Trans- } \\
\text { porting. } \\
18: 27 \\
19: 50 \\
29: 15 \\
29: 29 \\
37: 58 \\
38: 09 \\
47: 10 \\
47: 15 \\
56: 14 \\
56: 57 \\
\text { under eacl } \\
\text { ce was con } \\
\text { ding poin } \\
15: 25 \text { to } \\
\text {, etc. } \\
\text { nerally m } \\
\text { obtaining } \\
\text { on differen }\end{array}$ & $\begin{array}{l}\text { Dump- } \\
\text { ing. } \\
18: 50 \\
20: 30 \\
29: 22 \\
29: 35 \\
38: 09 \\
38: 20 \\
47: 15 \\
47: 20 \\
56: 27 \\
57: 12 \\
\text { h heading } \\
\text { npleted, i } \\
\text { t was fro } \\
15: 37 ; \text { tr } \\
\text { ade in g } \\
\text { the aver }\end{array}$ & $\begin{array}{l}\text { Trans- } \\
\text { porting } \\
\text { empty. } \\
20: 58 \\
22: 13 \\
32: 13 \\
32: 49 \\
40: 20 \\
40: 30 \\
49: 50 \\
50: 20 \\
58: 40 \\
59: 50 \\
\text { is that } \\
\text { e., Line } \\
\text { m } 13: 30 \\
\text { ansport- } \\
\\
\text { oups of } \\
\text { ages. } \\
\text { in order }\end{array}$ & $\begin{array}{l}\text { Fair. } \\
\text { Date, June } 20 . \\
\text { Sand and some loam. } \\
\text { Loads fair }-3 \text { to yard, } \\
\text { average. } \\
\text { Gang: } 10 \text { scrapers. } \\
1 \text { snatch. } \\
11 \text { loader. } \\
11 \text { dumpman. } \\
1 \text { foreman. } \\
1 \text { waterboy. } \\
\text { Road to dump better con- } \\
\text { dition today and driv- } \\
\text { ers walk. } \\
\text { Turn = } 12 \times 2.6=30^{\prime} \\
\text { D = } 240 \times 2.6=625^{\prime} \\
\text { D' } 225 \times 2.6=585^{\prime} \\
\text { Noted on several ocea- } \\
\text { sions less time oecupied } \\
\text { to dump, as drivers } \\
\text { mount scrapers without } \\
\text { stopping them. }\end{array}$ \\
\hline
\end{tabular}

Fig. 26-Cample Page from Inspector's Note Book Showing Method of Keeping Notes in the Field

A sample page from the inspeetor's note book is given by Fig. 26, showing how the notes were entered in the field, and there follows in Fig. 27 a typieal report, made up at night by the inspector, of his conclusions, with the result of observations. These notes were taken by an expert observer, and would not be easy to duplicate by the ordinary timekeeper; hut sufficintly good notes for maintaining excellent control over the work "an le made by a man of limited experience, once the prineiples are thomomb molerstord. The main essential is that the observer shall be absolutely unliased. Ife shall have no interest whatever in the quality or quantity of the work done and should confine himself solely to the accurate report of the facts. From these daily reports, eovering a period of several weeks, the data were tabulated, and 
work was done, but similar charts may be made to suit any given conditions.

The above is but a short abstract of the complete article by R. T. Dana, published in Engineering and Contracting, June 3, 1914, and the writer would refer all interested in these charts to the original article which is well worth their study, before undertaking similar methods.

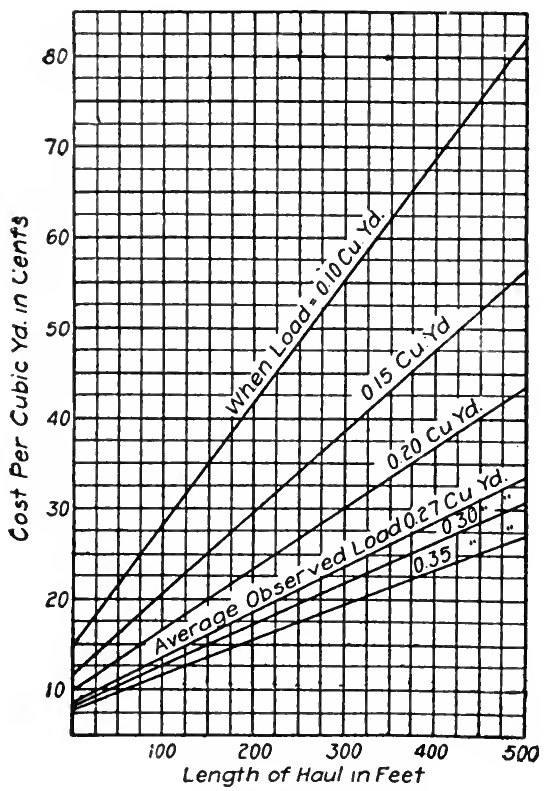

Fig. 28-Curves Showing Cost per Cubic Yard of Handling Loam, Sand, Etc., with Fresno Scrapers for Various Sizes of Load and Length of Haul

A Method of Determining Time of Performance of Work with Special Application to Grading. The time factor in construction work is a variable which is dependent upon many conditions and is often of considerable importance. It appears that a practical method of approximating the number of working days to be allowed for a given contract is hy carcfully segregating, analyzing and averaging portions of similar work performed under approximately the same conditions.

In a paper before the Society of Inunicipal Engineers of the City of Now York, Mr. (i. L. Bennett, efficiency engineer for the New York Board of Estimate and Apportionment, presented a general discussion of this subject with a detailed method of handling grading work, which is in use by Mr. R. Il. (iillespie, chief engineer of sewers and highways for the Borongh of Bronx, Now York. While the methods and costs given apply directly to New York ('ity conditions, the method of approaching the problem is applicable to any locality.

Contracts commonly arise from demands which in themselves are either urgent and set for a particular time of fulfillment or are more com- 


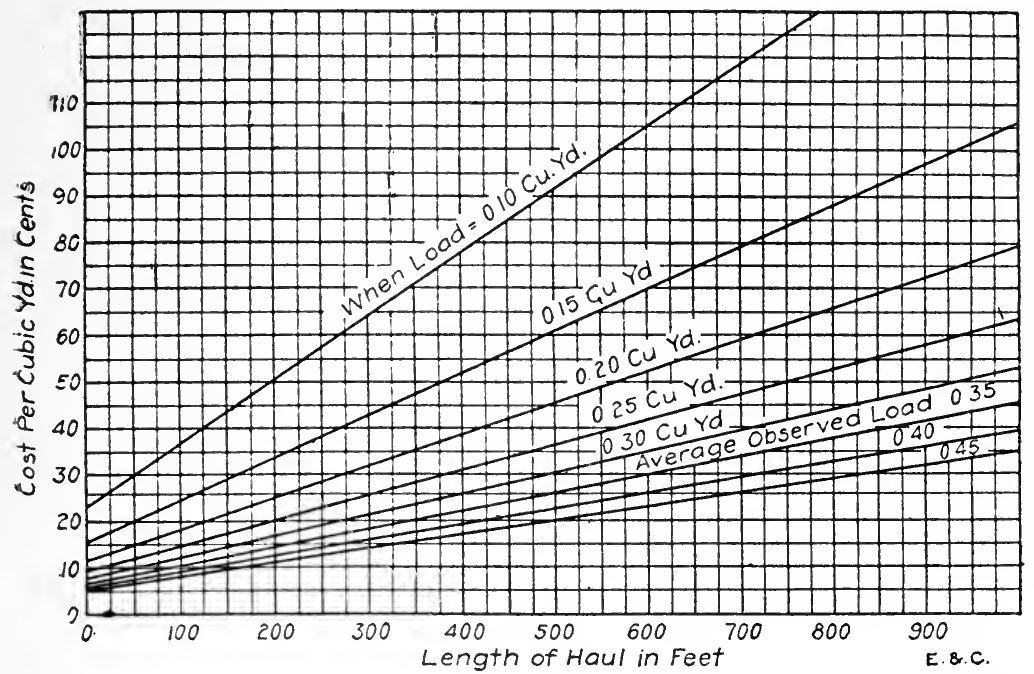

Fig. 29-Curves Showing Costs Per Cubic Yard of Handling Loam and Loam Clay with Wheel Scrapers for Various Sizes of Load and Length of Haul

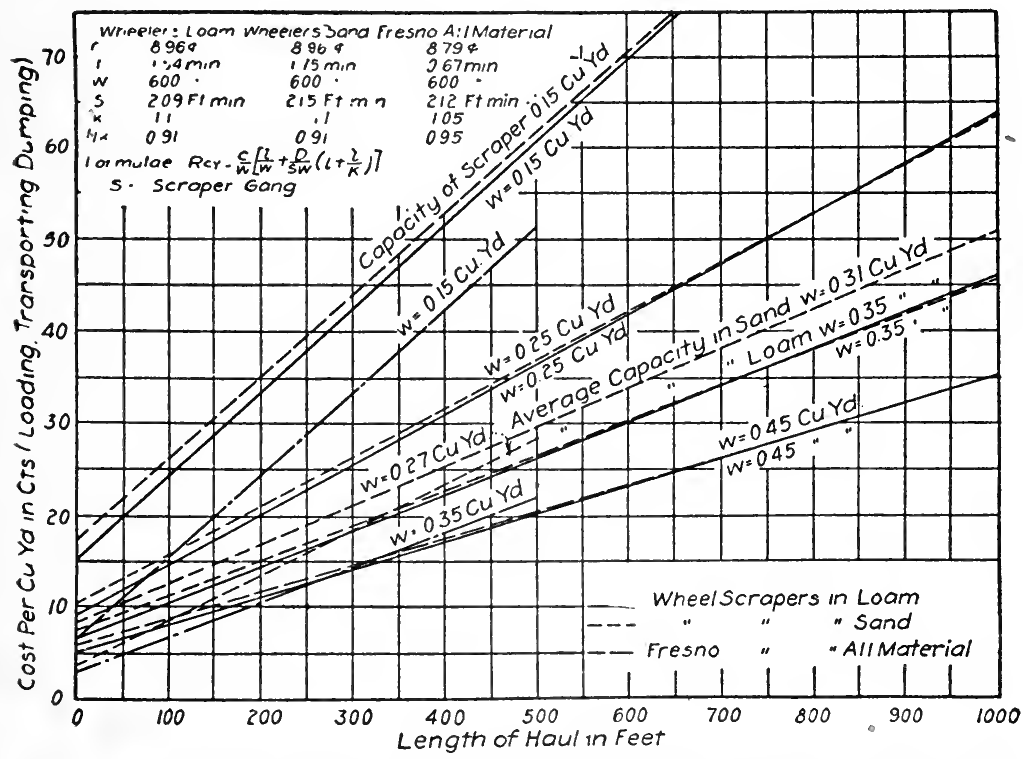

Fig. 30-Diagram Comparing Economy Up to 275 Ft. Haul of Fresno and Wheel Scrapers 
plaisant as to time, requiring only ultimate completion within rather widetime limits.

Contracts of the former type, for emergency work or for supplying necessary links in larger schemes, can, in proportion to their needs, afford to sacrifice economy for dispatch. Contracts of the latter type, which includes a majority of street improvement work, can properly aftord to disregard time as such and to seek economy of total costs alone.

Definition of Contractor's Economical Time.-There are comparatively few pieces of work which can only be economically accomplished by the use of some one particular equipment and of some one particular method. In general, there are a number of equipments and methods which, depending upon the genius of those in control for management under the conditions obtained will yield economical results, but will require somewhat different times for completion. Leaving out of account variations in required time due to such causes there is for each sort of work some number of working days, more than which could not economically be used by the contractor. Thus, on a pick and shovel job, the employment of less than a certain number of men would not be economical because of the cost of the foreman and superintendence; similarly, the use of more than a certain number of working days by a contractor would be uneconomical. The same thing applies to any job for which an equipment and force are provided sufficient to complete that job in one of the perhaps several most economical ways so far as contractor's costs are concerned; and to this somewhat varying time may be applied the term, "Contractor's Economical Time."

Equipment Warranted.-It is to be recognized that small total contract quantities, in general, only warrant the employment of light and easily moved equipments and that as the quantities become larger more and still more effective equipments are warranted.

But this may be modined considerably by the amount of work of this nature done or yet to be done, in the locality of the contract in question. Thus a rather small contract for rock excavation eould properly be given a shorter contract time in the northern part of Greater New York where rock excavation is constantly in progress than it could somewhere out on the shores of Long Island or southern New Jersey.

Relation of Labor Cost to Equipment.- The amount of equipment to be employed is scriously effected by the costs of labor and the ease of procuring equipment. Where satisfactory labor is expensive or difficult to procure, contractors will, in general, employ machinery of a type which otherwise would only be used on much larger contracts, resulting, of course, in a shorter contractor's economical time. Where machinery (an be easily hired, cfunipment will often be used on small jobs such as could not otherwise be afforded. This affects also very large jobs. for which, where no satisfactory disposal can be made of worn machinery, equipment is often provicled only in such quantities that it shall all be practically worn out when the job is completed.

I) finition of Total Cost.- The total cost of work rather than the cost to the contractor is the matter which interests the engineer in his capacity of manager for the party contracting for the work. And the total cost is, of eourse, the cont ract price plus the costs of surveys and designs, plus the eosts of inspection, superintendence and interest on the moneys 
invested by partial payments or otherwise, all of which latter vary nearly directly with the time taken for the work.

Two Methods of Determining Time.-Two methods of ascertaining the time to be allowed were open:

(a) By the balancing of inspection, superintendence, interest and similar time charges against the increased costs of obtaining and operating equipment of more capacity than are required to complete the contract in the contractor's economical time. That point on the curve which gives the least total cost, is the correct time to allow for the contract.

(b) By plotting the times allowed on previously completed contracts composed mainly of one kind of work and which had, in the judgment of the engineers in charge thereof, been prosecuted vigorously and with adequate equipment, a series of curves of quantity with reference to time can be drawn for each kind of work, each curve recognizing in its equation some particular controlling factor of variation. Having such curves and knowing the total quantity of work to be done, the proper contract time can be ascertained and the results combined to give the time for a contract including various kinds of work.

In view of the large number of assumptions which must be made and the work involved the first method has been discarded in favor of the second.

RULES FOR INTERPRETING CURVES USED IN METHOD B. FIGS. 31, 32, AND 33.

Determination of Contract Time.-To determine the number of days which shall be written into any contract for regulating and grading as the number of working days to be allowed, the curves hereto attached are to be used in accordance with the rules herein given.

Explanation.-The work to be done will consist of items of rock or earth excavation or of filling or of both, of curb, of flagging, and of bridge-stones. These may or may not be accompanied by walls of dry rubble, rubble in mortar, or concrete; by pipe drains; by inlets; by receiving basins; by manholes; by piles; and by special constructions.

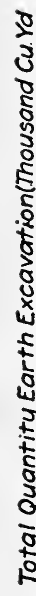

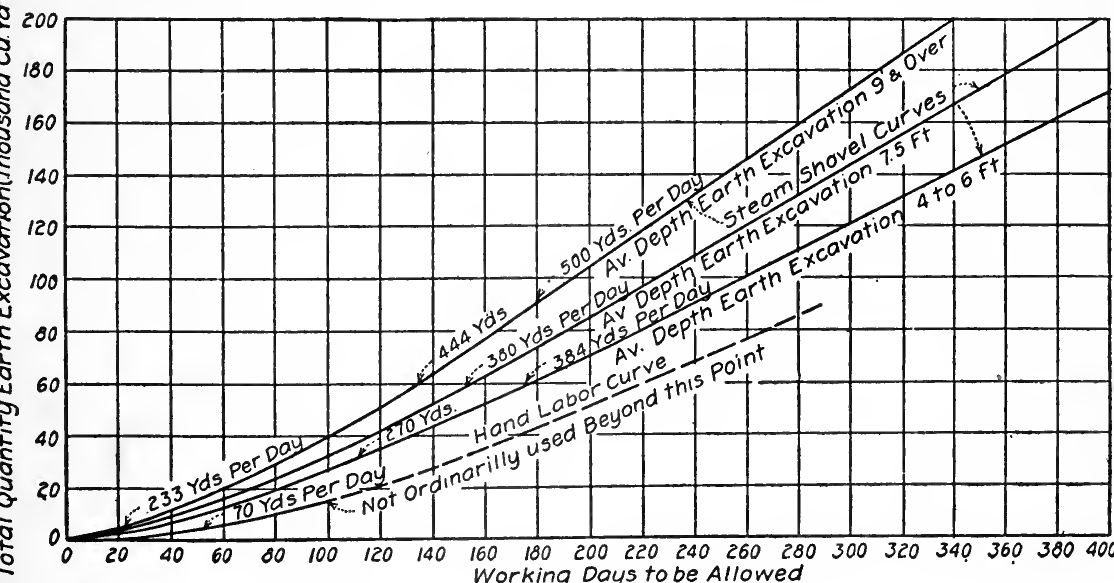

Fig. 31-Curve for Earth Excavation 
Items Which Govern.-Ordinarily, work on more than one of these items can be prosecuted at the same time. Care is therefore required to so use the information available of the special conditions surrounding a proposed work as to eliminate from consideration all of those items and all parts of items which can properly be done during the progress of

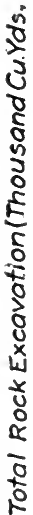

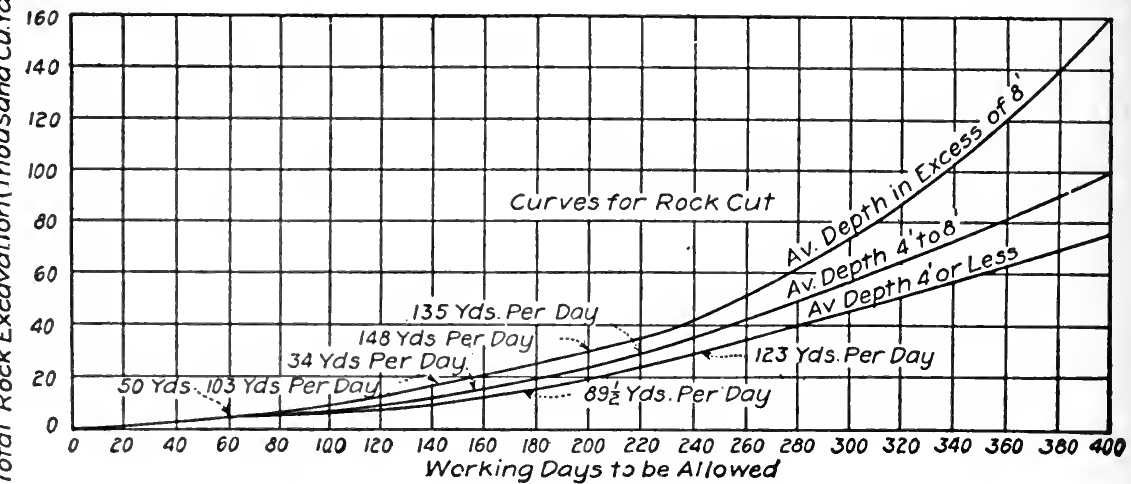

Fig. 32-Curve for Rock Excavation

some other item. There will thus be left as the determining factor in the required time for such work one, or, more rarely, two main items which can not be done simultaneously and some preliminary and subsequent parts of items.

The main items are liable to be earth and rock excavation or filling.

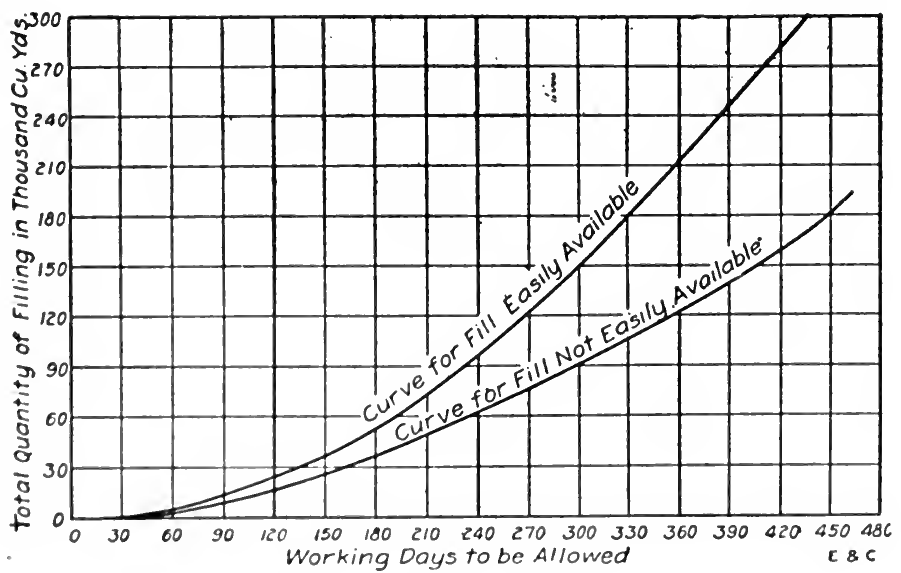

Fig. 33-Curve for Filling

The preliminary parts of items: the excavation of sufficient earth to permit earth and rock exavation to progress simultaneously: the building of sufficient of the pipe lrains, inlets, manholes or basins to permit such building and the filline to progress simultaneously or the building of sufficient walls, in those few cases where such wall is necessary before 
any filling can be done, to permit such wall building and filling to progress simultaneously. The subsequent parts of items: special structures which can not be completed until after the main item is completed; curb, flagging or bridgestone.

The curves have been drawn to recognize, in view of the total quantity of an item to be done, the equipment and force which should be used.

They show, accordingly each for its condition, the amount of time for any total quantity which should be consumed in completing that quantity.

Rule 1.- If the time for a part of an item has to be estimated, it is to be taken therefore at the same rate of accomplishment per day as is the total quantity.

Rule 2.-Where conditions are clearly intermediate between those shown by the curves, interpolation is permissible, but where doubt exists it is preferable as making for lower costs to take that nearest diagrammed condition which gives the longer contract time.

Rule 3.-Where a part of an item comes clearly under one condition recognized in the curves and the remainder as clearly under another, unless the equipment and force which should properly be used for doing these two parts is widely different, the time for the two parts, each taken at the same rate of accomplishment as if the total quantity came under that part's condition, shall be summed to give the time for that item.

Rule 4.-If the equipment, etc., should properly be different, the time for each part of the item is to be taken as if the quantity for this part item were a total quantity and the times so obtained, summed to give the total time for the item.

Note.-It is to be remembered that the contract provides that allowances of time for delays occasioned by the weather, or by any act or omission on the city's part, are to be made in addition to the number of working days. No consideration need be given in their determination to any conditions arising from such causes.

The curves represent average good practice as determined from the records of many contracts. They do not represent the greatest progress which can be made under good management, and if, therefore, conditions arise not provided for in these curves, such as inability to attack the work in more than a few points, unless the condition is very severe, no additional working days are to be allowed as a total.

Rule 5.-For time necessary to get the work started after being ordered ahead and for stopping, after completion, 10 working days are to be allowed as a total.

The following examples are given to illustrate the use of the curves.

Example 1.-On a contract with a center line length of 8,900 ft. and a. street width of $100 \mathrm{ft}$. there are the following items and quantities:

Earth excavation ..................... 88,000 cu. yds. Rock excavation ..................... 26,700 cu. yds.

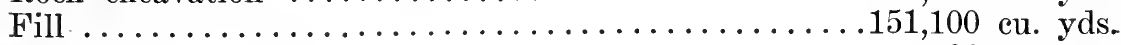
Dry rubble masonry....................... 700 cu. yds.

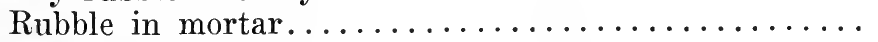

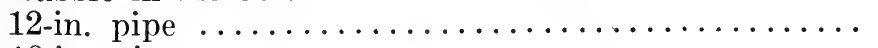
18-in. pipe $\ldots \ldots \ldots \ldots \ldots \ldots \ldots \ldots \ldots \ldots \ldots \ldots$ $25 \mathrm{cu}$. yds. 100 lin. ft. 575 lin. ft. 
Manholes

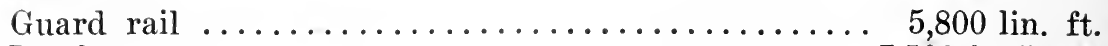

Lumber . ........................

Filling.-It is known that over a portion of the work where filling is required the street is located on a swamp where settlement will, in all probability, take place. Assume that this settlement will amount to $30,000 \mathrm{cu}$. yds. Then the total filling required to complete the work would be $151,100+30,000=181,100 \mathrm{cu}$. yds.

The sum of the earth and rock exeavation amounts to $114,700 \mathrm{cu}$. yds. It should be assumed that the entire excavation is to be applied to making the fill so the material can be eonsidered as easily available.

The balance of the material required for filling (66,400 eu. yds.) must be obtained from outside sources. It is further known that the swamp section of the street is near tide water where material can be obtained by scows. This material, so obtained, should be classed as material " easily available." Even though the doek or nearest obtainable landing may be at some distance from the street under eonsideration, and especially in view of the possibility of obtaining and placing this filling during the progress of the grading on other portions of the work, it should be classed as "easily available."

An examination of the filling diagram will therefore indicate that eonsidering 181,100 eu. yds. as "easily available," 332 days should be allowed, and adding to this 10 days for starting and stopping, we have 342 days, or say 345 days for the contract time.

Excatation.-If, on the other hand, we consider the excavation and know that the earth eutting averages from 4 to $6 \mathrm{ft}$. in depth, and the rock 4 to $8 \mathrm{ft}$. in depth, and that $10,000 \mathrm{cu}$. yds. of earth must be excavated before rock excavation ean begin, and that thereafter both will be earried on simultaneously, we will obtain from the eurves the following:

$10,000 \mathrm{cu}$. yds. earth excavation (at 88,000 rate) $\ldots \ldots \ldots \ldots 27$ days 26,700 cu. yds. rock excavation............................. days

Starting and stopping. $\ldots \ldots \ldots \ldots \ldots \ldots \ldots \ldots \ldots \frac{239 \text { days }}{10 \text { days }}$

If we consider only the earth excavation, and assume that while same is in progress the rock will be excavated, we have from eurves the following:

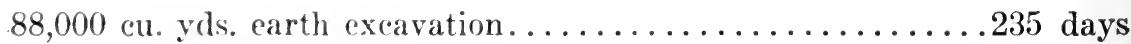

Starting and stopping....................... 10 days

Total time required............................ days

It is evident from the above that the filling required on the work controls and that the contract time should be fixed at 345 days. 


\section{Example 2-}

Earth excavation ..................... 1,000 cu. yds. Rock excavation ...................... $500 \mathrm{cu}$. yds. Filling .............................

In this example the excavation is plainly not to be considered. The filling if easily available will by the curves require 268 days. If not easily available, filling will require 357 days.

To either of these 10 days should be added for starting and stopping, making either 278 days which call 280 days, or 367 days which call 370 days.

\section{Example 3-}

Earth exeavation ....................... 6,000 cu. yds. Rock excavation ....................... 6,000 cu. yds.

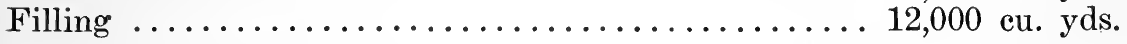

If earth overlays rock, the quantity of earth which must be removed to permit rock and earth excavation to progress simultaneously, must be determined from a knowledge of the local conditions. If these conditions show that say, $35 \%$ of the earth has to be removed before the rock excavation can be properly commenced, and that the rock has an average depth of four feet or less, the times required for excavation will be:

For earth $35 \%$ of the 64 days required by curve for hand labor for $6,000 \mathrm{yds}$.

For rock 114 days required by curve for $6,000 \mathrm{yds}$. of average depth four feet or less.

The sum of these two, plus 10 days for starting and stopping equals 146 days which eall 150 days.

The filling, which is all easily available, would only require 90 days.

Therefore the contract time for this job would be 150 days.

If earth and rock are in separate cuts and separately approachable so that the two sorts of excavation can properly progress simultaneously, the earth excavation need not be considered. The filling will, of course, not be the determining factor and the rock excavation will be. Under these conditions, the contract time should be for rock excavation, 114 days plus 10 days for starting and stopping equals 124 days, say 125 days.

\section{Example 4-}

Earth excavation .....................20,000 cu. yds.

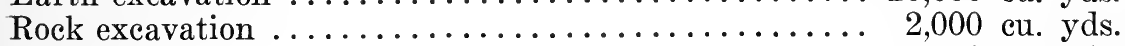
Filling ............................. 3,000 cu. yds.

In this case, the earth overlies subgrade rock throughout most of the work.

The rock excavation remaining to be finished after the earth excavation is completed will amount to about $18 \%$ of the total rock excavation.

The filling will not determine the required time.

The earth excavation being all in shallow cut will be taken out by hand labor, thus requiring 118 days.

The 2,000 yd. rock, $4 \mathrm{ft}$. cut or less, would require 36 days, and 18\% of this would require 6 days. 
The sum of the above plus 10 days start and stop equals 134 days, which call 135 days.

\section{Example 5-}

Earth excavation .................... 1,000 cu. yds.

Rock excavation .................... 6,000 cu. yds.

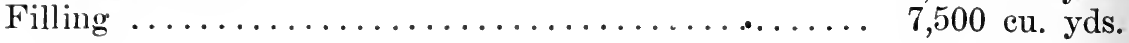

Here, the 1,000 cu. yds. earth overlies the 6,000 cu. yds. rock and the conditions show that only a very little earth will be taken off, say, $200 \mathrm{yds}$. before the rock is commenced. Two hundred yards earth should require about one-fifth of the 24 days required by hand labor curve for the total 1,000 yds., say 5 days.

Six thousand cubic yards rock, of a four-foot or less depth of cut, requires by the curve 114 days.

The sum of the above plus 10 days for start and stopping, equals 129 . days which call 130 days, contract time.

Example 6-

Earth excavation ....................... 30,000 cu. yds. Rock excavation ................... 45,000 cu. yds. Filling (easily available)................. 120,000 cu. yds.

The filling will require by the curve 268 days.

The earth and rock excavation are such that they can be prosecuted quite simultaneously. The rock excavation will therefore control and has an average depth of $6 \mathrm{ft}$., requiring by the curve 270 days, so that. 270 days is good for either.

Therefore 270 plus 10 days stop and start gives 280 days as the contract time.

The application of this method to others of the more usual types of municipal work is obvious and in some cases is under way.

The confidence of contractors in general in the absolute fairness and in the knowledge of the engineer, will, perhaps as much as any other factor, tend to lower the costs of work to be done. And to this end a uniform method of figuring the contract time rather than guessing at it, will, it is believed, contribute in no small degree.

It is probahle that the curves herein given will require modification in some instances to make them fit to the experience of other localities, and it is possible that a further study of data similar to that upon which these curves are hased may lead to somewhat higher or lower averages of performance, resulting in shorter or longer contract time.

The above is from Enginering and Contracting. May 13, 1914.

Manholes. The following article by John Wilson, is from Engineering and Contracting. December 9, 1908. Fig. 34 is for estimating the quantities of materials in manholes; and, at given prices of materials and labor, the cost of the manhole can likewise be ascertained. The diagram shown is for a 4 -ft. manhole.

Having the depth of the manhole given, the number of brick, the amomnt of sand, cement, mortar, the eost of labor, and total cost of manhole complete, plus $15 \%$ profit, may be taken from the diagram. 
Thus for a 15-ft. manhole, follow vertical line to intersection with brick curve, thence horizontally to left read 2,600. From the intersections of the last horizontal line with the sand, mortar and cement curves, respectively, read vertically above $1.88 \mathrm{cu}$. yds. of sand, 2.22 cu. yds. of mortar and 4.6 barrels of cement. To ascertain the cost, follow vertical line from bottom to intersection with the cost curves, and

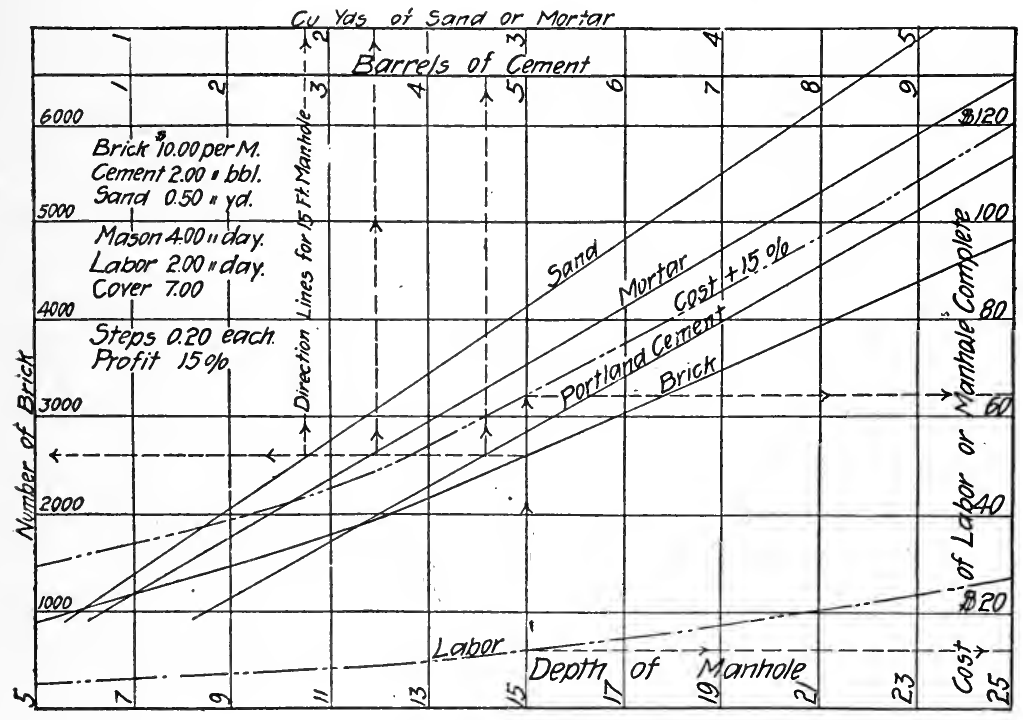

Fig. 34-Diagram for Estimating Cost of Manholes

read horizontally to right, cost plus $15 \%, \$ 64$, of which the cost of labor alone is $\$ 12.50$.

The curves allow for a double layer of brick in the bottom and the outside of the manhole to be well plastered. It is an easy matter to draw similar curves to meet local conditions and thus secure a very ready method of making estimates.

Estimating Highway Bridges. The following is from Engineering ing Record, August 7, 1915 :

Helpful diagrams for use in the selection of the most economical type and in making estimates for highway bridges have been plotted by G. F. Burch, assistant bridge engineer, Illinois Highway Department, based upon the standard plans of bridges prepared by that department and the quantities of material in many standard designs. These diagrams, which were published in the June issue of "Illinois Highways," give the weight of steel and the amount of concrete in steel truss spans from 50 to $160 \mathrm{ft}$. long, with a 4 -in. concrete floor, in reinforced-concrete girder spans from 30 to $60 \mathrm{ft}$. long, and in reinforced-concrete slabs from 5 to $30 \mathrm{ft}$. long. In addition curves are given for the amount of concrete in the abutments, both plain and reinforced.

The curves given are for quantities only. The cost of labor and 
material must be determined for each and every job and applied to the given quantities, which are intended primarily for use in the field.

The steel trusses are of the ordinary Pratt truss type with parallel chords and riveted connections. The design provides for a 4-in. concrete floor, with a wearing surface assumed to weigh not less than $50 \mathrm{lb}$. per square foot. On account of the weight and rigidity of the conerete floor no allowance is made for impact. Floor systems are designed to carry a 15-ton traction engine in addition to the dead load. Trusses are designed to carry a uniform load of $100 \mathrm{lb}$. per square foot of road surface for spans from 50 to $150 \mathrm{ft}$., and a uniform load of $85 \mathrm{lb}$. for spans exceeding $150 \mathrm{ft}$. long. The usual A. R. E. A. unit stresses are used in the design. Pony trusses are used for spans of from 50 to $85 \mathrm{ft}$., and through trusses for spans of from 90 to $160 \mathrm{ft}$.

Fig. 35 gives the eurves for the weight of structural steel, and the yardage of concrete in fioors, for 16 and 18 - $\mathrm{ft}$. roadways. The break in the steel curves is at the point where the change is made from low to. high trusses.

Reinforeed-concrete through girders are used for spans of from 30 to $60 \mathrm{ft}$. This type of structure is designed to earry either a uniform load of $125 \mathrm{lb}$. per square foot, or an engine load of 24 tons. The design provides for a wearing surface weighing $50 \mathrm{lb}$. per square foot. As free expansion and contraction are allowed by the cast-iron rockers placed under each girder at one end of the spans, the allowable unit. stresses used are quite high. Designs are figured for a steel stress of $16,000 \mathrm{lb}$. per square inch and a compression stress in the concrete of approximately 1,000 lb. per square inch. A maximum unit shear of $120 \mathrm{lb}$. per square inch is allowed. Stirrups are provided for all shear in excess of $40 \mathrm{lb}$. per square inch. Fig. 36 shows curves giving the quantities of concrete and reinforeing steel for 16,18 and 20 -ft. roadways.

For spans less than $30 \mathrm{ft}$. the slab type of construction has been found to be somewhat cheaper than the girder type, due to the fact that the arrangement of the steel is much simpler and less steel is used per eubic yard of concrete. It has not been found practicable to make provision for free expansion of slabs. Accordingly, a stress of 12,000 lb. per square inch of reinforeing steel is allowed for dead-load and live-load stresses. The concrete stress is $800 \mathrm{lb}$. per square inch. Slabs are designed for the same live load as girders. Fig. 37 gives the quantities. of concrete and steel in slabs having clear roadways of $16,18,20$, and 24 ft., for spans of from 5 to $30 \mathrm{ft}$.

In preparing eurves to show the quantities in abutments it was found that there were many variables which might be considered, but which if used would produce such complex formulas as to make the eurves of little use in the field. It was found that curves giving reliable results might be obtained by plotting the cubic yards of concrete in two abutments against a formuia which represented a measure of the quantities desired. The variables in this formula are $I$, height of abutment from bottom of foundation to top of roadway; $R$, clear width of roadway on superstructure, and $\mathrm{I}$, length of average wing wall. For plain concrete abutments the best results were obtained by using the term $H^{2}(R+2 W)$.

Plain conerete abutments for steel bridges are designed with a footing width of one-third of the height over all, and the thickness of the footing: 


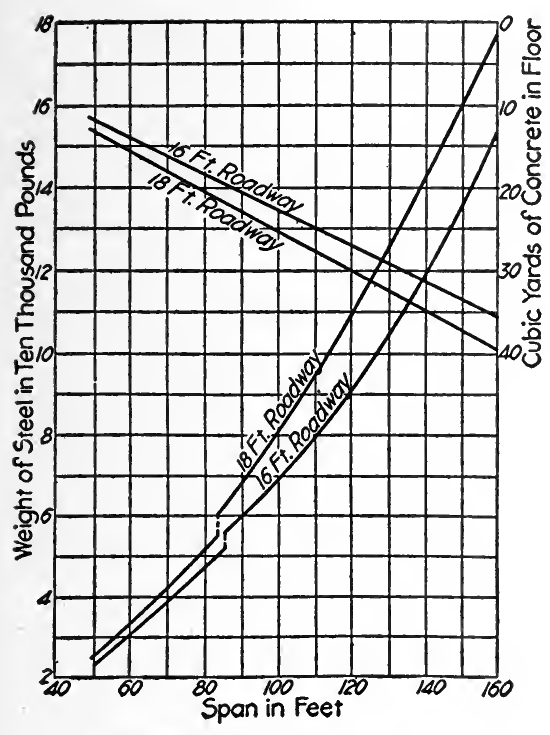

Fig. 35-Steel Truss Superstructures

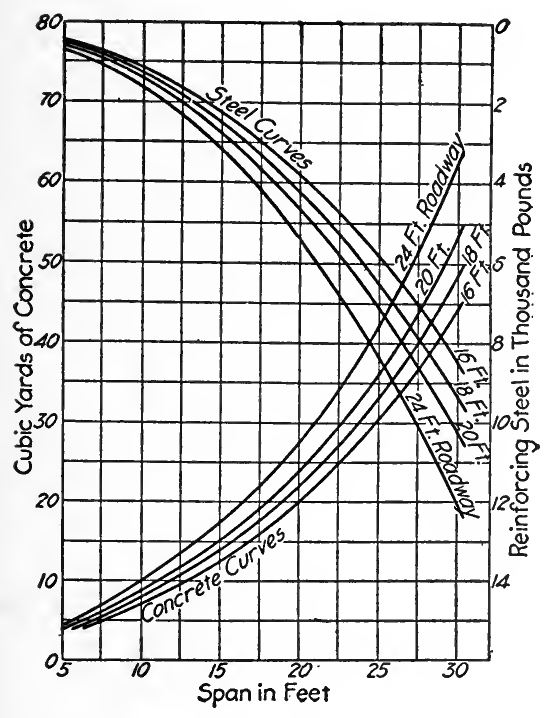

Fig. 37-Reinforced-Concrete Slab Superstructures

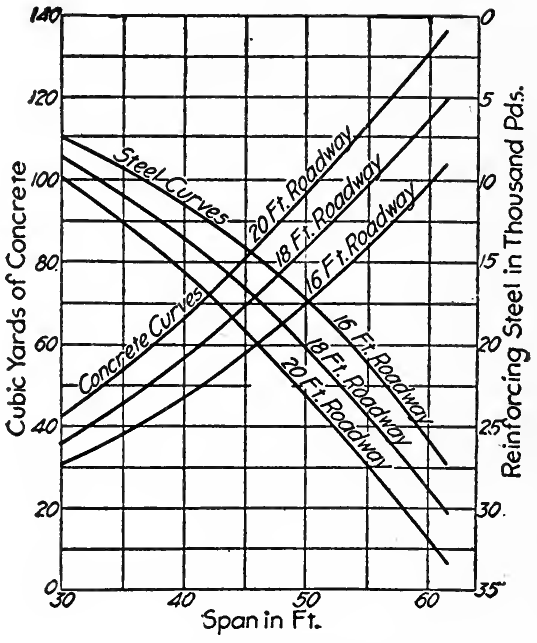

Fig. 36-Reinforced Concrete Through Girder Superstructures

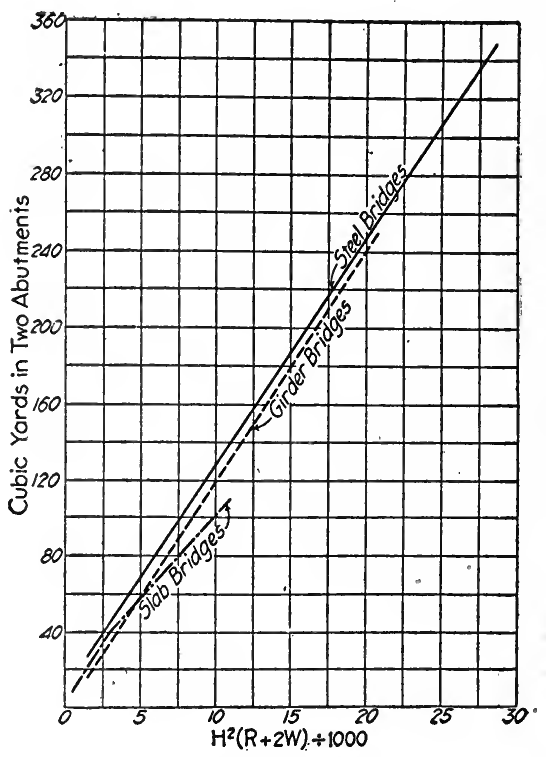

Fig. 38-Plain Concrete Abutments for Three Types of Bridges 
is usually from 18 to $24 \mathrm{in}$. The width of the base of the abutment and wing walls at the top of the footing is made approximately one-quarter of the height of the walls. The back of the abutment wall is vertical and the face of the wall is battered to a top width of from 30 to $38 \mathrm{in.}$ The wing walls are battered on both sides and have a top width of $12 \mathrm{in}$. Fig. 38 shows the curves from which the yardage of plain concrete abutments for steel bridges may be obtained. When field measurements are made to determine the necessary height of abutments, and the width of roadway is deeided upon, it is easy to estimate the length of wing walls which will be required. These figures are then used in the formula and the yardage of eonerete is read directly from the curve.

The design of plain concrete abutments for girder bridges is similar to the design for steel bridges, except that the wing walls are battered on the face side only, and the top width of the abutment wall is $18 \mathrm{in}$. Fig. 38 shows the eurve from which quantities for this type of abutment are obtained. Plain concrete abutments for slab bridges differ slightly from the preceding design. The width of footing on the abutment wall is limited only by the safe bearing eapacity of the soil, with a minimum of $3 \mathrm{ft}$. This width may sometimes be less than one-third of the height. This is deemed to be safe on aceount of the restraining effects of the superstructure. The top width of the abutment wall is $12 \mathrm{in}$. and the curve for estimating the yardage is shown in Fig. 38.

When these eurves are used for plans based on the Illinois Highway Department's standards, the maximum error for superstuctures is only about $3 \frac{1}{2} \%$ and for substructures about $5 \%$.

Following, is a list of articles containing further illustrations of the use of graphie charts of the class discussed in this chapter and pertaining to kindred subjects.

Upon request, the Codex Book Company, Ine., 19 William Street, New York, will be glad to proeure, where possible, copies of magazines containing the articles needed, only charging the "old magazine" price, plus postage.

"Cost of Girder and Slab Concrete Bridges," B. II. Piepmeier, Engineering Record, February 3, 1912.

"Estimating C'urves for" Standard Bridges of the Illinois Highway Dept.," Engincering and Contracting, Feb. 10, 1915.

"Railway Ditehing 'Trains," Engineering News, March 16, 1916.

"Aids to Estimating the Cost of Vitrified P'ipe Sewers," W. G. Kirehoffer, Enginerring Neu's, Oct. 26, 1916.

"Estimating Building ('osts," W. P. Anderson, Concrete, Nov., 1917.

"Graphical Solution of Transmission Line Problems," T. A. Wilkinson, Elcitrical World, August 12, 1916.

"Graphic Nothorls of Analysis in The Design and Operation of Steam Power Plants," R. .J. S. Pigott, Journal Amer. Soc. of Mechanical Enginurs, Dee., 1916.

“Motor I rive for Oprerating Steel Rolling Mill," William Knight, Electrical World, Sept. 3(1), 1916.

"Graphical Solution of Illumination Problems," N. S. Dickinson, Electrical World, August 17, 1918. 
"Chart for Calculating Hoisting Ropes," The Engineering and Mining Journal, March 22, 1913.

"Charts for Estimating the Strength of Bolts," William F. Fisher, Power, Jan. 11, 1916.

"Charts for the Design of Helical Springs," M. M. Brayton, American Machinist, June 13, 1913.

“Charts for Broach Design,"' L. A. Williams, American Machinist, April 19, 1917.

"Calculating Ratings for Elevator Motors," Electrical World, June 30, 1917.

"Data for Bulkhead Construction," F. K. Ruprecht, International Marine Engineering, July, 1917.

“Economic Conduit Location," Engineering Record, Jan. 17, 1914.

"Economical Section of Water Conduit for Power Development," Cary T. Hutchinson, Mechanical Engineering, April, 1919.

"Mass Diagram for Power and Water-supply Computations," W. L. Butcher, Engineering News, Aug. 24, 1916.

"An Additive Method of Determining Storm Water Run-off," Engineering Record, March 6, 1909.

"Design Charts for Open Flumes with Catenary Section," Ernest L. Robinson, Engineering News, March 23, 1916.

"Designing Steel Pipe for Minimum Weight of Metal Consistent with Safety," E. R. Bowen, Engineering Record, Dec. 20, 1913.

"Selection of Stacks and Breechings," B. S. Nelson, Power, March 31, 1914.

"Chart for Proportioning Trunk Main Systems," M. William Ehrlich, Metal Worker, February 16, 1917.

“Determining Gas-Passage Areas," William Carl Stripe, Power, July 31, 1917.

"Flow of Steam in Pipes," D. E. Foster, Power, March 17, 1914.

"'Size of Steam Pipes from Chart," W. L. Durand, Power, January 6, 1914.

"Capitalized Value of One-tenth of an Inch of Vacuum," C. H. Baker, Power, Dec. 9, 1917.

"Reciprocating Parts," Hubert L. Watson, Machinery, August, 1916.

"Useful Diagrams for Computing Sizes of Cross-Compound Pumping Engines," Electrical World, Oct. 14, 1916.

"Visits of Inspector Brown-XXIV (Safe working pressure of Boilers)," J. E. Terman, Power, March 13, 1917.

"Diagrams Facilitate Design of Office Building and Mill Building Columns Under Eccentric Loads," W. S. Wolf, Engineering NewsRecord, June 28, 1917.

“Diagrams for Reinforced Concrete Beams," H. O. Schermerhorn, Engineering Record, March 6, 1909.

"Design of Solenoids and Plunger Magnets," George L. Hedges, Electrical World, Oct. 20, 1917.

"Importance of Flexibility of Boiler Control," Victor B. Phillips, Electrical World, Oct. 20, 1917.

"New Forms of Co-ordinate Paper and Map Marking Devices," Industrial Management, March, 1917. 
“Nomography I-III,” F. Leigh Martineau and A. Marshall Arter, Automotive Industries, May 16, 23, 30, 1918.

"Selecting Economical Type of Riveted Joint for Steel Pipes and Stand-pipes,'” Frank II. Carter, Engineering Record, Oct. 14, 1916.

"Cost Accounting to Aid Production," G. Charter Harrison, Industrial Management, April, 1919.

“A Handy Foundation Chart for Gas Engines," N. G. Near, American Gas Engineering Journal, August 4, 1917.

“Estimating Heat Value of Fuel," Power, June 10, 1913. 


\section{CHAPTER XVIII}

\section{Miscellaneous Uses of Charts}

In this chapter are grouped several examples of charts made and used for a variety of purposes not included in the preceding pages.

Corrections for Sag in Long Steel Tapes. The following, by W. H. Rayner, is from Engineering and Mining Journal, August 11, 1917 :

In Fig. 1 are given the corrections for sag under various conditions of weight, pull and length, for tapes from 100 to $500 \mathrm{ft}$. in length. In this diagram use has been made of the nomograph to deal with the four variables involved. For any given tape the weight is a constant, hence the relation between weight and pull may be expressed as a ratio which $W$

becomes one variable $\frac{-}{P}$ in the nomograph. The units for the different quantities are shown on their corresponding ordinate lines in the diagram.

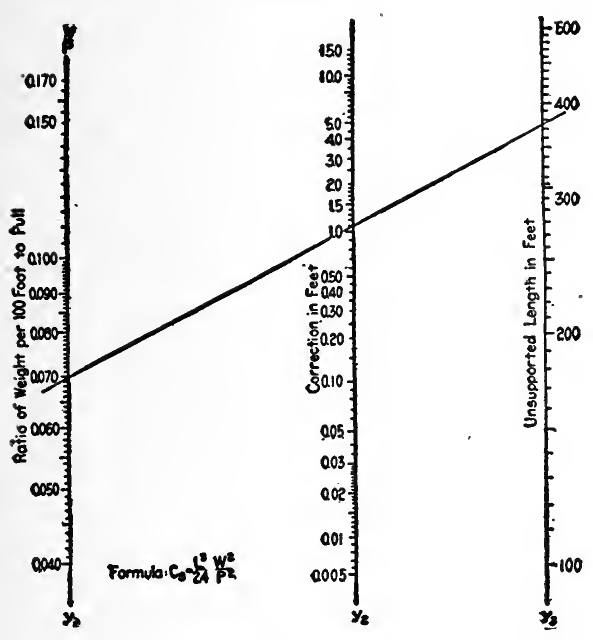

Fig. 1-Determining Correction for Sag in Steel Tapes

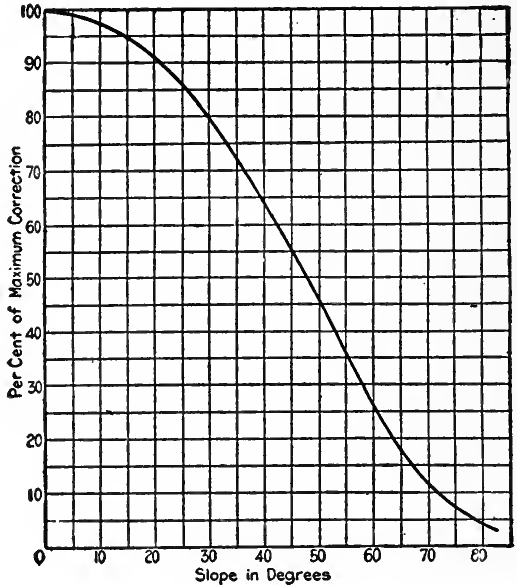

Fig. 2-For Determining Correction for Sag in Steel Tapes on Slope

The use of the nomograph is very simple, and one example will be given to illustrate it. A $500-\mathrm{ft}$. tape weighs $7 \mathrm{lb}$., or $1.4 \mathrm{lb}$. per $100 \mathrm{ft}$. A distance is measured with a pull of $20 \mathrm{lb}$. where the unsupported length is $375 \mathrm{ft}$. What is the correction for sag in this case? Solution: The ratio of weight per $100 \mathrm{ft}$. to pull $\left(\frac{W}{P}\right)$ equals $\frac{1.4}{20}$ or seventy hundredths. The unsupported length $L$ is $375 \mathrm{ft}$. Place a straight-edge across the 
nomograph to intersect these two quantities on their corresponding ordinate axes, and the middle ordinate is intersected at a point which reads 1.08 as the correction due to sag alone.

The effect of slope on sag corrections is shown in Fig. 2. This curve has been prepared from data given by E. V. Huntington and shows the percentage of maximum correction for the various slopes. For example, suppose that the above measurement had been made on a $20^{\circ}$ slope. From the curve it is seen that the correction for sag on a $20^{\circ}$ slope is $91 \%$ of the maximum and accordingly the final correction due to sag. is $91 \%$ of $1.08 \mathrm{ft}$. or $0.98 \mathrm{ft}$.

It is possible that some engineers in the field would like to make use of such a diagram to a larger scale; hence, the following directions are: given. First, construct three vertical lines (ordinate axes), which we may consider as being numbered $y_{1}, y_{2}$ and $y_{3}$ from left to right. The:

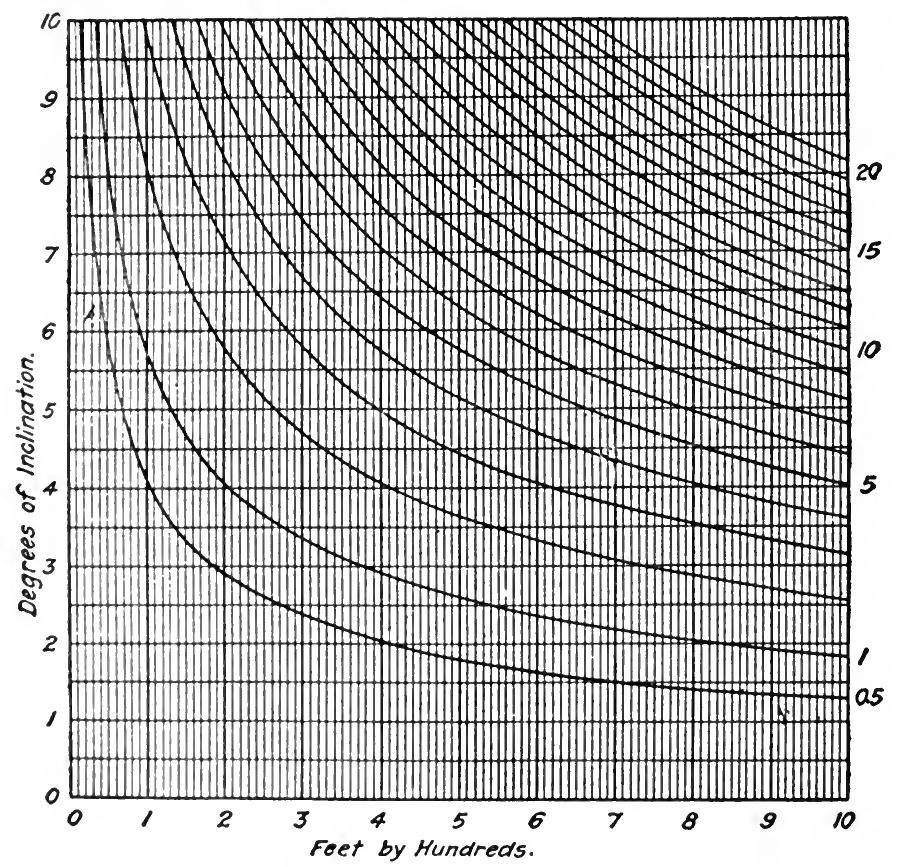

Fig. 3-Stadia Reduction

intervals $y_{1}$ to $y_{2}$ and $y_{1}$ to $y_{3}$ must be in the exact ratio of 3.6 to 6 . second, plot to any seale and beginning at any point on $y_{1}$ the logarithms of the numbers 40 to 170 , and to the same scale beginning at any point on $y_{3}$ plot the logarithms of numbers from 100 to 500 . This means that the linear distance from 100 to 170 on $y_{1}$ must be exactly the same as the distance from 100 to ijo on $y_{3}$. Third, solve the given equation, $I^{3} W^{2}$

$C_{\mathrm{s}}=-$ - for a number of selected conditions to give values of the cor$2+\Gamma^{2}$

rection which will be evenly distributed over the ordinate $y_{2}$. Fourth, 
subdivide this ordinate to any further degree desired and number the seale accordingly.

Stadia Corrections. Mining and Scientific Press, August 25, 1917, published the following by Earl Glass :

Fig. 3 may be used for computing horizontal distances from stadiareadings and observed angles of inclination, the curves giving the correction to be subtracted. The horizontal distance of the observation will be found at the lower edge of the chart, and the degrees of inclination of the angles of elevation or depression appear at the left. Find the intersection of these two lines, and follow the curve from this point of intersection to the right-hand edge of the chart where the correction will be figure found on the margin. Thus, for a stadia-reading of $100 \mathrm{ft}$., and an angle of inclination of $8^{\circ}$, the correction is $2 \mathrm{ft}$, , and the horizontal distance is the difference between the reading and the correction, or $98 \mathrm{ft}$. For a stadia-reading of $250 \mathrm{ft}$. and an angle of $3^{\circ} 40 \mathrm{ft}$., the correction is $1 \mathrm{ft}$., and the horizontal distance is $249 \mathrm{ft}$.

Designing, Drawing, etc. The following, from an article by R. T. Dana, published in Engineering News-Record, October 25, 1917, describes some uses to which rectilinear charts have been put:

The use of a standard cross-section paper for the plotting of curves which were to be blueprinted was found to be so convenient for other uses that in the writer's office it has practically supplanted other methods, except for maps and plans of large structures on which many of the details, such as rivet spacing, etc., require the use of extensive sheets. The success obtained resulted in the development of a set of methods employing a specially ruled paper for nearly all of the work for which, previously, the ordinary kind of tracing cloth, cross-section sheets and profile rolls have been employed.

The principal objections to the use of tracing cloth are that it turns opaque with age, and stretches unequally in various directions. Sometimes this stretching is considerable, and will vary with the weather. Furthermore it is ruined by water, so that if left by an open window, in a damp atmosphere, or over a radiator from which the steam is escaping, an expensive drawing may easily be spoiled.

Nor can it be satisfactorily used in a typewriter. Since it is easily cracked, it cannot be folded without injury, and hence is not conveniently portable. The small red ants, which infest the foundations and walls of buildings in hot climates, eat holes in the paper in endeavoring to get the sizing. Where these ants are present it is not safe to leave a tracing on the drawing table overnight.

Another objection to the usual systems lies in the fact that small tracings are of a good many different sizes, and are difficult to file satisfactorily. They must be kept flat and, when filed in large envelopes or folded in a sheet of heavy paper, are not readily at hand when wanted. They often become turned around in the drawer, the title of the desired sheet is seldom where one expects to find it; and consequently a certain amount of time is lost.

The ordinary cross-section sheets which are ruled to tenths of an inch have been found to have the following objertions: (1) The paper is so thick that they cannot be satisfactorily blucprinted. (2) They are of an inconvenient size-too large for the letter file and too small for the 
map file. (3) They are too stiff to lie flat and are constantly curling. (4) The ruling is not convenient for the listing of tabular data on the same sheet with the cross-sections, the lines being so close together

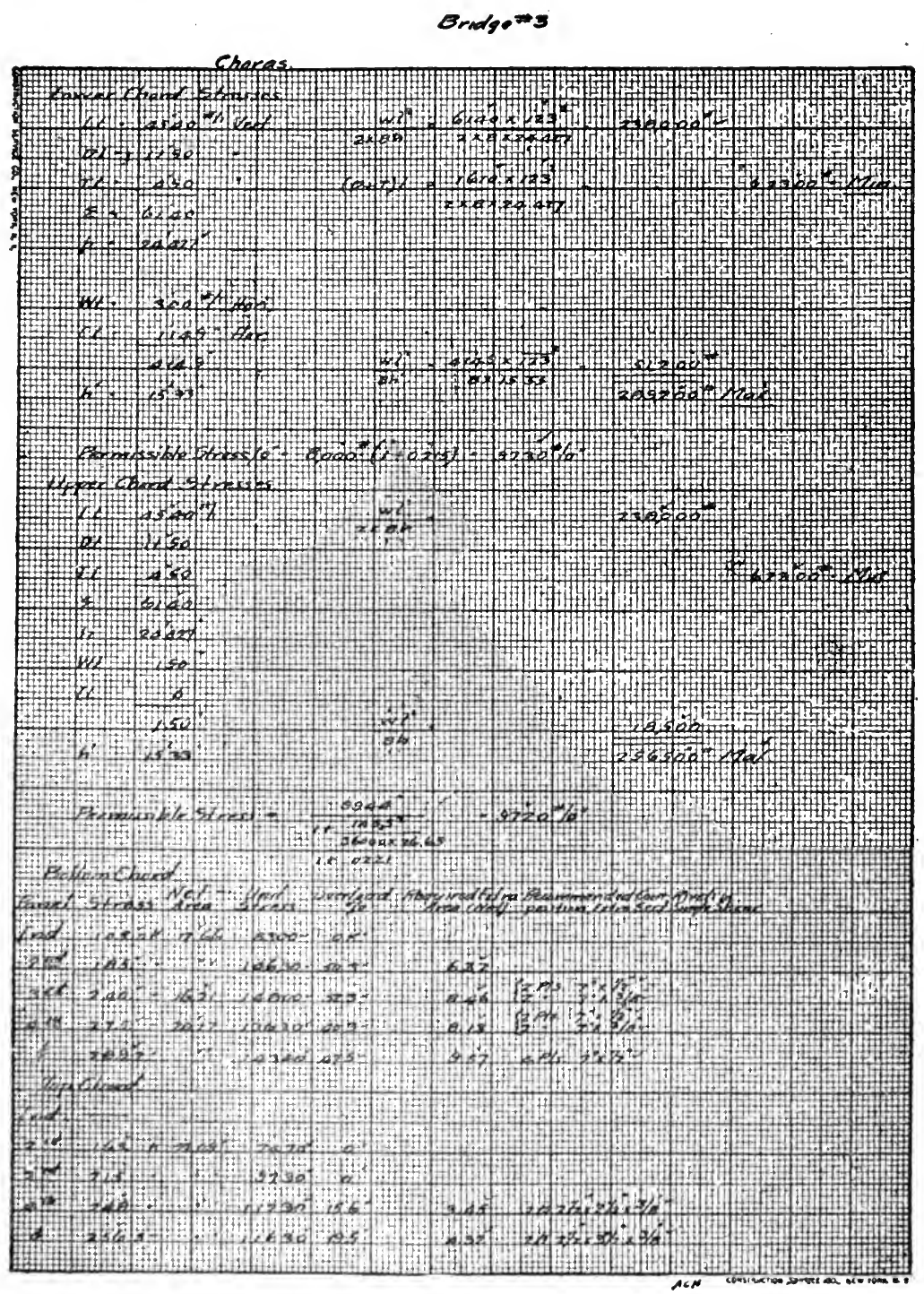

Fig. 4

(1/10 in.) as to offer no gruide for any but the smallest figures, whereas the inch lines are either too near together or too far apart to be used as columm rulings. (5) The sheets cannot be satisfactorily folded on 
account of their thickness and stiffness, and when rolled for the sake of portability they acquire the rolling habit and object to lying flat ever after. (6) The use of a scale of $10 \mathrm{ft} .=1 \mathrm{in}$. for plotting cross-sections is not satisfactory, since the eye does not accurately interpolate between

\section{sridge -3}

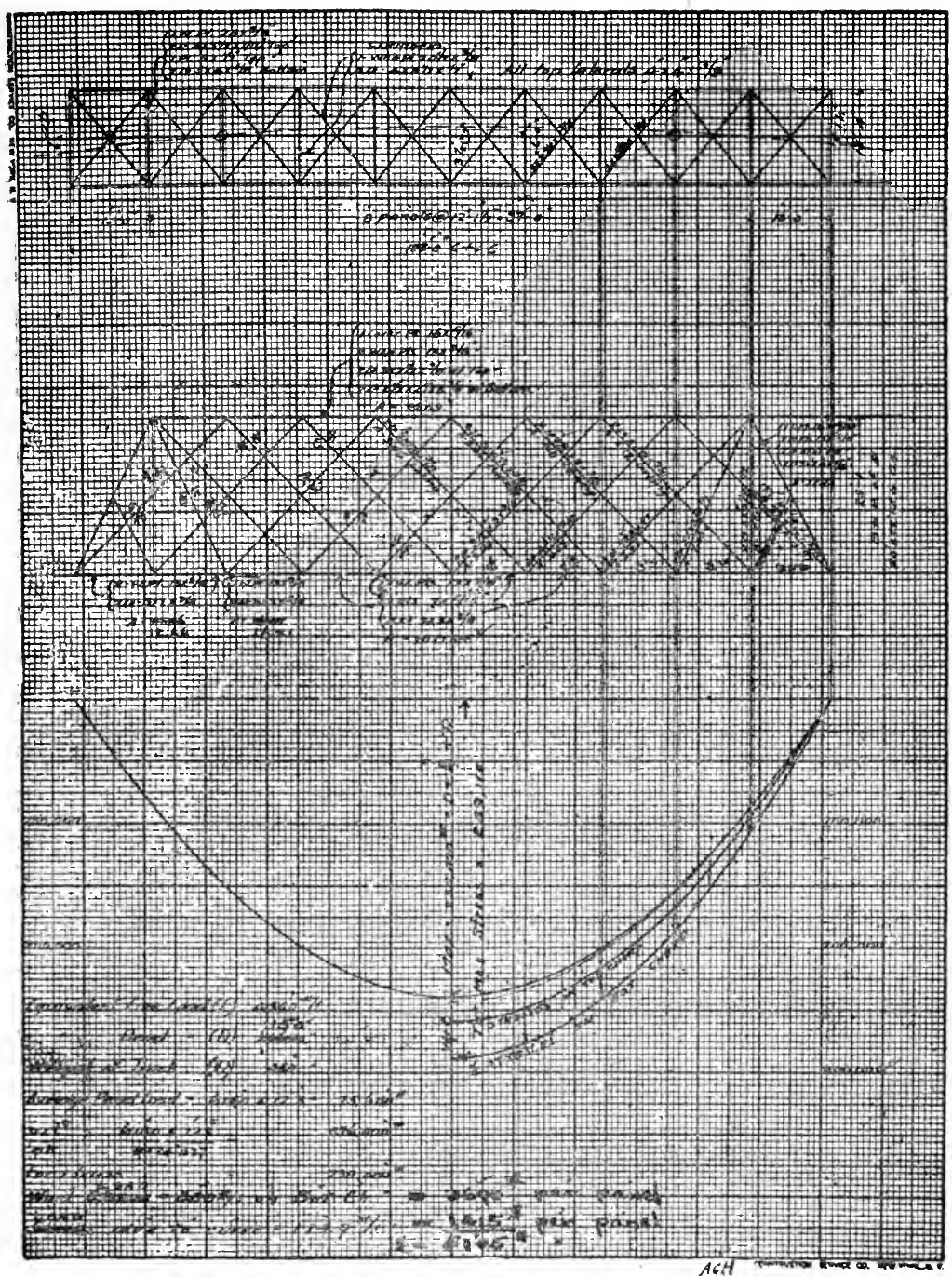

Fig. 5

the 1/10-in. lines; consequently the plotting is actually accurate to the nearest $2100-i n$., ordinarily representing $2 / 10$ of a foot. The same degree of accuracy in plotting can be accomplished on a paper ruled to about half this scale. 
In the case of profile paper we found that for most purposes there were the following objections: (1) It eannot be blueprinted unless tissue papers are used, and these are easily destroyed by eareless handling, or even by heary gusts of wind when they are not properly tacked down to

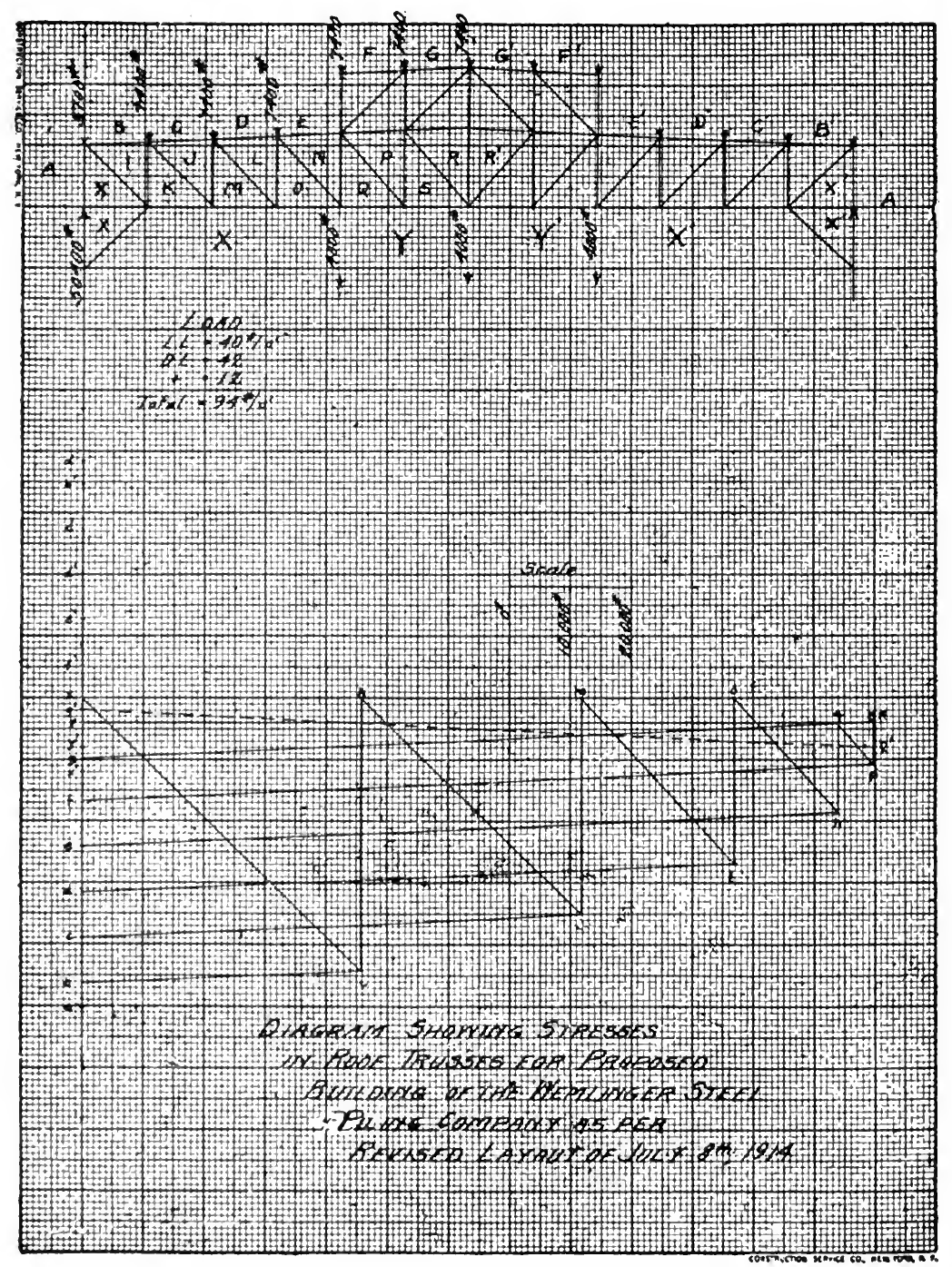

Fig. 6

the drawing paper. (2) It does not take erasure well. (3) The horizontal spacing of the heavy lines (4 in.) is inconveniently great for the plotting of $100 \mathrm{ft}$. stations, and the $1 / 10$-in. space is a little too small for the same purpose whre two or more pluses must be entered between two 100 ft. stations. The approximate location of the pluses eannot be marked 
closer than to the nearest 10 or $15 \mathrm{ft}$. except with the use of a scale, which involves a serious waste of time. (4) The vertical spacing is usually not convenient, the heavy lines indicating feet or $10 \mathrm{ft}$., etc., and the light ones feet, or $1 / 5 \mathrm{ft}$. To obtain greater precision than $1 / 10 \mathrm{ft}$., a scale of $1 \mathrm{ft}$. per in. must be used, involving a value for the smallest space of $1 / 25 \mathrm{ft}$., which is neither necessary nor convenient. (5) The profiles when rolled up are most inconvenient to carry about, and they occupy a very large space in the files, due largely to their shape. (6) In making use of them much time is lost, rolling them back from end to end to find the point wanted where the profiles cover a considerable length of line.

The inconveniences mentioned before came to mind with such monotonous regularity that after a number of years it was decided to experiment with sheets of paper $8 \frac{1}{2} \mathrm{in}$. wide by $11 \mathrm{in}$. long-the size of a standard letter head-punched in the left-hand margin for filing in a loose-leaf book, and ruled to $1 / 20$-in. with every fifth line heavy and every tenth line extra heavy.

In making detail sketches dimensions are furnished by the lines on the paper instead of by drawing lines and marking out points with a scale as when sketching on blank sheets. Sketches can be made in this manner very much more rapidly, and with the same degree of accuracy, as in the old way.

The record thus made on a durable grade of paper is as nearly permanent as anything of this sort can be. It will probably be as fresh and clear and legible fifty years hence as the day after it was made. The sheets may be filed in a standard letter file with the papers belonging to their particular piece of work, or they may be filed in loose-leaf books or bound in permanent form with special binders. All these methods have been used interchangeably.

For use as cross-section paper these sheets, containing an area of $30,000 \mathrm{sq}$. ft. on a $1 / 20$ th scale, are sufficiently accurate for most purposes, and ean be plotted to the nearest $0.2 \mathrm{ft}$.

For surveys involving a very large number of sections it has been suggested that blueprints from these sheets on thick paper be used, determining the areas by cutting out the sections and weighing each on a chemical balance. Cross-section paper is sometimes objected to for plotting sections which are to be planimetered, as its shrinkage necessitates a planimeter setting that is difficult to determine. Where the weighing method is employed shrinkage is of no importance as the section is to be compared by weight to a unit section which has shrunk uniformly.

For use as profile paper these plates have been entirely satisfactory, each sheet running for 20 stations horizontally and for 150 vertically to an accuracy of $0.2 \mathrm{ft}$. or $15 \mathrm{ft}$. vertically with an accuracy of $0.02 \mathrm{ft}$. When so used it has the advantage that mass diagrams and cross-sections can be placed on the same sheet, together with the tabular data accompanying the latter.

This paper has been used a great deal for progress diagrams, for which a 31-day month may be listed the short way of the paper, reading vertically when these sheets are filed in a loose-leaf book, and allowing a range for plotting of from 0 to 20 or any multiple thereof.

The original purpose for which the paper was designed was the plot- 
ting of eurves, and for this the $1 / 20$-in. ruling has turned out to be very well adapted, since across the paper it runs from 0 to 150 and lengthwise from 0 to 200 , thus giving a range sufficiently great for almost any kind of work.

One of the most convenient uses of the paper, and perhaps the last one which ordinarily would suggest itself, is for stress diagrams and equilibrium polygons in graphical statics. It would appear that small diagrams on this paper could not be made to sufficiently great precision to give a satisfactory check, but experience with it has shown that this is not the case. If the points are set with eare, and the parallel lines ruled with a pair of small but accurate triangles and a wedge-pointed pencil, the resultant precision of the work is remarkable. It is entirely possible to obtain results within $1 / 5$ of $1 \%$, which is satisfactory for most purposes. Very long lines on tracing cloth will be found not to be as accurate as they appear.

A convenient size of the equilibrium polygon is half the length of the paper or 1,000 divisions. Working to one-fifth of one division for half of this length gives an average error of one in five thousand. This is very much smaller than the error in the setting of the rolls for the manufacture of struetural steel shapes, and therefore any greater degree of refinement than this is a bit ridiculous.

The paper is used very largely in computing and tabulating figures, as will be noted by reference to Figs. 4,5 and 6 which have been taken more or less at random from the files of this office. It is often convenient to typewrite titles and notes on this paper rather than taking the trouble to letter them on freehand. When this is done, a piece of carbon paper placed underneath the section paper with the carbon side up will so strengthen the imprint of the type that most excellent blueprints are obtained.

A curious feature of this paper is that it can be used as a protractor, and is more accurate than the ordinary paper protractors on the market.

Use of Rectilinear Chart in Mechanical Drawing. What follows is from an article by Clarence E. Howell, published in Industrial Arts Magazine, May, 1919.

In mechanical drawing it is possible for a student to make a most excellent and commendable drawing, so far as the finished product is concerned, without really knowing very much about what he has accomplished or how he has done it.

It is, therefore, essential that we should know whether the student is really securing a definite, usable understanding of the problems presented, or whether he is merely becoming an expert copyist. Many a student seems perfectly elear on the points presented at the time of the demonstration, and can go through the entire problem with facility, but a few days or a few weeks later when he meets the same problem in class work or in a test, he falls down completely on it. Such lapses in essential knowledge, oecurring habitually and among a wide diversity of students, can constitute nothing less than a sovere critieism of our teaching methods. Often the things forgotten are the very ones the student should know above all others, indeed must know, if he is to carry away a usable knowledge from his school work.

The only plausible reason for these apparently complete lapses in 
memory is either that the pupil never really understood the problem in the first place, or else that he was not drilled upon it sufficiently to impress it indelibly upon his mind.

The ordinary method of school drawing does not provide either a quick and economical way for testing the ground-knowledge of theory beneath the delineation, or a convenient and easy scheme for drill in this theory.

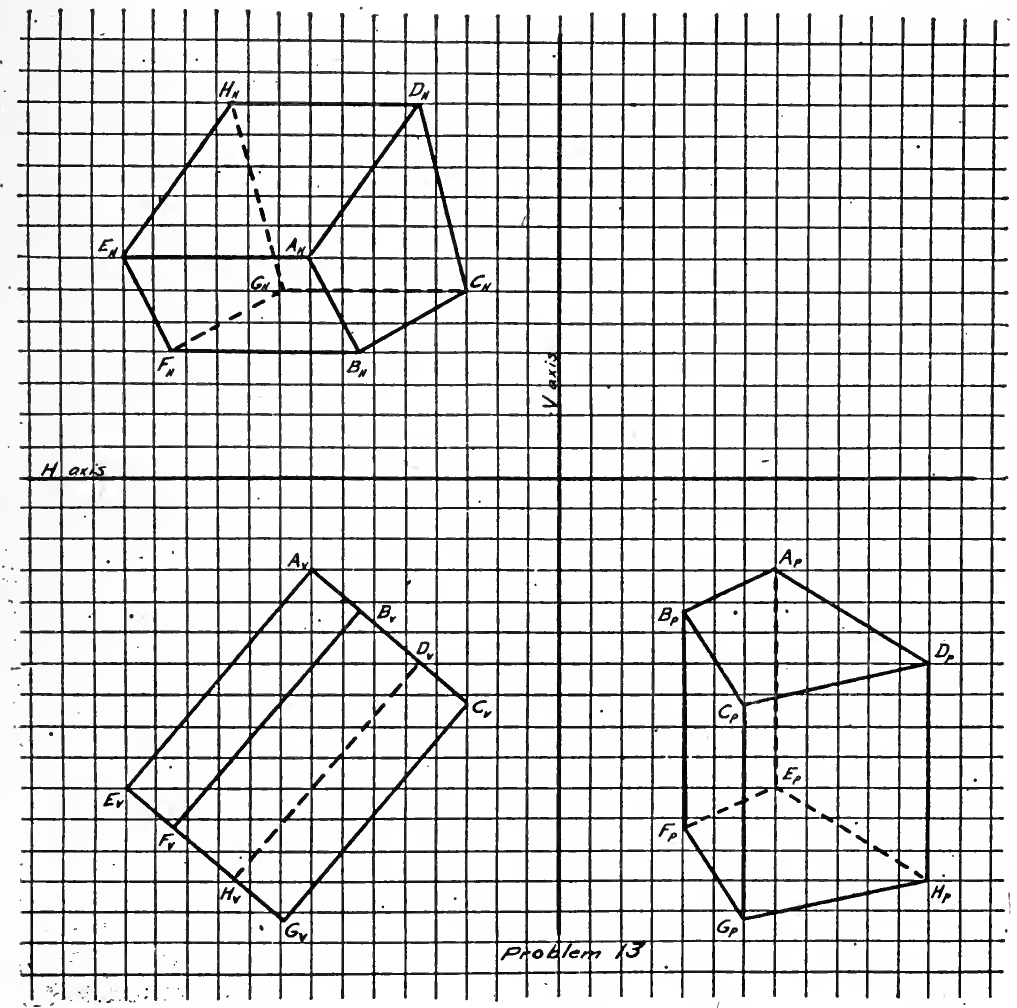

Fig. 7-Typical Supplementary Problem

The following plan, worked out to meet this situation, has been used successfully in actual classroom practice. This plan contemplates a series of short, rapid repetitions of essential problems connected with the regular drawing work, and is based upon the supposition that, in this instance, accuracy and appearance may be made secondary to speed and results.

Problems, Figs. 7 and 8, such as these should be introduced into odd moments of the regular class periods, to be worked out by the students with pencil, triangles, scale and compass, on 8 in. $x 11$ in. sheets of cheap grade $1 / 8$-in. cross-section paper, without the aid of the tee square. The paper should not be tacked to the drawing board, and the section lines of 
the graph paper should be used as guide lines whenever possible. By these methods a good deal may be accomplished in a very few minutes, provided the pupil really knows what he is about. If he does not, this type of work will soon show it, and his ease can then be given the individual eoaching which it demands. Assurance that practically every member of the class knows the theory, its use, and its application under varying conditions, may be obtained by repeating different applications of the same problem for successive days. The pupils may work on their

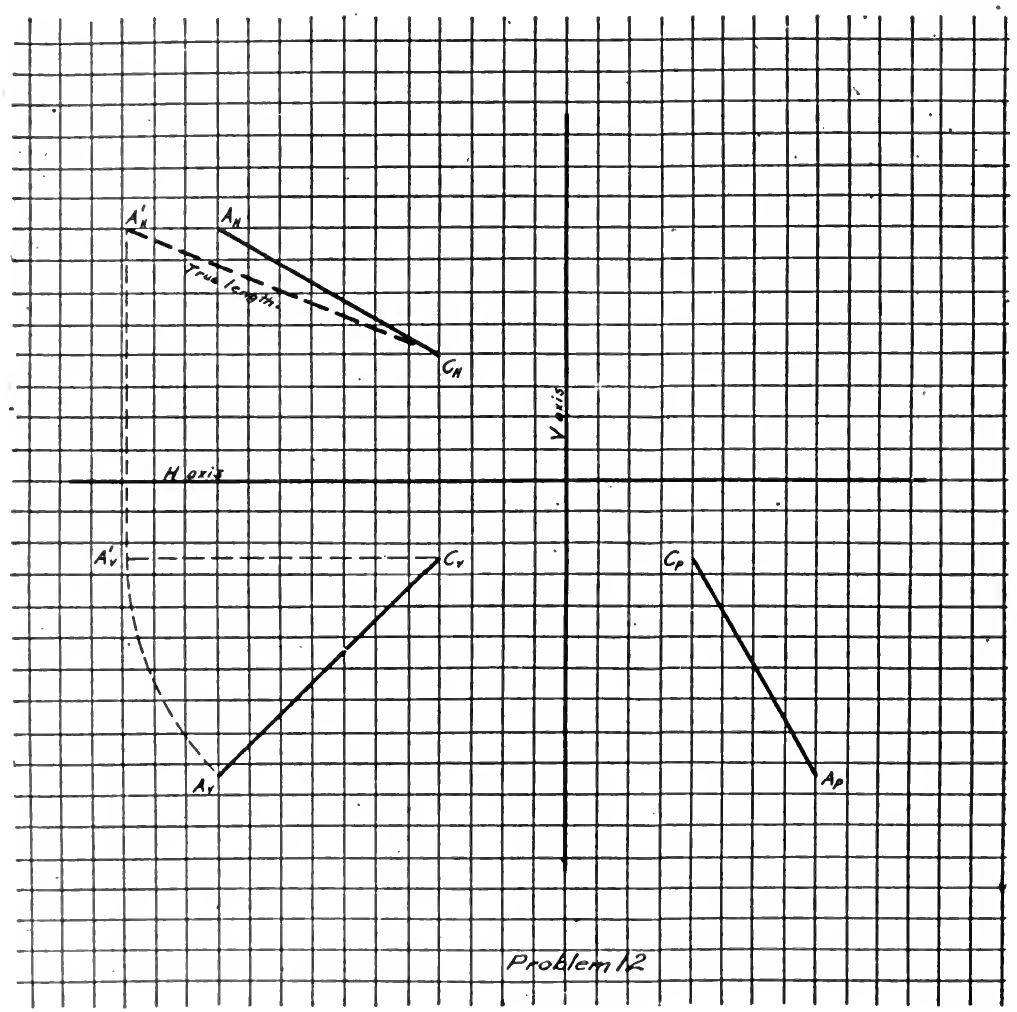

Fig. 8-One of the Problems

own desks without in any way disturbing their regular drawings. They can resume the latter the minute they have finished the special problem without waiting for the teacher to reach them. Thus those who solve the problem easily (an go on without waiting for the slow ones.

Excellent training will result from the placing and determining of various views with reference to the size of the paper, and to the $\mathrm{H}$ and the V Planes. It will be well, therefore, to emphasize this by leaving the locations and the sizes optional in as many cases as possible.

Several semesters' trial showed that a much more usable type of instruction was being created than under the old system where the stress 
was put on absolutely accurate, finished work to the exclusion of the fundamentals of theory.

Mapping. The use of Polar Charts for military mapping is described by Major E. F. Robinson on page 265 of his book, "Military Preparedness and the Engineer," as follows:

Machine gun companies and troops are now equipped with a self-contained range finder, consisting of a tube about $3 \mathrm{ft}$. long and $4 \mathrm{in}$. in diam., mounted at its center upon a light tripod. In one side, near the ends, are two windows, containing prisms. These refract the fields of view to the center, where they are seen superimposed through an eyepiece in the center of one side, opposite the objective windows. An ad-

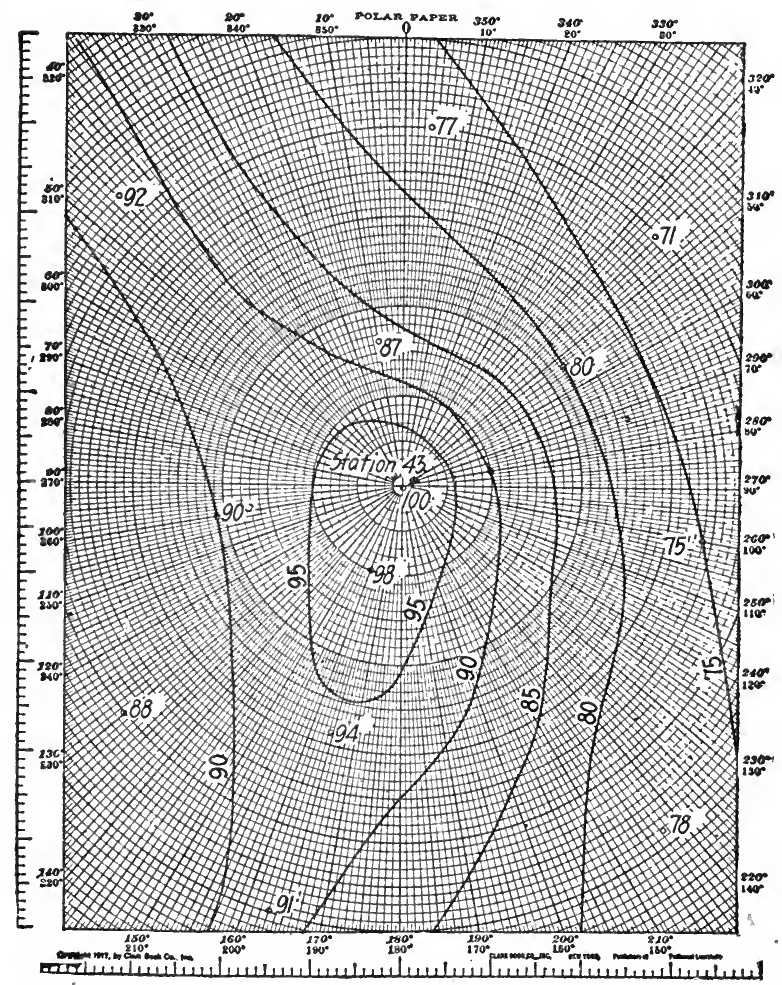

Fig. 10-Speeds Up Interpolation-Increases Accuracy

justing roller controls the angle of the end prisms, and consequently the position of the images in front of the eye-piece. A sliding scale is actuated by the same roller. When the two images, one from each end prism. appear in conjunction, the range may be read upon the scale. An accuracy of $0.5 \%$ may be attained with this three-foot base up to 1,500 yds. or more.

The use of this instrument for topographical work was first suggested to the writer by an infantry officer of the regular service. Upon investigating, it was found to be feasible for very rapid work in a level coun- 


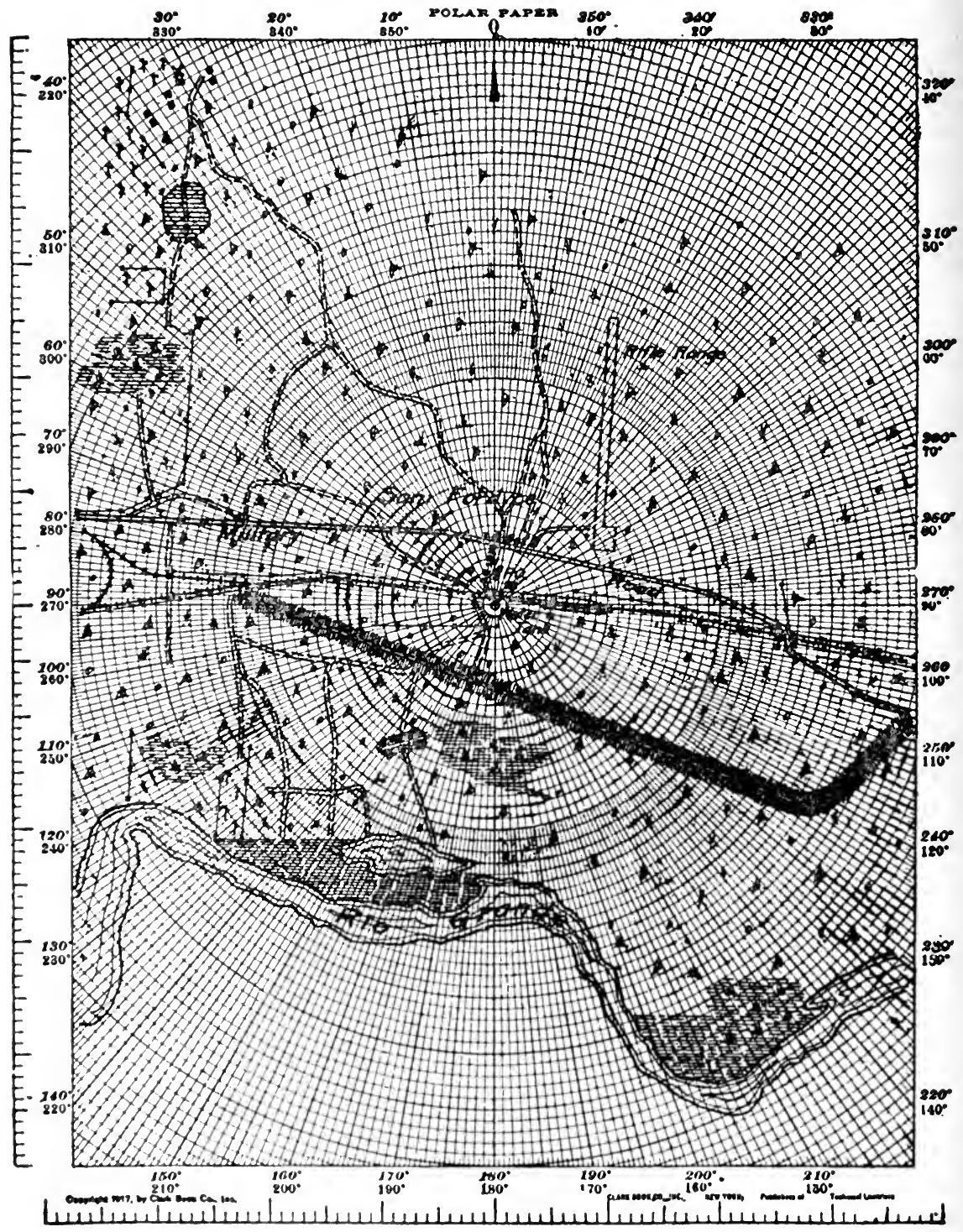

Fig. 9 
try. The observer takes his post upon a building or other elevated point, reads distances by the range finder and directions by a prismatic compass. It is simply the stadia method without the rodman. The use of polar co-ordinate paper, as shown in Fig. 9, is a great aid to rapid plotting.

Plotting Stadia Notes. Chas. R. Thomas is author of the following, published in Engineering News-Record, June 28, 1917 :

By plotting stadia notes on separate sheets such as are shown in Fig. 10 and tracing the finished map from these sheets, the cost of plotting stadia notes has been materially reduced and the speed almost doubled. Plotting topography by this method becomes independent of drafting-room equipments, a lead pencil being the only tool required.

The method of procedure is to sketch the topography in heavy pencil lines and either paste the sheets together for the draftsman or send them to him separately. The sheets are placed under the tracing cloth, and the finished tracing is made directly from them. The paper used for ordinary work is $8 \frac{1}{2} \times 11 \mathrm{in}$. in size. Using a scale. of $100 \mathrm{ft}$. to the inch, a length of $1,000 \mathrm{ft}$. and a width of $600 \mathrm{ft}$. of topography may be plotted on each sheet. Topography may be plotted by surveyors on rainy days, either in the fiela or in the office, several men working independently on separate parts of the notes. It is remarkable how much the use of a graduated paper speeds up the interpolation of contours and increases the accuracy of scaling.

Reconnaissance Mapping. Fig. 11 and the following short quotation from an article in Engineering and Contracting, April 29, 1914, show how admirably decimal rectilinear charts may be adapted to preliminary survey work.

In mapping the reconnaissance work the drawings were made to conform as nearly as possible to the working plans for the finished road. By doing this they showed the more essential features, such as profile, alignment and drainage of the road, and were more easily handled by the officials to whom they were submitted.

A fair sample of this portion of the work is shown in Figure 11-a portion of the reconnaissance made in Clare county. The small sketch shows the routes followed. In this county there were several different roads which could be taken and used as a portion of the trunk line highway. The heavy line shows the one in preference. These maps were drawn to such a scale (1,000 ft. to an inch) that ten miles of road could be conveniently placed on a sheet $20 \times 36$ ins. in size. The width of the road was exaggerated in order that such things as cuts, fills, ditches, etc., occurring within the limits of the road could be shown clearly. All information gathered was placed on, or adjacent to, the plan of the road except the profile, which was shown below.

Zones. Polar charts are especially useful for mapping of zones, such as fare zones with respect to a central point, residence zones, population density zones, etc. An example is shown in Fig. 12, taken from Electric Railway Journal, which gives the rush hour passenger traffic outbound from the one-mile zone, the width of the black lines showing relative number of passengers carried.

Chart of Angles. Fig. 13 by H. Hodgkinson, printed in American Machinist, January 16, 1919, shows a use for polar paper which would 

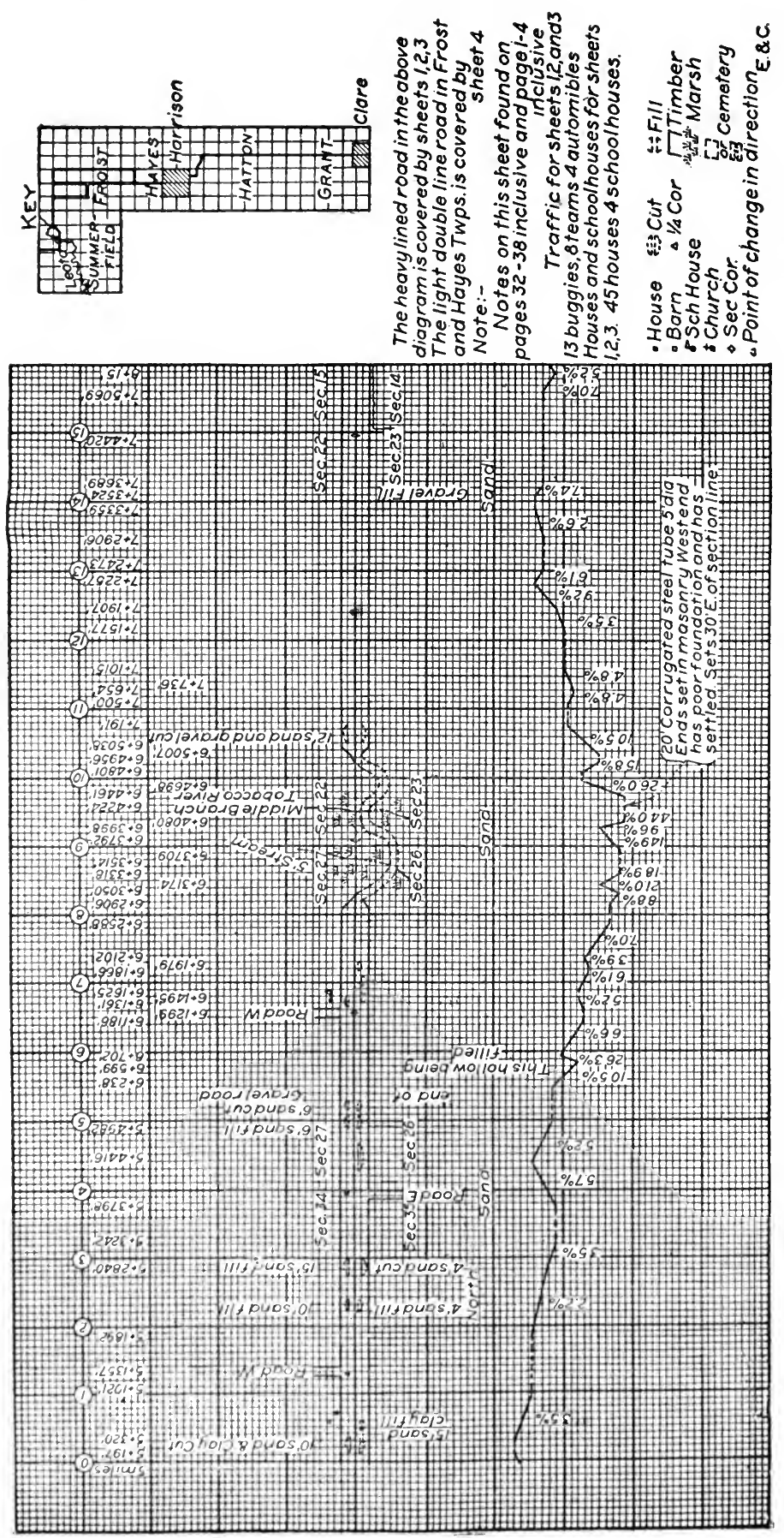


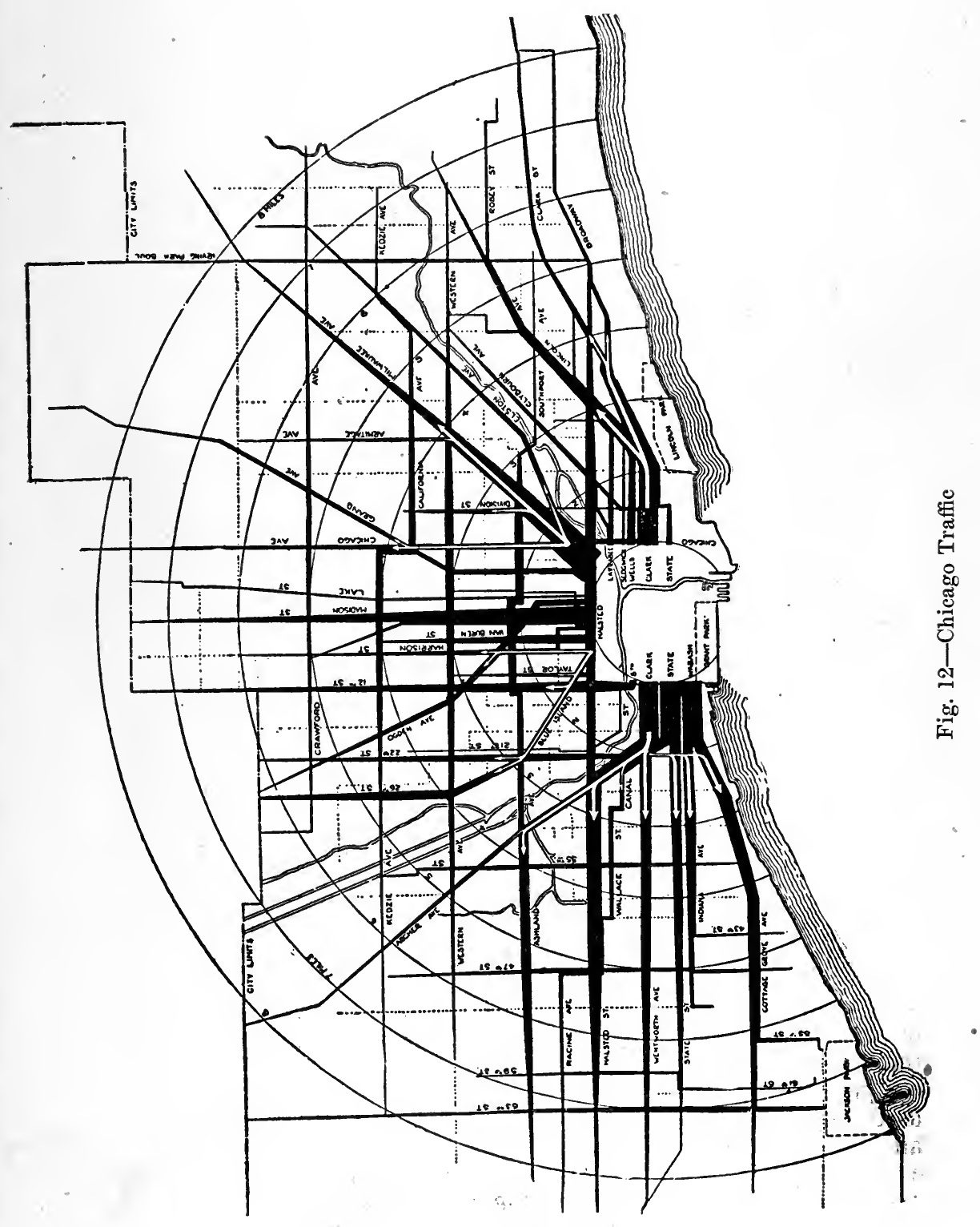


obviate the necessity of repeated reference to tables by those who had reason to use these data frequently.

Notations. The following is from System, the magazine of Business, April, 1918. See Fig. 14.

"Salary increases," says one manager, "are in my estimation about as good an indication as I can have of an employee's ability. I take up the subject of salary increases every six months, and give raises wherever they seem to be justified. And I keep a simple, graphic record that lets me know if any employee is not going ahead as he should.'

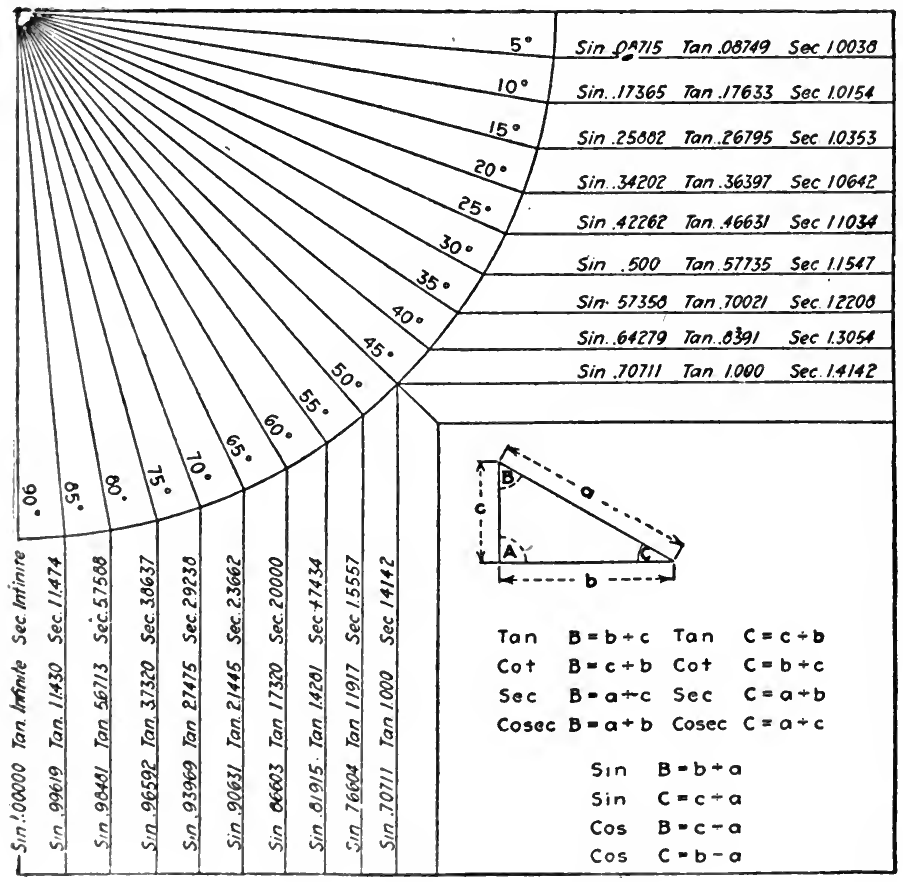

Fig. 13-A Chart of Angles

Demonstration. One of the functions of any ehart might be said to be demonstration - to show how a collection of data, a formula, a series of tests, etc., appear graphically-but frequently the only person to whom the demonstration is given is the one who makes the chart, or perhaps comparatively few persons. Charts may be used - in fact, should be used much inore frequently than they are- to demonstrate facts broadly and generally, as, for example, by an instructor as an adjunct to his classroom lectures. A good example of the use of charts for demonstration is where a city is endeavoring to raise a given sum of money for some purpose- $\$ 250,000$ for new grounds, and the erection and maintenance of buildings, equipment, ete. for the Y. M. C. A.- and in some prominent place in the eity a large elock is erected having its dial divided up into 25 parts, each representing $\$ 10,000$. Then as the money 
is pledged the hands of the clock are moved forward, one showing the total to date and the other the largest amount collected in one day, or some other such arrangement. The thermometer type of chart is also frequently seen upon similar occasions, the "mercury" going up day by day as new funds are obtained.

Frequently in government exhibits large wall charts, usually the bar or polar segment type, are posted to demonstrate certain comparisons, relative values, etc. The engineer makes frequent use of this method in his reports to his clients as well as many accountants and statisticians, but

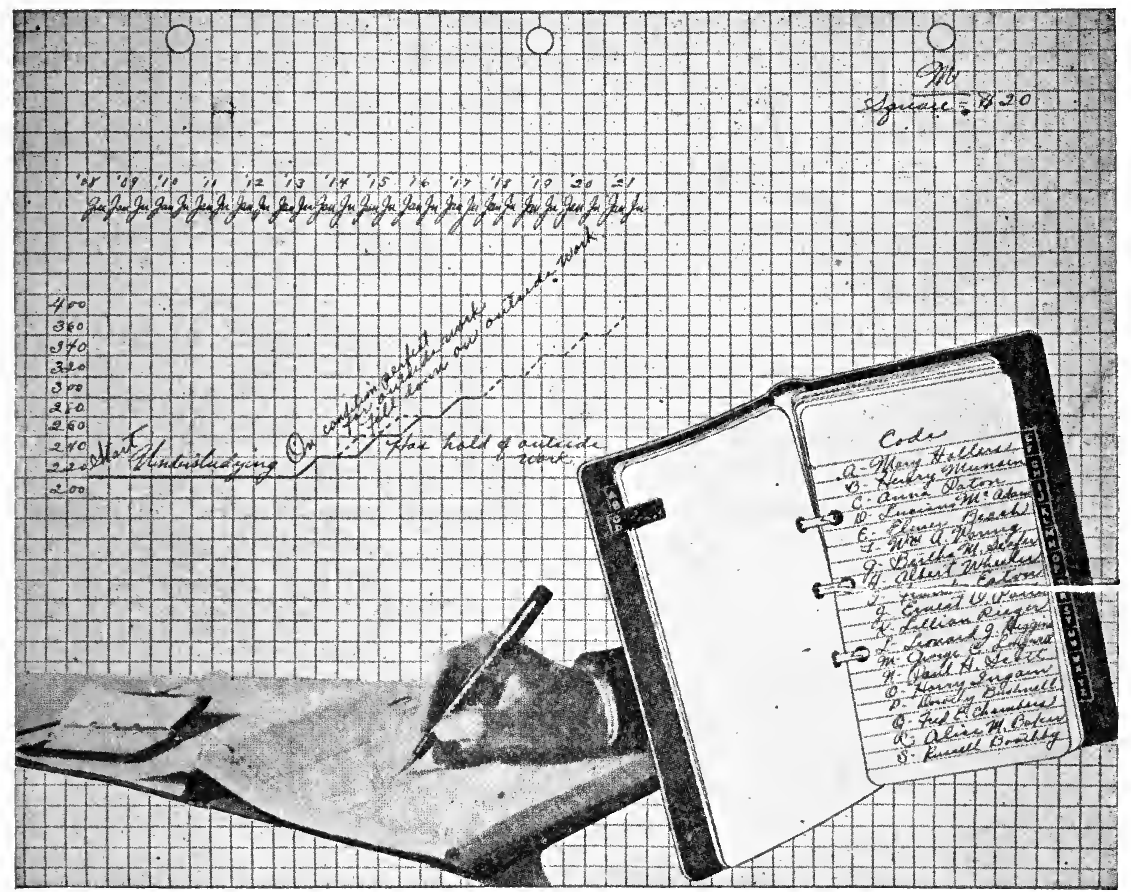

Fig. 14. Sample Page from "Salary Book." Full Line: Actual Course Man's Salary Has Taken. Dotted Line: Course Manager Would Normally Have Expected It to Take

the vast majority, both individuals and corporations, who have some message, either for a comparatively few, such as stockholders, directors, prospective clients, etc., or for the world at large, have not yet come to appreciate this admirable method for picturing the facts they desire to present It is well recognized by advertising people that an illustration or diagram will command attention when a sheet full of printed matter will not, and the endeavor of a good advertising man is to find a picture that will attract and at the same time illustrate the matter of his "ad." A chart picturing the facts described at length in a report or prospectus does just that.

A good example of the use of charts for this purpose is given in the following from Engineering and Contracting, January 10, 1917 : 


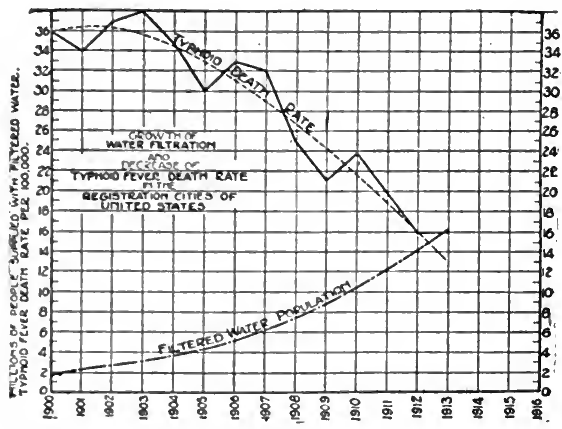

Fig. 15-Relation of Water Filtration to Typhoid

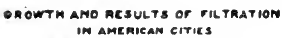

in amenican cities

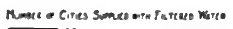

In 1904

In 1914

$\ln 1904$
i. 194

In 1904

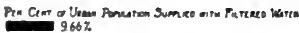

in 194

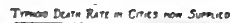
2.19.4

Fig. 17-Growth and Results of Filtration
Average Tramoio fever Deatm Rate Five Vears berore aro five Vears atree filtration

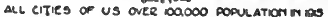

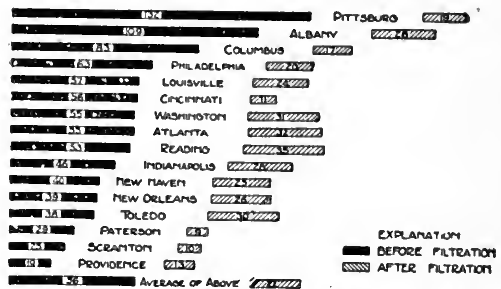

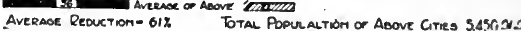
Fig. 16-Typhoid Death Rate in American Cities

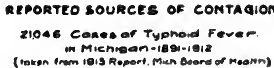

WATER AND ice

OUTSIOE JURISDICTIONS $4367: 207 \%$

TRACEO TO PORMER CASE IN SAME LOCALITY DS91:142\%

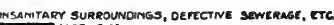

MUL ANO OTHER POCOS

íles $18904 \%$

WFECTEO MOUSES, CLOTHING, ETC

Fic. 18-Sources of Typhoid Fever Cases in Michigan

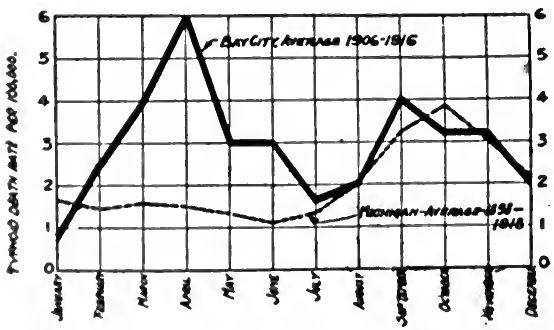

Fig. 19-Typhoid in Bay City and Michigan 
An expenditure of $\$ 837,000$ for water works improvements for the city of Bay City, Mich., is recommended in a report submitted recently to the city officials by Burns \& McDonnell, consulting engineers; Kansas City, Mo. The project, in brief, provides for the consolidation of the two old water works plants, both of which are using water without purification, the installation of new pumps, the laying of a new discharge line to the city, improvements to the distributing system and the erection of a filtration plant. The two pumping plants, under the proposed plan, would be consolidated at the present West Side station. A new intake line slightly larger than the old steel pipe line would be constructed to take water from Saginaw Bay.

The report of the engineers was prepared primarily with the object in view of making it readable and understandable by the average layman and voter. Much of the engineering data in the report are given in the form of graphical diagrams, charts, tables, etc.

Stereopticon slides have been made of the charts, and in the campaign for the bond issue for the improvements it is proposed to use them to illustrate to the citizens the need of water purification and other improvements. It is planned to hold public community center meetings all over town in order that the voters may be well informed on the question, as it is believed that only a lack of knowledge of the conditions would cause an adverse vote against the project.

Several of the charts are reproduced herewith, Figs. 15-19. Fig. 18, showing the sources of contagion in over 21,000 cases of typhoid occurring in the state of Michigan during the period from 1891 to 1912, is of particular interest in showing the larger percentage of sources of contagion from water supply and the rather small percentage due to milk, flies, etc. This was prepared from the records of the State Board of Health.

Bay City has had frequent epidemics of typhoid fever, one occurring recently with approximately 160 cases, with 10 deaths. The prevalence of typhoid is not surprising when it is considered that out of 40 sewer outlets 38 discharge into the river above one of the water works intakes, one outlet being only $100 \mathrm{ft}$. from the intake.

Fig. 20 was gotten up before the present daylight-saving law was passed to demonstrate what its effect would be upon the working-day.

Reduction in Cost of Electric Lighting Graphically Shown. The following is from Electrical Review, June 16, 1917, and is an excellent example of the use of a chart for demonstration:

One of the strong talking points of the electric lighting salesman, when he is required to show economy, is the steady reduction in both the cost of electrical energy and the cost of electric lamps, which together have resulted in a very marked and quite steadily rising increase in the amount of light obtainable for a dollar or a cent, whichever unit is chosen. Such curves have been used in the publicity work of various central stations as well as lamp and fixture-equipment companies, but probably have never been shown as a complete and self-contained set of curves as that illustrated herewith. Fig. 21. It has been prepared by the National Lamp Works of General Electric Company, Cleveland, O.

This chart is described as a photostat or composition curve, which shows at a glance valuable data that otherwise would require numerous 
and rather intricate tables. Curve $A$ shows the cost of energy plotted in cents per kilowatt-hour according to scale shown at the extreme right end" of the sheet. In curve $B$ the same data are given for the special con-

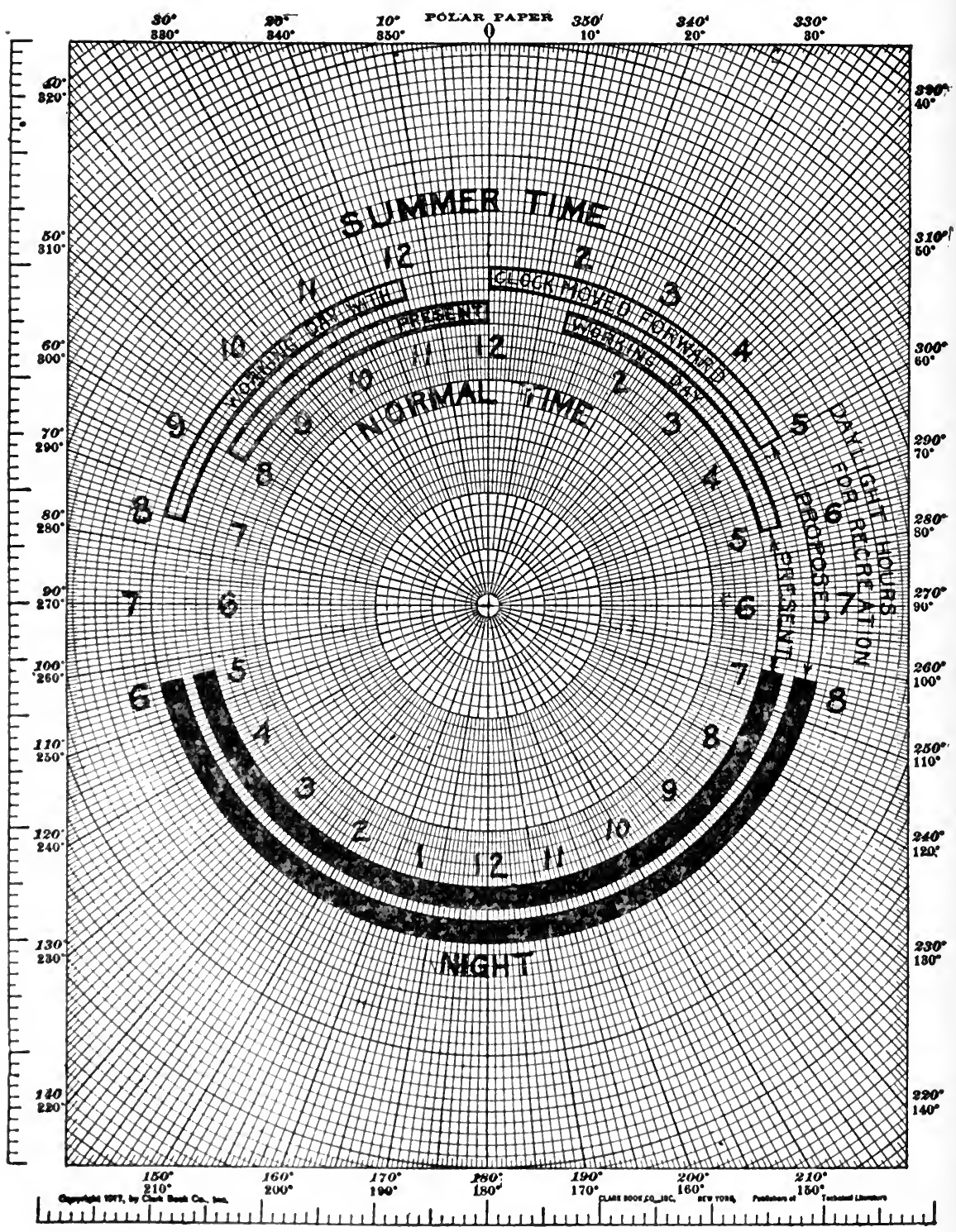

Fig. 20

ditions obtaining in the city of Chicago. Curve $C$ shows the changes in list prices of lamps, which have been considered as standards. Between 1907 and 1908 there is a hrok in the curve, due to a change from the use 
of the 50-watt Gem lamps, common to that time, to the 40-watt Mazda lamps, which replace the older units. The portion of the curve covering the use of the Mazda lamps has therefore been specially labeled as Curve $C^{1}$. It really is an extension of the broken curve $C$.

The curve obtained based on these two curves is the heavy curve shown as $D$. This gives the spherical candle-power-hours obtained for one cent, in accordance with the scale at the left edge of the sheet. The spherical candle-hours available are based on the cost of operation of the particular lamp that might be considered standard during the corresponding year.

Comparison of Fare Schedules. From Electric Railway Journal, August 11, 1917, is taken the following with respect to hearings upon an increase in fares for the Boston \& Worcester Street Railway. This is another demonstration chart.

The company presented a chart, Fig. 22, showing in graphic form the tariffs existing between all fare points on the basis of the present and the

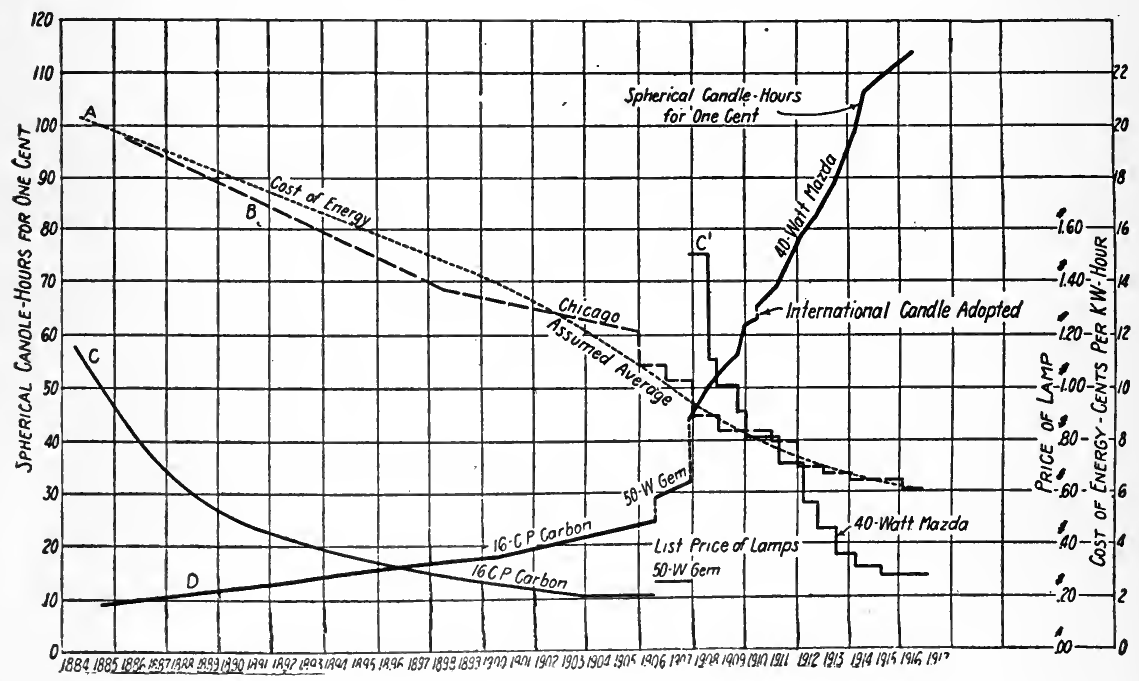

Fig. 21

proposed fares. Under the proposed schedule there will be twenty-nine fare points on the main line in the 31.15-mile trip from Chestnut Hill to the terminus in Shrewsbury. These will be twenty-nine 6-cent minimum rides. At present there are twenty-three 6-cent and six 12-cent minimum fares on the main line between the points mentioned. The new schedule shows many reductions in fare as well as increases, for the company plans to equalize the rates and the distances to which they apply.

Elecric Car vs. Jitney Operation. What follows is from an article in Electric Railway Journal, December 11, 1915, and shows the use of a chart for demonstration purposes by a railway company before the city council:

The data contained in Fig. 23 were submitted before the council by the railway company, the record of the number of cars in operation being 
submitted to show that the street railway company had not been able to decrease its service notwithstanding the jitney's spasmodic inroads into its gross receipts.

Growth of a Company. The following, from Electrical World, June 9, 1919, illustrates a chart used for demonstration purposes by a company to its stockholders.

A ten-year review dealing with the present status and prospects of the Consolidated Gas, Electric Light \& Power Company of Baltimore has been issued to shareholders, with a letter from President Herbert A. Wagner. See Fig. 24.

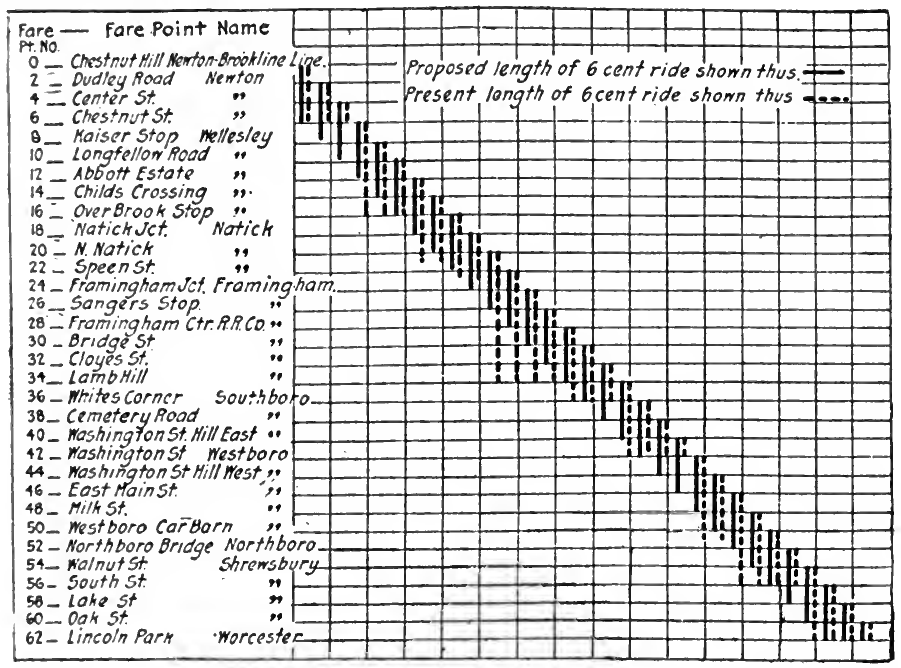

Fig. 22-Showing Inequalities of Present 6-Cent Fare Compared with Proposed 6-Cent Fare

Cost of Living. The following, from Gas Age, July 2, 1917, shows another use for a demonstration ehart.

The increasing price for living necessities has become a very real issue and when the (ity of Duluth Water and Light Department issued its annual report it included a chart, Fig. 25, which illustrated to the people of that city how the cost of water and gas had gone down while other necessities had been skyrocketing.

Conversion Chart. For one who has constant use for the metric and U. S. systems of linear measurement a conversion ehart may be made from a strip of millimeter paper and a strip of 20 divisions per inch or 16 divisions per ineh for decimal or non-decimal conversion. See Fig. 26.

Integration. ('ross-section paper presents a means for roughly determining the areas of irregular figures. Either one of two methods may be used with equally alecurate results. 1. Erect ordinates equally spaced eutting the bounding lines of the figure. Between them draw horizontal lines cutting the bounding line in such a way that the area above the bounding line and the horizontal is equal to the area below the bounding line and the horizontal. 'Then, the area of the irregular figure is the sum 
of the areas of the rectangles formed by the ordinates and horizontals. This would be the-sum of the heights of the rectangles multiplied by the distance apart that they are. 2. Draw the irregular figure on crosssection paper. Count the number of cross-section paper squares within the bounding line of the irregular figure. Estimate the fractional parts

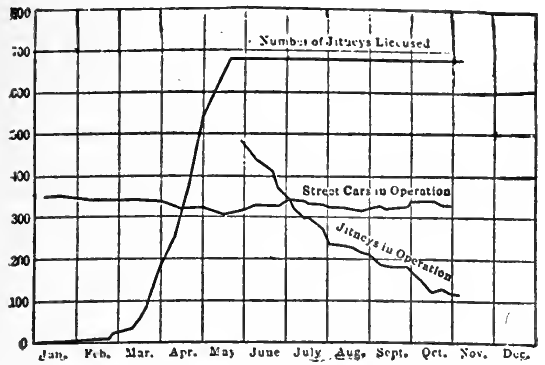

Fig. 23-Graphic Record of Jitney Movement

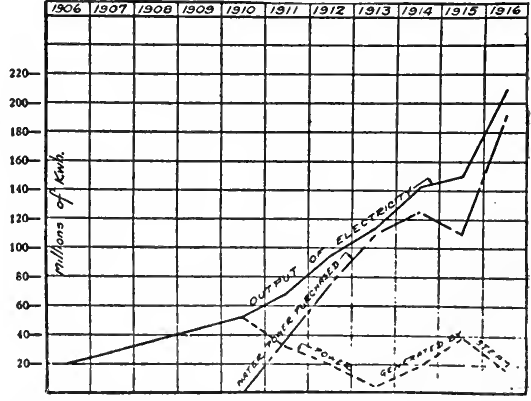

Fig. 24-Showing Growth of Baltimore Company

of squares cut by the bounding line, add these together and add their sum to the total above. The result is the area in units of the dimensions of the squares. Of course, the smaller the squares the less chance there is for error in estimating the fractions. Twenty divisions per inch paper is especially well adapted for this method of figuring areas.

Cross-Section Lines on Blackboards and their Illumination. Paul F. Gaehr is authority for the following in a letter to Science, March 14, 1919 :

Those who wish cross-section rulings on blackboards temporarily, thus leaving the board free for other work after the curve-plotting is finished, can do so by a simple device. On a sheet of white paper make a ruling of lines, $2 \mathrm{~cm}$. apart, the whole grid being $16 \times 24 \mathrm{~cm}$., and the lines not quite one mm. thick. Take a photograph of this, making the camera image the size of a lantern-slide. Mount the negative in a lantern, pro-

\section{Inches}

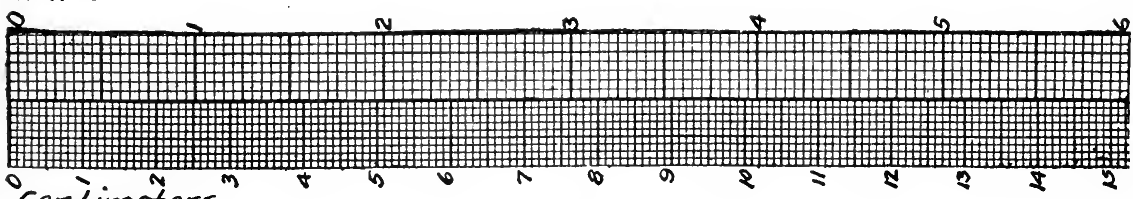

"Centimêters"

Fig. 26

jecting the image on the blackboard. A lantern equipped with a 400 watt Mazda lamp will make the lines sufficiently visible for plotting even in a well-lighted room. The lines are erased by turning off the lamp.

The lines on charts already ruled could be inked in heavier to serve this purpose admirably.

Following is a list of articles containing further illustrations of the use 


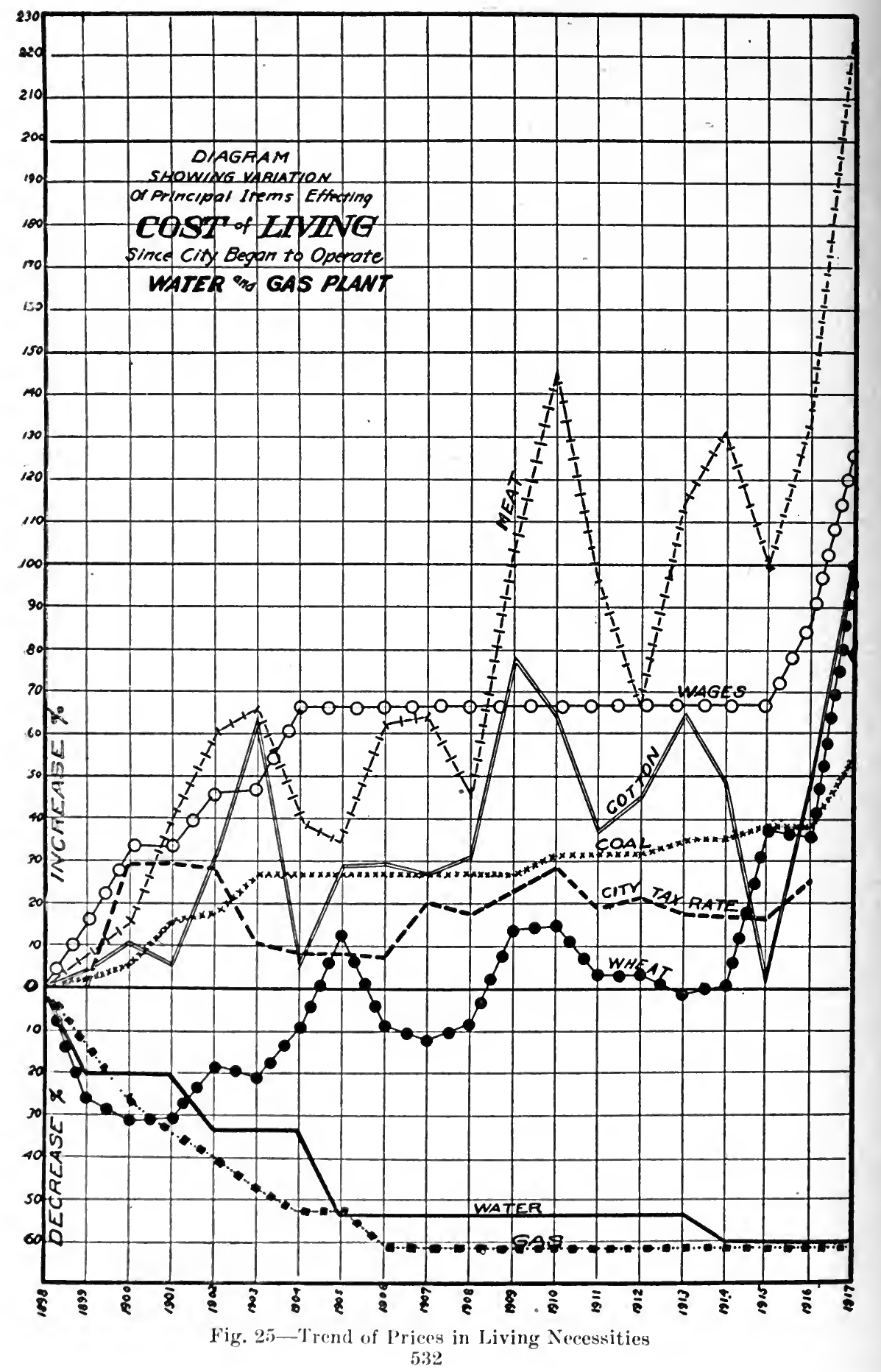


of graphic charts of the class discussed in this chapter and pertaining to kindred subjects.

Upon request, the Codex Book Company, Inc., 19 William Street, New York, will be glad to procure, where possible, copies of magazines containing the articles needed, only charging the "old magazine" price, plus postagie.

"Progress of Electrification of the Chicago, Milwaukee and St. Paul Railway," Railway Electrical Engineer, Nov., 1915.

“'St. Paul Equipment," Electrical Railway Journal, July 21, 1917.

“'Single Fare Zones,' Electrical Railway Journal, Aug. 5, 1916.

"Standard Railway Property Maps and Profiles Prescribed by the Interstate Commerce Commission," Engineering and Contracting, March 4, 1914.

“Determining First Cost of Edison Tube System,' Julian Loebenstein, Electrical World, Oct. 7, 1916.

"A Method of Studying Edison Distribution Systems," C. E. Bennett, Electrical World, Aug. 19, 1916.

"Proposed Dividend-Type Domestic Service Rate," W. W. Briggs, Electrical World, Oct. 28, 1916.

"Central-Station Load Curves Changing," H. S. Knowlton, Power, July 10, 1917.

"Stable Costs," Engineering News, July 9, 1914.

"The Sale of Current to Municipally Owned Distributing Systems by Central Stations,"' W. Rawson Collier, Electric Review \& Western Electrician, Nov. 13, 1915.

"Discrimination in Rates for Electricity," Henry D. Jackson, Power, March 31, 1914.

"Distribution of Employees in Departments of a Central Station," Electrical World, Oct. 28, 1916.

"Appraisement of Small Electric Properties," Edwin D. Dreyfus, Electrical Review and Western Electrician, March 6, 1915.

"Measuring Root-Mean-Square-Current," Electrical Age, April, 1917. "Chart for Converting Avoirdupois Pounds Into Short and Long Tons," Coal Age, Aug. 4, 1917.

"War-Time Cost of Power-House Coal," Electric Railway Journal, Aug. 11, 1917.

"Cost of Electric Light and the High Cost of Living," Electrical Re. view, Sept. 7, 1918.

"Graphic Aid in Figuring Power Bills," R. W. Parker, Electric World, June 17, 1916.

"Co-ordinating Power with Heating," letter from George H. Gibson, Power, Oct. 2, 1917.

"Methods for Figuring Interest," Engineering Record, May 16, 1914.

"Boiler Horsepower Saved by a Feed-Water Heater," W. F. Schaphorst, Engineering and Mining Journal, March 24, 1917.

"The Loss of Coal through Grates," Carleton W. Hubbard, Power, Feb. 27, 1917.

"Making the Valuation Records Permanent," James G. Wishart, Engineering News-Record, Oct. 11, 1917.

"City Manager's Chart Convention Program," Engineering Record, Dec. 9, 1916. 
"The Miami Valley Flood-Protection Work," Engineering News, Jan. $25,1914$.

"Devices for Measuring Water Level in Well and Discharge of Pump," April 11, 1917.

"Use of Water Meters," Engineering News-Record, May 24, 1917.

"Data on Rope Drive for Rolling Mills, Blast Furnace and Steel Plant," Aug., 1917.

"A Calibration Curve for Horizontal Cylindrical Tanks," Engineering News, April 29, 1915.

"Chart for Laying Out Curves by Tangent Offset," P. R. Keller, Engineering and Contracting, April 3, 1918.

"Chart for Finding Air Consumption of Drills," Robert S. Lewis, Engineering and Mining Journal, Feb. 24, 1917. 


\section{INDEX}

A.

Abscissas, definition of ........ 9

Accident prevention .......155, 159

Accidents, fatigue and ......... 159

Accuracy of charts .......... 6

Accuracy of data essential ....... 54

Advertising, charts for ......... 525

proper time for ........... 101

Alignment charts

$\ldots \ldots 5,37,39,48,374,432,435$

Analysis, graphic ..........189, 194

Analysis of food ........... 30

Angles, chart of ............ 524

Area charts, avoidance of ...... 56

Areas, determining by cross-section paper .............. 531

Arithmetical computation ........ 342

Arithmetical progression ........ 13

Artificial light and egg production. 315

Automobile design and manufacture 326

Automobile operating costs ...... 186

Axes, definition ............ 9

B.

"Bar" charts ..........11, 58, 60

Barges, cost of repairs .......... 185

Beams, bending moments ....... 447

rectangular ............. 406

reinforced concrete .......... 404

steel, deflection of ..........4 408

Bearings, friction of lineshaft..... 321

Belt conveyors .............. 274

Belts, conveyor ............441

Benefits, employees' .......... 153

Blackboard work, chart device for.. 531

Blue prints of charts....56, 57, 77, 511

Boiler design ............. 455

Boiler efficiency ............. 275

Bond and stock markets, trend of . . 326

Bonus and piece work rates. $145,149,153$

Bridge construction, progress in... 241

Bridge floors, concrete ......... 445

Bridges, estimating highway ..... 503

Building, reinforced concrete factory ............. 181

Buildings, cost of mill .......... 484

Business death-rate ........... 327

Buying, chart analysis aid in ..... 98

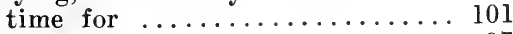
to prevent over ........... 97

C.

Calculations, chemical ........415

Census, traffic ............. 318

Centrifugal pumps ............ 268
PAGE of $\ldots \ldots \ldots \ldots \ldots \ldots \ldots \ldots 8,410$

Charts that mislead ........55, 72

Chemical calculations .........4 415

Chimney sizes ............. 468

Choice of paper .........371, 410

Circle, plotting equation of ..... 342

Coal cost vs. selling price ........ 216

Collections, keeping tabs on ...... 107

Color of ruling .........56, 70

Column design ......377, 449, 451, 454

Commodities, prices of ......326, 532

Comparative statistics, use of semilogarithmic chart best for.17, 20

Computation, arithmetical and geometrical ............. 342

logarithmic paper for ........ 353

Concrete beam, formula for....381, 404

Concrete bridge floors ..........445

Concrete, effects of freezing temperatüres . ............ 313

Concrete floor ${ }^{*}$ cost ........... 181

Concretè mixtures ............ 32

Concrete, reinforced, for factory buildings ........... 181

steel reinforcement $\ldots \ldots \ldots \ldots \ldots 183$

Conductors, electrical ........ 477

Construction, cost of building..... 181

cost of hydroelectric plant ...... 178

dam, powerhouse, etc. ........ 229

pole line ................ 192

progress in bridge .......... 241

Construction of alignment charts. 39,48

Contagion, sources of ........ 527

Conversion chart ..........417, 530

Conveyor belt, plies of, required...4 481

Conveyors, belt ........... 274

Co-ordinates, polar .......... 26

rectangular $\ldots \ldots \ldots \ldots \ldots, \quad 9$

Copper transmission lines ....... 477

Correct relationship best obtained by semi-logarithmic chart.17, 20

Corrections for $\operatorname{sag} \ldots \ldots \ldots \ldots \ldots 509$

Corrections, stadia ............. 511

Cost analysis .........194, 201, 205

Cost, direct labor ............ 93

Cost of living vs. wages ......... 159

Costs, manufacturing ......82, 92, 194

Cumulative curve .......83, 92, 159

Current densities .......... 477

Curve plotting .............. 342

Cutting time, for determining .... 368

D.

Daily curves .............. 84

Daily Record-Semi-logarithmic chart 17

Dam construction ............ 229 
Daylight-saving. demonstration of.. PAGE

Death-rate, business ............ 327

Demand, keepıng in touch with.... 98 prices of steel reflect ......... 327

Demonstration increases production 129

Demonstration, types of charts for $\ldots \ldots \ldots \ldots \ldots \ldots 24,532$

Design and manufacture, automobile 329

Designing $\ldots \ldots \ldots \ldots \ldots \ldots 445,511$

Dividends ............... 81

Dividing a scale logarithmically, method of ..........51, 52

Drawing ................. 511 mechanical ............. 516

Dump wagons, train vs. team drawn 316

E.

Earnings ............... 194 predicting of $\ldots \ldots \ldots \ldots \ldots \ldots 332$

Earth, transportation of .......490

Eccentric loading of columns ..... 449

Economy of electric lighting, chart showing .............. 529

Efficiency, record of ......... 147 selling ............... 107

Egg production and light ..... 315

Electric car vs. jitney ......... 529

Electric lighting, cost of ....... 527

Electric railway investments vs. traffic density ...........205

Electric railway maintenance .... 282

Electric railway transportation ... 284

Electric service, cost of ....... 209

Electrical conductors, economical size of $\ldots \ldots \ldots \ldots \ldots 476$

Electrical machinery, cost of ..... 174

Electrical plants ............ 177

Flectricity, cost of .........212

Elimination of waste ........ 83

Employees' benefits ........... 153

Employees, prevention of accident to $\ldots \ldots \ldots \ldots \ldots \ldots \ldots, 159$ and wages $\ldots \ldots \ldots \ldots \ldots \ldots$ 15\%)

Employment department, chart records for ............

Employment methorls ......... 121

Engine design .............. 464

Engine frame loads ..........464

Engine requirements, scheduling... 251

Engine test, gasoline ......... 320

Engineering socicties, trends of membership ..............331

Equation, plotting an ......... 342

Fssential facts to investigate .... 79

Estimates, printing .......... 489

Estimating ............. 445

Excavation, earth ........... 494 rock ................. 494

Expenditure, labor material and plant .................241

Experiments and tests, results of . . 309

Exponent, definition of ....... 12
F.

PAGE:

Factor, definition of $\ldots \ldots \ldots \ldots, 12$

Fare schedules, comparison of .... 530 .

Fatigue and accidents ......... 159

Fatigue study .............. 130 .

Feed water heater, economics of... 173.

Filing of charts ............ 511

Floor beams, bending moments ... 447

estimated cost ........... 449

Floor costs ............... 181

Floors, concrete bridge ......... 445 .

Flow of water in pipes ....... 47

Fluctuations in output ......... 332 .

Food analysis ............... 426

Formulas, nomographic solutions of 374

Formulas to fit curves, logarithmic paper for finding ........ 363

Freight statistics ............. 23 .

Friction of lineshaft bearings .... 321

Fuel cost of heat and power ...... 171

Functions of charts, the principal.. 2:

Furnace efficiency ............ 275 .

Future traffic, predicting ........

G.

Gas, pressure curve .......... 342

Gas company statistics ........ 57

Gasoline engine test ......... 320.

Geometrical computation ........ 342 .

Geometrical progression ......... 13.

General characteristics of charts... 6 .

General principles .........54, 67

Generating plants, cost of ....... 177

Going value ............... 336 .

Grading, cost of ........... 490

estimate of time for .........494

Growth of company ........... 530

H.

Health records ............ 527

Heat and power, cost of ....... 171

Heater, feed water ........... 173:

Hens, artificial light for ....... 315

Highway bridges, estimating . . . . . 503:

Hooke's law ................. 309

Horsepower, locomotive ... . . . . . 362:

Hotel operating .............. 304

Hydraulie formulas, logarithmic

charts for ............ 367

Hydroelectric plant cost ........ 178

Hyperbola, plotting equation of... 342:

I.

Ille time, reducing $\ldots \ldots \ldots \ldots 162^{\circ}$

Impact formula ............. 379

Inefliciency, record of ......... 147

Integration, chart method .......530

Interpolation .........349, 373, 521

Inventories ............. 83

Isometric charts ........... 27 
Isometric plotting

\section{$\mathrm{J}$.}

Jitney vs. electric car

$\mathrm{K}$.

Keying of charts .........56, 70 Kinds of charts, the principal.... 4

$\mathrm{L}$.

Labor conditions

Labor, expense, direct ............ indirect $\ldots \ldots \ldots \ldots \ldots \ldots . . .93$

letting the men know what they are doing .............. 129

maintenance $. . \ldots \ldots \ldots \ldots \ldots 11,159$

output $\ldots \ldots \ldots \ldots \ldots \ldots \ldots \ldots .141$

overtime problem ........... 232

prediction of demand ........2 237

schedule and progress chart..... 237

scheduling requirements ....... 232

turnover ......83,108,141, 153, 159

cost of ............. 1108

Law of variation shown by curve. 348

Leaking steam, cost of ......... 171

Lettering charts .........57, 68

Light and power service ........ 193

Light distribution ........... 315

Light, effect on egg production .... 315

Lighti cost of electric ........ 527

Lineshaft bearings, friction of ... 321

Living, cost of ........... 532

Load, eccentric, for columns ..... 449

Loads, engine frame ......... 464

safe, on piles ............. 351

Locomotive horsepower ........ 362

Locomotive requirements .......251

Logarithmic chart ......4, 12, 14, 424

Logarithmic graduation of a scale

............... 51, 52

Logarithmic paper for computing $\ldots \ldots \ldots \ldots \ldots 353,362,423$

Logarithms, explanation of ....12, 13 graphic use of $\ldots \ldots \ldots \ldots \ldots \ldots 51$

\section{M.}

Machinery, electrical, cost of ..... 174

Machines in operation .......120, 162

Mains, trunk ............ 466

Maintenance, electric railway .... 281

track ...............179, 180

Management ............. 78

Managerial problems, graphic analvsis ..............189, 194

Manholes, estimated cost of ..... 502

Manufacturing costs ...82, 92, 194, 201

Mapping, military ............ 519

Mapping reconnaissance ........ 521

Markets, trend of bond and stock markets .............. 320

Material costs .............. 82
Materials, expenditures for $\ldots . \ldots .241$ strength of ................ 309

Mechanical drawing, charts in .... 516

Membership trends ........... 330

Method of making chart ....... 10

Mill buildings, cost of ........484

Mixtures, chart for .........435

gas and chemical .......... 32

Motor cars, cost of operation ...... 187

Motor truck performance ......163, 164

Motor truck transportation ...... 218

N.

Negative direction, definition .... 9

Nomographic charts ..........5, 37

Nomographic solutions, of formulas

.............. 374, 432

Notations .................. 524

Number of trains vs. passing points ..............4 474

\section{o.}

Operating characteristics of machines .....262, 268, 274, 275

Operating characteristics of plants $\ldots \ldots \ldots \ldots 276,277,282,305$

Optical illusions ........... 55

Ordinates, definition of ........ 9

Organization .............. 95

Origin, definition of $\ldots \ldots \ldots \ldots .9$

Output, labor ............. 141

fluctuations in ............. 332

weekly and monthly ......... 159

Overbuying, to prevent ....... 97

Overtime problem ............ 232

\section{P.}

Paint, life of ............. 282

Paper for charts ........... 511

Parabola; plotting equation of ... 342

Payroll curves ............. 239

Piece work rates $\ldots \ldots \ldots \ldots 145,149,153$

Piles, cost of driving ......... 185

safe load on ..............351

Pin moments, chart for ......... 375

Pipes, flow of steam in ........466

flow of water ............ 47

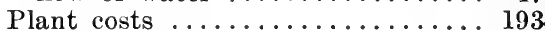

hydroelectric ........... 178

Plant, refrigerating ......... 277

Plant valuation, unit prices for.... 337

Plants, electrical, selection of engines for $\ldots \ldots \ldots \ldots \ldots, 177$

operating characteristics ...... 277

Plate, strength of .......... 455

Plotting, curve ..........58, 342

Plotting a point, definition ....... 9

Plotting an equation ......... 342

Plotting formulas .......... 363

Plowing, cost of ...........223

Polar eharts ........ 25, 519, 520

Polar ehart for computing ...... 414 
Polar co-ordinates $\ldots \ldots \ldots \ldots \ldots .26$

Poles, cost of ..............204

Population chart ............ 75

Population, probable .......... 334

Positive direction, definition .... 9

Power, cost of ............ 171

definition of ........... 12

purchased and isolated plant .... 193

Power consumers, revenue chart... 191

Power house construction ........ 229

Prediction, statistical .......326, 350

Preparing of charts ....56, $70,71,77$

Pressures, allowable for engines .. 464 boiler ................... 455

distribution of soil ......... 310

Prices of commodities ......... 326

Prices of steel reflect demand .... 327

Printing estimates ............ 489

Problems solved by plotting equation ............... 346

Production, effect of fatigue ..... 130

weekly and monthly ......... 159

increased by charts ......... 129

Production control, graphic ..... 258

Profits ................. 81

Profits, analyzing costs to increase 194

Progress chart .......235, 246, 247

Progress chart of bonus ........ 153

Prospects, demonstration of ..... 530

Pulley sizes .............. 480

Pulp problems ............ 437

Pumps, centrifugal .......... 268

cost of steam ........... 177

R.

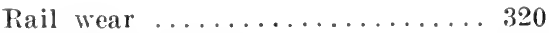

Railway accidents .......... 22

Railway fare schedules ........ 529

Railway freight statistics ....... 23

Railway investment and traflic density ............ 205

Railway maintenance, cost of . .179, 180

Railway maintenance, electric .... 282

Railway meeting points ....... 474

Railway records ............. 529

Railway scheduling . . . . . . . . 24!

Railway string-lining curves ..... 476

Railway track elevation ........ 231

Railway transportation, electric .. 284

Railway zones ............. 523

Rate sotting and dolay allowances. 130

Rates, bonus and piece work

Rations trilinear wart for . . . . 149, 1533

Rectangular co-ordinates .......4, 8

Piectilinear chart, nse of ......9, 511

Reflector, distribution curses for... 3it

liefrigerating plant .......... 277

lelative comparisons, use of semilogarithmic whart best for.17, 20

Reinforcement rosts . . . . . . 18:3

Repairs, cost of .......... 185

to automolile, cost of ....... 186
Research and managerial economics PAGE

Resistances, in parallel ........ 353

Rest periods, effect of ......... 130

Retaining walls, cost of ........ 488

Rivets, stress on ..........455

Rivets for eccentric load ....... 399

Room centers, calculation of ..... 414

Root, definition of $\ldots \ldots \ldots \ldots \ldots 13$

Rope, size required ......... 482

S.

Safety work .........83, 155, 159

Sag, corrections for . . . . . . . . 509

Salary increases ........... 524

Sales ............... 82

Sales, as affected by crop yield..... 100

as affected by fashion ........ 100

as affected by prosperity ...... 100

as affected by shopping habits... 101

as affected by weather ......... 99

Sales and collections ............ 107

Sales chart, method of making .... 10

Sales, cost, standard rs. actual... 103

Sales curves ............... 18

Sales manager, graphs for ...... 106

Salesmen, cliart to excite interest of 106 efficiency of ............. 107 records of performance ....... 105

Scale, definition ............ 68

Scale range of logarithmic chart... 15

Schedule and progress chart ...... 237

Scheduling, railway ........... 249

Scheduling and construction work $\ldots \ldots \ldots \ldots \ldots \ldots 29,237,241$

Scheduling equipment requirements 251

Seheduling labor requirements .... 232

Scheduling merchant ships ...... 251

Scheduling track work ......... 231

Scrapers for earth transportation .. 490

Selling, what it should cost ..... 103

Selling price vs. cost of coal ..... 215

Semi-logarithmic charts ......5, 17

Sewer progress ehart .......... 245

Ships, scheduling merchant .....251

Societies, trends of membership .. 331

Soil pressure, distribution of . . . . 310

Spacing of railway stops ........ 249

Squirrel-eage motors, performance of $\ldots \ldots \ldots \ldots \ldots \ldots \ldots 262$

Stalbility of working force ....... 111

Stacks, dimensions of ........ 468

Stadia corrections ........... 511

Stadia notes, plotting of ....... 521

Standard vs. actual performance.90, 94

Standards for graphic presentation $.7,56,71$

Statistics, gas company ......... 58

Steam, cost of coal for ........ 167

cost of leaking ............. 171

expansion of .............. 348

Steam pipes .............. 466

Steel prices reflect demand ..... 327

Steel reinforcement, cost of ..... 183 
Stenographer's ability ......... ${ }_{96}^{\text {PAGE }}$

Stock and bond markets, trend of. . 326

Stoker installations, comparisons of 275

Straight line, plotting equation of. 342

Strength of materials ......... 309

Stress, boiler .............. 455

column .............377, 451

String-lining curves ......... 476

Supervision, hotel ........... 304

Surveys, chart for ........414, 522

T.

Tapes, correction for sag ...... 509

Tendencies ..................... 326

Tests and experiments, results of. . 309

Timbers, measurement of .......4 411

Time, determining cutting ....... 368

Time of performance, method of determining .............494

Time study ................ 130,162

Time study on automatic machines. 139

Traffic, predicting future ....... 335

Traffic census ............... 318

Traffic density and revenue ...... 205

Traffic zones .............. 523

Train vs. team drawn dump wagons 316

Trains, meeting points of ....... 474

Transformers for induction motors. 262

Transmission, iron-wire ....... 205

Transmission line construction .... 229

Transmission line, copper ....... 477

Transmission line progress chart.. 247

Transportation, electric railway .. 284 estimating cost of ......... 490 motor truck .............. 218

Transportation of material .....4490

Trend of bond and stock markets . 326

Trend of prices ............. 532

Trends $\ldots \ldots \ldots \ldots \ldots \ldots \ldots \ldots . \ldots \ldots 26$
Trilinear chart $\ldots \ldots \ldots \ldots \ldots \ldots . \quad 30$

Trilinear chart for coal analysis .. 34

Trilinear charts for mixtures .....

Trilinear chart for rationing .... 30

Truck efficiency ............. 218

Truck performance .......... 163

Trucking, economy of centralized .. 164

Turnover labor $\ldots \ldots 108,111,121,141,153,159$ cost of .............. 118

Typewriting, record of ....... 95

Typhoid, sources of $\ldots \ldots \ldots \ldots \ldots 527$

L.

Unit prices of material for valuation ................ 337

V.

Value, going ............ 336 Valuation of plant, unit prices for. 337

W.

Wage payments, bonus and piece work .........145, 149, 153

Wages vs. cost of living ....... 159

Wagons, tests of dump ........ 316

Walls, cost of retaining ....... 488

Waste, elimination of ........ 83

Water, flow of $\ldots . \ldots \ldots \ldots \ldots \ldots 47$

Water consumption, predicted ... 336

Wear, proportion of rail ....... 320

Williams-Hazen formula ....... 385

$\mathrm{Z}$.

Zero line should appear ....... 72

Zones $\ldots \ldots \ldots \ldots \ldots \ldots \ldots \ldots .521$ 


\section{Codex Data Papers}

Codex Data Papers are indispensable for enabling you to readily visualize, in the shape of a simple curve, diagram or chart, just what is going on in your organization. Use Codex Data Papers to plot daily, weekly or monthly comparisons of (1) orders received, shipped and in production; (2) labor and material costs with selling prices; (3) gross earnings, operating expenses, deductions and surplus; (4) promise dates with delivery dates.

Use Codex Data Papers to show (1) relative locations, to scale, of actual or proposed equipment installations, or of building sites; (2) layouts for signal-call fire systems, etc.; (3) detail sketches of buildings, machine parts, etc.

Use Codex Data Papers for (1) records of individual performance; (2) diagrams of piece-rate and bonus sustems; (3) record of labor turnover; (4) vacation charts; (5) organization charts; (6) progress diagrams.

Codex Data Papers have many unique features: correspondence size, $81 / 2 \times 11$ in.; multiple thereof, $17 \times 11$ in.; note book size, $4 \frac{11}{4} \times 7 \frac{1 / 4}{\text { in.; }}$ accurately ruled on tough, light, translucent, bond paper; put up in pads of 100 sheets, bound on top and righthand edge; make excellent blue prints; and are very reasonabie in price.

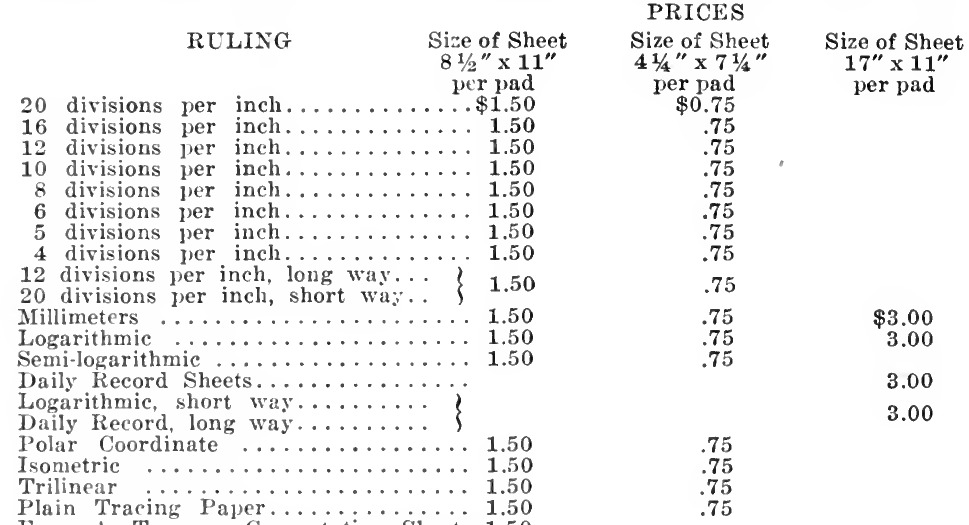

Form 1, Traverse Computation Sheets 1.50

Form 13, Traverse Computation Sheets 1.50

Forms for Computation of Farthwork 1.50

$$
\text { All of the above prices f. o. b. United States }
$$

Upon request we will be glad to panch pads in accordance with your specifications.

Let us supply your loose-leaf ring binders-stiff, or flexible covers; canvas, imitation leather, or best quality cowhde; also indexes with linen or leather tan. Write for prices. S siecial discounts on lots of 6 or over.

A strikingly effective labor-saving device that we handle is the FITTING CALCULATOR for computing the loss of power in electrical conductors. It consists of 3 circular slide rules, by the

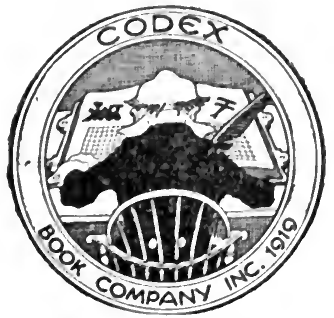
setting of which, with necessary tables of constants, the solutions are obtained. A copy will be mailed to you on approval, to be returned within ten days if it does not appear, on inspection, to be worth many times its price of \$IO.OO.

CODE, $\mathrm{X}$ BOOK CO., Inc.

\section{William St.}

New York City 


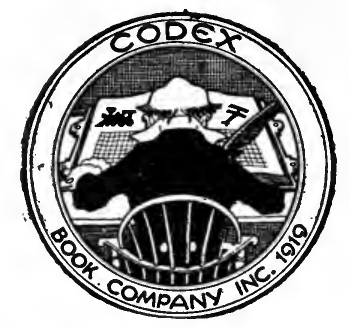

\section{When You Want An Engineering Book How Much Does It Cost You NOW?}

The price of the book - yes, and then some!

Have you ever considered what small units of your time are worth? WE HAVE!

If your salary is $\$ 25.00$ per week, one minute costs over 1 cent. " " " "\$50.00 " " " " " " " " 2 cents.

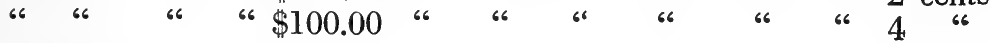

Therefore it costs you:

? to find a technical book store?

? to find a clerk in the store?

? for the clerk to find the book (if they have it)?

? for the store to get the book (if they haven't it)?

? grand total,- anywhere from 15 minutes to 15 days, representing a very respectable sum, whether your time is worth 1 cent or 4 !

In contrast with the above, you can have the book put on your desk by the Codex Book Company, Inc., for 2 minutes of your time plus a l-cent stamp, or a total of ? cents.

Our CODEX INDEX comprises several hundred Engineering Books specially chosen by unbiased experts from the lists of all publishers. Each book is the best on its subject.

To anyone on our mailing list, to any member of the National Engineering Societies, or to any other furnishing satisfactory references, we will send any book listed in the CODEX INDEX for 10 days' free examination.

Write for the CODEX INDEX today

CODEX B00K C0., Inc., 19 William St., NEW YORK CITY 


\section{Do You Know that the}

\section{GRAPHIC \\ CHART}

Is the Most Effective Means for Giving You Control of Your

OPERATING COSTS?

SALES EXPENSES?

MACHINE OUTPUT?

LABOR TURNOVER?

And 101 other elements of your business?

Do you know what kind of chart is the most efficient for YOUR SPECIAL PURPOSE and business?

Can you make up such a chart without spending a week or a month to study the subject? and then, after it is made up, is it clear and convincing?

If your answer to these three questions is YES, you ought to be on the staff of the Construction Service Company.

If your answer is $\mathrm{NO}$, you should employ the CONSTRUCTION SERVICE COMPANY to design and produce the charts best adapted to your special requirements.

CONSTRUC'TION SERVICE COMPANY 15 WILLIAM STREET NEW YORK, N. Y. 



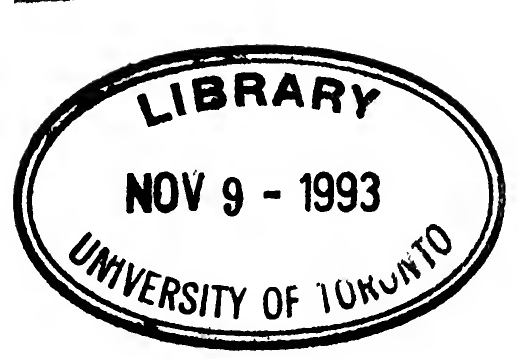




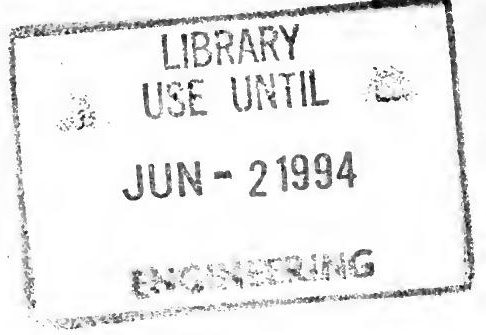


